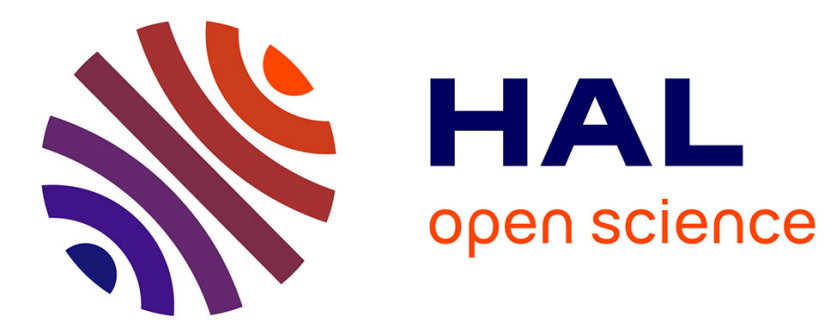

\title{
The Meteoritical Bulletin, No. 107
}

Jérôme Gattacceca, Francis Mccubbin, Audrey Bouvier, Jeffrey Grossman

\section{To cite this version:}

Jérôme Gattacceca, Francis Mccubbin, Audrey Bouvier, Jeffrey Grossman. The Meteoritical Bulletin, No. 107. Meteoritics and Planetary Science, 2020, 10.1111/maps.13440 . insu-02467538

\section{HAL Id: insu-02467538 https://hal-insu.archives-ouvertes.fr/insu-02467538}

Submitted on 22 Oct 2021

HAL is a multi-disciplinary open access archive for the deposit and dissemination of scientific research documents, whether they are published or not. The documents may come from teaching and research institutions in France or abroad, or from public or private research centers.
L'archive ouverte pluridisciplinaire HAL, est destinée au dépôt et à la diffusion de documents scientifiques de niveau recherche, publiés ou non, émanant des établissements d'enseignement et de recherche français ou étrangers, des laboratoires publics ou privés. 


\section{Meteoritics \& Planetary SCIENCE}

The Meteoritical Bulletin, No. 107

\begin{tabular}{|c|c|}
\hline Journal: & Meteoritics \& Planetary Science \\
\hline Manuscript ID & Draft \\
\hline Manuscript Type: & Report \\
\hline $\begin{array}{r}\text { Date Submitted by the } \\
\text { Author: }\end{array}$ & $n / a$ \\
\hline Complete List of Authors: & $\begin{array}{l}\text { Gattacceca, Jérôme; CEREGE, } \\
\text { McCubbin, Francis; NASA Johnson Space Center } \\
\text { Bouvier, Audrey; Universitat Bayreuth, Bayerisches Geo-Institut } \\
\text { Grossman, Jeffrey; NASA Headquarters }\end{array}$ \\
\hline Keywords: & Classification, Meteorite fall, Meteorite classification \\
\hline
\end{tabular}

\section{SCHOLARONE ${ }^{\text {m }}$ \\ Manuscripts}




\title{
The Meteoritical Bulletin, No. 107
}

Jérôme Gattacceca ${ }^{1}$, Francis M. McCubbin², Audrey Bouvier ${ }^{3}$, Jeffrey Grossman ${ }^{4}$

${ }^{1}$ CNRS, Aix Marseille Univ, IRD, Coll France, INRA, CEREGE, Aix-en-Provence, France

${ }^{2}$ NASA Johnson Space Center, Mail Code XI, 2101 NASA Parkway, Houston, TX 77058, USA.

${ }^{3}$ Universität Bayreuth, Bayerisches Geoinstitut, Bayreuth, Germany

${ }^{4}$ NASA Headquarters, Washington, DC, USA

\begin{abstract}
Meteoritical Bulletin 107 contains 2714 meteorites including 16 falls (Aba Panu, Ablaketka, Andila, Gueltat Zemmour, Hamburg, Karimati, Mahbas Arraid, Mangui, Mazichuan, Mukundpura, Ozerki, Parauapebas, Renchen, San Pedro de Urabá, Sokoto, Tintigny), with 2226 Ordinary chondrites, 168 HED achondrites, 132 Carbonaceous chondrites (including $41 \mathrm{CM}, 34 \mathrm{CV}, 26 \mathrm{CO}, 21 \mathrm{CK}, 4 \mathrm{CR}, 5$ ungrouped), 43 Ureilites, 30 Iron meteorites (including 2 ungrouped), 29 Lunar meteorites, 22 Martian meteorites, 16 Primitive achondrites (including 3 brachinites), 12 Rumuruti chondrites, 9 Enstatite chondrites, 7 Ungrouped achondrites, 6 Pallasites, 5 Mesosiderites, 3 Enstatite achondrites, 3 Ungrouped chondrites, and 2 Angrites. 1569 meteorites are from Antarctica, 835 from Africa, 206 from South America, 62 from Asia, 21 from North America, 11 from unknown locations, 8 from Europe (including one from Russia), and 1 from Oceania.
\end{abstract}

\section{Trends and specificities}

Meteoritical Bulletin 107 (MB107) contains the 2714 meteorites reported to and accepted by the Nomenclature Committee of the Meteoritical Society in 2018. This number is the second highest ever after a peak in MB102 with 3141 meteorites. The number of NWA meteorites reaches a new peak with 799 meteorites (Figure 1). Antarctic and NWA meteorites make up $58 \%$ and $29 \%$ of the total number of meteorites in MB107, respectively. As for the last few years, Chile, with 200 meteorites is now the main meteorite provider outside of NWA and Antarctica.

The 2714 meteorites in MB107 total over 2.3 tons of material, including 6 meteorites over $50 \mathrm{~kg}$. Of particular significance for 2017 is the large number of Lunar meteorites, with 29 meteorites totaling over $68 \mathrm{~kg}$, with 10 stones over $1 \mathrm{~kg}$ and 2 stones over $10 \mathrm{~kg}$. (Figure 3). All these lunar meteorites are from NW Africa (Algeria, Mali, Mauritania, Morocco, Western Sahara) and coordinates are known for 2 of them (Aridal 017 and Errachidia). Many of these stones are likely paired.

A similar surge in Martian meteorites is observed with 22 meteorites totaling over 15 $\mathrm{kg}$, including 3 meteorites over $2 \mathrm{~kg}$ (Figure 2). Again, most of these meteorites are from NW Africa (19 out of 22). 17 are shergottites, 1 is a nakhlite, and the remaining 4 (totaling 30g and including Rabt Sbayta 010 for which coordinates are available) are polymict breccias paired with NWA 7034 .

The total numbers of Lunar and Martian meteorites published until and including MB107 are 383 and 239, respectively, and growing at an increasing rate (Figure 3).

\section{Notable meteorites}


Four non-ordinary chondrite falls are reported: Mazichuan (diogenite), Mukundpura (CM2), Sokoto (Iron, IIIAB), and Tintigny (polymict eucrite). Another notable fall is Aba Panu, a $160 \mathrm{~kg} \mathrm{L3}$ from Nigeria. Other notable meteorites include NWA 12322 (35 kg CV3), NWA 11610 (28 kg CO3), NWA 12320 (4 kg angrite).

\section{Novelties in the Meteorite Bulletin Database}

Strewnfield data can now be plotted into Google Earth from the Meteorite Database. Example for Sutter's Mill meteorite is shown in Figure 4. Data are available in the Meteorite Bulletin Database by following the strewnfield link. We encourage submission of location coordinates for past and future strewnfields.

\section{Alphabetical text entries for non-Antarctic meteorites}

See online version of this article

\section{New Dense Collection Areas (DCA)}

In 2018, 24 new DCA were created, including 13 in China. See online version of this article for a list of DCA approved in 2018. A full list of all approved DCAs can be found at https://www.lpi.usra.edu/meteor/DenseAreas.php.

\section{Listing of institutes and collections}

Eight new type specimen repositories were approved (see online version of this article for a complete list).

In accordance with $\S 7.1$ of the Guidelines for Meteorite Nomenclature, type specimens of all new meteorites "... must be deposited in institutions that have well-curated meteorite collections and long-standing commitments to such curation." The minimum mass of a type specimen should be $20 \%$ of the total mass or $20 \mathrm{~g}$, whichever is the lesser amount. For larger meteorites, the Nomenclature Committee now strongly recommend the following: for 0.4 to $10 \mathrm{~kg}$ as at least $5 \%$ of total mass, and for meteorites $>10 \mathrm{~kg}$ as at least $500 \mathrm{~g}$. However, these larger type specimens requirements are mandatory for meteorites declared to be in Special Pairing Groups (\$4.2c).

\section{Reclassifications}

Sixteen meteorites have been reclassified in MB107. Eleven of these are Martian meteorites, likely paired with NWA 7034, that are now classified as "Martian (polymict breccia". See online version of this article for a complete list of reclassifications.

\section{Bibliography}

See online version of this article.

\section{SUPPORTING INFORMATION}

Additional supporting information may be found in the online version of this article:

- Online supplement 1: Table of Data including Antarctic meteorites

- Online supplement 2: Table of Corrections

- Data S1. A complete copy of entire Meteoritical Society Bulletin can be found in the supplementary information of this article as well as on the Meteoritical Bulletin Archive 
page at http://meteoriticalsociety.org/?page_id=57. Information about the approved meteorites can be obtained from the Meteoritical Bulletin Database (MBD) available online at https://www.lpi.usra.edu/meteor/.

\section{Figure captions}

Figure 1: Number of meteorites from Antarctica, NWA, and other areas reported in the last 12 Meteoritical Bulletins.

Figure 2: Number of Lunar and Martian meteorites reported in the last 12 Meteoritical Bulletins.

Figure 3: Cumulative number of Lunar and Martian meteorites with time.

Figure 4: Sutter's Mill strewnfield as extracted from the Meteoritical Bulletin Database. Each stone is numbered with full details (mass and coordinates) available in the database. 


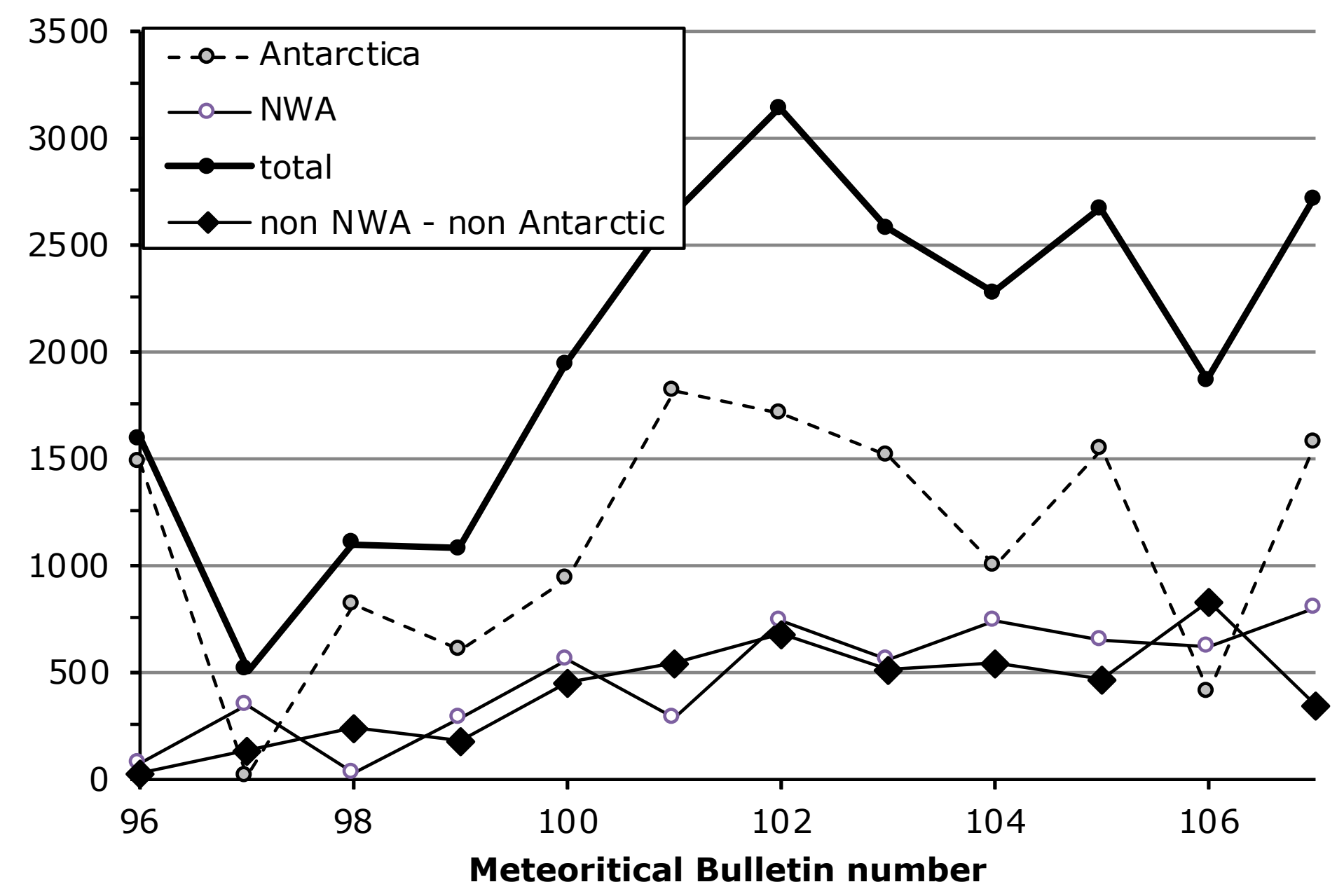




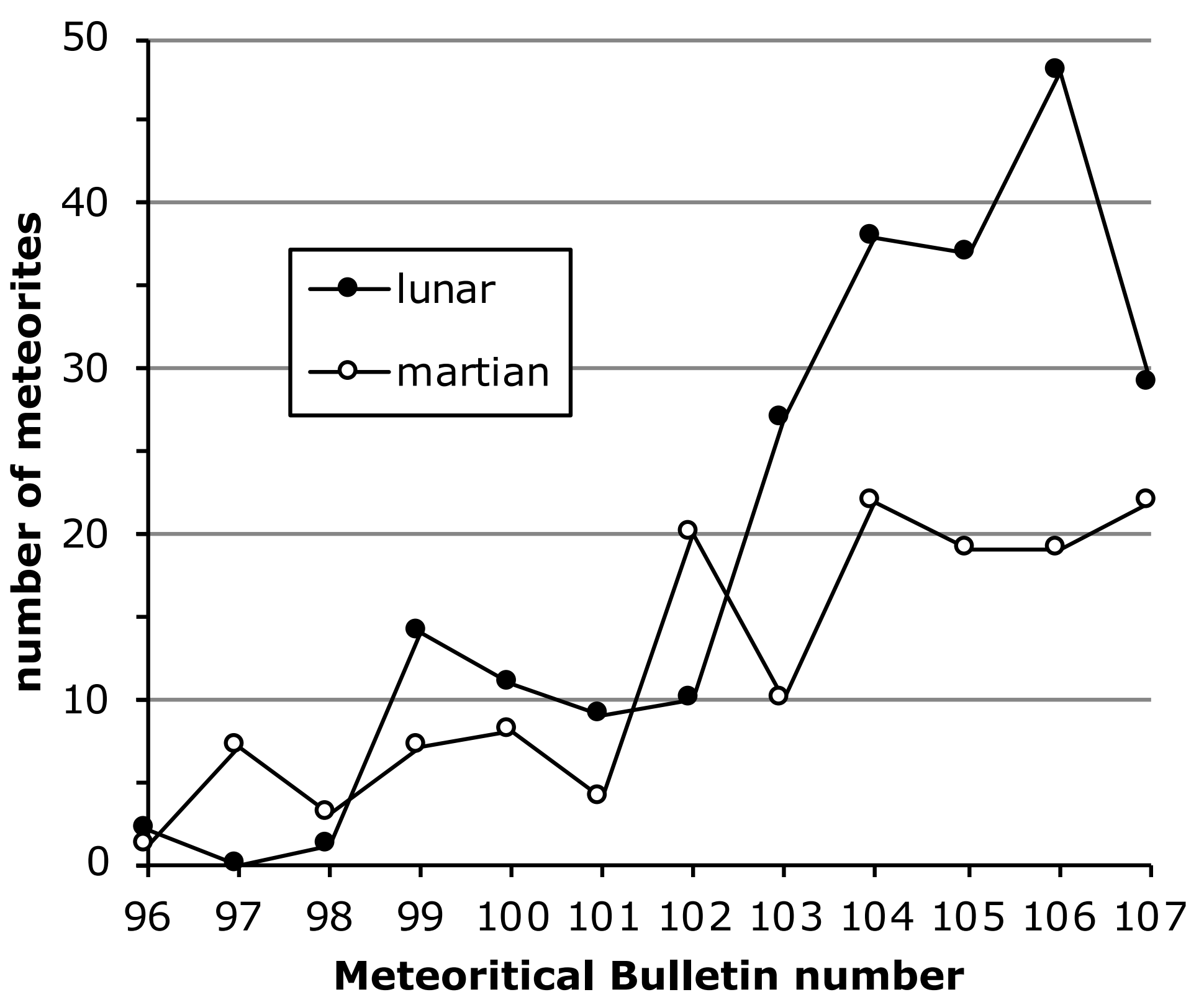




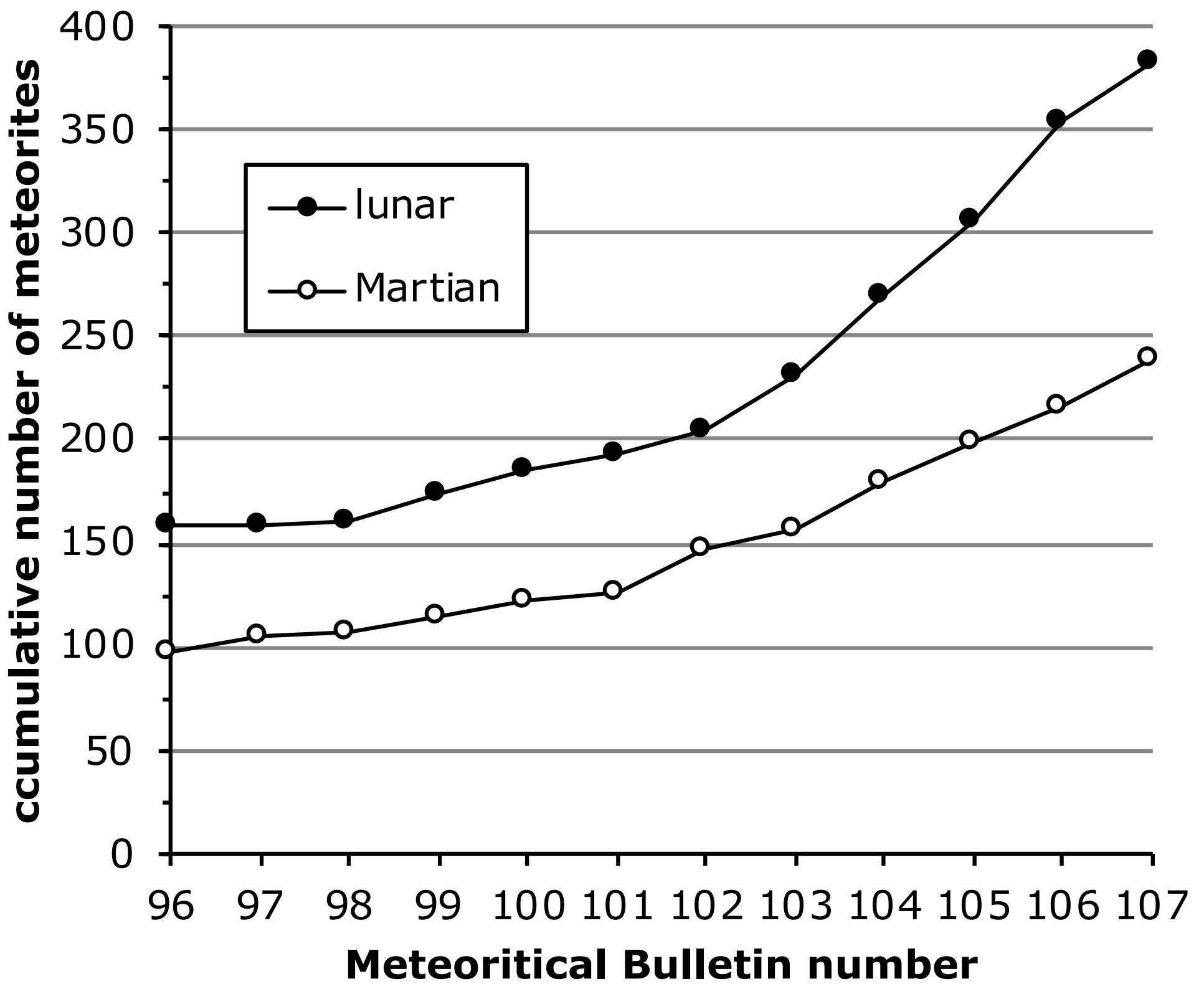





\begin{tabular}{|c|c|c|c|c|c|}
\hline db_id & db_name & db_class & db_country & tbl_mass & TKW (kg) \\
\hline 67412 & Aydar & Acapulcoite & Morocco & 533 & 0.533 \\
\hline 68489 & Northwest Africa 12199 & Acapulcoite & Western Sahi & 499 & 0.499 \\
\hline 67569 & Elephant Moraine 14074 & Acapulcoite & Antarctica & 14.4 & 0.0144 \\
\hline 67401 & Northwest Africa 11739 & \multicolumn{2}{|c|}{ Achondrite-pr (Northwest A1 } & 20 & 0.02 \\
\hline 67542 & Northwest Africa 11562 & \multicolumn{2}{|c|}{ Achondrite-ur(Northwest A1 } & 1361 & 1.361 \\
\hline 68624 & Northwest Africa 12338 & \multicolumn{2}{|c|}{ Achondrite-ur(Northwest Al } & 1126.5 & 1.1265 \\
\hline 66411 & Northwest Africa 11575 & \multicolumn{2}{|c|}{ Achondrite-ur Mali } & 598 & 0.598 \\
\hline 66865 & Northwest Africa 11112 & \multicolumn{2}{|c|}{ Achondrite-ur Morocco } & 528 & 0.528 \\
\hline 68468 & Northwest Africa 12217 & \multicolumn{2}{|c|}{ Achondrite-ur(Northwest Al } & 148 & 0.148 \\
\hline 67852 & Northwest Africa 11916 & \multicolumn{2}{|c|}{ Achondrite-ur(Northwest A1 } & 10.4 & 0.0104 \\
\hline 66390 & Northwest Africa 11558 & \multicolumn{2}{|c|}{ Achondrite-ur(Northwest At } & 3.92 & 0.00392 \\
\hline 68635 & Northwest Africa 12320 & Angrite & Mauritania & 4459 & 4.459 \\
\hline 68323 & Northwest Africa 12004 & Angrite & Morocco & 183 & 0.183 \\
\hline 66406 & Northwest Africa 11573 & Aubrite & (Northwest A1 & 107 & 0.107 \\
\hline 67546 & Northwest Africa 11756 & Brachinite & Morocco & 840 & 0.84 \\
\hline 52068 & Northwest Africa 6308 & Brachinite & (Northwest Al & 600 & 0.6 \\
\hline 51432 & Northwest Africa 6023 & \multicolumn{2}{|c|}{ Brachinite-an(Northwest A } & 72 & 0.072 \\
\hline 67850 & Northwest Africa 8781 & C-ung & (Northwest Al & 380 & 0.38 \\
\hline 67058 & Asuka 09535 & C-ung & Antarctica & 2.703 & 0.002703 \\
\hline 67378 & Asuka 09003 & C-ung & Antarctica & 1.696 & 0.001696 \\
\hline 66389 & Northwest Africa 11556 & C2-ung & (Northwest Al & 61 & 0.061 \\
\hline 67405 & Northwest Africa 11750 & C3.00-ung & Morocco & 8.49 & 0.00849 \\
\hline 67057 & Asuka 09505 & $\mathrm{CH} 3$ & Antarctica & 0.85 & 0.00085 \\
\hline 68609 & Northwest Africa 12273 & \multicolumn{2}{|c|}{ Chondrite-un!(Northwest A1 } & 280 & 0.28 \\
\hline 68620 & Sierra Gorda 009 & \multicolumn{2}{|c|}{ Chondrite-un! Chile } & 240 & 0.24 \\
\hline 67875 & Dominion Range 14080 & \multicolumn{2}{|c|}{ Chondrite-un! Antarctica } & 10.34 & 0.01034 \\
\hline 66830 & Northwest Africa 11708 & CK3 & Morocco & 1104 & 1.104 \\
\hline 66464 & Northwest Africa 11607 & CK3-6 & (Northwest Al & 450 & 0.45 \\
\hline 66864 & Northwest Africa 11707 & CK4 & Morocco & 453 & 0.453 \\
\hline 67590 & Northwest Africa 11808 & CK4 & (Northwest Al & 412 & 0.412 \\
\hline 66797 & Northwest Africa 11670 & CK4 & (Northwest At & 154 & 0.154 \\
\hline 68115 & \multicolumn{2}{|c|}{ Grosvenor Mountains 17169 CK4 } & Antarctica & 78.959 & 0.078959 \\
\hline 54694 & Northwest Africa 7055 & $\mathrm{CK} 4 / 5$ & (Northwest Al & 26.93 & 0.02693 \\
\hline 68613 & Northwest Africa 12286 & CK5 & (Northwest A1 & 795 & 0.795 \\
\hline 66863 & Northwest Africa 11706 & CK5 & Morocco & 116 & 0.116 \\
\hline 67493 & Northwest Africa 11764 & CK5 & (Northwest A1 & 113 & 0.113 \\
\hline 68108 & \multicolumn{2}{|c|}{ Grosvenor Mountains 17059 CK5 } & Antarctica & 102.442 & 0.102442 \\
\hline 67641 & Northwest Africa 11836 & CK5 & (Northwest Al & 70 & 0.07 \\
\hline 68413 & Northwest Africa 12192 & CK5 & Algeria & 42.7 & 0.0427 \\
\hline 67562 & Elephant Moraine 14007 & CK5 & Antarctica & 11.4 & 0.0114 \\
\hline 67502 & Northwest Africa 11773 & CK6 & (Northwest Al & 890 & 0.89 \\
\hline 66402 & Northwest Africa 11565 & CK6 & (Northwest Al & 399.8 & 0.3998 \\
\hline 66475 & Northwest Africa 11636 & CK6 & (Northwest Al & 152.5 & 0.1525 \\
\hline 68419 & Northwest Africa 12200 & CK6 & Mauritania & 76 & 0.076 \\
\hline
\end{tabular}




\begin{tabular}{|c|c|c|c|c|c|}
\hline 67584 & San Juan 085 & CK6 & Chile & 18 & 0.018 \\
\hline 67218 & Asuka 12267 & CK6 & Antarctica & 17.219 & 0.017219 \\
\hline 67494 & Northwest Africa 11765 & CK6 & (Northwest At & 15 & 0.015 \\
\hline 67187 & Asuka 12236 & $\mathrm{CM}$ & Antarctica & 93.65 & 0.09365 \\
\hline 67199 & Asuka 12248 & $\mathrm{CM}$ & Antarctica & 52.912 & 0.052912 \\
\hline 67350 & Asuka 12408 & $\mathrm{CM}$ & Antarctica & 10.216 & 0.010216 \\
\hline 36665 & Yamato 980039 & $\mathrm{CM}$ & Antarctica & 7.683 & 0.007683 \\
\hline 36663 & Yamato 980037 & $\mathrm{CM}$ & Antarctica & 2.675 & 0.002675 \\
\hline 36737 & Yamato 980111 & $\mathrm{CM}$ & Antarctica & 2.579 & 0.002579 \\
\hline 36664 & Yamato 980038 & $\mathrm{CM}$ & Antarctica & 2.56 & 0.00256 \\
\hline 36710 & Yamato 980084 & $\mathrm{CM}$ & Antarctica & 2.538 & 0.002538 \\
\hline 36709 & Yamato 980083 & $\mathrm{CM}$ & Antarctica & 2.447 & 0.002447 \\
\hline 36825 & Yamato 980199 & $\mathrm{CM}$ & Antarctica & 2.313 & 0.002313 \\
\hline 36988 & Yamato 980363 & $\mathrm{CM}$ & Antarctica & 2.092 & 0.002092 \\
\hline 36869 & Yamato 980243 & $\mathrm{CM}$ & Antarctica & 1.733 & 0.001733 \\
\hline 36715 & Yamato 980089 & $\mathrm{CM}$ & Antarctica & 1.336 & 0.001336 \\
\hline 36777 & Yamato 980151 & $\mathrm{CM}$ & Antarctica & 1.002 & 0.001002 \\
\hline 67123 & Asuka 10139 & $\mathrm{CM}$ & Antarctica & 0.83 & 0.00083 \\
\hline 66791 & Northwest Africa 11254 & CM-an & (Northwest At & 18 & 0.018 \\
\hline 68631 & Northwest Africa 12328 & CM1 & (Northwest Al & 43.2 & 0.0432 \\
\hline 66795 & Mukundpura & CM2 & India & 2000 & 2 \\
\hline 68329 & Northwest Africa 12184 & CM2 & (Northwest At & 220 & 0.22 \\
\hline 67568 & Elephant Moraine 14068 & CM2 & Antarctica & 185.6 & 0.1856 \\
\hline 66806 & Northwest Africa 11699 & CM2 & (Northwest Al & 75.6 & 0.0756 \\
\hline 67745 & Northwest Africa 11889 & CM2 & (Northwest At & 75 & 0.075 \\
\hline 68124 & Northwest Africa 11965 & CM2 & (Northwest At & 57.3 & 0.0573 \\
\hline 68103 & Grosvenor Mountains 17004 & $\mathrm{CM} 2$ & Antarctica & 52.933 & 0.052933 \\
\hline 67561 & Reckling Peak 14005 & $\mathrm{CM} 2$ & Antarctica & 33.4 & 0.0334 \\
\hline 68614 & Northwest Africa 12287 & CM2 & (Northwest At & 33 & 0.033 \\
\hline 67555 & Northwest Africa 11791 & $\mathrm{CM} 2$ & (Northwest At & 17.1 & 0.0171 \\
\hline 67705 & Northwest Africa 11781 & $\mathrm{CM} 2$ & (Northwest At & 15.5 & 0.0155 \\
\hline 68101 & Amundsen Glacier 17290 & CM2 & Antarctica & 14.393 & 0.014393 \\
\hline 67816 & Northwest Africa 11919 & $\mathrm{CM} 2$ & (Northwest Al & 11 & 0.011 \\
\hline 68040 & Miller Range 15094 & $\mathrm{CM} 2$ & Antarctica & 10.636 & 0.010636 \\
\hline 66446 & Northwest Africa 11588 & CM2 & (Northwest At & 10.3 & 0.0103 \\
\hline 67557 & Northwest Africa 11793 & $\mathrm{CM} 2$ & (Northwest Al & 10.1 & 0.0101 \\
\hline 67556 & Northwest Africa 11792 & $\mathrm{CM} 2$ & (Northwest At & 6 & 0.006 \\
\hline 67397 & Northwest Africa 11733 & CM2 & (Northwest At & 3.3 & 0.0033 \\
\hline 67635 & Northwest Africa 11841 & $\mathrm{CM} 2$ & (Northwest At & 2.2 & 0.0022 \\
\hline 67633 & Northwest Africa 11839 & $\mathrm{CM} 2$ & (Northwest At & 1.84 & 0.00184 \\
\hline 67634 & Northwest Africa 11840 & CM2 & (Northwest At & 1.8 & 0.0018 \\
\hline 67563 & Elephant Moraine 14013 & CM2 & Antarctica & 0.3 & 0.0003 \\
\hline 66794 & Northwest Africa 11638 & CM2.0 & (Northwest At & 9.7 & 0.0097 \\
\hline 67396 & Northwest Africa 11732 & CM2.0 & (Northwest At & 3.8 & 0.0038 \\
\hline 67321 & Asuka 12376 & $\mathrm{CO}$ & Antarctica & 129.97 & 0.12997 \\
\hline
\end{tabular}




\begin{tabular}{|c|c|c|c|c|c|}
\hline 36666 & Yamato 980040 & $\mathrm{CO}$ & Antarctica & 14.932 & 0.014932 \\
\hline 67060 & Asuka 09551 & $\mathrm{CO}$ & Antarctica & 2.306 & 0.002306 \\
\hline 67048 & Asuka 09471 & $\mathrm{CO}$ & Antarctica & 0.821 & 0.000821 \\
\hline 66468 & Northwest Africa 11610 & $\mathrm{CO} 3$ & (Northwest At & 28208 & 28.208 \\
\hline 66394 & Northwest Africa 11564 & $\mathrm{CO} 3$ & (Northwest A 1 & 3130 & 3.13 \\
\hline 53737 & Northwest Africa 6827 & $\mathrm{CO} 3$ & (Northwest At & 1289 & 1.289 \\
\hline 67746 & El Médano 389 & $\mathrm{CO} 3$ & Chile & 1186 & 1.186 \\
\hline 68298 & Northwest Africa 11996 & $\mathrm{CO} 3$ & (Northwest A 1 & 960 & 0.96 \\
\hline 66866 & Northwest Africa 11113 & $\mathrm{CO} 3$ & Morocco & 470 & 0.47 \\
\hline 66412 & Calama 005 & $\mathrm{CO} 3$ & Chile & 380 & 0.38 \\
\hline 67747 & El Médano 390 & $\mathrm{CO} 3$ & Chile & 132 & 0.132 \\
\hline 68470 & Northwest Africa 12219 & $\mathrm{CO} 3$ & (Northwest At & 129 & 0.129 \\
\hline 68628 & Northwest Africa 12367 & $\mathrm{CO} 3$ & (Northwest A 1 & 92.8 & 0.0928 \\
\hline 68627 & Northwest Africa 12366 & $\mathrm{CO} 3$ & (Northwest Al & 81.5 & 0.0815 \\
\hline 57789 & Dhofar 1762 & $\mathrm{CO} 3$ & Oman & 81 & 0.081 \\
\hline 68626 & Northwest Africa 12365 & $\mathrm{CO} 3$ & (Northwest A 1 & 56.1 & 0.0561 \\
\hline 32164 & Northwest Africa 1292 & $\mathrm{CO} 3$ & (Northwest Al & 56 & 0.056 \\
\hline 68519 & Northwest Africa 11521 & $\mathrm{CO} 3$ & Morocco & 50 & 0.05 \\
\hline 67849 & Northwest Africa 8676 & $\mathrm{CO} 3$ & (Northwest A 1 & 45 & 0.045 \\
\hline 67636 & Northwest Africa 11844 & $\mathrm{CO} 3$ & (Northwest A 1 & 35 & 0.035 \\
\hline 66391 & Northwest Africa 11559 & $\mathrm{CO} 3$ & Algeria & 33.9 & 0.0339 \\
\hline 68469 & Northwest Africa 12218 & $\mathrm{CO} 3$ & (Northwest A 1 & 27 & 0.027 \\
\hline 67730 & Northwest Africa 11790 & $\mathrm{CO} 3$ & (Northwest Al & 7.2 & 0.0072 \\
\hline 67788 & Northwest Africa 11906 & CO3.0 & (Northwest A 1 & 84.8 & 0.0848 \\
\hline 67407 & Northwest Africa 11751 & CO3.05 & (Northwest A 1 & 168.7 & 0.1687 \\
\hline 68417 & Northwest Africa 12197 & CR2 & Western Sahi & 487 & 0.487 \\
\hline 68109 & Grosvenor Mountains 17063 & 3 CR2 & Antarctica & 388.3 & 0.3883 \\
\hline 68110 & Grosvenor Mountains 1706 & 4 CR2 & Antarctica & 226.4 & 0.2264 \\
\hline 67631 & Northwest Africa 11561 & CR6 & (Northwest At & 5746 & 5.746 \\
\hline 68637 & Northwest Africa 12322 & CV3 & Niger & 35300 & 35.3 \\
\hline 67787 & Northwest Africa 11904 & CV3 & (Northwest At & 4000 & 4 \\
\hline 67399 & Northwest Africa 11737 & CV3 & (Northwest At & 2550 & 2.55 \\
\hline 68330 & Northwest Africa 12187 & CV3 & (Northwest A 1 & 2007 & 2.007 \\
\hline 60989 & Northwest Africa 8406 & CV3 & (Northwest At & 944 & 0.944 \\
\hline 68607 & Northwest Africa 12268 & CV3 & (Northwest At & 743 & 0.743 \\
\hline 68477 & Northwest Africa 12229 & CV3 & (Northwest A 1 & 675 & 0.675 \\
\hline 68642 & Northwest Africa 12343 & CV3 & (Northwest A 1 & 526 & 0.526 \\
\hline 68623 & Catalina 300 & CV3 & Chile & 380 & 0.38 \\
\hline 45608 & Northwest Africa 4669 & CV3 & (Northwest A 1 & 369 & 0.369 \\
\hline 68315 & Northwest Africa 12013 & CV3 & (Northwest At & 332.1 & 0.3321 \\
\hline 67830 & Northwest Africa 11957 & CV3 & (Northwest At & 251 & 0.251 \\
\hline 68416 & Northwest Africa 12196 & CV3 & Western Sahi & 226 & 0.226 \\
\hline 67497 & Northwest Africa 11768 & CV3 & (Northwest A 1 & 183 & 0.183 \\
\hline 66447 & Northwest Africa 11589 & CV3 & (Northwest At & 148.9 & 0.1489 \\
\hline 67646 & Northwest Africa 11850 & CV3 & (Northwest Al & 147 & 0.147 \\
\hline
\end{tabular}




\begin{tabular}{|c|c|c|c|c|c|}
\hline 68327 & Northwest Africa 12180 & CV3 & (Northwest Al & 126 & 0.126 \\
\hline 67402 & Northwest Africa 11740 & CV3 & (Northwest Al & 106.9 & 0.1069 \\
\hline 68503 & Northwest Africa 11977 & $\mathrm{CV} 3$ & (Northwest Al & 72.9 & 0.0729 \\
\hline 33145 & Northwest Africa 2637 & CV3 & (Northwest A 1 & 71.5 & 0.0715 \\
\hline 67637 & Northwest Africa 11845 & CV3 & (Northwest Al & 69 & 0.069 \\
\hline 68494 & Northwest Africa 12247 & CV3 & Algeria & 59.3 & 0.0593 \\
\hline 67823 & Northwest Africa 11946 & CV3 & (Northwest Al & 59 & 0.059 \\
\hline 68471 & Northwest Africa 12221 & CV3 & (Northwest Al & 55 & 0.055 \\
\hline 67499 & Northwest Africa 11770 & CV3 & (Northwest Al & 49 & 0.049 \\
\hline 67318 & Asuka 12373 & CV3 & Antarctica & 31.379 & 0.031379 \\
\hline 67495 & Northwest Africa 11766 & CV3 & (Northwest Al & 30 & 0.03 \\
\hline 45762 & Northwest Africa 4838 & CV3 & (Northwest Al & 28 & 0.028 \\
\hline 67793 & Northwest Africa 11937 & CV3 & (Northwest Al & 25.1 & 0.0251 \\
\hline 68049 & Miller Range 15254 & CV3 & Antarctica & 21.468 & 0.021468 \\
\hline 68052 & Miller Range 15257 & CV3 & Antarctica & 11.258 & 0.011258 \\
\hline 68050 & Miller Range 15255 & CV3 & Antarctica & 7.492 & 0.007492 \\
\hline 67650 & Northwest Africa 11854 & CV3 & (Northwest Al & 6.87 & 0.00687 \\
\hline 36803 & Yamato 980177 & CV3 & Antarctica & 1.959 & 0.001959 \\
\hline 66392 & Northwest Africa 11560 & Diogenite & (Northwest Al & 13060 & 13.06 \\
\hline 66831 & Mazichuan & Diogenite & China & 3282 & 3.282 \\
\hline 66803 & Northwest Africa 11696 & Diogenite & (Northwest At & 2706 & 2.706 \\
\hline 66793 & Erg Chech 001 & Diogenite & Algeria & 1266 & 1.266 \\
\hline 67822 & Northwest Africa 11945 & Diogenite & (Northwest Al & 1176 & 1.176 \\
\hline 66839 & Northwest Africa 11741 & Diogenite & (Northwest A 1 & 773 & 0.773 \\
\hline 67798 & Northwest Africa 11909 & Diogenite & (Northwest A 1 & 589.6 & 0.5896 \\
\hline 67658 & Northwest Africa 11857 & Diogenite & (Northwest At & 580 & 0.58 \\
\hline 67744 & Northwest Africa 11888 & Diogenite & (Northwest Al & 307 & 0.307 \\
\hline 67167 & Asuka 12144 & Diogenite & Antarctica & 143.03 & 0.14303 \\
\hline 41757 & Yamato 000995 & Diogenite & Antarctica & 134.75 & 0.13475 \\
\hline 68530 & Los Vientos 250 & Diogenite & Chile & 98.8 & 0.0988 \\
\hline 68116 & Grosvenor Mountains 17175 & Diogenite & Antarctica & 94.864 & 0.094864 \\
\hline 68606 & Northwest Africa 12267 & Diogenite & (Northwest At & 86 & 0.086 \\
\hline 68493 & Northwest Africa 12245 & Diogenite & (Northwest A 1 & 80.77 & 0.08077 \\
\hline 43638 & Yamato 002876 & Diogenite & Antarctica & 61.05 & 0.06105 \\
\hline 67500 & Northwest Africa 11771 & Diogenite & (Northwest At & 50 & 0.05 \\
\hline 28993 & Yamato 794039 & Diogenite & Antarctica & 42.72 & 0.04272 \\
\hline 43639 & Yamato 002877 & Diogenite & Antarctica & 34.652 & 0.034652 \\
\hline 67678 & Northwest Africa 11833 & Diogenite & (Northwest At & 29 & 0.029 \\
\hline 67169 & Asuka 12158 & Diogenite & Antarctica & 17.899 & 0.017899 \\
\hline 27187 & Yamato 791838 & Diogenite & Antarctica & 16 & 0.016 \\
\hline 41070 & Yamato 000305 & Diogenite & Antarctica & 12.414 & 0.012414 \\
\hline 41594 & Yamato 000832 & Diogenite & Antarctica & 11.689 & 0.011689 \\
\hline 41022 & Yamato 000257 & Diogenite & Antarctica & 3.136 & 0.003136 \\
\hline 67588 & Northwest Africa 11805 & \multicolumn{2}{|c|}{ Diogenite-pm(Northwest At } & 109.4 & 0.1094 \\
\hline 68544 & Caleta el Cobre 025 & EH3 & Chile & 777 & 0.777 \\
\hline
\end{tabular}




\begin{tabular}{|c|c|c|c|c|c|}
\hline 67196 & Asuka 12245 & EL4 & Antarctica & 14.084 & 0.014084 \\
\hline 68529 & Caleta el Cobre 024 & EL6 & Chile & 15900 & 15.9 \\
\hline 68528 & El Médano 394 & EL6 & Chile & 3079 & 3.079 \\
\hline 66488 & Northwest Africa 11667 & EL6 & (Northwest A1 & 682 & 0.682 \\
\hline 67395 & Northwest Africa 11731 & EL6 & (Northwest A1 & 500 & 0.5 \\
\hline 67157 & Asuka 12077 & EL6 & Antarctica & 185.43 & 0.18543 \\
\hline 66381 & MacKay Glacier 14005 & EL6 & Antarctica & 172.1 & 0.1721 \\
\hline 66801 & Northwest Africa 11679 & EL6 & Morocco & 98 & 0.098 \\
\hline 68132 & Northwest Africa 11354 & Enst achon-u & Morocco & 15000 & 15 \\
\hline 67869 & Northwest Africa 11998 & Eucrite & (Northwest A1 & 2241 & 2.241 \\
\hline 66448 & Northwest Africa 11591 & Eucrite & Algeria & 1549 & 1.549 \\
\hline 67566 & Elephant Moraine 14056 & Eucrite & Antarctica & 1220 & 1.22 \\
\hline 67564 & Elephant Moraine 14049 & Eucrite & Antarctica & 1121 & 1.121 \\
\hline 33346 & Northwest Africa 2887 & Eucrite & (Northwest Al & 887 & 0.887 \\
\hline 68541 & Dhofar 2093 & Eucrite & Oman & 764 & 0.764 \\
\hline 67596 & Northwest Africa 11777 & Eucrite & (Northwest Al & 705 & 0.705 \\
\hline 66387 & Dhofar 2092 & Eucrite & Oman & 440 & 0.44 \\
\hline 66812 & Northwest Africa 11710 & Eucrite & (Northwest Al & 394 & 0.394 \\
\hline 68126 & Northwest Africa 11999 & Eucrite & (Northwest A1 & 294.42 & 0.29442 \\
\hline 67825 & Northwest Africa 11949 & Eucrite & (Northwest At & 280 & 0.28 \\
\hline 67821 & Northwest Africa 11928 & Eucrite & (Northwest At & 235 & 0.235 \\
\hline 67642 & Northwest Africa 11837 & Eucrite & (Northwest Al & 218 & 0.218 \\
\hline 67558 & Northwest Africa 11794 & Eucrite & (Northwest A1 & 206.5 & 0.2065 \\
\hline 42127 & Yamato 001365 & Eucrite & Antarctica & 206.1 & 0.2061 \\
\hline 66811 & Northwest Africa 11709 & Eucrite & Morocco & 180 & 0.18 \\
\hline 66382 & Northwest Africa 11550 & Eucrite & (Northwest At & 159.4 & 0.1594 \\
\hline 68612 & Northwest Africa 12283 & Eucrite & (Northwest Al & 145.5 & 0.1455 \\
\hline 67657 & Northwest Africa 11856 & Eucrite & (Northwest Al & 137 & 0.137 \\
\hline 66384 & Northwest Africa 11552 & Eucrite & (Northwest At & 109.2 & 0.1092 \\
\hline 66451 & Northwest Africa 11594 & Eucrite & Algeria & 106.77 & 0.10677 \\
\hline 66444 & Northwest Africa 11586 & Eucrite & Algeria & 91.5 & 0.0915 \\
\hline 67179 & Asuka 12223 & Eucrite & Antarctica & 75.47 & 0.07547 \\
\hline 66457 & Northwest Africa 11600 & Eucrite & Algeria & 75.24 & 0.07524 \\
\hline 66452 & Northwest Africa 11595 & Eucrite & Algeria & 71.3 & 0.0713 \\
\hline 67824 & Northwest Africa 11947 & Eucrite & (Northwest At & 70 & 0.07 \\
\hline 67791 & Northwest Africa 11933 & Eucrite & (Northwest Al & 65.7 & 0.0657 \\
\hline 67632 & Malotas (b) & Eucrite & Argentina & 62.4 & 0.0624 \\
\hline 68617 & Northwest Africa 12290 & Eucrite & (Northwest At & 60 & 0.06 \\
\hline 41892 & Yamato 001130 & Eucrite & Antarctica & 59.25 & 0.05925 \\
\hline 4607 & Asuka 881898 & Eucrite & Antarctica & 55.82 & 0.05582 \\
\hline 66453 & Northwest Africa 11596 & Eucrite & Algeria & 51.93 & 0.05193 \\
\hline 67149 & Asuka 12043 & Eucrite & Antarctica & 49.815 & 0.049815 \\
\hline 66450 & Northwest Africa 11593 & Eucrite & Algeria & 44.37 & 0.04437 \\
\hline 67236 & Asuka 12285 & Eucrite & Antarctica & 38.049 & 0.038049 \\
\hline 68476 & Northwest Africa 12226 & Eucrite & (Northwest At & 38 & 0.038 \\
\hline
\end{tabular}


67319

66449

66459

66458

4196

41994

4587

66460

67349

44116

67131

36941

36815

36962

67567

66837

66413

66785

67586

68408

67491

50794

67492

33043

66415

68605

67398

67550

66840

68641

68473

68326

33153

67831

67826

66805

67551

67790

Northwest Africa 11597

Northwest Africa 11747

Asuka 881689

Northwest Africa 11748

Northwest Africa 11599

Asuka 12282

Northwest Africa 12293

Asuka 12374

Northwest Africa 11592

Northwest Africa 11602

Northwest Africa 11601

Asuka 881487

Yamato 001232

Asuka 881878

Northwest Africa 11603

Asuka 12407

Yamato 003354

Asuka 10228

Yamato 980315

Yamato 980189

Yamato 980337

Elephant Moraine 14066

Northwest Africa 11729

Northwest Africa 8671

Northwest Africa 8681

Northwest Africa 11803

Northwest Africa 12182

Northwest Africa 11762

Northwest Africa 5908

Northwest Africa 11763

Northwest Africa 2426

Northwest Africa 11576

Northwest Africa 12265

Northwest Africa 11734

Northwest Africa 11785

Northwest Africa 11742

Northwest Africa 12342

Northwest Africa 12223

Northwest Africa 12011

Northwest Africa 2650

Northwest Africa 11958

Northwest Africa 11953

Northwest Africa 11698

Northwest Africa 11786

Calama 014
Eucrite

Algeria

0.03474

Eucrite

(Northwest At

31.97

0.03197

Eucrite

Antarctica

29.962

0.029962

Eucrite

(Northwest At

27.5

0.0275

Eucrite

Eucrite

Algeria

22.07

0.02207

Eucrite

Antarctica

21.33

0.02133

Eucrite

Eucrite

(Northwest A

20

0.02

Antarctica

18.323

0.018323

Eucrite

Eucrite

Algeria

17.88

0.01788

Algeria

17.86

0.01786

Algeria

16.85

0.01685

Eucrite

Antarctica

12.921

0.012921

Eucrite

Antarctica

10.557

0.010557

Eucrite

Antarctica

10.53

0.01053

Eucrite

Algeria

10.1

0.0101

Eucrite

Antarctica

6.919

0.006919

Eucrite

Antarctica

2.369

0.002369

Eucrite

Antarctica

1.936

0.001936

Eucrite

Antarctica

1.298

0.001298

Eucrite

Antarctica

1.219

0.001219

Eucrite

Antarctica

0.425

0.000425

Eucrite

Antarctica

0.4

0.0004

Eucrite-an

Mauritania

345

0.345

Eucrite-an (Northwest Al

164.2

0.1642

Eucrite-br

(Northwest At

590

0.59

Eucrite-br Algeria

442

0.442

Eucrite-br

(Northwest Al

122.5

0.1225

Eucrite-br (Northwest Al

42

0.042

Eucrite-br (Northwest Al

20.3

0.0203

Eucrite-br (Northwest Al

14

0.014

Eucrite-cm (Northwest A1

630

0.63

Eucrite-melt $k$ (Northwest Al

18000

18

Eucrite-melt $k$ (Northwest Al

4433

4.433

Eucrite-melt $k$ (Northwest At

1010

1.01

Eucrite-melt $k$ (Northwest $A t$

822

0.822

Eucrite-melt $k$ (Northwest Al

650

0.65

Eucrite-melt $k$ (Northwest $A t$

526

0.526

Eucrite-melt $k$ (Northwest $A t$

327

0.327

Eucrite-mmic (Northwest Al

2835

2.835

Eucrite-mmic Morocco

2224

2.224

Eucrite-mmic (Northwest At

2112

2.112

Eucrite-mmic (Northwest Al

1160

1.16

Eucrite-mmic (Northwest At

832.4

0.8324

Eucrite-mmic (Northwest At

417

0.417

Eucrite-mmic Chile

251

0.251 
Northwest Africa 12230

Eucrite-pmict(Northwest A 1

1024

Eucrite-pmict(Northwest At

1015.8

1.0158

68625

67679

Northwest Africa 12339

Eucrite-pmict(Northwest A1

634

0.634

66800

68643

68414

Northwest Africa 11861

Eucrite-pmict(Northwest At

538

0.538

Northwest Africa 11677

Eucrite-pmict(Northwest A1

499.2

0.4992

Northwest Africa 12344

Eucrite-pmict(Northwest A 1

456

0.456

67868

68475

68492

67543

67792

68532

68114

68117

68490

68621

68639

68107

68415

68121

Northwest Africa 12193

Eucrite-pmictWestern Sahi

241

0.241

\section{Tintigny}

Eucrite-pmict Belgium

210

0.21

Northwest Africa 12225

Eucrite-pmict(Northwest At

169

0.169

Northwest Africa 12243

Eucrite-pmict Mauritania

164

0.164

Northwest Africa 11753

Eucrite-pmict Morocco

159

0.159

Northwest Africa 11934

Eucrite-pmict(Northwest A1

151.9

0.1519

Northwest Africa 12282

Eucrite-pmict(Northwest A1

141.5

0.1415

Grosvenor Mountains 17168 Eucrite-pmict Antarctica

133.858

0.133858

Grosvenor Mountains 17176 Eucrite-pmict Antarctica

131.112

0.131112

Northwest Africa 12202

Eucrite-pmictWestern Sahi

110

0.11

Northwest Africa 12326

Eucrite-pmict(Northwest At

76.1

0.0761

Northwest Africa 12340

Eucrite-pmict(Northwest At

74

0.074

Grosvenor Mountains 17049 Eucrite-pmict Antarctica

Northwest Africa 12195

Eucrite-pmict Algeria

61.353

0.061353

Grosvenor Mountains 17211 Eucrite-pmict Antarctica

68104

66484

68113

Grosvenor Mountains 17006 Eucrite-pmict Antarctica

Northwest Africa 11647

Eucrite-pmict(Northwest At

61

0.061

47.3

0.0473

40.372

0.040372

68118

68106

68622

Grosvenor Mountains 17099 Eucrite-pmict Antarctica

39

0.039

26.701

0.026701

Grosvenor Mountains 17189 Eucrite-pmict Antarctica

25.324

0.025324

23.531

0.023531

67789

68105

67859

67643

67406

68120

68603

68420

68111

68119

67795

68112

67144

66901

Grosvenor Mountains 1704
Northwest Africa 12327

Eucrite-pmict(Northwest A1

21.6

0.0216

Errachidia 002

Eucrite-pmict Morocco

21.26

0.02126

Grosvenor Mountains 17039 Eucrite-pmict Antarctica

8.269

0.008269

\section{Northwest Africa 11969}

Eucrite-unbr (Northwest AI

942

0.942

Northwest Africa 11846

Eucrite-unbr (Northwest At

Northwest Africa 11749

Eucrite-unbr Morocco

754

0.754

460

0.46

Grosvenor Mountains 17210 Eucrite-unbr Antarctica

184.233

0.184233

101.4

0.1014

$$
\text { Northwest Africa } 12201
$$

Eucrite-unbr Western Sah:

81

0.081

Grosvenor Mountains 17070 Eucrite-unbr Antarctica

64.631

0.064631

Grosvenor Mountains 17200 Eucrite-unbr Antarctica

46.365

0.046365

$$
\text { Northwest Africa } 11897
$$

Eucrite-unbr (Northwest At

43.9

0.0439

Grosvenor Mountains 17098 Eucrite-unbr Antarctica

38.752

0.038752

Asuka 12036

$\mathrm{H}$

Antarctica

70.24

0.07024

66403

Asuka 09242

$\mathrm{H}$

Antarctica

2.524

0.002524

66397

$\mathrm{H} / \mathrm{L} 4$

(Northwest At

75.04

0.07504

Northwest Africa 11579

$\mathrm{H} \sim 4$

(Northwest At

1247.7

1.2477




\begin{tabular}{|c|c|c|c|c|c|}
\hline 67801 & Northwest Africa 11972 & $\mathrm{H} \sim 5$ & Morocco & 2880 & 2.88 \\
\hline 66396 & Rabt Sbayta 009 & $\mathrm{H} \sim 5$ & Western Sahi & 2112.6 & 2.1126 \\
\hline 68129 & Alatage Mountain 044 & $\mathrm{H} \sim 5$ & China & 709 & 0.709 \\
\hline 67802 & Northwest Africa 11973 & $\mathrm{H} \sim 5$ & (Northwest A 1 & 334.8 & 0.3348 \\
\hline 68437 & Northwest Africa 12300 & $\mathrm{H} \sim 5$ & (Northwest A 1 & 273 & 0.273 \\
\hline 68659 & Northwest Africa 12383 & $\mathrm{H} \sim 5$ & (Northwest Al & 209.65 & 0.20965 \\
\hline 67800 & Northwest Africa 11971 & $\mathrm{H} \sim 5$ & (Northwest At & 105 & 0.105 \\
\hline 67803 & Northwest Africa 11974 & $\mathrm{H} \sim 5$ & (Northwest A 1 & 73.7 & 0.0737 \\
\hline 68431 & Loulan Yizhi 008 & $\mathrm{H} \sim 5$ & China & 29.3 & 0.0293 \\
\hline 68658 & Northwest Africa 12382 & $\mathrm{H} \sim 5$ & (Northwest At & 29.02 & 0.02902 \\
\hline 68438 & Lop Nur 003 & $\mathrm{H} \sim 5$ & China & 27.2 & 0.0272 \\
\hline 68651 & Qira 001 & $\mathrm{H} \sim 5$ & China & 8.03 & 0.00803 \\
\hline 68436 & Northwest Africa 12299 & $\mathrm{H} \sim 6$ & Morocco & 4500 & 4.5 \\
\hline 66398 & Northwest Africa 11580 & $\mathrm{H} \sim 6$ & (Northwest A 1 & 1518.9 & 1.5189 \\
\hline 68648 & Jinchang 001 & $\mathrm{H} \sim 6$ & China & 813 & 0.813 \\
\hline 68502 & Mount Yirtkuq Bulak 001 & $\mathrm{H} \sim 6$ & China & 540 & 0.54 \\
\hline 67805 & Northwest Africa 11979 & $\mathrm{H} \sim 6$ & (Northwest A 1 & 78.1 & 0.0781 \\
\hline 66401 & Northwest Africa 11583 & $\mathrm{H} \sim 6$ & Morocco & 24 & 0.024 \\
\hline 31833 & Ouargla 001 & H3 & Algeria & 2705 & 2.705 \\
\hline 67692 & Al Khuwaymat 001 & H3 & Libya & 1870.3 & 1.8703 \\
\hline 67758 & Northwest Africa 11936 & H3 & (Northwest Al & 1429.5 & 1.4295 \\
\hline 67592 & Taltal 001 & $\mathrm{H} 3$ & Chile & 1186 & 1.186 \\
\hline 31625 & Northwest Africa 432 & $\mathrm{H} 3$ & (Northwest A 1 & 839 & 0.839 \\
\hline 66825 & El Médano 377 & H3 & Chile & 405 & 0.405 \\
\hline 67749 & Northwest Africa 11923 & $\mathrm{H} 3$ & (Northwest Al & 252 & 0.252 \\
\hline 67416 & Reckling Peak 14003 & H3 & Antarctica & 231 & 0.231 \\
\hline 67710 & Stewart Valley 024 & $\mathrm{H} 3$ & United States & 158.2 & 0.1582 \\
\hline 67274 & Asuka 12327 & $\mathrm{H} 3$ & Antarctica & 156.7 & 0.1567 \\
\hline 67750 & Northwest Africa 11924 & $\mathrm{H} 3$ & (Northwest Al & 125 & 0.125 \\
\hline 67807 & Sahara 98063 & $\mathrm{H} 3$ & (Sahara) & 84 & 0.084 \\
\hline 67272 & Asuka 12323 & $\mathrm{H} 3$ & Antarctica & 60.59 & 0.06059 \\
\hline 67383 & Scarham Creek & $\mathrm{H} 3$ & United States & 52.1 & 0.0521 \\
\hline 67276 & Asuka 12330 & $\mathrm{H} 3$ & Antarctica & 42.665 & 0.042665 \\
\hline 67418 & Reckling Peak 14006 & $\mathrm{H} 3$ & Antarctica & 22.6 & 0.0226 \\
\hline 67510 & Northwest Africa 11810 & $\mathrm{H} 3$ & (Northwest A 1 & 21 & 0.021 \\
\hline 67509 & Northwest Africa 11806 & $\mathrm{H} 3$ & (Northwest At & 12 & 0.012 \\
\hline 67213 & Asuka 12262 & $\mathrm{H} 3$ & Antarctica & 5.182 & 0.005182 \\
\hline 67471 & Elephant Moraine 14058 & $\mathrm{H} 3$ & Antarctica & 0.7 & 0.0007 \\
\hline 67704 & Northwest Africa 11895 & H3-5 & (Sahara) & 148 & 0.148 \\
\hline 66854 & Yucca 050 & H3-5 & United States & 34.3 & 0.0343 \\
\hline 67748 & Yucca 052 & H3-5 & United States & 23.3 & 0.0233 \\
\hline 66853 & Yucca 049 & H3-5 & United States & 20.8 & 0.0208 \\
\hline 66850 & Yucca 046 & H3-5 & United States & 11.1 & 0.0111 \\
\hline 67391 & Catalina 257 & H3-6 & Chile & 42 & 0.042 \\
\hline 66849 & Yucca 045 & H3-6 & United States & 39.7 & 0.0397 \\
\hline
\end{tabular}




\begin{tabular}{|c|c|c|c|c|c|}
\hline 66852 & Yucca 048 & H3-6 & United States & 20.9 & 0.0209 \\
\hline 66851 & Yucca 047 & H3-6 & United States & 11.9 & 0.0119 \\
\hline 67983 & Dominion Range 14429 & $\mathrm{H} 3.5$ & Antarctica & 40.145 & 0.040145 \\
\hline 68006 & Dominion Range 14491 & H3.5 & Antarctica & 18.83 & 0.01883 \\
\hline 66834 & Northwest Africa 11700 & $\mathrm{H} 4$ & (Northwest Al & 102300 & 102.3 \\
\hline 67840 & Chug Chug 003 & $\mathrm{H} 4$ & Chile & 8918 & 8.918 \\
\hline 68515 & Northwest Africa 12350 & $\mathrm{H} 4$ & Morocco & 6629.8 & 6.6298 \\
\hline 66848 & Qatar 013 & $\mathrm{H} 4$ & Qatar & 5090 & 5.09 \\
\hline 67702 & Sahara 98001 & $\mathrm{H} 4$ & (Sahara) & 4450 & 4.45 \\
\hline 67385 & El Médano 380 & $\mathrm{H} 4$ & Chile & 2670 & 2.67 \\
\hline 66846 & Qatar 011 & $\mathrm{H} 4$ & Qatar & 2590 & 2.59 \\
\hline 68322 & Northwest Africa 12240 & $\mathrm{H} 4$ & Mauritania & 1808.4 & 1.8084 \\
\hline 68319 & Northwest Africa 12235 & $\mathrm{H} 4$ & Morocco & 1740 & 1.74 \\
\hline 68381 & Paposo 043 & $\mathrm{H} 4$ & Chile & 1614 & 1.614 \\
\hline 67172 & Asuka 12211 & $\mathrm{H} 4$ & Antarctica & 1371.7 & 1.3717 \\
\hline 68263 & Northwest Africa 12152 & $\mathrm{H} 4$ & Algeria & 1091.74 & 1.09174 \\
\hline 66772 & Hamburg & $\mathrm{H} 4$ & United States & 1000 & 1 \\
\hline 68547 & Los Vientos 259 & $\mathrm{H} 4$ & Chile & 981 & 0.981 \\
\hline 68261 & Northwest Africa 12150 & $\mathrm{H} 4$ & Algeria & 976.43 & 0.97643 \\
\hline 31782 & Northwest Africa 660 & $\mathrm{H} 4$ & (Northwest At & 668 & 0.668 \\
\hline 67757 & Northwest Africa 11935 & $\mathrm{H} 4$ & (Northwest A1 & 662.1 & 0.6621 \\
\hline 67259 & Asuka 12309 & $\mathrm{H} 4$ & Antarctica & 587.77 & 0.58777 \\
\hline 68266 & Northwest Africa 12155 & $\mathrm{H} 4$ & Algeria & 553.74 & 0.55374 \\
\hline 31646 & Northwest Africa 453 & $\mathrm{H} 4$ & (Northwest A1 & 539 & 0.539 \\
\hline 68514 & Northwest Africa 12349 & $\mathrm{H} 4$ & Morocco & 522.1 & 0.5221 \\
\hline 67696 & Jabal Zaltan 003 & $\mathrm{H} 4$ & Libya & 504.9 & 0.5049 \\
\hline 43244 & Yamato 002482 & $\mathrm{H} 4$ & Antarctica & 503.35 & 0.50335 \\
\hline 68422 & Lenghu 003 & $\mathrm{H} 4$ & China & 446 & 0.446 \\
\hline 68363 & Los Vientos 239 & $\mathrm{H} 4$ & Chile & 445 & 0.445 \\
\hline 31628 & Northwest Africa 435 & $\mathrm{H} 4$ & (Northwest At & 428 & 0.428 \\
\hline 68134 & Northwest Africa 12023 & $\mathrm{H} 4$ & Algeria & 408.46 & 0.40846 \\
\hline 67328 & Asuka 12383 & $\mathrm{H} 4$ & Antarctica & 406.04 & 0.40604 \\
\hline 31645 & Northwest Africa 452 & $\mathrm{H} 4$ & (Northwest Al & 373 & 0.373 \\
\hline 68138 & Northwest Africa 12027 & $\mathrm{H} 4$ & Algeria & 358.74 & 0.35874 \\
\hline 66669 & Dominion Range 14461 & $\mathrm{H} 4$ & Antarctica & 345.91 & 0.34591 \\
\hline 67698 & Jabal Zaltan 005 & $\mathrm{H} 4$ & Libya & 336 & 0.336 \\
\hline 67575 & Northwest Africa 11819 & $\mathrm{H} 4$ & (Northwest At & 330 & 0.33 \\
\hline 67727 & Calama 016 & $\mathrm{H} 4$ & Chile & 291 & 0.291 \\
\hline 67147 & Asuka 12040 & $\mathrm{H} 4$ & Antarctica & 280.89 & 0.28089 \\
\hline 68143 & Northwest Africa 12032 & $\mathrm{H} 4$ & Algeria & 276.91 & 0.27691 \\
\hline 68226 & Northwest Africa 12115 & $\mathrm{H} 4$ & Algeria & 273.2 & 0.2732 \\
\hline 67762 & Northwest Africa 11941 & $\mathrm{H} 4$ & (Northwest At & 266.8 & 0.2668 \\
\hline 67164 & Asuka 12136 & $\mathrm{H} 4$ & Antarctica & 266.69 & 0.26669 \\
\hline 68223 & Northwest Africa 12112 & $\mathrm{H} 4$ & Algeria & 250.67 & 0.25067 \\
\hline 68139 & Northwest Africa 12028 & $\mathrm{H} 4$ & Algeria & 241.17 & 0.24117 \\
\hline
\end{tabular}




\begin{tabular}{|c|c|c|c|c|c|}
\hline 68140 & Northwest Africa 12029 & $\mathrm{H} 4$ & Algeria & 221.83 & 0.22183 \\
\hline 68376 & Los Vientos 253 & $\mathrm{H} 4$ & Chile & 220 & 0.22 \\
\hline 67638 & Northwest Africa 11830 & $\mathrm{H} 4$ & (Northwest A 1 & 218 & 0.218 \\
\hline 68150 & Northwest Africa 12039 & $\mathrm{H} 4$ & Algeria & 199.18 & 0.19918 \\
\hline 31515 & Northwest Africa 317 & $\mathrm{H} 4$ & Algeria & 197.74 & 0.19774 \\
\hline 68399 & Northwest Africa 12251 & $\mathrm{H} 4$ & (Northwest A1 & 182 & 0.182 \\
\hline 68135 & Northwest Africa 12024 & $\mathrm{H} 4$ & Algeria & 181.04 & 0.18104 \\
\hline 68228 & Northwest Africa 12117 & $\mathrm{H} 4$ & Algeria & 176.82 & 0.17682 \\
\hline 68336 & Catalina 281 & $\mathrm{H} 4$ & Chile & 171 & 0.171 \\
\hline 68189 & Northwest Africa 12078 & $\mathrm{H} 4$ & Algeria & 167.26 & 0.16726 \\
\hline 67184 & Asuka 12233 & $\mathrm{H} 4$ & Antarctica & 149.07 & 0.14907 \\
\hline 67700 & Jabal Zaltan 007 & $\mathrm{H} 4$ & Libya & 147.5 & 0.1475 \\
\hline 67690 & Los Vientos 230 & $\mathrm{H} 4$ & Chile & 147 & 0.147 \\
\hline 68142 & Northwest Africa 12031 & $\mathrm{H} 4$ & Algeria & 138.44 & 0.13844 \\
\hline 67265 & Asuka 12316 & $\mathrm{H} 4$ & Antarctica & 135.64 & 0.13564 \\
\hline 68279 & Northwest Africa 12168 & $\mathrm{H} 4$ & Algeria & 131.65 & 0.13165 \\
\hline 68535 & Coipuno 003 & $\mathrm{H} 4$ & Chile & 130 & 0.13 \\
\hline 31516 & Northwest Africa 318 & $\mathrm{H} 4$ & Algeria & 127.73 & 0.12773 \\
\hline 68153 & Northwest Africa 12042 & $\mathrm{H} 4$ & Algeria & 125.53 & 0.12553 \\
\hline 68404 & Northwest Africa 12257 & $\mathrm{H} 4$ & (Northwest A 1 & 122 & 0.122 \\
\hline 67278 & Asuka 12332 & $\mathrm{H} 4$ & Antarctica & 119.55 & 0.11955 \\
\hline 68196 & Northwest Africa 12085 & $\mathrm{H} 4$ & Algeria & 119.31 & 0.11931 \\
\hline 68167 & Northwest Africa 12056 & $\mathrm{H} 4$ & Algeria & 118 & 0.118 \\
\hline 67315 & Asuka 12370 & $\mathrm{H} 4$ & Antarctica & 112.42 & 0.11242 \\
\hline 67703 & Sahara 98050 & $\mathrm{H} 4$ & (Sahara) & 106 & 0.106 \\
\hline 66548 & Dominion Range 14129 & $\mathrm{H} 4$ & Antarctica & 105.84 & 0.10584 \\
\hline 68227 & Northwest Africa 12116 & $\mathrm{H} 4$ & Algeria & 105.71 & 0.10571 \\
\hline 67180 & Asuka 12226 & $\mathrm{H} 4$ & Antarctica & 103.96 & 0.10396 \\
\hline 68209 & Northwest Africa 12098 & $\mathrm{H} 4$ & Algeria & 96.96 & 0.09696 \\
\hline 67153 & Asuka 12053 & $\mathrm{H} 4$ & Antarctica & 96.67 & 0.09667 \\
\hline 66496 & Northwest Africa 11680 & $\mathrm{H} 4$ & (Northwest A 1 & 96 & 0.096 \\
\hline 68148 & Northwest Africa 12037 & $\mathrm{H} 4$ & Algeria & 94.22 & 0.09422 \\
\hline 67159 & Asuka 12092 & $\mathrm{H} 4$ & Antarctica & 91.49 & 0.09149 \\
\hline 66508 & Northwest Africa 11692 & $\mathrm{H} 4$ & (Northwest A 1 & 88 & 0.088 \\
\hline 68146 & Northwest Africa 12035 & $\mathrm{H} 4$ & Algeria & 87.3 & 0.0873 \\
\hline 67966 & Dominion Range 14412 & $\mathrm{H} 4$ & Antarctica & 82.589 & 0.082589 \\
\hline 68182 & Northwest Africa 12071 & $\mathrm{H} 4$ & Algeria & 80.38 & 0.08038 \\
\hline 67695 & Jabal Zaltan 002 & $\mathrm{H} 4$ & Libya & 78.7 & 0.0787 \\
\hline 68270 & Northwest Africa 12159 & $\mathrm{H} 4$ & Algeria & 78.44 & 0.07844 \\
\hline 67361 & Asuka 12419 & $\mathrm{H} 4$ & Antarctica & 77.77 & 0.07777 \\
\hline 68252 & Northwest Africa 12141 & $\mathrm{H} 4$ & Algeria & 76.87 & 0.07687 \\
\hline 68168 & Northwest Africa 12057 & $\mathrm{H} 4$ & Algeria & 75.87 & 0.07587 \\
\hline 67181 & Asuka 12227 & $\mathrm{H} 4$ & Antarctica & 71.75 & 0.07175 \\
\hline 67191 & Asuka 12240 & $\mathrm{H} 4$ & Antarctica & 62.75 & 0.06275 \\
\hline 67139 & Asuka 12026 & $\mathrm{H} 4$ & Antarctica & 62.58 & 0.06258 \\
\hline
\end{tabular}




\begin{tabular}{|c|c|c|c|c|c|}
\hline 67182 & Asuka 12230 & $\mathrm{H} 4$ & Antarctica & 62.42 & 0.06242 \\
\hline 31514 & Northwest Africa 316 & $\mathrm{H} 4$ & Algeria & 60.25 & 0.06025 \\
\hline 67193 & Asuka 12242 & $\mathrm{H} 4$ & Antarctica & 56.58 & 0.05658 \\
\hline 67195 & Asuka 12244 & $\mathrm{H} 4$ & Antarctica & 56.162 & 0.056162 \\
\hline 68272 & Northwest Africa 12161 & $\mathrm{H} 4$ & Algeria & 55.04 & 0.05504 \\
\hline 67377 & Asuka 12436 & $\mathrm{H} 4$ & Antarctica & 51.125 & 0.051125 \\
\hline 42361 & Yamato 001599 & $\mathrm{H} 4$ & Antarctica & 49.567 & 0.049567 \\
\hline 67342 & Asuka 12399 & $\mathrm{H} 4$ & Antarctica & 48.987 & 0.048987 \\
\hline 67867 & Northwest Africa 12019 & $\mathrm{H} 4$ & (Northwest At & 48.89 & 0.04889 \\
\hline 67185 & Asuka 12234 & $\mathrm{H} 4$ & Antarctica & 46.912 & 0.046912 \\
\hline 67203 & Asuka 12252 & $\mathrm{H} 4$ & Antarctica & 46.292 & 0.046292 \\
\hline 66760 & Northwest Africa 11662 & $\mathrm{H} 4$ & (Northwest Al & 45.32 & 0.04532 \\
\hline 68208 & Northwest Africa 12097 & $\mathrm{H} 4$ & Algeria & 45.06 & 0.04506 \\
\hline 68224 & Northwest Africa 12113 & $\mathrm{H} 4$ & Algeria & 42.75 & 0.04275 \\
\hline 67333 & Asuka 12388 & $\mathrm{H} 4$ & Antarctica & 41.447 & 0.041447 \\
\hline 68275 & Northwest Africa 12164 & $\mathrm{H} 4$ & Algeria & 39.28 & 0.03928 \\
\hline 67202 & Asuka 12251 & $\mathrm{H} 4$ & Antarctica & 39.052 & 0.039052 \\
\hline 68247 & Northwest Africa 12136 & $\mathrm{H} 4$ & Algeria & 38.45 & 0.03845 \\
\hline 67292 & Asuka 12346 & $\mathrm{H} 4$ & Antarctica & 38.235 & 0.038235 \\
\hline 67275 & Asuka 12329 & $\mathrm{H} 4$ & Antarctica & 38.202 & 0.038202 \\
\hline 66573 & Dominion Range 14185 & $\mathrm{H} 4$ & Antarctica & 36.72 & 0.03672 \\
\hline 67876 & Dominion Range 14188 & $\mathrm{H} 4$ & Antarctica & 35.45 & 0.03545 \\
\hline 24804 & Yamato 74426 & $\mathrm{H} 4$ & Antarctica & 29.8 & 0.0298 \\
\hline 66645 & Dominion Range 14377 & $\mathrm{H} 4$ & Antarctica & 28.96 & 0.02896 \\
\hline 67989 & Dominion Range 14435 & $\mathrm{H} 4$ & Antarctica & 27.631 & 0.027631 \\
\hline 67261 & Asuka 12311 & $\mathrm{H} 4$ & Antarctica & 26.803 & 0.026803 \\
\hline 67249 & Asuka 12299 & $\mathrm{H} 4$ & Antarctica & 21.635 & 0.021635 \\
\hline 67324 & Asuka 12379 & $\mathrm{H} 4$ & Antarctica & 19.583 & 0.019583 \\
\hline 68278 & Northwest Africa 12167 & $\mathrm{H} 4$ & Algeria & 17.77 & 0.01777 \\
\hline 42059 & Yamato 001297 & $\mathrm{H} 4$ & Antarctica & 17.747 & 0.017747 \\
\hline 67269 & Asuka 12320 & $\mathrm{H} 4$ & Antarctica & 17.355 & 0.017355 \\
\hline 67208 & Asuka 12257 & $\mathrm{H} 4$ & Antarctica & 16.946 & 0.016946 \\
\hline 42191 & Yamato 001429 & $\mathrm{H} 4$ & Antarctica & 15.737 & 0.015737 \\
\hline 67277 & Asuka 12331 & $\mathrm{H} 4$ & Antarctica & 14.944 & 0.014944 \\
\hline 66518 & Dominion Range 14037 & $\mathrm{H} 4$ & Antarctica & 14.5 & 0.0145 \\
\hline 43242 & Yamato 002480 & $\mathrm{H} 4$ & Antarctica & 14.491 & 0.014491 \\
\hline 67189 & Asuka 12238 & $\mathrm{H} 4$ & Antarctica & 14.185 & 0.014185 \\
\hline 68302 & Calama 019 & $\mathrm{H} 4$ & Chile & 12.5 & 0.0125 \\
\hline 67305 & Asuka 12360 & $\mathrm{H} 4$ & Antarctica & 10.903 & 0.010903 \\
\hline 67284 & Asuka 12338 & $\mathrm{H} 4$ & Antarctica & 10.667 & 0.010667 \\
\hline 67183 & Asuka 12232 & $\mathrm{H} 4$ & Antarctica & 10.578 & 0.010578 \\
\hline 27088 & Yamato 791739 & $\mathrm{H} 4$ & Antarctica & 8.42 & 0.00842 \\
\hline 66661 & Dominion Range 14453 & $\mathrm{H} 4$ & Antarctica & 7.08 & 0.00708 \\
\hline 40813 & Yamato 000046 & $\mathrm{H} 4$ & Antarctica & 6.248 & 0.006248 \\
\hline 67078 & Asuka 09625 & $\mathrm{H} 4$ & Antarctica & 5.958 & 0.005958 \\
\hline
\end{tabular}




\begin{tabular}{|c|c|c|c|c|c|}
\hline 67005 & Asuka 09390 & $\mathrm{H} 4$ & Antarctica & 5.827 & 0.005827 \\
\hline 67097 & Asuka 09653 & $\mathrm{H} 4$ & Antarctica & 5.766 & 0.005766 \\
\hline 67081 & Asuka 09629 & $\mathrm{H} 4$ & Antarctica & 5.727 & 0.005727 \\
\hline 66984 & Asuka 09353 & $\mathrm{H} 4$ & Antarctica & 5.687 & 0.005687 \\
\hline 66976 & Asuka 09339 & $\mathrm{H} 4$ & Antarctica & 5.64 & 0.00564 \\
\hline 67071 & Asuka 09611 & $\mathrm{H} 4$ & Antarctica & 5.529 & 0.005529 \\
\hline 66921 & Asuka 09275 & $\mathrm{H} 4$ & Antarctica & 5.486 & 0.005486 \\
\hline 67032 & Asuka 09437 & $\mathrm{H} 4$ & Antarctica & 5.394 & 0.005394 \\
\hline 66924 & Asuka 09278 & $\mathrm{H} 4$ & Antarctica & 5.37 & 0.00537 \\
\hline 67085 & Asuka 09633 & $\mathrm{H} 4$ & Antarctica & 5.337 & 0.005337 \\
\hline 67098 & Asuka 09654 & $\mathrm{H} 4$ & Antarctica & 5.259 & 0.005259 \\
\hline 67052 & Asuka 09485 & $\mathrm{H} 4$ & Antarctica & 5.21 & 0.00521 \\
\hline 67049 & Asuka 09472 & $\mathrm{H} 4$ & Antarctica & 5.095 & 0.005095 \\
\hline 66922 & Asuka 09276 & $\mathrm{H} 4$ & Antarctica & 5.012 & 0.005012 \\
\hline 66783 & Northwest Africa 11743 & $\mathrm{H} 4$ & Morocco & 5 & 0.005 \\
\hline 41256 & Yamato 000491 & $\mathrm{H} 4$ & Antarctica & 4.993 & 0.004993 \\
\hline 41068 & Yamato 000303 & $\mathrm{H} 4$ & Antarctica & 4.981 & 0.004981 \\
\hline 41066 & Yamato 000301 & $\mathrm{H} 4$ & Antarctica & 4.888 & 0.004888 \\
\hline 41246 & Yamato 000481 & $\mathrm{H} 4$ & Antarctica & 4.884 & 0.004884 \\
\hline 41134 & Yamato 000369 & $\mathrm{H} 4$ & Antarctica & 4.788 & 0.004788 \\
\hline 40999 & Yamato 000234 & $\mathrm{H} 4$ & Antarctica & 4.758 & 0.004758 \\
\hline 67101 & Asuka 09657 & $\mathrm{H} 4$ & Antarctica & 4.676 & 0.004676 \\
\hline 41347 & Yamato 000582 & $\mathrm{H} 4$ & Antarctica & 4.606 & 0.004606 \\
\hline 67103 & Asuka 09659 & $\mathrm{H} 4$ & Antarctica & 4.53 & 0.00453 \\
\hline 43985 & Yamato 003223 & $\mathrm{H} 4$ & Antarctica & 4.438 & 0.004438 \\
\hline 41376 & Yamato 000612 & $\mathrm{H} 4$ & Antarctica & 4.433 & 0.004433 \\
\hline 40952 & Yamato 000187 & $\mathrm{H} 4$ & Antarctica & 4.425 & 0.004425 \\
\hline 66926 & Asuka 09280 & $\mathrm{H} 4$ & Antarctica & 4.386 & 0.004386 \\
\hline 67197 & Asuka 12246 & $\mathrm{H} 4$ & Antarctica & 4.36 & 0.00436 \\
\hline 67084 & Asuka 09632 & $\mathrm{H} 4$ & Antarctica & 4.353 & 0.004353 \\
\hline 41037 & Yamato 000272 & $\mathrm{H} 4$ & Antarctica & 4.297 & 0.004297 \\
\hline 66898 & Asuka 09233 & $\mathrm{H} 4$ & Antarctica & 4.257 & 0.004257 \\
\hline 41379 & Yamato 000615 & $\mathrm{H} 4$ & Antarctica & 4.25 & 0.00425 \\
\hline 40943 & Yamato 000178 & $\mathrm{H} 4$ & Antarctica & 4.245 & 0.004245 \\
\hline 40945 & Yamato 000180 & $\mathrm{H} 4$ & Antarctica & 4.243 & 0.004243 \\
\hline 40968 & Yamato 000203 & $\mathrm{H} 4$ & Antarctica & 4.096 & 0.004096 \\
\hline 66929 & Asuka 09283 & $\mathrm{H} 4$ & Antarctica & 4.057 & 0.004057 \\
\hline 43853 & Yamato 003091 & $\mathrm{H} 4$ & Antarctica & 3.983 & 0.003983 \\
\hline 67107 & Asuka 09663 & $\mathrm{H} 4$ & Antarctica & 3.938 & 0.003938 \\
\hline 41532 & Yamato 000769 & $\mathrm{H} 4$ & Antarctica & 3.844 & 0.003844 \\
\hline 41104 & Yamato 000339 & $\mathrm{H} 4$ & Antarctica & 3.74 & 0.00374 \\
\hline 66904 & Asuka 09248 & $\mathrm{H} 4$ & Antarctica & 3.739 & 0.003739 \\
\hline 41140 & Yamato 000375 & $\mathrm{H} 4$ & Antarctica & 3.721 & 0.003721 \\
\hline 67109 & Asuka 09665 & $\mathrm{H} 4$ & Antarctica & 3.706 & 0.003706 \\
\hline 41572 & Yamato 000810 & $\mathrm{H} 4$ & Antarctica & 3.688 & 0.003688 \\
\hline
\end{tabular}




\begin{tabular}{|c|c|c|c|c|c|}
\hline 43884 & Yamato 003122 & $\mathrm{H} 4$ & Antarctica & 3.646 & 0.003646 \\
\hline 67079 & Asuka 09626 & $\mathrm{H} 4$ & Antarctica & 3.642 & 0.003642 \\
\hline 67022 & Asuka 09411 & $\mathrm{H} 4$ & Antarctica & 3.641 & 0.003641 \\
\hline 41505 & Yamato 000741 & $\mathrm{H} 4$ & Antarctica & 3.615 & 0.003615 \\
\hline 40981 & Yamato 000216 & $\mathrm{H} 4$ & Antarctica & 3.606 & 0.003606 \\
\hline 66972 & Asuka 09333 & $\mathrm{H} 4$ & Antarctica & 3.602 & 0.003602 \\
\hline 41163 & Yamato 000398 & $\mathrm{H} 4$ & Antarctica & 3.543 & 0.003543 \\
\hline 67030 & Asuka 09429 & $\mathrm{H} 4$ & Antarctica & 3.514 & 0.003514 \\
\hline 66903 & Asuka 09247 & $\mathrm{H} 4$ & Antarctica & 3.499 & 0.003499 \\
\hline 67035 & Asuka 09444 & $\mathrm{H} 4$ & Antarctica & 3.465 & 0.003465 \\
\hline 41338 & Yamato 000573 & $\mathrm{H} 4$ & Antarctica & 3.46 & 0.00346 \\
\hline 43784 & Yamato 003022 & $\mathrm{H} 4$ & Antarctica & 3.455 & 0.003455 \\
\hline 66934 & Asuka 09288 & $\mathrm{H} 4$ & Antarctica & 3.454 & 0.003454 \\
\hline 41311 & Yamato 000546 & $\mathrm{H} 4$ & Antarctica & 3.401 & 0.003401 \\
\hline 66928 & Asuka 09282 & $\mathrm{H} 4$ & Antarctica & 3.4 & 0.0034 \\
\hline 66925 & Asuka 09279 & $\mathrm{H} 4$ & Antarctica & 3.394 & 0.003394 \\
\hline 41212 & Yamato 000447 & $\mathrm{H} 4$ & Antarctica & 3.309 & 0.003309 \\
\hline 67104 & Asuka 09660 & $\mathrm{H} 4$ & Antarctica & 3.283 & 0.003283 \\
\hline 67113 & Asuka 09669 & $\mathrm{H} 4$ & Antarctica & 3.245 & 0.003245 \\
\hline 67105 & Asuka 09661 & $\mathrm{H} 4$ & Antarctica & 3.205 & 0.003205 \\
\hline 67108 & Asuka 09664 & $\mathrm{H} 4$ & Antarctica & 3.204 & 0.003204 \\
\hline 41554 & Yamato 000791 & $\mathrm{H} 4$ & Antarctica & 3.203 & 0.003203 \\
\hline 66931 & Asuka 09285 & $\mathrm{H} 4$ & Antarctica & 3.189 & 0.003189 \\
\hline 67020 & Asuka 09407 & $\mathrm{H} 4$ & Antarctica & 3.188 & 0.003188 \\
\hline 67110 & Asuka 09666 & $\mathrm{H} 4$ & Antarctica & 3.181 & 0.003181 \\
\hline 40976 & Yamato 000211 & $\mathrm{H} 4$ & Antarctica & 3.176 & 0.003176 \\
\hline 41535 & Yamato 000772 & $\mathrm{H} 4$ & Antarctica & 3.146 & 0.003146 \\
\hline 41136 & Yamato 000371 & $\mathrm{H} 4$ & Antarctica & 3.136 & 0.003136 \\
\hline 66977 & Asuka 09340 & $\mathrm{H} 4$ & Antarctica & 3.123 & 0.003123 \\
\hline 67086 & Asuka 09634 & $\mathrm{H} 4$ & Antarctica & 3.121 & 0.003121 \\
\hline 66930 & Asuka 09284 & $\mathrm{H} 4$ & Antarctica & 3.091 & 0.003091 \\
\hline 41526 & Yamato 000763 & $\mathrm{H} 4$ & Antarctica & 3.088 & 0.003088 \\
\hline 41348 & Yamato 000583 & $\mathrm{H} 4$ & Antarctica & 3.083 & 0.003083 \\
\hline 41346 & Yamato 000581 & $\mathrm{H} 4$ & Antarctica & 3.051 & 0.003051 \\
\hline 43980 & Yamato 003218 & $\mathrm{H} 4$ & Antarctica & 3.033 & 0.003033 \\
\hline 67100 & Asuka 09656 & $\mathrm{H} 4$ & Antarctica & 3.009 & 0.003009 \\
\hline 66907 & Asuka 09252 & $\mathrm{H} 4$ & Antarctica & 2.921 & 0.002921 \\
\hline 66927 & Asuka 09281 & $\mathrm{H} 4$ & Antarctica & 2.916 & 0.002916 \\
\hline 42891 & Yamato 002129 & $\mathrm{H} 4$ & Antarctica & 2.906 & 0.002906 \\
\hline 67111 & Asuka 09667 & $\mathrm{H} 4$ & Antarctica & 2.856 & 0.002856 \\
\hline 67106 & Asuka 09662 & $\mathrm{H} 4$ & Antarctica & 2.832 & 0.002832 \\
\hline 67034 & Asuka 09440 & $\mathrm{H} 4$ & Antarctica & 2.799 & 0.002799 \\
\hline 66935 & Asuka 09289 & $\mathrm{H} 4$ & Antarctica & 2.797 & 0.002797 \\
\hline 67112 & Asuka 09668 & $\mathrm{H} 4$ & Antarctica & 2.759 & 0.002759 \\
\hline 66895 & Asuka 09230 & $\mathrm{H} 4$ & Antarctica & 2.748 & 0.002748 \\
\hline
\end{tabular}




\begin{tabular}{|c|c|c|c|c|c|}
\hline 66968 & Asuka 09325 & $\mathrm{H} 4$ & Antarctica & 2.731 & 0.002731 \\
\hline 67117 & Asuka 09673 & $\mathrm{H} 4$ & Antarctica & 2.634 & 0.002634 \\
\hline 40776 & Yamato 000008 & $\mathrm{H} 4$ & Antarctica & 2.594 & 0.002594 \\
\hline 67120 & Asuka 09678 & $\mathrm{H} 4$ & Antarctica & 2.558 & 0.002558 \\
\hline 66896 & Asuka 09231 & $\mathrm{H} 4$ & Antarctica & 2.506 & 0.002506 \\
\hline 66932 & Asuka 09286 & $\mathrm{H} 4$ & Antarctica & 2.496 & 0.002496 \\
\hline 66933 & Asuka 09287 & $\mathrm{H} 4$ & Antarctica & 2.481 & 0.002481 \\
\hline 67115 & Asuka 09671 & $\mathrm{H} 4$ & Antarctica & 2.445 & 0.002445 \\
\hline 66894 & Asuka 09228 & $\mathrm{H} 4$ & Antarctica & 2.432 & 0.002432 \\
\hline 67083 & Asuka 09631 & $\mathrm{H} 4$ & Antarctica & 2.253 & 0.002253 \\
\hline 67114 & Asuka 09670 & $\mathrm{H} 4$ & Antarctica & 2.163 & 0.002163 \\
\hline 66936 & Asuka 09290 & $\mathrm{H} 4$ & Antarctica & 2.085 & 0.002085 \\
\hline 66916 & Asuka 09263 & $\mathrm{H} 4$ & Antarctica & 2.063 & 0.002063 \\
\hline 67118 & Asuka 09674 & $\mathrm{H} 4$ & Antarctica & 1.946 & 0.001946 \\
\hline 66939 & Asuka 09293 & $\mathrm{H} 4$ & Antarctica & 1.94 & 0.00194 \\
\hline 67080 & Asuka 09628 & $\mathrm{H} 4$ & Antarctica & 1.932 & 0.001932 \\
\hline 66937 & Asuka 09291 & $\mathrm{H} 4$ & Antarctica & 1.927 & 0.001927 \\
\hline 66940 & Asuka 09294 & $\mathrm{H} 4$ & Antarctica & 1.891 & 0.001891 \\
\hline 66941 & Asuka 09295 & $\mathrm{H} 4$ & Antarctica & 1.794 & 0.001794 \\
\hline 67072 & Asuka 09612 & $\mathrm{H} 4$ & Antarctica & 1.702 & 0.001702 \\
\hline 66945 & Asuka 09299 & $\mathrm{H} 4$ & Antarctica & 1.692 & 0.001692 \\
\hline 66944 & Asuka 09298 & $\mathrm{H} 4$ & Antarctica & 1.672 & 0.001672 \\
\hline 67010 & Asuka 09395 & $\mathrm{H} 4$ & Antarctica & 1.652 & 0.001652 \\
\hline 66950 & Asuka 09304 & $\mathrm{H} 4$ & Antarctica & 1.632 & 0.001632 \\
\hline 67073 & Asuka 09613 & $\mathrm{H} 4$ & Antarctica & 1.616 & 0.001616 \\
\hline 66965 & Asuka 09321 & $\mathrm{H} 4$ & Antarctica & 1.559 & 0.001559 \\
\hline 66946 & Asuka 09300 & $\mathrm{H} 4$ & Antarctica & 1.507 & 0.001507 \\
\hline 67119 & Asuka 09677 & $\mathrm{H} 4$ & Antarctica & 1.497 & 0.001497 \\
\hline 66899 & Asuka 09234 & $\mathrm{H} 4$ & Antarctica & 1.494 & 0.001494 \\
\hline 67013 & Asuka 09398 & $\mathrm{H} 4$ & Antarctica & 1.436 & 0.001436 \\
\hline 66942 & Asuka 09296 & $\mathrm{H} 4$ & Antarctica & 1.374 & 0.001374 \\
\hline 67088 & Asuka 09636 & $\mathrm{H} 4$ & Antarctica & 1.366 & 0.001366 \\
\hline 66943 & Asuka 09297 & $\mathrm{H} 4$ & Antarctica & 1.317 & 0.001317 \\
\hline 67087 & Asuka 09635 & $\mathrm{H} 4$ & Antarctica & 1.263 & 0.001263 \\
\hline 42884 & Yamato 002122 & $\mathrm{H} 4$ & Antarctica & 1.167 & 0.001167 \\
\hline 67050 & Asuka 09477 & $\mathrm{H} 4$ & Antarctica & 1.165 & 0.001165 \\
\hline 67074 & Asuka 09614 & $\mathrm{H} 4$ & Antarctica & 1.09 & 0.00109 \\
\hline 66961 & Asuka 09315 & $\mathrm{H} 4$ & Antarctica & 1.075 & 0.001075 \\
\hline 66964 & Asuka 09320 & $\mathrm{H} 4$ & Antarctica & 1.044 & 0.001044 \\
\hline 67016 & Asuka 09401 & $\mathrm{H} 4$ & Antarctica & 1.02 & 0.00102 \\
\hline 67127 & Asuka 10206 & $\mathrm{H} 4$ & Antarctica & 0.88 & 0.00088 \\
\hline 67077 & Asuka 09623 & $\mathrm{H} 4$ & Antarctica & 0.857 & 0.000857 \\
\hline 66717 & Miller Range 15105 & $\mathrm{H} 4$ & Antarctica & 0.85 & 0.00085 \\
\hline 66955 & Asuka 09309 & $\mathrm{H} 4$ & Antarctica & 0.847 & 0.000847 \\
\hline 67129 & Asuka 10216 & $\mathrm{H} 4$ & Antarctica & 0.813 & 0.000813 \\
\hline
\end{tabular}




\begin{tabular}{|c|c|c|c|c|c|}
\hline 67082 & Asuka 09630 & $\mathrm{H} 4$ & Antarctica & 0.705 & 0.000705 \\
\hline 43371 & Yamato 002609 & $\mathrm{H} 4$ & Antarctica & 0.681 & 0.000681 \\
\hline 43891 & Yamato 003129 & $\mathrm{H} 4$ & Antarctica & 0.656 & 0.000656 \\
\hline 66957 & Asuka 09311 & $\mathrm{H} 4$ & Antarctica & 0.605 & 0.000605 \\
\hline 66956 & Asuka 09310 & $\mathrm{H} 4$ & Antarctica & 0.525 & 0.000525 \\
\hline 43553 & Yamato 002791 & $\mathrm{H} 4$ & Antarctica & 0.516 & 0.000516 \\
\hline 43369 & Yamato 002607 & $\mathrm{H} 4$ & Antarctica & 0.511 & 0.000511 \\
\hline 42639 & Yamato 001877 & $\mathrm{H} 4$ & Antarctica & 0.461 & 0.000461 \\
\hline 43334 & Yamato 002572 & $\mathrm{H} 4$ & Antarctica & 0.438 & 0.000438 \\
\hline 42640 & Yamato 001878 & $\mathrm{H} 4$ & Antarctica & 0.422 & 0.000422 \\
\hline 42693 & Yamato 001931 & $\mathrm{H} 4$ & Antarctica & 0.417 & 0.000417 \\
\hline 43288 & Yamato 002526 & $\mathrm{H} 4$ & Antarctica & 0.399 & 0.000399 \\
\hline 42739 & Yamato 001977 & $\mathrm{H} 4$ & Antarctica & 0.386 & 0.000386 \\
\hline 66959 & Asuka 09313 & $\mathrm{H} 4$ & Antarctica & 0.375 & 0.000375 \\
\hline 66960 & Asuka 09314 & $\mathrm{H} 4$ & Antarctica & 0.338 & 0.000338 \\
\hline 42638 & Yamato 001876 & $\mathrm{H} 4$ & Antarctica & 0.308 & 0.000308 \\
\hline 43382 & Yamato 002620 & $\mathrm{H} 4$ & Antarctica & 0.306 & 0.000306 \\
\hline 43240 & Yamato 002478 & $\mathrm{H} 4$ & Antarctica & 0.225 & 0.000225 \\
\hline 68520 & Parauapebas & $\mathrm{H} 4-5$ & Brazil & 272.3 & 0.2723 \\
\hline 67190 & Asuka 12239 & $\mathrm{H} 4-6$ & Antarctica & 52.553 & 0.052553 \\
\hline 66405 & Northwest Africa 11572 & H4-melt brec & (Northwest At & 897.52 & 0.89752 \\
\hline 66810 & Northwest Africa 11705 & $\mathrm{H} 4 / 5$ & (Northwest A 1 & 11036 & 11.036 \\
\hline 66845 & Qatar 010 & $\mathrm{H} 4 / 5$ & Qatar & 9264 & 9.264 \\
\hline 66847 & Qatar 012 & $\mathrm{H} 4 / 5$ & Qatar & 2431 & 2.431 \\
\hline 68395 & San Juan 099 & $\mathrm{H} 4 / 5$ & Chile & 1224 & 1.224 \\
\hline 68386 & Paposo 048 & $\mathrm{H} 4 / 5$ & Chile & 1089 & 1.089 \\
\hline 67660 & Paposo 041 & $\mathrm{H} 4 / 5$ & Chile & 860 & 0.86 \\
\hline 68347 & Catalina 292 & $\mathrm{H} 4 / 5$ & Chile & 325 & 0.325 \\
\hline 68352 & Catalina 297 & $\mathrm{H} 4 / 5$ & Chile & 180 & 0.18 \\
\hline 68390 & San Juan 094 & $\mathrm{H} 4 / 5$ & Chile & 115 & 0.115 \\
\hline 68504 & Northwest Africa 12354 & $\mathrm{H} 4 / 5$ & (Northwest Al & 94.1 & 0.0941 \\
\hline 67613 & Northwest Africa 11862 & $\mathrm{H} 4 / 5$ & (Northwest A 1 & 30.38 & 0.03038 \\
\hline 68338 & Catalina 283 & H5 & Chile & 47000 & 47 \\
\hline 67459 & Elephant Moraine 14043 & H5 & Antarctica & 36700 & 36.7 \\
\hline 67384 & El Médano 379 & H5 & Chile & 21300 & 21.3 \\
\hline 68619 & Maryborough & H5 & Australia & 17000 & 17 \\
\hline 67448 & Elephant Moraine 14032 & H5 & Antarctica & 11200 & 11.2 \\
\hline 67524 & El Médano 384 & H5 & Chile & 10440 & 10.44 \\
\hline 67874 & Dominion Range 14001 & H5 & Antarctica & 8205 & 8.205 \\
\hline 68629 & Ablaketka & H5 & Kazakhstan & 5500 & 5.5 \\
\hline 67846 & Northwest Africa 12001 & $\mathrm{H} 5$ & Morocco & 5230 & 5.23 \\
\hline 66756 & Northwest Africa 11658 & H5 & (Northwest A 1 & 5000 & 5 \\
\hline 68534 & Northwest Africa 12369 & H5 & (Northwest Al & 4685 & 4.685 \\
\hline 68311 & Northwest Africa 12215 & $\mathrm{H} 5$ & (Northwest A 1 & 4235 & 4.235 \\
\hline 66748 & Dar al Gani 1069 & $\mathrm{H} 5$ & Libya & 3958 & 3.958 \\
\hline
\end{tabular}




\begin{tabular}{|c|c|c|c|c|c|}
\hline 68660 & Northwest Africa 12385 & H5 & Morocco & 3463 & 3.463 \\
\hline 68550 & Los Vientos 261 & H5 & Chile & 3189 & 3.189 \\
\hline 68310 & Northwest Africa 12211 & H5 & (Northwest Al & 2729 & 2.729 \\
\hline 67514 & Northwest Africa 11814 & H5 & (Northwest Al & 2510 & 2.51 \\
\hline 68317 & Hami 007 & H5 & China & 2500 & 2.5 \\
\hline 68518 & Northwest Africa 12353 & H5 & Morocco & 1423 & 1.423 \\
\hline 67844 & Chug Chug 005 & H5 & Chile & 1295 & 1.295 \\
\hline 66438 & Northwest Africa 11642 & H5 & (Northwest Al & 1259 & 1.259 \\
\hline 68512 & Northwest Africa 12347 & H5 & Morocco & 1242.9 & 1.2429 \\
\hline 32062 & Northwest Africa 1089 & H5 & (Northwest Al & 1136 & 1.136 \\
\hline 67753 & Northwest Africa 11929 & H5 & (Northwest Al & 964 & 0.964 \\
\hline 42158 & Yamato 001396 & H5 & Antarctica & 922.7 & 0.9227 \\
\hline 67517 & Los Vientos 221 & H5 & Chile & 825 & 0.825 \\
\hline 67711 & Northwest Africa 11778 & H5 & (Northwest Al & 767.5 & 0.7675 \\
\hline 66510 & Northwest Africa 11694 & H5 & (Northwest At & 751 & 0.751 \\
\hline 68264 & Northwest Africa 12153 & H5 & Algeria & 677.79 & 0.67779 \\
\hline 67728 & Calama 017 & $\mathrm{H} 5$ & Chile & 668 & 0.668 \\
\hline 68283 & Northwest Africa 12172 & H5 & Algeria & 649.57 & 0.64957 \\
\hline 67659 & Los Vientos 227 & H5 & Chile & 646 & 0.646 \\
\hline 68549 & Los Vientos 260 & H5 & Chile & 646 & 0.646 \\
\hline 31644 & Northwest Africa 451 & H5 & (Northwest Al & 620 & 0.62 \\
\hline 67156 & Asuka 12060 & H5 & Antarctica & 612.5 & 0.6125 \\
\hline 68321 & Northwest Africa 12239 & H5 & (Northwest Al & 586 & 0.586 \\
\hline 68066 & Miller Range 15383 & H5 & Antarctica & 557.7 & 0.5577 \\
\hline 68265 & Northwest Africa 12154 & H5 & Algeria & 510.99 & 0.51099 \\
\hline 68304 & Sierra Gorda 007 & H5 & Chile & 502 & 0.502 \\
\hline 68516 & Northwest Africa 12351 & H5 & Morocco & 498.3 & 0.4983 \\
\hline 68508 & Northwest Africa 12359 & H5 & (Northwest A 1 & 486.2 & 0.4862 \\
\hline 66814 & Stewart Valley 023 & H5 & United States & 485 & 0.485 \\
\hline 67468 & Elephant Moraine 14054 & H5 & Antarctica & 463 & 0.463 \\
\hline 68358 & Los Vientos 234 & H5 & Chile & 461.9 & 0.4619 \\
\hline 68400 & Northwest Africa 12253 & H5 & (Northwest A 1 & 454 & 0.454 \\
\hline 68133 & Northwest Africa 12022 & H5 & Algeria & 439.34 & 0.43934 \\
\hline 66822 & El Médano 374 & H5 & Chile & 436 & 0.436 \\
\hline 67140 & Asuka 12027 & H5 & Antarctica & 427.83 & 0.42783 \\
\hline 68359 & Los Vientos 235 & H5 & Chile & 422.9 & 0.4229 \\
\hline 68236 & Northwest Africa 12125 & H5 & Algeria & 422.78 & 0.42278 \\
\hline 68194 & Northwest Africa 12083 & $\mathrm{H} 5$ & Algeria & 399.41 & 0.39941 \\
\hline 67697 & Jabal Zaltan 004 & H5 & Libya & 397.9 & 0.3979 \\
\hline 67176 & Asuka 12219 & H5 & Antarctica & 393.4 & 0.3934 \\
\hline 67716 & Chug Chug 002 & H5 & Chile & 388 & 0.388 \\
\hline 67706 & Qatar 014 & H5 & Qatar & 378 & 0.378 \\
\hline 68355 & El Médano 393 & H5 & Chile & 332 & 0.332 \\
\hline 67537 & El Médano 385 & H5 & Chile & 301 & 0.301 \\
\hline 67580 & Northwest Africa 11824 & H5 & (Northwest A 1 & 301 & 0.301 \\
\hline
\end{tabular}




\begin{tabular}{|c|c|c|c|c|c|}
\hline 67610 & Hermenegildo & H5 & Brazil & 300 & 0.3 \\
\hline 67574 & Northwest Africa 11818 & H5 & (Northwest Al & 278 & 0.278 \\
\hline 67530 & Catalina 267 & H5 & Chile & 267 & 0.267 \\
\hline 67521 & Catalina 262 & H5 & Chile & 255 & 0.255 \\
\hline 67538 & El Médano 386 & H5 & Chile & 253 & 0.253 \\
\hline 66499 & Northwest Africa 11683 & H5 & (Northwest At & 243 & 0.243 \\
\hline 67313 & Asuka 12368 & H5 & Antarctica & 231.36 & 0.23136 \\
\hline 67724 & Calama 012 & H5 & Chile & 227 & 0.227 \\
\hline 68237 & Northwest Africa 12126 & H5 & Algeria & 226.76 & 0.22676 \\
\hline 68191 & Northwest Africa 12080 & H5 & Algeria & 226.45 & 0.22645 \\
\hline 66823 & El Médano 375 & H5 & Chile & 223 & 0.223 \\
\hline 67581 & Northwest Africa 11825 & $\mathrm{H} 5$ & (Northwest A1 & 218.4 & 0.2184 \\
\hline 68517 & Northwest Africa 12352 & H5 & Morocco & 204.5 & 0.2045 \\
\hline 67178 & Asuka 12221 & H5 & Antarctica & 196.29 & 0.19629 \\
\hline 67891 & Dominion Range 14234 & H5 & Antarctica & 193.87 & 0.19387 \\
\hline 67534 & Catalina 270 & H5 & Chile & 193 & 0.193 \\
\hline 68357 & Los Vientos 233 & H5 & Chile & 192 & 0.192 \\
\hline 68260 & Northwest Africa 12149 & H5 & Algeria & 191.04 & 0.19104 \\
\hline 68239 & Northwest Africa 12128 & H5 & Algeria & 186.91 & 0.18691 \\
\hline 67665 & El Médano 388 & H5 & Chile & 185 & 0.185 \\
\hline 68144 & Northwest Africa 12033 & H5 & Algeria & 182.13 & 0.18213 \\
\hline 68505 & Northwest Africa 12356 & H5 & (Northwest A1 & 181.6 & 0.1816 \\
\hline 67701 & Jabal Zaltan 008 & H5 & Libya & 176.9 & 0.1769 \\
\hline 68185 & Northwest Africa 12074 & $\mathrm{H} 5$ & Algeria & 175.43 & 0.17543 \\
\hline 68301 & Calate 015 & H5 & Chile & 175 & 0.175 \\
\hline 68155 & Northwest Africa 12044 & $\mathrm{H} 5$ & Algeria & 173.59 & 0.17359 \\
\hline 68187 & Northwest Africa 12076 & H5 & Algeria & 173.54 & 0.17354 \\
\hline 68234 & Northwest Africa 12123 & H5 & Algeria & 171.34 & 0.17134 \\
\hline 68218 & Northwest Africa 12107 & H5 & Algeria & 166.55 & 0.16655 \\
\hline 68286 & Northwest Africa 12175 & H5 & Algeria & 164.82 & 0.16482 \\
\hline 67173 & Asuka 12212 & $\mathrm{H} 5$ & Antarctica & 164.57 & 0.16457 \\
\hline 68141 & Northwest Africa 12030 & H5 & Algeria & 161.01 & 0.16101 \\
\hline 66821 & Paposo 039 & H5 & Chile & 160.3 & 0.1603 \\
\hline 66816 & Ravar 003 & H5 & Iran & 154 & 0.154 \\
\hline 66815 & Gandom Beryan 010 & $\mathrm{H} 5$ & Iran & 150 & 0.15 \\
\hline 68366 & Los Vientos 242 & H5 & Chile & 147 & 0.147 \\
\hline 67535 & Catalina 271 & $\mathrm{H} 5$ & Chile & 142 & 0.142 \\
\hline 67577 & Northwest Africa 11821 & $\mathrm{H} 5$ & (Northwest At & 142 & 0.142 \\
\hline 67812 & Northwest Africa 11912 & $\mathrm{H} 5$ & (Northwest Al & 142 & 0.142 \\
\hline 68303 & Sierra Gorda 006 & $\mathrm{H} 5$ & Chile & 142 & 0.142 \\
\hline 68385 & Paposo 047 & H5 & Chile & 140 & 0.14 \\
\hline 68356 & Los Vientos 232 & H5 & Chile & 139 & 0.139 \\
\hline 67578 & Northwest Africa 11822 & H5 & (Northwest Al & 138.2 & 0.1382 \\
\hline 66501 & Northwest Africa 11685 & $\mathrm{H} 5$ & (Northwest At & 138 & 0.138 \\
\hline 66862 & Sayh al Uhaymir 606 & H5 & Oman & 136.502 & 0.136502 \\
\hline
\end{tabular}




\begin{tabular}{|c|c|c|c|c|c|}
\hline 67672 & Northwest Africa 11885 & $\mathrm{H} 5$ & (Northwest Al & 135 & 0.135 \\
\hline 67673 & Northwest Africa 8282 & H5 & (Northwest Al & 134 & 0.134 \\
\hline 68149 & Northwest Africa 12038 & H5 & Algeria & 131.94 & 0.13194 \\
\hline 68235 & Northwest Africa 12124 & H5 & Algeria & 130.3 & 0.1303 \\
\hline 67219 & Asuka 12268 & H5 & Antarctica & 128.51 & 0.12851 \\
\hline 67162 & Asuka 12117 & H5 & Antarctica & 127.91 & 0.12791 \\
\hline 67683 & Catalina 272 & $\mathrm{H} 5$ & Chile & 123 & 0.123 \\
\hline 68245 & Northwest Africa 12134 & H5 & Algeria & 122.71 & 0.12271 \\
\hline 66671 & Dominion Range 14463 & H5 & Antarctica & 122.65 & 0.12265 \\
\hline 67523 & Los Vientos 223 & H5 & Chile & 120 & 0.12 \\
\hline 68244 & Northwest Africa 12133 & H5 & Algeria & 119.15 & 0.11915 \\
\hline 67380 & Wood River & H5 & Canada & 118 & 0.118 \\
\hline 68274 & Northwest Africa 12163 & H5 & Algeria & 117.44 & 0.11744 \\
\hline 68340 & Catalina 285 & H5 & Chile & 117 & 0.117 \\
\hline 68147 & Northwest Africa 12036 & H5 & Algeria & 116.48 & 0.11648 \\
\hline 67452 & Elephant Moraine 14036 & H5 & Antarctica & 115.4 & 0.1154 \\
\hline 67325 & Asuka 12380 & H5 & Antarctica & 114.53 & 0.11453 \\
\hline 66829 & Catalina 255 & H5 & Chile & 112.1 & 0.1121 \\
\hline 68269 & Northwest Africa 12158 & H5 & Algeria & 111.79 & 0.11179 \\
\hline 68284 & Northwest Africa 12173 & H5 & Algeria & 109.91 & 0.10991 \\
\hline 68277 & Northwest Africa 12166 & $\mathrm{H} 5$ & Algeria & 109.38 & 0.10938 \\
\hline 67331 & Asuka 12386 & H5 & Antarctica & 100.73 & 0.10073 \\
\hline 67993 & Dominion Range 14439 & $\mathrm{H} 5$ & Antarctica & 100.154 & 0.100154 \\
\hline 68157 & Northwest Africa 12046 & H5 & Algeria & 99.9 & 0.0999 \\
\hline 68190 & Northwest Africa 12079 & H5 & Algeria & 97.72 & 0.09772 \\
\hline 67271 & Asuka 12322 & H5 & Antarctica & 97.43 & 0.09743 \\
\hline 66673 & Dominion Range 14465 & $\mathrm{H} 5$ & Antarctica & 96.57 & 0.09657 \\
\hline 67571 & Northwest Africa 11816 & H5 & (Northwest A 1 & 93 & 0.093 \\
\hline 67666 & Catalina 277 & H5 & Chile & 92.3 & 0.0923 \\
\hline 67467 & Elephant Moraine 14052 & H5 & Antarctica & 92.2 & 0.0922 \\
\hline 67267 & Asuka 12318 & H5 & Antarctica & 91.71 & 0.09171 \\
\hline 68151 & Northwest Africa 12040 & H5 & Algeria & 91.58 & 0.09158 \\
\hline 66505 & Northwest Africa 11689 & $\mathrm{H} 5$ & (Northwest Al & 90 & 0.09 \\
\hline 67177 & Asuka 12220 & H5 & Antarctica & 88.41 & 0.08841 \\
\hline 68163 & Northwest Africa 12052 & $\mathrm{H} 5$ & Algeria & 85 & 0.085 \\
\hline 67961 & Dominion Range 14407 & H5 & Antarctica & 84.25 & 0.08425 \\
\hline 67460 & Elephant Moraine 14044 & H5 & Antarctica & 83 & 0.083 \\
\hline 66574 & Dominion Range 14186 & $\mathrm{H} 5$ & Antarctica & 82.56 & 0.08256 \\
\hline 67582 & Northwest Africa 11826 & H5 & (Northwest A 1 & 82.4 & 0.0824 \\
\hline 66674 & Dominion Range 14466 & H5 & Antarctica & 82.15 & 0.08215 \\
\hline 67138 & Asuka 12024 & H5 & Antarctica & 80.1 & 0.0801 \\
\hline 67813 & San Juan 089 & H5 & Chile & 78 & 0.078 \\
\hline 67137 & Asuka 12021 & H5 & Antarctica & 77.03 & 0.07703 \\
\hline 67752 & Northwest Africa 11926 & H5 & (Northwest A 1 & 76 & 0.076 \\
\hline 66819 & El-Shaikh Fadl 010 & H5 & Egypt & 75.7 & 0.0757 \\
\hline
\end{tabular}




\begin{tabular}{|c|c|c|c|c|c|}
\hline 66843 & Dueodde & H5 & Denmark & 75 & 0.075 \\
\hline 68175 & Northwest Africa 12064 & H5 & Algeria & 71.18 & 0.07118 \\
\hline 68271 & Northwest Africa 12160 & H5 & Algeria & 70.68 & 0.07068 \\
\hline 67158 & Asuka 12091 & H5 & Antarctica & 68.8 & 0.0688 \\
\hline 68396 & San Juan 101 & H5 & Chile & 68.8 & 0.0688 \\
\hline 67572 & Kerman 204 & H5 & Iran & 68.2 & 0.0682 \\
\hline 68096 & Elephant Moraine 16016 & H5 & Antarctica & 67.903 & 0.067903 \\
\hline 68174 & Northwest Africa 12063 & H5 & Algeria & 67.77 & 0.06777 \\
\hline 67533 & Catalina 269 & H5 & Chile & 67 & 0.067 \\
\hline 67686 & San Juan 088 & H5 & Chile & 66.6 & 0.0666 \\
\hline 66817 & El-Shaikh Fadl 008 & H5 & Egypt & 66.5 & 0.0665 \\
\hline 68305 & Sierra Gorda 008 & H5 & Chile & 66 & 0.066 \\
\hline 67962 & Dominion Range 14408 & H5 & Antarctica & 63.981 & 0.063981 \\
\hline 67755 & Northwest Africa 11931 & H5 & (Northwest Al & 63 & 0.063 \\
\hline 68178 & Northwest Africa 12067 & H5 & Algeria & 62.14 & 0.06214 \\
\hline 67217 & Asuka 12266 & H5 & Antarctica & 61.04 & 0.06104 \\
\hline 68246 & Northwest Africa 12135 & H5 & Algeria & 61.04 & 0.06104 \\
\hline 68373 & Los Vientos 249 & H5 & Chile & 61 & 0.061 \\
\hline 67160 & Asuka 12100 & H5 & Antarctica & 60.88 & 0.06088 \\
\hline 67392 & Catalina 258 & H5 & Chile & 60.4 & 0.0604 \\
\hline 67722 & Northwest Africa 11914 & H5 & (Northwest A1 & 60.4 & 0.0604 \\
\hline 67455 & Elephant Moraine 14039 & H5 & Antarctica & 59.9 & 0.0599 \\
\hline 66757 & Northwest Africa 11659 & H5 & (Northwest Al & 58.2 & 0.0582 \\
\hline 24430 & Yamato 74052 & H5 & Antarctica & 58.18 & 0.05818 \\
\hline 67222 & Asuka 12271 & H5 & Antarctica & 57.919 & 0.057919 \\
\hline 66818 & El-Shaikh Fadl 009 & $\mathrm{H} 5$ & Egypt & 56.3 & 0.0563 \\
\hline 68173 & Northwest Africa 12062 & H5 & Algeria & 56.26 & 0.05626 \\
\hline 67293 & Asuka 12347 & H5 & Antarctica & 55.996 & 0.055996 \\
\hline 67221 & Asuka 12270 & $\mathrm{H} 5$ & Antarctica & 55.421 & 0.055421 \\
\hline 67721 & Northwest Africa 11913 & H5 & (Northwest At & 54.7 & 0.0547 \\
\hline 68342 & Catalina 287 & $\mathrm{H} 5$ & Chile & 54 & 0.054 \\
\hline 67964 & Dominion Range 14410 & H5 & Antarctica & 53.889 & 0.053889 \\
\hline 66503 & Northwest Africa 11687 & H5 & (Northwest Al & 52 & 0.052 \\
\hline 67393 & Catalina 259 & H5 & Chile & 51 & 0.051 \\
\hline 66516 & Dominion Range 14035 & $\mathrm{H} 5$ & Antarctica & 50.55 & 0.05055 \\
\hline 67893 & Dominion Range 14250 & H5 & Antarctica & 49.702 & 0.049702 \\
\hline 67583 & Northwest Africa 11827 & $\mathrm{H} 5$ & (Northwest Al & 49 & 0.049 \\
\hline 68276 & Northwest Africa 12165 & $\mathrm{H} 5$ & Algeria & 48.16 & 0.04816 \\
\hline 68213 & Northwest Africa 12102 & H5 & Algeria & 46.28 & 0.04628 \\
\hline 68287 & Northwest Africa 12176 & $\mathrm{H} 5$ & Algeria & 46.03 & 0.04603 \\
\hline 67427 & Elephant Moraine 14010 & H5 & Antarctica & 44.2 & 0.0442 \\
\hline 67515 & Los Vientos 219 & H5 & Chile & 44 & 0.044 \\
\hline 68166 & Northwest Africa 12055 & H5 & Algeria & 43.82 & 0.04382 \\
\hline 68217 & Northwest Africa 12106 & $\mathrm{H} 5$ & Algeria & 43.74 & 0.04374 \\
\hline 67998 & Dominion Range 14473 & H5 & Antarctica & 43.6 & 0.0436 \\
\hline
\end{tabular}




\begin{tabular}{|c|c|c|c|c|c|}
\hline 67669 & Northwest Africa 11883 & H5 & (Northwest At & 43.3 & 0.0433 \\
\hline 67262 & Asuka 12312 & H5 & Antarctica & 42.963 & 0.042963 \\
\hline 67239 & Asuka 12289 & H5 & Antarctica & 42.112 & 0.042112 \\
\hline 67327 & Asuka 12382 & H5 & Antarctica & 41.812 & 0.041812 \\
\hline 68172 & Northwest Africa 12061 & H5 & Algeria & 41.5 & 0.0415 \\
\hline 66520 & Dominion Range 14039 & H5 & Antarctica & 40.34 & 0.04034 \\
\hline 67214 & Asuka 12263 & H5 & Antarctica & 40.074 & 0.040074 \\
\hline 67457 & Elephant Moraine 14041 & H5 & Antarctica & 39 & 0.039 \\
\hline 67311 & Asuka 12366 & H5 & Antarctica & 38.66 & 0.03866 \\
\hline 66423 & Northwest Africa 11623 & H5 & (Northwest At & 37.59 & 0.03759 \\
\hline 68161 & Northwest Africa 12050 & H5 & Algeria & 36.93 & 0.03693 \\
\hline 67528 & Catalina 265 & H5 & Chile & 36.9 & 0.0369 \\
\hline 67229 & Asuka 12278 & H5 & Antarctica & 36.676 & 0.036676 \\
\hline 68285 & Northwest Africa 12174 & H5 & Algeria & 36.34 & 0.03634 \\
\hline 68067 & Miller Range 15390 & H5 & Antarctica & 36.15 & 0.03615 \\
\hline 68029 & Miller Range 15082 & H5 & Antarctica & 35.858 & 0.035858 \\
\hline 68423 & Red Dry Lake 062 & H5 & United States & 35.85 & 0.03585 \\
\hline 67238 & Asuka 12288 & H5 & Antarctica & 35.503 & 0.035503 \\
\hline 68211 & Northwest Africa 12100 & H5 & Algeria & 33 & 0.033 \\
\hline 67897 & Dominion Range 14254 & H5 & Antarctica & 32.339 & 0.032339 \\
\hline 68004 & Dominion Range 14479 & H5 & Antarctica & 31.43 & 0.03143 \\
\hline 67250 & Asuka 12300 & H5 & Antarctica & 31.131 & 0.031131 \\
\hline 68159 & Northwest Africa 12048 & H5 & Algeria & 30.78 & 0.03078 \\
\hline 68254 & Northwest Africa 12143 & H5 & Algeria & 30.37 & 0.03037 \\
\hline 68200 & Northwest Africa 12089 & H5 & Algeria & 30.1 & 0.0301 \\
\hline 68065 & Miller Range 15379 & H5 & Antarctica & 29.798 & 0.029798 \\
\hline 68219 & Northwest Africa 12108 & H5 & Algeria & 28.58 & 0.02858 \\
\hline 68056 & Miller Range 15370 & H5 & Antarctica & 28.343 & 0.028343 \\
\hline 68102 & Grosvenor Mountains 17001 & $\mathrm{H} 5$ & Antarctica & 28.215 & 0.028215 \\
\hline 68202 & Northwest Africa 12091 & H5 & Algeria & 27.91 & 0.02791 \\
\hline 67425 & Elephant Moraine 14008 & H5 & Antarctica & 27.3 & 0.0273 \\
\hline 67527 & Catalina 264 & H5 & Chile & 27 & 0.027 \\
\hline 68169 & Northwest Africa 12058 & H5 & Algeria & 27 & 0.027 \\
\hline 67280 & Asuka 12334 & H5 & Antarctica & 26.762 & 0.026762 \\
\hline 67895 & Dominion Range 14252 & H5 & Antarctica & 26.192 & 0.026192 \\
\hline 66593 & Dominion Range 14207 & H5 & Antarctica & 26.03 & 0.02603 \\
\hline 67426 & Elephant Moraine 14009 & H5 & Antarctica & 26 & 0.026 \\
\hline 68165 & Northwest Africa 12054 & H5 & Algeria & 25.76 & 0.02576 \\
\hline 68248 & Northwest Africa 12137 & H5 & Algeria & 25.32 & 0.02532 \\
\hline 68374 & Los Vientos 251 & H5 & Chile & 25 & 0.025 \\
\hline 67281 & Asuka 12335 & H5 & Antarctica & 23.504 & 0.023504 \\
\hline 68063 & Miller Range 15377 & H5 & Antarctica & 23.306 & 0.023306 \\
\hline 68012 & Dominion Range 14497 & H5 & Antarctica & 22.5 & 0.0225 \\
\hline 68256 & Northwest Africa 12145 & H5 & Algeria & 22.3 & 0.0223 \\
\hline 68171 & Northwest Africa 12060 & H5 & Algeria & 22.07 & 0.02207 \\
\hline
\end{tabular}




\begin{tabular}{|c|c|c|c|c|c|}
\hline 25607 & Yamato 790258 & H5 & Antarctica & 21.56 & 0.02156 \\
\hline 67341 & Asuka 12398 & H5 & Antarctica & 21.206 & 0.021206 \\
\hline 67975 & Dominion Range 14421 & H5 & Antarctica & 20.346 & 0.020346 \\
\hline 67215 & Asuka 12264 & H5 & Antarctica & 20.238 & 0.020238 \\
\hline 41340 & Yamato 000575 & H5 & Antarctica & 20.016 & 0.020016 \\
\hline 67294 & Asuka 12348 & H5 & Antarctica & 19.819 & 0.019819 \\
\hline 67911 & Dominion Range 14268 & H5 & Antarctica & 19.439 & 0.019439 \\
\hline 67909 & Dominion Range 14266 & H5 & Antarctica & 19.055 & 0.019055 \\
\hline 67987 & Dominion Range 14433 & H5 & Antarctica & 18.928 & 0.018928 \\
\hline 67248 & Asuka 12298 & H5 & Antarctica & 18.887 & 0.018887 \\
\hline 68389 & San Juan 093 & H5 & Chile & 18.1 & 0.0181 \\
\hline 67898 & Dominion Range 14255 & H5 & Antarctica & 17.966 & 0.017966 \\
\hline 67428 & Elephant Moraine 14011 & H5 & Antarctica & 17.2 & 0.0172 \\
\hline 24438 & Yamato 74060 & H5 & Antarctica & 17.1 & 0.0171 \\
\hline 68280 & Northwest Africa 12169 & H5 & Algeria & 16.94 & 0.01694 \\
\hline 41620 & Yamato 000858 & H5 & Antarctica & 16.784 & 0.016784 \\
\hline 68042 & Miller Range 15096 & H5 & Antarctica & 16.58 & 0.01658 \\
\hline 68064 & Miller Range 15378 & H5 & Antarctica & 16.525 & 0.016525 \\
\hline 68075 & Miller Range 15398 & H5 & Antarctica & 16.5 & 0.0165 \\
\hline 67478 & Elephant Moraine 14065 & H5 & Antarctica & 16.3 & 0.0163 \\
\hline 67516 & Los Vientos 220 & H5 & Chile & 16.2 & 0.0162 \\
\hline 67242 & Asuka 12292 & H5 & Antarctica & 16.046 & 0.016046 \\
\hline 67227 & Asuka 12276 & H5 & Antarctica & 15.868 & 0.015868 \\
\hline 66860 & Calate 013 & H5 & Chile & 15.6 & 0.0156 \\
\hline 68348 & Catalina 293 & H5 & Chile & 14 & 0.014 \\
\hline 68059 & Miller Range 15373 & H5 & Antarctica & 13.786 & 0.013786 \\
\hline 67917 & Dominion Range 14294 & H5 & Antarctica & 13.738 & 0.013738 \\
\hline 41561 & Yamato 000798 & H5 & Antarctica & 12.943 & 0.012943 \\
\hline 67279 & Asuka 12333 & H5 & Antarctica & 12.699 & 0.012699 \\
\hline 67310 & Asuka 12365 & H5 & Antarctica & 12.362 & 0.012362 \\
\hline 25541 & Yamato 790192 & H5 & Antarctica & 12.17 & 0.01217 \\
\hline 66660 & Dominion Range 14452 & H5 & Antarctica & 11.99 & 0.01199 \\
\hline 67906 & Dominion Range 14263 & H5 & Antarctica & 11.922 & 0.011922 \\
\hline 68037 & Miller Range 15091 & H5 & Antarctica & 11.888 & 0.011888 \\
\hline 66575 & Dominion Range 14187 & H5 & Antarctica & 11.78 & 0.01178 \\
\hline 67904 & Dominion Range 14261 & H5 & Antarctica & 11.599 & 0.011599 \\
\hline 68215 & Northwest Africa 12104 & H5 & Algeria & 11.58 & 0.01158 \\
\hline 67330 & Asuka 12385 & H5 & Antarctica & 11.533 & 0.011533 \\
\hline 68152 & Northwest Africa 12041 & H5 & Algeria & 11.35 & 0.01135 \\
\hline 68206 & Northwest Africa 12095 & H5 & Algeria & 11.03 & 0.01103 \\
\hline 68255 & Northwest Africa 12144 & H5 & Algeria & 10.93 & 0.01093 \\
\hline 67470 & Elephant Moraine 14057 & H5 & Antarctica & 10.9 & 0.0109 \\
\hline 68221 & Northwest Africa 12110 & H5 & Algeria & 10.88 & 0.01088 \\
\hline 66662 & Dominion Range 14454 & H5 & Antarctica & 10.76 & 0.01076 \\
\hline 41748 & Yamato 000986 & H5 & Antarctica & 10.62 & 0.01062 \\
\hline
\end{tabular}




\begin{tabular}{|c|c|c|c|c|c|}
\hline 67479 & Elephant Moraine 14067 & H5 & Antarctica & 10.5 & 0.0105 \\
\hline 42770 & Yamato 002008 & H5 & Antarctica & 10.415 & 0.010415 \\
\hline 66495 & Calama 007 & H5 & Chile & 10 & 0.01 \\
\hline 67420 & Elephant Moraine 14002 & $\mathrm{H} 5$ & Antarctica & 10 & 0.01 \\
\hline 68380 & Los Vientos 257 & H5 & Chile & 10 & 0.01 \\
\hline 41008 & Yamato 000243 & H5 & Antarctica & 9.887 & 0.009887 \\
\hline 41044 & Yamato 000279 & $\mathrm{H} 5$ & Antarctica & 9.852 & 0.009852 \\
\hline 68205 & Northwest Africa 12094 & H5 & Algeria & 9.27 & 0.00927 \\
\hline 67223 & Asuka 12272 & H5 & Antarctica & 9.268 & 0.009268 \\
\hline 2422 & Asuka 87065 & $\mathrm{H} 5$ & Antarctica & 9.147 & 0.009147 \\
\hline 41977 & Yamato 001215 & H5 & Antarctica & 9.063 & 0.009063 \\
\hline 2438 & Asuka 87081 & H5 & Antarctica & 9.03 & 0.00903 \\
\hline 68210 & Northwest Africa 12099 & H5 & Algeria & 8.84 & 0.00884 \\
\hline 66614 & Dominion Range 14248 & H5 & Antarctica & 8.56 & 0.00856 \\
\hline 67244 & Asuka 12294 & H5 & Antarctica & 8.519 & 0.008519 \\
\hline 2430 & Asuka 87073 & $\mathrm{H} 5$ & Antarctica & 8.372 & 0.008372 \\
\hline 66529 & Dominion Range 14089 & H5 & Antarctica & 8.3 & 0.0083 \\
\hline 27919 & Yamato 792570 & H5 & Antarctica & 7.63 & 0.00763 \\
\hline 41017 & Yamato 000252 & H5 & Antarctica & 7.63 & 0.00763 \\
\hline 67320 & Asuka 12375 & H5 & Antarctica & 7.34 & 0.00734 \\
\hline 40890 & Yamato 000125 & H5 & Antarctica & 6.878 & 0.006878 \\
\hline 67525 & Los Vientos 224 & H5 & Chile & 6.4 & 0.0064 \\
\hline 68536 & Calama 021 & H5 & Chile & 6.3 & 0.0063 \\
\hline 42451 & Yamato 001689 & H5 & Antarctica & 6.218 & 0.006218 \\
\hline 27921 & Yamato 792572 & H5 & Antarctica & 6.1 & 0.0061 \\
\hline 27915 & Yamato 792566 & H5 & Antarctica & 6.08 & 0.00608 \\
\hline 43558 & Yamato 002796 & H5 & Antarctica & 5.971 & 0.005971 \\
\hline 27920 & Yamato 792571 & $\mathrm{H} 5$ & Antarctica & 5.14 & 0.00514 \\
\hline 66982 & Asuka 09347 & H5 & Antarctica & 4.988 & 0.004988 \\
\hline 42550 & Yamato 001788 & H5 & Antarctica & 4.769 & 0.004769 \\
\hline 43837 & Yamato 003075 & H5 & Antarctica & 4.76 & 0.00476 \\
\hline 41573 & Yamato 000811 & H5 & Antarctica & 4.758 & 0.004758 \\
\hline 66979 & Asuka 09344 & H5 & Antarctica & 4.748 & 0.004748 \\
\hline 42165 & Yamato 001403 & H5 & Antarctica & 4.554 & 0.004554 \\
\hline 66908 & Asuka 09253 & H5 & Antarctica & 4.507 & 0.004507 \\
\hline 66962 & Asuka 09316 & H5 & Antarctica & 4.475 & 0.004475 \\
\hline 43782 & Yamato 003020 & H5 & Antarctica & 4.403 & 0.004403 \\
\hline 66868 & Asuka 09201 & H5 & Antarctica & 4.394 & 0.004394 \\
\hline 66923 & Asuka 09277 & H5 & Antarctica & 4.376 & 0.004376 \\
\hline 41422 & Yamato 000658 & H5 & Antarctica & 4.289 & 0.004289 \\
\hline 41507 & Yamato 000743 & H5 & Antarctica & 4.277 & 0.004277 \\
\hline 67102 & Asuka 09658 & H5 & Antarctica & 4.244 & 0.004244 \\
\hline 66871 & Asuka 09204 & H5 & Antarctica & 4.22 & 0.00422 \\
\hline 43987 & Yamato 003225 & $\mathrm{H} 5$ & Antarctica & 4.189 & 0.004189 \\
\hline 41400 & Yamato 000636 & $\mathrm{H} 5$ & Antarctica & 4.115 & 0.004115 \\
\hline
\end{tabular}




\begin{tabular}{|c|c|c|c|c|c|}
\hline 66869 & Asuka 09202 & $\mathrm{H} 5$ & Antarctica & 4.106 & 0.004106 \\
\hline 41183 & Yamato 000418 & H5 & Antarctica & 4.041 & 0.004041 \\
\hline 67099 & Asuka 09655 & H5 & Antarctica & 3.942 & 0.003942 \\
\hline 66980 & Asuka 09345 & H5 & Antarctica & 3.931 & 0.003931 \\
\hline 41131 & Yamato 000366 & $\mathrm{H} 5$ & Antarctica & 3.931 & 0.003931 \\
\hline 66985 & Asuka 09354 & H5 & Antarctica & 3.922 & 0.003922 \\
\hline 41531 & Yamato 000768 & $\mathrm{H} 5$ & Antarctica & 3.852 & 0.003852 \\
\hline 43989 & Yamato 003227 & H5 & Antarctica & 3.833 & 0.003833 \\
\hline 43850 & Yamato 003088 & $\mathrm{H} 5$ & Antarctica & 3.784 & 0.003784 \\
\hline 44008 & Yamato 003246 & H5 & Antarctica & 3.756 & 0.003756 \\
\hline 66667 & Dominion Range 14459 & H5 & Antarctica & 3.74 & 0.00374 \\
\hline 67006 & Asuka 09391 & H5 & Antarctica & 3.725 & 0.003725 \\
\hline 42733 & Yamato 001971 & $\mathrm{H} 5$ & Antarctica & 3.431 & 0.003431 \\
\hline 66995 & Asuka 09366 & H5 & Antarctica & 3.411 & 0.003411 \\
\hline 43874 & Yamato 003112 & $\mathrm{H} 5$ & Antarctica & 3.355 & 0.003355 \\
\hline 66988 & Asuka 09357 & H5 & Antarctica & 3.315 & 0.003315 \\
\hline 66999 & Asuka 09378 & H5 & Antarctica & 3.308 & 0.003308 \\
\hline 67000 & Asuka 09381 & $\mathrm{H} 5$ & Antarctica & 3.235 & 0.003235 \\
\hline 43498 & Yamato 002736 & H5 & Antarctica & 3.149 & 0.003149 \\
\hline 67076 & Asuka 09622 & H5 & Antarctica & 3.148 & 0.003148 \\
\hline 67440 & Elephant Moraine 14024 & $\mathrm{H} 5$ & Antarctica & 3.1 & 0.0031 \\
\hline 36656 & Yamato 980030 & $\mathrm{H} 5$ & Antarctica & 2.898 & 0.002898 \\
\hline 36839 & Yamato 980213 & H5 & Antarctica & 2.826 & 0.002826 \\
\hline 66915 & Asuka 09262 & $\mathrm{H} 5$ & Antarctica & 2.754 & 0.002754 \\
\hline 42062 & Yamato 001300 & H5 & Antarctica & 2.738 & 0.002738 \\
\hline 66983 & Asuka 09348 & $\mathrm{H} 5$ & Antarctica & 2.712 & 0.002712 \\
\hline 43793 & Yamato 003031 & $\mathrm{H} 5$ & Antarctica & 2.667 & 0.002667 \\
\hline 66997 & Asuka 09369 & H5 & Antarctica & 2.619 & 0.002619 \\
\hline 67008 & Asuka 09393 & $\mathrm{H} 5$ & Antarctica & 2.617 & 0.002617 \\
\hline 67017 & Asuka 09404 & H5 & Antarctica & 2.617 & 0.002617 \\
\hline 66938 & Asuka 09292 & H5 & Antarctica & 2.502 & 0.002502 \\
\hline 66909 & Asuka 09254 & $\mathrm{H} 5$ & Antarctica & 2.494 & 0.002494 \\
\hline 66910 & Asuka 09256 & H5 & Antarctica & 2.432 & 0.002432 \\
\hline 66912 & Asuka 09258 & H5 & Antarctica & 2.335 & 0.002335 \\
\hline 66969 & Asuka 09326 & $\mathrm{H} 5$ & Antarctica & 2.333 & 0.002333 \\
\hline 67225 & Asuka 12274 & H5 & Antarctica & 2.267 & 0.002267 \\
\hline 36932 & Yamato 980306 & H5 & Antarctica & 2.253 & 0.002253 \\
\hline 66878 & Asuka 09211 & $\mathrm{H} 5$ & Antarctica & 2.223 & 0.002223 \\
\hline 36695 & Yamato 980069 & H5 & Antarctica & 2.213 & 0.002213 \\
\hline 66978 & Asuka 09341 & H5 & Antarctica & 2.13 & 0.00213 \\
\hline 66981 & Asuka 09346 & H5 & Antarctica & 1.978 & 0.001978 \\
\hline 67116 & Asuka 09672 & H5 & Antarctica & 1.912 & 0.001912 \\
\hline 66885 & Asuka 09218 & H5 & Antarctica & 1.824 & 0.001824 \\
\hline 67095 & Asuka 09645 & $\mathrm{H} 5$ & Antarctica & 1.793 & 0.001793 \\
\hline 67053 & Asuka 09487 & H5 & Antarctica & 1.755 & 0.001755 \\
\hline
\end{tabular}




\begin{tabular}{|c|c|c|c|c|c|}
\hline 66948 & Asuka 09302 & $\mathrm{H} 5$ & Antarctica & 1.734 & 0.001734 \\
\hline 67018 & Asuka 09405 & $\mathrm{H} 5$ & Antarctica & 1.722 & 0.001722 \\
\hline 67009 & Asuka 09394 & H5 & Antarctica & 1.643 & 0.001643 \\
\hline 67011 & Asuka 09396 & $\mathrm{H} 5$ & Antarctica & 1.623 & 0.001623 \\
\hline 66966 & Asuka 09322 & $\mathrm{H} 5$ & Antarctica & 1.606 & 0.001606 \\
\hline 66949 & Asuka 09303 & H5 & Antarctica & 1.6 & 0.0016 \\
\hline 42890 & Yamato 002128 & $\mathrm{H} 5$ & Antarctica & 1.575 & 0.001575 \\
\hline 67014 & Asuka 09399 & H5 & Antarctica & 1.532 & 0.001532 \\
\hline 66887 & Asuka 09220 & $\mathrm{H} 5$ & Antarctica & 1.518 & 0.001518 \\
\hline 66902 & Asuka 09246 & $\mathrm{H} 5$ & Antarctica & 1.443 & 0.001443 \\
\hline 36906 & Yamato 980280 & H5 & Antarctica & 1.424 & 0.001424 \\
\hline 41416 & Yamato 000652 & $\mathrm{H} 5$ & Antarctica & 1.424 & 0.001424 \\
\hline 67015 & Asuka 09400 & $\mathrm{H} 5$ & Antarctica & 1.423 & 0.001423 \\
\hline 66967 & Asuka 09324 & H5 & Antarctica & 1.42 & 0.00142 \\
\hline 68092 & Miller Range 15536 & $\mathrm{H} 5$ & Antarctica & 1.41 & 0.00141 \\
\hline 66947 & Asuka 09301 & $\mathrm{H} 5$ & Antarctica & 1.373 & 0.001373 \\
\hline 66722 & Miller Range 15277 & H5 & Antarctica & 1.37 & 0.00137 \\
\hline 68087 & Miller Range 15531 & $\mathrm{H} 5$ & Antarctica & 1.37 & 0.00137 \\
\hline 67012 & Asuka 09397 & $\mathrm{H} 5$ & Antarctica & 1.367 & 0.001367 \\
\hline 67033 & Asuka 09438 & H5 & Antarctica & 1.355 & 0.001355 \\
\hline 67096 & Asuka 09646 & $\mathrm{H} 5$ & Antarctica & 1.335 & 0.001335 \\
\hline 68089 & Miller Range 15533 & $\mathrm{H} 5$ & Antarctica & 1.31 & 0.00131 \\
\hline 66883 & Asuka 09216 & H5 & Antarctica & 1.278 & 0.001278 \\
\hline 66911 & Asuka 09257 & $\mathrm{H} 5$ & Antarctica & 1.278 & 0.001278 \\
\hline 36669 & Yamato 980043 & H5 & Antarctica & 1.267 & 0.001267 \\
\hline 66951 & Asuka 09305 & $\mathrm{H} 5$ & Antarctica & 1.194 & 0.001194 \\
\hline 36660 & Yamato 980034 & H5 & Antarctica & 1.169 & 0.001169 \\
\hline 67091 & Asuka 09641 & H5 & Antarctica & 1.157 & 0.001157 \\
\hline 67264 & Asuka 12314 & $\mathrm{H} 5$ & Antarctica & 1.137 & 0.001137 \\
\hline 42889 & Yamato 002127 & H5 & Antarctica & 1.108 & 0.001108 \\
\hline 67417 & Reckling Peak 14004 & H5 & Antarctica & 1.1 & 0.0011 \\
\hline 67090 & Asuka 09640 & H5 & Antarctica & 1.087 & 0.001087 \\
\hline 67094 & Asuka 09644 & H5 & Antarctica & 1.086 & 0.001086 \\
\hline 66886 & Asuka 09219 & H5 & Antarctica & 1.056 & 0.001056 \\
\hline 67093 & Asuka 09643 & H5 & Antarctica & 1.04 & 0.00104 \\
\hline 66888 & Asuka 09221 & H5 & Antarctica & 1.03 & 0.00103 \\
\hline 66889 & Asuka 09222 & H5 & Antarctica & 1.02 & 0.00102 \\
\hline 66954 & Asuka 09308 & $\mathrm{H} 5$ & Antarctica & 1.008 & 0.001008 \\
\hline 36706 & Yamato 980080 & H5 & Antarctica & 0.994 & 0.000994 \\
\hline 36784 & Yamato 980158 & H5 & Antarctica & 0.969 & 0.000969 \\
\hline 67126 & Asuka 10152 & H5 & Antarctica & 0.957 & 0.000957 \\
\hline 67036 & Asuka 09449 & H5 & Antarctica & 0.826 & 0.000826 \\
\hline 67023 & Asuka 09415 & H5 & Antarctica & 0.82 & 0.00082 \\
\hline 66953 & Asuka 09307 & $\mathrm{H} 5$ & Antarctica & 0.801 & 0.000801 \\
\hline 68093 & Miller Range 15538 & H5 & Antarctica & 0.79 & 0.00079 \\
\hline
\end{tabular}




\begin{tabular}{|c|c|c|c|c|c|}
\hline 66952 & Asuka 09306 & H5 & Antarctica & 0.751 & 0.000751 \\
\hline 42886 & Yamato 002124 & H5 & Antarctica & 0.694 & 0.000694 \\
\hline 66737 & Elephant Moraine 16211 & H5 & Antarctica & 0.651 & 0.000651 \\
\hline 42892 & Yamato 002130 & H5 & Antarctica & 0.644 & 0.000644 \\
\hline 66891 & Asuka 09224 & H5 & Antarctica & 0.581 & 0.000581 \\
\hline 42623 & Yamato 001861 & H5 & Antarctica & 0.578 & 0.000578 \\
\hline 67092 & Asuka 09642 & H5 & Antarctica & 0.52 & 0.00052 \\
\hline 67125 & Asuka 10148 & H5 & Antarctica & 0.507 & 0.000507 \\
\hline 66958 & Asuka 09312 & H5 & Antarctica & 0.393 & 0.000393 \\
\hline 42586 & Yamato 001824 & H5 & Antarctica & 0.385 & 0.000385 \\
\hline 66890 & Asuka 09223 & H5 & Antarctica & 0.37 & 0.00037 \\
\hline 43164 & Yamato 002402 & H5 & Antarctica & 0.35 & 0.00035 \\
\hline 42740 & Yamato 001978 & H5 & Antarctica & 0.349 & 0.000349 \\
\hline 67466 & Elephant Moraine 14051 & H5 & Antarctica & 0.3 & 0.0003 \\
\hline 43621 & Yamato 002859 & H5 & Antarctica & 0.298 & 0.000298 \\
\hline 67489 & Beryozovka & H5-6 & Kazakhstan & 35650 & 35.65 \\
\hline 67839 & Metameur 012 & H5-6 & Tunisia & 74.2 & 0.0742 \\
\hline 67738 & Paposo 040 & \multicolumn{2}{|c|}{ H5-melt brecı Chile } & 23300 & 23.3 \\
\hline 67817 & Northwest Africa 11920 & \multicolumn{2}{|c|}{ H5-melt brecı(Northwest A1 } & 4 & 0.004 \\
\hline 68345 & Catalina 290 & $\mathrm{H} 5 / 6$ & Chile & 544 & 0.544 \\
\hline 67685 & Los Vientos 229 & $\mathrm{H} 5 / 6$ & Chile & 69.3 & 0.0693 \\
\hline 68634 & Gumu & $\mathrm{H} 6$ & China & 39000 & 39 \\
\hline 32061 & Northwest Africa 1088 & $\mathrm{H} 6$ & (Northwest At & 21000 & 21 \\
\hline 67718 & Northwest Africa 11903 & $\mathrm{H} 6$ & (Northwest Al & 8000 & 8 \\
\hline 66750 & Northwest Africa 11652 & $\mathrm{H} 6$ & (Northwest Al & 7674 & 7.674 \\
\hline 67838 & Northwest Africa 11995 & $\mathrm{H} 6$ & (Northwest A 1 & 7210 & 7.21 \\
\hline 45606 & Northwest Africa 4667 & $\mathrm{H} 6$ & (Northwest Al & 5000 & 5 \\
\hline 68335 & Catalina 280 & $\mathrm{H} 6$ & Chile & 4370 & 4.37 \\
\hline 67662 & San Juan 087 & $\mathrm{H} 6$ & Chile & 4210 & 4.21 \\
\hline 68316 & Coyote Dry Lake 349 & $\mathrm{H} 6$ & United States & 4000 & 4 \\
\hline 66437 & Catalina 254 & $\mathrm{H} 6$ & Chile & 2243 & 2.243 \\
\hline 43816 & Yamato 003054 & $\mathrm{H} 6$ & Antarctica & 1712.9 & 1.7129 \\
\hline 66504 & Northwest Africa 11688 & $\mathrm{H} 6$ & (Northwest Al & 1375 & 1.375 \\
\hline 67505 & Calama 010 & $\mathrm{H} 6$ & Chile & 1248.18 & 1.24818 \\
\hline 66751 & Northwest Africa 11653 & $\mathrm{H} 6$ & (Northwest A 1 & 1130 & 1.13 \\
\hline 68369 & Los Vientos 245 & $\mathrm{H} 6$ & Chile & 1118 & 1.118 \\
\hline 67175 & Asuka 12216 & $\mathrm{H} 6$ & Antarctica & 1043.3 & 1.0433 \\
\hline 67606 & Northwest Africa 11855 & $\mathrm{H} 6$ & (Northwest Al & 980 & 0.98 \\
\hline 68361 & Los Vientos 237 & $\mathrm{H} 6$ & Chile & 920 & 0.92 \\
\hline 68548 & Northwest Africa 12330 & $\mathrm{H} 6$ & (Northwest Al & 915 & 0.915 \\
\hline 68343 & Catalina 288 & $\mathrm{H} 6$ & Chile & 880 & 0.88 \\
\hline 43106 & Yamato 002344 & $\mathrm{H} 6$ & Antarctica & 676.8 & 0.6768 \\
\hline 68351 & Catalina 296 & $\mathrm{H} 6$ & Chile & 642 & 0.642 \\
\hline 67488 & Northwest Africa 11798 & $\mathrm{H} 6$ & (Northwest A 1 & 510 & 0.51 \\
\hline 67351 & Asuka 12409 & $\mathrm{H} 6$ & Antarctica & 496.69 & 0.49669 \\
\hline
\end{tabular}




\begin{tabular}{|c|c|c|c|c|c|}
\hline 31624 & Northwest Africa 431 & $\mathrm{H} 6$ & (Northwest A1 & 492 & 0.492 \\
\hline 68367 & Los Vientos 243 & $\mathrm{H} 6$ & Chile & 489 & 0.489 \\
\hline 67861 & Northwest Africa 12010 & $\mathrm{H} 6$ & (Northwest A 1 & 483 & 0.483 \\
\hline 67593 & Diego de Almagro 001 & $\mathrm{H} 6$ & Chile & 430 & 0.43 \\
\hline 68364 & Los Vientos 240 & $\mathrm{H} 6$ & Chile & 310 & 0.31 \\
\hline 67170 & Asuka 12201 & $\mathrm{H} 6$ & Antarctica & 286.05 & 0.28605 \\
\hline 68645 & Calama 023 & $\mathrm{H} 6$ & Chile & 284.04 & 0.28404 \\
\hline 68273 & Northwest Africa 12162 & $\mathrm{H} 6$ & Algeria & 281.64 & 0.28164 \\
\hline 68216 & Northwest Africa 12105 & $\mathrm{H} 6$ & Algeria & 270.13 & 0.27013 \\
\hline 68353 & Catalina 298 & $\mathrm{H} 6$ & Chile & 260 & 0.26 \\
\hline 68498 & Northwest Africa 12336 & $\mathrm{H} 6$ & (Northwest A 1 & 258 & 0.258 \\
\hline 68646 & Calama 024 & $\mathrm{H} 6$ & Chile & 252.95 & 0.25295 \\
\hline 67297 & Asuka 12351 & $\mathrm{H} 6$ & Antarctica & 242.87 & 0.24287 \\
\hline 66627 & Dominion Range 14301 & $\mathrm{H} 6$ & Antarctica & 211.53 & 0.21153 \\
\hline 67866 & Northwest Africa 12018 & $\mathrm{H} 6$ & (Northwest A 1 & 192.43 & 0.19243 \\
\hline 67536 & Los Vientos 226 & $\mathrm{H} 6$ & Chile & 182 & 0.182 \\
\hline 68229 & Northwest Africa 12118 & $\mathrm{H} 6$ & Algeria & 178.49 & 0.17849 \\
\hline 68282 & Northwest Africa 12171 & $\mathrm{H} 6$ & Algeria & 175.59 & 0.17559 \\
\hline 68160 & Northwest Africa 12049 & $\mathrm{H} 6$ & Algeria & 172.94 & 0.17294 \\
\hline 68199 & Northwest Africa 12088 & $\mathrm{H} 6$ & Algeria & 171.97 & 0.17197 \\
\hline 68241 & Northwest Africa 12130 & $\mathrm{H} 6$ & Algeria & 169.77 & 0.16977 \\
\hline 68195 & Northwest Africa 12084 & $\mathrm{H} 6$ & Algeria & 164.95 & 0.16495 \\
\hline 67889 & Dominion Range 14232 & $\mathrm{H} 6$ & Antarctica & 161.46 & 0.16146 \\
\hline 67308 & Asuka 12363 & $\mathrm{H} 6$ & Antarctica & 159.19 & 0.15919 \\
\hline 68230 & Northwest Africa 12119 & $\mathrm{H} 6$ & Algeria & 147.97 & 0.14797 \\
\hline 68362 & Los Vientos 238 & $\mathrm{H} 6$ & Chile & 140 & 0.14 \\
\hline 67972 & Dominion Range 14418 & $\mathrm{H} 6$ & Antarctica & 134.868 & 0.134868 \\
\hline 68250 & Northwest Africa 12139 & $\mathrm{H} 6$ & Algeria & 134.72 & 0.13472 \\
\hline 68183 & Northwest Africa 12072 & $\mathrm{H} 6$ & Algeria & 128.72 & 0.12872 \\
\hline 67371 & Asuka 12429 & $\mathrm{H} 6$ & Antarctica & 128.2 & 0.1282 \\
\hline 68201 & Northwest Africa 12090 & $\mathrm{H} 6$ & Algeria & 118.4 & 0.1184 \\
\hline 67970 & Dominion Range 14416 & $\mathrm{H} 6$ & Antarctica & 104.266 & 0.104266 \\
\hline 67579 & Northwest Africa 11823 & $\mathrm{H} 6$ & (Northwest A 1 & 103.4 & 0.1034 \\
\hline 68186 & Northwest Africa 12075 & $\mathrm{H} 6$ & Algeria & 101.97 & 0.10197 \\
\hline 66563 & Dominion Range 14175 & $\mathrm{H} 6$ & Antarctica & 96.42 & 0.09642 \\
\hline 68188 & Northwest Africa 12077 & $\mathrm{H} 6$ & Algeria & 89.17 & 0.08917 \\
\hline 68290 & Northwest Africa 12179 & $\mathrm{H} 6$ & Algeria & 88.71 & 0.08871 \\
\hline 67168 & Asuka 12154 & $\mathrm{H} 6$ & Antarctica & 87.71 & 0.08771 \\
\hline 68158 & Northwest Africa 12047 & $\mathrm{H} 6$ & Algeria & 85.94 & 0.08594 \\
\hline 66567 & Dominion Range 14179 & $\mathrm{H} 6$ & Antarctica & 83.13 & 0.08313 \\
\hline 67295 & Asuka 12349 & $\mathrm{H} 6$ & Antarctica & 79.45 & 0.07945 \\
\hline 68242 & Northwest Africa 12131 & $\mathrm{H} 6$ & Algeria & 77.52 & 0.07752 \\
\hline 66600 & Dominion Range 14224 & $\mathrm{H} 6$ & Antarctica & 77.064 & 0.077064 \\
\hline 67230 & Asuka 12279 & $\mathrm{H} 6$ & Antarctica & 72.98 & 0.07298 \\
\hline 68240 & Northwest Africa 12129 & $\mathrm{H} 6$ & Algeria & 68.15 & 0.06815 \\
\hline
\end{tabular}




\begin{tabular}{|c|c|c|c|c|c|}
\hline 67220 & Asuka 12269 & $\mathrm{H} 6$ & Antarctica & 66.81 & 0.06681 \\
\hline 67204 & Asuka 12253 & $\mathrm{H} 6$ & Antarctica & 66.05 & 0.06605 \\
\hline 67959 & Dominion Range 14405 & $\mathrm{H} 6$ & Antarctica & 66.002 & 0.066002 \\
\hline 67881 & Dominion Range 14213 & $\mathrm{H} 6$ & Antarctica & 63.269 & 0.063269 \\
\hline 68156 & Northwest Africa 12045 & $\mathrm{H} 6$ & Algeria & 62.69 & 0.06269 \\
\hline 68154 & Northwest Africa 12043 & $\mathrm{H} 6$ & Algeria & 61.61 & 0.06161 \\
\hline 66546 & Dominion Range 14126 & $\mathrm{H} 6$ & Antarctica & 59.06 & 0.05906 \\
\hline 68232 & Northwest Africa 12121 & $\mathrm{H} 6$ & Algeria & 58.55 & 0.05855 \\
\hline 66747 & Istifane 005 & $\mathrm{H} 6$ & Morocco & 58 & 0.058 \\
\hline 67207 & Asuka 12256 & $\mathrm{H} 6$ & Antarctica & 57.54 & 0.05754 \\
\hline 68179 & Northwest Africa 12068 & $\mathrm{H} 6$ & Algeria & 56.63 & 0.05663 \\
\hline 66568 & Dominion Range 14180 & $\mathrm{H} 6$ & Antarctica & 55.91 & 0.05591 \\
\hline 68251 & Northwest Africa 12140 & $\mathrm{H} 6$ & Algeria & 55.7 & 0.0557 \\
\hline 67253 & Asuka 12303 & $\mathrm{H} 6$ & Antarctica & 55.653 & 0.055653 \\
\hline 67296 & Asuka 12350 & $\mathrm{H} 6$ & Antarctica & 54.004 & 0.054004 \\
\hline 67240 & Asuka 12290 & $\mathrm{H} 6$ & Antarctica & 52.105 & 0.052105 \\
\hline 68249 & Northwest Africa 12138 & $\mathrm{H} 6$ & Algeria & 51.66 & 0.05166 \\
\hline 67150 & Asuka 12044 & $\mathrm{H} 6$ & Antarctica & 51.424 & 0.051424 \\
\hline 66706 & Dominion Range 14539 & $\mathrm{H} 6$ & Antarctica & 49.54 & 0.04954 \\
\hline 66507 & Northwest Africa 11691 & $\mathrm{H} 6$ & Mali & 49 & 0.049 \\
\hline 68055 & Miller Range 15357 & $\mathrm{H} 6$ & Antarctica & 48.785 & 0.048785 \\
\hline 66569 & Dominion Range 14181 & $\mathrm{H} 6$ & Antarctica & 44.96 & 0.04496 \\
\hline 66597 & Dominion Range 14221 & $\mathrm{H} 6$ & Antarctica & 44.183 & 0.044183 \\
\hline 68198 & Northwest Africa 12087 & $\mathrm{H} 6$ & Algeria & 44.15 & 0.04415 \\
\hline 67862 & Northwest Africa 12014 & $\mathrm{H} 6$ & (Northwest A1 & 43 & 0.043 \\
\hline 66533 & Dominion Range 14103 & $\mathrm{H} 6$ & Antarctica & 42.312 & 0.042312 \\
\hline 67759 & Northwest Africa 11938 & $\mathrm{H} 6$ & (Northwest Al & 41.9 & 0.0419 \\
\hline 66540 & Dominion Range 14120 & $\mathrm{H} 6$ & Antarctica & 41.44 & 0.04144 \\
\hline 67992 & Dominion Range 14438 & $\mathrm{H} 6$ & Antarctica & 41.315 & 0.041315 \\
\hline 67520 & Catalina 261 & $\mathrm{H} 6$ & Chile & 41 & 0.041 \\
\hline 68214 & Northwest Africa 12103 & $\mathrm{H} 6$ & Algeria & 40.22 & 0.04022 \\
\hline 68197 & Northwest Africa 12086 & $\mathrm{H} 6$ & Algeria & 39.77 & 0.03977 \\
\hline 68164 & Northwest Africa 12053 & $\mathrm{H} 6$ & Algeria & 37.84 & 0.03784 \\
\hline 68053 & Miller Range 15258 & $\mathrm{H} 6$ & Antarctica & 36.636 & 0.036636 \\
\hline 66425 & Northwest Africa 11625 & $\mathrm{H} 6$ & (Northwest A 1 & 36.41 & 0.03641 \\
\hline 67205 & Asuka 12254 & $\mathrm{H} 6$ & Antarctica & 35.441 & 0.035441 \\
\hline 68076 & Miller Range 15399 & $\mathrm{H} 6$ & Antarctica & 34.51 & 0.03451 \\
\hline 67485 & Mount DeWitt 14001 & $\mathrm{H} 6$ & Antarctica & 33.7 & 0.0337 \\
\hline 68071 & Miller Range 15394 & $\mathrm{H} 6$ & Antarctica & 32.9 & 0.0329 \\
\hline 30183 & Yamato 86677 & $\mathrm{H} 6$ & Antarctica & 31.81 & 0.03181 \\
\hline 66580 & Dominion Range 14194 & $\mathrm{H} 6$ & Antarctica & 31.703 & 0.031703 \\
\hline 66654 & Dominion Range 14396 & $\mathrm{H} 6$ & Antarctica & 31.64 & 0.03164 \\
\hline 68035 & Miller Range 15089 & $\mathrm{H} 6$ & Antarctica & 30.965 & 0.030965 \\
\hline 68034 & Miller Range 15088 & $\mathrm{H} 6$ & Antarctica & 30.538 & 0.030538 \\
\hline 66541 & Dominion Range 14121 & $\mathrm{H} 6$ & Antarctica & 30.48 & 0.03048 \\
\hline
\end{tabular}




\begin{tabular}{|c|c|c|c|c|c|}
\hline 67715 & Northwest Africa 11879 & $\mathrm{H} 6$ & (Northwest At & 28.95 & 0.02895 \\
\hline 67031 & Asuka 09430 & $\mathrm{H} 6$ & Antarctica & 28.836 & 0.028836 \\
\hline 67252 & Asuka 12302 & $\mathrm{H} 6$ & Antarctica & 28.816 & 0.028816 \\
\hline 66514 & Dominion Range 14033 & $\mathrm{H} 6$ & Antarctica & 28.24 & 0.02824 \\
\hline 66855 & Yucca 051 & $\mathrm{H} 6$ & United States & 27.8 & 0.0278 \\
\hline 68068 & Miller Range 15391 & $\mathrm{H} 6$ & Antarctica & 27.79 & 0.02779 \\
\hline 67257 & Asuka 12307 & $\mathrm{H} 6$ & Antarctica & 27.789 & 0.027789 \\
\hline 67210 & Asuka 12259 & $\mathrm{H} 6$ & Antarctica & 27.728 & 0.027728 \\
\hline 67322 & Asuka 12377 & $\mathrm{H} 6$ & Antarctica & 27.619 & 0.027619 \\
\hline 68030 & Miller Range 15083 & $\mathrm{H} 6$ & Antarctica & 27.598 & 0.027598 \\
\hline 67234 & Asuka 12283 & $\mathrm{H} 6$ & Antarctica & 26.259 & 0.026259 \\
\hline 27209 & Yamato 791860 & $\mathrm{H} 6$ & Antarctica & 25.16 & 0.02516 \\
\hline 67290 & Asuka 12344 & $\mathrm{H} 6$ & Antarctica & 24.638 & 0.024638 \\
\hline 66519 & Dominion Range 14038 & $\mathrm{H} 6$ & Antarctica & 24.49 & 0.02449 \\
\hline 67976 & Dominion Range 14422 & $\mathrm{H} 6$ & Antarctica & 24.271 & 0.024271 \\
\hline 68176 & Northwest Africa 12065 & $\mathrm{H} 6$ & Algeria & 23.78 & 0.02378 \\
\hline 67901 & Dominion Range 14258 & $\mathrm{H} 6$ & Antarctica & 23.193 & 0.023193 \\
\hline 67287 & Asuka 12341 & $\mathrm{H} 6$ & Antarctica & 22.842 & 0.022842 \\
\hline 66653 & Dominion Range 14395 & $\mathrm{H} 6$ & Antarctica & 22.53 & 0.02253 \\
\hline 68069 & Miller Range 15392 & $\mathrm{H} 6$ & Antarctica & 22.48 & 0.02248 \\
\hline 66610 & Dominion Range 14244 & $\mathrm{H} 6$ & Antarctica & 22.43 & 0.02243 \\
\hline 67899 & Dominion Range 14256 & $\mathrm{H} 6$ & Antarctica & 21.926 & 0.021926 \\
\hline 67251 & Asuka 12301 & $\mathrm{H} 6$ & Antarctica & 21.476 & 0.021476 \\
\hline 68072 & Miller Range 15395 & $\mathrm{H} 6$ & Antarctica & 21.45 & 0.02145 \\
\hline 68204 & Northwest Africa 12093 & $\mathrm{H} 6$ & Algeria & 21.21 & 0.02121 \\
\hline 68039 & Miller Range 15093 & $\mathrm{H} 6$ & Antarctica & 21.073 & 0.021073 \\
\hline 67283 & Asuka 12337 & $\mathrm{H} 6$ & Antarctica & 20.892 & 0.020892 \\
\hline 67483 & Elephant Moraine 14072 & $\mathrm{H} 6$ & Antarctica & 20.2 & 0.0202 \\
\hline 66647 & Dominion Range 14379 & $\mathrm{H} 6$ & Antarctica & 19.894 & 0.019894 \\
\hline 67198 & Asuka 12247 & $\mathrm{H} 6$ & Antarctica & 19.25 & 0.01925 \\
\hline 67984 & Dominion Range 14430 & $\mathrm{H} 6$ & Antarctica & 18.95 & 0.01895 \\
\hline 66609 & Dominion Range 14243 & $\mathrm{H} 6$ & Antarctica & 18.58 & 0.01858 \\
\hline 66590 & Dominion Range 14204 & $\mathrm{H} 6$ & Antarctica & 18.46 & 0.01846 \\
\hline 68225 & Northwest Africa 12114 & $\mathrm{H} 6$ & Algeria & 17.97 & 0.01797 \\
\hline 68074 & Miller Range 15397 & $\mathrm{H} 6$ & Antarctica & 17.9 & 0.0179 \\
\hline 68047 & Miller Range 15252 & $\mathrm{H} 6$ & Antarctica & 17.858 & 0.017858 \\
\hline 66716 & Dominion Range 14559 & $\mathrm{H} 6$ & Antarctica & 17.54 & 0.01754 \\
\hline 68043 & Miller Range 15097 & $\mathrm{H} 6$ & Antarctica & 16.096 & 0.016096 \\
\hline 67908 & Dominion Range 14265 & $\mathrm{H} 6$ & Antarctica & 15.701 & 0.015701 \\
\hline 67211 & Asuka 12260 & $\mathrm{H} 6$ & Antarctica & 15.69 & 0.01569 \\
\hline 66603 & Dominion Range 14227 & $\mathrm{H} 6$ & Antarctica & 15.45 & 0.01545 \\
\hline 67228 & Asuka 12277 & $\mathrm{H} 6$ & Antarctica & 14.953 & 0.014953 \\
\hline 66578 & Dominion Range 14191 & $\mathrm{H} 6$ & Antarctica & 14.95 & 0.01495 \\
\hline 68073 & Miller Range 15396 & $\mathrm{H} 6$ & Antarctica & 14.63 & 0.01463 \\
\hline 67231 & Asuka 12280 & $\mathrm{H} 6$ & Antarctica & 14.619 & 0.014619 \\
\hline
\end{tabular}




\begin{tabular}{|c|c|c|c|c|c|}
\hline 68193 & Northwest Africa 12082 & $\mathrm{H} 6$ & Algeria & 14.56 & 0.01456 \\
\hline 68346 & Catalina 291 & $\mathrm{H} 6$ & Chile & 14 & 0.014 \\
\hline 67950 & Dominion Range 14357 & $\mathrm{H} 6$ & Antarctica & 13.987 & 0.013987 \\
\hline 68220 & Northwest Africa 12109 & $\mathrm{H} 6$ & Algeria & 13.96 & 0.01396 \\
\hline 66710 & Dominion Range 14553 & $\mathrm{H} 6$ & Antarctica & 13.47 & 0.01347 \\
\hline 66639 & Dominion Range 14364 & $\mathrm{H} 6$ & Antarctica & 13.367 & 0.013367 \\
\hline 68058 & Miller Range 15372 & $\mathrm{H} 6$ & Antarctica & 12.891 & 0.012891 \\
\hline 68041 & Miller Range 15095 & $\mathrm{H} 6$ & Antarctica & 12.489 & 0.012489 \\
\hline 68048 & Miller Range 15253 & $\mathrm{H} 6$ & Antarctica & 11.99 & 0.01199 \\
\hline 67949 & Dominion Range 14356 & $\mathrm{H} 6$ & Antarctica & 11.982 & 0.011982 \\
\hline 68051 & Miller Range 15256 & $\mathrm{H} 6$ & Antarctica & 11.18 & 0.01118 \\
\hline 68045 & Miller Range 15250 & $\mathrm{H} 6$ & Antarctica & 11.156 & 0.011156 \\
\hline 67268 & Asuka 12319 & $\mathrm{H} 6$ & Antarctica & 10.865 & 0.010865 \\
\hline 68243 & Northwest Africa 12132 & $\mathrm{H} 6$ & Algeria & 10.82 & 0.01082 \\
\hline 43110 & Yamato 002348 & $\mathrm{H} 6$ & Antarctica & 10.288 & 0.010288 \\
\hline 66613 & Dominion Range 14247 & $\mathrm{H} 6$ & Antarctica & 10.02 & 0.01002 \\
\hline 27904 & Yamato 792555 & $\mathrm{H} 6$ & Antarctica & 9.97 & 0.00997 \\
\hline 2420 & Asuka 87063 & $\mathrm{H} 6$ & Antarctica & 9.636 & 0.009636 \\
\hline 67288 & Asuka 12342 & $\mathrm{H} 6$ & Antarctica & 9.597 & 0.009597 \\
\hline 2417 & Asuka 87060 & $\mathrm{H} 6$ & Antarctica & 9.078 & 0.009078 \\
\hline 2658 & Asuka 87301 & $\mathrm{H} 6$ & Antarctica & 9.055 & 0.009055 \\
\hline 66659 & Dominion Range 14451 & $\mathrm{H} 6$ & Antarctica & 9.03 & 0.00903 \\
\hline 2459 & Asuka 87102 & $\mathrm{H} 6$ & Antarctica & 8.995 & 0.008995 \\
\hline 67285 & Asuka 12339 & $\mathrm{H} 6$ & Antarctica & 8.963 & 0.008963 \\
\hline 66605 & Dominion Range 14229 & $\mathrm{H} 6$ & Antarctica & 8.777 & 0.008777 \\
\hline 2381 & Asuka 87024 & $\mathrm{H} 6$ & Antarctica & 8.651 & 0.008651 \\
\hline 68238 & Northwest Africa 12127 & $\mathrm{H} 6$ & Algeria & 7.89 & 0.00789 \\
\hline 66582 & Dominion Range 14196 & $\mathrm{H} 6$ & Antarctica & 7.539 & 0.007539 \\
\hline 67161 & Asuka 12108 & $\mathrm{H} 6$ & Antarctica & 7.233 & 0.007233 \\
\hline 68350 & Catalina 295 & $\mathrm{H} 6$ & Chile & 7 & 0.007 \\
\hline 36686 & Yamato 980060 & $\mathrm{H} 6$ & Antarctica & 6.974 & 0.006974 \\
\hline 43435 & Yamato 002673 & $\mathrm{H} 6$ & Antarctica & 6.818 & 0.006818 \\
\hline 67241 & Asuka 12291 & $\mathrm{H} 6$ & Antarctica & 6.694 & 0.006694 \\
\hline 67258 & Asuka 12308 & $\mathrm{H} 6$ & Antarctica & 6.345 & 0.006345 \\
\hline 43787 & Yamato 003025 & $\mathrm{H} 6$ & Antarctica & 6.215 & 0.006215 \\
\hline 66615 & Dominion Range 14249 & $\mathrm{H} 6$ & Antarctica & 5.61 & 0.00561 \\
\hline 68258 & Northwest Africa 12147 & $\mathrm{H} 6$ & Algeria & 5.36 & 0.00536 \\
\hline 67444 & Elephant Moraine 14028 & $\mathrm{H} 6$ & Antarctica & 5 & 0.005 \\
\hline 40962 & Yamato 000197 & $\mathrm{H} 6$ & Antarctica & 4.957 & 0.004957 \\
\hline 43916 & Yamato 003154 & $\mathrm{H} 6$ & Antarctica & 4.913 & 0.004913 \\
\hline 66986 & Asuka 09355 & $\mathrm{H} 6$ & Antarctica & 4.902 & 0.004902 \\
\hline 43105 & Yamato 002343 & $\mathrm{H} 6$ & Antarctica & 4.726 & 0.004726 \\
\hline 43942 & Yamato 003180 & $\mathrm{H} 6$ & Antarctica & 4.704 & 0.004704 \\
\hline 43890 & Yamato 003128 & $\mathrm{H} 6$ & Antarctica & 4.665 & 0.004665 \\
\hline 66867 & Asuka 09200 & $\mathrm{H} 6$ & Antarctica & 4.629 & 0.004629 \\
\hline
\end{tabular}




\begin{tabular}{|c|c|c|c|c|c|}
\hline 42405 & Yamato 001643 & $\mathrm{H} 6$ & Antarctica & 4.533 & 0.004533 \\
\hline 43845 & Yamato 003083 & $\mathrm{H} 6$ & Antarctica & 4.532 & 0.004532 \\
\hline 66987 & Asuka 09356 & $\mathrm{H} 6$ & Antarctica & 4.524 & 0.004524 \\
\hline 66991 & Asuka 09361 & $\mathrm{H} 6$ & Antarctica & 4.492 & 0.004492 \\
\hline 68044 & Miller Range 15098 & $\mathrm{H} 6$ & Antarctica & 4.436 & 0.004436 \\
\hline 66998 & Asuka 09376 & H6 & Antarctica & 4.272 & 0.004272 \\
\hline 43780 & Yamato 003018 & H6 & Antarctica & 4.25 & 0.00425 \\
\hline 66897 & Asuka 09232 & $\mathrm{H} 6$ & Antarctica & 4.217 & 0.004217 \\
\hline 67462 & Elephant Moraine 14046 & $\mathrm{H} 6$ & Antarctica & 4.2 & 0.0042 \\
\hline 68257 & Northwest Africa 12146 & H6 & Algeria & 4.16 & 0.00416 \\
\hline 66989 & Asuka 09358 & $\mathrm{H} 6$ & Antarctica & 4.153 & 0.004153 \\
\hline 67235 & Asuka 12284 & $\mathrm{H} 6$ & Antarctica & 4.077 & 0.004077 \\
\hline 43915 & Yamato 003153 & H6 & Antarctica & 4.057 & 0.004057 \\
\hline 67038 & Asuka 09451 & $\mathrm{H} 6$ & Antarctica & 4.056 & 0.004056 \\
\hline 43939 & Yamato 003177 & $\mathrm{H} 6$ & Antarctica & 3.942 & 0.003942 \\
\hline 41286 & Yamato 000521 & $\mathrm{H} 6$ & Antarctica & 3.926 & 0.003926 \\
\hline 43798 & Yamato 003036 & $\mathrm{H} 6$ & Antarctica & 3.897 & 0.003897 \\
\hline 41059 & Yamato 000294 & $\mathrm{H} 6$ & Antarctica & 3.895 & 0.003895 \\
\hline 67051 & Asuka 09483 & $\mathrm{H} 6$ & Antarctica & 3.854 & 0.003854 \\
\hline 43896 & Yamato 003134 & $\mathrm{H} 6$ & Antarctica & 3.83 & 0.00383 \\
\hline 40950 & Yamato 000185 & $\mathrm{H} 6$ & Antarctica & 3.787 & 0.003787 \\
\hline 66870 & Asuka 09203 & $\mathrm{H} 6$ & Antarctica & 3.565 & 0.003565 \\
\hline 43007 & Yamato 002245 & $\mathrm{H} 6$ & Antarctica & 3.56 & 0.00356 \\
\hline 43926 & Yamato 003164 & $\mathrm{H} 6$ & Antarctica & 3.504 & 0.003504 \\
\hline 67237 & Asuka 12286 & $\mathrm{H} 6$ & Antarctica & 3.327 & 0.003327 \\
\hline 67286 & Asuka 12340 & $\mathrm{H} 6$ & Antarctica & 3.316 & 0.003316 \\
\hline 36882 & Yamato 980256 & $\mathrm{H} 6$ & Antarctica & 3.288 & 0.003288 \\
\hline 43906 & Yamato 003144 & $\mathrm{H} 6$ & Antarctica & 3.166 & 0.003166 \\
\hline 43947 & Yamato 003185 & $\mathrm{H} 6$ & Antarctica & 3.152 & 0.003152 \\
\hline 66990 & Asuka 09360 & $\mathrm{H} 6$ & Antarctica & 3.084 & 0.003084 \\
\hline 43935 & Yamato 003173 & $\mathrm{H} 6$ & Antarctica & 3.037 & 0.003037 \\
\hline 36850 & Yamato 980224 & $\mathrm{H} 6$ & Antarctica & 2.999 & 0.002999 \\
\hline 36752 & Yamato 980126 & $\mathrm{H} 6$ & Antarctica & 2.982 & 0.002982 \\
\hline 41284 & Yamato 000519 & $\mathrm{H} 6$ & Antarctica & 2.935 & 0.002935 \\
\hline 66872 & Asuka 09205 & $\mathrm{H} 6$ & Antarctica & 2.854 & 0.002854 \\
\hline 67007 & Asuka 09392 & H6 & Antarctica & 2.791 & 0.002791 \\
\hline 41332 & Yamato 000567 & $\mathrm{H} 6$ & Antarctica & 2.755 & 0.002755 \\
\hline 43747 & Yamato 002985 & $\mathrm{H} 6$ & Antarctica & 2.699 & 0.002699 \\
\hline 66993 & Asuka 09363 & $\mathrm{H} 6$ & Antarctica & 2.642 & 0.002642 \\
\hline 68259 & Northwest Africa 12148 & $\mathrm{H} 6$ & Algeria & 2.62 & 0.00262 \\
\hline 67003 & Asuka 09385 & $\mathrm{H} 6$ & Antarctica & 2.603 & 0.002603 \\
\hline 67474 & Elephant Moraine 14061 & $\mathrm{H} 6$ & Antarctica & 2.6 & 0.0026 \\
\hline 66875 & Asuka 09208 & $\mathrm{H} 6$ & Antarctica & 2.588 & 0.002588 \\
\hline 66873 & Asuka 09206 & $\mathrm{H} 6$ & Antarctica & 2.522 & 0.002522 \\
\hline 66992 & Asuka 09362 & $\mathrm{H} 6$ & Antarctica & 2.462 & 0.002462 \\
\hline
\end{tabular}




\begin{tabular}{|c|c|c|c|c|c|}
\hline 36844 & Yamato 980218 & $\mathrm{H} 6$ & Antarctica & 2.453 & 0.002453 \\
\hline 66879 & Asuka 09212 & $\mathrm{H} 6$ & Antarctica & 2.379 & 0.002379 \\
\hline 66880 & Asuka 09213 & $\mathrm{H} 6$ & Antarctica & 2.286 & 0.002286 \\
\hline 66874 & Asuka 09207 & $\mathrm{H} 6$ & Antarctica & 2.179 & 0.002179 \\
\hline 43898 & Yamato 003136 & $\mathrm{H} 6$ & Antarctica & 2.137 & 0.002137 \\
\hline 66994 & Asuka 09364 & $\mathrm{H} 6$ & Antarctica & 2.136 & 0.002136 \\
\hline 67456 & Elephant Moraine 14040 & $\mathrm{H} 6$ & Antarctica & 2.1 & 0.0021 \\
\hline 67039 & Asuka 09454 & $\mathrm{H} 6$ & Antarctica & 2.047 & 0.002047 \\
\hline 66973 & Asuka 09335 & $\mathrm{H} 6$ & Antarctica & 2.041 & 0.002041 \\
\hline 66877 & Asuka 09210 & $\mathrm{H} 6$ & Antarctica & 1.928 & 0.001928 \\
\hline 66876 & Asuka 09209 & $\mathrm{H} 6$ & Antarctica & 1.799 & 0.001799 \\
\hline 66884 & Asuka 09217 & $\mathrm{H} 6$ & Antarctica & 1.753 & 0.001753 \\
\hline 67475 & Elephant Moraine 14062 & $\mathrm{H} 6$ & Antarctica & 1.7 & 0.0017 \\
\hline 67037 & Asuka 09450 & $\mathrm{H} 6$ & Antarctica & 1.65 & 0.00165 \\
\hline 36764 & Yamato 980138 & $\mathrm{H} 6$ & Antarctica & 1.616 & 0.001616 \\
\hline 66881 & Asuka 09214 & $\mathrm{H} 6$ & Antarctica & 1.59 & 0.00159 \\
\hline 36627 & Yamato 980001 & $\mathrm{H} 6$ & Antarctica & 1.551 & 0.001551 \\
\hline 66882 & Asuka 09215 & $\mathrm{H} 6$ & Antarctica & 1.521 & 0.001521 \\
\hline 36811 & Yamato 980185 & $\mathrm{H} 6$ & Antarctica & 1.503 & 0.001503 \\
\hline 66974 & Asuka 09336 & $\mathrm{H} 6$ & Antarctica & 1.46 & 0.00146 \\
\hline 68088 & Miller Range 15532 & $\mathrm{H} 6$ & Antarctica & 1.45 & 0.00145 \\
\hline 67025 & Asuka 09418 & $\mathrm{H} 6$ & Antarctica & 1.386 & 0.001386 \\
\hline 66723 & Miller Range 15292 & $\mathrm{H} 6$ & Antarctica & 1.37 & 0.00137 \\
\hline 66721 & Miller Range 15272 & $\mathrm{H} 6$ & Antarctica & 1.36 & 0.00136 \\
\hline 67301 & Asuka 12356 & $\mathrm{H} 6$ & Antarctica & 1.33 & 0.00133 \\
\hline 66893 & Asuka 09227 & $\mathrm{H} 6$ & Antarctica & 1.32 & 0.00132 \\
\hline 67464 & Elephant Moraine 14048 & $\mathrm{H} 6$ & Antarctica & 1.3 & 0.0013 \\
\hline 67476 & Elephant Moraine 14063 & $\mathrm{H} 6$ & Antarctica & 1.3 & 0.0013 \\
\hline 68078 & Miller Range 15417 & $\mathrm{H} 6$ & Antarctica & 1.2 & 0.0012 \\
\hline 36937 & Yamato 980311 & $\mathrm{H} 6$ & Antarctica & 1.188 & 0.001188 \\
\hline 66718 & Miller Range 15153 & $\mathrm{H} 6$ & Antarctica & 1.16 & 0.00116 \\
\hline 67458 & Elephant Moraine 14042 & $\mathrm{H} 6$ & Antarctica & 1.1 & 0.0011 \\
\hline 66743 & Elephant Moraine 16218 & $\mathrm{H} 6$ & Antarctica & 1.071 & 0.001071 \\
\hline 66732 & Elephant Moraine 16206 & $\mathrm{H} 6$ & Antarctica & 1.06 & 0.00106 \\
\hline 36821 & Yamato 980195 & $\mathrm{H} 6$ & Antarctica & 1.044 & 0.001044 \\
\hline 66739 & Elephant Moraine 16214 & $\mathrm{H} 6$ & Antarctica & 1.037 & 0.001037 \\
\hline 67124 & Asuka 10144 & $\mathrm{H} 6$ & Antarctica & 0.983 & 0.000983 \\
\hline 66905 & Asuka 09250 & $\mathrm{H} 6$ & Antarctica & 0.92 & 0.00092 \\
\hline 67026 & Asuka 09419 & $\mathrm{H} 6$ & Antarctica & 0.919 & 0.000919 \\
\hline 66744 & Elephant Moraine 16219 & $\mathrm{H} 6$ & Antarctica & 0.889 & 0.000889 \\
\hline 36957 & Yamato 980332 & $\mathrm{H} 6$ & Antarctica & 0.832 & 0.000832 \\
\hline 66742 & Elephant Moraine 16217 & $\mathrm{H} 6$ & Antarctica & 0.813 & 0.000813 \\
\hline 67482 & Elephant Moraine 14071 & $\mathrm{H} 6$ & Antarctica & 0.8 & 0.0008 \\
\hline 66970 & Asuka 09330 & $\mathrm{H} 6$ & Antarctica & 0.775 & 0.000775 \\
\hline 43860 & Yamato 003098 & $\mathrm{H} 6$ & Antarctica & 0.774 & 0.000774 \\
\hline
\end{tabular}




\section{5}

36971

66741

43929

66735

66975

67024

43932

68086

68082

66740

66731

43899

43104

68090

43341

43613

43913

66892

68080

66471

67785

68600

68291

68412

68632

66813

67656

66383

68421

66470

68474

68488

68496

67870

67640

68601

66455

66462

66461

66463

67872

67871

67625
68481
Miller Range $15477 \quad$ H6

Yamato $980346 \quad \mathrm{H6}$

Elephant Moraine $16216 \quad$ H6

Yamato 003167

Asuka 09337

Asuka 09417

Yamato 003170

Miller Range 15478

Miller Range 15432

Elephant Moraine 16215

Elephant Moraine 16205

Yamato 003137

Yamato 002342

Miller Range 15534

Yamato 002579

Yamato 002851

Yamato 003151

Asuka 09225

Miller Range 15430

Northwest Africa 11613

Northwest Africa 12236

Northwest Africa 11899

Northwest Africa 12227

Northwest Africa 11964

Northwest Africa 12190

Twayla 001

Northwest Africa 11712

Northwest Africa 11858

Northwest Africa 11551

Northwest Africa 12203

Northwest Africa 11612

Northwest Africa 12224

Northwest Africa 12194

Northwest Africa 12250

Northwest Africa 11997

Northwest Africa 11834

Northwest Africa 12231

Northwest Africa 11598

Northwest Africa 11605

Northwest Africa 11604

Northwest Africa 11606

Grosvenor Mountains 17102Iron, IAB corr Antarctica

Grosvenor Mountains 17051 Iron, IAB corr Antarctica

Nova 027

$\mathrm{H} 6$

$\mathrm{H} 6$

$\mathrm{H} 6$

$\mathrm{H} 6$

$\mathrm{H} 6$

$\mathrm{H} 6$

$\mathrm{H} 6$

$\mathrm{H} 6$

$\mathrm{H} 6$

$\mathrm{H} 6$

$\mathrm{H} 6$

$\mathrm{H} 6$

$\mathrm{H} 6$

$\mathrm{H} 6$

$\mathrm{H} 6$

$\mathrm{H} 6$

$\mathrm{H} 6$

$\mathrm{H} 7$

$\mathrm{H} 7$

Howardite

Howardite

Howardite

Howardite

Howardite

Howardite

Howardite

Howardite

Howardite

Howardite

Howardite

Howardite

Howardite

Howardite

Howardite

Howardite

Howardite

Howardite

Howardite

Algeria

Algeria

Morocco

Algeria

Algeria
Elephant Moraine 16209

(Northwest At

(Northwest A1

(Northwest A

Grosvenor Mountains 17102 Iron, IAB com

59

60
Iron, IAB-MG (unknown)
0.76

0.00076

$0.674 \quad 0.000674$

$0.672 \quad 0.000672$

$0.658 \quad 0.000658$

$0.628 \quad 0.000628$

$0.624 \quad 0.000624$

$0.555 \quad 0.000555$

$0.542 \quad 0.000542$

$0.54 \quad 0.00054$

$0.53 \quad 0.00053$

$0.522 \quad 0.000522$

$0.456 \quad 0.000456$

$0.437 \quad 0.000437$

$0.405 \quad 0.000405$

$0.39 \quad 0.00039$

$0.323 \quad 0.000323$

$0.311 \quad 0.000311$

$0.271 \quad 0.000271$

$0.244 \quad 0.000244$

0.17

0.00017

354

0.354

21.03

0.02103

16000

16

1780

1.78

1.25

1250

1.1465

500

0.5

500

0.5

453.5

0.4535

446.9

0.4469

293

0.293

274

0.274

230

0.23

85.1

0.0851

42.7

0.0427

30.2

0.0302

15

0.015

13

0.013

3.9

0.0039

2.13

0.00213

2.04

0.00204

1.71

0.00171

4959.5

4.9595

2805.95

2.80595

120000 


\begin{tabular}{|c|c|c|c|c|c|}
\hline 67621 & Northwest Africa 11859 & \multicolumn{2}{|c|}{ Iron, IAB-MG(Northwest AI } & 78000 & 78 \\
\hline 67628 & Nova 021 & \multicolumn{2}{|c|}{ Iron, IAB-MG (unknown) } & 62000 & 62 \\
\hline 67855 & Sauceda de la Borda & \multicolumn{2}{|c|}{ Iron, IAB-MG Mexico } & 41000 & 41 \\
\hline 67627 & Nova 026 & \multicolumn{2}{|c|}{ Iron, IAB-MG (unknown) } & 18200 & 18.2 \\
\hline 66486 & Northwest Africa 11649 & \multicolumn{2}{|c|}{ Iron, IAB-MG Morocco } & 17400 & 17.4 \\
\hline 67608 & Entrèves & \multicolumn{2}{|c|}{ Iron, IAB-MG Italy } & 17300 & 17.3 \\
\hline 66476 & Nagara & \multicolumn{2}{|c|}{ Iron, IAB-MG Japan } & 16243 & 16.243 \\
\hline 67837 & Yuli & \multicolumn{2}{|c|}{ Iron, IAB-MG China } & 10000 & 10 \\
\hline 67676 & Nova 025 & \multicolumn{2}{|c|}{ Iron, IAB-MG (unknown) } & 9370 & 9.37 \\
\hline 67731 & Nova 028 & \multicolumn{2}{|c|}{ Iron, IAB-MG (unknown) } & 3530 & 3.53 \\
\hline 67622 & Nova 022 & \multicolumn{2}{|c|}{ Iron, IAB-MG (unknown) } & 2150 & 2.15 \\
\hline 67626 & Elmore County & \multicolumn{2}{|c|}{ Iron, IAB-MGUnited States } & 578 & 0.578 \\
\hline 67620 & Nova 020 & \multicolumn{2}{|c|}{ Iron, IAB-MG (unknown) } & 538 & 0.538 \\
\hline 67623 & Nova 023 & \multicolumn{2}{|c|}{ Iron, IAB-MG (unknown) } & 537 & 0.537 \\
\hline 67624 & Nova 024 & \multicolumn{2}{|c|}{ Iron, IAB-MG (unknown) } & 110 & 0.11 \\
\hline 66428 & Northwest Africa 11577 & \multicolumn{2}{|c|}{ Iron, IAB-sHL Morocco } & 86 & 0.086 \\
\hline 66478 & Northwest Africa 11637 & Iron, IIAB & Morocco & 4368 & 4.368 \\
\hline 68314 & Northwest Africa 12000 & Iron, IIAB & Morocco & 225 & 0.225 \\
\hline 67806 & Caçapava do Sul & Iron, IID & Brazil & 27000 & 27 \\
\hline 67601 & Sokoto & Iron, IIIAB & Nigeria & 30000 & 30 \\
\hline 67381 & Dahongliuxia & Iron, IIIAB & China & 15600 & 15.6 \\
\hline 67851 & Ilafegh 018 & Iron, IIIAB & Algeria & 11100 & 11.1 \\
\hline 68465 & Bañobárez & Iron, IIIAB & Spain & 132 & 0.132 \\
\hline 66724 & Elephant Moraine 16045 & Iron, IIIAB & Antarctica & 105.047 & 0.105047 \\
\hline 67382 & Alatage Mountain 043 & Iron, IVB & China & 9800 & 9.8 \\
\hline 66792 & Jeminay & Iron, ungrou & China & 6427 & 6.427 \\
\hline 67873 & \multicolumn{3}{|c|}{ Grosvenor Mountains 17195 Iron, ungroup Antarctica } & 75.77 & 0.07577 \\
\hline 66545 & Dominion Range 14125 & L-imp melt & Antarctica & 71.31 & 0.07131 \\
\hline 67712 & Northwest Africa 11832 & L-melt rock & (Northwest Al & 290 & 0.29 \\
\hline 68501 & Nanbaxian 001 & $\mathrm{~L} \sim 4$ & China & 3784 & 3.784 \\
\hline 68533 & Ganq 003 & $\mathrm{~L} \sim 5$ & China & 2200 & 2.2 \\
\hline 68434 & Northwest Africa 12297 & $L \sim 5$ & (Northwest Al & 110.5 & 0.1105 \\
\hline 68500 & Da Qaidam 001 & $\mathrm{~L} \sim 6$ & China & 17500 & 17.5 \\
\hline 68523 & Ganq 002 & $\mathrm{~L} \sim 6$ & China & 5340 & 5.34 \\
\hline 68522 & Ganq 001 & $\mathrm{~L} \sim 6$ & China & 3907 & 3.907 \\
\hline 66399 & Northwest Africa 11581 & $\mathrm{~L} \sim 6$ & (Northwest A 1 & 2076 & 2.076 \\
\hline 68650 & Hoboksar 001 & $\mathrm{~L} \sim 6$ & China & 1500 & 1.5 \\
\hline 68652 & Turpan 001 & $\mathrm{~L} \sim 6$ & China & 1500 & 1.5 \\
\hline 68649 & Dunhuang 001 & $\mathrm{~L} \sim 6$ & China & 1427 & 1.427 \\
\hline 66395 & Northwest Africa 11578 & $\mathrm{~L} \sim 6$ & (Northwest At & 983.6 & 0.9836 \\
\hline 68430 & Hami 008 & $\mathrm{~L} \sim 6$ & China & 589.4 & 0.5894 \\
\hline 66400 & Northwest Africa 11582 & $\mathrm{~L} \sim 6$ & Mauritania & 564.5 & 0.5645 \\
\hline 68499 & Weiya 001 & $\mathrm{~L} \sim 6$ & China & 201 & 0.201 \\
\hline 68439 & Xinjiang 011 & $L \sim 6$ & China & 89.5 & 0.0895 \\
\hline 68432 & Xinjiang 009 & $\mathrm{~L} \sim 6$ & China & 60.3 & 0.0603 \\
\hline
\end{tabular}




\begin{tabular}{|c|c|c|c|c|c|}
\hline 68657 & Northwest Africa 12381 & $\mathrm{~L} \sim 6$ & (Northwest Al & 21.7 & 0.0217 \\
\hline 67799 & Aba Panu & L3 & Nigeria & 160000 & 160 \\
\hline 67486 & Northwest Africa 11782 & L3 & Mali & 25700 & 25.7 \\
\hline 66774 & Northwest Africa 11722 & L3 & (Northwest Al & 7210 & 7.21 \\
\hline 68424 & Northwest Africa 12285 & L3 & (Northwest Al & 4710 & 4.71 \\
\hline 68297 & Northwest Africa 11994 & L3 & (Northwest A 1 & 2160 & 2.16 \\
\hline 68482 & Northwest Africa 12324 & L3 & (Northwest Al & 1840 & 1.84 \\
\hline 68551 & Northwest Africa 12331 & L3 & (Northwest Al & 753 & 0.753 \\
\hline 68640 & Northwest Africa 12341 & L3 & (Northwest A 1 & 719 & 0.719 \\
\hline 67667 & Northwest Africa 11881 & L3 & (Northwest Al & 590 & 0.59 \\
\hline 66435 & Northwest Africa 11634 & L3 & (Northwest Al & 527 & 0.527 \\
\hline 66436 & Northwest Africa 11635 & L3 & (Northwest Al & 505 & 0.505 \\
\hline 67585 & Northwest Africa 11802 & L3 & (Northwest A 1 & 446.5 & 0.4465 \\
\hline 67171 & Asuka 12205 & L3 & Antarctica & 400.95 & 0.40095 \\
\hline 51835 & Northwest Africa 6263 & L3 & (Northwest At & 347 & 0.347 \\
\hline 66490 & Northwest Africa 11672 & L3 & (Northwest A 1 & 282 & 0.282 \\
\hline 67560 & Northwest Africa 11796 & L3 & (Northwest At & 233 & 0.233 \\
\hline 66409 & San Juan de Allende & L3 & Mexico & 200 & 0.2 \\
\hline 67761 & Northwest Africa 11940 & L3 & (Northwest A 1 & 180 & 0.18 \\
\hline 66781 & Northwest Africa 11735 & L3 & (Northwest A 1 & 163 & 0.163 \\
\hline 67135 & Asuka 12018 & L3 & Antarctica & 157.98 & 0.15798 \\
\hline 66765 & Northwest Africa 11713 & L3 & (Northwest A 1 & 80 & 0.08 \\
\hline 66766 & Northwest Africa 11714 & L3 & (Northwest At & 75 & 0.075 \\
\hline 66782 & Northwest Africa 11736 & L3 & (Northwest Al & 71.3 & 0.0713 \\
\hline 67751 & Northwest Africa 11925 & L3 & (Northwest A 1 & 67 & 0.067 \\
\hline 67611 & Northwest Africa 11829 & L3 & (Northwest Al & 52 & 0.052 \\
\hline 67612 & Northwest Africa 11835 & L3 & (Northwest At & 42.5 & 0.0425 \\
\hline 32398 & Northwest Africa 1723 & L3 & Morocco & 28 & 0.028 \\
\hline 66431 & Northwest Africa 11630 & L3 & (Northwest At & 18 & 0.018 \\
\hline 67434 & Elephant Moraine 14018 & L3 & Antarctica & 5.7 & 0.0057 \\
\hline 67001 & Asuka 09383 & L3 & Antarctica & 5.694 & 0.005694 \\
\hline 67027 & Asuka 09420 & L3 & Antarctica & 4.489 & 0.004489 \\
\hline 66963 & Asuka 09319 & L3 & Antarctica & 3.979 & 0.003979 \\
\hline 41494 & Yamato 000730 & L3 & Antarctica & 3.714 & 0.003714 \\
\hline 43805 & Yamato 003043 & L3 & Antarctica & 3.434 & 0.003434 \\
\hline 43990 & Yamato 003228 & L3 & Antarctica & 3.302 & 0.003302 \\
\hline 67075 & Asuka 09621 & L3 & Antarctica & 3.033 & 0.003033 \\
\hline 67028 & Asuka 09422 & L3 & Antarctica & 2.927 & 0.002927 \\
\hline 66920 & Asuka 09271 & L3 & Antarctica & 2.156 & 0.002156 \\
\hline 67128 & Asuka 10214 & L3 & Antarctica & 2.003 & 0.002003 \\
\hline 66913 & Asuka 09259 & L3 & Antarctica & 1.689 & 0.001689 \\
\hline 67061 & Asuka 09555 & L3 & Antarctica & 1.611 & 0.001611 \\
\hline 66918 & Asuka 09266 & L3 & Antarctica & 1.475 & 0.001475 \\
\hline 66914 & Asuka 09260 & L3 & Antarctica & 1.47 & 0.00147 \\
\hline 67029 & Asuka 09423 & L3 & Antarctica & 1.458 & 0.001458 \\
\hline
\end{tabular}




\begin{tabular}{|c|c|c|c|c|c|}
\hline 66906 & Asuka 09251 & L3 & Antarctica & 1.044 & 0.001044 \\
\hline 31849 & Northwest Africa 787 & L3-6 & (Northwest At & 30000 & 30 \\
\hline 68293 & Northwest Africa 11978 & L3-6 & (Northwest Al & 1159 & 1.159 \\
\hline 67819 & Northwest Africa 11922 & L3-6 & (Northwest Al & 138 & 0.138 \\
\hline 66410 & Northwest Africa 11574 & L3.10 & (Northwest A 1 & 750 & 0.75 \\
\hline 66544 & Dominion Range 14124 & L3.5 & Antarctica & 58.55 & 0.05855 \\
\hline 66587 & Dominion Range 14201 & L3.6 & Antarctica & 16.8 & 0.0168 \\
\hline 66522 & Dominion Range 14082 & L3.6 & Antarctica & 9.29 & 0.00929 \\
\hline 66601 & Dominion Range 14225 & L3.8 & Antarctica & 71.004 & 0.071004 \\
\hline 67670 & Northwest Africa 11884 & L4 & Morocco & 8015 & 8.015 \\
\hline 68655 & Gueltat Zemmour & L4 & Morocco & 8000 & 8 \\
\hline 67629 & Northwest Africa 11872 & L4 & (Northwest Al & 6220 & 6.22 \\
\hline 66777 & Northwest Africa 11725 & L4 & (Northwest A1 & 2703 & 2.703 \\
\hline 68513 & Northwest Africa 12348 & L4 & Morocco & 2347.5 & 2.3475 \\
\hline 66780 & Northwest Africa 11728 & L4 & (Northwest Al & 2157 & 2.157 \\
\hline 66491 & Northwest Africa 11674 & L4 & (Northwest A1 & 1536 & 1.536 \\
\hline 66493 & Northwest Africa 11678 & L4 & (Northwest Al & 1110 & 1.11 \\
\hline 32064 & Northwest Africa 1091 & L4 & (Northwest Al & 850 & 0.85 \\
\hline 68337 & Catalina 282 & L4 & Chile & 556 & 0.556 \\
\hline 67767 & Northwest Africa 11959 & L4 & (Northwest Al & 519.3 & 0.5193 \\
\hline 68136 & Northwest Africa 12025 & L4 & Algeria & 362.15 & 0.36215 \\
\hline 67469 & Elephant Moraine 14055 & L4 & Antarctica & 361 & 0.361 \\
\hline 68231 & Northwest Africa 12120 & L4 & Algeria & 355.04 & 0.35504 \\
\hline 66509 & Northwest Africa 11693 & L4 & (Northwest Al & 330 & 0.33 \\
\hline 67316 & Asuka 12371 & L4 & Antarctica & 189.62 & 0.18962 \\
\hline 67573 & Northwest Africa 11817 & L4 & (Northwest At & 188.1 & 0.1881 \\
\hline 66498 & Northwest Africa 11682 & L4 & (Northwest At & 163 & 0.163 \\
\hline 67323 & Asuka 12378 & L4 & Antarctica & 160.6 & 0.1606 \\
\hline 66758 & Northwest Africa 11660 & L4 & (Northwest At & 158 & 0.158 \\
\hline 32072 & Northwest Africa 1099 & L4 & (Northwest A1 & 140 & 0.14 \\
\hline 66672 & Dominion Range 14464 & L4 & Antarctica & 123.57 & 0.12357 \\
\hline 67836 & Northwest Africa 11986 & L4 & (Northwest At & 105.4 & 0.1054 \\
\hline 67145 & Asuka 12037 & L4 & Antarctica & 77.45 & 0.07745 \\
\hline 67655 & Northwest Africa 11878 & L4 & (Northwest At & 75.52 & 0.07552 \\
\hline 67148 & Asuka 12042 & L4 & Antarctica & 74.44 & 0.07444 \\
\hline 66675 & Dominion Range 14467 & L4 & Antarctica & 68.81 & 0.06881 \\
\hline 67615 & Northwest Africa 11864 & L4 & (Northwest A 1 & 65.31 & 0.06531 \\
\hline 67996 & Dominion Range 14471 & L4 & Antarctica & 44.84 & 0.04484 \\
\hline 66512 & Dominion Range 14031 & L4 & Antarctica & 41.66 & 0.04166 \\
\hline 66677 & Dominion Range 14469 & L4 & Antarctica & 37.31 & 0.03731 \\
\hline 66828 & Sayh al Uhaymir 605 & L4 & Oman & 26.24 & 0.02624 \\
\hline 66513 & Dominion Range 14032 & L4 & Antarctica & 25.37 & 0.02537 \\
\hline 66786 & MacKay Glacier 14006 & L4 & Antarctica & 15.7 & 0.0157 \\
\hline 67618 & Northwest Africa 11869 & L4 & (Northwest Al & 15.66 & 0.01566 \\
\hline 67317 & Asuka 12372 & L4 & Antarctica & 14.872 & 0.014872 \\
\hline
\end{tabular}




\begin{tabular}{|c|c|c|c|c|c|}
\hline 66691 & Dominion Range 14523 & L4 & Antarctica & 14.49 & 0.01449 \\
\hline 67256 & Asuka 12306 & L4 & Antarctica & 12.826 & 0.012826 \\
\hline 67291 & Asuka 12345 & L4 & Antarctica & 8.93 & 0.00893 \\
\hline 67200 & Asuka 12249 & L4 & Antarctica & 6.787 & 0.006787 \\
\hline 44107 & Yamato 003345 & L4 & Antarctica & 4.566 & 0.004566 \\
\hline 41092 & Yamato 000327 & L4 & Antarctica & 4.466 & 0.004466 \\
\hline 66971 & Asuka 09332 & L4 & Antarctica & 4.382 & 0.004382 \\
\hline 42126 & Yamato 001364 & L4 & Antarctica & 4.17 & 0.00417 \\
\hline 44131 & Yamato 003369 & L4 & Antarctica & 3.905 & 0.003905 \\
\hline 41139 & Yamato 000374 & L4 & Antarctica & 3.751 & 0.003751 \\
\hline 41159 & Yamato 000394 & L4 & Antarctica & 3.715 & 0.003715 \\
\hline 44133 & Yamato 003371 & L4 & Antarctica & 2.716 & 0.002716 \\
\hline 44112 & Yamato 003350 & L4 & Antarctica & 2.173 & 0.002173 \\
\hline 67059 & Asuka 09544 & L4 & Antarctica & 1.335 & 0.001335 \\
\hline 36659 & Yamato 980033 & L4 & Antarctica & 1.272 & 0.001272 \\
\hline 66789 & MacKay Glacier 14018 & L4 & Antarctica & 0.5 & 0.0005 \\
\hline 66726 & Elephant Moraine 16197 & L4 & Antarctica & 0.498 & 0.000498 \\
\hline 68122 & Nova 054 & L4-6 & (Unknown) & & \\
\hline 68328 & Northwest Africa 12181 & L4-melt brecc & (Northwest Al & 930 & 0.93 \\
\hline 66778 & Northwest Africa 11726 & L4/5 & (Northwest Al & 4470 & 4.47 \\
\hline 67663 & Caleta el Cobre 023 & L4/5 & Chile & 1500 & 1.5 \\
\hline 66416 & Northwest Africa 11609 & $\mathrm{~L} 4 / 5$ & (Northwest Al & 253 & 0.253 \\
\hline 66427 & Northwest Africa 11627 & L4/5 & (Northwest At & 68.82 & 0.06882 \\
\hline 66407 & Northwest Africa 11584 & L5 & (Northwest Al & 56970 & 56.97 \\
\hline 68383 & Paposo 045 & L5 & Chile & 13245 & 13.245 \\
\hline 68511 & Northwest Africa 12346 & L5 & Morocco & 12600 & 12.6 \\
\hline 66442 & Realicó & L5 & Argentina & 12000 & 12 \\
\hline 66827 & Los Vientos 213 & L5 & Chile & 7935 & 7.935 \\
\hline 68480 & Northwest Africa 12301 & L5 & (Northwest Al & 7500 & 7.5 \\
\hline 68633 & Wuerhe & L5 & China & 6000 & 6 \\
\hline 68382 & Paposo 044 & L5 & Chile & 5458 & 5.458 \\
\hline 66779 & Northwest Africa 11727 & L5 & (Northwest At & 3620 & 3.62 \\
\hline 67386 & El Médano 381 & L5 & Chile & 3485 & 3.485 \\
\hline 68320 & Northwest Africa 12238 & L5 & (Northwest Al & 2491.2 & 2.4912 \\
\hline 68540 & Sayh al Uhaymir 607 & L5 & Oman & 2452 & 2.452 \\
\hline 67713 & Ngare Sero & L5 & Tanzania & 2233 & 2.233 \\
\hline 66826 & El Médano 378 & L5 & Chile & 2201 & 2.201 \\
\hline 67605 & Northwest Africa 11847 & L5 & (Northwest Al & 2098 & 2.098 \\
\hline 68426 & Northwest Africa 12292 & L5 & (Northwest Al & 1957 & 1.957 \\
\hline 32068 & Northwest Africa 1095 & L5 & (Northwest Al & 1800 & 1.8 \\
\hline 66841 & Northwest Africa 11744 & L5 & (Northwest Al & 1566.1 & 1.5661 \\
\hline 66775 & Northwest Africa 11723 & L5 & (Northwest Al & 1522 & 1.522 \\
\hline 68521 & Northwest Africa 11980 & L5 & (Northwest At & 1370 & 1.37 \\
\hline 67841 & Chug Chug 004 & L5 & Chile & 1080 & 1.08 \\
\hline 67506 & Sierra Gorda 001 & L5 & Chile & 1013 & 1.013 \\
\hline
\end{tabular}




\begin{tabular}{|c|c|c|c|c|c|}
\hline 68598 & Karimati & L5 & India & 930 & 0.93 \\
\hline 67484 & Elephant Moraine 14073 & L5 & Antarctica & 796 & 0.796 \\
\hline 68384 & Paposo 046 & L5 & Chile & 678 & 0.678 \\
\hline 67699 & Jabal Zaltan 006 & L5 & Libya & 659.4 & 0.6594 \\
\hline 68267 & Northwest Africa 12156 & L5 & Algeria & 606.82 & 0.60682 \\
\hline 68538 & Chug Chug 006 & L5 & Chile & 594 & 0.594 \\
\hline 68268 & Northwest Africa 12157 & L5 & Algeria & 571.94 & 0.57194 \\
\hline 66842 & Northwest Africa 11745 & L5 & (Northwest A1 & 554.16 & 0.55416 \\
\hline 68177 & Northwest Africa 12066 & L5 & Algeria & 518.83 & 0.51883 \\
\hline 67508 & Chug Chug 001 & L5 & Chile & 514 & 0.514 \\
\hline 67299 & Asuka 12354 & L5 & Antarctica & 482.17 & 0.48217 \\
\hline 31796 & Northwest Africa 674 & L5 & (Northwest Al & 430 & 0.43 \\
\hline 67576 & Northwest Africa 11820 & L5 & (Northwest Al & 401 & 0.401 \\
\hline 66668 & Dominion Range 14460 & L5 & Antarctica & 380.76 & 0.38076 \\
\hline 68137 & Northwest Africa 12026 & L5 & Algeria & 347.67 & 0.34767 \\
\hline 68145 & Northwest Africa 12034 & L5 & Algeria & 342.12 & 0.34212 \\
\hline 68405 & Northwest Africa 12258 & L5 & (Northwest At & 328 & 0.328 \\
\hline 67764 & Northwest Africa 11943 & L5 & (Northwest Al & 321 & 0.321 \\
\hline 67165 & Asuka 12138 & L5 & Antarctica & 298.6 & 0.2986 \\
\hline 67887 & Dominion Range 14230 & L5 & Antarctica & 288.53 & 0.28853 \\
\hline 68181 & Northwest Africa 12070 & L5 & Algeria & 277.86 & 0.27786 \\
\hline 68095 & Elephant Moraine 16002 & L5 & Antarctica & 259.4 & 0.2594 \\
\hline 68406 & Northwest Africa 12259 & L5 & (Northwest At & 223 & 0.223 \\
\hline 68427 & Northwest Africa 12294 & L5 & (Northwest Al & 206 & 0.206 \\
\hline 67834 & Northwest Africa 11982 & L5 & (Northwest Al & 194 & 0.194 \\
\hline 67691 & Catalina 276 & L5 & Chile & 186 & 0.186 \\
\hline 66440 & Northwest Africa 11646 & L5 & (Northwest Al & 184.2 & 0.1842 \\
\hline 68407 & Northwest Africa 12260 & L5 & (Northwest Al & 179 & 0.179 \\
\hline 67842 & Sierra Gorda 003 & L5 & Chile & 154 & 0.154 \\
\hline 67694 & Jabal Zaltan 001 & L5 & Libya & 151.3 & 0.1513 \\
\hline 66858 & Northwest Africa 11760 & L5 & (Northwest At & 126 & 0.126 \\
\hline 66856 & Northwest Africa 11758 & L5 & (Northwest At & 124.4 & 0.1244 \\
\hline 68184 & Northwest Africa 12073 & L5 & Algeria & 120.07 & 0.12007 \\
\hline 67845 & Sierra Gorda 005 & L5 & Chile & 112 & 0.112 \\
\hline 67671 & Catalina 279 & L5 & Chile & 105 & 0.105 \\
\hline 66466 & Northwest Africa 11666 & L5 & (Northwest At & 101 & 0.101 \\
\hline 67967 & Dominion Range 14413 & L5 & Antarctica & 99.891 & 0.099891 \\
\hline 66560 & Dominion Range 14172 & L5 & Antarctica & 99.03 & 0.09903 \\
\hline 66759 & Northwest Africa 11661 & L5 & (Northwest At & 84.9 & 0.0849 \\
\hline 66572 & Dominion Range 14184 & L5 & Antarctica & 83.03 & 0.08303 \\
\hline 66632 & Dominion Range 14307 & L5 & Antarctica & 74.832 & 0.074832 \\
\hline 67963 & Dominion Range 14409 & L5 & Antarctica & 73.13 & 0.07313 \\
\hline 66562 & Dominion Range 14174 & L5 & Antarctica & 72.32 & 0.07232 \\
\hline 66421 & Northwest Africa 11621 & L5 & (Northwest At & 65.65 & 0.06565 \\
\hline 67955 & Dominion Range 14401 & L5 & Antarctica & 64.509 & 0.064509 \\
\hline
\end{tabular}




\begin{tabular}{|c|c|c|c|c|c|}
\hline 66755 & Northwest Africa 11657 & L5 & (Northwest A1 & 63.8 & 0.0638 \\
\hline 66565 & Dominion Range 14177 & L5 & Antarctica & 61.89 & 0.06189 \\
\hline 66676 & Dominion Range 14468 & L5 & Antarctica & 61.08 & 0.06108 \\
\hline 66754 & Northwest Africa 11656 & L5 & (Northwest A 1 & 59 & 0.059 \\
\hline 66598 & Dominion Range 14222 & L5 & Antarctica & 57.549 & 0.057549 \\
\hline 68300 & Calate 014 & L5 & Chile & 55 & 0.055 \\
\hline 66559 & Dominion Range 14171 & L5 & Antarctica & 52.14 & 0.05214 \\
\hline 68552 & Machuca 003 & L5 & Chile & 52 & 0.052 \\
\hline 67979 & Dominion Range 14425 & L5 & Antarctica & 49.982 & 0.049982 \\
\hline 66764 & Northwest Africa 11711 & L5 & (Northwest A 1 & 48.8 & 0.0488 \\
\hline 67936 & Dominion Range 14343 & L5 & Antarctica & 48.451 & 0.048451 \\
\hline 66679 & Dominion Range 14511 & L5 & Antarctica & 47.32 & 0.04732 \\
\hline 67883 & Dominion Range 14215 & L5 & Antarctica & 46.992 & 0.046992 \\
\hline 66820 & El Médano 373 & L5 & Chile & 46 & 0.046 \\
\hline 68388 & San Juan 092 & L5 & Chile & 44.7 & 0.0447 \\
\hline 68032 & Miller Range 15085 & L5 & Antarctica & 42.171 & 0.042171 \\
\hline 67886 & Dominion Range 14218 & L5 & Antarctica & 41.805 & 0.041805 \\
\hline 66561 & Dominion Range 14173 & L5 & Antarctica & 40.32 & 0.04032 \\
\hline 67603 & Sweihan & L5 & United Arab E & 40 & 0.04 \\
\hline 66564 & Dominion Range 14176 & L5 & Antarctica & 39.1 & 0.0391 \\
\hline 66680 & Dominion Range 14512 & L5 & Antarctica & 38.668 & 0.038668 \\
\hline 67958 & Dominion Range 14404 & L5 & Antarctica & 36.534 & 0.036534 \\
\hline 68024 & Dominion Range 14509 & L5 & Antarctica & 33.466 & 0.033466 \\
\hline 66646 & Dominion Range 14378 & L5 & Antarctica & 33.377 & 0.033377 \\
\hline 67991 & Dominion Range 14437 & L5 & Antarctica & 32.904 & 0.032904 \\
\hline 66539 & Dominion Range 14109 & L5 & Antarctica & 32.753 & 0.032753 \\
\hline 66526 & Dominion Range 14086 & L5 & Antarctica & 32.19 & 0.03219 \\
\hline 67619 & Northwest Africa 11870 & L5 & (Northwest A 1 & 30.91 & 0.03091 \\
\hline 67988 & Dominion Range 14434 & L5 & Antarctica & 30.193 & 0.030193 \\
\hline 68212 & Northwest Africa 12101 & L5 & Algeria & 30.16 & 0.03016 \\
\hline 66591 & Dominion Range 14205 & L5 & Antarctica & 29.48 & 0.02948 \\
\hline 66702 & Dominion Range 14535 & L5 & Antarctica & 27.91 & 0.02791 \\
\hline 67990 & Dominion Range 14436 & L5 & Antarctica & 27.561 & 0.027561 \\
\hline 67982 & Dominion Range 14428 & L5 & Antarctica & 27.068 & 0.027068 \\
\hline 67944 & Dominion Range 14351 & L5 & Antarctica & 26.878 & 0.026878 \\
\hline 68288 & Northwest Africa 12177 & L5 & Algeria & 26.49 & 0.02649 \\
\hline 66570 & Dominion Range 14182 & L5 & Antarctica & 25.89 & 0.02589 \\
\hline 66685 & Dominion Range 14517 & L5 & Antarctica & 24.937 & 0.024937 \\
\hline 66555 & Dominion Range 14136 & L5 & Antarctica & 24.86 & 0.02486 \\
\hline 67879 & Dominion Range 14211 & L5 & Antarctica & 24.675 & 0.024675 \\
\hline 66644 & Dominion Range 14376 & L5 & Antarctica & 23.992 & 0.023992 \\
\hline 67981 & Dominion Range 14427 & L5 & Antarctica & 22.324 & 0.022324 \\
\hline 68013 & Dominion Range 14498 & L5 & Antarctica & 21.65 & 0.02165 \\
\hline 68061 & Miller Range 15375 & L5 & Antarctica & 20.581 & 0.020581 \\
\hline 66703 & Dominion Range 14536 & L5 & Antarctica & 20.55 & 0.02055 \\
\hline
\end{tabular}




\begin{tabular}{|c|c|c|c|c|c|}
\hline 68070 & Miller Range 15393 & L5 & Antarctica & 20.54 & 0.02054 \\
\hline 66524 & Dominion Range 14084 & L5 & Antarctica & 20.08 & 0.02008 \\
\hline 66695 & Dominion Range 14528 & L5 & Antarctica & 19.34 & 0.01934 \\
\hline 68038 & Miller Range 15092 & L5 & Antarctica & 18.727 & 0.018727 \\
\hline 68057 & Miller Range 15371 & L5 & Antarctica & 18.278 & 0.018278 \\
\hline 66699 & Dominion Range 14532 & L5 & Antarctica & 16.96 & 0.01696 \\
\hline 66701 & Dominion Range 14534 & L5 & Antarctica & 16.1 & 0.0161 \\
\hline 66586 & Dominion Range 14200 & L5 & Antarctica & 15.88 & 0.01588 \\
\hline 66606 & Dominion Range 14240 & L5 & Antarctica & 15.87 & 0.01587 \\
\hline 66707 & Dominion Range 14550 & L5 & Antarctica & 15.72 & 0.01572 \\
\hline 66637 & Dominion Range 14362 & L5 & Antarctica & 15.354 & 0.015354 \\
\hline 68054 & Miller Range 15299 & L5 & Antarctica & 15.297 & 0.015297 \\
\hline 67953 & Dominion Range 14375 & L5 & Antarctica & 14.466 & 0.014466 \\
\hline 41021 & Yamato 000256 & L5 & Antarctica & 14.461 & 0.014461 \\
\hline 67915 & Dominion Range 14292 & L5 & Antarctica & 14.307 & 0.014307 \\
\hline 68025 & Dominion Range 14527 & L5 & Antarctica & 14.11 & 0.01411 \\
\hline 67928 & Dominion Range 14315 & L5 & Antarctica & 13.15 & 0.01315 \\
\hline 67920 & Dominion Range 14297 & L5 & Antarctica & 11.052 & 0.011052 \\
\hline 41750 & Yamato 000988 & L5 & Antarctica & 10.295 & 0.010295 \\
\hline 66585 & Dominion Range 14199 & L5 & Antarctica & 9.759 & 0.009759 \\
\hline 67877 & Dominion Range 14192 & L5 & Antarctica & 9.445 & 0.009445 \\
\hline 27103 & Yamato 791754 & L5 & Antarctica & 9.25 & 0.00925 \\
\hline 67243 & Asuka 12293 & L5 & Antarctica & 8.849 & 0.008849 \\
\hline 67289 & Asuka 12343 & L5 & Antarctica & 8.27 & 0.00827 \\
\hline 27104 & Yamato 791755 & L5 & Antarctica & 7.7 & 0.0077 \\
\hline 66665 & Dominion Range 14457 & L5 & Antarctica & 7.11 & 0.00711 \\
\hline 66581 & Dominion Range 14195 & L5 & Antarctica & 6.196 & 0.006196 \\
\hline 66861 & San Juan 084 & L5 & Chile & 5 & 0.005 \\
\hline 44222 & Yamato 003460 & L5 & Antarctica & 4.761 & 0.004761 \\
\hline 44115 & Yamato 003353 & L5 & Antarctica & 4.655 & 0.004655 \\
\hline 44209 & Yamato 003447 & L5 & Antarctica & 4.534 & 0.004534 \\
\hline 44218 & Yamato 003456 & L5 & Antarctica & 4.511 & 0.004511 \\
\hline 44117 & Yamato 003355 & L5 & Antarctica & 4.484 & 0.004484 \\
\hline 67002 & Asuka 09384 & L5 & Antarctica & 4.322 & 0.004322 \\
\hline 44300 & Yamato 003538 & L5 & Antarctica & 4.112 & 0.004112 \\
\hline 44211 & Yamato 003449 & L5 & Antarctica & 4.096 & 0.004096 \\
\hline 44215 & Yamato 003453 & L5 & Antarctica & 4.057 & 0.004057 \\
\hline 44095 & Yamato 003333 & L5 & Antarctica & 3.943 & 0.003943 \\
\hline 44227 & Yamato 003465 & L5 & Antarctica & 3.864 & 0.003864 \\
\hline 44212 & Yamato 003450 & L5 & Antarctica & 3.566 & 0.003566 \\
\hline 44279 & Yamato 003517 & L5 & Antarctica & 3.388 & 0.003388 \\
\hline 44128 & Yamato 003366 & L5 & Antarctica & 3.374 & 0.003374 \\
\hline 41253 & Yamato 000488 & L5 & Antarctica & 3.363 & 0.003363 \\
\hline 41052 & Yamato 000287 & L5 & Antarctica & 3.279 & 0.003279 \\
\hline 44207 & Yamato 003445 & L5 & Antarctica & 3.273 & 0.003273 \\
\hline
\end{tabular}




\begin{tabular}{|c|c|c|c|c|c|}
\hline 41392 & Yamato 000628 & L5 & Antarctica & 3.269 & 0.003269 \\
\hline 44024 & Yamato 003262 & L5 & Antarctica & 3.231 & 0.003231 \\
\hline 44223 & Yamato 003461 & L5 & Antarctica & 3.112 & 0.003112 \\
\hline 41434 & Yamato 000670 & L5 & Antarctica & 3.046 & 0.003046 \\
\hline 44186 & Yamato 003424 & L5 & Antarctica & 3.031 & 0.003031 \\
\hline 44063 & Yamato 003301 & L5 & Antarctica & 2.915 & 0.002915 \\
\hline 44228 & Yamato 003466 & L5 & Antarctica & 2.858 & 0.002858 \\
\hline 44122 & Yamato 003360 & L5 & Antarctica & 2.742 & 0.002742 \\
\hline 44261 & Yamato 003499 & L5 & Antarctica & 2.611 & 0.002611 \\
\hline 44130 & Yamato 003368 & L5 & Antarctica & 2.515 & 0.002515 \\
\hline 44104 & Yamato 003342 & L5 & Antarctica & 2.503 & 0.002503 \\
\hline 36810 & Yamato 980184 & L5 & Antarctica & 2.486 & 0.002486 \\
\hline 44189 & Yamato 003427 & L5 & Antarctica & 2.351 & 0.002351 \\
\hline 44020 & Yamato 003258 & L5 & Antarctica & 2.348 & 0.002348 \\
\hline 44202 & Yamato 003440 & L5 & Antarctica & 2.147 & 0.002147 \\
\hline 44028 & Yamato 003266 & L5 & Antarctica & 2.052 & 0.002052 \\
\hline 36986 & Yamato 980361 & L5 & Antarctica & 1.996 & 0.001996 \\
\hline 44221 & Yamato 003459 & L5 & Antarctica & 1.875 & 0.001875 \\
\hline 44114 & Yamato 003352 & L5 & Antarctica & 1.818 & 0.001818 \\
\hline 44311 & Yamato 003549 & L5 & Antarctica & 1.814 & 0.001814 \\
\hline 44120 & Yamato 003358 & L5 & Antarctica & 1.752 & 0.001752 \\
\hline 44307 & Yamato 003545 & L5 & Antarctica & 1.644 & 0.001644 \\
\hline 66788 & MacKay Glacier 14011 & L5 & Antarctica & 1.6 & 0.0016 \\
\hline 44226 & Yamato 003464 & L5 & Antarctica & 1.426 & 0.001426 \\
\hline 44225 & Yamato 003463 & L5 & Antarctica & 1.402 & 0.001402 \\
\hline 68091 & Miller Range 15535 & L5 & Antarctica & 1.4 & 0.0014 \\
\hline 44206 & Yamato 003444 & L5 & Antarctica & 1.385 & 0.001385 \\
\hline 36638 & Yamato 980012 & L5 & Antarctica & 1.321 & 0.001321 \\
\hline 66719 & Miller Range 15222 & L5 & Antarctica & 1.26 & 0.00126 \\
\hline 44065 & Yamato 003303 & L5 & Antarctica & 1.212 & 0.001212 \\
\hline 44179 & Yamato 003417 & L5 & Antarctica & 1.191 & 0.001191 \\
\hline 44066 & Yamato 003304 & L5 & Antarctica & 1.176 & 0.001176 \\
\hline 36911 & Yamato 980285 & L5 & Antarctica & 1.174 & 0.001174 \\
\hline 66730 & Elephant Moraine 16204 & L5 & Antarctica & 1.08 & 0.00108 \\
\hline 44201 & Yamato 003439 & L5 & Antarctica & 1.045 & 0.001045 \\
\hline 43475 & Yamato 002713 & L5 & Antarctica & 0.935 & 0.000935 \\
\hline 66727 & Elephant Moraine 16198 & L5 & Antarctica & 0.857 & 0.000857 \\
\hline 68079 & Miller Range 15418 & L5 & Antarctica & 0.85 & 0.00085 \\
\hline 44310 & Yamato 003548 & L5 & Antarctica & 0.836 & 0.000836 \\
\hline 44064 & Yamato 003302 & L5 & Antarctica & 0.789 & 0.000789 \\
\hline 36958 & Yamato 980333 & L5 & Antarctica & 0.721 & 0.000721 \\
\hline 44074 & Yamato 003312 & L5 & Antarctica & 0.721 & 0.000721 \\
\hline 44070 & Yamato 003308 & L5 & Antarctica & 0.699 & 0.000699 \\
\hline 44219 & Yamato 003457 & L5 & Antarctica & 0.677 & 0.000677 \\
\hline 66725 & Elephant Moraine 16173 & L5 & Antarctica & 0.663 & 0.000663 \\
\hline
\end{tabular}




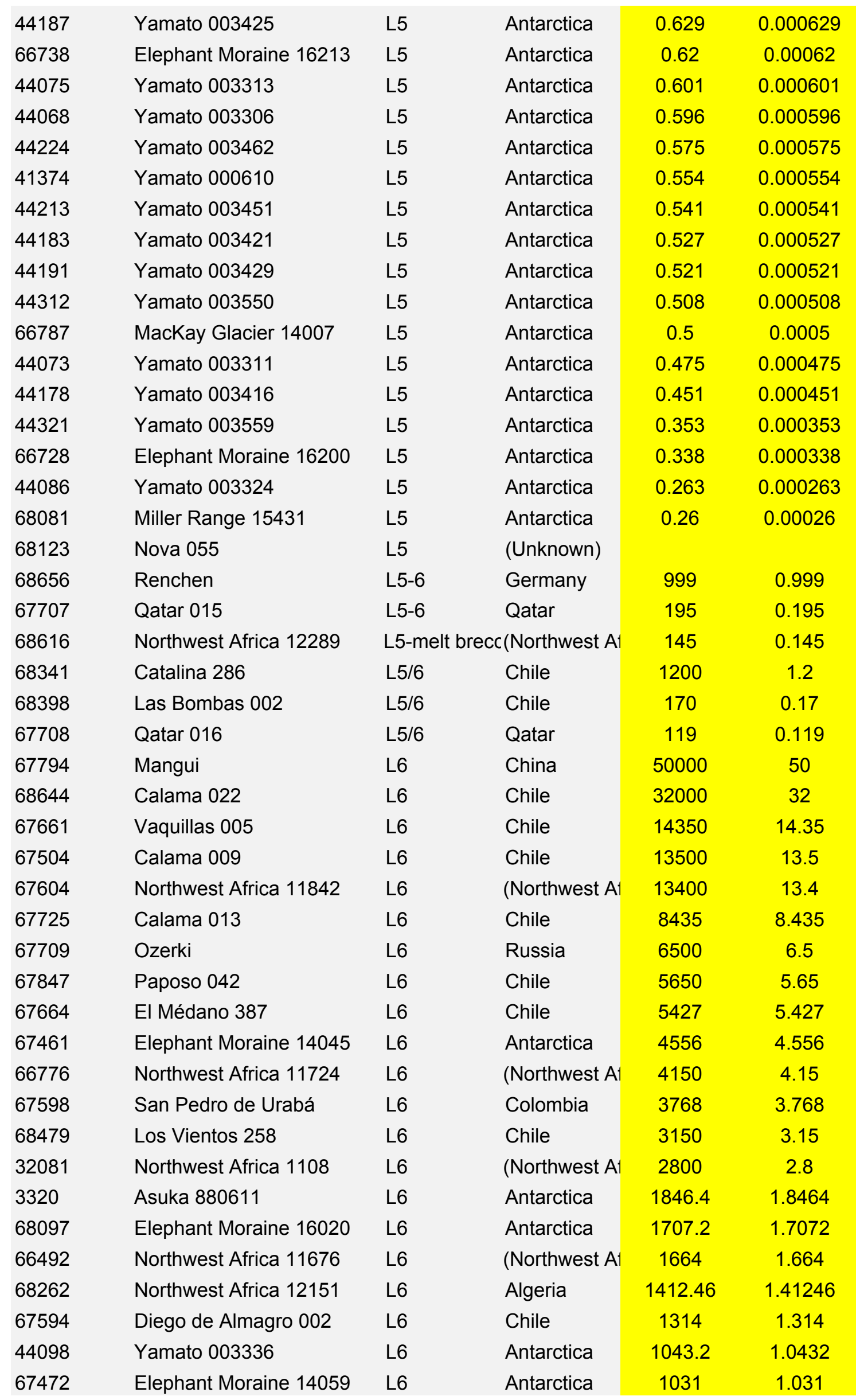




\begin{tabular}{|c|c|c|c|c|c|}
\hline 67595 & Las Bombas 001 & L6 & Chile & 1005 & 1.005 \\
\hline 41929 & Yamato 001167 & L6 & Antarctica & 1002.2 & 1.0022 \\
\hline 67848 & El Médano 391 & L6 & Chile & 984 & 0.984 \\
\hline 31791 & Northwest Africa 669 & L6 & (Northwest At & 908 & 0.908 \\
\hline 31793 & Northwest Africa 671 & L6 & (Northwest Al & 876 & 0.876 \\
\hline 67754 & Northwest Africa 11930 & L6 & (Northwest At & 864 & 0.864 \\
\hline 67473 & Elephant Moraine 14060 & L6 & Antarctica & 849 & 0.849 \\
\hline 31629 & Northwest Africa 436 & L6 & (Northwest Al & 841 & 0.841 \\
\hline 43185 & Yamato 002423 & L6 & Antarctica & 758.1 & 0.7581 \\
\hline 67411 & Northwest Africa 11779 & L6 & (Northwest At & 668.9 & 0.6689 \\
\hline 68334 & Altamira 002 & L6 & Chile & 566 & 0.566 \\
\hline 66502 & Northwest Africa 11686 & L6 & (Northwest A1 & 559 & 0.559 \\
\hline 68344 & Catalina 289 & L6 & Chile & 545 & 0.545 \\
\hline 68654 & Andila & L6 & Chad & 500 & 0.5 \\
\hline 68371 & Los Vientos 247 & L6 & Chile & 424 & 0.424 \\
\hline 67174 & Asuka 12214 & L6 & Antarctica & 413.64 & 0.41364 \\
\hline 67726 & Calama 015 & L6 & Chile & 373 & 0.373 \\
\hline 66497 & Northwest Africa 11681 & L6 & (Northwest Al & 368 & 0.368 \\
\hline 66422 & Northwest Africa 11622 & L6 & (Northwest A1 & 361.47 & 0.36147 \\
\hline 66485 & Mürtschenstock & L6 & Switzerland & 354.5 & 0.3545 \\
\hline 67329 & Asuka 12384 & L6 & Antarctica & 348.16 & 0.34816 \\
\hline 67511 & Los Vientos 218 & L6 & Chile & 326 & 0.326 \\
\hline 68393 & San Juan 097 & L6 & Chile & 313 & 0.313 \\
\hline 67414 & Reckling Peak 14001 & L6 & Antarctica & 304 & 0.304 \\
\hline 68207 & Northwest Africa 12096 & L6 & Algeria & 283.91 & 0.28391 \\
\hline 66670 & Dominion Range 14462 & L6 & Antarctica & 280.69 & 0.28069 \\
\hline 67373 & Asuka 12432 & L6 & Antarctica & 273.14 & 0.27314 \\
\hline 67487 & Northwest Africa 11797 & L6 & (Northwest A1 & 267 & 0.267 \\
\hline 68192 & Northwest Africa 12081 & L6 & Algeria & 265 & 0.265 \\
\hline 67134 & Asuka 12014 & L6 & Antarctica & 259.37 & 0.25937 \\
\hline 66434 & Northwest Africa 11633 & L6 & (Northwest Al & 255 & 0.255 \\
\hline 67888 & Dominion Range 14231 & L6 & Antarctica & 244.62 & 0.24462 \\
\hline 68180 & Northwest Africa 12069 & L6 & Algeria & 243.15 & 0.24315 \\
\hline 67652 & Northwest Africa 11875 & L6 & (Northwest At & 235.19 & 0.23519 \\
\hline 68483 & Northwest Africa 12325 & L6 & (Northwest Al & 228 & 0.228 \\
\hline 66626 & Dominion Range 14300 & L6 & Antarctica & 218.71 & 0.21871 \\
\hline 67518 & Los Vientos 222 & L6 & Chile & 206 & 0.206 \\
\hline 68377 & Los Vientos 254 & L6 & Chile & 199 & 0.199 \\
\hline 66494 & Calama 006 & L6 & Chile & 191 & 0.191 \\
\hline 68098 & Elephant Moraine 16021 & L6 & Antarctica & 183.169 & 0.183169 \\
\hline 67763 & Northwest Africa 11942 & L6 & (Northwest At & 182.9 & 0.1829 \\
\hline 67394 & Catalina 260 & L6 & Chile & 181 & 0.181 \\
\hline 67166 & Asuka 12142 & L6 & Antarctica & 171.68 & 0.17168 \\
\hline 67843 & Sierra Gorda 004 & L6 & Chile & 170 & 0.17 \\
\hline 31647 & Northwest Africa 454 & L6 & (Northwest A1 & 168 & 0.168 \\
\hline
\end{tabular}




\begin{tabular}{|c|c|c|c|c|c|}
\hline 67890 & Dominion Range 14233 & L6 & Antarctica & 164.1 & 0.1641 \\
\hline 67136 & Asuka 12020 & L6 & Antarctica & 163.04 & 0.16304 \\
\hline 66506 & Northwest Africa 11690 & L6 & (Northwest At & 163 & 0.163 \\
\hline 67132 & Asuka 12012 & L6 & Antarctica & 162.5 & 0.1625 \\
\hline 67531 & Los Vientos 225 & L6 & Chile & 161 & 0.161 \\
\hline 67151 & Asuka 12046 & L6 & Antarctica & 160.87 & 0.16087 \\
\hline 67760 & Northwest Africa 11939 & L6 & (Northwest Al & 152.4 & 0.1524 \\
\hline 67465 & Elephant Moraine 14050 & L6 & Antarctica & 146.3 & 0.1463 \\
\hline 67232 & Asuka 12281 & L6 & Antarctica & 135.4 & 0.1354 \\
\hline 67835 & Northwest Africa 11984 & L6 & (Northwest Al & 134.6 & 0.1346 \\
\hline 67206 & Asuka 12255 & L6 & Antarctica & 130.41 & 0.13041 \\
\hline 68391 & San Juan 095 & L6 & Chile & 129.4 & 0.1294 \\
\hline 67969 & Dominion Range 14415 & L6 & Antarctica & 128.801 & 0.128801 \\
\hline 67224 & Asuka 12273 & L6 & Antarctica & 126.11 & 0.12611 \\
\hline 68370 & Los Vientos 246 & L6 & Chile & 123 & 0.123 \\
\hline 67263 & Asuka 12313 & L6 & Antarctica & 119.83 & 0.11983 \\
\hline 68365 & Los Vientos 241 & L6 & Chile & 117 & 0.117 \\
\hline 67526 & Catalina 263 & L6 & Chile & 116 & 0.116 \\
\hline 68368 & Los Vientos 244 & L6 & Chile & 110 & 0.11 \\
\hline 67968 & Dominion Range 14414 & L6 & Antarctica & 100.68 & 0.10068 \\
\hline 33223 & Northwest Africa 2730 & L6 & (Northwest At & 100 & 0.1 \\
\hline 67971 & Dominion Range 14417 & L6 & Antarctica & 96.903 & 0.096903 \\
\hline 66542 & Dominion Range 14122 & L6 & Antarctica & 95.83 & 0.09583 \\
\hline 67453 & Elephant Moraine 14037 & L6 & Antarctica & 93.8 & 0.0938 \\
\hline 36780 & Yamato 980154 & L6 & Antarctica & 93.31 & 0.09331 \\
\hline 67387 & Los Vientos 214 & L6 & Chile & 93.1 & 0.0931 \\
\hline 66543 & Dominion Range 14123 & L6 & Antarctica & 92.66 & 0.09266 \\
\hline 67432 & Elephant Moraine 14016 & L6 & Antarctica & 90.6 & 0.0906 \\
\hline 66596 & Dominion Range 14220 & L6 & Antarctica & 87.186 & 0.087186 \\
\hline 67687 & Catalina 273 & L6 & Chile & 86 & 0.086 \\
\hline 68537 & Sierra Gorda 011 & L6 & Chile & 84 & 0.084 \\
\hline 67212 & Asuka 12261 & L6 & Antarctica & 83.12 & 0.08312 \\
\hline 67973 & Dominion Range 14419 & L6 & Antarctica & 81.815 & 0.081815 \\
\hline 67617 & Northwest Africa 11868 & L6 & (Northwest At & 80.15 & 0.08015 \\
\hline 67390 & Los Vientos 217 & L6 & Chile & 79 & 0.079 \\
\hline 66633 & Dominion Range 14308 & L6 & Antarctica & 76.049 & 0.076049 \\
\hline 67447 & Elephant Moraine 14031 & L6 & Antarctica & 75.6 & 0.0756 \\
\hline 66566 & Dominion Range 14178 & L6 & Antarctica & 74.96 & 0.07496 \\
\hline 67999 & Dominion Range 14474 & L6 & Antarctica & 72.51 & 0.07251 \\
\hline 67960 & Dominion Range 14406 & L6 & Antarctica & 71.72 & 0.07172 \\
\hline 67312 & Asuka 12367 & L6 & Antarctica & 71.5 & 0.0715 \\
\hline 67654 & Northwest Africa 11877 & L6 & (Northwest Al & 71.38 & 0.07138 \\
\hline 67529 & Catalina 266 & L6 & Chile & 69.9 & 0.0699 \\
\hline 67346 & Asuka 12404 & L6 & Antarctica & 69.75 & 0.06975 \\
\hline 67954 & Dominion Range 14400 & L6 & Antarctica & 65.518 & 0.065518 \\
\hline
\end{tabular}




\begin{tabular}{|c|c|c|c|c|c|}
\hline 67435 & Elephant Moraine 14019 & L6 & Antarctica & 64.7 & 0.0647 \\
\hline 67892 & Dominion Range 14235 & L6 & Antarctica & 63.72 & 0.06372 \\
\hline 66657 & Dominion Range 14399 & L6 & Antarctica & 60.93 & 0.06093 \\
\hline 68000 & Dominion Range 14475 & L6 & Antarctica & 60.43 & 0.06043 \\
\hline 68001 & Dominion Range 14476 & L6 & Antarctica & 60.16 & 0.06016 \\
\hline 67729 & Calama 018 & L6 & Chile & 60 & 0.06 \\
\hline 68170 & Northwest Africa 12059 & L6 & Algeria & 59.52 & 0.05952 \\
\hline 68003 & Dominion Range 14478 & L6 & Antarctica & 58.71 & 0.05871 \\
\hline 26079 & Yamato 790730 & L6 & Antarctica & 58.32 & 0.05832 \\
\hline 67532 & Catalina 268 & L6 & Chile & 58 & 0.058 \\
\hline 67956 & Dominion Range 14402 & L6 & Antarctica & 57.619 & 0.057619 \\
\hline 66532 & Dominion Range 14102 & L6 & Antarctica & 55.38 & 0.05538 \\
\hline 67965 & Dominion Range 14411 & L6 & Antarctica & 53.791 & 0.053791 \\
\hline 66857 & Northwest Africa 11759 & L6 & (Northwest At & 52.2 & 0.0522 \\
\hline 67937 & Dominion Range 14344 & L6 & Antarctica & 50.959 & 0.050959 \\
\hline 67141 & Asuka 12029 & L6 & Antarctica & 50.53 & 0.05053 \\
\hline 66687 & Dominion Range 14519 & L6 & Antarctica & 50.173 & 0.050173 \\
\hline 67878 & Dominion Range 14210 & L6 & Antarctica & 48.399 & 0.048399 \\
\hline 67674 & Northwest Africa 8285 & L6 & (Northwest A1 & 48 & 0.048 \\
\hline 66558 & Dominion Range 14139 & L6 & Antarctica & 47.05 & 0.04705 \\
\hline 68100 & Elephant Moraine 16043 & L6 & Antarctica & 47.04 & 0.04704 \\
\hline 68394 & San Juan 098 & L6 & Chile & 45 & 0.045 \\
\hline 67938 & Dominion Range 14345 & L6 & Antarctica & 44.888 & 0.044888 \\
\hline 66652 & Dominion Range 14394 & L6 & Antarctica & 44.72 & 0.04472 \\
\hline 66634 & Dominion Range 14309 & L6 & Antarctica & 44.139 & 0.044139 \\
\hline 68002 & Dominion Range 14477 & L6 & Antarctica & 43.11 & 0.04311 \\
\hline 66547 & Dominion Range 14128 & L6 & Antarctica & 42.99 & 0.04299 \\
\hline 67957 & Dominion Range 14403 & L6 & Antarctica & 41.377 & 0.041377 \\
\hline 68125 & Willcox Playa 011 & L6 & United States & 41 & 0.041 \\
\hline 67941 & Dominion Range 14348 & L6 & Antarctica & 40.59 & 0.04059 \\
\hline 67326 & Asuka 12381 & L6 & Antarctica & 40.343 & 0.040343 \\
\hline 67332 & Asuka 12387 & L6 & Antarctica & 40.291 & 0.040291 \\
\hline 68349 & Catalina 294 & L6 & Chile & 40.1 & 0.0401 \\
\hline 67282 & Asuka 12336 & L6 & Antarctica & 39.521 & 0.039521 \\
\hline 66684 & Dominion Range 14516 & L6 & Antarctica & 39.384 & 0.039384 \\
\hline 67934 & Dominion Range 14341 & L6 & Antarctica & 39.217 & 0.039217 \\
\hline 67246 & Asuka 12296 & L6 & Antarctica & 39.179 & 0.039179 \\
\hline 67522 & El Médano 383 & L6 & Chile & 39 & 0.039 \\
\hline 68253 & Northwest Africa 12142 & L6 & Algeria & 38.47 & 0.03847 \\
\hline 66537 & Dominion Range 14107 & L6 & Antarctica & 37.319 & 0.037319 \\
\hline 68062 & Miller Range 15376 & L6 & Antarctica & 36.611 & 0.036611 \\
\hline 67340 & Asuka 12397 & L6 & Antarctica & 36.144 & 0.036144 \\
\hline 66649 & Dominion Range 14391 & L6 & Antarctica & 36.12 & 0.03612 \\
\hline 66577 & Dominion Range 14190 & L6 & Antarctica & 36.032 & 0.036032 \\
\hline 67480 & Elephant Moraine 14069 & L6 & Antarctica & 36 & 0.036 \\
\hline
\end{tabular}




\begin{tabular}{|c|c|c|c|c|c|}
\hline 66683 & Dominion Range 14515 & L6 & Antarctica & 35.984 & 0.035984 \\
\hline 67943 & Dominion Range 14350 & L6 & Antarctica & 35.852 & 0.035852 \\
\hline 66531 & Dominion Range 14101 & L6 & Antarctica & 35.31 & 0.03531 \\
\hline 67339 & Asuka 12396 & L6 & Antarctica & 35.225 & 0.035225 \\
\hline 67995 & Dominion Range 14470 & L6 & Antarctica & 35.16 & 0.03516 \\
\hline 67980 & Dominion Range 14426 & L6 & Antarctica & 35.112 & 0.035112 \\
\hline 67689 & Catalina 275 & L6 & Chile & 34.8 & 0.0348 \\
\hline 66557 & Dominion Range 14138 & L6 & Antarctica & 34.63 & 0.03463 \\
\hline 67675 & Northwest Africa 8286 & L6 & (Northwest A 1 & 34 & 0.034 \\
\hline 67985 & Dominion Range 14431 & L6 & Antarctica & 33.987 & 0.033987 \\
\hline 67940 & Dominion Range 14347 & L6 & Antarctica & 33.898 & 0.033898 \\
\hline 66538 & Dominion Range 14108 & L6 & Antarctica & 33.249 & 0.033249 \\
\hline 67896 & Dominion Range 14253 & L6 & Antarctica & 33.229 & 0.033229 \\
\hline 67933 & Dominion Range 14340 & L6 & Antarctica & 33.113 & 0.033113 \\
\hline 66686 & Dominion Range 14518 & L6 & Antarctica & 32.429 & 0.032429 \\
\hline 67978 & Dominion Range 14424 & L6 & Antarctica & 32.146 & 0.032146 \\
\hline 67304 & Asuka 12359 & L6 & Antarctica & 32.074 & 0.032074 \\
\hline 68397 & San Juan 102 & L6 & Chile & 32 & 0.032 \\
\hline 67914 & Dominion Range 14291 & L6 & Antarctica & 31.977 & 0.031977 \\
\hline 66511 & Dominion Range 14030 & L6 & Antarctica & 31.88 & 0.03188 \\
\hline 66530 & Dominion Range 14100 & L6 & Antarctica & 31.461 & 0.031461 \\
\hline 66698 & Dominion Range 14531 & L6 & Antarctica & 31.36 & 0.03136 \\
\hline 66656 & Dominion Range 14398 & L6 & Antarctica & 31.11 & 0.03111 \\
\hline 67986 & Dominion Range 14432 & L6 & Antarctica & 31.067 & 0.031067 \\
\hline 67902 & Dominion Range 14259 & L6 & Antarctica & 31.06 & 0.03106 \\
\hline 66534 & Dominion Range 14104 & L6 & Antarctica & 30.852 & 0.030852 \\
\hline 68233 & Northwest Africa 12122 & L6 & Algeria & 30.81 & 0.03081 \\
\hline 68007 & Dominion Range 14492 & L6 & Antarctica & 30.71 & 0.03071 \\
\hline 66525 & Dominion Range 14085 & L6 & Antarctica & 30.7 & 0.0307 \\
\hline 67454 & Elephant Moraine 14038 & L6 & Antarctica & 30.5 & 0.0305 \\
\hline 66521 & Dominion Range 14081 & L6 & Antarctica & 30.45 & 0.03045 \\
\hline 67894 & Dominion Range 14251 & L6 & Antarctica & 30.263 & 0.030263 \\
\hline 66553 & Dominion Range 14134 & L6 & Antarctica & 30.11 & 0.03011 \\
\hline 66550 & Dominion Range 14131 & L6 & Antarctica & 30.01 & 0.03001 \\
\hline 66441 & Northwest Africa 11648 & L6 & (Northwest Al & 30 & 0.03 \\
\hline 66535 & Dominion Range 14105 & L6 & Antarctica & 29.744 & 0.029744 \\
\hline 67882 & Dominion Range 14214 & L6 & Antarctica & 29.675 & 0.029675 \\
\hline 68060 & Miller Range 15374 & L6 & Antarctica & 29.383 & 0.029383 \\
\hline 67348 & Asuka 12406 & L6 & Antarctica & 28.726 & 0.028726 \\
\hline 66681 & Dominion Range 14513 & L6 & Antarctica & 28.481 & 0.028481 \\
\hline 67947 & Dominion Range 14354 & L6 & Antarctica & 28.381 & 0.028381 \\
\hline 66678 & Dominion Range 14510 & L6 & Antarctica & 28.124 & 0.028124 \\
\hline 67314 & Asuka 12369 & L6 & Antarctica & 28.007 & 0.028007 \\
\hline 68162 & Northwest Africa 12051 & L6 & Algeria & 27.77 & 0.02777 \\
\hline 67913 & Dominion Range 14290 & L6 & Antarctica & 27.251 & 0.027251 \\
\hline
\end{tabular}


$67884 \quad$ Dominion Range $14216 \quad$ L6

67885 Dominion Range 14217 L6

66651 Dominion Range 14393 L6

66536

66648

66650

66655

68021

67463

67942

67997

66704

68354

67921

67923

66579

66705

67614

68031

67977

66527

67952

67880

67616

66608

66611

67939

67924

67912

66426

68222

67270

68015

67450

66658

67910

67925

67926

66700

66607

66551

67974

67688

67946

68033
Dominion Range 14106

Dominion Range 14390

Dominion Range 14392

Dominion Range 14397

Dominion Range 14506

Elephant Moraine 14047

Dominion Range 14349

Dominion Range 14472

Dominion Range 14537

El Médano 392

Dominion Range 14298

Dominion Range 14310

Dominion Range 14193

Dominion Range 14538

Northwest Africa 11863

Miller Range 15084

Dominion Range 14423

Dominion Range 14087

Dominion Range 14368

Dominion Range 14212

Northwest Africa 11865

Dominion Range 14242

Dominion Range 14245

Dominion Range 14346

Dominion Range 14311

Dominion Range 14269

Northwest Africa 11626

Northwest Africa 12111

Asuka 12321

Dominion Range 14500

Elephant Moraine 14034

Dominion Range 14450

Dominion Range 14267

Dominion Range 14312

Dominion Range 14313

Dominion Range 14533

Dominion Range 14241

Dominion Range 14132

Dominion Range 14420

Catalina 274

Dominion Range 14353

Miller Range 15087

\section{6
6}

L6

L6

L6

L6

L6 L6 L6 L6 L6 L6 L6 L6 L6 L6 L6

L6

L6

L6

L6

L6

L6

L6

L6

L6

L6

L6

L6

L6

L6

L6

L6

L6

L6

L6

L6

L6

L6

L6

L6

L6

L6

\begin{tabular}{|c|c|c|}
\hline Antarctica & 27.106 & 0.027106 \\
\hline Antarctica & 27.096 & 0.027096 \\
\hline Antarctica & 27.09 & 0.02709 \\
\hline Antarctica & 26.979 & 0.026979 \\
\hline Antarctica & 26.66 & 0.02666 \\
\hline Antarctica & 26.52 & 0.02652 \\
\hline Antarctica & 26.48 & 0.02648 \\
\hline Antarctica & 26.455 & 0.026455 \\
\hline Antarctica & 26.4 & 0.0264 \\
\hline Antarctica & 26.291 & 0.026291 \\
\hline Antarctica & 26.11 & 0.02611 \\
\hline Antarctica & 26.09 & 0.02609 \\
\hline Chile & 26 & 0.026 \\
\hline Antarctica & 25.743 & 0.025743 \\
\hline Antarctica & 25.278 & 0.025278 \\
\hline Antarctica & 25.186 & 0.025186 \\
\hline Antarctica & 25.12 & 0.02512 \\
\hline (Northwest Al & 24.92 & 0.02492 \\
\hline Antarctica & 24.85 & 0.02485 \\
\hline Antarctica & 24.475 & 0.024475 \\
\hline Antarctica & 24.46 & 0.02446 \\
\hline Antarctica & 23.887 & 0.023887 \\
\hline Antarctica & 23.509 & 0.023509 \\
\hline (Northwest A1 & 23.48 & 0.02348 \\
\hline Antarctica & 23.19 & 0.02319 \\
\hline Antarctica & 23.12 & 0.02312 \\
\hline Antarctica & 23.062 & 0.023062 \\
\hline Antarctica & 23.055 & 0.023055 \\
\hline Antarctica & 22.804 & 0.022804 \\
\hline (Northwest Al & 22.73 & 0.02273 \\
\hline Algeria & 22.49 & 0.02249 \\
\hline Antarctica & 22.367 & 0.022367 \\
\hline Antarctica & 21.98 & 0.02198 \\
\hline Antarctica & 21.8 & 0.0218 \\
\hline Antarctica & 21.44 & 0.02144 \\
\hline Antarctica & 21.307 & 0.021307 \\
\hline Antarctica & 20.873 & 0.020873 \\
\hline Antarctica & 20.598 & 0.020598 \\
\hline Antarctica & 20.58 & 0.02058 \\
\hline Antarctica & 20.47 & 0.02047 \\
\hline Antarctica & 20.31 & 0.02031 \\
\hline Antarctica & 20.093 & 0.020093 \\
\hline Chile & 20 & 0.02 \\
\hline Antarctica & 19.662 & 0.019662 \\
\hline Antarctica & 19.597 & 0.019597 \\
\hline
\end{tabular}




\begin{tabular}{|c|c|c|c|c|c|}
\hline 67309 & Asuka 12364 & L6 & Antarctica & 19.428 & 0.019428 \\
\hline 66549 & Dominion Range 14130 & L6 & Antarctica & 19.33 & 0.01933 \\
\hline 68022 & Dominion Range 14507 & L6 & Antarctica & 19.293 & 0.019293 \\
\hline 67424 & Elephant Moraine 14006 & L6 & Antarctica & 19 & 0.019 \\
\hline 66697 & Dominion Range 14530 & L6 & Antarctica & 18.87 & 0.01887 \\
\hline 66682 & Dominion Range 14514 & L6 & Antarctica & 18.548 & 0.018548 \\
\hline 68018 & Dominion Range 14503 & L6 & Antarctica & 18.51 & 0.01851 \\
\hline 66688 & Dominion Range 14520 & L6 & Antarctica & 18.27 & 0.01827 \\
\hline 66713 & Dominion Range 14556 & L6 & Antarctica & 18.13 & 0.01813 \\
\hline 68387 & San Juan 091 & L6 & Chile & 18.1 & 0.0181 \\
\hline 67916 & Dominion Range 14293 & L6 & Antarctica & 18.076 & 0.018076 \\
\hline 68016 & Dominion Range 14501 & L6 & Antarctica & 18.055 & 0.018055 \\
\hline 66554 & Dominion Range 14135 & L6 & Antarctica & 18.04 & 0.01804 \\
\hline 67922 & Dominion Range 14299 & L6 & Antarctica & 17.966 & 0.017966 \\
\hline 68020 & Dominion Range 14505 & L6 & Antarctica & 17.842 & 0.017842 \\
\hline 67918 & Dominion Range 14295 & L6 & Antarctica & 17.726 & 0.017726 \\
\hline 67927 & Dominion Range 14314 & L6 & Antarctica & 17.513 & 0.017513 \\
\hline 67370 & Asuka 12428 & L6 & Antarctica & 17.255 & 0.017255 \\
\hline 66583 & Dominion Range 14197 & L6 & Antarctica & 17.157 & 0.017157 \\
\hline 68028 & Dominion Range 14562 & L6 & Antarctica & 17.133 & 0.017133 \\
\hline 68378 & Los Vientos 255 & L6 & Chile & 17 & 0.017 \\
\hline 67306 & Asuka 12361 & L6 & Antarctica & 16.926 & 0.016926 \\
\hline 67930 & Dominion Range 14317 & L6 & Antarctica & 16.705 & 0.016705 \\
\hline 67443 & Elephant Moraine 14027 & L6 & Antarctica & 16.7 & 0.0167 \\
\hline 67507 & Sierra Gorda 002 & L6 & Chile & 16.4 & 0.0164 \\
\hline 68313 & Troy Dry Lake & L6 & United States & 16.3 & 0.0163 \\
\hline 68005 & Dominion Range 14490 & L6 & Antarctica & 16.29 & 0.01629 \\
\hline 67951 & Dominion Range 14358 & L6 & Antarctica & 16.209 & 0.016209 \\
\hline 68019 & Dominion Range 14504 & L6 & Antarctica & 16.092 & 0.016092 \\
\hline 67684 & Los Vientos 228 & L6 & Chile & 15.8 & 0.0158 \\
\hline 66696 & Dominion Range 14529 & L6 & Antarctica & 15.63 & 0.01563 \\
\hline 67945 & Dominion Range 14352 & L6 & Antarctica & 15.329 & 0.015329 \\
\hline 68017 & Dominion Range 14502 & L6 & Antarctica & 15.128 & 0.015128 \\
\hline 68036 & Miller Range 15090 & L6 & Antarctica & 15.084 & 0.015084 \\
\hline 67931 & Dominion Range 14318 & L6 & Antarctica & 14.912 & 0.014912 \\
\hline 68011 & Dominion Range 14496 & L6 & Antarctica & 14.86 & 0.01486 \\
\hline 66711 & Dominion Range 14554 & L6 & Antarctica & 14.75 & 0.01475 \\
\hline 68281 & Northwest Africa 12170 & L6 & Algeria & 14.71 & 0.01471 \\
\hline 67900 & Dominion Range 14257 & L6 & Antarctica & 14.357 & 0.014357 \\
\hline 66589 & Dominion Range 14203 & L6 & Antarctica & 14.34 & 0.01434 \\
\hline 66640 & Dominion Range 14365 & L6 & Antarctica & 14.304 & 0.014304 \\
\hline 66592 & Dominion Range 14206 & L6 & Antarctica & 14.2 & 0.0142 \\
\hline 67451 & Elephant Moraine 14035 & L6 & Antarctica & 14.1 & 0.0141 \\
\hline 67919 & Dominion Range 14296 & L6 & Antarctica & 14.015 & 0.014015 \\
\hline 66556 & Dominion Range 14137 & L6 & Antarctica & 13.87 & 0.01387 \\
\hline
\end{tabular}


67369

Asuka 12427

67907

Dominion Range 14264

L6

66576

Dominion Range 14189

66693

Dominion Range 14525

66643

Dominion Range 14369

66595

68009

Dominion Range 14209

68010

68026

Dominion Range 14494

Dominion Range 14495

68379

Dominion Range 14560

66689

66694

Los Vientos 256

68023

Dominion Range 14521

L6 L6

L6

L6

L6

L6

L6

L6

L6

L6

67932

66690

68372

67298

66623

66636

66515

66588

66708

67413

68008

67929

66638

68014

66612

66692

66715

67446

66635

67903

66552

66663

67388

67226

3897

66528

66664

66594

40934

67430

66641

67245

Dominion Range 14526

L6

L6

L6

Dominion Range 14319

Dominion Range 14522

Los Vientos 248

Asuka 12353

Dominion Range 14278

Dominion Range 14361

Dominion Range 14034

Dominion Range 14202

Dominion Range 14551

Calama 008

Dominion Range 14493

Dominion Range 14316

Dominion Range 14363

Dominion Range 14499

Dominion Range 14246

Dominion Range 14524

Dominion Range 14558

Elephant Moraine 14030

Dominion Range 14360

Dominion Range 14260

Dominion Range 14133

Dominion Range 14455

Los Vientos 215

Asuka 12275

Asuka 881188

Dominion Range 14088

Dominion Range 14456

Dominion Range 14208

Yamato 000169

Elephant Moraine 14014

Dominion Range 14366

Asuka 12295
Antarctica

Antarctica

Antarctica

Antarctica

Antarctica

Antarctica

Antarctica

Antarctica

Antarctica

Chile

Antarctica

Antarctica

Antarctica

Antarctica

Antarctica

Chile

Antarctica

Antarctica

Antarctica

Antarctica

Antarctica

Antarctica

Chile

Antarctica

Antarctica

Antarctica

Antarctica

Antarctica

Antarctica

Antarctica

Antarctica

Antarctica

Antarctica

Antarctica

Antarctica

Chile

Antarctica

Antarctica

Antarctica

Antarctica

Antarctica

Antarctica

Antarctica

Antarctica

Antarctica
13.821

13.742

13.63

13.52

13.469

13.42

13.28

13.27

13.268

13.2

13.08

12.92

12.874

12.388

12.08

12

11.973

11.936

11.825

11.65

11.54

11.29

11.23

11.19

11.129

10.956

10.83

0.01083

10.68

0.01068

10.55

0.01055

10.24

0.01024

10.2

0.0102

10.154

0.010154

9.975

0.009975

9.92

0.00992

9.92

0.00992

9.9

0.0099

9.839

0.009839

9.688

0.009688

9.6

0.0096

9.19

0.00919

9.14

0.00914

9.08

0.00908

9

0.009

8.923

0.008923

8.372 0.008372 


\begin{tabular}{|c|c|c|c|c|c|}
\hline 2440 & Asuka 87083 & L6 & Antarctica & 8.355 & 0.008355 \\
\hline 67437 & Elephant Moraine 14021 & L6 & Antarctica & 8.1 & 0.0081 \\
\hline 67255 & Asuka 12305 & L6 & Antarctica & 8.041 & 0.008041 \\
\hline 66642 & Dominion Range 14367 & L6 & Antarctica & 8 & 0.008 \\
\hline 66709 & Dominion Range 14552 & L6 & Antarctica & 7.8 & 0.0078 \\
\hline 67247 & Asuka 12297 & L6 & Antarctica & 7.527 & 0.007527 \\
\hline 66616 & Dominion Range 14271 & L6 & Antarctica & 7.451 & 0.007451 \\
\hline 66584 & Dominion Range 14198 & L6 & Antarctica & 7.386 & 0.007386 \\
\hline 68027 & Dominion Range 14561 & L6 & Antarctica & 7.176 & 0.007176 \\
\hline 67905 & Dominion Range 14262 & L6 & Antarctica & 7.146 & 0.007146 \\
\hline 67445 & Elephant Moraine 14029 & L6 & Antarctica & 6.7 & 0.0067 \\
\hline 67948 & Dominion Range 14355 & L6 & Antarctica & 6.607 & 0.006607 \\
\hline 66714 & Dominion Range 14557 & L6 & Antarctica & 6.44 & 0.00644 \\
\hline 67300 & Asuka 12355 & L6 & Antarctica & 6.361 & 0.006361 \\
\hline 67422 & Elephant Moraine 14004 & L6 & Antarctica & 6 & 0.006 \\
\hline 68375 & Los Vientos 252 & L6 & Chile & 6 & 0.006 \\
\hline 66712 & Dominion Range 14555 & L6 & Antarctica & 5.99 & 0.00599 \\
\hline 66996 & Asuka 09367 & L6 & Antarctica & 5.572 & 0.005572 \\
\hline 67438 & Elephant Moraine 14022 & L6 & Antarctica & 5.3 & 0.0053 \\
\hline 42930 & Yamato 002168 & L6 & Antarctica & 5.187 & 0.005187 \\
\hline 67064 & Asuka 09564 & L6 & Antarctica & 5.165 & 0.005165 \\
\hline 27021 & Yamato 791672 & L6 & Antarctica & 5.13 & 0.00513 \\
\hline 67519 & El Médano 382 & L6 & Chile & 5.1 & 0.0051 \\
\hline 44301 & Yamato 003539 & L6 & Antarctica & 4.989 & 0.004989 \\
\hline 44121 & Yamato 003359 & L6 & Antarctica & 4.93 & 0.00493 \\
\hline 44127 & Yamato 003365 & L6 & Antarctica & 4.875 & 0.004875 \\
\hline 41250 & Yamato 000485 & L6 & Antarctica & 4.812 & 0.004812 \\
\hline 25111 & Yamato 75070 & L6 & Antarctica & 4.75 & 0.00475 \\
\hline 41606 & Yamato 000844 & L6 & Antarctica & 4.733 & 0.004733 \\
\hline 44056 & Yamato 003294 & L6 & Antarctica & 4.69 & 0.00469 \\
\hline 44265 & Yamato 003503 & L6 & Antarctica & 4.596 & 0.004596 \\
\hline 44136 & Yamato 003374 & L6 & Antarctica & 4.529 & 0.004529 \\
\hline 44242 & Yamato 003480 & L6 & Antarctica & 4.438 & 0.004438 \\
\hline 41859 & Yamato 001097 & L6 & Antarctica & 4.41 & 0.00441 \\
\hline 44253 & Yamato 003491 & L6 & Antarctica & 4.376 & 0.004376 \\
\hline 44292 & Yamato 003530 & L6 & Antarctica & 4.375 & 0.004375 \\
\hline 43848 & Yamato 003086 & L6 & Antarctica & 4.359 & 0.004359 \\
\hline 44054 & Yamato 003292 & L6 & Antarctica & 4.276 & 0.004276 \\
\hline 67021 & Asuka 09410 & L6 & Antarctica & 4.228 & 0.004228 \\
\hline 43781 & Yamato 003019 & L6 & Antarctica & 4.185 & 0.004185 \\
\hline 44216 & Yamato 003454 & L6 & Antarctica & 4.176 & 0.004176 \\
\hline 44091 & Yamato 003329 & L6 & Antarctica & 4.162 & 0.004162 \\
\hline 44126 & Yamato 003364 & L6 & Antarctica & 4.135 & 0.004135 \\
\hline 44026 & Yamato 003264 & L6 & Antarctica & 4.099 & 0.004099 \\
\hline 44278 & Yamato 003516 & L6 & Antarctica & 4.086 & 0.004086 \\
\hline
\end{tabular}




\begin{tabular}{|c|c|c|c|c|c|}
\hline 43856 & Yamato 003094 & L6 & Antarctica & 3.998 & 0.003998 \\
\hline 67439 & Elephant Moraine 14023 & L6 & Antarctica & 3.9 & 0.0039 \\
\hline 36891 & Yamato 980265 & L6 & Antarctica & 3.9 & 0.0039 \\
\hline 66919 & Asuka 09270 & L6 & Antarctica & 3.841 & 0.003841 \\
\hline 66523 & Dominion Range 14083 & L6 & Antarctica & 3.83 & 0.00383 \\
\hline 43801 & Yamato 003039 & L6 & Antarctica & 3.811 & 0.003811 \\
\hline 44025 & Yamato 003263 & L6 & Antarctica & 3.756 & 0.003756 \\
\hline 44125 & Yamato 003363 & L6 & Antarctica & 3.731 & 0.003731 \\
\hline 44055 & Yamato 003293 & L6 & Antarctica & 3.718 & 0.003718 \\
\hline 67431 & Elephant Moraine 14015 & L6 & Antarctica & 3.6 & 0.0036 \\
\hline 44236 & Yamato 003474 & L6 & Antarctica & 3.565 & 0.003565 \\
\hline 44245 & Yamato 003483 & L6 & Antarctica & 3.549 & 0.003549 \\
\hline 67054 & Asuka 09490 & L6 & Antarctica & 3.523 & 0.003523 \\
\hline 44241 & Yamato 003479 & L6 & Antarctica & 3.491 & 0.003491 \\
\hline 67436 & Elephant Moraine 14020 & L6 & Antarctica & 3.4 & 0.0034 \\
\hline 44052 & Yamato 003290 & L6 & Antarctica & 3.375 & 0.003375 \\
\hline 44271 & Yamato 003509 & L6 & Antarctica & 3.319 & 0.003319 \\
\hline 41387 & Yamato 000623 & L6 & Antarctica & 3.296 & 0.003296 \\
\hline 44027 & Yamato 003265 & L6 & Antarctica & 3.261 & 0.003261 \\
\hline 44232 & Yamato 003470 & L6 & Antarctica & 3.257 & 0.003257 \\
\hline 43844 & Yamato 003082 & L6 & Antarctica & 3.251 & 0.003251 \\
\hline 44129 & Yamato 003367 & L6 & Antarctica & 3.238 & 0.003238 \\
\hline 67442 & Elephant Moraine 14026 & L6 & Antarctica & 3.2 & 0.0032 \\
\hline 44264 & Yamato 003502 & L6 & Antarctica & 3.173 & 0.003173 \\
\hline 44283 & Yamato 003521 & L6 & Antarctica & 3.161 & 0.003161 \\
\hline 44199 & Yamato 003437 & L6 & Antarctica & 3.143 & 0.003143 \\
\hline 67055 & Asuka 09492 & L6 & Antarctica & 3.131 & 0.003131 \\
\hline 44240 & Yamato 003478 & L6 & Antarctica & 3.086 & 0.003086 \\
\hline 43914 & Yamato 003152 & L6 & Antarctica & 3.075 & 0.003075 \\
\hline 44250 & Yamato 003488 & L6 & Antarctica & 3.019 & 0.003019 \\
\hline 68339 & Catalina 284 & L6 & Chile & 3 & 0.003 \\
\hline 67004 & Asuka 09386 & L6 & Antarctica & 2.974 & 0.002974 \\
\hline 44123 & Yamato 003361 & L6 & Antarctica & 2.932 & 0.002932 \\
\hline 44119 & Yamato 003357 & L6 & Antarctica & 2.922 & 0.002922 \\
\hline 67423 & Elephant Moraine 14005 & L6 & Antarctica & 2.9 & 0.0029 \\
\hline 67449 & Elephant Moraine 14033 & L6 & Antarctica & 2.9 & 0.0029 \\
\hline 36855 & Yamato 980229 & L6 & Antarctica & 2.9 & 0.0029 \\
\hline 41666 & Yamato 000904 & L6 & Antarctica & 2.892 & 0.002892 \\
\hline 44246 & Yamato 003484 & L6 & Antarctica & 2.882 & 0.002882 \\
\hline 67066 & Asuka 09601 & L6 & Antarctica & 2.814 & 0.002814 \\
\hline 36960 & Yamato 980335 & L6 & Antarctica & 2.81 & 0.00281 \\
\hline 44288 & Yamato 003526 & L6 & Antarctica & 2.747 & 0.002747 \\
\hline 44139 & Yamato 003377 & L6 & Antarctica & 2.676 & 0.002676 \\
\hline 36926 & Yamato 980300 & L6 & Antarctica & 2.654 & 0.002654 \\
\hline 67056 & Asuka 09493 & L6 & Antarctica & 2.627 & 0.002627 \\
\hline
\end{tabular}




\begin{tabular}{|c|c|c|c|c|c|}
\hline 44204 & Yamato 003442 & L6 & Antarctica & 2.609 & 0.002609 \\
\hline 67062 & Asuka 09557 & L6 & Antarctica & 2.603 & 0.002603 \\
\hline 67429 & Elephant Moraine 14012 & L6 & Antarctica & 2.6 & 0.0026 \\
\hline 36970 & Yamato 980345 & L6 & Antarctica & 2.585 & 0.002585 \\
\hline 36729 & Yamato 980103 & L6 & Antarctica & 2.569 & 0.002569 \\
\hline 36645 & Yamato 980019 & L6 & Antarctica & 2.565 & 0.002565 \\
\hline 36935 & Yamato 980309 & L6 & Antarctica & 2.564 & 0.002564 \\
\hline 67441 & Elephant Moraine 14025 & L6 & Antarctica & 2.5 & 0.0025 \\
\hline 67065 & Asuka 09600 & L6 & Antarctica & 2.482 & 0.002482 \\
\hline 36914 & Yamato 980288 & L6 & Antarctica & 2.474 & 0.002474 \\
\hline 36912 & Yamato 980286 & L6 & Antarctica & 2.39 & 0.00239 \\
\hline 44092 & Yamato 003330 & L6 & Antarctica & 2.377 & 0.002377 \\
\hline 44269 & Yamato 003507 & L6 & Antarctica & 2.339 & 0.002339 \\
\hline 44263 & Yamato 003501 & L6 & Antarctica & 2.323 & 0.002323 \\
\hline 44134 & Yamato 003372 & L6 & Antarctica & 2.303 & 0.002303 \\
\hline 44247 & Yamato 003485 & L6 & Antarctica & 2.287 & 0.002287 \\
\hline 44326 & Yamato 003564 & L6 & Antarctica & 2.287 & 0.002287 \\
\hline 44230 & Yamato 003468 & L6 & Antarctica & 2.232 & 0.002232 \\
\hline 36897 & Yamato 980271 & L6 & Antarctica & 2.221 & 0.002221 \\
\hline 44291 & Yamato 003529 & L6 & Antarctica & 2.221 & 0.002221 \\
\hline 67481 & Elephant Moraine 14070 & L6 & Antarctica & 2.2 & 0.0022 \\
\hline 36940 & Yamato 980314 & L6 & Antarctica & 2.18 & 0.00218 \\
\hline 36862 & Yamato 980236 & L6 & Antarctica & 2.174 & 0.002174 \\
\hline 44285 & Yamato 003523 & L6 & Antarctica & 2.171 & 0.002171 \\
\hline 36921 & Yamato 980295 & L6 & Antarctica & 2.147 & 0.002147 \\
\hline 44284 & Yamato 003522 & L6 & Antarctica & 2.133 & 0.002133 \\
\hline 44272 & Yamato 003510 & L6 & Antarctica & 2.121 & 0.002121 \\
\hline 67040 & Asuka 09458 & L6 & Antarctica & 2.106 & 0.002106 \\
\hline 36794 & Yamato 980168 & L6 & Antarctica & 2.104 & 0.002104 \\
\hline 67419 & Elephant Moraine 14001 & L6 & Antarctica & 2.1 & 0.0021 \\
\hline 44231 & Yamato 003469 & L6 & Antarctica & 2.082 & 0.002082 \\
\hline 67089 & Asuka 09637 & L6 & Antarctica & 2.073 & 0.002073 \\
\hline 36888 & Yamato 980262 & L6 & Antarctica & 2.052 & 0.002052 \\
\hline 36842 & Yamato 980216 & L6 & Antarctica & 2.009 & 0.002009 \\
\hline 44124 & Yamato 003362 & L6 & Antarctica & 2 & 0.002 \\
\hline 36903 & Yamato 980277 & L6 & Antarctica & 1.975 & 0.001975 \\
\hline 67067 & Asuka 09602 & L6 & Antarctica & 1.961 & 0.001961 \\
\hline 44244 & Yamato 003482 & L6 & Antarctica & 1.956 & 0.001956 \\
\hline 36826 & Yamato 980200 & L6 & Antarctica & 1.952 & 0.001952 \\
\hline 44195 & Yamato 003433 & L6 & Antarctica & 1.895 & 0.001895 \\
\hline 44267 & Yamato 003505 & L6 & Antarctica & 1.889 & 0.001889 \\
\hline 36875 & Yamato 980249 & L6 & Antarctica & 1.867 & 0.001867 \\
\hline 42885 & Yamato 002123 & L6 & Antarctica & 1.851 & 0.001851 \\
\hline 44308 & Yamato 003546 & L6 & Antarctica & 1.849 & 0.001849 \\
\hline 44293 & Yamato 003531 & L6 & Antarctica & 1.837 & 0.001837 \\
\hline
\end{tabular}




\begin{tabular}{|c|c|c|c|c|c|}
\hline 44184 & Yamato 003422 & L6 & Antarctica & 1.835 & 0.001835 \\
\hline 67019 & Asuka 09406 & L6 & Antarctica & 1.832 & 0.001832 \\
\hline 36657 & Yamato 980031 & L6 & Antarctica & 1.816 & 0.001816 \\
\hline 44257 & Yamato 003495 & L6 & Antarctica & 1.806 & 0.001806 \\
\hline 36671 & Yamato 980045 & L6 & Antarctica & 1.741 & 0.001741 \\
\hline 36847 & Yamato 980221 & L6 & Antarctica & 1.731 & 0.001731 \\
\hline 36860 & Yamato 980234 & L6 & Antarctica & 1.723 & 0.001723 \\
\hline 44268 & Yamato 003506 & L6 & Antarctica & 1.709 & 0.001709 \\
\hline 44273 & Yamato 003511 & L6 & Antarctica & 1.709 & 0.001709 \\
\hline 66917 & Asuka 09264 & L6 & Antarctica & 1.678 & 0.001678 \\
\hline 44274 & Yamato 003512 & L6 & Antarctica & 1.665 & 0.001665 \\
\hline 67042 & Asuka 09460 & L6 & Antarctica & 1.596 & 0.001596 \\
\hline 44314 & Yamato 003552 & L6 & Antarctica & 1.487 & 0.001487 \\
\hline 44252 & Yamato 003490 & L6 & Antarctica & 1.47 & 0.00147 \\
\hline 44256 & Yamato 003494 & L6 & Antarctica & 1.449 & 0.001449 \\
\hline 36892 & Yamato 980266 & L6 & Antarctica & 1.421 & 0.001421 \\
\hline 67260 & Asuka 12310 & L6 & Antarctica & 1.397 & 0.001397 \\
\hline 44297 & Yamato 003535 & L6 & Antarctica & 1.392 & 0.001392 \\
\hline 44313 & Yamato 003551 & L6 & Antarctica & 1.367 & 0.001367 \\
\hline 36854 & Yamato 980228 & L6 & Antarctica & 1.322 & 0.001322 \\
\hline 44138 & Yamato 003376 & L6 & Antarctica & 1.29 & 0.00129 \\
\hline 44205 & Yamato 003443 & L6 & Antarctica & 1.282 & 0.001282 \\
\hline 44254 & Yamato 003492 & L6 & Antarctica & 1.273 & 0.001273 \\
\hline 44111 & Yamato 003349 & L6 & Antarctica & 1.255 & 0.001255 \\
\hline 44260 & Yamato 003498 & L6 & Antarctica & 1.251 & 0.001251 \\
\hline 44316 & Yamato 003554 & L6 & Antarctica & 1.251 & 0.001251 \\
\hline 68077 & Miller Range 15416 & L6 & Antarctica & 1.25 & 0.00125 \\
\hline 44229 & Yamato 003467 & L6 & Antarctica & 1.25 & 0.00125 \\
\hline 44286 & Yamato 003524 & L6 & Antarctica & 1.236 & 0.001236 \\
\hline 44243 & Yamato 003481 & L6 & Antarctica & 1.204 & 0.001204 \\
\hline 44315 & Yamato 003553 & L6 & Antarctica & 1.186 & 0.001186 \\
\hline 67041 & Asuka 09459 & L6 & Antarctica & 1.168 & 0.001168 \\
\hline 67043 & Asuka 09461 & L6 & Antarctica & 1.147 & 0.001147 \\
\hline 44290 & Yamato 003528 & L6 & Antarctica & 1.143 & 0.001143 \\
\hline 67130 & Asuka 10221 & L6 & Antarctica & 1.111 & 0.001111 \\
\hline 67063 & Asuka 09558 & L6 & Antarctica & 1.089 & 0.001089 \\
\hline 36628 & Yamato 980002 & L6 & Antarctica & 1.076 & 0.001076 \\
\hline 68094 & Miller Range 15567 & L6 & Antarctica & 1.07 & 0.00107 \\
\hline 36829 & Yamato 980203 & L6 & Antarctica & 1.041 & 0.001041 \\
\hline 44328 & Yamato 003566 & L6 & Antarctica & 1.036 & 0.001036 \\
\hline 67068 & Asuka 09604 & L6 & Antarctica & 1.02 & 0.00102 \\
\hline 68084 & Miller Range 15436 & L6 & Antarctica & 1.01 & 0.00101 \\
\hline 42913 & Yamato 002151 & L6 & Antarctica & 0.999 & 0.000999 \\
\hline 36974 & Yamato 980349 & L6 & Antarctica & 0.969 & 0.000969 \\
\hline 44248 & Yamato 003486 & L6 & Antarctica & 0.967 & 0.000967 \\
\hline
\end{tabular}




\begin{tabular}{|c|c|c|c|c|c|}
\hline 67121 & Asuka 10062 & L6 & Antarctica & 0.888 & 0.000888 \\
\hline 44097 & Yamato 003335 & L6 & Antarctica & 0.873 & 0.000873 \\
\hline 44197 & Yamato 003435 & L6 & Antarctica & 0.857 & 0.000857 \\
\hline 67045 & Asuka 09463 & L6 & Antarctica & 0.854 & 0.000854 \\
\hline 44289 & Yamato 003527 & L6 & Antarctica & 0.837 & 0.000837 \\
\hline 66733 & Elephant Moraine 16207 & L6 & Antarctica & 0.835 & 0.000835 \\
\hline 44317 & Yamato 003555 & L6 & Antarctica & 0.826 & 0.000826 \\
\hline 44259 & Yamato 003497 & L6 & Antarctica & 0.821 & 0.000821 \\
\hline 36915 & Yamato 980289 & L6 & Antarctica & 0.815 & 0.000815 \\
\hline 67046 & Asuka 09464 & L6 & Antarctica & 0.795 & 0.000795 \\
\hline 44319 & Yamato 003557 & L6 & Antarctica & 0.783 & 0.000783 \\
\hline 44281 & Yamato 003519 & L6 & Antarctica & 0.775 & 0.000775 \\
\hline 44135 & Yamato 003373 & L6 & Antarctica & 0.77 & 0.00077 \\
\hline 44084 & Yamato 003322 & L6 & Antarctica & 0.768 & 0.000768 \\
\hline 44069 & Yamato 003307 & L6 & Antarctica & 0.752 & 0.000752 \\
\hline 44275 & Yamato 003513 & L6 & Antarctica & 0.748 & 0.000748 \\
\hline 67069 & Asuka 09605 & L6 & Antarctica & 0.744 & 0.000744 \\
\hline 67044 & Asuka 09462 & L6 & Antarctica & 0.728 & 0.000728 \\
\hline 44203 & Yamato 003441 & L6 & Antarctica & 0.701 & 0.000701 \\
\hline 67421 & Elephant Moraine 14003 & L6 & Antarctica & 0.7 & 0.0007 \\
\hline 43858 & Yamato 003096 & L6 & Antarctica & 0.674 & 0.000674 \\
\hline 44071 & Yamato 003309 & L6 & Antarctica & 0.657 & 0.000657 \\
\hline 44188 & Yamato 003426 & L6 & Antarctica & 0.648 & 0.000648 \\
\hline 44266 & Yamato 003504 & L6 & Antarctica & 0.585 & 0.000585 \\
\hline 44276 & Yamato 003514 & L6 & Antarctica & 0.585 & 0.000585 \\
\hline 44277 & Yamato 003515 & L6 & Antarctica & 0.58 & 0.00058 \\
\hline 44329 & Yamato 003567 & L6 & Antarctica & 0.521 & 0.000521 \\
\hline 66720 & Miller Range 15225 & L6 & Antarctica & 0.52 & 0.00052 \\
\hline 44255 & Yamato 003493 & L6 & Antarctica & 0.493 & 0.000493 \\
\hline 67070 & Asuka 09606 & L6 & Antarctica & 0.48 & 0.00048 \\
\hline 67047 & Asuka 09470 & L6 & Antarctica & 0.459 & 0.000459 \\
\hline 36630 & Yamato 980004 & L6 & Antarctica & 0.445 & 0.000445 \\
\hline 66729 & Elephant Moraine 16202 & L6 & Antarctica & 0.444 & 0.000444 \\
\hline 44113 & Yamato 003351 & L6 & Antarctica & 0.444 & 0.000444 \\
\hline 36894 & Yamato 980268 & L6 & Antarctica & 0.43 & 0.00043 \\
\hline 36816 & Yamato 980190 & L6 & Antarctica & 0.427 & 0.000427 \\
\hline 66736 & Elephant Moraine 16210 & L6 & Antarctica & 0.425 & 0.000425 \\
\hline 66734 & Elephant Moraine 16208 & L6 & Antarctica & 0.419 & 0.000419 \\
\hline 36959 & Yamato 980334 & L6 & Antarctica & 0.416 & 0.000416 \\
\hline 44078 & Yamato 003316 & L6 & Antarctica & 0.406 & 0.000406 \\
\hline 44320 & Yamato 003558 & L6 & Antarctica & 0.406 & 0.000406 \\
\hline 66900 & Asuka 09237 & L6 & Antarctica & 0.404 & 0.000404 \\
\hline 44172 & Yamato 003410 & L6 & Antarctica & 0.401 & 0.000401 \\
\hline 43232 & Yamato 002470 & L6 & Antarctica & 0.384 & 0.000384 \\
\hline 44193 & Yamato 003431 & L6 & Antarctica & 0.362 & 0.000362 \\
\hline
\end{tabular}




\begin{tabular}{|c|c|c|c|c|c|}
\hline 43814 & Yamato 003052 & L6 & Antarctica & 0.353 & 0.000353 \\
\hline 44152 & Yamato 003390 & L6 & Antarctica & 0.351 & 0.000351 \\
\hline 44185 & Yamato 003423 & L6 & Antarctica & 0.348 & 0.000348 \\
\hline 44162 & Yamato 003400 & L6 & Antarctica & 0.345 & 0.000345 \\
\hline 44169 & Yamato 003407 & L6 & Antarctica & 0.341 & 0.000341 \\
\hline 44044 & Yamato 003282 & L6 & Antarctica & 0.336 & 0.000336 \\
\hline 40937 & Yamato 000172 & L6 & Antarctica & 0.329 & 0.000329 \\
\hline 44174 & Yamato 003412 & L6 & Antarctica & 0.314 & 0.000314 \\
\hline 44046 & Yamato 003284 & L6 & Antarctica & 0.309 & 0.000309 \\
\hline 43686 & Yamato 002924 & L6 & Antarctica & 0.304 & 0.000304 \\
\hline 44077 & Yamato 003315 & L6 & Antarctica & 0.247 & 0.000247 \\
\hline 44233 & Yamato 003471 & L6 & Antarctica & 0.204 & 0.000204 \\
\hline 44082 & Yamato 003320 & L6 & Antarctica & 0.175 & 0.000175 \\
\hline 44087 & Yamato 003325 & L6 & Antarctica & 0.165 & 0.000165 \\
\hline 67645 & Northwest Africa 11849 & L6-an & (Northwest At & 335.4 & 0.3354 \\
\hline 67739 & Vaquillas 006 & \multicolumn{2}{|c|}{ L6-melt brecc Chile } & 4400 & 4.4 \\
\hline 67677 & San Juan 086 & \multicolumn{2}{|c|}{ L6-melt brecc Chile } & 490 & 0.49 \\
\hline 67389 & Los Vientos 216 & \multicolumn{2}{|c|}{ L6-melt brecc Chile } & 24 & 0.024 \\
\hline 66474 & Northwest Africa 11617 & $\mathrm{LL}(\mathrm{L}) 3$ & (Northwest At & 1200 & 1.2 \\
\hline 66469 & Northwest Africa 11611 & $\mathrm{LL}(\mathrm{L}) 3$ & (Northwest Al & 232 & 0.232 \\
\hline 68128 & Kumtag 040 & LL 4 & China & 560 & 0.56 \\
\hline 68292 & Northwest Africa 11975 & LL 5 & (Northwest At & 95.8 & 0.0958 \\
\hline 68429 & Alaer 003 & LL 6 & China & 85.4 & 0.0854 \\
\hline 68433 & Xinjiang 010 & LL 6 & China & 71.5 & 0.0715 \\
\hline 68653 & Yangguanzhen 001 & LL 6 & China & 8.66 & 0.00866 \\
\hline 66489 & Northwest Africa 11669 & LL3 & (Northwest At & 23000 & 23 \\
\hline 67720 & Northwest Africa 11908 & LL3 & (Northwest At & 12338 & 12.338 \\
\hline 68599 & Northwest Africa 12009 & LL3 & (Northwest At & 5751 & 5.751 \\
\hline 66417 & Northwest Africa 11615 & LL3 & (Northwest At & 3114 & 3.114 \\
\hline 67857 & Northwest Africa 11967 & LL3 & (Northwest At & 2347.3 & 2.3473 \\
\hline 67570 & Gandom Beryan 011 & LL3 & Iran & 2067 & 2.067 \\
\hline 67719 & Northwest Africa 11907 & LL3 & (Northwest Al & 1326.7 & 1.3267 \\
\hline 68294 & Northwest Africa 11991 & LL3 & (Northwest At & 1020 & 1.02 \\
\hline 68497 & Northwest Africa 12284 & LL3 & (Northwest At & 832.5 & 0.8325 \\
\hline 68295 & Northwest Africa 11992 & LL3 & (Northwest Al & 337 & 0.337 \\
\hline 67827 & Northwest Africa 11954 & LL3 & (Northwest At & 270 & 0.27 \\
\hline 68425 & Northwest Africa 12291 & LL3 & (Northwest At & 259 & 0.259 \\
\hline 68318 & Northwest Africa 12234 & LL3 & Algeria & 257 & 0.257 \\
\hline 67599 & Scottsdale & LL3 & United States & 210.8 & 0.2108 \\
\hline 67152 & Asuka 12050 & LL3 & Antarctica & 183.05 & 0.18305 \\
\hline 68331 & Northwest Africa 12244 & LL3 & Morocco & 174.8 & 0.1748 \\
\hline 67512 & Northwest Africa 11812 & LL3 & (Northwest Al & 144.6 & 0.1446 \\
\hline 32069 & Northwest Africa 1096 & LL3 & (Northwest At & 120 & 0.12 \\
\hline 66408 & Northwest Africa 11590 & LL3 & (Northwest At & 105.5 & 0.1055 \\
\hline 67804 & Northwest Africa 11976 & LL3 & (Northwest Al & 78.6 & 0.0786 \\
\hline
\end{tabular}




\begin{tabular}{|c|c|c|c|c|c|}
\hline 68299 & Northwest Africa 12185 & LL3 & (Northwest Al & 45 & 0.045 \\
\hline 68539 & Coya Sur 004 & LL3 & Chile & 40 & 0.04 \\
\hline 67347 & Asuka 12405 & LL3 & Antarctica & 32.443 & 0.032443 \\
\hline 68485 & Northwest Africa 12281 & LL3 & (Northwest At & 30.6 & 0.0306 \\
\hline 67513 & Northwest Africa 11813 & LL3 & (Northwest Al & 25.3 & 0.0253 \\
\hline 67477 & Elephant Moraine 14064 & LL3 & Antarctica & 23 & 0.023 \\
\hline 67433 & Elephant Moraine 14017 & LL3 & Antarctica & 11.8 & 0.0118 \\
\hline 67630 & Northwest Africa 11873 & LL3-6 & (Northwest At & 7630 & 7.63 \\
\hline 67639 & Northwest Africa 11831 & LL3-6 & (Northwest Al & 65 & 0.065 \\
\hline 67408 & Northwest Africa 11752 & LL3.05 & (Northwest At & 39.5 & 0.0395 \\
\hline 67797 & Northwest Africa 11905 & LL3.10-5 & (Northwest At & 6974 & 6.974 \\
\hline 67740 & Catalina 278 & LL3.4 & Chile & 10720 & 10.72 \\
\hline 66571 & Dominion Range 14183 & LL3.7 & Antarctica & 20.26 & 0.02026 \\
\hline 66666 & Dominion Range 14458 & LL3.7 & Antarctica & 9.51 & 0.00951 \\
\hline 66749 & Northwest Africa 11651 & LL4 & (Northwest Al & 7250 & 7.25 \\
\hline 67668 & Northwest Africa 11882 & LL4 & (Northwest At & 1200 & 1.2 \\
\hline 68360 & Los Vientos 236 & LL4 & Chile & 1073 & 1.073 \\
\hline 68401 & Northwest Africa 12254 & LL4 & (Northwest At & 427 & 0.427 \\
\hline 67765 & Northwest Africa 11944 & LL4 & (Northwest At & 397.2 & 0.3972 \\
\hline 67693 & Al Khuwaymat 002 & LL4 & Libya & 274 & 0.274 \\
\hline 67865 & Northwest Africa 12017 & LL4 & (Northwest Al & 130.78 & 0.13078 \\
\hline 66465 & Northwest Africa 11665 & LL4 & (Northwest At & 108.4 & 0.1084 \\
\hline 67146 & Asuka 12038 & LL4 & Antarctica & 81.98 & 0.08198 \\
\hline 36611 & Belgica 9819 & LL4 & Antarctica & 80.76 & 0.08076 \\
\hline 67335 & Asuka 12392 & LL4 & Antarctica & 67.72 & 0.06772 \\
\hline 67360 & Asuka 12418 & LL4 & Antarctica & 53.29 & 0.05329 \\
\hline 67935 & Dominion Range 14342 & LL4 & Antarctica & 42.705 & 0.042705 \\
\hline 67336 & Asuka 12393 & LL4 & Antarctica & 38.633 & 0.038633 \\
\hline 67337 & Asuka 12394 & LL4 & Antarctica & 28.981 & 0.028981 \\
\hline 67358 & Asuka 12416 & LL4 & Antarctica & 28.652 & 0.028652 \\
\hline 67357 & Asuka 12415 & LL4 & Antarctica & 27.419 & 0.027419 \\
\hline 67359 & Asuka 12417 & LL4 & Antarctica & 24.169 & 0.024169 \\
\hline 67651 & Northwest Africa 11874 & LL4 & (Northwest At & 19.75 & 0.01975 \\
\hline 66517 & Dominion Range 14036 & LL4 & Antarctica & 17.89 & 0.01789 \\
\hline 66790 & MacKay Glacier 14020 & LL4 & Antarctica & 2.9 & 0.0029 \\
\hline 68083 & Miller Range 15433 & LL4 & Antarctica & 1.04 & 0.00104 \\
\hline 67723 & Northwest Africa 11915 & LL4-6 & (Northwest At & 718 & 0.718 \\
\hline 68509 & Northwest Africa 12361 & LL4-6 & (Northwest Al & 583 & 0.583 \\
\hline 67766 & Northwest Africa 11948 & LL4-6 & (Northwest At & 260 & 0.26 \\
\hline 67133 & Asuka 12013 & LL4-6 & Antarctica & 164.61 & 0.16461 \\
\hline 66439 & Northwest Africa 11645 & LL4-6 & (Northwest At & 154.5 & 0.1545 \\
\hline 67307 & Asuka 12362 & LL4-6 & Antarctica & 80.52 & 0.08052 \\
\hline 66430 & Northwest Africa 11629 & LL4-6 & (Northwest Al & 61 & 0.061 \\
\hline 67338 & Asuka 12395 & LL4-6 & Antarctica & 37.259 & 0.037259 \\
\hline 31631 & Northwest Africa 438 & LL5 & (Northwest Al & 3000 & 3 \\
\hline
\end{tabular}




\begin{tabular}{|c|c|c|c|c|c|}
\hline 66482 & Northwest Africa 11643 & LL5 & (Northwest At & 2428 & 2.428 \\
\hline 68510 & Northwest Africa 12337 & LL5 & (Northwest Al & 1269 & 1.269 \\
\hline 67155 & Asuka 12059 & LL5 & Antarctica & 381.62 & 0.38162 \\
\hline 67302 & Asuka 12357 & LL5 & Antarctica & 348.31 & 0.34831 \\
\hline 66762 & Northwest Africa 11664 & LL5 & (Northwest Al & 262 & 0.262 \\
\hline 68099 & Elephant Moraine 16040 & LL5 & Antarctica & 218.38 & 0.21838 \\
\hline 67717 & Calama 011 & LL5 & Chile & 181 & 0.181 \\
\hline 66630 & Dominion Range 14304 & LL5 & Antarctica & 160.89 & 0.16089 \\
\hline 66424 & Northwest Africa 11624 & LL5 & (Northwest Al & 147.1 & 0.1471 \\
\hline 68306 & Calama 020 & LL5 & Chile & 144 & 0.144 \\
\hline 68203 & Northwest Africa 12092 & LL5 & Algeria & 136.64 & 0.13664 \\
\hline 67303 & Asuka 12358 & LL5 & Antarctica & 126.26 & 0.12626 \\
\hline 67365 & Asuka 12423 & LL5 & Antarctica & 99.17 & 0.09917 \\
\hline 66631 & Dominion Range 14306 & LL5 & Antarctica & 74.465 & 0.074465 \\
\hline 68403 & Northwest Africa 12256 & LL5 & (Northwest Al & 72.4 & 0.0724 \\
\hline 67356 & Asuka 12414 & LL5 & Antarctica & 46.888 & 0.046888 \\
\hline 66602 & Dominion Range 14226 & LL5 & Antarctica & 43.339 & 0.043339 \\
\hline 66824 & El Médano 376 & LL5 & Chile & 38 & 0.038 \\
\hline 67364 & Asuka 12422 & LL5 & Antarctica & 26.639 & 0.026639 \\
\hline 66418 & Northwest Africa 11618 & LL5 & (Northwest At & 26.13 & 0.02613 \\
\hline 67372 & Asuka 12431 & LL5 & Antarctica & 19.572 & 0.019572 \\
\hline 66624 & Dominion Range 14279 & LL5 & Antarctica & 13.818 & 0.013818 \\
\hline 67334 & Asuka 12391 & LL5 & Antarctica & 11.376 & 0.011376 \\
\hline 67366 & Asuka 12424 & LL5 & Antarctica & 8.065 & 0.008065 \\
\hline 66618 & Dominion Range 14273 & LL5 & Antarctica & 7.001 & 0.007001 \\
\hline 66859 & Catalina 256 & LL5 & Chile & 5.1 & 0.0051 \\
\hline 67344 & Asuka 12402 & LL5 & Antarctica & 4.67 & 0.00467 \\
\hline 36605 & Belgica 9813 & LL5 & Antarctica & 3.587 & 0.003587 \\
\hline 68441 & Northwest Africa 11811 & LL5-an & (Northwest Al & 182.4 & 0.1824 \\
\hline 68507 & Northwest Africa 12358 & LL5/6 & (Northwest At & 69.6 & 0.0696 \\
\hline 68506 & Northwest Africa 12357 & LL5/6 & (Northwest Al & 62 & 0.062 \\
\hline 68602 & Mahbas Arraid & LL6 & Western Sahi & 5500 & 5.5 \\
\hline 67600 & Northwest Africa 11838 & LL6 & (Northwest Al & 2489 & 2.489 \\
\hline 67864 & Northwest Africa 12016 & LL6 & (Northwest Al & 1035.47 & 1.03547 \\
\hline 67863 & Northwest Africa 12015 & LL6 & (Northwest Al & 1025.11 & 1.02511 \\
\hline 66420 & Northwest Africa 11620 & LL6 & (Northwest Al & 724.22 & 0.72422 \\
\hline 68127 & Northwest Africa 12020 & LL6 & (Northwest At & 576 & 0.576 \\
\hline 66752 & Northwest Africa 11654 & LL6 & (Northwest Al & 570 & 0.57 \\
\hline 67163 & Asuka 12129 & LL6 & Antarctica & 468.39 & 0.46839 \\
\hline 67142 & Asuka 12030 & LL6 & Antarctica & 337.58 & 0.33758 \\
\hline 66500 & Northwest Africa 11684 & LL6 & (Northwest At & 304 & 0.304 \\
\hline 67368 & Asuka 12426 & LL6 & Antarctica & 281.4 & 0.2814 \\
\hline 67756 & Northwest Africa 11932 & LL6 & (Northwest At & 250 & 0.25 \\
\hline 66419 & Northwest Africa 11619 & LL6 & (Northwest At & 216.93 & 0.21693 \\
\hline 67653 & Northwest Africa 11876 & LL6 & (Northwest At & 203.44 & 0.20344 \\
\hline
\end{tabular}




\begin{tabular}{|c|c|c|c|c|c|}
\hline 67353 & Asuka 12411 & LL6 & Antarctica & 186.87 & 0.18687 \\
\hline 67154 & Asuka 12058 & LL6 & Antarctica & 161.31 & 0.16131 \\
\hline 68392 & San Juan 096 & LL6 & Chile & 157 & 0.157 \\
\hline 68324 & Northwest Africa 12007 & LL6 & (Northwest At & 155.6 & 0.1556 \\
\hline 66628 & Dominion Range 14302 & LL6 & Antarctica & 150.64 & 0.15064 \\
\hline 66753 & Northwest Africa 11655 & LL6 & (Northwest Al & 149 & 0.149 \\
\hline 66432 & Northwest Africa 11631 & LL6 & (Northwest At & 145 & 0.145 \\
\hline 66767 & Northwest Africa 11715 & LL6 & (Northwest Al & 136 & 0.136 \\
\hline 66629 & Dominion Range 14303 & LL6 & Antarctica & 133.43 & 0.13343 \\
\hline 67143 & Asuka 12032 & LL6 & Antarctica & 121.02 & 0.12102 \\
\hline 67355 & Asuka 12413 & LL6 & Antarctica & 119.5 & 0.1195 \\
\hline 66761 & Northwest Africa 11663 & LL6 & (Northwest At & 104 & 0.104 \\
\hline 68402 & Northwest Africa 12255 & LL6 & (Northwest A 1 & 102.1 & 0.1021 \\
\hline 68428 & Northwest Africa 12295 & LL6 & (Northwest Al & 99 & 0.099 \\
\hline 67186 & Asuka 12235 & LL6 & Antarctica & 93.89 & 0.09389 \\
\hline 67354 & Asuka 12412 & LL6 & Antarctica & 88.02 & 0.08802 \\
\hline 68435 & Northwest Africa 12298 & LL6 & (Northwest Al & 87.6 & 0.0876 \\
\hline 66768 & Northwest Africa 11716 & LL6 & (Northwest At & 78.7 & 0.0787 \\
\hline 67833 & Northwest Africa 11963 & LL6 & (Northwest At & 73 & 0.073 \\
\hline 67362 & Asuka 12420 & LL6 & Antarctica & 71.46 & 0.07146 \\
\hline 66599 & Dominion Range 14223 & LL6 & Antarctica & 64.981 & 0.064981 \\
\hline 66433 & Northwest Africa 11632 & LL6 & (Northwest A 1 & 61 & 0.061 \\
\hline 67363 & Asuka 12421 & LL6 & Antarctica & 57.966 & 0.057966 \\
\hline 67352 & Asuka 12410 & LL6 & Antarctica & 51.003 & 0.051003 \\
\hline 67376 & Asuka 12435 & LL6 & Antarctica & 50.285 & 0.050285 \\
\hline 67201 & Asuka 12250 & LL6 & Antarctica & 45.093 & 0.045093 \\
\hline 67343 & Asuka 12401 & LL6 & Antarctica & 39.869 & 0.039869 \\
\hline 67375 & Asuka 12434 & LL6 & Antarctica & 35.703 & 0.035703 \\
\hline 67194 & Asuka 12243 & LL6 & Antarctica & 34.533 & 0.034533 \\
\hline 67345 & Asuka 12403 & LL6 & Antarctica & 34.092 & 0.034092 \\
\hline 66429 & Northwest Africa 11628 & LL6 & (Northwest Al & 33 & 0.033 \\
\hline 66763 & Northwest Africa 11702 & LL6 & Mauritania & 33 & 0.033 \\
\hline 67266 & Asuka 12317 & LL6 & Antarctica & 30.565 & 0.030565 \\
\hline 68289 & Northwest Africa 12178 & LL6 & Algeria & 27.51 & 0.02751 \\
\hline 67254 & Asuka 12304 & LL6 & Antarctica & 23.685 & 0.023685 \\
\hline 66771 & Northwest Africa 11719 & LL6 & (Northwest At & 23.5 & 0.0235 \\
\hline 67209 & Asuka 12258 & LL6 & Antarctica & 21.831 & 0.021831 \\
\hline 66769 & Northwest Africa 11717 & LL6 & (Northwest A 1 & 21.5 & 0.0215 \\
\hline 43960 & Yamato 003198 & LL6 & Antarctica & 21.03 & 0.02103 \\
\hline 67216 & Asuka 12265 & LL6 & Antarctica & 18.957 & 0.018957 \\
\hline 67192 & Asuka 12241 & LL6 & Antarctica & 15.067 & 0.015067 \\
\hline 67188 & Asuka 12237 & LL6 & Antarctica & 14.465 & 0.014465 \\
\hline 66621 & Dominion Range 14276 & LL6 & Antarctica & 13.744 & 0.013744 \\
\hline 66622 & Dominion Range 14277 & LL6 & Antarctica & 13.683 & 0.013683 \\
\hline 66770 & Northwest Africa 11718 & LL6 & (Northwest At & 12.3 & 0.0123 \\
\hline
\end{tabular}




\begin{tabular}{|c|c|c|c|c|c|}
\hline 66617 & Dominion Range 14272 & LL6 & Antarctica & 10.907 & 0.010907 \\
\hline 66620 & Dominion Range 14275 & LL6 & Antarctica & 10.253 & 0.010253 \\
\hline 26889 & Yamato 791540 & LL6 & Antarctica & 9.95 & 0.00995 \\
\hline 2671 & Asuka 87314 & LL6 & Antarctica & 8.446 & 0.008446 \\
\hline 68046 & Miller Range 15251 & LL6 & Antarctica & 8.337 & 0.008337 \\
\hline 67367 & Asuka 12425 & LL6 & Antarctica & 6.593 & 0.006593 \\
\hline 66619 & Dominion Range 14274 & LL6 & Antarctica & 6.39 & 0.00639 \\
\hline 66604 & Dominion Range 14228 & LL6 & Antarctica & 5.52 & 0.00552 \\
\hline 67374 & Asuka 12433 & LL6 & Antarctica & 5.397 & 0.005397 \\
\hline 67994 & Dominion Range 14445 & LL6 & Antarctica & 5.091 & 0.005091 \\
\hline 36964 & Yamato 980339 & LL6 & Antarctica & 4.626 & 0.004626 \\
\hline 43961 & Yamato 003199 & LL6 & Antarctica & 4.151 & 0.004151 \\
\hline 36917 & Yamato 980291 & LL6 & Antarctica & 1.803 & 0.001803 \\
\hline 36672 & Yamato 980046 & LL6 & Antarctica & 1.419 & 0.001419 \\
\hline 43614 & Yamato 002852 & LL6 & Antarctica & 0.32 & 0.00032 \\
\hline 67415 & Reckling Peak 14002 & LL6 & Antarctica & 0.2 & 0.0002 \\
\hline 67808 & Northwest Africa 11776 & LL6-an & (Northwest A1 & 100.7 & 0.1007 \\
\hline 66838 & Northwest Africa 11730 & \multicolumn{2}{|c|}{ LL6-melt brec(Northwest AI } & 315.4 & 0.3154 \\
\hline 68525 & Northwest Africa 12272 & LL7 & (Northwest Al & 153 & 0.153 \\
\hline 67741 & Los Vientos 231 & LL7 & Chile & 127 & 0.127 \\
\hline 67648 & Northwest Africa 11852 & LL7 & (Northwest At & 45 & 0.045 \\
\hline 67644 & Northwest Africa 11848 & Lodranite & (Northwest Al & 2064 & 2.064 \\
\hline 67559 & Northwest Africa 11795 & Lodranite & (Northwest At & 1270 & 1.27 \\
\hline 67832 & Northwest Africa 11960 & Lodranite & (Northwest Al & 1265 & 1.265 \\
\hline 67786 & Northwest Africa 11901 & Lodranite & Western Sahi & 662 & 0.662 \\
\hline 67860 & Northwest Africa 11970 & Lodranite & (Northwest A 1 & 180.9 & 0.1809 \\
\hline 42382 & Yamato 001620 & Lodranite & Antarctica & 9.533 & 0.009533 \\
\hline 42084 & Yamato 001322 & Lodranite & Antarctica & 5.232 & 0.005232 \\
\hline 66625 & Dominion Range 14289 & Lodranite & Antarctica & 3.42 & 0.00342 \\
\hline 66473 & Northwest Africa 11616 & Lunar & (Northwest Al & 2550.8 & 2.5508 \\
\hline 67854 & Northwest Africa 11962 & Lunar & (Northwest Al & 85.8 & 0.0858 \\
\hline 67597 & Northwest Africa 11801 & Lunar & Algeria & 67.05 & 0.06705 \\
\hline 67742 & Northwest Africa 11886 & Lunar & (Northwest Al & 36 & 0.036 \\
\hline 67496 & Northwest Africa 11767 & Lunar & (Northwest At & 15 & 0.015 \\
\hline 68325 & Northwest Africa 12008 & \multicolumn{2}{|c|}{ Lunar (basalt Morocco } & 577 & 0.577 \\
\hline 67552 & Northwest Africa 11787 & \multicolumn{2}{|c|}{ Lunar (feldsp Mauritania } & 23500 & 23.5 \\
\hline 67553 & Northwest Africa 11788 & \multicolumn{2}{|c|}{ Lunar (feldsp Mali } & 10441 & 10.441 \\
\hline 66745 & Northwest Africa 11303 & \multicolumn{2}{|c|}{ Lunar (feldsp (Northwest A } & 6000 & 6 \\
\hline 67554 & Northwest Africa 11789 & \multicolumn{2}{|c|}{ Lunar (feldsp Mauritania } & 5492 & 5.492 \\
\hline 67609 & Northwest Africa 11828 & \multicolumn{2}{|c|}{ Lunar (feldsp (Northwest A1 } & 5340 & 5.34 \\
\hline 68546 & Northwest Africa 12368 & \multicolumn{2}{|c|}{ Lunar (feldsp (Northwest At } & 1868 & 1.868 \\
\hline 68610 & Northwest Africa 12279 & \multicolumn{2}{|c|}{ Lunar (feldsp Algeria } & 1830 & 1.83 \\
\hline 68526 & Northwest Africa 12270 & \multicolumn{2}{|c|}{ Lunar (feldsp Mauritania } & 1300 & 1.3 \\
\hline 33042 & Northwest Africa 2425 & \multicolumn{2}{|c|}{ lunar (feldsp. (Northwest Al } & 810 & 0.81 \\
\hline 67784 & Northwest Africa 11898 & \multicolumn{2}{|c|}{ Lunar (feldsp Mali } & 767.8 & 0.7678 \\
\hline
\end{tabular}




\begin{tabular}{|c|c|c|c|c|}
\hline 66487 & Aridal 017 & Lunar (feldsp Western Sahi & 590 & 0.59 \\
\hline 67858 & Northwest Africa 11968 & Lunar (Feldsk (Northwest Al & 427.8 & 0.4278 \\
\hline 66802 & Northwest Africa 11695 & Lunar (feldsp Algeria & 350 & 0.35 \\
\hline 67647 & Northwest Africa 11851 & Lunar (feldsp Mali & 227 & 0.227 \\
\hline 67856 & Northwest Africa 11966 & Lunar (Feldsk(Northwest A1 & 157.13 & 0.15713 \\
\hline 66414 & Errachidia & Lunar (feldsp Morocco & 156 & 0.156 \\
\hline 66746 & Northwest Africa 11517 & Lunar (feldsp (Northwest Al & 88 & 0.088 \\
\hline 67591 & Northwest Africa 11809 & Lunar (feldsp (Northwest A1 & 67 & 0.067 \\
\hline 67548 & Northwest Africa 11783 & Lunar (Feldsr(Northwest Al & 65 & 0.065 \\
\hline 66393 & Northwest Africa 11563 & Lunar (feldsp (Northwest A1 & 54.5 & 0.0545 \\
\hline 68495 & Northwest Africa 12248 & Lunar (feldsp Mauritania & 37.3 & 0.0373 \\
\hline 67681 & Northwest Africa 11871 & Lunar (feldsp Algeria & 21.9 & 0.0219 \\
\hline 66808 & Northwest Africa 11703 & Lunar (gabbrı(Northwest Al & 5309.1 & 5.3091 \\
\hline 67379 & Caleta el Cobre 022 & Martian (nakr Chile & 474 & 0.474 \\
\hline 67783 & Northwest Africa 11896 & Martian (polyı)(Northwest At & 14 & 0.014 \\
\hline 67818 & Northwest Africa 11921 & Martian (polyı)(Northwest Al & 7.7 & 0.0077 \\
\hline 67853 & Rabt Sbayta 010 & Martian (polyıWestern Sahi & 6.1 & 0.0061 \\
\hline 68472 & Northwest Africa 12222 & Martian (polyı(Northwest Al & 1.9 & 0.0019 \\
\hline 68545 & Northwest Africa 12335 & Martian (sher (Northwest Al & 3970.7 & 3.9707 \\
\hline 66832 & Sueilila 002 & Martian (sherWestern Sahi & 3694 & 3.694 \\
\hline 68604 & Northwest Africa 12262 & Martian (sher (Northwest Al & 3294 & 3.294 \\
\hline 68608 & Northwest Africa 12269 & Martian (sher (Northwest At & 1679 & 1.679 \\
\hline 68491 & Northwest Africa 12241 & Martian (sher Algeria & 1150 & 1.15 \\
\hline 68638 & Northwest Africa 12323 & Martian (sher (Northwest At & 447 & 0.447 \\
\hline 67828 & Northwest Africa 11955 & Martian (sher (Northwest Al & 272 & 0.272 \\
\hline 68466 & Northwest Africa 12210 & Martian (sher (Northwest Al & 151 & 0.151 \\
\hline 68611 & Northwest Africa 12280 & Martian (sher Algeria & 79 & 0.079 \\
\hline 33041 & Northwest Africa 2424 & Martian (sher (Northwest Al & 58 & 0.058 \\
\hline 67587 & Northwest Africa 11804 & Martian (sher (Northwest Al & 34 & 0.034 \\
\hline 67273 & Asuka 12325 & Martian (sher Antarctica & 28.022 & 0.028022 \\
\hline 67680 & Northwest Africa 11866 & Martian (sher (Northwest At & 23.3 & 0.0233 \\
\hline 66796 & Northwest Africa 11668 & Martian (sher (Northwest At & 20 & 0.02 \\
\hline 67410 & Ramlat Fasad 057 & Martian (sher Oman & 13.049 & 0.013049 \\
\hline 66844 & Northwest Africa 11746 & Martian (sher (Northwest At & 9.4 & 0.0094 \\
\hline 68309 & Northwest Africa 12002 & Martian (sher Morocco & 5 & 0.005 \\
\hline 67490 & Northwest Africa 11761 & Mesosiderite (Northwest Al & 2258 & 2.258 \\
\hline 67540 & Northwest Africa 11774 & Mesosiderite Mauritania & 439.2 & 0.4392 \\
\hline 67649 & Northwest Africa 11853 & Mesosiderite (Northwest At & 270 & 0.27 \\
\hline 67743 & Northwest Africa 11887 & Mesosiderite (Northwest Al & 61 & 0.061 \\
\hline 68467 & Northwest Africa 12212 & Mesosiderite (Northwest At & 7.5 & 0.0075 \\
\hline 67814 & Jiddat al Harasis 846 & Oman & 260.1 & 0.2601 \\
\hline 68410 & Northwest Africa 12186 & (Northwest Al & 682 & 0.682 \\
\hline 49377 & Northwest Africa 5693 & (Northwest At & 70.7 & 0.0707 \\
\hline 68615 & Northwest Africa 12288 & (Northwest At & 29 & 0.029 \\
\hline 67809 & Chasseron & Pallasite, PM Switzerland & 4.867 & 0.004867 \\
\hline
\end{tabular}




\begin{tabular}{|c|c|c|c|c|c|}
\hline 66835 & Northwest Africa 11720 & \multicolumn{2}{|c|}{ Pallasite, PM (Northwest At } & 230 & 0.23 \\
\hline 67732 & La'gad 002 & \multicolumn{2}{|c|}{ Pallasite, PM Western Sahi } & 16.63 & 0.01663 \\
\hline 66836 & Northwest Africa 11721 & R3 & (Northwest A 1 & 5345 & 5.345 \\
\hline 67820 & Northwest Africa 11927 & R3 & (Northwest Al & 592 & 0.592 \\
\hline 67498 & Northwest Africa 11769 & R3 & (Northwest At & 210 & 0.21 \\
\hline 66445 & Northwest Africa 11587 & R3-5 & (Northwest At & 714 & 0.714 \\
\hline 67714 & Northwest Africa 11860 & R3-5 & (Northwest A 1 & 520 & 0.52 \\
\hline 66472 & Northwest Africa 11614 & R3-5 & (Northwest At & 314 & 0.314 \\
\hline 67607 & Northwest Africa 8791 & R3-5 & (Northwest Al & 241 & 0.241 \\
\hline 66804 & Northwest Africa 11697 & R3-6 & (Northwest At & 882.4 & 0.8824 \\
\hline 67682 & Northwest Africa 11880 & R3.5-4 & (Northwest At & 32.87 & 0.03287 \\
\hline 66833 & Northwest Africa 11304 & R4 & (Northwest At & 920 & 0.92 \\
\hline 66404 & Northwest Africa 11571 & $\mathrm{R} 4$ & (Northwest Al & 504.25 & 0.50425 \\
\hline 67501 & Northwest Africa 11772 & R5 & (Northwest Al & 38 & 0.038 \\
\hline 67735 & Northwest Africa 11892 & Ureilite & (Northwest At & 2131.5 & 2.1315 \\
\hline 67810 & Northwest Africa 11950 & Ureilite & (Northwest A 1 & 1645 & 1.645 \\
\hline 67547 & Northwest Africa 11757 & Ureilite & Morocco & 1600 & 1.6 \\
\hline 68527 & Northwest Africa 12271 & Ureilite & (Northwest A 1 & 925 & 0.925 \\
\hline 67829 & Northwest Africa 11956 & Ureilite & (Northwest At & 720 & 0.72 \\
\hline 66481 & Northwest Africa 11641 & Ureilite & (Northwest Al & 650 & 0.65 \\
\hline 66479 & Northwest Africa 11639 & Ureilite & (Northwest At & 583 & 0.583 \\
\hline 68487 & Northwest Africa 12191 & Ureilite & Western Sahi & 574 & 0.574 \\
\hline 67736 & Northwest Africa 11893 & Ureilite & (Northwest At & 525.7 & 0.5257 \\
\hline 68418 & Northwest Africa 12198 & Ureilite & Western Sahi & 506 & 0.506 \\
\hline 66480 & Northwest Africa 11640 & Ureilite & (Northwest At & 500 & 0.5 \\
\hline 68524 & Northwest Africa 12237 & Ureilite & (Northwest At & 478.8 & 0.4788 \\
\hline 66386 & Jiddat al Harasis 1101 & Ureilite & Oman & 460 & 0.46 \\
\hline 67796 & Northwest Africa 11900 & Ureilite & Western Sahi & 457 & 0.457 \\
\hline 68411 & Northwest Africa 12189 & Ureilite & Algeria & 435 & 0.435 \\
\hline 66798 & Northwest Africa 11671 & Ureilite & (Northwest At & 393 & 0.393 \\
\hline 66799 & Northwest Africa 11675 & Ureilite & (Northwest Al & 391.1 & 0.3911 \\
\hline 66385 & Jiddat al Harasis 1100 & Ureilite & Oman & 365 & 0.365 \\
\hline 67544 & Northwest Africa 11754 & Ureilite & Morocco & 304 & 0.304 \\
\hline 67734 & Northwest Africa 11891 & Ureilite & (Northwest At & 289.7 & 0.2897 \\
\hline 67602 & Northwest Africa 11815 & Ureilite & (Northwest Al & 279 & 0.279 \\
\hline 67565 & Elephant Moraine 14053 & Ureilite & Antarctica & 252.3 & 0.2523 \\
\hline 68296 & Northwest Africa 11993 & Ureilite & (Northwest At & 245 & 0.245 \\
\hline 67549 & Northwest Africa 11784 & Ureilite & (Northwest At & 234.6 & 0.2346 \\
\hline 66477 & Jiddat al Harasis 1103 & Ureilite & Oman & 225.8 & 0.2258 \\
\hline 68486 & Northwest Africa 12188 & Ureilite & Mauritania & 209 & 0.209 \\
\hline 67811 & Northwest Africa 11951 & Ureilite & (Northwest Al & 205 & 0.205 \\
\hline 68636 & Northwest Africa 12321 & Ureilite & (Northwest At & 194 & 0.194 \\
\hline 68409 & Northwest Africa 12183 & Ureilite & (Northwest At & 167 & 0.167 \\
\hline 68531 & San Juan 100 & Ureilite & Chile & 160.7 & 0.1607 \\
\hline 67733 & Northwest Africa 11890 & Ureilite & (Northwest Al & 150.2 & 0.1502 \\
\hline
\end{tabular}




\begin{tabular}{llll|c|c}
66388 & Jiddat al Harasis 1102 & Ureilite & Oman & 95 & 0.095 \\
67545 & Northwest Africa 11755 & Ureilite & Morocco & 83 & 0.083 \\
67737 & Northwest Africa 11894 & Ureilite & (Northwest A1 & 72.3 & 0.0723 \\
66443 & Northwest Africa 11585 & Ureilite & Morocco & 70 & 0.07 \\
67503 & Northwest Africa 11775 & Ureilite & Morocco & 63 & 0.063 \\
67400 & Northwest Africa 11738 & Ureilite & (Northwest A1 & 57 & 0.057 \\
66467 & Northwest Africa 11608 & Ureilite & (Northwest A1 & 53 & 0.053 \\
66483 & Northwest Africa 11644 & Ureilite & (Northwest A1 & 41.2 & 0.0412 \\
66773 & Ramlat as Sahmah 530 & Ureilite & Oman & 30 & 0.03 \\
41378 & Yamato 000614 & Ureilite & Antarctica & 4.684 & 0.004684 \\
67122 & Asuka 10104 & Ureilite & Antarctica & 0.766 & 0.000766 \\
67539 & Northwest Africa 11511 & Ureilite-an & Algeria & 527 & 0.527 \\
67815 & Northwest Africa 11917 & Winonaite & (Northwest A1 & 304.3 & 0.3043 \\
67589 & Northwest Africa 11807 & Winonaite & (Northwest A1 & 172.5 & 0.1725
\end{tabular}




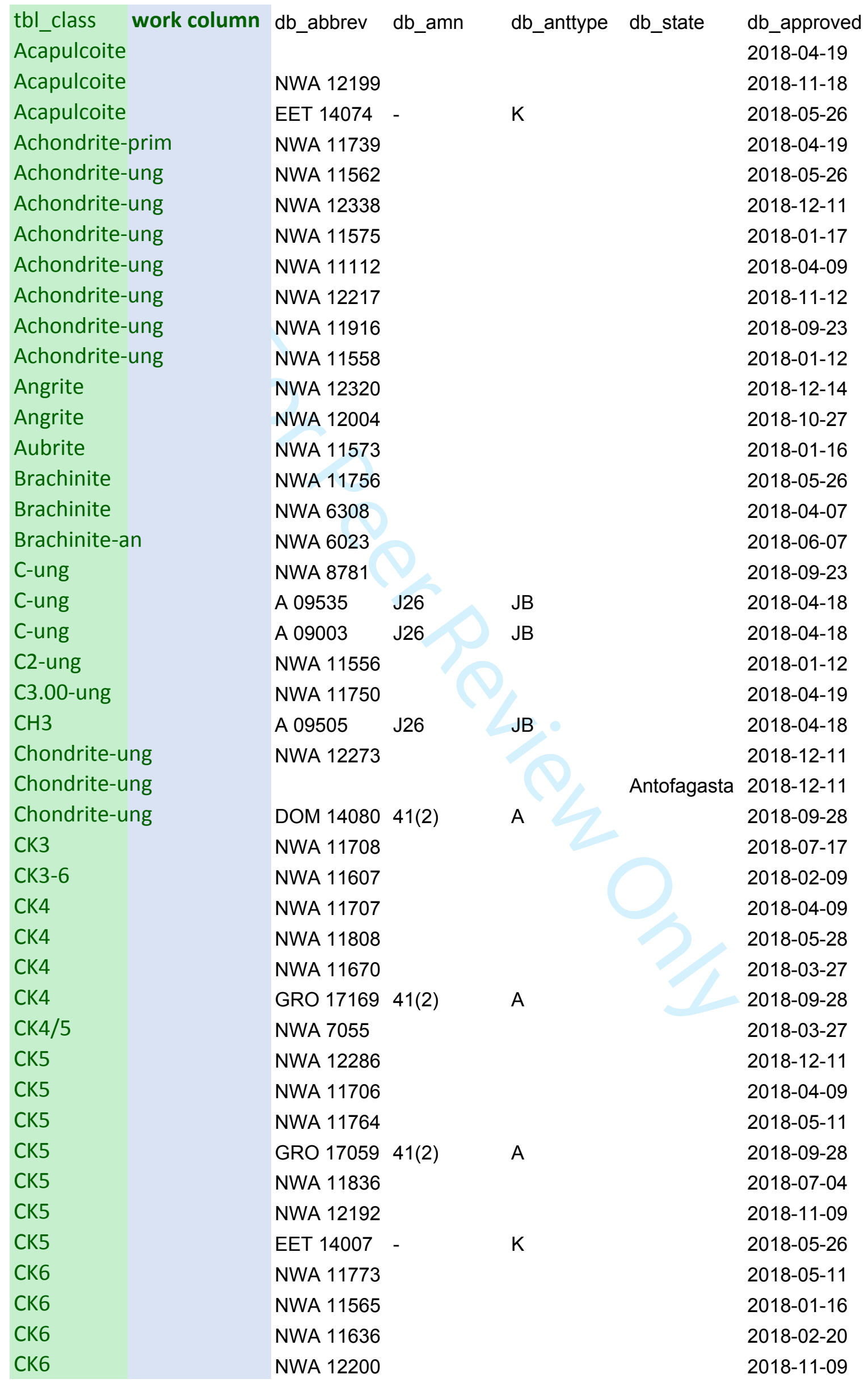




\begin{tabular}{|c|c|c|c|c|c|}
\hline CK6 & SJ 085 & & & Antofagasta & $2018-05-26$ \\
\hline CK6 & A 12267 & J26 & $\mathrm{JB}$ & & $2018-04-18$ \\
\hline CK6 & NWA 11765 & & & & 2018-05-11 \\
\hline $\mathrm{CM}$ & A 12236 & J26 & JB & & 2018-04-18 \\
\hline $\mathrm{CM}$ & A 12248 & $\mathrm{~J} 26$ & $\mathrm{JB}$ & & 2018-04-18 \\
\hline CM & A 12408 & J26 & $\mathrm{JB}$ & & 2018-04-18 \\
\hline CM & Y-980039 & J26 & $\mathrm{J}$ & & $2018-04-18$ \\
\hline CM & Y-980037 & $\mathrm{J} 26$ & $J$ & & 2018-04-18 \\
\hline CM & Y-980111 & J26 & J & & 2018-04-18 \\
\hline CM & Y-980038 & J26 & $\mathrm{J}$ & & $2018-04-18$ \\
\hline $\mathrm{CM}$ & Y-980084 & $\mathrm{J} 26$ & $J$ & & 2018-04-18 \\
\hline CM & Y-980083 & J26 & $J$ & & 2018-04-18 \\
\hline CM & Y-980199 & $\mathrm{J} 26$ & $\mathrm{~J}$ & & $2018-04-18$ \\
\hline $\mathrm{CM}$ & Y-980363 & $\mathrm{J} 26$ & J & & 2018-04-18 \\
\hline CM & Y-980243 & J26 & $J$ & & 2018-04-18 \\
\hline $\mathrm{CM}$ & Y-980089 & $\mathrm{J} 26$ & $J$ & & 2018-04-18 \\
\hline $\mathrm{CM}$ & Y-980151 & $\mathrm{J} 26$ & $J$ & & 2018-04-18 \\
\hline CM & A 10139 & J26 & $\mathrm{JB}$ & & 2018-04-18 \\
\hline CM-an & NWA 11254 & & & & 2018-03-27 \\
\hline CM1 & NWA 12328 & & & & 2018-12-24 \\
\hline $\mathrm{CM} 2$ & & & & Rajasthan & 2018-03-27 \\
\hline $\mathrm{CM} 2$ & NWA 12184 & & & & $2018-10-27$ \\
\hline $\mathrm{CM} 2$ & EET 14068 & - & K & & $2018-05-26$ \\
\hline $\mathrm{CM} 2$ & NWA 11699 & & & & 2018-03-27 \\
\hline $\mathrm{CM} 2$ & NWA 11889 & & & & 2018-08-03 \\
\hline $\mathrm{CM} 2$ & NWA 11965 & & & & 2018-10-06 \\
\hline $\mathrm{CM} 2$ & GRO 17004 & $41(2)$ & $A$ & & $2018-09-28$ \\
\hline $\mathrm{CM} 2$ & RKP 14005 & - & $\mathrm{K}$ & & 2018-05-26 \\
\hline $\mathrm{CM} 2$ & NWA 12287 & & & & $2018-12-11$ \\
\hline $\mathrm{CM} 2$ & NWA 11791 & & & & $2018-05-26$ \\
\hline $\mathrm{CM} 2$ & NWA 11781 & & & & $2018-07-20$ \\
\hline $\mathrm{CM} 2$ & AMU 17290 & $41(2)$ & $A$ & & 2018-09-28 \\
\hline $\mathrm{CM} 2$ & NWA 11919 & & & & 2018-09-15 \\
\hline $\mathrm{CM} 2$ & MIL 15094 & $41(2)$ & $A$ & & 2018-09-28 \\
\hline $\mathrm{CM} 2$ & NWA 11588 & & & & 2018-02-03 \\
\hline $\mathrm{CM} 2$ & NWA 11793 & & & & $2018-05-26$ \\
\hline $\mathrm{CM} 2$ & NWA 11792 & & & & $2018-05-26$ \\
\hline $\mathrm{CM} 2$ & NWA 11733 & & & & 2018-04-19 \\
\hline $\mathrm{CM} 2$ & NWA 11841 & & & & 2018-07-04 \\
\hline $\mathrm{CM} 2$ & NWA 11839 & & & & 2018-07-04 \\
\hline $\mathrm{CM} 2$ & NWA 11840 & & & & 2018-07-04 \\
\hline $\mathrm{CM} 2$ & EET 14013 & - & $\mathrm{K}$ & & $2018-05-26$ \\
\hline CM2.0 & NWA 11638 & & & & 2018-03-27 \\
\hline CM2.0 & NWA 11732 & & & & 2018-04-19 \\
\hline $\mathrm{CO}$ & A 12376 & $\mathrm{~J} 26$ & $\mathrm{JB}$ & & 2018-04-18 \\
\hline
\end{tabular}


2018-04-18

2018-04-18

2018-04-18

2018-02-20

2018-01-12

2018-03-27

Antofagasta 2018-08-03

2018-10-09

2018-04-09

Antofagasta 2018-01-17

Antofagasta 2018-08-03

2018-11-12

2018-12-11

2018-12-11

Zufar 2018-11-12

2018-12-11

2018-12-14

2018-12-02

2018-09-23

2018-07-04

2018-01-12

2018-11-12

2018-08-03

2018-08-31

2018-04-19

2018-11-09

2018-09-28

2018-09-28

2018-07-04

2018-12-14

2018-08-31

2018-04-19

2018-10-27

2018-06-07

2018-12-11

2018-11-13

2018-12-14

Antofagasta 2018-12-11

2018-12-11

2018-10-20

2018-09-15

2018-11-09

2018-05-11

2018-02-03

2018-07-04 


\begin{tabular}{|c|c|c|c|}
\hline CV3 & NWA 12180 & & $2018-10-27$ \\
\hline CV3 & NWA 11740 & & 2018-04-19 \\
\hline CV3 & NWA 11977 & & $2018-11-27$ \\
\hline CV3 & NWA 2637 & & $2018-05-28$ \\
\hline CV3 & NWA 11845 & & 2018-07-04 \\
\hline CV3 & NWA 12247 & & $2018-11-24$ \\
\hline CV3 & NWA 11946 & & 2018-09-15 \\
\hline CV3 & NWA 12221 & & $2018-11-12$ \\
\hline CV3 & NWA 11770 & & $2018-05-11$ \\
\hline CV & A $12373 \quad J 26$ & $J B$ & 2018-04-18 \\
\hline CV3 & NWA 11766 & & $2018-05-11$ \\
\hline CV3 & NWA 4838 & & $2018-06-07$ \\
\hline CV3 & NWA 11937 & & 2018-08-31 \\
\hline CV3 & MIL 15254 & $A$ & $2018-09-28$ \\
\hline CV3 & MIL 15257 & A & $2018-09-28$ \\
\hline CV3 & MIL 15255 & A & $2018-09-28$ \\
\hline CV3 & NWA 11854 & & 2018-07-04 \\
\hline $\mathrm{CV}$ & Y-980177 J26 & $\mathrm{J}$ & $2018-04-18$ \\
\hline Diogenite & NWA 11560 & & 2018-01-12 \\
\hline Diogenite & & Shaanxi & 2018-04-01 \\
\hline Diogenite & NWA 11696 & & $2018-03-27$ \\
\hline Diogenite & EC 001 & Adrar & 2018-03-27 \\
\hline Diogenite & NWA 11945 & & $2018-09-15$ \\
\hline Diogenite & NWA 11741 & & 2018-04-07 \\
\hline Diogenite & NWA 11909 & & 2018-09-02 \\
\hline Diogenite & NWA 11857 & & 2018-07-08 \\
\hline Diogenite & NWA 11888 & & 2018-08-03 \\
\hline Diogenite & A $12144 \quad J 26$ & $J B$ & 2018-04-18 \\
\hline Diogenite & Y-000995 & $\mathrm{J}$ & $2018-04-18$ \\
\hline Diogenite & LoV 250 & Antofagasta & 2018-12-02 \\
\hline Diogenite & GRO $1717541(2)$ & A & $2018-09-28$ \\
\hline Diogenite & NWA 12267 & & $2018-12-11$ \\
\hline Diogenite & NWA 12245 & & 2018-11-24 \\
\hline Diogenite & Y-002876 J26 & $\mathrm{J}$ & 2018-04-18 \\
\hline Diogenite & NWA 11771 & & 2018-05-11 \\
\hline Diogenite & Y-794039 J26 & $\mathrm{J}$ & 2018-04-18 \\
\hline Diogenite & Y-002877 & $\mathrm{J}$ & 2018-04-18 \\
\hline Diogenite & NWA 11833 & & $2018-07-17$ \\
\hline Diogenite & A $12158 \quad J 26$ & JB & 2018-04-18 \\
\hline Diogenite & Y-791838 & $J$ & 2018-04-18 \\
\hline Diogenite & Y-000305 & $\mathrm{J}$ & 2018-04-18 \\
\hline Diogenite & Y-000832 & $J$ & 2018-04-18 \\
\hline Diogenite & Y-000257 & $\mathrm{J}$ & 2018-04-18 \\
\hline Diogenite-pm & NWA 11805 & & $2018-05-28$ \\
\hline EH3 & $\mathrm{CeC} 025$ & Antofagasta & $2018-12-11$ \\
\hline
\end{tabular}


A $12245 \quad$ J26

JB

2018-04-18

EL6

CeC 024

EL6

EM 394

EL6

NWA 11667

EL6

NWA 11731

EL6

EL6

A 12077

J26

MCY 14005 -

JB

EL6

NWA 11679

Enst achon-ung

NWA 11354

eucrite

NWA 11998

Eucrite

NWA 11591

Eucrite

Eucrite

EET 14056 -

Eucrite

EET 14049 -

Eucrite

NWA 2887

Eucrite

Dho 2093

Eucrite

NWA 11777

Eucrite

Dho 2092

Eucrite

NWA 11710

Eucrite

Eucrite

NWA 11999

NWA 11949

Eucrite

NWA 11928

Eucrite

Eucrite

Eucrite

Eucrite

Eucrite

Eucrite

Eucrite

Eucrite

Eucrite

Eucrite

Eucrite

Eucrite

Eucrite

Eucrite

Eucrite

Eucrite

NWA 11837

NWA 11794

Y-001365 J26

NWA 11709

NWA 11550

NWA 12283

NWA 11856

NWA 11552

NWA 11594

NWA 11586

A $12223 \quad$ J26

NWA 11600

NWA 11595

NWA 11947

NWA 11933

Eucrite

Eucrite

Eucrite

Eucrite

Eucrite

Eucrite

Eucrite

NWA 12290

$\mathrm{K}$

$\mathrm{K}$

Antofagasta 2018-12-11

Antofagasta 2018-12-02

2018-02-20

2018-04-19

2018-04-18

2018-01-05

2018-03-27

2018-10-08

2018-09-27

2018-02-09

2018-05-26

2018-05-26

2018-05-11

Zufar 2018-12-02

2018-06-07

Zufar 2018-01-08

2018-03-27

2018-10-06

2018-09-15

2018-09-15

2018-07-04

2018-05-26

2018-04-18

2018-03-27

2018-01-05

2018-12-11

2018-07-08

2018-01-05

2018-02-09

2018-02-03

2018-04-18

2018-02-09

2018-02-09

2018-09-15

2018-08-31

Santiago del 2018-07-04

2018-12-11

Y-001130 J26

2018-04-18

A-881898 J26

2018-04-18

NWA 11596

2018-02-09

A $12043 \quad J 26 \quad J B$

2018-04-18

NWA 11593

2018-02-09

A $12285 \quad J 26 \quad J B$

2018-04-18

NWA 12226 


\begin{tabular}{|c|c|c|c|}
\hline \multirow{2}{*}{$\begin{array}{l}\text { Eucrite } \\
\text { Eucrite }\end{array}$} & \multicolumn{2}{|l|}{ NWA 11597} & 2018-02-09 \\
\hline & NWA 11747 & & 2018-04-19 \\
\hline Eucrite & A-881689 J26 & $\mathrm{J}$ & 2018-04-18 \\
\hline Eucrite & NWA 11748 & & 2018-04-19 \\
\hline Eucrite & NWA 11599 & & 2018-02-09 \\
\hline Eucrite & A $12282 \quad J 26$ & JB & 2018-04-18 \\
\hline Eucrite & NWA 12293 & & $2018-12-11$ \\
\hline Eucrite & A $12374 \quad$ J26 & JB & 2018-04-18 \\
\hline Eucrite & NWA 11592 & & 2018-02-09 \\
\hline Eucrite & NWA 11602 & & 2018-02-09 \\
\hline Eucrite & NWA 11601 & & 2018-02-09 \\
\hline Eucrite & A-881487 J26 & $J$ & 2018-04-18 \\
\hline Eucrite & Y-001232 & $J$ & 2018-04-18 \\
\hline Eucrite & A-881878 & $\mathrm{J}$ & 2018-04-18 \\
\hline Eucrite & NWA 11603 & & 2018-02-09 \\
\hline Eucrite & A $12407 \quad J 26$ & JB & 2018-04-18 \\
\hline Eucrite & Y-003354 & $J$ & 2018-04-18 \\
\hline Eucrite & A 10228 & $J B$ & 2018-04-18 \\
\hline Eucrite & Y-980315 & $J$ & 2018-04-18 \\
\hline Eucrite & Y-980189 & $J$ & 2018-04-18 \\
\hline Eucrite & Y-980337 & $\mathrm{J}$ & 2018-04-18 \\
\hline Eucrite & EET 14066 - & $\mathrm{K}$ & 2018-05-26 \\
\hline Eucrite-an & NWA 11729 & & 2018-04-07 \\
\hline Eucrite & NWA 8671 & & 2018-01-19 \\
\hline Eucrite-br & NWA 8681 & & 2018-03-13 \\
\hline Eucrite-br & NWA 11803 & & $2018-05-28$ \\
\hline Eucrite-br & NWA 12182 & & 2018-11-09 \\
\hline Eucrite-br & NWA 11762 & & 2018-05-11 \\
\hline Eucrite-br & NWA 5908 & & 2018-03-27 \\
\hline Eucrite-br & NWA 11763 & & 2018-05-11 \\
\hline Eucrite-cm & NWA 2426 & & 2018-05-11 \\
\hline Eucrite-melt breccia & NWA 11576 & & 2018-01-22 \\
\hline Eucrite-melt breccia & NWA 12265 & & $2018-12-11$ \\
\hline Eucrite-melt breccia & NWA 11734 & & 2018-04-19 \\
\hline Eucrite-melt breccia & NWA 11785 & & 2018-05-26 \\
\hline Eucrite-melt breccia & NWA 11742 & & 2018-04-07 \\
\hline Eucrite-melt breccia & NWA 12342 & & $2018-12-14$ \\
\hline Eucrite-melt breccia & NWA 12223 & & 2018-11-13 \\
\hline Eucrite-mmict & NWA 12011 & & $2018-10-27$ \\
\hline Eucrite-mmict & NWA 2650 & & 2018-08-03 \\
\hline Eucrite-mmict & NWA 11958 & & 2018-09-15 \\
\hline Eucrite-mmict & NWA 11953 & & 2018-09-15 \\
\hline Eucrite-mmict & NWA 11698 & & 2018-03-27 \\
\hline Eucrite-mmict & NWA 11786 & & $2018-05-26$ \\
\hline Eucrite-mmict & & & 2018-08-31 \\
\hline
\end{tabular}




\begin{tabular}{|c|c|c|c|c|}
\hline Eucrite-pmict & LoV 212 & & Antofagasta & 2018-04-19 \\
\hline Eucrite-pmict & NWA 11704 & & & 2018-03-27 \\
\hline Eucrite-pmict & NWA 12230 & & & 2018-11-13 \\
\hline Eucrite-pmict & NWA 11701 & & & 2018-03-27 \\
\hline Eucrite-pmict & NWA 12339 & & & $2018-12-11$ \\
\hline Eucrite-pmict & NWA 11861 & & & $2018-07-17$ \\
\hline Eucrite-pmict & NWA 11677 & & & 2018-03-27 \\
\hline Eucrite-pmict & NWA 12344 & & & 2018-12-14 \\
\hline Eucrite-pmict & NWA 12193 & & & 2018-11-09 \\
\hline Eucrite-pmict & & & Luxembourg & $2018-09-27$ \\
\hline Eucrite-pmict & NWA 12225 & & & 2018-11-13 \\
\hline Eucrite-pmict & NWA 12243 & & & 2018-11-24 \\
\hline Eucrite-pmict & NWA 11753 & & & $2018-05-26$ \\
\hline Eucrite-pmict & NWA 11934 & & & 2018-08-31 \\
\hline Eucrite-pmict & NWA 12282 & & & $2018-12-02$ \\
\hline Eucrite-pmict & GRO $17168 \quad 41(2)$ & $A$ & & $2018-09-28$ \\
\hline Eucrite-pmict & GRO $17176 \quad 41(2)$ & $A$ & & $2018-09-28$ \\
\hline Eucrite-pmict & NWA 12202 & & & $2018-11-18$ \\
\hline Eucrite-pmict & NWA 12326 & & & $2018-12-11$ \\
\hline Eucrite-pmict & NWA 12340 & & & 2018-12-14 \\
\hline Eucrite-pmict & GRO $1704941(2)$ & $A$ & & $2018-09-28$ \\
\hline Eucrite-pmict & NWA 12195 & & & 2018-11-09 \\
\hline Eucrite-pmict & GRO $1721141(2)$ & $A$ & & $2018-09-28$ \\
\hline Eucrite-pmict & GRO $1700641(2)$ & A & & $2018-09-28$ \\
\hline Eucrite-pmict & NWA 11647 & & & $2018-02-20$ \\
\hline Eucrite-pmict & GRO 17099 41(2) & $A$ & & $2018-09-28$ \\
\hline Eucrite-pmict & GRO 17189 41(2) & $A$ & & $2018-09-28$ \\
\hline Eucrite-pmict & GRO 17048 41(2) & $A$ & & $2018-09-28$ \\
\hline Eucrite-pmict & NWA 12327 & & & $2018-12-11$ \\
\hline Eucrite-pmict & & & Draâ-Tafilale & $2018-08-31$ \\
\hline Eucrite-pmict & GRO 17039 41(2) & $A$ & & $2018-09-28$ \\
\hline Eucrite-unbr & NWA 11969 & & & 2018-09-23 \\
\hline Eucrite-unbr & NWA 11846 & & & 2018-07-04 \\
\hline Eucrite-unbr & NWA 11749 & & & 2018-04-19 \\
\hline Eucrite-unbr & GRO $1721041(2)$ & $A$ & & $2018-09-28$ \\
\hline Eucrite-unbr & NWA 12246 & & & $2018-12-11$ \\
\hline Eucrite-unbr & NWA 12201 & & & 2018-11-09 \\
\hline Eucrite-unbr & GRO 17070 41(2) & $A$ & & $2018-09-28$ \\
\hline Eucrite-unbr & GRO $1720041(2)$ & $A$ & & $2018-09-28$ \\
\hline Eucrite-unbr & NWA 11897 & & & 2018-09-02 \\
\hline Eucrite-unbr & GRO 17098 41(2) & $A$ & & $2018-09-28$ \\
\hline $\mathrm{H}$ & A $12036 \quad J 26$ & $\mathrm{JB}$ & & 2018-04-18 \\
\hline $\mathrm{H}$ & A 09242 & $J B$ & & 2018-04-18 \\
\hline H/L4 & NWA 11570 & & & 2018-01-16 \\
\hline $\mathrm{H}^{\sim 4}$ & NWA 11579 & & & 2018-01-15 \\
\hline
\end{tabular}




\begin{tabular}{|c|c|c|c|c|c|}
\hline $\mathrm{H}^{\sim 5}$ & NWA 11972 & & & & 2018-09-02 \\
\hline $\mathrm{H}^{\sim} 5$ & & & & Rio de Oro & 2018-01-15 \\
\hline $\mathrm{H}^{\sim} 5$ & AM 044 & & & Xinjiang & 2018-10-06 \\
\hline $\mathrm{H}^{\sim} 5$ & NWA 11973 & & & & 2018-09-02 \\
\hline $\mathrm{H}^{\sim} 5$ & NWA 1230 & & & & 2018-11-09 \\
\hline $\mathrm{H} \sim 5$ & NWA 12383 & & & & $2018-12-24$ \\
\hline $\mathrm{H}^{\sim} 5$ & NWA 1197 & & & & 2018-09-02 \\
\hline $\mathrm{H}^{\sim 5}$ & NWA 11974 & & & & 2018-09-02 \\
\hline $\mathrm{H}^{\sim} 5$ & & & & Xinjiang & 2018-11-09 \\
\hline $\mathrm{H}^{\sim} 5$ & NWA 12382 & & & & $2018-12-24$ \\
\hline $\mathrm{H}^{\sim 5}$ & & & & Xinjiang & 2018-11-09 \\
\hline $\mathrm{H}^{\sim} 5$ & $N$ & & & Xinjiang & $2018-12-16$ \\
\hline $\mathrm{H}^{\sim} 6$ & NWA 12299 & & & & 2018-11-09 \\
\hline $\mathrm{H}^{\sim} 6$ & NWA 11580 & & & & 2018-01-15 \\
\hline $\mathrm{H}^{\sim} 6$ & & & & Gansu & $2018-12-16$ \\
\hline $\mathrm{H}^{\sim} 6$ & MYB 001 & & & XinJiang & 2018-11-24 \\
\hline $\mathrm{H}^{\sim} 6$ & NWA $1197 \mathrm{~s}$ & & & & 2018-09-02 \\
\hline $\mathrm{H} \sim 6$ & NWA 1158 & & & & $2018-01-15$ \\
\hline H3 & & & & Ouargla & $2018-06-10$ \\
\hline H3 & & & & Ajdabiya & 2018-07-17 \\
\hline H3 & NWA 11936 & & & & 2018-08-03 \\
\hline H3 & & & & Antofagasta & $2018-05-28$ \\
\hline H3 & NWA 432 & & & & $2018-10-20$ \\
\hline H3 & EM 377 & & & Antofagasta & $2018-03-27$ \\
\hline H3 & NWA 11923 & & & & 2018-08-03 \\
\hline H3 & RKP 14003 & - & K & & $2018-04-23$ \\
\hline H3 & StV 024 & & & Nevada & $2018-07-20$ \\
\hline H3 & A 12327 & $\mathrm{~J} 26$ & $J B$ & & $2018-04-18$ \\
\hline H3 & NWA 1192 & & & & 2018-08-03 \\
\hline H3 & & & & & 2018-09-15 \\
\hline H3 & A 12323 & $\mathrm{~J} 26$ & $\mathrm{JB}$ & & 2018-04-18 \\
\hline H3 & & & & Alabama & 2018-04-19 \\
\hline H3 & A 12330 & $\mathrm{~J} 26$ & $\mathrm{JB}$ & & $2018-04-18$ \\
\hline H3 & RKP 14006 & - & $\mathrm{K}$ & & $2018-04-23$ \\
\hline H3 & NWA 11810 & & & & 2018-05-11 \\
\hline H3 & NWA 1180 & & & & 2018-05-11 \\
\hline H3 & A 12262 & $\mathrm{~J} 26$ & $\mathrm{JB}$ & & 2018-04-18 \\
\hline H3 & EET 14058 & - & $\mathrm{K}$ & & $2018-04-23$ \\
\hline H3-5 & NWA 1189 & & & & $2018-07-20$ \\
\hline H3-5 & & & & Arizona & 2018-04-07 \\
\hline H3-5 & & & & Arizona & 2018-08-03 \\
\hline H3-5 & & & & Arizona & 2018-04-07 \\
\hline H3-5 & & & & Arizona & 2018-04-07 \\
\hline H3-6 & & & & Antofagasta & 2018-04-19 \\
\hline H3-6 & & & & Arizona & 2018-04-07 \\
\hline
\end{tabular}




\begin{tabular}{|c|c|c|c|c|c|}
\hline H3-6 & & & & Arizona & 2018-04-07 \\
\hline H3-6 & & & & Arizona & 2018-04-07 \\
\hline H3.5 & DOM 1442 & $41(2)$ & A & & $2018-09-28$ \\
\hline H3.5 & DOM 1449 & $41(2)$ & A & & $2018-09-28$ \\
\hline $\mathrm{H} 4$ & NWA 1170 & & & & 2018-04-07 \\
\hline $\mathrm{H} 4$ & & & & Antofagasta & 2018-09-15 \\
\hline $\mathrm{H} 4$ & NWA 1235 & & & & $2018-11-27$ \\
\hline $\mathrm{H} 4$ & & & & Jarayan al Be & ع 2018-04-07 \\
\hline $\mathrm{H} 4$ & & & & & $2018-07-20$ \\
\hline $\mathrm{H} 4$ & EM 380 & & & Antofagasta & 2018-04-19 \\
\hline $\mathrm{H} 4$ & & & & Ash Shamal & 2018-04-07 \\
\hline $\mathrm{H} 4$ & NWA 1224 & & & & $2018-10-20$ \\
\hline $\mathrm{H} 4$ & NWA 1223 & & & & $2018-10-20$ \\
\hline $\mathrm{H} 4$ & 38 & & & Antofagasta & 2018-11-08 \\
\hline H4 & A 12211 & J26 & $\mathrm{JB}$ & & 2018-04-18 \\
\hline $\mathrm{H} 4$ & NWA 1215 & & & & $2018-10-08$ \\
\hline $\mathrm{H} 4$ & & & & Michigan & 2018-03-02 \\
\hline H4 & LoV 259 & & & Antofagasta & $2018-12-06$ \\
\hline $\mathrm{H} 4$ & NWA 1215 & & & & $2018-10-08$ \\
\hline $\mathrm{H} 4$ & NWA 660 & & & & $2018-10-20$ \\
\hline H4 & NWA 1193 & & & & 2018-08-03 \\
\hline $\mathrm{H} 4$ & A 12309 & J26 & $\mathrm{JB}$ & & 2018-04-18 \\
\hline $\mathrm{H} 4$ & NWA 1215 & & & & $2018-10-08$ \\
\hline H4 & NWA 453 & & & & $2018-10-20$ \\
\hline $\mathrm{H} 4$ & NWA 1234 & & & & $2018-11-27$ \\
\hline $\mathrm{H} 4$ & & & & Ajdabiya & $2018-07-17$ \\
\hline H4 & Y-002482 & J26 & $\mathrm{J}$ & & 2018-04-18 \\
\hline $\mathrm{H} 4$ & & & & QingHai & 2018-11-09 \\
\hline $\mathrm{H} 4$ & LoV 239 & & & Antofagasta & $2018-11-08$ \\
\hline H4 & NWA 435 & & & & $2018-10-20$ \\
\hline $\mathrm{H} 4$ & NWA 1202 & & & & $2018-10-08$ \\
\hline $\mathrm{H} 4$ & А 12383 & J26 & $\mathrm{JB}$ & & 2018-04-18 \\
\hline H4 & NWA 452 & & & & $2018-10-20$ \\
\hline $\mathrm{H} 4$ & NWA 1202 & & & & $2018-10-08$ \\
\hline $\mathrm{H} 4$ & DOM 1446 & $41(1)$ & A & & $2018-02-23$ \\
\hline H4 & & & & Ajdabiya & 2018-07-17 \\
\hline $\mathrm{H} 4$ & NWA 1181 & & & & $2018-05-26$ \\
\hline $\mathrm{H} 4$ & & & & Antofagasta & 2018-07-24 \\
\hline H4 & A 12040 & $\mathrm{~J} 26$ & $\mathrm{JB}$ & & 2018-04-18 \\
\hline $\mathrm{H} 4$ & NWA 1203 & & & & $2018-10-08$ \\
\hline H4 & NWA 1211 & & & & 2018-10-08 \\
\hline H4 & NWA 1194 & & & & 2018-08-03 \\
\hline $\mathrm{H} 4$ & A 12136 & J26 & JB & & $2018-04-18$ \\
\hline $\mathrm{H} 4$ & NWA 1211 & & & & 2018-10-08 \\
\hline $\mathrm{H} 4$ & NWA 1202 & & & & 2018-10-08 \\
\hline
\end{tabular}




\begin{tabular}{|c|c|c|c|c|c|}
\hline H4 & NWA 1202 & & & & $2018-10-08$ \\
\hline $\mathrm{H} 4$ & LoV 253 & & & Antofagasta & 2018-11-08 \\
\hline $\mathrm{H} 4$ & NWA 1183 & & & & 2018-07-04 \\
\hline $\mathrm{H} 4$ & NWA 1203 & & & & 2018-10-08 \\
\hline $\mathrm{H} 4$ & NWA 317 & & & & $2018-10-20$ \\
\hline $\mathrm{H} 4$ & NWA 1225 & & & Morocco, Lae & a 2018-11-08 \\
\hline $\mathrm{H} 4$ & NWA 1202 & & & & $2018-10-08$ \\
\hline $\mathrm{H} 4$ & NWA 1211 & & & & $2018-10-08$ \\
\hline H4 & & & & Antofagasta & $2018-11-08$ \\
\hline $\mathrm{H} 4$ & NWA 1207 & & & & $2018-10-08$ \\
\hline $\mathrm{H} 4$ & A 12233 & $\mathrm{~J} 26$ & $\mathrm{JB}$ & & 2018-04-18 \\
\hline $\mathrm{H} 4$ & & & & Ajdabiya & 2018-07-17 \\
\hline $\mathrm{H} 4$ & LoV 230 & & & Antofagasta & $2018-07-17$ \\
\hline $\mathrm{H} 4$ & NWA 1203 & & & & $2018-10-08$ \\
\hline H4 & A 12316 & $\mathrm{~J} 26$ & $\mathrm{JB}$ & & 2018-04-18 \\
\hline $\mathrm{H} 4$ & NWA 1216 & & & & 2018-10-08 \\
\hline $\mathrm{H} 4$ & & & & Antofagasta & 2018-12-02 \\
\hline H4 & NWA 318 & & & & $2018-10-20$ \\
\hline $\mathrm{H} 4$ & NWA 1204 & & & & 2018-10-08 \\
\hline $\mathrm{H} 4$ & NWA 1225 & & & & 2018-11-08 \\
\hline $\mathrm{H} 4$ & A 12332 & J26 & $\mathrm{JB}$ & & $2018-04-18$ \\
\hline $\mathrm{H} 4$ & NWA 1208 & & & & 2018-10-08 \\
\hline $\mathrm{H} 4$ & NWA 1205 & & & & $2018-10-08$ \\
\hline $\mathrm{H} 4$ & A 12370 & J26 & JB & & 2018-04-18 \\
\hline $\mathrm{H} 4$ & & & & & $2018-07-20$ \\
\hline $\mathrm{H} 4$ & DOM 1412 & $41(1)$ & A & & $2018-02-23$ \\
\hline H4 & NWA 1211 & & & & $2018-10-08$ \\
\hline $\mathrm{H} 4$ & A 12226 & $\mathrm{~J} 26$ & $\mathrm{JB}$ & & $2018-04-18$ \\
\hline $\mathrm{H} 4$ & NWA 1209 & & & & $2018-10-08$ \\
\hline H4 & A 12053 & $\mathrm{~J} 26$ & $\mathrm{JB}$ & & 2018-04-18 \\
\hline $\mathrm{H} 4$ & NWA 1168 & & & & $2018-02-20$ \\
\hline H4 & NWA 1203 & & & & 2018-10-08 \\
\hline H4 & A 12092 & $\mathrm{~J} 26$ & $\mathrm{JB}$ & & 2018-04-18 \\
\hline $\mathrm{H} 4$ & NWA 1169 & & & & $2018-02-20$ \\
\hline H4 & NWA 1203 & & & & $2018-10-08$ \\
\hline H4 & DOM 1441 & $41(2)$ & $A$ & & $2018-09-28$ \\
\hline $\mathrm{H} 4$ & NWA 1207 & & & & $2018-10-08$ \\
\hline H4 & & & & Ajdabiya & $2018-07-17$ \\
\hline H4 & NWA 1215 & & & & 2018-10-08 \\
\hline $\mathrm{H} 4$ & A 12419 & J26 & $\mathrm{JB}$ & & $2018-04-18$ \\
\hline H4 & NWA 1214 & & & & $2018-10-08$ \\
\hline H4 & NWA 1205 & & & & 2018-10-08 \\
\hline $\mathrm{H} 4$ & A 12227 & $\mathrm{~J} 26$ & $\mathrm{JB}$ & & 2018-04-18 \\
\hline $\mathrm{H} 4$ & A 12240 & J26 & $J B$ & & $2018-04-18$ \\
\hline H4 & A 12026 & J26 & JB & & 2018-04-18 \\
\hline
\end{tabular}




\begin{tabular}{|c|c|c|c|c|}
\hline $\mathrm{H} 4$ & A 12230 & J26 & $J B$ & 2018-04-18 \\
\hline $\mathrm{H} 4$ & NWA 316 & & & $2018-10-20$ \\
\hline $\mathrm{H} 4$ & A 12242 & J26 & $J B$ & 2018-04-18 \\
\hline $\mathrm{H} 4$ & A 12244 & $\mathrm{~J} 26$ & $J B$ & 2018-04-18 \\
\hline $\mathrm{H} 4$ & NWA 12161 & & & 2018-10-08 \\
\hline $\mathrm{H} 4$ & A 12436 & $\mathrm{~J} 26$ & $J B$ & 2018-04-18 \\
\hline $\mathrm{H} 4$ & Y-001599 & $\mathrm{J} 26$ & $J$ & 2018-04-18 \\
\hline $\mathrm{H} 4$ & A 12399 & $\mathrm{~J} 26$ & $J B$ & 2018-04-18 \\
\hline $\mathrm{H} 4$ & NWA 12019 & & & 2018-09-23 \\
\hline $\mathrm{H} 4$ & A 12234 & $\mathrm{~J} 26$ & $J B$ & 2018-04-18 \\
\hline $\mathrm{H} 4$ & A 12252 & $\mathrm{~J} 26$ & $J B$ & 2018-04-18 \\
\hline $\mathrm{H} 4$ & NWA 11662 & & & $2018-02-24$ \\
\hline $\mathrm{H} 4$ & NWA 12097 & & & $2018-10-08$ \\
\hline $\mathrm{H} 4$ & NWA 12113 & & & 2018-10-08 \\
\hline $\mathrm{H} 4$ & A 12388 & $\mathrm{~J} 26$ & $J B$ & 2018-04-18 \\
\hline $\mathrm{H} 4$ & NWA 12164 & & & 2018-10-08 \\
\hline $\mathrm{H} 4$ & A 12251 & $\mathrm{~J} 26$ & $\mathrm{JB}$ & 2018-04-18 \\
\hline $\mathrm{H} 4$ & NWA 12136 & & & $2018-10-08$ \\
\hline $\mathrm{H} 4$ & A 12346 & $\mathrm{~J} 26$ & $J B$ & 2018-04-18 \\
\hline $\mathrm{H} 4$ & A 12329 & $\mathrm{~J} 26$ & $J B$ & 2018-04-18 \\
\hline $\mathrm{H} 4$ & DOM 14185 & $41(1)$ & $A$ & $2018-02-23$ \\
\hline $\mathrm{H} 4$ & DOM 14188 & $41(2)$ & $A$ & 2018-09-28 \\
\hline $\mathrm{H} 4$ & Y-74426 & $\mathrm{J} 26$ & $J$ & 2018-04-18 \\
\hline $\mathrm{H} 4$ & DOM 14377 & $41(1)$ & A & $2018-02-23$ \\
\hline $\mathrm{H} 4$ & DOM 14435 & $41(2)$ & A & 2018-09-28 \\
\hline $\mathrm{H} 4$ & A 12311 & $\mathrm{~J} 26$ & $\mathrm{JB}$ & 2018-04-18 \\
\hline $\mathrm{H} 4$ & A 12299 & J26 & $J B$ & 2018-04-18 \\
\hline $\mathrm{H} 4$ & A 12379 & $\mathrm{~J} 26$ & $J B$ & 2018-04-18 \\
\hline $\mathrm{H} 4$ & NWA 12167 & & & 2018-10-08 \\
\hline $\mathrm{H} 4$ & Y-001297 & J26 & $\mathrm{J}$ & 2018-04-18 \\
\hline $\mathrm{H} 4$ & A 12320 & $\mathrm{~J} 26$ & $\mathrm{JB}$ & 2018-04-18 \\
\hline $\mathrm{H} 4$ & A 12257 & $\mathrm{~J} 26$ & $\mathrm{JB}$ & 2018-04-18 \\
\hline $\mathrm{H} 4$ & Y-001429 & J26 & $\mathrm{J}$ & $2018-04-18$ \\
\hline $\mathrm{H} 4$ & A 12331 & $\mathrm{~J} 26$ & $J B$ & 2018-04-18 \\
\hline $\mathrm{H} 4$ & DOM 14037 & $41(1)$ & $A$ & 2018-02-23 \\
\hline $\mathrm{H} 4$ & Y-002480 & J26 & $J$ & 2018-04-18 \\
\hline $\mathrm{H} 4$ & A 12238 & $\mathrm{~J} 26$ & $\mathrm{JB}$ & $2018-04-18$ \\
\hline $\mathrm{H} 4$ & & & Antofagasta & 2018-10-09 \\
\hline $\mathrm{H} 4$ & A 12360 & J26 & $\mathrm{JB}$ & 2018-04-18 \\
\hline $\mathrm{H} 4$ & A 12338 & $\mathrm{~J} 26$ & $\mathrm{JB}$ & 2018-04-18 \\
\hline $\mathrm{H} 4$ & A 12232 & $\mathrm{~J} 26$ & $J B$ & 2018-04-18 \\
\hline $\mathrm{H} 4$ & Y-791739 & J26 & $J$ & $2018-04-18$ \\
\hline $\mathrm{H} 4$ & DOM 14453 & $41(1)$ & $A$ & $2018-02-23$ \\
\hline $\mathrm{H} 4$ & Y-000046 & $\mathrm{J} 26$ & $\mathrm{~J}$ & 2018-04-18 \\
\hline $\mathrm{H} 4$ & A 09625 & $\mathrm{~J} 26$ & $J B$ & 2018-04-18 \\
\hline
\end{tabular}




\begin{tabular}{|c|c|c|c|c|}
\hline H4 & A 09390 & J26 & JB & 2018-04-18 \\
\hline $\mathrm{H} 4$ & A 09653 & J26 & $J B$ & 2018-04-18 \\
\hline $\mathrm{H} 4$ & A 09629 & J26 & $\mathrm{JB}$ & 2018-04-18 \\
\hline H4 & A 09353 & $\mathrm{~J} 26$ & $J B$ & 2018-04-18 \\
\hline $\mathrm{H} 4$ & A 09339 & J26 & $J B$ & 2018-04-18 \\
\hline $\mathrm{H} 4$ & A 09611 & $\mathrm{~J} 26$ & $\mathrm{JB}$ & 2018-04-18 \\
\hline $\mathrm{H} 4$ & A 09275 & $\mathrm{~J} 26$ & $J B$ & 2018-04-18 \\
\hline H4 & A 09437 & J26 & $J B$ & 2018-04-18 \\
\hline $\mathrm{H} 4$ & A 09278 & J26 & $\mathrm{JB}$ & 2018-04-18 \\
\hline $\mathrm{H} 4$ & A 09633 & $\mathrm{~J} 26$ & $J B$ & 2018-04-18 \\
\hline H4 & A 09654 & J26 & JB & 2018-04-18 \\
\hline $\mathrm{H} 4$ & A 09485 & $\mathrm{~J} 26$ & $\mathrm{JB}$ & 2018-04-18 \\
\hline $\mathrm{H} 4$ & A 09472 & $\mathrm{~J} 26$ & $\mathrm{JB}$ & 2018-04-18 \\
\hline H4 & A 09276 & $\mathrm{~J} 26$ & $\mathrm{JB}$ & 2018-04-18 \\
\hline $\mathrm{H} 4$ & NWA 11743 & & & 2018-03-10 \\
\hline H4 & $Y-000491$ & $\mathrm{~J} 26$ & $J$ & 2018-04-18 \\
\hline $\mathrm{H} 4$ & $Y-000303$ & $\mathrm{~J} 26$ & $\mathrm{~J}$ & 2018-04-18 \\
\hline H4 & Y-000301 & J26 & $\mathrm{J}$ & 2018-04-18 \\
\hline $\mathrm{H} 4$ & Y-000481 & J26 & $\mathrm{J}$ & 2018-04-18 \\
\hline $\mathrm{H} 4$ & Y-000369 & $\mathrm{J} 26$ & $\mathrm{~J}$ & 2018-04-18 \\
\hline $\mathrm{H} 4$ & Y-000234 & $\mathrm{J} 26$ & $J$ & 2018-04-18 \\
\hline $\mathrm{H} 4$ & A 09657 & $\mathrm{~J} 26$ & $\mathrm{JB}$ & 2018-04-18 \\
\hline $\mathrm{H} 4$ & Y-000582 & $\mathrm{J} 26$ & $J$ & 2018-04-18 \\
\hline H4 & А 09659 & J26 & JB & 2018-04-18 \\
\hline $\mathrm{H} 4$ & Y-003223 & J26 & $\mathrm{J}$ & 2018-04-18 \\
\hline $\mathrm{H} 4$ & Y-000612 & $\mathrm{J} 26$ & $J$ & 2018-04-18 \\
\hline H4 & Y-000187 & J26 & $J$ & 2018-04-18 \\
\hline $\mathrm{H} 4$ & A 09280 & J26 & $\mathrm{JB}$ & 2018-04-18 \\
\hline $\mathrm{H} 4$ & A 12246 & J26 & $\mathrm{JB}$ & 2018-04-18 \\
\hline H4 & A 09632 & $\mathrm{~J} 26$ & JB & 2018-04-18 \\
\hline $\mathrm{H} 4$ & Y-000272 & J26 & $\mathrm{J}$ & 2018-04-18 \\
\hline $\mathrm{H} 4$ & A 09233 & $\mathrm{~J} 26$ & $\mathrm{JB}$ & 2018-04-18 \\
\hline H4 & Y-000615 & J26 & $J$ & 2018-04-18 \\
\hline $\mathrm{H} 4$ & Y-000178 & J26 & $\mathrm{J}$ & $2018-04-18$ \\
\hline $\mathrm{H} 4$ & Y-000180 & $\mathrm{J} 26$ & $\mathrm{~J}$ & 2018-04-18 \\
\hline H4 & Y-000203 & J26 & $J$ & 2018-04-18 \\
\hline $\mathrm{H} 4$ & A 09283 & J26 & $J B$ & 2018-04-18 \\
\hline $\mathrm{H} 4$ & Y-003091 & $\mathrm{J} 26$ & $J$ & 2018-04-18 \\
\hline H4 & A 09663 & J26 & $J B$ & 2018-04-18 \\
\hline $\mathrm{H} 4$ & Y-000769 & J26 & $\mathrm{J}$ & 2018-04-18 \\
\hline $\mathrm{H} 4$ & Y-000339 & $\mathrm{J} 26$ & $J$ & 2018-04-18 \\
\hline $\mathrm{H} 4$ & A 09248 & J26 & $\mathrm{JB}$ & 2018-04-18 \\
\hline $\mathrm{H} 4$ & Y-000375 & J26 & $\mathrm{J}$ & $2018-04-18$ \\
\hline $\mathrm{H} 4$ & A 09665 & J26 & $J B$ & 2018-04-18 \\
\hline H4 & Y-000810 & J26 & $\mathrm{J}$ & 2018-04-18 \\
\hline
\end{tabular}




\begin{tabular}{|c|c|c|c|c|}
\hline $\mathrm{H} 4$ & Y-003122 & $\mathrm{J} 26$ & $J$ & 2018-04-18 \\
\hline $\mathrm{H} 4$ & A 09626 & $\mathrm{~J} 26$ & $\mathrm{JB}$ & 2018-04-18 \\
\hline $\mathrm{H} 4$ & A 09411 & J26 & $\mathrm{JB}$ & 2018-04-18 \\
\hline $\mathrm{H} 4$ & Y-000741 & $\mathrm{J} 26$ & $J$ & 2018-04-18 \\
\hline $\mathrm{H} 4$ & Y-000216 & $\mathrm{J} 26$ & $J$ & 2018-04-18 \\
\hline $\mathrm{H} 4$ & A 09333 & $\mathrm{~J} 26$ & $J B$ & 2018-04-18 \\
\hline H4 & Y-000398 & J26 & $J$ & $2018-04-18$ \\
\hline $\mathrm{H} 4$ & A 09429 & $\mathrm{~J} 26$ & $J B$ & $2018-04-18$ \\
\hline $\mathrm{H} 4$ & A 09247 & $\mathrm{~J} 26$ & $\mathrm{JB}$ & 2018-04-18 \\
\hline $\mathrm{H} 4$ & A 09444 & $\mathrm{~J} 26$ & $J B$ & 2018-04-18 \\
\hline $\mathrm{H} 4$ & Y-000573 & $\mathrm{J} 26$ & $J$ & $2018-04-18$ \\
\hline $\mathrm{H} 4$ & Y-003022 & J26 & $J$ & 2018-04-18 \\
\hline H4 & A 09288 & $\mathrm{~J} 26$ & $\mathrm{JB}$ & $2018-04-18$ \\
\hline $\mathrm{H} 4$ & Y-000546 & $\mathrm{J} 26$ & $\mathrm{~J}$ & 2018-04-18 \\
\hline $\mathrm{H} 4$ & A 09282 & $\mathrm{~J} 26$ & $\mathrm{JB}$ & 2018-04-18 \\
\hline $\mathrm{H} 4$ & A 09279 & $\mathrm{~J} 26$ & $\mathrm{JB}$ & 2018-04-18 \\
\hline $\mathrm{H} 4$ & Y-000447 & $\mathrm{J} 26$ & $J$ & 2018-04-18 \\
\hline $\mathrm{H} 4$ & A 09660 & J26 & $J B$ & 2018-04-18 \\
\hline H4 & A 09669 & J26 & $J B$ & 2018-04-18 \\
\hline $\mathrm{H} 4$ & A 09661 & $\mathrm{~J} 26$ & $\mathrm{JB}$ & 2018-04-18 \\
\hline $\mathrm{H} 4$ & A 09664 & $\mathrm{~J} 26$ & $\mathrm{JB}$ & 2018-04-18 \\
\hline $\mathrm{H} 4$ & Y-000791 & $\mathrm{J} 26$ & $J$ & 2018-04-18 \\
\hline $\mathrm{H} 4$ & A 09285 & $\mathrm{~J} 26$ & $\mathrm{JB}$ & 2018-04-18 \\
\hline $\mathrm{H} 4$ & A 09407 & J26 & JB & 2018-04-18 \\
\hline H4 & A 09666 & $\mathrm{~J} 26$ & JB & $2018-04-18$ \\
\hline $\mathrm{H} 4$ & Y-000211 & $\mathrm{J} 26$ & $J$ & 2018-04-18 \\
\hline $\mathrm{H} 4$ & Y-000772 & J26 & $\mathrm{J}$ & 2018-04-18 \\
\hline $\mathrm{H} 4$ & Y-000371 & $\mathrm{J} 26$ & $J$ & 2018-04-18 \\
\hline $\mathrm{H} 4$ & A 09340 & $\mathrm{~J} 26$ & $\mathrm{JB}$ & 2018-04-18 \\
\hline $\mathrm{H} 4$ & A 09634 & J26 & $\mathrm{JB}$ & 2018-04-18 \\
\hline $\mathrm{H} 4$ & A 09284 & $\mathrm{~J} 26$ & $\mathrm{JB}$ & 2018-04-18 \\
\hline $\mathrm{H} 4$ & Y-000763 & $\mathrm{J} 26$ & $J$ & 2018-04-18 \\
\hline $\mathrm{H} 4$ & Y-000583 & J26 & $\mathrm{J}$ & $2018-04-18$ \\
\hline $\mathrm{H} 4$ & Y-000581 & $\mathrm{J} 26$ & $\mathrm{~J}$ & 2018-04-18 \\
\hline $\mathrm{H} 4$ & Y-003218 & $\mathrm{J} 26$ & $J$ & 2018-04-18 \\
\hline $\mathrm{H} 4$ & А 09656 & J26 & $J B$ & 2018-04-18 \\
\hline $\mathrm{H} 4$ & A 09252 & $\mathrm{~J} 26$ & $\mathrm{JB}$ & 2018-04-18 \\
\hline $\mathrm{H} 4$ & A 09281 & $\mathrm{~J} 26$ & $J B$ & 2018-04-18 \\
\hline $\mathrm{H} 4$ & Y-002129 & J26 & $\mathrm{J}$ & $2018-04-18$ \\
\hline $\mathrm{H} 4$ & A 09667 & $\mathrm{~J} 26$ & $\mathrm{JB}$ & 2018-04-18 \\
\hline $\mathrm{H} 4$ & A 09662 & $\mathrm{~J} 26$ & $J B$ & 2018-04-18 \\
\hline $\mathrm{H} 4$ & A 09440 & J26 & $J B$ & 2018-04-18 \\
\hline $\mathrm{H} 4$ & A 09289 & $\mathrm{~J} 26$ & $\mathrm{JB}$ & $2018-04-18$ \\
\hline $\mathrm{H} 4$ & A 09668 & $\mathrm{~J} 26$ & $J B$ & 2018-04-18 \\
\hline $\mathrm{H} 4$ & A 09230 & $\mathrm{~J} 26$ & $J B$ & 2018-04-18 \\
\hline
\end{tabular}




\begin{tabular}{|c|c|c|c|c|}
\hline $\mathrm{H} 4$ & A 09325 & J26 & JB & 2018-04-18 \\
\hline $\mathrm{H} 4$ & A 09673 & $\mathrm{~J} 26$ & $\mathrm{JB}$ & $2018-04-18$ \\
\hline $\mathrm{H} 4$ & Y-000008 & $\mathrm{J} 26$ & $\mathrm{~J}$ & 2018-04-18 \\
\hline $\mathrm{H} 4$ & A 09678 & $\mathrm{~J} 26$ & $J B$ & 2018-04-18 \\
\hline $\mathrm{H} 4$ & A 09231 & $\mathrm{~J} 26$ & $J B$ & $2018-04-18$ \\
\hline $\mathrm{H} 4$ & A 09286 & $\mathrm{~J} 26$ & $\mathrm{JB}$ & $2018-04-18$ \\
\hline $\mathrm{H} 4$ & A 09287 & $\mathrm{~J} 26$ & $J B$ & 2018-04-18 \\
\hline $\mathrm{H} 4$ & A 09671 & J26 & $J B$ & 2018-04-18 \\
\hline H4 & A 09228 & $\mathrm{~J} 26$ & $\mathrm{JB}$ & $2018-04-18$ \\
\hline H4 & A 09631 & $\mathrm{~J} 26$ & $J B$ & 2018-04-18 \\
\hline $\mathrm{H} 4$ & A 09670 & $\mathrm{~J} 26$ & $J B$ & 2018-04-18 \\
\hline H4 & A 09290 & $\mathrm{~J} 26$ & $\mathrm{JB}$ & 2018-04-18 \\
\hline $\mathrm{H} 4$ & A 09263 & $\mathrm{~J} 26$ & $J B$ & 2018-04-18 \\
\hline $\mathrm{H} 4$ & A 09674 & $\mathrm{~J} 26$ & $\mathrm{JB}$ & $2018-04-18$ \\
\hline H4 & A 09293 & $\mathrm{~J} 26$ & $\mathrm{JB}$ & 2018-04-18 \\
\hline $\mathrm{H} 4$ & A 09628 & $\mathrm{~J} 26$ & $J B$ & 2018-04-18 \\
\hline H4 & A 09291 & $\mathrm{~J} 26$ & $J B$ & $2018-04-18$ \\
\hline H4 & A 09294 & J26 & JB & 2018-04-18 \\
\hline $\mathrm{H} 4$ & A 09295 & J26 & $\mathrm{JB}$ & 2018-04-18 \\
\hline H4 & A 09612 & $\mathrm{~J} 26$ & $\mathrm{JB}$ & $2018-04-18$ \\
\hline H4 & А 09299 & $\mathrm{~J} 26$ & JB & 2018-04-18 \\
\hline $\mathrm{H} 4$ & A 09298 & J26 & $J B$ & 2018-04-18 \\
\hline $\mathrm{H} 4$ & A 09395 & $\mathrm{~J} 26$ & JB & $2018-04-18$ \\
\hline H4 & A 09304 & J26 & $\mathrm{JB}$ & 2018-04-18 \\
\hline $\mathrm{H} 4$ & A 09613 & J26 & $\mathrm{JB}$ & 2018-04-18 \\
\hline $\mathrm{H} 4$ & A 09321 & $\mathrm{~J} 26$ & $\mathrm{JB}$ & $2018-04-18$ \\
\hline H4 & A 09300 & $\mathrm{~J} 26$ & $J B$ & 2018-04-18 \\
\hline $\mathrm{H} 4$ & A 09677 & J26 & $\mathrm{JB}$ & 2018-04-18 \\
\hline $\mathrm{H} 4$ & A 09234 & $\mathrm{~J} 26$ & $\mathrm{JB}$ & $2018-04-18$ \\
\hline H4 & A 09398 & J26 & JB & 2018-04-18 \\
\hline $\mathrm{H} 4$ & А 09296 & $\mathrm{~J} 26$ & $\mathrm{JB}$ & 2018-04-18 \\
\hline H4 & A 09636 & $\mathrm{~J} 26$ & $\mathrm{JB}$ & 2018-04-18 \\
\hline H4 & A 09297 & J26 & $J B$ & 2018-04-18 \\
\hline $\mathrm{H} 4$ & A 09635 & J26 & $\mathrm{JB}$ & 2018-04-18 \\
\hline $\mathrm{H} 4$ & Y-002122 & $\mathrm{J} 26$ & $\mathrm{~J}$ & $2018-04-18$ \\
\hline H4 & A 09477 & J26 & $J B$ & 2018-04-18 \\
\hline $\mathrm{H} 4$ & A 09614 & J26 & $J B$ & $2018-04-18$ \\
\hline $\mathrm{H} 4$ & A 09315 & J26 & $\mathrm{JB}$ & 2018-04-18 \\
\hline H4 & A 09320 & J26 & $J B$ & $2018-04-18$ \\
\hline $\mathrm{H} 4$ & A 09401 & J26 & $\mathrm{JB}$ & 2018-04-18 \\
\hline $\mathrm{H} 4$ & A 10206 & J26 & $\mathrm{JB}$ & 2018-04-18 \\
\hline H4 & A 09623 & J26 & $\mathrm{JB}$ & 2018-04-18 \\
\hline $\mathrm{H} 4$ & MIL 15105 & $41(1)$ & $A$ & $2018-02-23$ \\
\hline $\mathrm{H} 4$ & A 09309 & J26 & $\mathrm{JB}$ & 2018-04-18 \\
\hline H4 & A 10216 & J26 & $J B$ & 2018-04-18 \\
\hline
\end{tabular}




\begin{tabular}{|c|c|c|c|c|c|}
\hline H4 & A 09630 & J26 & $J B$ & & 2018-04-18 \\
\hline $\mathrm{H} 4$ & Y-002609 & $\mathrm{J} 26$ & $J$ & & 2018-04-18 \\
\hline H4 & Y-003129 & J26 & $J$ & & 2018-04-18 \\
\hline $\mathrm{H} 4$ & A 09311 & J26 & $\mathrm{JB}$ & & 2018-04-18 \\
\hline $\mathrm{H} 4$ & A 09310 & $\mathrm{~J} 26$ & $J B$ & & 2018-04-18 \\
\hline H4 & Y-002791 & J26 & $J$ & & 2018-04-18 \\
\hline $\mathrm{H} 4$ & Y-002607 & J26 & $\mathrm{J}$ & & 2018-04-18 \\
\hline $\mathrm{H} 4$ & Y-001877 & $\mathrm{J} 26$ & $J$ & & 2018-04-18 \\
\hline H4 & Y-002572 & J26 & $J$ & & 2018-04-18 \\
\hline $\mathrm{H} 4$ & Y-001878 & J26 & $J$ & & 2018-04-18 \\
\hline $\mathrm{H} 4$ & Y-001931 & $\mathrm{J} 26$ & $J$ & & 2018-04-18 \\
\hline $\mathrm{H} 4$ & Y-002526 & J26 & $J$ & & 2018-04-18 \\
\hline $\mathrm{H} 4$ & Y-001977 & J26 & $J$ & & 2018-04-18 \\
\hline $\mathrm{H} 4$ & A 09313 & $\mathrm{~J} 26$ & $J B$ & & 2018-04-18 \\
\hline H4 & A 09314 & J26 & $J B$ & & 2018-04-18 \\
\hline $\mathrm{H} 4$ & Y-001876 & J26 & $J$ & & 2018-04-18 \\
\hline $\mathrm{H} 4$ & Y-002620 & $\mathrm{J} 26$ & $J$ & & 2018-04-18 \\
\hline H4 & Y-002478 & J26 & $J$ & & 2018-04-18 \\
\hline $\mathrm{H} 4-5$ & & & & Para & 2018-12-02 \\
\hline H4-6 & A 12239 & J26 & $\mathrm{JB}$ & & 2018-04-18 \\
\hline H4-melt breccia & NWA 11572 & & & & 2018-01-16 \\
\hline $\mathrm{H} 4 / 5$ & NWA 11705 & & & & $2018-03-27$ \\
\hline $\mathrm{H} 4 / 5$ & & & & Jarayan al $B c$ & ع 2018-04-07 \\
\hline $\mathrm{H} 4 / 5$ & & & & Jarayan al $B \hat{c}$ & ¿ 2018-04-07 \\
\hline $\mathrm{H} 4 / 5$ & SJ 099 & & & Antofagasta & 2018-11-08 \\
\hline $\mathrm{H} 4 / 5$ & & & & Antofagasta & 2018-11-08 \\
\hline $\mathrm{H} 4 / 5$ & & & & Antofagasta & 2018-07-08 \\
\hline $\mathrm{H} 4 / 5$ & & & & Antofagasta & 2018-11-08 \\
\hline $\mathrm{H} 4 / 5$ & & & & Antofagasta & 2018-11-08 \\
\hline $\mathrm{H} 4 / 5$ & SJ 094 & & & Antofagasta & 2018-11-08 \\
\hline $\mathrm{H} 4 / 5$ & NWA 12354 & & & & $2018-11-27$ \\
\hline $\mathrm{H} 4 / 5$ & NWA 11862 & & & & $2018-06-22$ \\
\hline H5 & & & & Antofagasta & 2018-11-08 \\
\hline H5 & EET 14043 & - & $\mathrm{K}$ & & $2018-04-23$ \\
\hline H5 & EM 379 & & & Antofagasta & 2018-04-19 \\
\hline H5 & & & & Victoria & $2018-12-11$ \\
\hline H5 & EET 14032 & - & $\mathrm{K}$ & & 2018-04-23 \\
\hline H5 & EM 384 & & & Antofagasta & 2018-05-11 \\
\hline H5 & DOM 14001 & $41(2)$ & $A$ & & $2018-09-28$ \\
\hline H5 & & & & East Kazakhs & ؟2018-12-11 \\
\hline H5 & NWA 12001 & & & & 2018-09-15 \\
\hline H5 & NWA 11658 & & & & $2018-02-24$ \\
\hline H5 & NWA 12369 & & & & $2018-12-02$ \\
\hline H5 & NWA 12215 & & & & $2018-10-16$ \\
\hline H5 & DaG 1069 & & & Al Jufrah & $2018-02-24$ \\
\hline
\end{tabular}




\begin{tabular}{|c|c|c|c|c|c|}
\hline H5 & NWA 1238 & & & & $2018-12-24$ \\
\hline H5 & LoV 261 & & & Antofagasta & 2018-12-06 \\
\hline H5 & NWA 1221 & & & & $2018-10-16$ \\
\hline H5 & NWA 11814 & & & & 2018-05-11 \\
\hline H5 & & & & Xinjiang & $2018-10-20$ \\
\hline H5 & NWA 12353 & & & & $2018-11-27$ \\
\hline H5 & & & & Antofagasta & 2018-09-15 \\
\hline H5 & NWA 11642 & & & & $2018-01-27$ \\
\hline H5 & NWA 12347 & & & & $2018-11-27$ \\
\hline H5 & NWA 1089 & & & & $2018-10-20$ \\
\hline H5 & NWA 1192 & & & & 2018-08-03 \\
\hline H5 & Y-001396 & $\mathrm{J} 26$ & $\mathrm{~J}$ & & 2018-04-18 \\
\hline H5 & LoV 221 & & & Antofagasta & 2018-05-11 \\
\hline H5 & NWA 11778 & & & & $2018-07-24$ \\
\hline H5 & NWA 1169 & & & & $2018-02-20$ \\
\hline H5 & NWA 12153 & & & & $2018-10-08$ \\
\hline H5 & 1 & & & Antofagasta & 2018-07-24 \\
\hline H5 & NWA 12172 & & & & $2018-10-08$ \\
\hline H5 & LoV 227 & & & Antofagasta & $2018-07-08$ \\
\hline H5 & LoV 260 & & & Antofagasta & $2018-12-06$ \\
\hline H5 & NWA 451 & & & & $2018-10-20$ \\
\hline H5 & A 12060 & J26 & $\mathrm{JB}$ & & $2018-04-18$ \\
\hline H5 & NWA 12239 & & & & $2018-10-20$ \\
\hline H5 & MIL 15383 & $41(2)$ & A & & $2018-09-28$ \\
\hline H5 & NWA 1215 & & & & $2018-10-08$ \\
\hline H5 & & & & Antofagasta & 2018-10-09 \\
\hline H5 & NWA 1235 & & & & $2018-11-27$ \\
\hline H5 & NWA 1235 & & & & $2018-11-27$ \\
\hline H5 & StV 023 & & & Nevada & 2018-03-27 \\
\hline H5 & EET 14054 & - & $\mathrm{K}$ & & $2018-04-23$ \\
\hline H5 & LoV 234 & & & Antofagasta & 2018-11-08 \\
\hline H5 & NWA 12253 & & & Morocco, Dal & | 2018-11-08 \\
\hline H5 & NWA 12022 & & & & $2018-10-08$ \\
\hline H5 & EM 374 & & & Antofagasta & 2018-03-27 \\
\hline H5 & A 12027 & J26 & $\mathrm{JB}$ & & $2018-04-18$ \\
\hline H5 & LoV 235 & & & Antofagasta & 2018-11-08 \\
\hline H5 & NWA 1212 & & & & $2018-10-08$ \\
\hline H5 & NWA 12083 & & & & $2018-10-08$ \\
\hline H5 & & & & Ajdabiya & $2018-07-17$ \\
\hline H5 & A 12219 & J26 & $\mathrm{JB}$ & & 2018-04-18 \\
\hline H5 & & & & Antofagasta & $2018-07-24$ \\
\hline H5 & & & & Jarayan al Ba & ع 2018-07-20 ع \\
\hline H5 & EM 393 & & & Antofagasta & 2018-11-08 \\
\hline H5 & EM 385 & & & Antofagasta & 2018-05-11 \\
\hline H5 & NWA 1182 & & & & $2018-05-26$ \\
\hline
\end{tabular}


EM 386

$\mathrm{H} 5$

NWA 11683

H5

A 12368 J26

JB

H5

NWA 12126

NWA 12080

EM 375

H5

NWA 11825

NWA 12352

A $12221 \quad J 26$

H5

$\mathrm{H} 5$

$\mathrm{H} 5$

DOM 14234 41(2)

JB

A

LoV 233

NWA 12149

NWA 12128

EM 388

NWA 12033

NWA 12356

NWA 12074

NWA 12044

NWA 12076

NWA 12123

NWA 12107

NWA 12175

A $12212 \quad J 26 \quad J B$

NWA 12030

LoV 242

NWA 11821

NWA 11912

H5

$\mathrm{H} 5$

H5

LoV 232

H5

NWA 11822

NWA 11685

SaU 606

Rio Grande d 2018-06-22

2018-05-26

Antofagasta 2018-05-11

Antofagasta 2018-05-11

Antofagasta 2018-05-11

2018-02-20

2018-04-18

Antofagasta 2018-07-24

2018-10-08

2018-10-08

Antofagasta 2018-03-27

2018-05-26

2018-11-27

2018-04-18

2018-09-28

Antofagasta 2018-05-11

Antofagasta 2018-11-08

2018-10-08

2018-10-08

Antofagasta 2018-07-08

2018-10-08

2018-11-27

Ajdabiya 2018-07-17

2018-10-08

Antofagasta 2018-10-09

2018-10-08

2018-10-08

2018-10-08

2018-10-08

2018-10-08

2018-04-18

2018-10-08

Antofagasta 2018-03-27

Kerman 2018-03-27

Kerman 2018-03-27

Antofagasta 2018-11-08

Antofagasta 2018-05-11

2018-05-26

2018-09-15

Antofagasta 2018-10-09

Antofagasta 2018-11-08

Antofagasta 2018-11-08

2018-05-26

2018-02-20

Al Wusta 2018-04-07 


\begin{tabular}{|c|c|c|c|c|c|}
\hline H5 & NWA 11885 & & & & $2018-07-08$ \\
\hline H5 & NWA 8282 & & & & $2018-07-17$ \\
\hline H5 & NWA 12038 & & & & 2018-10-08 \\
\hline H5 & NWA 12124 & & & & 2018-10-08 \\
\hline H5 & A 12268 & $\mathrm{~J} 26$ & $\mathrm{JB}$ & & 2018-04-18 \\
\hline H5 & A 12117 & $\mathrm{~J} 26$ & $\mathrm{JB}$ & & 2018-04-18 \\
\hline H5 & & & & Antofagasta & $2018-07-17$ \\
\hline H5 & NWA 12134 & & & & $2018-10-08$ \\
\hline H5 & DOM 14463 & $41(1)$ & $A$ & & $2018-02-23$ \\
\hline H5 & LoV 223 & & & Antofagasta & $2018-05-11$ \\
\hline H5 & NWA 12133 & & & & $2018-10-08$ \\
\hline H5 & Prang & & & Saskatchewa & 2018-04-19 \\
\hline H5 & NWA 12163 & & & & 2018-10-08 \\
\hline H5 & 38 & & & Antofagasta & 2018-11-08 \\
\hline H5 & NWA 12036 & & & & 2018-10-08 \\
\hline H5 & EET 14036 & - & $\mathrm{K}$ & & 2018-04-23 \\
\hline H5 & A 12380 & $\mathrm{~J} 26$ & $\mathrm{JB}$ & & $2018-04-18$ \\
\hline H5 & & & & Antofagasta & 2018-03-27 \\
\hline H5 & NWA 12158 & & & & 2018-10-08 \\
\hline H5 & NWA 12173 & & & & 2018-10-08 \\
\hline H5 & NWA 12166 & & & & 2018-10-08 \\
\hline H5 & A 12386 & $\mathrm{~J} 26$ & $\mathrm{JB}$ & & 2018-04-18 \\
\hline H5 & DOM 14439 & $41(2)$ & $A$ & & 2018-09-28 \\
\hline H5 & NWA 12046 & & & & 2018-10-08 \\
\hline H5 & NWA 12079 & & & & 2018-10-08 \\
\hline H5 & A 12322 & $\mathrm{~J} 26$ & $\mathrm{JB}$ & & 2018-04-18 \\
\hline H5 & DOM 14465 & $41(1)$ & $A$ & & $2018-02-23$ \\
\hline H5 & NWA 11816 & & & & $2018-05-26$ \\
\hline H5 & & & & Antofagasta & 2018-07-08 \\
\hline H5 & EET 14052 & - & K & & $2018-04-23$ \\
\hline H5 & A 12318 & $\mathrm{~J} 26$ & $\mathrm{JB}$ & & 2018-04-18 \\
\hline H5 & NWA 12040 & & & & 2018-10-08 \\
\hline H5 & NWA 11689 & & & & $2018-02-20$ \\
\hline H5 & A 12220 & $\mathrm{~J} 26$ & $\mathrm{JB}$ & & 2018-04-18 \\
\hline H5 & NWA 12052 & & & & 2018-10-08 \\
\hline H5 & DOM 14407 & $41(2)$ & $A$ & & $2018-09-28$ \\
\hline H5 & EET 14044 & - & $\mathrm{K}$ & & 2018-04-23 \\
\hline H5 & DOM 14186 & $41(1)$ & $A$ & & $2018-02-23$ \\
\hline H5 & NWA 11826 & & & & $2018-05-26$ \\
\hline H5 & DOM 14466 & $41(1)$ & $A$ & & $2018-02-23$ \\
\hline H5 & A 12024 & $\mathrm{~J} 26$ & $J B$ & & 2018-04-18 \\
\hline H5 & SJ 089 & & & Antofagasta & 2018-09-15 \\
\hline H5 & A 12021 & $\mathrm{~J} 26$ & $\mathrm{JB}$ & & $2018-04-18$ \\
\hline H5 & NWA 11926 & & & & 2018-08-03 \\
\hline H5 & ESF 010 & & & Al Bahr al Ah & 2018-03-27 \\
\hline
\end{tabular}


2018-04-07

2018-10-08

2018-10-08

A $12091 \quad$ J26 JB

SJ 101

EET 16016 41(2)

A NWA 12063

SJ 088

ESF 008

DOM 14408 41(2)

NWA 11931

A

NWA 12067

A $12266 \quad$ J26

NWA 12135

LoV 249

A $12100 \quad$ J26

NWA 11914

EET 14039 - $\quad \mathrm{K}$

NWA 11659

Y-74052 J26

A 12271 J26

ESF 009

NWA 12062

A $12347 \quad$ J26

A $12270 \quad$ J26

NWA 11913

DOM 14410 41(2)

NWA 11687

DOM 14035 41(1)

DOM 14250 41(2)

NWA 11827

NWA 12165

NWA 12102

NWA 12176

EET 14010 -

LoV 219

NWA 12055

NWA 12106

DOM 14473 41(2)

2018-04-18

Antofagasta 2018-11-08

Kerman 2018-05-26

2018-09-28

2018-10-08

Antofagasta 2018-05-11

Antofagasta 2018-07-17

Al Bahr al Ah 2018-03-27

Antofagasta 2018-10-09

2018-09-28

2018-08-03

2018-10-08

2018-04-18

2018-10-08

Antofagasta 2018-11-08

2018-04-18

Antofagasta 2018-04-19

2018-07-24

2018-04-23

2018-02-24

2018-04-18

2018-04-18

Al Bahr al Ah 2018-03-27

2018-10-08

2018-04-18

2018-04-18

2018-07-24

Antofagasta 2018-11-08

2018-09-28

2018-02-20

Antofagasta 2018-04-19

2018-02-23

2018-09-28

2018-05-26

2018-10-08

2018-10-08

2018-10-08

2018-04-23

Antofagasta 2018-05-11

2018-10-08

2018-10-08

2018-09-28 


\begin{tabular}{|c|c|c|c|c|c|}
\hline H5 & NWA 11883 & & & & $2018-07-08$ \\
\hline H5 & A 12312 & $\mathrm{~J} 26$ & $\mathrm{JB}$ & & $2018-04-18$ \\
\hline H5 & A 12289 & J26 & $\mathrm{JB}$ & & 2018-04-18 \\
\hline H5 & A 12382 & J26 & $\mathrm{JB}$ & & $2018-04-18$ \\
\hline H5 & NWA 12061 & & & & 2018-10-08 \\
\hline H5 & DOM 14039 & $41(1)$ & $A$ & & $2018-02-23$ \\
\hline H5 & A 12263 & J26 & $\mathrm{JB}$ & & $2018-04-18$ \\
\hline H5 & EET 14041 & - & $\mathrm{K}$ & & 2018-04-23 \\
\hline H5 & A 12366 & J26 & $\mathrm{JB}$ & & 2018-04-18 \\
\hline H5 & NWA 11623 & & & & 2018-01-24 \\
\hline H5 & NWA 12050 & & & & 2018-10-08 \\
\hline H5 & 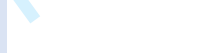 & & & Antofagasta & $2018-05-11$ \\
\hline H5 & A 12278 & J26 & $\mathrm{JB}$ & & $2018-04-18$ \\
\hline H5 & NWA 12174 & & & & 2018-10-08 \\
\hline H5 & MIL 15390 & $41(2)$ & $A$ & & $2018-09-28$ \\
\hline H5 & MIL 15082 & $41(2)$ & $A$ & & $2018-09-28$ \\
\hline H5 & RdDL 062 & & & Arizona & 2018-11-09 \\
\hline H5 & A 12288 & $\mathrm{~J} 26$ & $J B$ & & 2018-04-18 \\
\hline H5 & NWA 12100 & & & & $2018-10-08$ \\
\hline H5 & DOM 14254 & $41(2)$ & A & & $2018-09-28$ \\
\hline H5 & DOM 14479 & $41(2)$ & $A$ & & $2018-09-28$ \\
\hline H5 & A 12300 & $\mathrm{~J} 26$ & $\mathrm{JB}$ & & $2018-04-18$ \\
\hline H5 & NWA 12048 & & & & 2018-10-08 \\
\hline H5 & NWA 12143 & & & & $2018-10-08$ \\
\hline H5 & NWA 12089 & & & & 2018-10-08 \\
\hline H5 & MIL 15379 & $41(2)$ & A & & $2018-09-28$ \\
\hline H5 & NWA 12108 & & & & 2018-10-08 \\
\hline H5 & MIL 15370 & $41(2)$ & A & & 2018-09-28 \\
\hline H5 & GRO 17001 & $41(2)$ & A & & $2018-09-28$ \\
\hline H5 & NWA 12091 & & & & $2018-10-08$ \\
\hline H5 & EET 14008 & - & $\mathrm{K}$ & & 2018-04-23 \\
\hline H5 & & & & Antofagasta & $2018-05-11$ \\
\hline H5 & NWA 12058 & & & & $2018-10-08$ \\
\hline H5 & A 12334 & $\mathrm{~J} 26$ & $J B$ & & $2018-04-18$ \\
\hline H5 & DOM 14252 & $41(2)$ & $A$ & & $2018-09-28$ \\
\hline H5 & DOM 14207 & $41(1)$ & A & & $2018-02-23$ \\
\hline H5 & EET 14009 & - & $\mathrm{K}$ & & 2018-04-23 \\
\hline H5 & NWA 12054 & & & & 2018-10-08 \\
\hline H5 & NWA 12137 & & & & 2018-10-08 \\
\hline H5 & LoV 251 & & & Antofagasta & $2018-11-08$ \\
\hline H5 & A 12335 & $\mathrm{~J} 26$ & $\mathrm{JB}$ & & $2018-04-18$ \\
\hline H5 & MIL 15377 & $41(2)$ & $A$ & & $2018-09-28$ \\
\hline H5 & DOM 14497 & $41(2)$ & $A$ & & $2018-09-28$ \\
\hline H5 & NWA 12145 & & & & $2018-10-08$ \\
\hline H5 & NWA 12060 & & & & $2018-10-08$ \\
\hline
\end{tabular}




$\begin{array}{llll}\text { H5 } & \text { Y-790258 } & \text { J26 } & \text { J } \\ \text { H5 } & \text { A 12398 } & \text { J26 } & \text { J } \\ \text { H5 } & \text { DOM 14421 } & 41(2) & \text { A } \\ \text { H5 } & \text { A 12264 } & \text { J26 } & \text { J } \\ \text { H5 } & \text { Y-000575 } & \text { J26 } & \text { J } \\ \text { H5 } & \text { A 12348 } & \text { J26 } & \text { J } \\ \text { H5 } & \text { DOM 14268 } & 41(2) & \text { A } \\ \text { H5 } & \text { DOM 14266 } & 41(2) & \text { A } \\ \text { H5 } & \text { DOM 14433 } & 41(2) & \text { A } \\ \text { H5 } & \text { A 12298 } & \text { J26 } & \text { J }\end{array}$

J

JB

A

JB

$J$

JB

A

A

A

JB

SJ 093

H5

$\mathrm{H} 5$

$\mathrm{H} 5$

$\mathrm{H} 5$

H5

$\mathrm{H} 5$

$\mathrm{H} 5$

$\mathrm{H} 5$

H5

H5

H5

H5

H5

H5

$\mathrm{H} 5$

H5

$\mathrm{H} 5$

H5

$\mathrm{H} 5$

H5

$\mathrm{H} 5$

$\mathrm{H} 5$

H5
DOM 14255 41(2)

EET 14011 -

Y-74060 J26

NWA 12169

Y-000858 J26

MIL 15096 41(2)

MIL 15378 41(2)

MIL 15398 41(2)

EET 14065 -

LoV 220

A $12292 \quad \mathrm{~J} 26$

A 12276

J26

A

$\mathrm{K}$

$\mathrm{J}$

$J$

A

A

A

$\mathrm{K}$

JB

$\mathrm{JB}$

A

MIL 15373 41(2)

DOM 14294 41(2)

Y-000798 J26

A $12333 \quad$ J26

A $12365 \quad J 26$

Y-790192 J26

DOM 14452 41(1)

DOM 14263 41(2)

MIL 15091 41(2)

DOM 14261 41(2)

NWA 12104

A $12385 \quad J 26$

NWA 12041

NWA 12095

NWA 12144

EET 14057 -

NWA 12110

DOM 14454 41(1)

Y-000986 J26
DOM 14187 41(1)
2018-04-18

2018-04-18

2018-09-28

2018-04-18

2018-04-18

2018-04-18

2018-09-28

2018-09-28

2018-09-28

2018-04-18

Antofagasta 2018-11-08

2018-09-28

2018-04-23

2018-04-18

2018-10-08

2018-04-18

2018-09-28

2018-09-28

2018-09-28

2018-04-23

Antofagasta 2018-05-11

2018-04-18

2018-04-18

Antofagasta 2018-04-07

Antofagasta 2018-11-08

2018-09-28

2018-09-28

2018-04-18

2018-04-18

2018-04-18

2018-04-18

2018-02-23

2018-09-28

2018-09-28

2018-02-23

2018-09-28

2018-10-08

2018-04-18

2018-10-08

2018-10-08

2018-10-08

2018-04-23

2018-10-08

2018-02-23

2018-04-18 


\begin{tabular}{|c|c|c|c|c|c|}
\hline H5 & EET 14067 & - & $\mathrm{K}$ & & 2018-04-23 \\
\hline H5 & Y-002008 & $\mathrm{J} 26$ & $J$ & & 2018-04-18 \\
\hline H5 & & & & Antofagasta & $2018-02-20$ \\
\hline H5 & EET 14002 & - & $\mathrm{K}$ & & $2018-04-23$ \\
\hline H5 & LoV 257 & & & Antofagasta & 2018-11-08 \\
\hline H5 & $Y-000243$ & J26 & $\mathrm{J}$ & & 2018-04-18 \\
\hline H5 & Y-000279 & J26 & $J$ & & 2018-04-18 \\
\hline H5 & NWA 12094 & & & & $2018-10-08$ \\
\hline H5 & A 12272 & J26 & $\mathrm{JB}$ & & $2018-04-18$ \\
\hline H5 & A-87065 & $\mathrm{J} 26$ & $\mathrm{~J}$ & & 2018-04-18 \\
\hline H5 & Y-001215 & $\mathrm{J} 26$ & $J$ & & $2018-04-18$ \\
\hline H5 & A-87081 & J26 & $J$ & & $2018-04-18$ \\
\hline H5 & NWA 12099 & & & & $2018-10-08$ \\
\hline H5 & DOM 14248 & $41(1)$ & $A$ & & $2018-02-23$ \\
\hline H5 & A 12294 & $\mathrm{~J} 26$ & $\mathrm{JB}$ & & $2018-04-18$ \\
\hline H5 & A-87073 & $\mathrm{J} 26$ & $J$ & & 2018-04-18 \\
\hline H5 & DOM 14089 & $41(1)$ & $A$ & & $2018-02-23$ \\
\hline H5 & Y-792570 & J26 & $\mathrm{J}$ & & $2018-04-18$ \\
\hline H5 & Y-000252 & $\mathrm{J} 26$ & $J$ & & $2018-04-18$ \\
\hline H5 & A 12375 & $\mathrm{~J} 26$ & $\mathrm{JB}$ & & 2018-04-18 \\
\hline H5 & Y-000125 & $\mathrm{J} 26$ & $J$ & & $2018-04-18$ \\
\hline H5 & LoV 224 & & & Antofagasta & 2018-05-11 \\
\hline H5 & & & & Antofagasta & $2018-12-02$ \\
\hline H5 & Y-001689 & J26 & J & & 2018-04-18 \\
\hline H5 & Y-792572 & $\mathrm{J} 26$ & J & & 2018-04-18 \\
\hline H5 & Y-792566 & $\mathrm{J} 26$ & $J$ & & 2018-04-18 \\
\hline H5 & Y-002796 & J26 & $\mathrm{J}$ & & 2018-04-18 \\
\hline H5 & Y-792571 & $\mathrm{J} 26$ & $J$ & & 2018-04-18 \\
\hline H5 & A 09347 & $\mathrm{~J} 26$ & $\mathrm{JB}$ & & 2018-04-18 \\
\hline H5 & Y-001788 & J26 & $J$ & & 2018-04-18 \\
\hline H5 & Y-003075 & $\mathrm{J} 26$ & $J$ & & $2018-04-18$ \\
\hline H5 & $Y-000811$ & $\mathrm{~J} 26$ & $J$ & & $2018-04-18$ \\
\hline H5 & A 09344 & J26 & $\mathrm{JB}$ & & 2018-04-18 \\
\hline H5 & Y-001403 & $\mathrm{J} 26$ & $J$ & & $2018-04-18$ \\
\hline H5 & A 09253 & $\mathrm{~J} 26$ & $\mathrm{JB}$ & & 2018-04-18 \\
\hline H5 & A 09316 & J26 & $\mathrm{JB}$ & & 2018-04-18 \\
\hline H5 & Y-003020 & $\mathrm{J} 26$ & $J$ & & $2018-04-18$ \\
\hline H5 & A 09201 & $\mathrm{~J} 26$ & $\mathrm{JB}$ & & $2018-04-18$ \\
\hline H5 & A 09277 & $\mathrm{~J} 26$ & $\mathrm{JB}$ & & 2018-04-18 \\
\hline H5 & Y-000658 & $\mathrm{J} 26$ & $J$ & & $2018-04-18$ \\
\hline H5 & $Y-000743$ & $\mathrm{~J} 26$ & $J$ & & $2018-04-18$ \\
\hline H5 & A 09658 & $\mathrm{~J} 26$ & $\mathrm{JB}$ & & $2018-04-18$ \\
\hline H5 & A 09204 & J26 & $\mathrm{JB}$ & & 2018-04-18 \\
\hline H5 & Y-003225 & $\mathrm{J} 26$ & $J$ & & $2018-04-18$ \\
\hline H5 & Y-000636 & $\mathrm{J} 26$ & $\mathrm{~J}$ & & $2018-04-18$ \\
\hline
\end{tabular}




\begin{tabular}{|c|c|c|}
\hline H5 & A 09202 & J26 \\
\hline H5 & Y-000418 & $\mathrm{J} 26$ \\
\hline H5 & A 09655 & J26 \\
\hline H5 & A 09345 & $\mathrm{~J} 26$ \\
\hline H5 & Y-000366 & J26 \\
\hline H5 & A 09354 & J26 \\
\hline H5 & Y-000768 & $\mathrm{J} 26$ \\
\hline H5 & Y-003227 & J26 \\
\hline H5 & Y-003088 & J26 \\
\hline H5 & Y-003246 & J26 \\
\hline H5 & DOM 14459 & $41(1)$ \\
\hline H5 & A 09391 & $\mathrm{~J} 26$ \\
\hline H5 & Y-001971 & J26 \\
\hline H5 & A 09366 & J26 \\
\hline H5 & Y-003112 & J26 \\
\hline H5 & A 09357 & J26 \\
\hline H5 & A 09378 & $\mathrm{~J} 26$ \\
\hline H5 & A 09381 & J26 \\
\hline H5 & Y-002736 & $\mathrm{J} 26$ \\
\hline H5 & A 09622 & J26 \\
\hline H5 & EET 14024 & - \\
\hline H5 & Y-980030 & $\mathrm{J} 26$ \\
\hline H5 & Y-980213 & J26 \\
\hline H5 & A 09262 & $\mathrm{~J} 26$ \\
\hline H5 & Y-001300 & $\mathrm{J} 26$ \\
\hline H5 & A 09348 & J26 \\
\hline H5 & Y-003031 & J26 \\
\hline H5 & A 09369 & $\mathrm{~J} 26$ \\
\hline H5 & A 09393 & J26 \\
\hline H5 & A 09404 & J26 \\
\hline H5 & A 09292 & J26 \\
\hline H5 & A 09254 & J26 \\
\hline H5 & A 09256 & J26 \\
\hline H5 & A 09258 & J26 \\
\hline H5 & A 09326 & J26 \\
\hline H5 & A 12274 & $\mathrm{~J} 26$ \\
\hline H5 & Y-980306 & J26 \\
\hline H5 & A 09211 & J26 \\
\hline H5 & Y-980069 & J26 \\
\hline H5 & A 09341 & J26 \\
\hline H5 & A 09346 & J26 \\
\hline H5 & A 09672 & J26 \\
\hline H5 & A 09218 & J26 \\
\hline H5 & A 09645 & $\mathrm{~J} 26$ \\
\hline H5 & A 09487 & $\mathrm{~J} 26$ \\
\hline
\end{tabular}

2018-04-18

2018-04-18

2018-04-18

2018-04-18

2018-04-18

2018-04-18

2018-04-18

2018-04-18

2018-04-18

2018-04-18

2018-02-23

2018-04-18

2018-04-18

2018-04-18

2018-04-18

2018-04-18

2018-04-18

2018-04-18

2018-04-18

2018-04-18

2018-04-23

2018-04-18

2018-04-18

2018-04-18

2018-04-18

2018-04-18

2018-04-18

2018-04-18

2018-04-18

2018-04-18

2018-04-18

2018-04-18

2018-04-18

2018-04-18

2018-04-18

2018-04-18

2018-04-18

2018-04-18

2018-04-18

2018-04-18

2018-04-18

2018-04-18

2018-04-18

2018-04-18

2018-04-18 


\begin{tabular}{|c|c|c|c|c|}
\hline H5 & A 09302 & $\mathrm{~J} 26$ & $\mathrm{JB}$ & 2018-04-18 \\
\hline H5 & A 09405 & $\mathrm{~J} 26$ & $\mathrm{JB}$ & 2018-04-18 \\
\hline H5 & A 09394 & $\mathrm{~J} 26$ & $\mathrm{JB}$ & 2018-04-18 \\
\hline H5 & A 09396 & $\mathrm{~J} 26$ & $J B$ & 2018-04-18 \\
\hline H5 & A 09322 & $\mathrm{~J} 26$ & $\mathrm{JB}$ & 2018-04-18 \\
\hline H5 & А 09303 & $\mathrm{~J} 26$ & $\mathrm{JB}$ & 2018-04-18 \\
\hline H5 & Y-002128 & J26 & $\mathrm{J}$ & $2018-04-18$ \\
\hline H5 & A 09399 & $\mathrm{~J} 26$ & $\mathrm{JB}$ & 2018-04-18 \\
\hline H5 & A 09220 & J26 & $\mathrm{JB}$ & $2018-04-18$ \\
\hline H5 & A 09246 & J26 & $\mathrm{JB}$ & $2018-04-18$ \\
\hline H5 & Y-980280 & $\mathrm{J} 26$ & $J$ & 2018-04-18 \\
\hline H5 & Y-000652 & $\mathrm{J} 26$ & $J$ & 2018-04-18 \\
\hline H5 & A 09400 & $\mathrm{~J} 26$ & $\mathrm{JB}$ & $2018-04-18$ \\
\hline H5 & A 09324 & $\mathrm{~J} 26$ & $\mathrm{JB}$ & 2018-04-18 \\
\hline H5 & MIL 15536 & $41(2)$ & $A$ & $2018-09-28$ \\
\hline H5 & A 09301 & $\mathrm{~J} 26$ & $\mathrm{JB}$ & 2018-04-18 \\
\hline H5 & MIL 15277 & $41(1)$ & $A$ & 2018-02-23 \\
\hline H5 & MIL 15531 & $41(2)$ & $A$ & $2018-09-28$ \\
\hline H5 & A 09397 & $\mathrm{~J} 26$ & $J B$ & 2018-04-18 \\
\hline H5 & A 09438 & $\mathrm{~J} 26$ & $\mathrm{JB}$ & 2018-04-18 \\
\hline H5 & A 09646 & $\mathrm{~J} 26$ & $\mathrm{JB}$ & 2018-04-18 \\
\hline H5 & MIL 15533 & $41(2)$ & $A$ & 2018-09-28 \\
\hline H5 & A 09216 & $\mathrm{~J} 26$ & $\mathrm{JB}$ & 2018-04-18 \\
\hline H5 & A 09257 & J26 & $\mathrm{JB}$ & 2018-04-18 \\
\hline H5 & Y-980043 & $\mathrm{J} 26$ & $\mathrm{~J}$ & 2018-04-18 \\
\hline H5 & A 09305 & $\mathrm{~J} 26$ & $\mathrm{JB}$ & 2018-04-18 \\
\hline H5 & Y-980034 & J26 & $\mathrm{J}$ & 2018-04-18 \\
\hline H5 & A 09641 & $\mathrm{~J} 26$ & $\mathrm{JB}$ & 2018-04-18 \\
\hline H5 & A 12314 & $\mathrm{~J} 26$ & $\mathrm{JB}$ & 2018-04-18 \\
\hline H5 & Y-002127 & J26 & $J$ & 2018-04-18 \\
\hline H5 & RKP 14004 & - & $\mathrm{K}$ & 2018-04-23 \\
\hline H5 & A 09640 & $\mathrm{~J} 26$ & $\mathrm{JB}$ & 2018-04-18 \\
\hline H5 & A 09644 & J26 & $\mathrm{JB}$ & $2018-04-18$ \\
\hline H5 & A 09219 & $\mathrm{~J} 26$ & $J B$ & 2018-04-18 \\
\hline H5 & A 09643 & $\mathrm{~J} 26$ & $\mathrm{JB}$ & 2018-04-18 \\
\hline H5 & A 09221 & J26 & $\mathrm{JB}$ & 2018-04-18 \\
\hline H5 & A 09222 & $\mathrm{~J} 26$ & $\mathrm{JB}$ & 2018-04-18 \\
\hline H5 & A 09308 & $\mathrm{~J} 26$ & $J B$ & 2018-04-18 \\
\hline H5 & Y-980080 & J26 & $J$ & $2018-04-18$ \\
\hline H5 & Y-980158 & $\mathrm{J} 26$ & $J$ & 2018-04-18 \\
\hline H5 & A 10152 & $\mathrm{~J} 26$ & $J B$ & 2018-04-18 \\
\hline H5 & A 09449 & J26 & $\mathrm{JB}$ & $2018-04-18$ \\
\hline H5 & A 09415 & $\mathrm{~J} 26$ & $\mathrm{JB}$ & 2018-04-18 \\
\hline H5 & A 09307 & $\mathrm{~J} 26$ & $\mathrm{JB}$ & 2018-04-18 \\
\hline H5 & MIL 15538 & $41(2)$ & $A$ & 2018-09-28 \\
\hline
\end{tabular}


H5

$\mathrm{H} 5$

H5

$\mathrm{H} 5$

H5

$\mathrm{H} 5$

H5

$\mathrm{H} 5$

H5

H5

H5

$\mathrm{H} 5$

H5

H5

$\mathrm{H} 5$

H5-6

H5-6

H5-melt breccia

H5-melt breccia

$\mathrm{H} 5 / 6$

$\mathrm{H} 5 / 6$

$\mathrm{H} 6$

H6

H6

H6

H6

H6

$\mathrm{H} 6$

H6

H6

H6

$\mathrm{H} 6$

H6

H6

H6

$\mathrm{H} 6$

H6

H6

H6

H6

H6

H6

$\mathrm{H} 6$

H6

H6

$\begin{array}{lll}\text { A } 09306 & \text { J26 } & \text { JB } \\ \text { Y-002124 } & \text { J26 } & \text { J } \\ \text { EET 16211 } & 41(1) & \text { A } \\ \text { Y-002130 } & \text { J26 } & \text { J } \\ \text { A 09224 } & \text { J26 } & \text { JB } \\ \text { Y-001861 } & \text { J26 } & \text { J } \\ \text { A 09642 } & \text { J26 } & \text { JB } \\ \text { A 10148 } & \text { J26 } & \text { JB } \\ \text { A 09312 } & \text { J26 } & \text { JB } \\ \text { Y-001824 } & \text { J26 } & \text { J } \\ \text { A 09223 } & \text { J26 } & \text { JB } \\ \text { Y-002402 } & \text { J26 } & \text { J } \\ \text { Y-001978 } & \text { J26 } & \text { J } \\ \text { EET 14051 } & - & \text { K } \\ \text { Y-002859 } & \text { J26 } & \text { J } \\ & & \end{array}$

NWA 11920

LoV 229

NWA 1088

NWA 11903

NWA 11652

NWA 11995

NWA 4667

SJ 087

CyDL 349

Y-003054 J26

NWA 11688

NWA 11653

LoV 245

A $12216 \quad$ J26

NWA 11855

LoV 237

NWA 12330

Y-002344 J26

NWA 11798

A $12409 \quad$ J26
$\mathrm{JB}$

A

$\mathrm{JB}$

$\mathrm{JB}$

$J B$

$\mathrm{JB}$

$J$

$J B$

J

K

$\mathrm{J}$
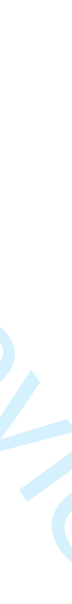

JB

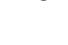

J

JB

2018-04-18

2018-04-18

2018-02-23

2018-04-18

2018-04-18

2018-04-18

2018-04-18

2018-04-18

2018-04-18

2018-04-18

2018-04-18

2018-04-18

2018-04-18

2018-04-23

2018-04-18

Pavlodar 2018-05-11

Madaniyin 2018-09-15

Antofagasta 2018-08-03

2018-09-15

Antofagasta 2018-11-08

Antofagasta 2018-07-17

Tibet 2018-12-14

2018-10-20

2018-07-24

2018-02-24

2018-09-15

2018-08-03

Antofagasta 2018-11-08

Antofagasta 2018-07-08

California $\quad$ 2018-10-20

Antofagasta 2018-01-27

2018-04-18

2018-02-20

Antofagasta 2018-05-11

2018-02-24

Antofagasta 2018-11-08

2018-04-18

2018-06-16

Antofagasta 2018-11-08

2018-12-06

Antofagasta 2018-11-08

2018-04-18

Antofagasta 2018-11-08

2018-04-25 


\begin{tabular}{|c|c|c|c|c|c|}
\hline H6 & NWA 431 & & & & $2018-10-20$ \\
\hline H6 & LoV 243 & & & Antofagasta & 2018-11-08 \\
\hline H6 & NWA 12010 & & & & $2018-09-23$ \\
\hline H6 & DdA 001 & & & Atacama & $2018-05-28$ \\
\hline H6 & LoV 240 & & & Antofagasta & 2018-11-08 \\
\hline H6 & A 12201 & J26 & $\mathrm{JB}$ & & 2018-04-18 \\
\hline H6 & & & & Antofagasta & $2018-12-14$ \\
\hline H6 & NWA 12162 & & & & 2018-10-08 \\
\hline H6 & NWA 12105 & & & & $2018-10-08$ \\
\hline H6 & & & & Antofagasta & 2018-11-08 \\
\hline H6 & NWA 12336 & & & & 2018-11-24 \\
\hline H6 & Prang & & & Antofagasta & $2018-12-14$ \\
\hline H6 & A 12351 & $\mathrm{~J} 26$ & $\mathrm{JB}$ & & 2018-04-18 \\
\hline H6 & DOM 14301 & $41(1)$ & $A$ & & $2018-02-23$ \\
\hline H6 & NWA 12018 & & & & $2018-09-23$ \\
\hline H6 & LoV 226 & & & Antofagasta & 2018-05-11 \\
\hline H6 & NWA 12118 & & & & 2018-10-08 \\
\hline H6 & NWA 12171 & & & & $2018-10-08$ \\
\hline H6 & NWA 12049 & & & & $2018-10-08$ \\
\hline H6 & NWA 12088 & & & & 2018-10-08 \\
\hline H6 & NWA 12130 & & & & $2018-10-08$ \\
\hline H6 & NWA 12084 & & & & $2018-10-08$ \\
\hline H6 & DOM 14232 & $41(2)$ & A & & $2018-09-28$ \\
\hline H6 & A 12363 & $\mathrm{~J} 26$ & JB & & 2018-04-18 \\
\hline H6 & NWA 12119 & & & & 2018-10-08 \\
\hline H6 & LoV 238 & & & Antofagasta & 2018-11-08 \\
\hline H6 & DOM 14418 & $41(2)$ & A & & $2018-09-28$ \\
\hline H6 & NWA 12139 & & & & 2018-10-08 \\
\hline H6 & NWA 12072 & & & & $2018-10-08$ \\
\hline H6 & A 12429 & J26 & $\mathrm{JB}$ & & 2018-04-18 \\
\hline H6 & NWA 12090 & & & & 2018-10-08 \\
\hline H6 & DOM 14416 & $41(2)$ & $A$ & & $2018-09-28$ \\
\hline H6 & NWA 11823 & & & & $2018-05-26$ \\
\hline H6 & NWA 12075 & & & & 2018-10-08 \\
\hline H6 & DOM 14175 & $41(1)$ & $A$ & & $2018-02-23$ \\
\hline H6 & NWA 12077 & & & & $2018-10-08$ \\
\hline H6 & NWA 12179 & & & & $2018-10-08$ \\
\hline H6 & A 12154 & $\mathrm{~J} 26$ & $\mathrm{JB}$ & & 2018-04-18 \\
\hline H6 & NWA 12047 & & & & $2018-10-08$ \\
\hline H6 & DOM 14179 & $41(1)$ & $A$ & & $2018-02-23$ \\
\hline H6 & A 12349 & $\mathrm{~J} 26$ & $J B$ & & 2018-04-18 \\
\hline H6 & NWA 12131 & & & & $2018-10-08$ \\
\hline H6 & DOM 14224 & $41(1)$ & $A$ & & $2018-02-23$ \\
\hline H6 & A 12279 & $\mathrm{~J} 26$ & $\mathrm{JB}$ & & 2018-04-18 \\
\hline H6 & NWA 12129 & & & & 2018-10-08 \\
\hline
\end{tabular}




$\begin{array}{lll}\text { A 12269 } & \text { J26 } & \text { JB } \\ \text { A 12253 } & \text { J26 } & \text { JB } \\ \text { DOM 14405 } & 41(2) & \text { A } \\ \text { DOM 14213 } & 41(2) & \text { A }\end{array}$

DOM 14126 41(1)

NWA 12121

A $12256 \quad$ J26

NWA 12068

DOM 14180 41(1)

NWA 12140

A $12303 \quad J 26$

A $12350 \quad J 26$

A $12290 \quad$ J26

NWA 12138

A $12044 \quad$ J26

DOM 14539 41(1)

NWA 11691

MIL 15357 41(2)

DOM 14181 41(1)

DOM 14221 41(1)

NWA 12087

NWA 12014

DOM 14103 41(1)

NWA 11938

DOM 14120 41(1)

DOM 14438 41(2)

NWA 12103

NWA 12086

NWA 12053

MIL 15258 41(2)

NWA 11625

A $12254 \quad J 26$

MIL 15399 41(2)

DEW 14001 -

MIL 15394 41(2)

Y-86677 J26

DOM 14194 41(1)

DOM 14396 41(1)

MIL 15089 41(2)

MIL 15088 41(2)

DOM 14121 41(1)
A

JB

JB

A

A

JB

A

JB

$J B$

$\mathrm{JB}$

JB

A

A

A

A

A

A

A

$J B$

A

$\mathrm{K}$

A

$\mathrm{J}$

A

A

A

A

A
2018-04-18

2018-04-18

2018-09-28

2018-09-28

2018-10-08

2018-10-08

2018-02-23

2018-10-08

South 2018-02-24

2018-04-18

2018-10-08

2018-02-23

2018-10-08

2018-04-18

2018-04-18

2018-04-18

2018-10-08

2018-04-18

2018-02-23

2018-02-20

2018-09-28

2018-02-23

2018-02-23

2018-10-08

2018-09-23

2018-02-23

2018-08-03

2018-02-23

2018-09-28

Antofagasta 2018-05-11

2018-10-08

2018-10-08

2018-10-08

2018-09-28

2018-01-24

2018-04-18

2018-09-28

2018-04-23

2018-09-28

2018-04-18

2018-02-23

2018-02-23

2018-09-28

2018-09-28

2018-02-23 


\begin{tabular}{|c|c|c|c|c|c|}
\hline H6 & NWA 11879 & & & & $2018-07-24$ \\
\hline H6 & A 09430 & $\mathrm{~J} 26$ & $\mathrm{JB}$ & & 2018-04-18 \\
\hline H6 & A 12302 & $\mathrm{~J} 26$ & $\mathrm{JB}$ & & 2018-04-18 \\
\hline H6 & DOM 14033 & $41(1)$ & $A$ & & $2018-02-23$ \\
\hline H6 & & & & Arizona & 2018-04-07 \\
\hline H6 & MIL 15391 & $41(2)$ & A & & $2018-09-28$ \\
\hline H6 & A 12307 & $\mathrm{~J} 26$ & $J B$ & & 2018-04-18 \\
\hline H6 & A 12259 & $\mathrm{~J} 26$ & $J B$ & & 2018-04-18 \\
\hline H6 & A 12377 & $\mathrm{~J} 26$ & $\mathrm{JB}$ & & 2018-04-18 \\
\hline H6 & MIL 15083 & $41(2)$ & $A$ & & $2018-09-28$ \\
\hline H6 & A 12283 & J26 & JB & & 2018-04-18 \\
\hline H6 & Y-791860 & $\mathrm{J} 26$ & $J$ & & 2018-04-18 \\
\hline H6 & A 12344 & $\mathrm{~J} 26$ & $\mathrm{JB}$ & & 2018-04-18 \\
\hline H6 & DOM 14038 & $41(1)$ & $A$ & & $2018-02-23$ \\
\hline H6 & DOM 14422 & $41(2)$ & A & & $2018-09-28$ \\
\hline H6 & NWA 12065 & & & & $2018-10-08$ \\
\hline H6 & DOM 14258 & $41(2)$ & $A$ & & $2018-09-28$ \\
\hline H6 & A 12341 & $\mathrm{~J} 26$ & JB & & 2018-04-18 \\
\hline H6 & DOM 14395 & $41(1)$ & $A$ & & $2018-02-23$ \\
\hline H6 & MIL 15392 & $41(2)$ & $A$ & & $2018-09-28$ \\
\hline H6 & DOM 14244 & $41(1)$ & $A$ & & $2018-02-23$ \\
\hline H6 & DOM 14256 & $41(2)$ & A & & $2018-09-28$ \\
\hline H6 & A 12301 & $\mathrm{~J} 26$ & JB & & 2018-04-18 \\
\hline H6 & MIL 15395 & $41(2)$ & A & & $2018-09-28$ \\
\hline H6 & NWA 12093 & & & & $2018-10-08$ \\
\hline H6 & MIL 15093 & $41(2)$ & $A$ & & $2018-09-28$ \\
\hline H6 & A 12337 & $\mathrm{~J} 26$ & $J B$ & & 2018-04-18 \\
\hline H6 & EET 14072 & - & $\mathrm{K}$ & & 2018-04-23 \\
\hline H6 & DOM 14379 & $41(1)$ & $A$ & & $2018-02-23$ \\
\hline H6 & A 12247 & $\mathrm{~J} 26$ & JB & & 2018-04-18 \\
\hline H6 & DOM 14430 & $41(2)$ & $A$ & & $2018-09-28$ \\
\hline H6 & DOM 14243 & $41(1)$ & A & & $2018-02-23$ \\
\hline H6 & DOM 14204 & $41(1)$ & $A$ & & $2018-02-23$ \\
\hline H6 & NWA 12114 & & & & $2018-10-08$ \\
\hline H6 & MIL 15397 & $41(2)$ & $A$ & & $2018-09-28$ \\
\hline H6 & MIL 15252 & $41(2)$ & $A$ & & $2018-09-28$ \\
\hline H6 & DOM 14559 & $41(1)$ & $A$ & & $2018-02-23$ \\
\hline H6 & MIL 15097 & $41(2)$ & A & & $2018-09-28$ \\
\hline H6 & DOM 14265 & $41(2)$ & $A$ & & $2018-09-28$ \\
\hline H6 & A 12260 & J26 & $\mathrm{JB}$ & & 2018-04-18 \\
\hline H6 & DOM 14227 & $41(1)$ & $A$ & & $2018-02-23$ \\
\hline H6 & A 12277 & $\mathrm{~J} 26$ & $\mathrm{JB}$ & & 2018-04-18 \\
\hline H6 & DOM 14191 & $41(1)$ & $A$ & & $2018-02-23$ \\
\hline H6 & MIL 15396 & $41(2)$ & $A$ & & $2018-09-28$ \\
\hline H6 & A 12280 & $\mathrm{~J} 26$ & JB & & 2018-04-18 \\
\hline
\end{tabular}


2018-10-08

H6

H6

H6

H6

H6

DOM 14357 41(2)

NWA 12109

DOM 14553 41(1)

DOM 14364 41(1)

MIL 15372 41(2)

MIL 15095 41(2)

MIL 15253 41(2)

DOM 14356 41(2)

MIL 15256 41(2)

MIL 15250 41(2)

A $12319 \quad$ J26

NWA 12132

Y-002348 J26

DOM 14247 41(1)

Y-792555 J26

A-87063 J26

A 12342 J26

A-87060 J26

A-87301 J26

DOM 14451 41(1)

A-87102 J26

A $12339 \quad$ J26

DOM 14229 41(1)

A-87024 J26

NWA 12127

DOM 14196 41(1)

A $12108 \quad$ J26

Y-980060 J26

Y-002673 J26

A $12291 \quad J 26$

A $12308 \quad \mathrm{~J} 26$

Y-003025 J26

DOM 14249 41(1)

NWA 12147

EET 14028 -

$\mathrm{Y}-000197 \quad \mathrm{~J} 26$

Y-003154 J26

A $09355 \quad J 26$

$\mathrm{Y}-002343 \quad \mathrm{~J} 26$

$\mathrm{Y}-003180 \quad \mathrm{~J} 26$

$\mathrm{Y}-003128 \quad \mathrm{~J} 26$

A $09200 \quad J 26$
A

A

A

A

A

A

A

A

A

$J B$

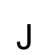

A

$\mathrm{J}$

$J$

JB

$\mathrm{J}$

$\mathrm{J}$

A

$\mathrm{J}$

JB

A

J

A

JB

J

$\mathrm{J}$

$\mathrm{JB}$

$\mathrm{JB}$

$\mathrm{J}$

A

K

J

$\mathrm{J}$

JB

$\mathrm{J}$

J

$\mathrm{J}$

JB
Antofagasta 2018-11-08

2018-09-28

2018-10-08

2018-02-23

2018-02-23

2018-09-28

2018-09-28

2018-09-28

2018-09-28

2018-09-28

2018-09-28

2018-04-18

2018-10-08

2018-04-18

2018-02-23

2018-04-18

2018-04-18

2018-04-18

2018-04-18

2018-04-18

2018-02-23

2018-04-18

2018-04-18

2018-02-23

2018-04-18

2018-10-08

2018-02-23

2018-04-18

Antofagasta 2018-11-08

2018-04-18

2018-04-18

2018-04-18

2018-04-18

2018-04-18

2018-02-23

2018-10-08

2018-04-23

2018-04-18

2018-04-18

2018-04-18

2018-04-18

2018-04-18

2018-04-18

2018-04-18 


\begin{tabular}{|c|c|c|c|c|}
\hline H6 & Y-001643 & $\mathrm{J} 26$ & $J$ & 2018-04-18 \\
\hline H6 & Y-003083 & $\mathrm{J} 26$ & $\mathrm{~J}$ & 2018-04-18 \\
\hline H6 & A 09356 & $\mathrm{~J} 26$ & $\mathrm{JB}$ & 2018-04-18 \\
\hline H6 & A 09361 & $\mathrm{~J} 26$ & $J B$ & 2018-04-18 \\
\hline H6 & MIL 15098 & $41(2)$ & $A$ & $2018-09-28$ \\
\hline H6 & A 09376 & $\mathrm{~J} 26$ & $\mathrm{JB}$ & 2018-04-18 \\
\hline H6 & Y-003018 & J26 & $\mathrm{J}$ & $2018-04-18$ \\
\hline H6 & A 09232 & $\mathrm{~J} 26$ & $\mathrm{JB}$ & 2018-04-18 \\
\hline H6 & EET 14046 & - & $\mathrm{K}$ & 2018-04-23 \\
\hline H6 & NWA 12146 & & & $2018-10-08$ \\
\hline H6 & A 09358 & $\mathrm{~J} 26$ & $\mathrm{JB}$ & 2018-04-18 \\
\hline H6 & A 12284 & $\mathrm{~J} 26$ & $\mathrm{JB}$ & 2018-04-18 \\
\hline H6 & Y-003153 & J26 & $\mathrm{J}$ & $2018-04-18$ \\
\hline H6 & A 09451 & $\mathrm{~J} 26$ & $\mathrm{JB}$ & 2018-04-18 \\
\hline H6 & Y-003177 & $\mathrm{J} 26$ & $\mathrm{~J}$ & 2018-04-18 \\
\hline H6 & $Y-000521$ & $\mathrm{~J} 26$ & $J$ & 2018-04-18 \\
\hline H6 & Y-003036 & $\mathrm{J} 26$ & $J$ & 2018-04-18 \\
\hline H6 & Y-000294 & J26 & $\mathrm{J}$ & 2018-04-18 \\
\hline H6 & A 09483 & $\mathrm{~J} 26$ & $J B$ & 2018-04-18 \\
\hline H6 & Y-003134 & $\mathrm{J} 26$ & $J$ & 2018-04-18 \\
\hline H6 & Y-000185 & $\mathrm{J} 26$ & $J$ & 2018-04-18 \\
\hline H6 & A 09203 & $\mathrm{~J} 26$ & $\mathrm{JB}$ & 2018-04-18 \\
\hline H6 & Y-002245 & $\mathrm{J} 26$ & $J$ & 2018-04-18 \\
\hline H6 & Y-003164 & J26 & $\mathrm{J}$ & 2018-04-18 \\
\hline H6 & A 12286 & $\mathrm{~J} 26$ & $J B$ & 2018-04-18 \\
\hline H6 & A 12340 & $\mathrm{~J} 26$ & $\mathrm{JB}$ & 2018-04-18 \\
\hline H6 & Y-980256 & J26 & $J$ & 2018-04-18 \\
\hline H6 & Y-003144 & $\mathrm{J} 26$ & $J$ & 2018-04-18 \\
\hline H6 & Y-003185 & $\mathrm{J} 26$ & $J$ & 2018-04-18 \\
\hline H6 & A 09360 & J26 & $\mathrm{JB}$ & 2018-04-18 \\
\hline H6 & $Y-003173$ & $\mathrm{~J} 26$ & $\mathrm{~J}$ & 2018-04-18 \\
\hline H6 & Y-980224 & $\mathrm{J} 26$ & $J$ & 2018-04-18 \\
\hline H6 & Y-980126 & J26 & $\mathrm{J}$ & $2018-04-18$ \\
\hline H6 & Y-000519 & $\mathrm{J} 26$ & $J$ & 2018-04-18 \\
\hline H6 & A 09205 & $\mathrm{~J} 26$ & $\mathrm{JB}$ & 2018-04-18 \\
\hline H6 & A 09392 & J26 & $\mathrm{JB}$ & 2018-04-18 \\
\hline $\mathrm{H} 6$ & Y-000567 & $\mathrm{J} 26$ & $J$ & 2018-04-18 \\
\hline H6 & Y-002985 & $\mathrm{J} 26$ & $J$ & 2018-04-18 \\
\hline H6 & A 09363 & J26 & $\mathrm{JB}$ & $2018-04-18$ \\
\hline $\mathrm{H} 6$ & NWA 12148 & & & 2018-10-08 \\
\hline H6 & A 09385 & $\mathrm{~J} 26$ & $J B$ & 2018-04-18 \\
\hline H6 & EET 14061 & - & $\mathrm{K}$ & $2018-04-23$ \\
\hline H6 & A 09208 & $\mathrm{~J} 26$ & $\mathrm{JB}$ & 2018-04-18 \\
\hline H6 & A 09206 & $\mathrm{~J} 26$ & $\mathrm{JB}$ & 2018-04-18 \\
\hline H6 & A 09362 & $\mathrm{~J} 26$ & $\mathrm{JB}$ & 2018-04-18 \\
\hline
\end{tabular}


2018-04-18

A $09212 \quad J 26 \quad J B$

2018-04-18

H6

A $09213 \quad J 26 \quad J B$

2018-04-18

H6

A $09207 \quad \mathrm{~J} 26$

2018-04-18

Y-003136 J26

2018-04-18

$\mathrm{H} 6$

A $09364 \quad J 26$

2018-04-18

EET 14040 -

2018-04-23

A $09454 \quad J 26 \quad J B$

2018-04-18

A $09335 \quad J 26$

2018-04-18

A $09210 \quad J 26$

2018-04-18

A $09209 \quad J 26 \quad J B$

2018-04-18

A $09217 \quad J 26 \quad J B$

2018-04-18

EET 14062 - K

2018-04-23

A $09450 \quad J 26 \quad J B$

2018-04-18

2018-04-18

2018-04-18

2018-04-18

2018-04-18

2018-04-18

2018-04-18

2018-09-28

2018-04-18

2018-02-23

2018-02-23

2018-04-18

2018-04-18

2018-04-23

2018-04-23

2018-09-28

2018-04-18

2018-02-23

2018-04-23

2018-02-23

2018-02-23

2018-04-18

2018-02-23

2018-04-18

2018-04-18

2018-04-18

2018-02-23

2018-04-18

2018-02-23

2018-04-23

2018-04-18

2018-04-18 


\begin{tabular}{|c|c|c|c|c|}
\hline H6 & MIL 15477 & $41(2)$ & $A$ & $2018-09-28$ \\
\hline H6 & Y-980346 & $\mathrm{J} 26$ & J & 2018-04-18 \\
\hline H6 & EET 16216 & $41(1)$ & A & $2018-02-23$ \\
\hline H6 & Y-003167 & $\mathrm{J} 26$ & $\mathrm{~J}$ & 2018-04-18 \\
\hline H6 & EET 16209 & $41(1)$ & $A$ & $2018-02-23$ \\
\hline H6 & A 09337 & $\mathrm{~J} 26$ & $\mathrm{JB}$ & 2018-04-18 \\
\hline H6 & A 09417 & $\mathrm{~J} 26$ & $J B$ & 2018-04-18 \\
\hline H6 & Y-003170 & $\mathrm{J} 26$ & $J$ & $2018-04-18$ \\
\hline H6 & MIL 15478 & $41(2)$ & $A$ & $2018-09-28$ \\
\hline H6 & MIL 15432 & $41(2)$ & A & $2018-09-28$ \\
\hline H6 & EET 16215 & $41(1)$ & $A$ & $2018-02-23$ \\
\hline H6 & EET 16205 & $41(1)$ & $A$ & $2018-02-23$ \\
\hline H6 & Y-003137 & $\mathrm{J} 26$ & $J$ & 2018-04-18 \\
\hline H6 & Y-002342 & $\mathrm{J} 26$ & $J$ & $2018-04-18$ \\
\hline H6 & MIL 15534 & $41(2)$ & $A$ & $2018-09-28$ \\
\hline H6 & $Y-002579$ & $\mathrm{~J} 26$ & $J$ & 2018-04-18 \\
\hline H6 & $Y-002851$ & $\mathrm{~J} 26$ & $J$ & $2018-04-18$ \\
\hline H6 & $Y-003151$ & J26 & $\mathrm{J}$ & 2018-04-18 \\
\hline H6 & A 09225 & $\mathrm{~J} 26$ & $J B$ & 2018-04-18 \\
\hline H6 & MIL 15430 & $41(2)$ & $A$ & $2018-09-28$ \\
\hline $\mathrm{H} 7$ & NWA 11613 & & & $2018-02-20$ \\
\hline $\mathrm{H} 7$ & NWA 12236 & & & $2018-11-14$ \\
\hline Howardite & NWA 11899 & & & 2018-08-31 \\
\hline Howardite & NWA 12227 & & & $2018-12-11$ \\
\hline Howardite & NWA 11964 & & & 2018-10-09 \\
\hline Howardite & NWA 12190 & & & 2018-11-09 \\
\hline Howardite & & & & II 2018-12-14 \\
\hline Howardite & NWA 11712 & & & 2018-03-27 \\
\hline Howardite & NWA 11858 & & & $2018-07-08$ \\
\hline Howardite & NWA 11551 & & & 2018-01-05 \\
\hline Howardite & NWA 12203 & & & 2018-11-09 \\
\hline Howardite & NWA 11612 & & & $2018-02-20$ \\
\hline Howardite & NWA 12224 & & & $2018-11-13$ \\
\hline Howardite & NWA 12194 & & & 2018-11-18 \\
\hline Howardite & NWA 12250 & & & $2018-11-24$ \\
\hline Howardite & NWA 11997 & & & $2018-09-27$ \\
\hline Howardite & NWA 11834 & & & 2018-07-04 \\
\hline Howardite & NWA 12231 & & & $2018-12-11$ \\
\hline Howardite & NWA 11598 & & & 2018-02-09 \\
\hline Howardite & NWA 11605 & & & 2018-02-09 \\
\hline Howardite & NWA 11604 & & & 2018-02-09 \\
\hline Howardite & NWA 11606 & & & 2018-02-09 \\
\hline Iron, IAB complex & GRO 17102 & $41(2)$ & $A$ & $2018-09-28$ \\
\hline Iron, IAB complex & GRO 17051 & $41(2)$ & $A$ & 2018-09-28 \\
\hline Iron, IAB-MG & & & & $2018-06-30$ \\
\hline
\end{tabular}




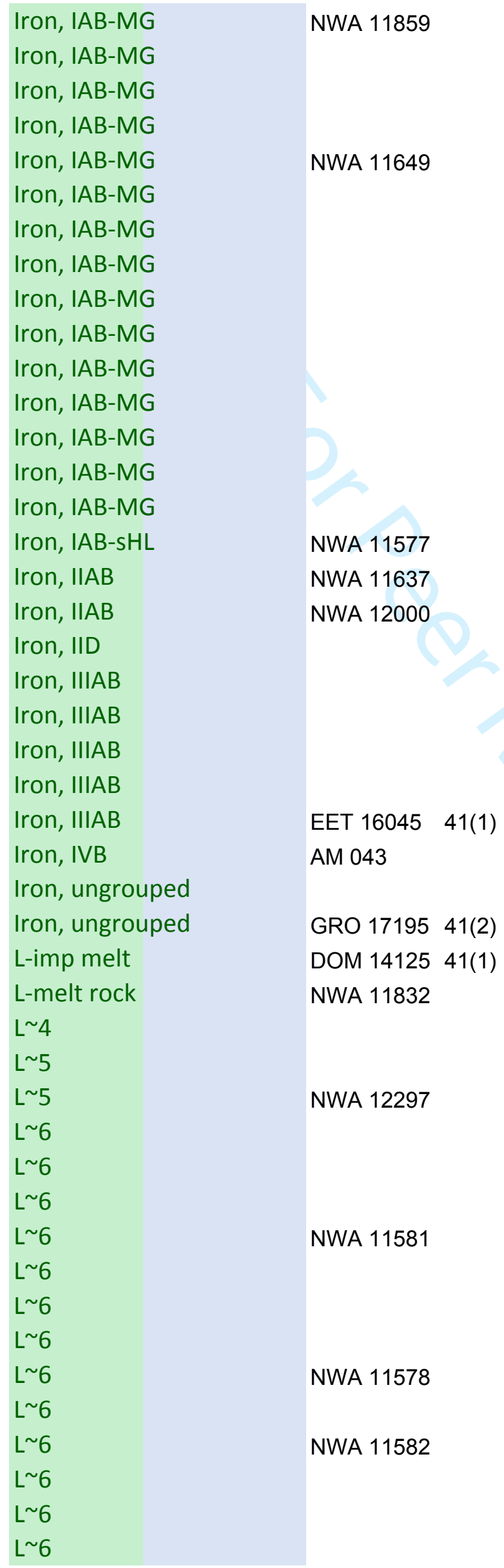

2018-06-30

2018-06-30

Zacatecas 2018-09-23

2018-06-30

2018-02-20

Valle d'Aosta 2018-06-22

Chubu 2018-02-20

Xinjiang 2018-09-15

2018-07-17

2018-08-03

2018-06-30

2018-06-30

2018-06-30

2018-06-30

2018-06-30

2018-01-24

2018-02-20

2018-10-20

Rio Grande d 2018-09-02

Sokoto 2018-06-16

Xinjiang 2018-04-19

Adrar 2018-09-23

Castilla y Leo 2018-11-12

2018-02-23

Xinjiang 2018-04-19

Xinjiang 2018-03-27

2018-09-28

2018-02-23

2018-07-24

Qinghai 2018-11-24

Qinghai 2018-12-02

2018-11-09

Qinghai 2018-11-24

Qinghai 2018-12-02

Qinghai 2018-12-02

2018-01-15

Xinjiang 2018-12-16

Xinjiang 2018-12-16

Gansu 2018-12-16

2018-01-15

Xinjiang 2018-11-09

2018-01-15

XinJiang 2018-11-24

Xinjiang 2018-11-09

Xinjiang 2018-11-09 


\begin{tabular}{|c|c|c|c|c|c|}
\hline L 6 & NWA 12381 & & & & $2018-12-24$ \\
\hline L3 & & & & Oyo & 2018-09-02 \\
\hline L3 & NWA 11782 & & & & 2018-04-25 \\
\hline L3 & NWA 11722 & & & & 2018-03-07 \\
\hline L3 & NWA 12285 & & & & 2018-11-09 \\
\hline L3 & NWA 11994 & & & & 2018-10-09 \\
\hline L3 & NWA 12324 & & & & 2018-11-14 \\
\hline L3 & NWA 12331 & & & & $2018-12-06$ \\
\hline L3 & NWA 12341 & & & & $2018-12-14$ \\
\hline L3 & NWA 11881 & & & & 2018-07-08 \\
\hline L3 & NWA 11634 & & & & 2018-01-24 \\
\hline L3 & NWA 11635 & & & & 2018-01-24 \\
\hline L3 & NWA 11802 & & & & $2018-05-28$ \\
\hline L3 & A 12205 & $\mathrm{~J} 26$ & JB & & 2018-04-18 \\
\hline L3 & NWA 6263 & & & & $2018-05-28$ \\
\hline L3 & NWA 11672 & & & & $2018-02-20$ \\
\hline L3 & NWA 11796 & & & & $2018-05-26$ \\
\hline L3 & & & & Chihuahua & $2018-01-17$ \\
\hline L3 & NWA 11940 & & & & 2018-08-03 \\
\hline L3 & NWA 11735 & & & & 2018-03-07 \\
\hline L3 & A 12018 & $\mathrm{~J} 26$ & JB & & 2018-04-18 \\
\hline L3 & NWA 11713 & & & & $2018-02-24$ \\
\hline L3 & NWA 11714 & & & & 2018-02-24 \\
\hline L3 & NWA 11736 & & & & 2018-03-07 \\
\hline L3 & NWA 11925 & & & & 2018-08-03 \\
\hline L3 & NWA 11829 & & & & $2018-06-22$ \\
\hline L3 & NWA 11835 & & & & 2018-06-22 \\
\hline L3 & NWA 1723 & & & & 2018-12-02 \\
\hline L3 & NWA 11630 & & & & 2018-01-24 \\
\hline L3 & EET 14018 & - & $\mathrm{K}$ & & 2018-04-23 \\
\hline L3 & A 09383 & $\mathrm{~J} 26$ & $\mathrm{JB}$ & & 2018-04-18 \\
\hline L3 & A 09420 & $\mathrm{~J} 26$ & $\mathrm{JB}$ & & 2018-04-18 \\
\hline L3 & A 09319 & $\mathrm{~J} 26$ & $\mathrm{JB}$ & & 2018-04-18 \\
\hline L3 & Y-000730 & $\mathrm{J} 26$ & $J$ & & 2018-04-18 \\
\hline L3 & Y-003043 & $\mathrm{J} 26$ & $J$ & & 2018-04-18 \\
\hline L3 & Y-003228 & $\mathrm{J} 26$ & $\mathrm{~J}$ & & 2018-04-18 \\
\hline L3 & A 09621 & $\mathrm{~J} 26$ & $\mathrm{JB}$ & & 2018-04-18 \\
\hline L3 & A 09422 & $\mathrm{~J} 26$ & $\mathrm{JB}$ & & 2018-04-18 \\
\hline L3 & A 09271 & $\mathrm{~J} 26$ & $\mathrm{JB}$ & & 2018-04-18 \\
\hline L3 & A 10214 & $\mathrm{~J} 26$ & $\mathrm{JB}$ & & 2018-04-18 \\
\hline L3 & A 09259 & $\mathrm{~J} 26$ & $\mathrm{JB}$ & & 2018-04-18 \\
\hline L3 & A 09555 & J26 & $\mathrm{JB}$ & & 2018-04-18 \\
\hline L3 & A 09266 & $\mathrm{~J} 26$ & $J B$ & & 2018-04-18 \\
\hline L3 & A 09260 & $\mathrm{~J} 26$ & $\mathrm{JB}$ & & 2018-04-18 \\
\hline L3 & A 09423 & $\mathrm{~J} 26$ & $J B$ & & 2018-04-18 \\
\hline
\end{tabular}


A $09251 \quad J 26 \quad J B$

2018-04-18

NWA 787

2018-03-27

L3-6

NWA 11978

2018-10-09

L3-6

NWA 11922

2018-09-15

L3.10

NWA 11574

2018-01-17

L3.5

L3.6

DOM 14124 41(1)

A

2018-02-23

DOM 14201 41(1)

A

2018-02-23

L3.6

DOM 14082 41(1)

A

2018-02-23

L3.8

DOM 14225 41(1)

A

2018-02-23

L4

NWA 11884

L4

NWA 11872

NWA 11725

NWA 12348

NWA 11728

NWA 11674

NWA 11678

NWA 1091

2018-07-08

Boujdour

2018-12-24

2018-06-30

2018-03-07

2018-11-27

2018-03-07

2018-02-20

2018-02-20

2018-10-20

Antofagasta 2018-11-08

NWA 11959

2018-08-03

2018-10-08

2018-04-23

2018-10-08

2018-02-20

2018-04-18

2018-05-26

2018-02-20

2018-04-18

2018-02-24

2018-10-20

2018-02-23

2018-09-15

2018-04-18

2018-07-04

2018-04-18

2018-02-23

2018-06-22

2018-09-28

DOM 14471 41(2)

A

2018-02-23

DOM 14031 41(1)

2018-02-23

L4

DOM 14469 41(1)

SaU 605

A

2018-03-27

DOM 14032 41(1)

Al Wusta

2018-02-23

2018-03-13

2018-06-22

NWA 11869

2018-04-18 


\begin{tabular}{|c|c|c|c|c|c|}
\hline L4 & DOM 14523 & $41(1)$ & A & & $2018-02-23$ \\
\hline L4 & A 12306 & $\mathrm{~J} 26$ & $J B$ & & 2018-04-18 \\
\hline L4 & A 12345 & J26 & JB & & 2018-04-18 \\
\hline L4 & A 12249 & J26 & $J B$ & & 2018-04-18 \\
\hline L4 & Y-003345 & $\mathrm{J} 26$ & $J$ & & 2018-04-18 \\
\hline L4 & Y-000327 & J26 & $J$ & & 2018-04-18 \\
\hline L4 & A 09332 & J26 & $J B$ & & 2018-04-18 \\
\hline L4 & Y-001364 & $\mathrm{J} 26$ & $J$ & & 2018-04-18 \\
\hline L4 & Y-003369 & J26 & $J$ & & 2018-04-18 \\
\hline L4 & Y-000374 & J26 & $J$ & & 2018-04-18 \\
\hline L4 & Y-000394 & $\mathrm{J} 26$ & $J$ & & 2018-04-18 \\
\hline L4 & Y-003371 & J26 & $J$ & & 2018-04-18 \\
\hline L4 & $\mathrm{Y}-003350$ & J26 & $J$ & & 2018-04-18 \\
\hline L4 & A 09544 & $\mathrm{~J} 26$ & $J B$ & & 2018-04-18 \\
\hline L4 & Y-980033 & J26 & $J$ & & 2018-04-18 \\
\hline L4 & MCY 14018 & - & $P$ & & 2018-03-13 \\
\hline \multirow[t]{2}{*}{ L4 } & EET 16197 & $41(1)$ & A & & $2018-02-23$ \\
\hline & & & & & 2018-10-02 \\
\hline L4-melt breccia & NWA 12181 & & & & $2018-10-27$ \\
\hline $\mathrm{L} 4 / 5$ & NWA 11726 & & & & 2018-03-07 \\
\hline L4/5 & $\mathrm{CeC} 023$ & & & Antofagasta & 2018-07-08 \\
\hline L4/5 & NWA 11609 & & & & 2018-01-22 \\
\hline L4/5 & NWA 11627 & & & & $2018-01-24$ \\
\hline L5 & NWA 11584 & & & & 2018-01-16 \\
\hline L5 & & & & Antofagasta & 2018-11-08 \\
\hline L5 & NWA 12346 & & & & $2018-11-27$ \\
\hline L5 & & & & La Pampa & $2018-02-03$ \\
\hline L5 & LoV 213 & & & Antofagasta & 2018-03-27 \\
\hline L5 & NWA 12301 & & & & 2018-11-13 \\
\hline L5 & & & & Xinjiang & $2018-12-14$ \\
\hline L5 & & & & Antofagasta & 2018-11-08 \\
\hline L5 & NWA 11727 & & & & 2018-03-07 \\
\hline L5 & EM 381 & & & Antofagasta & 2018-04-19 \\
\hline L5 & NWA 12238 & & & & $2018-10-20$ \\
\hline L5 & SaU 607 & & & Al Wusta & $2018-12-02$ \\
\hline L5 & & & & Arusha & 2018-07-24 \\
\hline L5 & EM 378 & & & Antofagasta & 2018-03-27 \\
\hline L5 & NWA 11847 & & & & 2018-06-16 \\
\hline L5 & NWA 12292 & & & & 2018-11-09 \\
\hline L5 & NWA 1095 & & & & $2018-10-20$ \\
\hline L5 & NWA 11744 & & & & 2018-04-07 \\
\hline L5 & NWA 11723 & & & & 2018-03-07 \\
\hline L5 & NWA 11980 & & & & $2018-12-02$ \\
\hline L5 & & & & Antofagasta & 2018-09-15 \\
\hline L5 & & & & Antofagasta & 2018-05-11 \\
\hline
\end{tabular}


2018-04-23

NWA 12156

NWA 12157

NWA 11745

NWA 12066

Uttar Pradest 2018-12-11

A $12354 \quad$ J26

JB

NWA 674

NWA 11820

DOM 14460 41(1)

A

NWA 12026

NWA 12034

NWA 12258

NWA 11943

A 12138 J26

DOM 14230 41(2)

JB

NWA 12070

A

EET 16002 41(2)

A

NWA 12259

NWA 12294

NWA 11982

NWA 11646

NWA 12260

NWA 11760

NWA 11758

NWA 12073

NWA 11666

DOM 14413 41(2)

A

DOM 14172 41(1)

A

NWA 11661

DOM 14184 41(1)

A

DOM 14307 41(1)

A

DOM 14409 41(2)

A

DOM 14174 41(1)

NWA 11621

A

Antofagasta 2018-11-08

Ajdabiya 2018-07-17

2018-10-08

Antofagasta 2018-12-02

2018-10-08

2018-04-07

2018-10-08

Antofagasta 2018-05-11

2018-04-18

2018-10-20

2018-05-26

2018-02-23

2018-10-08

2018-10-08

2018-11-08

2018-08-03

2018-04-18

2018-09-28

2018-10-08

2018-09-28

2018-11-08

2018-11-09

2018-09-15

Antofagasta 2018-07-17

2018-01-27

2018-11-08

Antofagasta 2018-09-15

Ajdabiya $\quad$ 2018-07-17

2018-04-07

2018-04-07

2018-10-08

Antofagasta 2018-09-15

Antofagasta 2018-07-08

2018-02-09

2018-09-28

2018-02-23

2018-02-24

2018-02-23

2018-02-23

2018-09-28

2018-02-23

2018-01-24

DOM 14401 41(2)

A

2018-09-28 


\begin{tabular}{|c|c|c|c|c|}
\hline L5 & NWA 11657 & & & 2018-02-24 \\
\hline L5 & DOM 14177 41(1) & A & & $2018-02-23$ \\
\hline L5 & DOM 14468 41(1) & A & & $2018-02-23$ \\
\hline L5 & NWA 11656 & & & 2018-02-24 \\
\hline L5 & DOM 14222 41(1) & A & & $2018-02-23$ \\
\hline L5 & & & Antofagasta & 2018-10-09 \\
\hline L5 & DOM 14171 41(1) & A & & $2018-02-23$ \\
\hline L5 & & & Antofagasta & 2018-12-06 \\
\hline L5 & DOM 14425 41(2) & A & & $2018-09-28$ \\
\hline L5 & NWA 11711 & & & 2018-02-24 \\
\hline L5 & DOM 14343 41(2) & A & & $2018-09-28$ \\
\hline L5 & DOM 14511 41(1) & A & & $2018-02-23$ \\
\hline L5 & DOM 14215 41(2) & A & & $2018-09-28$ \\
\hline L5 & EM 373 & & Antofagasta & 2018-03-27 \\
\hline L5 & SJ 092 & & Antofagasta & 2018-11-08 \\
\hline L5 & MIL 15085 & A & & $2018-09-28$ \\
\hline L5 & DOM 14218 41(2) & A & & $2018-09-28$ \\
\hline L5 & DOM 14173 41(1) & A & & $2018-02-23$ \\
\hline L5 & & & Abu Zaby & $2018-06-16$ \\
\hline L5 & DOM 14176 41(1) & A & & $2018-02-23$ \\
\hline L5 & DOM 14512 41(1) & A & & $2018-02-23$ \\
\hline L5 & DOM 14404 41(2) & A & & $2018-09-28$ \\
\hline L5 & DOM 14509 41(2) & A & & 2018-09-28 \\
\hline L5 & DOM 14378 41(1) & A & & $2018-02-23$ \\
\hline L5 & DOM 14437 41(2) & A & & 2018-09-28 \\
\hline L5 & DOM 14109 41(1) & A & & $2018-02-23$ \\
\hline L5 & DOM 14086 41(1) & A & & $2018-02-23$ \\
\hline L5 & NWA 11870 & & & 2018-06-22 \\
\hline L5 & DOM 14434 41(2) & A & & $2018-09-28$ \\
\hline L5 & NWA 12101 & & & $2018-10-08$ \\
\hline L5 & DOM 14205 41(1) & A & & $2018-02-23$ \\
\hline L5 & DOM 14535 41(1) & A & & $2018-02-23$ \\
\hline L5 & DOM 14436 41(2) & A & & $2018-09-28$ \\
\hline L5 & DOM 14428 41(2) & A & & $2018-09-28$ \\
\hline L5 & DOM $1435141(2)$ & A & & $2018-09-28$ \\
\hline L5 & NWA 12177 & & & $2018-10-08$ \\
\hline L5 & DOM 14182 41(1) & A & & $2018-02-23$ \\
\hline L5 & DOM 14517 41(1) & A & & $2018-02-23$ \\
\hline L5 & DOM 14136 41(1) & A & & $2018-02-23$ \\
\hline L5 & DOM $1421141(2)$ & A & & $2018-09-28$ \\
\hline L5 & DOM 14376 41(1) & A & & $2018-02-23$ \\
\hline L5 & DOM 14427 41(2) & A & & 2018-09-28 \\
\hline L5 & DOM 14498 41(2) & A & & 2018-09-28 \\
\hline L5 & MIL $15375 \quad 41(2)$ & A & & $2018-09-28$ \\
\hline L5 & DOM 14536 41(1) & A & & 2018-02-23 \\
\hline
\end{tabular}


MIL 15393 41(2)

A

2018-09-28

DOM 14084 41(1)

2018-02-23

DOM 14528 41(1)

A

2018-02-23

MIL 15092 41(2)

A

2018-09-28

MIL $15371 \quad 41(2)$

A

2018-09-28

DOM 14532 41(1)

A

2018-02-23

DOM 14534 41(1)

A

2018-02-23

DOM 14200 41(1)

A

2018-02-23

DOM 14240 41(1)

A

2018-02-23

DOM 14550 41(1)

A

2018-02-23

DOM 14362 41(1)

2018-02-23

MIL 15299 41(2)

A

2018-09-28

DOM 14375 41(2)

Y-000256 J26

2018-09-28

2018-04-18

DOM 14292 41(2)

2018-09-28

DOM 14527 41(2)

DOM 14315 41(2)

2018-09-28

2018-09-28

DOM 14297 41(2)

2018-09-28

Y-000988 J26

2018-04-18

DOM 14199 41(1)

2018-02-23

DOM 14192 41(2)

2018-09-28

Y-791754 J26

2018-04-18

A $12293 \quad J 26$

2018-04-18

A $12343 \quad \mathrm{~J} 26$

Y-791755 J26

2018-04-18

2018-04-18

DOM 14457 41(1)

2018-02-23

DOM 14195 41(1)

SJ 084

Y-003460 J26

2018-02-23

$\mathrm{Y}-003353 \quad \mathrm{~J} 26$

$\mathrm{Y}-003447 \quad \mathrm{~J} 26$

Y-003456 J26

$\mathrm{Y}-003355 \quad \mathrm{~J} 26$

A $09384 \quad J 26$

2018-04-07

2018-04-18

2018-04-18

2018-04-18

2018-04-18

2018-04-18

2018-04-18

$\mathrm{Y}-003538 \quad \mathrm{~J} 26$

2018-04-18

Y-003449 J26

2018-04-18

Y-003453 J26

2018-04-18

Y-003333 J26

2018-04-18

Y-003465 J26

2018-04-18

$\mathrm{Y}-003450 \quad \mathrm{~J} 26$

2018-04-18

$\mathrm{Y}-003517 \quad \mathrm{~J} 26$

2018-04-18

$\mathrm{Y}-003366 \quad \mathrm{~J} 26$

2018-04-18

$\mathrm{Y}-000488 \quad \mathrm{~J} 26$

2018-04-18

$\mathrm{Y}-000287 \quad \mathrm{~J} 26$

2018-04-18

Y-003445 J26

2018-04-18 


\begin{tabular}{|c|c|c|c|c|}
\hline L5 & Y-000628 & $\mathrm{J} 26$ & $J$ & 2018-04-18 \\
\hline L5 & Y-003262 & $\mathrm{J} 26$ & $J$ & 2018-04-18 \\
\hline L5 & Y-003461 & J26 & $J$ & 2018-04-18 \\
\hline L5 & Y-000670 & $\mathrm{J} 26$ & $J$ & 2018-04-18 \\
\hline L5 & Y-003424 & $\mathrm{J} 26$ & $J$ & 2018-04-18 \\
\hline L5 & Y-003301 & $\mathrm{J} 26$ & $\mathrm{~J}$ & 2018-04-18 \\
\hline L5 & Y-003466 & J26 & $\mathrm{J}$ & $2018-04-18$ \\
\hline L5 & Y-003360 & $\mathrm{J} 26$ & $J$ & 2018-04-18 \\
\hline L5 & Y-003499 & $\mathrm{J} 26$ & $\mathrm{~J}$ & 2018-04-18 \\
\hline L5 & Y-003368 & $\mathrm{J} 26$ & $J$ & 2018-04-18 \\
\hline L5 & Y-003342 & $\mathrm{J} 26$ & $J$ & 2018-04-18 \\
\hline L5 & Y-980184 & J26 & $J$ & 2018-04-18 \\
\hline L5 & Y-003427 & $\mathrm{J} 26$ & $\mathrm{~J}$ & $2018-04-18$ \\
\hline L5 & Y-003258 & $\mathrm{J} 26$ & $J$ & 2018-04-18 \\
\hline L5 & Y-003440 & J26 & $J$ & 2018-04-18 \\
\hline L5 & Y-003266 & $\mathrm{J} 26$ & $J$ & 2018-04-18 \\
\hline L5 & Y-980361 & $\mathrm{J} 26$ & $J$ & 2018-04-18 \\
\hline L5 & Y-003459 & J26 & $\mathrm{J}$ & 2018-04-18 \\
\hline L5 & Y-003352 & $\mathrm{J} 26$ & $J$ & 2018-04-18 \\
\hline L5 & Y-003549 & $\mathrm{J} 26$ & $J$ & 2018-04-18 \\
\hline L5 & Y-003358 & $\mathrm{J} 26$ & $\mathrm{~J}$ & $2018-04-18$ \\
\hline L5 & Y-003545 & $\mathrm{J} 26$ & $J$ & 2018-04-18 \\
\hline L5 & MCY 14011 & - & $P$ & 2018-03-13 \\
\hline L5 & Y-003464 & J26 & $\mathrm{J}$ & 2018-04-18 \\
\hline L5 & Y-003463 & $\mathrm{J} 26$ & $J$ & 2018-04-18 \\
\hline L5 & MIL 15535 & $41(2)$ & A & 2018-09-28 \\
\hline L5 & Y-003444 & J26 & $\mathrm{J}$ & 2018-04-18 \\
\hline L5 & Y-980012 & $\mathrm{J} 26$ & $J$ & 2018-04-18 \\
\hline L5 & MIL 15222 & $41(1)$ & $A$ & 2018-02-23 \\
\hline L5 & Y-003303 & J26 & $\mathrm{J}$ & 2018-04-18 \\
\hline L5 & Y-003417 & $\mathrm{J} 26$ & $J$ & 2018-04-18 \\
\hline L5 & Y-003304 & $\mathrm{J} 26$ & $J$ & 2018-04-18 \\
\hline L5 & Y-980285 & $\mathrm{J} 26$ & $J$ & $2018-04-18$ \\
\hline L5 & EET 16204 & $41(1)$ & $A$ & $2018-02-23$ \\
\hline L5 & Y-003439 & $\mathrm{J} 26$ & $\mathrm{~J}$ & 2018-04-18 \\
\hline L5 & Y-002713 & $\mathrm{J} 26$ & $J$ & 2018-04-18 \\
\hline L5 & EET 16198 & $41(1)$ & $A$ & $2018-02-23$ \\
\hline L5 & MIL 15418 & $41(2)$ & $A$ & 2018-09-28 \\
\hline L5 & Y-003548 & J26 & $J$ & $2018-04-18$ \\
\hline L5 & Y-003302 & $\mathrm{J} 26$ & $J$ & 2018-04-18 \\
\hline L5 & Y-980333 & $\mathrm{J} 26$ & $J$ & 2018-04-18 \\
\hline L5 & Y-003312 & $\mathrm{J} 26$ & $J$ & 2018-04-18 \\
\hline L5 & Y-003308 & $\mathrm{J} 26$ & $J$ & 2018-04-18 \\
\hline L5 & Y-003457 & $\mathrm{J} 26$ & $J$ & 2018-04-18 \\
\hline L5 & EET 16173 & $41(1)$ & $A$ & $2018-02-23$ \\
\hline
\end{tabular}


L5-6

\section{L5-melt breccia}

L5/6

L5/6

L5/6

$\begin{array}{lll}\text { Y-003425 } & \text { J26 } & \text { J } \\ \text { EET 16213 } & 41(1) & \text { A } \\ \text { Y-003313 } & \text { J26 } & \text { J } \\ \text { Y-003306 } & \text { J26 } & \text { J } \\ \text { Y-003462 } & \text { J26 } & \text { J } \\ \text { Y-000610 } & \text { J26 } & \text { J } \\ \text { Y-003451 } & \text { J26 } & \text { J } \\ \text { Y-003421 } & \text { J26 } & \text { J } \\ \text { Y-003429 } & \text { J26 } & \text { J } \\ \text { Y-003550 } & \text { J26 } & \text { J } \\ \text { MCY 14007 } & - & \text { P } \\ \text { Y-003311 } & \text { J26 } & \text { J } \\ \text { Y-003416 } & \text { J26 } & \text { J } \\ \text { Y-003559 } & \text { J26 } & \text { J } \\ \text { EET 16200 } & 41(1) & \text { A } \\ \text { Y-003324 } & \text { J26 } & \text { J } \\ \text { MIL 15431 } & 41(2) & \text { A }\end{array}$

NWA 12289

NWA 11842

\section{EM 387}

EET 14045 - K
NWA 11724

LoV 258

NWA 1108

A-880611 J26

EET 16020 41(2)

NWA 11676

NWA 12151

DdA 002

Y-003336 J26

EET 14059 -
2018-04-18

2018-02-23

2018-04-18

2018-04-18

2018-04-18

2018-04-18

2018-04-18

2018-04-18

2018-04-18

2018-04-18

2018-03-13

2018-04-18

2018-04-18

2018-04-18

2018-02-23

2018-04-18

2018-09-28

2018-10-02

Baden-Wurtt 6 2018-12-24

Al Jumayliyar 2018-07-20

2018-12-11

Antofagasta 2018-11-08

Antofagasta 2018-11-08 Jarayan al Be 2018-07-20 Yunnan 2018-08-31

Antofagasta 2018-12-14

Antofagasta 2018-07-08

Antofagasta 2018-05-11 2018-06-16

Antofagasta 2018-07-24 Lipetskaya ot 2018-07-20 Antofagasta 2018-09-15 Antofagasta 2018-07-08 2018-04-23 2018-03-07

Antioquia 2018-06-07

Antofagasta 2018-11-13 2018-10-20 2018-04-18 2018-09-28 2018-02-20 2018-10-08

Atacama 2018-05-28 2018-04-18 2018-04-23 
Y-001167 J26

$\mathrm{J}$

EM 391

NWA 669

NWA 671

NWA 11930

EET 14060 -

NWA 436

Y-002423 J26

NWA 11779

NWA 11686

LoV 247

A $12214 \quad$ J26

JB

Antofagasta 2018-05-28

2018-04-18

Antofagasta 2018-09-15

2018-10-20

2018-10-20

2018-08-03

2018-04-23

2018-10-20

2018-04-18

2018-04-19

Antofagasta 2018-11-08

2018-02-20

Antofagasta 2018-11-08

Ouaddai 2018-12-24

Antofagasta 2018-11-08

2018-04-18

NWA 11681

2018-07-24

2018-02-20

2018-01-24

Glarus 2018-02-20

2018-04-18

A $12384 \quad$ J26

JB

LoV 218

SJ 097

RKP 14001 - K

NWA 12096

DOM 14462 41(1)

A

Antofagasta

$\begin{array}{ll}\text { Antofagasta } & 2018-05-11 \\ \text { Antofagasta } & 2018-11-08\end{array}$

2018-04-23

2018-10-08

2018-02-23

A $12432 \quad$ J26 JB

2018-04-18

NWA 11797

2018-04-25

NWA 12081

2018-10-08

A $12014 \quad$ J26 JB

2018-04-18

NWA 11633

2018-01-24

2018-09-28

2018-10-08

2018-07-04

2018-11-14

2018-02-23

L6

DOM 14231 41(2)

A

NWA 12069

NWA 11875

NWA 12325

DOM 14300 41(1)

A

Antofagasta 2018-05-11

LoV 222

LoV 254

Antofagasta 2018-02-20

EET $16021 \quad 41(2)$

2018-09-28

2018-08-03

NWA 11942

A

A $12142 \quad J 26$

JB

Antofagasta 2018-04-19

2018-04-18

Antofagasta 2018-09-15

NWA 454

2018-10-20 
DOM 14233 41(2)

A

2018-09-28

A $12020 \quad$ J26

JB

2018-04-18

NWA 11690

2018-02-20

A $12012 \quad$ J26

JB

2018-04-18

LoV 225

JB

A $12046 \quad J 26$

2018-05-11

NWA 11939

2018-04-18

2018-08-03

EET 14050 - K

2018-04-23

A $12281 \quad$ J26

$\mathrm{JB}$

2018-04-18

NWA 11984

2018-09-15

A $12255 \quad J 26 \quad J B$

2018-04-18

SJ 095

A

DOM 14415 41(2)

A $12273 \quad J 26$

JB

LoV 246

A $12313 \quad$ J26

JB

LoV 241

LoV 244

A

DOM 14414 41(2)

NWA 2730

DOM 14417 41(2)

A

DOM 14122 41(1)

A

EET 14037 -

Y-980154 J26

LoV 214

DOM 14123 41(1)

EET 14016 -

$\begin{array}{ll}\text { Antofagasta } & 2018-11-08 \\ & 2018-09-28\end{array}$

2018-04-18

Antofagasta 2018-11-08

2018-04-18

Antofagasta 2018-11-08

Antofagasta 2018-05-11

Antofagasta 2018-11-08

2018-09-28

2018-11-09

2018-09-28

2018-02-23

2018-04-23

2018-04-18

Antofagasta 2018-04-19

2018-02-23

2018-04-23

DOM 14220 41(1)

A

2018-02-23

A

Antofagasta 2018-07-17

Antofagasta 2018-12-02

A $12261 \quad J 26$

$J B$

2018-04-18

DOM 14419 41(2)

A

2018-09-28

2018-06-22

NWA 11868

2018-04-19

DOM 14308 41(1)

A

2018-02-23

EET 14031 -

2018-04-23

DOM 14178 41(1)

$\mathrm{K}$

2018-02-23

DOM 14474 41(2)

2018-09-28

DOM 14406 41(2)

2018-09-28

A $12367 \quad$ J26

NWA 11877

2018-04-18

2018-07-04

A $12404 \quad$ J26

JB

2018-05-11

2018-04-18

DOM 14400 41(2)

A

2018-09-28 


\begin{tabular}{|c|c|c|c|c|c|}
\hline L6 & EET 14019 & - & $\mathrm{K}$ & & $2018-04-23$ \\
\hline L6 & DOM 14235 & $41(2)$ & $A$ & & $2018-09-28$ \\
\hline L6 & DOM 14399 & $41(1)$ & $A$ & & $2018-02-23$ \\
\hline L6 & DOM 14475 & $41(2)$ & A & & 2018-09-28 \\
\hline L6 & DOM 14476 & $41(2)$ & $A$ & & $2018-09-28$ \\
\hline L6 & & & & Antofagasta & $2018-07-24$ \\
\hline L6 & NWA 12059 & & & & 2018-10-08 \\
\hline L6 & DOM 14478 & $41(2)$ & A & & $2018-09-28$ \\
\hline L6 & Y-790730 & J26 & $\mathrm{J}$ & & 2018-04-18 \\
\hline L6 & & & & Antofagasta & $2018-05-11$ \\
\hline L6 & DOM 14402 & $41(2)$ & $A$ & & $2018-09-28$ \\
\hline L6 & DOM 14102 & $41(1)$ & $A$ & & $2018-02-23$ \\
\hline L6 & DOM 14411 & $41(2)$ & $A$ & & $2018-09-28$ \\
\hline L6 & NWA 11759 & & & & 2018-04-07 \\
\hline L6 & DOM 14344 & $41(2)$ & A & & $2018-09-28$ \\
\hline L6 & A 12029 & $\mathrm{~J} 26$ & $\mathrm{JB}$ & & 2018-04-18 \\
\hline L6 & DOM 14519 & $41(1)$ & $A$ & & $2018-02-23$ \\
\hline L6 & DOM 14210 & $41(2)$ & $A$ & & $2018-09-28$ \\
\hline L6 & NWA 8285 & & & & 2018-07-17 \\
\hline L6 & DOM 14139 & $41(1)$ & A & & $2018-02-23$ \\
\hline L6 & EET 16043 & $41(2)$ & $A$ & & $2018-09-28$ \\
\hline L6 & SJ 098 & & & Antofagasta & $2018-11-08$ \\
\hline L6 & DOM 14345 & $41(2)$ & A & & $2018-09-28$ \\
\hline L6 & DOM 14394 & $41(1)$ & A & & $2018-02-23$ \\
\hline L6 & DOM 14309 & $41(1)$ & A & & $2018-02-23$ \\
\hline L6 & DOM 14477 & $41(2)$ & A & & 2018-09-28 \\
\hline L6 & DOM 14128 & $41(1)$ & $A$ & & $2018-02-23$ \\
\hline L6 & DOM 14403 & $41(2)$ & A & & 2018-09-28 \\
\hline L6 & & & & Arizona & 2018-10-06 \\
\hline L6 & DOM 14348 & $41(2)$ & $A$ & & $2018-09-28$ \\
\hline L6 & A 12381 & $\mathrm{~J} 26$ & $\mathrm{JB}$ & & 2018-04-18 \\
\hline L6 & A 12387 & $\mathrm{~J} 26$ & $\mathrm{JB}$ & & 2018-04-18 \\
\hline L6 & & & & Antofagasta & 2018-11-08 \\
\hline L6 & A 12336 & J26 & $\mathrm{JB}$ & & $2018-04-18$ \\
\hline L6 & DOM 14516 & $41(1)$ & $A$ & & $2018-02-23$ \\
\hline L6 & DOM 14341 & $41(2)$ & $A$ & & $2018-09-28$ \\
\hline L6 & A 12296 & $\mathrm{~J} 26$ & $\mathrm{JB}$ & & $2018-04-18$ \\
\hline L6 & EM 383 & & & Antofagasta & 2018-05-11 \\
\hline L6 & NWA 12142 & & & & $2018-10-08$ \\
\hline L6 & DOM 14107 & $41(1)$ & $A$ & & $2018-02-23$ \\
\hline L6 & MIL 15376 & $41(2)$ & $A$ & & $2018-09-28$ \\
\hline L6 & A 12397 & $\mathrm{~J} 26$ & $\mathrm{JB}$ & & 2018-04-18 \\
\hline L6 & DOM 14391 & $41(1)$ & $A$ & & $2018-02-23$ \\
\hline L6 & DOM 14190 & $41(1)$ & A & & $2018-02-23$ \\
\hline L6 & EET 14069 & - & $\mathrm{K}$ & & 2018-04-23 \\
\hline
\end{tabular}


DOM 14515 41(1)

A

2018-02-23

DOM 14350 41(2)

A

2018-09-28

DOM 14101 41(1)

2018-02-23

A $12396 \quad$ J26

A

2018-04-18

DOM 14470 41(2)

JB

2018-09-28

DOM 14426 41(2)

A

2018-09-28

DOM 14138 41(1)

A

NWA 8286

Antofagasta 2018-07-17

DOM 14431 41(2)

A

2018-07-17

DOM 14347 41(2)

2018-09-28

DOM 14108 41(1)

A

2018-09-28

DOM 14253 41(2)

A

2018-02-23

DOM 14340 41(2)

2018-09-28

A

2018-09-28

DOM 14518 41(1)

A

2018-02-23

DOM 14424 41(2)

A

2018-09-28

A $12359 \quad J 26$

A

2018-04-18

SJ 102

DOM 14291 41(2)

A

2018-11-08

DOM 14030 41(1)

2018-09-28

DOM 14100 41(1)

A

2018-02-23

A

2018-02-23

DOM 14531 41(1)

A

2018-02-23

DOM 14398 41(1)

A

2018-02-23

DOM 14432 41(2)

A

2018-09-28

DOM 14259 41(2)

A

2018-09-28

DOM 14104 41(1)

A

2018-02-23

NWA 12122

2018-10-08

DOM 14492 41(2)

A

2018-09-28

DOM 14085 41(1)

EET 14038 -

A

2018-02-23

2018-04-23

DOM 14081 41(1)

2018-02-23

DOM 14251 41(2)

2018-09-28

DOM 14134 41(1)

2018-02-23

DOM 14131 41(1)

2018-02-23

NWA 11648

2018-01-29

DOM 14105 41(1)

2018-02-23

DOM 14214 41(2)

2018-09-28

MIL 15374 41(2)

A $12406 \quad$ J26

DOM 14513 41(1)

2018-09-28

DOM 14354 41(2)

DOM 14510 41(1)

2018-04-18

JB

2018-02-23

A

2018-09-28

L6

A $12369 \quad$ J26

2018-02-23

L6

L6

NWA 12051

2018-04-18

2018-10-08

DOM 14290 41(2)

2018-09-28 


\begin{tabular}{|c|c|}
\hline L6 & DOM $1421641(2)$ \\
\hline L6 & DOM $1421741(2)$ \\
\hline L6 & DOM 14393 41(1) \\
\hline L6 & DOM 14106 41(1) \\
\hline L6 & DOM 14390 41(1) \\
\hline L6 & DOM 14392 41(1) \\
\hline L6 & DOM 14397 41(1) \\
\hline L6 & DOM 14506 41(2) \\
\hline L6 & EET 14047 - \\
\hline L6 & DOM 14349 41(2) \\
\hline L6 & DOM $1447241(2)$ \\
\hline L6 & DOM $1453741(1)$ \\
\hline
\end{tabular}

A

2018-09-28

A

2018-09-28

A

2018-02-23

A

2018-02-23

A

2018-02-23

A

2018-02-23

A

2018-02-23

A

2018-09-28

$\mathrm{K}$

2018-04-23

A

2018-09-28

A

2018-09-28

A

2018-02-23

Antofagasta 2018-11-08

DOM 14298 41(2)

A

2018-09-28

DOM 14310 41(2)

A

2018-09-28

DOM 14193 41(1)

A

2018-02-23

DOM 14538 41(1)

A

2018-02-23

NWA 11863

2018-06-22

MIL 15084 41(2)

A

2018-09-28

DOM 14423 41(2)

2018-09-28

DOM 14087 41(1)

A

2018-02-23

DOM 14368 41(2)

2018-09-28

DOM 14212 41(2)

NWA 11865

2018-09-28

2018-06-22

DOM 14242 41(1)

A

2018-02-23

DOM 14245 41(1)

A

2018-02-23

DOM 14346 41(2)

DOM 14311 41(2)

DOM 14269 41(2)

NWA 11626

NWA 12111

A $12321 \quad$ J26

A

2018-09-28

A

2018-09-28

A

2018-09-28

2018-01-24

2018-10-08

2018-04-18

DOM 14500 41(2)

EET 14034 -

2018-09-28

2018-04-23

DOM 14450 41(1)

2018-02-23

DOM 14267 41(2)

2018-09-28

DOM 14312 41(2)

2018-09-28

DOM 14313 41(2)

2018-09-28

DOM 14533 41(1)

2018-02-23

DOM 14241 41(1)

2018-02-23

DOM 14132 41(1)

2018-02-23

DOM 14420 41(2)

2018-09-28

L6

A

2018-07-17

DOM 14353 41(2)

2018-09-28

MIL $15087 \quad 41(2)$

A

2018-09-28 
A $12364 \quad J 26$

JB

2018-04-18

A

2018-02-23

DOM 14507 41(2)

A

2018-09-28

EET 14006 -

2018-04-23

DOM 14530 41(1)

$\mathrm{K}$

2018-02-23

DOM 14514 41(1)

2018-02-23

DOM 14503 41(2)

A

2018-09-28

DOM 14520 41(1)

2018-02-23

DOM 14556 41(1)

2018-02-23

SJ 091

DOM 14293 41(2)

A

DOM 14501 41(2)

A

Antofagasta 2018-11-08

DOM 14135 41(1)

A

2018-09-28

DOM 14299 41(2)

2018-02-23

DOM 14505 41(2)

A

2018-09-28

2018-09-28

DOM 14295 41(2)

A

2018-09-28

DOM 14314 41(2)

2018-09-28

A $12428 \quad J 26$

A

2018-04-18

DOM 14197 41(1)

JB

2018-02-23

DOM 14562 41(2)

LoV 255

A $12361 \quad J 26$

DOM 14317 41(2)

EET 14027 -

A

2018-09-28

Antofagasta 2018-11-08

2018-04-18

2018-09-28

2018-04-23

$\mathrm{K}$

$\begin{array}{ll}\text { Antofagasta } & 2018-05-11 \\ \text { California } & 2018-10-20\end{array}$

DOM 14490 41(2)

A

2018-09-28

DOM 14358 41(2)

A

2018-09-28

DOM 14504 41(2)

LoV 228

DOM 14529 41(1)

A

2018-09-28

L6

DOM 14352 41(2)

A

2018-07-17

2018-02-23

2018-09-28

DOM 14502 41(2)

A

2018-09-28

MIL 15090 41(2)

2018-09-28

DOM 14318 41(2)

2018-09-28

DOM 14496 41(2)

2018-09-28

DOM 14554 41(1)

2018-02-23

NWA 12170

2018-10-08

DOM 14257 41(2)

2018-09-28

DOM 14203 41(1)

2018-02-23

DOM 14365 41(1)

2018-02-23

DOM 14206 41(1)

2018-02-23

EET 14035 -

2018-04-23

DOM 14296 41(2)

2018-09-28

DOM 14137 41(1)

2018-02-23 


\begin{tabular}{|c|c|c|c|c|c|}
\hline L6 & A 12427 & J26 & $\mathrm{JB}$ & & 2018-04-18 \\
\hline L6 & DOM 14264 & $41(2)$ & $A$ & & $2018-09-28$ \\
\hline L6 & DOM 14189 & $41(1)$ & $A$ & & $2018-02-23$ \\
\hline L6 & DOM 14525 & $41(1)$ & $A$ & & 2018-02-23 \\
\hline L6 & DOM 14369 & $41(1)$ & $A$ & & $2018-02-23$ \\
\hline L6 & DOM 14209 & $41(1)$ & $A$ & & $2018-02-23$ \\
\hline L6 & DOM 14494 & $41(2)$ & $A$ & & 2018-09-28 \\
\hline L6 & DOM 14495 & $41(2)$ & $A$ & & $2018-09-28$ \\
\hline L6 & DOM 14560 & $41(2)$ & $A$ & & $2018-09-28$ \\
\hline L6 & LoV 256 & & & Antofagasta & 2018-11-08 \\
\hline L6 & DOM 14521 & $41(1)$ & $A$ & & $2018-02-23$ \\
\hline L6 & DOM 14526 & $41(1)$ & $A$ & & $2018-02-23$ \\
\hline L6 & DOM 14508 & $41(2)$ & $A$ & & $2018-09-28$ \\
\hline L6 & DOM 14319 & $41(2)$ & $A$ & & $2018-09-28$ \\
\hline L6 & DOM 14522 & $41(1)$ & $A$ & & $2018-02-23$ \\
\hline L6 & LoV 248 & & & Antofagasta & 2018-11-08 \\
\hline L6 & A 12353 & J26 & $\mathrm{JB}$ & & 2018-04-18 \\
\hline L6 & DOM 14278 & $41(1)$ & A & & $2018-02-23$ \\
\hline L6 & DOM 14361 & $41(1)$ & A & & $2018-02-23$ \\
\hline L6 & DOM 14034 & $41(1)$ & $A$ & & $2018-02-23$ \\
\hline L6 & DOM 14202 & $41(1)$ & $A$ & & $2018-02-23$ \\
\hline L6 & DOM 14551 & $41(1)$ & $A$ & & $2018-02-23$ \\
\hline L6 & & & & Antofagasta & 2018-04-19 \\
\hline L6 & DOM 14493 & $41(2)$ & A & & $2018-09-28$ \\
\hline L6 & DOM 14316 & $41(2)$ & A & & $2018-09-28$ \\
\hline L6 & DOM 14363 & $41(1)$ & A & & $2018-02-23$ \\
\hline L6 & DOM 14499 & $41(2)$ & $A$ & & $2018-09-28$ \\
\hline L6 & DOM 14246 & $41(1)$ & $A$ & & $2018-02-23$ \\
\hline L6 & DOM 14524 & $41(1)$ & $A$ & & $2018-02-23$ \\
\hline L6 & DOM 14558 & $41(1)$ & A & & $2018-02-23$ \\
\hline L6 & EET 14030 & - & $\mathrm{K}$ & & $2018-04-23$ \\
\hline L6 & DOM 14360 & $41(1)$ & $A$ & & $2018-02-23$ \\
\hline L6 & DOM 14260 & $41(2)$ & $A$ & & 2018-09-28 \\
\hline L6 & DOM 14133 & $41(1)$ & $A$ & & $2018-02-23$ \\
\hline L6 & DOM 14455 & $41(1)$ & $A$ & & $2018-02-23$ \\
\hline L6 & LoV 215 & & & Antofagasta & 2018-04-19 \\
\hline L6 & A 12275 & J26 & JB & & 2018-04-18 \\
\hline L6 & A-881188 & $\mathrm{J} 26$ & $J$ & & 2018-04-18 \\
\hline L6 & DOM 14088 & $41(1)$ & $A$ & & $2018-02-23$ \\
\hline L6 & DOM 14456 & $41(1)$ & $A$ & & $2018-02-23$ \\
\hline L6 & DOM 14208 & $41(1)$ & $A$ & & $2018-02-23$ \\
\hline L6 & Y-000169 & J26 & $J$ & & 2018-04-18 \\
\hline L6 & EET 14014 & - & $\mathrm{K}$ & & $2018-04-23$ \\
\hline L6 & DOM 14366 & $41(1)$ & $A$ & & $2018-02-23$ \\
\hline L6 & A 12295 & $\mathrm{~J} 26$ & $J B$ & & 2018-04-18 \\
\hline
\end{tabular}


A-87083 J26 J

2018-04-18

EET 14021 -

2018-04-23

A $12305 \quad J 26$

JB

2018-04-18

DOM 14367 41(1)

A

2018-02-23

DOM 14552 41(1)

2018-02-23

A $12297 \quad$ J26

A

2018-04-18

DOM 14271 41(1)

JB

2018-02-23

DOM 14198 41(1)

2018-02-23

DOM 14561 41(2)

2018-09-28

DOM 14262 41(2)

EET 14029 -

2018-09-28

2018-04-23

DOM 14355 41(2)

2018-09-28

DOM 14557 41(1)

2018-02-23

A 12355 J26 JB

2018-04-18

EET 14004 -

2018-04-23

LoV 252

DOM 14555 41(1)

A

A $09367 \quad$ J26

JB

2018-11-08

2018-02-23

2018-04-18

2018-04-23

2018-04-18

Y-002168 J26

2018-04-18

2018-04-18

Y-791672 J26

EM 382

Y-003539 J26

Y-003359 J26

Y-003365 J26

Y-000485 J26

Y-75070 J26

Y-000844 J26

Y-003294 J26

Y-003503 J26

Y-003374 J26

Y-003480 J26

Y-001097 J26

Y-003491 J26

Y-003530 J26

Y-003086 J26

Y-003292 J26

A $09410 \quad J 26$

Y-003019 J26

Y-003454 J26

Y-003329 J26

Y-003364 J26

Y-003264 J26

Y-003516 J26

2018-05-11

2018-04-18

2018-04-18

2018-04-18

2018-04-18

2018-04-18

2018-04-18

2018-04-18

2018-04-18

2018-04-18

2018-04-18

2018-04-18

2018-04-18

2018-04-18

2018-04-18

2018-04-18

2018-04-18

2018-04-18

2018-04-18

2018-04-18

2018-04-18

2018-04-18

2018-04-18 


\begin{tabular}{|c|c|c|c|c|}
\hline L6 & Y-003094 & $\mathrm{J} 26$ & $J$ & 2018-04-18 \\
\hline L6 & EET 14023 & - & $\mathrm{K}$ & 2018-04-23 \\
\hline L6 & Y-980265 & $\mathrm{J} 26$ & $J$ & 2018-04-18 \\
\hline L6 & A 09270 & $\mathrm{~J} 26$ & $J B$ & 2018-04-18 \\
\hline L6 & DOM 14083 & $41(1)$ & $A$ & $2018-02-23$ \\
\hline L6 & Y-003039 & J26 & J & 2018-04-18 \\
\hline L6 & Y-003263 & $\mathrm{J} 26$ & $J$ & 2018-04-18 \\
\hline L6 & Y-003363 & $\mathrm{J} 26$ & $J$ & 2018-04-18 \\
\hline L6 & Y-003293 & $\mathrm{J} 26$ & $J$ & 2018-04-18 \\
\hline L6 & EET 14015 & - & $\mathrm{K}$ & $2018-04-23$ \\
\hline L6 & Y-003474 & $\mathrm{J} 26$ & $J$ & 2018-04-18 \\
\hline L6 & Y-003483 & $\mathrm{J} 26$ & $J$ & 2018-04-18 \\
\hline L6 & A 09490 & $\mathrm{~J} 26$ & $\mathrm{JB}$ & $2018-04-18$ \\
\hline L6 & Y-003479 & $\mathrm{J} 26$ & $J$ & 2018-04-18 \\
\hline L6 & EET 14020 & - & $\mathrm{K}$ & $2018-04-23$ \\
\hline L6 & Y-003290 & $\mathrm{J} 26$ & $\mathrm{~J}$ & 2018-04-18 \\
\hline L6 & Y-003509 & $\mathrm{J} 26$ & $J$ & 2018-04-18 \\
\hline L6 & Y-000623 & J26 & $\mathrm{J}$ & 2018-04-18 \\
\hline L6 & Y-003265 & $\mathrm{J} 26$ & $J$ & 2018-04-18 \\
\hline L6 & Y-003470 & $\mathrm{J} 26$ & $J$ & 2018-04-18 \\
\hline L6 & Y-003082 & $\mathrm{J} 26$ & $\mathrm{~J}$ & 2018-04-18 \\
\hline L6 & Y-003367 & $\mathrm{J} 26$ & $J$ & 2018-04-18 \\
\hline L6 & EET 14026 & - & $\mathrm{K}$ & $2018-04-23$ \\
\hline L6 & Y-003502 & J26 & $\mathrm{J}$ & 2018-04-18 \\
\hline L6 & Y-003521 & $\mathrm{J} 26$ & $J$ & 2018-04-18 \\
\hline L6 & Y-003437 & $\mathrm{J} 26$ & $J$ & 2018-04-18 \\
\hline L6 & A 09492 & J26 & $J B$ & $2018-04-18$ \\
\hline L6 & $Y-003478$ & $\mathrm{~J} 26$ & $J$ & 2018-04-18 \\
\hline L6 & Y-003152 & $\mathrm{J} 26$ & $J$ & 2018-04-18 \\
\hline L6 & Y-003488 & $\mathrm{J} 26$ & $\mathrm{~J}$ & 2018-04-18 \\
\hline L6 & & & & $2018-11-08$ \\
\hline L6 & A 09386 & $\mathrm{~J} 26$ & $J B$ & $2018-04-18$ \\
\hline L6 & Y-003361 & J26 & J & 2018-04-18 \\
\hline L6 & Y-003357 & $\mathrm{J} 26$ & $J$ & 2018-04-18 \\
\hline L6 & EET 14005 & - & $\mathrm{K}$ & 2018-04-23 \\
\hline L6 & EET 14033 & - & $\mathrm{K}$ & $2018-04-23$ \\
\hline L6 & Y-980229 & $\mathrm{J} 26$ & $J$ & 2018-04-18 \\
\hline L6 & Y-000904 & $\mathrm{J} 26$ & $J$ & 2018-04-18 \\
\hline L6 & Y-003484 & J26 & $J$ & 2018-04-18 \\
\hline L6 & A 09601 & $\mathrm{~J} 26$ & $J B$ & 2018-04-18 \\
\hline L6 & Y-980335 & $\mathrm{J} 26$ & $J$ & 2018-04-18 \\
\hline L6 & Y-003526 & J26 & $\mathrm{J}$ & 2018-04-18 \\
\hline L6 & Y-003377 & $\mathrm{J} 26$ & $J$ & 2018-04-18 \\
\hline L6 & Y-980300 & $\mathrm{J} 26$ & $J$ & 2018-04-18 \\
\hline L6 & A 09493 & $\mathrm{~J} 26$ & $J B$ & 2018-04-18 \\
\hline
\end{tabular}


Y-003442 J26 J

2018-04-18

A $09557 \quad J 26$

2018-04-18

EET 14012 -

JB

2018-04-23

Y-980345 J26

2018-04-18

$\mathrm{Y}-980103 \quad \mathrm{~J} 26$

2018-04-18

Y-980019 J26

2018-04-18

Y-980309 J26

2018-04-18

EET 14025 - $\mathrm{K}$

2018-04-23

A $09600 \quad$ J26

2018-04-18

2018-04-18

2018-04-18

2018-04-18

2018-04-18

2018-04-18

2018-04-18

2018-04-18

2018-04-18

2018-04-18

2018-04-18

2018-04-18

2018-04-23

2018-04-18

2018-04-18

2018-04-18

2018-04-18

2018-04-18

2018-04-18

2018-04-18

2018-04-18

2018-04-23

2018-04-18

2018-04-18

2018-04-18

2018-04-18

2018-04-18

2018-04-18

2018-04-18

2018-04-18

2018-04-18

2018-04-18

2018-04-18

2018-04-18

2018-04-18

2018-04-18

2018-04-18 


\begin{tabular}{|c|c|c|c|c|}
\hline L6 & Y-003422 & $\mathrm{J} 26$ & $J$ & 2018-04-18 \\
\hline L6 & A 09406 & $\mathrm{~J} 26$ & $\mathrm{JB}$ & 2018-04-18 \\
\hline L6 & Y-980031 & J26 & $\mathrm{J}$ & 2018-04-18 \\
\hline L6 & Y-003495 & $\mathrm{J} 26$ & $J$ & 2018-04-18 \\
\hline L6 & Y-980045 & $\mathrm{J} 26$ & $J$ & 2018-04-18 \\
\hline L6 & Y-980221 & $\mathrm{J} 26$ & $\mathrm{~J}$ & 2018-04-18 \\
\hline L6 & Y-980234 & $\mathrm{J} 26$ & $J$ & 2018-04-18 \\
\hline L6 & Y-003506 & $\mathrm{J} 26$ & $J$ & 2018-04-18 \\
\hline L6 & Y-003511 & J26 & $J$ & $2018-04-18$ \\
\hline L6 & A 09264 & $\mathrm{~J} 26$ & $\mathrm{JB}$ & 2018-04-18 \\
\hline L6 & Y-003512 & $\mathrm{J} 26$ & $J$ & 2018-04-18 \\
\hline L6 & A 09460 & $\mathrm{~J} 26$ & $\mathrm{JB}$ & 2018-04-18 \\
\hline L6 & Y-003552 & $\mathrm{J} 26$ & $J$ & $2018-04-18$ \\
\hline L6 & Y-003490 & $\mathrm{J} 26$ & $J$ & 2018-04-18 \\
\hline L6 & Y-003494 & $\mathrm{J} 26$ & $J$ & 2018-04-18 \\
\hline L6 & Y-980266 & $\mathrm{J} 26$ & $J$ & 2018-04-18 \\
\hline L6 & A 12310 & $\mathrm{~J} 26$ & $\mathrm{JB}$ & 2018-04-18 \\
\hline L6 & Y-003535 & J26 & $\mathrm{J}$ & 2018-04-18 \\
\hline L6 & Y-003551 & $\mathrm{J} 26$ & $J$ & 2018-04-18 \\
\hline L6 & Y-980228 & $\mathrm{J} 26$ & $J$ & 2018-04-18 \\
\hline L6 & Y-003376 & $\mathrm{J} 26$ & $J$ & 2018-04-18 \\
\hline L6 & Y-003443 & $\mathrm{J} 26$ & $J$ & 2018-04-18 \\
\hline L6 & Y-003492 & $\mathrm{J} 26$ & $J$ & 2018-04-18 \\
\hline L6 & Y-003349 & J26 & J & 2018-04-18 \\
\hline L6 & Y-003498 & $\mathrm{J} 26$ & $J$ & 2018-04-18 \\
\hline L6 & Y-003554 & $\mathrm{J} 26$ & $J$ & 2018-04-18 \\
\hline L6 & MIL 15416 & $41(2)$ & $A$ & $2018-09-28$ \\
\hline L6 & Y-003467 & $\mathrm{J} 26$ & $J$ & 2018-04-18 \\
\hline L6 & Y-003524 & $\mathrm{J} 26$ & $J$ & 2018-04-18 \\
\hline L6 & Y-003481 & J26 & $\mathrm{J}$ & 2018-04-18 \\
\hline L6 & Y-003553 & $\mathrm{J} 26$ & $J$ & 2018-04-18 \\
\hline L6 & A 09459 & $\mathrm{~J} 26$ & $\mathrm{JB}$ & 2018-04-18 \\
\hline L6 & A 09461 & J26 & $\mathrm{JB}$ & $2018-04-18$ \\
\hline L6 & Y-003528 & $\mathrm{J} 26$ & $\mathrm{~J}$ & 2018-04-18 \\
\hline L6 & A 10221 & $\mathrm{~J} 26$ & $\mathrm{JB}$ & 2018-04-18 \\
\hline L6 & A 09558 & $\mathrm{~J} 26$ & $\mathrm{JB}$ & 2018-04-18 \\
\hline L6 & Y-980002 & $\mathrm{J} 26$ & $\mathrm{~J}$ & 2018-04-18 \\
\hline L6 & MIL 15567 & $41(2)$ & $A$ & 2018-09-28 \\
\hline L6 & Y-980203 & $\mathrm{J} 26$ & $J$ & $2018-04-18$ \\
\hline L6 & Y-003566 & $\mathrm{J} 26$ & $J$ & 2018-04-18 \\
\hline L6 & A 09604 & $\mathrm{~J} 26$ & $J B$ & 2018-04-18 \\
\hline L6 & MIL 15436 & $41(2)$ & $A$ & $2018-09-28$ \\
\hline L6 & Y-002151 & $\mathrm{J} 26$ & $J$ & 2018-04-18 \\
\hline L6 & Y-980349 & $\mathrm{J} 26$ & $J$ & 2018-04-18 \\
\hline L6 & Y-003486 & $\mathrm{J} 26$ & $J$ & 2018-04-18 \\
\hline
\end{tabular}


A $10062 \quad J 26$

JB

2018-04-18

Y-003335 J26

2018-04-18

Y-003435 J26

2018-04-18

A $09463 \quad J 26$

2018-04-18

Y-003527 J26

2018-04-18

EET 16207 41(1)

2018-02-23

Y-003555 J26

2018-04-18

Y-003497 J26

2018-04-18

Y-980289 J26

2018-04-18

A $09464 \quad J 26$

2018-04-18

Y-003557 J26

2018-04-18

Y-003519 J26

2018-04-18

Y-003373 J26

2018-04-18

2018-04-18

2018-04-18

2018-04-18

2018-04-18

2018-04-18

2018-04-18

2018-04-23

2018-04-18

2018-04-18

2018-04-18

2018-04-18

2018-04-18

2018-04-18

2018-04-18

2018-02-23

2018-04-18

2018-04-18

2018-04-18

2018-04-18

2018-02-23

2018-04-18

2018-04-18

2018-04-18

2018-02-23

2018-02-23

2018-04-18

2018-04-18

2018-04-18

2018-04-18

2018-04-18

2018-04-18

2018-04-18 


\begin{tabular}{|c|c|c|c|c|c|}
\hline L6 & Y-003052 & J26 & $J$ & & 2018-04-18 \\
\hline L6 & Y-003390 & $\mathrm{J} 26$ & $J$ & & 2018-04-18 \\
\hline L6 & Y-003423 & J26 & $\mathrm{J}$ & & 2018-04-18 \\
\hline L6 & Y-003400 & $\mathrm{J} 26$ & $J$ & & 2018-04-18 \\
\hline L6 & Y-003407 & $\mathrm{J} 26$ & $J$ & & 2018-04-18 \\
\hline L6 & Y-003282 & J26 & $J$ & & 2018-04-18 \\
\hline L6 & Y-000172 & J26 & $\mathrm{J}$ & & 2018-04-18 \\
\hline L6 & Y-003412 & $\mathrm{J} 26$ & $J$ & & 2018-04-18 \\
\hline L6 & Y-003284 & J26 & $J$ & & 2018-04-18 \\
\hline L6 & Y-002924 & J26 & $J$ & & 2018-04-18 \\
\hline L6 & Y-003315 & $\mathrm{J} 26$ & $J$ & & 2018-04-18 \\
\hline L6 & Y-003471 & J26 & $J$ & & 2018-04-18 \\
\hline L6 & Y-003320 & $\mathrm{J} 26$ & $J$ & & 2018-04-18 \\
\hline L6 & Y-003325 & $\mathrm{J} 26$ & $J$ & & 2018-04-18 \\
\hline L6-an & NWA 1184 & & & & 2018-07-04 \\
\hline L6-melt breccia & 0 & & & Antofagasta & 2018-08-03 \\
\hline L6-melt breccia & SJ 086 & & & Antofagasta & 2018-07-17 \\
\hline L6-melt breccia & LoV 216 & & & Antofagasta & 2018-04-19 \\
\hline $\operatorname{LL}(\mathrm{L}) 3$ & NWA 1161 & & & & $2018-02-20$ \\
\hline LL(L)3 & NWA 1161 & & & & $2018-02-20$ \\
\hline LL $\sim 4$ & & & & Xinjiang & $2018-10-06$ \\
\hline LL 5 & NWA 1197 & & & & 2018-10-09 \\
\hline LL 6 & & & & Xinjiang & 2018-11-09 \\
\hline LL $\sim 6$ & & & & Xinjiang & 2018-11-09 \\
\hline LL 6 & & & & Gansu & $2018-12-16$ \\
\hline LL3 & NWA 1166 & & & & $2018-02-20$ \\
\hline LL3 & NWA 1190 & & & & 2018-07-24 \\
\hline LL3 & NWA 1200 & & & & 2018-12-11 \\
\hline LL3 & NWA 1161 & & & & 2018-01-22 \\
\hline LL3 & NWA 1196 & & & & $2018-09-23$ \\
\hline LL3 & & & & Kerman & $2018-05-26$ \\
\hline LL3 & NWA 1190 & & & & $2018-07-24$ \\
\hline LL3 & NWA 1199 & & & & $2018-10-09$ \\
\hline LL3 & NWA 1228 & & & & $2018-11-24$ \\
\hline LL3 & NWA 1199 & & & & 2018-10-09 \\
\hline LL3 & NWA 1195 & & & & 2018-09-15 \\
\hline LL3 & NWA 1229 & & & & 2018-11-09 \\
\hline LL3 & NWA 1223 & & & & $2018-10-20$ \\
\hline LL3 & & & & Arizona & $2018-06-07$ \\
\hline LL3 & A 12050 & $\mathrm{~J} 26$ & $\mathrm{JB}$ & & 2018-04-18 \\
\hline LL3 & NWA 1224 & & & & $2018-10-27$ \\
\hline LL3 & NWA 1181 & & & & 2018-05-11 \\
\hline LL3 & NWA 1096 & & & & $2018-10-20$ \\
\hline LL3 & NWA 1159 & & & & $2018-01-16$ \\
\hline LL3 & NWA 1197 & & & & 2018-09-02 \\
\hline
\end{tabular}


NWA 12185

2018-10-09

CS 004

LL3

A 12405

J26

JB

LL3

NWA 12281

LL3

NWA 11813

LL3

EET 14064 -

K

EET 14017 -

$\mathrm{K}$

Antofagasta 2018-12-02

2018-04-18

2018-11-17

2018-05-11

LL3

LL3-6

NWA 11873

LL3-6

NWA 11831

LL3.05

NWA 11752

LL3.10-5

NWA 11905

2018-04-23

2018-04-23

2018-06-30

2018-07-04

2018-04-19

2018-09-02

LL3.4

LL3.7

DOM 14183 41(1)

A

DOM 14458 41(1)

A

LL4

NWA 11651

NWA 11882

LoV 236

NWA 12254

NWA 11944

NWA 12017

NWA 11665

A $12038 \quad J 26$

JB

B-9819 J26

$J$

A $12392 \quad J 26$

$\mathrm{JB}$

A $12418 \quad J 26 \quad J B$

$\begin{array}{rr}\text { Antofagasta } & 2018-08-03 \\ & 2018-02-23\end{array}$

2018-02-23

2018-02-24

2018-07-08

Antofagasta 2018-11-08

Morocco, Dat 2018-11-08

2018-08-03

Ajdabiya $\quad$ 2018-07-17

2018-09-23

2018-02-09

2018-04-18

2018-04-18

2018-04-18

2018-04-18

DOM 14342 41(2) A

2018-09-28

A $12393 \quad J 26 \quad J B$

2018-04-18

A $12394 \quad J 26$

2018-04-18

A $12416 \quad$ J26 JB

2018-04-18

A $12415 \quad J 26 \quad J B$

2018-04-18

A $12417 \quad$ J26 JB

2018-04-18

NWA 11874

2018-07-04

2018-02-23

DOM 14036 41(1) A

MCY 14020 - $P$

2018-03-13

MIL 15433 41(2) A

2018-09-28

2018-07-24

2018-11-27

2018-08-03

2018-04-18

A $12013 \quad J 26 \quad J B$

2018-01-27

NWA 11645

2018-04-18

A $12362 \quad J 26 \quad J B$

2018-01-24

LL4-6

NWA 11629

2018-04-18

A $12395 \quad J 26 \quad J B$

2018-10-20 


\begin{tabular}{|c|c|c|c|c|c|}
\hline LL5 & NWA 11643 & & & & $2018-02-20$ \\
\hline LL5 & NWA 12337 & & & & $2018-11-27$ \\
\hline LL5 & A 12059 & $\mathrm{~J} 26$ & $\mathrm{JB}$ & & $2018-04-18$ \\
\hline LL5 & A 12357 & $\mathrm{~J} 26$ & $J B$ & & $2018-04-18$ \\
\hline LL5 & NWA 11664 & & & & $2018-02-24$ \\
\hline LL5 & EET 16040 & $41(2)$ & $A$ & & $2018-09-28$ \\
\hline LL5 & & & & Antofagasta & $2018-07-24$ \\
\hline LL5 & DOM 14304 & $41(1)$ & $A$ & & $2018-02-23$ \\
\hline LL5 & NWA 11624 & & & & $2018-01-24$ \\
\hline LL5 & & & & Antofagasta & 2018-10-09 \\
\hline LL5 & NWA 12092 & & & & $2018-10-08$ \\
\hline LL5 & A 12358 & J26 & $\mathrm{JB}$ & & 2018-04-18 \\
\hline LL5 & A 12423 & $\mathrm{~J} 26$ & $\mathrm{JB}$ & & $2018-04-18$ \\
\hline LL5 & DOM 14306 & $41(1)$ & $A$ & & $2018-02-23$ \\
\hline LL5 & NWA 12256 & & & & 2018-11-08 \\
\hline LL5 & A 12414 & $\mathrm{~J} 26$ & $J B$ & & 2018-04-18 \\
\hline LL5 & DOM 14226 & $41(1)$ & $A$ & & $2018-02-23$ \\
\hline LL5 & EM 376 & & & Antofagasta & 2018-03-27 \\
\hline LL5 & A 12422 & $\mathrm{~J} 26$ & $J B$ & & 2018-04-18 \\
\hline LL5 & NWA 11618 & & & & 2018-01-24 \\
\hline LL5 & A 12431 & $\mathrm{~J} 26$ & $\mathrm{JB}$ & & 2018-04-18 \\
\hline LL5 & DOM 14279 & $41(1)$ & $A$ & & $2018-02-23$ \\
\hline LL5 & A 12391 & $\mathrm{~J} 26$ & $\mathrm{JB}$ & & 2018-04-18 \\
\hline LL5 & A 12424 & $\mathrm{~J} 26$ & $\mathrm{JB}$ & & $2018-04-18$ \\
\hline LL5 & DOM 14273 & $41(1)$ & A & & $2018-02-23$ \\
\hline LL5 & & & & Antofagasta & 2018-04-07 \\
\hline LL5 & A 12402 & $\mathrm{~J} 26$ & $\mathrm{JB}$ & & 2018-04-18 \\
\hline LL5 & B-9813 & $\mathrm{J} 26$ & $\mathrm{~J}$ & & 2018-04-18 \\
\hline LL5-an & NWA 11811 & & & & 2018-11-09 \\
\hline LL5/6 & NWA 12358 & & & & $2018-11-27$ \\
\hline LL5/6 & NWA 12357 & & & & $2018-11-27$ \\
\hline LL6 & & & & Guelmim Sm & :2018-12-11 \\
\hline LL6 & NWA 11838 & & & & 2018-06-07 \\
\hline LL6 & NWA 12016 & & & & 2018-09-23 \\
\hline LL6 & NWA 12015 & & & & $2018-09-23$ \\
\hline LL6 & NWA 11620 & & & & 2018-01-24 \\
\hline LL6 & NWA 12020 & & & & 2018-10-06 \\
\hline LL6 & NWA 11654 & & & & $2018-02-24$ \\
\hline LL6 & A 12129 & $\mathrm{~J} 26$ & $\mathrm{JB}$ & & $2018-04-18$ \\
\hline LL6 & A 12030 & $\mathrm{~J} 26$ & $J B$ & & 2018-04-18 \\
\hline LL6 & NWA 11684 & & & & $2018-02-20$ \\
\hline LL6 & A 12426 & $\mathrm{~J} 26$ & $J B$ & & $2018-04-18$ \\
\hline LL6 & NWA 11932 & & & & 2018-08-03 \\
\hline LL6 & NWA 11619 & & & & 2018-01-24 \\
\hline LL6 & NWA 11876 & & & & 2018-07-04 \\
\hline
\end{tabular}


A $12411 \quad J 26$

JB

2018-04-18

LL6

A $12058 \quad J 26$

JB

LL6

SJ 096

LL6

NWA 12007

LL6

DOM 14302 41(1)

A

LL6

LL6

LL6

LL6

LL6

LL6

LL6

LL6

LL6

LL6

LL6

LL6

LL6

LL6

LL6

LL6

LL6

LL6

LL6

LL6

LL6

LL6

LL6

LL6

LL6

LL6

LL6

LL6

LL6

LL6

LL6

LL6

LL6

LL6

LL6

LL6

LL6

LL6

LL6

LL6

NWA 11655

NWA 11631

NWA 11715

DOM 14303 41(1)

A

A $12032 \quad J 26$

A $12413 \quad J 26$

NWA 11663

NWA 12255

NWA 12295

A $12235 \quad J 26$

A $12412 \quad J 26$

NWA 12298

NWA 11716

NWA 11963

A $12420 \quad$ J26

$J B$

DOM 14223 41(1)

NWA 11632

A $12421 \quad J 26$

A $12410 \quad J 26$

A $12435 \quad J 26$

A $12250 \quad \mathrm{~J} 26$

A $12401 \quad J 26$

A $12434 \quad \mathrm{~J} 26$

A $12243 \quad \mathrm{~J} 26$

A $12403 \quad J 26$

NWA 11628

NWA 11702

A $12317 \quad$ J26

NWA 12178

A $12304 \quad J 26$

NWA 11719

A $12258 \quad$ J26

NWA 11717

Y-003198 J26

A $12265 \quad J 26$

A $12241 \quad \mathrm{~J} 26$

A $12237 \quad \mathrm{~J} 26$

DOM 14276 41(1)

DOM 14277 41(1)

NWA 11718
2018-04-18

Antofagasta 2018-11-08

2018-10-27

2018-02-23

2018-02-24

2018-01-24

2018-02-24

2018-02-23

2018-04-18

2018-04-18

2018-02-24

2018-11-08

2018-11-09

2018-04-18

2018-04-18

2018-11-09

2018-02-24

2018-09-15

2018-04-18

2018-02-23

2018-01-24

2018-04-18

2018-04-18

2018-04-18

2018-04-18

2018-04-18

2018-04-18

2018-04-18

2018-04-18

2018-01-24

2018-02-24

2018-04-18

2018-10-08

2018-04-18

2018-02-24

2018-04-18

2018-02-24

2018-04-18

2018-04-18

2018-04-18

2018-04-18

2018-02-23

2018-02-23

2018-02-24 


\begin{tabular}{|c|c|c|c|c|c|}
\hline LL6 & DOM 14272 & $41(1)$ & A & & $2018-02-23$ \\
\hline LL6 & DOM 14275 & $41(1)$ & A & & $2018-02-23$ \\
\hline LL6 & Y-791540 & $\mathrm{J} 26$ & $J$ & & 2018-04-18 \\
\hline LL6 & A-87314 & $\mathrm{J} 26$ & $\mathrm{~J}$ & & 2018-04-18 \\
\hline LL6 & MIL 15251 & $41(2)$ & A & & $2018-09-28$ \\
\hline LL6 & A 12425 & $\mathrm{~J} 26$ & $J B$ & & 2018-04-18 \\
\hline LL6 & DOM 14274 & $41(1)$ & $A$ & & $2018-02-23$ \\
\hline LL6 & DOM 14228 & $41(1)$ & A & & $2018-02-23$ \\
\hline LL6 & A 12433 & $\mathrm{~J} 26$ & JB & & 2018-04-18 \\
\hline LL6 & DOM 14445 & $41(2)$ & $A$ & & 2018-09-28 \\
\hline LL6 & Y-980339 & $\mathrm{J} 26$ & $\mathrm{~J}$ & & $2018-04-18$ \\
\hline LL6 & Y-003199 & $\mathrm{J} 26$ & $J$ & & 2018-04-18 \\
\hline LL6 & Y-980291 & $\mathrm{J} 26$ & $\mathrm{~J}$ & & 2018-04-18 \\
\hline LL6 & Y-980046 & $\mathrm{J} 26$ & $\mathrm{~J}$ & & $2018-04-18$ \\
\hline LL6 & Y-002852 & J26 & $\mathrm{J}$ & & 2018-04-18 \\
\hline LL6 & RKP 14002 & - & K & & $2018-04-23$ \\
\hline LL6-an & NWA 11776 & & & & 2018-09-15 \\
\hline LL6-melt breccia & NWA 11730 & & & & 2018-04-07 \\
\hline LL7 & NWA 12272 & & & & 2018-12-02 \\
\hline LL7 & LoV 231 & & & Antofagasta & 2018-08-03 \\
\hline LL7 & NWA 11852 & & & & 2018-07-04 \\
\hline Lodranite & NWA 11848 & & & & 2018-07-04 \\
\hline Lodranite & NWA 11795 & & & & $2018-05-26$ \\
\hline Lodranite & NWA 11960 & & & & 2018-09-15 \\
\hline Lodranite & NWA 11901 & & & & 2018-08-31 \\
\hline Lodranite & NWA 11970 & & & & $2018-09-23$ \\
\hline Lodranite & Y-001620 & J26 & $J$ & & 2018-04-18 \\
\hline Lodranite & Y-001322 & $\mathrm{J} 26$ & $\mathrm{~J}$ & & 2018-04-18 \\
\hline Lodranite & DOM 14289 & $41(1)$ & A & & $2018-02-23$ \\
\hline Lunar & NWA 11616 & & & & $2018-02-20$ \\
\hline Lunar & NWA 11962 & & & & $2018-09-23$ \\
\hline Lunar & NWA 11801 & & & & 2018-06-07 \\
\hline Lunar & NWA 11886 & & & & 2018-08-03 \\
\hline Lunar & NWA 11767 & & & & 2018-05-11 \\
\hline Lunar (basalt) & NWA 12008 & & & & $2018-10-27$ \\
\hline Lunar (feldsp. breccia) & NWA 11787 & & & & $2018-05-26$ \\
\hline Lunar (feldsp. breccia) & NWA 11788 & & & & $2018-05-26$ \\
\hline Lunar (feldsp. breccia) & NWA 11303 & & & & 2018-02-24 \\
\hline Lunar (feldsp. breccia) & NWA 11789 & & & & $2018-05-26$ \\
\hline Lunar (feldsp. breccia) & NWA 11828 & & & & $2018-06-22$ \\
\hline Lunar (feldsp. breccia) & NWA 12368 & & & & 2018-12-06 \\
\hline Lunar (feldsp. breccia) & NWA 12279 & & & Tindouf & 2018-12-11 \\
\hline Lunar (feldsp. breccia) & NWA 12270 & & & & 2018-12-02 \\
\hline lunar (feldsp. breccia) & NWA 2425 & & & & $2018-05-11$ \\
\hline Lunar (feldsp. breccia) & NWA 11898 & & & & 2018-08-31 \\
\hline
\end{tabular}


Rio de Oro

NWA 11968

NWA 11695

NWA 11851

NWA 11966

Lunar (Feldsp. Breccia)

NWA 11966

NWA 11517

NWA 11809

NWA 11783

NWA 11563

NWA 12248

NWA 11871

NWA 11703

CeC 022

NWA 11896

NWA 11921

Martian (basaltic breccia)

Martian (basaltic breccia)

Martian (shergottite)

Martian (shergottite)

Martian (shergottite)

Martian (shergottite)

Martian (shergottite)

Martian (shergottite)

Martian (shergottite)

Martian (shergottite)

Martian (shergottite)

Martian (shergottite)

Martian (shergottite)

Martian (shergottite)

Martian (shergottite)

Martian (shergottite)

Martian (shergottite)

Martian (shergottite)

Martian (shergottite)

Mesosiderite

Mesosiderite

Mesosiderite

Mesosiderite

Mesosiderite

OC3

Pallasite

Pallasite

Pallasite

Pallasite, PMG
NWA 12222

NWA 12335

NWA 12262

NWA 12269

NWA 12241

NWA 12323

NWA 11955

NWA 12210

NWA 12280

NWA 2424

NWA 11804

A $12325 \quad$ J26

NWA 11866

NWA 11668

NWA 11746

NWA 12002

NWA 11761

NWA 11774

NWA 11853

NWA 11887

NWA 12212

$\mathrm{JaH} 846$

NWA 12186

NWA 5693

NWA 12288

2018-02-20

2018-09-23

2018-03-27

2018-07-04

2018-09-23

2018-01-22

2018-02-24

2018-05-28

2018-05-26

2018-01-12

2018-11-24

2018-07-17

2018-03-27

Antofagasta 2018-04-10

2018-08-31

2018-09-15

Rio de Oro 2018-09-23

2018-11-12

2018-12-06

Rio de Oro 2018-04-07

2018-12-11

2018-12-11

2018-11-24

2018-12-14

2018-09-15

2018-11-12

Tindouf 2018-12-11

2018-05-11

2018-05-28

2018-04-18

2018-07-17

2018-03-27

2018-04-19

2018-04-07

2018-10-16

2018-05-11

2018-05-12

2018-07-04

2018-08-03

2018-11-12

Al Wusta 2018-09-15

2018-11-09

2018-08-31

2018-12-11

Vaud 2018-09-15 


\begin{tabular}{|c|c|c|c|}
\hline Pallasite, PMG-an & NWA 11720 & & 2018-04-07 \\
\hline Pallasite, PMG-an & & Saguia el $\mathrm{Ha}$ & 1) 2018-08-03 \\
\hline R3 & NWA 11721 & & 2018-04-07 \\
\hline R3 & NWA 11927 & & $2018-09-15$ \\
\hline R3 & NWA 11769 & & 2018-05-11 \\
\hline R3-5 & NWA 11587 & & $2018-02-03$ \\
\hline R3-5 & NWA 11860 & & $2018-07-24$ \\
\hline R3-5 & NWA 11614 & & $2018-02-20$ \\
\hline R3-5 & NWA 8791 & & $2018-06-22$ \\
\hline R3-6 & NWA 11697 & & $2018-03-27$ \\
\hline R3.5-4 & NWA 11880 & & $2018-07-17$ \\
\hline R4 & NWA 11304 & & $2018-04-07$ \\
\hline R4 & NWA 11571 & & $2018-01-16$ \\
\hline R5 & NWA 11772 & & 2018-05-11 \\
\hline Ureilite & NWA 11892 & & $2018-08-03$ \\
\hline Ureilite & NWA 11950 & & $2018-09-15$ \\
\hline Ureilite & NWA 11757 & & $2018-05-26$ \\
\hline Ureilite & NWA 12271 & & $2018-12-02$ \\
\hline Ureilite & NWA 11956 & & $2018-09-15$ \\
\hline Ureilite & NWA 11641 & & $2018-02-20$ \\
\hline Ureilite & NWA 11639 & & $2018-02-20$ \\
\hline Ureilite & NWA 12191 & & $2018-11-18$ \\
\hline Ureilite & NWA 11893 & & $2018-08-03$ \\
\hline Ureilite & NWA 12198 & & 2018-11-09 \\
\hline Ureilite & NWA 11640 & & $2018-02-20$ \\
\hline Ureilite & NWA 12237 & & $2018-12-02$ \\
\hline Ureilite & JaH 1101 & Al Wusta & 2018-01-08 \\
\hline Ureilite & NWA 11900 & & 2018-09-02 \\
\hline Ureilite & NWA 12189 & & 2018-11-09 \\
\hline Ureilite & NWA 11671 & & $2018-03-27$ \\
\hline Ureilite & NWA 11675 & & $2018-03-27$ \\
\hline Ureilite & JaH 1100 & Al Wusta & 2018-01-08 \\
\hline Ureilite & NWA 11754 & & $2018-05-26$ \\
\hline Ureilite & NWA 11891 & & $2018-08-03$ \\
\hline Ureilite & NWA 11815 & & $2018-06-16$ \\
\hline Ureilite & EET 14053 & & $2018-05-26$ \\
\hline Ureilite & NWA 11993 & & 2018-10-09 \\
\hline Ureilite & NWA 11784 & & $2018-05-26$ \\
\hline Ureilite & JaH 1103 & Al Wusta & $2018-02-20$ \\
\hline Ureilite & NWA 12188 & & $2018-11-18$ \\
\hline Ureilite & NWA 11951 & & $2018-09-15$ \\
\hline Ureilite & NWA 12321 & & $2018-12-14$ \\
\hline Ureilite & NWA 12183 & & 2018-11-09 \\
\hline Ureilite & SJ 100 & Antofagasta & $2018-12-02$ \\
\hline Ureilite & NWA 11890 & & $2018-08-03$ \\
\hline
\end{tabular}




\begin{tabular}{|c|c|}
\hline Ureilite & $\mathrm{JaH} 1102$ \\
\hline Ureilite & NWA 11755 \\
\hline Ureilite & NWA 11894 \\
\hline Ureilite & NWA 11585 \\
\hline Ureilite & NWA 11775 \\
\hline Ureilite & NWA 11738 \\
\hline Ureilite & NWA 11608 \\
\hline Ureilite & NWA 11644 \\
\hline Ureilite & RaS 530 \\
\hline Ureilite & Y-000614 \\
\hline Ureilite & A 10104 \\
\hline Ureilite-an & NWA 11511 \\
\hline Winonaite & NWA 11917 \\
\hline Winonaite & NWA 11807 \\
\hline
\end{tabular}

$\begin{array}{cr}\text { Al Wusta } & 2018-01-08 \\ & 2018-05-26 \\ 2018-08-03 \\ 2018-02-03 \\ 2018-05-11 \\ 2018-04-19 \\ \\ 2018-02-20 \\ \text { AI Wusta } & 2018-02-20 \\ & 2018-03-02 \\ & 2018-04-18 \\ & 2018-04-18 \\ & 2018-09-12 \\ 2018-05-28\end{array}$




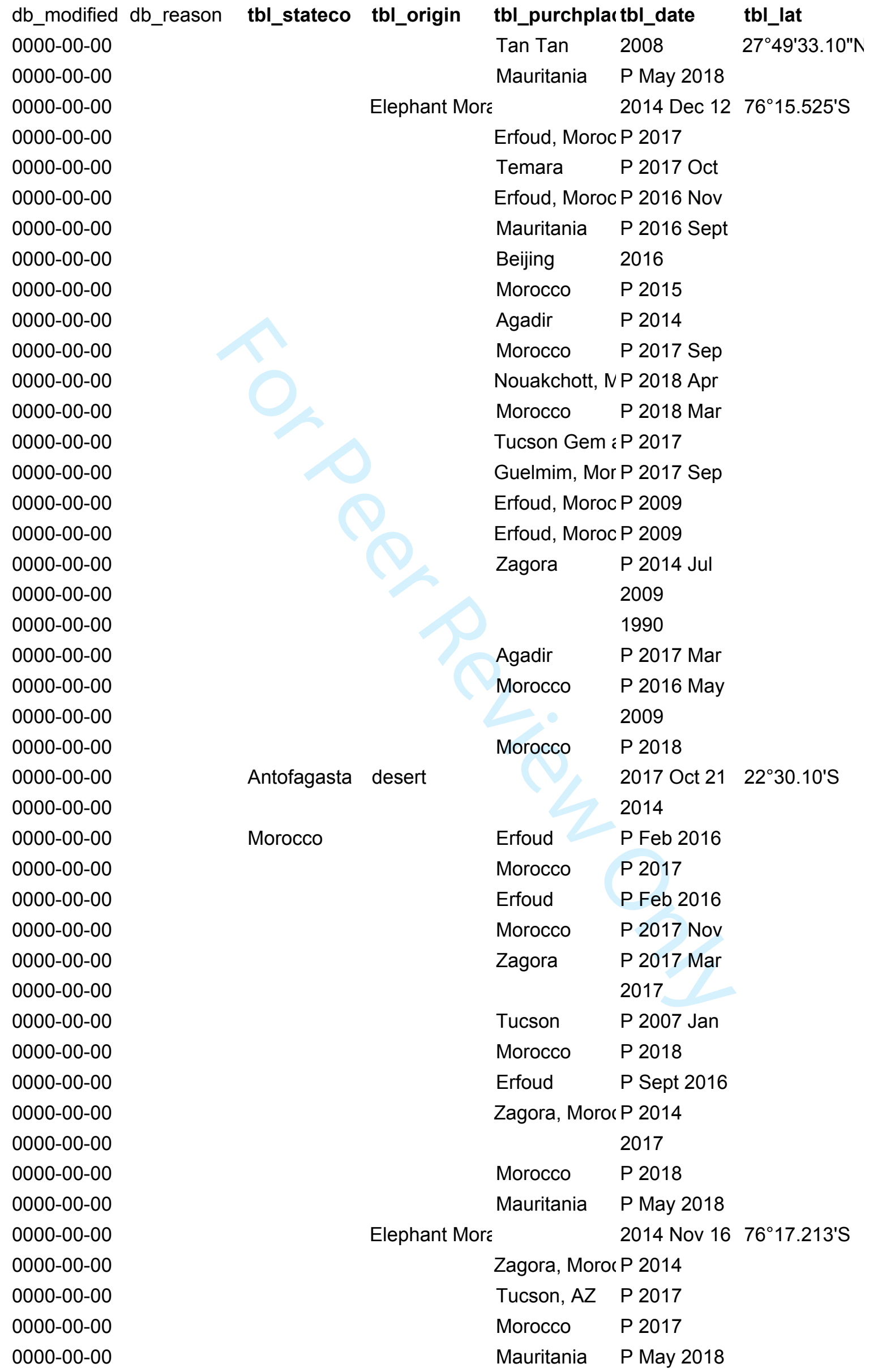


0000-00-00

0000-00-00

0000-00-00

0000-00-00

0000-00-00

0000-00-00

0000-00-00

0000-00-00

0000-00-00

0000-00-00

0000-00-00

0000-00-00

0000-00-00

0000-00-00

0000-00-00

0000-00-00

0000-00-00

0000-00-00

0000-00-00

0000-00-00

0000-00-00

0000-00-00

0000-00-00

0000-00-00

0000-00-00

0000-00-00

0000-00-00

0000-00-00

0000-00-00

0000-00-00

0000-00-00

0000-00-00

0000-00-00

0000-00-00

0000-00-00

0000-00-00

0000-00-00

0000-00-00

0000-00-00

0000-00-00

0000-00-00

0000-00-00

0000-00-00

0000-00-00

0000-00-00

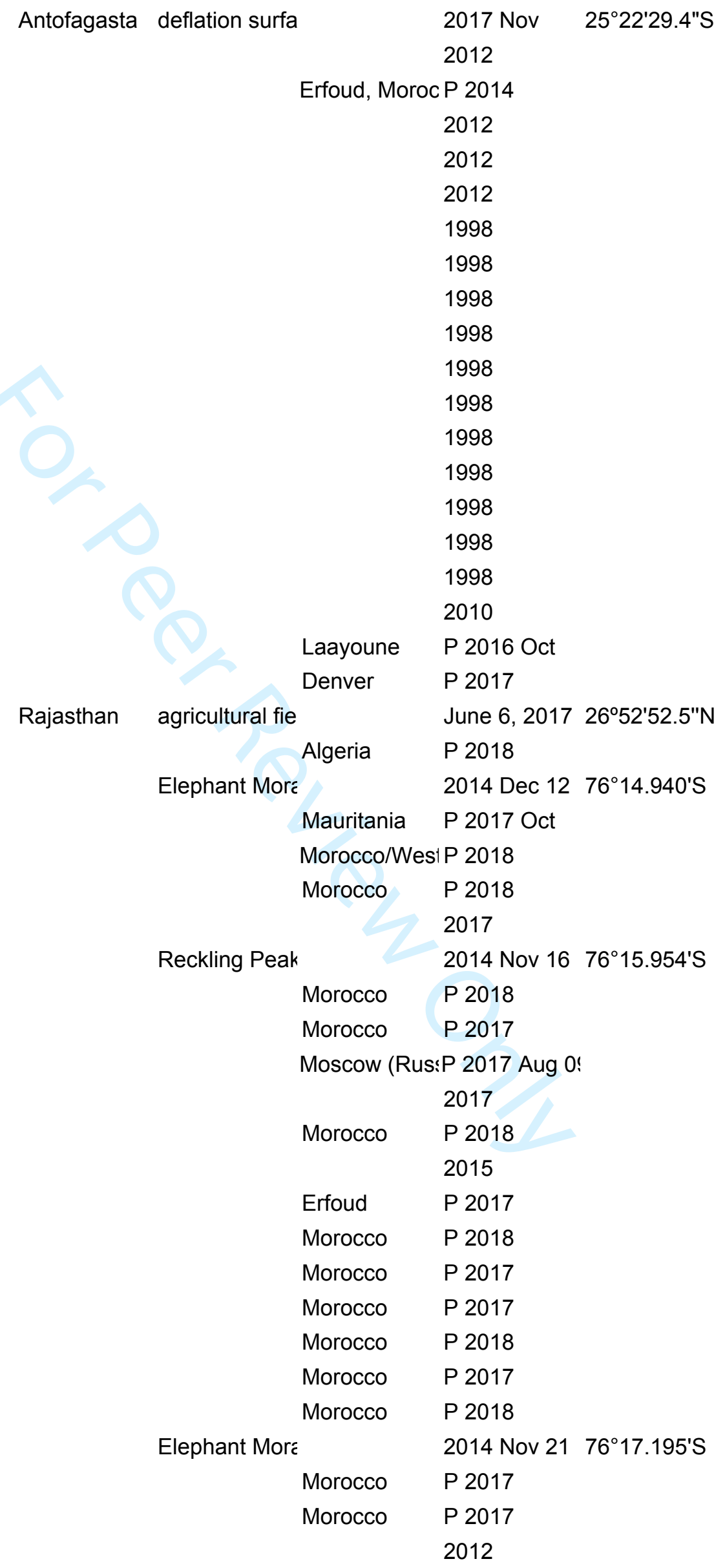




\begin{tabular}{|c|c|c|c|c|c|}
\hline 0000-00-00 & & & & 1998 & \\
\hline 0000-00-00 & & & & 2009 & \\
\hline 0000-00-00 & & & & 2009 & \\
\hline 0000-00-00 & & & Morocco & P 2017 Jun & \\
\hline 0000-00-00 & & & Mauritania & P 2017 Sep & \\
\hline 0000-00-00 & & & Erfoud, Mor & P 2008 & \\
\hline $0000-00-00$ & Antofagasta & deflation surfa & & 2016 Nov 04 & $\sim 24^{\circ} 51^{\prime} \mathrm{S}$ \\
\hline 0000-00-00 & & & Tinduf, Alge & eP 2017 Apr & \\
\hline 0000-00-00 & & & Beijing & 2016 & \\
\hline 0000-00-00 & Antofagasta & Desert & & 25 March 201 & $22^{\circ} 23.56^{\prime} \mathrm{S}$ \\
\hline 0000-00-00 & Antofagasta & deflation surfa & & 2017 May 17 & $\sim 24^{\circ} 51^{\prime} \mathrm{S}$ \\
\hline 0000-00-00 & & & Ensisheim, & P 2007 Jun & \\
\hline 0000-00-00 & & & Zagora (Mo & (P April 2018 & \\
\hline 0000-00-00 & & & Zagora, Mo & (P April 2018 & \\
\hline 0000-00-00 & Zufar & & Poland & P 2018 Feb & $18.888^{\circ} \mathrm{N}$ \\
\hline $0000-00-00$ & & & Laayoune, I & D April 2018 & \\
\hline $0000-00-00$ & & & Erfoud & P Aug 2001 & \\
\hline 0000-00-00 & & Desert & Ensisheim, & P 2017 & \\
\hline 0000-00-00 & & & Laayoune & P 2014 May & \\
\hline 0000-00-00 & & & Morocco & P 2018 & \\
\hline 0000-00-00 & & & Mauritania & P 2017 Jun & \\
\hline 0000-00-00 & & & Ensisheim, & P 2007 Jun & \\
\hline 0000-00-00 & & & Morocco & P 2018 & \\
\hline 0000-00-00 & & & Algeria & P 2018 & \\
\hline 0000-00-00 & & & Erfoud & P 2017 & \\
\hline 0000-00-00 & & & Mauritania & P May 2018 & \\
\hline 0000-00-00 & & & & 2017 & \\
\hline 0000-00-00 & & & & 2017 & \\
\hline 0000-00-00 & & & France & P 2016 Dec & \\
\hline 0000-00-00 & & & Temara & P 2018 Aug & \\
\hline 0000-00-00 & & & Morocco & P 2018 & \\
\hline 0000-00-00 & & & Erfoud, Mor & P 2017 & \\
\hline $0000-00-00$ & & & Morocco & P 2018 & \\
\hline 0000-00-00 & & & Rissani & P 2013 Feb & \\
\hline 0000-00-00 & & & Morocco & P 2018 Jun & \\
\hline 0000-00-00 & & & Tucson Gen & \& 2017 & \\
\hline 0000-00-00 & & & Ouarzatate, & A P 2018 Mar & \\
\hline 0000-00-00 & Antofagasta & deflation surfa & & 2018 March & $25^{\circ} 14^{\prime} \mathrm{S}$ \\
\hline $0000-00-00$ & & & Erfoud & P 2006 & \\
\hline $0000-00-00$ & & & Morocco & P 2018 & \\
\hline $0000-00-00$ & & & Zagora & P 2018 Mar & \\
\hline $0000-00-00$ & & & Mauritania & P May 2018 & \\
\hline 0000-00-00 & & & Erfoud, Mor & P 2013 & \\
\hline 0000-00-00 & & & Erfoud & P 2017 & \\
\hline 0000-00-00 & & & Erfoud & P 2017 Sep & \\
\hline
\end{tabular}




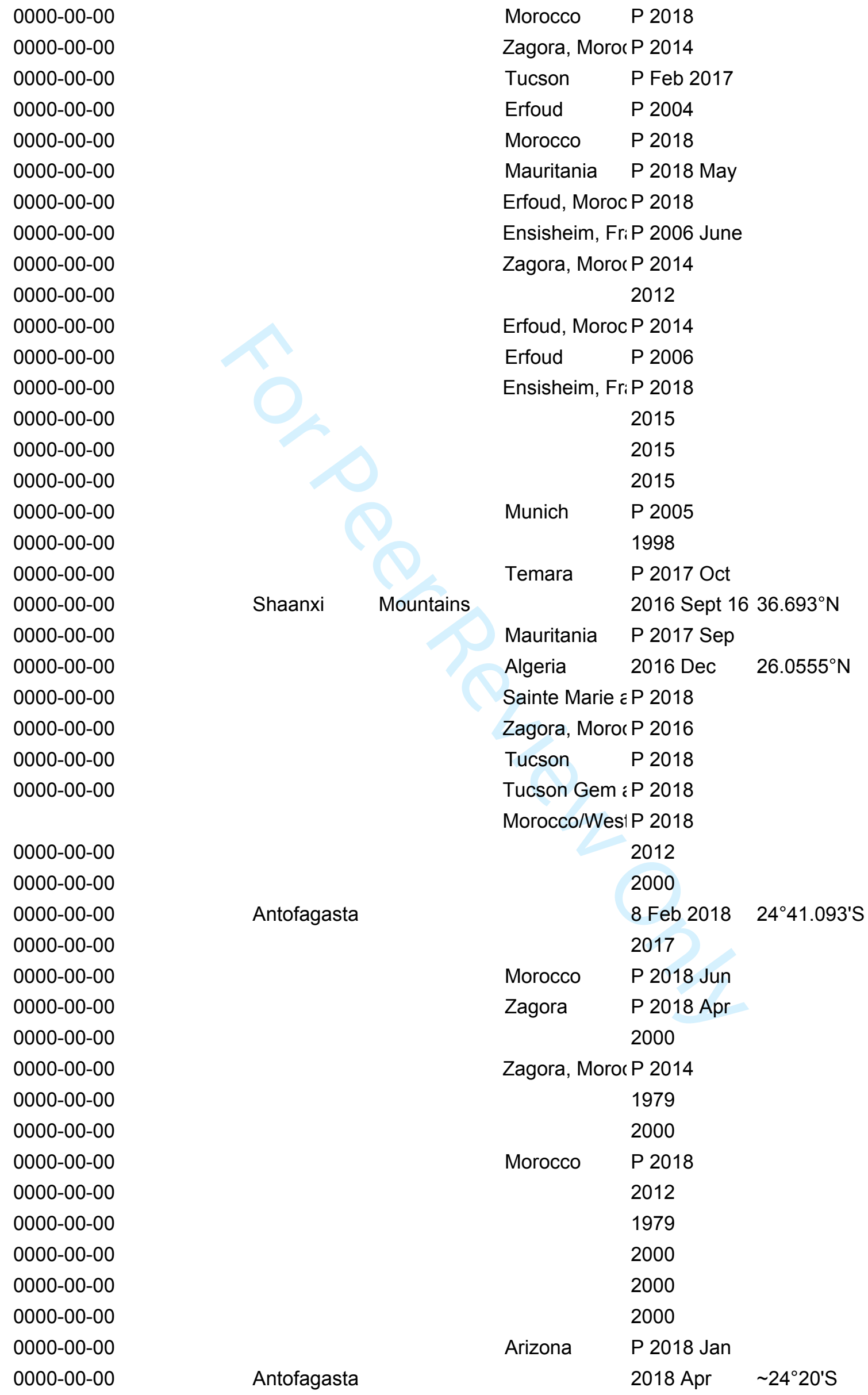




\begin{tabular}{|c|c|c|c|c|}
\hline 0000-00-00 & & & 2012 & \\
\hline $0000-00-00$ & Antofagasta & & 2018 Apr & $\sim 24^{\circ} 20^{\prime} \mathrm{S}$ \\
\hline 0000-00-00 & Antofagasta & & 2018 Apr & $24^{\circ} 51^{\prime} \mathrm{S}$ \\
\hline 0000-00-00 & & Morocco & P 2017 & \\
\hline 0000-00-00 & & Morocco & P 2017 & \\
\hline 0000-00-00 & & & 2012 & \\
\hline $0000-00-00$ & & & 2015 Jan 15 & $77^{\circ} 13.981 ' S$ \\
\hline 0000-00-00 & Morocco, Laa! & Genova & P May 2014 & \\
\hline 0000-00-00 & & Beijing Ch & P Feb. 2017 & \\
\hline 0000-00-00 & & Tucson G & P 2017 & \\
\hline 0000-00-00 & & Morocco & P 2016 May 1 & \\
\hline 0000-00-00 & Elephant Morć & & 2014 Dec 12 & $76^{\circ} 12.637^{\prime} \mathrm{S}$ \\
\hline 0000-00-00 & Elephant Morć & & 2014 Dec 12 & $76^{\circ} 15.926^{\prime} S$ \\
\hline 0000-00-00 & & Tucson & P 2016 & \\
\hline 0000-00-00 & Zufar & & 2014 & $18^{\circ} 34.398^{\prime} \mathrm{N}$ \\
\hline 0000-00-00 & & Tucson G & P 2017 & \\
\hline 0000-00-00 & Zufar & & 15 Feb 2015 & $18^{\circ} 45.30^{\prime} \mathrm{N}$ \\
\hline 0000-00-00 & & Munich, G & $P 2017$ & \\
\hline $0000-00-00$ & & Tucson G & P 2011 Feb & \\
\hline 0000-00-00 & & Laayoune & P 2018 & \\
\hline 0000-00-00 & & Erfoud, Mc & P 2018 & \\
\hline 0000-00-00 & & Morocco & P 2018 & \\
\hline 0000-00-00 & & Morocco & P 2017 & \\
\hline $0000-00-00$ & & & 2000 & \\
\hline 0000-00-00 & & Erfoud & P June 2016 & \\
\hline $0000-00-00$ & & Tucson & P 2017 Jan $2 \varsigma$ & \\
\hline 0000-00-00 & & Agadir, Mc & P 2018 & \\
\hline 0000-00-00 & & Tucson G & $P 2017$ & \\
\hline $0000-00-00$ & & internet & P 2016 Nov 16 & \\
\hline 0000-00-00 & & Morocco & P 2016 May 1 & \\
\hline $0000-00-00$ & & Morocco & P 2016 May & \\
\hline 0000-00-00 & & & 2012 & \\
\hline $0000-00-00$ & & Morocco & P 2016 May 1 & \\
\hline 0000-00-00 & & Morocco & P 2016 May 1 & \\
\hline 0000-00-00 & & Erfoud, Mc & P 2018 & \\
\hline 0000-00-00 & & Ensisheim & P 2018 & \\
\hline $0000-00-00$ & Santiago del EDepartment of & & 22 Jun 1931 & $28^{\circ} 56^{\prime} \mathrm{S}$ \\
\hline 0000-00-00 & & Layaoune & P 2018 & \\
\hline $0000-00-00$ & & & 2000 & \\
\hline $0000-00-00$ & & & 1988 & \\
\hline 0000-00-00 & & Morocco & P 2016 May 1 & \\
\hline $0000-00-00$ & & & 2012 & \\
\hline 0000-00-00 & & Morocco & P 2016 May 1 & \\
\hline 0000-00-00 & & & 2012 & \\
\hline 0000-00-00 & & Hamburg, & P 2007 Dec & \\
\hline
\end{tabular}


0000-00-00

0000-00-00

0000-00-00

0000-00-00

0000-00-00

0000-00-00

0000-00-00

0000-00-00

0000-00-00

0000-00-00

0000-00-00

0000-00-00

0000-00-00

0000-00-00

0000-00-00

0000-00-00

0000-00-00

0000-00-00

0000-00-00

0000-00-00

0000-00-00

0000-00-00

0000-00-00

2018-06-08 Reclassified

0000-00-00

0000-00-00

0000-00-00

0000-00-00

0000-00-00

0000-00-00

0000-00-00

0000-00-00

0000-00-00

0000-00-00

0000-00-00

0000-00-00

0000-00-00

0000-00-00

0000-00-00

0000-00-00

0000-00-00

0000-00-00

0000-00-00

0000-00-00

0000-00-00

$\begin{array}{ll}\text { Morocco } & \text { P 2016 May 1 } \\ \text { Tucson } & \text { P 2011 } \\ & 1988 \\ \text { Tucson } & \text { P 2011 } \\ \text { Morocco } & \text { P 2016 May 1 } \\ & 2012\end{array}$

Layaoune, Mo P 2018

2012

Morocco P 2016 May 1

Morocco P 2016 May 1

Morocco P 2016 May 1

1988

2000

1988

Morocco P 2016 May 1

2012

2000

2010

1998

1998

1998

Elephant Morc

Mauritania

2014 Dec 12 76¹5.492'S

Morocco P 2014 May

Ensisheim P 2014 Jun

Morocco P 2017 Aug

Tagant, Mauri P 2017

Zagora, Moroc P 2014

Munich, Germ P 2007

Zagora, Moroc P 2014

Tucson P 2016

Morocco P 2017

Rissani P 2015 Jan

Erfoud, Moroc P 2017

Morocco P 22 May 201

Quarzazate, N P 2016

Guelmim, Mor P 2018 Aug

Sainte-Marie-¿P 2005 Jun

Tucson P 2018 Jan

Erfoud 2004

Tucson P $2018 \mathrm{Feb}$

Morocco P 2018 Feb

Tucson P $2017 \mathrm{Feb}$

Morocco P 30 Aug 201!

Antofagasta

11 Feb $2018 \quad 22^{\circ} 26.27^{\prime} S$ 


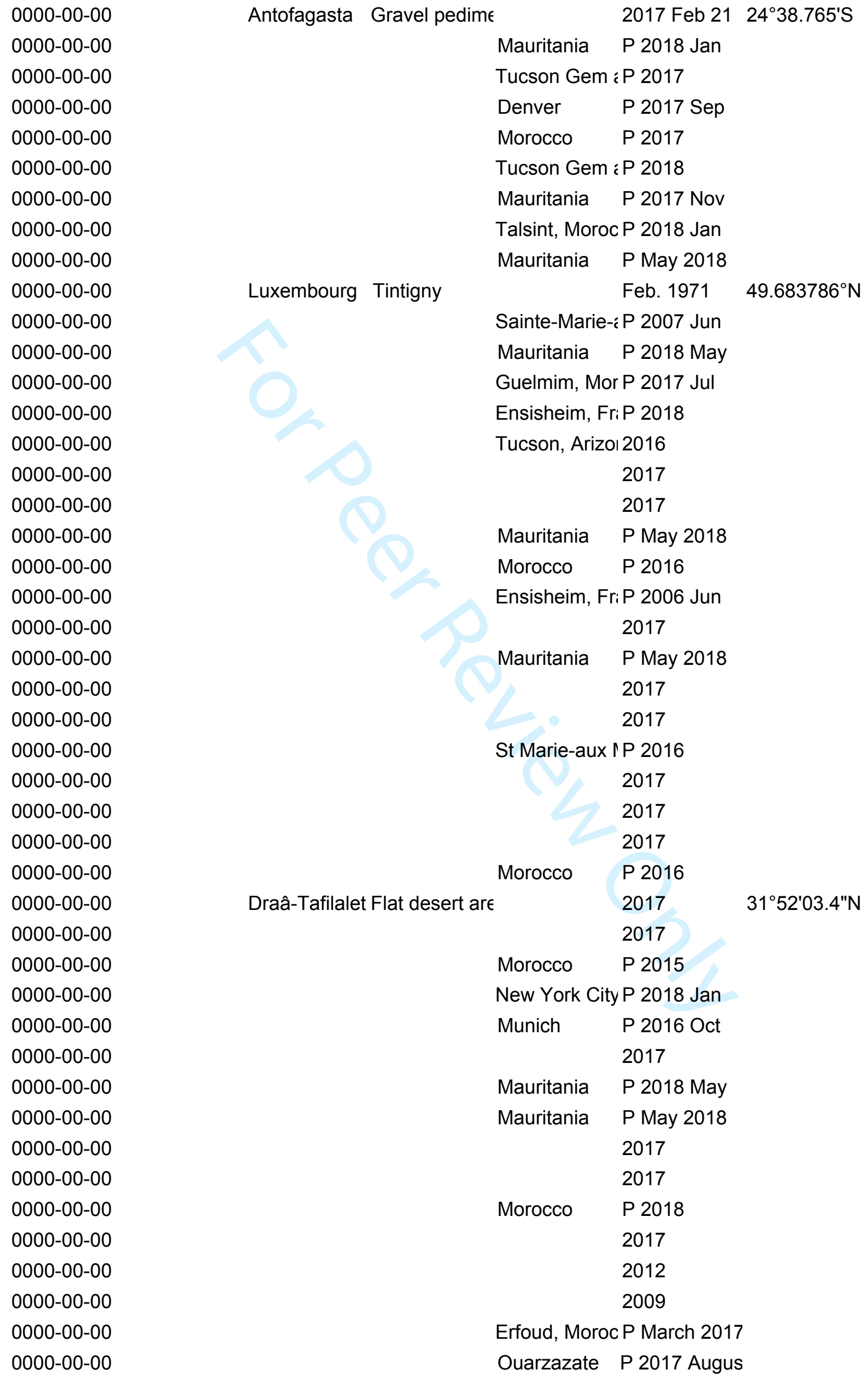




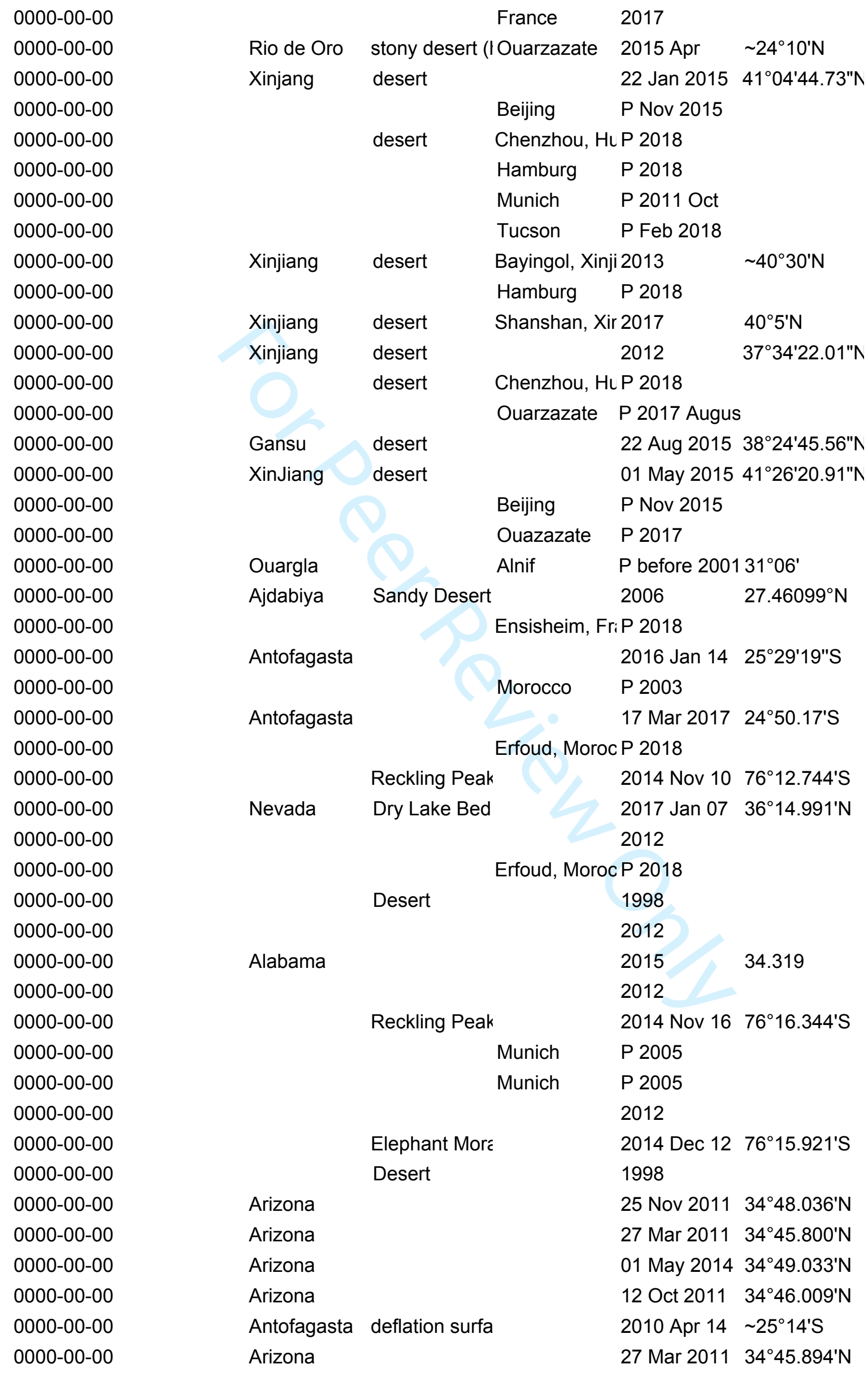




\begin{tabular}{|c|c|c|c|c|c|c|}
\hline 0000-00-00 & & Arizona & & & 23 Sep 2012 & $34^{\circ} 49.402^{\prime} \mathrm{N}$ \\
\hline $0000-00-00$ & & Arizona & & & 24 Nov 2011 & $34^{\circ} 49.281^{\prime} \mathrm{N}$ \\
\hline $0000-00-00$ & & & & & 2014 & \\
\hline $0000-00-00$ & & & & & 2014 & \\
\hline $0000-00-00$ & & & & Temara & P 2017 Nov & \\
\hline 0000-00-00 & & Antofagasta & plain & & 2018 Apr 5 & $22^{\circ} 29.931^{\prime} \mathrm{S}$ \\
\hline $0000-00-00$ & & & & Quartzite, & ZP 2006 & \\
\hline $0000-00-00$ & & Jarayan al $\mathrm{B}$ & Desert & & 13 Feb 2018 & $24^{\circ} 52.27^{\prime} \mathrm{N}$ \\
\hline 0000-00-00 & & & Desert & & 1998 & \\
\hline 0000-00-00 & & Antofagasta & deflation surfa & & 2017 Mar & $24^{\circ} 43^{\prime} 37.02^{\prime \prime S}$ \\
\hline $0000-00-00$ & & Ash Shamal & Desert & & 6 Feb 2018 & $25^{\circ} 58.36^{\prime} \mathrm{N}$ \\
\hline 2018-11-06 & Corrections tc & 6 & & CFMS sh & r 2017 & \\
\hline 0000-00-00 & & & & Ebay; S. & P Jan. 2017 & \\
\hline 0000-00-00 & & Antofagasta & & & 26 Feb 2016 & $25^{\circ} 00.560^{\prime} \mathrm{S}$ \\
\hline 0000-00-00 & & & & & 2012 & \\
\hline 0000-00-00 & & & & Morocco & P 2016 May 0 & \\
\hline 0000-00-00 & & Michigan & & & 2018 Jan 16 & $42^{\circ} 26.82^{\prime} \mathrm{N}$ \\
\hline $0000-00-00$ & & Antofagasta & granodiorite $p$ & & 2017 Feb 21 & $24^{\circ} 38^{\prime} 57.2^{\prime \prime S}$ \\
\hline 0000-00-00 & & & & Morocco & P 2016 May 0 & \\
\hline 0000-00-00 & & & & Erfoud, M & c P 2001 & \\
\hline 0000-00-00 & & & & Ensishein & P 2018 & \\
\hline 0000-00-00 & & & & & 2012 & \\
\hline 0000-00-00 & & & & Morocco & P 2016 May 0 & \\
\hline $0000-00-00$ & & & & Morocco & P 2003 & \\
\hline 0000-00-00 & & & & Erfoud, $\mathrm{M}$ & cP 2017 Feb & \\
\hline 0000-00-00 & & Ajdabiya & Arid Plateau & & 2006 & $28.17254^{\circ} \mathrm{N}$ \\
\hline 0000-00-00 & & & & & 2000 & \\
\hline 0000-00-00 & & QingHai & Qaidam Basin & & 20 Aug 2017 & $38.0713^{\circ} \mathrm{N}$ \\
\hline 0000-00-00 & & Antofagasta & & & 21 Feb 2017 & $24^{\circ} 42.610^{\prime} \mathrm{S}$ \\
\hline 0000-00-00 & & & & Morocco & P 2003 & \\
\hline 0000-00-00 & & & & Morocco & P 2016 May 0 & \\
\hline 0000-00-00 & & & & & 2012 & \\
\hline 0000-00-00 & & & & Morocco & P 2003 & \\
\hline 0000-00-00 & & & & Morocco & P 2016 May 0 & \\
\hline 0000-00-00 & & & & & 2014 & \\
\hline 0000-00-00 & & Ajdabiya & Arid Plateau & & 2006 & $28.25716^{\circ} \mathrm{N}$ \\
\hline 0000-00-00 & & & & Bologna & P March 2016 & \\
\hline 0000-00-00 & & Antofagasta & & & 17 March 201 & $22^{\circ} 26.38^{\prime} S$ \\
\hline 0000-00-00 & & & & & 2012 & \\
\hline 0000-00-00 & & & & Morocco & P 2016 May 0 & \\
\hline $0000-00-00$ & & & & Morocco & P 2016 May 0 & \\
\hline 0000-00-00 & & & & Sainte $\mathrm{Ma}$ & є P 2018 & \\
\hline 0000-00-00 & & & & & 2012 & \\
\hline $0000-00-00$ & & & & Morocco & P 2016 May 0 & \\
\hline $0000-00-00$ & & & & Morocco & P 2016 May 0 & \\
\hline
\end{tabular}


0000-00-00

0000-00-00

0000-00-00

0000-00-00

0000-00-00

0000-00-00

0000-00-00

0000-00-00

0000-00-00

0000-00-00

0000-00-00

0000-00-00

0000-00-00

0000-00-00

0000-00-00

0000-00-00

0000-00-00

0000-00-00

0000-00-00

0000-00-00

0000-00-00

0000-00-00

0000-00-00

0000-00-00

0000-00-00

0000-00-00

0000-00-00

0000-00-00

0000-00-00

0000-00-00

0000-00-00

0000-00-00

0000-00-00

0000-00-00

0000-00-00

0000-00-00

0000-00-00

0000-00-00

0000-00-00

0000-00-00

0000-00-00

0000-00-00

0000-00-00

0000-00-00

0000-00-00

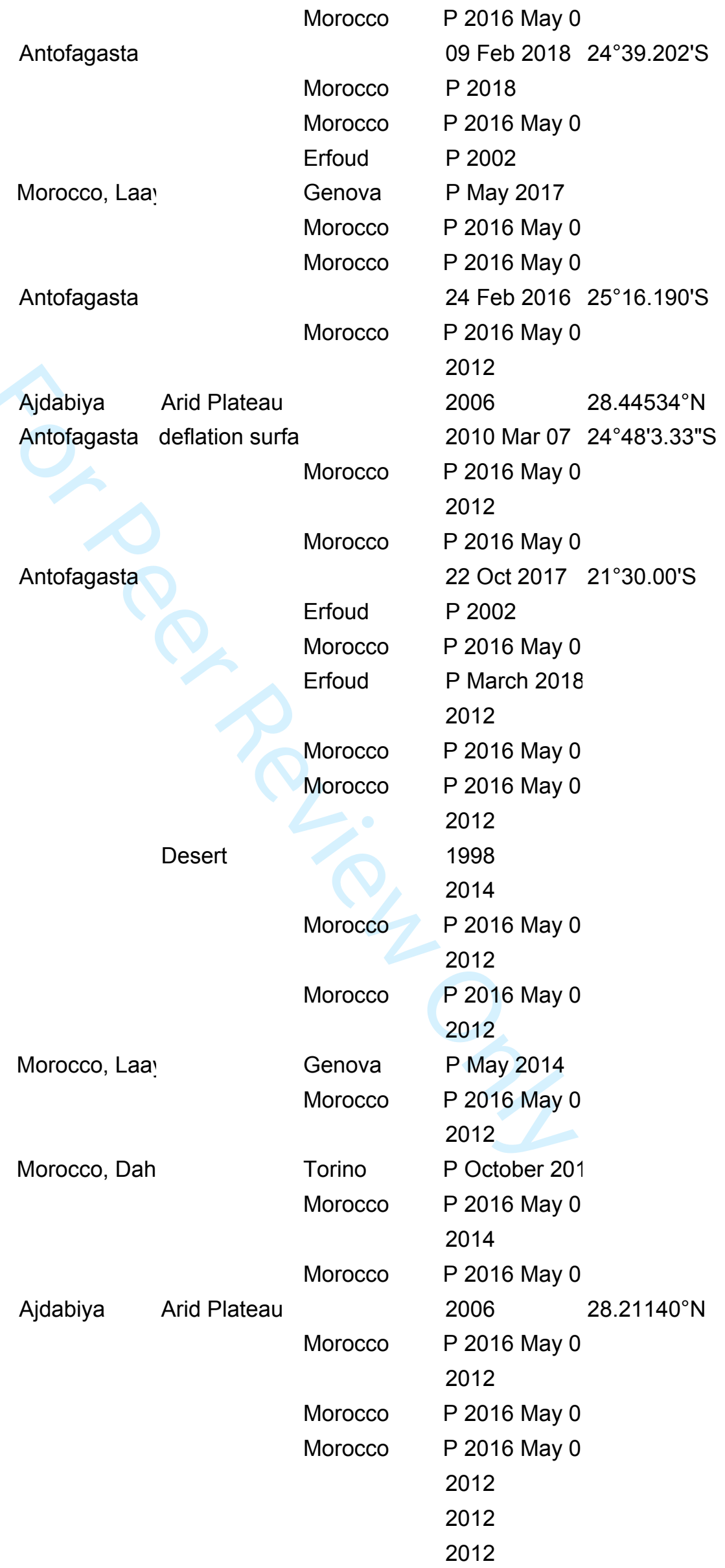




\begin{tabular}{|c|c|c|c|}
\hline $0000-00-00$ & & & 2012 \\
\hline 0000-00-00 & & Erfoud & P 2002 \\
\hline 0000-00-00 & & & 2012 \\
\hline 0000-00-00 & & & 2012 \\
\hline 0000-00-00 & & Morocco & P 2016 May 0 \\
\hline $0000-00-00$ & & & 2012 \\
\hline 0000-00-00 & & & 2000 \\
\hline 0000-00-00 & & & 2012 \\
\hline 0000-00-00 & & Bessey & P 2012 \\
\hline 0000-00-00 & & & 2012 \\
\hline 0000-00-00 & & & 2012 \\
\hline 0000-00-00 & & Verona & P 2016 \\
\hline 0000-00-00 & & Morocco & P 2016 May 0 \\
\hline 0000-00-00 & & Morocco & P 2016 May 0 \\
\hline 0000-00-00 & & & 2012 \\
\hline 0000-00-00 & & Morocco & P 2016 May 0 \\
\hline 0000-00-00 & & & 2012 \\
\hline $0000-00-00$ & & Morocco & P 2016 May 0 \\
\hline $0000-00-00$ & & & 2012 \\
\hline 0000-00-00 & & & 2012 \\
\hline 0000-00-00 & & & 2014 \\
\hline 0000-00-00 & & & 2014 \\
\hline 0000-00-00 & & & 1974 \\
\hline 0000-00-00 & & & 2014 \\
\hline 0000-00-00 & & & 2014 \\
\hline $0000-00-00$ & & & 2012 \\
\hline $0000-00-00$ & & & 2012 \\
\hline $0000-00-00$ & & & 2012 \\
\hline 0000-00-00 & & Morocco & P 2016 May 0 \\
\hline 0000-00-00 & & & 2000 \\
\hline 0000-00-00 & & & 2012 \\
\hline 0000-00-00 & & & 2012 \\
\hline $0000-00-00$ & & & 2000 \\
\hline 0000-00-00 & & & 2012 \\
\hline $0000-00-00$ & & & 2014 \\
\hline 0000-00-00 & & & 2000 \\
\hline 0000-00-00 & & & 2012 \\
\hline 0000-00-00 & Antofagasta & & 21 Mar $201722^{\circ} 21.63^{\prime S}$ \\
\hline $0000-00-00$ & & & 2012 \\
\hline 0000-00-00 & & & 2012 \\
\hline $0000-00-00$ & & & 2012 \\
\hline $0000-00-00$ & & & 1979 \\
\hline 0000-00-00 & & & 2014 \\
\hline $0000-00-00$ & & & 2000 \\
\hline 0000-00-00 & & & 2009 \\
\hline
\end{tabular}


0000-00-00

2009

$0000-00-00 \quad 2009$

$0000-00-00 \quad 2009$

0000-00-00 2009

0000-00-00 2009

0000-00-00 2009

0000-00-00 2009

0000-00-00 2009

0000-00-00 2009

$0000-00-00 \quad 2009$

0000-00-00 2009

0000-00-00 2009

$0000-00-00 \quad 2009$

0000-00-00 2009

0000-00-00

Marrakech P May 2014

2000

2000

0000-00-00

2000

0000-00-00

2000

0000-00-00

2000

0000-00-00 2000

$0000-00-00 \quad 2009$

$0000-00-00 \quad 2000$

0000-00-00 2009

$0000-00-00 \quad 2000$

$0000-00-00 \quad 2000$

$0000-00-00 \quad 2000$

$0000-00-00 \quad 2009$

0000-00-00 2012

0000-00-00 2009

$0000-00-00 \quad 2000$

0000-00-00 2009

$0000-00-00 \quad 2000$

$0000-00-00 \quad 2000$

$0000-00-00 \quad 2000$

$0000-00-00 \quad 2000$

$0000-00-00 \quad 2009$

0000-00-00 2000

0000-00-00 2009

0000-00-00 2000

$0000-00-00 \quad 2000$

$0000-00-00 \quad 2009$

$0000-00-00 \quad 2000$

$0000-00-00 \quad 2009$

0000-00-00 2000 


\begin{tabular}{|c|c|}
\hline $0000-00-00$ & 2000 \\
\hline 0000-00-00 & 2009 \\
\hline 0000-00-00 & 2009 \\
\hline $0000-00-00$ & 2000 \\
\hline $0000-00-00$ & 2000 \\
\hline 0000-00-00 & 2009 \\
\hline $0000-00-00$ & 2000 \\
\hline $0000-00-00$ & 2009 \\
\hline $0000-00-00$ & 2009 \\
\hline $0000-00-00$ & 2009 \\
\hline $0000-00-00$ & 2000 \\
\hline $0000-00-00$ & 2000 \\
\hline $0000-00-00$ & 2009 \\
\hline $0000-00-00$ & 2000 \\
\hline 0000-00-00 & 2009 \\
\hline $0000-00-00$ & 2009 \\
\hline $0000-00-00$ & 2000 \\
\hline $0000-00-00$ & 2009 \\
\hline 0000-00-00 & 2009 \\
\hline $0000-00-00$ & 2009 \\
\hline 0000-00-00 & 2009 \\
\hline 0000-00-00 & 2000 \\
\hline $0000-00-00$ & 2009 \\
\hline $0000-00-00$ & 2009 \\
\hline 0000-00-00 & 2009 \\
\hline $0000-00-00$ & 2000 \\
\hline $0000-00-00$ & 2000 \\
\hline 0000-00-00 & 2000 \\
\hline $0000-00-00$ & 2009 \\
\hline $0000-00-00$ & 2009 \\
\hline $0000-00-00$ & 2009 \\
\hline 0000-00-00 & 2000 \\
\hline $0000-00-00$ & 2000 \\
\hline $0000-00-00$ & 2000 \\
\hline 0000-00-00 & 2000 \\
\hline 0000-00-00 & 2009 \\
\hline $0000-00-00$ & 2009 \\
\hline 0000-00-00 & 2009 \\
\hline 0000-00-00 & 2000 \\
\hline $0000-00-00$ & 2009 \\
\hline $0000-00-00$ & 2009 \\
\hline 0000-00-00 & 2009 \\
\hline $0000-00-00$ & 2009 \\
\hline $0000-00-00$ & 2009 \\
\hline $0000-00-00$ & 2009 \\
\hline
\end{tabular}


0000-00-00

2009

0000-00-00 2009

$0000-00-00 \quad 2000$

0000-00-00 2009

0000-00-00 2009

0000-00-00 2009

0000-00-00 2009

$0000-00-00 \quad 2009$

0000-00-00 2009

0000-00-00 2009

0000-00-00 2009

0000-00-00 2009

$0000-00-00 \quad 2009$

$0000-00-00 \quad 2009$

0000-00-00 2009

0000-00-00 2009

$0000-00-00 \quad 2009$

0000-00-00 2009

$0000-00-00 \quad 2009$

$0000-00-00 \quad 2009$

0000-00-00 2009

$0000-00-00 \quad 2009$

$0000-00-00 \quad 2009$

$0000-00-00 \quad 2009$

$0000-00-00 \quad 2009$

0000-00-00 2009

0000-00-00 2009

$0000-00-00 \quad 2009$

0000-00-00 2009

$0000-00-00 \quad 2009$

$0000-00-00 \quad 2009$

0000-00-00 2009

$0000-00-00 \quad 2009$

$0000-00-00 \quad 2009$

0000-00-00 2000

$0000-00-00 \quad 2009$

$0000-00-00 \quad 2009$

0000-00-00 2009

$0000-00-00 \quad 2009$

0000-00-00 2009

0000-00-00 2010

$0000-00-00 \quad 2009$

0000-00-00 2015

0000-00-00 2009

0000-00-00 2010 


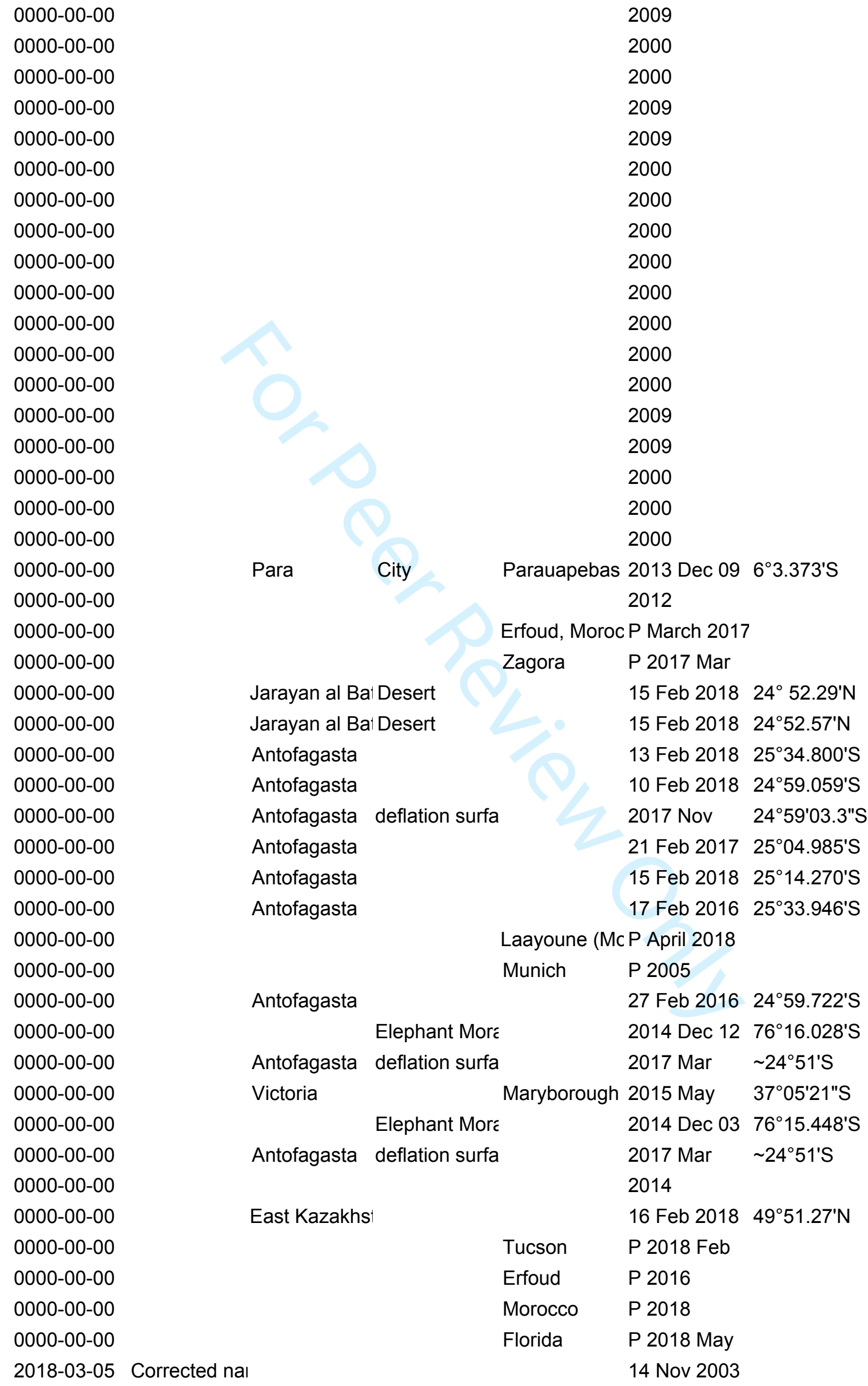


0000-00-00

0000-00-00

0000-00-00

0000-00-00

0000-00-00

0000-00-00

0000-00-00

0000-00-00

0000-00-00

0000-00-00

0000-00-00

0000-00-00

0000-00-00

0000-00-00

0000-00-00

0000-00-00

0000-00-00

0000-00-00

0000-00-00

0000-00-00

0000-00-00

0000-00-00

0000-00-00

0000-00-00

0000-00-00

0000-00-00

0000-00-00

0000-00-00

0000-00-00

0000-00-00

0000-00-00

0000-00-00

0000-00-00

0000-00-00

0000-00-00

0000-00-00

0000-00-00

0000-00-00

0000-00-00

0000-00-00

0000-00-00

0000-00-00

0000-00-00

0000-00-00

0000-00-00

Changsha, Cr unknown

\begin{tabular}{|c|c|c|c|}
\hline Antofagasta & granodiorite $p_{1}$ & 2017 Feb 21 & $24^{\circ} 40^{\prime} 05.3^{\prime \prime S}$ \\
\hline & Morocco & P 2018 Apr & \\
\hline & Zagora & P 2016 & \\
\hline Xinjiang & a sigle piece v Beijing & 2013 Oct 12 & $42^{\circ} 08^{\prime} 4.55^{\prime \prime} \mathrm{N}$ \\
\hline & Erfoud, $\mathrm{M}$ & c P 2017 Feb & \\
\hline Antofagasta & & 10 Apr 2018 & $22^{\circ} 29.79^{\prime} \mathrm{S}$ \\
\hline & Morocco & P 2017 & \\
\hline & Erfoud, $\mathrm{M}$ & c P 2017 Feb & \\
\hline & Erfoud, $\mathrm{M}$ & c P 2003 & \\
\hline & Erfoud, M & c P 2018 & \\
\hline & & 2000 & \\
\hline Antofagasta & deflation surfa & 2012 July 7 & $\sim 24^{\circ} 41^{\prime} S$ \\
\hline & Morocco, & giP June 2013 & \\
\hline Morocco, Dah & Torino & P October 201 & \\
\hline & Morocco & P 2016 May 0 & \\
\hline Antofagasta & & 21 March 201 & $22^{\circ} 26.44 ' S$ \\
\hline & Morocco & P 2016 May 0 & \\
\hline Antofagasta & deflation surfa & 2017 Nov & $24^{\circ} 34^{\prime} 00.6^{\prime \prime S}$ \\
\hline Antofagasta & & 2017 Nov & $24^{\circ} 41^{\prime} \mathrm{S}$ \\
\hline & Morocco & P 2003 & \\
\hline & & 2012 & \\
\hline & & P 2017 & \\
\hline & & 2015 & \\
\hline & Morocco & P 2016 May 0 & \\
\hline tofagasta & & 31 Mar 2018 & $22^{\circ} 30.59^{\prime} S$ \\
\hline
\end{tabular}

Erfoud, Moroc P 2017 Feb

Zagora (Moror P April 2018

$\begin{aligned} & \text { Nevada } \text { Dry Lake Bed } \\ & \text { Elephant More }\end{aligned}$

Antofagasta

Morocco, Dah

Antofagasta

Antofagasta

Ajdabiya Arid Plateau
Antofagasta
Jarayan al Bat Desert
Antofagasta
Antofagasta deflation surfa

2017 Jan $6 \quad 36^{\circ} 14.06^{\prime} \mathrm{N}$

2014 Dec 12 76¹3.852'S

27 Feb $2016 \quad 24^{\circ} 37.727^{\prime} \mathrm{S}$

$\begin{array}{ll}\text { Genova } & \text { P May } 2017 \\ \text { Morocco } & \text { P 2016 May } 0\end{array}$

16 Mar 2017 2443.64'S

2012

28 Feb 2016 243․689'S

Morocco P 2016 May 0

Morocco P 2016 May 0

2006

$28.28916^{\circ} \mathrm{N}$

2012

6 Apr $2018 \quad 22^{\circ} 29.47^{\prime} \mathrm{S}$

06 March 201:24 $53.30^{\prime} \mathrm{N}$

10 Feb $201824^{\circ} 46.928^{\prime} S$

2015 Jan $03 \sim 24^{\circ} 51^{\prime} S$

Bologna P March 2016 


\begin{tabular}{|c|c|c|c|c|}
\hline 0000-00-00 & Rio Grande dc sand & \multirow[b]{2}{*}{ Bologna } & 2004 & $33^{\circ} 39.07^{\prime} \mathrm{S}$ \\
\hline 0000-00-00 & & & \multicolumn{2}{|c|}{ P March 2016} \\
\hline 0000-00-00 & \multicolumn{2}{|c|}{ deflation surfa } & $2010 \mathrm{Jul} 05$ & $\sim 25^{\circ} 14^{\prime} S$ \\
\hline $0000-00-00$ & \multicolumn{2}{|l|}{ deflation surfa } & 2017 Nov 13 & $\sim 25^{\circ} 14^{\prime} S$ \\
\hline 0000-00-00 & \multicolumn{2}{|l|}{ deflation surfa } & 2011 Nov 12 & $\sim 24^{\circ} 51^{\prime} \mathrm{S}$ \\
\hline 0000-00-00 & \multirow[t]{2}{*}{ Morocco, Dah } & Bologna & P March 2016 & \\
\hline 0000-00-00 & & & 2012 & \\
\hline 0000-00-00 & \multicolumn{2}{|l|}{ Antofagasta } & 19 Nov 2017 & $22^{\circ} 26.02^{\prime} S$ \\
\hline 0000-00-00 & & Morocco & P 2016 May 0 & \\
\hline 0000-00-00 & & Morocco & P 2016 May 0 & \\
\hline 0000-00-00 & \multicolumn{2}{|l|}{ Antofagasta } & 16 Mar 2017 & $24^{\circ} 42.15^{\prime} S$ \\
\hline 0000-00-00 & & Bologna & P March 2016 & \\
\hline 0000-00-00 & & \multicolumn{3}{|c|}{ Erfoud, Moroc P 2017 Feb } \\
\hline 0000-00-00 & & & 2012 & \\
\hline 0000-00-00 & & & 2014 & \\
\hline 0000-00-00 & Antofagasta deflation surfa & deflation surfa & 2011 Nov 12 & $\sim 25^{\circ} 14^{\prime} \mathrm{S}$ \\
\hline 0000-00-00 & \multirow[t]{3}{*}{ Antofagasta } & & 27 Feb 2016 & $24^{\circ} 41.635^{\prime} \mathrm{S}$ \\
\hline 0000-00-00 & & Morocco & P 2016 May 0 & \\
\hline 0000-00-00 & & Morocco & P 2016 May 0 & \\
\hline 0000-00-00 & \multicolumn{2}{|c|}{ Antofagasta deflation surfa } & 2017 Jun 19 & $24^{\circ} 49^{\prime} 02.1^{\prime \prime S}$ \\
\hline 0000-00-00 & & Morocco & P 2016 May 0 & \\
\hline 0000-00-00 & \multicolumn{4}{|c|}{ Laayoune (Mc P April 2018} \\
\hline 0000-00-00 & \multirow{2}{*}{ Arid Plateau } & & 2006 & $28.30210^{\circ} \mathrm{N}$ \\
\hline 0000-00-00 & & Morocco & P 2016 May 0 & \\
\hline 0000-00-00 & \multirow[t]{8}{*}{ Antofagasta } & & 21 Oct 2016 & $21^{\circ} 35.80^{\prime} S$ \\
\hline 0000-00-00 & & Morocco & P 2016 May 0 & \\
\hline 0000-00-00 & & Morocco & P 2016 May 0 & \\
\hline 0000-00-00 & & Morocco & P 2016 May 0 & \\
\hline 0000-00-00 & & Morocco & P 2016 May 0 & \\
\hline 0000-00-00 & & Morocco & P 2016 May 0 & \\
\hline 0000-00-00 & & & 2012 & \\
\hline 0000-00-00 & & Morocco & P 2016 May 0 & \\
\hline 0000-00-00 & Antofagasta & & 16 Mar 2017 & $24^{\circ} 55.18^{\prime} \mathrm{S}$ \\
\hline 0000-00-00 & Kerman & & 02 April 2016 & $31^{\circ} 58.18^{\prime} \mathrm{N}$ \\
\hline 0000-00-00 & Kerman & & 01 April 2016 & $31^{\circ} 52.29^{\prime} \mathrm{N}$ \\
\hline 0000-00-00 & Antofagasta & & 22 Feb 2017 & $24^{\circ} 41.570^{\prime} \mathrm{S}$ \\
\hline 0000-00-00 & \multicolumn{2}{|l|}{ deflation surfa } & 2014 Dec 19 & $\sim 25^{\circ} 14^{\prime} \mathrm{S}$ \\
\hline 0000-00-00 & & Bologna & P March 2016 & \\
\hline 0000-00-00 & \multicolumn{4}{|c|}{ Tucson, ArizoıP February 20} \\
\hline 0000-00-00 & \multicolumn{2}{|l|}{ Antofagasta } & 31 Mar 2018 & $22^{\circ} 30.66^{\prime} \mathrm{S}$ \\
\hline 0000-00-00 & \multicolumn{2}{|l|}{ Antofagasta } & 17 Feb 2017 & $24^{\circ} 56.893^{\prime} \mathrm{S}$ \\
\hline 0000-00-00 & Antofagasta & & 27 Feb 2016 & $24^{\circ} 40.200^{\prime} \mathrm{S}$ \\
\hline 0000-00-00 & & Bologna & P March 2016 & \\
\hline $0000-00-00$ & Morocco, Laa! & Bologna & P March 2016 & \\
\hline $0000-00-00$ & Desert plain & & 12.02 .17 & $20^{\circ} 20.555^{\prime} \mathrm{N}$ \\
\hline
\end{tabular}




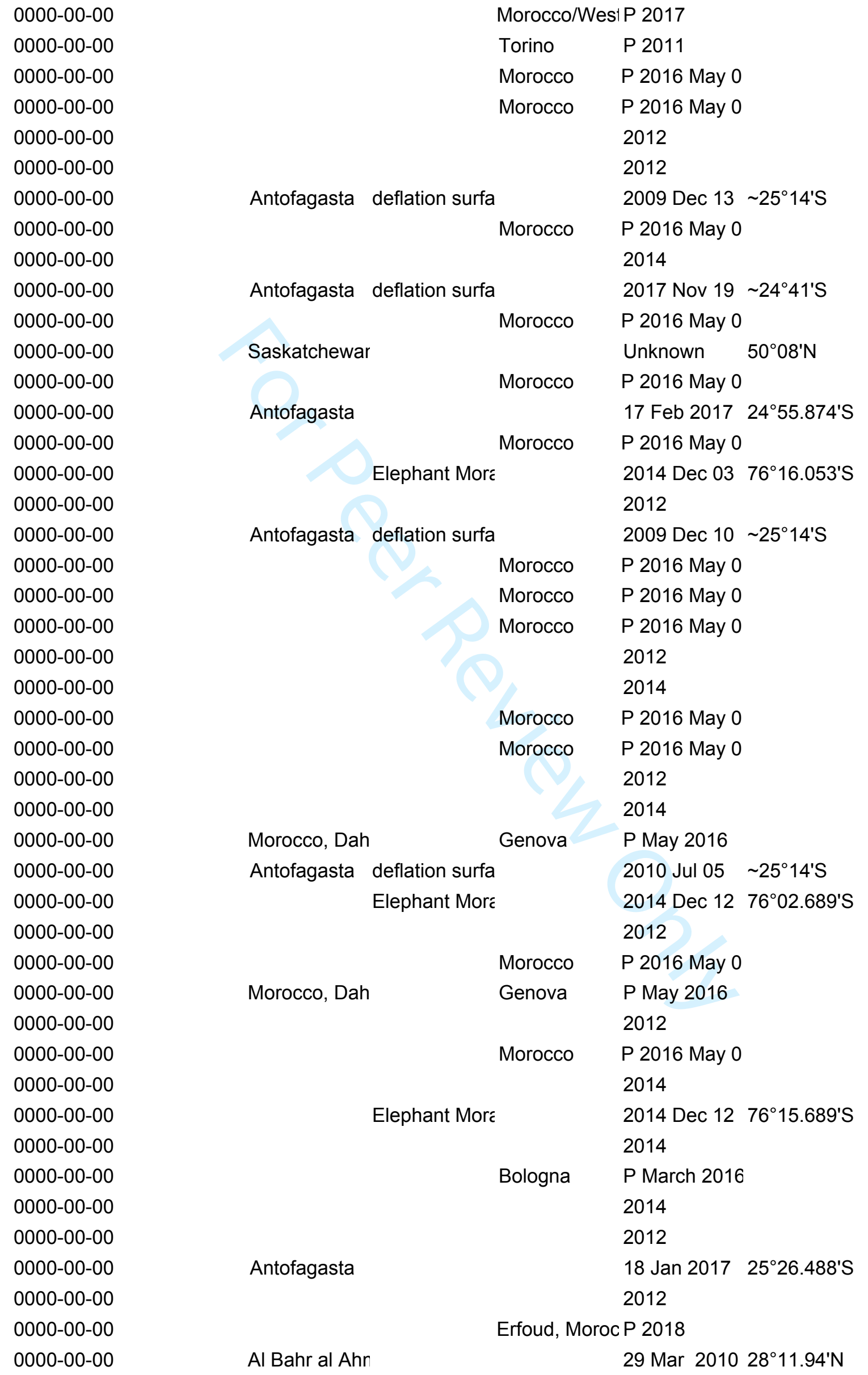




\begin{tabular}{|c|c|c|c|c|c|}
\hline 0000-00-00 & & & & \multicolumn{2}{|c|}{2017 July $1754^{\circ} 59^{\prime} 24^{\prime \prime N}$} \\
\hline 0000-00-00 & & & Morocco & \multicolumn{2}{|c|}{ P 2016 May 0} \\
\hline 0000-00-00 & & & Morocco & \multicolumn{2}{|l|}{ P 2016 May 0} \\
\hline 0000-00-00 & & & & \multicolumn{2}{|l|}{2012} \\
\hline 0000-00-00 & Antofagasta & & & 14 Feb 2018 & $25^{\circ} 29.963^{\prime} \mathrm{S}$ \\
\hline 0000-00-00 & Kerman & & Bologna & \multicolumn{2}{|c|}{ P March $201630^{\circ} 23.99^{\prime} \mathrm{N}$} \\
\hline 0000-00-00 & & & & \multicolumn{2}{|c|}{2016} \\
\hline 0000-00-00 & & & Morocco & \multicolumn{2}{|l|}{ P 2016 May 0} \\
\hline 0000-00-00 & Antofagasta & \multicolumn{2}{|c|}{ deflation surfa } & & $\sim 25^{\circ} 14^{\prime} S$ \\
\hline 0000-00-00 & Antofagasta & \multicolumn{2}{|l|}{ deflation surfa } & 2010 Feb 16 & $25^{\circ} 18^{\prime} 43.31 " \mathrm{~S}$ \\
\hline $0000-00-00$ & Al Bahr al Ahn & & & 27 Mar 2010 & $28^{\circ} 13.23^{\prime} \mathrm{N}$ \\
\hline 0000-00-00 & Antofagasta & & & \multicolumn{2}{|c|}{10 Apr $201822^{\circ} 30.15^{\prime} S$} \\
\hline 0000-00-00 & & & & \multicolumn{2}{|l|}{2014} \\
\hline 0000-00-00 & & & Erfoud, $\mathrm{M}$ & \multicolumn{2}{|l|}{$P 2018$} \\
\hline 0000-00-00 & & & Morocco & \multicolumn{2}{|l|}{ P 2016 May 0} \\
\hline 0000-00-00 & & & & \multicolumn{2}{|l|}{2012} \\
\hline 0000-00-00 & & & Morocco & \multicolumn{2}{|l|}{ P 2016 May 0} \\
\hline 0000-00-00 & Antofagasta & & & 09 Feb 2018 & $24^{\circ} 40.875^{\prime} \mathrm{S}$ \\
\hline 0000-00-00 & & & & \multicolumn{2}{|c|}{2012} \\
\hline 0000-00-00 & Antofagasta & deflation surfa & & 2010 Jul 04 & $\sim 25^{\circ} 14^{\prime} \mathrm{S}$ \\
\hline 0000-00-00 & & & E-bay & \multicolumn{2}{|l|}{ P 2013} \\
\hline 0000-00-00 & \multicolumn{3}{|c|}{ Elephant Morć } & 2014 Dec 12 & $76^{\circ} 15.759^{\prime} S$ \\
\hline 0000-00-00 & & & Bologna & \multicolumn{2}{|l|}{ P $3 / 2015$} \\
\hline 0000-00-00 & & & & \multicolumn{2}{|l|}{1974} \\
\hline 0000-00-00 & & & & \multicolumn{2}{|l|}{2012} \\
\hline 0000-00-00 & Al Bahr al Ahn & & & 26 Feb 2010 & $28^{\circ} 12.94^{\prime} \mathrm{N}$ \\
\hline 0000-00-00 & & & Morocco & P 2016 May 0 & \\
\hline 0000-00-00 & & & & 2012 & \\
\hline 0000-00-00 & & & & 2012 & \\
\hline 0000-00-00 & & & E-bay & P 2013 & \\
\hline 0000-00-00 & Antofagasta & & & 17 Feb 2017 & $24^{\circ} 57.191^{\prime} \mathrm{S}$ \\
\hline 0000-00-00 & & & & 2014 & \\
\hline 0000-00-00 & Morocco, Sme & & Bologna & P March 2016 & \\
\hline 0000-00-00 & Antofagasta & deflation surfa & & 2014 Dec 20 & $\sim 25^{\circ} 14^{\prime} \mathrm{S}$ \\
\hline 0000-00-00 & & & & 2014 & \\
\hline 0000-00-00 & & & & 2014 & \\
\hline 0000-00-00 & & & Bologna & P March 2016 & \\
\hline 0000-00-00 & & & Morocco & P 2016 May 0 & \\
\hline 0000-00-00 & & & Morocco & P 2016 May 0 & \\
\hline 0000-00-00 & & & Morocco & P 2016 May 0 & \\
\hline 0000-00-00 & & Elephant Morć & & 2014 Nov 16 & $76^{\circ} 17.133^{\prime} S$ \\
\hline 0000-00-00 & Antofagasta & deflation surfa & & 2014 & $\sim 24^{\circ} 41^{\prime} \mathrm{S}$ \\
\hline 0000-00-00 & & & Morocco & P 2016 May 0 & \\
\hline 0000-00-00 & & & Morocco & P 2016 May 0 & \\
\hline 0000-00-00 & & & & 2014 & \\
\hline
\end{tabular}


0000-00-00

0000-00-00

0000-00-00

0000-00-00

0000-00-00

0000-00-00

0000-00-00

0000-00-00

0000-00-00

0000-00-00

0000-00-00

0000-00-00

0000-00-00

0000-00-00

0000-00-00

0000-00-00

0000-00-00

0000-00-00

0000-00-00

0000-00-00

0000-00-00

0000-00-00

0000-00-00

0000-00-00

0000-00-00

0000-00-00

0000-00-00

0000-00-00

0000-00-00

0000-00-00

0000-00-00

0000-00-00

0000-00-00

0000-00-00

0000-00-00

0000-00-00

0000-00-00

0000-00-00

0000-00-00

0000-00-00

0000-00-00

0000-00-00

0000-00-00

0000-00-00

0000-00-00

\begin{tabular}{|c|c|c|c|}
\hline & & Zagora & P 2018 \\
\hline & & & 2012 \\
\hline & & & 2012 \\
\hline & & & 2012 \\
\hline & & Morocco & P 2016 May 0 \\
\hline & & & 2014 \\
\hline & & & 2012 \\
\hline & Elephant Moré & & 2014 Dec 12 76¹5.654'S \\
\hline & & & 2012 \\
\hline & & Tucson (Said & P Jan 2010 \\
\hline & & Morocco & P 2016 May 0 \\
\hline Antofagasta & deflation surfa & & 2010 Jul $04 \quad \sim 25^{\circ} 14$ 'S \\
\hline & & & 2012 \\
\hline & & Morocco & P 2016 May 0 \\
\hline & & & 2015 \\
\hline & & & 2015 \\
\hline Arizona & dry lake & & 2005 Sep $0335^{\circ} 39.05^{\prime} \mathrm{N}$ \\
\hline & & & 2012 \\
\hline & & Morocco & P 2016 May 0 \\
\hline & & & 2014 \\
\hline & & & 2014 \\
\hline & & & 2012 \\
\hline & & Morocco & P 2016 May 0 \\
\hline & & Morocco & P 2016 May 0 \\
\hline & & Morocco & P 2016 May 0 \\
\hline & & & 2015 \\
\hline & & Morocco & P 2016 May 0 \\
\hline & & & 2015 \\
\hline & & & 2017 \\
\hline & & Morocco & P 2016 May 0 \\
\hline & Elephant Morć & & 2014 Nov $1676^{\circ} 16.407$ 'S \\
\hline Antofagasta & deflation surfa & & 2009 Dec $18 \sim 25^{\circ} 14^{\prime} S$ \\
\hline & & Morocco & P 2016 May 0 \\
\hline & & & 2012 \\
\hline & & & 2014 \\
\hline & & & 2014 \\
\hline & Elephant Morć & & 2014 Nov $1676^{\circ} 17.133^{\prime} S$ \\
\hline & & Morocco & P 2016 May 0 \\
\hline & & Morocco & P 2016 May 0 \\
\hline Antofagasta & & & 09 Feb $201824^{\circ} 40.892^{\prime} S$ \\
\hline & & & 2012 \\
\hline & & & 2015 \\
\hline & & & 2014 \\
\hline & & Morocco & P 2016 May 0 \\
\hline & & Morocco & P 2016 May 0 \\
\hline
\end{tabular}




\begin{tabular}{|c|c|c|c|c|}
\hline 0000-00-00 & & & 1979 & \\
\hline $0000-00-00$ & & & 2012 & \\
\hline 0000-00-00 & & & 2014 & \\
\hline $0000-00-00$ & & & 2012 & \\
\hline 0000-00-00 & & & 2000 & \\
\hline 0000-00-00 & & & 2012 & \\
\hline $0000-00-00$ & & & 2014 & \\
\hline 0000-00-00 & & & 2014 & \\
\hline 0000-00-00 & & & 2014 & \\
\hline 0000-00-00 & & & 2012 & \\
\hline 0000-00-00 & Antofagasta & & 03 Feb 2015 & $25^{\circ} 34.691^{\prime} \mathrm{S}$ \\
\hline $0000-00-00$ & & & 2014 & \\
\hline 0000-00-00 & & Elephant Moré & 2014 Nov 16 & $76^{\circ} 17.644^{\prime} \mathrm{S}$ \\
\hline 0000-00-00 & & & 1974 & \\
\hline 0000-00-00 & & Morocco & P 2016 May 0 & \\
\hline 0000-00-00 & & & 2000 & \\
\hline 0000-00-00 & & & 2015 & \\
\hline $0000-00-00$ & & & 2015 & \\
\hline $0000-00-00$ & & & 2015 & \\
\hline 0000-00-00 & & Elephant Moré & 2014 Dec 12 & $76^{\circ} 15.332^{\prime} \mathrm{S}$ \\
\hline 0000-00-00 & Antofagasta & deflation surfa & 2014 & $\sim 24^{\circ} 41^{\prime} \mathrm{S}$ \\
\hline 0000-00-00 & & & 2012 & \\
\hline 0000-00-00 & & & 2012 & \\
\hline 0000-00-00 & Antofagasta & & 25 Oct 2016 & $21^{\circ} 34.33^{\prime} \mathrm{S}$ \\
\hline 0000-00-00 & Antofagasta & & 14 Feb 2018 & $25^{\circ} 17.895^{\prime} S$ \\
\hline $0000-00-00$ & & & 2015 & \\
\hline $0000-00-00$ & & & 2014 & \\
\hline 0000-00-00 & & & 2000 & \\
\hline 0000-00-00 & & & 2012 & \\
\hline 0000-00-00 & & & 2012 & \\
\hline 0000-00-00 & & & 1979 & \\
\hline 0000-00-00 & & & 2014 & \\
\hline 0000-00-00 & & & 2014 & \\
\hline 0000-00-00 & & & 2015 & \\
\hline $0000-00-00$ & & & 2014 & \\
\hline 0000-00-00 & & & 2014 & \\
\hline 0000-00-00 & & Morocco & P 2016 May 0 & \\
\hline $0000-00-00$ & & & 2012 & \\
\hline 0000-00-00 & & Morocco & P 2016 May 0 & \\
\hline $0000-00-00$ & & Morocco & P 2016 May 0 & \\
\hline 0000-00-00 & & Morocco & P 2016 May 0 & \\
\hline $0000-00-00$ & & Elephant Moré & 2014 Dec 12 & $76^{\circ} 15.899^{\prime} S$ \\
\hline 0000-00-00 & & Morocco & P 2016 May 0 & \\
\hline 0000-00-00 & & & 2014 & \\
\hline 0000-00-00 & & & 2000 & \\
\hline
\end{tabular}


0000-00-00

0000-00-00

0000-00-00

0000-00-00

0000-00-00

0000-00-00

0000-00-00

0000-00-00

0000-00-00

0000-00-00

0000-00-00

0000-00-00

0000-00-00

0000-00-00

0000-00-00

0000-00-00

0000-00-00

0000-00-00

0000-00-00

0000-00-00

0000-00-00

0000-00-00

0000-00-00

0000-00-00

0000-00-00

0000-00-00

0000-00-00

0000-00-00

0000-00-00

0000-00-00

0000-00-00

0000-00-00

0000-00-00

0000-00-00

0000-00-00

0000-00-00

0000-00-00

0000-00-00

0000-00-00

0000-00-00

0000-00-00

0000-00-00

0000-00-00

0000-00-00

0000-00-00

\section{Elephant Mora}

Antofagasta

Elephant Moré

Antofagasta

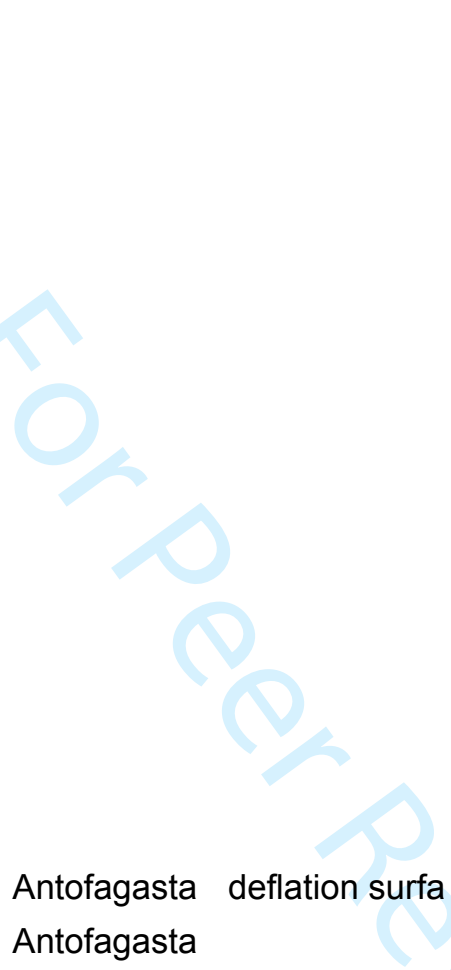

Antofagasta
2014 Dec 12 76¹5.177'S

2000

17 Mar $201722^{\circ} 19.26^{\prime} S$

2014 Nov 16 76¹7.625'S

18 Feb $201824^{\circ} 41.972^{\prime} S$

2000

2000

Morocco P 2016 May 0

2012

1987

2000

1987

Morocco P 2016 May 0

2014

2012

1987

2014

1979

2000

2012

2000

2010 Jan $22 \sim 24^{\circ} 41^{\prime} S$

21 Mar $201722^{\circ} 21.37 ' S$

2000

1979

1979

2000

1979

2009

2000

2000

2000

2009

2000

2009

2009

2000

2009

2009

2000

2000

2009

2009

2000

2000 


\begin{tabular}{|c|c|}
\hline 0000-00-00 & 2009 \\
\hline $0000-00-00$ & 2000 \\
\hline 0000-00-00 & 2009 \\
\hline 0000-00-00 & 2009 \\
\hline 0000-00-00 & 2000 \\
\hline 0000-00-00 & 2009 \\
\hline 0000-00-00 & 2000 \\
\hline 0000-00-00 & 2000 \\
\hline 0000-00-00 & 2000 \\
\hline $0000-00-00$ & 2000 \\
\hline 0000-00-00 & 2014 \\
\hline 0000-00-00 & 2009 \\
\hline $0000-00-00$ & 2000 \\
\hline $0000-00-00$ & 2009 \\
\hline 0000-00-00 & 2000 \\
\hline 0000-00-00 & 2009 \\
\hline 0000-00-00 & 2009 \\
\hline $0000-00-00$ & 2009 \\
\hline 0000-00-00 & 2000 \\
\hline $0000-00-00$ & 2009 \\
\hline $0000-00-00$ & 2014 Nov $2176^{\circ} 17.163 ' S$ \\
\hline 0000-00-00 & 1998 \\
\hline 0000-00-00 & 1998 \\
\hline 0000-00-00 & 2009 \\
\hline 0000-00-00 & 2000 \\
\hline 0000-00-00 & 2009 \\
\hline $0000-00-00$ & 2000 \\
\hline $0000-00-00$ & 2009 \\
\hline 0000-00-00 & 2009 \\
\hline 0000-00-00 & 2009 \\
\hline 0000-00-00 & 2009 \\
\hline 0000-00-00 & 2009 \\
\hline 0000-00-00 & 2009 \\
\hline $0000-00-00$ & 2009 \\
\hline 0000-00-00 & 2009 \\
\hline 0000-00-00 & 2012 \\
\hline 0000-00-00 & 1998 \\
\hline $0000-00-00$ & 2009 \\
\hline 0000-00-00 & 1998 \\
\hline 0000-00-00 & 2009 \\
\hline 0000-00-00 & 2009 \\
\hline 0000-00-00 & 2009 \\
\hline 0000-00-00 & 2009 \\
\hline 0000-00-00 & 2009 \\
\hline 0000-00-00 & 2009 \\
\hline
\end{tabular}


0000-00-00

2009

$0000-00-00 \quad 2009$

$0000-00-00 \quad 2009$

0000-00-00 2009

0000-00-00 2009

0000-00-00 2009

0000-00-00 2000

0000-00-00 2009

0000-00-00 2009

0000-00-00 2009

0000-00-00 1998

0000-00-00 2000

$0000-00-00 \quad 2009$

0000-00-00 2009

0000-00-00 2015

0000-00-00 2009

$0000-00-00 \quad 2015$

0000-00-00 2015

0000-00-00 2009

$0000-00-00 \quad 2009$

0000-00-00 2009

0000-00-00 2015

0000-00-00 2009

0000-00-00 2009

0000-00-00 1998

0000-00-00 2009

0000-00-00 1998

$0000-00-00 \quad 2009$

0000-00-00 2012

0000-00-00 2000

0000-00-00

0000-00-00

Reckling Peak

2014 Nov $1076^{\circ} 12.376^{\prime} S$

2009

2009

$0000-00-00$

2009

$0000-00-00$

2009

$0000-00-00 \quad 2009$

0000-00-00 2009

0000-00-00 2009

0000-00-00 1998

0000-00-00 1998

0000-00-00 2010

0000-00-00 2009

0000-00-00 2009

0000-00-00 2009

0000-00-00 2015 


\begin{tabular}{|c|c|c|c|c|c|}
\hline $0000-00-00$ & & & & 2009 & \\
\hline 0000-00-00 & & & & 2000 & \\
\hline 0000-00-00 & & & & 2016 & \\
\hline 0000-00-00 & & & & 2000 & \\
\hline 0000-00-00 & & & & 2009 & \\
\hline 0000-00-00 & & & & 2000 & \\
\hline 0000-00-00 & & & & 2009 & \\
\hline 0000-00-00 & & & & 2010 & \\
\hline 0000-00-00 & & & & 2009 & \\
\hline 0000-00-00 & & & & 2000 & \\
\hline 0000-00-00 & & & & 2009 & \\
\hline 0000-00-00 & & & & 2000 & \\
\hline 0000-00-00 & & & & 2000 & \\
\hline 0000-00-00 & & Elephant Morć & & 2014 Dec 12 & $76^{\circ} 14.962^{\prime} S$ \\
\hline 0000-00-00 & & & & 2000 & \\
\hline 0000-00-00 & Pavlodar & & & August 2013 & $52^{\circ} 39.86^{\prime} \mathrm{N}$ \\
\hline 0000-00-00 & Madaniyin & Desert & & $2018 \mathrm{Mar}$ & $33^{\circ} 19^{\prime} 50.07$ \\
\hline 0000-00-00 & Antofagasta & deflation surfa & & 2017 Nov & $24^{\circ} 56^{\prime} 32.9^{\prime \prime S}$ \\
\hline 0000-00-00 & & & Zagora, M & (P 2018 & \\
\hline 0000-00-00 & Antofagasta & & & 20 Feb 2017 & $25^{\circ} 12.962^{\prime} \mathrm{S}$ \\
\hline 0000-00-00 & Antofagasta & deflation surfa & & 2010 Jan 19 & $\sim 24^{\circ} 41^{\prime} S$ \\
\hline 0000-00-00 & Tibet & & Beijing & June 25,2018 & $833^{\circ} 10.17^{\prime} \mathrm{N}$ \\
\hline $0000-00-00$ & & & Erfoud, Mc & $P 2003$ & \\
\hline 0000-00-00 & & & Morocco & P 2016 & \\
\hline $0000-00-00$ & & & Erfoud & P 2017 & \\
\hline 0000-00-00 & & & Alicante, $\varsigma$ & P 2012 & \\
\hline 0000-00-00 & & & Rissani & P 2004 & \\
\hline $0000-00-00$ & Antofagasta & & & 31 Jan 2015 & $25^{\circ} 12.068 ' S$ \\
\hline 0000-00-00 & Antofagasta & deflation surfa & & 2017 Nov & $25^{\circ} 24^{\prime} 09.0^{\prime \prime S}$ \\
\hline 0000-00-00 & California & buried under c & & 2015 Dec 12 & $35^{\circ} 3.396^{\prime} \mathrm{N}$ \\
\hline 0000-00-00 & Antofagasta & & & 24 Mar 2016 & $25^{\circ} 13^{\prime} 40^{\prime \prime} \mathrm{S}$ \\
\hline $0000-00-00$ & & & & 2000 & \\
\hline 0000-00-00 & Morocco, Our: & & Genova & P May 2016 & \\
\hline 0000-00-00 & Antofagasta & limestone defl & & 2017 Oct 15 & $22^{\circ} 24.643^{\prime} \mathrm{S}$ \\
\hline $0000-00-00$ & & & Erfoud & P 2017 & \\
\hline $0000-00-00$ & Antofagasta & & & 22 Feb 2017 & $24^{\circ} 41.170^{\prime} \mathrm{S}$ \\
\hline $0000-00-00$ & & & & 2012 & \\
\hline 0000-00-00 & & & Munich & P 2017 Oct & \\
\hline $0000-00-00$ & Antofagasta & & & 21 Feb 2017 & $24^{\circ} 45.942^{\prime} \mathrm{S}$ \\
\hline $0000-00-00$ & & & Western S & P 2018 & \\
\hline $0000-00-00$ & Antofagasta & & & 20 Feb 2017 & $25^{\circ} 13.711$ 'S \\
\hline $0000-00-00$ & & & & 2000 & \\
\hline 0000-00-00 & Antofagasta & & & 15 Feb 2018 & $25^{\circ} 14.262^{\prime S}$ \\
\hline 0000-00-00 & & near Dakhla & Morocco & P 2018 & \\
\hline $0000-00-00$ & & & & 2012 & \\
\hline
\end{tabular}


0000-00-00

0000-00-00

0000-00-00

0000-00-00

0000-00-00

0000-00-00

0000-00-00

0000-00-00

0000-00-00

0000-00-00

0000-00-00

0000-00-00

0000-00-00

0000-00-00

0000-00-00

0000-00-00

0000-00-00

0000-00-00

0000-00-00

0000-00-00

0000-00-00

0000-00-00

0000-00-00

0000-00-00

0000-00-00

0000-00-00

0000-00-00

0000-00-00

0000-00-00

0000-00-00

0000-00-00

0000-00-00

0000-00-00

0000-00-00

0000-00-00

0000-00-00

0000-00-00

0000-00-00

0000-00-00

0000-00-00

0000-00-00

0000-00-00

0000-00-00

0000-00-00

0000-00-00

\begin{tabular}{|c|c|c|c|}
\hline \multirow{3}{*}{ Antofagasta } & Morocco & P 2003 & \\
\hline & & 22 Feb 2017 & $24^{\circ} 41.386^{\prime} \mathrm{S}$ \\
\hline & Morocco & P 2018 Apr & \\
\hline \multicolumn{2}{|l|}{ Atacama } & 2017 Nov 10 & $26^{\circ} 17^{\prime} 55^{\prime \prime S}$ \\
\hline \multirow[t]{2}{*}{ Antofagasta } & & 22 Feb 2017 & $24^{\circ} 39.042^{\prime} \mathrm{S}$ \\
\hline & & 2012 & \\
\hline \multirow[t]{3}{*}{ Antofagasta limestone defl } & & 19 Oct 2017 & $22^{\circ} 28.050^{\prime} \mathrm{S}$ \\
\hline & Morocco & P 2016 May 0 & \\
\hline & Morocco & P 2016 May 0 & \\
\hline \multirow[t]{2}{*}{ Antofagasta } & & 15 Feb 2018 & $25^{\circ} 13.153^{\prime} \mathrm{S}$ \\
\hline & & P 2017 & \\
\hline \multirow[t]{4}{*}{ Antofagasta limestone defl } & & 19 Oct 2017 & $22^{\circ} 27.549^{\prime} S$ \\
\hline & & 2012 & \\
\hline & & 2014 & \\
\hline & Tucson G & ¿P 2012 Feb & \\
\hline \multirow[t]{10}{*}{ Antofagasta deflation surfa } & & 2014 Dec 21 & $\sim 24^{\circ} 41^{\prime} \mathrm{S}$ \\
\hline & Morocco & P 2016 May 0 & \\
\hline & Morocco & P 2016 May 0 & \\
\hline & Morocco & P 2016 May 0 & \\
\hline & Morocco & P 2016 May 0 & \\
\hline & Morocco & P 2016 May 0 & \\
\hline & Morocco & P 2016 May 0 & \\
\hline & & 2014 & \\
\hline & & 2012 & \\
\hline & Morocco & P 2016 May 0 & \\
\hline \multirow[t]{20}{*}{ Antofagasta } & & 21 Feb 2017 & $24^{\circ} 44.527^{\prime} \mathrm{S}$ \\
\hline & & 2014 & \\
\hline & Morocco & P 2016 May 0 & \\
\hline & Morocco & P 2016 May 0 & \\
\hline & & 2012 & \\
\hline & Morocco & P 2016 May 0 & \\
\hline & & 2014 & \\
\hline & Bologna & P March 2016 & \\
\hline & Morocco & P 2016 May 0 & \\
\hline & & 2014 & \\
\hline & Morocco & P 2016 May 0 & \\
\hline & Morocco & P 2016 May 0 & \\
\hline & & 2012 & \\
\hline & Morocco & P 2016 May 0 & \\
\hline & & 2014 & \\
\hline & & 2012 & \\
\hline & Morocco & P 2016 May 0 & \\
\hline & & 2014 & \\
\hline & & 2012 & \\
\hline & Morocco & P 2016 May 0 & \\
\hline
\end{tabular}




\begin{tabular}{|c|c|c|c|c|c|}
\hline 0000-00-00 & & & & 2012 & \\
\hline $0000-00-00$ & & & & 2012 & \\
\hline 0000-00-00 & & & & 2014 & \\
\hline 0000-00-00 & & & & 2014 & \\
\hline 0000-00-00 & & & Morocco & P 2016 May 0 & \\
\hline 0000-00-00 & & & Morocco & P 2016 May 0 & \\
\hline 0000-00-00 & & & & 2014 & \\
\hline 0000-00-00 & & & Morocco & P 2016 May 0 & \\
\hline 0000-00-00 & South & Gravel & & 2017 Mar & $31^{\circ} 01.55^{\prime} \mathrm{N}$ \\
\hline 0000-00-00 & & & & 2012 & \\
\hline 0000-00-00 & & & Morocco & P 2016 May 0 & \\
\hline 0000-00-00 & & & & 2014 & \\
\hline 0000-00-00 & & & Morocco & P 2016 May 0 & \\
\hline $0000-00-00$ & & & & 2012 & \\
\hline 0000-00-00 & & & & 2012 & \\
\hline 0000-00-00 & & & & 2012 & \\
\hline 0000-00-00 & & & Morocco & P 2016 May 0 & \\
\hline 0000-00-00 & & & & 2012 & \\
\hline 0000-00-00 & & & & 2014 & \\
\hline 0000-00-00 & Mali & & Torino & P October 201 & \\
\hline 0000-00-00 & & & & 2015 & \\
\hline 0000-00-00 & & & & 2014 & \\
\hline 0000-00-00 & & & & 2014 & \\
\hline $0000-00-00$ & & & Morocco & P 2016 May 0 & \\
\hline 0000-00-00 & & & & P 2017 & \\
\hline 0000-00-00 & & & & 2014 & \\
\hline $0000-00-00$ & & & Sainte Marie & e P 2018 & \\
\hline $0000-00-00$ & & & & 2014 & \\
\hline 0000-00-00 & & & & 2014 & \\
\hline 0000-00-00 & Antofagasta & deflation surfa & & 2017 Nov 12 & $\sim 25^{\circ} 14^{\prime} S$ \\
\hline 0000-00-00 & & & Morocco & P 2016 May 0 & \\
\hline 0000-00-00 & & & Morocco & P 2016 May 0 & \\
\hline 0000-00-00 & & & Morocco & P 2016 May 0 & \\
\hline 0000-00-00 & & & & 2015 & \\
\hline 0000-00-00 & & & Tucson (Said & P Jan 2012 & \\
\hline 0000-00-00 & & & & 2012 & \\
\hline $0000-00-00$ & & & & 2015 & \\
\hline 0000-00-00 & & Mt. DeWitt & & 2014 Dec 11 & $77^{\circ} 12.239^{\prime} \mathrm{S}$ \\
\hline 0000-00-00 & & & & 2015 & \\
\hline 0000-00-00 & & & & 1986 & \\
\hline 0000-00-00 & & & & 2014 & \\
\hline 0000-00-00 & & & & 2014 & \\
\hline 0000-00-00 & & & & 2015 & \\
\hline 0000-00-00 & & & & 2015 & \\
\hline 0000-00-00 & & & & 2014 & \\
\hline
\end{tabular}




\begin{tabular}{|c|c|c|c|}
\hline 0000-00-00 & & \multicolumn{2}{|c|}{ Said Hadnany P 2011 Feb } \\
\hline 0000-00-00 & & & 2009 \\
\hline 0000-00-00 & & & 2012 \\
\hline 0000-00-00 & & & 2014 \\
\hline 0000-00-00 & Arizona & & 11 Nov $201134^{\circ} 48.990^{\prime} \mathrm{N}$ \\
\hline 0000-00-00 & & & 2015 \\
\hline 0000-00-00 & & & 2012 \\
\hline 0000-00-00 & & & 2012 \\
\hline 0000-00-00 & & & 2012 \\
\hline 0000-00-00 & & & 2015 \\
\hline 0000-00-00 & & & 2012 \\
\hline 0000-00-00 & & & 1979 \\
\hline 0000-00-00 & & & 2012 \\
\hline 0000-00-00 & & & 2014 \\
\hline 0000-00-00 & & & 2014 \\
\hline 0000-00-00 & & Morocco & P 2016 May 0 \\
\hline 0000-00-00 & & & 2014 \\
\hline 0000-00-00 & & & 2012 \\
\hline 0000-00-00 & & & 2014 \\
\hline 0000-00-00 & & & 2015 \\
\hline 0000-00-00 & & & 2014 \\
\hline 0000-00-00 & & & 2014 \\
\hline 0000-00-00 & & & 2012 \\
\hline 0000-00-00 & & & 2015 \\
\hline 0000-00-00 & & Morocco & P 2016 May 0 \\
\hline 0000-00-00 & & & 2015 \\
\hline 0000-00-00 & & & 2012 \\
\hline 0000-00-00 & & & 2014 Dec 12 76¹5.270'S \\
\hline 0000-00-00 & & & 2014 \\
\hline 0000-00-00 & & & 2012 \\
\hline 0000-00-00 & & & 2014 \\
\hline 0000-00-00 & & & 2014 \\
\hline 0000-00-00 & & & 2014 \\
\hline 0000-00-00 & & Morocco & P 2016 May 0 \\
\hline 0000-00-00 & & & 2015 \\
\hline 0000-00-00 & & & 2015 \\
\hline 0000-00-00 & & & 2014 \\
\hline 0000-00-00 & & & 2015 \\
\hline 0000-00-00 & & & 2014 \\
\hline 0000-00-00 & & & 2012 \\
\hline 0000-00-00 & & & 2014 \\
\hline 0000-00-00 & & & 2012 \\
\hline 0000-00-00 & & & 2014 \\
\hline 0000-00-00 & & & 2015 \\
\hline 0000-00-00 & & & 2012 \\
\hline
\end{tabular}




\begin{tabular}{|c|c|c|c|}
\hline 0000-00-00 & & Morocco & P 2016 May 0 \\
\hline $0000-00-00$ & Antofagasta & & 21 Feb $201725^{\circ} 04.950 ' S$ \\
\hline 0000-00-00 & & & 2014 \\
\hline 0000-00-00 & & Morocco & P 2016 May 0 \\
\hline 0000-00-00 & & & 2014 \\
\hline 0000-00-00 & & & 2014 \\
\hline 0000-00-00 & & & 2015 \\
\hline $0000-00-00$ & & & 2015 \\
\hline 0000-00-00 & & & 2015 \\
\hline 0000-00-00 & & & 2014 \\
\hline $0000-00-00$ & & & 2015 \\
\hline 0000-00-00 & & & 2015 \\
\hline $0000-00-00$ & & & 2012 \\
\hline 0000-00-00 & & Morocco & P 2016 May 0 \\
\hline $0000-00-00$ & & & 2000 \\
\hline 0000-00-00 & & & 2014 \\
\hline 0000-00-00 & & & 1979 \\
\hline 0000-00-00 & & & 1987 \\
\hline 0000-00-00 & & & 2012 \\
\hline 0000-00-00 & & & 1987 \\
\hline 0000-00-00 & & & 1987 \\
\hline 0000-00-00 & & & 2014 \\
\hline $0000-00-00$ & & & 1987 \\
\hline $0000-00-00$ & & & 2012 \\
\hline 0000-00-00 & & & 2014 \\
\hline 0000-00-00 & & & 1987 \\
\hline $0000-00-00$ & & Morocco & P 2016 May 0 \\
\hline 0000-00-00 & & & 2014 \\
\hline $0000-00-00$ & & & 2012 \\
\hline $0000-00-00$ & Antofagasta & & 15 Feb 2018 25⒕789'S \\
\hline $0000-00-00$ & & & 1998 \\
\hline 0000-00-00 & & & 2000 \\
\hline 0000-00-00 & & & 2012 \\
\hline $0000-00-00$ & & & 2012 \\
\hline 0000-00-00 & & & 2000 \\
\hline 0000-00-00 & & & 2014 \\
\hline 0000-00-00 & & Morocco & P 2016 May 0 \\
\hline $0000-00-00$ & & & 2014 Nov $2176^{\circ} 17.270 ' S$ \\
\hline $0000-00-00$ & & & 2000 \\
\hline 0000-00-00 & & & 2000 \\
\hline $0000-00-00$ & & & 2009 \\
\hline 0000-00-00 & & & 2000 \\
\hline 0000-00-00 & & & 2000 \\
\hline 0000-00-00 & & & 2000 \\
\hline 0000-00-00 & & & 2009 \\
\hline
\end{tabular}




\begin{tabular}{|c|c|c|}
\hline 0000-00-00 & & 2000 \\
\hline $0000-00-00$ & & 2000 \\
\hline $0000-00-00$ & & 2009 \\
\hline $0000-00-00$ & & 2009 \\
\hline 0000-00-00 & & 2015 \\
\hline 0000-00-00 & & 2009 \\
\hline 0000-00-00 & & 2000 \\
\hline $0000-00-00$ & & 2009 \\
\hline 0000-00-00 & Elephant Morć & 2014 Dec 12 76¹5.507'S \\
\hline 0000-00-00 & Morocco & P 2016 May 0 \\
\hline $0000-00-00$ & & 2009 \\
\hline 0000-00-00 & & 2012 \\
\hline $0000-00-00$ & & 2000 \\
\hline $0000-00-00$ & & 2009 \\
\hline 0000-00-00 & & 2000 \\
\hline $0000-00-00$ & & 2000 \\
\hline 0000-00-00 & & 2000 \\
\hline 0000-00-00 & & 2000 \\
\hline 0000-00-00 & & 2009 \\
\hline 0000-00-00 & & 2000 \\
\hline 0000-00-00 & & 2000 \\
\hline $0000-00-00$ & & 2009 \\
\hline $0000-00-00$ & & 2000 \\
\hline 0000-00-00 & & 2000 \\
\hline 0000-00-00 & & 2012 \\
\hline 0000-00-00 & & 2012 \\
\hline $0000-00-00$ & & 1998 \\
\hline 0000-00-00 & & 2000 \\
\hline 0000-00-00 & & 2000 \\
\hline $0000-00-00$ & & 2009 \\
\hline $0000-00-00$ & & 2000 \\
\hline 0000-00-00 & & 1998 \\
\hline $0000-00-00$ & & 1998 \\
\hline 0000-00-00 & & 2000 \\
\hline 0000-00-00 & & 2009 \\
\hline 0000-00-00 & & 2009 \\
\hline 0000-00-00 & & 2000 \\
\hline 0000-00-00 & & 2000 \\
\hline $0000-00-00$ & & 2009 \\
\hline $0000-00-00$ & Morocco & P 2016 May 0 \\
\hline 0000-00-00 & & 2009 \\
\hline 0000-00-00 & Elephant Morć & 2014 Dec $1276^{\circ} 15.602 ' S$ \\
\hline 0000-00-00 & & 2009 \\
\hline 0000-00-00 & & 2009 \\
\hline 0000-00-00 & & 2009 \\
\hline
\end{tabular}




\begin{tabular}{|c|c|c|}
\hline $0000-00-00$ & & 1998 \\
\hline $0000-00-00$ & & 2009 \\
\hline $0000-00-00$ & & 2009 \\
\hline $0000-00-00$ & & 2009 \\
\hline 0000-00-00 & & 2000 \\
\hline 0000-00-00 & & 2009 \\
\hline 0000-00-00 & Elephant Moré & 2014 Dec 12 76¹5.663'S \\
\hline 0000-00-00 & & 2009 \\
\hline 0000-00-00 & & 2009 \\
\hline 0000-00-00 & & 2009 \\
\hline 0000-00-00 & & 2009 \\
\hline $0000-00-00$ & & 2009 \\
\hline 0000-00-00 & Elephant Morć & 2014 Dec $1276^{\circ} 15.553^{\prime} S$ \\
\hline 0000-00-00 & & 2009 \\
\hline 0000-00-00 & & 1998 \\
\hline 0000-00-00 & & 2009 \\
\hline 0000-00-00 & & 1998 \\
\hline 0000-00-00 & & 2009 \\
\hline $0000-00-00$ & & 1998 \\
\hline 0000-00-00 & & 2009 \\
\hline 0000-00-00 & & 2015 \\
\hline 0000-00-00 & & 2009 \\
\hline 0000-00-00 & & 2015 \\
\hline $0000-00-00$ & & 2015 \\
\hline 0000-00-00 & & 2012 \\
\hline 0000-00-00 & & 2009 \\
\hline 0000-00-00 & Elephant Moré & 2014 Dec 12 76¹5.349'S \\
\hline $0000-00-00$ & Elephant Morć & 2014 Dec 12 76¹5.280'S \\
\hline 0000-00-00 & & 2015 \\
\hline $0000-00-00$ & & 1998 \\
\hline 0000-00-00 & & 2015 \\
\hline 0000-00-00 & Elephant Moré & 2014 Dec 12 76¹5.573'S \\
\hline 0000-00-00 & & 2016 \\
\hline 0000-00-00 & & 2016 \\
\hline 0000-00-00 & & 1998 \\
\hline 0000-00-00 & & 2016 \\
\hline 0000-00-00 & & 2010 \\
\hline 0000-00-00 & & 2009 \\
\hline $0000-00-00$ & & 2009 \\
\hline 0000-00-00 & & 2016 \\
\hline $0000-00-00$ & & 1998 \\
\hline 0000-00-00 & & 2016 \\
\hline 0000-00-00 & Elephant Moré & 2014 Dec $1276^{\circ} 15.554 ' S$ \\
\hline 0000-00-00 & & 2009 \\
\hline $0000-00-00$ & & 2000 \\
\hline
\end{tabular}




\begin{tabular}{|c|c|c|c|}
\hline 0000-00-00 & & 2015 & \\
\hline 0000-00-00 & & 1998 & \\
\hline 0000-00-00 & & 2016 & \\
\hline 0000-00-00 & & 2000 & \\
\hline 0000-00-00 & & 2016 & \\
\hline 0000-00-00 & & 2009 & \\
\hline 0000-00-00 & & 2009 & \\
\hline 0000-00-00 & & 2000 & \\
\hline 0000-00-00 & & 2015 & \\
\hline 0000-00-00 & & 2015 & \\
\hline 0000-00-00 & & 2016 & \\
\hline 0000-00-00 & & 2016 & \\
\hline 0000-00-00 & & 2000 & \\
\hline 0000-00-00 & & 2000 & \\
\hline 0000-00-00 & & 2015 & \\
\hline 0000-00-00 & & 2000 & \\
\hline 0000-00-00 & & 2000 & \\
\hline 0000-00-00 & & 2000 & \\
\hline 0000-00-00 & & 2009 & \\
\hline 0000-00-00 & & 2015 & \\
\hline 0000-00-00 & Temara & P 2017 Aug & \\
\hline 0000-00-00 & Tucson Gem & ¿P 2018 Jan & \\
\hline 0000-00-00 & Mali & P 2017 & \\
\hline 0000-00-00 & & April 2018 & \\
\hline 0000-00-00 & Morocco & P 2017 & \\
\hline 0000-00-00 & Mauritania & P May 2018 & \\
\hline 0000-00-00 & Saguia el Handry river (wadi Ouarzazate & $2014 \mathrm{Dec}$ & $\sim 26^{\circ} 50.2^{\prime} \mathrm{N}$ \\
\hline 0000-00-00 & Morocco & P 2017 & \\
\hline 0000-00-00 & Munich & P 2008 & \\
\hline 0000-00-00 & Tucson & P 2017 Jan 30 & \\
\hline 0000-00-00 & Mauritania & P May 2018 & \\
\hline 0000-00-00 & Temara & P 2017 Aug & \\
\hline 0000-00-00 & Sainte-Marie & $\therefore P 2006$ Jun & \\
\hline 0000-00-00 & Mauritania & P May 2018 & \\
\hline 0000-00-00 & Mauritania & P 2018 May & \\
\hline 0000-00-00 & online & P July 2018 & \\
\hline 0000-00-00 & Morocco & P 2018 & \\
\hline 0000-00-00 & & P 2018 & \\
\hline 0000-00-00 & Morocco & P 2016 May 1 & \\
\hline 0000-00-00 & Morocco & P 2016 May 1 & \\
\hline 0000-00-00 & Morocco & P 2016 May 1 & \\
\hline 0000-00-00 & Morocco & P 2016 May 1 & \\
\hline 0000-00-00 & & 2017 & \\
\hline 0000-00-00 & & 2017 & \\
\hline 0000-00-00 & & P 2005 & \\
\hline
\end{tabular}




\begin{tabular}{|c|c|c|c|c|c|}
\hline 0000-00-00 & & & & P 2017 & \\
\hline 0000-00-00 & & & & P 2016 & \\
\hline 0000-00-00 & Zacatecas & & & October, 2016 & $622^{\circ} 49^{\prime} 15.73 " \mathrm{~N}$ \\
\hline 0000-00-00 & & & & P 2005 & \\
\hline 0000-00-00 & & & Munich & P 2005 & \\
\hline 0000-00-00 & Valle d'Aosta & & & May 1987 & $45^{\circ} 49.14^{\prime} \mathrm{N}$ \\
\hline 0000-00-00 & Chubu & & & 2012 Oct & $35^{\circ} 26^{\prime} 33.9^{\prime \prime} \mathrm{N}$ \\
\hline 0000-00-00 & Xinjiang & & & $2015 \mathrm{Dec}$ & $41.3^{\circ} \mathrm{N}$ \\
\hline 0000-00-00 & & & & P 2004 & \\
\hline 0000-00-00 & & & & P 2004 & \\
\hline 0000-00-00 & & & & P 2013 & \\
\hline 0000-00-00 & & & & 1940 & \\
\hline 0000-00-00 & & & & P 2014 & \\
\hline 0000-00-00 & & & & P 2014 & \\
\hline 0000-00-00 & & & & P 2010 & \\
\hline 0000-00-00 & & & Ouarzazate & P 2017 Jan & \\
\hline 0000-00-00 & & & Chenzhou & P May 2017 & \\
\hline 0000-00-00 & & & Chenzhou Cit & ? P May 2017 & \\
\hline 0000-00-00 & Rio Grande d & c Countryside & & about 1908 & $30^{\circ} 41^{\prime} 34.9 " \mathrm{~S}$ \\
\hline 0000-00-00 & Sokoto & & & 10 Jan 2008 & $13^{\circ} 0.3^{\prime} \mathrm{N}$ \\
\hline 0000-00-00 & Xinjiang & & Hami & 1999 & $44^{\circ} 10^{\prime} 20^{\prime \prime}$ \\
\hline 0000-00-00 & Adrar & Limestone pec & & $2001 \mathrm{Dec}$ & $21^{\circ} 20^{\prime} 31.93 " \mathrm{~N}$ \\
\hline 0000-00-00 & Salamanca & & & 1981 & $40^{\circ} 51.04 ' \mathrm{~N}$ \\
\hline 0000-00-00 & & & & 2016 & \\
\hline 0000-00-00 & Xinjiang & & Urumqi & 2013 & $41^{\circ} 03^{\prime} 22^{\prime \prime} \mathrm{N}$ \\
\hline 0000-00-00 & Xinjiang & desert & & 6 June 2017 & $47^{\circ} 52^{\prime} 59^{\prime \prime} \mathrm{N}$ \\
\hline 0000-00-00 & & & & 2017 & \\
\hline 0000-00-00 & & & & 2014 & \\
\hline 0000-00-00 & & & Morocco & P 2018 & \\
\hline 0000-00-00 & Qinghai & desert & & 07 June 2016 & $37^{\circ} 59^{\prime} 2.07^{\prime \prime} \mathrm{N}$ \\
\hline 0000-00-00 & Qinghai & desert & & 16 Sept 2015 & ה $37^{\circ} 39^{\prime} 35.17^{\prime \prime}$ \\
\hline 0000-00-00 & & desert & Beijing & P 2015 & \\
\hline 0000-00-00 & Qinghai & desert & & 13 July 2015 & $37^{\circ} 49^{\prime} 2.34^{\prime \prime} \mathrm{N}$ \\
\hline 0000-00-00 & Qinghai & desert & & 10 Mar 2014 & $37^{\circ} 34^{\prime} 54.59 " \mathrm{~N}$ \\
\hline 0000-00-00 & Qinghai & desert & & 03 Nov 2013 & $37^{\circ} 29^{\prime} 12.31^{\prime \prime}$ \\
\hline 0000-00-00 & & & Ouarzazate & P 2017 Augus & \\
\hline 2019-01-15 & Updated coor Xinjiang & desert & & 2018 Jun & $46^{\circ} 49^{\prime} 51.07 " \mathrm{~N}$ \\
\hline 0000-00-00 & Xinjiang & desert & & 26 Nov 2013 & $42^{\circ} 33^{\prime} 43.03^{\prime \prime} \mathrm{N}$ \\
\hline 0000-00-00 & Gansu & desert & & 16 Sept 2018 & $40^{\circ} 13^{\prime} 12.56 " \mathrm{~N}$ \\
\hline 0000-00-00 & & & Ouarzazate & P 2017 March & \\
\hline 0000-00-00 & Xinjiang & desert & & 16 Nov 2016 & $42^{\circ} 27^{\prime} 51.71 " \mathrm{~N}$ \\
\hline 0000-00-00 & & & ebay & 2017 & \\
\hline 0000-00-00 & XinJiang & desert & & 20 Aug 2012 & $41^{\circ} 43^{\prime} 1.04^{\prime \prime} \mathrm{N}$ \\
\hline 0000-00-00 & Xinjiang & desert & Urumqi, Xinjia & 2014 & \\
\hline $0000-00-00$ & Xinjiang & desert & Hami, Xinjians & CP 2013 & \\
\hline
\end{tabular}


0000-00-00

0000-00-00

0000-00-00

0000-00-00

0000-00-00

0000-00-00

0000-00-00

0000-00-00

0000-00-00

0000-00-00

0000-00-00

0000-00-00

0000-00-00

0000-00-00

0000-00-00

0000-00-00

0000-00-00

0000-00-00

0000-00-00

0000-00-00

0000-00-00

0000-00-00

0000-00-00

0000-00-00

0000-00-00

0000-00-00

0000-00-00

0000-00-00

0000-00-00

0000-00-00

0000-00-00

0000-00-00

0000-00-00

0000-00-00

0000-00-00

0000-00-00

0000-00-00

0000-00-00

0000-00-00

0000-00-00

0000-00-00

0000-00-00

0000-00-00

0000-00-00

0000-00-00
Hamburg P 2018

Oyo

19 April 2018 8¹6'55.83"N

Mali P 2018

Munich P 2005

Morocco P 2018

Tinduf, Algerie P 2017 Jan

Morocco P 2017

Zagora P March 2018

Guelmim, Mor P 2018 Aug

Zagora P 2018

Hamburg, Ger P 2017

Hamburg, Ger P 2017

Morocco P 2017 Jun

2012

Northwest Afri Erfoud P 2010

Morocco P 2017 Jun

Tindouf, Alger P 2018

Chihuahua

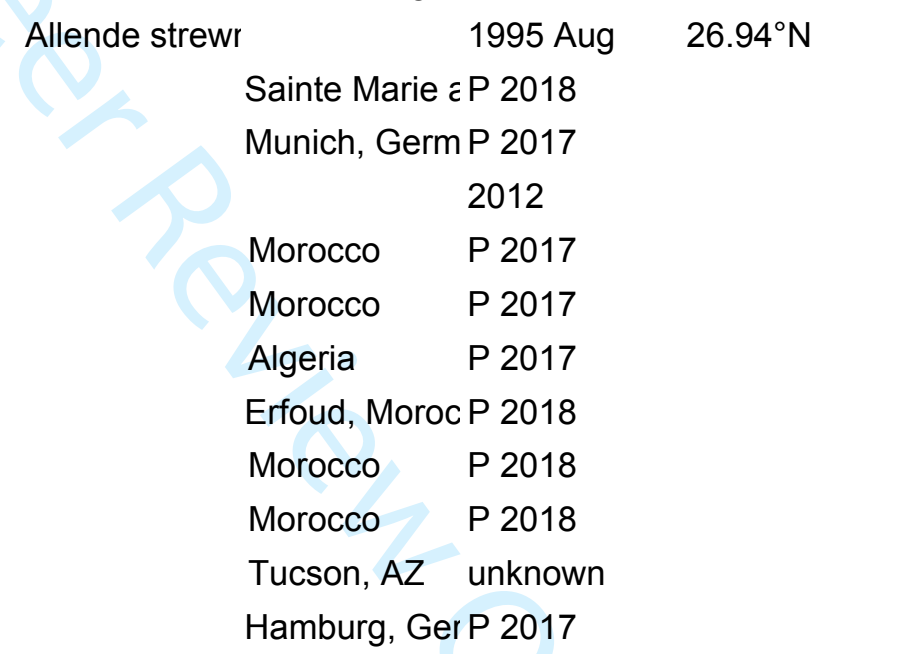

Elephant Moré

2014 Nov $2176^{\circ} 17.125^{\prime S}$

2009

2009

2009

2000

2000

2000

2009

2009

2009

2010

2009

2009

2009

2009

2009 


\begin{tabular}{|c|c|c|c|c|}
\hline $0000-00-00$ & & & & 2009 \\
\hline 0000-00-00 & & & Casablanca & P 2001 Mar \\
\hline 0000-00-00 & & & Beijing & P Nov. 2015 \\
\hline 0000-00-00 & & & Erfoud, Moro & $P 2018$ \\
\hline 0000-00-00 & & & Morocco & P 2017 \\
\hline 0000-00-00 & & & & 2014 \\
\hline 0000-00-00 & & & & 2014 \\
\hline 0000-00-00 & & & & 2014 \\
\hline 0000-00-00 & & & & 2014 \\
\hline 0000-00-00 & & & Sainte-Marie & Jun 2017 \\
\hline 2018-02-24 & changed to $\mathrm{c}$ Boujdour & Gueltat Zemm & & 21 Aug $201825^{\circ} 05^{\prime} 32.0^{\prime \prime} \mathrm{N}$ \\
\hline 0000-00-00 & & & Ensisheim, $F$ & P 2007 June \\
\hline 0000-00-00 & & & Munich & P 2005 \\
\hline 0000-00-00 & & & Erfoud, Moro & P 2017 Feb \\
\hline 0000-00-00 & & & Munich & P 2005 \\
\hline 0000-00-00 & & & Temara & P 2017 Nov \\
\hline 0000-00-00 & & & Temara & P 2017 Nov \\
\hline 0000-00-00 & & & Erfoud, Moro & $P 2003$ \\
\hline 0000-00-00 & Antofagasta & & & 25 Feb $201625^{\circ} 08.980^{\prime} S$ \\
\hline 0000-00-00 & & & Morocco & P 2018 May \\
\hline 0000-00-00 & & & Morocco & P 2016 May 0 \\
\hline 0000-00-00 & & Elephant Morc & & 2014 Dec $1276^{\circ} 16.246^{\prime} S$ \\
\hline 0000-00-00 & & & Morocco & P 2016 May 0 \\
\hline 0000-00-00 & Morocco, Dah & & Torino & P October 201 \\
\hline 0000-00-00 & & & & 2012 \\
\hline 0000-00-00 & & & Bologna & P March 2016 \\
\hline 0000-00-00 & Morocco, Laa! & & Bologna & P March 2016 \\
\hline 0000-00-00 & & & & 2012 \\
\hline 0000-00-00 & & & Bologna & P 3/2015 \\
\hline 0000-00-00 & & & Erfoud, Moro & $P 2003$ \\
\hline 0000-00-00 & & & & 2014 \\
\hline 0000-00-00 & & & Munich & P 2005 \\
\hline 0000-00-00 & & & & 2012 \\
\hline 0000-00-00 & & & Said Hadnan & /P 2011 Feb \\
\hline 0000-00-00 & & & & 2012 \\
\hline 0000-00-00 & & & & 2014 \\
\hline 0000-00-00 & & & Munich & P 2005 \\
\hline 0000-00-00 & & & & 2014 \\
\hline 0000-00-00 & & & & 2014 \\
\hline 0000-00-00 & & & & 2014 \\
\hline 0000-00-00 & Al Wusta & & & $20^{\circ} 32^{\prime} 37.51 " \mathrm{~N}$ \\
\hline 0000-00-00 & & & & 2014 \\
\hline 0000-00-00 & & & & 2015 Jan $17 \quad 77^{\circ} 13.645^{\prime} S$ \\
\hline 0000-00-00 & & & Munich & P 2005 \\
\hline 0000-00-00 & & & & 2012 \\
\hline
\end{tabular}


0000-00-00

2014

0000-00-00

2012

0000-00-00

2012

0000-00-00

2012

0000-00-00

2000

0000-00-00

2000

0000-00-00

2009

0000-00-00

2000

0000-00-00

2000

0000-00-00

2000

0000-00-00

2000

0000-00-00

2000

0000-00-00

2000

0000-00-00

2009

0000-00-00

0000-00-00

0000-00-00

1998

2015 Jan $20 \quad 77^{\circ} 14.453^{\prime} S$

2016

0000-00-00

0000-00-00

0000-00-00

0000-00-00

0000-00-00

0000-00-00

0000-00-00

0000-00-00

0000-00-00

0000-00-00

0000-00-00

0000-00-00

0000-00-00

0000-00-00

0000-00-00

0000-00-00

0000-00-00

0000-00-00

0000-00-00

0000-00-00

0000-00-00

0000-00-00

0000-00-00

0000-00-00

0000-00-00

0000-00-00

0000-00-00

0000-00-00

Antofagasta
La Pampa Realico
Antofagasta
Xinjiang
Antofagasta
Antofagasta deflation surfa
Al Wusta
Arusha
Antofagasta
Antofagasta
Antofagasta




\begin{tabular}{|c|c|c|c|c|c|}
\hline 0000-00-00 & Uttar Pradesh & hKarimati villag & & 28 May 2009 & $25^{\circ} 54^{\prime} \mathrm{N}$ \\
\hline 0000-00-00 & & Elephant Moré & & 2014 Dec 12 & $76^{\circ} 15.853^{\prime S}$ \\
\hline 0000-00-00 & Antofagasta & & & 16 Feb 2017 & $24^{\circ} 56.977^{\prime} \mathrm{S}$ \\
\hline 0000-00-00 & Ajdabiya & Arid Plateau & & 2006 & $28.25710^{\circ} \mathrm{N}$ \\
\hline 0000-00-00 & & & Morocco & P 2016 May 0 & \\
\hline 0000-00-00 & Antofagasta & & & 29 Mar 2018 & $22^{\circ} 29.50^{\prime} \mathrm{S}$ \\
\hline 0000-00-00 & & & Morocco & P 2016 May 0 & \\
\hline 0000-00-00 & & & Morocco & P 2004 & \\
\hline 0000-00-00 & & & Morocco & P 2016 May 0 & \\
\hline 0000-00-00 & Antofagasta & & & 24 Mar 2017 & $22^{\circ} 29.25^{\prime} \mathrm{S}$ \\
\hline 0000-00-00 & & & & 2012 & \\
\hline 0000-00-00 & & & Erfoud, Moroc & $P 2001$ & \\
\hline 0000-00-00 & & & Bologna & P March 2016 & \\
\hline 0000-00-00 & & & & 2014 & \\
\hline 0000-00-00 & & & Morocco & P 2016 May 0 & \\
\hline 0000-00-00 & & & Morocco & P 2016 May 0 & \\
\hline 0000-00-00 & & & Erfoud & P March 2018 & \\
\hline 0000-00-00 & & & Sainte Marie $\varepsilon$ & $E P 2018$ & \\
\hline 0000-00-00 & & & & 2012 & \\
\hline 0000-00-00 & & & & 2014 & \\
\hline 0000-00-00 & & & Morocco & P 2016 May 0 & \\
\hline 0000-00-00 & & & & 2016 & \\
\hline 0000-00-00 & & & Erfoud & P March 2018 & \\
\hline 0000-00-00 & & & Layaoune, Mo & P 2018 & \\
\hline 0000-00-00 & & & Munich & P 2005 & \\
\hline 0000-00-00 & Antofagasta & deflation surfa & & 2010 Mar 21 & $\sim 25^{\circ} 14^{\prime} S$ \\
\hline 0000-00-00 & & & Munich, Germ & P 2015 & \\
\hline 0000-00-00 & & & Erfoud & P March 2018 & \\
\hline 0000-00-00 & Antofagasta & & & 06 Apr 2018 & $22^{\circ} 30.32^{\prime} \mathrm{S}$ \\
\hline 0000-00-00 & Ajdabiya & Arid Plateau & & 2006 & $28.28583^{\circ} \mathrm{N}$ \\
\hline 0000-00-00 & & & Alexander's K & P 31 Mar 201 & \\
\hline 0000-00-00 & & & St. Marie-aux- & - P 2011 & \\
\hline 0000-00-00 & & & Morocco & P 2016 May 0 & \\
\hline 0000-00-00 & Antofagasta & & & 06 Apr 2018 & $22^{\circ} 30.32^{\prime} \mathrm{S}$ \\
\hline 0000-00-00 & Antofagasta & Gravel pedim€ & & 2017 Feb 17 & $24^{\circ} 54^{\prime} 21.2^{\prime \prime S}$ \\
\hline 0000-00-00 & & & Munich, Germ & P 2015 & \\
\hline 0000-00-00 & & & & 2014 & \\
\hline 0000-00-00 & & & & 2014 & \\
\hline 0000-00-00 & & & Verona & P 2015 & \\
\hline 0000-00-00 & & & & 2014 & \\
\hline 0000-00-00 & & & & 2014 & \\
\hline 0000-00-00 & & & & 2014 & \\
\hline 0000-00-00 & & & & 2014 & \\
\hline 0000-00-00 & & & Tucson (Said & P Jan 2012 & \\
\hline 0000-00-00 & & & & 2014 & \\
\hline
\end{tabular}




\begin{tabular}{|c|c|c|c|c|}
\hline $0000-00-00$ & & Bologna & P 2017 & \\
\hline $0000-00-00$ & & & 2014 & \\
\hline $0000-00-00$ & & & 2014 & \\
\hline $0000-00-00$ & & Bologna & P 2017 & \\
\hline 0000-00-00 & & & 2014 & \\
\hline 0000-00-00 & Antofagasta & & 20 Oct 2016 & $21^{\circ} 32.79 ' \mathrm{~S}$ \\
\hline 0000-00-00 & & & 2014 & \\
\hline $0000-00-00$ & Antofagasta & & 2017 Apr 28 & $22^{\circ} 44^{\prime} 59.9^{\prime \prime S}$ \\
\hline 0000-00-00 & & & 2014 & \\
\hline 0000-00-00 & & Morocco & P 2017 & \\
\hline $0000-00-00$ & & & 2014 & \\
\hline 0000-00-00 & & & 2014 & \\
\hline $0000-00-00$ & & & 2014 & \\
\hline 0000-00-00 & Antofagasta & & 15 Mar 2017 & $24^{\circ} 47.73^{\prime} \mathrm{S}$ \\
\hline 0000-00-00 & Antofagasta & & 02 Feb 2015 & $25^{\circ} 34.466^{\prime} S$ \\
\hline 0000-00-00 & & & 2015 & \\
\hline 0000-00-00 & & & 2014 & \\
\hline 0000-00-00 & & & 2014 & \\
\hline 2018-06-17 & corrected ma: Abu Zaby & & 2017 Feb & $24^{\circ} 25.42^{\prime} \mathrm{N}$ \\
\hline 0000-00-00 & & & 2014 & \\
\hline 0000-00-00 & & & 2014 & \\
\hline 0000-00-00 & & & 2014 & \\
\hline 0000-00-00 & & & 2014 & \\
\hline 0000-00-00 & & & 2014 & \\
\hline $0000-00-00$ & & & 2014 & \\
\hline 0000-00-00 & & & 2014 & \\
\hline $0000-00-00$ & & & 2014 & \\
\hline 0000-00-00 & & Munich & P 2005 & \\
\hline 0000-00-00 & & & 2014 & \\
\hline $0000-00-00$ & & Morocco & P 2016 May 0 & \\
\hline $0000-00-00$ & & & 2014 & \\
\hline 0000-00-00 & & & 2014 & \\
\hline 0000-00-00 & & & 2014 & \\
\hline $0000-00-00$ & & & 2014 & \\
\hline 0000-00-00 & & & 2014 & \\
\hline 0000-00-00 & & Morocco & P 2016 May 0 & \\
\hline 0000-00-00 & & & 2014 & \\
\hline 0000-00-00 & & & 2014 & \\
\hline 0000-00-00 & & & 2014 & \\
\hline $0000-00-00$ & & & 2014 & \\
\hline 0000-00-00 & & & 2014 & \\
\hline 0000-00-00 & & & 2014 & \\
\hline 0000-00-00 & & & 2014 & \\
\hline 0000-00-00 & & & 2015 & \\
\hline $0000-00-00$ & & & 2014 & \\
\hline
\end{tabular}




\begin{tabular}{|c|c|c|}
\hline 0000-00-00 & & 2015 \\
\hline 0000-00-00 & & 2014 \\
\hline 0000-00-00 & & 2014 \\
\hline $0000-00-00$ & & 2015 \\
\hline $0000-00-00$ & & 2015 \\
\hline 0000-00-00 & & 2014 \\
\hline 0000-00-00 & & 2014 \\
\hline 0000-00-00 & & 2014 \\
\hline 0000-00-00 & & 2014 \\
\hline 0000-00-00 & & 2014 \\
\hline $0000-00-00$ & & 2014 \\
\hline 0000-00-00 & & 2015 \\
\hline 0000-00-00 & & 2014 \\
\hline 0000-00-00 & & 2000 \\
\hline 0000-00-00 & & 2014 \\
\hline 0000-00-00 & & 2014 \\
\hline 0000-00-00 & & 2014 \\
\hline 0000-00-00 & & 2014 \\
\hline $0000-00-00$ & & 2000 \\
\hline 0000-00-00 & & 2014 \\
\hline 0000-00-00 & & 2014 \\
\hline 0000-00-00 & & 1979 \\
\hline 0000-00-00 & & 2012 \\
\hline 0000-00-00 & & 2012 \\
\hline 0000-00-00 & & 1979 \\
\hline 0000-00-00 & & 2014 \\
\hline $0000-00-00$ & & 2014 \\
\hline 0000-00-00 & Antofagasta & 25 Oct $201625^{\circ} 34.11 ' S$ \\
\hline 0000-00-00 & & 2000 \\
\hline $0000-00-00$ & & 2000 \\
\hline $0000-00-00$ & & 2000 \\
\hline 0000-00-00 & & 2000 \\
\hline 0000-00-00 & & 2000 \\
\hline 0000-00-00 & & 2009 \\
\hline 0000-00-00 & & 2000 \\
\hline 0000-00-00 & & 2000 \\
\hline $0000-00-00$ & & 2000 \\
\hline $0000-00-00$ & & 2000 \\
\hline $0000-00-00$ & & 2000 \\
\hline $0000-00-00$ & & 2000 \\
\hline 0000-00-00 & & 2000 \\
\hline $0000-00-00$ & & 2000 \\
\hline $0000-00-00$ & & 2000 \\
\hline $0000-00-00$ & & 2000 \\
\hline 0000-00-00 & & 2000 \\
\hline
\end{tabular}


0000-00-00

2000

$0000-00-00 \quad 2000$

$0000-00-00 \quad 2000$

0000-00-00 2000

$0000-00-00 \quad 2000$

0000-00-00 2000

0000-00-00 2000

0000-00-00 2000

0000-00-00 2000

$0000-00-00 \quad 2000$

$0000-00-00 \quad 2000$

0000-00-00 1998

$0000-00-00 \quad 2000$

$0000-00-00 \quad 2000$

0000-00-00 2000

0000-00-00 2000

0000-00-00 1998

0000-00-00 2000

$0000-00-00 \quad 2000$

$0000-00-00 \quad 2000$

0000-00-00 2000

$0000-00-00 \quad 2000$

0000-00-00 2015 Jan $18 \quad 77^{\circ} 14.442 ' S$

0000-00-00 2000

$0000-00-00 \quad 2000$

0000-00-00 2015

$0000-00-00 \quad 2000$

0000-00-00 1998

0000-00-00 2015

0000-00-00 2000

$0000-00-00 \quad 2000$

0000-00-00 2000

0000-00-00 1998

$0000-00-00 \quad 2016$

$0000-00-00 \quad 2000$

0000-00-00 2000

$0000-00-00 \quad 2016$

0000-00-00 2015

0000-00-00 2000

0000-00-00 2000

0000-00-00 1998

$0000-00-00 \quad 2000$

$0000-00-00 \quad 2000$

$0000-00-00 \quad 2000$

0000-00-00 2016 


\begin{tabular}{|c|c|c|c|c|}
\hline 0000-00-00 & & & 2000 & \\
\hline 0000-00-00 & & & 2016 & \\
\hline 0000-00-00 & & & 2000 & \\
\hline 0000-00-00 & & & 2000 & \\
\hline 0000-00-00 & & & 2000 & \\
\hline 0000-00-00 & & & 2000 & \\
\hline 0000-00-00 & & & 2000 & \\
\hline 0000-00-00 & & & 2000 & \\
\hline 0000-00-00 & & & 2000 & \\
\hline 0000-00-00 & & & 2000 & \\
\hline 0000-00-00 & & & 2015 Jan 17 & $77^{\circ} 13.641 ' \mathrm{~S}$ \\
\hline 0000-00-00 & & & 2000 & \\
\hline 0000-00-00 & & & 2000 & \\
\hline 0000-00-00 & & & 2000 & \\
\hline 0000-00-00 & & & 2016 & \\
\hline 0000-00-00 & & & 2000 & \\
\hline 0000-00-00 & & & 2015 & \\
\hline 0000-00-00 & Baden-Wurtte & Field & 10 July 2018 & $48^{\circ} 35^{\prime} 17.998 \varepsilon$ \\
\hline 0000-00-00 & Al Jumayliyar & h Desert & 03 March 201 & $25^{\circ} 30.44^{\prime} \mathrm{N}$ \\
\hline 0000-00-00 & & & CP 2018 & \\
\hline 0000-00-00 & Antofagasta & & 17 Feb 2017 & $24^{\circ} 56.256^{\prime} \mathrm{S}$ \\
\hline 0000-00-00 & Antofagasta & & 31 Jan 12 & $25^{\circ} 51.490^{\prime} \mathrm{S}$ \\
\hline 0000-00-00 & Jarayan al Ba & Desert & 20 April 2018 & $24^{\circ} 55.17^{\prime} \mathrm{N}$ \\
\hline 0000-00-00 & Yunnan & farming field & 1 June 2018 & $22^{\circ} 2^{\prime} 6 " \mathrm{~N}$ \\
\hline 0000-00-00 & Antofagasta & plain & 2017 Oct 15 & $22^{\circ} 18.192^{\prime} S$ \\
\hline 0000-00-00 & Antofagasta & deflation surfa & 2017 Nov & $25^{\circ} 21^{\prime} 58.1^{\prime \prime S}$ \\
\hline 0000-00-00 & Antofagasta & limestone defl & 2017 Oct 13 & $22^{\circ} 24.200^{\prime} S$ \\
\hline 0000-00-00 & & & P 2018 & \\
\hline 0000-00-00 & Antofagasta & & 28 Jan 2018 & $22^{\circ} 26.15^{\prime} \mathrm{S}$ \\
\hline 0000-00-00 & Lipetskaya ob & plain, plowed I & 21 June 2018 & $52^{\circ} 48.084^{\prime} \mathrm{N}$ \\
\hline 0000-00-00 & Antofagasta & & 2017 Nov 11 & $25^{\circ} 4.155^{\prime} \mathrm{S}$ \\
\hline 0000-00-00 & Antofagasta & deflation surfa & 2017 May 17 & $24^{\circ} 49^{\prime} 54.1^{\prime \prime S}$ \\
\hline 0000-00-00 & & Elephant Moré & 2014 Dec 12 & $76^{\circ} 15.683^{\prime} S$ \\
\hline 0000-00-00 & & & P 2005 & \\
\hline 0000-00-00 & Antioquia & & 16 Feb 2017 & $8^{\circ} 16^{\prime} 44.39 " \mathrm{~N}$ \\
\hline 0000-00-00 & Antofagasta & & 2017 Nov & $24^{\circ} 41^{\prime} \mathrm{S}$ \\
\hline 0000-00-00 & & & CP 2003 & \\
\hline 0000-00-00 & & & 1988 & \\
\hline 0000-00-00 & & & 2016 & \\
\hline 0000-00-00 & & & P 2005 & \\
\hline 0000-00-00 & & & P 2016 May 0 & \\
\hline 0000-00-00 & Atacama & & 2016 Jan 17 & $26^{\circ} 20^{\prime} 52^{\prime \prime} \mathrm{S}$ \\
\hline 0000-00-00 & & & 2000 & \\
\hline 0000-00-00 & & Elephant Morc & 2014 Dec 12 & $76^{\circ} 15.878^{\prime S}$ \\
\hline
\end{tabular}


0000-00-00

0000-00-00

0000-00-00

0000-00-00

0000-00-00

0000-00-00

0000-00-00

0000-00-00

0000-00-00

0000-00-00

0000-00-00

0000-00-00

0000-00-00

0000-00-00

0000-00-00

0000-00-00

0000-00-00

0000-00-00

0000-00-00

0000-00-00

0000-00-00

0000-00-00

0000-00-00

0000-00-00

0000-00-00

0000-00-00

0000-00-00

0000-00-00

0000-00-00

0000-00-00

0000-00-00

0000-00-00

0000-00-00

0000-00-00

0000-00-00

0000-00-00

0000-00-00

0000-00-00

0000-00-00

0000-00-00

0000-00-00

0000-00-00

0000-00-00

0000-00-00

Antofagasta

Antofagasta

Antofagasta

Antofagasta

Antofagasta

Antofagasta

Antofagasta

Antofagasta $\begin{array}{lll}\text { Antofagasta } & 2017 \text { Nov } 8 \quad 25^{\circ} 466^{\prime} 27^{\prime \prime S} \\ & 2000 \\ \text { Antofagasta } & 2017 \text { Nov } 13 \quad 24^{\circ} 45.242 \text { 'S }\end{array}$

Erfoud, Moroc P 2001

Erfoud, Moroc P 2001

Erfoud, Moroc P 2018

Elephant Moré

2014 Dec 12 76¹5.784'S

Morocco P 2003

2000

Morocco P 2012

Morocco, Smé

Ouaddai fields

02 Feb 2015 254․689'S

Bologna P March 2016

20 Feb 2017 25⒔012'S

2014 Aug $2112.79^{\circ} \mathrm{N}$

08 Feb $201824^{\circ} 37.402^{\prime} \mathrm{S}$

2012

Morocco, Laa!

11 Feb $201822^{\circ} 26.29^{\prime} S$

Bologna P March 2016

Tucson (Said P Jan 2015

Glarus Rocky mounte

2017 Jul $17 \quad 47^{\circ} 4.285^{\prime} \mathrm{N}$

2012

Antofagasta deflation surfa

Reckling Peak

2015 Oct $30 \sim 24^{\circ} 41^{\prime} \mathrm{S}$

18 Feb $201625^{\circ} 32.674$ 'S

2014 Nov $1076^{\circ} 12.782^{\prime S}$

Morocco $\quad$ P 2016 May 0

2014

2012

Tindouf, Alger P 2018

Morocco P 2016 May 0

2012

Hamburg, Ger P 2017

2014

Morocco P 2016 May 0

Said Hadnany P 2011 Feb

Morocco P 2018

2014

Antofagasta deflation surfa

$2012 \quad 24^{\circ} 41^{\prime} \mathrm{S}$

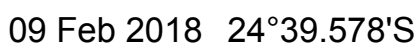
15 Mar $201722^{\circ} 22.87^{\prime} S$ 2016

Sainte Marie є P 2018

Antofagasta deflation surfa

2014 Dec $20 \sim 25^{\circ} 14$ 'S

2012

06 Apr 2018 22³0.18'S

Morocco P 2003 


\begin{tabular}{|c|c|c|c|c|c|}
\hline 0000-00-00 & & & & 2014 & \\
\hline $0000-00-00$ & & & & 2012 & \\
\hline 0000-00-00 & Morocco, Dah & & Genova & P May 2016 & \\
\hline 0000-00-00 & & & & 2012 & \\
\hline 0000-00-00 & Antofagasta & deflation surfa & & 2010 Sep 30 & $\sim 24^{\circ} 41^{\prime} \mathrm{S}$ \\
\hline 0000-00-00 & & & & 2012 & \\
\hline 0000-00-00 & & & Sainte $\mathrm{M}$ & E 2018 & \\
\hline 0000-00-00 & & Elephant Moré & & 2014 Dec 12 & $76^{\circ} 15.040^{\prime} \mathrm{S}$ \\
\hline 0000-00-00 & & & & 2012 & \\
\hline 0000-00-00 & & & Munich & P 2005 & \\
\hline 0000-00-00 & & & & 2012 & \\
\hline 0000-00-00 & Antofagasta & & & 17 Feb 2016 & $25^{\circ} 32.700^{\prime} \mathrm{S}$ \\
\hline 0000-00-00 & & & & 2014 & \\
\hline 0000-00-00 & & & & 2012 & \\
\hline 0000-00-00 & Antofagasta & & & 22 Feb 2017 & $24^{\circ} 42.402^{\prime} \mathrm{S}$ \\
\hline 0000-00-00 & & & & 2012 & \\
\hline 0000-00-00 & Antofagasta & & & 22 Feb 2017 & $24^{\circ} 40.457^{\prime} \mathrm{S}$ \\
\hline 0000-00-00 & Antofagasta & deflation surfa & & 2010 Mar 06 & $\sim 25^{\circ} 14^{\prime} S$ \\
\hline 0000-00-00 & Antofagasta & & & 22 Feb 2017 & $24^{\circ} 41.412^{\prime} \mathrm{S}$ \\
\hline 0000-00-00 & & & & 2014 & \\
\hline 0000-00-00 & & & Erfoud & P 2004 & \\
\hline 0000-00-00 & & & & 2014 & \\
\hline $0000-00-00$ & & & & 2014 & \\
\hline 0000-00-00 & & Elephant Moré & & $2014 \mathrm{Dec} 12$ & $76^{\circ} 16.015^{\prime S}$ \\
\hline 0000-00-00 & & & & 1998 & \\
\hline 0000-00-00 & Antofagasta & deflation surfa & & $2009 \operatorname{Dec} 28$ & $\sim 24^{\circ} 41^{\prime} S$ \\
\hline $0000-00-00$ & & & & 2014 & \\
\hline 0000-00-00 & & Elephant Morć & & 2014 Nov 21 & $76^{\circ} 16.800^{\prime} \mathrm{S}$ \\
\hline 0000-00-00 & & & & 2014 & \\
\hline 0000-00-00 & Antofagasta & deflation surfa & & 2010 Mar 06 & $\sim 25^{\circ} 14^{\prime} S$ \\
\hline $0000-00-00$ & Antofagasta & & & 1 Apr 2018 & $22^{\circ} 30.58^{\prime} \mathrm{S}$ \\
\hline 0000-00-00 & & & & 2012 & \\
\hline 0000-00-00 & & & & 2014 & \\
\hline 0000-00-00 & & & Munich & P 2005 & \\
\hline 0000-00-00 & Antofagasta & deflation surfa & & 2015 Jan 03 & $\sim 24^{\circ} 41^{\prime} S$ \\
\hline 0000-00-00 & & & & 2014 & \\
\hline 0000-00-00 & & Elephant Morć & & 2014 Nov 21 & $76^{\circ} 16.968^{\prime} \mathrm{S}$ \\
\hline 0000-00-00 & & & & 2014 & \\
\hline 0000-00-00 & & & & 2014 & \\
\hline 0000-00-00 & & & & 2014 & \\
\hline 0000-00-00 & & & & 2012 & \\
\hline 0000-00-00 & & & Said Hac & P 2008 Feb & \\
\hline 0000-00-00 & Antofagasta & deflation surfa & & 2010 Jul 05 & $\sim 25^{\circ} 14^{\prime} S$ \\
\hline $0000-00-00$ & & & & 2012 & \\
\hline 0000-00-00 & & & & 2014 & \\
\hline
\end{tabular}


0000-00-00

0000-00-00

0000-00-00

0000-00-00

0000-00-00

0000-00-00

0000-00-00

0000-00-00

0000-00-00

0000-00-00

0000-00-00

0000-00-00

0000-00-00

0000-00-00

0000-00-00

0000-00-00

0000-00-00

0000-00-00

0000-00-00

0000-00-00

0000-00-00

0000-00-00

0000-00-00

0000-00-00

0000-00-00

0000-00-00

0000-00-00

0000-00-00

0000-00-00

0000-00-00

0000-00-00

0000-00-00

0000-00-00

0000-00-00

0000-00-00

0000-00-00

0000-00-00

0000-00-00

0000-00-00

0000-00-00

0000-00-00

0000-00-00

0000-00-00

0000-00-00

0000-00-00

\section{Elephant Morє}

2014 Nov 21 76¹7.319'S

2014

2014

2014

2014

Antofagasta

29 April $201822^{\circ} 26.11$ 'S

Morocco $\quad$ P 2016 May 0

2014

1979

Antofagasta deflation surfa

2010 Jul $05 \quad 25^{\circ} 14^{\prime} S$

2014

2014

2014

Alexander's KIP 31 Mar 201

2014

2012

2014

2014

Torino P 2011

2014

2016

24 Feb $2016 \quad 25^{\circ} 21.102^{\prime} S$

2014

2014

2014

2014

2014

2014

Arizona Willcox Playa

2018 Jan $2232^{\circ} 7.0^{\prime} \mathrm{N}$

2014

2012

2012

Antofagasta

14 Feb $201825^{\circ} 14.820^{\prime} S$

2012

2014

2014

2012

Antofagasta deflation surfa

2017 Nov 13 24²3.293'S

P 2016 May 0

2014

2015

2012

2014

2014

Elephant Mor: 


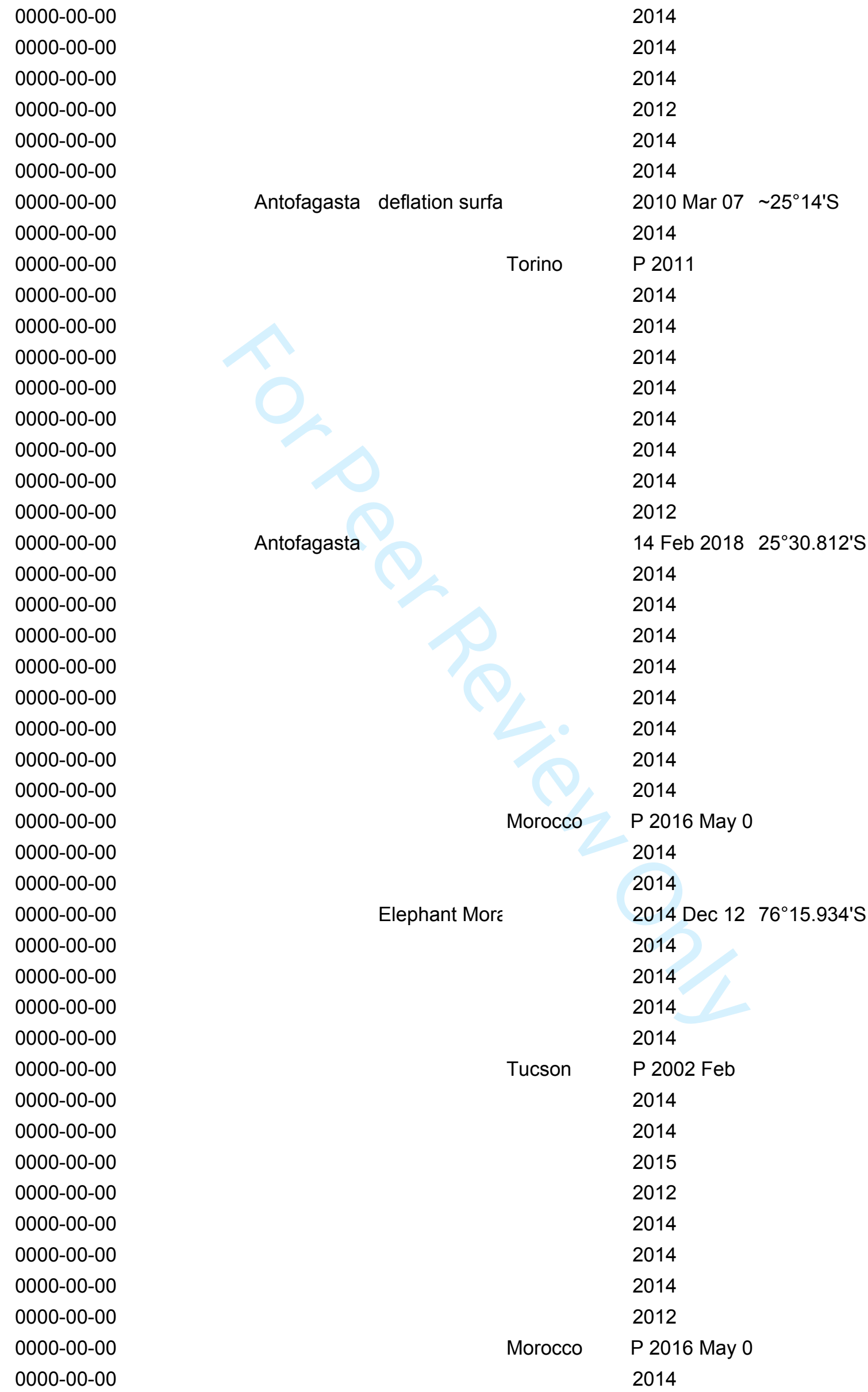




\begin{tabular}{|c|c|c|c|}
\hline 0000-00-00 & & & 2014 \\
\hline $0000-00-00$ & & & 2014 \\
\hline $0000-00-00$ & & & 2014 \\
\hline $0000-00-00$ & & & 2014 \\
\hline 0000-00-00 & & & 2014 \\
\hline 0000-00-00 & & & 2014 \\
\hline 0000-00-00 & & & 2014 \\
\hline $0000-00-00$ & & & 2014 \\
\hline 0000-00-00 & & Elephant Morć & 2014 Dec 12 76¹5.350'S \\
\hline 0000-00-00 & & & 2014 \\
\hline $0000-00-00$ & & & 2014 \\
\hline 0000-00-00 & & & 2014 \\
\hline $0000-00-00$ & Antofagasta & & 10 Feb $201824^{\circ} 47.015^{\prime} \mathrm{S}$ \\
\hline $0000-00-00$ & & & 2014 \\
\hline 0000-00-00 & & & 2014 \\
\hline $0000-00-00$ & & & 2014 \\
\hline 0000-00-00 & & & 2014 \\
\hline 0000-00-00 & & Munich & P 2005 \\
\hline 0000-00-00 & & & 2015 \\
\hline $0000-00-00$ & & & 2014 \\
\hline 0000-00-00 & & & 2014 \\
\hline 0000-00-00 & & & 2014 \\
\hline $0000-00-00$ & & & 2014 \\
\hline 0000-00-00 & & Munich & P 2005 \\
\hline 0000-00-00 & & & 2014 \\
\hline 0000-00-00 & & & 2014 \\
\hline $0000-00-00$ & & & 2014 \\
\hline 0000-00-00 & & & 2014 \\
\hline $0000-00-00$ & & & 2014 \\
\hline $0000-00-00$ & & Tucson (Said & P Jan 2013 \\
\hline $0000-00-00$ & & Morocco & P 2016 May 0 \\
\hline 0000-00-00 & & & 2012 \\
\hline $0000-00-00$ & & & 2014 \\
\hline 0000-00-00 & & Elephant Morć & 2014 Dec 03 76¹6.510'S \\
\hline 0000-00-00 & & & 2014 \\
\hline 0000-00-00 & & & 2014 \\
\hline 0000-00-00 & & & 2014 \\
\hline 0000-00-00 & & & 2014 \\
\hline $0000-00-00$ & & & 2014 \\
\hline $0000-00-00$ & & & 2014 \\
\hline 0000-00-00 & & & 2014 \\
\hline $0000-00-00$ & & & 2014 \\
\hline 0000-00-00 & Antofagasta & deflation surfa & 2010 Mar $06 \sim 25^{\circ} 14^{\prime} S$ \\
\hline 0000-00-00 & & & 2014 \\
\hline $0000-00-00$ & & & 2015 \\
\hline
\end{tabular}




\begin{tabular}{|c|c|c|c|c|}
\hline 0000-00-00 & & & 2012 & \\
\hline $0000-00-00$ & & & 2014 & \\
\hline 0000-00-00 & & & 2014 & \\
\hline 0000-00-00 & & Elephant Morć & 2014 Nov 16 & $76^{\circ} 17.332^{\prime} \mathrm{S}$ \\
\hline 0000-00-00 & & & 2014 & \\
\hline 0000-00-00 & & & 2014 & \\
\hline 0000-00-00 & & & 2014 & \\
\hline 0000-00-00 & & & 2014 & \\
\hline 0000-00-00 & & & 2014 & \\
\hline 0000-00-00 & Antofagasta & & 03 Feb 2015 & $25^{\circ} 34.478^{\prime} \mathrm{S}$ \\
\hline 0000-00-00 & & & 2014 & \\
\hline 0000-00-00 & & & 2014 & \\
\hline $0000-00-00$ & & & 2014 & \\
\hline $0000-00-00$ & & & 2014 & \\
\hline 0000-00-00 & & & 2014 & \\
\hline 0000-00-00 & & & 2014 & \\
\hline 0000-00-00 & & & 2014 & \\
\hline 0000-00-00 & & & 2012 & \\
\hline 0000-00-00 & & & 2014 & \\
\hline 0000-00-00 & & & 2014 & \\
\hline 0000-00-00 & Antofagasta & & 09 Feb 2018 & $24^{\circ} 41.581^{\prime} \mathrm{S}$ \\
\hline 0000-00-00 & & & 2012 & \\
\hline $0000-00-00$ & & & 2014 & \\
\hline 0000-00-00 & & Elephant Moré & 2014 Nov 21 & $76^{\circ} 16.804^{\prime} \mathrm{S}$ \\
\hline 0000-00-00 & Antofagasta & & 24 Mar 2017 & $22^{\circ} 30.27^{\prime} \mathrm{S}$ \\
\hline 0000-00-00 & California & On the margin & 2017 Apr 7 & $34^{\circ} 48.53^{\prime} \mathrm{N}$ \\
\hline 0000-00-00 & & & 2014 & \\
\hline 0000-00-00 & & & 2014 & \\
\hline 0000-00-00 & & & 2014 & \\
\hline $0000-00-00$ & Antofagasta & deflation surfa & 2009 Dec 28 & $\sim 24^{\circ} 41^{\prime} S$ \\
\hline $0000-00-00$ & & & 2014 & \\
\hline 0000-00-00 & & & 2014 & \\
\hline 0000-00-00 & & & 2014 & \\
\hline 0000-00-00 & & & 2015 & \\
\hline 0000-00-00 & & & 2014 & \\
\hline 0000-00-00 & & & 2014 & \\
\hline 0000-00-00 & & & 2014 & \\
\hline 0000-00-00 & & Morocco & P 2016 May 0 & \\
\hline 0000-00-00 & & & 2014 & \\
\hline $0000-00-00$ & & & 2014 & \\
\hline 0000-00-00 & & & 2014 & \\
\hline 0000-00-00 & & & 2014 & \\
\hline 0000-00-00 & & Elephant Moré & $2014 \mathrm{Dec} 03$ & $76^{\circ} 16.035^{\prime S}$ \\
\hline 0000-00-00 & & & 2014 & \\
\hline $0000-00-00$ & & & 2014 & \\
\hline
\end{tabular}




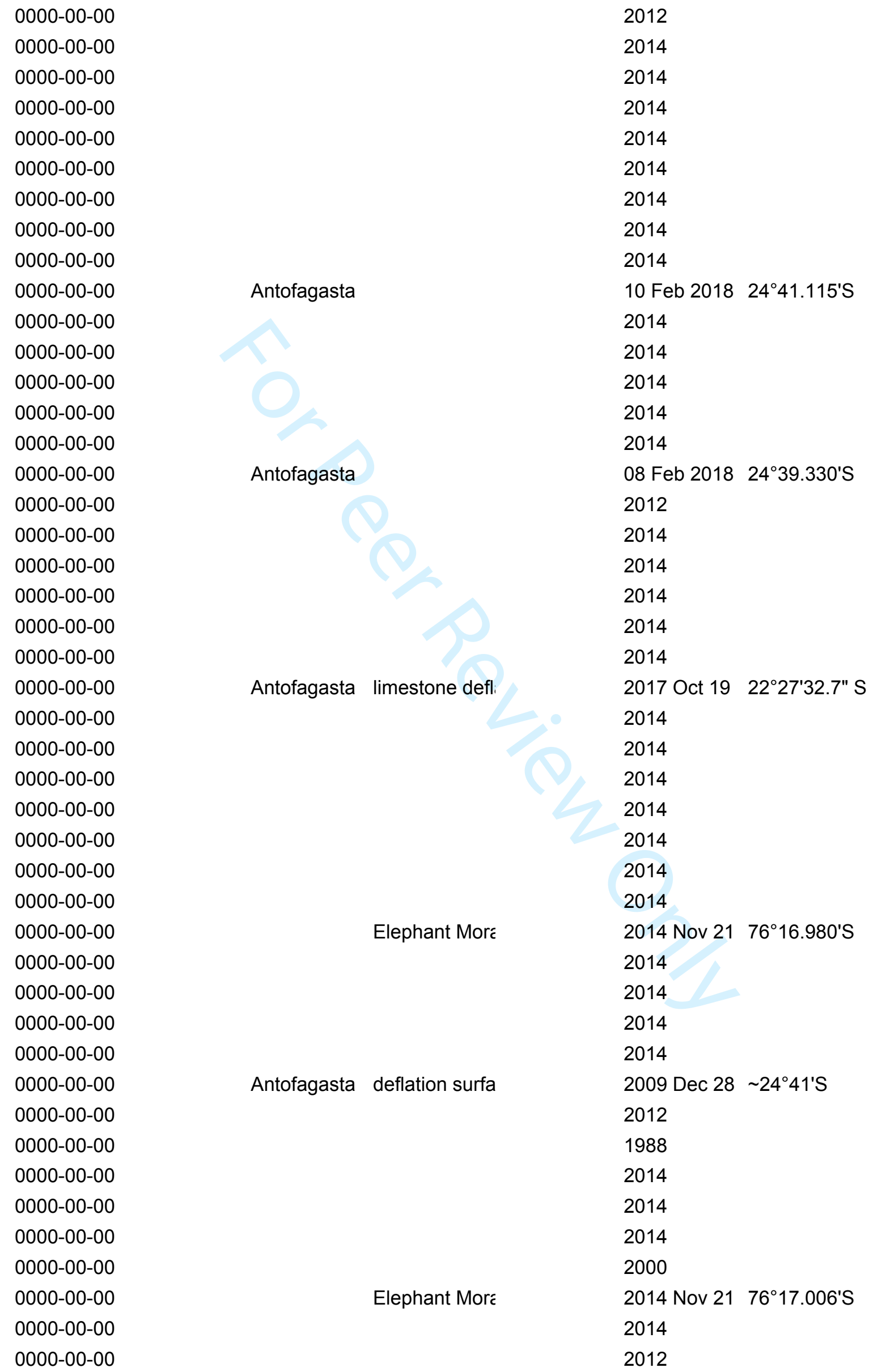




\begin{tabular}{|c|c|c|c|}
\hline $0000-00-00$ & & 1987 & \\
\hline $0000-00-00$ & Elephant Moré & 2014 Nov 21 & $76^{\circ} 17.222^{\prime} \mathrm{S}$ \\
\hline 0000-00-00 & & 2012 & \\
\hline 0000-00-00 & & 2014 & \\
\hline $0000-00-00$ & & 2014 & \\
\hline 0000-00-00 & & 2012 & \\
\hline 0000-00-00 & & 2014 & \\
\hline 0000-00-00 & & 2014 & \\
\hline 0000-00-00 & & 2014 & \\
\hline 0000-00-00 & & 2014 & \\
\hline 0000-00-00 & Elephant Moré & 2014 Nov 21 & $76^{\circ} 17.187^{\prime} S$ \\
\hline 0000-00-00 & & 2014 & \\
\hline 0000-00-00 & & 2014 & \\
\hline $0000-00-00$ & & 2012 & \\
\hline 0000-00-00 & Elephant Morć & 2014 Nov 16 & $76^{\circ} 17.132 ' S$ \\
\hline 0000-00-00 & & 09 Feb 2018 & $24^{\circ} 39.621^{\prime} S$ \\
\hline 0000-00-00 & & 2014 & \\
\hline 0000-00-00 & & 2009 & \\
\hline 0000-00-00 & Elephant Morć & 2014 Nov 21 & $76^{\circ} 17.176^{\prime S}$ \\
\hline 0000-00-00 & & 2000 & \\
\hline 0000-00-00 & & 2009 & \\
\hline 0000-00-00 & & 1979 & \\
\hline 0000-00-00 & deflation surfa & 2017 Nov 14 & $24^{\circ} 46.125^{\prime} \mathrm{S}$ \\
\hline 0000-00-00 & & 2000 & \\
\hline 0000-00-00 & & 2000 & \\
\hline 0000-00-00 & & 2000 & \\
\hline $0000-00-00$ & & 2000 & \\
\hline $0000-00-00$ & & 1975 & \\
\hline 0000-00-00 & & 2000 & \\
\hline 0000-00-00 & & 2000 & \\
\hline 0000-00-00 & & 2000 & \\
\hline 0000-00-00 & & 2000 & \\
\hline 0000-00-00 & & 2000 & \\
\hline $0000-00-00$ & & 2000 & \\
\hline 0000-00-00 & & 2000 & \\
\hline $0000-00-00$ & & 2000 & \\
\hline $0000-00-00$ & & 2000 & \\
\hline 0000-00-00 & & 2000 & \\
\hline $0000-00-00$ & & 2009 & \\
\hline 0000-00-00 & & 2000 & \\
\hline 0000-00-00 & & 2000 & \\
\hline 0000-00-00 & & 2000 & \\
\hline 0000-00-00 & & 2000 & \\
\hline 0000-00-00 & & 2000 & \\
\hline 0000-00-00 & & 2000 & \\
\hline
\end{tabular}


0000-00-00

0000-00-00

0000-00-00

0000-00-00

0000-00-00

0000-00-00

0000-00-00

0000-00-00

0000-00-00

0000-00-00

0000-00-00

0000-00-00

0000-00-00

0000-00-00

0000-00-00

0000-00-00

0000-00-00

0000-00-00

0000-00-00

0000-00-00

0000-00-00

0000-00-00

0000-00-00

0000-00-00

0000-00-00

0000-00-00

0000-00-00

0000-00-00

0000-00-00

0000-00-00

0000-00-00

0000-00-00

0000-00-00

0000-00-00

0000-00-00

0000-00-00

0000-00-00

0000-00-00

0000-00-00

0000-00-00

0000-00-00

0000-00-00

0000-00-00

0000-00-00

0000-00-00
2000

Elephant Morc

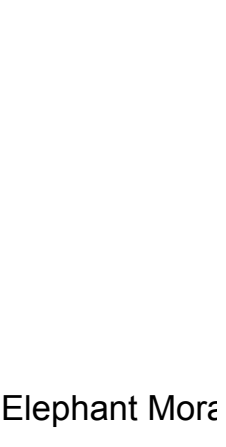

Elephant Morc

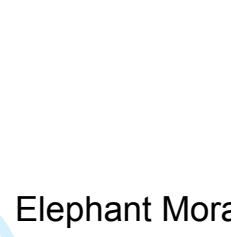

Elephant More

Antofagasta

Elephant Mor

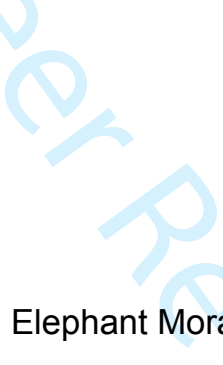

Elephant Moré

Elephant Morє
2014 Nov $2176^{\circ} 17.166^{\prime} S$

1998

2009

2014

2000

2000

2000

2000

2014 Nov 21 76¹6.805'S

2000

2000

2009

2000

2014 Nov 21 76¹7.092'S

2000

2000

2000

2000

2000

2000

2000

2014 Nov $2176^{\circ} 16.865^{\prime} S$

2000

2000

2000

2009

2000

2000

2000

17 Feb $201724^{\circ} 55.315^{\prime} S$

2009

2000

2000

2014 Nov 16 76¹7.370'S

2014 Dec 03 76¹6.563'S

1998

2000

2000

2009

1998

2000

2000

1998

2009 


\begin{tabular}{|c|c|c|c|}
\hline $0000-00-00$ & & 2000 & \\
\hline 0000-00-00 & & 2009 & \\
\hline 0000-00-00 & Elephant Moré & 2014 Nov 21 & $76^{\circ} 17.284^{\prime} S$ \\
\hline 0000-00-00 & & 1998 & \\
\hline $0000-00-00$ & & 1998 & \\
\hline $0000-00-00$ & & 1998 & \\
\hline 0000-00-00 & & 1998 & \\
\hline 0000-00-00 & Elephant Moré & 2014 Nov 21 & $76^{\circ} 17.125^{\prime} S$ \\
\hline $0000-00-00$ & & 2009 & \\
\hline $0000-00-00$ & & 1998 & \\
\hline 0000-00-00 & & 1998 & \\
\hline 0000-00-00 & & 2000 & \\
\hline 0000-00-00 & & 2000 & \\
\hline 0000-00-00 & & 2000 & \\
\hline 0000-00-00 & & 2000 & \\
\hline 0000-00-00 & & 2000 & \\
\hline 0000-00-00 & & 2000 & \\
\hline 0000-00-00 & & 2000 & \\
\hline $0000-00-00$ & & 1998 & \\
\hline 0000-00-00 & & 2000 & \\
\hline 0000-00-00 & Elephant Moré & 2014 Dec 12 & $76^{\circ} 15.320^{\prime S}$ \\
\hline 0000-00-00 & & 1998 & \\
\hline 0000-00-00 & & 1998 & \\
\hline 0000-00-00 & & 2000 & \\
\hline 0000-00-00 & & 1998 & \\
\hline 0000-00-00 & & 2000 & \\
\hline $0000-00-00$ & & 2000 & \\
\hline $0000-00-00$ & & 2009 & \\
\hline 0000-00-00 & & 1998 & \\
\hline 0000-00-00 & Elephant Moré & 2014 Nov 16 & $76^{\circ} 17.758^{\prime S}$ \\
\hline 0000-00-00 & & 2000 & \\
\hline $0000-00-00$ & & 2009 & \\
\hline $0000-00-00$ & & 1998 & \\
\hline $0000-00-00$ & & 1998 & \\
\hline $0000-00-00$ & & 2000 & \\
\hline 0000-00-00 & & 1998 & \\
\hline 0000-00-00 & & 2009 & \\
\hline $0000-00-00$ & & 2000 & \\
\hline $0000-00-00$ & & 1998 & \\
\hline 0000-00-00 & & 2000 & \\
\hline 0000-00-00 & & 2000 & \\
\hline 0000-00-00 & & 1998 & \\
\hline 0000-00-00 & & 2000 & \\
\hline $0000-00-00$ & & 2000 & \\
\hline 0000-00-00 & & 2000 & \\
\hline
\end{tabular}


0000-00-00

2000

0000-00-00 2009

0000-00-00 1998

0000-00-00 2000

0000-00-00 1998

0000-00-00 1998

0000-00-00 1998

$0000-00-00 \quad 2000$

0000-00-00 2000

$0000-00-00 \quad 2009$

0000-00-00 2000

0000-00-00 2009

$0000-00-00 \quad 2000$

$0000-00-00 \quad 2000$

0000-00-00 2000

0000-00-00 1998

$0000-00-00 \quad 2012$

0000-00-00 2000

$0000-00-00 \quad 2000$

0000-00-00 1998

0000-00-00 2000

$0000-00-00 \quad 2000$

$0000-00-00 \quad 2000$

$0000-00-00 \quad 2000$

$0000-00-00 \quad 2000$

$0000-00-00 \quad 2000$

$0000-00-00 \quad 2015$

$0000-00-00 \quad 2000$

$0000-00-00 \quad 2000$

$0000-00-00 \quad 2000$

$0000-00-00 \quad 2000$

0000-00-00 2009

$0000-00-00 \quad 2009$

$0000-00-00 \quad 2000$

0000-00-00 2010

$0000-00-00 \quad 2009$

0000-00-00 1998

0000-00-00 2015

0000-00-00 1998

0000-00-00 2000

0000-00-00 2009

0000-00-00 2015

0000-00-00 2000

0000-00-00 1998

0000-00-00 2000 


\begin{tabular}{|c|c|}
\hline $0000-00-00$ & 2010 \\
\hline 0000-00-00 & 2000 \\
\hline $0000-00-00$ & 2000 \\
\hline 0000-00-00 & 2009 \\
\hline 0000-00-00 & 2000 \\
\hline $0000-00-00$ & 2016 \\
\hline 0000-00-00 & 2000 \\
\hline 0000-00-00 & 2000 \\
\hline 0000-00-00 & 1998 \\
\hline $0000-00-00$ & 2009 \\
\hline $0000-00-00$ & 2000 \\
\hline 0000-00-00 & 2000 \\
\hline 0000-00-00 & 2000 \\
\hline 0000-00-00 & 2000 \\
\hline 0000-00-00 & 2000 \\
\hline $0000-00-00$ & 2000 \\
\hline 0000-00-00 & 2009 \\
\hline $0000-00-00$ & 2009 \\
\hline 0000-00-00 & 2000 \\
\hline $0000-00-00$ & 2014 Nov $1676^{\circ} 17.517 ' S$ \\
\hline 0000-00-00 & 2000 \\
\hline 0000-00-00 & 2000 \\
\hline 0000-00-00 & 2000 \\
\hline 0000-00-00 & 2000 \\
\hline 0000-00-00 & 2000 \\
\hline 0000-00-00 & 2000 \\
\hline $0000-00-00$ & 2000 \\
\hline $0000-00-00$ & 2015 \\
\hline 0000-00-00 & 2000 \\
\hline $0000-00-00$ & 2009 \\
\hline 0000-00-00 & 2009 \\
\hline $0000-00-00$ & 1998 \\
\hline 0000-00-00 & 2016 \\
\hline $0000-00-00$ & 2000 \\
\hline $0000-00-00$ & 1998 \\
\hline 0000-00-00 & 1998 \\
\hline 0000-00-00 & 2016 \\
\hline $0000-00-00$ & 2016 \\
\hline 0000-00-00 & 1998 \\
\hline 0000-00-00 & 2000 \\
\hline 0000-00-00 & 2000 \\
\hline $0000-00-00$ & 2009 \\
\hline 0000-00-00 & 2000 \\
\hline $0000-00-00$ & 2000 \\
\hline 0000-00-00 & 2000 \\
\hline
\end{tabular}




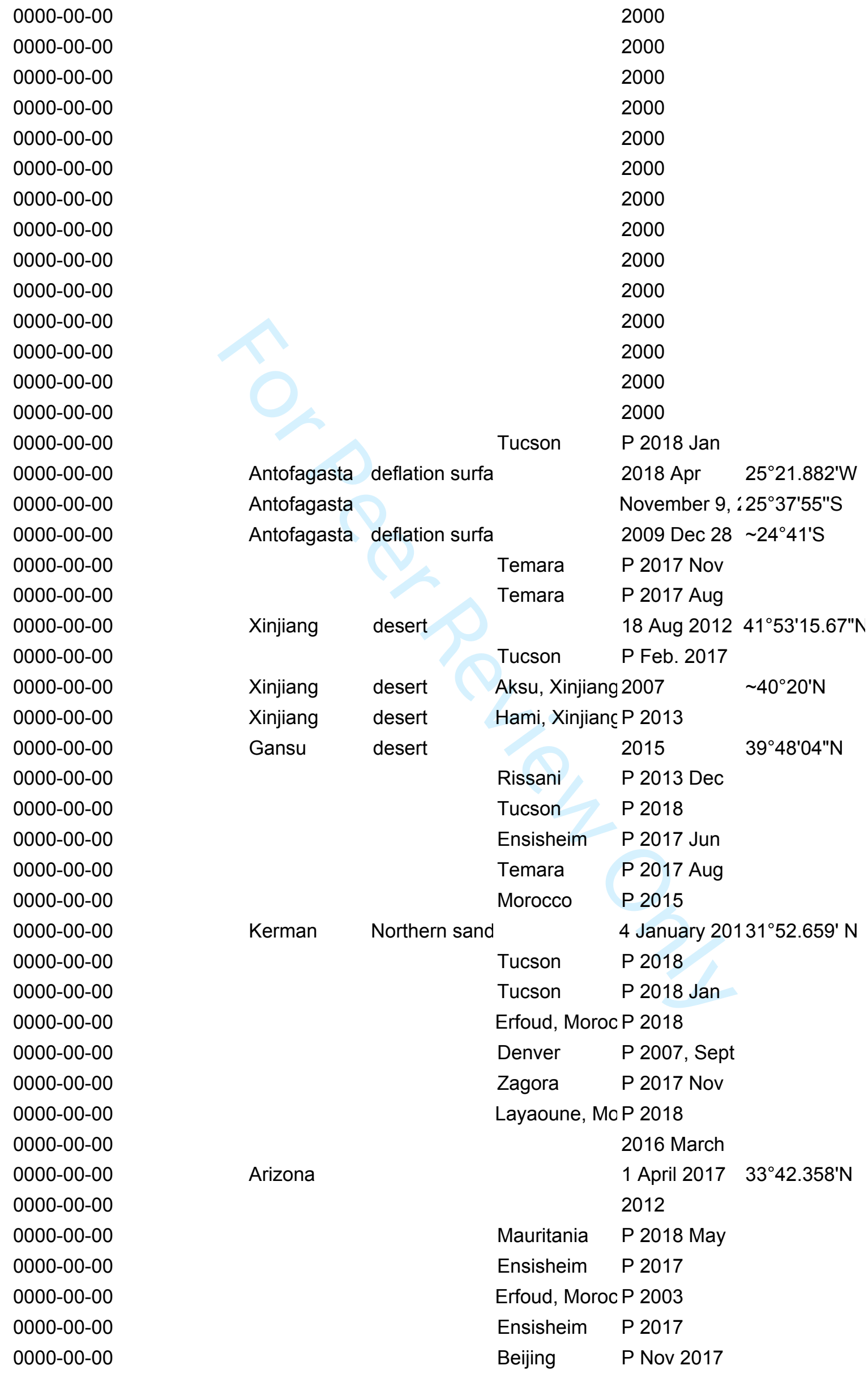




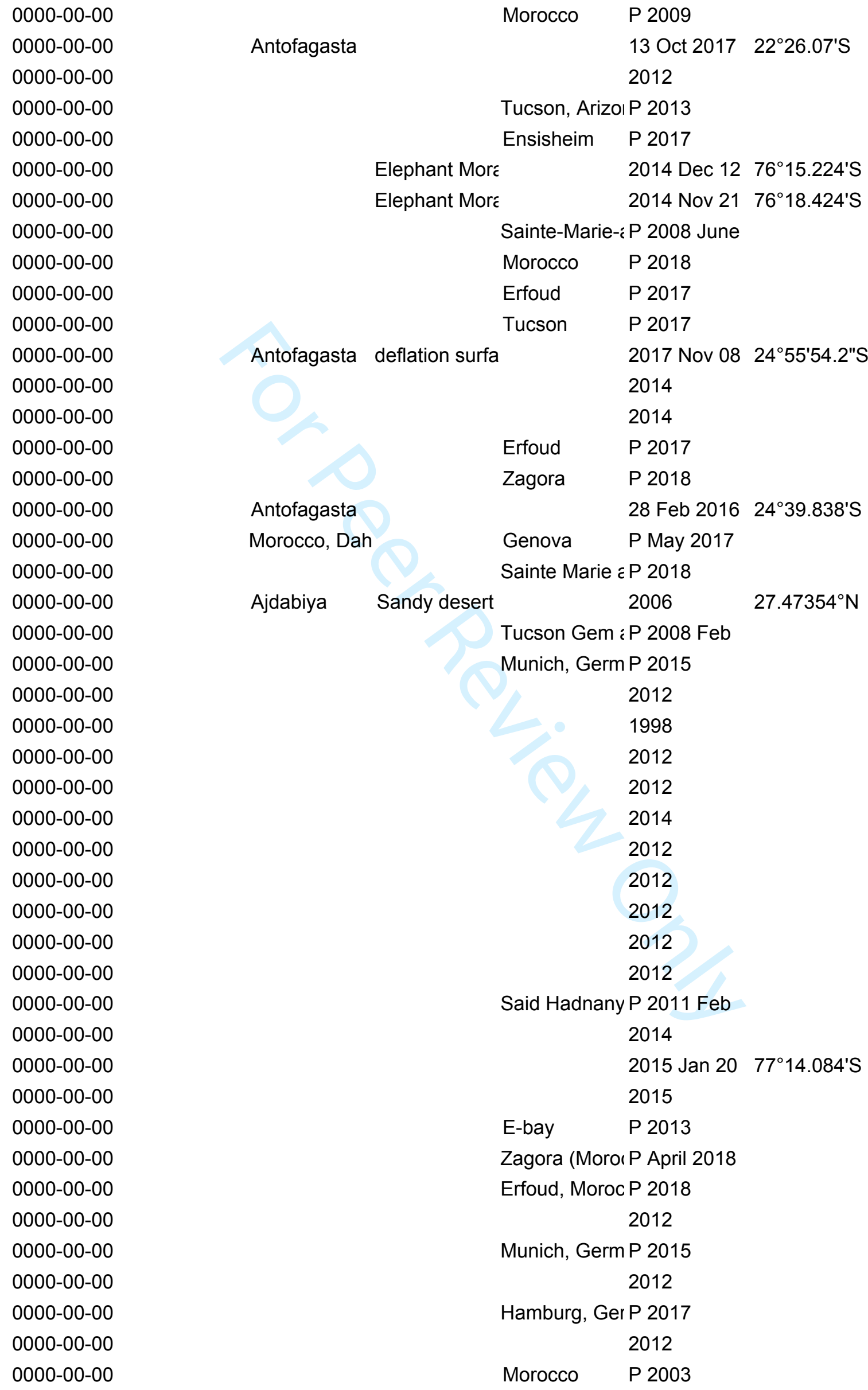


0000-00-00

0000-00-00

0000-00-00

0000-00-00

0000-00-00

0000-00-00

0000-00-00

0000-00-00

0000-00-00

0000-00-00

0000-00-00

0000-00-00

0000-00-00

0000-00-00

0000-00-00

0000-00-00

0000-00-00

0000-00-00

0000-00-00

0000-00-00

0000-00-00

0000-00-00

0000-00-00

0000-00-00

0000-00-00

0000-00-00

0000-00-00

0000-00-00

0000-00-00

0000-00-00

0000-00-00

0000-00-00

0000-00-00

0000-00-00

0000-00-00

0000-00-00

0000-00-00

0000-00-00

0000-00-00

0000-00-00

0000-00-00

0000-00-00

0000-00-00

0000-00-00

0000-00-00
Tinghir, Moro P 2017

$\begin{array}{ll}\text { Morocco } & \text { P } 2015 \text { Apr } \\ & 2012 \\ & 2012\end{array}$

Erfoud P 2016

2016

Antofagasta

16 Oct $2017 \quad 22^{\circ} 18.65^{\prime} \mathrm{S}$

2014

Tucson (Said P Jan 2009

15 Oct $201722^{\circ} 18.21^{\prime} \mathrm{S}$

Morocco P 2016 May 0

2012

2012

2014

Erfoud P March 2016

2012

2014

17 Mar $201724^{\circ} 52.25^{\prime} \mathrm{S}$

2012

Tucson (Said P Jan 2012

2012

2014

2012

2012

2014

24 Oct $2016 \quad 25^{\circ} 3.75^{\prime} \mathrm{S}$

2012

1998

Erfoud

P 2017

Zagora (Moror P April 2018

Zagora (Moror P April 2018

Guelmim Sma

2013 Dec $9 \quad 27^{\circ} 38.333^{\prime} \mathrm{N}$

2017

Tucson Gem ‘P 2005 Feb

Tucson Gem ‘P 2007 Feb

Tucson (Said P Jan 2011

Tinduf, Algerie P 2017 Dec

Erfoud P 2017

2012

2012

Morocco, Sme

Bologna P March 2016

2012

Erfoud, Moroc P 2018

Tucson (Said P Jan 2008

Said Hadnany P 2011 Feb 


\begin{tabular}{|c|c|c|c|}
\hline 0000-00-00 & & & 2012 \\
\hline 0000-00-00 & & & 2012 \\
\hline 0000-00-00 & Antofagasta & & 17 Feb $201625^{\circ} 32.669 ' S$ \\
\hline 0000-00-00 & & Tucson & P 2018 Feb \\
\hline 0000-00-00 & & & 2014 \\
\hline 0000-00-00 & & Erfoud & P 2017 \\
\hline $0000-00-00$ & & Hamburg, G & r P 2017 \\
\hline 0000-00-00 & & Morocco & P 2017 \\
\hline 0000-00-00 & & & 2014 \\
\hline 0000-00-00 & & & 2012 \\
\hline 0000-00-00 & & & 2012 \\
\hline 0000-00-00 & & Erfoud & P 2016 \\
\hline 0000-00-00 & & Erfoud & P March 2016 \\
\hline 0000-00-00 & & Layaoune, $\mathrm{N}$ & P 2018 \\
\hline 0000-00-00 & & & 2012 \\
\hline $0000-00-00$ & & & 2012 \\
\hline 0000-00-00 & desert & Beijing & P 2015 \\
\hline 0000-00-00 & & Morocco & P 2017 \\
\hline 0000-00-00 & Sahara & Tucson, AZ & $\mathrm{P} 02 / 12 / 2015$ \\
\hline 0000-00-00 & & & 2012 \\
\hline 0000-00-00 & & & 2014 \\
\hline 0000-00-00 & & Hamburg, G & r P 2017 \\
\hline $0000-00-00$ & & & 2012 \\
\hline 0000-00-00 & & & 2012 \\
\hline 0000-00-00 & & & 2012 \\
\hline 0000-00-00 & & & 2012 \\
\hline 0000-00-00 & & & 2012 \\
\hline 0000-00-00 & & & 2012 \\
\hline 0000-00-00 & & & 2012 \\
\hline 0000-00-00 & & & 2012 \\
\hline 0000-00-00 & & Hamburg, G & r P 2017 \\
\hline $0000-00-00$ & & Mauritania & P 2017 Sep \\
\hline 0000-00-00 & & & 2012 \\
\hline $0000-00-00$ & & Morocco & P 2016 May 0 \\
\hline 0000-00-00 & & & 2012 \\
\hline 0000-00-00 & & Morocco & P 2017 \\
\hline 0000-00-00 & & & 2012 \\
\hline 0000-00-00 & & Morocco & P 2017 \\
\hline 0000-00-00 & & & 2000 \\
\hline 0000-00-00 & & & 2012 \\
\hline $0000-00-00$ & & & 2012 \\
\hline 0000-00-00 & & & 2012 \\
\hline 0000-00-00 & & & 2014 \\
\hline 0000-00-00 & & & 2014 \\
\hline 0000-00-00 & & Morocco & P 2017 \\
\hline
\end{tabular}




\begin{tabular}{|c|c|c|c|}
\hline 0000-00-00 & & & 2014 \\
\hline 0000-00-00 & & & 2014 \\
\hline 0000-00-00 & & & 1979 \\
\hline 0000-00-00 & & & 1987 \\
\hline 0000-00-00 & & & 2015 \\
\hline 0000-00-00 & & & 2012 \\
\hline 0000-00-00 & & & 2014 \\
\hline 0000-00-00 & & & 2014 \\
\hline 0000-00-00 & & & 2012 \\
\hline 0000-00-00 & & & 2014 \\
\hline 0000-00-00 & & & 1998 \\
\hline 0000-00-00 & & & 2000 \\
\hline 0000-00-00 & & & 1998 \\
\hline 0000-00-00 & & & 1998 \\
\hline 0000-00-00 & & & 2000 \\
\hline 0000-00-00 & & Reckling Peak & 2014 Nov $1076^{\circ} 12.608^{\prime} \mathrm{S}$ \\
\hline 0000-00-00 & & Tucson Gem & ¿P 2017 \\
\hline 0000-00-00 & & Morocco & P 2017 \\
\hline 0000-00-00 & & Guelmim, Mo & rP 2017 Oct \\
\hline 0000-00-00 & Antofagasta & Gravel pedim€ & 2017 Feb $2124^{\circ} 39.116^{\prime} S$ \\
\hline 0000-00-00 & & Tucson & P 2018 Feb \\
\hline 0000-00-00 & & Nouadhibou, & P 2018 Jan \\
\hline 0000-00-00 & & Tindouf, Algeı & r P 2018 \\
\hline 0000-00-00 & & Morocco & P 2018 Apr \\
\hline 0000-00-00 & & Western Sahe & aP 2017 \\
\hline 0000-00-00 & & Tucson & 2015 \\
\hline 0000-00-00 & & & 2000 \\
\hline 0000-00-00 & & & 2000 \\
\hline 0000-00-00 & & & 2014 \\
\hline 0000-00-00 & & Mauritania & P 2017 Mar \\
\hline 0000-00-00 & & Vienna & P 2013 \\
\hline 0000-00-00 & & Morocco & P 2016 May 1 \\
\hline 0000-00-00 & & Erfoud & P 2018 March \\
\hline 0000-00-00 & & Erfoud, Moroc & c P 2012 \\
\hline 0000-00-00 & & Zagora & P 2018 Aug \\
\hline 0000-00-00 & & Mauritania & P 2017 \\
\hline 0000-00-00 & & Mali & P 2017 \\
\hline 0000-00-00 & & Mauritania & P 2017 Mar \\
\hline 0000-00-00 & & Mauritania & P 2017 \\
\hline 0000-00-00 & & Morroco & 2017 \\
\hline 0000-00-00 & & Morocco & P 2018 \\
\hline 0000-00-00 & Tindouf & Tindouf Hama Denver, USA & 2016 Oct 10 \\
\hline 0000-00-00 & & Mauritania & P 2018 \\
\hline 0000-00-00 & & Tucson & P 2016 \\
\hline 0000-00-00 & & Mali & P 2018 \\
\hline
\end{tabular}




\begin{tabular}{|c|c|c|c|c|c|}
\hline $0000-00-00$ & Rio de Oro & & Denver & P 2016 Sep & $25.634^{\circ} \mathrm{N}$ \\
\hline $0000-00-00$ & & & Morocco & P 2015 & \\
\hline 0000-00-00 & & & Morocco & P 2017 & \\
\hline $0000-00-00$ & & & Mali & P 2018 Mar & \\
\hline $0000-00-00$ & & & Morocco & P 2015 & \\
\hline 0000-00-00 & & Desert & & April 2016 & $31^{\circ} 41.28102 ' \uparrow$ \\
\hline 0000-00-00 & & & St. Marie aux & P 2017 & \\
\hline $0000-00-00$ & & & Erfoud & P 2017 Sep & \\
\hline 0000-00-00 & & & Morocco & P 20 Sep 201، & \\
\hline 0000-00-00 & & & Morocco & P 2016 Jun & \\
\hline $0000-00-00$ & & & Mauritania & P 2018 May & \\
\hline $0000-00-00$ & & & Gladstone, Or & P 2018 May & \\
\hline 0000-00-00 & & & Erfoud & P 2015 Apr & \\
\hline 0000-00-00 & Antofagasta & deflation surfa & & 2016 Nov & $\sim 24^{\circ} 20^{\prime} S$ \\
\hline 2018-11-16 & Updated clas: & & Ensisheim & P 2017 & \\
\hline 2018-11-16 & Updated clas: & & Zagora, Moroc & (P 2018 & \\
\hline 2018-11-16 & Updated clas: Rio de Oro & & Zagora & 2017 Dec 8 & 24.135944 \\
\hline 2018-11-16 & Updated clas: & & Guelmim, Mor & P 2018 Mar & \\
\hline 0000-00-00 & & & Morocco & P 2018 & \\
\hline 0000-00-00 & Rio de Oro & Jrifiya & & 2014 May 25 & $24.732^{\circ} \mathrm{N}$ \\
\hline 0000-00-00 & & & Ouarzazate & P 2018 Jul & \\
\hline 0000-00-00 & & & Ouarzazate & P 2018 Jul & \\
\hline 0000-00-00 & & & Bordj Badji Mc & cP 2018 Aug & \\
\hline 0000-00-00 & & & Agadir & P 2018 Aug & \\
\hline 0000-00-00 & & & Zagora & P 2018 May & \\
\hline $0000-00-00$ & & & Zagora & P 2018 Jun & \\
\hline 0000-00-00 & Tindouf & Tindouf Hama & Denver, USA & 2016 Oct 10 & \\
\hline 0000-00-00 & & & Tucson & P 2016 & \\
\hline $0000-00-00$ & & & Mauritania & P 2018 Jan & \\
\hline 0000-00-00 & & & & 2012 & \\
\hline 0000-00-00 & & & Zagora & P 2018 May & \\
\hline 0000-00-00 & & & Tindouf, Alger & P 2018 & \\
\hline 0000-00-00 & Zufar & Desert plain & & 31.01 .16 & $19^{\circ} 0.580^{\prime} \mathrm{N}$ \\
\hline $0000-00-00$ & & & Morocco & P 2015 & \\
\hline $0000-00-00$ & & & France & P 2017 & \\
\hline 0000-00-00 & & & St. Marie-aux- & - P June 2016 & \\
\hline 0000-00-00 & & & Zagora & P 2018 Jan & \\
\hline 0000-00-00 & & & Agadir & P 2011 Apr & \\
\hline 0000-00-00 & & & Erfoud & P 2015 & \\
\hline 0000-00-00 & & & Munich & P 2005 & \\
\hline 0000-00-00 & Al Wusta & & & 2011 & $19.839^{\circ} \mathrm{N}$ \\
\hline 0000-00-00 & & & Algeria & P 2018 & \\
\hline $0000-00-00$ & & & Erfoud, Moroc & $P 2007$ & \\
\hline $0000-00-00$ & & & Morocco & P 2017 & \\
\hline $0000-00-00$ & Vaud & Pasturage & & 1959 & $46^{\circ} 51.07^{\prime} \mathrm{N}$ \\
\hline
\end{tabular}


0000-00-00

0000-00-00

0000-00-00

0000-00-00

0000-00-00

0000-00-00

0000-00-00

0000-00-00

0000-00-00

0000-00-00

0000-00-00

0000-00-00

0000-00-00

0000-00-00

0000-00-00

0000-00-00

0000-00-00

0000-00-00

0000-00-00

0000-00-00

0000-00-00

0000-00-00

0000-00-00

0000-00-00

0000-00-00

0000-00-00

0000-00-00

0000-00-00

0000-00-00

0000-00-00

0000-00-00

0000-00-00

0000-00-00

0000-00-00

0000-00-00

0000-00-00

0000-00-00

0000-00-00

0000-00-00

0000-00-00

0000-00-00

0000-00-00

0000-00-00

0000-00-00

0000-00-00
Tucson

P 2017 Jan

Saguia el Han

2004

$27^{\circ} 14^{\prime} 25.7^{\prime \prime} \mathrm{N}$

Mauritania P 2017 Dec

Erfoud, Moroc P 2018

Zagora, Moroc P 2014

Tucson Gem :P 2017

Tucson Gem ‘P 2017

Morocco P 2017 Sep

Ensisheim P 2011 Jun

Zagora P $2017 \mathrm{Nov}$

Tucson Gem :P 2018 Jan

Morocco P 2017 April

Erfoud, Moroc P March 2017

Zagora, Moroc P 2014

Erfoud, Moroc P Nov 2016

Tucson P 2007 Jan

Guelmim, Mor P 2017 Sep

Morocco P 2018

Munich P 2015 Oct

Mali $\quad P 2017$

Morocco P 2017

Mauritania P May 2018

Erfoud, Moroc P Feb 2017

Mauritania P May 2018

Morocco P 2017

Tucson P 2017

Al Wusta

15 Feb $201519^{\circ} 44.58^{\prime} \mathrm{N}$

Western Saha P 2017

Mauritania P May 2018

Temara P 2017 Aug

Zagora P $2017 \mathrm{Mar}$

Al Wusta

15 Feb $201519^{\circ} 42.31^{\prime} \mathrm{N}$

Guelmim, Mor P 2017 Jul

Erfoud, Moroc P Nov 2016

Tindouf, Alger P 2017 Dec

Elephant Morć

2014 Dec 12 76¹7.138'S

Denver P2006 Sept

Morocco P 27 Jul 2015

Al Wusta $\quad$ MC 154

2014 May $7 \quad 19^{\circ} 46.660^{\prime} \mathrm{N}$

Mauritania P May 2018

Tucson P 2014 Jan

Erfoud P 2004 Oct

Morocco P 2018

14 Feb 2018 2529.875'S

Antofagasta 


\begin{tabular}{|c|c|c|c|}
\hline $0000-00-00$ & Al Wusta & & 15 Feb $201519^{\circ} 25.34^{\prime} \mathrm{N}$ \\
\hline $0000-00-00$ & & \multicolumn{2}{|c|}{ Missour, Moro P 2017 Sep } \\
\hline $0000-00-00$ & & Morocco & P 2016 \\
\hline $0000-00-00$ & North West Af & Ouarzazate & P October 201 \\
\hline 0000-00-00 & & \multicolumn{2}{|c|}{ Malaga, Spair P 2018 Jan } \\
\hline 0000-00-00 & & \multicolumn{2}{|c|}{ Zagora, Moroc P 2012} \\
\hline $0000-00-00$ & & Temara & P 2017 Aug \\
\hline $0000-00-00$ & & Morocco & P 2017 \\
\hline 0000-00-00 & Al Wusta & & 20 Apr $201420^{\circ} 34.104^{\prime} \mathrm{N}$ \\
\hline 0000-00-00 & & & 2000 \\
\hline $0000-00-00$ & & & 2010 \\
\hline 2018-08-03 & reclassified u: & Mauritania & P 2017 Jun \\
\hline 0000-00-00 & & \multicolumn{2}{|c|}{ Tucson, Arizoı P 2012} \\
\hline 2018-08-13 & reclassified $b$ & Tucson & P 2018 Jan \\
\hline
\end{tabular}




\begin{tabular}{|c|c|c|c|c|c|c|}
\hline tbl_long & tbl_pieces & tbl_shock & tbl_weath & tbl_fa & tbl_fs & tbl_wo \\
\hline \multicolumn{3}{|c|}{$10^{\circ} 311^{\prime} 0.40 " W 21$} & high & \multicolumn{3}{|c|}{$10.9 \pm 0.0(\mathrm{~N}=29.8 \pm 0.0(\mathrm{~N}=2) 1.5 \pm 0.3$} \\
\hline & 2 & low & moderate & $12.0 \pm 0.1$ & $10.4 \pm 0.1$ & $4.5 \pm 0.1$ \\
\hline \multirow[t]{22}{*}{$156^{\circ} 34.277^{\prime} \mathrm{E}$} & & & & $10.4 \pm 0.5$ & $10.4 \pm 0.3$ & $2.1 \pm 0.2$ \\
\hline & 1 & low & low & \multicolumn{3}{|c|}{$31.2 \pm 0.1(n=1.11 .2 \pm 0.5(n=1.43 .4 \pm 0.6(n=1}$. \\
\hline & 1 & high & low & $1.0-1.1$ & $0.9-1.0$ & $1.0-2.3$ \\
\hline & 1 & moderate & low & \multicolumn{3}{|c|}{$70.0 \pm 0.2(\mathrm{~N}=140.5 \pm 3.3(\mathrm{~N}=210.6 \pm 4.1(\mathrm{~N}=2$} \\
\hline & 1 & moderate & low & \multicolumn{3}{|c|}{$28.3 \pm 3.3,24.47 .3 \pm 1.6,30.0 \pm$} \\
\hline & 2 & low & weak & \multicolumn{3}{|c|}{$37.3 \pm 0.6(n=129.3 \pm 0.2(n=13.1 \pm 0.1 \quad(n=13$} \\
\hline & 1 & high & low & $9.6 \pm 2.5$ & $19.3 \pm 0.8$ & $2.6 \pm 0.4$ \\
\hline & 1 & & moderate & & \multicolumn{2}{|c|}{$38.7 \pm 3.8(\mathrm{~N}=86.9 \pm 2.0$} \\
\hline & 2 & low & low & & $5.1-78.7$ & $38.8-15.4$ \\
\hline & several & low & low & $39.6-79.7$ & $20.5-45.9$ & $51.6-52.8$ \\
\hline & 1 & low & low & $36.6-84.1$ & $20.9-46.7$ & $52.0-52.9$ \\
\hline & 3 & low & moderate & & $0.2 \pm 0.2$ & $0.4 \pm 0.1$ \\
\hline & 1 & low & high & 26.8 & $22.1 ; 9.1$ & $2.1 ; 43.6$ \\
\hline & 1 & low & moderate & 28.5 & 25.4 & 2.2 \\
\hline & 1 & low & moderate & 32.3 & 11.4 & 43.3 \\
\hline & several & S2 & low & $1.1-70.7$ & $1.0-7.0$ & $1.2-0.4$ \\
\hline & 1 & & B & \multicolumn{3}{|c|}{$11.4(0.6-49.91 .6(0.8-3.2)$} \\
\hline & 1 & & B & \multicolumn{3}{|c|}{$19.3(0.5-31.517 .1(0.9-9.5)$} \\
\hline & 1 & S1 & low & $1.2-51.0$ & $0.9-1.0$ & $1.0-3.6$ \\
\hline & 1 & & minimal & \multicolumn{3}{|c|}{$11.9 \pm 17.1(\mathrm{~N}=3.2 \pm 2.2(\mathrm{~N}=3) 3.8 \pm 1.1$} \\
\hline & 1 & & $B$ & \multicolumn{3}{|c|}{$13.5(1.1-45.33 .7(2.0-5.5)$} \\
\hline & 1 & low & low & $26.2 \pm 3.3$ & $15.3 \pm 1.8$ & $0.7 \pm 0.2$ \\
\hline \multirow[t]{15}{*}{$69^{\circ} 08.20^{\prime} \mathrm{W}$} & 3 & S1 & moderate & \multicolumn{3}{|c|}{$0.47 \pm 0.10(\mathrm{~N}=1.42 \pm 0.39(\mathrm{~N}=0.88 \pm 0.63(\mathrm{~N}=$} \\
\hline & 1 & & C & 15 & $5-14$ & \\
\hline & 1 & S3 & W1 & \multicolumn{3}{|c|}{$1.7 \pm 0.2(n=6 ; 2.5 \pm 0.2(n=152.1 \pm 0.1 ; 39.0 \pm$} \\
\hline & 1 & S1 & low & $22.4 ; 29.6$ & $16.3 ; 26.9$ & $0.9 ; 1.1$ \\
\hline & 1 & S3 & W1 & \multicolumn{3}{|c|}{$28.0 \pm 1.2(n=123.7 \pm 1.0(n=41.7 \pm 0.2$} \\
\hline & 1 & S2 & low & $34.2-34.3$ & $9.3-9.8$ & $46.5-46.0$ \\
\hline & 1 & S2 & low & $32.1-32.4$ & $26.7-29.7$ & $0.8-1.8$ \\
\hline & 1 & & $A / B$ & & $11-15$ & \\
\hline & 1 & S2 & low & $30.9-31.1$ & $25.4-26.3$ & $0.5-0.9$ \\
\hline & 1 & S1 & W2 & 29.9 & $25.7 ; 7.0$ & $0.8 ; 47.5$ \\
\hline & 1 & S3 & W1 & \multicolumn{3}{|c|}{$34.0 \pm 1.3(n=713.5 \pm 0.4(n=649.3 \pm 1.3$} \\
\hline & 1 & S2 & low & \multicolumn{3}{|c|}{$33.4 \pm 0.3(n=126.8 \pm 0.2(n=90.8 \pm 0.1(n=9)$} \\
\hline & 1 & & $\mathrm{~B}$ & 31 & 23 & \\
\hline & 1 & S2 & W3 & 31.1 & $25.8 ; 11.3$ & $1.0 ; 48.2$ \\
\hline & 1 & low & moderate & $29.9 \pm 1.0$ & $25.4 \pm 1.0$ & $0.7 \pm 0.0$ \\
\hline $156^{\circ} 26.081^{\prime} \mathrm{E}$ & 1 & & & $29.8 \pm 0.2$ & $25.5 \pm 0.7$ & $0.6 \pm 0.04$ \\
\hline & 1 & S2 & W1 & \multicolumn{3}{|c|}{$33.2 \pm 0.2(n=1$} \\
\hline & 1 & S2 & W4 & $30.1 \pm 0.5(r$ & $2.5(n=1)$ & $0.6(n=1)$ \\
\hline & 16 & S1 & W2 & \multicolumn{3}{|c|}{$29.5 \pm 0.2(n=125.3 \pm 0.5(n=31.6 \pm 0.6(n=3)$} \\
\hline & 1 & moderate & moderate & \multicolumn{3}{|l|}{$27.2 \pm 0.9$} \\
\hline
\end{tabular}




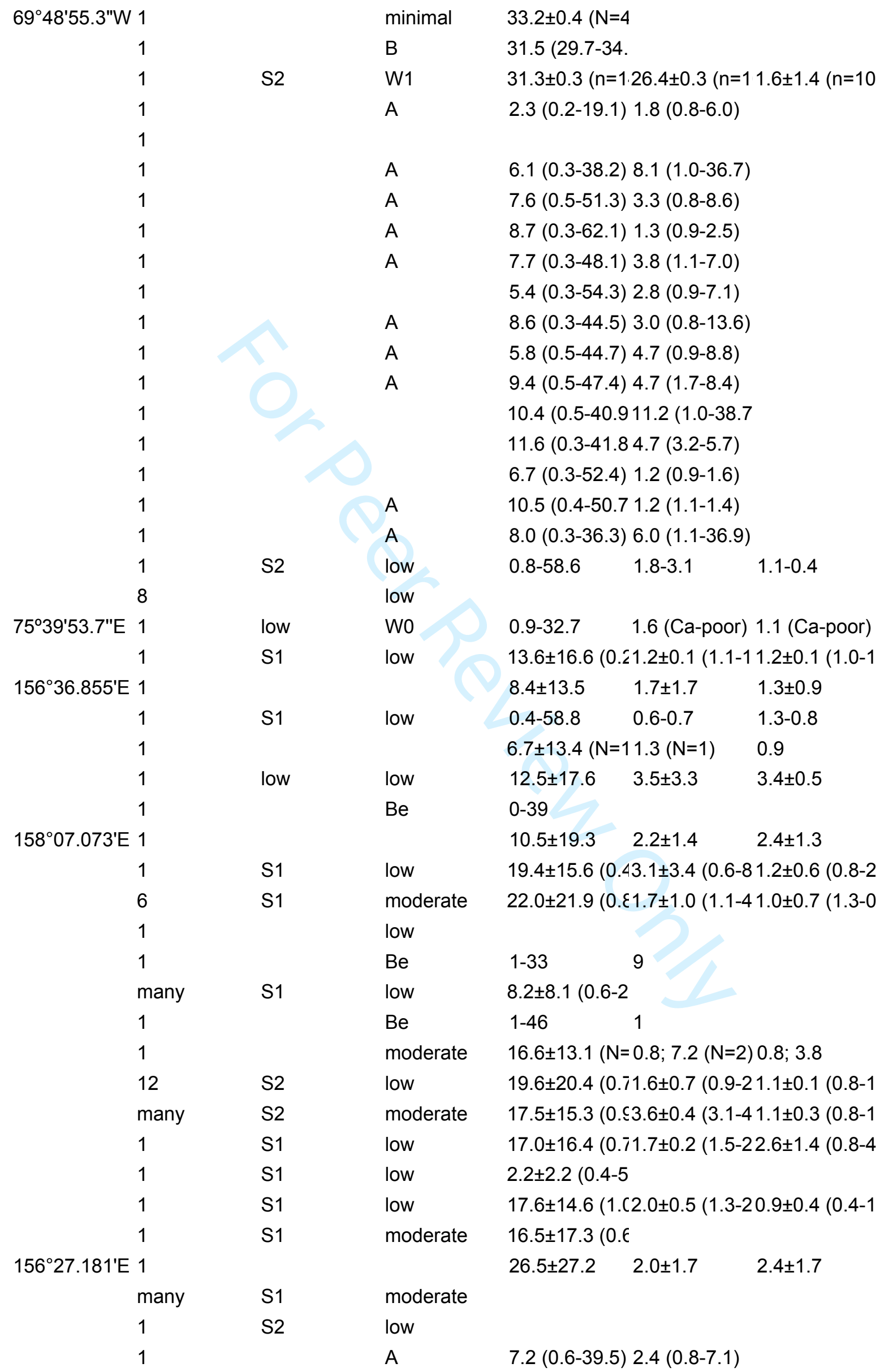




\begin{tabular}{|c|c|c|c|c|c|c|}
\hline & 1 & & A & \multicolumn{3}{|c|}{$15.4(0.3-60.43 .2(0.9-11.2)$} \\
\hline & 1 & & $A$ & \multicolumn{3}{|c|}{$13.9(0.2-54.411 .0(0.7-29.4$} \\
\hline & 1 & & B & $3.4(0.4-8.4)$ & \multicolumn{2}{|c|}{$1.6(0.7-4.0)$} \\
\hline & 1 & S2 & low & $0.2-41.0$ & 2.3-23.2 & $0.8-0.9$ \\
\hline & 1 & S2 & low & $1.2-64.7$ & $0.6-27.5$ & $0.8-3.0$ \\
\hline & 25 & S1 & W1 & \multicolumn{3}{|c|}{$16.7 \pm 15.0(1 . C 2.7 \pm 0.8(2.0-41.0 \pm 0.1(0.7-1$} \\
\hline \multirow[t]{3}{*}{$\sim 70^{\circ} 32^{\prime} \mathrm{W}$} & 1 & & minimal & \multicolumn{3}{|c|}{$16.8 \pm 18.2(\mathrm{~N}=$} \\
\hline & Many & S1 & W2 & $36.0 \pm 8.0$ & $5.7 \pm 3.1$ & $2.8 \pm 1.4$ \\
\hline & 1 & low & weak & \multicolumn{3}{|c|}{$5.0 \pm 2.6(n=161.9 \pm 0.9(n=151.9 \pm 1.4(n=15$} \\
\hline $68^{\circ} 38.61^{\prime} \mathrm{W}$ & 1 & & & \multicolumn{3}{|c|}{$22.2 \pm 18.4(\mathrm{~N}=11.6 \pm 10.8(\mathrm{~N}=1.8 \pm 1.4(\mathrm{~N}=2 \mathrm{C}$} \\
\hline \multirow[t]{4}{*}{$\sim 70^{\circ} 32^{\prime} \mathrm{W}$} & 1 & & moderate & \multicolumn{2}{|c|}{$18.0 \pm 15.6(\mathrm{~N}=1.0(\mathrm{~N}=1)$} & 4.0 \\
\hline & 1 & S1 & Low & $21.5 \pm 14.3$ & $3.6 \pm 3.0$ & $1.9 \pm 1.1$ \\
\hline & 1 & S2 & moderate & \multicolumn{3}{|c|}{$10.3 \pm 14.5(n=6.2 \pm 12.0(n=11.5 \pm 1.0$} \\
\hline & 1 & S1 & moderate & \multicolumn{3}{|c|}{$11.9 \pm 14.0(n=2.3 \pm 1.3(n=121.7 \pm 1.5$} \\
\hline \multirow[t]{23}{*}{$54.467^{\circ} \mathrm{E}$} & 1 & S2 & low & $1.7-60.7$ & $0.6-36.6$ & $0.9-2.8$ \\
\hline & 1 & S2 & strong & \multicolumn{3}{|c|}{$8.9 \pm 9.8(n=217.2 \pm 13.3(n=11.3 \pm 1.1$} \\
\hline & 1 & S2 & & $0.5-39.9$ & $1.4 ; 6.5$ & $2.9 ; 5.7$ \\
\hline & 1 & S2 & low & \multicolumn{3}{|c|}{$18.7 \pm 14.6(n=4.8 \pm 3.4(n=242.7 \pm 2.2 \quad(n=24$} \\
\hline & 1 & S2 & W2 & $0.7-43.4$ & $0.9-6.3$ & $0.9-4.4$ \\
\hline & 1 & S2 & moderate & \multicolumn{3}{|c|}{$20.2 \pm 16.8(2 . c 1.5 \pm 2.9(1.3-21.6 \pm 0.9(0.8-3$} \\
\hline & 1 & S2 & low & $0.9-41.5$ & $12.6-23.8$ & $2.1-0.6$ \\
\hline & 1 & S1 & Low & $12.3 \pm 16.7$ & $2.7 \pm 2.4$ & $1.7 \pm 0.9$ \\
\hline & 2 & S2 & moderate & \multicolumn{3}{|c|}{$15.0 \pm 17.6(0 . \subseteq 1.5 \pm 0.4(0.9-21.6 \pm 1.2(0.8-4$} \\
\hline & 1 & low & low & $14.0 \pm 14.6$ & $2.3 \pm 1.2$ & $1.7 \pm 1.4$ \\
\hline & 1 & & moderate & \multicolumn{3}{|c|}{$10.4 \pm 10.0(N=3.6 \pm 1.4(N=4) 3.8 \pm 2.0$} \\
\hline & 1 & low & moderate & $3.3 \pm 3.3$ & $1.3 \pm 0.4$ & $1.9 \pm 1.3$ \\
\hline & 1 & & B & $9-38$ & $12-17$ & \\
\hline & 1 & & $B$ & $1-36$ & $9-19$ & \\
\hline & 1 & low & low & $29.0-29.8$ & 23.3-23.6; 1 & $3.8-3.6 ; 41.8-$ \\
\hline & 2 & S2 & moderate & $0.2-45.3$ & $0.7-0.9 ; 2.5$ & $1.1 ; 36.0$ \\
\hline & 1 & low & moderate & $2.8 \pm 2.7$ & $4.5 \pm 7.7$ & $1.2 \pm 0.5$ \\
\hline & 16 & s2 & moderate & \multicolumn{3}{|c|}{$6.8 \pm 4.7(1.4-11.6 \pm 0.5(0.8-20.7 \pm 0.2(0.4-1$} \\
\hline & 1 & S2 & strong & \multicolumn{3}{|c|}{$16.6 \pm 18.2(0.52 .2 \pm 0.4(1.8-31.1 \pm 0.1(0.9-1$} \\
\hline & 1 & S2 & low & $0.4-16.8$ & $0.8-13.8$ & $0.9-3.5$ \\
\hline & 3 & low & low & $0.8-53.0$ & $0.7-2.4$ & $1.0-1.2$ \\
\hline & 1 & low & moderate & $11.3 \pm 15.6$ & $1.4 \pm 0.4$ & $1.1 \pm 0.8$ \\
\hline & many & S3 & moderate & 7.4 & 1.8 & 1.6 \\
\hline \multirow[t]{8}{*}{$69^{\circ} 43^{\prime} \mathrm{W}$} & 1 & & moderate & \multicolumn{2}{|c|}{$4.73 \pm 6.1(n=610.0(n=1)$} & $0.6(n=1)$ \\
\hline & 1 & S1 & moderate & \multicolumn{3}{|c|}{6.7 (chondrule } \\
\hline & 1 & S2 & high & $3.2 \pm 2.5 ; 11$. & $1.1 \pm 0.3$ & $1.8 \pm 1.8$ \\
\hline & several & S2 & moderate & $1.2-58.9$ & $1.1-1.2$ & $0.7-4.2$ \\
\hline & 1 & low & moderate & $4.4 \pm 4.1$ & $1.1 \pm 0.3$ & $0.9 \pm 0.2$ \\
\hline & 5 & S2 & high & $5.5 \pm 4.9(0.6$ & $1.2 \pm 0.2(1.0$ & $1.0 \pm 0.1(0.9-1$ \\
\hline & 1 & & low & $10.0 \pm 10.5(\wedge$ & $=1.2 \pm 0.4(\mathrm{~N}=$ & $0.9 \pm 0.1$ \\
\hline & 1 & s2 & low & $0.6-58.7$ & $1.7-4.1$ & $1.2-1.3$ \\
\hline
\end{tabular}




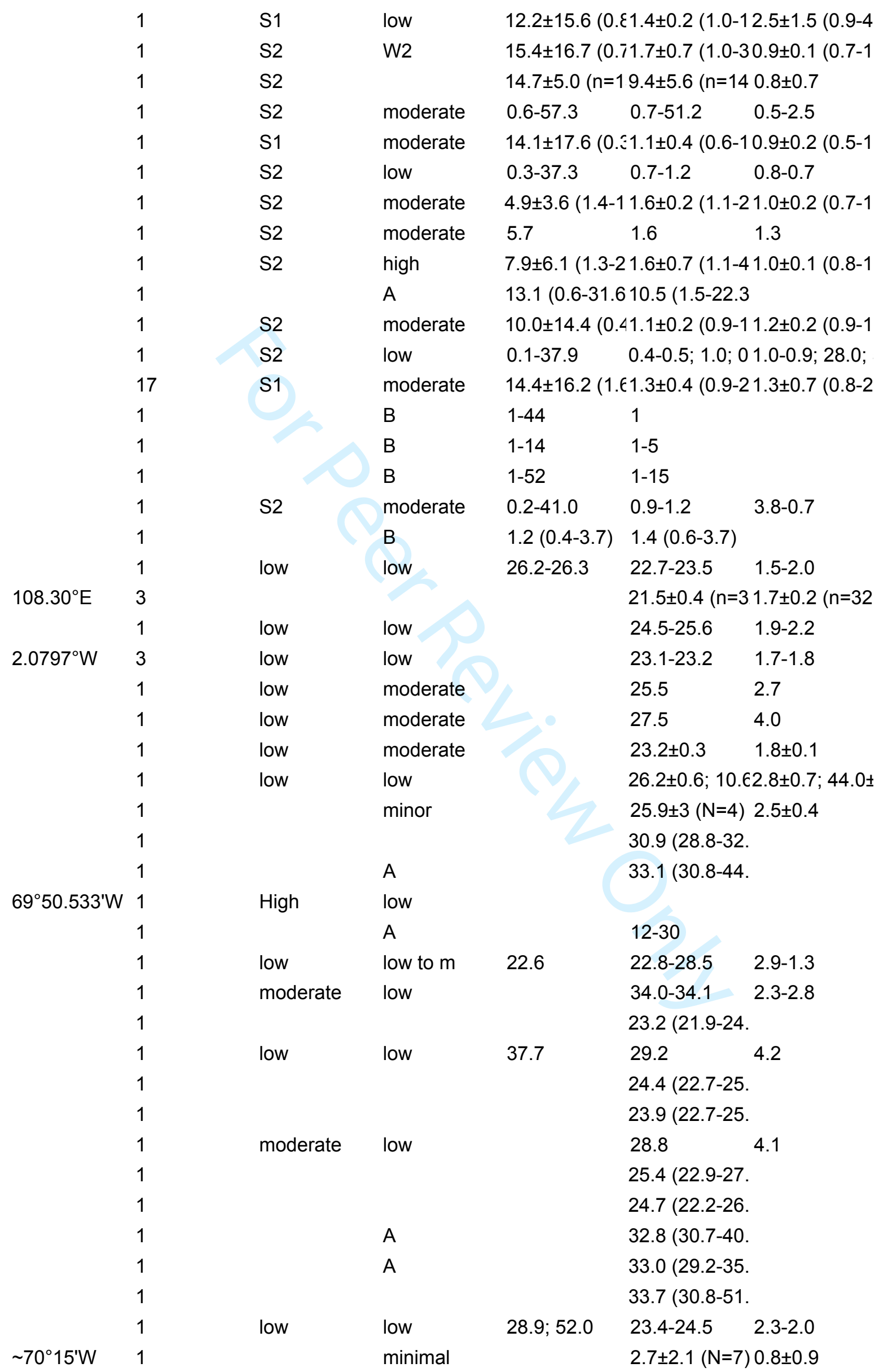




\begin{tabular}{|c|c|c|c|c|c|}
\hline & 1 & & B & \multicolumn{2}{|c|}{$1.0(0.0-5.0)$} \\
\hline$\sim 70^{\circ} 15^{\prime} \mathrm{W}$ & 1 & & minimal & \multicolumn{2}{|c|}{$0.4 \pm 0.1(\mathrm{~N}=3) 1.4 \pm 0.0$} \\
\hline \multirow[t]{4}{*}{$70^{\circ} 32^{\prime} \mathrm{W}$} & 2 & & minimal & \multicolumn{2}{|c|}{$0.3 \pm 0.0(\mathrm{~N}=3) 1.6 \pm 0.0$} \\
\hline & 1 & S2 & W2 & \multicolumn{2}{|c|}{$0.4 \pm 0.1(n=171.4 \pm 0.2(n=17$} \\
\hline & 1 & S1 & W4 & \multicolumn{2}{|c|}{$0.4 \pm 0.1(n=171.6 \pm 0.1(n=17$} \\
\hline & 1 & & A & \multicolumn{2}{|c|}{$0.4(0.1-0.9)$} \\
\hline \multirow[t]{5}{*}{$158^{\circ} 13.450^{\prime}$} & 1 & & & & \\
\hline & 1 & & & 0.3 & 1.6 \\
\hline & 1 & low & W1 & \multicolumn{2}{|c|}{$0.1 \pm 0.2(n=180.1 \pm 0.2(n=191.4 \pm 0.3(n=19$} \\
\hline & 1 & moderate & low & \multicolumn{2}{|c|}{$43.9 \pm 2.2 ; 61.71 .7 \pm 0.6 ; 44.6 \pm$} \\
\hline & 1 & & & & \\
\hline $156^{\circ} 41.497^{\prime} \mathrm{E}$ & 1 & & & $45.9 \pm 0.6$ & $1.7 \pm 0.6$ \\
\hline \multirow[t]{2}{*}{$156^{\circ} 38.370^{\prime} \mathrm{E}$} & 1 & & & $37.3 \pm 14.8$ & $3.1 \pm 1.7$ \\
\hline & 3 & moderate & moderate & \multicolumn{2}{|c|}{$49 \pm 13(N=10) 17 \pm 16$} \\
\hline \multirow[t]{2}{*}{$54^{\circ} 8.889^{\prime} \mathrm{E}$} & 4 & & low & \multicolumn{2}{|c|}{$56.8 \pm 3.3(n=17.2 \pm 4.4$} \\
\hline & 1 & moderate & low & \multicolumn{2}{|c|}{$61.2 \pm 0.5 ; 26.42 .5 \pm 0.4 ; 44.2 \pm$} \\
\hline \multirow[t]{20}{*}{$54^{\circ} 31.15^{\prime} \mathrm{E}$} & 3 & & & \multicolumn{2}{|c|}{$50.2 \pm 14.9(\mathrm{~N}=15.22 \pm 18.0(\mathrm{~N}$} \\
\hline & 1 & low & low & \multirow[t]{2}{*}{$58.3 ; 29.6$} & \multirow[t]{2}{*}{$3.9 ; 39.4$} \\
\hline & 1 & low & low & & \\
\hline & 1 & low & low & $57.7 ; 23.7$ & $1.9 ; 44.6$ \\
\hline & 1 & moderate & low & $35.6 ; 45.0$ & $6.3 ; 31.5$ \\
\hline & 1 & low & moderate & $62.6 ; 34.4$ & $6.6 ; 39.6$ \\
\hline & 11 & moderate & moderate & $61.6 ; 26.8$ & $2.4 ; 43.3$ \\
\hline & 1 & & $A$ & \multicolumn{2}{|c|}{$62.7(60.8-64$} \\
\hline & 1 & medium & medium & \multicolumn{2}{|c|}{$36.6 \pm 1.1(n=63.3 \pm 0.2 ; 44.4 \pm$} \\
\hline & 1 & & minor & $60.0 \pm 1.7$ & $2.1 \pm 0.8$ \\
\hline & 4 & low & low & $60.3 ; 26.7$ & $2.4 ; 43.4$ \\
\hline & 1 & low & low & & $59.4 \pm 0.6 ; 25.82 .0 \pm 0.2 ; 43.4 \pm$ \\
\hline & 1 & & moderate & $66.1 \pm 1.7$ & \multirow[t]{3}{*}{$4.9 \pm 0.3$} \\
\hline & 1 & & & & \\
\hline & 1 & & & & \\
\hline & 1 & & A & $47.4(46.2$ & \\
\hline & 1 & & & & \\
\hline & 1 & & & & \\
\hline & 1 & low & low & $34.2 ; 60.7$ & $9.3 ; 24.5$ \\
\hline & 1 & low & low & $58.5 ; 29.1$ & $2.8 ; 40.5$ \\
\hline $63^{\circ} 14^{\prime} \mathrm{W}$ & 1 & minimal & & & \\
\hline & 1 & strong & moderate & $60.3 ; 26.3$ & $2.6 ; 42.9$ \\
\hline & 1 & & $A$ & $46.9(32.8$ & \\
\hline & 1 & & & $61.1(58.4$ & \\
\hline & 1 & & & & \\
\hline & 1 & & & $40.3(39.1$ & \\
\hline & 1 & & & & \\
\hline & 1 & & & $40.3(39.4$ & \\
\hline & 1 & low & low & $58.3 ; 35.3$ & $6.7 ; 34.4$ \\
\hline
\end{tabular}


1
$46.5 \pm 8.3(\mathrm{~N}=48.4 \pm 0.9$

39.8 (38.6-41.

$58.1 \pm 1.3(\mathrm{~N}=36.6 \pm 1.7$

66.6 (64.0-68.

$60.3 ; 26.4 \quad 2.1 ; 42.8$

$61.6(60.6-63$.

$64.1(62.0-65$.

A

$62.9(40.3-65$.

61.5 (58.4-66.

68.2

25.3 (23.6-29. 21.7 (20.5-24.

58.5 (56.4-60.

47.9 (46.2-48.

38.1 (29.1-42.

48.9 (46.4-49.

$35.3 \pm 13.7 \quad 2.5 \pm 1.3$

73.9-74.1

69.5-69.6 1.9-1.7

47.9-49.1; 36. 5.5-4.4; 10.8 61.0-62.2; 26.4.4-2.1; 43.6-८ $61.1 ; 27.3 \quad 2.1 ; 42.4$ 42.3; $29.6 \quad 4.1 ; 37.5$ $42.3 ; 25.2 \quad 3.9 ; 39.6$ $42.1 ; 27.1 \quad 2.1 ; 40.8$ $60.9 ; 26.7 \quad 2.4 ; 42.6$ $31 \pm 9(\mathrm{~N}=45) \quad 18 \pm 17$ $62.1 \pm 1.1,28.32 .7 \pm 0.8,42.2 \pm$ $\begin{array}{lll}80.4-80.8 & 31.2-58.7 & 12.0-19.6\end{array}$ $60.8 ; 27.6 \quad 2.8 ; 42.9$ $53.1,22.6 \quad 1.9,44.1$ $59.9 ; 27.8 \quad 4.0 ; 42.1$ $59.8 ; 26.7 \quad 3.5 ; 43.2$ $63.3 ; 27.8 \quad 2.4 ; 43.2$ $59.0-59.4 ; 24.1 .8-1.5 ; 43.3-4$ 58.3-61.4; 26.6.5-3.0; 44.1-4 57.9-60.6; 26. 4.0-2.1; 41.8-4 60.8-62.5 3.6-1.8 $59.0 \pm 2.1,27.65 .2 \pm 2.5,42.4 \pm$ 


\begin{tabular}{|c|c|c|c|c|c|c|}
\hline \multirow[t]{9}{*}{$69^{\circ} 53.048^{\prime} \mathrm{W}$} & \multicolumn{2}{|l|}{2} & \multicolumn{4}{|l|}{ minimal } \\
\hline & many & moderate & low & & $58.5 ; 28.1$ & $4.6 ; 41.0$ \\
\hline & 34 & moderate & low & $43.5 \pm 6.5$ & \multicolumn{2}{|c|}{$51.4 \pm 9.8 ; 26.25 .8 \pm 2.3 ; 40.5 \pm$} \\
\hline & 2 & high & low & $84.5-85.6$ & $61.4-61.5$ & $2.8-2.0$ \\
\hline & 1 & moderate & low & & \multicolumn{2}{|c|}{$33.0 \pm 3.9(N=138.1 \pm 5.3(N=1$} \\
\hline & 1 & moderate & low & & \multicolumn{2}{|c|}{$49.7 \pm 7.6 ; 42 . c 2.5 \pm 0.7 ; 12.2 \pm$} \\
\hline & 1 & low & low & & $61.2 ; 26.0$ & $1.5 ; 42.9$ \\
\hline & many & strong & moderate & 75.0 & $64.5 ; 30.8$ & $2.4 ; 42.5$ \\
\hline & 1 & high & moderate & & $48.1 \pm 14.0$ & $10.7 \pm 7.3$ \\
\hline \multirow[t]{20}{*}{$5.532957^{\circ} \mathrm{E}$} & 1 & & & & & \\
\hline & 1 & moderate & moderate & & $26.7 ; 56.0$ & $43.0 ; 5.2$ \\
\hline & 1 & low & low & $42.3-51.0$ & \multicolumn{2}{|c|}{$27.9-29.0 ; 43.2 .1-3.6 ; 2.6-5$} \\
\hline & 1 & moderate & low & & \multicolumn{2}{|c|}{$47.8 ; 20.2 ; 29.2 .1 ; 42.2 ; 3.7$} \\
\hline & 3 & low & low & & \multicolumn{2}{|c|}{$37.6 ; 34.4 ; 29.5 .3 ; 36.3 ; 3.3$} \\
\hline & 1 & low & low & & & \\
\hline & 1 & & $A / B$ & & \multicolumn{2}{|l|}{$28-64$} \\
\hline & 1 & & $A / B$ & & \multicolumn{2}{|l|}{$27-63$} \\
\hline & 1 & high & low & & $48.3 \pm 11.6$ & $9.3 \pm 9.2$ \\
\hline & 2 & low & low & & $55.1 ; 31.6$ & $3.4 ; 34.9$ \\
\hline & 2 & high & moderate & & $45.6 ; 27.3$ & $4.7 ; 40.1$ \\
\hline & 1 & & $\mathrm{~A} / \mathrm{B}$ & & \multicolumn{2}{|l|}{$27-65$} \\
\hline & 1 & high & low & & $42.6 \pm 11.4$ & $9.8 \pm 9.1$ \\
\hline & 1 & & $A / B$ & & \multicolumn{2}{|l|}{$28-65$} \\
\hline & 1 & & $A / B$ & & \multicolumn{2}{|l|}{$28-64$} \\
\hline & 1 & low & low & & \multicolumn{2}{|c|}{$26.7 ; 43.4 ; 21.2 .9 ; 2.9 ; 40.0$} \\
\hline & 1 & & $A$ & & \multicolumn{2}{|l|}{$20-52$} \\
\hline & 1 & & A & & \multicolumn{2}{|l|}{$26-60$} \\
\hline & 1 & & $A / B$ & & \multicolumn{2}{|l|}{$29-66$} \\
\hline & 2 & moderate & low & & $41.1 ; 21.0$ & $2.6 ; 41.9$ \\
\hline \multirow[t]{16}{*}{$4^{\circ} 09^{\prime} 55.2^{\prime \prime W}$} & 2 & & low & & \multicolumn{2}{|c|}{$49.6 \pm 12.9(2716.64 \pm 13.8$ (2 } \\
\hline & 1 & & $A / B$ & & \multicolumn{2}{|c|}{$27-62$} \\
\hline & 1 & moderate & low & & \multicolumn{2}{|c|}{$59.4 \pm 1.0,33.73 .5 \pm 1.6,35.2 \pm$} \\
\hline & 1 & low & low & & & \\
\hline & 3 & & minimal & & $63.0 \pm 0.3($ & $62.2 \pm 0.4$ \\
\hline & 1 & & $A / B$ & & $25-60$ & \\
\hline & 1 & low & low & $80.5-82.6$ & 30.2-30.7; & $5.8-6.1 ; 3.9-2$ \\
\hline & 1 & low & moderate & & $57.1 \pm 1.4$ & $53.8 \pm 2.4,41.5 \pm$ \\
\hline & 1 & & A & & $29-60$ & \\
\hline & 1 & & $\mathrm{~A} / \mathrm{B}$ & & $25-61$ & \\
\hline & 1 & moderate & low & & $45.5 \pm 8.4$ & $5.2 \pm 2.0$ \\
\hline & 1 & & $\mathrm{~A} / \mathrm{B}$ & & $24-60$ & \\
\hline & 1 & & B & 20.8 (19.2- & $17.6(15.6$ & \\
\hline & 1 & & B & 20.2 & $.18 .5(15.0$ & \\
\hline & 1 & S4 & W5 & $21.7 \pm 1.0(r$ & $117.6 \pm 6.6$ & $1.2 \pm 0.8(n=6)$ \\
\hline & 1 & & W4 & & & \\
\hline
\end{tabular}




\begin{tabular}{|c|c|c|c|c|c|c|}
\hline & 1 & & W0/1 & & & \\
\hline$\sim 14^{\circ} 24^{\prime} \mathrm{W}$ & 23 & & W2 & & & \\
\hline \multirow[t]{6}{*}{$92^{\circ} 01^{\prime 2} 24.76 " \mathrm{E}$} & & & W3 & & & \\
\hline & 1 & & W2 & & & \\
\hline & 1 & & W1 & & & \\
\hline & 1 & & W2 & & & \\
\hline & 1 & & W1 to W4 & & & \\
\hline & 1 & & W3 & & & \\
\hline \multirow[t]{2}{*}{$\sim 89^{\circ} 54^{\prime} \mathrm{E}$} & 1 & & W4 & & & \\
\hline & 1 & & W4 & & & \\
\hline $90^{\circ} 12^{\prime} \mathrm{E}$ & 1 & & W1 & & & \\
\hline \multirow[t]{3}{*}{$80^{\circ} 23^{\prime} 33.46 " \mathrm{E}$} & & & W5 & & & \\
\hline & 1 & & Wo & & & \\
\hline & 1 & & W3 & & & \\
\hline \multicolumn{2}{|c|}{$102^{\circ} 2^{\prime} 48.71 " \mathrm{E} 1$} & & W2 & & & \\
\hline \multirow[t]{3}{*}{$90^{\circ} 42^{\prime} 25.67 " \mathrm{E}$} & & & W3 & & & \\
\hline & 1 & & W2 & & & \\
\hline & 1 & & W1 & & & \\
\hline $5^{\circ} 11^{\prime}$ & 5 & S1 & W2 & $19.4 \pm 1.5(n=$ & $16.4 \pm 0.5$ & $31.2 \pm 0.2$ \\
\hline \multirow[t]{2}{*}{$22.36440^{\circ} \mathrm{E}$} & 1 & S3 & W1 & $18.2 \pm 7.2(n=$ & $18.0 \pm 9.1(n$ & $42.4 \pm 1.6(n=4)$ \\
\hline & 5 & S2 & W1 & $17.3 \pm 7.5(0.3$ & $-13.1 \pm 4.7(2$ & $-0.9 \pm 0.7(0.3-1$ \\
\hline \multirow[t]{2}{*}{$70^{\circ} 02^{\prime} 28^{\prime \prime W}$} & 1 & S2 & W1 & $14.5 \pm 8.0(4.0$ & $-10.9 \pm 7.1(2$ & $3-0.8 \pm 0.3(0.3-1$ \\
\hline & 1 & S1 & W5 & 18.1 & & \\
\hline \multirow[t]{2}{*}{$70^{\circ} 23.02^{\prime} \mathrm{W}$} & 1 & S2 & W2 & $18.5 \pm 2.4(\mathrm{~N}=$ & $120.8 \pm 2.7(\mathrm{~N}$ & $12.1 \pm 1.6(\mathrm{~N}=15$ \\
\hline & 1 & S2 & W3 & $14.6 \pm 9.5(0.8$ & $-11.4 \pm 7.21$. & $11.5 \pm 1.2(0.4-4$ \\
\hline \multicolumn{2}{|c|}{$158^{\circ} 32.205^{\prime} E 1$} & S4 & W1 & $11.3 \pm 10.9$ & $19.2 \pm 7.4$ & $2.1 \pm 1.8$ \\
\hline \multicolumn{2}{|c|}{$116^{\circ} 10.319^{\prime} \mathrm{W} 1$} & S1 & W5 & \multicolumn{3}{|c|}{$18.0 \pm 0.2(n=116.1 \pm 0.2(n=1.1 .5 \pm 0.1$} \\
\hline & 1 & & B & \multicolumn{3}{|c|}{$19.0(17.4-24.16 .6(15.4-18$} \\
\hline & 1 & S1 & W3 & \multicolumn{3}{|c|}{$11.8 \pm 8.3(0.2-10.9 \pm 7.0(1.0-1.4 \pm 1.5(0.3-4$} \\
\hline & 1 & S2 & W2 & \multicolumn{3}{|c|}{$19.6 \pm 4.3(n=212.3 \pm 5.7(n=21.3 \pm 1.2(n=20$} \\
\hline & 1 & & B & \multicolumn{3}{|c|}{$15.5(3.7-28.99 .0(1.9-27.5)$} \\
\hline \multirow[t]{2}{*}{-85.979} & 1 & S2 & W3 & $14.7 \pm 4.0$ & $10.8 \pm 8.8$ & $0.9 \pm 0.5$ \\
\hline & 1 & & C & \multicolumn{3}{|c|}{$15.2(14.8-15.13 .7(10.4-17$} \\
\hline \multicolumn{2}{|c|}{$158^{\circ} 08.727^{\prime} \mathrm{E} 1$} & S2 & W1 & $20.6 \pm 7.9$ & $17.5 \pm 7.1$ & $1.1 \pm 1.1$ \\
\hline & 1 & S2 & W1 & $2.0-37.0$ & $2.1-31.3$ & $0.6-1.7$ \\
\hline & 1 & S2 & W2 & $0.8-23.1$ & $1.4-28.6$ & $0.7-1.8$ \\
\hline & 1 & & B & \multicolumn{3}{|c|}{$20.2(0.6-29.011 .4(2.1-25.6$} \\
\hline \multicolumn{2}{|c|}{$156^{\circ} 34.723^{\prime} \mathrm{E} 1$} & S2 & W2 & $14.9 \pm 5.3$ & $12.2 \pm 5.2$ & $1.3 \pm 1.3$ \\
\hline & 1 & S2 & W2 & \multicolumn{3}{|c|}{$17.6 \pm 2.5(n=915.7 \pm 3.4(n=11.4 \pm 0.5(n=11$} \\
\hline \multicolumn{2}{|c|}{$114^{\circ} 15.346^{\prime} \mathrm{W} 1$} & S3 & W1 & $18.7 \pm 2.2$ & $15.2 \pm 3.6$ & $1.6 \pm 0.8$ \\
\hline \multicolumn{2}{|c|}{$114^{\circ} 14.349^{\prime} \mathrm{W} 1$} & S3 & W2 & $17.0 \pm 6.1 ; 18$ & 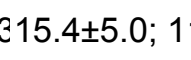 & $51.4 \pm 0.9 ; 1.1 \pm 1$ \\
\hline $114^{\circ} 16.238^{\prime} \mathrm{W}$ & & S4 & W2 & $19.5 \pm 5.1$ & $15.6 \pm 6.4$ & $1.2 \pm 1.1$ \\
\hline $114^{\circ} 14.415^{\prime} \mathrm{W}$ & & S3 & W1 & $20.1 \pm 6.0$ & $15.6 \pm 5.3$ & $1.2 \pm 1.0$ \\
\hline$\sim 69^{\circ} 43^{\prime} \mathrm{W}$ & 1 & & W1 & $18.2 \pm 1.9(\mathrm{~N}=$ & $616.9 \pm 0.5(\mathrm{~N}$ & $31.0 \pm 0.2$ \\
\hline $114^{\circ} 14.101^{\prime} \mathrm{W}$ & 1 & S3 & W2 & $17.7 \pm 9.2 ; 19$ & $615.4 \pm 6.5 ; 1$ & $61.2 \pm 1.0 ; 1.3 \pm C$ \\
\hline
\end{tabular}




\begin{tabular}{|c|c|c|c|c|c|c|}
\hline \multicolumn{2}{|c|}{$114^{\circ} 16.573^{\prime} \mathrm{W} 1$} & S3 & W1 & $17.6 \pm 9.6$ & $13.9 \pm 4.8$ & $1.4 \pm 0.7$ \\
\hline \multicolumn{2}{|c|}{$114^{\circ} 16.655^{\prime} \mathrm{W} 1$} & S3 & W1 & $19.2 \pm 7.0$ & $13.1 \pm 5.4$ & $0.9 \pm 0.8$ \\
\hline & 1 & & B & $5-44$ & $8-25$ & \\
\hline & 1 & & A & $9-41$ & $2-22$ & \\
\hline & many & S2 & W2 & $18.8-18.9$ & $15.5-17.0$ & $0.5-1.2$ \\
\hline \multirow[t]{2}{*}{$69^{\circ} 8.683^{\prime} \mathrm{W}$} & 6 & S1 & W1 & $19.86 \pm 1.57$ & 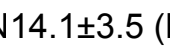 & $31.42 \pm 1.26(\mathrm{~N}=$ \\
\hline & 1 & S2 & W1 & $19.1 \pm 0.5$ & $116.2 \pm 0.1$ & $11.0 \pm 0.3(\mathrm{~N}=10$ \\
\hline \multirow[t]{2}{*}{$51^{\circ} 05.25^{\prime} \mathrm{E}$} & 25 & S3 & W4 & $17.7 \pm 0.3$ & $15.8 \pm 0.4$ & $12.0 \pm 1.1$ \\
\hline & 1 & S2 & W3 & $17.0 \pm 0.4$ & $15.2 \pm 0.2$ & $11.1 \pm 0.2(n=19$ \\
\hline \multicolumn{2}{|c|}{ 7008'42.18"V 1} & & W1 & $17.6 \pm 0.2$ & $315.8 \pm 0.4$ & $91.1 \pm 0.6$ \\
\hline \multirow[t]{3}{*}{$51^{\circ} 03.56^{\prime} \mathrm{E}$} & 1 & S2 & W3-4 & $17.5 \pm 0.4$ & $15.6 \pm 0.3$ & $91.1 \pm 0.2$ \\
\hline & 8 & S2 & W1 & $19.0 \pm 2.9$ & $17.6 \pm 3.0$ & $11.1 \pm 0.5(n=18$ \\
\hline & 1 & & W4 & $18.1 \pm 1.1$ & $116.4 \pm 0.9$ & $11.5 \pm 0.4$ \\
\hline \multirow[t]{3}{*}{$70^{\circ} 08.670^{\prime} \mathrm{W}$} & 3 & S3 & W2 & $17.8 \pm 0.5$ & $115.7 \pm 0.5$ & $11.0 \pm 0.5(\mathrm{~N}=16$ \\
\hline & 1 & & B & $19.2(18.1$ & $17.3(16.3$ & \\
\hline & 1 & S2 & W2 & $17.79 \pm 0.23$ & $16.02 \pm 0.1$ & $n 0.94 \pm 0.14(n=$ \\
\hline $83^{\circ} 50.5^{\prime} \mathrm{W}$ & 20 & S2 & wo & $18.7 \pm 0.7$ & $16.3 \pm 0.4$ & [1.3 \pm 0.1 (1SD; \\
\hline \multicolumn{2}{|c|}{$069^{\circ} 52^{\prime} 45.5^{\prime \prime} \vee 1$} & & W1 & $19.0 \pm 0.5$ & $16.8 \pm 0.4$ & $23.0 \pm 2.28$ \\
\hline & 1 & S2 & W2 & $18.84 \pm 0.5$ & $16.91 \pm 0.2$ & $n 1.11 \pm 0.35(n=$ \\
\hline & 1 & S4 & W1 & 18.2 & & \\
\hline & 1 & S2 & W2 & $17.3 \pm 0.3$ & $14.3 \pm 2.3$ & $-0.7 \pm 0.7(0.2-3$ \\
\hline & 1 & & $B$ & 18.9 (16.9 & 16.7 (14.9 & \\
\hline & 1 & S2 & W3 & $20.99 \pm 3.6$ & $16.78 \pm 0.3$ & $n 0.94 \pm 0.27(n=$ \\
\hline & 1 & S2 & W5 & 17.4 & & \\
\hline & 92 & S2 & W4 & $19.5 \pm 0.1$ & $116.3 \pm 1.4($ & $11.4 \pm 1.1(\mathrm{~N}=10$ \\
\hline \multirow[t]{2}{*}{$20.36420^{\circ} \mathrm{E}$} & 1 & S1 & W3 & $17.6 \pm 0.2$ & $15.8 \pm 0.4$ & $41.3 \pm 0.2(n=4)$ \\
\hline & 1 & & $B$ & $18.3(17.4$ & $16.1(14.0$ & \\
\hline $93.0853^{\circ} \mathrm{E}$ & 1 & S2 & w3 & $19.3 \pm 1.02$ & $=17.4 \pm 1.09$ & $=1.84 \pm 0.97(n=$ \\
\hline \multirow[t]{7}{*}{$69^{\circ} 50.650^{\prime} \mathrm{W}$} & 2 & S3 & W1 & $19.9 \pm 0.5$ & $117.2 \pm 0.5$ & $11.2 \pm 0.5(\mathrm{~N}=17$ \\
\hline & 1 & S1 & W5 & 17.3 & & \\
\hline & 1 & S2 & W1 & $18.19 \pm 0.3$ & $16.05 \pm 0.5$ & $n 0.95 \pm 1.02(n=$ \\
\hline & 1 & & B & $18.7(18.1$ & $16.4(15.9$ & \\
\hline & 1 & S1 & W3 & 17.8 & & \\
\hline & 1 & S1 & W2 & $18.56 \pm 0.4$ & $=16.42 \pm 0.5$ & $=1.24 \pm 0.16(\mathrm{n}=$ \\
\hline & 1 & & $\mathrm{Ce}$ & 17 & 15 & \\
\hline \multirow[t]{2}{*}{$20.29086^{\circ} \mathrm{E}$} & 1 & S1 & W3/4 & $17.9 \pm 0.2$ & $17.1 \pm 1.7$ & $50.7 \pm 0.6(n=5)$ \\
\hline & 1 & S1 & W4 & $19.4 \pm 0.3$ & $17.5 \pm 0.3$ & $61.2 \pm 0.1$ \\
\hline \multirow[t]{8}{*}{$68^{\circ} 50.32^{\prime} \mathrm{W}$} & 3 & S2 & w2 & $18.3 \pm 0.4$ & $216.4 \pm 0.5$ & $21.4 \pm 0.4(N=22$ \\
\hline & 1 & & $C$ & $19.0(18.1$ & $17.1(14.6$ & \\
\hline & 1 & S3 & W3 & $18.88 \pm 0.6$ & $17.02 \pm 1$ & $: 1.34 \pm 0.96(n=$ \\
\hline & 1 & S2 & W3 & $18.22 \pm 0.4$ & $16.48 \pm 0.8$ & $0.77 \pm 0.34(n=$ \\
\hline & 1 & S1 & W3 & $17.4 \pm 0.4$ & $15.2 \pm 3.9$ & $-0.7 \pm 0.2(0.4-1$ \\
\hline & 1 & & $A$ & $19.1(17.5$ & $16.5(15.5$ & \\
\hline & 1 & S1 & w2 & $18.15 \pm 0.7$ & $15.46 \pm 0.2$ & $0.61 \pm 0.49(n=$ \\
\hline & 1 & S2 & W2 & $18.51 \pm 0.1$ & $16.28 \pm 0.2$ & $n 0.84 \pm 0.2(n=1$ \\
\hline
\end{tabular}




\begin{tabular}{|c|c|c|c|c|}
\hline & 1 & S2 & W2 & $18.51 \pm 0.79(n \cdot 16.23 \pm 0.19(n) 1.44 \pm 0.13(n=$ \\
\hline \multirow[t]{7}{*}{$69^{\circ} 52.244^{\prime} \mathrm{W}$} & 1 & S1 & W4 & $19.0 \pm 0.5(\mathrm{~N}=117.4 \pm 0.5(\mathrm{~N}=21.2 \pm 0.5(\mathrm{~N}=20$ \\
\hline & 1 & S1 & W3 & $17.0 \pm 0.2(n=1.13 .8 \pm 3.1(n=20.5 \pm 0.4(n=29$ \\
\hline & 1 & S2 & W1 & $19.29 \pm 1.37(n-16.73 \pm 0.62(n-0.97 \pm 0.27(n=$ \\
\hline & 1 & S2 & W5 & $17.6 \pm 0.4$ \\
\hline & 2 & S3 & W2 & $19.2 \pm 0.3(n=716.6 \pm 0.5(n=61.3 \pm 0.1$ \\
\hline & 1 & S2 & W1 & $18.09 \pm 0.56(n-15.88 \pm 0.26(n) 1.35 \pm 0.78(n=$ \\
\hline & 1 & S2 & W2 & $18.75 \pm 1.08(n-16.86 \pm 0.56(n-1.06 \pm 0.4(n=7$ \\
\hline \multirow[t]{3}{*}{$69^{\circ} 50.234^{\prime} \mathrm{W}$} & 1 & S1 & W2 & $20.0 \pm 0.5(\mathrm{~N}=116.8 \pm 0.5(\mathrm{~N}=11.1 \pm 0.5(\mathrm{~N}=15$ \\
\hline & 1 & S2 & W1 & $18.7 \pm 0.03(n=16.74 \pm 1.02(n 0.91 \pm 0.45(n=$ \\
\hline & 1 & & B & $19.3(18.3-23.17 .1(15.6-19$ \\
\hline $20.25766^{\circ} \mathrm{E}$ & 1 & S2 & W3/4 & $17.4 \pm 0.3(n=3 \quad 15 \pm 0.2(n=3) \quad 0.4 \pm 0.7(n=3)$ \\
\hline \multirow[t]{4}{*}{$69^{\circ} 43^{\prime} 16.02^{\prime \prime V}$} & & & W1 & \\
\hline & 1 & S2 & W2 & $17.98 \pm 0.17(n-16.21 \pm 0.31(n) 1.58 \pm 0.17(n=$ \\
\hline & 1 & & B & $18.4(17.1-20.16 .3$ (15.6-17. \\
\hline & 1 & S2 & W2 & $19.12 \pm 0.45(n \cdot 17.3 \pm 0.5(n=40.89 \pm 0.18(n=$ \\
\hline \multirow[t]{21}{*}{$69^{\circ} 2.02 \mathrm{~W}$} & 1 & S1 & W1 & $18.6 \pm 1.5(\mathrm{~N}=115.3 \pm 1.7(\mathrm{~N}=11.4 \pm 0.3(\mathrm{~N}=15$ \\
\hline & 1 & S2 & W5 & $17.6 \pm 0.1$ \\
\hline & 1 & S1 & W2 & $17.34 \pm 2.26(n-16.07 \pm 1.1(n=0.57 \pm 0.21(n=$ \\
\hline & 1 & S1 & W2 & $19.1 \pm 0.3(n=716.7 \pm 0.2(n=61.3 \pm 0.1$ \\
\hline & 1 & & B & $18.7(17.2-21.16 .7(15.8-18$ \\
\hline & 1 & S2 & W3 & $18.58 \pm 0.28(n-16.98 \pm 0.74(n-1.43 \pm 0.28(n=$ \\
\hline & 1 & S1 & W2 & $17.83 \pm 0.64(n \cdot 15.74 \pm 1(n=1 \leqslant 0.96 \pm 0.49(n=$ \\
\hline & 1 & & B & 19.4 (17.9-22. 16.8 (15.7-19. \\
\hline & 1 & S2 & W2 & $19.1 \pm 0.2(n=216.6 \pm 1.0(n=10.8 \pm 0.3(n=19$ \\
\hline & 1 & & $\mathrm{Be}$ & 18 \\
\hline & 1 & S2 & W2 & $16.94 \pm 0.24(n: 15.13 \pm 0.86(n: 0.43 \pm 0.13(n=$ \\
\hline & 1 & & B & $19.3(17.9-22.16 .8(14.8-17$ \\
\hline & 1 & S2 & W2 & $16.16 \pm 0.37(n-15.36 \pm 1.2(n=0.96 \pm 0.56(n=$ \\
\hline & 1 & & B & $19.0(15.1-20.16 .5(13.1-18$ \\
\hline & 1 & S1 & W4 & $19.7 \pm 0.2(n=1.17 .9 \pm 0.2(n=51.5 \pm 0.1$ \\
\hline & 1 & S2 & W3 & $18.4 \pm 0.19(n=16.42 \pm 0.4(n=0.72 \pm 0.29(n=$ \\
\hline & 1 & & B & $19.4(18.0-22.17 .3(15.3-21$ \\
\hline & 1 & S4 & W2 & $20.7 \pm 0.2(n=718.0 \pm 0.2(n=1.1 .2 \pm 0.2$ \\
\hline & 1 & S3 & W2 & $18.44 \pm 0.36(n-16.57 \pm 0.57(n-0.98 \pm 0.32(n=$ \\
\hline & 1 & & B & $19 \quad 17$ \\
\hline & 1 & S3 & W3 & $18.58 \pm 0.31(n-16.25 \pm 0.66(n-0.92 \pm 1.15(n=$ \\
\hline \multirow[t]{8}{*}{$20.36367^{\circ} \mathrm{E}$} & 1 & S2 & W3 & $17.7 \pm 0.3(n=515.8 \pm 0.4(n=31.4 \pm 0.3(n=3)$ \\
\hline & 1 & S1 & W3 & $17.35 \pm 0.23(n \cdot 18.01 \pm 0.87(n \cdot 1.23 \pm 1.37(n=$ \\
\hline & 1 & & B & $16.4(15.5-17.14 .9(13.8-16$ \\
\hline & 1 & S3 & W2 & $18.88 \pm 0.5(n=16.63 \pm 0.74(n) 1.9 \pm 1.02(n=6$ \\
\hline & 1 & S1 & W2 & $18.53 \pm 0.2(n=16.76 \pm 0.43(n) 1.16 \pm 0.26(n=$ \\
\hline & 1 & & B & $19.4(18.5-22.16 .8$ (15.3-20. \\
\hline & 1 & & B & $18.9(17.7-20.17 .2(16.1-19$. \\
\hline & 1 & & $\mathrm{~B}$ & $18.5(16.1-21.16 .0(14.8-17$. \\
\hline
\end{tabular}




\begin{tabular}{|c|c|c|c|}
\hline & 1 & B & 19.3 (18.0-23. 16.8 (13.4-19. \\
\hline & S1 & W5 & $18.9 \pm 0.2$ \\
\hline & 1 & B & $19.1(18.2-20.16 .6(15.5-18$ \\
\hline & 1 & $B$ & $19.5(18.1-24.16 .8(15.2-19$ \\
\hline & S2 & W2 & $18.25 \pm 0.24(n-16.66 \pm 0.77(n) 1.51 \pm 0.98(n=$ \\
\hline & 1 & B & $19.5(18.4-21.17 .4(15.4-24$ \\
\hline & 1 & B & $18.9(17.0-20.17 .1(16.2-18$. \\
\hline & 1 & $\mathrm{~B}$ & $18.5(18.0-19.16 .5(14.6-21$. \\
\hline & S3 & W2 & $18.7 \pm 1$ \\
\hline & 1 & B & $19.6(17.5-23.17 .5(16.3-21$. \\
\hline & 1 & $\mathrm{~B}$ & $19.7(17.9-23.16 .6(14.3-17$. \\
\hline & S1 & W4 & $18.1 \pm 0.1(n=818.2 \pm 0.1(n=72.4$ \\
\hline & S2 & W2 & $18.51 \pm 0.29(n) 16.51 \pm 0.78(n) 1.24 \pm 0.72(n=$ \\
\hline & S2 & w2 & $17.1 \pm 0.26(n=15.33 \pm 0.43(n) 0.7 \pm 0.39(n=6$ \\
\hline & 1 & B & $20.1(18.6-22.17 .9(15.9-22$ \\
\hline & S1 & W1 & $17.55 \pm 0.59(n: 15.27 \pm 0.25(n) 0.8 \pm 0.64(n=7$ \\
\hline & 1 & B & $19.2(17.8-21.17 .1(16.0-19$ \\
\hline & S2 & W3 & $18.88 \pm 0.35(n) 16.27 \pm 0.89(n) 1.38 \pm 1.56(n=$ \\
\hline & 1 & A & $19.7(18.6-24.17 .2(16.1-20$ \\
\hline & 1 & B & $18.6(17.3-19.16 .6(14.4-19$. \\
\hline & 1 & $\mathrm{Ce}$ & $17 \quad 15$ \\
\hline & 1 & $\mathrm{Ce}$ & $7-29$ \\
\hline & 1 & B & $19.7(18.0-24.17 .4(16.2-21$. \\
\hline & 1 & $\mathrm{~B} / \mathrm{C}$ & $17 \quad 15$ \\
\hline & 1 & $\mathrm{~B} / \mathrm{C}$ & $19 \quad 17$ \\
\hline & 1 & B & $19.1(18.1-22.16 .7(15.5-17$. \\
\hline & 1 & $\mathrm{~B}$ & $19.7(17.9-23.17 .3(15.2-20$. \\
\hline & 1 & $\mathrm{~B}$ & $19.4(17.9-23.16 .9(15.6-20$ \\
\hline & S4 & W2 & $19.41 \pm 0.36(n \cdot 17.26 \pm 0.55(n) 1.66 \pm 0.9(n=6$ \\
\hline & 1 & C & $19.3(17.8-21.17 .1(16.0-19$ \\
\hline & 1 & $\mathrm{~B}$ & $18.4(17.4-22.16 .3(14.5-21$. \\
\hline & 1 & B & $19.7(17.1-21.16 .8(13.0-18$. \\
\hline & 1 & B & $17.6(15.7-21.15 .4(12.1-18$ \\
\hline & 1 & B & $18.1(16.8-20.16 .9(13.6-29$. \\
\hline & 1 & $\mathrm{~B} / \mathrm{C}$ & $17 \quad 7-26$ \\
\hline & 1 & B & $20.5(18.3-23.16 .9(15.5-18$. \\
\hline & 1 & B & $20.0(18.6-22.17 .8(15.8-19$ \\
\hline $68^{\circ} 36.42^{\prime} \mathrm{W}$ & 1 & & $17.7 \pm 0.6(\mathrm{~N}=114.9 \pm 0.7(\mathrm{~N}=11.0 \pm 0.5(\mathrm{~N}=14$ \\
\hline & 1 & $\mathrm{~B}$ & $18.3(14.7-19.15 .9(12.2-17$ \\
\hline & 1 & B & $19.4(17.5-21.17 .1$ (15.5-18. \\
\hline & 1 & B & $18.6(16.5-20.16 .0(15.2-17$. \\
\hline & 1 & $\mathrm{~B}$ & $18.9(17.6-20.17 .0(16.3-20$. \\
\hline & 1 & $\mathrm{C}$ & $19 \quad 16$ \\
\hline & 1 & B & $19.7(17.6-25.17 .7(14.6-23$. \\
\hline & 1 & $\mathrm{~B}$ & $19.8(18.4-25.17 .6(15.9-20$. \\
\hline
\end{tabular}


B

B

B

B

B

B

B

B

B

C

B

B

B

B

W2

B

B

B

B

B

B

B

B

B

B

B

B

B

B

C

B

C

B

B

B

B

B

B

B

B

B

C

B

B

B
19.1 (18.0-20. 16.4 (13.1-18.

17.6 (16.9-18. 16.3 (15.2-19.

$19.2(18.4-20.17 .0(12.5-19$.

$18.9(17.8-20.17 .0(15.1-20$.

17.8 (16.9-20. 16.0 (14.8-19.

19.1 (18.5-19. 16.8 (15.8-19.

17.9 (16.5-21. 15.7 (14.1-18.

$17.6(15.7-20.15 .8(9.4-18.5$

18.0 (17.0-20. 16.1 (15.3-17.

$19.2(17.7-21.16 .7(14.1-21$.

$18.0(17.0-20.15 .8(15.2-16$.

19.3 (17.9-20. 17.0 (15.7-19.

19.7 (18.3-21. 17.1 (15.0-21.

$18.3(17.1-20.16 .2(14.7-18$.

$18.9 \pm 0.3(N=217.8 \pm 0.3 \quad(n=2 \cdot 0.8 \pm 0.1 \quad(n=24$

$19.3(18.1-22.17 .2(14.9-19$.

20.5 (17.8-24. 17.3 (16.8-18.

19.0 (17.6-20. 16.8 (16.1-18.

19.3 (18.5-21. 17.3 (16.0-18.

19.2 (18.0-21. 17.2 (15.5-21.

19.8 (18.0-24. 17.9 (16.6-19.

18.5 (17.0-20. 16.7 (16.0-20.

19.2 (18.1-21. 16.6 (15.6-17.

18.2 (17.0-21. 16.5 (14.8-22.

18.8 (17.7-21. 16.5 (14.9-17.

19.8 (19.0-21. 18.0 (17.0-19.

19.4 (18.2-21. 17.2 (15.8-19.

18.1 (16.2-21. 15.9 (14.5-17.

19.0 (18.3-22. 16.4 (14.9-18.

19.4 (18.1-22. 16.7 (15.3-17.

19.3 (18.3-21. 17.0 (15.8-20.

18.8 (18.1-19. 17.0 (15.9-20.

19.7 (18.2-22. 17.1 (15.9-19.

18.7 (17.2-21. 17.0 (15.7-19.

19.6 (18.3-23. 16.8 (15.4-18.

19.0 (17.9-20. 16.8 (15.9-18.

17.9 (16.3-19. 16.1 (14.4-19.

19.2 (18.5-20. 16.9 (15.8-19.

18.1 (16.3-20. 16.1 (14.5-17.

19.3 (17.7-21. 16.9 (15.2-20.

19.3 (18.2-22. 17.0 (15.0-20.

19.4 (18.4-22. 16.8 (15.0-18.

19.4 (18.1-21. 17.0 (15.4-19.

18.2 (17.1-21. 17.1 (14.7-23.

19.4 (18.7-20. 17.0 (15.8-17. 
C

B

B

B

B

B

B

B

C

B

C

B

B

B

B

B

C

A

B

B

B

B

B

A

B

B

B

B

B

C

B

B

B

B

B

A

B

B

B

B

B

B

B

B

B
20.0 (18.6-22. 17.1 (16.2-19.

20.5 (18.7-25. 17.6 (15.2-21.

18.8 (17.2-20. 16.9 (16.0-19.

19.5 (18.1-24. 17.4 (16.2-20.

19.5 (17.5-21. 17.2 (16.0-19.

18.7 (16.6-22. 16.5 (14.8-18.

17.8 (16.3-20. 15.6 (14.4-16.

19.2 (17.4-23. 17.2 (16.1-22.

19.3 (18.2-21. 17.1 (16.2-19.

19.5 (18.7-21. 17.2 (16.0-23.

18.8 (17.7-19. 16.6 (14.7-17.

19.8 (18.4-24. 17.9 (16.3-21.

17.9 (16.3-19. 16.6 (15.5-19.

20.2 (17.7-26. 18.0 (16.3-21.

17.7 (16.7-18. 16.0 (14.9-17.

17.6 (15.3-19. 16.0 (14.3-18.

20.0 (19.0-20. 17.3 (16.3-18.

17.9 (17.0-20. 16.6 (14.8-19.

18.2 (16.0-22. 16.3 (14.9-19.

17.9 (16.1-22. 16.5 (14.8-19.

17.8 (17.0-19. 16.3 (15.4-17.

18.9 (18.1-19. 16.9 (15.9-18.

17.8 (16.4-22. 16.3 (15.0-19.

19.3 (16.9-22. 17.6 (16.5-21.

18.1 (17.2-19. 16.3 (14.7-20.

19.1 (17.4-21. 16.8 (15.8-17.

19.2 (17.3-21. 16.8 (15.2-18.

19.3 (17.6-20. 16.9 (15.7-18.

18.2 (17.2-22. 16.2 (14.8-20.

19.0 (17.6-20. 16.6 (15.6-17.

18.0 (17.1-20. 16.1 (15.2-18.

18.4 (17.7-18. 16.5 (15.0-18.

18.0 (17.2-19. 15.7 (14.2-17.

19.6 (19.0-20. 17.5 (16.2-21.

18.8 (18.2-19. 16.7 (15.2-18.

18.3 (17.0-21. 17.0 (15.4-19.

17.8 (16.3-19. 16.0 (14.4-18.

17.8 (17.2-20. 16.2 (14.9-18.

19.1 (17.0-23. 16.8 (14.9-20.

17.7 (16.8-18. 16.1 (14.8-18.

17.9 (16.8-20. 16.4 (15.3-20.

18.8 (17.8-19. 16.6 (15.7-17.

17.6 (17.0-19. 15.8 (14.7-17.

18.5 (17.1-23. 17.0 (15.0-19.

19.1 (18.1-20. 16.6 (15.4-17. 
B

B

B

B

B

B

B

B

B

B

B

B

B

B

B

B

B

B

B

B

B

B

B

B

B

B

C

B

C

A

B

B

B

B

B

B

B

B

B

A/B

$B$

B

B

B

B

19.2 (17.6-23. 16.4 (15.0-17.

$18.3(17.1-20.16 .8$ (15.7-21.

17.8 (17.4-18. 16.0 (14.5-17.

18.0 (16.9-20. 16.4 (15.0-20.

19.3 (18.7-22. 17.0 (15.9-19.

18.0 (16.7-20. 16.5 (14.5-18.

18.1 (17.1-20. 16.5 (15.4-19.

17.8 (16.3-21. 16.1 (15.3-17.

18.9 (17.9-19. 16.8 (15.9-18.

18.0 (16.9-19. 15.9 (15.2-17.

18.2 (16.4-20. 16.7 (14.9-21.

18.0 (16.5-20. 16.7 (15.4-20.

17.8 (16.6-19. 16.1 (14.9-18.

18.8 (17.0-22. 16.0 (13.5-17.

18.4 (16.8-21. 16.4 (13.2-19.

19.2 (18.0-25. 17.0 (16.2-18.

18.3 (16.9-20. 16.1 (15.1-19.

18.0 (16.9-20. 16.9 (15.7-20.

18.0 (17.0-20. 16.9 (15.4-21.

19.4 (18.2-25. 17.1 (15.8-20.

17.8 (16.9-20. 16.4 (14.6-21.

18.4 (16.4-21. 15.8 (14.7-17.

20.0 (18.5-22. 17.3 (13.5-25.

18.3 (16.8-22. 16.7 (14.5-22.

19.5 (18.3-21. 17.1 (14.6-19.

17.9 (17.0-21. 16.1 (15.0-17.

18.4 (17.2-21. 16.8 (15.0-21.

17.7 (16.5-20. 16.1 (14.6-18.

18.9 (17.6-19. 17.4 (15.9-20.

19.6 (17.7-25. 17.3 (15.3-19.

17.8 (16.7-18. 15.7 (9.0-16.8

17.9 (16.4-19. 15.7 (14.8-16.

18.0 (16.9-22. 16.2 (15.3-18.

17.9 (17.1-19. 15.8 (14.8-16.

19.7 (18.2-25. 16.8 (16.0-18.

18.1 (16.6-19. 16.3 (15.1-18.

19.1 (18.2-22. 16.7 (15.4-18.

19.1 (17.8-21. 16.7 (15.6-18.

18.6 (16.7-22. 17.1 (15.3-20.

18.8 (13.7-20. 16.7 (12.1-20.

18.7 (17.1-21. 16.8 (14.8-19.

19.8 (18.6-22. 17.0 (15.8-19.

$20 \quad 8-20$

18.1 (16.5-23. 16.4 (15.4-20.

18.7 (17.1-20. 16.4 (15.4-17. 
20.1 (18.0-25. 17.2 (14.8-19.

18.9 (17.6-20. 17.1 (11.6-21.

17.4 (15.6-18. 15.1 (13.7-16.

$18.2(15.8-20.16 .3(15.0-19$.

$18.2(17.1-20.16 .8(15.2-20$.

19.3 (18.1-22. 17.1 (16.5-17.

18.5 (18.0-19. 16.8 (15.2-19.

19.5 (18.3-22. 17.4 (15.0-20.

19.7 (18.5-25. 17.6 (16.2-22.

20.2 (18.9-24. 17.6 (16.0-21.

19.9 (18.3-24. $18.4(16.5-22$.

19.9 (17.6-24. 17.3 (15.8-19.

19.8 (18.2-24. 17.2 (15.0-19.

18.2 (16.7-23. 16.1 (14.3-18.

$18.1(17.1-20.15 .8$ (14.4-18.

19.7 (18.3-22. 17.2 (15.9-19.

19.2 (18.2-21. 17.2 (16.0-19.

19.3 (18.6-22. 17.4 (15.8-20.

$49^{\circ} 53.347^{\prime} \mathrm{W} 2$

S4

C

$17.8-21.2(n=65.6-19.3(n=6,0.00-47.5 \quad(n=6$

W0

$19.3(17.6-20.15 .8)(2.8-19.8$

S5

B

$17.5 \pm 0.8(n=816.0 \pm 0.6(n=50.7 \pm 0.2 \quad(n=5)$

W1

15.6-15.7 14.0-14.1 0.6-0.7

$51^{\circ}$ 05.30'E 1

S2

W0/1

$17.7 \pm 0.2(n=115.6 \pm 0.3(n=1 \quad 0.8 \pm 0.3$

$51^{\circ} 04.24^{\prime} \mathrm{E} \quad 10$

W4

$17.7 \pm 0.2(n=115.4 \pm 1.0(n=11.7 \pm 0.8$

$69^{\circ} 44.571^{\prime} \mathrm{W} 1$

W4

$17.9 \pm 0.5(\mathrm{~N}=215.2 \pm 0.5(\mathrm{~N}=21.4 \pm 0.5(\mathrm{~N}=21$

$70^{\circ} 07.710^{\prime} \mathrm{W} 1$

W1

$18.2 \pm 0.5(\mathrm{~N}=215.4 \pm 0.5(\mathrm{~N}=21.3 \pm 0.5(\mathrm{~N}=23$

$70^{\circ} 10^{\prime} 52.7^{\prime \prime} \mathrm{W} 1$

$69^{\circ} 45.031^{\prime} \mathrm{W} 1$

W1

W1

$69^{\circ} 48.414^{\prime} \mathrm{W} 1$

W2

S1

$69^{\circ} 43.036^{\prime} \mathrm{W} 1$

W2

W2

W3

W2

6959.310'W many

W3

$156^{\circ} 36.238^{\prime} \mathrm{E} 1$

$\sim 70^{\circ} 32^{\prime} \mathrm{W} \quad 7$

1434ㄴ'32"E 1

$156^{\circ} 38.987^{\prime} \mathrm{E} 1$

$\sim 70^{\circ} 32^{\prime} \mathrm{W} \quad 2$

1

$82^{\circ} 48.89^{\prime} \mathrm{E} \quad 10$

S3

W1

W1

W1

W1

W1

B

WO

$18.7 \pm 0.5(\mathrm{~N}=115.4 \pm 0.5(\mathrm{~N}=11.0 \pm 0.5(\mathrm{~N}=19$ $19.6 \pm 0.5(\mathrm{~N}=117.7 \pm 0.5(\mathrm{~N}=11.2 \pm 0.5(\mathrm{~N}=18$

$19.7 \pm 0.5(\mathrm{~N}=116.5 \pm 0.5(\mathrm{~N}=11.0 \pm 0.5(\mathrm{~N}=17$

$18.6 \pm 0.3(n=516.3 \pm 0.3(n=51.1 \pm 0.1 \quad(n=5)$

18.9-19.1 16.3-17.2 1.0-0.7

$17.3 \pm 0.5(\mathrm{~N}=216.8 \pm 0.5(\mathrm{~N}=21.0 \pm 0.5(\mathrm{~N}=22$

$\begin{array}{lll}18.2 \pm 0.1 & 16.0 \pm 0.3 \quad 1.4 \pm 0.2\end{array}$

$18.5(\mathrm{~N}=1) \quad 16.7(\mathrm{~N}=1) \quad 0.8$

$19.3 \quad 17.1 \quad 1.4$

$\begin{array}{lll}17.9 \pm 0.2 & 15.7 \pm 0.2 \quad 1.5 \pm 0.2\end{array}$

$19.1(\mathrm{~N}=1) \quad 16.2(\mathrm{~N}=1) \quad 0.6$

$18 \quad 17$

W1.0 18.3 $\pm 0.3(n=4.16 .0 \pm 0.2(n=31.2 \pm 0.2(n=39$

W3 $20.8 \pm 0.2(n=717.2 \pm 0.1(n=71.6$

$\begin{array}{llll}\text { W2 } & 18.2 \pm 0.2 & 16.0 \pm 0.1 & 1.5 \pm 0.1\end{array}$

W1/2 19.5-19.9 16.8-16.9 1.1-1.0

W3 20.5 $\pm 0.2(n=717.1 \pm 0.1(n=62.0$ 


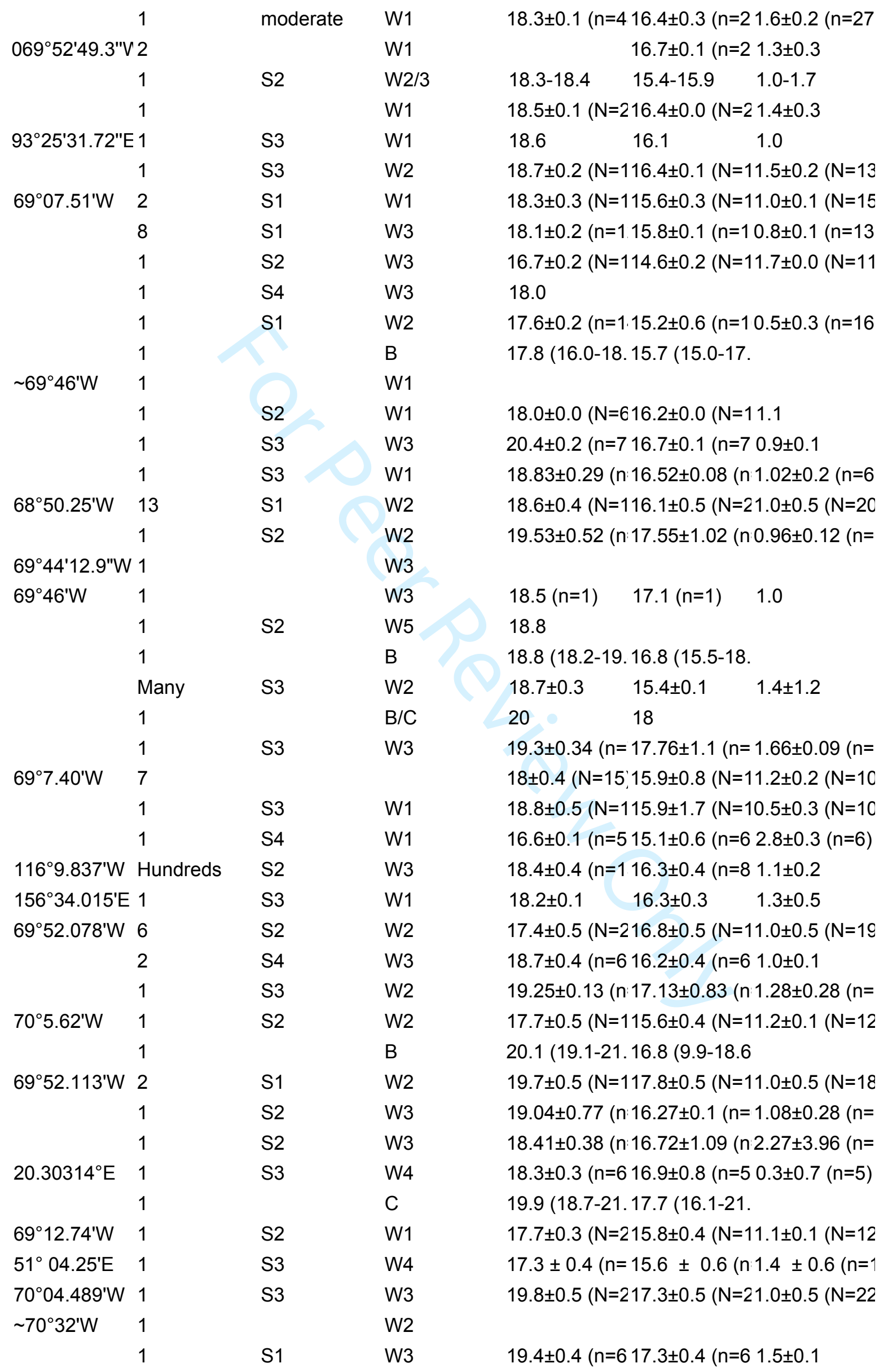




\begin{tabular}{|c|c|c|c|c|}
\hline \multirow[t]{2}{*}{$53^{\circ} 14.90^{\prime} \mathrm{W}$} & 1 & S4 & W2 & $19.3 \pm 0.6(N=: 17.3 \pm 0.9(N=01.14 \pm 0.15(n=$ \\
\hline & 1 & S1 & W2 & $20.2 \pm 0.3(n=617.6 \pm 0.2(n=71.8 \pm 0.1$ \\
\hline$\sim 69^{\circ} 43^{\prime} \mathrm{W}$ & 1 & & W1 & \\
\hline$\sim 69^{\circ} 43^{\prime} \mathrm{W}$ & 1 & & W1 & \\
\hline \multirow[t]{3}{*}{$\sim 70^{\circ} 32^{\prime} \mathrm{W}$} & 1 & & W1 & \\
\hline & 1 & S2 & W3 & $20.5 \pm 0.2(n=617.4 \pm 0.2(n=61.6 \pm 0.1$ \\
\hline & 1 & & B & $18.6(17.7-19.16 .8(16.2-18$. \\
\hline \multirow[t]{3}{*}{$68^{\circ} 50.59^{\prime} \mathrm{W}$} & 1 & S3 & W1 & $18.2 \pm 0.4(\mathrm{~N}=215.5 \pm 0.4(\mathrm{~N}=21.2 \pm 0.4(\mathrm{~N}=21$ \\
\hline & 1 & S4 & W2 & $19.58 \pm 0.52(n: 17.05 \pm 0.68$ (n $1.26 \pm 0.4(n=7$ \\
\hline & 1 & S4 & W2 & $19.38 \pm 0.78(n) 16.69 \pm 0.54(n) 1.47 \pm 0.67(n=$ \\
\hline \multirow[t]{5}{*}{$70^{\circ} 7.74^{\prime} \mathrm{W}$} & 1 & S3 & W1 & $18.6 \pm 0.6(\mathrm{~N}=116.3 \pm 0.3(\mathrm{~N}=11.1 \pm 0.3(\mathrm{~N}=12$ \\
\hline & 1 & S1 & W4 & $20.3 \pm 0.2(n=715.0 \pm 0.2(n=8 \quad 0.5 \pm 0.1$ \\
\hline & 1 & S2 & W1 & $17.7 \pm 0.2(\mathrm{~N}=115.5 \pm 0.2(\mathrm{~N}=11.4 \pm 0.1(\mathrm{~N}=10$ \\
\hline & 1 & & B & $19.8(18.7-21.17 .3(16.3-19$ \\
\hline & 1 & & C & 21 \\
\hline$\sim 69^{\circ} 43^{\prime} \mathrm{W}$ & 1 & & W2 & \\
\hline \multirow[t]{3}{*}{$69^{\circ} 54.795^{\prime} \mathrm{W}$} & 1 & S1 & W3 & $19.9 \pm 0.5(\mathrm{~N}=117.2 \pm 0.5(\mathrm{~N}=10.9 \pm 0.5(\mathrm{~N}=19$ \\
\hline & 1 & S3 & W1 & $17.53 \pm 0.11(n: 15.77 \pm 0.52(n: 1.62 \pm 0.83(n=$ \\
\hline & 1 & S2 & W2 & $18.47 \pm 0.88(n \cdot 16.4 \pm 0.5(n=60.88 \pm 0.31(n=$ \\
\hline \multirow[t]{3}{*}{$70^{\circ} 05^{\prime} 08.8^{\prime \prime} \mathrm{W}$} & & & W1 & \\
\hline & 1 & S2 & W1 & $18.65 \pm 0.36(n) 16.64 \pm 0.72(n) 1.23 \pm 0.96(n=$ \\
\hline & 1 & S2 & W4 & $18.5 \pm 0.3(n=516.4 \pm 0.2(n=41.5 \pm 0.2(n=4)$ \\
\hline \multirow[t]{2}{*}{$20.35023^{\circ} \mathrm{E}$} & 1 & S2 & W3 & $17.2 \pm 0.4(n=315.1 \pm 0.3(n=40.3 \pm 0.6(n=4)$ \\
\hline & 1 & S2 & W2 & $18.18 \pm 0.47(n) 16.09 \pm 0.71(n: 0.71 \pm 0.5(n=7$ \\
\hline \multirow[t]{8}{*}{$69^{\circ} 37.40^{\prime} \mathrm{W}$} & 15 & & & $17.2 \pm 0.3(\mathrm{~N}=115.1 \pm 0.2(\mathrm{~N}=10.8 \pm 0.3(\mathrm{~N}=13$ \\
\hline & 1 & S2 & W2 & $20.55 \pm 0.24(n-16.88 \pm 1.26(n-0.61 \pm 0.34(n=$ \\
\hline & 1 & S3 & W2 & $17.48 \pm 0.28(n) 15.75 \pm 0.85(n) 1.18 \pm 0.65(n=$ \\
\hline & 1 & S1 & W2 & $17.19 \pm 2.28(n \cdot 16.05 \pm 0.2(n=0.88 \pm 0.19(n=$ \\
\hline & 1 & S3 & W3 & $18.8 \pm 0.32(n=16.65 \pm 0.33(n) 1.11 \pm 0.31(n=$ \\
\hline & 1 & S1 & W2 & $18.9 \pm 0.36(n=16.81 \pm 0.22(n 0.83 \pm 0.23(n=$ \\
\hline & 1 & & B & $20.2(18.6-23.17 .8(16.1-19$ \\
\hline & 1 & S2 & W3 & $18.26 \pm 0.5(n=16.06 \pm 0.35(n \cdot 1.62 \pm 0.63(n=$ \\
\hline $70^{\circ} 13.92^{\prime} \mathrm{W}$ & 1 & S3 & W1 & $19.3 \pm 1.5(\mathrm{~N}=117.0 \pm 1.3(\mathrm{~N}=11.3 \pm 0.6(\mathrm{~N}=12$ \\
\hline $56^{\circ} 59.80^{\prime} \mathrm{N}$ & 1 & S2 & W3 & $17.8 \pm 0.9(\mathrm{~N}=515.1 \pm 1.1(\mathrm{~N}=51.2 \pm 0.5(\mathrm{~N}=5)$ \\
\hline $57^{\circ} 3.80$ & 1 & S1 & W3 & $18.9 \pm 0.8(\mathrm{~N}=116.6 \pm 0.9(\mathrm{~N}=11.1 \pm 0.4(\mathrm{~N}=10$ \\
\hline $69^{\circ} 55.317^{\prime} \mathrm{W}$ & 1 & S1 & W2 & $18.7 \pm 0.5(\mathrm{~N}=117.0 \pm 0.5(\mathrm{~N}=21.0 \pm 0.5(\mathrm{~N}=20$ \\
\hline \multirow[t]{3}{*}{$\sim 69^{\circ} 43^{\prime} \mathrm{W}$} & 1 & & W1 & \\
\hline & 1 & S3 & W2 & $19.1 \pm 0.3(n=716.6 \pm 0.3(n=71.3 \pm 0.2$ \\
\hline & 1 & S2 & W1 & $18.4 \pm 0.3(N=519.5 \pm 0.3(N=61.7 \pm 0.1$ \\
\hline $69^{\circ} 7.43^{\prime} \mathrm{W}$ & 5 & & & $17.6 \pm 0.2(N=115.4 \pm 0.1(N=11.1 \pm 0.2(N=13$ \\
\hline $70^{\circ} 00.557^{\prime} \mathrm{W}$ & 1 & S1 & W1 & $17.8 \pm 0.5(\mathrm{~N}=116.9 \pm 0.5(\mathrm{~N}=21.0 \pm 0.5(\mathrm{~N}=20$ \\
\hline \multirow[t]{3}{*}{$69^{\circ} 55.222^{\prime} \mathrm{W}$} & 1 & S2 & W2 & $18.9 \pm 0.5(\mathrm{~N}=117.7 \pm 0.5(\mathrm{~N}=11.0 \pm 0.5(\mathrm{~N}=16$ \\
\hline & 1 & S2 & W3 & $20.8 \pm 0.2(n=718.8 \pm 0.1(n=71.3 \pm 0.1$ \\
\hline & 1 & S3 & W3 & $20.5 \pm 0.1(n=817.4 \pm 0.2(n=51.2 \pm 0.1$ \\
\hline $56^{\circ} 49.427^{\prime} \mathrm{E}$ & 1 & S2 & W0 & $18.1 \pm 0.3(\mathrm{~N}=16.3 \pm 0.4(\mathrm{~N}=0.9 \pm 0.2(\mathrm{~N}=2$ \\
\hline
\end{tabular}




\begin{tabular}{|c|c|c|c|c|c|c|}
\hline & 1 & & \multicolumn{4}{|l|}{ W1 } \\
\hline & 1 & S1 & W2 & 16.2 & 14.3 & 1.3 \\
\hline & 1 & S1 & W2 & \multicolumn{3}{|c|}{$17.88 \pm 0.52(n) 15.89 \pm 0.5(n=1.49 \pm 1.19(n=$} \\
\hline & 1 & S3 & W2 & \multicolumn{3}{|c|}{$19.43 \pm 0.47(n) 16.17 \pm 1.09(n) 1.66 \pm 1.13(n=$} \\
\hline & 1 & & $\mathrm{~B}$ & \multicolumn{3}{|c|}{$18.5(17.8-19.16 .3$ (15.2-17. } \\
\hline & 1 & & C & \multicolumn{3}{|c|}{$19.9(18.3-22.17 .6(16.1-20}$. \\
\hline \multirow[t]{3}{*}{$\sim 69^{\circ} 43^{\prime} \mathrm{W}$} & 2 & & W3 & $19.1(\mathrm{~N}=1)$ & $18.5(\mathrm{~N}=1$ & 0.9 \\
\hline & 1 & S3 & W3 & \multicolumn{3}{|c|}{$17.17 \pm 0.46(n) 15.97 \pm 0.75(n: 0.8 \pm 0.68(n=5$} \\
\hline & 1 & & C & 17 & \multicolumn{2}{|c|}{15} \\
\hline \multirow[t]{2}{*}{$\sim 69^{\circ} 46^{\prime} \mathrm{W}$} & 1 & & W1 & & & \\
\hline & 1 & S3 & W2 & \multicolumn{3}{|c|}{$19.12 \pm 0.5(n=16.7 \pm 0.58(n=1.15 \pm 0.24(n=$} \\
\hline \multirow[t]{2}{*}{$106^{\circ} 13^{\prime} \mathrm{W}$} & 5 & s2 & W2 & \multicolumn{3}{|c|}{$20.0 \pm 1.2(n=16.6 \pm 0.9(n=1.1 \pm 0.1$} \\
\hline & 1 & S4 & W2 & \multicolumn{3}{|c|}{$19.73 \pm 0.4(n=17.45 \pm 0.28(n) 1.19 \pm 0.28(n=$} \\
\hline \multirow[t]{2}{*}{$69^{\circ} 58.263^{\prime} \mathrm{W}$} & 1 & S2 & W2 & \multicolumn{3}{|c|}{$19.8 \pm 0.5(\mathrm{~N}=117.2 \pm 0.5(\mathrm{~N}=11.0 \pm 0.5(\mathrm{~N}=18$} \\
\hline & 1 & S3 & W3 & \multicolumn{3}{|c|}{$18.13 \pm 0.3(n=16.56 \pm 0.58(n) 1.35 \pm 0.11(n=$} \\
\hline $156^{\circ} 30.735^{\prime} \mathrm{E}$ & 1 & S1 & W1 & $20.1 \pm 0.4$ & $17.7 \pm 0.6$ & $1.4 \pm 0.2$ \\
\hline & 1 & & B & \multicolumn{3}{|c|}{19.6 (17.7-22. 17.9 (15.5-25. } \\
\hline \multirow[t]{11}{*}{$\sim 69^{\circ} 43^{\prime} \mathrm{W}$} & 2 & & W2 & $19.2(\mathrm{~N}=1)$ & $17.1(\mathrm{~N}=1$ & 1.3 \\
\hline & 1 & S2 & W2 & \multicolumn{3}{|c|}{$19.81 \pm 0.36(n) 17.02 \pm 0.56(n) 1.07 \pm 0.36(n=$} \\
\hline & 1 & S1 & W2 & \multicolumn{3}{|c|}{$19.46 \pm 0.23(n-17.74 \pm 0.56(n) 1.16 \pm 0.07(n=$} \\
\hline & 1 & S2 & W3 & \multicolumn{3}{|c|}{$18.05 \pm 0.25(n) 16.94 \pm 1.28(n) 1.02 \pm 0.09(n=$} \\
\hline & 1 & & B & $17.3(16.0-2$ & $.15 .5(12 . \varsigma$ & \\
\hline & 1 & & $\mathrm{~B} / \mathrm{C}$ & 19 & 17 & \\
\hline & 1 & s2 & W3 & $18.8 \pm 0.42($ & $=16.81 \pm 0.7$ & $1.31 \pm 0.14(n=$ \\
\hline & 1 & s2 & W2 & $17.79 \pm 0.03$ & $15.7 \pm 0.18$ & $=1.32 \pm 0.12(n=$ \\
\hline & 1 & & $B$ & $19.4(18.4-2$ & $.16 .9(16.1$ & \\
\hline & 1 & & $\mathrm{C}$ & 17 & 15 & \\
\hline & 1 & S2 & w3 & $17.8 \pm 0.3(n$ & $314.1 \pm 0.5$ & $0.9 \pm 0.2$ \\
\hline$\sim 69^{\circ} 43^{\prime} \mathrm{W}$ & 1 & & W3 & & & \\
\hline $156^{\circ} 07.593^{\prime} E$ & 1 & S3 & W1 & $18.0 \pm 0.3$ & $15.9 \pm 0.1$ & $1.1 \pm 0.4$ \\
\hline & 1 & & $B$ & $18.6(17.7-2$ & $.16 .9(15.8$ & \\
\hline & 1 & S2 & W3 & $20.18 \pm 1.41$ & $16.63 \pm 0.8$ & $1.18 \pm 0.19(n=$ \\
\hline & 1 & S1 & w3 & $20.6 \pm 0.2(n$ & $17.7 \pm 0.2$ & $0.7 \pm 0.1$ \\
\hline & 1 & & $B$ & $19.4(18.1-2$ & $.17 .2(15.4$ & \\
\hline & 1 & S3 & W2 & $19.19 \pm 0.18$ & $17.41 \pm 0.6$ & $=1.38 \pm 0.27(n=$ \\
\hline & 1 & & $\mathrm{~B} / \mathrm{C}$ & 19 & 17 & \\
\hline $156^{\circ} 36.561^{\prime} \mathrm{E}$ & 1 & S2 & W1 & $18.1 \pm 0.2$ & $16.0 \pm 1.0$ & $1.1 \pm 0.2$ \\
\hline & 1 & & $B / C$ & 19 & 17 & \\
\hline & 1 & S2 & W3 & $20.0 \pm 0.3(n$ & $17.7 \pm 0.3$ & $1.4 \pm 0.2$ \\
\hline & 1 & & $\mathrm{C}$ & 17 & 15 & \\
\hline & 1 & & B & $18.4(17.0-2$ & $.16 .1(15.2$ & \\
\hline $69^{\circ} 53.232^{\prime} \mathrm{W}$ & 1 & S4 & W1 & $19.9 \pm 0.5$ & $17.8 \pm 0.7$ & $1.3 \pm 0.2$ \\
\hline & 1 & & $B$ & $18.2(17.0-1$ & $16.2(14.8$ & \\
\hline & 1 & S1 & W1 & $17.7 \pm 0.1(n$ & $15.5 \pm 0.3$ & $1.1 \pm 0.2(n=10$ \\
\hline $31^{\circ} 16.66^{\prime} \mathrm{E}$ & 1 & S3 & w3 & $18.5 \pm 0.1(\mathrm{~N}$ & $516.6 \pm 0.5$ & $51.1 \pm 0.2(\mathrm{~N}=5)$ \\
\hline
\end{tabular}




\begin{tabular}{|c|c|c|c|c|c|}
\hline \multirow[t]{4}{*}{$15^{\circ} 04^{\prime} 12^{\prime \prime} \mathrm{E}$} & 1 & & W1 & $19.4 \pm 0.4$ & $17.2 \pm 0.5$ \\
\hline & 1 & S2 & w2 & \multicolumn{2}{|c|}{$16.35 \pm 0.3(n=14.29 \pm 1.44(n) 1.78 \pm 0.31(n=$} \\
\hline & 1 & S2 & W3 & \multicolumn{2}{|c|}{$20.83 \pm 2.09$ (n 19.92 \pm 1.25 (n $1.55 \pm 1.94(n=$} \\
\hline & 1 & & $\mathrm{~B} / \mathrm{C}$ & \multicolumn{2}{|c|}{$20.1(18.5-22.17 .1(15.4-19$} \\
\hline $69^{\circ} 44.648^{\prime} \mathrm{W}$ & 1 & S2 & W3 & \multicolumn{2}{|c|}{$17.6 \pm 0.5(\mathrm{~N}=116.5 \pm 0.5(\mathrm{~N}=11.1 \pm 0.5(\mathrm{~N}=16$} \\
\hline \multirow[t]{3}{*}{$57^{\circ} 54.92^{\prime} \mathrm{E}$} & 1 & S3 & W3 & \multicolumn{2}{|c|}{$20.4 \pm 0.2(n=619.0 \pm 0.3(n=61.5 \pm 0.1$} \\
\hline & 1 & & $\mathrm{~B} / \mathrm{C}$ & 19 & 17 \\
\hline & 1 & S2 & W3 & \multicolumn{2}{|c|}{$19.24 \pm 0.94(n) 17.35 \pm 2.89$ (n $1.40 \pm 0.59(n=$} \\
\hline$\sim 69^{\circ} 43^{\prime} \mathrm{W}$ & 1 & & W1 & & \\
\hline \multicolumn{3}{|l|}{$69^{\circ} 48^{\prime} 58.51 " \vee 2$} & W3/4 & $19.7(\mathrm{~N}=1)$ & $16.8(\mathrm{~N}=1)$ \\
\hline $31^{\circ} 15.12$ 'E & 1 & S1 & W2 & \multicolumn{2}{|c|}{$18.3 \pm 0.6(\mathrm{~N}=516.3 \pm 0.3(\mathrm{~N}=51.3 \pm 0.3(\mathrm{~N}=5)$} \\
\hline \multirow{6}{*}{$69^{\circ} 7.97{ }^{\prime} \mathrm{W}$} & 1 & & & \multicolumn{2}{|c|}{$18.1 \pm 0.4(N=115.8 \pm 0.3(N=11.3 \pm 0.6(N=10$} \\
\hline & 1 & & $A / B$ & 19 & 17 \\
\hline & 1 & S2 & W1 & \multicolumn{2}{|c|}{$16.7 \pm 0.2(n=114.6 \pm 0.1(n=10.6 \pm 0.1 \quad(n=16$} \\
\hline & 1 & S2 & W2 & \multicolumn{2}{|c|}{$20.24 \pm 1.58(n-17.3 \pm 0.42(n=1.06 \pm 0.25(n=$} \\
\hline & 1 & & B & \multicolumn{2}{|c|}{$19.0(17.9-20.17 .6(15.8-20$} \\
\hline & 1 & S4 & W2 & \multicolumn{2}{|c|}{$19.06 \pm 0.18(n: 16.89 \pm 0.21(n: 0.21 \pm 0.21(n=$} \\
\hline \multirow[t]{2}{*}{$69^{\circ} 50.901 ' \mathrm{~W}$} & 1 & $\mathrm{~S} 1$ & W4 & \multicolumn{2}{|c|}{$20.6 \pm 0.5(\mathrm{~N}=118.0 \pm 0.5(\mathrm{~N}=10.9 \pm 0.5(\mathrm{~N}=15$} \\
\hline & 1 & & B & \multicolumn{2}{|c|}{$19.7(18.8-21.17 .6(15.5-19$} \\
\hline \multirow[t]{2}{*}{$\sim 69^{\circ} 43^{\prime} \mathrm{W}$} & 11 & & W3 & $19.6(\mathrm{~N}=1)$ & $17.2 \pm 0.2(\mathrm{~N}=21.1 \pm 0.0$ \\
\hline & 1 & S2 & W2 & $19.2 \pm 0.6$ & $17.1 \pm 0.8 \quad 1.2 \pm 0.3$ \\
\hline $156^{\circ} 34.601 ' \mathrm{E}$ & 1 & S2 & W1 & $20.1 \pm 0.3$ & $17.6 \pm 0.3 \quad 1.5 \pm 0.1$ \\
\hline & 1 & S1 & W3 & $19.1 \pm 0.1(n=$ & $17.0 \pm 0.1(n=71.3$ \\
\hline & 1 & & C & $19.1(17.8-2$ & $17.0(16.0-18$ \\
\hline & 1 & & B & $19.4(18.2-2$ & $16.8(15.8-19$ \\
\hline $31^{\circ} 14.66^{\prime} \mathrm{E}$ & 1 & S3 & W3 & $18.3 \pm 0.2(\mathrm{~N}=$ & $516.2 \pm 0.3(\mathrm{~N}=51.3 \pm 0.2(\mathrm{~N}=5)$ \\
\hline & 1 & S2 & W2 & $18.66 \pm 0.25$ & $16.33 \pm 0.66(n-1.74 \pm 1.76(n=$ \\
\hline & 1 & & B & $19.7(18.5-2$ & $17.6(16.0-20$ \\
\hline & 1 & & B & $19.4(17.9-2$ & $16.8(15.4-18$ \\
\hline & 1 & S2 & W4 & $19.8 \pm 0.2$ & $17.4 \pm 0.2 \quad 1.5 \pm 0.1$ \\
\hline $69^{\circ} 58.940^{\prime} \mathrm{W}$ & 2 & S2 & W2 & $20.2 \pm 0.5(\mathrm{~N}=$ & $118.1 \pm 0.5(\mathrm{~N}=11.1 \pm 0.5(\mathrm{~N}=17$ \\
\hline & 1 & & B & 20 & 18 \\
\hline & 1 & S2 & W2 & $20.5 \pm 0.1(n=$ & $17.8 \pm 0.2(n=7 \quad 1.3 \pm 0.2$ \\
\hline$\sim 69^{\circ} 43^{\prime} \mathrm{W}$ & 1 & & W1 & $18.5(\mathrm{~N}=1)$ & $16.1(\mathrm{~N}=1) \quad 1.1$ \\
\hline & 1 & & B & 18 & 16 \\
\hline & 1 & & B & 19 & 18 \\
\hline & 1 & S1 & W2 & $19.2 \pm 0.4(n=$ & $17.5 \pm 0.7(n=7 \quad 1.5 \pm 0.1$ \\
\hline & 1 & S3 & W2 & $19.56 \pm 0.49$ & $17.91 \pm 0.49(n-1.39 \pm 0.07(n=$ \\
\hline & 1 & S2 & W3 & $18.92 \pm 0.51$ & $16.56 \pm 0.66(n-1.09 \pm 0.25(n=$ \\
\hline & 1 & S2 & W2 & $19.45 \pm 0.44$ & $17.88 \pm 0.83(n-1.03 \pm 0.45(n=$ \\
\hline $156^{\circ} 27.409^{\prime} \mathrm{E}$ & 1 & S1 & W1 & $18.3 \pm 0.5$ & $16.2 \pm 0.3 \quad 1.4 \pm 0.2$ \\
\hline$\sim 69^{\circ} 46^{\prime} \mathrm{W}$ & 1 & & W1 & & \\
\hline & 1 & S3 & W3 & $18.45 \pm 0.34$ & $16.22 \pm 0.3(n=1.16 \pm 0.57(n=$ \\
\hline & 1 & S2 & W2 & $18.75 \pm 0.46$ & $17.02 \pm 0.56(n) 1.5 \pm 0.21(n=6$ \\
\hline & 1 & & $\mathrm{~B} / \mathrm{C}$ & 19 & 17 \\
\hline
\end{tabular}




\begin{tabular}{|c|c|c|c|c|c|}
\hline & 6 & & W3 & $18.7(\mathrm{~N}=1)$ & $16.4(\mathrm{~N}=1) \quad 1.4(\mathrm{~N}=1)$ \\
\hline & 1 & & $B$ & \multicolumn{2}{|c|}{19.8 (18.3-22. 17.5 (16.2-22. } \\
\hline & 1 & & B & \multicolumn{2}{|c|}{19.2 (18.2-20. 16.9 (14.6-18. } \\
\hline & 1 & & B & \multicolumn{2}{|c|}{$19.3(18.4-21.16 .9(15.6-21}$. \\
\hline & 1 & S3 & W2 & \multicolumn{2}{|c|}{$18.62 \pm 1.11(n \cdot 17.93 \pm 1.85(n) 0.75 \pm 0.40(n=$} \\
\hline & 1 & & $B / C$ & 18 & 16 \\
\hline & 1 & & B & \multicolumn{2}{|c|}{18.6 (17.3-21. 16.5 (15.1-19. } \\
\hline $156^{\circ} 34.675^{\prime} \mathrm{E}$ & 1 & S2 & W1 & $18.0 \pm 0.2$ & $16.0 \pm 0.2 \quad 1.0 \pm 0.3$ \\
\hline & 1 & & A & \multicolumn{2}{|c|}{18.0 (17.3-19. 16.3 (14.4-19. } \\
\hline & 1 & S2 & W2 & \multicolumn{2}{|c|}{16} \\
\hline & 1 & S3 & W2 & \multicolumn{2}{|c|}{$18.24 \pm 0.78(n \cdot 16.9 \pm 2.52(n=0.77 \pm 0.34(n=$} \\
\hline \multirow[t]{5}{*}{$\sim 69^{\circ} 43^{\prime} \mathrm{W}$} & 1 & & W1 & & \\
\hline & 1 & & C & \multicolumn{2}{|c|}{$18.9(17.8-20.17 .2(16.6-19$} \\
\hline & 1 & S3 & W1 & \multicolumn{2}{|c|}{$19.15 \pm 0.4(n=16.92 \pm 0.28(n) 0.88 \pm 0.19(n=$} \\
\hline & 1 & & C & 20 & 17 \\
\hline & 1 & & $B / C$ & 19 & 17 \\
\hline \multirow[t]{14}{*}{$114^{\circ} 1.66^{\prime} \mathrm{W}$} & 1 & S2 & W5 & \multicolumn{2}{|c|}{$18.40 \pm 0.3(\mathrm{~N}=16.6 \pm 0.2(\mathrm{~N}=11.5 \pm 0.2(\mathrm{~N}=13$} \\
\hline & 1 & & C & \multicolumn{2}{|c|}{19.1 (18.1-21.16.9 (15.2-18. } \\
\hline & 1 & S2 & W3 & \multicolumn{2}{|c|}{$19.9 \pm 0.65(n=15.6 \pm 1.47(n=0.67 \pm 0.59(n=$} \\
\hline & 1 & & B & \multicolumn{2}{|c|}{19} \\
\hline & 1 & & $\mathrm{~B} / \mathrm{C}$ & 19 & 17 \\
\hline & 1 & & B & \multicolumn{2}{|c|}{19.7 (18.3-25. $17.0(13.8-20}$. \\
\hline & 1 & S3 & W3 & \multicolumn{2}{|c|}{$18.77 \pm 0.4(n=16.09 \pm 3.17(n) 1.57 \pm 0.86(n=$} \\
\hline & 1 & S2 & W1 & \multicolumn{2}{|c|}{$18.57 \pm 0.24(n) 16.67 \pm 0.41(n) 1.46 \pm 0.15(n=$} \\
\hline & 1 & S2 & W3 & \multicolumn{2}{|c|}{$19.34 \pm 0.57(n \cdot 16.92 \pm 0.37(n \cdot 1.29 \pm 0.09(n=$} \\
\hline & 1 & & $B / C$ & 19 & 17 \\
\hline & 1 & S2 & W3 & $18.9 \pm 0.26($ & $=16.73 \pm 0.1(n=1.44 \pm 0.11(n=$ \\
\hline & 1 & & $\mathrm{~B} / \mathrm{Ce}$ & 19 & 17 \\
\hline & 1 & & $\mathrm{~B} / \mathrm{C}$ & 19 & 17 \\
\hline & 1 & S5 & W3 & $18.87 \pm 0.52$ & $17.14 \pm 0.66(n-1.53 \pm 1.07(n=$ \\
\hline $156^{\circ} 27.376^{\prime} \mathrm{E}$ & 1 & S1 & W1 & $18.2 \pm 0.2$ & $16.2 \pm 0.4$ \\
\hline$\sim 69^{\circ} 43^{\prime} \mathrm{W}$ & 1 & & W1 & & \\
\hline & 1 & S1 & W2 & $18.42 \pm 0.39$ & $16.38 \pm 0.67(n-1.04 \pm 0.72(n=$ \\
\hline & 1 & & C & $18.1(15.9-1$ & $15.9(15.5-16$ \\
\hline & 1 & & $B / C$ & 20 & 17 \\
\hline & 1 & & $\mathrm{Be}$ & 17 & 15 \\
\hline $156^{\circ} 27.409^{\prime} \mathrm{E}$ & 1 & S2 & W1 & $18.2 \pm 0.2$ & $16.1 \pm 0.3 \quad 1.2 \pm 0.2$ \\
\hline & 1 & S2 & W2 & $18.61 \pm 0.55$ & $16.38 \pm 0.48(n+1 \pm 0.53(n=13)$ \\
\hline & 1 & S1 & W3 & $18.78 \pm 0.21$ & $16.37 \pm 0.46(n-1.17 \pm 0.17(n=$ \\
\hline $69^{\circ} 51.070^{\prime} \mathrm{W}$ & 1 & S3 & W3 & $19.7 \pm 0.5(\mathrm{~N}$ & $117.1 \pm 0.5(\mathrm{~N}=11.0 \pm 0.5(\mathrm{~N}=14$ \\
\hline & 1 & & B & $18.0(16.0-2$ & $15.9(14.6-19$ \\
\hline & 1 & & $\mathrm{~B} / \mathrm{Ce}$ & 19 & 17 \\
\hline & 1 & & C & 19 & 18 \\
\hline & 1 & S1 & W2 & $18.56 \pm 0.15$ & $17.19 \pm 0.78(n-1.26 \pm 0.29(n=$ \\
\hline & 1 & S3 & W2 & $17.4 \pm 2.9(\mathrm{n}$ & $16.15 \pm 0.56(n-1.05 \pm 0.24(n=$ \\
\hline
\end{tabular}




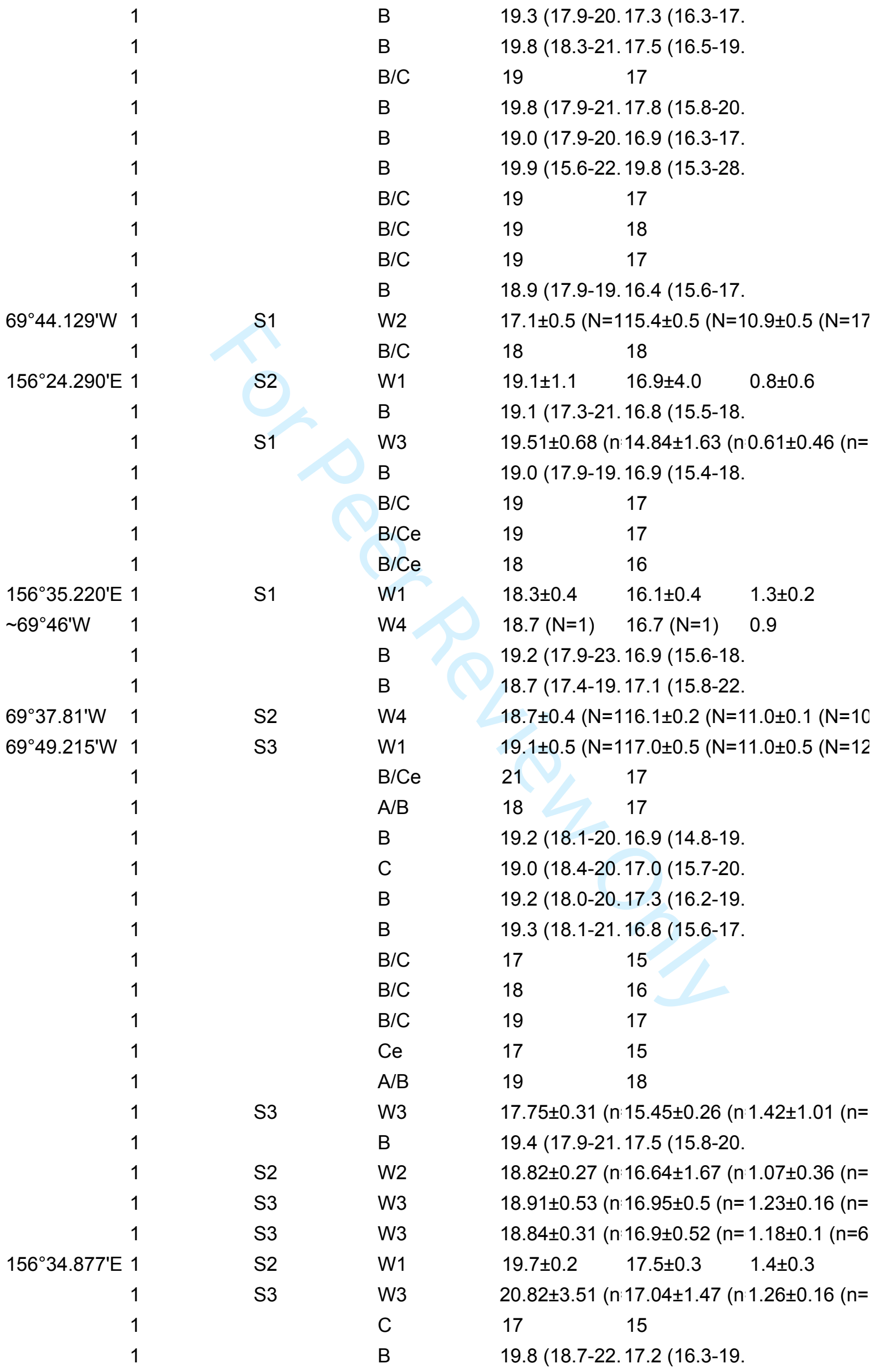




\begin{tabular}{|c|c|c|c|c|c|c|}
\hline \multicolumn{2}{|c|}{$156^{\circ} 36.723^{\prime} \mathrm{E} 1$} & \multirow[t]{2}{*}{ S2 } & W1 & $18.5 \pm 0.1$ & $16.4 \pm 0.2$ & $1.0 \pm 0.3$ \\
\hline & 1 & & $\mathrm{~B}$ & \multicolumn{3}{|c|}{$18.6(17.3-22.16 .9(15.7-21$} \\
\hline $68^{\circ} 40.52^{\prime} \mathrm{W}$ & 1 & S1 & W3 & \multicolumn{3}{|c|}{$19.0 \pm 0.2(\mathrm{~N}=114.9 \pm 0.2(\mathrm{~N}=11.1 \pm 0.2(\mathrm{~N}=13$} \\
\hline $156^{\circ} 25.443^{\prime} \mathrm{E}$ & 1 & S1 & W1 & $18.5 \pm 0.2$ & $16.3 \pm 0.2$ & $1.5 \pm 0.2$ \\
\hline \multirow[t]{17}{*}{$69^{\circ} 50.770^{\prime} \mathrm{W}$} & 1 & S2 & W4 & \multicolumn{3}{|c|}{$17.4 \pm 0.5(\mathrm{~N}=116.2 \pm 0.5(\mathrm{~N}=11.1 \pm 0.5(\mathrm{~N}=13$} \\
\hline & 1 & & $\mathrm{~B}$ & \multicolumn{3}{|c|}{$19.1(18.4-20.16 .8(15.6-17}$. \\
\hline & 1 & & B & \multicolumn{3}{|c|}{$19.2(18.2-21.17 .2(16.6-18}$. \\
\hline & 1 & S2 & W3 & \multicolumn{3}{|c|}{$18.55 \pm 0.72(n: 16.31 \pm 0.51(n) 1.34 \pm 0.28(n=$} \\
\hline & 1 & & B & \multicolumn{3}{|c|}{$18.9(17.2-21.16 .0(14.7-17}$. \\
\hline & 1 & & C & \multicolumn{3}{|c|}{18.8 (17.7-19. 16.7 (14.6-18. } \\
\hline & 1 & & B & \multicolumn{3}{|c|}{$18.9(17.8-20.16 .7(15.6-20}$. \\
\hline & 1 & & B & \multicolumn{3}{|c|}{$17.2(15.8-21.15 .7(13.8-19}$. \\
\hline & 1 & S3 & W3 & \multicolumn{3}{|c|}{$18.38 \pm 0.32(n-16.4 \pm 0.45(n=1.77 \pm 0.63(n=$} \\
\hline & 1 & & $\mathrm{~B} / \mathrm{C}$ & 17 & 15 & \\
\hline & 1 & & B & \multicolumn{3}{|c|}{$19.0(17.9-19.16 .5$ (15.7-17. } \\
\hline & 1 & & B & \multicolumn{3}{|c|}{19.9 (18.7-24. 17.7 (16.0-22. } \\
\hline & 1 & & C & 17 & 15 & \\
\hline & 1 & & B & \multicolumn{3}{|c|}{$19.5(18.8-20.17 .0(16.2-17}$. \\
\hline & 1 & & $\mathrm{~B}$ & \multicolumn{3}{|c|}{$19.4(17.4-22.17 .1(16.1-20}$. \\
\hline & 1 & & A & \multicolumn{3}{|c|}{$19.9(18.3-23.17 .0(12.2-21}$. \\
\hline & 1 & & $\mathrm{~B}$ & \multicolumn{3}{|c|}{$19.3(18.3-21.17 .1(15.7-22}$. \\
\hline$\sim 69^{\circ} 46^{\prime} \mathrm{W}$ & 1 & & W2 & & & \\
\hline \multirow[t]{23}{*}{$68^{\circ} 36.42^{\prime} \mathrm{W}$} & 1 & S1 & W2 & \multicolumn{3}{|c|}{$18.5 \pm 0.2(\mathrm{~N}=116.2 \pm 0.1(\mathrm{~N}=11.2 \pm 0.2(\mathrm{~N}=13$} \\
\hline & 1 & & B & \multicolumn{3}{|c|}{$19.2(18.0-21.16 .6(15.5-17$} \\
\hline & 1 & & $\mathrm{~B}$ & $19.4(18$ & $.17 .1(16$. & \\
\hline & 1 & & B & $19.5(18$. & $17.3(15$. & \\
\hline & 1 & & B & $19.7(18$. & 17.0 & \\
\hline & 1 & & B & 19.3 & 17.2 (16. & \\
\hline & 1 & & B & 18.7 (17. & $16.5(14.7$ & \\
\hline & 1 & & B & 19.0 (18. & 16.8 & \\
\hline & 1 & & B & 18.9 & $.16 .7(14$. & \\
\hline & 1 & & B & 19.7 (18. & 16.9 & \\
\hline & 1 & & B & 19.7 (18. & $17.2(14$. & \\
\hline & 1 & & $A$ & 17.2 & 7.15 .4 & \\
\hline & 1 & & B & 19.3 & 2. $17.1(15$ & \\
\hline & 1 & & C & 18.9 (17. & . 15. & \\
\hline & 1 & & B & 19.6 (18. & 3. 17.1 (16. & \\
\hline & 1 & & B & 18.3 & 9. $16.4(15$ & \\
\hline & 1 & & B & 17.6 & $1.15 .9(14$. & \\
\hline & 1 & & B & 20.1 & $2.17 .5(16$ & \\
\hline & 1 & & B & 18.9 & ). 16.5 & \\
\hline & 1 & & B & 17.8 & ). 15.9 (14. & \\
\hline & 1 & & B & 19.4 & $.17 .2(16$ & \\
\hline & 1 & & $\mathrm{~B} / \mathrm{C}$ & 18.6 & ). 16.3 & \\
\hline & 1 & & B & $19.8(18$ & $2.17 .4(16$. & \\
\hline
\end{tabular}


A/B

A

C

B

C

B

C

B

B

B

W2

B/C

C

B

A

C

\section{B}

B

B

B

B

C

C

C

B

B

B

B

B

B

B

B

B

B

B
19.5 (17.9-23. 17.0 (16.1-18.

19.1 (17.3-20. 16.9 (16.2-18.

18.1 (17.1-20. 16.7 (15.5-19.

19.4 (18.2-22. 16.5 (14.3-17.

18.9 (17.7-19. 16.7 (15.6-18.

19.1 (18.0-23. 17.0 (15.9-20.

19.9 (18.1-21. 17.2 (15.7-19.

18.7 (17.7-20. 16.4 (15.0-19.

19.6 (18.4-20. 17.5 (16.5-20.

19.2 (18.1-21. 17.3 (16.6-20.

17

19.5 (17.7-21. 17.2 (3.6-27.3

20.0 (18.7-22. 16.9 (16.1-17.

18.6 (16.9-21. 16.4 (15.2-21.

19.0 (17.3-22. 17.1 (15.7-21.

19.1 (17.1-21. 17.1 (15.6-20.

18.7 (18.3-19. 16.7 (15.5-17.

18.5 (17.5-19. 16.5 (15.8-18.

19.3 (17.7-21. 16.8 (14.2-19.

20.0 (18.5-21. 18.1 (16.8-21.

$18.2 \pm 0.2 \quad 16.4 \pm 0.4 \quad 1.5 \pm 0.3$

19.3 (18.2-23. 16.5 (15.5-17.

19.1 (18.3-20. 16.9 (15.8-17.

$19.6(18.1-22.17 .8$ (15.8-23.

19.7 (18.6-22. 17.5 (16.1-20.

19.7 (18.0-24. 17.4 (14.8-21.

19.4 (18.3-20. 17.5 (16.4-19.

19.1 (17.8-22. 16.9 (15.3-18.

19.4 (16.5-22. 17.0 (15.2-19.

18.7 (17.5-21. 16.6 (15.9-17.

18.1 (16.6-21. 16.2 (15.1-19.

19.0 (16.7-21. 16.9 (15.4-18.

19.0 (17.7-24. 16.5 (14.8-18.

18.7 (18.1-20. 16.5 (14.8-17.

18.4 (16.5-21. 16.4 (15.7-18.

20.8 (18.5-23. 17.7 (10.7-26.

19.4 (17.5-21. 16.9 (14.5-18.

19.4 (18.0-21. 16.8 (16.0-18.

19.0 (18.1-20. 16.9 (15.0-19.

18.3 (17.1-24. 15.9 (13.7-17.

19.0 (17.5-20. 16.9 (14.2-19.

18.2 (16.8-21. 16.4 (15.2-19.

19.0 (18.0-20. 16.5 (15.7-17.

17.6 (16.4-19. 15.8 (13.4-17.

18.3 (16.1-23. 16.7 (15.3-19. 
17.9 (16.6-21. 16.4 (14.7-18. 19.0 (17.7-22. 16.8 (14.8-19. 19.8 (18.7-22. 17.1 (11.2-18. 19.7 (18.4-21. 17.1 (12.3-19. 19.3 (18.2-23. 16.8 (15.7-18. 17.6 (16.5-20. 16.0 (15.0-16. 19.2 (17.9-23. 17.7 (15.6-21. 19.3 (15.1-23. 17.8 (15.2-26. 19.2 (18.4-20. 17.2 (15.9-21. 19.3 (18.0-22. 17.1 (16.1-21. 18.6 (17.8-21. 16.5 (15.5-17. 20.1 (18.7-22. 17.5 (16.1-18. 19.5 (17.4-24. 16.6 (5.5-25.3 19.4 (17.8-20. 17.5 (16.5-19. 20 17 18.1 (16.9-21. 16.5 (14.3-22. 16 15 19 17 19.5 (18.4-20. 17.1 (15.7-20. 17.0 (12.2-20. 16.2 (14.4-18. 18.0 (16.6-20. 16.0 (14.6-17. 19 17

18.7 (16.9-19. 17.1 (15.3-20. 19.2 (17.9-21. 16.9 (14.9-19. 19.1 (18.3-20. 16.8 (15.3-18. 18.2 (16.8-22. 16.4 (14.4-20. 19.4 (17.9-23. 16.8 (15.4-19. 19.4 (18.6-21. 16.8 (14.3-19. 18.6 (17.5-20. 16.5 (15.6-19. 18.3 (17.5-21. 16.2 (14.3-19. $18.9 \pm 0.4 \quad 16.6 \pm 0.3 \quad 1.3 \pm 0.2$ 19.3 (18.5-20. 16.8 (16.0-17. 17.9 (16.1-19. 16.0 (15.1-18. 19.1 (18.0-20. 16.6 (15.8-17. 18.1 (16.5-20. 16.3 (15.0-17. 18.8 (17.8-20. 16.5 (15.5-17. 18.7 (17.6-19. 16.7 (14.7-19. $17.9(16.8-20.16 .0(14.5-18$. 19.8 (18.7-23. 17.1 (15.3-18. 19.1 (17.2-21. 16.8 (15.8-18. 17.9 (17.1-21. 16.6 (15.0-21. 18.8 (17.8-22. 16.4 (15.5-18. 19.7 (18.3-23. 17.5 (16.3-20. 18.1 (16.4-22. 16.5 (15.4-18.

\section{B} $\mathrm{Ce}$ 
B

B

B

B

B

C

B

B

B

B

C

156 $40.593^{\prime} \mathrm{E} 1$

$76^{\circ} 55.26^{\prime} \mathrm{E} \quad 1$

10²5'10.17"E 4

7011'40.9"W 3

6947.183'W 2

$\sim 69^{\circ} 46^{\prime} \mathrm{W} \quad 1$

$85^{\circ} 41.86$ 'E 20

$69^{\circ} 43.577^{\prime} \mathrm{W} 1$

69³9'05.5"W 1

$116^{\circ} 46.096^{\prime} \mathrm{W}$ many

$69^{\circ} 49^{\prime} 14^{\prime \prime} \mathrm{W} \quad 1$

\section{1}

1

$68^{\circ} 34.622^{\prime}$ W 19

\section{1}

$69^{\circ} 55.177^{\prime} \mathrm{W} 3$

$\begin{array}{r}1 \\ 1 \\ \hline\end{array}$

$69^{\circ} 50.947$ 'W 1

$69^{\circ} 47.325^{\prime} \mathrm{W} 1$

6948.309'W 1
S2

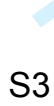

S3

S4

S4

S2

S2

S3

S2

S1

S1

S2

S4

S1

S3

S3

S3

S1

S4

S3

S2

S1

S1
18.0 (16.6-20. 16.6 (14.9-19.

19.4 (18.2-20. 17.1 (15.7-19.

17

15

19.5 (18.0-24. 17.2 (14.6-21.

18.7 (17.9-19. 16.5 (14.8-18.

19.6 (18.6-23. 16.8 (15.9-18.

19.2 (18.3-22. 16.9 (15.0-19.

19.0 (17.6-22. 16.5 (15.2-17.

17.5 (15.6-18. 16.4 (14.9-19.

19.5 (18.1-23. 17.5 (15.6-19.

19.8 (18.5-23. 17.0 (15.6-18.

18.4 (17.3-20. 17.0 (15.3-19.

19.4 (18.3-22. 17.3 (16.2-21.

$19.6 \pm 0.4 \quad 17.2 \pm 0.3 \quad 1.3 \pm 0.4$

19.4 (17.6-21. 17.2 (16.4-19.

$19.1 \pm 0.5(\mathrm{~N}=116.7 \pm 0.3(\mathrm{~N}=11.4 \pm 0.2(\mathrm{~N}=16$

$19.0 \pm 0.6(n=416.6 \pm 0.3(n=61.1 \pm 0.1(n=6)$

$20.9 \pm 0.3(n=1$.

18.6 $\pm 0.3(\mathrm{~N}=116.5 \pm 0.4(\mathrm{~N}=11.3 \pm 0.4(\mathrm{~N}=18$

$19.2(\mathrm{~N}=1) \quad 16.4(\mathrm{~N}=1) \quad 1.7$

$\begin{array}{lll}19.1 & 16.3 & 1.4\end{array}$

19.3

$19.2 \pm 0.1 \quad 17.0 \pm 0.2 \quad 1.3 \pm 0.3$

$20.8 \pm 0.1(n=717.1 \pm 0.1(n=83.3$

$19.5 \pm 0.4 \quad 17.0 \pm 0.3 \quad 1.3 \pm 0.2$

$19.9 \quad 17.7 \quad 1.5$

19.8 $\pm 0.3(\mathrm{~N}=218.1 \pm 0.4(\mathrm{~N}=21.2 \pm 0.4(\mathrm{~N}=22$

$20.1 \pm 0.11(\mathrm{~N}=19.25 \pm 0.23(\mathrm{~N} 2.29 \pm 0.07(\mathrm{~N}=$ $18.8 \pm 0.2(n=116.6 \pm 0.2(n=11.3 \pm 0.1(n=11$ 18.9 (18.3-19. 16.5 (15.7-17.

$19.5 \pm 0.1(n=816.8 \pm 0.1(n=81.1 \pm 0.1$

19.3 $\pm 0.4(\mathrm{~N}=217.0 \pm 0.3(\mathrm{~N}=21.5 \pm 0.2(\mathrm{~N}=25$

$19.4 \pm 0.1(n=817.4 \pm 0.1(n=71.2$

$18.8 \pm 0.3(\mathrm{~N}=116.7 \pm 0.4(\mathrm{~N}=11.0 \pm 0.4(\mathrm{~N}=19$

18.6 (17.5-21. 16.4 (15.5-20.

19.9-20.0 16.9-17.6 1.2-1.1

19.2 $\pm 0.3(\mathrm{~N}=216.8 \pm 0.4(\mathrm{~N}=21.0 \pm 0.4(\mathrm{~N}=20$

$18.6 \pm 0.9(n=617.0 \pm 0.7(n=50.9 \pm 0.7$

18.6 $\pm 0.3(\mathrm{~N}=216.5 \pm 0.4(\mathrm{~N}=21.1 \pm 0.4(\mathrm{~N}=21$ 18.6 (17.8-19. 16.4 (15.0-17.

$18.5 \pm 0.3(\mathrm{~N}=217.1 \pm 0.4(\mathrm{~N}=20.9 \pm 0.4(\mathrm{~N}=21$

18.3 $\pm 0.3(n=1.16 .3 \pm 0.1(n=1.1 .4 \pm 0.2(n=12$

19.5 (17.7-20. 17.1 (16.0-19. 


\begin{tabular}{|c|c|c|c|c|c|}
\hline \multirow{3}{*}{$69^{\circ} 54.539^{\prime} \mathrm{W}$} & 1 & S2 & W2 & \multicolumn{2}{|l|}{18.2} \\
\hline & 1 & S1 & w2 & \multicolumn{2}{|c|}{$18.0 \pm 0.3(\mathrm{~N}=216.6 \pm 0.4(\mathrm{~N}=21.1 \pm 0.4(\mathrm{~N}=22$} \\
\hline & 1 & S2 & W1 & $19.5-19.8$ & $16.9-17.4 \quad 1.5-1.3$ \\
\hline $70^{\circ} 08^{\prime} 43^{\prime \prime} \mathrm{W}$ & 1 & s2 & W2 & \multicolumn{2}{|c|}{$19.4 \pm 0.2(n=1.17 .1 \pm 0.1(n=11.4 \pm 0.2(n=13$} \\
\hline \multirow[t]{2}{*}{$69^{\circ} 56.696^{\prime} \mathrm{W}$} & 1 & S3 & W1 & \multicolumn{2}{|c|}{$18.3 \pm 0.3(\mathrm{~N}=216.3 \pm 0.4(\mathrm{~N}=20.9 \pm 0.4(\mathrm{~N}=22$} \\
\hline & 1 & & A & \multicolumn{2}{|c|}{$20.0(19.2-21.17 .5$ (16.3-18. } \\
\hline \multirow[t]{3}{*}{$68^{\circ} 38.786^{\prime} \mathrm{W}$} & 2 & S2 & W2 & \multicolumn{2}{|c|}{$19.44 \pm 0.18(\mathrm{~N} 17.28 \pm 0.35(\mathrm{~N} 1.45 \pm 0.14(\mathrm{~N}=$} \\
\hline & 1 & S2 & W3 & \multicolumn{2}{|c|}{$18.9 \pm 0.26(n=16.84 \pm 0.22(n) 1.41 \pm 0.09(n=$} \\
\hline & 1 & S2 & W2 & \multicolumn{2}{|c|}{$19.15 \pm 0.25(n: 16.93 \pm 0.24(n) 1.54 \pm 0.14(n=$} \\
\hline \multirow[t]{2}{*}{$69^{\circ} 47.512^{\prime} \mathrm{W}$} & 1 & s2 & w2 & \multicolumn{2}{|c|}{$19.1 \pm 0.3(\mathrm{~N}=116.8 \pm 0.4(\mathrm{~N}=10.9 \pm 0.4(\mathrm{~N}=16$} \\
\hline & 1 & S3 & W4 & $20.1 \pm 0.9$ & $18.9 \pm 0.8$ \\
\hline \multirow{4}{*}{$68^{\circ} 39.227^{\prime} \mathrm{W}$} & 2 & S2 & W2 & \multicolumn{2}{|c|}{$19.73 \pm 0.36(\mathrm{~N} 17.24 \pm 0.26(\mathrm{~N} 1.22 \pm 0.25(\mathrm{~N}=$} \\
\hline & 1 & & $\mathrm{~B}$ & \multicolumn{2}{|c|}{$19.5(18.8-19.17 .3(15.7-20$} \\
\hline & 1 & & B & 18 & 17 \\
\hline & 1 & S4 & W2 & $19.8 \pm 1$ & \\
\hline \multirow[t]{10}{*}{$\sim 69^{\circ} 46^{\prime} \mathrm{W}$} & 1 & & W3 & & \\
\hline & 1 & S2 & W2 & \multicolumn{2}{|c|}{$18.81 \pm 0.14(n \cdot 16.66 \pm 0.4(n=1.33 \pm 0.12(n=$} \\
\hline & 1 & S3 & W2 & \multicolumn{2}{|c|}{$20.06 \pm 0.32(n \cdot 17.87 \pm 0.47(n \cdot 1.24 \pm 0.27(n=$} \\
\hline & 1 & S2 & W2 & \multicolumn{2}{|c|}{$19.57 \pm 0.86(n-17.32 \pm 0.32(n \cdot 1.52 \pm 0.43(n=$} \\
\hline & 1 & S3 & w3 & \multicolumn{2}{|c|}{$19.22 \pm 0.2(n=17.6 \pm 1.55(n=1.45 \pm 0.19(n=$} \\
\hline & 1 & S1 & W2 & \multicolumn{2}{|c|}{$18.98 \pm 0.37(n \cdot 16.78 \pm 0.18(n \cdot 1.38 \pm 0.18(n=$} \\
\hline & 1 & S2 & W2 & \multicolumn{2}{|c|}{$18.78 \pm 0.68(n-16.79 \pm 1.67(n) 1.14 \pm 0.41(n=$} \\
\hline & 1 & & C & 19 & 17 \\
\hline & 1 & & $\mathrm{C}$ & \multicolumn{2}{|c|}{20.3 (18.9-22. 17.4 (16.3-21. } \\
\hline & 1 & S2 & W3 & $19.55 \pm 1.0$ & $17.04 \pm 0.41(n \cdot 1.72 \pm 1.46(n=$ \\
\hline $69^{\circ} 51.649^{\prime} \mathrm{W}$ & 1 & S3 & W1 & $19.1 \pm 0.3$ & $216.5 \pm 0.4(N=11.0 \pm 0.4(N=18$ \\
\hline & 1 & & $B$ & 19 & 18 \\
\hline & 1 & S2 & W2 & $19.19 \pm 0.2$ & $16.67 \pm 0.25(n-1.17 \pm 0.22(n=$ \\
\hline & 1 & S2 & W3 & $19.08 \pm 0.2$ & $17.05 \pm 0.18(n) 1.5 \pm 0.25 \quad(n=1$ \\
\hline & 1 & & C & $18.7(17.6$ & $.16 .3(14.8-16$. \\
\hline & 1 & S3 & W2 & $18.67 \pm 0.4$ & $16.46 \pm 0.51(n-1.11 \pm 0.23(n=$ \\
\hline & 1 & & $B / C$ & 18 & 17 \\
\hline & 1 & S1 & W4 & $20.8 \pm 0.3$ & $318.0 \pm 0.3(n=8 \quad 1.6 \pm 0.1$ \\
\hline & 1 & S3 & W3 & $19.11 \pm 0.6$ & $16.86 \pm 0.86(n-1.15 \pm 0.3(n=7$ \\
\hline & 1 & & C & 17 & 15 \\
\hline & 1 & S2 & W2 & $18.38 \pm 0.4$ & $16.46 \pm 0.36(n-1.23 \pm 0.14$ \\
\hline & 1 & S3 & W3 & $20.03 \pm 0.5$ & $17.96 \pm 0.76(n-1.37 \pm 0.11(n=$ \\
\hline & 1 & & $B$ & $19.3(18.2$ & $17.0(15.8-17$ \\
\hline & 1 & S3 & W2 & $19.41 \pm 1.7$ & $15.6 \pm 2.05(n=1.83 \pm 0.67$ \\
\hline & 1 & & C & 17 & 15 \\
\hline & 1 & & C & $18.5(18.0$ & $16.2(14.5-17$ \\
\hline & 1 & S1 & W2 & $18.75 \pm 0.3$ & $16.47 \pm 0.22(n-1.3 \pm 0.18(n=6$ \\
\hline & 1 & & $\mathrm{~B} / \mathrm{Ce}$ & 21 & 18 \\
\hline & 1 & & B & $19.4(18.1$ & $17.6(15.8-20$ \\
\hline & 1 & S2 & W3 & $19.81 \pm 2.7$ & $16.47 \pm 1.12(n-1.16 \pm 0.17(n=$ \\
\hline
\end{tabular}




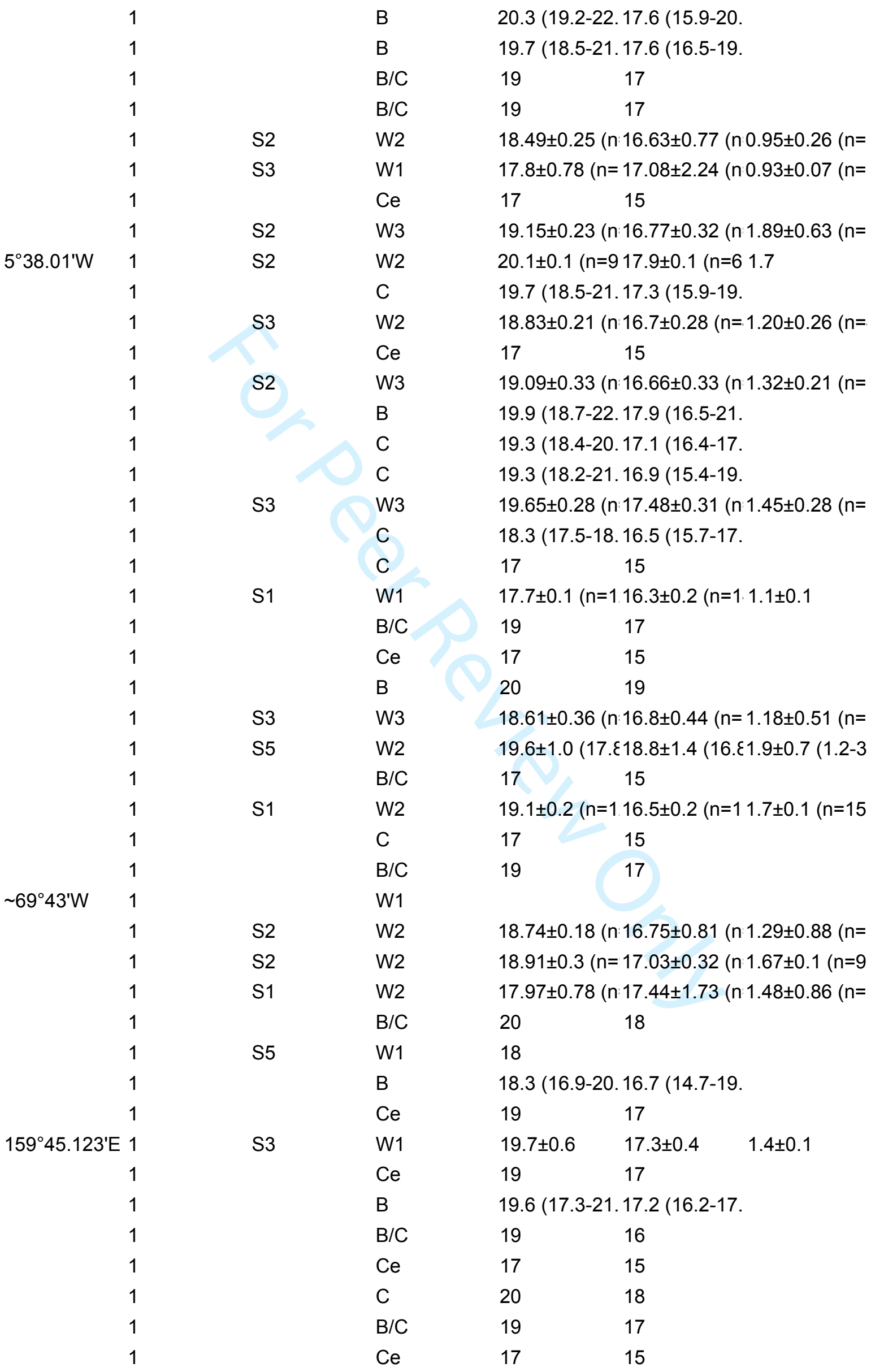




\begin{tabular}{|c|c|c|c|c|c|}
\hline 1 & S5 & W2 & \multicolumn{3}{|l|}{$20.0 \pm 1$} \\
\hline 1 & & $B$ & \multicolumn{3}{|c|}{$20.2(19.6-22.17 .8$ (16.8-20. } \\
\hline 1 & & C & \multicolumn{3}{|c|}{$18.4(17.7-21.16 .3(14.5-17$} \\
\hline 1 & & $\mathrm{C}$ & 18 & 16 & \\
\hline $114^{\circ} 16.151^{\prime} \mathrm{W} 1$ & S3 & W1 & $19.9 \pm 0.5$ & $17.3 \pm 0.5$ & $1.4 \pm 0.4$ \\
\hline 1 & & $\mathrm{Ce}$ & 19 & 18 & \\
\hline 1 & & $B$ & \multicolumn{3}{|c|}{$19.6(18.6-21.17 .5$ (15.8-19. } \\
\hline 1 & & B & \multicolumn{3}{|c|}{$20.3(18.6-23.18 .2$ (16.2-22. } \\
\hline 1 & & B & \multicolumn{3}{|c|}{20.0 (18.8-22. 17.6 (16.3-23. } \\
\hline 1 & & $\mathrm{~B} / \mathrm{C}$ & 18 & 16 & \\
\hline 1 & & B & \multicolumn{3}{|c|}{20.2 (18.7-24. 17.5 (16.1-18. } \\
\hline 1 & & B & \multicolumn{3}{|c|}{19.3 (18.2-20. 16.7 (15.4-17. } \\
\hline 1 & & B & \multicolumn{3}{|c|}{19.7 (18.6-22. 17.2 (16.3-18. } \\
\hline 1 & & $\mathrm{~B} / \mathrm{C}$ & 18 & \multicolumn{2}{|c|}{16} \\
\hline 1 & & $\mathrm{~B} / \mathrm{C}$ & 19 & \multicolumn{2}{|l|}{18} \\
\hline 1 & S1 & W1 & \multicolumn{3}{|c|}{$19.01 \pm 0.29(n \cdot 16.8 \pm 0.32(n=1.22 \pm 0.20(n=$} \\
\hline 1 & & $B / C$ & 20 & \multicolumn{2}{|c|}{17} \\
\hline 1 & & B & \multicolumn{3}{|c|}{$19.5(17.8-26.17 .6(16.0-20}$. \\
\hline 1 & & $\mathrm{Ce}$ & 18 & \multicolumn{2}{|c|}{16} \\
\hline 1 & & $\mathrm{Ce}$ & \multicolumn{3}{|l|}{19} \\
\hline 1 & & $\mathrm{C}$ & 18 & \multicolumn{2}{|l|}{16} \\
\hline 1 & & $\mathrm{~B} / \mathrm{C}$ & 20 & \multicolumn{2}{|l|}{19} \\
\hline 1 & & B & \multicolumn{3}{|c|}{19.5 (18.5-21. $17.2(14.8-19$} \\
\hline 1 & & $\mathrm{C}$ & 19 & \multicolumn{2}{|c|}{17} \\
\hline 1 & S4 & W2 & \multicolumn{3}{|c|}{$18.95 \pm 0.35(n-17.03 \pm 0.38(n) 1.31 \pm 0.11(n=$} \\
\hline 1 & & $\mathrm{~B} / \mathrm{C}$ & 19 & \multicolumn{2}{|c|}{17} \\
\hline 1 & & B & \multicolumn{3}{|c|}{$19.0(17.4-21.17 .1(15.5-20}$. \\
\hline $156^{\circ} 39.601^{\prime} E 1$ & S1 & W1 & $20.0 \pm 0.5$ & $17.2 \pm 0.4$ & $1.3 \pm 0.3$ \\
\hline 1 & & $\mathrm{~B} / \mathrm{C}$ & 17 & \multicolumn{2}{|l|}{15} \\
\hline 1 & & B & $19.7(19.0$ & 17.3 & \\
\hline 1 & & $\mathrm{~B} / \mathrm{C}$ & 19 & 18 & \\
\hline 1 & & C & 19 & 16 & \\
\hline 1 & & $\mathrm{Ce}$ & 18 & 16 & \\
\hline 1 & S3 & W2 & $19.97 \pm 0.2$ & $17.42 \pm 0$ & $1.49 \pm 0.18(n=$ \\
\hline 1 & & $\mathrm{C}$ & 18 & & \\
\hline 1 & & $\mathrm{~B} / \mathrm{C}$ & 18 & 16 & \\
\hline 1 & & $C$ & 18 & 16 & \\
\hline 1 & & $B / C$ & 19 & 18 & \\
\hline 1 & & $\mathrm{~B} / \mathrm{C}$ & 19 & 17 & \\
\hline 1 & & B & $21.4(18.5$ & 17.7 & \\
\hline 1 & & $\mathrm{~B} / \mathrm{Ce}$ & 19 & 16 & \\
\hline 1 & & B & $19.7(18.5$ & 17.3 & \\
\hline 1 & & $\mathrm{~B} / \mathrm{Ce}$ & 17 & 15 & \\
\hline 1 & & $\mathrm{Ce}$ & 19 & 17 & \\
\hline 1 & & $B$ & $20.3(18.4$ & 17.9 & \\
\hline
\end{tabular}




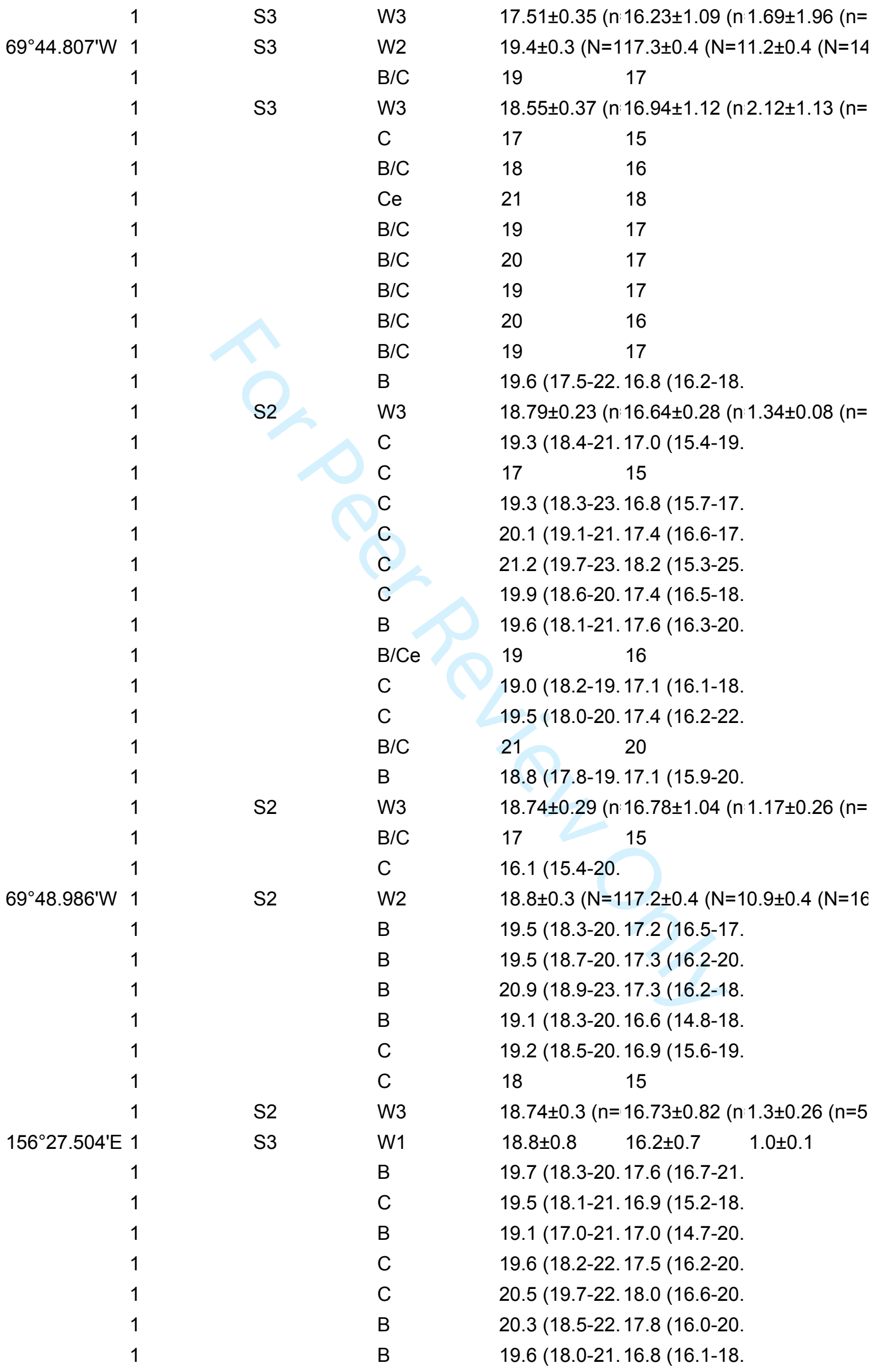


C

B

B

$\mathrm{B} / \mathrm{C}$

B

B

B

S1

S2

W1

W3

B

B

B

C

C

B

B

B

B

B

B

B

B

B

C

C

B

B

C

B

B

B

B

B

B

B

B

B

B

W3

$\mathrm{B} / \mathrm{C}$

W1

B

B

B
19.9 (18.3-22. 17.7 (16.1-19.

19.7 (18.4-20. 17.4 (16.0-18.

$19.3(18.2-22.17 .4(15.8-20$.

19.7 (18.4-23. 17.0 (15.8-18.

20

17

19.4 (18.1-25. 17.1 (15.8-20.

19.4 (18.6-20. 17.1 (16.1-17.

19.4 (18.3-20. 17.4 (16.5-20.

$19.3 \pm 0.1 \quad 16.6 \pm 0.6 \quad 1.4 \pm 0.1$

$19.28 \pm 0.59$ (n $16.68 \pm 0.29$ (n $1.43 \pm 0.62(n=$ 19.0 (17.9-21. 17.0 (15.7-21.

19.6 (18.1-22. 16.9 (15.9-17.

19.4 (18.4-20. 16.9 (15.1-20.

18.7 (17.2-20. 16.7 (15.1-20.

20.1 (19.4-21. 17.9 (16.2-20.

18.9 (17.3-20. 17.0 (16.0-18.

19.1 (18.0-19. 17.1 (16.6-17.

19.3 (18.3-19. 17.4 (16.6-19.

19.6 (18.2-22. 17.5 (15.4-21.

17.3 (16.3-17. 15.4 (14.1-19.

19.7 (19.0-22. 17.2 (15.3-18.

19.3 (18.3-20. 16.9 (15.7-19.

19.6 (18.2-23. 17.7 (15.8-23.

20.3 (18.9-23. 17.2 (15.4-19.

19.4 (18.8-20. 17.2 (16.3-17.

19.3 (18.1-21. 17.3 (15.7-21.

19.4 (17.8-21. 17.3 (16.6-18.

18.9 (17.3-20. 16.8 (15.3-19.

19.8 (18.5-21. 16.8 (15.5-18.

19.5 (17.7-23. 17.2 (15.6-23.

20.3 (18.7-24. 17.8 (16.7-19.

19.9 (18.6-22. 17.1 (16.2-18.

19.3 (18.4-22. 16.7 (15.0-19.

20.1 (18.9-21. 17.8 (16.9-19.

19.5 (18.1-24. 17.5 (16.2-20.

19.7 (18.3-24. 16.8 (14.6-19.

20.0 (19.1-21. 17.6 (16.3-18.

18.9 (17.4-20. 16.6 (14.6-18.

19.2 (18.1-21. 16.6 (15.3-18.

$19.09 \pm 0.23(n-17.12 \pm 0.38$ (n $1.4 \pm 0.15(n=5$

19.4 (18.3-22. 17.3 (14.5-20.

$19.1 \pm 0.2 \quad 16.7 \pm 0.1 \quad 1.2 \pm 0.4$

19.5 (18.9-22. 17.0 (12.7-20.

18.9 (17.7-20. 17.1 (14.9-20.

19.2 (18.1-22. 17.4 (15.2-21. 


\begin{tabular}{|c|c|c|c|c|c|}
\hline 1 & & C & \multicolumn{3}{|c|}{18.5 (17.8-19. 16.4 (16.0-16. } \\
\hline 1 & & B & \multicolumn{3}{|c|}{$19.5(17.8-22.17 .1$ (15.7-18. } \\
\hline 1 & & B & \multicolumn{3}{|c|}{$19.5(18.3-21.17 .3(15.6-20}$. \\
\hline 1 & & B & \multicolumn{3}{|c|}{$19.3(18.6-21.17 .2(16.0-19}$. \\
\hline 1 & & B & \multicolumn{3}{|c|}{$18.9(17.5-19.16 .9$ (16.2-18. } \\
\hline 1 & & B & \multicolumn{3}{|c|}{$19.1(17.5-20.17 .2(15.5-22}$. \\
\hline $156^{\circ} 34.660^{\prime} \mathrm{E}$ & S1 & W1 & $19.0 \pm 0.04$ & $17.0 \pm 0.2$ & $1.5 \pm 0.3$ \\
\hline 1 & & $\mathrm{~B}$ & \multicolumn{3}{|c|}{$19.2(18.1-21.17 .0(15.8-19$} \\
\hline 1 & & B & \multicolumn{3}{|c|}{$20.2(19.1-22.17 .8$ (16.3-21. } \\
\hline 1 & & B & \multicolumn{3}{|c|}{$19.2(17.6-21.17 .1$ (15.1-21. } \\
\hline 1 & & B & \multicolumn{3}{|c|}{$19.0(18.2-19.16 .8$ (16.1-17. } \\
\hline 1 & & B & \multicolumn{3}{|c|}{$19.0(17.6-21.16 .5(14.6-18$} \\
\hline $156^{\circ} 35.571^{\prime} \mathrm{E}$ & S1 & W1 & $19.2 \pm 0.2$ & $16.8 \pm 0.2$ & $1.4 \pm 0.2$ \\
\hline 1 & & C & \multicolumn{3}{|c|}{$18.6(17.2-23.16 .5(15.8-17$} \\
\hline 1 & & B & \multicolumn{3}{|c|}{$19.9(18.4-21.17 .6(15.7-21}$. \\
\hline 1 & & B & \multicolumn{3}{|c|}{19.4 (18.4-21. 17.3 (15.8-19. } \\
\hline 1 & & C & \multicolumn{3}{|c|}{$19.0(17.5-22.16 .8$ (15.9-19. } \\
\hline 1 & & B & \multicolumn{3}{|c|}{$19.4(17.5-21.16 .9$ (15.7-20. } \\
\hline 1 & & B & \multicolumn{3}{|c|}{19.9 (19.1-23. 17.4 (16.5-17. } \\
\hline 1 & & B & \multicolumn{3}{|c|}{$20.6(19.2-27.17 .2(16.2-17$} \\
\hline 1 & & $\mathrm{Ce}$ & 19 & 18 & \\
\hline 1 & & C & \multicolumn{3}{|c|}{19.4 (18.1-22. 16.9 (15.4-19. } \\
\hline 1 & & $\mathrm{Ce}$ & 17 & 15 & \\
\hline 1 & & C & \multicolumn{3}{|c|}{$16 \quad 15$} \\
\hline 1 & & B & \multicolumn{3}{|c|}{$19.8(18.6-21.17 .0$ (15.9-18. } \\
\hline 1 & & $\mathrm{~B}$ & \multicolumn{3}{|c|}{19.5 (18.4-20. 16.7 (16.0-17. } \\
\hline $156^{\circ} 36.976^{\prime} \mathrm{E}$ & S1 & W1 & $19.3 \pm 0.2$ & $16.9 \pm 0.2$ & $1.4 \pm 0.2$ \\
\hline $156^{\circ} 35.709^{\prime} \mathrm{E}$ & S1 & W1 & $19.2 \pm 0.3$ & $17.0 \pm 0.1$ & $1.3 \pm 0.2$ \\
\hline 1 & & C & 18 & 17 & \\
\hline 1 & & C & \multicolumn{3}{|c|}{$19.1(17.9-22.16 .9(16.4-17$} \\
\hline 1 & & C & 17 & 15 & \\
\hline $156^{\circ} 34.702^{\prime} \mathrm{E}$ & S1 & W1 & $19.1 \pm 0.2$ & $16.9 \pm 0.4$ & $1.2 \pm 0.2$ \\
\hline 1 & & & 17 & 15 & \\
\hline 1 & & & 17 & 15 & \\
\hline 1 & & C & $19.2(18.5$ & $17.1(16.3$ & \\
\hline 1 & & & 17 & 15 & \\
\hline 1 & & B & $20.2(18.9$ & $17.5(16.8$ & \\
\hline 1 & & C & $19.5(18.7$ & $17.0(16.1$ & \\
\hline 1 & & C & $19.1(18.1$ & $16.6(15.6$ & \\
\hline 1 & & & 17 & 15 & \\
\hline 1 & & $B$ & $20.3(19.0$ & $17.8(16.4$ & \\
\hline 1 & & & 17 & 15 & \\
\hline $156^{\circ} 37.010^{\prime} \mathrm{E}$ & S1 & W1 & $19.0 \pm 0.3$ & $16.7 \pm 0.2$ & $1.2 \pm 0.2$ \\
\hline 1 & & B & $19.6(18.5$ & $17.2(15.2$ & \\
\hline 1 & & $\mathrm{C}$ & $18.8(17.0$ & 16.6 & \\
\hline
\end{tabular}




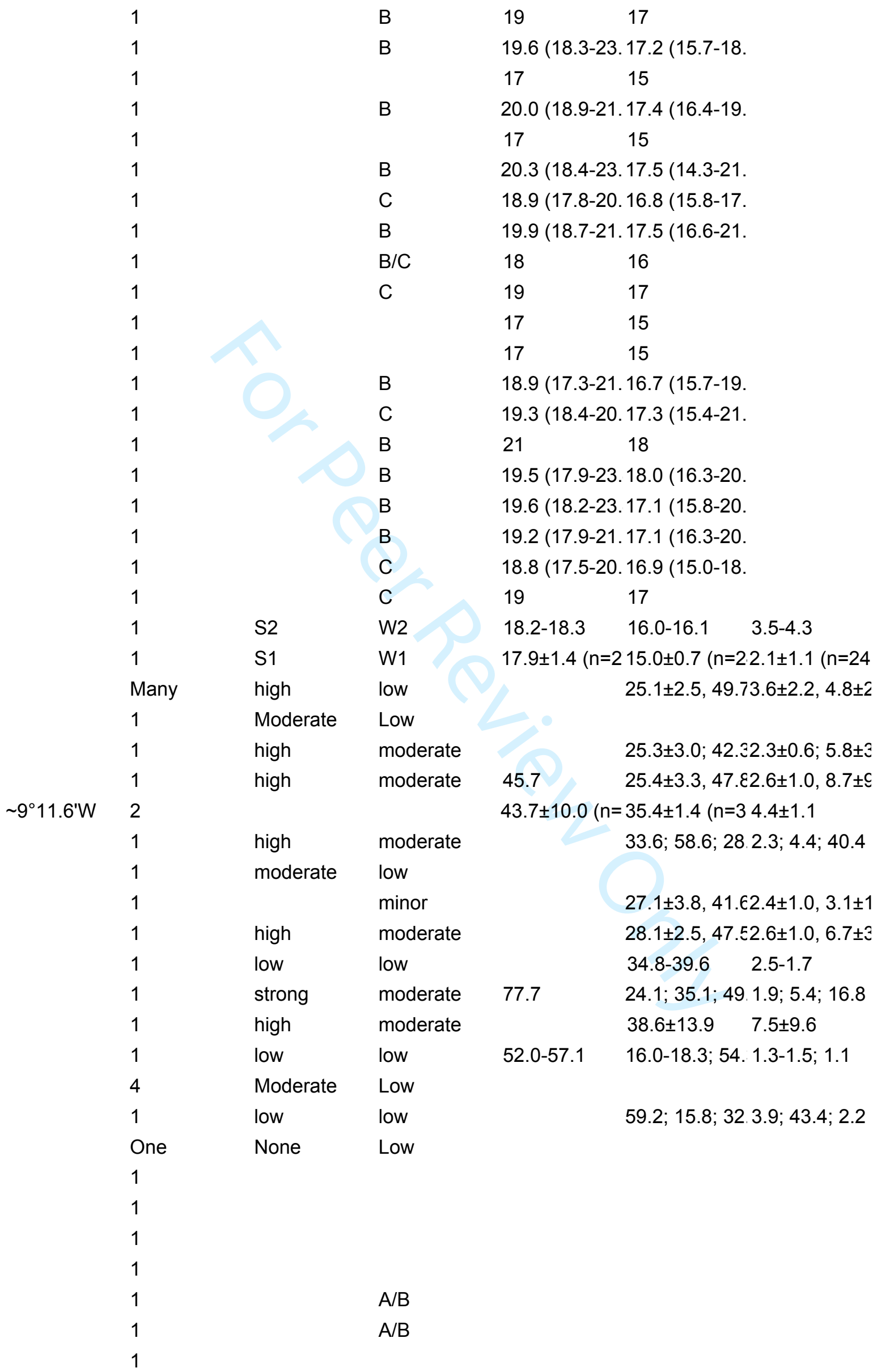




\begin{tabular}{|c|c|c|c|}
\hline \multicolumn{4}{|c|}{1} \\
\hline & 1 & \multirow{3}{*}{ medium } & \multirow{3}{*}{ low } \\
\hline \multicolumn{2}{|c|}{$102^{\circ} 31 ' 28.99 " 1$} & & \\
\hline & 1 & & \\
\hline & 1 & moderate & low \\
\hline $06^{\circ} 54.82^{\prime} \mathrm{E}$ & 1 & & medium \\
\hline \multicolumn{2}{|c|}{$136^{\circ} 46^{\prime} 51.5^{\prime \prime E} 2$} & & \\
\hline \multirow[t]{11}{*}{$86.25^{\circ} \mathrm{E}$} & 1 & & \\
\hline & 1 & & \\
\hline & 1 & & \\
\hline & 1 & & \\
\hline & 1 & & \\
\hline & 1 & & \\
\hline & 1 & & \\
\hline & 1 & & \\
\hline & 2 & low & low \\
\hline & 3 & low & low \\
\hline & 1 & moderate & low \\
\hline \multicolumn{3}{|c|}{$53^{\circ} 39^{\prime} 05.1 " \mathrm{~W} 1$} & \\
\hline $5^{\circ} 14.85^{\prime} \mathrm{E}$ & 1 & low & low \\
\hline 91²42'30" & 1 & & \\
\hline $0^{\circ} 49^{\prime} 26.02 " \mathrm{E}$ & 1 & & minimal \\
\hline \multirow[t]{2}{*}{$6^{\circ} 36.46^{\prime} \mathrm{W}$} & 1 & & moderate \\
\hline & 1 & & \\
\hline $92^{\circ} 06^{\prime} 00^{\prime \prime} \mathrm{E}$ & 4 & & \\
\hline \multirow[t]{4}{*}{$85^{\circ} 54^{\prime} 22^{\prime \prime} \mathrm{E}$} & 1 & high & low \\
\hline & 1 & & $A / B$ \\
\hline & 1 & & $C$ \\
\hline & 1 & S4 & W1 \\
\hline $94^{\circ} 18^{\prime} 6.72 " \mathrm{E}$ & 1 & & W3 \\
\hline $92^{\circ} 15^{\prime} 36.22 "$ & $=1$ & & W3/4 \\
\hline & 1 & & wo \\
\hline $95^{\circ} 23^{\prime} 25.53^{\prime \prime}$ & 2 & & W3 \\
\hline $92^{\circ} 14^{\prime} 45.81^{\prime \prime}$ & $=1$ & & W1 \\
\hline $92^{\circ} 18^{\prime} 37.84^{\prime \prime}$ & $=1$ & & w3 \\
\hline & 1 & & W4 \\
\hline $85^{\circ} 42^{\prime} 4.33^{\prime \prime} \mathrm{E}$ & many & & W1 \\
\hline $89^{\circ} 00^{\prime} 36.02^{\prime \prime}$ & 1 & & W3 \\
\hline $94^{\circ} 23^{\prime} 55.97^{\prime \prime}$ & $=9$ & & W3 \\
\hline & 1 & & W4 \\
\hline $93^{\circ} 13^{\prime} 68.47^{\prime \prime}$ & $=3$ & & w3 \\
\hline & 1 & & W1 \\
\hline $94^{\circ} 14^{\prime} 30.94^{\prime \prime}$ & $=1$ & & W4 \\
\hline & 1 & & W4 \\
\hline & 1 & & W4 \\
\hline
\end{tabular}




\begin{tabular}{|c|c|c|c|c|c|c|}
\hline \multirow{2}{*}{ 3॰34'1.72"E } & \multicolumn{2}{|l|}{1} & \multicolumn{4}{|l|}{ W2 } \\
\hline & Many & S4 & Wo & $24.3 \pm 5.7$ & $17.0 \pm 11.6$ & $2.0 \pm 1.9$ \\
\hline & 1 & S2 & W2 & $24.7 \pm 5.5$ & $19.8 \pm 5.6$ & $1.2 \pm 0.7$ \\
\hline & 1 & S2 & $\mathrm{W} 1 / 2$ & $6.3-43.5$ & $2.1-19.5$ & $0.3-0.4$ \\
\hline & 1 & S1 & W3 & \multicolumn{3}{|c|}{$14.0 \pm 9.6(0.6-12.3 \pm 7.8(3.3-1.1 \pm 1.3(0.1-3$} \\
\hline & 1 & S4 & W2 & $22.1 \pm 3.1$ & $11.6 \pm 5.2$ & $1.0 \pm 1.1$ \\
\hline & 1 & S2 & W2 & \multicolumn{3}{|c|}{$14.9 \pm 9.1(5.2-12.3 \pm 8.1(3.1-0.8 \pm 0.5(0.4-2$} \\
\hline & 1 & & W2 & \multicolumn{3}{|c|}{$25.4 \pm 2.5(n=514.4 \pm 4.7(n=40.6 \pm 3.32$} \\
\hline & 5 & S1 & W4 & \multicolumn{3}{|c|}{$18.4 \pm 12.3(n=7.3 \pm 6.4(n=220.7 \pm 0.5$} \\
\hline & many & & W2 & \multicolumn{3}{|c|}{$22.7 \pm 8.2(\mathrm{~N}=714.8 \pm 10.3(\mathrm{~N}=0.5 \pm 0.2$} \\
\hline & 4 & S1 & W2 & \multicolumn{3}{|c|}{$23.4 \pm 2.8(15.514 .4 \pm 6.5(2.4-1.0 \pm 0.7(0.3-3$} \\
\hline & 5 & S1 & W2 & \multicolumn{3}{|c|}{$23.2 \pm 4.4(10 . \varepsilon 15.0 \pm 6.9(5.9-0.7 \pm 0.7(0.2-4$} \\
\hline & 2 & S2 & W1 & $0.6-81.6$ & $1.5-23.3$ & $0.2-3.4$ \\
\hline & 1 & & B & \multicolumn{3}{|c|}{$24.5(13.3-27.21 .0(12.5-23$} \\
\hline & 3 & S2 & W2 & $4.7-26.5$ & $9.2-12.9$ & $0.9-1.1$ \\
\hline & 1 & S2 & W0/1 & $1.8-64.0$ & $3.3-18.8$ & $0.2-2.0$ \\
\hline & 1 & S1 & W2 & \multicolumn{3}{|c|}{$14.5 \pm 9.4(0.8-12.6 \pm 8.0(1.4-1.3 \pm 0.9(0.5-4$} \\
\hline \multirow[t]{12}{*}{$105.30^{\circ} \mathrm{W}$} & 1 & S3 & W1 & $22.1 \pm 7.5$ & $11.6 \pm 8.2$ & $0.9 \pm 0.8$ \\
\hline & 1 & S1 & W2 & \multicolumn{3}{|c|}{$14.8 \pm 9.6(0.8-13.1 \pm 7.3(3.8-0.7 \pm 0.4(0.3-1$} \\
\hline & 1 & S2 & W2 & \multicolumn{3}{|c|}{$13.7 \pm 8.2(2.4-11.2 \pm 8.2(2.1-0.7 \pm 0.3(0.1-1$} \\
\hline & 1 & & B & \multicolumn{3}{|c|}{$24.1(0.8-30.213 .7(5.0-21.4$} \\
\hline & 1 & S4 & W2 & \multicolumn{3}{|c|}{$22.7 \pm 8.1(1.5-15.6 \pm 6.4(5.6-0.8 \pm 0.6(0.3-1$} \\
\hline & 1 & S1 & W2 & \multicolumn{3}{|c|}{$15.6 \pm 10.1(0 . \subseteq 11.1 \pm 7.7(1.4-0.6 \pm 0.3(0.2-1$} \\
\hline & 1 & S2 & W2 & \multicolumn{3}{|c|}{$15.3 \pm 8.8(3.5-12.5 \pm 7.4(2.8-0.9 \pm 0.6(0.2-1$} \\
\hline & 1 & S4 & W2 & \multicolumn{3}{|c|}{$13.0 \pm 8.3(0.5-11.3 \pm 7.5(2.5-0.8 \pm 0.4(0.4-1$} \\
\hline & 1 & S2 & W1 & \multicolumn{3}{|c|}{$11.9 \pm 11.3(0 . \varepsilon 12.1 \pm 7.8(2.1-0.6 \pm 0.3(0.3-1$} \\
\hline & 1 & S2 & W1 & \multicolumn{3}{|c|}{$13.4 \pm 10.9(0.411 .8 \pm 7.8(1.5-0.9 \pm 0.8(0.2-2$} \\
\hline & 1 & S2 & W2 & $22.9(\mathrm{~N}=17$ & $19.4(\mathrm{~N}=1$ & 2 \\
\hline & 1 & S1 & W2 & \multicolumn{3}{|c|}{$16.1 \pm 10.7(0.713 .1 \pm 7.5(5.3-1.1 \pm 1.2(0.2-4$} \\
\hline $156^{\circ} 29.040^{\prime} \mathrm{E}$ & 1 & S2 & W1 & $17.3 \pm 9.2$ & $14.9 \pm 7.1$ & $1.1 \pm 1.1$ \\
\hline & 1 & & B & $21.4(0.9-30$ & $216.9(3.2-3$ & \\
\hline & 1 & & B & $11.4(1.3-24$ & $311.6(1.5-2$ & \\
\hline & 1 & & B & $21.8(1.6-44$ & $9.7(2.3-4$ & \\
\hline & 1 & & B & $26.3(25.2-2$ & $20.3(4.8-2$ & \\
\hline & 1 & & $B$ & $25.2(23.1-3$ & 17.0 (6.9-2 & \\
\hline & 1 & & $B / C$ & $16.5(0.7-28$ & $911.2(1.1-2$ & \\
\hline & 1 & & A & $24.1(15.7-2$ & $18.8(6.4-2$ & \\
\hline & 1 & & B & $24.6(19.6-2$ & $18.2(6.3-2$ & \\
\hline & 1 & & A & $24.1(14.2-2$ & $.19 .1(10.3$ & \\
\hline & 1 & & A & 28.9 (2.3-84 & 12.5 & \\
\hline & 1 & & $B$ & $25.5(24.1-3$ & $19.7(7.7-3$ & \\
\hline & 1 & & A & $24.4(12.0-2$ & $15.9(7.6-$ & \\
\hline & 1 & & A & $23.5(16.0-2$ & $15.4(2.8-2$ & \\
\hline & 1 & & B & $24.9(23.3-2$ & $18.0(9.2-3$ & \\
\hline & 1 & & $A$ & $24.0(10.1-2$ & $17.8(9.0-2$ & \\
\hline
\end{tabular}




\begin{tabular}{|c|c|c|c|c|c|c|}
\hline \multicolumn{3}{|c|}{1} & B & \multicolumn{3}{|c|}{24.6 (20.1-27. 19.6 (8.9-26.5 } \\
\hline & many & S2 & W1 & \multicolumn{2}{|c|}{ 24.3-27.3 $(\mathrm{N}=20.4(\mathrm{~N}=2)$} & 1.1 \\
\hline & 1 & S3 & W4 & \multicolumn{3}{|c|}{$23.2 \pm 2.0(n=217.0 \pm 5.6(n=11.5 \pm 1.4$} \\
\hline & 2 & S4 & W1 & $14.9 ; 25.2$ & $12.5 ; 20.0$ & $0.5 ; 1.9$ \\
\hline & 1 & S2 & W2 & $16.4 \pm 12.5$ & $13.0 \pm 10.6$ & $1.8 \pm 1.9$ \\
\hline & 1 & & $B / C$ & $0-48$ & $16-24$ & \\
\hline & 1 & & $\mathrm{Be}$ & $1-30$ & $2-24$ & \\
\hline & 1 & & $\mathrm{~B} / \mathrm{C}$ & $0-34$ & $5-19$ & \\
\hline & 1 & & $\mathrm{~B} / \mathrm{Ce}$ & $18-27$ & $6-28$ & \\
\hline & 1 & & W1 & $25.2(\mathrm{~N}=1)$ & $21.1(N=1$ & 2.42 \\
\hline $12^{\circ} 37^{\prime} 23.9^{\prime \prime} \mathrm{W}$ & & S2 & wo & \multicolumn{3}{|c|}{$26.0 \pm 0.7(n=120.7 \pm 0.8(n=71.1 \pm 0.5(N=7)$} \\
\hline & 1 & S4 & W1 & \multicolumn{3}{|c|}{$24.5 \pm 0.5(23 . \varepsilon 24.0 \pm 3.1(14.20 .5 \pm 0.3(0.2-1$} \\
\hline & 1 & S2 & W1 & $24.9-25.0$ & $20.5-20.6$ & $1.6-4.0$ \\
\hline & 1 & S4 & w3 & \multicolumn{3}{|c|}{$25.0 \pm 2.0(\mathrm{~N}=120.5 \pm 0.6(\mathrm{~N}=11.5 \pm 0.2(\mathrm{~N}=10$} \\
\hline & 1 & S2 & W1 & $24.5-24.9$ & 19.4-20.5 & $0.5-1.2$ \\
\hline & 1 & S2 & W1 & $24.9-25.3$ & 20.6-20.8 & $0.9-0.7$ \\
\hline & 1 & S2 & W2 & $25.5-25.6$ & 20.9-21.0 & $1.4-1.5$ \\
\hline & 4 & S4 & W2 & \multicolumn{3}{|l|}{23.9} \\
\hline $69^{\circ} 41.730^{\prime} \mathrm{W}$ & 1 & S1 & W3 & \multicolumn{3}{|c|}{$24.6 \pm 0.7(\mathrm{~N}=121.4 \pm 0.6(\mathrm{~N}=11.5 \pm 0.5(\mathrm{~N}=15$} \\
\hline & 1 & S2 & W2 & $23.5-23.8$ & $19.8-20.5$ & $1.3-1.0$ \\
\hline & 1 & S2 & W1 & \multicolumn{3}{|c|}{$23.96 \pm 1.16(n \cdot 20.26 \pm 0.71(n) 1.57 \pm 1.55(n=$} \\
\hline $157^{\circ} 12.207^{\prime} \mathrm{E}$ & 1 & S2 & W1 & $25.3 \pm 0.4$ & $21.2 \pm 0.3$ & $1.4 \pm 0.3$ \\
\hline & 1 & S1 & W2 & \multicolumn{3}{|c|}{$24.79 \pm 0.24(n \cdot 16.4 \pm 0.5(n=61.47 \pm 1.07(n=$} \\
\hline & 1 & S4 & W2 & \multicolumn{3}{|c|}{$22.2 \pm 2.8(n=721.7 \pm 3.3(n=70.8 \pm 0.1$} \\
\hline & 1 & & B & \multicolumn{3}{|c|}{$25.0(23.5-27.21 .1(19.9-22$} \\
\hline & 1 & S2 & W1 & \multicolumn{3}{|c|}{$26.0 \pm 0.3(n=722.3 \pm 0.2(n=61.5 \pm 0.1$} \\
\hline & 1 & S4 & W2 & \multicolumn{3}{|c|}{$26.3 \pm 0.3(n=621.6 \pm 0.3(n=50.8 \pm 0.1$} \\
\hline & 1 & & A & \multicolumn{3}{|c|}{$25.5(24.1-29.21 .4(19.5-23$} \\
\hline & 1 & S1 & W3 & \multicolumn{3}{|c|}{$26.1 \pm 0.2(n=821.9 \pm 0.2(n=81.6$} \\
\hline & 1 & S4 & W1 & \multicolumn{3}{|c|}{24.0} \\
\hline & 1 & & $\mathrm{Be}$ & \multicolumn{3}{|l|}{23} \\
\hline & 1 & S2 & W2 & $24.5-24.7$ & $19.6-20.9$ & $1.0-1.6$ \\
\hline & 1 & & B & \multicolumn{3}{|c|}{24.3 (20.0-25. 19.3 (12.6-21. } \\
\hline & 1 & S2 & W2 & \multicolumn{3}{|c|}{$26.2 \pm 1$} \\
\hline & 1 & & B & \multicolumn{3}{|c|}{24.9 (24.1-27. 21.4 (20.2-24. } \\
\hline & 1 & & $\mathrm{Be}$ & 23 & \multicolumn{2}{|l|}{19} \\
\hline & 1 & S2 & W1/2 & $24.8-25.1$ & 18.9-20.6 & $1.1-0.5$ \\
\hline & 1 & & $B / C$ & 25 & 21 & \\
\hline & 1 & & B & 25 & 21 & \\
\hline & 1 & & $\mathrm{~B} / \mathrm{Ce}$ & 23 & 19 & \\
\hline $56^{\circ} 40^{\prime} 35.98^{\prime \prime} \mathrm{E}$ & & & W1 & $24.2 \pm 1.1(\mathrm{~N}$ & $316.2 \pm 6.5$ & $11.0 \pm 0.8$ \\
\hline & 1 & & $A / B$ & 22 & 19 & \\
\hline $158^{\circ} 09.197{ }^{\prime} \mathrm{E}$ & 1 & S3 & W1 & $21.6 \pm 1.0(\mathrm{~N}$ & $18.4 \pm 1.2$ & 81.4 \\
\hline & 1 & S4 & W1/2 & $23.5-23.7$ & $20.0-20.2$ & $3.4-1.5$ \\
\hline & 1 & & $\mathrm{~B}$ & $25.8(24.4-2$ & $21.8(20.2$ & \\
\hline
\end{tabular}




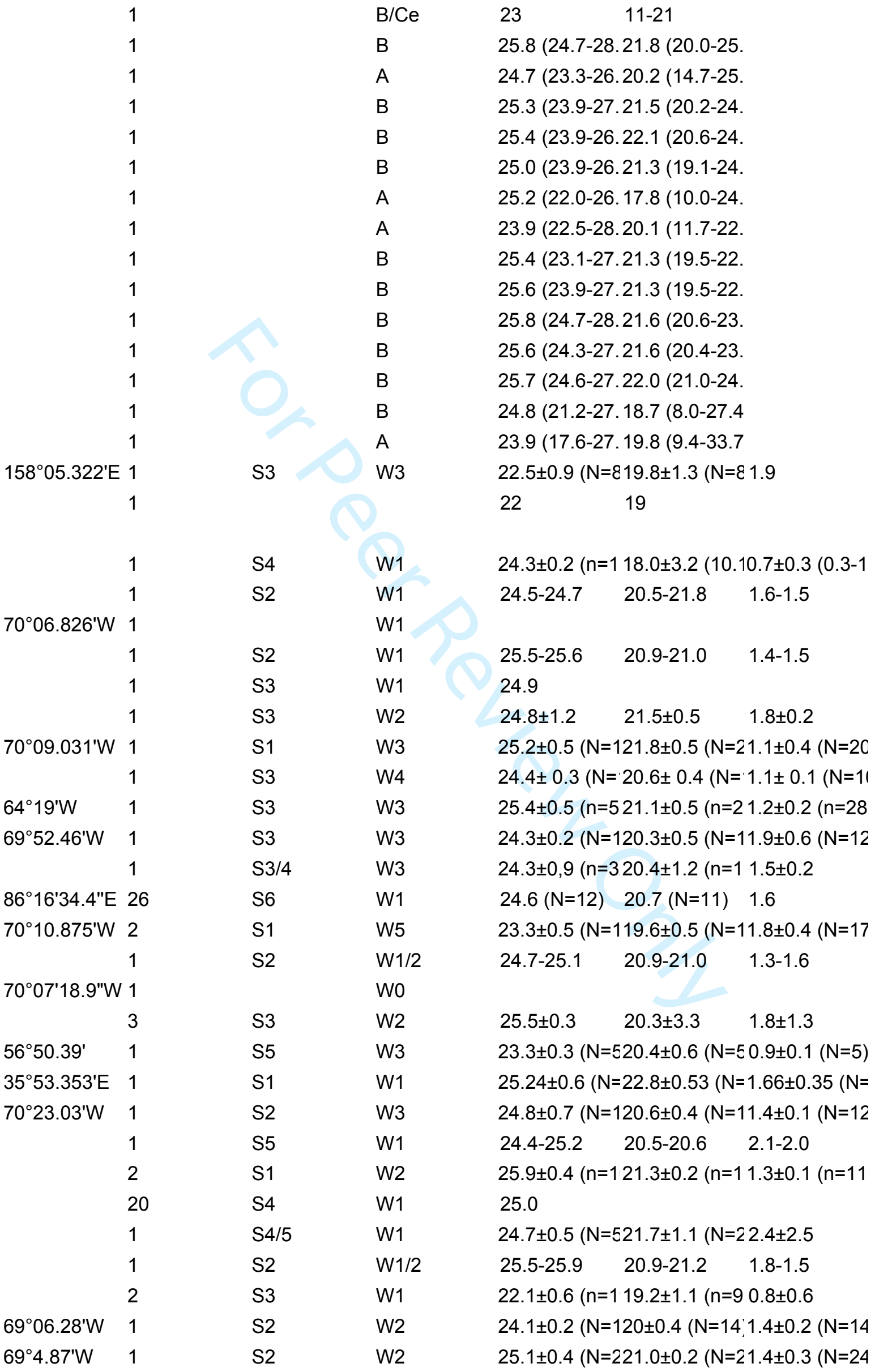




\begin{tabular}{|c|c|c|c|c|c|c|}
\hline $80^{\circ} 10^{\prime} 31 " \mathrm{E}$ & 1 & $\mathrm{~S} 2 / 3$ & W0 & \multicolumn{3}{|c|}{$25.3 \pm 0.6(n=1.22 .2 \pm 1.0(n=11.0$} \\
\hline $156^{\circ} 34.258^{\prime} \mathrm{E}$ & & S2 & W1 & $24.9 \pm 0.3$ & $21.0 \pm 0.5$ & $1.5 \pm 0.2$ \\
\hline $70^{\circ} 02.304^{\prime} \mathrm{W}$ & 1 & S1 & W3 & \multicolumn{3}{|c|}{$25.5 \pm 0.4(\mathrm{~N}=222.2 \pm 0.4(\mathrm{~N}=21.7 \pm 0.3(\mathrm{~N}=20$} \\
\hline \multirow{2}{*}{$20.29093^{\circ} \mathrm{E}$} & 1 & S4 & W4 & \multicolumn{3}{|c|}{$24.0 \pm 1(n=3) \quad 20.3 \pm 0.1(n=30.8 \pm 0.7(n=3)$} \\
\hline & 1 & S2 & W2 & \multicolumn{3}{|c|}{$24.53 \pm 0.4(n=20.97 \pm 0.72(n) 1.56 \pm 0.34(n=$} \\
\hline \multirow[t]{4}{*}{$69^{\circ} 5.23^{\prime} \mathrm{W}$} & 1 & S2 & W2 & \multicolumn{3}{|c|}{$23.5 \pm 0.4(\mathrm{~N}=119.8 \pm 0.3(\mathrm{~N}=11.5 \pm 0.2(\mathrm{~N}=13$} \\
\hline & 1 & S1 & W2 & \multicolumn{3}{|c|}{$24.42 \pm 0.25(n 21.76 \pm 1.5(n=1.28 \pm 0.4(n=8$} \\
\hline & 1 & $S 4 / 5$ & W1 & \multicolumn{3}{|c|}{$25.5 \pm 0.8(N=122.2 \pm 0.7 \quad 1.7 \pm 0.2$} \\
\hline & 1 & S2 & W2 & \multicolumn{3}{|c|}{$23.79 \pm 0.44(n \cdot 20.4 \pm 0.12(n=20.4 \pm 0.12(n=$} \\
\hline \multirow[t]{16}{*}{$69^{\circ} 6.34^{\prime} \mathrm{W}$} & 1 & S2 & W2 & \multicolumn{3}{|c|}{$24.7 \pm 0.3(N=120.4 \pm 0.4(N=11.7 \pm 0.3(N=12$} \\
\hline & 1 & & B & \multicolumn{3}{|c|}{$25.4(24.2-27.21 .8(20.0-24$} \\
\hline & 1 & S2 & W4 & \multicolumn{3}{|c|}{25.6} \\
\hline & 1 & S1 & W2 & \multicolumn{3}{|c|}{$26.6 \pm 0.2(n=821.6 \pm 0.4(n=81.4 \pm 0.2$} \\
\hline & 1 & & $\mathrm{~B} / \mathrm{Ce}$ & 23 & 19 & \\
\hline & 1 & S3 & W2 & \multicolumn{3}{|c|}{$25.24 \pm 0.82(n \cdot 20.6 \pm 0.24(n=1.43 \pm 0.26(n=$} \\
\hline & 1 & S3 & W1 & \multicolumn{3}{|c|}{$25.09 \pm 0.23(n 21.23 \pm 0.31(n) 1.67 \pm 0.33(n=$} \\
\hline & 1 & S3 & W3 & \multicolumn{3}{|c|}{$24.7 \pm 0.5(n=620.5 \pm 0.3(n=71.6 \pm 0.1$} \\
\hline & 1 & S2 & W1 & \multicolumn{3}{|c|}{$23.3 \pm 0.2(n=1.19 .4 \pm 0.2(n=11.4 \pm 0.1(n=13$} \\
\hline & 1 & & C & \multicolumn{3}{|c|}{$25.2(24.0-26.21 .3(20.1-23$} \\
\hline & 1 & & $\mathrm{~B} / \mathrm{C}$ & \multicolumn{3}{|c|}{25} \\
\hline & 1 & S2 & W1 & \multicolumn{3}{|c|}{$24.94 \pm 0.31(n \cdot 22.06 \pm 0.69(n) 1.4 \pm 0.31(n=9$} \\
\hline & 1 & & $\mathrm{~B} / \mathrm{C}$ & 26 & 21 & \\
\hline & 1 & S2 & W2 & \multicolumn{3}{|c|}{$25.4 \pm 0.3(n=521.2 \pm 0.3(n=61.9 \pm 0.2$} \\
\hline & 1 & S2 & W2 & $25.5 \pm 0.4(n=$ & $121.0 \pm 0.2(n=$ & $11.0 \pm 0.4(n=12$ \\
\hline & 1 & S2 & W2 & $24.6-25.5$ & $20.4-20.7$ & $1.5-1.0$ \\
\hline$\sim 69^{\circ} 43^{\prime} \mathrm{W}$ & 1 & & W1 & $25.2(N=1)$ & $21.3 \pm 0.2(\mathrm{~N}=$ & $41.8 \pm 0.2$ \\
\hline & 2 & S2 & W1 & $24.0 \pm 0.2(n=$ & $120.5 \pm 0.2(n=$ & $1.1 .7 \pm 0.1 \quad(n=14$ \\
\hline & 1 & S2 & W1 & $25.3 \pm 0.4(n=$ & $519.5 \pm 0.5(n=$ & $71.8 \pm 0.1$ \\
\hline $69^{\circ} 08.94^{\prime} \mathrm{W}$ & 1 & S2 & W4 & $24.2 \pm 0.4(\mathrm{~N}$ & $=119.9 \pm 0.3(N=$ & $11.6 \pm 0.2(\mathrm{~N}=15$ \\
\hline $20.31391^{\circ} \mathrm{E}$ & 1 & & W2/3 & $24.7 \pm 1.4(n=$ & 1.22.1 $\pm 2.6(n=$ & $50.3 \pm 0.7(n=5)$ \\
\hline & 1 & S2 & W3 & $24.2 \pm 0.2(\mathrm{~N}$ & $=120.7 \pm 0.4(\mathrm{~N}=$ & $11.5 \pm 0.2(\mathrm{~N}=10$ \\
\hline & 4 & S3 & W1 & $24.6 \pm 0.3(\mathrm{~N}$ & $=520.6 \pm 0.5(\mathrm{~N}=$ & $51.6 \pm 0.1(N=5)$ \\
\hline & 1 & S2 & W2 & $24.94 \pm 1.1(r$ & $=20.86 \pm 1.11($ & $1.31 \pm 0.21(n=$ \\
\hline $69^{\circ} 08.98^{\prime} \mathrm{W}$ & 1 & S2 & W4 & $24.4 \pm 0.2(\mathrm{~N}$ & $=120.2 \pm 0.2(\mathrm{~N}=$ & $11.5 \pm 0.2(\mathrm{~N}=15$ \\
\hline $069^{\circ} 59^{\prime} 35.6 " \mathrm{~V}$ & & & W2 & $24.7 \pm 0.2(\mathrm{~N}$ & $=221.2(\mathrm{~N}=1)$ & 1.4 \\
\hline & 1 & S2 & W2 & $25.2 \pm 0.1(n=$ & $1.21 .1 \pm 0.2(n=$ & $1.1 .7 \pm 0.4(n=18$ \\
\hline & 1 & & $\mathrm{~B} / \mathrm{C}$ & 25 & 21 & \\
\hline & 1 & & $A / B$ & 22 & 19 & \\
\hline & 1 & S3 & W4 & $26.0 \pm 0.2(n=$ & $621.7 \pm 0.2(n=$ & 51.3 \\
\hline & 1 & & $\mathrm{Be}$ & 22 & 19 & \\
\hline & 1 & & $B / C$ & 21 & 19 & \\
\hline & 1 & & B & 24 & 21 & \\
\hline & 1 & & $A / B$ & 22 & 19 & \\
\hline & 1 & S1 & W2 & 23 & & \\
\hline & 1 & & $\mathrm{~B} / \mathrm{C}$ & 25 & 21 & \\
\hline
\end{tabular}




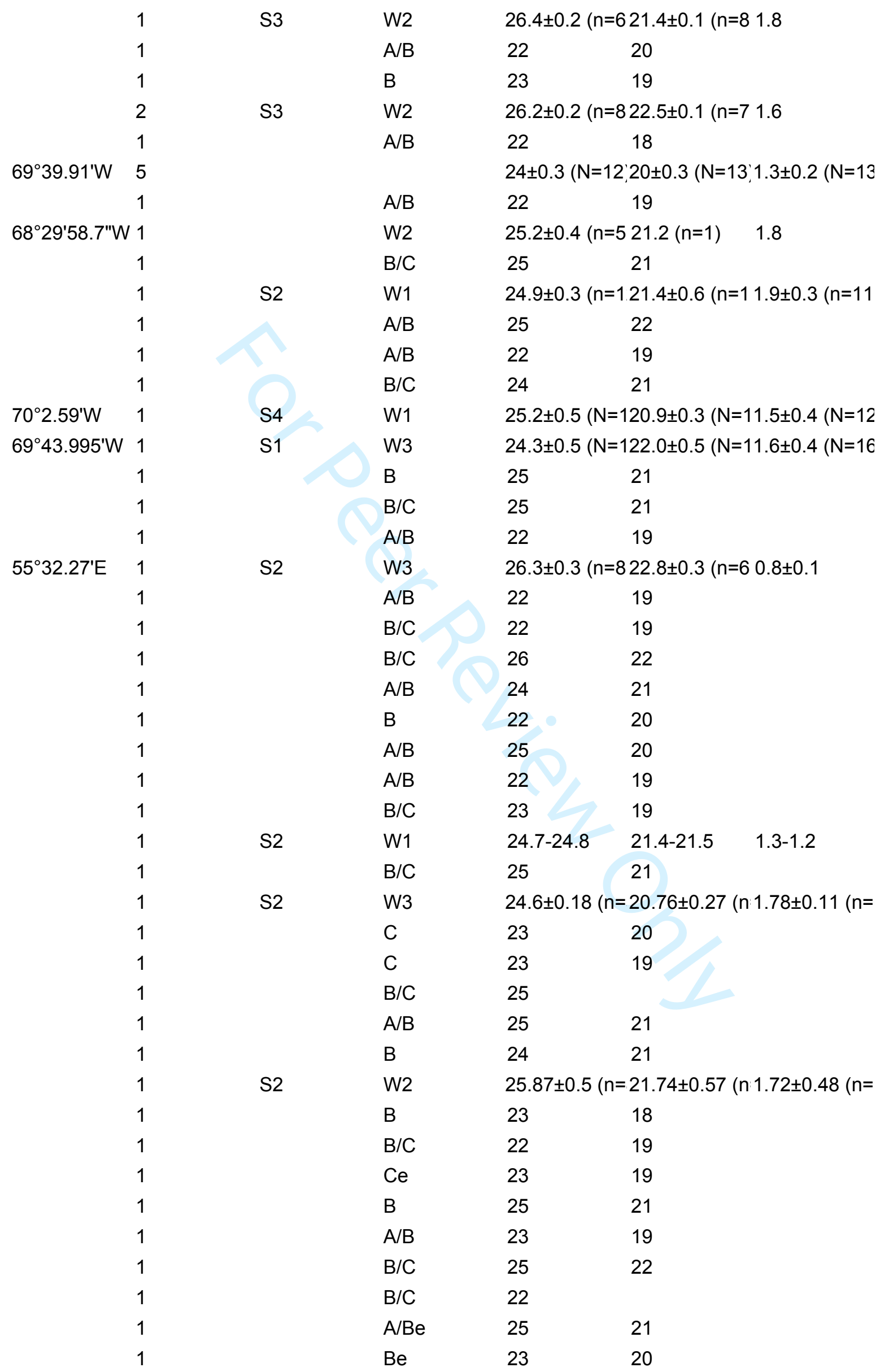




\begin{tabular}{|c|c|c|c|c|}
\hline & 1 & $\mathrm{~B} / \mathrm{C}$ & 24 & 21 \\
\hline & 1 & $\mathrm{~B}$ & 23 & 19 \\
\hline & 1 & B & 22 & 19 \\
\hline & 1 & $\mathrm{~B} / \mathrm{Ce}$ & 22 & 20 \\
\hline & 1 & $\mathrm{Be}$ & 25 & 21 \\
\hline & 1 & $\mathrm{~B} / \mathrm{Ce}$ & 22 & 19 \\
\hline & 1 & $\mathrm{C}$ & 22 & 19 \\
\hline & 1 & $\mathrm{Ce}$ & 23 & 19 \\
\hline & 1 & $A / B$ & 23 & 19 \\
\hline & 1 & $\mathrm{~B} / \mathrm{C}$ & 22 & 19 \\
\hline & 1 & $\mathrm{~B} / \mathrm{C}$ & 23 & 18 \\
\hline & 1 & $A$ & 25 & 21 \\
\hline & 1 & $\mathrm{~B} / \mathrm{C}$ & 25 & 21 \\
\hline & 1 & $A$ & 25.8 & $3.21 .8(20.9-22$ \\
\hline & 1 & $\mathrm{~B} / \mathrm{C}$ & 25 & 22 \\
\hline & 1 & $\mathrm{Be}$ & 25 & \\
\hline & 1 & $\mathrm{~B} / \mathrm{C}$ & 24 & 22 \\
\hline & 1 & $A / B$ & 24 & 21 \\
\hline & 1 & A & 25.5 & $3.22 .2(20.0-25$ \\
\hline & 1 & $\mathrm{~B} / \mathrm{C}$ & 22 & \\
\hline & 1 & $A / B$ & 25 & 20 \\
\hline & 1 & $A$ & 24.4 & $5.21 .3(20.3-23$ \\
\hline & 1 & B & 24.8 & $7.21 .0(19.9-22$ \\
\hline & 1 & A & 26.6 & $9.22 .4(21.5-23$ \\
\hline & 1 & B & 25.4 & 7.21 .7 (20.4-24. \\
\hline & 1 & C & 24 & \\
\hline & 1 & $\mathrm{~B} / \mathrm{C}$ & 23 & 19 \\
\hline $69^{\circ} 55.64^{\prime} \mathrm{W}$ & 1 & W4 & $24 . c$ & $=120.6 \pm 0.3(\mathrm{~N}=11.3 \pm 0.2(\mathrm{~N}=10$ \\
\hline & 1 & $B$ & 25.6 & $8.21 .8(20.0-26$ \\
\hline & 1 & B & 25.2 & $0.21 .4(20.3-22$ \\
\hline & 1 & B & 25.7 & $7.21 .5(20.4-23$ \\
\hline & 1 & B & 25.7 & $7.22 .1(20.6-27$ \\
\hline & 1 & B & 25.4 & $8.22 .0(20.4-24$ \\
\hline & 1 & A & 24.7 & 5. $21.2(19.6-23$ \\
\hline & 1 & A & 25.5 & $9.21 .5(19.8-22$ \\
\hline & 1 & B & 25.6 & $0.21 .8(20.0-27$ \\
\hline & 1 & B & 25.3 & $8.22 .4(20.7-24$ \\
\hline & 1 & B & 25.6 & $7.21 .2(20.2-22$ \\
\hline & 1 & B & 25.3 & $7.22 .0(19.8-24$. \\
\hline & 1 & B & 25.5 & 6.21 .7 (20.0-24. \\
\hline & 1 & B & 25.6 & $6.21 .9(20.6-24$. \\
\hline & 1 & A & 25.3 & $8.21 .6(20.8-23$. \\
\hline & 1 & B & 26.0 & $0.21 .9(20.3-23$ \\
\hline & 1 & B & $25 . c$ & $8.22 .0(20.0-26$. \\
\hline & 1 & B & 25.4 & 7.21 .7 (20.7-23. \\
\hline
\end{tabular}









\begin{tabular}{|c|c|c|c|c|c|c|}
\hline \multirow{2}{*}{\multicolumn{2}{|c|}{$\begin{array}{l}1 \\
1\end{array}$}} & & \multirow[t]{2}{*}{ A } & \multicolumn{3}{|c|}{$25.2(24.2-27.21 .6(20.2-24$} \\
\hline & & & & 23 & \multicolumn{2}{|l|}{20} \\
\hline & 1 & & B & \multicolumn{3}{|c|}{$25.4(24.4-26.21 .0$ (19.1-23. } \\
\hline & 1 & & B & \multicolumn{3}{|c|}{$25.1(22.6-27.21 .2(20.4-21$} \\
\hline & 1 & & B & \multicolumn{3}{|c|}{25.2 (24.3-28. 21.3 (19.1-23. } \\
\hline & 1 & & A & \multicolumn{3}{|c|}{26.2 (24.3-28. 22.3 (20.1-23. } \\
\hline & 1 & & B & \multicolumn{3}{|c|}{25.5 (23.9-29. 21.8 (20.5-24. } \\
\hline & 1 & & B & \multicolumn{3}{|c|}{$25.9(24.8-28.22 .3(21.2-25}$. \\
\hline & 1 & & A & \multicolumn{3}{|c|}{25.0 (22.3-27. 21.6 (19.8-24. } \\
\hline & 1 & & B & \multicolumn{3}{|c|}{26.3 (24.6-29. 22.5 (20.8-26. } \\
\hline $158^{\circ} 09.196^{\prime} \mathrm{E}$ & 1 & S4 & W1 & \multicolumn{3}{|c|}{$22.0 \pm 0.7(\mathrm{~N}=818.6 \pm 0.8(\mathrm{~N}=82$} \\
\hline & 1 & & B & \multicolumn{3}{|c|}{25.1 (23.9-27. 21.2 (20.2-21. } \\
\hline & 1 & & A & \multicolumn{3}{|c|}{26.1 (24.8-28. 22.7 (21.1-25. } \\
\hline & 1 & & B & \multicolumn{3}{|c|}{25.5 (24.4-28. 21.7 (20.8-25. } \\
\hline & 1 & & & 23 & \multicolumn{2}{|c|}{19} \\
\hline & 1 & & B & \multicolumn{3}{|c|}{$25.6(24.1-27.22 .2$ (20.8-24. } \\
\hline & 1 & & $\mathrm{~B} / \mathrm{C}$ & 25 & \multicolumn{2}{|c|}{20} \\
\hline $8^{\circ} 0^{\prime} 00.00^{\prime \prime} \mathrm{E}$ & 5 & S4 & wo & \multicolumn{3}{|c|}{$26.0 \pm 0.5(n=\Sigma 21.9 \pm 0.4(n=<1.4 \pm 0.3(n=2$} \\
\hline $50^{\circ} 55.33^{\prime} \mathrm{E}$ & 1 & S4 & W4 & \multirow{2}{*}{\multicolumn{3}{|c|}{$24.1 \pm 0.7(n=20.2 \pm 0.4(n 2.1 \pm 0.9(n=$}} \\
\hline & 1 & S2, melt & W3 & & & \\
\hline $69^{\circ} 58.341^{\prime} \mathrm{W}$ & 5 & S3 & W3 & \multicolumn{3}{|c|}{$24.7 \pm 0.4(\mathrm{~N}=220.1 \pm 0.4(\mathrm{~N}=11.0 \pm 0.3(\mathrm{~N}=18$} \\
\hline $70^{\circ} 21.911^{\prime} \mathrm{W}$ & 1 & S1 & W2 & \multicolumn{3}{|c|}{$24.2 \pm 0.5(\mathrm{~N}=120.8 \pm 0.5(\mathrm{~N}=11.2 \pm 0.4(\mathrm{~N}=10$} \\
\hline $51^{\circ} 07.54^{\prime} \mathrm{E}$ & 1 & S4 & W3-4 & \multicolumn{3}{|c|}{$25.0 \pm 04(n=\varepsilon 21.5 \pm 0.9(n 1.3 \pm 0.1(n=$} \\
\hline $100^{\circ} 10^{\prime} 29 " \mathrm{E}$ & hundreds & S5 & wo & $25.0 \pm 0.6$ & $21.1 \pm 0.3($ & $61.8 \pm 0.3$ \\
\hline $68^{\circ} 33.0557^{\prime} \mathrm{W}$ & many & $\mathrm{s} 1$ & W1 & $25.4 \pm 0.6$ & $20.7 \pm 0.7$ & $21.5 \pm 0.3(N=21$ \\
\hline $69^{\circ} 29^{\prime} 19.8^{\prime \prime} \mathrm{W}$ & many & & W1 & & & \\
\hline $68^{\circ} 37.632^{\prime} \mathrm{W}$ & & S3/4 & $\mathrm{W} 1 / 2$ & $25.7 \pm 0.4$ & $121.5 \pm 0.3$ & $31.4 \pm 0.3(N=32$ \\
\hline & 1 & S2 & W2 & $25.0 \pm 0.4$ & $21.1 \pm 0.2(r$ & $11.6 \pm 0.2(n=11$ \\
\hline $68^{\circ} 51.25^{\prime} \mathrm{W}$ & 964 & S2 & W2 & $25.4 \pm 0.5$ & $21.2 \pm 0.5$ & $11.6 \pm 0.3(N=18$ \\
\hline $38^{\circ} 10.268^{\prime} \mathrm{E}$ & $>91$ & $\mathrm{~S} 4 / 5$ & wo & $25.6 \pm 0.3$ & $21.4 \pm 0.2$ & $41.61 \pm 0.26(\mathrm{~N}=$ \\
\hline $70^{\circ} 11.315^{\prime} \mathrm{W}$ & 1 & S3 & w2 & $24.3 \pm 0.2$ & $20.7 \pm 0.3(r$ & $11.5 \pm 0.2(n=11$ \\
\hline $70^{\circ} 03^{\prime} 15.2^{\prime \prime} \mathrm{W}$ & 1 & & wo & & & \\
\hline $156^{\circ} 36.560^{\prime} \mathrm{E}$ & 1 & $\mathrm{~S} 1$ & W1 & $25.3 \pm 0.2$ & $21.5 \pm 0.1$ & $1.5 \pm 0.2$ \\
\hline & 1 & S2 & W2/3 & $25.2-25.3$ & $21.6-21.8$ & $1.7-1.1$ \\
\hline $76^{\circ} 22^{\prime} 41.67^{\prime \prime V}$ & & S3 & wo & $25.1 \pm 0.3$ & $21.4 \pm 1.2$ & $1.5 \pm 0.2$ \\
\hline $69^{\circ} 46^{\prime} \mathrm{W}$ & 9 & & w2 & & & \\
\hline & 25 & S3 & w2 & 23.7 & & \\
\hline & 1 & & A & $25.5(24.3$ & $21.7(19.7$ & \\
\hline & 1 & & $A$ & 24 & 21 & \\
\hline & 1 & S2 & w2 & $26.1-26.2$ & $20.9-21.2$ & $1.3-1.5$ \\
\hline & 1 & S3 & W3 & $25.16 \pm 0.4$ & $21.1 \pm 0.13$ & $=1.63 \pm 0.19(n=$ \\
\hline $70^{\circ} 08^{\prime} 55^{\prime \prime} \mathrm{W}$ & 1 & S2 & w2 & $25.1 \pm 0.2$ & $20.9 \pm 0.1(r$ & $11.6 \pm 0.2(n=16$ \\
\hline & 1 & & $B$ & $25.6(24.0$ & $22.0(20.8$ & \\
\hline $156^{\circ} 34.859^{\prime} \mathrm{E}$ & & S1 & W1 & $25.5 \pm 0.1$ & $21.3 \pm 0.1$ & $1.8 \pm 0.3$ \\
\hline
\end{tabular}




\begin{tabular}{|c|c|c|c|c|}
\hline \multirow[t]{2}{*}{$70^{\circ} 19^{\prime} 32^{\prime \prime} \mathrm{W}$} & 1 & S2 & W2 & $24.6 \pm 0.2(n=121.1 \pm 0.6(n=1.1 .6 \pm 0.2(n=14$ \\
\hline & 1 & & A & $25.0(24.7-25.21 .4(20.5-23$ \\
\hline \multirow[t]{4}{*}{$70^{\circ} 21.871^{\prime} \mathrm{W}$} & 1 & S3 & W2 & $24.1 \pm 0.6(n=1.20 .6 \pm 0.4(n=11.7 \pm 0.1 \quad(n=13$ \\
\hline & 1 & S2 & W3 & 25.4 \\
\hline & 1 & S4 & W2 & 25.3 \\
\hline & 1 & S2 & W3 & $24.7 \pm 0.3(n=1.20 .6 \pm 0.1(n=1.1 .8 \pm 0.1(n=10$ \\
\hline \multirow[t]{4}{*}{$156^{\circ} 35.092^{\prime} \mathrm{E}$} & 1 & S1 & W1 & $25.3 \pm 0.2 \quad 21.1 \pm 0.1 \quad 1.7 \pm 0.2$ \\
\hline & 1 & S3 & W5 & 21.1 \\
\hline & 1 & & B & 24.9 (23.7-27. 20.7 (19.4-22. \\
\hline & 1 & S1 & W1 & 19.4 \\
\hline \multirow[t]{2}{*}{$69^{\circ} 49.774^{\prime} \mathrm{W}$} & 5 & S1 & W2 & $23.9 \pm 0.5(N=120.8 \pm 0.5(N=11.8 \pm 0.4(N=16$ \\
\hline & 1 & S1 & W4 & $26.4 \pm 0.2(n=822.4 \pm 0.3(n=1.1 .4 \pm 0.2$ \\
\hline $69^{\circ} 47.012^{\prime} \mathrm{W}$ & 2 & S1 & W3 & $24.6 \pm 0.4(N=121.6 \pm 0.4(N=11.6 \pm 0.3(N=19$ \\
\hline $21.20^{\circ} \mathrm{E}$ & several & S1 & Wo & $25.5 \pm 0.2(\mathrm{~N}=321.8 \pm 0.1(\mathrm{~N}=31.8 \pm 0.3$ \\
\hline \multirow[t]{2}{*}{$69^{\circ} 48.772^{\prime} \mathrm{W}$} & 3 & S2 & W2 & $25.4 \pm 0.4(\mathrm{~N}=122.3 \pm 0.4(\mathrm{~N}=12.2 \pm 0.3(\mathrm{~N}=19$ \\
\hline & 1 & & B & 24.8 (19.9-28. 20.9 (19.1-23. \\
\hline \multirow[t]{3}{*}{$68^{\circ} 51.27 \mathrm{~W}$} & 5 & S3 & W1 & $25.8 \pm 0.5(\mathrm{~N}=221.5 \pm 0.5(\mathrm{~N}=21.5 \pm 0.3(\mathrm{~N}=21$ \\
\hline & 1 & S3 & W2 & $25.8 \pm 0.3(n=721.7 \pm 0.3(n=11.6 \pm 0.2$ \\
\hline & 1 & S2 & W2 & 27 \\
\hline \multirow[t]{2}{*}{$9^{\circ} 9.023^{\prime} \mathrm{E}$} & 1 & S4 & W1 & 24.4 $\pm 0.4(24 . C 20.6 \pm 0.4(20.21 .5 \pm 0.3(0.9-2$ \\
\hline & 1 & & B & $25.9(24.4-28.21 .3(19.9-23$ \\
\hline$\sim 69^{\circ} 46^{\prime} \mathrm{W}$ & 1 & & W3 & $21.1(\mathrm{~N}=1) \quad 1.8$ \\
\hline $69^{\circ} 42.679^{\prime} \mathrm{W}$ & 1 & S4 & W2 & $23.5 \pm 0.5(\mathrm{~N}=121.0 \pm 0.5(\mathrm{~N}=11.1 \pm 0.4(\mathrm{~N}=19$ \\
\hline \multirow[t]{13}{*}{$158^{\circ} 28.320^{\prime} \mathrm{E}$} & 1 & S4 & W1 & $24.9 \pm 0.3 \quad 21.2 \pm 0.4 \quad 1.5 \pm 0.2$ \\
\hline & 1 & S3 & W2 & $23.52 \pm 0.98(n \cdot 20.75 \pm 1.82(n) 1.5 \pm 0.3(n=7)$ \\
\hline & 1 & & $\mathrm{~A} / \mathrm{B}$ & $23 \quad 19$ \\
\hline & 1 & & B & $25.4(24.4-26.21 .9(20.2-28$ \\
\hline & 1 & S3 & W2 & $24.6 \pm 0.1(n=1.20 .9 \pm 0.2(n=11.6 \pm 0.2(n=13$ \\
\hline & 1 & S3 & W3 & $25.1 \pm 0.63(n=21.57 \pm 0.92(n) 1.84 \pm 0.11(n=$ \\
\hline & 1 & & B & $25.7(24.1-29.22 .1(20.7-24$ \\
\hline & 1 & S3 & W2 & $24.2 \pm 0.2(n=1.20 .6 \pm 0.2(n=11.6 \pm 0.1(n=13$ \\
\hline & 1 & & $\mathrm{~A} / \mathrm{B}$ & $24 \quad 21$ \\
\hline & 1 & S2 & W2 & $25.16 \pm 0.01(n \cdot 21.31 \pm 0.35(n) 1.58 \pm 0.25(n=$ \\
\hline & 1 & S2 & W2 & $25.0 \pm 1$ \\
\hline & 1 & S2 & W2 & $23.7 \pm 0.4(n=1.20 .0 \pm 0.5(n=11.4 \pm 0.2(n=13$ \\
\hline & 1 & & B & $23 \quad 19$ \\
\hline$\sim 69^{\circ} 46^{\prime} \mathrm{W}$ & 1 & & W2 & $25.1 \pm 0.3(N=321.6 \pm 0.2(N=21.2 \pm 0.0$ \\
\hline $69^{\circ} 58.006^{\prime} \mathrm{W}$ & 2 & S2 & W2 & $24.4 \pm 0.4(\mathrm{~N}=120.8 \pm 0.4(\mathrm{~N}=11.4 \pm 0.3(\mathrm{~N}=16$ \\
\hline \multirow[t]{3}{*}{$68^{\circ} 38.36^{\prime} \mathrm{W}$} & 2 & S3 & W2 & $24.8 \pm 0.5(\mathrm{~N}=220.8 \pm 0.7(\mathrm{~N}=21.4 \pm 0.3(\mathrm{~N}=21$ \\
\hline & 1 & & $A / B$ & $25 \quad 21$ \\
\hline & 1 & S2 & W2 & $23.8 \pm 0.4(n=120.5 \pm 0.8(n=1.1 .6 \pm 0.4(n=14$ \\
\hline \multirow[t]{2}{*}{$\sim 69^{\circ} 43^{\prime} \mathrm{W}$} & 7 & & W2 & $25.0(\mathrm{~N}=1) \quad 21.1(\mathrm{~N}=1) \quad 1.4$ \\
\hline & 1 & & B & $25.2(24.2-26.21 .7$ (20.1-24. \\
\hline \multirow[t]{2}{*}{$69^{\circ} 08.81^{\prime} \mathrm{W}$} & 1 & S2 & W2 & $23.9 \pm 0.2(N=119.7 \pm 0.2(N=11.6 \pm 0.2(N=15$ \\
\hline & 1 & S4 & W1 & 24.2 \\
\hline
\end{tabular}




\begin{tabular}{|c|c|c|c|c|c|}
\hline & 1 & A & 26 & \multicolumn{2}{|l|}{21} \\
\hline & 1 & A & \multicolumn{3}{|c|}{25.5 (23.4-29. 21.4 (18.9-24. } \\
\hline & S4 & W2 & \multicolumn{3}{|c|}{$25.6 \pm 0.3(n=122.5 \pm 0.3(n=61.1 \pm 0.1$} \\
\hline & 1 & $\mathrm{~B}$ & \multicolumn{3}{|c|}{25.5 (23.6-27. 21.7 (19.8-23. } \\
\hline$\sim 69^{\circ} 46^{\prime} \mathrm{W}$ & 1 & W2 & & & \\
\hline & 1 & B & \multicolumn{3}{|c|}{$25.0(23.8-25.21 .1(20.3-21}$. \\
\hline & S2 & W1 & \multicolumn{3}{|c|}{$23.7 \pm 0.1(n=119.8 \pm 0.2(n=11.7 \pm 0.2(n=11$} \\
\hline $156^{\circ} 40.126^{\prime} \mathrm{E}$ & S2 & W1 & $25.2 \pm 0.3$ & $21.2 \pm 0.2$ & $1.5 \pm 0.1$ \\
\hline & 1 & $B$ & \multicolumn{3}{|c|}{$25.4(23.6-26.22 .2(20.3-24$} \\
\hline & S2 & W2 & $25.0-25.5$ & $21.0-21.2$ & 2.1-1.9 \\
\hline & 1 & $\mathrm{~B}$ & \multicolumn{3}{|c|}{$25.3(23.3-28.22 .0(20.2-24$} \\
\hline $69^{\circ} 42.886^{\prime} \mathrm{W}$ & S1 & W3 & \multicolumn{3}{|c|}{$24.6 \pm 0.5(\mathrm{~N}=121.8 \pm 0.5(\mathrm{~N}=11.7 \pm 0.4(\mathrm{~N}=17$} \\
\hline & 1 & $\mathrm{~B} / \mathrm{C}$ & 25 & \multicolumn{2}{|l|}{21} \\
\hline & 1 & B & \multicolumn{3}{|c|}{$25.1(24.0-28.21 .5(20.2-23$} \\
\hline $69^{\circ} 52.279^{\prime} \mathrm{W}$ & S1 & W3 & \multicolumn{3}{|c|}{$24.3 \pm 0.4(\mathrm{~N}=121.9 \pm 0.4(\mathrm{~N}=11.5 \pm 0.3(\mathrm{~N}=19$} \\
\hline & 1 & $\mathrm{~B}$ & \multicolumn{3}{|c|}{$24.9(23.2-28.21 .2(20.2-25$} \\
\hline $69^{\circ} 58.551^{\prime} \mathrm{W}$ & S2 & W2 & \multicolumn{3}{|c|}{ 23.3 $\pm 0.5(\mathrm{~N}=120.4 \pm 0.5(\mathrm{~N}=11.3 \pm 0.4(\mathrm{~N}=14$} \\
\hline$\sim 69^{\circ} 43^{\prime} \mathrm{W}$ & 1 & W1 & & & \\
\hline \multirow[t]{5}{*}{$69^{\circ} 54.506^{\prime} \mathrm{W}$} & S2 & W2 & \multicolumn{3}{|c|}{ 23.1 $1 \pm 0.5(\mathrm{~N}=120.8 \pm 0.5(\mathrm{~N}=11.5 \pm 0.4(\mathrm{~N}=16$} \\
\hline & 1 & $\mathrm{~B} / \mathrm{C}$ & \multicolumn{3}{|c|}{$23 \quad 21$} \\
\hline & $\mathrm{S} 2$ & W1/2 & $24.8-24.9$ & $20.6-20.9$ & \multirow[t]{3}{*}{$1.5-1.4$} \\
\hline & 1 & $\mathrm{~B} / \mathrm{C}$ & 24 & 21 & \\
\hline & 1 & $\mathrm{~B}$ & 23 & 19 & \\
\hline \multirow{2}{*}{$156^{\circ} 34.262^{\prime} \mathrm{E}$} & $\mathrm{S} 2$ & W1 & $25.2 \pm 0.3$ & $21.1 \pm 0.3$ & $1.6 \pm 0.2$ \\
\hline & 1 & A & \multicolumn{3}{|c|}{$25.6(24.4-27.22 .1(20.5-26$} \\
\hline \multirow[t]{2}{*}{$\sim 69^{\circ} 46^{\prime} \mathrm{W}$} & 1 & W2 & $25.1(N=1)$ & $20.9(\mathrm{~N}=1)$ & 1.4 \\
\hline & 1 & $A / B$ & 22 & 19 & \\
\hline $156^{\circ} 31.487^{\prime} \mathrm{E}$ & S4 & W1 & $25.2 \pm 0.4$ & $21.1 \pm 0.3$ & $1.5 \pm 0.3$ \\
\hline & 1 & $\mathrm{~B} / \mathrm{C}$ & 22 & 19 & \\
\hline$\sim 69^{\circ} 43^{\prime} \mathrm{W}$ & 1 & W3 & $25.7(\mathrm{~N}=1)$ & $20.5(\mathrm{~N}=1)$ & 3.4 \\
\hline $69^{\circ} 7.75^{\prime} \mathrm{W}$ & S2 & W2 & $23.7 \pm 0.2$ & $119.9 \pm 0.2$ & $11.6 \pm 0.1(\mathrm{~N}=12$ \\
\hline & 1 & $\mathrm{~B}$ & $26.0(24.9$ & $22.3(20.8$ & \\
\hline & 1 & $\mathrm{~A} / \mathrm{B}$ & 24 & 21 & \\
\hline & $\mathrm{S} 2$ & W1 & $24.8-24.9$ & $20.8-21.8$ & $1.0-0.9$ \\
\hline$\sim 69^{\circ} 46^{\prime} \mathrm{W}$ & 1 & W2 & $25.4 \pm 0.0$ & $21.8 \pm 0.3$ & $21.3 \pm 0.2$ \\
\hline & 1 & $\mathrm{~B}$ & 23 & 20 & \\
\hline $156^{\circ} 30.985^{\prime} \mathrm{E}$ & S4 & W1 & $25.0 \pm 0.2$ & $21.0 \pm 0.1$ & $1.7 \pm 0.3$ \\
\hline & 1 & $\mathrm{~A} / \mathrm{B}$ & 22 & 20 & \\
\hline & 1 & $\mathrm{~A} / \mathrm{Be}$ & 24 & 20 & \\
\hline & 1 & $\mathrm{~A} / \mathrm{B}$ & 25 & & \\
\hline & 1 & A & 24.8 (23.9- & 21.1 (20.3- & \\
\hline & $\mathrm{S} 2$ & W1 & $24.8 \pm 1$ & & \\
\hline$\sim 69^{\circ} 43^{\prime} \mathrm{W}$ & 1 & W1 & & & \\
\hline & 1 & $B$ & $26.0(24.6-$ & $22.4(20.4$ & \\
\hline & 1 & $A / B$ & 25 & 21 & \\
\hline
\end{tabular}




\begin{tabular}{|c|c|c|c|c|c|}
\hline \multicolumn{2}{|l|}{$156^{\circ} 28.475^{\prime} E 1$} & \multirow[t]{5}{*}{ S4 } & W1 & $25.0 \pm 0.4$ & $21.1 \pm 0.2$ \\
\hline & 1 & & $\mathrm{~B}$ & 24 & 21 \\
\hline & 1 & & $A / B$ & 22 & 19 \\
\hline & 1 & & $A / B$ & 24 & 21 \\
\hline & 1 & & $B$ & 25 & \\
\hline \multirow[t]{4}{*}{$68^{\circ} 50.31^{\prime} \mathrm{W}$} & 1 & S2 & W2 & \multicolumn{2}{|c|}{$25.1 \pm 0.6(\mathrm{~N}=121.2 \pm 0.5(\mathrm{~N}=11.4 \pm 0.3(\mathrm{~N}=17$} \\
\hline & 1 & S3 & W2 & \multicolumn{2}{|c|}{$24.57 \pm 0.41(n 21.08 \pm 0.8(n=1.31 \pm 0.21(n=$} \\
\hline & 1 & & $\mathrm{~B} / \mathrm{C}$ & 25 & 21 \\
\hline & 1 & & B & \multicolumn{2}{|c|}{$26.0(24.4-33.21 .5(20.5-23}$. \\
\hline \multirow[t]{12}{*}{$\sim 69^{\circ} 43^{\prime} \mathrm{W}$} & 1 & & W3 & & \\
\hline & 1 & & $\mathrm{~B} / \mathrm{C}$ & 24 & 22 \\
\hline & 1 & & $A / B$ & 23 & 19 \\
\hline & 1 & & $A / B$ & 25 & 21 \\
\hline & 1 & S2 & W1 & \multicolumn{2}{|c|}{$25.7 \pm 0.8(\mathrm{~N}=121.4 \pm 0.3(\mathrm{~N}=11.4 \pm 0.3(\mathrm{~N}=12$} \\
\hline & 1 & & $B / C$ & 24 & 21 \\
\hline & 1 & & B & \multicolumn{2}{|c|}{$26.0(23.8-29.21 .5(20.2-23}$. \\
\hline & 1 & & $\mathrm{~A} / \mathrm{Be}$ & \multicolumn{2}{|c|}{22} \\
\hline & 1 & & $A / B$ & 25 & 21 \\
\hline & 1 & S1 & W1 & 22.5 & 19.5 \\
\hline & 1 & & B & 23 & 19 \\
\hline & 1 & & $\mathrm{~A} / \mathrm{B}$ & \multicolumn{2}{|l|}{25} \\
\hline \multirow{7}{*}{$69^{\circ} 47.217^{\prime} \mathrm{W}$} & 1 & S1 & W3 & \multicolumn{2}{|c|}{$24.3 \pm 0.5(\mathrm{~N}=121.5 \pm 0.5(\mathrm{~N}=11.5 \pm 0.4(\mathrm{~N}=18$} \\
\hline & 1 & & $\mathrm{~B} / \mathrm{C}$ & 24 & 21 \\
\hline & 1 & & $\mathrm{~B} / \mathrm{C}$ & 22 & 19 \\
\hline & 1 & & B & 23 & 19 \\
\hline & 1 & & $B / C$ & 24 & 21 \\
\hline & 1 & & C & 23 & 19 \\
\hline & 1 & & $A / B$ & 25 & 21 \\
\hline \multirow[t]{4}{*}{$109^{\circ} 53.3^{\prime} \mathrm{W}$} & 1 & S4 & W2 & \multicolumn{2}{|c|}{$25.1 \pm 0.2(N=422.4 \pm 0.2(N=53.0 \pm 0.1$} \\
\hline & 1 & & $\mathrm{~A} / \mathrm{Be}$ & 24 & 20 \\
\hline & 1 & & B & $25.6(24.4$ & $21.8(19.6-24$ \\
\hline & 1 & & $A$ & $25.4(24.7$ & $21.3(20.5-22$ \\
\hline $69^{\circ} 49.008^{\prime} \mathrm{W}$ & 1 & S3 & W3 & $25.5 \pm 0.4$ & $122.3 \pm 0.4(\mathrm{~N}=11.5 \pm 0.3(\mathrm{~N}=13$ \\
\hline & 1 & & B & $25.1(24.0$ & $21.2(19.9-23$ \\
\hline & 1 & & $B / C$ & 23 & 19 \\
\hline & 1 & & $\mathrm{~B} / \mathrm{C}$ & 25 & 21 \\
\hline & 1 & & B & $25.3(24$ & $21.7(20.5-25$ \\
\hline $70^{\circ} 11.445^{\prime} \mathrm{W}$ & 1 & & W1 & $24.9(\mathrm{~N}=$ & $21.4(\mathrm{~N}=1) \quad 1.1$ \\
\hline & 1 & S2 & W2 & $25.32 \pm 0.3$ & $21.34 \pm 0.36(n-1.93 \pm 0.18(n=$ \\
\hline & 1 & & $\mathrm{~A} / \mathrm{B}$ & 22 & 19 \\
\hline & 1 & & $\mathrm{Be}$ & 24 & \\
\hline & 1 & & $B$ & $26.1(24.5$ & 21.7 (20.8-22. \\
\hline & 1 & & $B / C$ & 23 & 19 \\
\hline & 1 & & $A / B$ & 22 & 19 \\
\hline $156^{\circ} 39.795^{\prime} \mathrm{E}$ & 1 & S4 & W1 & $25.0 \pm 0.9$ & $20.7 \pm 0.6$ \\
\hline
\end{tabular}




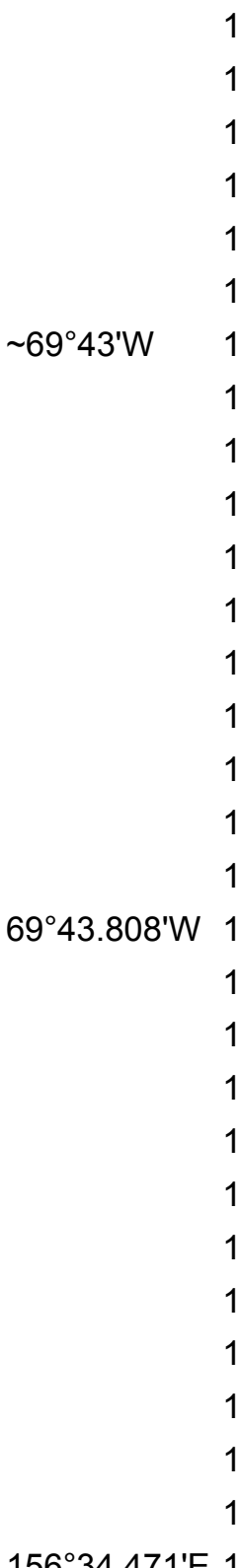

S2

$\begin{array}{lll}\text { B/Ce } & 23 & 19 \\ \mathrm{~B} / \mathrm{C} & 25 & 21 \\ \mathrm{~A} / \mathrm{B} & 23 & 19\end{array}$

C

25.2 (23.7-27. 21.0 (20.0-21.

B

24

21

A/B

25

22

W1

B 23

WO

22.3

19.8 1.8

$\mathrm{A} / \mathrm{B}$

24

22

$\mathrm{B} / \mathrm{C}$

25

21

$\mathrm{B} / \mathrm{C}$

22

19

A/B

25

21

A/B

25

20

$\mathrm{A} / \mathrm{B}$

22

19

$\mathrm{A} / \mathrm{B}$

24

21

B

$25.0(23.7-25.21 .0(19.7-23$.

S3

W2

25.3 $\pm 0.4(\mathrm{~N}=122.0 \pm 0.4(\mathrm{~N}=11.4 \pm 0.3(\mathrm{~N}=15$

$\mathrm{B} / \mathrm{C}$

25

22

B

23

19

A/B

23

19

$\mathrm{B} / \mathrm{C}$

22

19

B

23

19

B/C

25

22

B

25

21

B/C

22

19

S4

W2

$B / C$

$25.72 \pm 0.54(n 21.59 \pm 0.45(n 1.77 \pm 0.31(n=$

\section{$156^{\circ} 34.471$ 'E 1}

S4

$\mathrm{B} / \mathrm{C}$

25

22

W1

C

$\mathrm{B} / \mathrm{C}$

$\mathrm{Ce}$

B

S5

W1

$\mathrm{A} / \mathrm{B}$

A/B

22

19

$25.1 \pm 0.3$

$21.3 \pm 0.2$

$1.6 \pm 0.2$

22

19

25

21

22

19

23

19

$24.8 \pm 0.2$

$21.1 \pm 0.2$

$1.5 \pm 0.3$

22

19

B

24

22

C

24

20

A/B

26.2 (23.9-29. 21.5 (19.9-22.

$A / B$

$\mathrm{A} / \mathrm{B}$

A

W2

A/B
22

25

23

21

24.9 (24.3-26. 21.0 (19.5-22.

$24.54 \pm 0.3(n=22.16 \pm 1.29(n) 1.58 \pm 0.19(n=$ 24
21 


\begin{tabular}{|c|c|c|c|c|c|}
\hline & 1 & B & 24 & 21 & \\
\hline & 1 & $B$ & 25 & 20 & \\
\hline & 1 & $\mathrm{C}$ & 23 & 19 & \\
\hline & 1 & $\mathrm{~B} / \mathrm{C}$ & 22 & 19 & \\
\hline & 1 & $B$ & 22 & 19 & \\
\hline & 1 & C & 23 & 19 & \\
\hline & 1 & $\mathrm{~B}$ & 22 & 19 & \\
\hline & 1 & $\mathrm{~A} / \mathrm{B}$ & 25 & 22 & \\
\hline $156^{\circ} 36.976^{\prime} \mathrm{E}$ & S4 & W1 & $24.8 \pm 0.4$ & $21.1 \pm 0.2$ & $1.4 \pm 0.2$ \\
\hline & 1 & $B$ & 24 & 21 & \\
\hline & 1 & $\mathrm{~B} / \mathrm{C}$ & 25 & 21 & \\
\hline & 1 & $\mathrm{~B} / \mathrm{C}$ & 22 & 19 & \\
\hline $70^{\circ} 04.500^{\prime} \mathrm{W}$ & $1 \quad$ S5 & W2 & $23.9 \pm 0.5$ & $120.9 \pm 0.5($ & $1.3 \pm 0.4(\mathrm{~N}=14$ \\
\hline & 1 & $\mathrm{~A} / \mathrm{B}$ & 25 & 21 & \\
\hline & 1 & $\mathrm{~A} / \mathrm{B}$ & 25 & 21 & \\
\hline & 1 & $\mathrm{~B} / \mathrm{C}$ & 23 & 19 & \\
\hline & 1 & C & 23 & 19 & \\
\hline & $\mathrm{S} 2$ & W1 & $25.0-25.8$ & $20.6-21.1$ & $2.0-2.5$ \\
\hline & 1 & $\mathrm{~A} / \mathrm{B}$ & 25 & 22 & \\
\hline & 1 & B & 24 & 22 & \\
\hline & 1 & $\mathrm{~B} / \mathrm{C}$ & 22 & 19 & \\
\hline & 1 & $\mathrm{~B} / \mathrm{C}$ & 23 & 19 & \\
\hline & 1 & $\mathrm{~B}$ & 25 & 21 & \\
\hline & S2 & W2 & $24.5-25.0$ & $21.6-21.7$ & $1.6-1.9$ \\
\hline & 1 & $\mathrm{~B} / \mathrm{Ce}$ & 23 & 19 & \\
\hline & 1 & C & 23 & 19 & \\
\hline & 1 & $\mathrm{~B} / \mathrm{C}$ & 25 & 21 & \\
\hline & 1 & B & 25 & 21 & \\
\hline & 1 & $\mathrm{~B} / \mathrm{C}$ & 25 & 21 & \\
\hline & S4 & W2 & 26 & & \\
\hline & S3 & W2 & $25.1 \pm 0.27$ & $21.4 \pm 0.29$ & $1.62 \pm 0.35(n=$ \\
\hline & 1 & $\mathrm{~B}$ & 24.5 (22.9 & $20.9(20.2$ & \\
\hline & 1 & $\mathrm{~A} / \mathrm{B}$ & 25 & 22 & \\
\hline $156^{\circ} 30.635^{\prime} \mathrm{E}$ & S4 & W1 & $25.1 \pm 0.4$ & $21.4 \pm 0.2$ & $1.6 \pm 0.2$ \\
\hline & 1 & $\mathrm{~B}$ & 22 & 20 & \\
\hline & 1 & $B$ & 25 & 21 & \\
\hline & 1 & B & 25 & & \\
\hline & 1 & $\mathrm{~A} / \mathrm{B}$ & 25 & 20 & \\
\hline & 1 & $\mathrm{C}$ & 22 & 19 & \\
\hline & 1 & $\mathrm{C}$ & 23 & 19 & \\
\hline & 1 & $\mathrm{Be}$ & 22 & 19 & \\
\hline & 1 & $\mathrm{~B} / \mathrm{C}$ & 23 & 20 & \\
\hline$\sim 69^{\circ} 43^{\prime} \mathrm{W}$ & 1 & W3 & & & \\
\hline & 1 & $A / B$ & 24 & 21 & \\
\hline & 1 & $A / B$ & 25 & 22 & \\
\hline
\end{tabular}




\begin{tabular}{|c|c|c|c|c|c|}
\hline & 1 & B & \multicolumn{3}{|c|}{25.4 (24.3-27. 21.3 (20.7-22. } \\
\hline & 1 & B & 23 & \multicolumn{2}{|c|}{19} \\
\hline & 1 & $\mathrm{~B} / \mathrm{C}$ & 25 & & \\
\hline $156^{\circ} 25.913^{\prime} E$ & S4 & W1 & $25.2 \pm 0.3$ & $21.6 \pm 0.3$ & $1.3 \pm 0.3$ \\
\hline & 1 & $A / B$ & 22 & \multicolumn{2}{|l|}{19} \\
\hline & 1 & $A / B$ & 23 & \multicolumn{2}{|l|}{19} \\
\hline & 1 & $B$ & 25 & \multicolumn{2}{|l|}{22} \\
\hline & 1 & B & 23 & \multicolumn{2}{|l|}{19} \\
\hline & 1 & B & 23 & & \\
\hline $69^{\circ} 43.993^{\prime} \mathrm{W}$ & S3 & W2 & \multicolumn{3}{|c|}{$24.9 \pm 0.5(\mathrm{~N}=121.7 \pm 0.5(\mathrm{~N}=11.4 \pm 0.4(\mathrm{~N}=11$} \\
\hline & 1 & $A / B$ & 25 & \multicolumn{2}{|c|}{21} \\
\hline & 1 & B & 24 & \multicolumn{2}{|l|}{21} \\
\hline & 1 & $\mathrm{~B} / \mathrm{Ce}$ & 23 & \multicolumn{2}{|l|}{19} \\
\hline & 1 & $A / B$ & & \multicolumn{2}{|l|}{20} \\
\hline & 1 & $B / C$ & 24 & & \\
\hline & 1 & $B / C$ & 25 & & \\
\hline & 1 & $B / C$ & 25 & \multicolumn{2}{|l|}{21} \\
\hline & 1 & C & \multicolumn{3}{|c|}{$25.2(23.8-26.21 .2(20.3-21}$. \\
\hline & 1 & $\mathrm{~B} / \mathrm{C}$ & 22 & \multicolumn{2}{|c|}{19} \\
\hline & 1 & $A / B$ & 25 & \multicolumn{2}{|l|}{21} \\
\hline $69^{\circ} 56.768^{\prime} \mathrm{W}$ & $\mathrm{S} 2$ & W2 & \multicolumn{3}{|c|}{$24.5 \pm 0.4(\mathrm{~N}=120.7 \pm 0.4(\mathrm{~N}=11.4 \pm 0.3(\mathrm{~N}=15$} \\
\hline & 1 & B & \multicolumn{3}{|c|}{$25.7(24.7-28.21 .7(20.4-25$} \\
\hline & 1 & B & 24 & \multicolumn{2}{|c|}{21} \\
\hline $156^{\circ} 28.048^{\prime} \mathrm{E}$ & S3 & W1 & $25.8 \pm 0.5$ & $21.4 \pm 0.1$ & $1.5 \pm 0.1$ \\
\hline $69^{\circ} 1.85^{\prime} \mathrm{W}$ & S3 & W2 & \multicolumn{3}{|c|}{$25.2 \pm 0.9(\mathrm{~N}=120.9 \pm 0.9(\mathrm{~N}=11.6 \pm 0.3(\mathrm{~N}=12$} \\
\hline $116^{\circ} 33.05^{\prime} \mathrm{W}$ & S4 & W2 & \multicolumn{3}{|c|}{$24.7 \pm 0.2(\mathrm{~N}=222.3 \pm 0.2(\mathrm{~N}=22.7 \pm 0.2$} \\
\hline & 1 & B & 25 & \multicolumn{2}{|l|}{21} \\
\hline & 1 & $B / C$ & 24 & \multicolumn{2}{|l|}{21} \\
\hline & 1 & B & \multicolumn{3}{|l|}{25} \\
\hline$\sim 69^{\circ} 46^{\prime} \mathrm{W}$ & 1 & W2 & $25.7(\mathrm{~N}=1)$ & \multicolumn{2}{|l|}{$20.9(\mathrm{~N}=1)$} \\
\hline & 1 & B & 22 & 19 & \\
\hline & 1 & $B / C$ & 24 & 20 & \\
\hline & 1 & B & 25 & & \\
\hline & 1 & $\mathrm{~B} / \mathrm{C}$ & 22 & 18 & \\
\hline & 1 & $B / C$ & 24 & 21 & \\
\hline & 1 & $B / C$ & 25 & 21 & \\
\hline & 1 & B & 23 & 19 & \\
\hline & S2 & W3 & $25.99 \pm 0.51$ & $25.29 \pm 3.78$ & $1.84 \pm 0.02(n=$ \\
\hline & 1 & B & 25 & 21 & \\
\hline & 1 & $A / B$ & 25 & 21 & \\
\hline & 1 & $B / C$ & 23 & 19 & \\
\hline & 1 & C & 23 & 20 & \\
\hline $156^{\circ} 31.227^{\prime} \mathrm{E}$ & S4 & W1 & $25.0 \pm 0.3$ & $21.5 \pm 0.2$ & $1.4 \pm 0.2$ \\
\hline & 1 & $B / C$ & 25 & 21 & \\
\hline & 1 & C & 23 & 19 & \\
\hline
\end{tabular}




\begin{tabular}{|c|c|c|c|c|c|}
\hline & 1 & C & \multicolumn{3}{|c|}{$25.6(24.5-27.22 .0(21.1-23}$. \\
\hline & 1 & $\mathrm{~B}$ & 25 & \multicolumn{2}{|c|}{21} \\
\hline & 1 & $A / B$ & 22 & \multicolumn{2}{|l|}{19} \\
\hline & 1 & $\mathrm{Ce}$ & 23 & \multicolumn{2}{|l|}{19} \\
\hline & 1 & $A / B$ & 23 & \multicolumn{2}{|l|}{19} \\
\hline & 1 & C & 22 & \multicolumn{2}{|l|}{19} \\
\hline & 1 & $\mathrm{~B} / \mathrm{C}$ & 24 & \multicolumn{2}{|l|}{21} \\
\hline & 1 & $\mathrm{~B} / \mathrm{C}$ & 25 & \multicolumn{2}{|l|}{21} \\
\hline & 1 & $B / C$ & 25 & \multicolumn{2}{|l|}{21} \\
\hline \multirow{6}{*}{$69^{\circ} 55.044^{\prime} \mathrm{W}$} & $\mathrm{S} 1$ & W2 & \multicolumn{3}{|c|}{$23.3 \pm 0.5(\mathrm{~N}=119.8 \pm 0.5(\mathrm{~N}=11.6 \pm 0.4(\mathrm{~N}=16$} \\
\hline & 1 & B & 23 & \multicolumn{2}{|c|}{19} \\
\hline & 1 & B & 22 & \multicolumn{2}{|l|}{19} \\
\hline & 1 & B & 25 & \multicolumn{2}{|l|}{21} \\
\hline & 1 & $\mathrm{Be}$ & 25 & \multicolumn{2}{|l|}{21} \\
\hline & 1 & C & 23 & \multicolumn{2}{|l|}{19} \\
\hline \multirow[t]{7}{*}{$69^{\circ} 49.813^{\prime} \mathrm{W}$} & $\mathrm{S} 2$ & W2 & \multicolumn{3}{|c|}{$23.8 \pm 0.5(\mathrm{~N}=119.0 \pm 0.5(\mathrm{~N}=11.7 \pm 0.4(\mathrm{~N}=15$} \\
\hline & 1 & B & \multicolumn{3}{|c|}{$25.3(24.3-27.21 .4(20.2-22$} \\
\hline & 1 & $\mathrm{~B} / \mathrm{C}$ & 22 & \multicolumn{2}{|c|}{19} \\
\hline & 1 & $B / C$ & 23 & \multicolumn{2}{|l|}{19} \\
\hline & 1 & C & 22 & \multicolumn{2}{|l|}{19} \\
\hline & 1 & C & 22 & \multicolumn{2}{|l|}{19} \\
\hline & 1 & $\mathrm{~B}$ & 23 & \multicolumn{2}{|l|}{19} \\
\hline $68^{\circ} 39^{\prime} 12^{\prime \prime} \mathrm{W}$ & S2 & W2 & $25.59 \pm 0.50$ & $21.72 \pm 0.28$ & $1.61 \pm 0.21(\mathrm{~N}=$ \\
\hline & 1 & $\mathrm{~B} / \mathrm{C}$ & 25 & 21 & \\
\hline & 1 & $A / B$ & 24 & 21 & \\
\hline & 1 & $B / C$ & 22 & 19 & \\
\hline & 1 & $\mathrm{Ce}$ & 25 & 21 & \\
\hline & 1 & $\mathrm{C}$ & 25 & 21 & \\
\hline & 1 & $A / B$ & 22 & 19 & \\
\hline & 1 & $\mathrm{~B} / \mathrm{C}$ & 22 & 20 & \\
\hline $156^{\circ} 30.790^{\prime} \mathrm{E}$ & S4 & W1 & $25.2 \pm 0.8$ & $20.6 \pm 0.9$ & $2.8 \pm 2.4$ \\
\hline & 1 & $\mathrm{Be}$ & 25 & 21 & \\
\hline & 1 & $B$ & 24 & 21 & \\
\hline & 1 & $\mathrm{Ce}$ & 22 & 19 & \\
\hline & 1 & $\mathrm{C}$ & 23 & 19 & \\
\hline$\sim 69^{\circ} 46^{\prime} \mathrm{W}$ & 1 & W2 & $25.7(\mathrm{~N}=1)$ & $20.8(\mathrm{~N}=1)$ & 1.9 \\
\hline & 1 & B & $25.3(24.4-2$ & 21.4 (20.3-2 & \\
\hline & 1 & $B$ & $25.0(24.2-2$ & $21.3(20.6-$ & \\
\hline & 1 & $B / C$ & 22 & 19 & \\
\hline & 1 & $A / B$ & 23 & 19 & \\
\hline & 1 & $\mathrm{Ce}$ & 25 & 21 & \\
\hline & 1 & B & $25.3(23.7-2$ & 21.7 (20.4-? & \\
\hline $156^{\circ} 27.828^{\prime} \mathrm{E}$ & S4 & W1 & $24.7 \pm 0.2$ & $20.5 \pm 0.1$ & $1.5 \pm 0.2$ \\
\hline & 1 & $B / C$ & 25 & 21 & \\
\hline & 1 & B & $25.2(22.4-2$ & $21.7(20.5-2$ & \\
\hline
\end{tabular}




\begin{tabular}{|c|c|c|c|c|c|}
\hline 1 & & $\mathrm{~B}$ & \multicolumn{3}{|c|}{$25.5(24.5-28.21 .9(20.7-25$} \\
\hline $156^{\circ} 26.695^{\prime} \mathrm{E} 1$ & S4 & W1 & $24.8 \pm 0.4$ & $21.6 \pm 0.7$ & $1.4 \pm 0.2$ \\
\hline 1 & & $A$ & \multicolumn{3}{|c|}{24.5 (23.0-25. 20.6 (19.1-21. } \\
\hline 1 & & $\mathrm{~B} / \mathrm{C}$ & 22 & \multicolumn{2}{|c|}{20} \\
\hline 1 & & $B$ & 23 & \multicolumn{2}{|l|}{19} \\
\hline 1 & & B & \multicolumn{3}{|c|}{$25.2(23.8-26.21 .5$ (20.4-23. } \\
\hline 1 & & $\mathrm{~B} / \mathrm{C}$ & 21 & \multicolumn{2}{|c|}{19} \\
\hline 1 & & $\mathrm{~B} / \mathrm{C}$ & 22 & \multicolumn{2}{|l|}{19} \\
\hline 1 & & $\mathrm{~B} / \mathrm{C}$ & 25 & \multicolumn{2}{|l|}{22} \\
\hline 1 & & B & 24 & \multicolumn{2}{|l|}{21} \\
\hline $156^{\circ} 27.893^{\prime}$ E 1 & S4 & W1 & $25.1 \pm 0.8$ & $21.1 \pm 0.3$ & $1.5 \pm 0.3$ \\
\hline 1 & & $\mathrm{~B} / \mathrm{C}$ & 25 & \multicolumn{2}{|l|}{21} \\
\hline 1 & & $\mathrm{~B}$ & 23 & \multicolumn{2}{|l|}{19} \\
\hline 1 & & $A$ & \multicolumn{3}{|c|}{25.4 (24.6-26. 21.5 (20.6-22. } \\
\hline $156^{\circ} 26.552^{\prime} \mathrm{E} 1$ & S4 & W1 & $25.6 \pm 0.6$ & $21.2 \pm 0.2$ & $1.6 \pm 0.1$ \\
\hline $69^{\circ} 51.844^{\prime} \mathrm{W} 1$ & S5 & W3 & \multicolumn{3}{|c|}{$23.4 \pm 0.4(\mathrm{~N}=120.9 \pm 0.4(\mathrm{~N}=11.9 \pm 0.3(\mathrm{~N}=15$} \\
\hline 1 & & B & 23 & \multicolumn{2}{|c|}{19} \\
\hline 1 & & B & \multicolumn{3}{|c|}{$25.5(23.9-29.22 .3$ (20.2-24. } \\
\hline $156^{\circ} 26.757^{\prime} \mathrm{E} 1$ & S4 & W1 & $25.4 \pm 0.7$ & $21.1 \pm 0.3$ & $1.6 \pm 0.1$ \\
\hline 1 & & A & \multicolumn{3}{|c|}{$23.0(21.7-25.20 .2(18.4-23$} \\
\hline 1 & & $A$ & \multicolumn{3}{|c|}{$25.2(24.3-27.21 .8(20.1-24}$. \\
\hline 1 & & B & \multicolumn{3}{|c|}{24.9 (23.9-26. $21.7(19.6-25}$. \\
\hline $70^{\circ} 21.673^{\prime} \mathrm{W} 1$ & & W2 & \\
\hline 1 & & B & \multicolumn{3}{|c|}{$26.2(24.7-30.23 .1$ (19.5-29. } \\
\hline 1 & & B & \multicolumn{3}{|c|}{$24.7(23.4-27.20 .9(20.3-22}$. \\
\hline 1 & & B & \multicolumn{3}{|c|}{$25.6(23.8-27.21 .7(20.7-23}$. \\
\hline 1 & & B & \multicolumn{3}{|c|}{$26.1(23.9-27.22 .0(19.9-26}$. \\
\hline 1 & & B & \multicolumn{3}{|c|}{$25.6(24.5-27.21 .7(20.5-23}$. \\
\hline 1 & & B & $25.4(24 . C$ & $21.2(19$ & \\
\hline 1 & & A & $25.1(23.6$ & $21.0(19$ & \\
\hline 1 & & B & 25.3 & 21.8 & \\
\hline 1 & & B & 25.3 & 21.8 & \\
\hline 1 & & B & 25.1 & $21.5(20$ & \\
\hline 1 & & A & 26.6 & $22.8(21$ & \\
\hline 1 & & A & 25.9 & $21.9(20$ & \\
\hline 1 & & B & $25.7(24.3$ & $22.2(20$ & \\
\hline 1 & & B & $25.2(23.8$ & $21.1(20$ & \\
\hline 1 & & A & 25.2 & 21.5 & \\
\hline 1 & & B & 25.4 & 21.8 & \\
\hline 1 & & B & $26.6(25.8$ & $21.8(20$ & \\
\hline 1 & & B & $25.7(24.5$ & $21.7(20$ & \\
\hline 1 & & $A$ & 25.4 & $21.9(20$ & \\
\hline 1 & & B & $25.8(24.3$ & 21.8 (19. & \\
\hline 1 & & B & 25.1 & 21.2 (19. & \\
\hline 1 & & $A$ & 25.6 & $21.6(20$ & \\
\hline
\end{tabular}




\begin{tabular}{|c|c|c|c|c|}
\hline 1 & & B & \multicolumn{2}{|c|}{$25.2(24.2-27.21 .5$ (21.1-22. } \\
\hline $156^{\circ} 26.801^{\prime} \mathrm{E} 1$ & S4 & W1 & $24.8 \pm 0.1 \quad 20.8 \pm 0.3$ & $1.4 \pm 0.1$ \\
\hline 1 & & $\mathrm{~B}$ & \multicolumn{2}{|c|}{25.4 (24.3-27. 21.5 (20.6-22. } \\
\hline 1 & & $\mathrm{~B}$ & \multicolumn{2}{|c|}{$25.6(24.1-29.21 .4(20.4-23}$. \\
\hline 1 & & C & \multicolumn{2}{|c|}{21} \\
\hline 1 & & $\mathrm{~B}$ & \multicolumn{2}{|c|}{25.5 (24.4-28. 21.5 (20.0-24. } \\
\hline 1 & & B & \multicolumn{2}{|c|}{$25.3(22.3-28.21 .6(20.9-25}$. \\
\hline 1 & & $\mathrm{~B}$ & \multicolumn{2}{|c|}{$25.4(23.5-27.21 .8(20.5-23}$. \\
\hline 1 & & $\mathrm{~B}$ & \multicolumn{2}{|c|}{25.3 (24.1-26. 21.2 (19.6-23. } \\
\hline $156^{\circ} 31.060^{\prime} \mathrm{E}$ & S4 & W1 & $25.0 \pm 0.2 \quad 21.0 \pm 0.3$ & $1.6 \pm 0.3$ \\
\hline 1 & & $\mathrm{~B}$ & \multicolumn{2}{|c|}{$25.2(23.4-27.21 .6(19.9-25$} \\
\hline 1 & & B & \multicolumn{2}{|c|}{$25.6(23.6-28.20 .9$ (19.6-22. } \\
\hline 1 & & $\mathrm{~B}$ & \multicolumn{2}{|c|}{$26.4(24.1-31.22 .1(20.4-24}$. \\
\hline 1 & & $\mathrm{~B}$ & \multicolumn{2}{|c|}{25.5 (24.3-27. 21.6 (19.5-25. } \\
\hline $156^{\circ} 29.957^{\prime} \mathrm{E}$ & S4 & W1 & $24.6 \pm 0.1 \quad 20.8 \pm 0.7$ & $1.4 \pm 0.1$ \\
\hline 1 & & B & \multicolumn{2}{|c|}{$25.8(24.4-27.21 .5(19.7-25$} \\
\hline 1 & & $A / B$ & \multicolumn{2}{|c|}{25.3 (23.5-28. 21.6 (20.0-24. } \\
\hline 1 & & B & \multicolumn{2}{|c|}{$25.4(24.2-27.21 .3(19.4-22}$. \\
\hline 1 & & B & \multicolumn{2}{|c|}{$25.4(24.2-30.21 .4(20.6-23}$. \\
\hline 1 & & B & \multicolumn{2}{|c|}{$25.4(23.9-27.22 .0(20.6-23}$. \\
\hline 1 & & B & \multicolumn{2}{|c|}{$25.5(24.7-29.21 .7(20.1-24}$. \\
\hline 1 & & $\mathrm{~B}$ & \multicolumn{2}{|c|}{25.5 (23.8-28. 21.4 (19.9-24. } \\
\hline $156^{\circ} 27.564^{\prime} \mathrm{E}$ & S4 & W1 & $24.9 \pm 0.6 \quad 20.8 \pm 0.1$ & $1.4 \pm 0.3$ \\
\hline 1 & & $A / B$ & \multicolumn{2}{|c|}{$25.5(24.2-28.22 .0(19.6-26$} \\
\hline 1 & & B & \multicolumn{2}{|c|}{$25.7(24.2-28.21 .7(20.5-25$} \\
\hline 1 & & $\mathrm{~B}$ & \multicolumn{2}{|c|}{$25.4(23.4-28.21 .9$ (20.6-25. } \\
\hline 1 & & $\mathrm{~B}$ & \multicolumn{2}{|c|}{26.3 (24.3-30. 21.9 (20.0-27. } \\
\hline 1 & & $\mathrm{~B}$ & \multicolumn{2}{|c|}{25.6 (23.9-30. 21.9 (19.5-26. } \\
\hline 1 & & A & $25.3(24.0-29.21 .2(20.0$ & \\
\hline 1 & & $A$ & $25.3(23.8-26.22 .0(20.5$ & \\
\hline $69^{\circ} 58.022^{\prime} \mathrm{W}$ & S1 & W2 & $23.5 \pm 0.5(N=121.4 \pm 0.5$ & $11.5 \pm 0.4(N=14$ \\
\hline 1 & & A & $24.6(22.8-25.21 .0(20.1$ & \\
\hline 1 & & B & $25.7(24.0-28.21 .6(20.7$ & \\
\hline 1 & & $A$ & $25.8(24.4-28.21 .7(20.1$ & \\
\hline $156^{\circ} 25.744^{\prime} \mathrm{E}$ & S4 & W1 & $24.9 \pm 0.7 \quad 20.8 \pm 0.3$ & $1.3 \pm 0.3$ \\
\hline $156^{\circ} 30.605^{\prime} \mathrm{E}$ & S4 & W1 & $25.0 \pm 0.4$ & $2.5 \pm 2.3$ \\
\hline 1 & & B & $25.7(24.9-27.21 .9(21.0$ & \\
\hline 1 & & B & $25.8(24.5-30.22 .1(20.4$ & \\
\hline 1 & & B & $25.5(23.8-28.21 .1(19.9$ & \\
\hline 1 & & $\mathrm{~B}$ & $25.1(22.1-30.21 .8(20.8$ & \\
\hline 1 & & B & $25.9(25.1-27.21 .9(21.3$ & \\
\hline 1 & & B & $25.9(24.4-27.21 .9(20.2$ & \\
\hline 1 & & $\mathrm{~B}$ & $25.8(24.5-29.22 .2(21.0$ & \\
\hline 1 & & B & $25.9(24.7-28.21 .6(20.0$ & \\
\hline 1 & & $\mathrm{~B}$ & $25.5(24.5-27.21 .1(20.0$ & \\
\hline
\end{tabular}




\begin{tabular}{|c|c|c|c|c|c|}
\hline 1 & & B & \multicolumn{3}{|c|}{25.9 (23.9-29. 22.0 (20.4-24. } \\
\hline 1 & & A & \multicolumn{3}{|c|}{25.7 (24.8-27. 22.2 (21.5-23. } \\
\hline $156^{\circ} 27.039^{\prime} \mathrm{E} 1$ & S3 & W1 & $24.6 \pm 0.2$ & $20.4 \pm 0.4$ & $1.6 \pm 0.2$ \\
\hline 1 & & $\mathrm{~B}$ & \multicolumn{3}{|c|}{$25.1(23.5-28.21 .2(20.2-22}$. \\
\hline 1 & & B & \multicolumn{3}{|c|}{25.8 (23.8-29. 21.7 (20.9-23. } \\
\hline 1 & & B & \multicolumn{3}{|c|}{$25.3(24.0-26.21 .3(19.9-24}$. \\
\hline 1 & & B & \multicolumn{3}{|c|}{$25.7(24.7-27.21 .5$ (21.0-22. } \\
\hline $156^{\circ} 26.951^{\prime} E 1$ & S4 & W1 & $25.3 \pm 1.2$ & $21.0 \pm 0.2$ & $1.4 \pm 0.1$ \\
\hline 1 & & B & \multicolumn{3}{|c|}{$25.3(23.0-32.22 .4(20.9-26$} \\
\hline 1 & & B & \multicolumn{3}{|c|}{$25.7(24.6-27.21 .9$ (21.0-22. } \\
\hline 1 & & B & \multicolumn{3}{|c|}{25.9 (24.8-29. 22.3 (21.1-24. } \\
\hline 1 & & B & \multicolumn{3}{|c|}{$25.5(23.3-27.22 .1$ (20.4-24. } \\
\hline 1 & & A & \multicolumn{3}{|c|}{$25.4(23.8-27.21 .5(20.3-24}$. \\
\hline 1 & & A & \multicolumn{3}{|c|}{$25.4(23.0-26.21 .7$ (20.3-24. } \\
\hline 1 & & A & \multicolumn{3}{|c|}{25.5 (24.3-28. 21.8 (19.5-23. } \\
\hline 1 & & B & \multicolumn{3}{|c|}{$25.5(24.4-28.21 .5$ (19.9-23. } \\
\hline 1 & & B & \multicolumn{3}{|c|}{25.8 (23.9-28. 21.7 (20.5-23. } \\
\hline 1 & & B & \multicolumn{3}{|c|}{$25.3(23.9-27.22 .0(20.6-24}$. \\
\hline 1 & & B & \multicolumn{3}{|c|}{$25.8(24.5-28.22 .0(20.5-24}$. \\
\hline 1 & & $\mathrm{~B}$ & \multicolumn{3}{|c|}{25.7 (24.9-26. 21.9 (21.1-23. } \\
\hline $156^{\circ} 34.040^{\prime} \mathrm{E} 1$ & S4 & W1 & $24.7 \pm 0.2$ & $20.4 \pm 0.5$ & $1.6 \pm 0.2$ \\
\hline 1 & & $\mathrm{~B}$ & \multicolumn{3}{|c|}{$25.9(24.2-28.22 .0(21.1-23$} \\
\hline 1 & & B & \multicolumn{3}{|c|}{25.7 (24.5-27.21.5 (20.5-23. } \\
\hline 1 & & B & \multicolumn{3}{|c|}{$25.8(24.3-28.22 .1(20.6-24}$. \\
\hline 1 & & B & \multicolumn{3}{|c|}{$26.0(24.9-29.21 .8$ (20.1-24. } \\
\hline 1 & & B & \multicolumn{3}{|c|}{$26.0(24.9-29.21 .9$ (20.4-24. } \\
\hline 1 & & A & \multicolumn{3}{|c|}{25.9 (24.2-29. 21.7 (20.8-23. } \\
\hline 1 & & B & $25.2(23 . \&$ & $.21 .7(20.5$ & \\
\hline 1 & & B & $25.6(24$. & $21.5(20$ & \\
\hline $156^{\circ} 24.970^{\prime} \mathrm{E} 1$ & S2 & W1 & $24.4 \pm 1.0$ & $20.4 \pm 0.3$ & $1.4 \pm 0.2$ \\
\hline 1 & & $\mathrm{~B}$ & $25.3(23.3$ & $21.7(19$. & \\
\hline 1 & & B & $25.6(24$. & $.21 .8(20.6$ & \\
\hline 1 & & A & $25.9(25$. & $21.5(20.2$ & \\
\hline 1 & & B & $25.5(24.8$ & 21.4 & \\
\hline 1 & & B & $25.3(23.2$ & $22.0(20.6$ & \\
\hline 1 & & B & 25.6 & 22.3 & \\
\hline 1 & & B & 25.0 & $21.7(20$. & \\
\hline 1 & & B & 25.8 & 21.7 & \\
\hline 1 & & A & 25.8 & $.21 .4(20$. & \\
\hline 1 & & B & 25.6 & 21.3 & \\
\hline 1 & & A & 25.7 & 21.9 & \\
\hline 1 & & B & 25.6 & 21.5 & \\
\hline 1 & & A & 25.1 (23. & 21.9 & \\
\hline 1 & & B & 26.0 & $.22 .8(20$. & \\
\hline 1 & & B & 25.4 & $.21 .6(20$. & \\
\hline
\end{tabular}


B

B

B

A

A

B

A

A

A

B

A

B

$B$

A

A

B

B

B

A

B

B

B

A

B

A

B

$A / B$

A

B

B

B

B

B

A

B

A

B

C

A

B

B

$\mathrm{Be}$

B

B

A

25.5 (24.4-27. 21.6 (20.8-24.

24.8 (23.0-25. 20.8 (18.8-23.

24.8 (23.4-26. 21.4 (20.1-24.

25.6 (24.2-28. 21.4 (19.7-23.

25.3 (24.4-26. 21.7 (20.2-25.

26.0 (24.4-29. 21.7 (20.5-23.

26.0 (24.9-28. 22.0 (20.4-25.

25.4 (22.9-27. 22.2 (21.0-25.

25.5 (24.2-27. 21.5 (20.6-22.

25.8 (23.9-29. 22.4 (21.1-25.

25.4 (22.3-28. 21.8 (20.6-24.

26.3 (25.4-30. 22.1 (21.3-24.

25.8 (24.5-28. 21.7 (20.9-23.

25.7 (22.4-28. 22.0 (20.5-23.

25.7 (24.5-27. 21.9 (20.0-24.

25.7 (24.5-28. 21.6 (21.0-22.

25.4 (24.7-27. 21.6 (20.9-23.

25.7 (24.5-27. 22.2 (21.0-25.

25.4 (23.7-27. 21.3 (20.3-22.

25.6 (24.5-26. 21.7 (20.1-24.

25.2 (23.6-27. 21.4 (20.3-22.

25.3 (24.4-26. 22.1 (19.6-26.

25.8 (24.4-27. 21.9 (20.8-24.

25.9 (24.3-28. 22.0 (19.4-24.

25.9 (24.6-28. 22.0 (20.7-24.

26.0 (24.7-29. 22.5 (20.8-25.

$25 \quad 22$

25.3 (23.8-26. 21.7 (20.2-24.

25.5 (24.4-27. 21.8 (20.4-25.

25.3 (23.5-27. 21.5 (19.6-24.

26.2 (24.9-31. 22.4 (21.2-24.

25.9 (24.4-31. 21.1 (19.8-22.

25.7 (23.8-28. 21.4 (20.2-23.

25.9 (24.3-27. 21.7 (21.0-22.

25.5 (24.2-27. 21.8 (18.7-24.

26.0 (24.2-27. 21.8 (20.6-23.

25.3 (24.1-28. 21.3 (20.5-23.

26

22

25.6 (24.5-28. 21.8 (20.9-24.

26.2 (25.1-28. 21.6 (20.0-23.

25.6 (24.2-30. 21.5 (20.2-23.

25

22

25.5 (23.4-28. 21.3 (20.2-22.

25.2 (23.7-27. 21.8 (20.0-25.

25.8 (24.4-28. 21.8 (20.2-23. 
24.9 (23.7-26. 21.3 (20.3-22.

B

25.2 (24.3-27. 21.5 (20.1-23.

B

25.3 (24.0-27. 21.5 (20.2-23.

B

25.7 (24.4-28. 21.6 (19.7-25.

B

25.8 (24.8-26. 21.9 (20.7-24.

23

19

B

26.1 (25.0-29. 22.7 (22.1-24.

A

25.5 (24.4-29. 21.8 (20.3-25.

B

25.7 (24.6-27. 21.5 (21.1-22.

25.5 (23.4-28. 21.4 (19.9-23.

26.0 (24.5-27. 22.2 (20.9-24.

25.3 (22.0-27. 21.5 (19.9-22.

A

25.2 (22.5-27. 22.0 (19.6-24.

25.2 (22.9-27. 21.2 (20.2-23.

25.7 (23.2-29. 22.1 (21.0-26.

25.4 (21.2-29. 22.1 (20.5-25.

25.2 (22.2-26. 21.3 (20.4-22.

25.5 (24.4-29. 22.1 (19.8-25.

25.2 (24.1-27. 21.7 (20.1-24.

$24.8 \pm 0.6$

$21.2 \pm 0.8$

$1.4 \pm 0.1$

B

25.7 (24.6-27. 21.8 (20.6-23.

26.0 (24.2-28. 22.2 (20.5-25.

A

25.4 (24.4-27. 21.4 (20.2-22.

25.3 (23.3-27. 22.1 (20.1-24.

A

25.4 (24.1-29. 21.4 (20.8-22.

A

25.8 (24.2-28. 22.1 (20.6-25.

A

25.4 (23.9-27. 21.5 (20.3-24.

$\mathrm{B} / \mathrm{C}$

23

20

$\mathrm{B}$

25.5 (24.4-29. 22.1 (20.2-24.

B

25.1 (24.3-27. 21.6 (20.0-23.

B

25.1 (21.9-27. 21.1 (19.5-22.

A

25.2 (23.7-26. 21.2 (20.3-22.

23

19

A

25.5 (24.1-28. 21.8 (19.9-23.

B

25.0 (24.0-26. 21.1 (19.9-23.

B

26.0 (25.1-27. 22.0 (20.5-24.

22

19

25

21

B

25.6 (24.5-26. 21.9 (20.8-23.

B

25.6 (24.3-27. 21.9 (20.4-26.

B $\quad 25.7(23.9-30.22 .0(20.4-25$.

B $\quad 25.3(24.2-27.21 .8(20.5-23$.

A

25.9 (24.7-30. 21.7 (19.0-23.

A

25.5 (24.4-26. 21.3 (20.4-22.

A

$25.7(24.5-28.21 .6(20.8-23$. 


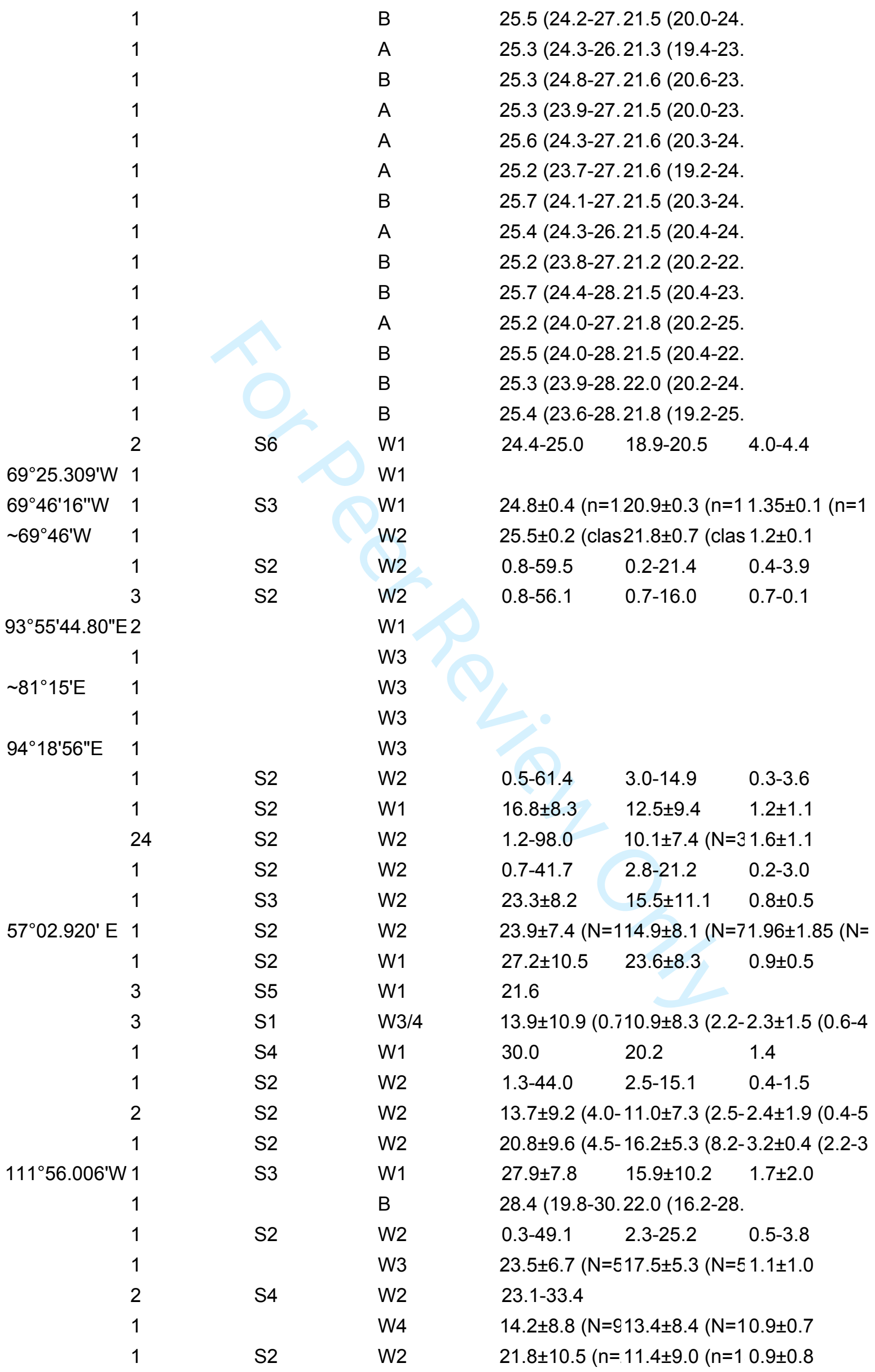




\begin{tabular}{|c|c|c|c|c|c|c|}
\hline & 1 & S2 & W1 & \multicolumn{3}{|c|}{$15.7 \pm 10.2(0 . € 11.0 \pm 8.1(1.4-0.7 \pm 0.8(0.2-3$} \\
\hline \multirow[t]{4}{*}{$69^{\circ} 39.16^{\prime} \mathrm{W}$} & 4 & S1 & W4 & \multicolumn{3}{|c|}{$26.1 \pm 14.9(\mathrm{~N}=15.6 \pm 7(\mathrm{~N}=35,1.6 \pm 1.6(\mathrm{~N}=35$} \\
\hline & 1 & & $A$ & \multicolumn{3}{|c|}{$28.8(26.8-30.21 .6(9.7-25.0$} \\
\hline & 1 & S1 & W2 & $20.0 \pm 6.2$ & $15.7 \pm 7.8$ & $1.4 \pm 1.2$ \\
\hline & 1 & & W3 & \multicolumn{3}{|c|}{$18.2 \pm 8.1(\mathrm{~N}=18.0 \pm 4.1(\mathrm{~N}=4) 2.3 \pm 1.8$} \\
\hline \multicolumn{2}{|c|}{$156^{\circ} 35.422^{\prime} \mathrm{E} 1$} & S2 & W1 & $18.8 \pm 9.2$ & $13.0 \pm 6.4$ & $1.4 \pm 1.6$ \\
\hline \multicolumn{2}{|c|}{ 156³1.991'E 1} & S1 & W1 & $13.8 \pm 10.9$ & $14.7 \pm 9.8$ & $9.2 \pm 12.9$ \\
\hline & 1 & S3 & W3 & $28.2 ; 29.3$ & $17.2 ; 23.6$ & $0.8 ; 1.5$ \\
\hline & 1 & S2 & W1 & $14.9 ; 30.7$ & $11.9 ; 25.0$ & $0.8 ; 2.1$ \\
\hline & 1 & & W3 & \multicolumn{3}{|c|}{$16.8 \pm 5.4(\mathrm{~N}=610.4 \pm 8.4(\mathrm{~N}=41.6 \pm 1.3$} \\
\hline & 1 & S3 & W2 & $14.0 \pm 11.0$ & $11.1 \pm 10.0$ & $1.1 \pm 0.8$ \\
\hline \multicolumn{2}{|c|}{$69^{\circ} 44^{\prime} 15.9 " W 365$} & & W2 & \multicolumn{3}{|c|}{$13.9 \pm 9.6(N=1$} \\
\hline & 1 & & $A$ & $15-31$ & \multicolumn{2}{|l|}{$5-26$} \\
\hline & 1 & & $A / B$ & $4-44$ & \multicolumn{2}{|l|}{$5-20$} \\
\hline & 1 & S2 & W3 & \multicolumn{3}{|c|}{$29.2 \pm 0.3(n=624.2 \pm 0.2(n=71.5$} \\
\hline & many & & W2 & \multicolumn{3}{|c|}{$29.1 \pm 0.2(N=223.7 \pm 0.3(N=20.9 \pm 0.1$} \\
\hline \multirow[t]{3}{*}{$69^{\circ} 51.574^{\prime} \mathrm{W}$} & 5 & S1 & W4 & \multicolumn{3}{|c|}{$28.7 \pm 0.5(\mathrm{~N}=222.6 \pm 0.5(\mathrm{~N}=21.8 \pm 0.5(\mathrm{~N}=22$} \\
\hline & 2 & S4 & W1 & \multicolumn{3}{|c|}{$27.8 \pm 0.4(n=823.5 \pm 2.2(n=50.6 \pm 0.1$} \\
\hline & 1 & S2 & W2 & \multicolumn{3}{|c|}{$27.6 \pm 0.4(n=120.3 \pm 3.2(9.6-0.4 \pm 0.1(0.2-0$} \\
\hline \multirow[t]{15}{*}{$22.38276^{\circ} \mathrm{E}$} & 1 & S3 & W2 & \multicolumn{3}{|c|}{$27.2 \pm 0.8(n=123.4 \pm 0.1(n=2$ n.d. $(n=2)$} \\
\hline & 1 & S3 & W3 & \multicolumn{3}{|l|}{$28.1 \pm 1$} \\
\hline & 1 & S2 & W2 & \multicolumn{3}{|c|}{$28.5 \pm 0.2(n=121.0 \pm 2.7(n=21.8 \pm 0.8(n=23$} \\
\hline & 1 & & B & \multicolumn{3}{|c|}{$26.8(25.2-30.20 .3(15.2-23$} \\
\hline & 1 & & B & $28.3(27.5-$ & $23.7(22.7-$ & \\
\hline & 1 & & A & $29.1(27.4-$ & $23.9(22.6-$ & \\
\hline & 1 & & A & $29.1(27.1-$ & $24.0(22.0-$ & \\
\hline & 1 & & B & 28 & 24 & \\
\hline & 1 & & A & 29.3 (28.3- & 24.0 (19.9- & \\
\hline & 1 & & A & 29.4 (28.8- & 22.9 (14.6- & \\
\hline & 1 & & A & 29.5 (28.2- & 24.1 (19.5- & \\
\hline & 1 & & A & $29.6(28.4-$ & 24.3 (22.5- & \\
\hline & 1 & & $A$ & 29.7 (28.9- & 23.6 (17.3- & \\
\hline & 1 & S1 & W2 & $28.2 \pm 1$ & & \\
\hline & 1 & & $\mathrm{~B} / \mathrm{C}$ & 30 & 24 & \\
\hline $158^{\circ} 07.606^{\prime} \mathrm{E}$ & 1 & S3 & W2 & $27.6 \pm 1.4$ & $23.0 \pm 0.6$ & 81.9 \\
\hline & 1 & & $A / B$ & 29 & 23 & \\
\hline & 1 & S2 & W3 & $30.2 \pm 0.4,3$ & $19.9 \pm 4.9,2$ & $=1.0 \pm 0.8,2.0 \pm C$ \\
\hline & 1 & S4 & W1 & $26.7 \pm 0.7(r$ & $23.7 \pm 6.5(n$ & $1.9 \pm 2.6(n=14$ \\
\hline & 1 & S2 & W2 & $27.3 ; 27.2$ & $20.6 ; 22.6$ & $1.2 ; 3.5$ \\
\hline & 1 & & B & 30.3 (27.0- & $24.2(20.6-$ & \\
\hline & 1 & S2 & W1 & $29.7 ; 30.0$ & $23.4 ; 25.2$ & $2.3 ; 2.8$ \\
\hline & 1 & & $B$ & 30.3 (19.9- & $25.5(15.6-$ & \\
\hline & 1 & S2 & W1 & $29.2 ; 30.0$ & $20.6 ; 24.2$ & $2.4 ; 3.5$ \\
\hline & 1 & & A & $29.6(28.5-$ & $22.5(20.8-$ & \\
\hline & 1 & $\mathrm{~S} 2$ & W1 & 26.6 & & \\
\hline
\end{tabular}




\begin{tabular}{|c|c|c|c|c|}
\hline & 72 & S4 & W1 & $26.7 \pm 0.2(n=1.21 .8 \pm 0.1(n=11.0 \pm 0.5(n=16$ \\
\hline & 1 & S2 & low & $28.2 \pm 0.4(\mathrm{~N}=123.1 \pm 0.4(\mathrm{~N}=11.3 \pm 0.1$ \\
\hline & 1 & & A & 29.7 (26.9-33. 23.6 (21.0-27. \\
\hline & 1 & & B & $29.0(23.7-32.23 .0(13.3-26$. \\
\hline & 1 & S1 & W1 & $33.0 \pm 0.3(n=627.0 \pm 0.3(n=71.7$ \\
\hline & 1 & & $A / B$ & $28 \quad 24$ \\
\hline $68^{\circ} 33.00^{\prime} \mathrm{W}$ & 1 & S2 & W2 & $29.0 \pm 1.0(\mathrm{~N}=123.9 \pm 0.7(\mathrm{~N}=11.6 \pm 0.3(\mathrm{~N}=13$ \\
\hline & 1 & & B & 27 \\
\hline & 1 & S2 & W2 & 28 \\
\hline $68^{\circ} 33.02^{\prime} \mathrm{W}$ & 1 & & & $30.8 \pm 1.5(\mathrm{~N}=124.3 \pm 0.4(\mathrm{~N}=12 \pm 0.2(\mathrm{~N}=10)$ \\
\hline & 1 & S2 & W3 & $27.63 \pm 0.57(n 23.5 \pm 1.71(n=2.08 \pm 0.14(n=$ \\
\hline & 1 & & A & $28.4(24.9-31.22 .5$ (19.6-25. \\
\hline & 1 & & B & $29.2(27.4-30.24 .4(21.7-25$. \\
\hline & 1 & & $\mathrm{~A} / \mathrm{Be}$ & $27 \quad 23$ \\
\hline & 1 & S3 & W1 & $33.0 \pm 0.5(n=627.0 \pm 0.5(n=71.6 \pm 0.1$ \\
\hline & 1 & & B & $29.4(28.7-30.23 .7(17.8-26$ \\
\hline & 1 & & $B / C$ & 24 \\
\hline $70^{\circ} 22.67^{\prime} \mathrm{W}$ & 1 & S1 & W2 & $27.4 \pm 0.3(\mathrm{~N}=122.5 \pm 0.4(\mathrm{~N}=11.5 \pm 0.5(\mathrm{~N}=12$ \\
\hline & 1 & & A & $30.0(29.1-31.25 .0(22.3-26$ \\
\hline & 2 & S2 & W2 & 28 \\
\hline & 1 & & A & $28.8(27.9-32.23 .5(20.7-26$. \\
\hline & 1 & & $B / C$ & 23 \\
\hline & 1 & & A & 29.8 (28.7-34.24.7 (23.4-26. \\
\hline & 1 & & B & $29.7(28.7-30.22 .7(14.8-25$ \\
\hline & 1 & & $B / C$ & 27 \\
\hline $69^{\circ} 55.64^{\prime} \mathrm{W}$ & 1 & S3 & W2 & $27.6 \pm 0.9(\mathrm{~N}=121.7 \pm 0.3(\mathrm{~N}=11.9 \pm 0.2(\mathrm{~N}=12$ \\
\hline & 1 & & $A$ & $29.6(28.6-30.23 .7(22.2-25$ \\
\hline & 1 & & B & $28.1(26.7-29.22 .9(21.7-23$. \\
\hline & 1 & & moderate & $33.5 \pm 0.9(\mathrm{~N}=826.4 \pm 0.2(\mathrm{~N}=51.6 \pm 0.1$ \\
\hline & 1 & S2 & W1-2 & $32.8 \pm 0.4(n=526.3 \pm 0.5(n=52.2 \pm 0.9(n=5)$ \\
\hline & 1 & S3 & W3-4 & $32.7 \pm 0.3(n=426.6 \pm 0.3(n=51.8 \pm 0.3(n=5)$ \\
\hline $9^{\circ} 28.124 ' \mathrm{~W}$ & Many & S4 & Wo & $26 \pm 0.4(n=15) 25 \pm 0.2(n=11) 2 \pm 0.3$ \\
\hline & 1 & S3 & W4 & 31.1 $\pm 0.7(N=527.7 \pm 0.6(N=53.2 \pm 0.8(N=51$ \\
\hline & 1 & S3 & W1 & $30.5 \pm 1$ \\
\hline & 1 & S4 & W1 & $28.8 \pm 1$ \\
\hline & 1 & S3 & W3 & 27 \\
\hline & 2 & S3 & W3 & $31.3 \pm 0.4$ \\
\hline & 1 & S1 & W1 & $33.0 \pm 0.3(n=727.0 \pm 0.2(n=81.9$ \\
\hline & 1 & & $A / B$ & 28.9 (26.1-32. $22.8(19.3-26$. \\
\hline & 1 & & B & 30.5 (26.7-33. 24.4 (19.5-26. \\
\hline & 1 & S3 & W2 & $26.4 \pm 0.2(n=523.6 \pm 0.3(n=61.6 \pm 0.1$ \\
\hline & 1 & & $B$ & $31.1(29.9-32.25 .4(24.8-25$ \\
\hline & 1 & S2 & W3 & $28.4 \pm 0.1(n=123.6 \pm 0.1(n=11.7 \pm 0.2(n=10$ \\
\hline & 2 & S3 & W1 & 29 \\
\hline & 1 & S3 & W4 & $27.9 \pm 1$ \\
\hline
\end{tabular}




\begin{tabular}{|c|c|c|c|c|c|}
\hline 1 & & A & $29.4(28.2$ & $.24 .2(22.3$ & \\
\hline 1 & & B & 30.5 (27.9 & $24.5(19.6$ & \\
\hline $69^{\circ} 42.890^{\prime} \mathrm{W} \quad 1$ & S2 & W4 & $30.8 \pm 0.3$ & $224.6 \pm 0.4$ & $21.8 \pm 0.4(N=22$ \\
\hline 1 & S2 & W1 & $33.0-33.1$ & 26.4-26.6 & $1.9-1.8$ \\
\hline 1 & & $\mathrm{Ce}$ & 27 & 23 & \\
\hline 1 & S1 & W1 & $32.6 \pm 0.2$ & $727.0 \pm 0.2$ & 71.7 \\
\hline 1 & S2 & W1 & $29.5 \pm 0.1$ & $124.9 \pm 0.2$ & $12.7 \pm 0.1(n=13$ \\
\hline 1 & S2 & W1 & $32.9 \pm 0.2$ & $126.6 \pm 0.2$ & $61.6 \pm 0.1(n=6)$ \\
\hline 1 & & C & 27 & 23 & \\
\hline 1 & & A & 30.7 (27.2 & $25.2(23.8$ & \\
\hline 1 & & B & $29.2(27.9$ & $.24 .4(21.2$ & \\
\hline 1 & S3 & w3 & $27.8 \pm 0.3$ & $722.1 \pm 0.2$ & 61.6 \\
\hline 1 & S3 & W3 & $33.0 \pm 0.3$ & $726.4 \pm 0.3$ & $71.6 \pm 0.1$ \\
\hline 2 & S1 & W3 & $29.8 \pm 0.2$ & $124.4 \pm 0.2$ & $11.8 \pm 0.2(n=11$ \\
\hline 1 & & $B$ & $32.2(30.6$ & $26.4(25.6$ & \\
\hline 1 & & A & $28.8(27.7$ & $.22 .2(13.7$ & \\
\hline 1 & & wo & & & \\
\hline 1 & S2 & W1 & $32.6 \pm 0.2$ & $126.2 \pm 0.2$ & $11.8 \pm 0.1(n=13$ \\
\hline 1 & S5 & W1 & $28.4 \pm 0.3$ & $123.3 \pm 0.2$ & $41.9 \pm 0.1(n=4)$ \\
\hline 1 & & B & 30.4 (28.6 & $.25 .0(24.3$ & \\
\hline 1 & & $B / C$ & 27 & 24 & \\
\hline 1 & S2 & W1 & $31.4 \pm 0.2$ & $1.25 .5 \pm 0.2$ & $11.7 \pm 0.1(n=13$ \\
\hline 1 & & $A$ & $29.6(28.8$ & $.24 .4(23.4$ & \\
\hline 1 & & A & $29.4(28.4$ & $.24 .2(22.3$ & \\
\hline 1 & & A & 29.3 (28.3 & $.24 .5(22.5$ & \\
\hline 1 & & B & $31.3(30.3$ & $.25 .6(23.4$ & \\
\hline 1 & & B & $30.0(29.2$ & $.24 .9(23.3$ & \\
\hline 1 & & B & 30.1 (29.3 & $.24 .3(21.4$ & \\
\hline 1 & & B & $31.0(26.9$ & $25.0(22.0$ & \\
\hline 1 & & $A$ & $29.5(29.1$ & $.24 .0(18.9$ & \\
\hline 1 & S2 & W1 & $32.1 \pm 0.2$ & $126.0 \pm 0.2$ & 1.2.1 $1 \pm 0.1(n=12$ \\
\hline 1 & S2 & Wo/1 & $29.7-30.0$ & $22.9-23.6$ & $2.7-3.9$ \\
\hline 1 & & $B$ & $31.6(30.5$ & $25.6(24.5$ & \\
\hline 1 & S4 & W1 & $25.74 \pm 0.5$ & $23.66 \pm 1.0$ & $1.53 \pm 0.27$ \\
\hline 1 & & $A$ & $29.7(26.4$ & $23.6(13.1$ & \\
\hline 1 & S3 & W2 & $32.4 \pm 0.2$ & $126.1 \pm 0.2$ & $1.1 .8 \pm 0.1(n=12$ \\
\hline 1 & & $\mathrm{~B}$ & $29.1(25.0$ & $23.1(12.9$ & \\
\hline 1 & S3 & W1 & $32.2 \pm 0.2$ & $1 \cdot 26.0 \pm 0.2$ & $1.2 .2 \pm 0.2(n=14$ \\
\hline 1 & & $B$ & $28.6(26.7$ & $23.1(22.4$ & \\
\hline 1 & & A & $31.6(30.5$ & $26.4(24.6$ & \\
\hline 1 & & B & $31.3(28.8$ & $24.3(20.0$ & \\
\hline 1 & & $B$ & $31.2(29.0$ & $25.4(24.5$ & \\
\hline 1 & & $A / B$ & 27 & 23 & \\
\hline 1 & & $\mathrm{~B} / \mathrm{C}$ & 27 & 23 & \\
\hline 1 & S4 & W2 & $33.0 \pm 0.4$ & $126.5 \pm 0.4$ & $12.3 \pm 0.3(n=15$ \\
\hline
\end{tabular}




\begin{tabular}{|c|c|c|c|c|c|c|}
\hline & 1 & & $\mathrm{~B} / \mathrm{C}$ & 27 & \multicolumn{2}{|l|}{23} \\
\hline & 1 & & $A / B$ & 27 & \multicolumn{2}{|l|}{23} \\
\hline & 1 & & A & \multicolumn{3}{|c|}{$29.8(28.1-34.24 .0(23.1-24$} \\
\hline & 1 & & A & \multicolumn{3}{|c|}{$31.0(29.5-34.24 .6(22.3-25}$. \\
\hline & 1 & & B & 29 & \multicolumn{2}{|c|}{23} \\
\hline & 1 & & A & \multicolumn{3}{|c|}{$29.6(28.1-30.24 .2(19.1-26}$. \\
\hline & 1 & & $\mathrm{~B} / \mathrm{C}$ & 27 & \multicolumn{2}{|c|}{23} \\
\hline & 1 & & $\mathrm{~A} / \mathrm{B}$ & 29 & \multicolumn{2}{|l|}{24} \\
\hline & 1 & & A & \multicolumn{3}{|c|}{$30.0(29.0-30.24 .3(19.8-26$} \\
\hline & 1 & & B & \multicolumn{3}{|c|}{28} \\
\hline & 1 & & A & \multicolumn{3}{|c|}{30.6 (28.9-32. 25.3 (23.0-26. } \\
\hline & 1 & & B & \multicolumn{3}{|c|}{$28.5(27.0-30.23 .3(21.7-25$} \\
\hline & 1 & & B & \multicolumn{3}{|c|}{31.9 (30.6-33. 25.7 (24.8-26. } \\
\hline & 1 & & A & \multicolumn{3}{|c|}{32.6 (30.7-33. 26.6 (25.7-28. } \\
\hline & 1 & & $A$ & \multicolumn{3}{|c|}{$30.3(29.3-31.25 .0(24.3-25$} \\
\hline $158^{\circ} 28.086^{\prime} E$ & 1 & $\mathrm{~S} 2$ & W1 & $31.4 \pm 0.8$ & $25.8 \pm 0.7$ & $1.4 \pm 0.2$ \\
\hline & 1 & S6 & W3 & $35.7 \pm 0.3$ & $26.6 \pm 0.4$ & $1.9 \pm 0.3$ \\
\hline & 1 & S4 & W2 & $32.7 \pm 0.2$ & $26.3 \pm 0.2$ & $1.8 \pm 0.3$ \\
\hline & 1 & S2 & W2 & $29.9 \pm 0.3$ & \multicolumn{2}{|c|}{$23.9 \pm 0.4 ; 12.84 .1 \pm 0.3 ; 38.5 \pm$} \\
\hline $69^{\circ} 53.708^{\prime} \mathrm{W}$ & 1 & & high & $31.1(\mathrm{~N}=1)$ & \multicolumn{2}{|c|}{$25.7 \pm 0.0(\mathrm{~N}=32.0 \pm 0.3$} \\
\hline & 1 & S2 & W0/1 & 29.6-29.8 & $23.7-23.8$ & $1.7-2.0$ \\
\hline & 1 & low & moderate & $10.7-10.8$ & $9.9-10.5$ & $1.5-2.7$ \\
\hline & 1 & moderate & strong & 10.7 & $10.3 ; 4.6$ & $2.2 ; 43.6$ \\
\hline & 1 & low & low & $10.4-10.6$ & $4.2-4.4$ & $45.1-45.5$ \\
\hline & Many & low & moderate & $11.0 \pm 0.1$ & $5.0 \pm 0.6$ & $41.4 \pm 2.9$ \\
\hline & 4 & low & low & $11.5-11.9$ & \multicolumn{2}{|c|}{$10.5-12.5 ; 4.3 \cdot 0.1-4.0 ; 39.7-$} \\
\hline & 1 & & A & \multicolumn{3}{|c|}{$12.4(9.2-13.211 .2(10.4-12$} \\
\hline & 1 & & $A$ & \multicolumn{3}{|c|}{$12.1(11.5-12.10 .7(9.5-12.5$} \\
\hline & 1 & & $\mathrm{Ce}$ & $12-13$ & \multicolumn{2}{|c|}{$5-12$} \\
\hline & 1 & high & low & $34.6-59.8$ & $26.4-39.4$ & $9.7-11.7$ \\
\hline & 1 & & Moderate & $12.0-61.1$ & \multicolumn{2}{|c|}{$10.7-69.9 \quad 0.9-44.9$} \\
\hline & 1 & & & & & \\
\hline & 1 & & minimal & & \multicolumn{2}{|c|}{$44.4 \pm 6.0(\mathrm{~N}=522.9 \pm 9.0$} \\
\hline & many & high & low & 31.6 & $25.6 ; 16.1$ & $10.7 ; 35.7$ \\
\hline & 1 & high & low & $39.0-90.0$ & $25.9-71.4$ & 28.6-23.1 \\
\hline & Many & high & moderate & $25.6 \pm 8.9$ & \multicolumn{2}{|c|}{$26.2 \pm 8.8,35 . \subseteq 6.2 \pm 2.5,26.9 \pm$} \\
\hline & Many & high & moderate & $31.2 \pm 9.4$ & $36.0 \pm 10.0$ & $22.2 \pm 8.8$ \\
\hline & several & low & low & $26.7-47.2$ & $24.1 ; 52.4$ & C $3.6 ; 18.8 ; 44.3$ \\
\hline & $\begin{array}{l}9 \\
\text { several }\end{array}$ & high & moderate & $31.7 \pm 6.3$ & $32.2 \pm 10.8$ & $12.9 \pm 8.2$ \\
\hline & 1 & high & moderate & $53.3 \pm 11.8,91$ & $.26 .0 \pm 1.3,3$ & $36.4 \pm 1.3,24.2 \pm$ \\
\hline & 1 & & & & & \\
\hline & 6 & high & moderate & $42.7 \pm 7.3$ & $37.0 \pm 8.0$ & $17.9 \pm 6.9$ \\
\hline & 1 & moderate & moderate & $28 \pm 5(N=13)$ & $29 \pm 11(N=$ & $16 \pm 16$ \\
\hline & Several & high & low & $25.0 \pm 6.4$ & $31.0 \pm 3.6 ; 2$ & $38.1 \pm 3.6 ; 35.0 \pm$ \\
\hline
\end{tabular}




\begin{tabular}{|c|c|c|c|c|c|c|}
\hline \multirow[t]{5}{*}{$13.193^{\circ} \mathrm{W}$} & 1 & low & low & $10.1-27.0$ & $20.9-21.7$ & $4.5-7.0$ \\
\hline & 1 & high & low & $37.4 \pm 9.5$ & $36.1 \pm 3.5,19$ & $c 5.4 \pm 1.3,33.3 \pm$ \\
\hline & Many & high & moderate & $35.7 \pm 10.2$ & $30.0 \pm 6.8,26$ & $710.1 \pm 7.6,37.0$ \\
\hline & 1 & moderate & low & $17.8-23.5$ & $25.4-37.2$ & $8.1-13.0$ \\
\hline & 1 & high & low & $35.8 \pm 5.0$ & $33.6 \pm 5.3,29$ & C12.6 $\pm 6.8,34.8$ \\
\hline \multicolumn{2}{|c|}{$4^{\circ} 11.16582^{\prime} \mathrm{W} 1$} & moderate & low & \multicolumn{3}{|c|}{32.6 (9.8-90.129.5 (15.6-507.9 (1.7-24.8, } \\
\hline & 4 & moderate & low & $18.6-53.0$ & \multicolumn{2}{|c|}{$16.4-42.5 ; 40.2 .9-5.8 ; 5.9-26$} \\
\hline & 1 & low & low & $30.0-41.3$ & $25.2-35.7$ & $7.9-4.2$ \\
\hline & Several & high & moderate & $28.1 \pm 4.8$ & $19.0 \pm 4.4$ & $23.1 \pm 17.6$ \\
\hline & 1 & low & low & $23.0-37.4$ & $29.5 ; 29.6 ; 2$ & $4.5 ; 7.4 ; 25.1$ \\
\hline & 1 & low & low & $13.1 ; 44.5$ & $32.0-38.2$ & $14.6-4.5$ \\
\hline & 1 & low & low & $19.7-51.6$ & $45.7-47.9$ & 3.6 \\
\hline & 1 & high & low & $32.1-32.4$ & $26.0-27.8$ & $9.7-5.7$ \\
\hline \multirow[t]{3}{*}{$\sim 70^{\circ} 15^{\prime} \mathrm{W}$} & 1 & low & minimal & \multirow{2}{*}{\multicolumn{3}{|c|}{$70.2 \pm 3.4(\mathrm{~N}=728.4 \pm 2.9(\mathrm{~N}=240.0 \pm 0.6$}} \\
\hline & 1 & & & & & \\
\hline & 6 & & low & & \multicolumn{2}{|c|}{$25.1 ; 39.9 ; 25.3 .6 ; 8.0 ; 36.7$} \\
\hline \multirow[t]{3}{*}{-14.763139} & 3 & high & low & & \multicolumn{2}{|c|}{$36.0 \pm 10.0,23.5 .0 \pm 2.3,40.3 \pm$} \\
\hline & 9 & moderate & low & & $37.6,33.5$ & $35.7,4.0$ \\
\hline & 6 & high & moderate & & \multicolumn{2}{|c|}{$48.0 \pm 10.7,29.16 .8 \pm 6.0,34.7$} \\
\hline \multirow[t]{13}{*}{$14.319^{\circ} \mathrm{W}$} & several & high & moderate & 19.1-48.7 & \multicolumn{2}{|c|}{$16.5-21.7 ; 32.2 .2-3.5 ; 11.0-$} \\
\hline & 1 & high & low & & $28.5-30.6$ & 28.2-23.7 \\
\hline & 1 & high & low & & $24.8-41.8$ & $31.4-24.3$ \\
\hline & many & low & low & $32.7-34.3$ & \multicolumn{2}{|c|}{$25.0-30.4 ; 17.5 .5-10.0 ; 32.7$} \\
\hline & 1 & high & low & & \multicolumn{2}{|c|}{$22.1 ; 29.9 ; 31.3 .1 ; 7.3 ; 13.7-$} \\
\hline & 1 & high & low & $49.3-49.4$ & $29.2-45.7$ & $10.4-8.1$ \\
\hline & 1 & high & low & $38.1-40.7$ & \multirow{2}{*}{\multicolumn{2}{|c|}{$24.7 ; 26.4-30.4 .1 ; 11.0-7.5$}} \\
\hline & 1 & & & & & \\
\hline & 1 & high & moderate & \multicolumn{3}{|c|}{$32+ \pm 8(N=82) 31 \pm 6(N=168) 15 \pm 9$} \\
\hline & several & high & low & 76.3 & \multicolumn{2}{|c|}{$26.1-45.0 ; 47.28 .6-30.8 ; 12}$. \\
\hline & 1 & & A & $37.1(33.9$ & $.25 .4(24.4-2$ & \\
\hline & several & high & low & & \multicolumn{2}{|c|}{$41.8-58.8 ; 24.11 .1-14.4 ; 33}$. \\
\hline & 4 & strong & low & & $53.7 ; 26.2$ & $14.3 ; 32.2$ \\
\hline \multirow[t]{8}{*}{$53^{\circ} 20.587^{\prime} \mathrm{E}$} & 1 & strong & low & \multicolumn{3}{|c|}{$40.8 \pm 3.5(N=29.8 \pm 3.1(N=12.1 \pm 8.1(N)=$} \\
\hline & 1 & High & Low & & \multicolumn{2}{|c|}{$50.5 \pm 14.8,29.16 .9 \pm 6.8,31.7$} \\
\hline & 1 & moderate & low & & & \\
\hline & 1 & & & & & \\
\hline & 1 & low & low & & $30.3-33.5$ & $3.6-5.3$ \\
\hline & many & low & moderate & & $15.8 ; 24.7 ; 4$ & 6. $1.1 ; 2.7 ; 2.8$ \\
\hline & 1 & & minimal & & $15.9(\mathrm{~N}=1)$ & 0.60 \\
\hline & 1 & low & low & & $32.7-32.9$ & $4.2-5.1$ \\
\hline \multirow[t]{4}{*}{$55.870^{\circ} \mathrm{E}$} & 1 & S2 & W2 & $0.6-38.1$ & $1.9-35.2$ & $0.5-4.4$ \\
\hline & 1 & low & moderate & \multicolumn{3}{|c|}{$12.9 \pm 0.1(n=1$} \\
\hline & 1 & low & high & 13.1 & & \\
\hline & many & S1 & strong & $12.9 \pm 0.2(r$ & & \\
\hline $6^{\circ} 32.32 \mathrm{E}$ & 1 & & W1 & $14.9 \pm 1.1$ & & \\
\hline
\end{tabular}




\begin{tabular}{|c|c|c|c|c|c|c|}
\hline \multirow{3}{*}{$8^{\circ} 50^{\prime} 49.5^{\prime \prime W}$} & \multirow{2}{*}{$\begin{array}{l}\text { several } \\
1\end{array}$} & \multirow[t]{2}{*}{ low } & low & \multicolumn{3}{|l|}{ 13.3-13.7 } \\
\hline & & & low & \multicolumn{3}{|c|}{$13.8 \pm 0.1(\mathrm{~N}=8$} \\
\hline & several & S2 & low & $7.0-57.4$ & 8.1-32.7 & $0.1-6.6$ \\
\hline & 1 & S3 & W2 & 26.3 & $18.7 ; 10.3$ & $1.5 ; 42.1$ \\
\hline & 1 & $\mathrm{~S} 2$ & W3 & \multicolumn{3}{|c|}{$36.0 \pm 4.8(26 . € 19.8 \pm 4.2(13 . € 0.7 \pm 0.3(0.2-1$} \\
\hline & 1 & S3 & W2 & \multicolumn{3}{|c|}{$38.9 \pm 0.3(n=611.1 \pm 0.9(n=645.9 \pm 1.0 ; 1.6 \pm$} \\
\hline & 1 & S2 & W2 & \multicolumn{3}{|c|}{$25.0 \pm 14.7 ; 41.13 .8 \pm 9.2 ; 36 . C 1.1 \pm 1.0 ; 2.2 \pm C$} \\
\hline & many & $\mathrm{S} 2$ & W1 & $2.9-42.4$ & $1.1-31.0$ & $0.3-2.2$ \\
\hline & 1 & S2 & W2 & 39.2-39.9 & $2.2-30.3$ & $0.2-3.2$ \\
\hline & 3 & S2 & low & $1.5-48.4$ & $1.9-14.5$ & $0.3-2.9$ \\
\hline & 1 & S2 & Wo & $8.3-43.1$ & 10.5-33.8 & $1.1-46.3$ \\
\hline & 2 & S2 & low & \multicolumn{3}{|c|}{$1.0 ; 39.1-41.64 .5 ; 16.3 ; 11 . C 0.4 ; 2.7 ; 45.5$} \\
\hline & 1 & S1 & W2 & \multicolumn{3}{|c|}{$37.2 \pm 1.7(n=122.1 \pm 1.4(n=241.6 \pm 1.6(n=2$} \\
\hline & 38 & S4 & W1 & 38.2 & $30.1 ; 12.0$ & $1.3 ; 44.3$ \\
\hline & 1 & low & low & \multicolumn{3}{|c|}{$21.6 \pm 0.1(\mathrm{~N}=111.5 \pm 2.4(\mathrm{~N}=17.9 \pm 1.6(\mathrm{~N}=10$} \\
\hline & 1 & & moderate & \multicolumn{3}{|c|}{$21.1 \pm 0.2(\mathrm{~N}=117.9 \pm 0.1(\mathrm{~N}=17.5 \pm 0.1(\mathrm{~N}=15$} \\
\hline & 1 & moderate & moderate & $21.0 ; 1.4$ & 18.0 & 7.2 \\
\hline & 1 & low & moderate & $20.8 \pm 0.1$ & \multicolumn{2}{|c|}{$17.4 \pm 0.2,10 . \subseteq 4.3 \pm 0.2,34.5 \pm$} \\
\hline & 1 & low & low & 10.8-11.0 & $11.0-11.1$ & 4.6-4.7 \\
\hline & 1 & low & moderate & \multicolumn{3}{|c|}{$20.0 \pm 0.1(n=1.17 .0 \pm 0.1(n=1.9 .1 \pm 0.1 \quad(n=12$} \\
\hline & 1 & low & moderate & \multicolumn{3}{|c|}{$25.5 \pm 0.1(n=121.0 \pm 0.3(n=1.4 .3 \pm 0.1 \quad(n=12$} \\
\hline & 1 & low & moderate & $15.4 \pm 0.1$ & $9.8 \pm 2.1$ & $7.0 \pm 1.8$ \\
\hline & 1 & low & low & \multicolumn{3}{|c|}{$16.0 \pm 0.1(\operatorname{cor} \in 13.7 \pm 0.1(13.47 .4 \pm 0.1(7.3-7$} \\
\hline & 1 & low & moderate & $21.6 \pm 0.3$ & $12.4 \pm 1.5$ & $9.8 \pm 3.0$ \\
\hline & 1 & low & moderate & \multicolumn{3}{|c|}{$20.9 \pm 0.1(n=1.13 .4 \pm 1.4(10 . \varepsilon 7.6 \pm 3.4(3.4-1$} \\
\hline & 1 & low & moderate & $22.0 \pm 0.2$ & $18.8 \pm 0.1$ & $6.0 \pm 0.2$ \\
\hline $56^{\circ} 41.22^{\prime} \mathrm{E}$ & 3 & S5 & W3 & \multicolumn{3}{|c|}{$23.8 \pm 3.0(\mathrm{~N}=415.8 \pm 2.0(\mathrm{~N}=67.5 \pm 1.7$} \\
\hline & 1 & low & moderate & $22.2 \pm 0.1$ & \multicolumn{2}{|c|}{$18.6 \pm 0.3,11.74 .2 \pm 0.1,34.9 \pm$} \\
\hline & 1 & low & moderate & $21.5 \pm 0.1$ & $17.8 \pm 0.1$ & $4.8 \pm 0.8$ \\
\hline & 1 & low & low & \multicolumn{3}{|l|}{$20.6-20.8$} \\
\hline & 1 & low & moderate & 19.6-19.9 & $13.0-15.5$ & 6.6-11.7 \\
\hline $56^{\circ} 35.64^{\prime} \mathrm{E}$ & 2 & & & \multicolumn{3}{|c|}{$21.8 \pm 2.0(\mathrm{~N}=215.5 \pm 2.8(\mathrm{~N}=28.0 \pm 2.9$} \\
\hline & 1 & moderate & moderate & $11.4 ; 6.6$ & $10.7 ; 6.6$ & $4.9 ; 36.2$ \\
\hline & 4 & low & low & \multicolumn{3}{|c|}{$19.7 \pm 0.1(\mathrm{~N}=116.7 \pm 0.2(\mathrm{~N}=17.6 \pm 0.1 \quad(\mathrm{~N}=10$} \\
\hline & 1 & moderate & high & \multicolumn{2}{|c|}{$18.3 \pm 0.2 ; 3.6 \pm 15.3 \pm 0.3$} & $8.1 \pm 0.3$ \\
\hline $156^{\circ} 35.726^{\prime} \mathrm{E}$ & 1 & low & W1 & $14.9 \pm 0.3$ & $12.8 \pm 0.2$ & $4.3 \pm 0.2$ \\
\hline & 1 & U-S4 & minor & $22.6,15.6$ & & \\
\hline & 1 & low & moderate & $12.0 \pm 3.5$ & $12.7 \pm 0.3$ & $\pm 4.9 \pm 0.0,35.4 \pm$ \\
\hline $56^{\circ} 32.840^{\prime} \mathrm{E}$ & 1 & moderate & moderate & $23.8 \pm 0.2($ & $114.3 \pm 1.4($ & 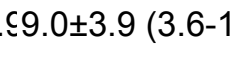 \\
\hline & 1 & low & moderate & $12.1 \pm 0.1$ & $11.1 \pm 1.6$ & $\pm 4.5 \pm 0.1,36.5 \pm$ \\
\hline & 1 & & moderate & $19.6 \pm 0.2($ & $117.1 \pm 0.1($ & $15.4 \pm 0.1(\mathrm{~N}=17$ \\
\hline & 1 & low & low & $21.9-22.2$ & 18.4-18.5 & $9.3-9.2$ \\
\hline & 1 & low & moderate & $21.3 \pm 0.1$ & $118.1 \pm 0.1$ & $18.9 \pm 0.1(n=13$ \\
\hline $69^{\circ} 44.639^{\prime} \mathrm{W}$ & 1 & medium & low & & & \\
\hline & 1 & low & low & $21.7 \pm 0.1$ & $112.6 \pm 2.5($ & $19.3 \pm 1.7(\mathrm{~N}=10$ \\
\hline
\end{tabular}




\section{1}

low (1)

1

2

1

1

2




\begin{tabular}{|c|c|c|c|c|}
\hline \multirow[t]{9}{*}{4.44} & \multicolumn{2}{|c|}{ Jean RedelspıJ. Gattacceca Mohamed Ma: } & \multicolumn{2}{|c|}{ Jean RedelspiJ. Gattacceca:Mohamed Ma: } \\
\hline & 21.1 & UNM & DPitt & C. Agee, UNN \\
\hline & 14.4 & KOPRI & KOPRI & C. Park, S. Y. J. H. Kim \\
\hline & 4.2 & MNB & Stefan Ralew & A. Greshake, I \\
\hline & 20.5 & UWB & Ke Zuokai & A. Irving and $\mathrm{F}$ \\
\hline & 24.0 & UWO & S. Tutorow & Z.G. Guo, UW \\
\hline & 22.1 & UNM & DPitt & C. Agee, UNN \\
\hline & 20 & PMO & Ke Zuokai & Li Y., PMO \\
\hline & 20.7 & UNM & Jay Piatek & C. Agee, UNN \\
\hline \multirow[t]{12}{*}{3.91} & 2.2 & CEREGE & \multicolumn{2}{|c|}{ Labenne Luc J. Gattacceca: Labenne Luc } \\
\hline & 2.56 & UWB & \multicolumn{2}{|c|}{ B. Hoefnagels A. Irving and $\subseteq$} \\
\hline & 20.6 & UWB & D. Pitt & A. Irving and $\leqq$ \\
\hline & 22.3 & UWB & \multicolumn{2}{|c|}{ B. Hoefnagels A. Irving and $\leqq$} \\
\hline & 20.6 & App & PARI & A. Love, App Anonymous \\
\hline & 20 & IfP, Münster & \multicolumn{2}{|c|}{ Nourddine AzıK. Metzler, IfP } \\
\hline & 27.2 & MNB & \multicolumn{2}{|c|}{ Marcin CimalєA. Greshake, I } \\
\hline & 14.4 & MNB & \multicolumn{2}{|c|}{ Stefan Ralew A. Greshake, I } \\
\hline & 20.1 & UWB & \multicolumn{2}{|c|}{ S. Ralew/D. PA. Irving and $\subseteq$} \\
\hline & 2.703 & NIPR & NIPR & A. Yamaguchi \\
\hline & 1.696 & NIPR & NIPR & A. Yamaguchi \\
\hline & 12.3 & UWB & A. Habibi & A. Irving and $\leqq$ \\
\hline \multirow[t]{4}{*}{4.03} & 1.69 & CEREGE & CEREGE & J. Gattacceca: \\
\hline & 0.85 & NIPR & NIPR & A. Yamaguchi \\
\hline & 23.2 & UNM & Jay Piatek & C. Agee, UNN \\
\hline & 30 & Vernad. & \multicolumn{2}{|c|}{ Anonymous o'M.A. Ivanova, Mr. M.E. Nepc } \\
\hline \multirow[t]{6}{*}{5.07} & 10.340 & JSC & JSC & SI \\
\hline & 24.0 & MSN-FI & \multicolumn{2}{|c|}{ Mimaghador V. Moggi Cecc } \\
\hline & 25.2 & MNB & \multicolumn{2}{|c|}{ Martin Goff A. Greshake, I } \\
\hline & 20.1 & MSN-FI & \multicolumn{2}{|c|}{ Mimaghador V. Moggi Cec } \\
\hline & 20.2 & UWB & \multicolumn{2}{|l|}{ M. Arjdal $\quad A$} \\
\hline & 21 & UWB & \multicolumn{2}{|c|}{ A. Aaronson A. Irving and $\leqslant$} \\
\hline \multirow[t]{5}{*}{4.58} & 78.959 & JSC & JSC & ANSMET \\
\hline & 26 & ROM & D. Gregory & A. Irving and $\varsigma$ \\
\hline & 20 & MNB & \multicolumn{2}{|c|}{ Philippe SchmA. Greshake, I } \\
\hline & 20 & MSN-FI & \multicolumn{2}{|c|}{ Mimaghador V. Moggi Cecc } \\
\hline & 20.1 & MNB & \multicolumn{2}{|c|}{ Stefan Ralew A. Greshake, I } \\
\hline \multirow[t]{8}{*}{4.54} & 102.442 & JSC & JSC & ANSMET \\
\hline & 14 & MNB & \multicolumn{2}{|c|}{ Philippe SchmA. Greshake, I } \\
\hline & 9 & UNM & Darryl Pitt & C. Agee, UNN \\
\hline & 11.4 & KOPRI & KOPRI & C. Park, S. Y. H. Yoo \\
\hline & 20 & MNB & Stefan Ralew & A. Greshake, I \\
\hline & 20.31 & UCLA & with owner (SI & A. Rubin, UCL \\
\hline & 20 & MNB & Vincent Gira & A. Greshake, I \\
\hline & 15.9 & UNM & Darryl Pitt & C. Agee, UNN \\
\hline
\end{tabular}


4.45

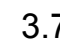

17.219

3.3

93.65

52.912

10.216

7.683

2.675

2.579

2.56

2.538

2.447

2.313

2.092

1.733

1.336

1.002

0.83

3.6

8.7

30.5

20

185.6

15.2

4.97

23.5

11.5

3.54

52.933

33.4

7

3.5

3.9

3.13

14.393

2.3

10.636

4.23
CEREGE

NIPR

MNB

NIPR

NIPR

NIPR

NIPR

NIPR

NIPR

NIPR

NIPR

NIPR

NIPR

NIPR

NIPR

NIPR

NIPR

NIPR

UWB

ASU

PRL

MNB

KOPRI

UWB

CEREGE

UNM

JSC

KOPRI

MNB

MNB

UrFU

JSC

MNB

JSC

CEREGE

MNB

MNB

MNB

0.7

0.44

0.38

0.36

0.3

2

0.8

129.97
MNB

MNB

MNB

KOPRI

MNB

MNB

NIPR
ArtMet collectiJ. Gattacceca anonymous

NIPR

A. Yamaguchi

Stefan Ralew A. Greshake, I

NIPR

A. Yamaguchi

NIPR

A. Yamaguchi

NIPR

A. Yamaguchi

NIPR

A. Yamaguchi

NIPR

A. Yamaguchi

NIPR

A. Yamaguchi

NIPR

A. Yamaguchi

NIPR

A. Yamaguchi

NIPR

A. Yamaguchi

NIPR

A. Yamaguchi

NIPR

A. Yamaguchi

NIPR

A. Yamaguchi

NIPR

A. Yamaguchi

NIPR

A. Yamaguchi

NIPR

A. Yamaguchi

D. Dickens

A. Irving and $\leqslant$

Ruben Garcia L. Garvie

GSI

A.D. Shukla aı A.D. Shukla

Marcin CimaleA. Greshake, I

KOPRI

C. Park, S. Y. S. Park

B. HoefnagelsA. Irving and $\leqslant$

R. Lenssen J. Gattacceca:

Aziz Habibi C. Agee, UNN

JSC

$\mathrm{SI}$

KOPRI

C. Park, S. Y J. I. Lee

Vincent Gira

A. Greshake, I

Vincent Gira

A. Greshake, I

A.N. ZavaritskS. V. Berzin a।

JSC

SI

ANSMET

Philippe SchrrA. Greshake, I

JSC

SI

ANSMET

Kuntz

J. Gattacceca:

Vincent Gira A. Greshake, I

Vincent Gira A. Greshake, I

Philippe SchrrA. Greshake, I

Vincent Gira A. Greshake, I

Vincent Gira

A. Greshake, I

Vincent Gira

A. Greshake, I

KOPRI

C. Park, S. Y. J. I. Lee

Vincent Gira A. Greshake, I

Philippe Schrr A. Greshake, I

NIPR

A. Yamaguchi 


\begin{tabular}{|c|c|c|c|c|}
\hline & 14.932 & NIPR & NIPR & A. Yamaguchi \\
\hline & 2.306 & NIPR & NIPR & A. Yamaguchi \\
\hline & 0.821 & NIPR & NIPR & A. Yamaguchi \\
\hline & 40.1 & UWB & K. Zuokai & A. Irving and $\subseteq$ \\
\hline & 24.05 & UWB & H. Naji & A. Irving and $\leqq$ \\
\hline & 20.4 & MNB & Stefan Ralew & A. Greshake, I \\
\hline 4.42 & 53 & CEREGE & MMC & J. Gattacceca:Rodrigo Martir \\
\hline & 20 & IfP & Manuel Nieto & K. Metzler, IfP \\
\hline & 27 & PMO & Ke Zuokai & Li Y., PMO \\
\hline & 76 & Vernad & Mr. Timur Kry: & :M. A. Ivanova:Mr. Timur Kry: \\
\hline 4.63 & 20.2 & CEREGE & MMC & J. Gattacceca.Rodrigo Martir \\
\hline & 20 & IfP & Anonymous & K. Metzler, IfP \\
\hline & 19.0 & IfP & Marc Jost and & dK.Klemm, Add \\
\hline & 17.1 & IfP & Marc Jost and & dK.Klemm, Add \\
\hline & 16.3 & UWB & M. Goff & A. Irving and $\leqq$ \\
\hline & 11.3 & IfP & Marc Jost and & dK.Klemm, Add \\
\hline & 21.7 & UCLA, PSF & G. and A. Hup & pA. Irving and $\subseteq$ \\
\hline & 10.1 & IfP & Sergey Vassili & liKerstin Klemn unknown \\
\hline & 9 & UWB & J. Higgins & A. Irving and $\leqq$ \\
\hline & 7 & MNB & Philippe Schm & rA. Greshake, I \\
\hline & 9.5 & UWB & DPitt & A. Irving and $\leqq$ \\
\hline & 5.5 & IfP & Anonymous & K. Metzler, IfP \\
\hline & 1.5 & MNB & Vincent Gira & A. Greshake, I \\
\hline & 17.3 & UNM & Matthew Strez & ¿C. Agee, UNN \\
\hline 4.69 & 21.10 & CEREGE & Kuntz & J. Gattacceca: \\
\hline & 22.3 & UNM & Darryl Pitt & C. Agee, UNN \\
\hline 4 & 388.300 & JSC & JSC & SI ANSMET \\
\hline 3.87 & 226.400 & JSC & JSC & $\begin{array}{ll}\text { SI } & \text { ANSMET }\end{array}$ \\
\hline & 53.0 & UWB & B. Hoefnagels & sA. Irving and $\subseteq$ \\
\hline & 42 & UWB & A. Aaronson & A. Irving \& P. I \\
\hline & 20.66 & UNM & Abdelhadi Aitr & rC. Agee, UNN \\
\hline & 20.1 & MNB & Stefan Ralew & A. Greshake, I \\
\hline & 22.4 & MNB & Philippe Schm & rA. Greshake, I \\
\hline & 24 & UWB & M. Jost & A. Irving and $\leqq$ \\
\hline & 24.5 & UWB & C. Zlimen & A. Irving and $\leqslant$ \\
\hline 3.91 & 28 & App & PARI & A. Love, App Unknown \\
\hline & 20 & IfP, Münster & Nourddine Azı & K. Metzler, IfP \\
\hline 4.39 & 23.00 & CEREGE & Labenne Luc & L. Krämer Ruş \\
\hline & 21.7 & NHMV & A. Pani & F. Brandstätte \\
\hline 4.35 & 42.38 & App & Blaine Reed & A. Love, App Anonymous \\
\hline & 21.82 & UWB & FKuntz & A. Irving and $\leqq$ \\
\hline & 21.2 & UNM & Darryl Pitt & C. Agee, UNN \\
\hline & 20.2 & MNB & Stefan Ralew & A. Greshake, I \\
\hline 3.91 & 22.64 & CEREGE & Kuntz & J. Gattacceca: \\
\hline & 21.62 & UWB & F. Kuntz & A. Irving and $\leqslant$ \\
\hline
\end{tabular}




\section{0}

20.4

14.6

14.4

13.8

15.8

11.9

11

9.8

31.379

6.2

28

5.8

21.468

11.258

7.492

6.87

1.959

20.2

36

24.8

21.6

24

20.6

20.1

20

3.32

30.9

143.03

134.75

21

2.52

94.864

18.1

16.32

61.05

10

42.72

34.652

5.9

17.899

16

12.414

11.689

3.136

21.1

5.24

MNB

MNB

Kiel

PSF

MNB

UWB

MNB

IfP

MNB

NIPR

MNB

ROM

MNB

JSC

JSC

JSC

PSF

NIPR

UWB

PMO

UWB

UWB

MNB

MNB

UNM

App

CEREGE

NIPR

NIPR

UPC

JSC

UWB

UWB

NIPR

MNB

NIPR

NIPR

MNB

NIPR

NIPR

NIPR

NIPR

NIPR

UWB
Martin Goff A. Greshake, I

Stefan Ralew A. Greshake, I

Li Bofang, BeiR. Bartoschen anonymous
A. Hupé
A. Irving and $\subseteq$

Philippe Schrr A. Greshake, I

DPitt

A. Irving and $\leqslant$

Harald StehlikA. Greshake, I

Anonymous K. Metzler, IfP

Stefan Ralew A. Greshake, I
NIPR
A. Yamaguchi

Stefan Ralew A. Greshake, I
ROM
A. Irving and $\leqslant$

Hanno Strufe A. Greshake, I

$\begin{array}{lll}\text { JSC } & \text { SI } & \text { ANSMET } \\ \text { JSC } & \text { SI } & \text { ANSMET } \\ \text { JSC } & \text { SI } & \text { ANSMET }\end{array}$

PSF A. Irving and $\leqslant$

NIPR A. Yamaguchi

Aaronson A. Irving \& P. I

Liu Yukun Wu Y., PMO

T. Boudreaux/A. Irving and $F$

J. Higgins A. Irving and $s$

Hanno Strufe A. Greshake, I

Marcin CimaleA. Greshake, I

MMGM C. Agee, UNN

Steve Arnold A. Love, App Unknown

R. Lenssen J. Gattacceca:

NIPR A. Yamaguchi

NIPR A. Yamaguchi

David Allepuz J. Llorca, UPCDavid Allepuz. JSC SI ANSMET

C. Zlimen A. Irving and $F$

F. Olsen/D. BíA. Irving and $\leqslant$

NIPR A. Yamaguchi

Stefan Ralew A. Greshake, I

NIPR A. Yamaguchi

NIPR A. Yamaguchi

Philippe SchrrA. Greshake, I

NIPR A. Yamaguchi

NIPR A. Yamaguchi

NIPR A. Yamaguchi

NIPR A. Yamaguchi

NIPR A. Yamaguchi

C. Colvin A. Irving and $\leqslant$

CEREGE ArtMet collectiJ. Gattacceca: anonymous 


\begin{tabular}{|c|c|c|c|c|}
\hline & 14.084 & NIPR & NIPR & A. Yamaguchi \\
\hline 5.36 & 32 & CEREGE & \multicolumn{2}{|c|}{ ArtMet collectiJ. Gattacceca anonymous } \\
\hline \multirow[t]{4}{*}{5.51} & 44 & CEREGE & \multicolumn{2}{|c|}{ ArtMet collectiJ. Gattacceca anonymous } \\
\hline & 20.3 & MNB & Said Yousfi & A. Greshake, I \\
\hline & 20 & MNB & \multicolumn{2}{|c|}{ Philippe SchrA. Greshake, I } \\
\hline & 185.43 & NIPR & NIPR & A. Yamaguchi \\
\hline \multirow[t]{8}{*}{5.51} & 54.8 & MNA-SI & MNA-SI & M.Salvini, M. ( Jacopo Nava \\
\hline & 19.6 & MSN-FI & Castellano & V. Moggi Cecc \\
\hline & 24 & PMO & Ke Zuokai & Wu Y., PMO \\
\hline & 35.6 & App & PARI & A. Love, App Unknown \\
\hline & 35.1 & GUT & GUT & B. Miao, L.Hui \\
\hline & 1220 & KOPRI & KOPRI & C. Park, S. Y. H. Yoo \\
\hline & 1121 & KOPRI & KOPRI & C. Park, S. Y. S. Han \\
\hline & 26.7 & UCLA & N. Gessler & P. Warren, UC \\
\hline \multirow[t]{31}{*}{2.88} & 20.0 & Kiel & Anonymous & R. Bartoschen Anonymous \\
\hline & 20 & App & PARI & A. Love, App Unknown \\
\hline & 440 & SQU & SQU & P. Hill, N. Ban \\
\hline & 20.7 & MNB & \multicolumn{2}{|c|}{ Philippe SchrA. Greshake, I } \\
\hline & 22.11 & ROM & Gregory & V. Di Cecco, F \\
\hline & 21.9 & MNB & \multicolumn{2}{|c|}{ Abdeltif Mech:A. Greshake, I } \\
\hline & 20 & MNB & \multicolumn{2}{|c|}{ Hichame Mim:A. Greshake, I } \\
\hline & 29.3 & MNB & \multicolumn{2}{|c|}{ Philippe SchmA. Greshake, I } \\
\hline & 20.3 & MNB & \multicolumn{2}{|c|}{ Vincent Gira A. Greshake, I } \\
\hline & 206.1 & NIPR & NIPR & A. Yamaguchi \\
\hline & 20.2 & MSN-FI & Cavaglià & V. Moggi Cecc \\
\hline & 157.8 & Cascadia & Cascadia & M. Hutson, A. \\
\hline & 22 & MNB & \multicolumn{2}{|c|}{ Hanno Strufe A. Greshake, I } \\
\hline & 20.0 & App & PARI & A. Love, App Unknown \\
\hline & 21.5 & Cascadia & \multicolumn{2}{|c|}{ Bastian BaeckM. Hutson, A. } \\
\hline & 34.6 & GUT & GUT & B. Miao, L.Hui \\
\hline & 21.8 & GUT & GUT & B. Miao, C. Zh \\
\hline & 75.47 & NIPR & NIPR & A. Yamaguchi \\
\hline & 16.2 & GUT & GUT & B. Miao, L. Hu \\
\hline & 25.3 & GUT & GUT & B. Miao, L.Hui \\
\hline & 14.2 & MNB & \multicolumn{2}{|c|}{ Harald StehlikA. Greshake, I } \\
\hline & 14.3 & MNB & \multicolumn{2}{|c|}{ Hanno Strufe A. Greshake, I } \\
\hline & 20 & MLP & \multicolumn{2}{|c|}{ MMG-CordobiJ. Roszjar, NFProf. Juan Ols } \\
\hline & 12.1 & MNB & \multicolumn{2}{|c|}{ Hichame Mim:A. Greshake, I } \\
\hline & 59.25 & NIPR & NIPR & A. Yamaguchi \\
\hline & 55.82 & NIPR & NIPR & A. Yamaguchi \\
\hline & 16.9 & GUT & GUT & B. Miao, L. Hu \\
\hline & 49.815 & NIPR & NIPR & A. Yamaguchi \\
\hline & 12.1 & GUT & GUT & B. Miao, L. Hu \\
\hline & 38.049 & NIPR & NIPR & A. Yamaguchi \\
\hline & 8 & IfP, $M \tilde{A}^{1} 1 / 4 r$ & Anonymous & K. Metzler, IfP \\
\hline
\end{tabular}




\begin{tabular}{|c|c|c|c|}
\hline 11.1 & GUT & GUT & B. Miao, L.Hui \\
\hline 6.7 & ROM & D. Gregory & L. F. White an \\
\hline 29.962 & NIPR & NIPR & A. Yamaguchi \\
\hline 6.1 & ROM & D. Gregory & L. F. White an \\
\hline 8.9 & GUT & GUT & B. Miao, L. Hu \\
\hline 21.33 & NIPR & NIPR & A. Yamaguchi \\
\hline 4.6 & MNB & \multicolumn{2}{|c|}{ Hichame Mim:A. Greshake, I } \\
\hline 18.323 & NIPR & NIPR & A. Yamaguchi \\
\hline 4.2 & GUT & GUT & B. Miao, L.Hui \\
\hline 10.8 & GUT & GUT & B. Miao, L. Hu \\
\hline 8.4 & GUT & GUT & B. Miao, L.Hui \\
\hline 12.921 & NIPR & NIPR & A. Yamaguchi \\
\hline 10.557 & NIPR & NIPR & A. Yamaguchi \\
\hline 10.53 & NIPR & NIPR & A. Yamaguchi \\
\hline 4.6 & GUT & GUT & B. Miao, L. Hu \\
\hline 6.919 & NIPR & NIPR & A. Yamaguchi \\
\hline 2.369 & NIPR & NIPR & A. Yamaguchi \\
\hline 1.936 & NIPR & NIPR & A. Yamaguchi \\
\hline 1.298 & NIPR & NIPR & A. Yamaguchi \\
\hline 1.219 & NIPR & NIPR & A. Yamaguchi \\
\hline 0.425 & NIPR & NIPR & A. Yamaguchi \\
\hline 0.4 & KOPRI & KOPRI & C. Park, S. Y. T. Kim \\
\hline 22.5 & UWB & R. Falls & A. Irving and $₫$ \\
\hline 20.1 & UWB & Hmani Inc. & A. Irving and $\subseteq$ \\
\hline 24.8 & UWB & S. Vasiliev & A. Irving and $\leqq$ \\
\hline 22 & UWB & G. Fujihara & A. Irving and $\leqq$ \\
\hline 20.3 & MNB & \multicolumn{2}{|c|}{ Marcin CimalEA. Greshake, I } \\
\hline 8.4 & MNB & \multicolumn{2}{|c|}{ Stefan Ralew A. Greshake, I } \\
\hline 4.2 & MNB & \multicolumn{2}{|c|}{ Jürgen NaubeA. Greshake, I } \\
\hline 2.8 & MNB & \multicolumn{2}{|c|}{ Stefan Ralew A. Greshake, I } \\
\hline 20 & UCLA & N. Gessler & P. Warren, UC \\
\hline 24.5 & UNM & \multicolumn{2}{|c|}{ Mohamed AidC. Agee, UNN } \\
\hline 20.6 & UWB & \multicolumn{2}{|c|}{ A. Aaronson A. Irving and $\subseteq$} \\
\hline 20 & MNB & \multicolumn{2}{|c|}{ Harald StehlikA. Greshake, I } \\
\hline 20 & UNM & Jay Piatek & C. Agee, UNN \\
\hline 24.4 & MNB & \multicolumn{2}{|c|}{ Marcin CimalєA. Greshake, I } \\
\hline 20 & IfP, Münster & \multicolumn{2}{|c|}{ Nourddine AzıK. Metzler, IfP } \\
\hline 20 & \multicolumn{2}{|c|}{ IfP, MÃ¹/4nster Anonymous } & K. Metzler, IfP \\
\hline 20.2 & UWB & MMGM & A. Irving and $s$ \\
\hline 23.1 & ROM & D. Schultz & T. Bunch and \\
\hline 25.5 & UWB & R. Falls & A. Irving and $s$ \\
\hline 46.5 & UWB & J. Phillips & A. Irving and $\leqq$ \\
\hline 25.5 & UWB & R. Falls & A. Irving and $\subseteq$ \\
\hline 22 & UNM & Jay Piatek & C. Agee, UNN \\
\hline 20 & UPC & \multicolumn{2}{|c|}{ Alfonso Viera J. Llorca, UPC Alfonso Viera } \\
\hline
\end{tabular}




\begin{tabular}{|c|c|c|c|c|c|}
\hline \multirow[t]{2}{*}{3.69} & 21 & CEREGE & \multicolumn{3}{|c|}{ Sergey VasilieJ. Gattacceca:Sergey Vasilie } \\
\hline & 26.6 & UWB & J. Higgins & A. Irving and $\subseteq$ & \\
\hline \multirow[t]{13}{*}{3.44} & 41.5 & App & J. Sinclair & A. Love, App & Unknown \\
\hline & 25.8 & UWB & R. Falls & A. Irving and $\leqslant$ & \\
\hline & 20.8 & UWO & R. Watts & Z.G. Guo, UW & \\
\hline & 20 & App & Steve Arnold & A. Love, App & Unknown \\
\hline & 21.1 & UWB & D. Pitt & A. Irving and $\leqslant$ & \\
\hline & 20 & IfP, Münster & \multicolumn{3}{|c|}{ Nourddine AzıK. Metzler, IfP } \\
\hline & 21.9 & UNM & Darryl Pitt & \multicolumn{2}{|l|}{ C. Agee, UNN } \\
\hline & 210 & RBINS & RBINS & \multicolumn{2}{|c|}{ H. PourkhorsaEudore Schmi } \\
\hline & 20 & IfP & Anonymous & \multicolumn{2}{|l|}{ K. Metzler, IfP } \\
\hline & 20.1 & UWB & DPitt & \multicolumn{2}{|l|}{ A. Irving and $\subseteq$} \\
\hline & 20 & IfP, Münster & \multicolumn{3}{|c|}{ Nourddine AzıK. Metzler, IfP } \\
\hline & 21.2 & MNB & \multicolumn{3}{|c|}{ Hanno Strufe A. Greshake, I } \\
\hline & 21.99 & ASU & \multicolumn{3}{|c|}{ Michael FarmıS. Dillon, E. D Anonymous } \\
\hline 2.69 & 133.858 & JSC & JSC & SI & ANSMET \\
\hline \multirow[t]{4}{*}{2.65} & 131.112 & JSC & JSC & SI & ANSMET \\
\hline & 20.4 & UNM & DPitt & \multicolumn{2}{|l|}{ C. Agee, UNN } \\
\hline & 19 & MNB & \multicolumn{3}{|c|}{ Franco VignatA. Greshake, I } \\
\hline & 15 & IfP, Münster & anonymous & \multicolumn{2}{|c|}{ K. Metzler, IfP } \\
\hline \multirow[t]{2}{*}{2.72} & 61.353 & JSC & JSC & SI & ANSMET \\
\hline & 12.5 & UNM & \multicolumn{3}{|c|}{ Darryl Pitt $\quad$ C. Agee, UNN } \\
\hline 2.81 & 47.300 & JSC & JSC & SI & ANSMET \\
\hline \multirow[t]{2}{*}{2.67} & 40.372 & JSC & JSC & SI & ANSMET \\
\hline & 8.6 & MNB & \multicolumn{3}{|c|}{ Jürgen NaubeA. Greshake, I } \\
\hline 2.02 & 26.701 & JSC & JSC & SI & ANSMET \\
\hline 2.71 & 25.324 & JSC & JSC & SI & ANSMET \\
\hline \multirow[t]{2}{*}{3.62} & 23.531 & JSC & JSC & $\mathrm{SI}$ & ANSMET \\
\hline & 6.7 & MNB & \multicolumn{3}{|c|}{ Franco VignatA. Greshake, I } \\
\hline 3.56 & 4.4 & FSAC & \multicolumn{3}{|c|}{ Private collect $\mathrm{H}$. Chennaoui Tarik Hezzi (E } \\
\hline \multirow[t]{3}{*}{2.89} & 8.269 & JSC & JSC & SI & ANSMET \\
\hline & 21 & UNM & Jay Piatek & C. Agee, UNN & \\
\hline & 20.6 & UWB & D. Pitt & A. Irving and $\subseteq$ & \\
\hline 2.64 & 23.9 & CEREGE & \multicolumn{3}{|c|}{ Andreas GrenJ. Gattacceca: } \\
\hline 2.61 & 184.233 & JSC & JSC & SI & ANSMET \\
\hline & 21.1 & UWB & DPitt & A. Irving and $\leqq$ & \\
\hline & 17.1 & UNM & Darryl Pitt & C. Agee, UNN & \\
\hline 2.72 & 64.631 & JSC & JSC & SI & ANSMET \\
\hline 2.64 & 46.365 & JSC & JSC & SI & ANSMET \\
\hline & 8.9 & UNM & Craig Zlimen & C. Agee, UNN & \\
\hline 2.59 & 38.752 & JSC & JSC & $\mathrm{SI}$ & ANSMET \\
\hline & 70.24 & NIPR & NIPR & A. Yamaguchi & \\
\hline & 2.524 & NIPR & NIPR & A. Yamaguchi & \\
\hline & 16.79 & UCLA & Sean Tutoron & A. Rubin, UCL & \\
\hline 4.74 & 24.3 & Kiel & Dieter Janek, & Bart & anonymous \\
\hline
\end{tabular}


F. Vignato, Ve Bart

anonymous

5.14

21

4.86

12

5.182

0.7

\section{Kiel}

Kiel

Kiel

Kiel

Kiel

Kiel

UCLA

OU

MNB

MNB

UCLA

Vernad

MNB

KOPRI

UCLA

NIPR

MNB

IfP

NIPR

UNM

NIPR

KOPRI

PSF

PSF

NIPR

KOPRI

IfP

\section{Cascadia}

Cascadia

Cascadia

Cascadia

CEREGE

Cascadia
Dieter Janek, Bart nomad group

Xu Jianjiang aR. Bartoscheu Xu Jianjiang

Li Bofang, BeiR. Bartoschen anonymous Wang Ziyao R. Bartoschen anonymous W. Kalisch $(B \in R$. Bartoschen anonymous anonymous Bart anonymous Li Bofang, BeiR. Bartoschen anonymous Wang Ziyao R. BartoscheuBi Chunlin ( $\mathrm{Ta}$ Joseph KaiserR. Bartoschen anonymous Wang Ziyao R. BartoscheuZhao Tailu (St BartoschewitzR. BartoschenBi Chunlin (Ta Wang Ziyao R. Bartoschen anonymous Dieter Janek, Bart anonymous Wang Ziyao R. Bartoschen Wang Ziyao Xu Jianjiang R. Bartoschen Xu Jianjiang Qu Pan, Beijir R. Bartoschen anonymous

A. Mazur Bart anonymous David GregoryA. Rubin, UCL Joe Bazyk R. Greenwooc Joe Bazyk Hanno Strufe A. Greshake, I Dario Ruiz Ansgar Gresh Dario Ruiz Nick Gessler A. Rubin, UCL

DMUH C. A. Lorenz, '

Hichame MimiA. Greshake, I

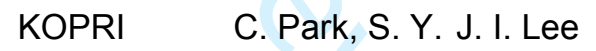

Ken Eltrich A.Rubin, UCL, Ken Eltrich NIPR A. Yamaguchi Hichame Mim:A. Greshake, I Labenne K. Klemm, A. NIPR A. Yamaguchi KD MeteoritesC. Agee, UNN Chris Whitten NIPR

A. Yamaguchi KOPRI C. Park, S. Y. J. I. Lee

PSF

A. Irving and $\leqslant$

PSF

A. Irving and $s$

NIPR

A. Yamaguchi

KOPRI

C. Park, S. Y. T. Kim unknown K. Klemm, A.

Cascadia M. Hutson ancWendy Woods

Cascadia

M. Hutson anc Jim Wooddell

Cascadia

M. Hutson anc Troy Ball

Cascadia

M. Hutson anc Jim Wooddell

Eric Christens J. Gattacceca Eric Christens

Cascadia M. Hutson anc Jim Wooddell 
Cascadia

M. Hutson anc Troy Ball

40.145

Cascadia

Cascadia

JSC

M. Hutson anc Jim Wooddell

3.97

18.830

29.2

20

206

28.3

27.8

5.10

30.3

20.1

53.3

21.88

33

1371.7

1091.74

22.7

5.44

23.78

976.43

136.65

5.42

26.4

587.77

553.74

28.4

23.2

87.2

503.35

223

22

26.4

408.46

406.04

21.94

358.74

5.2

345.91

23.3

29.8

24

280.89

276.91

273.2

4.95

JSC

JSC

\section{UWB}

\section{FMMR}

UWO

$$
\text { IfP }
$$

$$
\text { IfP }
$$

CEREGE

$$
\text { IfP }
$$

UCLA

\section{YPMNH}

UPC

$$
\text { NIPR }
$$

GUT

$\mathrm{FMNH}$

CEREGE

$$
\text { GUT }
$$

UCLA

MNB

$$
\text { NIPR }
$$

GUT

UCLA

$$
\text { UWO }
$$

$$
\text { OU }
$$

\section{NIPR}

GUT

UPC

\section{UCLA}

GUT

NIPR

$$
\text { UCLA }
$$

$$
\text { GUT }
$$

JSC

OU

MSN-FI

UPC

32.8

266.69

250.67

241.17

\section{NIPR}

GUT

\section{GUT}

MNB

NIPR

GUT

GUT
SI

ANSMET

SI

A. Aaronson A. Irving and $\varsigma$

Mikhail NeponPavel Yu. PlecMikhail Nepon

S. Tutorow M. Spencer, U

Finder K. Klemm andNasser Albuai

Labenne K. Klemm, A.

ArtMet collectiJ. Gattacceca anonymous

Finder K. Klemm andNasser Albuai

Michael SantcA. Rubin, UCL

WES K. Abe and J.

Ion Ander LanJ. Llorca, UPClon Ander Lan

NIPR

A. Yamaguchi

GUT

B. Miao, Z. Xic

Robert Ward P. Heck, Jenn Robert Ward

Andreas KoppL. Krämer RuçAndreas Kopp

GUT

B. Miao, Z. Xic

Michael CottinA. Rubin, UCL

Hanno Strufe A. Greshake, I

NIPR

A. Yamaguchi

GUT

B. Miao, Z. Xí

Nick Gessler A. Rubin, UCL

S. Tutorow T.V. Kizovski,

Joe Bazyk R. Greenwooc Joe Bazyk

NIPR

A. Yamaguchi

GUT

H. Chen, J. ZrY.Li, HY.Chen José Vicente (J. Llorca, UPCJosé Vicente ( Nick Gessler A. Rubin, UCL GUT B. Miao, Z. Xí

NIPR A. Yamaguchi

Nick Gessler A. Rubin, UCL

GUT $\quad$ B. Miao, Z. Xis

$\begin{array}{lll}\text { JSC } & \text { SI }\end{array}$

Joe Bazyk R. Greenwooc Joe Bazyk

Di Martino V. Moggi Cecc Anonymous

Alfonso Viera J. Llorca, UPC Alfonso Viera

NIPR

A. Yamaguchi

GUT

B. Miao, Z. Xic

GUT

B. Miao, Z. Xis

Hanno Strufe A. Greshake, I

NIPR

A. Yamaguchi

GUT

B. Miao, Z. Xiá

GUT

B. Miao, Z. Xis 


\begin{tabular}{|c|c|c|c|}
\hline 221.83 & GUT & GUT & B. Miao, Z. Xic \\
\hline 22 & UPC & \multicolumn{2}{|c|}{ José Vicente (J. Llorca, UPCJosé Vicente } \\
\hline 23.8 & MNB & \multicolumn{2}{|c|}{ Philippe SchrrA. Greshake, I } \\
\hline 199.18 & GUT & GUT & B. Miao, Z. Xić \\
\hline 42.53 & UCLA & \multicolumn{2}{|c|}{ Dean Bessey A. Rubin, UCL } \\
\hline 32.0 & MSN-FI & Castellano & Vanni Moggi C \\
\hline 181.04 & GUT & GUT & B. Miao, Z. Xić \\
\hline 176.82 & GUT & GUT & B. Miao, Z. Xiá \\
\hline 30 & UPC & \multicolumn{2}{|c|}{ Ion Ander LanJ. Llorca, UPClon Ander Lar } \\
\hline 167.26 & GUT & GUT & B. Miao, Z. Xić \\
\hline 149.07 & NIPR & NIPR & A. Yamaguchi \\
\hline 21.7 & OU & Joe Bazyk & R. Greenwooc Joe Bazyk \\
\hline 28 & CEREGE & \multicolumn{2}{|c|}{ Eric Christens J. Gattacceca Eric Christens } \\
\hline 138.44 & GUT & GUT & B. Miao, Z. Xić \\
\hline 135.64 & NIPR & NIPR & A. Yamaguchi \\
\hline 131.65 & GUT & GUT & B. Miao, Z. Xić \\
\hline 32.4 & Vernad & \multicolumn{2}{|c|}{ Mr. M. NepomLorenz C. A., 'Mr. M. Nepom } \\
\hline 21.64 & UCLA & \multicolumn{2}{|c|}{ Dean Bessey A. Rubin, UCL } \\
\hline 125.53 & GUT & GUT & B. Miao, Z. Xí́ \\
\hline 20.1 & MSN-FI & Mimaghador & Vanni Moggi C \\
\hline 119.55 & NIPR & NIPR & A. Yamaguchi \\
\hline 119.31 & GUT & GUT & B. Miao, Z. Xí̌ \\
\hline 118 & GUT & GUT & B. Miao, Z. Xić \\
\hline 112.42 & NIPR & NIPR & A. Yamaguchi \\
\hline 9.7 & IfP & Labenne & K. Klemm, A. \\
\hline 105.84 & JSC & JSC & SI ANSMET \\
\hline 105.71 & GUT & GUT & B. Miao, Z. Xiá \\
\hline 103.96 & NIPR & NIPR & A. Yamaguchi \\
\hline 96.96 & GUT & GUT & B. Miao, Z. Xié \\
\hline 96.67 & NIPR & NIPR & A. Yamaguchi \\
\hline 19.2 & MSN-FI & Castellano & V. Moggi Cecc \\
\hline 94.22 & GUT & GUT & B. Miao, Z. Xić \\
\hline 91.49 & NIPR & NIPR & A. Yamaguchi \\
\hline 17.7 & MSN-FI & Castellano & V. Moggi Cecr \\
\hline 87.3 & GUT & GUT & B. Miao, Z. Xič \\
\hline 82.589 & JSC & JSC & SI $\quad$ ANSMET \\
\hline 80.38 & GUT & GUT & B. Miao, Z. Xić \\
\hline 16 & OU & Joe Bazyk & R. Greenwooc Joe Bazyk \\
\hline 78.44 & GUT & GUT & B. Miao, Z. Xiš \\
\hline 77.77 & NIPR & NIPR & A. Yamaguchi \\
\hline 76.87 & GUT & GUT & B. Miao, Z. Xić \\
\hline 75.87 & GUT & GUT & B. Miao, Z. Xić \\
\hline 71.75 & NIPR & NIPR & A. Yamaguchi \\
\hline 62.75 & NIPR & NIPR & A. Yamaguchi \\
\hline 62.58 & NIPR & NIPR & A. Yamaguchi \\
\hline
\end{tabular}




$\begin{array}{llll}62.42 & \text { NIPR } & \text { NIPR } & \text { A. Yamaguchi } \\ 20 & \text { UCLA } & \text { Dean Bessey A. Rubin, UCL } \\ 56.58 & \text { NIPR } & \text { NIPR } & \text { A. Yamaguchi } \\ 56.162 & \text { NIPR } & \text { NIPR } & \text { A. Yamaguchi } \\ 55.04 & \text { GUT } & \text { GUT } & \text { B. Miao, Z. Xic } \\ 51.125 & \text { NIPR } & \text { NIPR } & \text { A. Yamaguchi } \\ 49.567 & \text { NIPR } & \text { NIPR } & \text { A. Yamaguchi } \\ 48.987 & \text { NIPR } & \text { NIPR } & \text { A. Yamaguchi } \\ 10.2 & \text { ROM } & \text { Gregory } & \text { S. Yokoyama, } \\ 46.912 & \text { NIPR } & \text { NIPR } & \text { A. Yamaguchi } \\ 46.292 & \text { NIPR } & \text { NIPR } & \text { A. Yamaguchi } \\ 12.4 & \text { MSN-FI } & \text { Cojocaru } & \text { V. Moggi Cece } \\ 45.06 & \text { GUT } & \text { GUT } & \text { B. Miao, Z. Xic } \\ 42.75 & \text { GUT } & \text { GUT } & \text { B. Miao, Z. Xic } \\ 41.447 & \text { NIPR } & \text { NIPR } & \text { A. Yamaguchi } \\ 39.28 & \text { GUT } & \text { GUT } & \text { B. Miao, Z. Xic } \\ 39.052 & \text { NIPR } & \text { NIPR } & \text { A. Yamaguchi } \\ 38.45 & \text { GUT } & \text { GUT } & \text { B. Miao, Z. Xic } \\ 38.235 & \text { NIPR } & \text { NIPR } & \text { A. Yamaguchi } \\ 38.202 & \text { NIPR } & \text { NIPR } & \text { A. Yamaguchi } \\ 36.72 & \text { JSC } & \text { JSC } & \text { SI } ~\end{array}$




\begin{tabular}{|c|c|c|c|}
\hline 5.827 & NIPR & NIPR & A. Yamaguchi \\
\hline 5.766 & NIPR & NIPR & A. Yamaguchi \\
\hline 5.727 & NIPR & NIPR & A. Yamaguchi \\
\hline 5.687 & NIPR & NIPR & A. Yamaguchi \\
\hline 5.64 & NIPR & NIPR & A. Yamaguchi \\
\hline 5.529 & NIPR & NIPR & A. Yamaguchi \\
\hline 5.486 & NIPR & NIPR & A. Yamaguchi \\
\hline 5.394 & NIPR & NIPR & A. Yamaguchi \\
\hline 5.37 & NIPR & NIPR & A. Yamaguchi \\
\hline 5.337 & NIPR & NIPR & A. Yamaguchi \\
\hline 5.259 & NIPR & NIPR & A. Yamaguchi \\
\hline 5.21 & NIPR & NIPR & A. Yamaguchi \\
\hline 5.095 & NIPR & NIPR & A. Yamaguchi \\
\hline 5.012 & NIPR & NIPR & A. Yamaguchi \\
\hline $4.94 \mathrm{~g}$ & NHMV & AUA & Ioannis Baziot \\
\hline 4.993 & NIPR & NIPR & A. Yamaguchi \\
\hline 4.981 & NIPR & NIPR & A. Yamaguchi \\
\hline 4.888 & NIPR & NIPR & A. Yamaguchi \\
\hline 4.884 & NIPR & NIPR & A. Yamaguchi \\
\hline 4.788 & NIPR & NIPR & A. Yamaguchi \\
\hline 4.758 & NIPR & NIPR & A. Yamaguchi \\
\hline 4.676 & NIPR & NIPR & A. Yamaguchi \\
\hline 4.606 & NIPR & NIPR & A. Yamaguchi \\
\hline 4.53 & NIPR & NIPR & A. Yamaguchi \\
\hline 4.438 & NIPR & NIPR & A. Yamaguchi \\
\hline 4.433 & NIPR & NIPR & A. Yamaguchi \\
\hline 4.425 & NIPR & NIPR & A. Yamaguchi \\
\hline 4.386 & NIPR & NIPR & A. Yamaguchi \\
\hline 4.36 & NIPR & NIPR & A. Yamaguchi \\
\hline 4.353 & NIPR & NIPR & A. Yamaguchi \\
\hline 4.297 & NIPR & NIPR & A. Yamaguchi \\
\hline 4.257 & NIPR & NIPR & A. Yamaguchi \\
\hline 4.25 & NIPR & NIPR & A. Yamaguchi \\
\hline 4.245 & NIPR & NIPR & A. Yamaguchi \\
\hline 4.243 & NIPR & NIPR & A. Yamaguchi \\
\hline 4.096 & NIPR & NIPR & A. Yamaguchi \\
\hline 4.057 & NIPR & NIPR & A. Yamaguchi \\
\hline 3.983 & NIPR & NIPR & A. Yamaguchi \\
\hline 3.938 & NIPR & NIPR & A. Yamaguchi \\
\hline 3.844 & NIPR & NIPR & A. Yamaguchi \\
\hline 3.74 & NIPR & NIPR & A. Yamaguchi \\
\hline 3.739 & NIPR & NIPR & A. Yamaguchi \\
\hline 3.721 & NIPR & NIPR & A. Yamaguchi \\
\hline 3.706 & NIPR & NIPR & A. Yamaguchi \\
\hline 3.688 & NIPR & NIPR & A. Yamaguchi \\
\hline
\end{tabular}




\begin{tabular}{|c|c|c|c|}
\hline 3.646 & NIPR & NIPR & A. Yamaguchi \\
\hline 3.642 & NIPR & NIPR & A. Yamaguchi \\
\hline 3.641 & NIPR & NIPR & A. Yamaguchi \\
\hline 3.615 & NIPR & NIPR & A. Yamaguchi \\
\hline 3.606 & NIPR & NIPR & A. Yamaguchi \\
\hline 3.602 & NIPR & NIPR & A. Yamaguchi \\
\hline 3.543 & NIPR & NIPR & A. Yamaguchi \\
\hline 3.514 & NIPR & NIPR & A. Yamaguchi \\
\hline 3.499 & NIPR & NIPR & A. Yamaguchi \\
\hline 3.465 & NIPR & NIPR & A. Yamaguchi \\
\hline 3.46 & NIPR & NIPR & A. Yamaguchi \\
\hline 3.455 & NIPR & NIPR & A. Yamaguchi \\
\hline 3.454 & NIPR & NIPR & A. Yamaguchi \\
\hline 3.401 & NIPR & NIPR & A. Yamaguchi \\
\hline 3.4 & NIPR & NIPR & A. Yamaguchi \\
\hline 3.394 & NIPR & NIPR & A. Yamaguchi \\
\hline 3.309 & NIPR & NIPR & A. Yamaguchi \\
\hline 3.283 & NIPR & NIPR & A. Yamaguchi \\
\hline 3.245 & NIPR & NIPR & A. Yamaguchi \\
\hline 3.205 & NIPR & NIPR & A. Yamaguchi \\
\hline 3.204 & NIPR & NIPR & A. Yamaguchi \\
\hline 3.203 & NIPR & NIPR & A. Yamaguchi \\
\hline 3.189 & NIPR & NIPR & A. Yamaguchi \\
\hline 3.188 & NIPR & NIPR & A. Yamaguchi \\
\hline 3.181 & NIPR & NIPR & A. Yamaguchi \\
\hline 3.176 & NIPR & NIPR & A. Yamaguchi \\
\hline 3.146 & NIPR & NIPR & A. Yamaguchi \\
\hline 3.136 & NIPR & NIPR & A. Yamaguchi \\
\hline 3.123 & NIPR & NIPR & A. Yamaguchi \\
\hline 3.121 & NIPR & NIPR & A. Yamaguchi \\
\hline 3.091 & NIPR & NIPR & A. Yamaguchi \\
\hline 3.088 & NIPR & NIPR & A. Yamaguchi \\
\hline 3.083 & NIPR & NIPR & A. Yamaguchi \\
\hline 3.051 & NIPR & NIPR & A. Yamaguchi \\
\hline 3.033 & NIPR & NIPR & A. Yamaguchi \\
\hline 3.009 & NIPR & NIPR & A. Yamaguchi \\
\hline 2.921 & NIPR & NIPR & A. Yamaguchi \\
\hline 2.916 & NIPR & NIPR & A. Yamaguchi \\
\hline 2.906 & NIPR & NIPR & A. Yamaguchi \\
\hline 2.856 & NIPR & NIPR & A. Yamaguchi \\
\hline 2.832 & NIPR & NIPR & A. Yamaguchi \\
\hline 2.799 & NIPR & NIPR & A. Yamaguchi \\
\hline 2.797 & NIPR & NIPR & A. Yamaguchi \\
\hline 2.759 & NIPR & NIPR & A. Yamaguchi \\
\hline 2.748 & NIPR & NIPR & A. Yamaguchi \\
\hline
\end{tabular}




\begin{tabular}{|c|c|c|c|}
\hline 2.731 & NIPR & NIPR & A. Yamaguchi \\
\hline 2.634 & NIPR & NIPR & A. Yamaguchi \\
\hline 2.594 & NIPR & NIPR & A. Yamaguchi \\
\hline 2.558 & NIPR & NIPR & A. Yamaguchi \\
\hline 2.506 & NIPR & NIPR & A. Yamaguchi \\
\hline 2.496 & NIPR & NIPR & A. Yamaguchi \\
\hline 2.481 & NIPR & NIPR & A. Yamaguchi \\
\hline 2.445 & NIPR & NIPR & A. Yamaguchi \\
\hline 2.432 & NIPR & NIPR & A. Yamaguchi \\
\hline 2.253 & NIPR & NIPR & A. Yamaguchi \\
\hline 2.163 & NIPR & NIPR & A. Yamaguchi \\
\hline 2.085 & NIPR & NIPR & A. Yamaguchi \\
\hline 2.063 & NIPR & NIPR & A. Yamaguchi \\
\hline 1.946 & NIPR & NIPR & A. Yamaguchi \\
\hline 1.94 & NIPR & NIPR & A. Yamaguchi \\
\hline 1.932 & NIPR & NIPR & A. Yamaguchi \\
\hline 1.927 & NIPR & NIPR & A. Yamaguchi \\
\hline 1.891 & NIPR & NIPR & A. Yamaguchi \\
\hline 1.794 & NIPR & NIPR & A. Yamaguchi \\
\hline 1.702 & NIPR & NIPR & A. Yamaguchi \\
\hline 1.692 & NIPR & NIPR & A. Yamaguchi \\
\hline 1.672 & NIPR & NIPR & A. Yamaguchi \\
\hline 1.652 & NIPR & NIPR & A. Yamaguchi \\
\hline 1.632 & NIPR & NIPR & A. Yamaguchi \\
\hline 1.616 & NIPR & NIPR & A. Yamaguchi \\
\hline 1.559 & NIPR & NIPR & A. Yamaguchi \\
\hline 1.507 & NIPR & NIPR & A. Yamaguchi \\
\hline 1.497 & NIPR & NIPR & A. Yamaguchi \\
\hline 1.494 & NIPR & NIPR & A. Yamaguchi \\
\hline 1.436 & NIPR & NIPR & A. Yamaguchi \\
\hline 1.374 & NIPR & NIPR & A. Yamaguchi \\
\hline 1.366 & NIPR & NIPR & A. Yamaguchi \\
\hline 1.317 & NIPR & NIPR & A. Yamaguchi \\
\hline 1.263 & NIPR & NIPR & A. Yamaguchi \\
\hline 1.167 & NIPR & NIPR & A. Yamaguchi \\
\hline 1.165 & NIPR & NIPR & A. Yamaguchi \\
\hline 1.09 & NIPR & NIPR & A. Yamaguchi \\
\hline 1.075 & NIPR & NIPR & A. Yamaguchi \\
\hline 1.044 & NIPR & NIPR & A. Yamaguchi \\
\hline 1.02 & NIPR & NIPR & A. Yamaguchi \\
\hline 0.88 & NIPR & NIPR & A. Yamaguchi \\
\hline 0.857 & NIPR & NIPR & A. Yamaguchi \\
\hline 0.85 & JSC & JSC & SI \\
\hline 0.847 & NIPR & NIPR & A. Yamaguchi \\
\hline 0.813 & NIPR & NIPR & A. Yamaguchi \\
\hline
\end{tabular}




\begin{tabular}{|c|c|c|c|}
\hline 0.705 & NIPR & NIPR & A. Yamaguchi \\
\hline 0.681 & NIPR & NIPR & A. Yamaguchi \\
\hline 0.656 & NIPR & NIPR & A. Yamaguchi \\
\hline 0.605 & NIPR & NIPR & A. Yamaguchi \\
\hline 0.525 & NIPR & NIPR & A. Yamaguchi \\
\hline 0.516 & NIPR & NIPR & A. Yamaguchi \\
\hline 0.511 & NIPR & NIPR & A. Yamaguchi \\
\hline 0.461 & NIPR & NIPR & A. Yamaguchi \\
\hline 0.438 & NIPR & NIPR & A. Yamaguchi \\
\hline 0.422 & NIPR & NIPR & A. Yamaguchi \\
\hline 0.417 & NIPR & NIPR & A. Yamaguchi \\
\hline 0.399 & NIPR & NIPR & A. Yamaguchi \\
\hline 0.386 & NIPR & NIPR & A. Yamaguchi \\
\hline 0.375 & NIPR & NIPR & A. Yamaguchi \\
\hline 0.338 & NIPR & NIPR & A. Yamaguchi \\
\hline 0.308 & NIPR & NIPR & A. Yamaguchi \\
\hline 0.306 & NIPR & NIPR & A. Yamaguchi \\
\hline 0.225 & NIPR & NIPR & A. Yamaguchi \\
\hline 34.8 & IGc/USP & \multicolumn{2}{|c|}{ Andre L. R. M M.E. ZucolottcI. S. Lima and } \\
\hline 52.553 & NIPR & NIPR & A. Yamaguchi \\
\hline 28.89 & UCLA & Sean Tutorov & NA. Rubin, UCL \\
\hline 26.7 & UWB & R. Falls & A. Irving and $\subseteq$ \\
\hline 24.8 & IfP & Finder & K. Klemm andNasser Albuai \\
\hline 36.2 & IfP & Finder & K. Klemm andNasser Albuai \\
\hline 39 & UPC & \multicolumn{2}{|c|}{ José Vicente (J. Llorca, UPCJosé Vicente ( } \\
\hline 23 & UPC & \multicolumn{2}{|c|}{ David Allepuz J. Llorca, UPCDavid Allepuz. } \\
\hline 24.8 & CEREGE & \multicolumn{2}{|c|}{ ArtMet collectiJ. Gattacceca: anonymous } \\
\hline 27 & UPC & \multicolumn{2}{|c|}{ José Vicente (J. Llorca, UPCJosé Vicente ( } \\
\hline 25 & UPC & \multicolumn{2}{|c|}{ José Vicente (J. Llorca, UPCJosé Vicente ( } \\
\hline 25 & UPC & \multicolumn{2}{|c|}{ Ion Ander LanJ. Llorca, UPClon Ander Lan } \\
\hline 25.6 & IfP & \multicolumn{2}{|c|}{ Marc Jost andK. Klemm and } \\
\hline 30.38 & PSF & PSF & A. Irving and $\subseteq$ \\
\hline 50 & UPC & \multicolumn{2}{|c|}{ José Vicente (J. Llorca, UPCJosé Vicente ( } \\
\hline 36700 & KOPRI & KOPRI & C. Park, S. Y. T. Kim \\
\hline 83.8 & CEREGE & \multicolumn{2}{|c|}{ ArtMet collectiJ. Gattacceca: anonymous } \\
\hline 15900 & Vic & Vic & W. Birch, Vic; David Cole \\
\hline 11200 & KOPRI & KOPRI & C. Park, S. Y. J. I. Lee \\
\hline 44.4 & CEREGE & \multicolumn{2}{|c|}{ ArtMet collectiJ. Gattacceca anonymous } \\
\hline 8205.000 & JSC & JSC & SI $\quad$ ANSMET \\
\hline 165.9 & Vernad & anonymous & Lorenz C. A., 'Mr. Alexander \\
\hline 71.8 & UAb & M. Ouzillou & C. Herd and L \\
\hline 21.1 & MSN-FI & Gal Hassin & V. Moggi Cecc \\
\hline 25 & UNM & Aaronson & C. Agee, UNN \\
\hline 122.1 & UWB & S. Lee & A. Irving and $\leqq$ \\
\hline 57.2 & MSN-FI & Tomelleri & V. Moggi CecrGiorgio Tomel \\
\hline
\end{tabular}




\subsection{1}

3463

36.8

20.6

5.47

25

90

20.5

191.7

23.6

54.0

32.90

5.06

62.6

5.40

922.7

\section{4}

120

21.0

677.79

21

\subsection{8}

649.57

4.90

36

34

38.6

612.5

20

5.21

557.700

510.99

103.17

32.2

26.7

20

463

37

24.0

439.34

60.6

427.83

30

422.78

399.41

45.9

393.4

91

23.2

65

5.04

22

20.1

\section{HMG}

CEREGE

UWB

CEREGE

IGGCAS

UWO

Vernad.

\section{MNB}

UWO

UCLA

MNB

NIPR

CEREGE

USil

MSN-FI

GUT

UPC

GUT

CEREGE

CEREGE

UCLA

NIPR

UNM

JSC

GUT

Vernad.

$$
\text { UWO }
$$

IfP

\section{UCLA}

\section{KOPRI}

UPC

MSN-FI

\section{GUT}

Vernad

NIPR

UPC

GUT

GUT

OU

NIPR

Vernad

IfP

UPC

CEREGE

MSN-FI
HMG

G. Wang, GIGUnknown

A.Koppelt andL. Krämer RuçA. Koppelt anc

T. Simard

A. Irving and $\subseteq$

Labenne Luc J. Gattacceca: Labenne Luc

Qiang Xu Y. Xu, N. Wan anonymous

S. Tutorow Z. Jiang, UWC

Ilya ChaplyginLorenz C. A., 'llya Chaplygin

anonymous A. Greshake, I

S. Tutorow J. Laughton, L

Michael CottinA. Rubin, UCL

Hichame Mim:A. Greshake, I

NIPR A. Yamaguchi

Labenne Luc J. Gattacceca: Labenne Luc

USil T.A. Przylibski

Castellano V. Moggi Cecc

GUT

B. Miao, Z. Xic

Alfonso Viera J. Llorca, UPC Alfonso Viera

GUT B. Miao, Z. Xić

ArtMet collectiJ. Gattacceca: anonymous

ArtMet collectiL. Krämer Rus

Nick Gessler A. Rubin, UCL

NIPR

A. Yamaguchi

Suzanne MorrC. Agee, UNN

$\begin{array}{ll}\text { JSC } & \text { SI } \quad \text { ANSMET } \\ \text { GUT } & \text { B. Miao, Z. Xic }\end{array}$

Mr. T. KryachlLorenz C. A., 'Mr. T. Kryachl

S. Tutorow C. Zhu, UWO

Marc Jost andK. Klemm and
Ken Eltrich
A. Rubin, UCL Ken Eltrich
KOPRI
C. Park, S. Y. H. Yoo

David Allepuz J. Llorca, UPC David Allepuz

Castellano Vanni Moggi $C$

GUT B. Miao, Z. Xía

DMUH C.A. Lorenz, I

NIPR A. Yamaguchi

David AllepuzJ. Llorca, UPCDavid Allepuz

GUT

B. Miao, Z. Xí

GUT

B. Miao, Z. Xić

Joe Bazyk R. Greenwooc Joe Bazyk

NIPR

A. Yamaguchi

Mr. T. KryachlC. A. Lorenz, 'Mr. T. Kryachl

Finder K. Klemm andNasser Albuai José Vicente (J. Llorca, UPCJosé Vicente ( Eric Christens J. Gattacceca: Eric Christens

Di Martino V. Moggi Cecc Anonymous 


\begin{tabular}{|c|c|c|c|c|}
\hline & 23 & MNRJ & \multicolumn{2}{|c|}{ Museu TancreM.E. ZucolottcCarlos Alberto } \\
\hline & 28.4 & MSN-FI & Di Martino & V. Moggi Cecc Anonymous \\
\hline 5.19 & 24 & CEREGE & \multicolumn{2}{|c|}{ Eric Christens J. Gattacceca: Eric Christens } \\
\hline 5.19 & 250 & CEREGE & CEREGE & H. PourkhorsaRomain Tartes \\
\hline \multirow[t]{10}{*}{5.15} & 23 & CEREGE & \multicolumn{2}{|c|}{ Eric Christens J. Gattacceca: Eric Christens } \\
\hline & 20.3 & MSN-FI & Castellano & V. Moggi Cecc \\
\hline & 231.36 & NIPR & NIPR & A. Yamaguchi \\
\hline & 24 & UPC & \multicolumn{2}{|c|}{ Alfonso Viera J. Llorca, UPC Alfonso Viera } \\
\hline & 226.76 & GUT & GUT & B. Miao, Z. Xić \\
\hline & 226.45 & GUT & GUT & B. Miao, Z. Xí̌ \\
\hline & 21.0 & Vernad & DMUH & C.A. Lorenz, l \\
\hline & 20.6 & MSN-FI & Di Martino & V. Moggi Cecc Anonymous \\
\hline & 30.2 & UWO & S. Tutorow & B. Borba de C \\
\hline & 196.29 & NIPR & NIPR & A. Yamaguchi \\
\hline 5.42 & 193.87 & JSC & JSC & ANSMET \\
\hline \multirow[t]{4}{*}{5.09} & 23 & CEREGE & \multicolumn{2}{|c|}{ Eric Christens J. Gattacceca:Eric Christens } \\
\hline & 27 & UPC & \multicolumn{2}{|c|}{ Ion Ander LanJ. Llorca, UPCIon Ander Lan } \\
\hline & 191.04 & GUT & GUT & B. Miao, Z. Xić \\
\hline & 186.91 & GUT & GUT & B. Miao, Z. Xić \\
\hline \multirow[t]{17}{*}{5.21} & 24 & CEREGE & \multicolumn{2}{|c|}{ Museo del meJ. Gattacceca:Rodrigo Martir } \\
\hline & 182.13 & GUT & GUT & B. Miao, Z. Xí \\
\hline & 29.6 & IfP & \multicolumn{2}{|c|}{ Marc Jost andK. Klemm and } \\
\hline & 24.1 & OU & Joe Bazyk & R. Greenwooc Joe Bazyk \\
\hline & 175.43 & GUT & GUT & B. Miao, Z. Xic \\
\hline & 40.7 & Vernad. & \multicolumn{2}{|c|}{ Mr. T. KryachlLorenz C. A., 'Mr. T. Kryachl } \\
\hline & 173.59 & GUT & GUT & B. Miao, Z. Xi \\
\hline & 173.54 & GUT & GUT & B. Miao, Z. Xí̌ \\
\hline & 171.34 & GUT & GUT & B. Miao, Z. Xis \\
\hline & 166.55 & GUT & GUT & B. Miao, Z. Xić \\
\hline & 164.82 & GUT & GUT & B. Miao, Z. Xić \\
\hline & 164.57 & NIPR & NIPR & A. Yamaguchi \\
\hline & 161.01 & GUT & GUT & B. Miao, Z. Xic \\
\hline & 28.7 & Vernad & DMUH & C.A. Lorenz, l \\
\hline & 29.3 & Vernad & \multicolumn{2}{|c|}{ Mr. T. V. Krya Lorenz C. A., 'Mr. T. V. Krya' } \\
\hline & 40.3 & Vernad & \multicolumn{2}{|c|}{ Mr. T. V. Krya Lorenz C. A., 'Mr. T. V. Krya' } \\
\hline & 23 & UPC & \multicolumn{2}{|c|}{ José Vicente (J. Llorca, UPCJosé Vicente ( } \\
\hline \multirow[t]{9}{*}{5.19} & 21 & CEREGE & \multicolumn{2}{|c|}{ Eric Christens J. Gattacceca:Eric Christens } \\
\hline & 20.1 & MSN-FI & Di Martino & V. Moggi Cecc Anonymous \\
\hline & 24.30 & UCLA & Fred Olsen & D. Sheikh, FS \\
\hline & 28.42 & Vernad. & \multicolumn{2}{|c|}{ Mr. T. KryachlLorenz C. A., 'Mr. T. Kryachl } \\
\hline & 33 & UPC & \multicolumn{2}{|c|}{ José Vicente (J. Llorca, UPCJosé Vicente ( } \\
\hline & 30 & UPC & \multicolumn{2}{|c|}{ Josep Maria EJ. Llorca, UPCJosep Maria E } \\
\hline & 21.1 & MSN-FI & Di Martino & V. Moggi Cecc Anonymous \\
\hline & 21.5 & MSN-FI & Castellano & V. Moggi Cecc \\
\hline & 136.502 & NMBE & NMBE & A. Rosén, E.GEdwin Gnos, E \\
\hline
\end{tabular}




\begin{tabular}{|c|c|c|c|c|}
\hline \multirow[t]{6}{*}{5.39} & 20.1 & CEREGE & R. Lenssen & J. Gattacceca: \\
\hline & 20.84 & MSP & Castellano & V. Moggi Cecc \\
\hline & 131.94 & GUT & GUT & B. Miao, Z. Xí \\
\hline & 130.3 & GUT & GUT & B. Miao, Z. Xić \\
\hline & 128.51 & NIPR & NIPR & A. Yamaguchi \\
\hline & 127.91 & NIPR & NIPR & A. Yamaguchi \\
\hline \multirow[t]{2}{*}{5.03} & 23 & CEREGE & \multicolumn{2}{|c|}{ Eric Christens J. Gattacceca: Eric Christens } \\
\hline & 122.71 & GUT & GUT & B. Miao, Z. Xić \\
\hline 5.14 & 122.65 & JSC & JSC & SI $\quad$ ANSMET \\
\hline \multirow{8}{*}{5.16} & 110 & CEREGE & CEREGE & H. Pourkhorsa Lydie Bonal \\
\hline & 119.15 & GUT & GUT & B. Miao, Z. Xí \\
\hline & 21 & ROM & owner & V. Di Cecco, I. \\
\hline & 117.44 & GUT & GUT & B. Miao, Z. Xić \\
\hline & 20 & UPC & \multicolumn{2}{|c|}{ José Vicente (J. Llorca, UPCJosé Vicente ( } \\
\hline & 116.48 & GUT & GUT & B. Miao, Z. Xis \\
\hline & 115.4 & KOPRI & KOPRI & C. Park, S. Y. J. I. Lee \\
\hline & 114.53 & NIPR & NIPR & A. Yamaguchi \\
\hline \multirow[t]{5}{*}{4.82} & 22 & CEREGE & \multicolumn{2}{|c|}{ Eric Christens H. PourkhorsaEric Christens } \\
\hline & 111.79 & GUT & GUT & B. Miao, Z. Xić \\
\hline & 109.91 & GUT & GUT & B. Miao, Z. Xié \\
\hline & 109.38 & GUT & GUT & B. Miao, Z. Xić \\
\hline & 100.73 & NIPR & NIPR & A. Yamaguchi \\
\hline \multirow[t]{4}{*}{5.20} & 100.154 & JSC & JSC & SI $\quad$ ANSMET \\
\hline & 99.9 & GUT & GUT & B. Miao, Z. Xić \\
\hline & 97.72 & GUT & GUT & B. Miao, Z. Xié \\
\hline & 97.43 & NIPR & NIPR & A. Yamaguchi \\
\hline \multirow[t]{2}{*}{5.05} & 96.57 & JSC & JSC & SI ANSMET \\
\hline & 18.6 & MSN-FI & Castellano & V. Moggi Cecc Anonymous \\
\hline \multirow[t]{7}{*}{5.09} & 19 & CEREGE & \multicolumn{2}{|c|}{ Eric Christens J. Gattacceca: Eric Christens } \\
\hline & 92.2 & KOPRI & KOPRI & C. Park, S. Y. H. Yoo \\
\hline & 91.71 & NIPR & NIPR & A. Yamaguchi \\
\hline & 91.58 & GUT & GUT & B. Miao, Z. Xiś \\
\hline & 18.1 & MSN-FI & Castellano & V. Moggi Cecc \\
\hline & 88.41 & NIPR & NIPR & A. Yamaguchi \\
\hline & 85 & GUT & GUT & B. Miao, Z. Xí \\
\hline \multirow[t]{2}{*}{4.99} & 84.250 & JSC & JSC & SI $\quad$ ANSMET \\
\hline & 83 & KOPRI & KOPRI & C. Park, S. Y. J. I. Lee \\
\hline \multirow[t]{2}{*}{5.07} & 82.56 & JSC & JSC & SI $\quad$ ANSMET \\
\hline & 21.3 & MSN-FI & Di Martino & V. Moggi Cecc Anonymous \\
\hline \multirow[t]{4}{*}{5.12} & 82.15 & JSC & JSC & SI $\quad$ ANSMET \\
\hline & 80.1 & NIPR & NIPR & A. Yamaguchi \\
\hline & 17.3 & Cascadia & \multicolumn{2}{|c|}{ Charles Lucas M. Hutson ancCharles and $\mathrm{N}$} \\
\hline & 77.03 & NIPR & NIPR & A. Yamaguchi \\
\hline \multirow[t]{2}{*}{5.17} & 21 & MNB & \multicolumn{2}{|c|}{ Hichame Mim:A. Greshake, I } \\
\hline & 16.3 & Vernad & anonymous & Lorenz C. A., 'anonymous \\
\hline
\end{tabular}


NHMD

A.N. Krot, D. V

70.68

\section{GUT}

GUT

B. Miao, Z. Xić

68.8

GUT

GUT

B. Miao, Z. Xí́

14

18.7

UPC

NIPR

A. Yamaguchi

David Allepuz J. Llorca, UPCDavid Allepuz.

67.903

MSN-FI

Di Martino

V. Moggi Cecc Anonymous

67.77

4.71

18

14

16.3

13.23

4.82

5.15

63.981

24.4

62.14

61.04

61.04

18

60.88

4.61

13

17.1

59.9

16.9

58.18

57.919

11.3

56.26

55.996

55.421

11.4

18

5.10

53.889

12.1

4.92

5.03

5.10

12

50.55

49.702

13

48.16

46.28

46.03

44.2

5.31

11

43.82

43.74

5.03

JSC

GUT

JSC

SI

GUT

B. Miao, Z. Xić

CEREGE Eric Christens J. Gattacceca: Eric Christens CEREGE

Vernad

Eric Christens J. Gattacceca Eric Christens anonymous Lorenz C. A., 'anonymous

Vernad. JSC

MNB

GUT

$$
\text { NIPR }
$$

GUT

UPC

$$
\text { NIPR }
$$

CEREGE

Cascadia

\section{KOPRI}

MSN-FI

\section{NIPR}

$$
\text { NIPR }
$$

Vernad

\section{GUT}

$$
\text { NIPR }
$$

$$
\text { NIPR }
$$

Cascadia

UPC

JSC

\section{MSN-FI}

CEREGE

$$
\text { JSC }
$$

JSC

MSN-FI

GUT

GUT

GUT

KOPRI

CEREGE

GUT

GUT

JSC
Mr. T. KryachlLorenz C.

JSC

$\mathrm{SI}$

Hichame Mim:A. Greshake, I

GUT

B. Miao, Z. Xić

NIPR

A. Yamaguchi

GUT

B. Miao, Z. Xí

José Vicente (J. Llorca, UPCJosé Vicente ( NIPR

A. Yamaguchi

Eric Christens J. Gattacceca:Eric Christens John A. Shea, M. Hutson, A.

KOPRI

C. Park, S. Y. J. I. Lee

Petriccione V. Moggi Cecc

NIPR A. Yamaguchi

NIPR

A. Yamaguchi

anonymous

Lorenz C. A., 'anonymous

GUT

B. Miao, Z. Xic

NIPR

A. Yamaguchi

NIPR

A. Yamaguchi

John A. Shea, M. Hutson anc

José Vicente (J. Llorca, UPC José Vicente (

JSC

SI

ANSMET

Castellano V. Moggi Cecc

Eric Christens J. Gattacceca:Eric Christens

JSC

SI

ANSMET

JSC

$\mathrm{SI}$

ANSMET

Di Martino V. Moggi Cecc Anonymous

GUT

B. Miao, Z. Xí́

GUT

B. Miao, Z. Xik

GUT

B. Miao, Z. Xí

KOPRI

C. Park, S. Y. J. I. Lee

Labenne Luc J. Gattacceca: Labenne Luc

GUT

B. Miao, Z. Xic

GUT

B. Miao, Z. Xić

JSC

ANSMET 


\begin{tabular}{|c|c|c|c|}
\hline 4.80 & CEREGE & \multicolumn{2}{|c|}{ Jean RedelspıJ. Gattacceca: } \\
\hline 42.963 & NIPR & NIPR & A. Yamaguchi \\
\hline 42.112 & NIPR & NIPR & A. Yamaguchi \\
\hline 41.812 & NIPR & NIPR & A. Yamaguchi \\
\hline 41.5 & GUT & GUT & B. Miao, Z. Xiá \\
\hline \multirow[t]{6}{*}{4.88} & JSC & JSC & ANSMET \\
\hline & NIPR & NIPR & A. Yamaguchi \\
\hline & KOPRI & KOPRI & C. Park, S. Y. J. I. Lee \\
\hline & NIPR & NIPR & A. Yamaguchi \\
\hline & ROM & Gregory & S. Yokoyama, \\
\hline & GUT & GUT & B. Miao, Z. Xié \\
\hline \multirow[t]{3}{*}{5.02} & CEREGE & \multicolumn{2}{|c|}{ Eric Christens J. Gattacceca:Eric Christens } \\
\hline & NIPR & NIPR & A. Yamaguchi \\
\hline & GUT & GUT & B. Miao, Z. Xić \\
\hline \multirow[t]{5}{*}{5.13} & JSC & JSC & SI $\quad$ ANSMET \\
\hline & JSC & JSC & ANSMET \\
\hline & UCLA & Verish & A. Rubin, UCL John P. Wolfe \\
\hline & NIPR & NIPR & A. Yamaguchi \\
\hline & GUT & GUT & B. Miao, Z. Xić \\
\hline 32.339 & JSC & JSC & SI $\quad$ ANSMET \\
\hline \multirow[t]{5}{*}{4.90} & JSC & JSC & ANSMET \\
\hline & NIPR & NIPR & A. Yamaguchi \\
\hline & GUT & GUT & B. Miao, Z. Xiś \\
\hline & GUT & GUT & B. Miao, Z. Xis \\
\hline & GUT & GUT & B. Miao, Z. Xić \\
\hline \multirow[t]{2}{*}{5.03} & JSC & JSC & ANSMET \\
\hline & GUT & GUT & B. Miao, Z. Xić \\
\hline 28.343 & JSC & JSC & ANSMET \\
\hline \multirow[t]{3}{*}{4.94} & JSC & JSC & SI $\quad$ ANSMET \\
\hline & GUT & GUT & B. Miao, Z. Xis \\
\hline & KOPRI & KOPRI & C. Park, S. Y. H. Yoo \\
\hline \multirow[t]{3}{*}{5.16} & CEREGE & \multicolumn{2}{|c|}{ Eric Christens J. Gattacceca:Eric Christens } \\
\hline & GUT & GUT & B. Miao, Z. Xici \\
\hline & NIPR & NIPR & A. Yamaguchi \\
\hline 26.192 & JSC & JSC & ANSMET \\
\hline \multirow[t]{6}{*}{5.03} & JSC & JSC & ANSMET \\
\hline & KOPRI & KOPRI & C. Park, S. Y. S. Park \\
\hline & GUT & GUT & B. Miao, Z. Xic \\
\hline & GUT & GUT & B. Miao, Z. Xic \\
\hline & UPC & \multicolumn{2}{|c|}{ José Vicente (J. Llorca, UPCJosé Vicente ( } \\
\hline & NIPR & NIPR & A. Yamaguchi \\
\hline 23.306 & JSC & JSC & ANSMET \\
\hline 22.500 & JSC & JSC & ANSMET \\
\hline 22.3 & GUT & GUT & B. Miao, Z. Xié \\
\hline 22.07 & GUT & GUT & B. Miao, Z. Xiá \\
\hline
\end{tabular}


21.56

21.206

4.81

5.17

5.21

5.11

5.04

4.79

5.02

4.51

5.15

5.01

5.15

5.06

4.99

5.18

11.58
11.533

11.35

11.03

10.93

10.9

10.88

5.24

\begin{abstract}
NIPR
\end{abstract}
NIPR

JSC

NIPR

NIPR

NIPR

JSC

JSC

JSC

NIPR

UPC

JSC

KOPRI

NIPR

GUT

NIPR

JSC

JSC

JSC

KOPRI

CEREGE

NIPR

NIPR

Vernad

UPC

JSC

JSC

NIPR

NIPR

NIPR

NIPR

JSC

JSC

JSC

JSC

JSC

GUT

NIPR

GUT

GUT

GUT

KOPRI

GUT

JSC

NIPR
NIPR

NIPR

JSC

NIPR

NIPR

NIPR

JSC

JSC

JSC

NIPR

Jordi Llorca

JSC

KOPRI

NIPR

GUT

NIPR

JSC

JSC

JSC

KOPRI

Labenne Luc

NIPR

NIPR

Vernad

José Vicente (J.

JSC

JSC

NIPR

NIPR

NIPR

NIPR

JSC

JSC

JSC

JSC

JSC

GUT

NIPR

GUT

GUT

GUT

KOPRI

GUT

JSC

NIPR
A. Yamaguchi

A. Yamaguchi

SI

ANSMET

A. Yamaguchi

A. Yamaguchi

A. Yamaguchi

SI

ANSMET

SI

ANSMET

SI

ANSMET

A. Yamaguchi

J. Llorca, UPC Jordi Llorca

$\mathrm{SI}$

ANSMET

C. Park, S. Y. J. I. Lee

A. Yamaguchi

B. Miao, Z. Xí

A. Yamaguchi

SI ANSMET

SI ANSMET

SI ANSMET

C. Park, S. Y. Justin

J. Gattacceca Labenne Luc

A. Yamaguchi

A. Yamaguchi

C. A. Lorenz, 'Mr. S. E. Boris

(J. Llorca, UPCJosé Vicente (

SI

ANSMET

ANSMET

A. Yamaguchi

A. Yamaguchi

A. Yamaguchi

A. Yamaguchi

SI

ANSMET

SI

ANSMET

$\mathrm{SI}$

ANSMET

SI

ANSMET

SI

ANSMET

B. Miao, Z. Xia

A. Yamaguchi

B. Miao, Z. Xic

B. Miao, Z. Xí

B. Miao, Z. Xíz

C. Park, S. Y. S. Park

B. Miao, Z. Xí

SI

ANSMET

A. Yamaguchi 


$\begin{array}{llll}10.5 & \text { KOPRI } & \text { KOPRI } & \text { C. Park, S. Y. S. Park } \\ 10.415 & \text { NIPR } & \text { NIPR } & \text { A. Yamaguchi }\end{array}$

\begin{tabular}{|c|c|c|c|c|}
\hline & 3.6 & Vernad. & T. Kryac & kLorenz C. A., 'T. Kryachko, E \\
\hline & 10 & KOPRI & KOPRI & C. Park, S. Y. J. I. Lee \\
\hline & 8 & UPC & José Vic & (J. Llorca, UPCJosé Vicente ( \\
\hline & 9.887 & NIPR & NIPR & A. Yamaguchi \\
\hline & 9.852 & NIPR & NIPR & A. Yamaguchi \\
\hline & 9.27 & GUT & GUT & B. Miao, Z. Xić \\
\hline & 9.268 & NIPR & NIPR & A. Yamaguchi \\
\hline & 9.147 & NIPR & NIPR & A. Yamaguchi \\
\hline & 9.063 & NIPR & NIPR & A. Yamaguchi \\
\hline & 9.03 & NIPR & NIPR & A. Yamaguchi \\
\hline & 8.84 & GUT & GUT & B. Miao, Z. Xié \\
\hline 5.21 & 8.56 & JSC & JSC & ANSMET \\
\hline & 8.519 & NIPR & NIPR & A. Yamaguchi \\
\hline & 8.372 & NIPR & NIPR & A. Yamaguchi \\
\hline 4.59 & 8.3 & JSC & JSC & SI $\quad$ ANSMET \\
\hline & 7.63 & NIPR & NIPR & A. Yamaguchi \\
\hline & 7.63 & NIPR & NIPR & A. Yamaguchi \\
\hline & 7.34 & NIPR & NIPR & A. Yamaguchi \\
\hline & 6.878 & NIPR & NIPR & A. Yamaguchi \\
\hline 5.02 & 2 & CEREGE & Eric Chr & J. Gattacceca:Eric Christens \\
\hline & 4.36 & Vernad & Mr. T. K & Horenz C. A., 'Mr. T. Kryachł \\
\hline & 6.218 & NIPR & NIPR & A. Yamaguchi \\
\hline & 6.1 & NIPR & NIPR & A. Yamaguchi \\
\hline & 6.08 & NIPR & NIPR & A. Yamaguchi \\
\hline & 5.971 & NIPR & NIPR & A. Yamaguchi \\
\hline & 5.14 & NIPR & NIPR & A. Yamaguchi \\
\hline & 4.988 & NIPR & NIPR & A. Yamaguchi \\
\hline & 4.769 & NIPR & NIPR & A. Yamaguchi \\
\hline & 4.76 & NIPR & NIPR & A. Yamaguchi \\
\hline & 4.758 & NIPR & NIPR & A. Yamaguchi \\
\hline & 4.748 & NIPR & NIPR & A. Yamaguchi \\
\hline & 4.554 & NIPR & NIPR & A. Yamaguchi \\
\hline & 4.507 & NIPR & NIPR & A. Yamaguchi \\
\hline & 4.475 & NIPR & NIPR & A. Yamaguchi \\
\hline & 4.403 & NIPR & NIPR & A. Yamaguchi \\
\hline & 4.394 & NIPR & NIPR & A. Yamaguchi \\
\hline & 4.376 & NIPR & NIPR & A. Yamaguchi \\
\hline & 4.289 & NIPR & NIPR & A. Yamaguchi \\
\hline & 4.277 & NIPR & NIPR & A. Yamaguchi \\
\hline & 4.244 & NIPR & NIPR & A. Yamaguchi \\
\hline & 4.22 & NIPR & NIPR & A. Yamaguchi \\
\hline & 4.189 & NIPR & NIPR & A. Yamaguchi \\
\hline & 4.115 & NIPR & NIPR & A. Yamaguchi \\
\hline
\end{tabular}




\begin{tabular}{|c|c|c|c|c|}
\hline & 4.106 & NIPR & NIPR & A. Yamaguchi \\
\hline & 4.041 & NIPR & NIPR & A. Yamaguchi \\
\hline & 3.942 & NIPR & NIPR & A. Yamaguchi \\
\hline & 3.931 & NIPR & NIPR & A. Yamaguchi \\
\hline & 3.931 & NIPR & NIPR & A. Yamaguchi \\
\hline & 3.922 & NIPR & NIPR & A. Yamaguchi \\
\hline & 3.852 & NIPR & NIPR & A. Yamaguchi \\
\hline & 3.833 & NIPR & NIPR & A. Yamaguchi \\
\hline & 3.784 & NIPR & NIPR & A. Yamaguchi \\
\hline & 3.756 & NIPR & NIPR & A. Yamaguchi \\
\hline 4.61 & 3.74 & JSC & JSC & SI $\quad$ ANSMET \\
\hline & 3.725 & NIPR & NIPR & A. Yamaguchi \\
\hline & 3.431 & NIPR & NIPR & A. Yamaguchi \\
\hline & 3.411 & NIPR & NIPR & A. Yamaguchi \\
\hline & 3.355 & NIPR & NIPR & A. Yamaguchi \\
\hline & 3.315 & NIPR & NIPR & A. Yamaguchi \\
\hline & 3.308 & NIPR & NIPR & A. Yamaguchi \\
\hline & 3.235 & NIPR & NIPR & A. Yamaguchi \\
\hline & 3.149 & NIPR & NIPR & A. Yamaguchi \\
\hline & 3.148 & NIPR & NIPR & A. Yamaguchi \\
\hline & 3.1 & KOPRI & KOPRI & C. Park, S. Y. B. W. Son \\
\hline & 2.898 & NIPR & NIPR & A. Yamaguchi \\
\hline & 2.826 & NIPR & NIPR & A. Yamaguchi \\
\hline & 2.754 & NIPR & NIPR & A. Yamaguchi \\
\hline & 2.738 & NIPR & NIPR & A. Yamaguchi \\
\hline & 2.712 & NIPR & NIPR & A. Yamaguchi \\
\hline & 2.667 & NIPR & NIPR & A. Yamaguchi \\
\hline & 2.619 & NIPR & NIPR & A. Yamaguchi \\
\hline & 2.617 & NIPR & NIPR & A. Yamaguchi \\
\hline & 2.617 & NIPR & NIPR & A. Yamaguchi \\
\hline & 2.502 & NIPR & NIPR & A. Yamaguchi \\
\hline & 2.494 & NIPR & NIPR & A. Yamaguchi \\
\hline & 2.432 & NIPR & NIPR & A. Yamaguchi \\
\hline & 2.335 & NIPR & NIPR & A. Yamaguchi \\
\hline & 2.333 & NIPR & NIPR & A. Yamaguchi \\
\hline & 2.267 & NIPR & NIPR & A. Yamaguchi \\
\hline & 2.253 & NIPR & NIPR & A. Yamaguchi \\
\hline & 2.223 & NIPR & NIPR & A. Yamaguchi \\
\hline & 2.213 & NIPR & NIPR & A. Yamaguchi \\
\hline & 2.13 & NIPR & NIPR & A. Yamaguchi \\
\hline & 1.978 & NIPR & NIPR & A. Yamaguchi \\
\hline & 1.912 & NIPR & NIPR & A. Yamaguchi \\
\hline & 1.824 & NIPR & NIPR & A. Yamaguchi \\
\hline & 1.793 & NIPR & NIPR & A. Yamaguchi \\
\hline & 1.755 & NIPR & NIPR & A. Yamaguchi \\
\hline
\end{tabular}




\begin{tabular}{|c|c|c|c|}
\hline 1.734 & NIPR & NIPR & A. Yamaguchi \\
\hline 1.722 & NIPR & NIPR & A. Yamaguchi \\
\hline 1.643 & NIPR & NIPR & A. Yamaguchi \\
\hline 1.623 & NIPR & NIPR & A. Yamaguchi \\
\hline 1.606 & NIPR & NIPR & A. Yamaguchi \\
\hline 1.6 & NIPR & NIPR & A. Yamaguchi \\
\hline 1.575 & NIPR & NIPR & A. Yamaguchi \\
\hline 1.532 & NIPR & NIPR & A. Yamaguchi \\
\hline 1.518 & NIPR & NIPR & A. Yamaguchi \\
\hline 1.443 & NIPR & NIPR & A. Yamaguchi \\
\hline 1.424 & NIPR & NIPR & A. Yamaguchi \\
\hline 1.424 & NIPR & NIPR & A. Yamaguchi \\
\hline 1.423 & NIPR & NIPR & A. Yamaguchi \\
\hline 1.42 & NIPR & NIPR & A. Yamaguchi \\
\hline 1.410 & JSC & JSC & SI $\quad$ ANSMET \\
\hline 1.373 & NIPR & NIPR & A. Yamaguchi \\
\hline 1.37 & JSC & JSC & ANSMET \\
\hline 1.370 & JSC & JSC & ANSMET \\
\hline 1.367 & NIPR & NIPR & A. Yamaguchi \\
\hline 1.355 & NIPR & NIPR & A. Yamaguchi \\
\hline 1.335 & NIPR & NIPR & A. Yamaguchi \\
\hline 1.310 & JSC & JSC & ANSMET \\
\hline 1.278 & NIPR & NIPR & A. Yamaguchi \\
\hline 1.278 & NIPR & NIPR & A. Yamaguchi \\
\hline 1.267 & NIPR & NIPR & A. Yamaguchi \\
\hline 1.194 & NIPR & NIPR & A. Yamaguchi \\
\hline 1.169 & NIPR & NIPR & A. Yamaguchi \\
\hline 1.157 & NIPR & NIPR & A. Yamaguchi \\
\hline 1.137 & NIPR & NIPR & A. Yamaguchi \\
\hline 1.108 & NIPR & NIPR & A. Yamaguchi \\
\hline 1.1 & KOPRI & KOPRI & C. Park, S. Y. S. Park \\
\hline 1.087 & NIPR & NIPR & A. Yamaguchi \\
\hline 1.086 & NIPR & NIPR & A. Yamaguchi \\
\hline 1.056 & NIPR & NIPR & A. Yamaguchi \\
\hline 1.04 & NIPR & NIPR & A. Yamaguchi \\
\hline 1.03 & NIPR & NIPR & A. Yamaguchi \\
\hline 1.02 & NIPR & NIPR & A. Yamaguchi \\
\hline 1.008 & NIPR & NIPR & A. Yamaguchi \\
\hline 0.994 & NIPR & NIPR & A. Yamaguchi \\
\hline 0.969 & NIPR & NIPR & A. Yamaguchi \\
\hline 0.957 & NIPR & NIPR & A. Yamaguchi \\
\hline 0.826 & NIPR & NIPR & A. Yamaguchi \\
\hline 0.82 & NIPR & NIPR & A. Yamaguchi \\
\hline 0.801 & NIPR & NIPR & A. Yamaguchi \\
\hline 0.790 & JSC & JSC & SI $\quad$ ANSMET \\
\hline
\end{tabular}




\begin{tabular}{|c|c|c|c|c|}
\hline & 0.751 & NIPR & NIPR & A. Yamaguchi \\
\hline & 0.694 & NIPR & NIPR & A. Yamaguchi \\
\hline & 0.651 & JSC & JSC & SI $\quad$ ANSMET \\
\hline & 0.644 & NIPR & NIPR & A. Yamaguchi \\
\hline & 0.581 & NIPR & NIPR & A. Yamaguchi \\
\hline & 0.578 & NIPR & NIPR & A. Yamaguchi \\
\hline & 0.52 & NIPR & NIPR & A. Yamaguchi \\
\hline & 0.507 & NIPR & NIPR & A. Yamaguchi \\
\hline & 0.393 & NIPR & NIPR & A. Yamaguchi \\
\hline & 0.385 & NIPR & NIPR & A. Yamaguchi \\
\hline & 0.37 & NIPR & NIPR & A. Yamaguchi \\
\hline & 0.35 & NIPR & NIPR & A. Yamaguchi \\
\hline & 0.349 & NIPR & NIPR & A. Yamaguchi \\
\hline & 0.3 & KOPRI & KOPRI & C. Park, S. Y. H. Yoo \\
\hline & 0.298 & NIPR & NIPR & A. Yamaguchi \\
\hline & 1468 & Vernad & DMUH & C. A. Lorenz, ' \\
\hline & 16.0 & IfP & Finder & K. Klemm, A. Ianonymous \\
\hline 5.22 & 77.4 & CEREGE & ArtMet collect & tiJ. Gattacceca anonymous \\
\hline 4.92 & 0.82 & MNB & Said Yousfi & A. Greshake, I \\
\hline & 37 & UPC & José Vicente & (J. Llorca, UPCJosé Vicente ( \\
\hline 4.66 & 21 & CEREGE & Eric Christens & S. Gattacceca:Eric Christens \\
\hline & 290 & IGGCAS & Pengli Chen & N. Wang, Y. XCa Rongpubu \\
\hline & 593.36 & UCLA & Michael Cottin & nA. Rubin, UCL \\
\hline & 25 & UNM & Dustin Dicken & C. Agee, UNN \\
\hline & 52.4 & MSN-FI & Tomelleri & V. Moggi Cece \\
\hline & 20 & IfP & Manuel Nieto & K. Metzler, IfP \\
\hline & 141 & NHMV & A. Pani & F. Brandstaett \\
\hline & 47 & UPC & Ion Ander Lan & nJ. Llorca, UPClon Ander Lan \\
\hline 5.04 & 54 & CEREGE & ArtMet collect & tiJ. Gattacceca anonymous \\
\hline & 36 & UCLA & Verish & D. Sheikh, FS Mark Bittmanr \\
\hline & 25.5 & MNB & Dario Ruiz & Ansgar Gresh Dario Ruiz \\
\hline & 1712.9 & NIPR & NIPR & A. Yamaguchi \\
\hline & 20.2 & MSN-FI & Castellano & V. Moggi Cecr \\
\hline & 1189.29 & UrFU & UrFU & V. V. SharygirUrFU meteorit \\
\hline & 30.2 & MSN-FI & Tomelleri & V. Moggi Cecr \\
\hline & 21 & UPC & José Vicente & (J. Llorca, UPCJosé Vicente ( \\
\hline & 1043.3 & NIPR & NIPR & A. Yamaguchi \\
\hline & 20.8 & UWB & S. Ralew & A. Irving and $\subseteq$ \\
\hline & 28 & UPC & José Vicente & (J. Llorca, UPCJosé Vicente ( \\
\hline 5.05 & 23.3 & CEREGE & R. Lenssen & L. Krämer Ruç \\
\hline & 78 & UPC & José Vicente & (J. Llorca, UPC José Vicente ( \\
\hline & 676.8 & NIPR & NIPR & A. Yamaguchi \\
\hline & 41 & UPC & José Vicente & (J. Llorca, UPCJosé Vicente ( \\
\hline & 29.2 & MNB & Ahmed Taleb & A. Greshake, IAhmed Taleb \\
\hline & 496.69 & NIPR & NIPR & A. Yamaguchi \\
\hline
\end{tabular}


$20.51 \quad$ UCLA Nick Gessler A. Rubin, UCL

$31 \quad$ UPC

$20.1 \quad$ UWB

$24.9 \quad$ MNB

$58 \quad$ UPC

$286.05 \quad$ NIPR

$249.36 \quad$ UrFU

281.64 GUT

$270.13 \quad$ GUT

33

UPC

258

\section{OAM}

213.8

UrFU

José Vicente (J. Llorca, UPCJosé Vicente (

242.87

NIPR

211.53

JSC

M. Goff

A. Irving and $\leqslant$

Dario Ruiz Ansgar Gresh Dario Ruiz

José Vicente (J. Llorca, UPCJosé Vicente (

NIPR

A. Yamaguchi

UrFU

Victor V. Shar UrFU meteorit

25.1

4.95

21

178.49

ROM

GUT

B. Miao, Z. Xic

GUT

B. Miao, Z. Xí

David Allepuz J. Llorca, UPCDavid Allepuz.

OAM L. Folco, UPis

UrFU Victor V. Shar UrFU meteorit

NIPR A. Yamaguchi

JSC SI ANSMET

Gregory S. Yokoyama,

CEREGE Eric Christens J. Gattacceca Eric Christens

175.59

GUT

GUT

B. Miao, Z. Xic

172.94

GUT

GUT

B. Miao, Z. Xic

171.97

GUT

GUT

B. Miao, Z. Xis

169.77

GUT

GUT

B. Miao, Z. Xis

164.95

GUT

B. Miao, Z. Xia

4.88

161.460

GUT

GUT

B. Miao, Z. Xía

159.19

JSC

JSC

SI

ANSMET

147.97

NIPR

NIPR

A. Yamaguchi

GUT

B. Miao, Z. Xí

20

GUT

5.12

134.868

UPC

José Vicente (J. Llorca, UPCJosé Vicente (

134.72

JSC

128.72

GUT

JSC

SI

ANSMET

GUT

B. Miao, Z. Xic

128.2

GUT

GUT

B. Miao, Z. Xis

118.4

NIPR

5.23

GUT

NIPR

A. Yamaguchi

GUT

B. Miao, Z. Xí

$104.266 \quad J S C$

JSC

SI

ANSMET

20.5

MSN-FI

101.97

GUT

Di Martino

V. Moggi Cecc Anonymous

GUT

B. Miao, Z. Xí

5.19

96.42

JSC

89.17

GUT

JSC

SI

ANSMET

88.71

$$
\text { GUT }
$$

GUT

B. Miao, Z. Xia

87.71

NIPR

GUT

B. Miao, Z. Xis

85.94

GUT

5.03

83.13

JSC

79.45

NIPR

77.52

GUT

5.38

77.064

JSC

72.98

NIPR

NIPR

A. Yamaguchi

GUT

B. Miao, Z. Xic

JSC

SI

ANSMET

NIPR

A. Yamaguchi

GUT

B. Miao, Z. Xí

JSC

SI

ANSMET

68.15

GUT

NIPR

A. Yamaguchi

GUT

B. Miao, Z. Xí 


\subsection{1}

66.05

66.002

63.269

62.69

61.61

5.2

5.15

5.24

4.90

5.07

5.09

5.16

5.30

5.1

5.05

5.10

59.06

58.55

11.7

57.54

56.63

55.91

55.7

55.653

54.004

52.105

51.66

51.424

49.54

12.0

48.785

44.96

44.183

44.15

26.9

42.312

9.6

41.44

41.315

40

40.22

39.77

37.84

5.10

36.636

8.2

35.441

5.05

34.510

33.7

4.88

32.900

31.81

5.04

5.07

31.703

31.64

30.965

30.538

30.48
NIPR

$$
\text { NIPR }
$$

JSC

JSC

GUT

GUT

JSC

GUT

MSN-FI

$$
\text { NIPR }
$$

GUT

JSC

GUT

NIPR

NIPR

NIPR

GUT

NIPR

JSC

MSN-F

JSC

JSC

JSC

GUT

UCLA

JSC

MNB

JSC

JSC

\section{CEREGE}

GUT

GUT

GUT

JSC

ROM

NIPR

JSC

KOPRI

JSC

NIPR

JSC

JSC

JSC

JSC

JSC
NIPR

NIPR

JSC

JSC

GUT

GUT

JSC

GUT

Jacques de la V. Moggi Cecc

NIPR

GUT

JSC

GUT

NIPR

NIPR

NIPR

GUT

NIPR

JSC

Castellano

JSC

JSC

JSC

GUT

UCLA

JSC

A. Yamaguchi

B. Miao, Z. Xí

SI

B. Miao, Z. Xiz

A. Yamaguchi

A. Yamaguchi

A. Yamaguchi

B. Miao, Z. Xía

A. Yamaguchi

SI

V. Moggi Cecc

SI

SI

SI

B. Miao, Z. Xic

C. Anderkin, L

Hanno Strufe A. Greshake, I

JSC SI ANSMET

JSC

SI

ANSMET

CEREGE H. Pourkhorsa J. Gattacceca

GUT

B. Miao, Z. Xía

GUT

B. Miao, Z. Xis

GUT

B. Miao, Z. Xí

JSC

SI

ANSMET

Gregory

S. Yokoyama,

NIPR

A. Yamaguchi

JSC

SI

ANSMET

KOPRI

C. Park, S. Y. T. Kim

JSC

SI

ANSMET

NIPR

A. Yamaguchi

JSC

SI

ANSMET

JSC

SI

ANSMET

JSC

SI

ANSMET

JSC

SI

ANSMET

JSC
SI

ANSMET 
5.8

$28.836 \quad$ NIPR

ROM

Gregory

J. Poitras and

NIPR

A. Yamaguchi

28.816

NIPR

NIPR

A. Yamaguchi

5.21

28.24

JSC

4.83

21.7

Cascadia

JSC

SI

ANSMET

27.790

JSC

Cascadia

M. Hutson anc Jim Wooddell

27.789

NIPR

JSC

SI

ANSMET

27.728

NIPR

NIPR

A. Yamaguchi

27.619

NIPR

NIPR

A. Yamaguchi

27.598

NIPR

A. Yamaguchi

26.259

JSC

JSC

SI

ANSMET

25.16

NIPR

NIPR

A. Yamaguchi

NIPR

A. Yamaguchi

24.638

NIPR

NIPR

A. Yamaguchi

4.75

24.49

JSC

JSC

SI

ANSMET

5.11

24.271

JSC

JSC

SI

ANSMET

23.78

GUT

GUT

B. Miao, Z. Xí

5.07

23.193

JSC

JSC

22.842

NIPR

NIPR

SI

ANSMET

5.01

22.53

JSC

JSC

A. Yamaguchi

4.87

22.480

JSC

JSC

SI

ANSMET

4.98

22.43

JSC

JSC

SI

ANSMET

4.99

21.926

JSC

JSC

SI

ANSMET

21.476

NIPR

NIPR

SI

ANSMET

5.09

21.450

JSC

21.21

GUT

JSC

A. Yamaguchi

SI

ANSMET

21.073

JSC

GUT

B. Miao, Z. Xic

20.892

NIPR

JSC

SI

ANSMET

20.2

KOPRI

NIPR

A. Yamaguchi

KOPRI

C. Park, S. Y. T. Kim

4.97

19.894

JSC

JSC

SI

ANSMET

5.09

19.25

NIPR

NIPR

A. Yamaguchi

18.950

JSC

JSC

SI

ANSMET

4.74

18.58

JSC

JSC

SI

ANSMET

4.86

18.46

JSC

JSC

SI

ANSMET

17.97

GUT

GUT

B. Miao, Z. Xić

5.11

JSC

JSC

SI

ANSMET

17.858

JSC

JSC

SI

ANSMET

4.9

17.54

JSC

JSC

SI

ANSMET

4.77

JSC

JSC

SI

ANSMET

5.02

15.701

JSC

JSC

SI

ANSMET

15.69

NIPR

NIPR

A. Yamaguchi

5.18

15.45

JSC

JSC

SI

ANSMET

14.953

NIPR

NIPR

A. Yamaguchi

5.01

14.95

JSC

JSC

SI

ANSMET

14.630

JSC

JSC

SI

ANSMET

14.619

NIPR

NIPR

A. Yamaguchi 


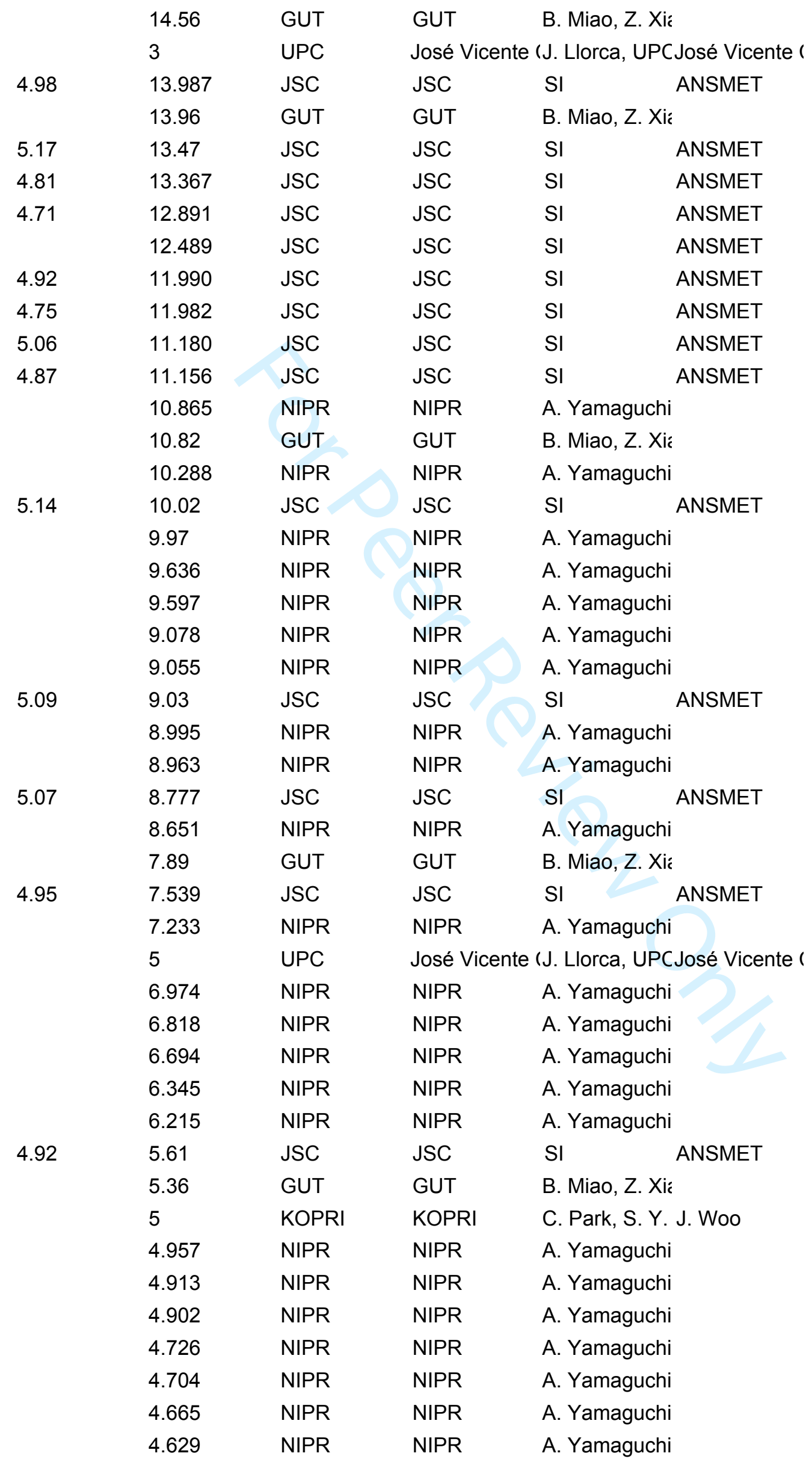




\begin{tabular}{|c|c|c|c|}
\hline 4.533 & NIPR & NIPR & A. Yamaguchi \\
\hline 4.532 & NIPR & NIPR & A. Yamaguchi \\
\hline 4.524 & NIPR & NIPR & A. Yamaguchi \\
\hline 4.492 & NIPR & NIPR & A. Yamaguchi \\
\hline 4.436 & JSC & JSC & SI $\quad$ ANSMET \\
\hline 4.272 & NIPR & NIPR & A. Yamaguchi \\
\hline 4.25 & NIPR & NIPR & A. Yamaguchi \\
\hline 4.217 & NIPR & NIPR & A. Yamaguchi \\
\hline 4.2 & KOPRI & KOPRI & C. Park, S. Y. J. I. Lee \\
\hline 4.16 & GUT & GUT & B. Miao, Z. Xić \\
\hline 4.153 & NIPR & NIPR & A. Yamaguchi \\
\hline 4.077 & NIPR & NIPR & A. Yamaguchi \\
\hline 4.057 & NIPR & NIPR & A. Yamaguchi \\
\hline 4.056 & NIPR & NIPR & A. Yamaguchi \\
\hline 3.942 & NIPR & NIPR & A. Yamaguchi \\
\hline 3.926 & NIPR & NIPR & A. Yamaguchi \\
\hline 3.897 & NIPR & NIPR & A. Yamaguchi \\
\hline 3.895 & NIPR & NIPR & A. Yamaguchi \\
\hline 3.854 & NIPR & NIPR & A. Yamaguchi \\
\hline 3.83 & NIPR & NIPR & A. Yamaguchi \\
\hline 3.787 & NIPR & NIPR & A. Yamaguchi \\
\hline 3.565 & NIPR & NIPR & A. Yamaguchi \\
\hline 3.56 & NIPR & NIPR & A. Yamaguchi \\
\hline 3.504 & NIPR & NIPR & A. Yamaguchi \\
\hline 3.327 & NIPR & NIPR & A. Yamaguchi \\
\hline 3.316 & NIPR & NIPR & A. Yamaguchi \\
\hline 3.288 & NIPR & NIPR & A. Yamaguchi \\
\hline 3.166 & NIPR & NIPR & A. Yamaguchi \\
\hline 3.152 & NIPR & NIPR & A. Yamaguchi \\
\hline 3.084 & NIPR & NIPR & A. Yamaguchi \\
\hline 3.037 & NIPR & NIPR & A. Yamaguchi \\
\hline 2.999 & NIPR & NIPR & A. Yamaguchi \\
\hline 2.982 & NIPR & NIPR & A. Yamaguchi \\
\hline 2.935 & NIPR & NIPR & A. Yamaguchi \\
\hline 2.854 & NIPR & NIPR & A. Yamaguchi \\
\hline 2.791 & NIPR & NIPR & A. Yamaguchi \\
\hline 2.755 & NIPR & NIPR & A. Yamaguchi \\
\hline 2.699 & NIPR & NIPR & A. Yamaguchi \\
\hline 2.642 & NIPR & NIPR & A. Yamaguchi \\
\hline 2.62 & GUT & GUT & B. Miao, Z. Xić \\
\hline 2.603 & NIPR & NIPR & A. Yamaguchi \\
\hline 2.6 & KOPRI & KOPRI & C. Park, S. Y. S. Park \\
\hline 2.588 & NIPR & NIPR & A. Yamaguchi \\
\hline 2.522 & NIPR & NIPR & A. Yamaguchi \\
\hline 2.462 & NIPR & NIPR & A. Yamaguchi \\
\hline
\end{tabular}




\begin{tabular}{|c|c|c|c|}
\hline 2.453 & NIPR & NIPR & A. Yamaguchi \\
\hline 2.379 & NIPR & NIPR & A. Yamaguchi \\
\hline 2.286 & NIPR & NIPR & A. Yamaguchi \\
\hline 2.179 & NIPR & NIPR & A. Yamaguchi \\
\hline 2.137 & NIPR & NIPR & A. Yamaguchi \\
\hline 2.136 & NIPR & NIPR & A. Yamaguchi \\
\hline 2.1 & KOPRI & KOPRI & C. Park, S. Y. J. I. Lee \\
\hline 2.047 & NIPR & NIPR & A. Yamaguchi \\
\hline 2.041 & NIPR & NIPR & A. Yamaguchi \\
\hline 1.928 & NIPR & NIPR & A. Yamaguchi \\
\hline 1.799 & NIPR & NIPR & A. Yamaguchi \\
\hline 1.753 & NIPR & NIPR & A. Yamaguchi \\
\hline 1.7 & KOPRI & KOPRI & C. Park, S. Y. S. Park \\
\hline 1.65 & NIPR & NIPR & A. Yamaguchi \\
\hline 1.616 & NIPR & NIPR & A. Yamaguchi \\
\hline 1.59 & NIPR & NIPR & A. Yamaguchi \\
\hline 1.551 & NIPR & NIPR & A. Yamaguchi \\
\hline 1.521 & NIPR & NIPR & A. Yamaguchi \\
\hline 1.503 & NIPR & NIPR & A. Yamaguchi \\
\hline 1.46 & NIPR & NIPR & A. Yamaguchi \\
\hline 1.450 & JSC & JSC & SI $\quad$ ANSMET \\
\hline 1.386 & NIPR & NIPR & A. Yamaguchi \\
\hline 1.37 & JSC & JSC & ANSMET \\
\hline 1.36 & JSC & JSC & SI $\quad$ ANSMET \\
\hline 1.33 & NIPR & NIPR & A. Yamaguchi \\
\hline 1.32 & NIPR & NIPR & A. Yamaguchi \\
\hline 1.3 & KOPRI & KOPRI & C. Park, S. Y. Z. Beach \\
\hline 1.3 & KOPRI & KOPRI & C. Park, S. Y. S. Park \\
\hline 1.200 & JSC & JSC & SI $\quad$ ANSMET \\
\hline 1.188 & NIPR & NIPR & A. Yamaguchi \\
\hline 1.16 & JSC & JSC & SI $\quad$ ANSMET \\
\hline 1.1 & KOPRI & KOPRI & C. Park, S. Y. J. I. Lee \\
\hline 1.071 & JSC & JSC & ANSMET \\
\hline 1.06 & JSC & JSC & SI $\quad$ ANSMET \\
\hline 1.044 & NIPR & NIPR & A. Yamaguchi \\
\hline 1.037 & JSC & JSC & SI $\quad$ ANSMET \\
\hline 0.983 & NIPR & NIPR & A. Yamaguchi \\
\hline 0.92 & NIPR & NIPR & A. Yamaguchi \\
\hline 0.919 & NIPR & NIPR & A. Yamaguchi \\
\hline 0.889 & JSC & JSC & SI $\quad$ ANSMET \\
\hline 0.832 & NIPR & NIPR & A. Yamaguchi \\
\hline 0.813 & JSC & JSC & SI $\quad$ ANSMET \\
\hline 0.8 & KOPRI & KOPRI & C. Park, S. Y. S. Han \\
\hline 0.775 & NIPR & NIPR & A. Yamaguchi \\
\hline 0.774 & NIPR & NIPR & A. Yamaguchi \\
\hline
\end{tabular}




\begin{tabular}{|c|c|c|c|c|c|}
\hline & 0.760 & JSC & JSC & SI & ANSMET \\
\hline & 0.674 & NIPR & NIPR & A. Yamaguchi & \\
\hline & 0.672 & JSC & JSC & SI & ANSMET \\
\hline & 0.658 & NIPR & NIPR & A. Yamaguchi & \\
\hline & 0.628 & JSC & JSC & $\mathrm{SI}$ & ANSMET \\
\hline & 0.624 & NIPR & NIPR & A. Yamaguchi & \\
\hline & 0.555 & NIPR & NIPR & A. Yamaguchi & \\
\hline & 0.542 & NIPR & NIPR & A. Yamaguchi & \\
\hline & 0.540 & JSC & JSC & SI & ANSMET \\
\hline & 0.530 & JSC & JSC & SI & ANSMET \\
\hline & 0.522 & JSC & JSC & SI & ANSMET \\
\hline & 0.456 & JSC & JSC & SI & ANSMET \\
\hline & 0.437 & NIPR & NIPR & A. Yamaguchi & \\
\hline & 0.405 & NIPR & NIPR & A. Yamaguchi & \\
\hline & 0.390 & JSC & JSC & SI & ANSMET \\
\hline & 0.323 & NIPR & NIPR & A. Yamaguchi & \\
\hline & 0.311 & NIPR & NIPR & A. Yamaguchi & \\
\hline & 0.271 & NIPR & NIPR & A. Yamaguchi & \\
\hline & 0.244 & NIPR & NIPR & A. Yamaguchi & \\
\hline & 0.170 & JSC & JSC & SI & ANSMET \\
\hline & 21.5 & UWB & A. Aaronson & A. Irving and $\subseteq$ & \\
\hline & 5.02 & ROM & GREGORY & V. Di Cecco, F & \\
\hline & 22.8 & UNM & Dustin Dicken & C. Agee, UNN & \\
\hline & 25.65 & UCLA & Matthew Stree & ¿D. Sheikh, FS & \\
\hline & 20.0 & UNM & Kai Ke & C. Agee, UNN & \\
\hline & 25.3 & UNM & Darryl Pitt & C. Agee, UNN & \\
\hline 3.34 & 20.03 & Kiel & Dieter Janek, & Bart & Lahsen Duine \\
\hline & 20 & MNB & Philippe Schm & rA. Greshake, I & \\
\hline & 21.3 & UAb & Joshua Tree $\wedge$ & ^C. Herd and J & \\
\hline & 20.0 & Cascadia & HMANI Inc & M. Hutson, A. & \\
\hline & 23.3 & UNM & Darryl Pitt & C. Agee, UNN & \\
\hline & 24 & UWB & A. Aaronson & A. Irving and & \\
\hline & 20 & IfP, Münster & Anonymous & K. Metzler, IfP & \\
\hline & 22.8 & UNM & DPitt & C. Agee, UNN & \\
\hline & 12.2 & UWB & DPitt & A. Irving and $\leqslant$ & \\
\hline & 8.98 & UCLA & D. Sheikh & D. Sheikh, FS & \\
\hline & 3 & MNB & Philippe Schm & rA. Greshake, I & \\
\hline & 3 & UCLA & Main mass wit & it C. Anderkin & \\
\hline & 1.5 & GUT & GUT & B. Miao, L.Hui & \\
\hline & 0.6 & GUT & GUT & B. Miao, L. Hu & \\
\hline & 1.2 & GUT & GUT & B. Miao, L. Hu & \\
\hline & 1.3 & GUT & GUT & B. Miao, L. Hu & \\
\hline & 4959.500 & SI & SI & SI & ANSMET \\
\hline & 2805.950 & SI & $\mathrm{SI}$ & SI & ANSMET \\
\hline & 1680 & UCLA & unknown & J.T. Wasson, I & \\
\hline
\end{tabular}


75.770

71.31

24.6

22.2

20.7

20.1

22.7

20.3

21.3

20.8

22.9

21.9

22.3

24.0

21.7

22.7

22.6

18.5

12.3

$\begin{array}{lll}\text { UCLA } & \text { unknown } & \text { J.T. Wasson, I } \\ \text { UCLA } & \text { unknown } & \text { J.T. Wasson, I } \\ \text { ASU } & \text { MFarmer } & \text { L. Garvie, ASIAlejandro Cor }\end{array}$

UCLA

Adam Hupé

J.T. Wasson, I

UAb Hollis Meteoril C. Herd, UAb

$$
\text { MSN-FI Tomelleri V. Moggi Cecc Anonymous }
$$

UTok and NIP Finder T. Mikouchi, UK. Mitsumura

PMO SSTM J.T. Wasson, IAnonymous

ASU unknown J.T. Wasson, I

ASU unknown J.T. Wasson, I

UCLA J. Utas J.T. Wasson, I

UCLA Fred B<span !J.T. Wasson, I

UCLA UCLA J.T. Wasson, I

NMBE NMBE J.T.Wasson, I

$\begin{array}{lll}\text { UCLA } & 110 \quad \text { J.T. Wasson, I }\end{array}$

\begin{abstract}
UAb
\end{abstract}
J. Shea
C. Herd, UAb

UAb

Wang Chen

C. Herd, UAb

UAb Zhang QingyaC. Herd and L

MNRJ/UFRJ UNIPAMPA J.T. Wasson, IElver Ubirajar: UAb Unnamed owr C. Herd, UAb

IGCAS Peng Wang Hao Peng, IGla local herder CEREGE Frankie Yu M. D'Orazio, J UPC MNCN Jordi Llorca (L

JSC JSC SI ANSMET

IGCAS Xinping Wen Hao Peng, IGla stone hunteI UAb

\section{SI}

JSC

MNB

Wang Ziyao

C. Herd, UAb Ye Erbo Lati

SI

SI

ANSMET

JSC SI ANSMET

Philippe SchrrA. Greshake, I

\section{Kiel}

Kiel

Kiel

Kiel

Kiel

Kiel

Kiel

Kiel

Kiel

Kiel

Kiel

Kiel

Kiel

Kiel

Kiel

Wang Ziyao

R. Bartoschen Xu Zhong

Liu Binghan R. BartoschenChen Wie (Ha

Wang Ziyao R. Bartoschen anonymous

Wang Ziyao R. Bartoschen Ma Yonglu Liu Binghan aıR. Bartoscheu Zhang Qun Liu Binghan aıR. Bartoschen Liu Binghan Dieter Janek, Bart anonymous Wang Ziyao a R. BartoscheuZijian Wang ( Wang Ziyao a R. BartoschenZijian Wang (l Wang Ziyao a R. BartoscheuLiu Binghan ( Dieter Janek, Bart anonymous Wang Ziyao R. BartoschevZijian Wang (l
A. Mazur
Bart anonymous Wang Ziyao R. Bartoschen Wang Ziyao Wang Ziyao R. Bartoschen anonymous Kiel 


\begin{tabular}{|c|c|c|c|c|}
\hline \multirow[t]{4}{*}{4.85} & 4.4 & Kiel & \multicolumn{2}{|c|}{ Ben Levi Kars R. Bartoschen anonymous } \\
\hline & 242 & ASU & \multicolumn{2}{|c|}{ Michael Farmı L. Garvie } \\
\hline & 39.4 & UNM & \multicolumn{2}{|c|}{ Abdelhadi Aitt C. Agee, UNN } \\
\hline & 7210 & PSF & PSF & A. Irving and $s$ \\
\hline \multirow[t]{2}{*}{4.36} & 20 & MNB & \multicolumn{2}{|c|}{ Philippe Schm A. Greshake, I } \\
\hline & 20 & IfP & \multicolumn{2}{|c|}{ Manuel Nieto K. Metzler, IfP } \\
\hline 4.63 & 26.5 & MNB & \multicolumn{2}{|c|}{ Franco VignatA. Greshake, I } \\
\hline \multirow[t]{2}{*}{4.45} & 23.13 & CEREGE & \multicolumn{2}{|c|}{ Fabien Kuntz L. Krämer Ruç } \\
\hline & 20 & IfP, Münster & \multicolumn{2}{|c|}{ Nourddine AzıK. Metzler, IfP } \\
\hline \multirow[t]{3}{*}{4.53} & 25 & CEREGE & \multicolumn{2}{|c|}{ Jean RedelspıJ. Gattacceca: } \\
\hline & 20.6 & MNB & \multicolumn{2}{|c|}{ Hanno Strufe A. Greshake, I } \\
\hline & 27.8 & MNB & \multicolumn{2}{|c|}{ Hanno Strufe A. Greshake, I } \\
\hline \multirow[t]{2}{*}{4.76} & 20.5 & UWB & G. Fujihara & A. Irving and \\
\hline & 400.95 & NIPR & \multicolumn{2}{|c|}{ NIPR A. Yamaguchi } \\
\hline 4.46 & 23 & UWB & \multicolumn{2}{|c|}{ C. Giessler A. Irving and $\leqslant$} \\
\hline \multirow[t]{3}{*}{4.73} & 21.8 & UWB & \multicolumn{2}{|c|}{ G. Fujihara A. Irving and $\subseteq$} \\
\hline & 20 & MNB & \multicolumn{2}{|c|}{ Salamu HamaA. Greshake, I } \\
\hline & 20.2 & Cascadia & \multicolumn{2}{|c|}{ Anonymous hiK. Farley, M. ł } \\
\hline \multirow[t]{6}{*}{4.59} & 22.5 & MNB & \multicolumn{2}{|c|}{ Hanno Strufe A. Greshake, I } \\
\hline & 26.8 & MNB & Hanno Strufe & A. Greshake, I \\
\hline & 157.98 & NIPR & NIPR & A. Yamaguchi \\
\hline & 16.5 & MNB & Philippe Sch & A. Greshake, I \\
\hline & 16.5 & MNB & Philippe Sch & A. Greshake, I \\
\hline & 15 & MNB & Alan Mazur & A. Greshake, I \\
\hline 4.61 & 17.7 & MNB & Hichame Min & A. Greshake, I \\
\hline & 10.4 & MNB & Philippe Sch & A. Greshake, I \\
\hline & 9.2 & MNB & Philippe Schı & A. Greshake, I \\
\hline & 6.5 & SI & M. Cottingha & M. Takahashi, unknown \\
\hline & 4 & MNB & Hanno Strufe & A. Greshake, I \\
\hline & 5.7 & KOPRI & KOPRI & C. Park, S. Y. H. Yoo \\
\hline & 5.694 & NIPR & NIPR & A. Yamaguchi \\
\hline & 4.489 & NIPR & NIPR & A. Yamaguchi \\
\hline & 3.979 & NIPR & NIPR & A. Yamaguchi \\
\hline & 3.714 & NIPR & NIPR & A. Yamaguchi \\
\hline & 3.434 & NIPR & NIPR & A. Yamaguchi \\
\hline & 3.302 & NIPR & NIPR & A. Yamaguchi \\
\hline & 3.033 & NIPR & NIPR & A. Yamaguchi \\
\hline & 2.927 & NIPR & NIPR & A. Yamaguchi \\
\hline & 2.156 & NIPR & NIPR & A. Yamaguchi \\
\hline & 2.003 & NIPR & NIPR & A. Yamaguchi \\
\hline & 1.689 & NIPR & NIPR & A. Yamaguchi \\
\hline & 1.611 & NIPR & NIPR & A. Yamaguchi \\
\hline & 1.475 & NIPR & NIPR & A. Yamaguchi \\
\hline & 1.47 & NIPR & NIPR & A. Yamaguchi \\
\hline & 1.458 & NIPR & NIPR & A. Yamaguchi \\
\hline
\end{tabular}




\begin{tabular}{|c|c|c|c|c|}
\hline & 1.044 & NIPR & NIPR & A. Yamaguchi \\
\hline & 90 & UCLA & M. Farmer & A. Irving and $\leqq$ \\
\hline 4.48 & 20.1 & Kiel & \multicolumn{2}{|c|}{ Li Bofang, BeiR. Bartoschen anonymous } \\
\hline \multirow[t]{2}{*}{4.45} & 24.6 & MNB & \multicolumn{2}{|c|}{ Hichame Mim:A. Greshake, I } \\
\hline & 29 & UNM & \multicolumn{2}{|c|}{ Marcin CimalzC. Agee, UNN } \\
\hline 4.61 & 58.55 & JSC & JSC & ANSMET \\
\hline 4.19 & 16.8 & JSC & JSC & ANSMET \\
\hline 3.9 & 9.29 & JSC & JSC & ANSMET \\
\hline \multirow[t]{2}{*}{5.08} & 71.004 & JSC & JSC & ANSMET \\
\hline & 25 & CEREGE & \multicolumn{2}{|c|}{ Andreas GrenJ. Gattacceca: } \\
\hline \multirow[t]{20}{*}{4.95} & 50.1 & FSAC & \multicolumn{2}{|c|}{ Aaronson, MoH. Chennaoui Mohamed Oui } \\
\hline & 20 & IfP & Anonymous & K. Metzler, IfP \\
\hline & 2703 & PSF & PSF & A. Irving and $\varsigma$ \\
\hline & 51.3 & UWO & S. Tutorow & G. Ibe, UWO \\
\hline & 2157 & PSF & PSF & A. Irving and $\subseteq$ \\
\hline & 21 & UWB & Aaronson & A. Irving and $\leqslant$ \\
\hline & 35.4 & UWB & A. Aaronson & A. Irving and $\leq$ \\
\hline & 61.10 & UCLA & \multicolumn{2}{|c|}{ Michael CottinA. Rubin, UCL } \\
\hline & 44 & UPC & \multicolumn{2}{|c|}{ David Allepuz J. Llorca, UPC David Allepuz } \\
\hline & 26.2 & UWB & \multicolumn{2}{|c|}{ B. Hoefnagels A. Irving and $\leqslant$} \\
\hline & 362.15 & GUT & GUT & B. Miao, Z. Xiá \\
\hline & 361 & KOPRI & KOPRI & C. Park, S. Y. H. Yoo \\
\hline & 355.04 & GUT & GUT & B. Miao, Z. Xič \\
\hline & 20.1 & MSN-FI & Castellano & V. Moggi Cecc \\
\hline & 189.62 & NIPR & NIPR & A. Yamaguchi \\
\hline & 20.1 & MSN-FI & Di Martino & V. Moggi Cecc Anonymous \\
\hline & 25.8 & MSN-FI & Castellano & V. Moggi Cecc \\
\hline & 160.6 & NIPR & NIPR & A. Yamaguchi \\
\hline & 20.5 & MSN-FI & Petriccione & V. Moggi Cecc \\
\hline & 25 & UCLA & Michael Cottir & nA. Rubin, UCL \\
\hline 4.65 & 123.57 & JSC & JSC & SI $\quad$ ANSMET \\
\hline & 105.4 & PSF & PSF & A. Irving and $\leqq$ \\
\hline & 77.45 & NIPR & NIPR & A. Yamaguchi \\
\hline & 16.27 & ROM & Gregory & J. Poitras and \\
\hline & 74.44 & NIPR & NIPR & A. Yamaguchi \\
\hline 4.64 & 68.81 & JSC & JSC & SI $\quad$ ANSMET \\
\hline & 65.31 & PSF & PSF & A. Irving and $\subseteq$ \\
\hline 4.37 & 44.840 & JSC & JSC & $\begin{array}{ll}\text { SI } & \text { ANSMET }\end{array}$ \\
\hline 4.42 & 41.66 & JSC & JSC & ANSMET \\
\hline 4.58 & 37.31 & JSC & JSC & SI $\quad$ ANSMET \\
\hline 4.71 & 6.38 & CEREGE & anonymous & J. Gattacceca: anonymous \\
\hline 4.86 & 25.37 & JSC & JSC & SI $\quad$ ANSMET \\
\hline & 15.7 & MNA-SI & MNA-SI & F. Focacci, M.Maurizio Gem \\
\hline & 15.66 & PSF & PSF & A. Irving and $\varsigma$ \\
\hline & 14.872 & NIPR & NIPR & A. Yamaguchi \\
\hline
\end{tabular}


4.5

$\begin{array}{llll}14.49 & \text { JSC } & \text { JSC } & \text { SI ANSMET } \\ 12.826 & \text { NIPR } & \text { NIPR } & \text { A. Yamaguchi } \\ 8.93 & \text { NIPR } & \text { NIPR } & \text { A. Yamaguchi } \\ 6.787 & \text { NIPR } & \text { NIPR } & \text { A. Yamaguchi } \\ 4.566 & \text { NIPR } & \text { NIPR } & \text { A. Yamaguchi } \\ 4.466 & \text { NIPR } & \text { NIPR } & \text { A. Yamaguchi } \\ 4.382 & \text { NIPR } & \text { NIPR } & \text { A. Yamaguchi } \\ 4.17 & \text { NIPR } & \text { NIPR } & \text { A. Yamaguchi } \\ 3.905 & \text { NIPR } & \text { NIPR } & \text { A. Yamaguchi } \\ 3.751 & \text { NIPR } & \text { NIPR } & \text { A. Yamaguchi } \\ 3.715 & \text { NIPR } & \text { NIPR } & \text { A. Yamaguchi } \\ 2.716 & \text { NIPR } & \text { NIPR } & \text { A. Yamaguchi } \\ 2.173 & \text { NIPR } & \text { NIPR } & \text { A. Yamaguchi } \\ 1.335 & \text { NIPR } & \text { NIPR } & \text { A. Yamaguchi } \\ 1.272 & \text { NIPR } & \text { NIPR } & \text { A. Yamaguchi } \\ 0.5 & \text { MNA-SI } & \text { MNA-SI } & \text { F. Focacci, M. Jacopo Nava } \\ 0.498 & \text { JSC } & \text { JSC } & \text { SI ANSMET }\end{array}$

\subsection{7}

35

4470

4.53

24

21.2

16

20.5

161

89.6

47.5

43.8

4.04

20.2

136

43

3620

4.98

23.5

30.6

663.5

106

115.5

20.1

MNB

PSF

CEREGE

PSF

SI

ANSMET

UWB

ArtMet collectiJ. Gattacceca anonymous

B. HoefnagelsA. Irving and $\leqslant$

ROM

Gregory

S. Yokoyama,

DPitt

C. Agee, UNN

José Vicente (J. Llorca, UPCJosé Vicente (

UPC

S. Tutorow

P. Reger, UW

Private

M. E. Varela, I unknown

DMUH

C.A. Lorenz, I

Fabien Kuntz R. Bartoschen Anonymous

Kiel

Pengli Chen aY. Xu, N. WanPengli Chen, I

IGGCAS

UPC

Ion Ander LanJ. Llorca, UPClon Ander Lan

PSF

PSF

A. Irving and $\leqslant$

\section{CEREGE}

UNM

ArtMet collectiJ. Gattacceca anonymous

Brad Kern

C. Agee, UNN

Vernad

Mr. E. ZakharıC. A. Lorenz, 'Mr. E. Zacharı

FMMR

$2127 \mathrm{~g}$ - Kirill P. Yu. PlechoiKirill Vlasov ar

Vernad

DMUH

C.A. Lorenz, I

42.9

UWB

DPitt

A. Irving and $\varsigma$

114.80

MNB

Hicha

miA. Greshake, I

1566.1

UCLA

Michae

OU

A. Rubin, UCL

1522

OU

PSF

Craig Walton,

4.94

20.1

PSF

Dieter Janek, Bart

anonymous

152.6

Kiel

Ilya ChaplyginLorenz C. A., 'llya Chaplygin

130.3

Vernad.

Vernad

Mr. T. KryachlC. A. Lorenz, 'Mr. T. Kryachl 


\begin{tabular}{|c|c|c|c|c|}
\hline & 930 & GSI & GSI & A. Dutta and [ \\
\hline & 796 & KOPRI & KOPRI & C. Park, S. Y. J. H. Kim \\
\hline & 79 & UPC & \multicolumn{2}{|c|}{ José Vicente (J. Llorca, UPC José Vicente } \\
\hline & 22.8 & OU & Joe Bazyk & R. Greenwooc Joe Bazyk \\
\hline & 606.82 & GUT & GUT & B. Miao, Z. Xic \\
\hline & 68.7 & Vernad & \multicolumn{2}{|c|}{ Mr. M. NepomLorenz C. A., 'Mr. M. Nepom } \\
\hline & 571.94 & GUT & GUT & B. Miao, Z. Xić \\
\hline & 554.16 & OU & OU & C. Walton, US \\
\hline & 518.83 & GUT & GUT & B. Miao, Z. Xí̌ \\
\hline & 24.2 & Vernad & DMUH & C. A. Lorenz, ' \\
\hline & 482.17 & NIPR & NIPR & A. Yamaguchi \\
\hline & 27.6 & UCLA & \multicolumn{2}{|c|}{ Michael CottinA. Rubin, UCL } \\
\hline & 20.2 & MSN-FI & Di Martino & V. Moggi Cecc Anonymous \\
\hline \multirow[t]{4}{*}{4.86} & 380.76 & JSC & JSC & ANSMET \\
\hline & 347.67 & GUT & GUT & B. Miao, Z. Xí̌ \\
\hline & 342.12 & GUT & GUT & B. Miao, Z. Xić \\
\hline & 20.2 & MSN-FI & Mimaghador & Vanni Moggi C \\
\hline \multirow[t]{2}{*}{4.92} & 35.6 & MNB & \multicolumn{2}{|c|}{ Hanno Strufe A. Greshake, I } \\
\hline & 298.6 & NIPR & NIPR & A. Yamaguchi \\
\hline \multirow[t]{2}{*}{4.83} & 288.530 & JSC & JSC & SI $\quad$ ANSMET \\
\hline & 277.86 & GUT & GUT & B. Miao, Z. Xik \\
\hline \multirow[t]{4}{*}{4.75} & 259.400 & JSC & JSC & SI $\quad$ ANSMET \\
\hline & 20 & MSN-FI & Mimaghador & Vanni Moggi C \\
\hline & 20.1 & MNB & \multicolumn{2}{|c|}{ Hichame MimiA. Greshake, I } \\
\hline & 194 & PSF & PSF & A. Irving and $\leqslant$ \\
\hline \multirow[t]{11}{*}{4.63} & 20 & CEREGE & \multicolumn{2}{|c|}{ Eric Christens J. Gattacceca:Eric Christens } \\
\hline & 25.4 & MNB & \multicolumn{2}{|c|}{ Juergen NaubA. Greshake, I } \\
\hline & 27 & MSN-FI & Mimaghador & Vanni Moggi C \\
\hline & 49.5 & Vernad. & \multicolumn{2}{|c|}{ Ilya ChaplyginLorenz C. A., 'Ilya Chaplygin } \\
\hline & 103 & OU & Joe Bazyk & R. Greenwooc Joe Bazyk \\
\hline & 26 & Vernad & DMUH & M. A. Ivanova: \\
\hline & 24.9 & Vernad & anonymous & C. A. Lorenz, 'anonymous \\
\hline & 120.07 & GUT & GUT & B. Miao, Z. Xis \\
\hline & 30.9 & Vernad. & \multicolumn{2}{|c|}{ Ilya ChaplyginLorenz C. A., 'Ilya Chaplygin } \\
\hline & 22.1 & CEREGE & Peter Buhl & J. Gattacceca, Peter Buhl \\
\hline & 23.9 & MNB & \multicolumn{2}{|c|}{ Jörgen NaubeA. Greshake, I } \\
\hline 4.60 & 99.891 & JSC & JSC & ANSMET \\
\hline \multirow[t]{2}{*}{4.77} & 99.03 & JSC & JSC & SI $\quad$ ANSMET \\
\hline & 17.85 & MSN-FI & Cojocaru & V. Moggi Cecc \\
\hline 4.74 & 83.03 & JSC & JSC & ANSMET \\
\hline 4.16 & 74.832 & JSC & JSC & ANSMET \\
\hline 4.63 & 73.130 & JSC & JSC & ANSMET \\
\hline \multirow[t]{2}{*}{4.63} & 72.32 & JSC & JSC & ANSMET \\
\hline & 13.5 & ROM & Gregory & J. Poitras, UT، \\
\hline 4.61 & 64.509 & JSC & JSC & SI $\quad$ ANSMET \\
\hline
\end{tabular}




\begin{tabular}{|c|c|c|c|c|c|}
\hline & 12.9 & MSN-FI & Cavagliã & \multicolumn{2}{|c|}{ V. Moggi Cecc } \\
\hline 4.81 & 61.89 & JSC & JSC & SI & ANSMET \\
\hline \multirow[t]{2}{*}{4.61} & 61.08 & JSC & JSC & SI & ANSMET \\
\hline & 23 & MSN-FI & CavagliÃ & \multicolumn{2}{|c|}{ V. Moggi Cecc } \\
\hline \multirow[t]{2}{*}{5.08} & 57.549 & JSC & JSC & $\mathrm{SI}$ & ANSMET \\
\hline & 12.5 & Vernad. & \multicolumn{3}{|c|}{ Mr. T. KryachlLorenz C. A., 'Mr. T. Kryachl } \\
\hline 4.67 & 52.14 & JSC & JSC & $\mathrm{SI}$ & ANSMET \\
\hline 4.66 & 11 & CEREGE & MMC & \multicolumn{2}{|c|}{ L. Krämer Ruş } \\
\hline \multirow[t]{2}{*}{4.41} & 49.982 & JSC & JSC & $\mathrm{SI}$ & ANSMET \\
\hline & 12.1 & MNB & \multicolumn{3}{|c|}{ Philippe SchrrA. Greshake, I } \\
\hline 4.85 & 48.451 & JSC & JSC & $\mathrm{SI}$ & ANSMET \\
\hline 4.56 & 47.32 & JSC & JSC & SI & ANSMET \\
\hline \multirow[t]{4}{*}{4.66} & 46.992 & JSC & JSC & SI & ANSMET \\
\hline & 9.5 & Vernad & DMUH & \multicolumn{2}{|c|}{ C.A. Lorenz, l } \\
\hline & 40 & UPC & Jordi Llorca & \multicolumn{2}{|c|}{ J. Llorca, UPC Jordi Llorca } \\
\hline & 42.171 & JSC & JSC & SI & ANSMET \\
\hline 4.66 & 41.805 & JSC & JSC & SI & ANSMET \\
\hline \multirow[t]{2}{*}{4.75} & 40.32 & JSC & JSC & SI & ANSMET \\
\hline & 8 & MSN-FI & OAM & \multicolumn{2}{|c|}{ V. Moggi Cecc Anonymous } \\
\hline 4.53 & 39.1 & JSC & JSC & SI & ANSMET \\
\hline 4.59 & 38.668 & JSC & JSC & SI & ANSMET \\
\hline 4.42 & 36.534 & JSC & JSC & SI & ANSMET \\
\hline 4.53 & 33.466 & JSC & JSC & SI & ANSMET \\
\hline 4.62 & 33.377 & JSC & JSC & $\mathrm{SI}$ & ANSMET \\
\hline 4.70 & 32.904 & JSC & JSC & SI & ANSMET \\
\hline 4.7 & 32.753 & JSC & JSC & SI & ANSMET \\
\hline \multirow[t]{2}{*}{4.72} & 32.19 & JSC & JSC & SI & ANSMET \\
\hline & 30.91 & PSF & PSF & \multicolumn{2}{|c|}{ A. Irving and $\leqslant$} \\
\hline \multirow[t]{2}{*}{4.53} & 30.193 & JSC & JSC & SI & ANSMET \\
\hline & 30.16 & GUT & GUT & \multicolumn{2}{|c|}{ B. Miao, Z. Xić } \\
\hline 4.42 & 29.48 & JSC & JSC & SI & ANSMET \\
\hline 4.52 & 27.91 & JSC & JSC & SI & ANSMET \\
\hline 4.63 & 27.561 & JSC & JSC & SI & ANSMET \\
\hline 4.56 & 27.068 & JSC & JSC & SI & ANSMET \\
\hline \multirow[t]{2}{*}{4.81} & 26.878 & JSC & JSC & SI & ANSMET \\
\hline & 26.49 & GUT & GUT & \multicolumn{2}{|c|}{ B. Miao, Z. Xić } \\
\hline 4.66 & 25.89 & JSC & JSC & SI & ANSMET \\
\hline 4.45 & 24.937 & JSC & JSC & SI & ANSMET \\
\hline 4.44 & 24.86 & JSC & JSC & SI & ANSMET \\
\hline 4.54 & 24.675 & JSC & JSC & SI & ANSMET \\
\hline 4.49 & 23.992 & JSC & JSC & SI & ANSMET \\
\hline 4.65 & 22.324 & JSC & JSC & SI & ANSMET \\
\hline 4.42 & 21.650 & JSC & JSC & SI & ANSMET \\
\hline 4.73 & 20.581 & JSC & JSC & SI & ANSMET \\
\hline 4.56 & 20.55 & JSC & JSC & SI & ANSMET \\
\hline
\end{tabular}




\begin{tabular}{|c|c|c|c|c|c|}
\hline 4.51 & 20.540 & JSC & JSC & SI & ANSMET \\
\hline 4.57 & 20.08 & JSC & JSC & SI & ANSMET \\
\hline \multirow[t]{2}{*}{4.51} & 19.34 & JSC & JSC & SI & ANSMET \\
\hline & 18.727 & JSC & JSC & SI & ANSMET \\
\hline 4.49 & 18.278 & JSC & JSC & SI & ANSMET \\
\hline 4.58 & 16.96 & JSC & JSC & SI & ANSMET \\
\hline 4.57 & 16.1 & JSC & JSC & $\mathrm{SI}$ & ANSMET \\
\hline 4.59 & 15.88 & JSC & JSC & SI & ANSMET \\
\hline 4.52 & 15.87 & JSC & JSC & SI & ANSMET \\
\hline 4.57 & 15.72 & JSC & JSC & SI & ANSMET \\
\hline 4.5 & 15.354 & JSC & JSC & SI & ANSMET \\
\hline 4.24 & 15.297 & JSC & JSC & SI & ANSMET \\
\hline \multirow[t]{2}{*}{4.52} & 14.466 & JSC & JSC & SI & ANSMET \\
\hline & 14.461 & NIPR & NIPR & \multicolumn{2}{|c|}{ A. Yamaguchi } \\
\hline 4.55 & 14.307 & JSC & JSC & SI & ANSMET \\
\hline 4.56 & 14.110 & JSC & JSC & $\mathrm{SI}$ & ANSMET \\
\hline 4.49 & 13.150 & JSC & JSC & $\mathrm{SI}$ & ANSMET \\
\hline \multirow[t]{2}{*}{4.54} & 11.052 & JSC & JSC & SI & ANSMET \\
\hline & 10.295 & NIPR & NIPR & \multicolumn{2}{|c|}{ A. Yamaguchi } \\
\hline 4.55 & 9.759 & JSC & JSC & $\mathrm{SI}$ & ANSMET \\
\hline \multirow[t]{5}{*}{4.65} & 9.445 & JSC & JSC & $\mathrm{SI}$ & ANSMET \\
\hline & 9.25 & NIPR & NIPR & \multicolumn{2}{|c|}{ A. Yamaguchi } \\
\hline & 8.849 & NIPR & NIPR & \multicolumn{2}{|c|}{ A. Yamaguchi } \\
\hline & 8.27 & NIPR & NIPR & \multicolumn{2}{|c|}{ A. Yamaguchi } \\
\hline & 7.7 & NIPR & NIPR & \multicolumn{2}{|c|}{ A. Yamaguchi } \\
\hline 4.64 & 7.11 & JSC & JSC & $\mathrm{SI}$ & ANSMET \\
\hline \multirow[t]{19}{*}{4.55} & 6.196 & JSC & JSC & SI & ANSMET \\
\hline & 3.4 & Vernad & Vernad & \multicolumn{2}{|c|}{ C. A. Lorenz, 'Mr. S. E. Boris } \\
\hline & 4.761 & NIPR & NIPR & \multicolumn{2}{|c|}{ A. Yamaguchi } \\
\hline & 4.655 & NIPR & NIPR & \multicolumn{2}{|c|}{ A. Yamaguchi } \\
\hline & 4.534 & NIPR & NIPR & \multicolumn{2}{|c|}{ A. Yamaguchi } \\
\hline & 4.511 & NIPR & NIPR & \multicolumn{2}{|c|}{ A. Yamaguchi } \\
\hline & 4.484 & NIPR & NIPR & \multicolumn{2}{|c|}{ A. Yamaguchi } \\
\hline & 4.322 & NIPR & NIPR & \multicolumn{2}{|c|}{ A. Yamaguchi } \\
\hline & 4.112 & NIPR & NIPR & \multicolumn{2}{|c|}{ A. Yamaguchi } \\
\hline & 4.096 & NIPR & NIPR & \multicolumn{2}{|c|}{ A. Yamaguchi } \\
\hline & 4.057 & NIPR & NIPR & \multicolumn{2}{|c|}{ A. Yamaguchi } \\
\hline & 3.943 & NIPR & NIPR & \multicolumn{2}{|c|}{ A. Yamaguchi } \\
\hline & 3.864 & NIPR & NIPR & \multicolumn{2}{|c|}{ A. Yamaguchi } \\
\hline & 3.566 & NIPR & NIPR & \multicolumn{2}{|c|}{ A. Yamaguchi } \\
\hline & 3.388 & NIPR & NIPR & A. & \\
\hline & 3.374 & NIPR & NIPR & A. & \\
\hline & 3.363 & NIPR & NIPR & A. & \\
\hline & 3.279 & NIPR & NIPR & A. & \\
\hline & 3.273 & NIPR & NIPR & A. & \\
\hline
\end{tabular}




\begin{tabular}{|c|c|c|c|}
\hline 3.269 & NIPR & NIPR & A. Yamaguchi \\
\hline 3.231 & NIPR & NIPR & A. Yamaguchi \\
\hline 3.112 & NIPR & NIPR & A. Yamaguchi \\
\hline 3.046 & NIPR & NIPR & A. Yamaguchi \\
\hline 3.031 & NIPR & NIPR & A. Yamaguchi \\
\hline 2.915 & NIPR & NIPR & A. Yamaguchi \\
\hline 2.858 & NIPR & NIPR & A. Yamaguchi \\
\hline 2.742 & NIPR & NIPR & A. Yamaguchi \\
\hline 2.611 & NIPR & NIPR & A. Yamaguchi \\
\hline 2.515 & NIPR & NIPR & A. Yamaguchi \\
\hline 2.503 & NIPR & NIPR & A. Yamaguchi \\
\hline 2.486 & NIPR & NIPR & A. Yamaguchi \\
\hline 2.351 & NIPR & NIPR & A. Yamaguchi \\
\hline 2.348 & NIPR & NIPR & A. Yamaguchi \\
\hline 2.147 & NIPR & NIPR & A. Yamaguchi \\
\hline 2.052 & NIPR & NIPR & A. Yamaguchi \\
\hline 1.996 & NIPR & NIPR & A. Yamaguchi \\
\hline 1.875 & NIPR & NIPR & A. Yamaguchi \\
\hline 1.818 & NIPR & NIPR & A. Yamaguchi \\
\hline 1.814 & NIPR & NIPR & A. Yamaguchi \\
\hline 1.752 & NIPR & NIPR & A. Yamaguchi \\
\hline 1.644 & NIPR & NIPR & A. Yamaguchi \\
\hline 1.6 & MNA-SI & MNA-SI & F. Focacci, M.Maurizio Gem \\
\hline 1.426 & NIPR & NIPR & A. Yamaguchi \\
\hline 1.402 & NIPR & NIPR & A. Yamaguchi \\
\hline 1.400 & JSC & JSC & SI ANSMET \\
\hline 1.385 & NIPR & NIPR & A. Yamaguchi \\
\hline 1.321 & NIPR & NIPR & A. Yamaguchi \\
\hline 1.26 & JSC & JSC & SI $\quad$ ANSMET \\
\hline 1.212 & NIPR & NIPR & A. Yamaguchi \\
\hline 1.191 & NIPR & NIPR & A. Yamaguchi \\
\hline 1.176 & NIPR & NIPR & A. Yamaguchi \\
\hline 1.174 & NIPR & NIPR & A. Yamaguchi \\
\hline 1.08 & JSC & JSC & SI $\quad$ ANSMET \\
\hline 1.045 & NIPR & NIPR & A. Yamaguchi \\
\hline 0.935 & NIPR & NIPR & A. Yamaguchi \\
\hline 0.857 & JSC & JSC & ANSMET \\
\hline 0.850 & JSC & JSC & ANSMET \\
\hline 0.836 & NIPR & NIPR & A. Yamaguchi \\
\hline 0.789 & NIPR & NIPR & A. Yamaguchi \\
\hline 0.721 & NIPR & NIPR & A. Yamaguchi \\
\hline 0.721 & NIPR & NIPR & A. Yamaguchi \\
\hline 0.699 & NIPR & NIPR & A. Yamaguchi \\
\hline 0.677 & NIPR & NIPR & A. Yamaguchi \\
\hline 0.663 & JSC & JSC & ANSMET \\
\hline
\end{tabular}




\begin{tabular}{|c|c|c|c|}
\hline 0.629 & NIPR & NIPR & A. Yamaguchi \\
\hline 0.62 & JSC & JSC & ANSMET \\
\hline 0.601 & NIPR & NIPR & A. Yamaguchi \\
\hline 0.596 & NIPR & NIPR & A. Yamaguchi \\
\hline 0.575 & NIPR & NIPR & A. Yamaguchi \\
\hline 0.554 & NIPR & NIPR & A. Yamaguchi \\
\hline 0.541 & NIPR & NIPR & A. Yamaguchi \\
\hline 0.527 & NIPR & NIPR & A. Yamaguchi \\
\hline 0.521 & NIPR & NIPR & A. Yamaguchi \\
\hline 0.508 & NIPR & NIPR & A. Yamaguchi \\
\hline 0.5 & MNA-SI & MNA-SI & F. Focacci, M. Jacopo Nava \\
\hline 0.475 & NIPR & NIPR & A. Yamaguchi \\
\hline 0.451 & NIPR & NIPR & A. Yamaguchi \\
\hline 0.353 & NIPR & NIPR & A. Yamaguchi \\
\hline 0.338 & JSC & JSC & ANSMET \\
\hline 0.263 & NIPR & NIPR & A. Yamaguchi \\
\hline 0.260 & JSC & JSC & ANSMET \\
\hline 20.9 & IfP & Finder & M. Patzek, A. anonymous \\
\hline 20.1 & IfP & Finder & K. Klemm andNasser Albuai \\
\hline 20 & MNB & Said Yousfi & A. Greshake, I \\
\hline 114 & UPC & \multirow{2}{*}{\multicolumn{2}{|c|}{$\begin{array}{l}\text { José Vicente (J. Llorca, UPCJosé Vicente ( } \\
\text { Ion Ander Lan J. Llorca, UPClon Ander Lan }\end{array}$}} \\
\hline 32 & UPC & & \\
\hline 20.2 & IfP & Finder & K. Klemm andNasser Albuai \\
\hline 80 & \multicolumn{3}{|c|}{ IGGCAS, PM(several Chine:S. Hu, Y. Lin e } \\
\hline 60 & FMMR & \multicolumn{2}{|c|}{ Timur KryachkPavel Yu. PlecTimur Kryachk } \\
\hline 80 & CEREGE & \multicolumn{2}{|c|}{ ArtMet collectiJ. Gattacceca: anonymous } \\
\hline 12512 & UrFU & UrFU & V. V. SharygirUrFU meteorit \\
\hline 244.4 & MNB & Martin Goff & A. Greshake, I \\
\hline 26 & UPC & \multicolumn{2}{|c|}{ Alfonso Viera J. Llorca, UPC Alfonso Viera } \\
\hline 798.6 & UrFU & \multicolumn{2}{|c|}{798.6 g (3 sar V. V. SharygirUrFU meteorit } \\
\hline 48.1 & MNB & \multicolumn{2}{|c|}{ Tomasz JakukAnsgar Gresh:Tomasz Jakuk } \\
\hline 49 & CEREGE & \multicolumn{2}{|c|}{ Museo del meJ. Gattacceca:Rodrigo Martir } \\
\hline 4556 & KOPRI & KOPRI & C. Park, S. Y. J. I. Lee \\
\hline 4150 & PSF & PSF & A. Irving and $\leqslant$ \\
\hline 32 & ASU & \multicolumn{2}{|c|}{ Michael FarmıL. Garvie, ASı Orlando Cuev } \\
\hline 25.7 & CEREGE & \multicolumn{2}{|c|}{ ArtMet collectiJ. Gattacceca, anonymous } \\
\hline 98.3 & UCLA & \multicolumn{2}{|c|}{ Michael CottinA. Rubin, UCL } \\
\hline 1846.4 & NIPR & NIPR & A. Yamaguchi \\
\hline 1707.200 & JSC & JSC & ANSMET \\
\hline 39.1 & PSF & \multicolumn{2}{|c|}{ Kenosha Publ A. Irving and $\leqslant$} \\
\hline 1412.46 & GUT & GUT & B. Miao, Z. Xić \\
\hline 27.2 & MNB & Dario Ruiz & Ansgar Gresh Dario Ruiz \\
\hline 1043.2 & NIPR & NIPR & A. Yamaguchi \\
\hline 1031 & KOPRI & KOPRI & C. Park, S. Y. S. Park \\
\hline
\end{tabular}




\begin{tabular}{|c|c|c|c|}
\hline 28.2 & MNB & Dario Ruiz & Ansgar Gresh Dario Ruiz \\
\hline 1002.2 & NIPR & NIPR & A. Yamaguchi \\
\hline \multirow[t]{3}{*}{4.62} & MNB & \multicolumn{2}{|c|}{ Tomasz JakukAnsgar Gresh Tomasz Jakuk } \\
\hline & UCLA & \multicolumn{2}{|c|}{ Michael CottinA. Rubin, UCL } \\
\hline & UCLA & \multicolumn{2}{|c|}{ Michael CottinA. Rubin, UCL } \\
\hline \multirow[t]{8}{*}{4.55} & MNB & \multicolumn{2}{|c|}{ Hichame Mim:A. Greshake, I } \\
\hline & KOPRI & KOPRI & C. Park, S. Y. S. Park \\
\hline & UCLA & Nick Gessler & A. Rubin, UCL \\
\hline & NIPR & NIPR & A. Yamaguchi \\
\hline & USil & Tomasz Jakı & KR. Kryza, T.A. \\
\hline & UPC & José Vicente & (J. Llorca, UPCJosé Vicente ( \\
\hline & MSN-FI & Castellano & V. Moggi Cecc \\
\hline & UPC & \multicolumn{2}{|c|}{ José Vicente (J. Llorca, UPC José Vicente } \\
\hline \multirow[t]{6}{*}{4.84} & CEREGE & \multicolumn{2}{|c|}{ with governor JC Doumnangvillagers and I } \\
\hline & UPC & \multicolumn{2}{|c|}{ José Vicente (J. Llorca, UPCJosé Vicente ( } \\
\hline & NIPR & NIPR & A. Yamaguchi \\
\hline & UPC & \multicolumn{2}{|c|}{ Alfonso Viera J. Llorca, UPC Alfonso Viera } \\
\hline & MSN-FI & Castellano & V. Moggi Cecc \\
\hline & ROM & Gregory & S. Yokoyama, \\
\hline \multirow[t]{6}{*}{4.70} & ETH & \multicolumn{2}{|c|}{ Naturwissens(E. Gnos, B. HıAndreas Stuck } \\
\hline & NIPR & NIPR & A. Yamaguchi \\
\hline & CEREGE & \multicolumn{2}{|c|}{ CEREGE J. Gattacceca:Bertrand Devc } \\
\hline & UPC & \multicolumn{2}{|c|}{ Francisco Gal J. Llorca, UPCFrancisco Gal' } \\
\hline & KOPRI & KOPRI & C. Park, S. Y. H. Yoo \\
\hline & GUT & GUT & B. Miao, Z. Xić \\
\hline \multirow[t]{6}{*}{4.63} & JSC & JSC & ANSMET \\
\hline & NIPR & NIPR & A. Yamaguchi \\
\hline & MNB & \multicolumn{2}{|c|}{ Salamu HamaA. Greshake, I } \\
\hline & GUT & GUT & B. Miao, Z. Xici \\
\hline & NIPR & NIPR & A. Yamaguchi \\
\hline & MNB & \multicolumn{2}{|c|}{ Hanno Strufe A. Greshake, I } \\
\hline \multirow[t]{3}{*}{4.81} & JSC & JSC & SI $\quad$ ANSMET \\
\hline & GUT & GUT & B. Miao, Z. Xić \\
\hline & ROM & Gregory & J. Poitras and \\
\hline 33.7 & MNB & \multicolumn{2}{|c|}{ Franco VignatA. Greshake, I } \\
\hline 218.71 & JSC & JSC & SI $\quad$ ANSMET \\
\hline 26 & CEREGE & \multicolumn{2}{|c|}{ Labenne Luc J. Gattacceca: Labenne Luc } \\
\hline 32 & UPC & \multicolumn{2}{|c|}{ José Vicente (J. Llorca, UPCJosé Vicente ( } \\
\hline 39.2 & Vernad & \multicolumn{2}{|c|}{ T. Kryachko, kLorenz C. A., 'T. Kryachko, E } \\
\hline 183.169 & JSC & JSC & SI $\quad$ ANSMET \\
\hline 25.8 & MNB & \multicolumn{2}{|c|}{ Hanno Strufe A. Greshake, I } \\
\hline 26 & CEREGE & \multicolumn{2}{|c|}{ Eric Christens J. Gattacceca: Eric Christens } \\
\hline 171.68 & NIPR & NIPR & A. Yamaguchi \\
\hline 38.0 & Vernad. & Ilya Chaplygi & nLorenz C. A., 'llya Chaplygin \\
\hline 22.45 & UCLA & Nick Gessler & A. Rubin, UCL \\
\hline
\end{tabular}




\begin{tabular}{|c|c|c|c|c|}
\hline \multirow[t]{4}{*}{4.63} & 164.100 & JSC & JSC & ANSMET \\
\hline & 163.04 & NIPR & NIPR & A. Yamaguchi \\
\hline & 20.1 & MSN-FI & Castellano & V. Moggi Cecc \\
\hline & 162.5 & NIPR & NIPR & A. Yamaguchi \\
\hline \multirow[t]{2}{*}{4.48} & 26 & CEREGE & \multicolumn{2}{|c|}{ Eric Christens J. Gattacceca: Eric Christens } \\
\hline & 160.87 & NIPR & NIPR & A. Yamaguchi \\
\hline \multirow[t]{6}{*}{4.78} & 22.3 & MNB & \multicolumn{2}{|c|}{ Hanno Strufe A. Greshake, I } \\
\hline & 146.3 & KOPRI & KOPRI & C. Park, S. Y. H. Yoo \\
\hline & 135.4 & NIPR & NIPR & A. Yamaguchi \\
\hline & 134.6 & PSF & PSF & A. Irving and $\varsigma$ \\
\hline & 130.41 & NIPR & NIPR & A. Yamaguchi \\
\hline & 33 & UPC & \multicolumn{2}{|c|}{ Josep Maria EJ. Llorca, UPCJosep Maria E } \\
\hline \multirow[t]{5}{*}{4.18} & 128.801 & JSC & JSC & SI $\quad$ ANSMET \\
\hline & 126.11 & NIPR & NIPR & A. Yamaguchi \\
\hline & 24 & UPC & \multicolumn{2}{|c|}{ José Vicente (J. Llorca, UPC José Vicente } \\
\hline & 119.83 & NIPR & NIPR & A. Yamaguchi \\
\hline & 22 & UPC & \multicolumn{2}{|c|}{ José Vicente (J. Llorca, UPCJosé Vicente ( } \\
\hline \multirow[t]{2}{*}{4.58} & 23 & CEREGE & \multicolumn{2}{|c|}{ Eric Christens J. Gattacceca: Eric Christens } \\
\hline & 20 & UPC & \multicolumn{2}{|c|}{ José Vicente (J. Llorca, UPCJosé Vicente ( } \\
\hline \multirow[t]{2}{*}{4.65} & 100.68 & JSC & JSC & ANSMET \\
\hline & 24.5 & PSF & PSF & A. Irving and $\subseteq$ \\
\hline 4.81 & 96.903 & JSC & JSC & SI $\quad$ ANSMET \\
\hline \multirow[t]{3}{*}{4.47} & 95.83 & JSC & JSC & ANSMET \\
\hline & 93.8 & KOPRI & KOPRI & C. Park, S. Y. J. I. Lee \\
\hline & 93.31 & NIPR & NIPR & A. Yamaguchi \\
\hline 4.70 & 21 & CEREGE & \multicolumn{2}{|c|}{ Eric Christens J. Gattacceca: Eric Christens } \\
\hline \multirow[t]{2}{*}{4.55} & 92.66 & JSC & JSC & SI $\quad$ ANSMET \\
\hline & 90.6 & KOPRI & KOPRI & C. Park, S. Y. Z. Beach \\
\hline 5.21 & 87.186 & JSC & JSC & SI $\quad$ ANSMET \\
\hline \multirow[t]{3}{*}{4.45} & 20 & CEREGE & \multicolumn{2}{|c|}{ Eric Christens J. Gattacceca:Eric Christens } \\
\hline & 16.9 & Vernad & \multicolumn{2}{|c|}{ Mr. T. KryachlLorenz C. A., 'Mr. T. Kryachl } \\
\hline & 83.12 & NIPR & NIPR & A. Yamaguchi \\
\hline \multirow[t]{2}{*}{4.77} & 81.815 & JSC & JSC & SI $\quad$ ANSMET \\
\hline & 80.15 & PSF & PSF & A. Irving and $\subseteq$ \\
\hline 4.20 & 19 & CEREGE & \multicolumn{2}{|c|}{ Eric Christens J. Gattacceca: Eric Christens } \\
\hline 4.77 & 76.049 & JSC & JSC & SI $\quad$ ANSMET \\
\hline & 75.6 & KOPRI & KOPRI & C. Park, S. Y. J. I. Lee \\
\hline 4.63 & 74.96 & JSC & JSC & SI $\quad$ ANSMET \\
\hline 4.68 & 72.510 & JSC & JSC & ANSMET \\
\hline 4.62 & 71.720 & JSC & JSC & SI $\quad$ ANSMET \\
\hline & 71.5 & NIPR & NIPR & A. Yamaguchi \\
\hline & 14.52 & ROM & Gregory & J. Poitras and \\
\hline 4.64 & 16 & CEREGE & Eric Christer & sJ. Gattacceca:Eric Christens \\
\hline & 69.75 & NIPR & NIPR & A. Yamaguchi \\
\hline 4.70 & 65.518 & JSC & JSC & SI $\quad$ ANSMET \\
\hline
\end{tabular}




\begin{tabular}{|c|c|c|c|c|}
\hline 64.7 & KOPRI & KOPRI & \multicolumn{2}{|c|}{ C. Park, S. Y. S. Park } \\
\hline 63.720 & JSC & JSC & SI & ANSMET \\
\hline 60.93 & JSC & JSC & SI & ANSMET \\
\hline 60.430 & JSC & JSC & $\mathrm{SI}$ & ANSMET \\
\hline 60.160 & JSC & JSC & SI & ANSMET \\
\hline 21 & UPC & \multicolumn{3}{|c|}{ Alfonso Viera J. Llorca, UPC Alfonso Viera } \\
\hline 59.52 & GUT & GUT & \multicolumn{2}{|c|}{ B. Miao, Z. Xić } \\
\hline 58.710 & JSC & JSC & SI & ANSMET \\
\hline 58.32 & NIPR & NIPR & \multicolumn{2}{|c|}{ A. Yamaguchi } \\
\hline 13 & CEREGE & \multicolumn{3}{|c|}{ Eric Christens J. Gattacceca: Eric Christens } \\
\hline 57.619 & JSC & JSC & $\mathrm{SI}$ & ANSMET \\
\hline 55.38 & JSC & JSC & $\mathrm{SI}$ & ANSMET \\
\hline 53.791 & JSC & JSC & $\mathrm{SI}$ & ANSMET \\
\hline 12.9 & Vernad & DMUH & \multicolumn{2}{|l|}{ M. A. Ivanova } \\
\hline 50.959 & JSC & JSC & SI & ANSMET \\
\hline 50.53 & NIPR & NIPR & \multicolumn{2}{|c|}{ A. Yamaguchi } \\
\hline 50.173 & JSC & JSC & SI & ANSMET \\
\hline 48.399 & JSC & JSC & $\mathrm{SI}$ & ANSMET \\
\hline 9.61 & MSP & Castellano & \multicolumn{2}{|c|}{ V. Moggi Cecr } \\
\hline 47.05 & JSC & JSC & SI & ANSMET \\
\hline 47.040 & JSC & JSC & $\mathrm{SI}$ & ANSMET \\
\hline 22 & UPC & \multicolumn{3}{|c|}{ Ion Ander LanJ. Llorca, UPClon Ander Lan } \\
\hline 44.888 & JSC & JSC & SI & ANSMET \\
\hline 44.72 & JSC & JSC & SI & ANSMET \\
\hline 44.139 & JSC & JSC & $\mathrm{SI}$ & ANSMET \\
\hline 43.110 & JSC & JSC & SI & ANSMET \\
\hline 42.99 & JSC & JSC & SI & ANSMET \\
\hline 41.377 & JSC & JSC & $\mathrm{SI}$ & ANSMET \\
\hline 8.2 & UCLA & \multicolumn{3}{|c|}{ Robert Verish D. Sheikh, FS } \\
\hline 40.590 & JSC & JSC & SI & ANSMET \\
\hline 40.343 & NIPR & NIPR & \multicolumn{2}{|c|}{ A. Yamaguchi } \\
\hline 40.291 & NIPR & NIPR & \multicolumn{2}{|l|}{ A. Yamaguchi } \\
\hline 11 & UPC & \multicolumn{3}{|c|}{ David AllepuzJ. Llorca, UPCDavid Allepuz. } \\
\hline 39.521 & NIPR & NIPR & \multicolumn{2}{|c|}{ A. Yamaguchi } \\
\hline 39.384 & JSC & JSC & SI & ANSMET \\
\hline 39.217 & JSC & JSC & SI & ANSMET \\
\hline 39.179 & NIPR & NIPR & \multicolumn{2}{|l|}{ A. Yamaguchi } \\
\hline 38 & CEREGE & CEREGE & \multicolumn{2}{|c|}{ H. Pourkhorsa J. Gattacceca } \\
\hline 38.47 & GUT & GUT & \multicolumn{2}{|c|}{ B. Miao, Z. Xic } \\
\hline 37.319 & JSC & JSC & SI & ANSMET \\
\hline 36.611 & JSC & JSC & SI & ANSMET \\
\hline 36.144 & NIPR & NIPR & \multicolumn{2}{|c|}{ A. Yamaguchi } \\
\hline 36.12 & JSC & JSC & $\mathrm{SI}$ & ANSMET \\
\hline 36.032 & JSC & JSC & SI & ANSMET \\
\hline 36 & KOPRI & KOPRI & \multicolumn{2}{|c|}{ C. Park, S. Y. S. Park } \\
\hline
\end{tabular}




\begin{tabular}{|c|c|c|c|c|c|}
\hline 4.84 & 35.984 & JSC & JSC & SI & ANSMET \\
\hline 4.56 & 35.852 & JSC & JSC & SI & ANSMET \\
\hline \multirow[t]{2}{*}{4.66} & 35.31 & JSC & JSC & SI & ANSMET \\
\hline & 35.225 & NIPR & NIPR & \multicolumn{2}{|c|}{ A. Yamaguchi } \\
\hline 4.58 & 35.160 & JSC & JSC & SI & ANSMET \\
\hline 4.51 & 35.112 & JSC & JSC & SI & ANSMET \\
\hline 4.45 & 9 & CEREGE & Eric Christen & J. Gattacceca: & Eric Christens \\
\hline \multirow[t]{2}{*}{4.55} & 34.63 & JSC & JSC & SI & ANSMET \\
\hline & 6.83 & MSP & Castellano & \multicolumn{2}{|l|}{ V. Moggi Cecc } \\
\hline 4.91 & 33.987 & JSC & JSC & SI & ANSMET \\
\hline 4.53 & 33.898 & JSC & JSC & SI & ANSMET \\
\hline 4.74 & 33.249 & JSC & JSC & SI & ANSMET \\
\hline 4.76 & 33.229 & JSC & JSC & SI & ANSMET \\
\hline 4.50 & 33.113 & JSC & JSC & SI & ANSMET \\
\hline 4.64 & 32.429 & JSC & JSC & SI & ANSMET \\
\hline \multirow[t]{3}{*}{4.77} & 32.146 & JSC & JSC & SI & ANSMET \\
\hline & 32.074 & NIPR & NIPR & \multicolumn{2}{|c|}{ A. Yamaguchi } \\
\hline & 7 & UPC & \multicolumn{3}{|c|}{ José Vicente (J. Llorca, UPC José Vicente ( } \\
\hline 4.46 & 31.977 & JSC & JSC & SI & ANSMET \\
\hline 4.52 & 31.88 & JSC & JSC & SI & ANSMET \\
\hline 4.66 & 31.461 & JSC & JSC & SI & ANSMET \\
\hline 4.46 & 31.36 & JSC & JSC & SI & ANSMET \\
\hline 4.53 & 31.11 & JSC & JSC & SI & ANSMET \\
\hline 4.53 & 31.067 & JSC & JSC & $\mathrm{SI}$ & ANSMET \\
\hline 4.73 & 31.060 & JSC & JSC & $\mathrm{SI}$ & ANSMET \\
\hline \multirow[t]{2}{*}{4.52} & 30.852 & JSC & JSC & SI & ANSMET \\
\hline & 30.81 & GUT & GUT & \multicolumn{2}{|l|}{ B. Miao, Z. Xiá } \\
\hline 4.41 & 30.710 & JSC & JSC & SI & ANSMET \\
\hline \multirow[t]{2}{*}{4.57} & 30.7 & JSC & JSC & SI & ANSMET \\
\hline & 30.5 & KOPRI & KOPRI & \multicolumn{2}{|c|}{ C. Park, S. Y. J. I. Lee } \\
\hline 4.75 & 30.45 & JSC & JSC & SI & ANSMET \\
\hline 4.72 & 30.263 & JSC & JSC & SI & ANSMET \\
\hline 4.45 & 30.11 & JSC & JSC & SI & ANSMET \\
\hline \multirow[t]{2}{*}{4.61} & 30.01 & JSC & JSC & SI & ANSMET \\
\hline & 28.7 & Cascadia & Cascadia & \multicolumn{2}{|c|}{ A. Ruzicka, K. } \\
\hline 4.62 & 29.744 & JSC & JSC & SI & ANSMET \\
\hline 4.53 & 29.675 & JSC & JSC & SI & ANSMET \\
\hline \multirow[t]{2}{*}{4.86} & 29.383 & JSC & JSC & SI & ANSMET \\
\hline & 28.726 & NIPR & NIPR & \multicolumn{2}{|l|}{ A. Yamaguchi } \\
\hline 4.5 & 28.481 & JSC & JSC & SI & ANSMET \\
\hline 4.59 & 28.381 & JSC & JSC & SI & ANSMET \\
\hline \multirow[t]{3}{*}{4.54} & 28.124 & JSC & JSC & SI & ANSMET \\
\hline & 28.007 & NIPR & NIPR & \multicolumn{2}{|c|}{ A. Yamaguchi } \\
\hline & 27.77 & GUT & GUT & \multicolumn{2}{|l|}{ B. Miao, Z. Xié } \\
\hline 4.44 & 27.251 & JSC & JSC & SI & ANSMET \\
\hline
\end{tabular}




$\begin{array}{llllll}4.57 & 27.106 & \text { JSC } & \text { JSC } & \text { SI } & \text { ANSMET } \\ 4.66 & 27.096 & \text { JSC } & \text { JSC } & \text { SI } & \text { ANSMET } \\ 4.53 & 27.09 & \text { JSC } & \text { JSC } & \text { SI } & \text { ANSMET } \\ 4.72 & 26.979 & \text { JSC } & \text { JSC } & \text { SI } & \text { ANSMET } \\ 4.51 & 26.66 & \text { JSC } & \text { JSC } & \text { SI } & \text { ANSMET } \\ 4.69 & 26.52 & \text { JSC } & \text { JSC } & \text { SI } & \text { ANSMET } \\ 4.56 & 26.48 & \text { JSC } & \text { JSC } & \text { SI } & \text { ANSMET } \\ 4.59 & 26.455 & \text { JSC } & \text { JSC } & \text { SI } & \text { ANSMET } \\ & 26.4 & \text { KOPRI } & \text { KOPRI } & \text { C. Park, S. Y. Z. Beach } \\ 4.49 & 26.291 & \text { JSC } & \text { JSC } & \text { SI } & \text { ANSMET } \\ 4.58 & 26.110 & \text { JSC } & \text { JSC } & \text { SI } & \text { ANSMET } \\ 4.51 & 26.09 & \text { JSC } & \text { JSC } & \text { SI } & \text { ANSMET }\end{array}$

4.59

6

José Vicente (J. Llorca, UPCJosé Vicente (

4.51

25.743

UPC

JSC SI

ANSMET

4.43

25.278

JSC

JSC

ANSMET

4.57

25.186

JSC

ANSMET

25.12

JSC

JSC

ANSMET

24.92

JSC

PSF

SI

$24.850 \quad J S C$

JSC

A. Irving and $s$

4.74

24.475 JSC

JSC

SI

ANSMET

4.63

24.46

JSC

JSC

SI

ANSMET

4.52

23.887

JSC

JSC

ANSMET

4.54

23.509

JSC

JSC

ANSMET

23.48

PSF

PSF

ANSMET

4.54

23.19

JSC

JSC

A. Irving and $\subseteq$

4.53

23.12

JSC

JSC

SI

ANSMET

4.54

23.062

JSC

JSC

SI

ANSMET

4.54

23.055

JSC

JSC

SI

ANSMET

4.65

22.804

JSC

JSC

ANSMET

4.5

ROM

Gregory

SI

ANSMET

22.49

GUT

GUT

S. Yokoyama,

$22.367 \quad$ NIPR

NIPR

B. Miao, Z. Xí

4.41

21.980

JSC

JSC

A. Yamaguchi

21.8

KOPRI

KOPRI

SI

ANSMET

4.72

21.44

JSC

JSC

C.

S. Park

4.55

21.307

JSC

JSC

SI

ANSMET

4.48

20.873

JSC

JSC

ANSMET

4.47

20.598

JSC

JSC

ANSMET

4.49

20.58

JSC

JSC

ANSMET

4.61

20.47

JSC

JSC

ANSMET

4.46

20.31

JSC

JSC

ANSMET

5.20

20.093

JSC

JSC

ANSMET

4.33

5

CEREGE

$S$

ANSMET

4.64

19.662

JSC

JSC

J. Gat

Eric Christens

19.597

JSC

JSC

SI

ANSMET

ANSMET 


\begin{tabular}{|c|c|c|c|c|c|}
\hline & 19.428 & NIPR & NIPR & \multicolumn{2}{|c|}{ A. Yamaguchi } \\
\hline 4.63 & 19.33 & JSC & JSC & $\mathrm{SI}$ & ANSMET \\
\hline \multirow[t]{2}{*}{4.60} & 19.293 & JSC & JSC & SI & ANSMET \\
\hline & 19 & KOPRI & KOPRI & \multicolumn{2}{|c|}{ C. Park, S. Y. H. Yoo } \\
\hline 4.63 & 18.87 & JSC & JSC & SI & ANSMET \\
\hline 4.58 & 18.548 & JSC & JSC & SI & ANSMET \\
\hline 4.53 & 18.510 & JSC & JSC & SI & ANSMET \\
\hline 4.67 & 18.27 & JSC & JSC & SI & ANSMET \\
\hline \multirow[t]{2}{*}{4.5} & 18.13 & JSC & JSC & SI & ANSMET \\
\hline & 7 & UPC & \multicolumn{3}{|c|}{ David Allepuz J. Llorca, UPCDavid Allepuz } \\
\hline 4.54 & 18.076 & JSC & JSC & SI & ANSMET \\
\hline 4.56 & 18.055 & JSC & JSC & SI & ANSMET \\
\hline 4.59 & 18.04 & JSC & JSC & SI & ANSMET \\
\hline 4.58 & 17.966 & JSC & JSC & SI & ANSMET \\
\hline 4.54 & 17.842 & JSC & JSC & SI & ANSMET \\
\hline 4.41 & 17.726 & JSC & JSC & SI & ANSMET \\
\hline \multirow[t]{2}{*}{4.46} & 17.513 & JSC & JSC & SI & ANSMET \\
\hline & 17.255 & NIPR & NIPR & \multicolumn{2}{|c|}{ A. Yamaguchi } \\
\hline 4.78 & 17.157 & JSC & JSC & SI & ANSMET \\
\hline \multirow[t]{3}{*}{4.56} & 17.133 & JSC & JSC & SI & ANSMET \\
\hline & 16 & UPC & \multicolumn{3}{|c|}{ José Vicente (J. Llorca, UPC José Vicente ( } \\
\hline & 16.926 & NIPR & NIPR & \multicolumn{2}{|c|}{ A. Yamaguchi } \\
\hline \multirow[t]{4}{*}{4.56} & 16.705 & JSC & JSC & SI & ANSMET \\
\hline & 16.7 & KOPRI & KOPRI & \multicolumn{2}{|c|}{ C. Park, S. Y. J. Woo } \\
\hline & 4.0 & Vernad & DMUH & \multicolumn{2}{|c|}{ C. A. Lorenz, ' } \\
\hline & 3.5 & UCLA & Verish & \multicolumn{2}{|c|}{ D. Sheikh, FS Mark Bittmanr } \\
\hline 4.59 & 16.290 & JSC & JSC & SI & ANSMET \\
\hline 4.61 & 16.209 & JSC & JSC & SI & ANSMET \\
\hline 4.60 & 16.092 & JSC & JSC & SI & ANSMET \\
\hline 4.74 & 4.2 & CEREGE & \multicolumn{3}{|c|}{ Eric Christens J. Gattacceca: Eric Christens } \\
\hline 4.58 & 15.63 & JSC & JSC & SI & ANSMET \\
\hline 4.42 & 15.329 & JSC & JSC & SI & ANSMET \\
\hline \multirow[t]{2}{*}{4.58} & 15.128 & JSC & JSC & SI & ANSMET \\
\hline & 15.084 & JSC & JSC & SI & ANSMET \\
\hline 4.67 & 14.912 & JSC & JSC & SI & ANSMET \\
\hline 4.53 & 14.860 & JSC & JSC & SI & ANSMET \\
\hline \multirow[t]{2}{*}{4.55} & 14.75 & JSC & JSC & SI & ANSMET \\
\hline & 14.71 & GUT & GUT & \multicolumn{2}{|c|}{ B. Miao, Z. Xiś } \\
\hline 4.68 & 14.357 & JSC & JSC & SI & ANSMET \\
\hline 4.85 & 14.34 & JSC & JSC & SI & ANSMET \\
\hline 4.79 & 14.304 & JSC & JSC & SI & ANSMET \\
\hline \multirow[t]{2}{*}{4.63} & 14.2 & JSC & JSC & SI & ANSMET \\
\hline & 14.1 & KOPRI & KOPRI & \multicolumn{2}{|c|}{ C. Park, S. Y. J. H. Kim } \\
\hline 4.52 & 14.015 & JSC & JSC & SI & ANSMET \\
\hline 4.55 & 13.87 & JSC & JSC & SI & ANSMET \\
\hline
\end{tabular}




\begin{tabular}{|c|c|c|c|c|c|}
\hline & 13.821 & NIPR & NIPR & \multicolumn{2}{|c|}{ A. Yamaguchi } \\
\hline 4.57 & 13.742 & JSC & JSC & SI & ANSMET \\
\hline 4.67 & 13.63 & JSC & JSC & SI & ANSMET \\
\hline 4.58 & 13.52 & JSC & JSC & SI & ANSMET \\
\hline 4.62 & 13.469 & JSC & JSC & SI & ANSMET \\
\hline 4.61 & 13.42 & JSC & JSC & SI & ANSMET \\
\hline 4.56 & 13.280 & JSC & JSC & SI & ANSMET \\
\hline 4.54 & 13.270 & JSC & JSC & SI & ANSMET \\
\hline \multirow[t]{2}{*}{4.55} & 13.268 & JSC & JSC & SI & ANSMET \\
\hline & 7 & UPC & \multicolumn{3}{|c|}{ David Allepuz J. Llorca, UPCDavid Allepuz. } \\
\hline 4.59 & 13.08 & JSC & JSC & $\mathrm{SI}$ & ANSMET \\
\hline 4.53 & 12.92 & JSC & JSC & SI & ANSMET \\
\hline 4.55 & 12.874 & JSC & JSC & SI & ANSMET \\
\hline 4.47 & 12.388 & JSC & JSC & SI & ANSMET \\
\hline \multirow[t]{3}{*}{4.57} & 12.08 & JSC & JSC & $\mathrm{SI}$ & ANSMET \\
\hline & 11 & UPC & \multicolumn{3}{|c|}{ José Vicente (J. Llorca, UPC José Vicente ( } \\
\hline & 11.973 & NIPR & NIPR & \multicolumn{2}{|c|}{ A. Yamaguchi } \\
\hline 5.08 & 11.936 & JSC & JSC & SI & ANSMET \\
\hline 4.57 & 11.825 & JSC & JSC & SI & ANSMET \\
\hline 4.51 & 11.65 & JSC & JSC & SI & ANSMET \\
\hline 4.52 & 11.54 & JSC & JSC & SI & ANSMET \\
\hline \multirow[t]{2}{*}{4.56} & 11.29 & JSC & JSC & SI & ANSMET \\
\hline & 8.4 & UrFU & \multicolumn{3}{|c|}{$8.4 \mathrm{~g}$ cut-off - V.V. Sharygin UrFU meteorit } \\
\hline 4.66 & 11.190 & JSC & JSC & SI & ANSMET \\
\hline 4.62 & 11.129 & JSC & JSC & SI & ANSMET \\
\hline 4.54 & 10.956 & JSC & JSC & SI & ANSMET \\
\hline 4.56 & 10.830 & JSC & JSC & SI & ANSMET \\
\hline 4.31 & 10.68 & JSC & JSC & SI & ANSMET \\
\hline 4.59 & 10.55 & JSC & JSC & SI & ANSMET \\
\hline \multirow[t]{2}{*}{4.55} & 10.24 & JSC & JSC & SI & ANSMET \\
\hline & 10.2 & KOPRI & KOPRI & \multicolumn{2}{|c|}{ C. Park, S. Y. H. Yoo } \\
\hline 4.67 & 10.154 & JSC & JSC & SI & ANSMET \\
\hline 4.52 & 9.975 & JSC & JSC & SI & ANSMET \\
\hline 4.57 & 9.92 & JSC & JSC & SI & ANSMET \\
\hline 4.81 & 9.92 & JSC & JSC & SI & ANSMET \\
\hline \multirow[t]{3}{*}{4.85} & 3 & CEREGE & \multicolumn{3}{|c|}{ Eric Christens H. PourkhorsaEric Christens } \\
\hline & 9.839 & NIPR & NIPR & \multicolumn{2}{|c|}{ A. Yamaguchi } \\
\hline & 9.688 & NIPR & NIPR & \multicolumn{2}{|c|}{ A. Yamaguchi } \\
\hline 4.67 & 9.6 & JSC & JSC & SI & ANSMET \\
\hline 4.74 & 9.19 & JSC & JSC & SI & ANSMET \\
\hline \multirow[t]{3}{*}{4.77} & 9.14 & JSC & JSC & SI & ANSMET \\
\hline & 9.08 & NIPR & NIPR & \multicolumn{2}{|c|}{ A. Yamaguchi } \\
\hline & 9 & KOPRI & KOPRI & \multicolumn{2}{|c|}{ C. Park, S. Y. J. I. Lee } \\
\hline \multirow[t]{2}{*}{4.37} & 8.923 & JSC & JSC & SI & ANSMET \\
\hline & 8.372 & NIPR & NIPR & \multicolumn{2}{|c|}{ A. Yamaguchi } \\
\hline
\end{tabular}




\begin{tabular}{|c|c|c|c|c|}
\hline & 8.355 & NIPR & NIPR & A. Yamaguchi \\
\hline & 8.1 & KOPRI & KOPRI & C. Park, S. Y. S. Park \\
\hline & 8.041 & NIPR & NIPR & A. Yamaguchi \\
\hline 4.56 & 8 & JSC & JSC & SI $\quad$ ANSMET \\
\hline \multirow[t]{2}{*}{4.66} & 7.8 & JSC & JSC & ANSMET \\
\hline & 7.527 & NIPR & NIPR & A. Yamaguchi \\
\hline 4.96 & 7.451 & JSC & JSC & SI $\quad$ ANSMET \\
\hline 4.27 & 7.386 & JSC & JSC & ANSMET \\
\hline 4.54 & 7.176 & JSC & JSC & ANSMET \\
\hline \multirow[t]{2}{*}{4.68} & 7.146 & JSC & JSC & SI $\quad$ ANSMET \\
\hline & 6.7 & KOPRI & KOPRI & C. Park, S. Y. J. Woo \\
\hline 4.70 & 6.607 & JSC & JSC & SI $\quad$ ANSMET \\
\hline \multirow[t]{4}{*}{4.5} & 6.44 & JSC & JSC & SI $\quad$ ANSMET \\
\hline & 6.361 & NIPR & NIPR & A. Yamaguchi \\
\hline & 6 & KOPRI & KOPRI & C. Park, S. Y. H. Yoo \\
\hline & 6 & UPC & \multicolumn{2}{|c|}{ José Vicente (J. Llorca, UPCJosé Vicente ( } \\
\hline \multirow[t]{6}{*}{4.57} & 5.99 & JSC & JSC & SI $\quad$ ANSMET \\
\hline & 5.572 & NIPR & NIPR & A. Yamaguchi \\
\hline & 5.3 & KOPRI & KOPRI & C. Park, S. Y. S. Park \\
\hline & 5.187 & NIPR & NIPR & A. Yamaguchi \\
\hline & 5.165 & NIPR & NIPR & A. Yamaguchi \\
\hline & 5.13 & NIPR & NIPR & A. Yamaguchi \\
\hline \multirow[t]{23}{*}{4.60} & 5 & CEREGE & CEREGE & H. Pourkhorsa Lydie Bonal \\
\hline & 4.989 & NIPR & NIPR & A. Yamaguchi \\
\hline & 4.93 & NIPR & NIPR & A. Yamaguchi \\
\hline & 4.875 & NIPR & NIPR & A. Yamaguchi \\
\hline & 4.812 & NIPR & NIPR & A. Yamaguchi \\
\hline & 4.75 & NIPR & NIPR & A. Yamaguchi \\
\hline & 4.733 & NIPR & NIPR & A. Yamaguchi \\
\hline & 4.69 & NIPR & NIPR & A. Yamaguchi \\
\hline & 4.596 & NIPR & NIPR & A. Yamaguchi \\
\hline & 4.529 & NIPR & NIPR & A. Yamaguchi \\
\hline & 4.438 & NIPR & NIPR & A. Yamaguchi \\
\hline & 4.41 & NIPR & NIPR & A. Yamaguchi \\
\hline & 4.376 & NIPR & NIPR & A. Yamaguchi \\
\hline & 4.375 & NIPR & NIPR & A. Yamaguchi \\
\hline & 4.359 & NIPR & NIPR & A. Yamaguchi \\
\hline & 4.276 & NIPR & NIPR & A. Yamaguchi \\
\hline & 4.228 & NIPR & NIPR & A. Yamaguchi \\
\hline & 4.185 & NIPR & NIPR & A. Yamaguchi \\
\hline & 4.176 & NIPR & NIPR & A. Yamaguchi \\
\hline & 4.162 & NIPR & NIPR & A. Yamaguchi \\
\hline & 4.135 & NIPR & NIPR & A. Yamaguchi \\
\hline & 4.099 & NIPR & NIPR & A. Yamaguchi \\
\hline & 4.086 & NIPR & NIPR & A. Yamaguchi \\
\hline
\end{tabular}




\begin{tabular}{|c|c|c|c|}
\hline 3.998 & NIPR & NIPR & A. Yamaguchi \\
\hline 3.9 & KOPRI & KOPRI & C. Park, S. Y. S. Park \\
\hline 3.9 & NIPR & NIPR & A. Yamaguchi \\
\hline 3.841 & NIPR & NIPR & A. Yamaguchi \\
\hline 3.83 & JSC & JSC & $\begin{array}{ll}\text { SI } & \text { ANSMET }\end{array}$ \\
\hline 3.811 & NIPR & NIPR & A. Yamaguchi \\
\hline 3.756 & NIPR & NIPR & A. Yamaguchi \\
\hline 3.731 & NIPR & NIPR & A. Yamaguchi \\
\hline 3.718 & NIPR & NIPR & A. Yamaguchi \\
\hline 3.6 & KOPRI & KOPRI & C. Park, S. Y. J. I. Lee \\
\hline 3.565 & NIPR & NIPR & A. Yamaguchi \\
\hline 3.549 & NIPR & NIPR & A. Yamaguchi \\
\hline 3.523 & NIPR & NIPR & A. Yamaguchi \\
\hline 3.491 & NIPR & NIPR & A. Yamaguchi \\
\hline 3.4 & KOPRI & KOPRI & C. Park, S. Y. B. W. Son \\
\hline 3.375 & NIPR & NIPR & A. Yamaguchi \\
\hline 3.319 & NIPR & NIPR & A. Yamaguchi \\
\hline 3.296 & NIPR & NIPR & A. Yamaguchi \\
\hline 3.261 & NIPR & NIPR & A. Yamaguchi \\
\hline 3.257 & NIPR & NIPR & A. Yamaguchi \\
\hline 3.251 & NIPR & NIPR & A. Yamaguchi \\
\hline 3.238 & NIPR & NIPR & A. Yamaguchi \\
\hline 3.2 & KOPRI & KOPRI & C. Park, S. Y. J. Woo \\
\hline 3.173 & NIPR & NIPR & A. Yamaguchi \\
\hline 3.161 & NIPR & NIPR & A. Yamaguchi \\
\hline 3.143 & NIPR & NIPR & A. Yamaguchi \\
\hline 3.131 & NIPR & NIPR & A. Yamaguchi \\
\hline 3.086 & NIPR & NIPR & A. Yamaguchi \\
\hline 3.075 & NIPR & NIPR & A. Yamaguchi \\
\hline 3.019 & NIPR & NIPR & A. Yamaguchi \\
\hline 2 & UPC & \multicolumn{2}{|c|}{ José Vicente (J. Llorca, UPCJosé Vicente ( } \\
\hline 2.974 & NIPR & NIPR & A. Yamaguchi \\
\hline 2.932 & NIPR & NIPR & A. Yamaguchi \\
\hline 2.922 & NIPR & NIPR & A. Yamaguchi \\
\hline 2.9 & KOPRI & KOPRI & C. Park, S. Y. H. Yoo \\
\hline 2.9 & KOPRI & KOPRI & C. Park, S. Y. H. Yoo \\
\hline 2.9 & NIPR & NIPR & A. Yamaguchi \\
\hline 2.892 & NIPR & NIPR & A. Yamaguchi \\
\hline 2.882 & NIPR & NIPR & A. Yamaguchi \\
\hline 2.814 & NIPR & NIPR & A. Yamaguchi \\
\hline 2.81 & NIPR & NIPR & A. Yamaguchi \\
\hline 2.747 & NIPR & NIPR & A. Yamaguchi \\
\hline 2.676 & NIPR & NIPR & A. Yamaguchi \\
\hline 2.654 & NIPR & NIPR & A. Yamaguchi \\
\hline 2.627 & NIPR & NIPR & A. Yamaguchi \\
\hline
\end{tabular}




\begin{tabular}{|c|c|c|c|}
\hline 2.609 & NIPR & NIPR & A. Yamaguchi \\
\hline 2.603 & NIPR & NIPR & A. Yamaguchi \\
\hline 2.6 & KOPRI & KOPRI & C. Park, S. Y. J. I. Lee \\
\hline 2.585 & NIPR & NIPR & A. Yamaguchi \\
\hline 2.569 & NIPR & NIPR & A. Yamaguchi \\
\hline 2.565 & NIPR & NIPR & A. Yamaguchi \\
\hline 2.564 & NIPR & NIPR & A. Yamaguchi \\
\hline 2.5 & KOPRI & KOPRI & C. Park, S. Y. B. W. Son \\
\hline 2.482 & NIPR & NIPR & A. Yamaguchi \\
\hline 2.474 & NIPR & NIPR & A. Yamaguchi \\
\hline 2.39 & NIPR & NIPR & A. Yamaguchi \\
\hline 2.377 & NIPR & NIPR & A. Yamaguchi \\
\hline 2.339 & NIPR & NIPR & A. Yamaguchi \\
\hline 2.323 & NIPR & NIPR & A. Yamaguchi \\
\hline 2.303 & NIPR & NIPR & A. Yamaguchi \\
\hline 2.287 & NIPR & NIPR & A. Yamaguchi \\
\hline 2.287 & NIPR & NIPR & A. Yamaguchi \\
\hline 2.232 & NIPR & NIPR & A. Yamaguchi \\
\hline 2.221 & NIPR & NIPR & A. Yamaguchi \\
\hline 2.221 & NIPR & NIPR & A. Yamaguchi \\
\hline 2.2 & KOPRI & KOPRI & C. Park, S. Y. S. Han \\
\hline 2.18 & NIPR & NIPR & A. Yamaguchi \\
\hline 2.174 & NIPR & NIPR & A. Yamaguchi \\
\hline 2.171 & NIPR & NIPR & A. Yamaguchi \\
\hline 2.147 & NIPR & NIPR & A. Yamaguchi \\
\hline 2.133 & NIPR & NIPR & A. Yamaguchi \\
\hline 2.121 & NIPR & NIPR & A. Yamaguchi \\
\hline 2.106 & NIPR & NIPR & A. Yamaguchi \\
\hline 2.104 & NIPR & NIPR & A. Yamaguchi \\
\hline 2.1 & KOPRI & KOPRI & C. Park, S. Y. J. I. Lee \\
\hline 2.082 & NIPR & NIPR & A. Yamaguchi \\
\hline 2.073 & NIPR & NIPR & A. Yamaguchi \\
\hline 2.052 & NIPR & NIPR & A. Yamaguchi \\
\hline 2.009 & NIPR & NIPR & A. Yamaguchi \\
\hline 2 & NIPR & NIPR & A. Yamaguchi \\
\hline 1.975 & NIPR & NIPR & A. Yamaguchi \\
\hline 1.961 & NIPR & NIPR & A. Yamaguchi \\
\hline 1.956 & NIPR & NIPR & A. Yamaguchi \\
\hline 1.952 & NIPR & NIPR & A. Yamaguchi \\
\hline 1.895 & NIPR & NIPR & A. Yamaguchi \\
\hline 1.889 & NIPR & NIPR & A. Yamaguchi \\
\hline 1.867 & NIPR & NIPR & A. Yamaguchi \\
\hline 1.851 & NIPR & NIPR & A. Yamaguchi \\
\hline 1.849 & NIPR & NIPR & A. Yamaguchi \\
\hline 1.837 & NIPR & NIPR & A. Yamaguchi \\
\hline
\end{tabular}




\begin{tabular}{|c|c|c|c|}
\hline 1.835 & NIPR & NIPR & A. Yamaguchi \\
\hline 1.832 & NIPR & NIPR & A. Yamaguchi \\
\hline 1.816 & NIPR & NIPR & A. Yamaguchi \\
\hline 1.806 & NIPR & NIPR & A. Yamaguchi \\
\hline 1.741 & NIPR & NIPR & A. Yamaguchi \\
\hline 1.731 & NIPR & NIPR & A. Yamaguchi \\
\hline 1.723 & NIPR & NIPR & A. Yamaguchi \\
\hline 1.709 & NIPR & NIPR & A. Yamaguchi \\
\hline 1.709 & NIPR & NIPR & A. Yamaguchi \\
\hline 1.678 & NIPR & NIPR & A. Yamaguchi \\
\hline 1.665 & NIPR & NIPR & A. Yamaguchi \\
\hline 1.596 & NIPR & NIPR & A. Yamaguchi \\
\hline 1.487 & NIPR & NIPR & A. Yamaguchi \\
\hline 1.47 & NIPR & NIPR & A. Yamaguchi \\
\hline 1.449 & NIPR & NIPR & A. Yamaguchi \\
\hline 1.421 & NIPR & NIPR & A. Yamaguchi \\
\hline 1.397 & NIPR & NIPR & A. Yamaguchi \\
\hline 1.392 & NIPR & NIPR & A. Yamaguchi \\
\hline 1.367 & NIPR & NIPR & A. Yamaguchi \\
\hline 1.322 & NIPR & NIPR & A. Yamaguchi \\
\hline 1.29 & NIPR & NIPR & A. Yamaguchi \\
\hline 1.282 & NIPR & NIPR & A. Yamaguchi \\
\hline 1.273 & NIPR & NIPR & A. Yamaguchi \\
\hline 1.255 & NIPR & NIPR & A. Yamaguchi \\
\hline 1.251 & NIPR & NIPR & A. Yamaguchi \\
\hline 1.251 & NIPR & NIPR & A. Yamaguchi \\
\hline 1.250 & JSC & JSC & $\begin{array}{ll}\text { SI } & \text { ANSMET }\end{array}$ \\
\hline 1.25 & NIPR & NIPR & A. Yamaguchi \\
\hline 1.236 & NIPR & NIPR & A. Yamaguchi \\
\hline 1.204 & NIPR & NIPR & A. Yamaguchi \\
\hline 1.186 & NIPR & NIPR & A. Yamaguchi \\
\hline 1.168 & NIPR & NIPR & A. Yamaguchi \\
\hline 1.147 & NIPR & NIPR & A. Yamaguchi \\
\hline 1.143 & NIPR & NIPR & A. Yamaguchi \\
\hline 1.111 & NIPR & NIPR & A. Yamaguchi \\
\hline 1.089 & NIPR & NIPR & A. Yamaguchi \\
\hline 1.076 & NIPR & NIPR & A. Yamaguchi \\
\hline 1.070 & JSC & JSC & SI $\quad$ ANSMET \\
\hline 1.041 & NIPR & NIPR & A. Yamaguchi \\
\hline 1.036 & NIPR & NIPR & A. Yamaguchi \\
\hline 1.02 & NIPR & NIPR & A. Yamaguchi \\
\hline 1.010 & JSC & JSC & SI $\quad$ ANSMET \\
\hline 0.999 & NIPR & NIPR & A. Yamaguchi \\
\hline 0.969 & NIPR & NIPR & A. Yamaguchi \\
\hline 0.967 & NIPR & NIPR & A. Yamaguchi \\
\hline
\end{tabular}




\begin{tabular}{|c|c|c|c|}
\hline 0.888 & NIPR & NIPR & A. Yamaguchi \\
\hline 0.873 & NIPR & NIPR & A. Yamaguchi \\
\hline 0.857 & NIPR & NIPR & A. Yamaguchi \\
\hline 0.854 & NIPR & NIPR & A. Yamaguchi \\
\hline 0.837 & NIPR & NIPR & A. Yamaguchi \\
\hline 0.835 & JSC & JSC & $\begin{array}{ll}\text { SI } & \text { ANSMET }\end{array}$ \\
\hline 0.826 & NIPR & NIPR & A. Yamaguchi \\
\hline 0.821 & NIPR & NIPR & A. Yamaguchi \\
\hline 0.815 & NIPR & NIPR & A. Yamaguchi \\
\hline 0.795 & NIPR & NIPR & A. Yamaguchi \\
\hline 0.783 & NIPR & NIPR & A. Yamaguchi \\
\hline 0.775 & NIPR & NIPR & A. Yamaguchi \\
\hline 0.77 & NIPR & NIPR & A. Yamaguchi \\
\hline 0.768 & NIPR & NIPR & A. Yamaguchi \\
\hline 0.752 & NIPR & NIPR & A. Yamaguchi \\
\hline 0.748 & NIPR & NIPR & A. Yamaguchi \\
\hline 0.744 & NIPR & NIPR & A. Yamaguchi \\
\hline 0.728 & NIPR & NIPR & A. Yamaguchi \\
\hline 0.701 & NIPR & NIPR & A. Yamaguchi \\
\hline 0.7 & KOPRI & KOPRI & C. Park, S. Y. H. Yoo \\
\hline 0.674 & NIPR & NIPR & A. Yamaguchi \\
\hline 0.657 & NIPR & NIPR & A. Yamaguchi \\
\hline 0.648 & NIPR & NIPR & A. Yamaguchi \\
\hline 0.585 & NIPR & NIPR & A. Yamaguchi \\
\hline 0.585 & NIPR & NIPR & A. Yamaguchi \\
\hline 0.58 & NIPR & NIPR & A. Yamaguchi \\
\hline 0.521 & NIPR & NIPR & A. Yamaguchi \\
\hline 0.52 & JSC & JSC & $\begin{array}{ll}\text { SI } & \text { ANSMET }\end{array}$ \\
\hline 0.493 & NIPR & NIPR & A. Yamaguchi \\
\hline 0.48 & NIPR & NIPR & A. Yamaguchi \\
\hline 0.459 & NIPR & NIPR & A. Yamaguchi \\
\hline 0.445 & NIPR & NIPR & A. Yamaguchi \\
\hline 0.444 & JSC & JSC & $\begin{array}{ll}\text { SI } & \text { ANSMET }\end{array}$ \\
\hline 0.444 & NIPR & NIPR & A. Yamaguchi \\
\hline 0.43 & NIPR & NIPR & A. Yamaguchi \\
\hline 0.427 & NIPR & NIPR & A. Yamaguchi \\
\hline 0.425 & JSC & JSC & $\begin{array}{ll}\text { SI } & \text { ANSMET }\end{array}$ \\
\hline 0.419 & JSC & JSC & SI $\quad$ ANSMET \\
\hline 0.416 & NIPR & NIPR & A. Yamaguchi \\
\hline 0.406 & NIPR & NIPR & A. Yamaguchi \\
\hline 0.406 & NIPR & NIPR & A. Yamaguchi \\
\hline 0.404 & NIPR & NIPR & A. Yamaguchi \\
\hline 0.401 & NIPR & NIPR & A. Yamaguchi \\
\hline 0.384 & NIPR & NIPR & A. Yamaguchi \\
\hline 0.362 & NIPR & NIPR & A. Yamaguchi \\
\hline
\end{tabular}




$\begin{array}{llll}0.353 & \text { NIPR } & \text { NIPR } & \text { A. Yamaguchi } \\ 0.351 & \text { NIPR } & \text { NIPR } & \text { A. Yamaguchi } \\ 0.348 & \text { NIPR } & \text { NIPR } & \text { A. Yamaguchi } \\ 0.345 & \text { NIPR } & \text { NIPR } & \text { A. Yamaguchi } \\ 0.341 & \text { NIPR } & \text { NIPR } & \text { A. Yamaguchi } \\ 0.336 & \text { NIPR } & \text { NIPR } & \text { A. Yamaguchi } \\ 0.329 & \text { NIPR } & \text { NIPR } & \text { A. Yamaguchi } \\ 0.314 & \text { NIPR } & \text { NIPR } & \text { A. Yamaguchi } \\ 0.309 & \text { NIPR } & \text { NIPR } & \text { A. Yamaguchi } \\ 0.304 & \text { NIPR } & \text { NIPR } & \text { A. Yamaguchi } \\ 0.247 & \text { NIPR } & \text { NIPR } & \text { A. Yamaguchi } \\ 0.204 & \text { NIPR } & \text { NIPR } & \text { A. Yamaguchi } \\ 0.175 & \text { NIPR } & \text { NIPR } & \text { A. Yamaguchi } \\ 0.165 & \text { NIPR } & \text { NIPR } & \text { A. Yamaguchi } \\ 20.4 & \text { UWB } & \text { G. Hupe } & \text { A. Irving and }\end{array}$

\begin{tabular}{|c|c|c|c|}
\hline & 20 & MNB & A. Greshake, I Dario Ruiz \\
\hline \multirow[t]{3}{*}{4.79} & 5.5 & CEREGE & Eric Christens J. Gattacceca:Eric Christens \\
\hline & 21.2 & UWB & A. Aaronson A. Irving and $s$ \\
\hline & 22 & UWB & A. Aaronson A. Irving and $\leqslant$ \\
\hline 4.44 & 22.7 & Kiel & Wang Ziyao R. Bartoschen Wang Ziyao \\
\hline 4.41 & 19.1 & Kiel & Li Bofang, BeiR. Bartoschen anonymous \\
\hline 4.44 & 18.1 & Kiel & Wang Ziyao R. BartoscheuFan Xingang ( \\
\hline 4.37 & 15.6 & Kiel & Wang Ziyao R. Bartoschen anonymous \\
\hline 4.08 & 1.77 & Kiel & BartoschewitzR. BartoscheuLiu Binghan ( \\
\hline \multirow[t]{2}{*}{3.22} & 26.4 & UWB & A. Aaronson A. Irving and $\leqq$ \\
\hline & 24.4 & UNM & C. Agee, UNN \\
\hline \multirow[t]{6}{*}{3.61} & 22.7 & UWB & A. Irving and $s$ \\
\hline & 32.8 & UWB & A. Irving and $\leqslant$ \\
\hline & 22.4 & UNM & C. Agee, UNN \\
\hline & 2056.6 & URFU & V.V. Sharygin.UrFU meteorit \\
\hline & 22.7 & UNM & C. Agee, UNN \\
\hline & 48.4 & Cascadia & Tobin $\quad$ M. Hutson, S. \\
\hline 4.08 & 21.3 & MNB & Hanno Strufe A. Greshake, I \\
\hline & 22.7 & Cascadia & Mr. Ronnie McM. Hutson, S. \\
\hline 3.80 & 22.41 & UWB & FKuntz $\quad$ A. Irving and $\leqslant$ \\
\hline \multirow[t]{5}{*}{4.18} & 21.3 & MNB & Hichame Mim:A. Greshake, I \\
\hline & 22.13 & UCLA & Matthew StreєD. Sheikh, FS \\
\hline & 23.5 & ASU & L. Garvie, ASIMr. Todd Dziu \\
\hline & 183.05 & NIPR & A. Yamaguchi \\
\hline & 25.6 & UWB & A. Irving and $\subseteq$ \\
\hline \multirow[t]{3}{*}{3.94} & 20.53 & CEREGE & Kuntz J. Gattacceca: \\
\hline & 20 & UCLA & Michael CottinA. Rubin, UCL \\
\hline & 20.49 & CEREGE & Kuntz J. Gattacceca \\
\hline 3.95 & 15.8 & Kiel & Li Bofang, BeiR. Bartoschen anonymous \\
\hline
\end{tabular}




\begin{tabular}{|c|c|c|c|c|}
\hline \multirow[t]{3}{*}{3.85} & 9 & MNB & \multicolumn{2}{|c|}{ Tomasz JakukA. Greshake, I } \\
\hline & 8.0 & Vernad & \multicolumn{2}{|c|}{ Mr. T. KryachłLorenz C. A., 'Mr. T. Kryachł } \\
\hline & 32.443 & NIPR & NIPR & A. Yamaguchi \\
\hline & 30.6 & ASU & ASU & Bercovici, $\mathrm{H}_{.}$, anonymous \\
\hline \multirow[t]{5}{*}{3.51} & 5.15 & CEREGE & Kuntz & J. Gattacceca: \\
\hline & 23 & KOPRI & KOPRI & C. Park, S. Y. S. Park \\
\hline & 11.8 & KOPRI & KOPRI & C. Park, S. Y. H. Yoo \\
\hline & 20 & IfP & Anonymous & K. Metzler, IfP \\
\hline & 13 & MNB & \multicolumn{2}{|c|}{ Philippe SchrA. Greshake, I } \\
\hline \multirow[t]{2}{*}{4.00} & 8.14 & CEREGE & Kuntz & J. Gattacceca: \\
\hline & 48.5 & UNM & \multicolumn{2}{|c|}{ Matthew MartiC. Agee, UNN } \\
\hline 3.93 & 182 & CEREGE & MMC & J. Gattacceca:Rodrigo Martir \\
\hline 3.94 & 20.26 & JSC & JSC & ANSMET \\
\hline \multirow[t]{2}{*}{4.65} & 9.51 & JSC & JSC & SI $\quad$ ANSMET \\
\hline & 32.4 & MSN-FI & Tomelleri & V. Moggi Cecr \\
\hline \multirow[t]{3}{*}{4.09} & 22 & CEREGE & \multicolumn{2}{|c|}{ Jean RedelspıJ. Gattacceca: } \\
\hline & 46 & UPC & \multicolumn{2}{|c|}{ Ion Ander LanJ. Llorca, UPClon Ander Lan } \\
\hline & 25.0 & MSN-FI & Castellano & Vanni Moggi C \\
\hline \multirow[t]{8}{*}{4.06} & 53.2 & MNB & \multicolumn{2}{|c|}{ Hanno Strufe A. Greshake, I } \\
\hline & 21.7 & OU & Joe Bazyk & R. Greenwooc Joe Bazyk \\
\hline & 21.0 & ROM & Gregory & S. Yokoyama, \\
\hline & 24.5 & MNB & \multicolumn{2}{|c|}{ Jörgen NaubeA. Greshake, I } \\
\hline & 81.98 & NIPR & NIPR & A. Yamaguchi \\
\hline & 80.76 & NIPR & NIPR & A. Yamaguchi \\
\hline & 67.72 & NIPR & NIPR & A. Yamaguchi \\
\hline & 53.29 & NIPR & NIPR & A. Yamaguchi \\
\hline \multirow[t]{7}{*}{4.23} & 42.705 & JSC & JSC & SI ANSMET \\
\hline & 38.633 & NIPR & NIPR & A. Yamaguchi \\
\hline & 28.981 & NIPR & NIPR & A. Yamaguchi \\
\hline & 28.652 & NIPR & NIPR & A. Yamaguchi \\
\hline & 27.419 & NIPR & NIPR & A. Yamaguchi \\
\hline & 24.169 & NIPR & NIPR & A. Yamaguchi \\
\hline & 5.81 & ROM & Gregory & J. Poitras and \\
\hline \multirow[t]{5}{*}{3.46} & 17.89 & JSC & JSC & SI $\quad$ ANSMET \\
\hline & 2.9 & MNA-SI & MNA-SI & F. Focacci, M. Gabriele Giuli \\
\hline & 1.040 & JSC & JSC & ANSMET \\
\hline & 83.5 & Cascadia & \multicolumn{2}{|c|}{ John A. Shea, M. Hutson anc } \\
\hline & 33.3 & IfP & \multicolumn{2}{|c|}{ Marc Jost andK. Klemm and } \\
\hline \multirow[t]{7}{*}{3.71} & 29.1 & MNB & \multicolumn{2}{|c|}{ Harald StehlikA. Greshake, I } \\
\hline & 164.61 & NIPR & NIPR & A. Yamaguchi \\
\hline & 23.3 & MNB & \multicolumn{2}{|c|}{ Juergen NaubA. Greshake, I } \\
\hline & 80.52 & NIPR & NIPR & A. Yamaguchi \\
\hline & 13.8 & MNB & \multicolumn{2}{|c|}{ Hanno Strufe A. Greshake, I } \\
\hline & 37.259 & NIPR & NIPR & A. Yamaguchi \\
\hline & 20 & UCLA & \multicolumn{2}{|c|}{ Nick Gessler A. Rubin, UCL } \\
\hline
\end{tabular}




\subsection{3}

20

MNB

anonymous

A. Greshake, I

42.7

$381.62 \quad$ NIPR

348.31

20.2

218.380

4.71

4.6

4.63

4.75

4.75

3.42

3.7

3.33

40.7

160.89

22.2

27

136.64

126.26

99.17

74.465

20.5

46.888

43.339

7.98

26.639

5.7

19.572

13.818

11.376

8.065

7.001

3.7

4.67

3.587

20.07

14.8

12.7

51

455.88

23.5

21.2

22.1

20

23.7

468.39

337.58

20.1

281.4

Vernad

$$
\text { JSC }
$$

ROM

Vernad.

GUT

NIPR

NIPR

JSC

MSN-FI

$$
\text { NIPR }
$$

JSC

Vernad

NIPR

ROM

NIPR

JSC

NIPR

NIPR

JSC

Vernad

$$
\text { NIPR }
$$

$$
\text { NIPR }
$$

CEREGE

$$
\text { IfP }
$$$$
\text { IfP }
$$

FSAC

UCLA

ROM

ROM

ROM

IfP

48.6

22.34

NIPR

MNB

ROM

ROM
UWO

$$
\text { NIPR }
$$

MSN-FI

JSC

S. Tutorow NIPR Z.G. Guo, UW NIPR

A. Yamaguchi

A. Yamaguchi

Mimaghador V. Moggi Cecc

JSC

SI

ANSMET

Mr. T. KryachlC. A. Lorenz, 'Mr. T. Kryacht

JSC SI

ANSMET

Gregory S. Yokoyama,

Mr. T. KryachłLorenz C. A., 'Mr. T. Kryachł

GUT $\quad$ B. Miao, Z. Xić

NIPR

A. Yamaguchi

NIPR

A. Yamaguchi

JSC

SI

ANSMET

Vignato

Vanni Moggi $C$

NIPR

A. Yamaguchi

JSC

SI

ANSMET

DMUH

C.A. Lorenz, I

NIPR

A. Yamaguchi

Gregory

J. Poitras, UT,

NIPR

A. Yamaguchi

JSC

SI

ANSMET

NIPR

A. Yamaguchi

NIPR

A. Yamaguchi

JSC

SI

ANSMET

Vernad

C. A. Lorenz, 'Mr. S. E. Boris

NIPR

A. Yamaguchi

NIPR

A. Yamaguchi

Kuntz

J. Gattacceca.

Marc Jost andK. Klemm and

Marc Jost andK. Klemm and

Aaras Jonikas H. Chennaoui A nomad girl $r$

Daniel SheikhD. Sheikh, UF

Gregory S. Yokoyama,

Gregory S. Yokoyama,

Gregory J. Poitras, UT,

Manuel Nieto K. Metzler, IfP

MSN-FI Tomelleri V. Moggi Cecc

$\begin{array}{lll}\text { NIPR } & \text { NIPR } & \text { A. Yamaguchi } \\ \text { NIPR } & \text { NIPR } & \text { A. Yamaguchi }\end{array}$

MSN-FI Castellano V. Moggi Cecc

NIPR

A. Yamaguchi

Hichame Mim:A. Greshake, I

Gregory

J. Poitras, UT,

Gregory

J. Poitras and 


$\begin{array}{llll}186.87 & \text { NIPR } & \text { NIPR } & \text { A. Yamaguchi } \\ 161.31 & \text { NIPR } & \text { NIPR } & \text { A. Yamaguchi }\end{array}$

\begin{tabular}{|c|c|c|c|c|}
\hline \multirow{4}{*}{4.62} & \multirow{3}{*}{$\begin{array}{l}21 \\
21.2 \\
150.64\end{array}$} & \multirow{3}{*}{$\begin{array}{l}\text { UPC } \\
\text { UWB } \\
\text { JSC }\end{array}$} & \multicolumn{2}{|c|}{ Francisco Gal'J. Llorca, UPCFrancisco Gal' } \\
\hline & & & \multicolumn{2}{|c|}{ B. Reed $\quad$ A. Irving and $\leq$} \\
\hline & & & JSC & SI $\quad$ ANSMET \\
\hline & 22 & MSN-FI & Tomelleri & V. Moggi Cecc \\
\hline & 20.6 & MNB & \multicolumn{2}{|c|}{ Hanno Strufe A. Greshake, I } \\
\hline & 22.2 & MNB & \multicolumn{2}{|c|}{ Philippe SchrA. Greshake, I } \\
\hline \multirow[t]{8}{*}{5} & 133.43 & JSC & JSC & SI $\quad$ ANSMET \\
\hline & 121.02 & NIPR & NIPR & A. Yamaguchi \\
\hline & 119.5 & NIPR & NIPR & A. Yamaguchi \\
\hline & 104.4 & MSN-FI & Mimaghador & V. Moggi Cecc \\
\hline & 24.7 & MSN-FI & Vignato & Vanni Moggi C \\
\hline & 19.9 & MNB & \multicolumn{2}{|c|}{ Hichame Mim:A. Greshake, I } \\
\hline & 93.89 & NIPR & NIPR & A. Yamaguchi \\
\hline & 88.02 & NIPR & NIPR & A. Yamaguchi \\
\hline \multirow[t]{4}{*}{3.49} & 18.2 & Kiel & Wang Ziyao & R. Bartoschen anonymous \\
\hline & 16 & MNB & \multicolumn{2}{|c|}{ Philippe SchrA. Greshake, I } \\
\hline & 17 & UCLA & Verish & A. Rubin, UCL \\
\hline & 71.46 & NIPR & NIPR & A. Yamaguchi \\
\hline
\end{tabular}

4.59

64.98

JSC

JSC

SI

ANSMET

15.1 MNB Hanno Strufe A. Greshake, I

$\begin{array}{llll}57.966 & \text { NIPR } & \text { NIPR } & \text { A. Yamaguchi }\end{array}$

$\begin{array}{llll}51.003 & \text { NIPR } & \text { NIPR } & \text { A. Yamaguchi }\end{array}$

$50.285 \quad$ NIPR $\quad$ NIPR $\quad$ A. Yamaguchi

$45.093 \quad$ NIPR $\quad$ NIPR $\quad$ A. Yamaguchi

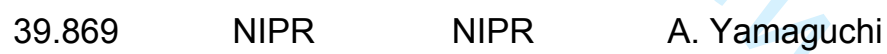

$35.703 \quad$ NIPR $\quad$ NIPR $\quad$ A. Yamaguchi

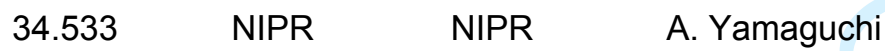

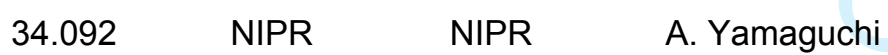

8.8 MNB Hanno Strufe A. Greshake, I

$8.4 \quad$ UWB J. Bliss A. Irving and 5

$30.565 \quad$ NIPR NIPR A. Yamaguchi

$\begin{array}{llll}27.51 & \text { GUT } & \text { GUT } & \text { B. Miao, Z. Xic }\end{array}$

$23.685 \quad$ NIPR $\quad$ NIPR $\quad$ A. Yamaguchi

4.7 MNB Philippe Schm A. Greshake, I

21.831 NIPR NIPR A. Yamaguchi

4.4 MNB Philippe SchrA. Greshake, I

21.03 NIPR NIPR A. Yamaguchi

18.957 NIPR NIPR $\quad$ A. Yamaguchi

15.067 NIPR NIPR $\quad$ A. Yamaguchi

$14.465 \quad$ NIPR $\quad$ NIPR $\quad$ A. Yamaguchi

$\begin{array}{llllll}4.83 & 13.744 & \text { JSC } & \text { JSC } & \text { SI } & \text { ANSMET }\end{array}$

$\begin{array}{llllll}4.59 & 13.683 & \text { JSC } & \text { JSC } & \text { SI } & \text { ANSMET }\end{array}$

$2.5 \quad$ MNB Philippe SchrrA. Greshake, I 


\begin{tabular}{|c|c|c|c|c|c|}
\hline 4.69 & 10.907 & JSC & JSC & SI & ANSMET \\
\hline \multirow[t]{3}{*}{4.78} & 10.253 & JSC & JSC & SI & ANSMET \\
\hline & 9.95 & NIPR & NIPR & \multicolumn{2}{|l|}{ A. Yamaguchi } \\
\hline & 8.446 & NIPR & NIPR & \multicolumn{2}{|l|}{ A. Yamaguchi } \\
\hline \multirow[t]{2}{*}{3.64} & 8.337 & JSC & JSC & SI & ANSMET \\
\hline & 6.593 & NIPR & NIPR & \multicolumn{2}{|l|}{ A. Yamaguchi } \\
\hline 4.64 & 6.39 & JSC & JSC & SI & ANSMET \\
\hline \multirow[t]{2}{*}{4.6} & 5.52 & JSC & JSC & SI & ANSMET \\
\hline & 5.397 & NIPR & NIPR & \multicolumn{2}{|l|}{ A. Yamaguchi } \\
\hline \multirow[t]{7}{*}{3.87} & 5.091 & JSC & JSC & $\mathrm{SI}$ & ANSMET \\
\hline & 4.626 & NIPR & NIPR & \multicolumn{2}{|c|}{ A. Yamaguchi } \\
\hline & 4.151 & NIPR & NIPR & \multicolumn{2}{|l|}{ A. Yamaguchi } \\
\hline & 1.803 & NIPR & NIPR & \multicolumn{2}{|l|}{ A. Yamaguchi } \\
\hline & 1.419 & NIPR & NIPR & \multicolumn{2}{|l|}{ A. Yamaguchi } \\
\hline & 0.32 & NIPR & NIPR & \multicolumn{2}{|l|}{ A. Yamaguchi } \\
\hline & 0.2 & KOPRI & KOPRI & \multicolumn{2}{|c|}{ C. Park, S. Y. C. H. Oh } \\
\hline \multirow[t]{3}{*}{3.88} & 20.0 & App & PARI & \multicolumn{2}{|c|}{ A. Love, App Unknown } \\
\hline & 34.8 & UNM & Larry Atkins & \multicolumn{2}{|c|}{ C. Agee, UNN } \\
\hline & 20 & IfP & Nourddine Az & K. Metzler, IfP & \\
\hline \multirow[t]{9}{*}{3.16} & 20 & CEREGE & Sergey Vasilie & $\in J$. Gattacceca: & Thomas Kurtz \\
\hline & 9.2 & UWB & F. Olsen & \multicolumn{2}{|l|}{ A. Irving and $\leqq$} \\
\hline & 28.8 & UWB & R. Chaoui & \multicolumn{2}{|l|}{ A. Irving and $\varsigma$} \\
\hline & 35 & MNB & Salamu Hama & \\
\hline & 24.1 & UWB & B. Hoefnagels & \multicolumn{2}{|c|}{$\begin{array}{l}\text { רaA. Greshake, } \\
\text { IsA. Irving and } \leqslant\end{array}$} \\
\hline & 22.9 & UNM & Dustin Dicken & \multicolumn{2}{|l|}{ nC. Agee, UNN } \\
\hline & 180.9 & ASU & ASU & \multicolumn{2}{|l|}{ E.T. Dunham, } \\
\hline & 9.533 & NIPR & NIPR & A. Yamaguchi & \\
\hline & 5.232 & NIPR & NIPR & A. Yamaguchi & \\
\hline 5.46 & 3.42 & JSC & JSC & SI & ANSMET \\
\hline & 20.9 & UWB & D. Pitt & A. Irving, S. KI & \\
\hline & 80.8 & NHMV & NHMV & F. Brandstätte & \\
\hline & 67.05 & GUT & GUT & B. Miao, H.Liu & \\
\hline 3.60 & 7.5 & CEREGE & Labenne Luc & J. Gattacceca: & \\
\hline & 3 & MNB & Stefan Ralew & A. Greshake, I & \\
\hline & 25.2 & UWB & B. Hoefnagels & sA. Irving and & \\
\hline & 21.25 & UNM & Dustin Dicken & C. Agee, UNN & \\
\hline & 28.9 & UNM & Dustin Dicken & C. Agee, UNN & \\
\hline & 20.0 & UWB & D. Dickens & A. Irving and & \\
\hline & 43.15 & UNM & Dustin Dicken & C. Agee, UNN & \\
\hline & 663 & Vernad & anonymous & F. Badyukov, ' & \\
\hline & 20.6 & UNM & Aaronson & C. Agee, UNN & \\
\hline & 37.9 & GUT & Guobing Zhon & nH. Chen, J. Zr & \\
\hline & 29.2 & UNM & Terry Boudrea & ¿C. Agee, UNN & \\
\hline & 21.2 & UCLA & N. Gessler & P. Warren, UC & \\
\hline & 21.6 & UNM & Dustin Dicken & C. Agee, UNN & \\
\hline
\end{tabular}




\begin{tabular}{|c|c|c|c|}
\hline 20.2 & ROM/UWB & D. Gregory & A. Irving and $\subseteq$ \\
\hline 20 & UNM & Jay Piatek & C. Agee, UNN \\
\hline 20.85 & CSFK & Z. Kereszty & C. Agee, UNN \\
\hline 22 & UWB & H. Naji & A. Irving \& P. 1 \\
\hline 20.1 & UNM & Jay Piatek & C. Agee, UNN \\
\hline 23.5 & IfP & Finder & A.-K. Kraemer anonymous \\
\hline 17.6 & App & J. Sinclair & A. Love, App Anonymous \\
\hline 14.11 & UWB & F. Kuntz & A. Irving and $\leqslant$ \\
\hline 13 & UNM & Jay Piatek & C. Agee, UNN \\
\hline 11.8 & UWB & \multicolumn{2}{|c|}{ B. HoefnagelsA. Irving and $\subseteq$} \\
\hline 9.5 & UWB & DPitt & A. Irving and $\varsigma$ \\
\hline 4.73 & UWB & J. Hum & A. Irving and $\leqq$ \\
\hline 20.1 & UWB & D. Pitt & A. Irving and $\leqq$ \\
\hline 21 & CEREGE & \multicolumn{2}{|c|}{ with anonymo J. Gattacceca anonymous } \\
\hline 2.90 & SI & \multicolumn{2}{|c|}{ Ahmed El GorN. G. Lunning } \\
\hline 1.55 & MNB & Said Yousfi & A. Greshake, I \\
\hline 1.3 & UNM & \multicolumn{2}{|c|}{ Jean RedelspiC. Agee, UNN } \\
\hline 0.38 & IfP & \multicolumn{2}{|c|}{ Nourddine AzıK. Metzler, IfP } \\
\hline 20.7 & UNM & \multicolumn{2}{|c|}{ John Higgins C. Agee, UNN } \\
\hline
\end{tabular}

41 $21 \mathrm{~g} \mathrm{UWB}, 20 \mathrm{D}$. Pitt; S. ArnıA. Irving and §Zaid Balli and UWB

A. Aaronson A. Irving, P. C

20.5

UWB

Aaronson

A. Irving, $P$. C

22.4

UWB

$\mathrm{N}$. Faraji and IA. Irving and $\leqslant$

22.1

UWB

B. HoefnagelsA. Irving and $\leqslant$

23.4

UWB

B. Hoefnagels A. Irving and $s$

20.5

UWB

B. Hoefnagels A. Irving and $s$

18.5

GUT

Guobing ZhonH. Chen, J. Zr

14.2

UCLA

N. Gessler

P. Warren, UC

6.88

UWB

J. Higgins

A. Irving and $\varsigma$

28.022

NIPR

NIPR

A. Yamaguchi

4.8

UWB

B. Hoefnagels A. Irving and $s$

4.2

MNB

13.049

1.9

NMBE

3.2

UNM

Salamu HamaA.

NMBE

F. Mechelke, Eddwin Gnos, E

Jay Piatek

C. Agee, UNN

103

$\mathrm{UAb}$

M. Ouzillou

C. Herd and L

24.1

Vernad

anonymous

C. A. Lorenz,'

21

UWB

R. Falls

A. Irving \& S. I

16

PSF

7.5

CEREGE

A. Debienne

A. Irving and $\leqslant$

Labenne Luc J. Gattacceca:

PSF

A. Irving and $s$

4.91

24.19

PSF

Anonymous

A. Irving and $s$

22.5

MNB

Philippe Schm

A. Greshake, I

14.4

MNB

Fabien Kuntz A. Greshake, I

5.8

MNB

Vincent Gira

A. Greshake, I

$4.8446 \mathrm{~g}$

UNIL

UNIL

B. Hofmann, MMr Reto Merlo 


\begin{tabular}{|c|c|c|c|c|c|}
\hline & 20.2 & UWB & M. Bandli & A. Irving and $\subseteq$ & \\
\hline & 16.63 & ASU & ASU & L. Garvie & Michael Farm \\
\hline & 25.3 & UWB & J. Phillips & A. Irving and $\leqslant$ & \\
\hline 2.91 & 31.2 & MNB & Hichame Mim & A. Greshake, I & \\
\hline & 20.3 & MNB & Stefan Ralew & A. Greshake, I & \\
\hline & 20.2 & App & PARI & A. Love, App & Anonymous \\
\hline & 20.0 & App & PARI & A. Love, App & Unknown \\
\hline & 25.7 & UWB & G. Fujihara & A. Irving and $\leqslant$ & \\
\hline & 21.2 & PSF & F. Kuntz & A. Irving and & \\
\hline & 20.5 & UWB & R. Falls & A. Irving and $\leqslant$ & \\
\hline & 32.87 & ROM & ROM & V. Di Cecco, F & \\
\hline & 21.2 & UWB & D. Dickens & A. Irving and $\subseteq$ & \\
\hline & 33.97 & UCLA & Sean Tutorov & NA. Rubin, UCL & \\
\hline & 7.6 & MNB & Stefan Ralew & A. Greshake, I & \\
\hline & 37.8 & UWO & S. Tutorow & M. Spencer, U & \\
\hline & 40 & ROM & Gregory & B. C. Hyde, R & \\
\hline & 20 & IfP, Münster & Nourddine $A z$ & K. Metzler, IfP & \\
\hline & 20.2 & UNM & Zouhair Fetto & C. Agee, UNN & \\
\hline & 27.8 & UWB & M. Jost & A. Irving and $\varsigma$ & \\
\hline & 20.4 & MNB & Marcin Cimal & ¿A. Greshake, I & \\
\hline & 24.1 & MNB & Marcin Cimal & ¿A. Greshake, I & \\
\hline & 20.1 & UNM & DPitt & C. Agee, UNN & \\
\hline & 21.0 & UWO & S. Tutorow & M. Spencer, C & \\
\hline & 20 & UNM & Darryl Pitt & C. Agee, UNN & \\
\hline & 20 & MNB & Marcin Cimal & ¿A. Greshake, I & \\
\hline & 20.2 & UNM & Suzanne Mor & rC. Agee, UNN & \\
\hline & 460 & SQU & SQU & P. Hill, N. Ban & \\
\hline & 20.7 & UNM & Dustin Dicker & C. Agee, UNN & \\
\hline & 21.1 & UNM & Darryl Pitt & C. Agee, UNN & \\
\hline & 20.6 & UWB & A. Aaronson & A. Irving and $\leqq$ & \\
\hline & 22.4 & UWB & R. Falls & A. Irving and $\varsigma$ & \\
\hline & 365 & SQU & SQU & P. Hill, N. Ban & \\
\hline & 20 & IfP, Münster & Nourddine Az & K. Metzler, IfP & \\
\hline & 20.1 & UWO & S. Tutorow & M. Spencer, U & \\
\hline & 20 & IfP & Manuel Nieto & K. Metzler, IfP & \\
\hline & 252.3 & KOPRI & KOPRI & C. Park, S. Y. & H. Yoo \\
\hline & 20.6 & Cascadia & Mr. Ronnie M & (A. Ruzicka, S. & \\
\hline & 20.2 & UNM & Jay Piatek & C. Agee, UNN & \\
\hline & 20 & MNB & Marcin Cimal & ¿A. Greshake, I & \\
\hline & 22.2 & UNM & DPitt & C. Agee, UNN & \\
\hline & 20 & ROM & Gregory & B. C. Hyde, R & \\
\hline & 20.1 & UWB & A. Aaronson & A. Irving and $\subseteq$ & \\
\hline & 20 & MNB & Marcin Cimal & ¿A. Greshake, I & \\
\hline & 21 & UPC & David Allepuz & zJ. Llorca, UPC & David Allepuz \\
\hline & 20.0 & UWO & S. Tutorow & M. Spencer, U & \\
\hline
\end{tabular}




$\begin{array}{llll}95 & \text { SQU } & \text { SQU } & \text { P. Hill, N. Ban } \\ 20 & \text { IfP, Münster } & \text { Nourddine AzıK. Metzler, IfP } \\ 15.3 & \text { UWO } & \text { S. DeBoer } & \text { M. Spencer, F } \\ 14.2 & \text { NHM } & \text { Morten Bilet } & \text { N. Rai, N. V. F } \\ 13 & \text { IfP, Münster } & \text { José Antonio } \text { :K. Metzler, IfP } \\ 11.4 & \text { MNB } & \text { Stefan Ralew A. Greshake, I } \\ 10.7 & \text { UWB } & \text { A. Aaronson } & \text { A. Irving and } \\ 8.3 & \text { MNB } & \text { anonymous } & \text { A. Greshake, I } \\ 6.6 & \text { MNB } & \text { Marcin CimaleA. Greshake, IMarcin Cimale } \\ 4.684 & \text { NIPR } & \text { NIPR } & \text { A. Yamaguchi } \\ 0.766 & \text { NIPR } & \text { NIPR } & \text { A. Yamaguchi } \\ 20.3 & \text { UWB } & \text { DPitt } & \text { A. Irving and } \\ 20.73 & \text { UCLA } & \text { Brad Kern } & \text { A. Rubin, UCL } \\ 20.2 & \text { UWB } & \text { D. Pitt } & \text { A. Irving and }\end{array}$




\section{tblcomment}

work name JR049; submitted by Jerome Gattaceca

Field name MVCH-360; submitted by C. Agee, UNM

Submitted by C. Park, KOPRI

Submitted by Ansgar Greshake

Work name M2049; submitted by A. Irving

G688; submitted by A. Bouvier

Submitted by C. Agee

Submitted by Hsu W.

Field name JP-94; submitted by C. Agee

work name LL2015-9; submitted by J. Gattacceca

Work name $\mathrm{BH} 127$; submitted by $\mathrm{A}$. Irving

Work name MVCH 338; submitted by A. Irving

Work name BH140; submitted by A. Irving

Submitted by Anthony Love

Submitted by K. Metzler, IfP

Submitted by Ansgar Greshake

Submitted by Ansgar Greshake

RC60.2; submitted by A. Irving

Y-82094 like; submitted by A. Yamaguchi

Y-82094 like; submitted by A. Yamaguchi

Work name AH17-30; submitted by A. Irving

work name CC1701; submitted by Jerome Gattacceca

Submitted by A. Yamaguchi

Field name ZF-1; submitted by C. Agee

Field names Calama 3-51, Misha 3-37 and KIR10; submitted by Marina Ivanova

Submitted by AMN

Submitted by Vanni Moggi Cecchi

Submitted by Ansgar Greshake

Submitted by Vanni Moggi Cecchi

Arj18-1; submitted by A. Irving

Work name M2014; submitted by A. Irving

Submitted by AMN

LM52939; submitted by A. Irving

Submitted by Ansgar Greshake

Submitted by Vanni Moggi Cecchi

Submitted by Ansgar Greshake

Submitted by AMN

Submitted by Ansgar Greshake

Field name MVCH-353; submitted by C. Agee, UNM

Submitted by C. Park, KOPRI

Submitted by Ansgar Greshake

Submitted by Alan Rubin

Submitted by Ansgar Greshake

Field name MVCH-361; submitted by C. Agee, UNM 
work name LM68 / D55; submitted by Jérôme Gattacceca

Submitted by A. Yamaguchi

Submitted by Ansgar Greshake

Submitted by A. Yamaguchi

Heavily altered; submitted by A. Yamaguchi

Submitted by A. Yamaguchi

Submitted by A. Yamaguchi

Submitted by A. Yamaguchi

Submitted by A. Yamaguchi

Submitted by A. Yamaguchi

Submitted by A. Yamaguchi

Submitted by A. Yamaguchi

Submitted by A. Yamaguchi

Submitted by A. Yamaguchi

Submitted by A. Yamaguchi

Submitted by A. Yamaguchi

Submitted by A. Yamaguchi

Submitted by A. Yamaguchi

Work name DD17-6; submitted by A. Irving

Submitted by L. Garvie

Submitted by A.D. Shukla, PRL

Algeria; submitted by Ansgar Greshake

Submitted by C. Park, KOPRI

Work name BH129; submitted by A. Irving

work name RL04; submitted by Jérôme Gattacceca

Submitted by C. Agee

Submitted by AMN

Submitted by C. Park, KOPRI

Submitted by Ansgar Greshake

Submitted by Ansgar Greshake

Submitted by Stepan Vasilevich Berzin

Submitted by AMN

Submitted by Ansgar Greshake

Submitted by AMN

work name K326; submitted by Jérôme Gattacceca

Submitted by Ansgar Greshake

Submitted by Ansgar Greshake

Submitted by Ansgar Greshake

Submitted by Ansgar Greshake

Submitted by Ansgar Greshake

Submitted by Ansgar Greshake

Submitted by C. Park, KOPRI

Submitted by Ansgar Greshake

Submitted by Ansgar Greshake

Submitted by A. Yamaguchi 
Submitted by A. Yamaguchi

Submitted by A. Yamaguchi

Submitted by A. Yamaguchi

Work name 507-A; submitted by A. Irving

Work name 400-A; submitted by A. Irving

Submitted by Ansgar Greshake

work name RM567; submitted by Jérôme Gattacceca

Submitted by K. Metzler, IfP

Submitted by Hsu W.

Submitted by M. A. Ivanova

work name RM598; submitted by Jérôme Gattacceca

Submitted by K. Metzler, IfP

Working No.: SM 1804-10; submitted by K. Klemm, IfP; submitted by Klemm K.

Working No.: SM 1804-9; submitted by K. Klemm, IfP; submitted by Klemm K.

Submitted by A. Irving

Working No.: SM 1804-6; submitted by K. Klemm, IfP; submitted by Klemm K.

Work name H128; submitted by A. Irving

Field name: SV2017-01; submitted by Samuel Ebert; submitted by Ebert S.

JH14-14; submitted by A. Irving

Submitted by Ansgar Greshake

Work name MVCH 303; submitted by A. Irving

Submitted by K. Metzler, IfP

Submitted by Ansgar Greshake

Field name MAS-2; submitted by C. Agee, UNM

work name K338; submitted by Jerome Gattacceca

Field name MVCH-358; submitted by C. Agee, UNM

Submitted by AMN

Submitted by AMN

Work name BH116; submitted by A. Irving

Work name M2169; submitted by A. Irving

Field name AB-73; submitted by C. Agee, UNM

Submitted by Ansgar Greshake

Morocco; submitted by Ansgar Greshake

SJS 13001; submitted by A. Irving

Work name ZLI03; submitted by A. Irving

Submitted by Anthony Love

Submitted by K. Metzler, IfP

Work name LL75; submitted by Lisa Krämer Ruggiu

Submitted by F. Brandstätter

Submitted by Anthony Love

Work name K386; submitted by A. Irving

Field name MVCH-357; submitted by C. Agee, UNM

Submitted by Ansgar Greshake

work name K352; submitted by Jérôme Gattacceca

Work name K373; submitted by A. Irving 
Morocco; submitted by Ansgar Greshake

Submitted by Ansgar Greshake

NWA-LB005; submitted by R. Bartoschewitz

Submitted by A. Irving

Submitted by Ansgar Greshake

Work name MVCH 369; submitted by A. Irving

Submitted by Ansgar Greshake

Submitted by K. Metzler, IfP

Submitted by Ansgar Greshake

Submitted by A. Yamaguchi

Submitted by Ansgar Greshake

Work name MC030; submitted by A. Irving

Submitted by Ansgar Greshake

Submitted by AMN

Submitted by AMN

Submitted by AMN

Work name CHC18018; submitted by A. Irving

Submitted by A. Yamaguchi

Work name M2036; submitted by A. Irving

Submitted by Hsu W.

Work name 349-A; submitted by A. Irving

Work name JH17-6; submitted by A. Irving

Submitted by Ansgar Greshake

Submitted by Ansgar Greshake

MVCH-336; submitted by C. Agee, UNM

Submitted by Anthony Love

work name RL02; submitted by Jérôme Gattacceca

Unbrecciated; submitted by A. Yamaguchi

Submitted by A. Yamaguchi

Field name: Met-2018-007; submitted by David Allepuz

Submitted by AMN

Work name ZLI02; submitted by A. Irving

Work name FO18-1; submitted by A. Irving

Submitted by A. Yamaguchi

Submitted by Ansgar Greshake

Submitted by A. Yamaguchi

Submitted by A. Yamaguchi

Submitted by Ansgar Greshake

Breccia; submitted by A. Yamaguchi

Submitted by A. Yamaguchi

An79.5-87.3; submitted by A. Yamaguchi

An90.6; submitted by A. Yamaguchi

Heavily shocked; submitted by A. Yamaguchi

CC18-1; submitted by A. Irving

work name LM79/E44; submitted by Jérôme Gattacceca 
$\mathrm{Si}=0.51 \mathrm{wt} \%$; submitted by A. Yamaguchi

work name LM77/E37; submitted by Jérôme Gattacceca

work name LM67/E1; submitted by Jérôme Gattacceca

Submitted by Ansgar Greshake

Submitted by Ansgar Greshake

$\mathrm{Si}=0.83 \mathrm{wt} \%$; submitted by A. Yamaguchi

Submitted by M.Salvini, M. Gemelli DST-PI

Submitted by Vanni Moggi Cecchi

Submitted by Hsu W.

Submitted by Anthony Love

Submitted by Bingkui Miao

Submitted by C. Park, KOPRI

Submitted by C. Park, KOPRI

Gessler's address: 2010 Calgary Ln, Los Angeles, CA 90077, USA; submitted by Paul Warren

Submitted by R. Bartoschewitz

Submitted by Anthony Love

Submitted by P. Hill

Submitted by Ansgar Greshake

Submitted by V. Di Cecco, ROM

Submitted by Ansgar Greshake

Submitted by Ansgar Greshake

Submitted by Ansgar Greshake

Submitted by Ansgar Greshake

An85.3-91.4; submitted by A. Yamaguchi

Submitted by Vanni Moggi Cecchi

Lab number CML0920; submitted by A. Ruzicka

Submitted by Ansgar Greshake

Submitted by Anthony Love

Lab number CML0943; submitted by A. Ruzicka

Submitted by Bingkui Miao

Submitted by Bing-kui Miao

An89.7-92.6; submitted by A. Yamaguchi

Submitted by Bingkui Miao

Submitted by Bingkui Miao

Submitted by Ansgar Greshake

Submitted by Ansgar Greshake

Submitted by Maria Eugenia Varela

Submitted by Ansgar Greshake

An84.5-91.9; submitted by A. Yamaguchi

An67.3-92.8; submitted by A. Yamaguchi

Submitted by Bingkui Miao

An92.3-94.2; submitted by A. Yamaguchi

Submitted by Bingkui Miao

An92.3-94.2; submitted by A. Yamaguchi

Submitted by K. Metzler, IfP 


\section{Submitted by Bingkui Miao}

Sample number LM58206 in the Royal Ontario Museum collection. Formerly named DGRO 33-16.; subr An92.4-94.1; submitted by A. Yamaguchi

Sample number LM58207 in the Royal Ontario Museum collection. Formerly named ROM060.; submitte Submitted by Bingkui Miao

An81.1-89.7; submitted by A. Yamaguchi

Submitted by Ansgar Greshake

An72.8-93.7; submitted by A. Yamaguchi

Submitted by Bingkui Miao

Submitted by Bingkui Miao

Submitted by Bingkui Miao

An85.4-89.3; submitted by A. Yamaguchi

An86.1-88.8; submitted by A. Yamaguchi

An82.3-91.6; submitted by A. Yamaguchi

Submitted by Bingkui Miao

Wo4.2-37.1En20.7-55.3, An78.4-86.6; submitted by A. Yamaguchi

Submitted by A. Yamaguchi

An75.3-86.6; submitted by A. Yamaguchi

An87.2-90.2; submitted by A. Yamaguchi

An79.3-94.9; submitted by A. Yamaguchi

An86.8-90.4; submitted by A. Yamaguchi

Submitted by C. Park, KOPRI

Work name RC97.1; submitted by A. Irving

OT15; submitted by A. Irving

SVMJ14-1; submitted by A. Irving

GF18-2; submitted by A. Irving

Submitted by Ansgar Greshake

Submitted by Ansgar Greshake

Submitted by Ansgar Greshake

Submitted by Ansgar Greshake

Gessler's address: 2010 Calgary Ln, Los Angeles, CA 90077, USA; submitted by Paul Warren

Field name AID 125; submitted by C. Agee, UNM

Work name M1796; submitted by A. Irving

Submitted by Ansgar Greshake

Field name JP-75; submitted by C. Agee, UNM

Submitted by Ansgar Greshake

Submitted by K. Metzler, IfP

Submitted by K. Metzler, IfP

Work name MVCH 340; submitted by A. Irving

Submitted by Aicheng Zhang

Work name BF18-24; submitted by A. Irving

Work name JP18-2; submitted by A. Irving

Work name BF17-15; submitted by A. Irving

Field name JP-76; submitted by C. Agee, UNM

Submitted by Jordi Llorca (UPC) 
work name ST15; submitted by Jerome Gattacceca

Work name JH18-1; submitted by A. Irving

Submitted by Anthony Love

Work name BF17-20; submitted by A. Irving

UWO-244; submitted by A. Bouvier

Submitted by Anthony Love

Work name MVCH 324; submitted by A. Irving

Submitted by K. Metzler, IfP

Field name MVCH-354; submitted by C. Agee, UNM

Submitted by Vinciane Debaille

Submitted by K. Metzler, IfP

Work name MVCH 365; submitted by A. Irving

Submitted by K. Metzler, IfP

Submitted by Ansgar Greshake

Submitted by L. Garvie

Submitted by AMN

Submitted by AMN

Field name MVCH-363; submitted by C. Agee, UNM

Submitted by Ansgar Greshake

Submitted by K. Metzler, IfP

Submitted by AMN

Field name MVCH-356; submitted by C. Agee, UNM

Submitted by AMN

Submitted by AMN

Submitted by Ansgar Greshake

Submitted by AMN

Submitted by AMN

Submitted by AMN

Submitted by Ansgar Greshake

Submitted by T. Shisseh

Submitted by AMN

Field name JP-401; submitted by C. Agee, UNM

Work name MVCH 330; submitted by A. Irving

work name AG001; submitted by Jerome Gattacccea

Submitted by AMN

Work name MVCH 367; submitted by A. Irving

Field name MVCH-362; submitted by $\mathrm{C}$. Agee, UNM

Submitted by AMN

Submitted by AMN

Field name DD-24; submitted by C. Agee, UNM

Submitted by AMN

Melt breccia; submitted by A. Yamaguchi

Breccia; submitted by A. Yamaguchi

Submitted by Alan Rubin

working name DJ2018/02; submitted by R. Bartoschewitz 
Working name NWA-FV01; submitted by R. Bartoschewitz working name DJ2017/17; submitted by R. Bartoschewitz working name WZ10; submitted by R. Bartoschewitz NWA-LB001; submitted by R. Bartoschewitz working name WZ38; submitted by R. Bartoschewitz NWA-WK01; submitted by R. Bartoschewitz Submitted by R. Bartoschewiz NWA-LB002; submitted by R. Bartoschewitz working name WZ18; submitted by R. Bartoschewitz NWA-JK01; submitted by R. Bartoschewitz working name WZ16; submitted by R. Bartoschewitz Submitted by R. Bartoschewitz working name WZ30; submitted by R. Bartoschewitz working name DJ2018/03; submitted by R. Bartoschewitz working name WZ05; submitted by R. Bartoschewitz working name WZ11; submitted by R. Bartoschewitz NWA-LB007; submitted by R. Bartoschewitz

NWA AM4; submitted by R. Bartoschewitz synonym Northwest Africa 736; submitted by Simone de Leuw work name B1; submitted by Richard Greenwood, Hélène Greenwood Submitted by Ansgar Greshake Submitted by Ansgar Greshake Submitted by Simone de Leuw Field name is 2-5; analyst S. E. Borisovsky (IGEM); Estimated subtype 3.9; submitted by C. A. Lorenz ( Submitted by Ansgar Greshake

Submitted by C. Park, KOPRI

Submitted by Ken Eltrich

Submitted by A. Yamaguchi

Submitted by Ansgar Greshake

Submitted by Ebert S.

Submitted by A. Yamaguchi

Submitted by C. Agee

Submitted by A. Yamaguchi

Submitted by C. Park, KOPRI

CHC18020; submitted by A. Irving

CHC18019; submitted by A. Irving

Submitted by A. Yamaguchi

Submitted by C. Park, KOPRI

work name Sahara 98050A; submitted by Ebert S.

Lab number CML0788; field number W232.; submitted by A. Ruzicka

Lab number CML0775; field number J25.; submitted by A. Ruzicka

Lab number CML0780; field number TB673.; submitted by A. Ruzicka

Lab number CML0777; field number J141.; submitted by A. Ruzicka

work name EJ492; submitted by Jérôme Gattacceca

Lab number CML0776; field number J32; submitted by A. Ruzicka 
Lab number CML0779; field number TB519.; submitted by A. Ruzicka

Lab number CML0778; field number J223.; submitted by A. Ruzicka

Submitted by AMN

Submitted by AMN

Work name M2081; submitted by A. Irving

Submitted by Pavel Yu. Plechov, pplechov@gmail.com

G709; submitted by A. Bouvier, UWO

Submitted by Ebert $\mathrm{S}$.

Submitted by Ebert $\mathrm{S}$.

work name LM47 / C12; Packed chondrules rimmed with opaques.; submitted by Jérôme Gattacceca

Submitted by Ebert $\mathrm{S}$.

Submitted by Simone de Leuw

Submitted by James P. Greenwood

Field name: Met-2016-029; submitted by David Allepuz

Submitted by A. Yamaguchi

In Chinese publications, its former name was M16214; submitted by Bingkui Miao

P.R. Heck acknowledges Robert Ward for donating his first meteorite to FMNH, Terry Boudreaux for sur

Work name AK26; submitted by Lisa Krämer Ruggiu

In Chinese publications, its former name was M16212; submitted by Bingkui Miao

Submitted by Simone de Leuw

Submitted by Ansgar Greshake

Submitted by A. Yamaguchi

In Chinese publications, its former name was M16218; submitted by Bingkui Miao

Submitted by Simone de Leuw

G706; submitted by A. Bouvier, UWO

work name E2; submitted by Richard Greenwood, Hélène Greenwood

Submitted by A. Yamaguchi

Submitted by Bingkui Miao

Field name: Met-2017-026; submitted by David Allepuz

Submitted by Simone de Leuw

In Chinese publications, its former name was M16031; submitted by Bingkui Miao

Submitted by A. Yamaguchi

Submitted by Simone de Leuw

In Chinese publications, its former name was M16035; submitted by Bingkui Miao

SEM; submitted by AMN

work name E4; submitted by Richard Greenwood, Hélène Greenwood

Submitted by Vanni Moggi Cecchi

Submitted by Jordi Llorca (UPC)

Submitted by A. Yamaguchi

In Chinese publications, its former name was M16046; submitted by Bingkui Miao

In Chinese publications, its former name was M16155; submitted by Bingkui Miao

Submitted by Ansgar Greshake

Submitted by A. Yamaguchi

In Chinese publications, its former name was M16152; submitted by Bingkui Miao

In Chinese publications, its former name was M16036; submitted by Bingkui Miao 
In Chinese publications, its former name was M16037; submitted by Bingkui Miao Field name: Met-2018-012; submitted by David Allepuz

Submitted by Ansgar Greshake

In Chinese publications, its former name was M16061; submitted by Bingkui Miao

Chondrules well defined. Olivine: undulose extinction, lacks planar fractures. A few low-Ca pyroxene phe Submitted by Vanni Moggi Cecchi

In Chinese publications, its former name was M16032; submitted by Bingkui Miao

In Chinese publications, its former name was M16157; submitted by Bingkui Miao

Field name: Met-2016-026; submitted by David Allepuz

In Chinese publications, its former name was M16117; submitted by Bingkui Miao

Submitted by A. Yamaguchi

work name E6; submitted by Richard Greenwood, Hélène Greenwood

work name EJ426; submitted by Jérôme Gattacceca

In Chinese publications, its former name was M16039; submitted by Bingkui Miao

Submitted by A. Yamaguchi

In Chinese publications, its former name was M16250; submitted by Bingkui Miao

The meteorite is brecciated. Field name is Calama 2-66; analyst T. Kryachko (Technograd, Moscow); su Chondrules are well defined. Olivine exhibits undulose extinction, but lacks planar fractures. Small chron In Chinese publications, its former name was M16064; submitted by Bingkui Miao

Submitted by Vanni Moggi Cecchi

Submitted by A. Yamaguchi

In Chinese publications, its former name was M16124; submitted by Bingkui Miao

In Chinese publications, its former name was M16078; submitted by Bingkui Miao

Submitted by A. Yamaguchi

work name Sahara 98050B; submitted by Ebert S.

Submitted by AMN

In Chinese publications, its former name was M16156; submitted by Bingkui Miao Breccia; submitted by A. Yamaguchi

In Chinese publications, its former name was M16137; submitted by Bingkui Miao

Submitted by A. Yamaguchi

Submitted by Vanni Moggi Cecchi

In Chinese publications, its former name was M16053; submitted by Bingkui Miao

Submitted by A. Yamaguchi

Submitted by Vanni Moggi Cecchi

In Chinese publications, its former name was M16049; submitted by Bingkui Miao

SEM; submitted by AMN

In Chinese publications, its former name was M16110; submitted by Bingkui Miao work name E1; submitted by Richard Greenwood, Hélène Greenwood

In Chinese publications, its former name was M16230; submitted by Bingkui Miao

Submitted by A. Yamaguchi

In Chinese publications, its former name was M16183; submitted by Bingkui Miao

In Chinese publications, its former name was M16079; submitted by Bingkui Miao

Submitted by A. Yamaguchi

Submitted by A. Yamaguchi

Submitted by A. Yamaguchi 
Submitted by A. Yamaguchi

Chondrules are well defined. Olivine exhibits sharp optical extinction. Very few low-Ca pyroxene grains $\epsilon$ Submitted by A. Yamaguchi

Submitted by A. Yamaguchi

In Chinese publications, its former name was M16236; submitted by Bingkui Miao

Submitted by A. Yamaguchi

Submitted by A. Yamaguchi

Submitted by A. Yamaguchi

Catalogued at the Royal Ontario Museum as LM58150; submitted by V. Di Cecco, ROM

Submitted by A. Yamaguchi

Submitted by A. Yamaguchi

Submitted by Vanni Moggi Cecchi

In Chinese publications, its former name was M16136; submitted by Bingkui Miao

In Chinese publications, its former name was M16153; submitted by Bingkui Miao

Submitted by A. Yamaguchi

In Chinese publications, its former name was M16244; submitted by Bingkui Miao

Submitted by A. Yamaguchi

In Chinese publications, its former name was M16177; submitted by Bingkui Miao

Submitted by A. Yamaguchi

Submitted by A. Yamaguchi

SEM; submitted by AMN

SEM; submitted by AMN

Submitted by A. Yamaguchi

SEM; submitted by AMN

SEM; submitted by AMN

Darkened; submitted by A. Yamaguchi

Submitted by A. Yamaguchi

Submitted by A. Yamaguchi

In Chinese publications, its former name was M16249; submitted by Bingkui Miao

Submitted by A. Yamaguchi

Darkened; submitted by A. Yamaguchi

Slightly brecciated; submitted by A. Yamaguchi

Darkened; submitted by A. Yamaguchi

Partly melted; submitted by A. Yamaguchi

Submitted by AMN

Submitted by A. Yamaguchi

Submitted by A. Yamaguchi

Field name is Calama 2-34; analyst T. Kryachko (Technograd, Moscow); submitted by Lorenz C.A., Verr

Submitted by A. Yamaguchi

Submitted by A. Yamaguchi

Submitted by A. Yamaguchi

Submitted by A. Yamaguchi

Submitted by AMN

Submitted by A. Yamaguchi

Submitted by A. Yamaguchi 
Slightly brecciated; submitted by A. Yamaguchi

Submitted by A. Yamaguchi

Breccia; submitted by A. Yamaguchi

Submitted by A. Yamaguchi

Submitted by A. Yamaguchi

Submitted by A. Yamaguchi

Submitted by A. Yamaguchi

Submitted by A. Yamaguchi

Submitted by A. Yamaguchi

Submitted by A. Yamaguchi

Submitted by A. Yamaguchi

Darkened; submitted by A. Yamaguchi

Darkened; submitted by A. Yamaguchi

Submitted by A. Yamaguchi

Submitted by loannis Baziotis

Submitted by A. Yamaguchi

Breccia; submitted by A. Yamaguchi

Submitted by A. Yamaguchi

Submitted by A. Yamaguchi

Submitted by A. Yamaguchi

Submitted by A. Yamaguchi

Submitted by A. Yamaguchi

Submitted by A. Yamaguchi

Submitted by A. Yamaguchi

Submitted by A. Yamaguchi

Submitted by A. Yamaguchi

Submitted by A. Yamaguchi

Submitted by A. Yamaguchi

Submitted by A. Yamaguchi

Submitted by A. Yamaguchi

Submitted by A. Yamaguchi

Submitted by A. Yamaguchi

Submitted by A. Yamaguchi

Submitted by A. Yamaguchi

Submitted by A. Yamaguchi

Submitted by A. Yamaguchi

Submitted by A. Yamaguchi

Shock vein; submitted by A. Yamaguchi

Submitted by A. Yamaguchi

Submitted by A. Yamaguchi

Submitted by A. Yamaguchi

Submitted by A. Yamaguchi

Submitted by A. Yamaguchi

Submitted by A. Yamaguchi

Submitted by A. Yamaguchi 
Submitted by A. Yamaguchi

Breccia; submitted by A. Yamaguchi

Submitted by A. Yamaguchi

Submitted by A. Yamaguchi

Submitted by A. Yamaguchi

Submitted by A. Yamaguchi

Submitted by A. Yamaguchi

Submitted by A. Yamaguchi

Submitted by A. Yamaguchi

Submitted by A. Yamaguchi

Darkened; submitted by A. Yamaguchi

Darkened; submitted by A. Yamaguchi

Submitted by A. Yamaguchi

Breccia; submitted by A. Yamaguchi

Submitted by A. Yamaguchi

Submitted by A. Yamaguchi

Submitted by A. Yamaguchi

Submitted by A. Yamaguchi

Submitted by A. Yamaguchi

Submitted by A. Yamaguchi

Submitted by A. Yamaguchi

Submitted by A. Yamaguchi

Submitted by A. Yamaguchi

Slightly brecciated; submitted by A. Yamaguchi

Submitted by A. Yamaguchi

Submitted by A. Yamaguchi

Submitted by A. Yamaguchi

Submitted by A. Yamaguchi

Submitted by A. Yamaguchi

Submitted by A. Yamaguchi

Submitted by A. Yamaguchi

Submitted by A. Yamaguchi

Submitted by A. Yamaguchi

Submitted by A. Yamaguchi

Submitted by A. Yamaguchi

Submitted by A. Yamaguchi

Submitted by A. Yamaguchi

Submitted by A. Yamaguchi

Submitted by A. Yamaguchi

Submitted by A. Yamaguchi

Submitted by A. Yamaguchi

Submitted by A. Yamaguchi

Submitted by A. Yamaguchi

Submitted by A. Yamaguchi

Submitted by A. Yamaguchi 
Submitted by A. Yamaguchi

Submitted by A. Yamaguchi

Darkened; submitted by A. Yamaguchi

Submitted by A. Yamaguchi

Submitted by A. Yamaguchi

Submitted by A. Yamaguchi

Submitted by A. Yamaguchi

Submitted by A. Yamaguchi

Submitted by A. Yamaguchi

Submitted by A. Yamaguchi

Submitted by A. Yamaguchi

Submitted by A. Yamaguchi

Submitted by A. Yamaguchi

Submitted by A. Yamaguchi

Submitted by A. Yamaguchi

Submitted by A. Yamaguchi

Submitted by A. Yamaguchi

Submitted by A. Yamaguchi

Submitted by A. Yamaguchi

Submitted by A. Yamaguchi

Submitted by A. Yamaguchi

Submitted by A. Yamaguchi

Slightly brecciated; submitted by A. Yamaguchi

Submitted by A. Yamaguchi

Submitted by A. Yamaguchi

Submitted by A. Yamaguchi

Submitted by A. Yamaguchi

Submitted by A. Yamaguchi

Submitted by A. Yamaguchi

Slightly brecciated; submitted by A. Yamaguchi

Submitted by A. Yamaguchi

Submitted by A. Yamaguchi

Submitted by A. Yamaguchi

Submitted by A. Yamaguchi

Submitted by A. Yamaguchi

Submitted by A. Yamaguchi

Submitted by A. Yamaguchi

Submitted by A. Yamaguchi

Submitted by A. Yamaguchi

Submitted by A. Yamaguchi

Submitted by A. Yamaguchi

Breccia; submitted by A. Yamaguchi

SEM; submitted by AMN

Submitted by A. Yamaguchi

Submitted by A. Yamaguchi 
Breccia; submitted by A. Yamaguchi

Submitted by A. Yamaguchi

Darkened; submitted by A. Yamaguchi

Submitted by A. Yamaguchi

Submitted by A. Yamaguchi

Submitted by A. Yamaguchi

Submitted by A. Yamaguchi

Submitted by A. Yamaguchi

Submitted by A. Yamaguchi

Submitted by A. Yamaguchi

Submitted by A. Yamaguchi

Submitted by A. Yamaguchi

Submitted by A. Yamaguchi

Submitted by A. Yamaguchi

Submitted by A. Yamaguchi

Submitted by A. Yamaguchi

Submitted by A. Yamaguchi

Submitted by A. Yamaguchi

Submitted by Daniel Atencio, Dorilia Cunha and Andre L. R. Moutinho

Genomict breccia; submitted by A. Yamaguchi

Submitted by Alan Rubin

Work name BF17-21; submitted by A. Irving

Submitted by Ebert $\mathrm{S}$.

Submitted by Ebert S.

Field name: Met-2018-026; submitted by David Allepuz

Breccia Field name: Met-2018-024; submitted by David Allepuz work name LM60/D26; submitted by Jérôme Gattacceca

Field name: Met-2017-023; submitted by David Allepuz

Field name: Met-2018-049; submitted by David Allepuz

Field name: Met-2016-013; submitted by David Allepuz

Working No.: SM 1804-4; submitted by K. Klemm, IfP; submitted by Klemm K.

Work name CHC18009; submitted by A. Irving

Field name: Met-2016-041; submitted by David Allepuz

Submitted by C. Park, KOPRI

work name LM33 / C10; Brecciated chondrite with type 3 and type 5 material.; submitted by Jérôme Gat

Submitted by W. Birch

Submitted by C. Park, KOPRI

work name LM45 / C08; submitted by J. Gattacceca

SEM; submitted by AMN

Submitted by C. A. Lorenz

Field number MMO-2018-3A; submitted by C. Herd

Submitted by Vanni Moggi Cecchi

M2159; submitted by C. Agee

Work name SLee18-1; submitted by A. Irving

Submitted by Vanni Moggi Cecchi 


\begin{abstract}
Submitted by Guiqin Wang Nengyong Hu
Work name MA002; submitted by Lisa Krämer Ruggiu

Work name TS18-1; submitted by A. Irving

work name LL21 (LAB060417a); submitted by J. Gattacceca
\end{abstract}

Submitted by Y. Xu, IGGCAS

G713; submitted by A. Bouvier, UWO

Field name is Calama032-Ch; analist S. E. Borisovsky (IGEM); submitted by Lorenz C.A., Vernad

plagioclase grain size about 15 um; submitted by Ansgar Greshake

G704; submitted by A. Bouvier, UWO

Submitted by Simone de Leuw

Brownish individual without fusion crust. plagioclase grain size about $25 \mu \mathrm{m}$; submitted by Ansgar Gresh

Submitted by A. Yamaguchi

work name LL2-3; submitted by J. Gattacceca

Submitted by Katarzyna Luszczek

Submitted by Vanni Moggi Cecchi

In Chinese publications, its former name was M16215; submitted by Bingkui Miao

Submitted by Jordi Llorca (UPC)

In Chinese publications, its former name was M16262; submitted by Bingkui Miao

work name LM53/D5; This ordinary chondrite is rather unusual with high porosity (15\%, determined by $p$ work name LM53/D5; has high porosity (15\%, determined by point counting, $\mathrm{N}=169)$; well-preserved chc Submitted by Simone de Leuw

Submitted by A. Yamaguchi

Field name SM-3; submitted by C. Agee, UNM

SEM; submitted by AMN

In Chinese publications, its former name was M16216; submitted by Bingkui Miao

Field name is Calama 4-16; analyst T. Kryachko (Technograd, Moscow); submitted by Lorenz C.A., Verr

G710; submitted by A. Bouvier, UWO

Working No.: SM 1804-16; submitted by K. Klemm, IfP; submitted by Klemm K.

Submitted by Ken Eltrich

Submitted by C. Park, KOPRI

Field name: Met-2016-045; submitted by David Allepuz

Submitted by Vanni Moggi Cecchi

In Chinese publications, its former name was M16030; submitted by Bingkui Miao

Field name is 2-1; analyst S. E. Borisovsky (IGEM); submitted by C. A. Lorenz (Vernad)

Darkened; submitted by A. Yamaguchi

Field name: Met-2016-047; submitted by David Allepuz

In Chinese publications, its former name was M16165; submitted by Bingkui Miao

In Chinese publications, its former name was M16122; submitted by Bingkui Miao

work name E3; submitted by Richard Greenwood, Hélène Greenwood

Submitted by A. Yamaguchi

Field name is Calama 3-57; analyst V. V. Kozlov (Oxford Instruments, Moscow); submitted by Lorenz C.

Submitted by Eber $\mathrm{S}$.

Field name: Met-2018-020; submitted by David Allepuz

work name EJ1125; submitted by J. Gattacceca

Submitted by Vanni Moggi Cecchi 
Submitted by Maria Elizabeth Zucolotto

Submitted by Vanni Moggi Cecchi

work name EJ615; submitted by J. Gattacceca

work name JG061; submitted by J. Gattacceca

work name EJ971; submitted by J. Gattacceca

Submitted by Vanni Moggi Cecchi

Submitted by A. Yamaguchi

Submitted by Jordi Llorca (UPC)

In Chinese publications, its former name was M16166; submitted by Bingkui Miao

In Chinese publications, its former name was M16119; submitted by Bingkui Miao

Field name is 2-3b; analyst S. E. Borisovsky (IGEM); submitted by C. A. Lorenz (Vernad)

Submitted by Vanni Moggi Cecchi

G711; submitted by A. Bouvier, UWO

Submitted by A. Yamaguchi

Submitted by AMN

work name EJ972; submitted by J. Gattacceca

Field name: Met-2016-040; submitted by David Allepuz

In Chinese publications, its former name was M16209; submitted by Bingkui Miao

In Chinese publications, its former name was M16168; submitted by Bingkui Miao work name RM615; submitted by Jérôme Gattacceca

In Chinese publications, its former name was M16047; submitted by Bingkui Miao

Working No.: SM 1804-8; submitted by K. Klemm, IfP; submitted by Klemm K. work name E7; submitted by Richard Greenwood, Hélène Greenwood

In Chinese publications, its former name was M16113; submitted by Bingkui Miao

Field name is Calate Q10; analyst T. Kryachko (Technograd, Moscow); submitted by Lorenz C.A., Verne In Chinese publications, its former name was M16066; submitted by Bingkui Miao

In Chinese publications, its former name was M16115; submitted by Bingkui Miao In Chinese publications, its former name was M16163; submitted by Bingkui Miao In Chinese publications, its former name was M16146; submitted by Bingkui Miao In Chinese publications, its former name was M16271; submitted by Bingkui Miao Submitted by A. Yamaguchi

In Chinese publications, its former name was M16038; submitted by Bingkui Miao Field name is 1-8; analyst S. E. Borisovsky (IGEM); submitted by C. A. Lorenz (Vernad) Field name is Irantown7; analyst N. N. Kononkova (Vernad); submitted by Lorenz C. A., Vernad Field name is Irantown6; analyst N. N. Kononkova (Vernad); submitted by Lorenz C. A., Vernad Field name: Met-2017-031; submitted by David Allepuz work name EJ1092. Shock veins. Chromite-plagioclase assemblages.; submitted by J. Gattacceca Submitted by Vanni Moggi Cecchi

Submitted by Daniel Sheikh

Field name is Calama 4-19; analyst T. Kryachko (Technograd, Moscow); submitted by Lorenz C.A., Verr Field name: Met-2017-016; submitted by David Allepuz

Field name: Met-2016-038; submitted by David Allepuz

Submitted by Vanni Moggi Cecchi

Submitted by Vanni Moggi Cecchi

Field number 1702_0369; submitted by Beda Hofmann 
work name RL06. Crusted stone. Cut surface reveals grey interior with abundant fresh metal.; submitted Submitted by Vanni Moggi Cecchi

In Chinese publications, its former name was M16060; submitted by Bingkui Miao

In Chinese publications, its former name was M16164; submitted by Bingkui Miao

Submitted by A. Yamaguchi

Submitted by A. Yamaguchi

work name EJ116; submitted by Jérôme Gattacceca

In Chinese publications, its former name was M16175; submitted by Bingkui Miao

SEM; submitted by AMN

work name JG142. Shock veins. Average plagioclase size below 50 um.; submitted by J. Gattacceca

In Chinese publications, its former name was M16173; submitted by Bingkui Miao

Submitted by V. Di Cecco

In Chinese publications, its former name was M16241; submitted by Bingkui Miao

Field name: Met-2017-009; submitted by David Allepuz

In Chinese publications, its former name was M16052; submitted by Bingkui Miao

Submitted by C. Park, KOPRI

Submitted by A. Yamaguchi

work name EJ057; Chondritic texture with average plagioclase size below 50 um.; submitted by Jerome In Chinese publications, its former name was M16229; submitted by Bingkui Miao

In Chinese publications, its former name was M16268; submitted by Bingkui Miao

In Chinese publications, its former name was M16247; submitted by Bingkui Miao

Submitted by A. Yamaguchi

SEM; submitted by AMN

In Chinese publications, its former name was M16068; submitted by Bingkui Miao

In Chinese publications, its former name was M16118; submitted by Bingkui Miao

Submitted by A. Yamaguchi

SEM; submitted by AMN

Submitted by Vanni Moggi Cecchi

work name EJ611; submitted by Gattacceca

Submitted by C. Park, KOPRI

Submitted by A. Yamaguchi

In Chinese publications, its former name was M16062; submitted by Bingkui Miao

Submitted by Vanni Moggi Cecchi

Submitted by A. Yamaguchi

In Chinese publications, its former name was M16074; submitted by Bingkui Miao

SEM; submitted by AMN

Submitted by C. Park, KOPRI

SEM; submitted by AMN

Submitted by Vanni Moggi Cecchi

SEM; submitted by AMN

Submitted by A. Yamaguchi

Lab number CML0948; submitted by A. Ruzicka

Submitted by A. Yamaguchi

Brownish individual partly covered with fusion crust. plagioclase grain size about $30 \mu \mathrm{m}$; submitted by $\mathrm{Ar}$ Field name is CH70ESF; analyst N. N. Kononkova (Vernad); submitted by Lorenz C. A., Vernad 
Submitted by M. Bizzarro, A.N. Krot, D. Wielandt

In Chinese publications, its former name was M16087; submitted by Bingkui Miao In Chinese publications, its former name was M16232; submitted by Bingkui Miao

Submitted by A. Yamaguchi

Field name: Met-2018-030; submitted by David Allepuz

Submitted by Vanni Moggi Cecchi

SEM; submitted by AMN

In Chinese publications, its former name was M16085; submitted by Bingkui Miao work name EJ166; submitted by J. Gattacceca

work name EJ322; submitted by Jérôme Gattacceca

Field name is CH40AT5ESF; analyst N. N. Kononkova (Vernad); submitted by Lorenz C. A., Vernad Field name is Calama 4-70; analyst T. Kryachko (Technograd, Moscow); submitted by Lorenz C.A., Verr SEM; submitted by AMN

Brownish individual without fusion crust. plagioclase grain size about $20 \mu \mathrm{m}$; submitted by Ansgar Gresh In Chinese publications, its former name was M16096; submitted by Bingkui Miao

Submitted by A. Yamaguchi

In Chinese publications, its former name was M16176; submitted by Bingkui Miao

Field name: Met-2018-008; submitted by David Allepuz

Submitted by A. Yamaguchi

work name EJ589. Average plagioclase size below 50 um. Chromite-plagioclase assemblages.; submitt Lab number CML0813; submitted by A. Ruzicka

Submitted by C. Park, KOPRI

Submitted by Vanni Moggi Cecchi

Submitted by A. Yamaguchi

Submitted by A. Yamaguchi

Field name is CH20G8ESF; analyst N. N. Kononkova (Vernad); submitted by Lorenz C. A., Vernad

In Chinese publications, its former name was M16084; submitted by Bingkui Miao

Submitted by A. Yamaguchi

Submitted by A. Yamaguchi

Lab number CML0812; submitted by A. Ruzicka

Field name: Met-2017-013; submitted by David Allepuz

SEM; submitted by AMN

Submitted by Vanni Moggi Cecchi

work name EJ1094; submitted by Jérôme Gattacceca

SEM; submitted by AMN

SEM; submitted by AMN

Submitted by Vanni Moggi Cecchi

In Chinese publications, its former name was M16245; submitted by Bingkui Miao

In Chinese publications, its former name was M16141; submitted by Bingkui Miao

In Chinese publications, its former name was M16283; submitted by Bingkui Miao

Submitted by C. Park, KOPRI

work name LL1-3a; submitted by J. Gattacceca

In Chinese publications, its former name was M16077; submitted by Bingkui Miao

In Chinese publications, its former name was M16145; submitted by Bingkui Miao

SEM; submitted by AMN 
work name JR052; Bought from Mohamed Elguirah in Zagora in 2018. Six identical looking fragments wi Submitted by A. Yamaguchi

Submitted by A. Yamaguchi

Submitted by A. Yamaguchi

In Chinese publications, its former name was M16083; submitted by Bingkui Miao

SEM; submitted by AMN

Submitted by A. Yamaguchi

Submitted by C. Park, KOPRI

Submitted by A. Yamaguchi

ROM Number M57519; No mosaicism, some oxidation with veining; submitted by V. Di Cecco, ROM

In Chinese publications, its former name was M16072; submitted by Bingkui Miao

work name EJ588. shock veins.; submitted by J. Gattacceca

Submitted by A. Yamaguchi

In Chinese publications, its former name was M16270; submitted by Bingkui Miao

SEM; submitted by AMN

Submitted by AMN

Fieldname = ReDL062; thin-section ID\# = RSV062; submitted by Daniel Sheikh

Submitted by A. Yamaguchi

In Chinese publications, its former name was M16139; submitted by Bingkui Miao

SEM; submitted by AMN

SEM; submitted by AMN

Submitted by A. Yamaguchi

In Chinese publications, its former name was M16070; submitted by Bingkui Miao

In Chinese publications, its former name was M16185; submitted by Bingkui Miao

In Chinese publications, its former name was M16128; submitted by Bingkui Miao

SEM; submitted by AMN

In Chinese publications, its former name was M16147; submitted by Bingkui Miao SEM; submitted by AMN

Submitted by AMN

In Chinese publications, its former name was M16130; submitted by Bingkui Miao Submitted by C. Park, KOPRI

work name EJ150; submitted by J. Gattacceca

In Chinese publications, its former name was M16080; submitted by Bingkui Miao Submitted by A. Yamaguchi

SEM; submitted by AMN

SEM; submitted by AMN

Submitted by C. Park, KOPRI

In Chinese publications, its former name was M16076; submitted by Bingkui Miao In Chinese publications, its former name was M16178; submitted by Bingkui Miao Field name: Met-2018-009; submitted by David Allepuz

Submitted by A. Yamaguchi

SEM; submitted by AMN

SEM; submitted by AMN

In Chinese publications, its former name was M16187; submitted by Bingkui Miao

In Chinese publications, its former name was M16082; submitted by Bingkui Miao 
Submitted by A. Yamaguchi

Submitted by A. Yamaguchi

SEM; submitted by AMN

Submitted by A. Yamaguchi

Submitted by A. Yamaguchi

Submitted by A. Yamaguchi

SEM; submitted by AMN

SEM; submitted by AMN

SEM; submitted by AMN

Submitted by A. Yamaguchi

Field name: Met-2015-033; submitted by David Allepuz

SEM; submitted by AMN

Submitted by C. Park, KOPRI

Breccia; submitted by A. Yamaguchi

In Chinese publications, its former name was M16254; submitted by Bingkui Miao

Submitted by A. Yamaguchi

SEM; submitted by AMN

SEM; submitted by AMN

SEM; submitted by AMN

Submitted by C. Park, KOPRI

work name LL1-6; submitted by J. Gattacceca

Submitted by A. Yamaguchi

Submitted by A. Yamaguchi

Field name is 7-1; analyst S. E. Borisovsky (IGEM); submitted by C. A. Lorenz (Vernad)

Field name: Met-2018-034; submitted by David Allepuz

SEM; submitted by AMN

SEM; submitted by AMN

Submitted by A. Yamaguchi

Submitted by A. Yamaguchi

Submitted by A. Yamaguchi

Submitted by A. Yamaguchi

SEM; submitted by AMN

SEM; submitted by AMN

SEM; submitted by AMN

SEM; submitted by AMN

SEM; submitted by AMN

In Chinese publications, its former name was M16143; submitted by Bingkui Miao

Submitted by A. Yamaguchi

In Chinese publications, its former name was M16063; submitted by Bingkui Miao

In Chinese publications, its former name was M16134; submitted by Bingkui Miao

In Chinese publications, its former name was M16186; submitted by Bingkui Miao

Submitted by C. Park, KOPRI

In Chinese publications, its former name was M16150; submitted by Bingkui Miao

SEM; submitted by AMN

Submitted by A. Yamaguchi 
Submitted by C. Park, KOPRI

Submitted by A. Yamaguchi

Field name is Calama18. Analyst V. V. Kozlov (Tescan Demo Centre, Moscow, Russia); submitted by Lc

Submitted by C. Park, KOPRI

Field name: Met-2018-066; submitted by David Allepuz

Submitted by A. Yamaguchi

Submitted by A. Yamaguchi

In Chinese publications, its former name was M16133; submitted by Bingkui Miao

Submitted by A. Yamaguchi

Submitted by A. Yamaguchi

Submitted by A. Yamaguchi

Submitted by A. Yamaguchi

In Chinese publications, its former name was M16138; submitted by Bingkui Miao

SEM; submitted by AMN

Submitted by A. Yamaguchi

Slightly brecciated; submitted by A. Yamaguchi

SEM; submitted by AMN

Submitted by A. Yamaguchi

Submitted by A. Yamaguchi

Submitted by A. Yamaguchi

Submitted by A. Yamaguchi

work name EJ310; submitted by J. Gattacceca

Field name is Calama 1-36; analyst T. Kryachko (Technograd, Moscow); submitted by Lorenz C.A., Verr

Submitted by A. Yamaguchi

Submitted by A. Yamaguchi

Submitted by A. Yamaguchi

Submitted by A. Yamaguchi

Submitted by A. Yamaguchi

Submitted by A. Yamaguchi

Submitted by A. Yamaguchi

Submitted by A. Yamaguchi

Submitted by A. Yamaguchi

Submitted by A. Yamaguchi

Submitted by A. Yamaguchi

Submitted by A. Yamaguchi

Submitted by A. Yamaguchi

Submitted by A. Yamaguchi

Submitted by A. Yamaguchi

Submitted by A. Yamaguchi

Submitted by A. Yamaguchi

Submitted by A. Yamaguchi

Submitted by A. Yamaguchi

Submitted by A. Yamaguchi

Submitted by A. Yamaguchi

Submitted by A. Yamaguchi 
Submitted by A. Yamaguchi

Submitted by A. Yamaguchi

Submitted by A. Yamaguchi

Submitted by A. Yamaguchi

Submitted by A. Yamaguchi

Submitted by A. Yamaguchi

Submitted by A. Yamaguchi

Submitted by A. Yamaguchi

Submitted by A. Yamaguchi

Submitted by A. Yamaguchi

SEM; submitted by AMN

Slightly brecciated; submitted by A. Yamaguchi

Submitted by A. Yamaguchi

Submitted by A. Yamaguchi

Darkened; submitted by A. Yamaguchi

Submitted by A. Yamaguchi

Submitted by A. Yamaguchi

Submitted by A. Yamaguchi

Submitted by A. Yamaguchi

Submitted by A. Yamaguchi

Submitted by C. Park, KOPRI

Submitted by A. Yamaguchi

Submitted by A. Yamaguchi

Submitted by A. Yamaguchi

Submitted by A. Yamaguchi

Submitted by A. Yamaguchi

Submitted by A. Yamaguchi

Submitted by A. Yamaguchi

Submitted by A. Yamaguchi

Submitted by A. Yamaguchi

Submitted by A. Yamaguchi

Submitted by A. Yamaguchi

Submitted by A. Yamaguchi

Submitted by A. Yamaguchi

Submitted by A. Yamaguchi

Submitted by A. Yamaguchi

Submitted by A. Yamaguchi

Submitted by A. Yamaguchi

Submitted by A. Yamaguchi

Submitted by A. Yamaguchi

Submitted by A. Yamaguchi

Submitted by A. Yamaguchi

Submitted by A. Yamaguchi

Submitted by A. Yamaguchi

Submitted by A. Yamaguchi 
Submitted by A. Yamaguchi

Submitted by A. Yamaguchi

Slightly brecciated; submitted by A. Yamaguchi

Submitted by A. Yamaguchi

Submitted by A. Yamaguchi

Submitted by A. Yamaguchi

Submitted by A. Yamaguchi

Slightly brecciated; submitted by A. Yamaguchi

Submitted by A. Yamaguchi

Submitted by A. Yamaguchi

Submitted by A. Yamaguchi

Submitted by A. Yamaguchi

Slightly brecciated; submitted by A. Yamaguchi

Submitted by A. Yamaguchi

SEM; submitted by AMN

Submitted by A. Yamaguchi

SEM; submitted by AMN

SEM; submitted by AMN

Submitted by A. Yamaguchi

Submitted by A. Yamaguchi

Submitted by A. Yamaguchi

SEM; submitted by AMN

Submitted by A. Yamaguchi

Submitted by A. Yamaguchi

Submitted by A. Yamaguchi

Submitted by A. Yamaguchi

Submitted by A. Yamaguchi

Submitted by A. Yamaguchi

Submitted by A. Yamaguchi

Submitted by A. Yamaguchi

Submitted by C. Park, KOPRI

Submitted by A. Yamaguchi

Submitted by A. Yamaguchi

Submitted by A. Yamaguchi

Submitted by A. Yamaguchi

Submitted by A. Yamaguchi

Submitted by A. Yamaguchi

Submitted by A. Yamaguchi

Submitted by A. Yamaguchi

Submitted by A. Yamaguchi

Submitted by A. Yamaguchi

Submitted by A. Yamaguchi

Submitted by A. Yamaguchi

Submitted by A. Yamaguchi

SEM; submitted by AMN 
Submitted by A. Yamaguchi

Large metal nodule; submitted by A. Yamaguchi

SEM; submitted by AMN

Submitted by A. Yamaguchi

Submitted by A. Yamaguchi

Submitted by A. Yamaguchi

Submitted by A. Yamaguchi

Submitted by A. Yamaguchi

Submitted by A. Yamaguchi

Submitted by A. Yamaguchi

Submitted by A. Yamaguchi

Slightly brecciated; submitted by A. Yamaguchi

Submitted by A. Yamaguchi

Submitted by C. Park, KOPRI

Submitted by A. Yamaguchi

analyst N.N. Kononkova, Vernad; submitted by C. A. Lorenz (Vernad)

breccia; submitted by Ebert S.

work name LM58/D24; Breccia with chondritic clasts in a melt rock matrix. The chondritic clasts have avi Submitted by Ansgar Greshake

Field name: Met-2017-021; submitted by David Allepuz

work name EJ242. High interchondrules porosity; submitted by Jérôme Gattacceca

Submitted by Y. Xu, IGGCAS

Submitted by Simone de Leuw

Field name DD-32; submitted by C. Agee, UNM

Submitted by Vanni Moggi Cecchi

Submitted by K. Metzler, IfP

Submitted by F. BrandstÃatter

Field name: Met-2015-020; submitted by David Allepuz

work name LM66/D49; submitted by Jérôme Gattacceca

Fieldname = UU151212V; thin-section ID = V-C1; submitted by Daniel Sheikh

plagioclase grain size about 60 um; submitted by Ansgar Greshake

Submitted by A. Yamaguchi

Submitted by Vanni Moggi Cecchi

Field name Kol-2; analyst V.V. Sharygin (SIGM and UrFU); submitted by Victor V. Sharygin, sharygin@i!

Submitted by Vanni Moggi Cecchi

Field name: Met-2017-034; submitted by David Allepuz

Submitted by A. Yamaguchi

Submitted by A. Irving

Field name: Met-2017-024; submitted by David Allepuz

Work name RL01; Highly recrystallized with relict chondrules. Plagioclase to $100 \mathrm{um}$, average 80um.; su

Field name: Met-2017-019; submitted by David Allepuz

Submitted by A. Yamaguchi

Field name: Met-2018-048; submitted by David Allepuz

Plagioclase grain size about $60 \mu \mathrm{m}$; submitted by Ansgar Greshake

Submitted by A. Yamaguchi 
Submitted by Simone de Leuw

Field name: Met-2017-032; submitted by David Allepuz

Work name MG18-1; submitted by A. Irving

plagioclase grain size about 70 um; submitted by Ansgar Greshake

Field name: Met-2017-028; submitted by David Allepuz

Submitted by A. Yamaguchi

Field name LAR-4; submitted by V.V. Sharygin (SIGM and UrFU)

In Chinese publications, its former name was M16239; submitted by Bingkui Miao

In Chinese publications, its former name was M16144; submitted by Bingkui Miao

Field name: Met-2018-051; submitted by David Allepuz

Submitted by Romano Serra (OAM)

Field name LAR-3; submitted by Victor V. Sharygin, SIGM and UrFU

Submitted by A. Yamaguchi

Submitted by AMN

Catalogued at the Royal Ontario Museum as LM58145; submitted by V. Di Cecco, ROM work name EJ1108; submitted by J. Gattacceca

In Chinese publications, its former name was M16158; submitted by Bingkui Miao

In Chinese publications, its former name was M16260; submitted by Bingkui Miao

In Chinese publications, its former name was M16071; submitted by Bingkui Miao

In Chinese publications, its former name was M16127; submitted by Bingkui Miao

In Chinese publications, its former name was M16170; submitted by Bingkui Miao

In Chinese publications, its former name was M16123; submitted by Bingkui Miao

SEM; submitted by AMN

Darkened; submitted by A. Yamaguchi

In Chinese publications, its former name was M16159; submitted by Bingkui Miao

Field name: Met-2017-025; submitted by David Allepuz

SEM; submitted by AMN

In Chinese publications, its former name was M16181; submitted by Bingkui Miao

In Chinese publications, its former name was M16111; submitted by Bingkui Miao

Submitted by A. Yamaguchi

In Chinese publications, its former name was M16129; submitted by Bingkui Miao SEM; submitted by AMN

Submitted by Vanni Moggi Cecchi

In Chinese publications, its former name was M16114; submitted by Bingkui Miao SEM; submitted by AMN

In Chinese publications, its former name was M16116; submitted by Bingkui Miao

In Chinese publications, its former name was M16310; submitted by Bingkui Miao

Submitted by A. Yamaguchi

In Chinese publications, its former name was M16069; submitted by Bingkui Miao

SEM; submitted by AMN

Submitted by A. Yamaguchi

In Chinese publications, its former name was M16171; submitted by Bingkui Miao

SEM; submitted by AMN

Submitted by A. Yamaguchi

In Chinese publications, its former name was M16169; submitted by Bingkui Miao 
Submitted by A. Yamaguchi

Submitted by A. Yamaguchi

SEM; submitted by AMN

SEM; submitted by AMN

In Chinese publications, its former name was M16067; submitted by Bingkui Miao

In Chinese publications, its former name was M16065; submitted by Bingkui Miao

SEM; submitted by AMN

In Chinese publications, its former name was M16161; submitted by Bingkui Miao

Submitted by Vanni Moggi Cecchi

Submitted by A. Yamaguchi

In Chinese publications, its former name was M16097; submitted by Bingkui Miao

SEM; submitted by AMN

In Chinese publications, its former name was M16182; submitted by Bingkui Miao

Submitted by A. Yamaguchi

Submitted by A. Yamaguchi

Submitted by A. Yamaguchi

In Chinese publications, its former name was M16180; submitted by Bingkui Miao

Submitted by A. Yamaguchi

SEM; submitted by AMN

Submitted by Vanni Moggi Cecchi

SEM; submitted by AMN

SEM; submitted by AMN

SEM; submitted by AMN

In Chinese publications, its former name was M16126; submitted by Bingkui Miao

Submitted by Christian Anderkin

SEM; submitted by AMN

Dark brownish individual lacking any fusion crust. plagioclase grain size about $60 \mu \mathrm{m}$; submitted by Ans? SEM; submitted by AMN

SEM; submitted by AMN

work name JG058. Shock veins. Average plagioclase size above 50 um.; submitted by J. Gattacceca

In Chinese publications, its former name was M16142; submitted by Bingkui Miao

In Chinese publications, its former name was M16125; submitted by Bingkui Miao

In Chinese publications, its former name was M16075; submitted by Bingkui Miao

SEM; submitted by AMN

ROM Number LM58184; Poorly defined chondrules, major mosaicism, minor oxidation; submitted by V.।

Submitted by A. Yamaguchi

SEM; submitted by AMN

Submitted by C. Park, KOPRI

SEM; submitted by AMN

Submitted by A. Yamaguchi

SEM; submitted by AMN

SEM; submitted by AMN

SEM; submitted by AMN

SEM; submitted by AMN

SEM; submitted by AMN 
ROM Number LM57102; Chrondrules barely discernable and broken up. Many olivine grains show mosii Submitted by A. Yamaguchi Submitted by A. Yamaguchi

SEM; submitted by AMN

Lab number CML0796; field number JW193.; submitted by A. Ruzicka

SEM; submitted by AMN

Submitted by A. Yamaguchi

Submitted by A. Yamaguchi

Submitted by A. Yamaguchi

SEM; submitted by AMN

Submitted by A. Yamaguchi

Submitted by A. Yamaguchi

Shock vein; submitted by A. Yamaguchi

SEM; submitted by AMN

SEM; submitted by AMN

In Chinese publications, its former name was M16089; submitted by Bingkui Miao SEM; submitted by AMN

Submitted by A. Yamaguchi

SEM; submitted by AMN

SEM; submitted by AMN

SEM; submitted by AMN

SEM; submitted by AMN

Submitted by A. Yamaguchi

SEM; submitted by AMN

In Chinese publications, its former name was M16132; submitted by Bingkui Miao

SEM; submitted by AMN

Submitted by A. Yamaguchi

Submitted by C. Park, KOPRI

SEM; submitted by AMN

Submitted by A. Yamaguchi

SEM; submitted by AMN

SEM; submitted by AMN

SEM; submitted by AMN

In Chinese publications, its former name was M16154; submitted by Bingkui Miao

SEM; submitted by AMN

SEM; submitted by AMN

SEM; submitted by AMN

SEM; submitted by AMN

SEM; submitted by AMN

Submitted by A. Yamaguchi

Submitted by AMN

Submitted by A. Yamaguchi

SEM; submitted by AMN

SEM; submitted by AMN

Submitted by A. Yamaguchi 
In Chinese publications, its former name was M16121; submitted by Bingkui Miao Field name: Met-2017-022; submitted by David Allepuz

SEM; submitted by AMN

In Chinese publications, its former name was M16148; submitted by Bingkui Miao SEM; submitted by AMN

SEM; submitted by AMN

SEM; submitted by AMN

SEM; submitted by AMN

SEM; submitted by AMN

SEM; submitted by AMN

SEM; submitted by AMN

SEM; submitted by AMN

Submitted by A. Yamaguchi

In Chinese publications, its former name was M16172; submitted by Bingkui Miao

Submitted by A. Yamaguchi

SEM; submitted by AMN

Submitted by A. Yamaguchi

Submitted by A. Yamaguchi

Submitted by A. Yamaguchi

Submitted by A. Yamaguchi

Submitted by A. Yamaguchi

SEM; submitted by AMN

Submitted by A. Yamaguchi

Submitted by A. Yamaguchi

SEM; submitted by AMN

Submitted by A. Yamaguchi

In Chinese publications, its former name was M16167; submitted by Bingkui Miao SEM; submitted by AMN

Large metal nodule; submitted by A. Yamaguchi

Field name: Met-2018-045; submitted by David Allepuz

Submitted by A. Yamaguchi

Submitted by A. Yamaguchi

Submitted by A. Yamaguchi

Submitted by A. Yamaguchi

Submitted by A. Yamaguchi

SEM; submitted by AMN

In Chinese publications, its former name was M16189; submitted by Bingkui Miao

Submitted by C. Park, KOPRI

Submitted by A. Yamaguchi

Shock vein; submitted by A. Yamaguchi

Submitted by A. Yamaguchi

Submitted by A. Yamaguchi

Shock vein; submitted by A. Yamaguchi

Large metal nodule; submitted by A. Yamaguchi

Submitted by A. Yamaguchi 
Submitted by A. Yamaguchi

Submitted by A. Yamaguchi

Submitted by A. Yamaguchi

Submitted by A. Yamaguchi

SEM; submitted by AMN

Submitted by A. Yamaguchi

Submitted by A. Yamaguchi

Submitted by A. Yamaguchi

Submitted by C. Park, KOPRI

In Chinese publications, its former name was M16188; submitted by Bingkui Miao

Submitted by A. Yamaguchi

Submitted by A. Yamaguchi

Submitted by A. Yamaguchi

Submitted by A. Yamaguchi

Submitted by A. Yamaguchi

Submitted by A. Yamaguchi

Submitted by A. Yamaguchi

Submitted by A. Yamaguchi

Submitted by A. Yamaguchi

Darkened; submitted by A. Yamaguchi

Submitted by A. Yamaguchi

Submitted by A. Yamaguchi

Submitted by A. Yamaguchi

Submitted by A. Yamaguchi

Submitted by A. Yamaguchi

Submitted by A. Yamaguchi

Submitted by A. Yamaguchi

Submitted by A. Yamaguchi

Submitted by A. Yamaguchi

Submitted by A. Yamaguchi

Shock vein; submitted by A. Yamaguchi

Submitted by A. Yamaguchi

Submitted by A. Yamaguchi

Submitted by A. Yamaguchi

Submitted by A. Yamaguchi

Submitted by A. Yamaguchi

Submitted by A. Yamaguchi

Submitted by A. Yamaguchi

Submitted by A. Yamaguchi

In Chinese publications, its former name was M16191; submitted by Bingkui Miao

Submitted by A. Yamaguchi

Submitted by C. Park, KOPRI

Submitted by A. Yamaguchi

Submitted by A. Yamaguchi

Submitted by A. Yamaguchi 
Submitted by A. Yamaguchi

Submitted by A. Yamaguchi

Submitted by A. Yamaguchi

Submitted by A. Yamaguchi

Submitted by A. Yamaguchi

Submitted by A. Yamaguchi

Submitted by C. Park, KOPRI

Submitted by A. Yamaguchi

Submitted by A. Yamaguchi

Submitted by A. Yamaguchi

Submitted by A. Yamaguchi

Submitted by A. Yamaguchi

Submitted by C. Park, KOPRI

Submitted by A. Yamaguchi

Submitted by A. Yamaguchi

Submitted by A. Yamaguchi

Submitted by A. Yamaguchi

Submitted by A. Yamaguchi

Submitted by A. Yamaguchi

Submitted by A. Yamaguchi

SEM; submitted by AMN

Submitted by A. Yamaguchi

SEM; submitted by AMN

SEM; submitted by AMN

Submitted by A. Yamaguchi

Submitted by A. Yamaguchi

Submitted by C. Park, KOPRI

Submitted by C. Park, KOPRI

SEM; submitted by AMN

Submitted by A. Yamaguchi

SEM; submitted by AMN

Submitted by C. Park, KOPRI

SEM; submitted by AMN

SEM; submitted by AMN

Submitted by A. Yamaguchi

SEM; submitted by AMN

Submitted by A. Yamaguchi

Submitted by A. Yamaguchi

Submitted by A. Yamaguchi

SEM; submitted by AMN

Submitted by A. Yamaguchi

SEM; submitted by AMN

Submitted by C. Park, KOPRI

Submitted by A. Yamaguchi

Submitted by A. Yamaguchi 
SEM; submitted by AMN

Submitted by A. Yamaguchi

SEM; submitted by AMN

Submitted by A. Yamaguchi

SEM; submitted by AMN

Submitted by A. Yamaguchi

Submitted by A. Yamaguchi

Shock vein; submitted by A. Yamaguchi

SEM; submitted by AMN

SEM; submitted by AMN

SEM; submitted by AMN

SEM; submitted by AMN

Submitted by A. Yamaguchi

Submitted by A. Yamaguchi

SEM; submitted by AMN

Submitted by A. Yamaguchi

Submitted by A. Yamaguchi

Submitted by A. Yamaguchi

Submitted by A. Yamaguchi

SEM; submitted by AMN

Work name M2047; submitted by A. Irving

Submitted by V. Di Cecco, ROM

Field name DD-26; submitted by C. Agee, UNM

Submitted by D. Sheikh

Submitted by C. Agee

Field name MVCH-351; submitted by C. Agee, UNM

working name DJ2017/12; submitted by R. Bartoschewitz

Submitted by Ansgar Greshake

Submitted by C. Herd

Lab number CML0928; submitted by A. Ruzicka

Field name MVCH-364; submitted by C. Agee, UNM

Work name M2046; submitted by A. Irving

Submitted by K. Metzler, IfP

Field name MVCH-355; submitted by C. Agee, UNM

Work name MVCH 375; submitted by A. Irving

Submitted by D. Sheikh

Submitted by Ansgar Greshake

Submitted by Christian Anderkin

Submitted by Bingkui Miao

Submitted by Bingkui Miao

Submitted by Bingkui Miao

Submitted by Bingkui Miao

Submitted by AMN

Submitted by AMN

Submitted by J.T. Wasson 
Submitted by J.T. Wasson

Submitted by J.T. Wasson

Submitted by L. Garvie

Submitted by J.T. Wasson

Submitted by C. Herd

Submitted by Vanni Moggi Cecchi

Submitted by T. Mikouchi, UTok

Submitted by J. T. Wasson and W. B. Xu

Submitted by J.T. Wasson

Submitted by J.T. Wasson

Submitted by J.T. Wasson

Submitted by J.T. Wasson

Submitted by J.T. Wasson

Submitted by J.T. Wasson

Submitted by J.T. Wasson

Submitted by C. Herd

Submitted by $\mathrm{C}$. Herd

Field number MMO-2018-3B; submitted by C. Herd

Submitted by M. Zucolotto

Submitted by C. Herd

Submitted by Shijie Li

Submitted by J. Gattacceca

Submitted by Jordi Llorca (UPC)

Submitted by AMN

Submitted by Shijie Li

Submitted by C. Herd

Submitted by AMN

Submitted by AMN

Submitted by Ansgar Greshake

working name WZ09; submitted by R. Bartoschewitz working name WZ12; submitted by R. Bartoschewitz working name WZ24; submitted by R. Bartoschewitz working name WZ08; submitted by R. Bartoschewitz working name WZ02; submitted by R. Bartoschewitz working name WZ01; submitted by R. Bartoschewitz working name DJ2018/04; submitted by R. Bartoschewitz working name WZ13; submitted by R. Bartoschewitz working name WZ14; submitted by R. Bartoschewitz working name WZ23; submitted by R. Bartoschewitz working name DJ2017/16; submitted by R. Bartoschewitz working name WZ15; submitted by R. Bartoschewitz NWA AM3; submitted by R. Bartoschewitz working name WZ07; submitted by R. Bartoschewitz working name WZ19; submitted by R. Bartoschewitz working name WZ20; submitted by R. Bartoschewitz 
NWA-BLK01; submitted by R. Bartoschewitz

Submitted by L. Garvie

Field name AB-220; submitted by C. Agee, UNM

Work name $\mathrm{CHC18001;} \mathrm{submitted} \mathrm{by} \mathrm{A.} \mathrm{Irving}$

Submitted by Ansgar Greshake

Submitted by K. Metzler, IfP

Submitted by Ansgar Greshake

Work name K382; submitted by Lisa Krämer Ruggiu

Submitted by K. Metzler, IfP

work name JR050; submitted by Gattacceca

mean chondrule diameter is about $0.6 \mathrm{~mm}$; submitted by Ansgar Greshake

mean chondrule diameter is about $0.7 \mathrm{~mm}$; submitted by Ansgar Greshake

GF18-1; submitted by A. Irving

Submitted by A. Yamaguchi

work name CG10-3; submitted by A. Irving

Work name GF17-3; submitted by A. Irving

Submitted by Ansgar Greshake

Lab number CML0115. Coordinates are 26.94+-0.03 deg N and 105.30+-0.04 deg W. The errors are +-

Submitted by Ansgar Greshake

Submitted by Ansgar Greshake

Submitted by A. Yamaguchi

Chondritic texture of partly deformed, closely packed chondrules (mean diam $\sim 0.7 \mathrm{~mm}$ ) in finer grained $\mathrm{r}$

Chondritic texture of sharply defined chondrules of various types $\sim 0.6$ in diameter set in a fine-grained $\mathrm{m}$

Submitted by Ansgar Greshake

Submitted by Ansgar Greshake

Submitted by Ansgar Greshake

Submitted by Ansgar Greshake

Submitted by M. Zolensky and M.Takahashi

mean chondrule diameter is about $0.6 \mathrm{~mm}$; submitted by Ansgar Greshake

Submitted by C. Park, KOPRI

Submitted by A. Yamaguchi

Submitted by A. Yamaguchi

Submitted by A. Yamaguchi

Submitted by A. Yamaguchi

Submitted by A. Yamaguchi

Submitted by A. Yamaguchi

Submitted by A. Yamaguchi

Submitted by A. Yamaguchi

Submitted by A. Yamaguchi

Submitted by A. Yamaguchi

Submitted by A. Yamaguchi

Submitted by A. Yamaguchi

Submitted by A. Yamaguchi

Submitted by A. Yamaguchi

Submitted by A. Yamaguchi 
Submitted by A. Yamaguchi

UCLA specimen \#1108; submitted by A. Irving

NWA-LB006; submitted by R. Bartoschewitz

Submitted by Ansgar Greshake

Field name MC 150; submitted by C. Agee, UNM

Submitted by AMN

Submitted by AMN

Submitted by AMN

Submitted by AMN

work name AG003. Fragments with reddish-brown fracture surfaces and dark brown fusion crust. Well d

Submitted by $\mathrm{H}$. Chennaoui Aoudjehane

Submitted by K. Metzler, IfP

Work name CHC18004; submitted by A. Irving

G705; submitted by A. Bouvier, UWO

Work name CHC18007; submitted by A. Irving

Work name M2076; submitted by A. Irving

Work name M2077; submitted by A. Irving

Submitted by Simone de Leuw

Field name: Met-2016-028; submitted by David Allepuz

Work name BH147; submitted by A. Irving

In Chinese publications, its former name was M16033; submitted by Bingkui Miao

Submitted by C. Park, KOPRI

In Chinese publications, its former name was M16160; submitted by Bingkui Miao

Submitted by Vanni Moggi Cecchi

Submitted by A. Yamaguchi

Submitted by Vanni Moggi Cecchi

Submitted by Vanni Moggi Cecchi

Submitted by A. Yamaguchi

Submitted by Vanni Moggi Cecchi

Submitted by Simone de Leuw

SEM; submitted by AMN

CHC18026; submitted by A. Irving

Submitted by A. Yamaguchi

ROM Number LM57442; Chondrules readily defined and have irregular fractures and grains showing un

Submitted by A. Yamaguchi

SEM; submitted by AMN

Work name CHC 18011; submitted by A. Irving

SEM; submitted by AMN

Submitted by AMN

SEM; submitted by AMN

work name F01-2014; submitted by Jerome Gattacceca

SEM; submitted by AMN

$3 \times 2 \times 1.5 \mathrm{~cm}$ irregualar shape stone, $60 \%$ crusted with bleached haloes, brown oxidised interior; submi Work name $\mathrm{CHC18016}$; submitted by A. Irving

Shock vein; submitted by $A$. Yamaguchi 
SEM; submitted by AMN

Submitted by A. Yamaguchi

Submitted by A. Yamaguchi

Submitted by A. Yamaguchi

Submitted by A. Yamaguchi

Submitted by A. Yamaguchi

Submitted by A. Yamaguchi

Submitted by A. Yamaguchi

Submitted by A. Yamaguchi

Submitted by A. Yamaguchi

Submitted by A. Yamaguchi

Submitted by A. Yamaguchi

Submitted by A. Yamaguchi

Submitted by A. Yamaguchi

Submitted by A. Yamaguchi

millimeter sized, crusted fragment; submitted by F.Focacci M. Gemelli, (DST-PI)

SEM; submitted by AMN

Morocco; submitted by Ansgar Greshake

Work name $\mathrm{CHC} 18005$; submitted by A. Irving

work name LM75/E28; submitted by Jérôme Gattacceca

Work name BH128; submitted by A. Irving

ROM Number LM57455, formerly DGR01114; Readily delineated chondrules, some undulatory extinctio

Field name MVCH314; submitted by C. Agee, UNM

Field name: Met-2016-032; submitted by David Allepuz

G701; submitted by A. Bouvier, UWO

Submitted by M. E. Varela

Field name is 2-24a; analyst S. E. Borisovsky (IGEM); submitted by C. A. Lorenz (Vernad)

working name K369; submitted by R. Bartoschewitz

Submitted by Y. Xu, IGGCAS

Field name: Met-2016-031; submitted by David Allepuz

Work name CHC18006; submitted by A. Irving

work name LM67 / D53; submitted by Jérôme Gattacceca

Field name SM-2; submitted by C. Agee, UNM

Field name is Om2011; analyst N. N. Kononkova (Vernad.); submitted by C. A. Lorenz (Vernad)

Field name Engare Sero; submitted by Pavel Yu.Plechov (FMMR)

Field name is 2-6; analyst S. E. Borisovsky (IGEM); submitted by C. A. Lorenz (Vernad)

Work name MVCH 331; submitted by A. Irving

plagioclase grain size about $15 \mu \mathrm{m}$; Two brownish individuals without fusion crust.; submitted by Ansgar

Submitted by Simone de Leuw

Work name M13; submitted by Craig Walton

Work name CHC18002; submitted by A. Irving

working name DJ2017/13; submitted by R. Bartoschewitz

Field name is Calama002-Ch; analist S. E. Borisovsky (IGEM); submitted by Lorenz C.A., Vernad

Field name is Calama50; analist V. V. Kozlov (Oxford Instruments, Moscow). A stone of irregular shape 
Submitted by Director General (DG), GSI.

Submitted by C. Park, KOPRI

Field name: Met-2017-006; submitted by David Allepuz

work name E5; submitted by Richard Greenwood, Hélène Greenwood

In Chinese publications, its former name was M16220; submitted by Bingkui Miao

Field name is Calama 3-Misha; analyst T. Kryachko (Technograd, Moscow); submitted by Lorenz C.A., I In Chinese publications, its former name was M16224; submitted by Bingkui Miao work name M15.; submitted by Craig Walton

In Chinese publications, its former name was M16091; submitted by Bingkui Miao

Field name is 2-23; analyst S. E. Borisovsky (IGEM); submitted by C. A. Lorenz (Vernad)

Submitted by A. Yamaguchi

Submitted by Simone de Leuw

Submitted by Vanni Moggi Cecchi

SEM; submitted by AMN

In Chinese publications, its former name was M16034; submitted by Bingkui Miao

In Chinese publications, its former name was M16048; submitted by Bingkui Miao

Submitted by Vanni Moggi Cecchi

Dark grayish individual with some fusion crust. plagioclase grain size about $30 \mu \mathrm{m}$; submitted by Ansgar Shock vein; submitted by A. Yamaguchi

SEM; submitted by AMN

In Chinese publications, its former name was M16109; submitted by Bingkui Miao

SEM; submitted by AMN

Submitted by Vanni Moggi Cecchi

plagioclase grain size about $20 \mu \mathrm{m}$; Brownish individual with some fusion crust.; submitted by Ansgar $\mathrm{Gr}$ CHC18022; submitted by A. Irving

work name EJ448; submitted by Jérôme Gattacceca

breccia; plagioclase grain size about 30 um; submitted by Ansgar Greshake

Submitted by Vanni Moggi Cecchi

Field name is Calama025-Ch; analist S. E. Borisovsky (IGEM); submitted by Lorenz C.A., Vernad work name D; submitted by Richard Greenwood, Hélène Greenwood

Field name is PET-2; analyst S. E. Borisovsky (IGEM); submitted by C. A. Lorenz (Vernad)

Field name is ?H2FM; analyst N. N. Kononkova (Vernad); submitted by C. A. Lorenz (Vernad)

In Chinese publications, its former name was M16112; submitted by Bingkui Miao

Field name is Calama027-Ch; analist S. E. Borisovsky (IGEM); submitted by Lorenz C.A., Vernad work name PB01. Found on grano-diorite pediment surface while meteorite prospecting with six-person Purchased from a Moroccan meteorite dealer at the mineral fair in Munich, Germany. Plagioclase grain s SEM; submitted by AMN

SEM; submitted by AMN

Submitted by Vanni Moggi Cecchi

SEM; submitted by AMN

SEM; submitted by AMN

SEM; submitted by AMN

SEM; submitted by AMN

ROM Number LM57439; submitted by V. Di Cecco, ROM

SEM; submitted by AMN 


\section{Submitted by Vanni Moggi Cecchi}

SEM; submitted by AMN

SEM; submitted by AMN

Submitted by Vanni Moggi Cecchi

SEM; submitted by AMN

Field name is Calate Q5; analyst T. Kryachko (Technograd, Moscow); submitted by Lorenz C.A., Vernac

SEM; submitted by AMN

Work name RM586; submitted by Lisa Krämer Ruggiu

SEM; submitted by AMN

plagioclase grain size about 30 um; submitted by Ansgar Greshake

SEM; submitted by AMN

SEM; submitted by AMN

SEM; submitted by AMN

Field name is 1-1; analyst S. E. Borisovsky (IGEM); submitted by C. A. Lorenz (Vernad)

Field name: Met-2015-024; submitted by David Allepuz

SEM; submitted by AMN

SEM; submitted by AMN

SEM; submitted by AMN

Submitted by Vanni Moggi Cecchi

SEM; submitted by AMN

SEM; submitted by AMN

SEM; submitted by AMN

SEM; submitted by AMN

SEM; submitted by AMN

SEM; submitted by AMN

SEM; submitted by AMN

SEM; submitted by AMN

Work name $\mathrm{CHC18017}$; submitted by A. Irving

SEM; submitted by AMN

In Chinese publications, its former name was M16140; submitted by Bingkui Miao

SEM; submitted by AMN

SEM; submitted by AMN

SEM; submitted by AMN

SEM; submitted by AMN

SEM; submitted by AMN

In Chinese publications, its former name was M16291; submitted by Bingkui Miao

SEM; submitted by AMN

SEM; submitted by AMN

SEM; submitted by AMN

SEM; submitted by AMN

SEM; submitted by AMN

SEM; submitted by AMN

SEM; submitted by AMN

SEM; submitted by AMN

SEM; submitted by AMN 
SEM; submitted by AMN

SEM; submitted by AMN

SEM; submitted by AMN

SEM; submitted by AMN

SEM; submitted by AMN

SEM; submitted by AMN

SEM; submitted by AMN

SEM; submitted by AMN

SEM; submitted by AMN

SEM; submitted by AMN

SEM; submitted by AMN

SEM; submitted by AMN

SEM; submitted by AMN

Submitted by A. Yamaguchi

SEM; submitted by AMN

Submitted by AMN

SEM; submitted by AMN

SEM; submitted by AMN

Submitted by A. Yamaguchi

SEM; submitted by AMN

SEM; submitted by AMN

Submitted by A. Yamaguchi

Submitted by A. Yamaguchi

Submitted by A. Yamaguchi

Submitted by A. Yamaguchi

SEM; submitted by AMN

SEM; submitted by AMN

Field name is 4-II; analyst S. E. Borisovsky (IGEM); submitted by C. A. Lorenz (Vernad)

Submitted by A. Yamaguchi

Submitted by A. Yamaguchi

Submitted by A. Yamaguchi

Submitted by A. Yamaguchi

Submitted by A. Yamaguchi

Submitted by A. Yamaguchi

Submitted by A. Yamaguchi

Submitted by A. Yamaguchi

Submitted by A. Yamaguchi

Submitted by A. Yamaguchi

Shock vein; submitted by A. Yamaguchi

Shock vein; submitted by A. Yamaguchi

Shock vein; submitted by A. Yamaguchi

Shock vein; submitted by A. Yamaguchi

Submitted by A. Yamaguchi

Submitted by A. Yamaguchi

Shock vein; submitted by A. Yamaguchi 
Submitted by A. Yamaguchi

Shock vein; submitted by A. Yamaguchi

Submitted by A. Yamaguchi

Submitted by A. Yamaguchi

Submitted by A. Yamaguchi

Submitted by A. Yamaguchi

Shock vein; submitted by A. Yamaguchi

Submitted by A. Yamaguchi

Submitted by A. Yamaguchi

Submitted by A. Yamaguchi

Submitted by A. Yamaguchi

Submitted by A. Yamaguchi

Submitted by A. Yamaguchi

Shock vein; submitted by A. Yamaguchi

Shock vein; submitted by A. Yamaguchi

Submitted by A. Yamaguchi

Submitted by A. Yamaguchi

Submitted by A. Yamaguchi

Submitted by A. Yamaguchi

Submitted by A. Yamaguchi

Submitted by A. Yamaguchi

Submitted by A. Yamaguchi

$1 \times 1 \times 1 \mathrm{~cm}$ rounded brownish stone with cracks, fully crusted; submitted by F.Focacci M. Gemelli, (DS1

Submitted by A. Yamaguchi

Submitted by A. Yamaguchi

SEM; submitted by AMN

Submitted by A. Yamaguchi

Submitted by A. Yamaguchi

SEM; submitted by AMN

Submitted by A. Yamaguchi

Submitted by A. Yamaguchi

Submitted by A. Yamaguchi

Submitted by A. Yamaguchi

SEM; submitted by AMN

Submitted by A. Yamaguchi

Submitted by A. Yamaguchi

SEM; submitted by AMN

SEM; submitted by AMN

Submitted by A. Yamaguchi

Submitted by A. Yamaguchi

Submitted by A. Yamaguchi

Submitted by A. Yamaguchi

Submitted by A. Yamaguchi

Submitted by A. Yamaguchi

SEM; submitted by AMN 
Submitted by A. Yamaguchi

SEM; submitted by AMN

Submitted by A. Yamaguchi

Submitted by A. Yamaguchi

Submitted by A. Yamaguchi

Submitted by A. Yamaguchi

Submitted by A. Yamaguchi

Submitted by A. Yamaguchi

Submitted by A. Yamaguchi

Submitted by A. Yamaguchi

$1 \times 0.5 \times 0.5 \mathrm{~cm}$ fully crusted rounded brownish stone; submitted by F.Focacci M. Gemelli, (DST-PI)

Submitted by A. Yamaguchi

Submitted by A. Yamaguchi

Submitted by A. Yamaguchi

SEM; submitted by AMN

Submitted by A. Yamaguchi

SEM; submitted by AMN

Submitted by Samuel Ebert; submitted by Ebert S.

shock veins, breccia; submitted by Eber $\mathrm{S}$.

Submitted by Ansgar Greshake

Field name: Met-2017-010; submitted by David Allepuz

Field name: CL-12-1 (meado); submitted by David Allepuz

Submitted by Eber $\mathrm{S}$.

Submitted by S. Hu,Y. Lin and J. Ji, IGGCAS ; Weibiao Hsu, PMO

Submitted by Pavel Yu. Plechov, pplechov@gmail.com

work name LM61/D35; Contains mm thick shock veins.; submitted by Jérôme Gattacceca

Field name KRUG-1; analyst V.V. Sharygin (SIGM and UrFU).; submitted by Victor V. Sharygin, sharygiı plagioclase grain size about $80 \mu \mathrm{m}$; Big brownish individual lacking any fusion crust.; submitted by Ansg: Submitted by Jordi Llorca (UPC)

Submitted by Victor V. Sharygin

plagioclase grain size about $80 \mu \mathrm{m}$; submitted by Ansgar Greshake

work name RM596; Shock veins containing metal and troilite droplets. Troilite polycristalline. Plagioclas $€$

Submitted by C. Park, KOPRI

Work name $\mathrm{CHC} 18003$; submitted by A. Irving

Submitted by L. Garvie

work name LM57/D19; submitted by Jérôme Gattacceca

Submitted by Simone de Leuw

Shock vein; submitted by A. Yamaguchi

SEM; submitted by AMN

Work name CHC18008; submitted by A. Irving

In Chinese publications, its former name was M16213; submitted by Bingkui Miao

plagioclase grain size about 60 um; submitted by Ansgar Greshake

Submitted by A. Yamaguchi

Submitted by C. Park, KOPRI 
plagioclase grain size about 70 um; submitted by Ansgar Greshake

Submitted by A. Yamaguchi

plagioclase grain size about $90 \mu \mathrm{m}$; Single stone with fusion crust.; submitted by Ansgar Greshake

Submitted by Simone de Leuw

Submitted by Simone de Leuw

Brownish individual without fusion crust. plagioclase grain size about $60 \mu \mathrm{m}$; submitted by Ansgar Gresh

Submitted by C. Park, KOPRI

Submitted by Simone de Leuw

Shock vein; submitted by A. Yamaguchi

Submitted by Katarzyna Luszczek

Field name: Met-2015-021; submitted by David Allepuz

Submitted by Vanni Moggi Cecchi

Field name: Met-2017-020; submitted by David Allepuz

Submitted by Pierre Rochette

Field name: Met-2018-003; submitted by David Allepuz

Submitted by A. Yamaguchi

Submitted by Jordi Llorca (UPC)

Submitted by Vanni Moggi Cecchi

ROM Number LM57453; No mosaicism, minor oxidation; submitted by V. Di Cecco, ROM

Submitted by Maria Schönbächler

Submitted by A. Yamaguchi

work name M640; Recrystallized chondritic texture with average plagiocalse size above 50 um.; submitte Field name: Met-2016-017; submitted by David Allepuz

Submitted by C. Park, KOPRI

In Chinese publications, its former name was M16135; submitted by Bingkui Miao

SEM; submitted by AMN

Submitted by A. Yamaguchi

Submitted by Ansgar Greshake

In Chinese publications, its former name was M16120; submitted by Bingkui Miao

Shock vein; submitted by A. Yamaguchi

plagioclase grain size about $70 \mu \mathrm{m}$; submitted by Ansgar Greshake

SEM; submitted by AMN

In Chinese publications, its former name was M16108; submitted by Bingkui Miao

ROM Number LM57439A; Chondrules very poorly defined. Minor undulatory extinction and very few sho plagioclase grain size about $60 \mu \mathrm{m}$; Brownish individual with some fusion crust.; submitted by Ansgar $\mathrm{Gr}$ SEM; submitted by AMN

work name LL14; submitted by J. Gattacceca

Field name: Met-2018-014; submitted by David Allepuz

Field name is Calama11. Analyst V. V. Kozlov (Tescan Demo Centre, Moscow, Russia); submitted by Lc SEM; submitted by AMN

Dark brownish individual without fusion crust. plagioclase grain size about $70 \mu \mathrm{m}$; submitted by Ansgar C work name EJ1098; submitted by Jérôme Gattacceca

Submitted by A. Yamaguchi

Field name is Calama023-Ch; analist S. E. Borisovsky (IGEM); submitted by Lorenz C.A., Vernad

Submitted by Simone de Leuw 
SEM; submitted by AMN

Submitted by A. Yamaguchi

Submitted by Vanni Moggi Cecchi

Submitted by A. Yamaguchi

work name EJ696; submitted by J. Gattacceca

Submitted by A. Yamaguchi

Dark grayish individual with some fusion crust. plagioclase grain size about $60 \mu \mathrm{m}$; submitted by Ansgar

Submitted by C. Park, KOPRI

Submitted by A. Yamaguchi

CHC18024; submitted by A. Irving

Submitted by A. Yamaguchi

Field name: Met-2016-015; submitted by David Allepuz

SEM; submitted by AMN

Submitted by A. Yamaguchi

Field name: Met-2017-036; submitted by David Allepuz

Submitted by A. Yamaguchi

Field name: Met-2017-030; submitted by David Allepuz

work name EJ397; submitted by J. Gattacceca

Field name: Met-2017-033; submitted by David Allepuz

SEM; submitted by AMN

Submitted by A. Irving

SEM; submitted by AMN

SEM; submitted by AMN

Submitted by C. Park, KOPRI

Breccia; submitted by A. Yamaguchi

work name EJ206; chondritic texture with average plagioclase size above 50 um. Shock veins. Troilite is SEM; submitted by AMN

Submitted by C. Park, KOPRI

SEM; submitted by AMN

work name EJ398; submitted by Jerome Gattacceca

Field name is Calama 3-30; analyst T. Kryachko (Technograd, Moscow); submitted by Lorenz C.A., Verr

Shock vein; submitted by A. Yamaguchi

SEM; submitted by AMN

Work name CHC18015; submitted by A. Irving

work name EJ1116; submitted by Jérôme Gattacceca

SEM; submitted by AMN

Submitted by C. Park, KOPRI

SEM; submitted by AMN

SEM; submitted by AMN

SEM; submitted by AMN

Submitted by A. Yamaguchi

ROM Number LM52582; Chondrules very poorly defined. Majority of matrix recrystallized. Undulatory ex work name EJ606. Average plagioclase size above 50 um. Shock veins.; submitted by J. Gattacceca

Shock vein; submitted by A. Yamaguchi

SEM; submitted by AMN 
Submitted by C. Park, KOPRI

SEM; submitted by AMN

SEM; submitted by AMN

SEM; submitted by AMN

SEM; submitted by AMN

Submitted by Jordi Llorca (UPC)

In Chinese publications, its former name was M16081; submitted by Bingkui Miao

SEM; submitted by AMN

Submitted by A. Yamaguchi

worj name EJ607; submitted by J. Gattacceca

SEM; submitted by AMN

SEM; submitted by AMN

SEM; submitted by AMN

Field name is 52.2; analyst S. E. Borisovsky (IGEM); submitted by C. A. Lorenz (Vernad)

SEM; submitted by AMN

Shock vein; submitted by A. Yamaguchi

SEM; submitted by AMN

SEM; submitted by AMN

Submitted by Vanni Moggi Cecchi

SEM; submitted by AMN

SEM; submitted by AMN

Field name: Met-2016-021; submitted by David Allepuz

SEM; submitted by AMN

SEM; submitted by AMN

SEM; submitted by AMN

SEM; submitted by AMN

SEM; submitted by AMN

SEM; submitted by AMN

Submitted by Daniel Sheikh

SEM; submitted by AMN

Submitted by A. Yamaguchi

Shock vein; submitted by A. Yamaguchi

Field name: Met-2018-044; submitted by David Allepuz

Submitted by A. Yamaguchi

SEM; submitted by AMN

SEM; submitted by AMN

Submitted by A. Yamaguchi

work name JG065. Shock veins. Average plagioclase size above 50 um.; submitted by J. Gattacceca

In Chinese publications, its former name was M16184; submitted by Bingkui Miao

SEM; submitted by AMN

SEM; submitted by AMN

Submitted by A. Yamaguchi

SEM; submitted by AMN

SEM; submitted by AMN

Submitted by C. Park, KOPRI 
SEM; submitted by AMN

SEM; submitted by AMN

SEM; submitted by AMN

Submitted by A. Yamaguchi

SEM; submitted by AMN

SEM; submitted by AMN

work name EJ419. Shock veins; submitted by Jérôme Gattacceca

SEM; submitted by AMN

Submitted by Vanni Moggi Cecchi

SEM; submitted by AMN

SEM; submitted by AMN

SEM; submitted by AMN

SEM; submitted by AMN

SEM; submitted by AMN

SEM; submitted by AMN

SEM; submitted by AMN

Submitted by A. Yamaguchi

Field name: Met-2018-029; submitted by David Allepuz

SEM; submitted by AMN

SEM; submitted by AMN

SEM; submitted by AMN

SEM; submitted by AMN

SEM; submitted by AMN

SEM; submitted by AMN

SEM; submitted by AMN

SEM; submitted by AMN

In Chinese publications, its former name was M16162; submitted by Bingkui Miao SEM; submitted by AMN

SEM; submitted by AMN

Submitted by C. Park, KOPRI

SEM; submitted by AMN

SEM; submitted by AMN

SEM; submitted by AMN

SEM; submitted by AMN

Lab number CML0015; submitted by Alex Ruzicka

SEM; submitted by AMN

SEM; submitted by AMN

SEM; submitted by AMN

Submitted by A. Yamaguchi

SEM; submitted by AMN

SEM; submitted by AMN

SEM; submitted by AMN

Submitted by A. Yamaguchi

In Chinese publications, its former name was M16073; submitted by Bingkui Miao

SEM; submitted by AMN 
SEM; submitted by AMN

SEM; submitted by AMN

SEM; submitted by AMN

SEM; submitted by AMN

SEM; submitted by AMN

SEM; submitted by AMN

SEM; submitted by AMN

SEM; submitted by AMN

Submitted by C. Park, KOPRI

SEM; submitted by AMN

SEM; submitted by AMN

SEM; submitted by AMN

Field name: Met-2018-019; submitted by David Allepuz

SEM; submitted by AMN

SEM; submitted by AMN

SEM; submitted by AMN

SEM; submitted by AMN

Work name CHC18010; submitted by A. Irving

SEM; submitted by AMN

SEM; submitted by AMN

SEM; submitted by AMN

SEM; submitted by AMN

SEM; submitted by AMN

Work name CHC18012; submitted by A. Irving

SEM; submitted by AMN

SEM; submitted by AMN

SEM; submitted by AMN

SEM; submitted by AMN

SEM; submitted by AMN

ROM Number LM57440, formerly DGR 00314; Poorly defined chondrules, some mosaicism, some oxide

In Chinese publications, its former name was M16151; submitted by Bingkui Miao

Large igneous clast; submitted by A. Yamaguchi

SEM; submitted by AMN

Submitted by C. Park, KOPRI

SEM; submitted by AMN

SEM; submitted by AMN

SEM; submitted by AMN

SEM; submitted by AMN

SEM; submitted by AMN

SEM; submitted by AMN

SEM; submitted by AMN

SEM; submitted by AMN

work name EJ401; submitted by Jérôme Gattacceca

SEM; submitted by AMN

SEM; submitted by AMN 
Submitted by A. Yamaguchi

SEM; submitted by AMN

SEM; submitted by AMN

Submitted by C. Park, KOPRI

SEM; submitted by AMN

SEM; submitted by AMN

SEM; submitted by AMN

SEM; submitted by AMN

SEM; submitted by AMN

Field name: Met-2015-027; submitted by David Allepuz

SEM; submitted by AMN

SEM; submitted by AMN

SEM; submitted by AMN

SEM; submitted by AMN

SEM; submitted by AMN

SEM; submitted by AMN

SEM; submitted by AMN

Submitted by A. Yamaguchi

SEM; submitted by AMN

SEM; submitted by AMN

Field name: Met-2018-015; submitted by David Allepuz

Submitted by A. Yamaguchi

SEM; submitted by AMN

Submitted by C. Park, KOPRI

Field name is 1-21c; analyst S. E. Borisovsky (IGEM); submitted by C. A. Lorenz (Vernad)

Submitted by Daniel Sheikh

SEM; submitted by AMN

SEM; submitted by AMN

SEM; submitted by AMN

work name EJ212; Shock veins; submitted by Jérôme Gattacceca

SEM; submitted by AMN

SEM; submitted by AMN

SEM; submitted by AMN

SEM; submitted by AMN

SEM; submitted by AMN

SEM; submitted by AMN

SEM; submitted by AMN

In Chinese publications, its former name was M16259; submitted by Bingkui Miao

SEM; submitted by AMN

Submitted by AMN

SEM; submitted by AMN

SEM; submitted by AMN

Submitted by C. Park, KOPRI

SEM; submitted by AMN

SEM; submitted by AMN 
Submitted by A. Yamaguchi

SEM; submitted by AMN

SEM; submitted by AMN

SEM; submitted by AMN

SEM; submitted by AMN

SEM; submitted by AMN

SEM; submitted by AMN

SEM; submitted by AMN

SEM; submitted by AMN

Field name: Met-2018-018; submitted by David Allepuz

SEM; submitted by AMN

SEM; submitted by AMN

SEM; submitted by AMN

SEM; submitted by AMN

SEM; submitted by AMN

Field name: Met-2018-004; submitted by David Allepuz

Submitted by A. Yamaguchi

SEM; submitted by AMN

SEM; submitted by AMN

SEM; submitted by AMN

SEM; submitted by AMN

SEM; submitted by AMN

Submitted by Victor V. Sharygin

SEM; submitted by AMN

SEM; submitted by AMN

SEM; submitted by AMN

SEM; submitted by AMN

Submitted by AMN

SEM; submitted by AMN

SEM; submitted by AMN

Submitted by C. Park, KOPRI

Submitted by AMN

SEM; submitted by AMN

SEM; submitted by AMN

SEM; submitted by AMN

work name EJ214. Abundant shock veins. Polycrystalline troilite.; submitted by Jérôme Gattacceca

Submitted by A. Yamaguchi

Submitted by A. Yamaguchi

SEM; submitted by AMN

SEM; submitted by AMN

Submitted by AMN

Submitted by A. Yamaguchi

Submitted by C. Park, KOPRI

Submitted by AMN

Shock vein; submitted by A. Yamaguchi 
Submitted by A. Yamaguchi

Submitted by C. Park, KOPRI

Submitted by A. Yamaguchi

SEM; submitted by AMN

SEM; submitted by AMN

Submitted by A. Yamaguchi

SEM; submitted by AMN

SEM; submitted by AMN

SEM; submitted by AMN

SEM; submitted by AMN

Submitted by C. Park, KOPRI

SEM; submitted by AMN

SEM; submitted by AMN

Submitted by A. Yamaguchi

Submitted by C. Park, KOPRI

Field name: Met-2018-011; submitted by David Allepuz

SEM; submitted by AMN

Breccia; submitted by A. Yamaguchi

Submitted by C. Park, KOPRI

Submitted by A. Yamaguchi

Submitted by A. Yamaguchi

Submitted by A. Yamaguchi

work name DM047; Average plagioclase size above 50 um.; submitted by J. Gattacceca

Submitted by A. Yamaguchi

Submitted by A. Yamaguchi

Submitted by A. Yamaguchi

Shock vein; submitted by A. Yamaguchi

Shock vein; submitted by A. Yamaguchi

Submitted by A. Yamaguchi

Submitted by A. Yamaguchi

Shock vein; submitted by A. Yamaguchi

Submitted by A. Yamaguchi

Shock vein; submitted by A. Yamaguchi

Submitted by A. Yamaguchi

Submitted by A. Yamaguchi

Submitted by A. Yamaguchi

Shock vein; submitted by A. Yamaguchi

Submitted by A. Yamaguchi

Submitted by A. Yamaguchi

Submitted by A. Yamaguchi

Submitted by A. Yamaguchi

Submitted by A. Yamaguchi

Submitted by A. Yamaguchi

Submitted by A. Yamaguchi

Submitted by A. Yamaguchi 
Shock vein; submitted by A. Yamaguchi

Submitted by C. Park, KOPRI

Submitted by A. Yamaguchi

Submitted by A. Yamaguchi

Submitted by AMN

Shock vein; submitted by A. Yamaguchi

Shock vein; submitted by A. Yamaguchi

Submitted by A. Yamaguchi

Submitted by A. Yamaguchi

Submitted by C. Park, KOPRI

Submitted by A. Yamaguchi

Submitted by A. Yamaguchi

Submitted by A. Yamaguchi

Submitted by A. Yamaguchi

Submitted by C. Park, KOPRI

Submitted by A. Yamaguchi

Submitted by A. Yamaguchi

Darkened; submitted by A. Yamaguchi

Shock vein; submitted by A. Yamaguchi

Shock vein; submitted by A. Yamaguchi

Shock vein; submitted by A. Yamaguchi

Submitted by A. Yamaguchi

Submitted by C. Park, KOPRI

Submitted by A. Yamaguchi

Shock vein; submitted by A. Yamaguchi

Shock vein; submitted by A. Yamaguchi

Submitted by A. Yamaguchi

Submitted by A. Yamaguchi

Submitted by A. Yamaguchi

Submitted by A. Yamaguchi

Field name: Met-2017-007; submitted by David Allepuz

Shock vein; submitted by A. Yamaguchi

Submitted by A. Yamaguchi

Submitted by A. Yamaguchi

Submitted by C. Park, KOPRI

Submitted by C. Park, KOPRI

Submitted by A. Yamaguchi

Submitted by A. Yamaguchi

Submitted by A. Yamaguchi

Submitted by A. Yamaguchi

Shock vein; submitted by A. Yamaguchi

Submitted by A. Yamaguchi

Submitted by A. Yamaguchi

Submitted by A. Yamaguchi

Submitted by A. Yamaguchi 
Submitted by A. Yamaguchi

Submitted by A. Yamaguchi

Submitted by C. Park, KOPRI

Submitted by A. Yamaguchi

Submitted by A. Yamaguchi

Submitted by A. Yamaguchi

Submitted by A. Yamaguchi

Submitted by C. Park, KOPRI

Submitted by A. Yamaguchi

Submitted by A. Yamaguchi

Shock vein; submitted by A. Yamaguchi

Submitted by A. Yamaguchi

Submitted by A. Yamaguchi

Submitted by A. Yamaguchi

Submitted by A. Yamaguchi

Submitted by A. Yamaguchi

Submitted by A. Yamaguchi

Submitted by A. Yamaguchi

Submitted by A. Yamaguchi

Submitted by A. Yamaguchi

Submitted by C. Park, KOPRI

Shock vein; submitted by A. Yamaguchi

Shock vein; submitted by A. Yamaguchi

Submitted by A. Yamaguchi

Submitted by A. Yamaguchi

Submitted by A. Yamaguchi

Submitted by A. Yamaguchi

Submitted by A. Yamaguchi

Submitted by A. Yamaguchi

Submitted by C. Park, KOPRI

Submitted by A. Yamaguchi

Submitted by A. Yamaguchi

Submitted by A. Yamaguchi

Submitted by A. Yamaguchi

Submitted by A. Yamaguchi

Submitted by A. Yamaguchi

Submitted by A. Yamaguchi

Submitted by A. Yamaguchi

Submitted by A. Yamaguchi

Submitted by A. Yamaguchi

Submitted by A. Yamaguchi

Shock vein; submitted by A. Yamaguchi

Submitted by A. Yamaguchi

Submitted by A. Yamaguchi

Submitted by A. Yamaguchi 
Submitted by A. Yamaguchi

Submitted by A. Yamaguchi

Shock vein; submitted by A. Yamaguchi

Submitted by A. Yamaguchi

Submitted by A. Yamaguchi

Submitted by A. Yamaguchi

Submitted by A. Yamaguchi

Submitted by A. Yamaguchi

Submitted by A. Yamaguchi

Shock vein; submitted by A. Yamaguchi

Shock vein; submitted by A. Yamaguchi

Submitted by A. Yamaguchi

Shock vein; submitted by A. Yamaguchi

Submitted by A. Yamaguchi

Submitted by A. Yamaguchi

Submitted by A. Yamaguchi

Submitted by A. Yamaguchi

Submitted by A. Yamaguchi

Submitted by A. Yamaguchi

Submitted by A. Yamaguchi

Submitted by A. Yamaguchi

Submitted by A. Yamaguchi

Submitted by A. Yamaguchi

Submitted by A. Yamaguchi

Submitted by A. Yamaguchi

Submitted by A. Yamaguchi

SEM; submitted by AMN

Submitted by A. Yamaguchi

Submitted by A. Yamaguchi

Submitted by A. Yamaguchi

Submitted by A. Yamaguchi

Submitted by A. Yamaguchi

Submitted by A. Yamaguchi

Submitted by A. Yamaguchi

Submitted by A. Yamaguchi

Submitted by A. Yamaguchi

Submitted by A. Yamaguchi

SEM; submitted by AMN

Submitted by A. Yamaguchi

Submitted by A. Yamaguchi

Submitted by A. Yamaguchi

SEM; submitted by AMN

Submitted by A. Yamaguchi

Submitted by A. Yamaguchi

Submitted by A. Yamaguchi 
Submitted by A. Yamaguchi

Submitted by A. Yamaguchi

Submitted by A. Yamaguchi

Submitted by A. Yamaguchi

Submitted by A. Yamaguchi

SEM; submitted by AMN

Submitted by A. Yamaguchi

Submitted by A. Yamaguchi

Submitted by A. Yamaguchi

Submitted by A. Yamaguchi

Submitted by A. Yamaguchi

Shock vein; submitted by A. Yamaguchi

Shock vein; submitted by A. Yamaguchi

Submitted by A. Yamaguchi

Submitted by A. Yamaguchi

Shock vein; submitted by A. Yamaguchi

Submitted by A. Yamaguchi

Submitted by A. Yamaguchi

Submitted by A. Yamaguchi

Submitted by C. Park, KOPRI

Submitted by A. Yamaguchi

Submitted by A. Yamaguchi

Submitted by A. Yamaguchi

Submitted by A. Yamaguchi

Submitted by A. Yamaguchi

Submitted by A. Yamaguchi

Submitted by A. Yamaguchi

SEM; submitted by AMN

Shock vein; submitted by A. Yamaguchi

Darkened; submitted by A. Yamaguchi

Submitted by A. Yamaguchi

Shock vein; submitted by A. Yamaguchi

SEM; submitted by AMN

Submitted by A. Yamaguchi

Submitted by A. Yamaguchi

Submitted by A. Yamaguchi

SEM; submitted by AMN

SEM; submitted by AMN

Submitted by A. Yamaguchi

Submitted by A. Yamaguchi

Submitted by A. Yamaguchi

Submitted by A. Yamaguchi

Submitted by A. Yamaguchi

Shock vein; submitted by A. Yamaguchi

Submitted by A. Yamaguchi 
Shock vein; submitted by A. Yamaguchi

Submitted by A. Yamaguchi

Submitted by A. Yamaguchi

Submitted by A. Yamaguchi

Submitted by A. Yamaguchi

Submitted by A. Yamaguchi

Submitted by A. Yamaguchi

Submitted by A. Yamaguchi

Submitted by A. Yamaguchi

Submitted by A. Yamaguchi

Submitted by A. Yamaguchi

Submitted by A. Yamaguchi

Shock vein; submitted by A. Yamaguchi

Submitted by A. Yamaguchi

Work name GH-469; submitted by A. Irving

work name LM72/E09; Breccia with chondritic clasts in a melt rock matrix. Chondritic clasts have plagio Submitted by Ansgar Greshake

work name EJ218. Type 6 chondritic clasts set in a melt rock matrix.; submitted by Jérôme Gattacceca

Work name M2061; submitted by A. Irving

Work name M2040; submitted by A. Irving

working name WZ06; submitted by R. Bartoschewitz

NWA-LB003; submitted by R. Bartoschewitz

working name WZ22; submitted by R. Bartoschewitz

working name WZ21; submitted by R. Bartoschewitz

Submitted by R. Bartoschewitz

Work name M1630; submitted by A. Irving

MVCH-333; submitted by C. Agee, UNM

Work name SJS18004; submitted by A. Irving

Work name M2056; submitted by A. Irving

Field name JP-90; submitted by C. Agee, UNM

Field name Past-4; analyst V.V. Sharygin (SIGM and UrFU); submitted by Victor Sharygin

MVCH-332; submitted by C. Agee, UNM

Specimen number CML0091.; submitted by Alex Ruzicka

Submitted by Ansgar Greshake

Specimen numbers RM-4 and CML0677.; submitted by Alex Ruzicka

Work name K372; submitted by A. Irving

Submitted by Ansgar Greshake

Submitted by Daniel Sheikh

Submitted by L. Garvie

Submitted by A. Yamaguchi

Work name MVCH 372; submitted by A. Irving

work name K360; submitted by J. Gattacceca

Submitted by Simone de Leuw

work name K366; submitted by Jérôme Gattacceca

NWA-LB004; submitted by R. Bartoschewitz 
Morocco; submitted by Ansgar Greshake

Field name is Calama 2-2; analyst T. Kryachko (Technograd, Moscow); submitted by Lorenz C.A., Verna Submitted by A. Yamaguchi

Submitted by L. Garvie

work name K368; submitted by J. Gattacceca

Submitted by C. Park, KOPRI

Submitted by C. Park, KOPRI

Submitted by K. Metzler, IfP

Submitted by Ansgar Greshake

work name K340; submitted by Jerome Gattacceca

Submitted by C. Agee

work name RM647; submitted by Gattacceca

Submitted by AMN

Submitted by AMN

Submitted by Vanni Moggi Cecchi

work name JR051; Bought from Mohamed Elguirah in Zagora in 2018. Gray stones, some with fusion $\mathrm{cr}$ Field name: Met-2016-048; submitted by David Allepuz

Submitted by Vanni Moggi Cecchi

Submitted by Ansgar Greshake

work name B2; submitted by Richard Greenwood, Hélène Greenwood

Catalogued at the Royal Ontario Museum as LM58174. Formerly DGR-031-16; submitted by V. Di Cecc

Purchased from a Moroccan meteorite dealer at the mineral fair in Munich, Germany. Mean chondrule di

Darkened; submitted by A. Yamaguchi

Submitted by A. Yamaguchi

Submitted by A. Yamaguchi

Submitted by A. Yamaguchi

SEM; submitted by AMN

Submitted by A. Yamaguchi

Submitted by A. Yamaguchi

Submitted by A. Yamaguchi

Submitted by A. Yamaguchi

Submitted by A. Yamaguchi

ROM Number LM57441; Chondrules not sharply but readily delineated. Undulatory extinction, but few st Submitted by AMN

$1 \times 1 \times 1 \mathrm{~cm}$ rounded stone, 90\% brown fusion crust, fresh interior; submitted by F.Focacci M. Gemelli, ( SEM; submitted by AMN

Lab number CML0817; submitted by A. Ruzicka

Breccia, shock veins; Working No.: SM 1804-18; submitted by K. Klemm, IfP; submitted by Klemm K.

Submitted by Ansgar Greshake

Genomict breccia; submitted by A. Yamaguchi

Submitted by Ansgar Greshake

Genomict breccia; submitted by A. Yamaguchi

Submitted by Ansgar Greshake

Genomict breccia; submitted by A. Yamaguchi

Submitted by Simone de Leuw 
Submitted by Ansgar Greshake

G671; submitted by A. Bouvier

Submitted by A. Yamaguchi

Submitted by A. Yamaguchi

Submitted by Vanni Moggi Cecchi

SEM; submitted by AMN

Field name is Calama 2-24; analyst V. V. Kozlov (Oxford Instruments, Moscow); submitted by Lorenz C..

SEM; submitted by AMN

ROM Number LM57445; Readily delineated chondrules, some undulatory extinction but no opaque shoc The meteorite is metamorphosed clastic breccia. Field name is Calama 3-20; analyst T. Kryachko (Tech In Chinese publications, its former name was M16131; submitted by Bingkui Miao

Submitted by A. Yamaguchi

Submitted by A. Yamaguchi

SEM; submitted by AMN

Submitted by Vanni Moggi Cecchi

Submitted by A. Yamaguchi

SEM; submitted by AMN

Field name is 2-4; analyst S. E. Borisovsky (IGEM); submitted by C. A. Lorenz (Vernad)

Submitted by A. Yamaguchi

ROM Number LM58185; Many chondrules defined, more "readily delineated." Some undulatory extinctiı Submitted by A. Yamaguchi

SEM; submitted by AMN

Submitted by A. Yamaguchi

Submitted by A. Yamaguchi

SEM; submitted by AMN

Field name is 5-1; analyst S. E. Borisovsky (IGEM); submitted by C. A. Lorenz (Vernad)

Submitted by $A$. Yamaguchi

Breccia; submitted by A. Yamaguchi

work name K331; submitted by J. Gattacceca

Working No.: SM 1804-15; submitted by K. Klemm, IfP; submitted by Klemm K.

Working No.: SM 1804-14; submitted by K. Klemm, IfP; submitted by Klemm K.

Submitted by $\mathrm{H}$. Chennaoui Aoudjehane

Submitted by Daniel Sheikh

Catalogued at the Royal Ontario Museum as LM58148; submitted by V. Di Cecco, ROM

Catalogued at the Royal Ontario Museum as LM58147; submitted by V. Di Cecco, ROM

ROM Number LM57444; submitted by V. Di Cecco, ROM

Submitted by K. Metzler, IfP

Submitted by Vanni Moggi Cecchi

Submitted by A. Yamaguchi

Submitted by A. Yamaguchi

Submitted by Vanni Moggi Cecchi

Submitted by A. Yamaguchi

Brownish individual without fusion crust. plagioclase grain size about $60 \mu \mathrm{m}$; submitted by Ansgar Gresh ROM Number LM58183; Some chondrules visible. Olivnies bear undulatory extinction and planar fractur ROM Number LM57446; Chondrules barely defined and matrix recrystallized. Largest grains show no sị 
Submitted by A. Yamaguchi
Submitted by A. Yamaguchi

Field name: Met-2016-016; submitted by David Allepuz

Work name BR 106; submitted by A. Irving

SEM; submitted by AMN

Submitted by Vanni Moggi Cecchi

breccia; plagioclase grain size about $80 \mu \mathrm{m}$; submitted by Ansgar Greshake

plagioclase grain size about 60 um; submitted by Ansgar Greshake

SEM; submitted by AMN

Submitted by A. Yamaguchi

Submitted by A. Yamaguchi

Submitted by Vanni Moggi Cecchi

Submitted by Vanni Moggi Cecchi

plagioclase grain size about $60 \mu \mathrm{m}$; Two brownish individuals with some fusion crust.; submitted by Ans!

Breccia; submitted by A. Yamaguchi

Breccia; submitted by A. Yamaguchi

working name WZ27; submitted by R. Bartoschewitz

plagioclase grain size about 80 um; submitted by Ansgar Greshake

Submitted by Simone de Leuw

Submitted by A. Yamaguchi

SEM; submitted by AMN

plagioclase grain size about $60 \mu \mathrm{m}$; submitted by Ansgar Greshake

Submitted by A. Yamaguchi

Submitted by A. Yamaguchi

Submitted by A. Yamaguchi

Breccia; submitted by A. Yamaguchi

Submitted by A. Yamaguchi

Submitted by A. Yamaguchi

Breccia; submitted by A. Yamaguchi

Breccia; submitted by A. Yamaguchi

breccia; plagioclase grain size about $60 \mu \mathrm{m}$; submitted by Ansgar Greshake

Work name JB17-1; submitted by A. Irving

Breccia; submitted by A. Yamaguchi

In Chinese publications, its former name was M16303; submitted by Bingkui Miao

Submitted by A. Yamaguchi

plagioclase grain size about 80 um; submitted by Ansgar Greshake

Submitted by A. Yamaguchi

plagioclase grain size about 60 um; contains shock veins; submitted by Ansgar Greshake

Shock vein; submitted by A. Yamaguchi

Submitted by A. Yamaguchi

Breccia; submitted by A. Yamaguchi

Breccia; submitted by A. Yamaguchi

SEM; submitted by AMN

SEM; submitted by AMN

plagioclase grain size about 60 um; submitted by Ansgar Greshake 
SEM; submitted by AMN

SEM; submitted by AMN

Submitted by A. Yamaguchi

Submitted by A. Yamaguchi

SEM; submitted by AMN

Submitted by A. Yamaguchi

SEM; submitted by AMN

SEM; submitted by AMN

Submitted by A. Yamaguchi

Submitted by AMN

Submitted by A. Yamaguchi

Shock vein; submitted by A. Yamaguchi

Submitted by A. Yamaguchi

Breccia; submitted by A. Yamaguchi

Submitted by A. Yamaguchi

Submitted by C. Park, KOPRI

Submitted by Anthony Love

Field name LA-4; submitted by C. Agee, UNM

Submitted by K. Metzler, IfP

work name ST11; submitted by Gattacceca

Work name F018-1; submitted by A. Irving

Work name RC99.2; submitted by A. Irving

Submitted by Ansgar Greshake

Work name BH146; submitted by A. Irving

Field name DD-28; submitted by C. Agee, UNM

Submitted by L. Garvie

Submitted by A. Yamaguchi

Submitted by A. Yamaguchi

Submitted by AMN

Work name MVCH 258; submitted by A. Irving

Submitted by F. Brandstätter

Submitted by Bingkui Miao

work name LL76; submitted by Jérôme Gattacceca

Submitted by Ansgar Greshake

Work name $\mathrm{BH} 155$; submitted by $\mathrm{A}$. Irving

Field name DD-20; submitted by C. Agee, UNM

Field name DD-21; submitted by C. Agee, UNM

Work name DD17-10; submitted by A. Irving

Field name DD-22; submitted by C. Agee, UNM

Submitted by Dmitry Badyukov

M2157; submitted by C. Agee

Submitted by Bingkui Miao

Field name MF-6; submitted by C. Agee, UNM

Gessler's address: 2010 Calgary Ln, Los Angeles, CA 90077, USA; submitted by Paul Warren

Field name DD-25; submitted by C. Agee, UNM 
Work name DG18-1; submitted by A. Irving

Field name M1820; submitted by C. Agee, UNM

Field name ZK-13; submitted by C. Agee, UNM

Work name HN-713; submitted by A. Irving

Field name JP-84; submitted by C. Agee, UNM

Work name: "Aoufous"; submitted by Ebert Samuel

Submitted by Anthony Love

K371; submitted by A. Irving

Field name JP-29; submitted by C. Agee, UNM

Work name $\mathrm{BH} 003$; submitted by A. Irving

Work name MVCH 371; submitted by A. Irving

Work name Hum18-1; submitted by A. Irving

Work name MAC 133; submitted by A. Irving

work name RM573; submitted by Jérôme Gattacceca

Submitted by Nicole G. Lunning

Submitted by Ansgar Greshake

Submitted by C. Agee

Submitted by K. Metzler, IfP

Field name Z-2; submitted by C. Agee

Submitted by A. Irving

Work name MA18-200; submitted by A. Irving

Work name MA18-201; submitted by A. Irving

Work name ML18-1; submitted by A. Irving

Work name $\mathrm{BH} 145$; submitted by A. Irving

Work name $\mathrm{BH} 141$; submitted by $\mathrm{A}$. Irving

Work name BH142; submitted by A. Irving

Submitted by Bingkui Miao

Gessler's address: 2010 Calgary Ln, Los Angeles, CA 90077, USA; submitted by Paul Warren

JH18-2; submitted by A. Irving

Shock vein, An52.5-55.4; submitted by A. Yamaguchi

Work name BH144; submitted by A. Irving

Submitted by Ansgar Greshake

Field number 1601_0094; submitted by Beda Hofmann

Fieldname JP-24; submitted by C. Agee

Field number MMO-2018-3C; submitted by C. Herd

Submitted by C. A. Lorenz (Vernad)

Work name BF18-22; submitted by A. Irving

Submitted by A. Irving

work name LL82; submitted by Jérôme Gattacceca

Work name E511; submitted by A. Irving

Work name MCZ1; submitted by A. Irving

Algeria; submitted by Ansgar Greshake

Submitted by Ansgar Greshake

Submitted by Ansgar Greshake

Submitted by Nicolas Meisser 
Work name B386; submitted by A. Irving

Provisional name was NWA 2683; submitted by L. Garvie

Work name JP18-1; submitted by A. Irving

Submitted by Ansgar Greshake

Submitted by Ansgar Greshake

Submitted by Anthony Love

Submitted by Anthony Love

Work name GF17-4; submitted by A. Irving

work name K189; submitted by A. Irving

Work name BF17-19; submitted by A. Irving

Sample registered at ROM as M58493; submitted by V. Di Cecco, ROM

Work name DD17-9; submitted by A. Irving

Submitted by Alan Rubin

Submitted by Ansgar Greshake

G692 as a field name.; submitted by A. Bouvier

Submitted by B. C. Hyde

Submitted by K. Metzler, IfP

Field name Z-3; submitted by C. Agee, UNM

Work name SJS18005; submitted by A. Irving

Submitted by Ansgar Greshake

Submitted by Ansgar Greshake

Field name MVCH-352; submitted by C. Agee, UNM

G703 as a field name.; submitted by A. Bouvier

Field name MVCH-359; submitted by C. Agee, UNM

Submitted by Ansgar Greshake

Field name SM-1; submitted by C. Agee, UNM

Submitted by $P$. Hill

Field name DD-27; submitted by C. Agee, UNM

Field name MVCH-350; submitted by C. Agee, UNM

Work name M2027; submitted by A. Irving

Work name BF17-16; submitted by A. Irving

Submitted by P. Hill

Submitted by K. Metzler, IfP

G691 as a field name.; submitted by A. Bouvier

Submitted by K. Metzler, IfP

Submitted by C. Park, KOPRI

Specimen numbers RM-8 and CML0681.; submitted by Alex Ruzicka

Field name JP-62; submitted by C. Agee, UNM

Submitted by Ansgar Greshake

Field name MVCH-349; submitted by C. Agee, UNM

Submitted by B. C. Hyde

Work name M365; submitted by A. Irving

Morocco; submitted by Ansgar Greshake

Field name: Met-2018-032; submitted by David Allepuz

G690 as a field name.; submitted by A. Bouvier 
Submitted by P. Hill

Submitted by K. Metzler, IfP

Submitted by A. Bouvier

Submitted by N. V. Almeida

Submitted by K. Metzler, IfP

Submitted by Ansgar Greshake

Work name M2019; submitted by A. Irving

Submitted by Ansgar Greshake

Submitted by Ansgar Greshake

Submitted by A. Yamaguchi

Submitted by A. Yamaguchi

Work name MVCH 296; submitted by A. Irving

Submitted by Alan Rubin

MVCH 335; submitted by A. Irving 
nitted by Lee White

ad by Lee White 
Vernad) 
sport, the Tawani Foundation for funding, Jim Holstein and Drew Carhart for help with sample prepar: 
enocrysts exhibit polysynthetic twinning. Weathering veins rim chondrules and traverse the sample.; ;

ıbmitted by Lorenz C.A., Vernad

nite veinlets occur. Weathering veins rim the chondrules and traverse the sample.; submitted by Simc 
exhibit polysynthetic twinning. Weathering veins rim the chondrules and traverse the sample.; submitt

10

nad 
lake

soint counting, $\mathrm{N}=169$ ) between well-preserved chondrules.; submitted by Jérôme Gattacceca गndrules.; submitted by Lisa Krämer Ruggiu

nad

A., Vernad 
I by Gattacceca

\section{Gattacceca}


nad

rake

ed by Jérôme Gattacceca 
'ith shiny fusion crust.; submitted by Gattacceca 
orenz C. A. (Vernad.)

nad 
erage plagioclase size $<50$ um. They contain veinlets of metal and sulfides.; submitted by Jérôme Ge

gm.nsc.ru

ıbmitted by Lisa Krämer Ruggiu 
GUT: Guilin Universiy of Technology. PRIC: Polar Research Institute of China 
gar Greshake

Di Cecco, ROM 
cism. Thin section has iron staining. More than $20 \%$ of the metal and sufides affected by oxidation.; s 
matrix that contains weakly altered FeNi metal.; submitted by Ansgar Greshake latrix of chondrule and mineral fragments and FeNi metal.; submitted by Ansgar Greshake 
lefined chondrules. Rare plagioclase to 5 um. Zoned pyroxenes.; submitted by Gattacccea

dulatory extinction. More than $20 \%$ of metal oxidized.; submitted by V. Di Cecco, ROM

itted by F.Focacci M. Gemelli, (DST-PI) 


\section{- Greshake}

has a smooth surface that is covered by dark-brown fusion crust.; submitted by C. A. Lorenz (Vernac 


\section{Vernad}

\section{Greshake}

reshake

team. Shock veins.; submitted by Gattacceca

size about 15 um.; submitted by Ansgar Greshake 
T-PI) 


\section{n@igm.nsc.ru}

ar Greshake

ə-chromite assemblages. A 3.5 metal nugget is visible in the studied polished section.; submitted by $\downarrow$ 
lake

ed by J. Gattacceca

sck veins are present. Some fractures affected by weathering.; submitted by V. Di Cecco, ROM reshake

orenz C. A. (Vernad.)

Greshake 


\section{Greshake}

; polycrystalline.; submitted by Jérôme Gattacceca

nad

stinction and mocaicism are present. Metal mostly unaltered with minor oxidation on rims.; submitted 
clase average size $>50 \mathrm{um}$. They contain veinlets of metal and sulfides, chromite-plagioclase asseml 
ad

ust. Millimeter-sized chondrules are visible on broken surfaces.; submitted by Gattacceca

zO, ROM

iameter is about $0.8 \mathrm{~mm}$; submitted by Ansgar Greshake

hock veins. Metal lightly oxidized.; submitted by V. Di Cecco, ROM

'DST-PI) 


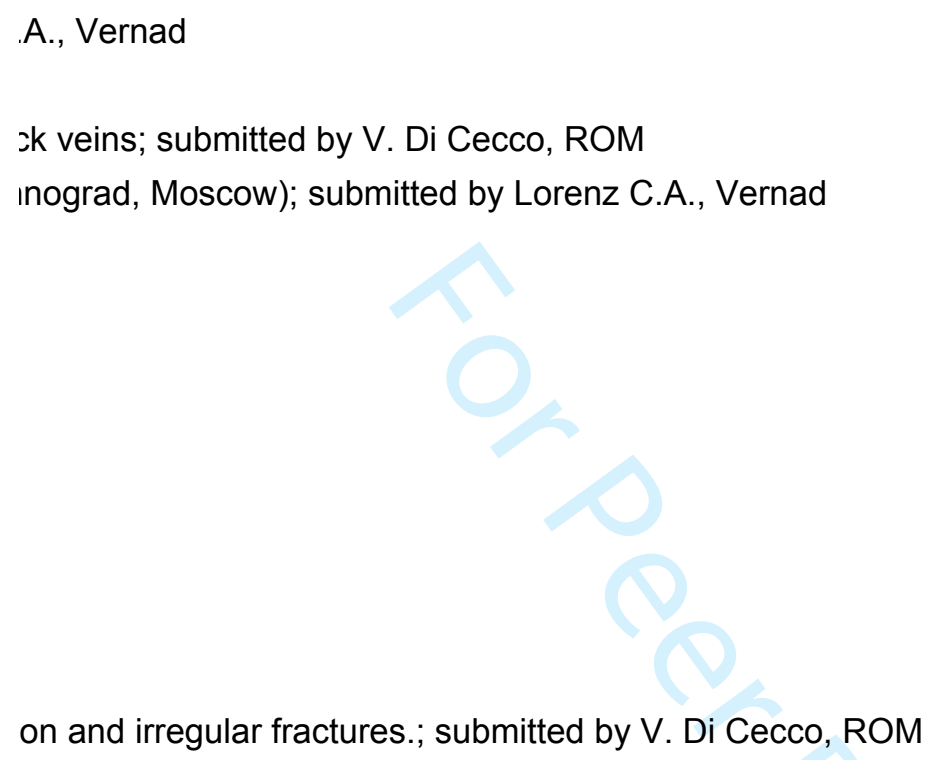

on and irregular fractures.; submitted by V. Di Cecco, ROM

\section{lake}

res.; submitted by V. Di Cecco, ROM

gns of undulatory extinction, and sample fairly weathered through its orange coloration and $<5 \%$ pres 


\section{gar Greshake}

20

22

23

24

25

26

27

28

29

30

31

32

33

34

35

36

37

38

39 
ation.; submitted by Philipp R. Heck, Jennika Greer 
submitted by Simone de Leuw

one de Leuw 
ted by Simone de Leuw 
łttacceca 
submitted by V. Di Cecco, ROM

10 


\section{lex Ruzicka}


blages, and eutectic metal/sulfide grains.; submitted by Jérôme Gattacceca 


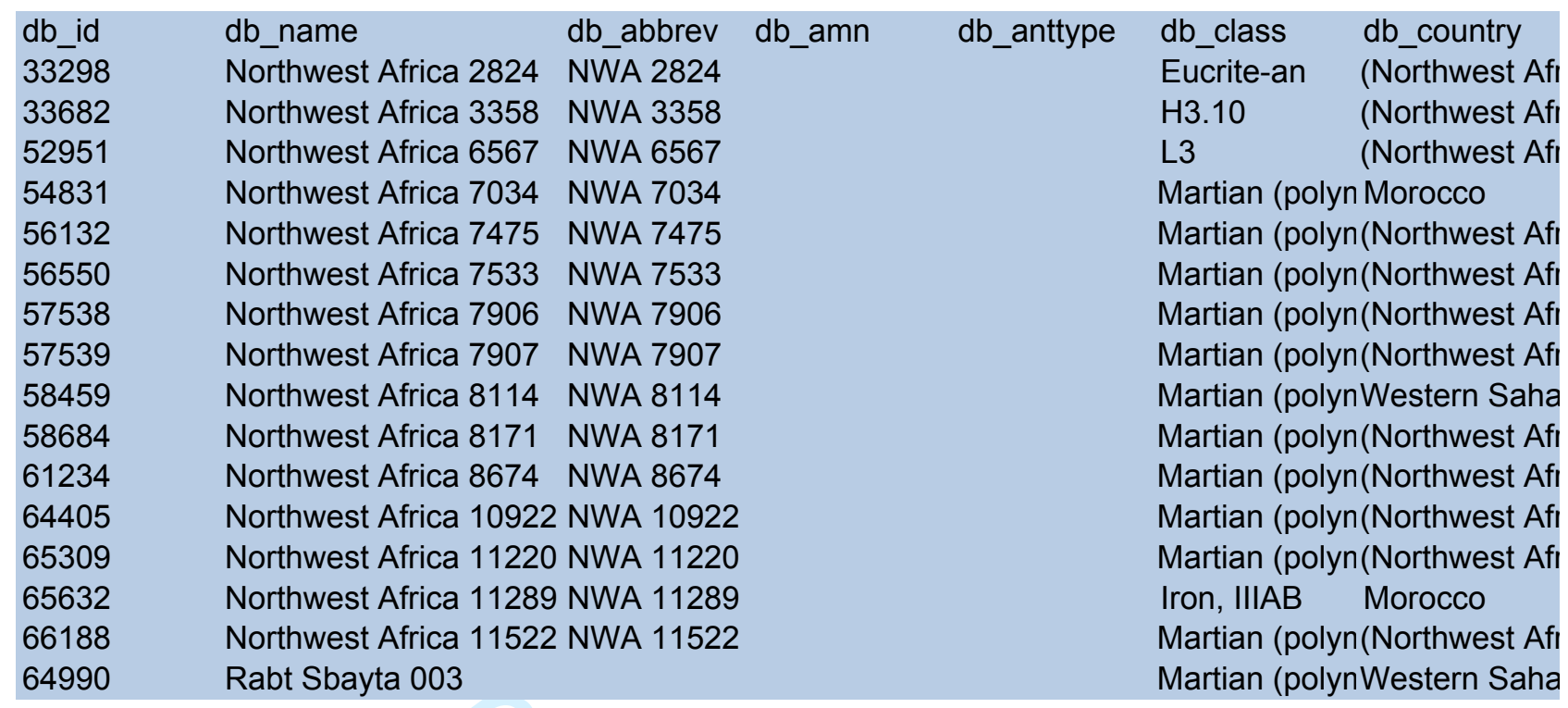




\begin{tabular}{|c|c|c|c|c|}
\hline \multirow[t]{9}{*}{ db_state } & db_approved & db_modified & db_reason & \multirow[t]{9}{*}{ tbl_stateco } \\
\hline & $2008-12-23$ & 2018-06-08 & Reclassified & \\
\hline & 2005-02-03 & 2018-06-08 & Reclassified & \\
\hline & 2013-09-06 & $2018-05-28$ & Reclassified from CV3 to L3 & \\
\hline & 2012-01-24 & 2018-11-16 & Updated class & \\
\hline & 2013-05-06 & 2018-11-16 & Updated class; see NWA 7034) & \\
\hline & 2012-12-11 & 2018-11-16 & Updated class; see NWA 7034) & \\
\hline & 2013-06-19 & 2018-11-16 & Updated class; see NWA 7034) & \\
\hline & 2013-06-19 & 2018-11-16 & Updated class; see NWA 7034) & \\
\hline \multirow[t]{7}{*}{ Rio de Oro } & 2013-12-10 & 2018-11-16 & Updated class; see NWA 7034) & \multirow[t]{7}{*}{ Rio de Oro } \\
\hline & 2014-01-23 & 2018-11-16 & Updated class; see NWA 7034) & \\
\hline & 2014-12-12 & 2018-11-16 & Updated class; see NWA 7034) & \\
\hline & 2016-12-03 & 2018-11-16 & Updated class; see NWA 7034) & \\
\hline & 2017-08-06 & 2018-11-16 & Updated class; see NWA 7034) & \\
\hline & $2017-06-28$ & 2018-06-16 & Reclassified by Wasson & \\
\hline & $2017-11-21$ & 2018-11-16 & Updated class; see NWA 7034) & \\
\hline Rio de Oro & 2017-03-03 & 2018-11-16 & Updated class; see NWA 7034) & Rio de Oro \\
\hline
\end{tabular}




\begin{tabular}{|c|c|c|c|c|c|c|}
\hline tbl_origin & tbl_purchpla & tbl_date & tbl_lat & tbl_long & tbl_mass & tbl_pieces \\
\hline & Morocco & 2005 & & & $48 \overline{5}$ & 1 \\
\hline & Sainte Marie & May 2001 & & & 1162 & 1 \\
\hline & Temara & P 2010 Nov & & & 6000 & 1 \\
\hline & Morocco & P 2011 & & & 319.8 & 1 \\
\hline & Morocco & P 2012 Sep & & & 80.2 & 1 \\
\hline & Agadir & P June 2012 & & & 84 & 1 \\
\hline & Morocco & P Jan 2013 & & & 47.68 & 1 \\
\hline & Morocco & P Jan 2013 & & & 29.94 & 1 \\
\hline & & Feb 2013 & $24^{\circ} 04^{\prime} 45.8^{\prime \prime N}$ & $14^{\circ} 47^{\prime} 12.4^{\prime \prime W}$ & 1.9 & 1 \\
\hline & Ensisheim, Fr & P June 2013 & & & 81.88 & 1 \\
\hline & Morocco & P 2012 & & & 12 & 1 \\
\hline & Morocco & P 2013 & & & 182 & 1 \\
\hline & Zagora, Moro & P Jan 2017 & & & 36.62 & 1 \\
\hline $50 \mathrm{~km}$ east of & Temara & P 2012 Oct & & & $4.6 \mathrm{~kg}$ & 1 \\
\hline & Morocco & P 2013 Nov & & & 3.2 & 1 \\
\hline & Taliouine & 2016 Nov & $24.122^{\circ} \mathrm{N}$ & $14.772^{\circ} \mathrm{W}$ & 20.57 & 1 \\
\hline
\end{tabular}




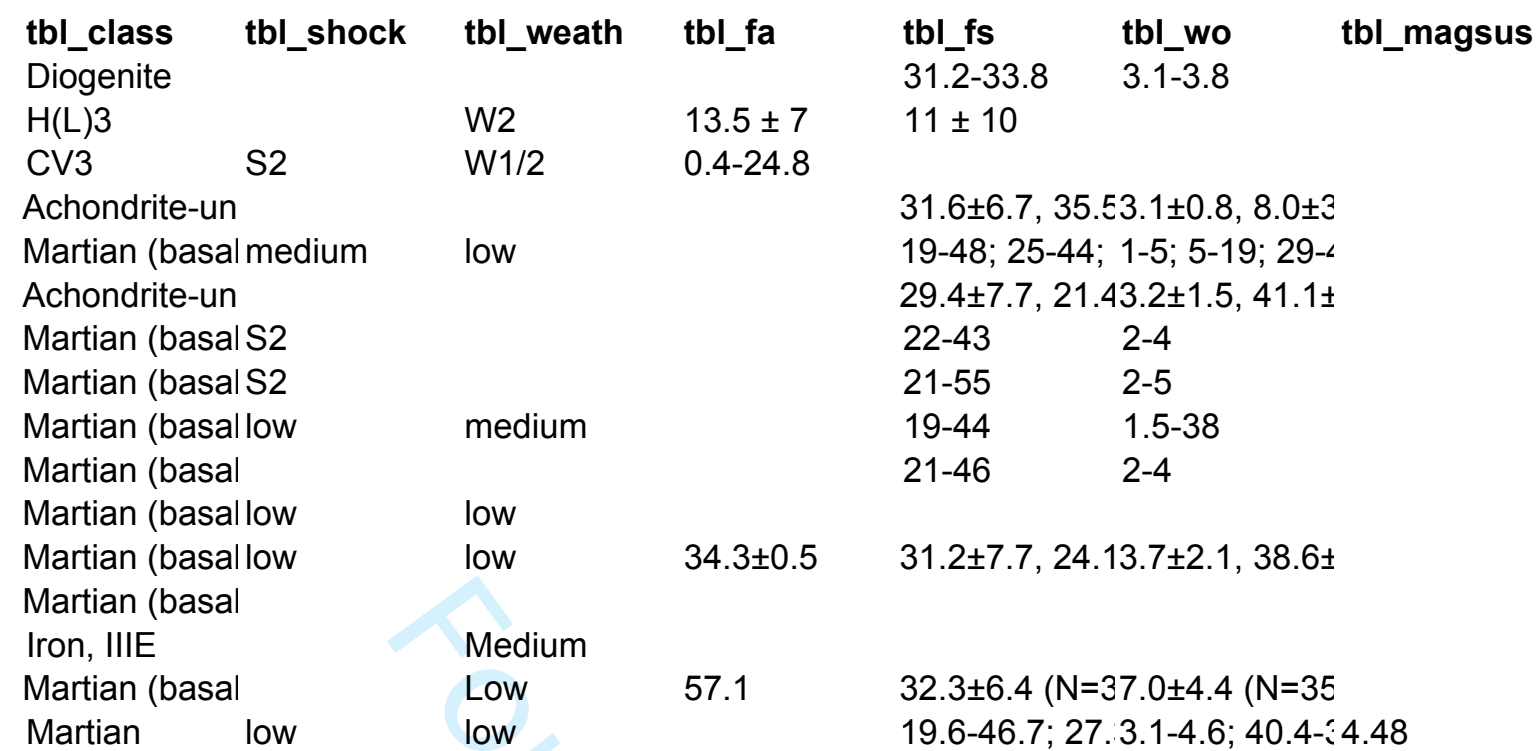


tbl_typespectbl_typespecltbl_mainmas: tbl_classifier tbl_finder 32 PSF anonymous T. Bunch and $23 \quad$ Mun $23 \quad$ UWB $30 \quad$ UNM $16.1 \quad$ UWB 17.1 10.50

8.20

1.9 16.50

2.5

22

7.46

93.0

0.948 4.2 Niemeier, Jorc Aaronson A. Irving and $\leqslant$ Jay Piatek

C. Agee L. Labenne

A. Wittmann, I Labenne Mété R. Hewins, B. Marc Jost

B. Hofmann N Marc Jost

B. Hofmann $\mathrm{N}$

NIME NMBE

ULei NMBE

UNM

UNM ULei Marc Jost, Be:B. Hofmann, $\Gamma$ Jay Piatek Jay Piatek

C. Agee, UNN NHM London Martin Goff A. M. Krzesins UWO NHM UWB
J. Bridges, UL
A. Aaronson
A. Bouvier, Ul Unknown
NHM
C. Smith, $\mathrm{NHN}$

B. Hoefnagels A. Irving and $\leqslant$ tblcomment

Highly shocked and brecciated c

Submitted by A. Irving

Submitted by C. Agee

Submitted by $A$. Irving

Submitted by B. Zanda

Submitted by Beda Hofmann

Submitted by Beda Hofmann

Submitted by John Bridges

Submitted by Beda Hofmann

Submitted by C. Agee

Field name BB-182; submitted $b$

Submitted by N. V. Almeida

Submitted by A. Bouvier, UWO

The specimen is texturally and $n$

BH040; submitted by A. Irving 
diogenite that is plagioclase rich

$$
\text { sy C. Agee }
$$

nineralogically very similar to NWA 7034 and its numerous pairs, including NWA 11220, which was a 
Iso classified at the NHM.; submitted by Caroline Smith 
The Meteoritical Bulletin, No. 107

\title{
TABLE OF CONTENTS
}

1. Alphabetical text entries for non-Antarctic meteorites

2. New Dense Collection Areas

3. Listing of institutes and collections

4. Bibliography

\section{Alphabetical text entries for non-Antarctic meteorites}

\author{
Aba Panu $\quad 8^{\circ} 16^{\prime} 55.83^{\prime \prime} \mathrm{N}, 3^{\circ} 34^{\prime} 1.72^{\prime \prime} \mathrm{E}$ \\ Oyo, Nigeria \\ Confirmed fall: 19 April 2018 \\ Classification: Ordinary chondrite (L3)
}

History: On the afternoon of 19 April 2018, a large fireball detonated over the Nigerian state of Oyo. This fireball was recorded by NASAs Center for Near Earth Object Studies ( $\underline{\mathrm{CNEOS}}$ ) as event 2018-04-19 14:02:27. The meteoroid entered at $20.9 \mathrm{~km} / \mathrm{s}$ and detonated at an altitude of $30 \mathrm{~km}$ at $7.5^{\prime} \mathrm{N}, 3.6^{\prime} \mathrm{E}$ releasing a calculated total impact energy of $0.23 \mathrm{kt}$. Many stones fell between the villages of Ipapo $\left(8^{\circ} 7^{\prime} 50.84^{\prime \prime} \mathrm{N}, 3^{\circ} 30^{\prime} 34.58^{\prime \prime} \mathrm{E}\right)$ and Tede to the north $\left(8^{\circ} 33^{\prime} 21.49^{\prime \prime} \mathrm{N}\right.$, $\left.3^{\circ} 26^{\prime} 46.31^{\prime \prime E}\right)$. Stone were collected at multiple locations. The meteorite is named for the village of Aba Panu near the center of the strewn-field: multiple kg-sized stones were found in and around this village. Current total known weight is near $160 \mathrm{~kg}$.

Physical characteristics: The stones are hard, range from $30 \mathrm{~g}$ to near $40 \mathrm{~kg}$, rounded with broad poorly developed regmaglypts, and largely lacking fusion crust. Fusion crust is occasionally preserved within the shallow regmaglypts. Most stones are greyish green and show areas of rounded to angular light-colored clasts. Largest clast $(5 \times 2 \mathrm{~cm})$ is angular, fine-grained, and achondritic. Observations from multiple slices show that clasts constitute roughly $<10$ areal $\%$.

Petrography: Visually the cut surfaces are dominated by a gray matrix, studded with well-developed chondrules and chondrule fragments. Some chondrules to 4 $\mathrm{mm}$ but the majority are 0.1 to $1 \mathrm{~mm}$ across. Observations on an $8 \times 7 \mathrm{~cm}$ slab shows the following clasts: L6 $(3 \times 1 \mathrm{~cm})$; L5 $(1.5 \times 1 \mathrm{~cm})$; and sub-rounded $1-\mathrm{cm}$ dark clast with sparse 200 micron chondrules. Shock veins are rare. Metal and sulfide occurs as: fined grained spheres in the matrix; armoring chondrules; and, occasional lumps to $5 \mathrm{~mm}$. Petrographic observations show a range of chondrule types dominated by PO, POP, RP, PP, and BO. Particularly evident in thin section are $\mathrm{BO}$ and $\mathrm{PO}$ chondrules with reddish purple (in plain polarized light) glass that is isotropic under crossed polars. The silicates are extensively shocked with olivine showing mosaicism and PDFs. SEM observations show that much of the metal/sulfide in the matrix occurs as fine-droplets, veins, and melt pods. Large FeNi grains are polycrystalline with each crystal showing a kamacite core, dark- 
etched martensitic inner rim, and outer Ni-rich rim. Troilite is dominantly single crystal, and occasionally polycrystalline. Sparse chromite grains.

Geochemistry: (L. Garvie, $A S U$ ) Olivine $\mathrm{Fa}_{24.3 \pm 5.7}, \mathrm{n}=18$, range $\mathrm{Fa}_{7.5-32.8}$. Mean $\mathrm{Cr}_{2} \mathrm{O}_{3}=0.08$, sigma- $\mathrm{Cr}_{2} \mathrm{O}_{3}=0.07$, range 0.02 to 0.3 . $\mathrm{FeO} / \mathrm{MnO}=55.8 \pm 12.6$. Low $\mathrm{Ca}$, pyroxene $\mathrm{Fs}_{17.0 \pm 11.6} \mathrm{Wo}_{2.0 \pm 1.9}$ range $\mathrm{Fs}_{2.4-49.2} \mathrm{Wo}_{0.3-6.0}, \mathrm{n}=17$. High Ca pyroxene $\mathrm{Fs}_{8.7} \mathrm{Wo}_{49.2}, \mathrm{n}=1$. Reddish purple, isotropic glass within $\mathrm{BO}$ and $\mathrm{PO}$ chondrules $\mathrm{Or}_{1.4 \pm 0.8} \mathrm{Ab}_{87.6 \pm 4.8}, \mathrm{n}=6, \mathrm{TiO}_{2}$ to $1 \mathrm{wt} \%$. Oxygen isotopes (K. Ziegler, $\left.U N M\right)$ : analyses of three subsamples by laser fluorination gave, respectively, $\delta^{17} \mathrm{O}=3.724$, $3.625,3.684 ; \delta^{18} \mathrm{O}=5.160,4.980,5.089 ; \Delta^{17} \mathrm{O}=1.000,0.996,0.997$ per mil.

Classification: Geochemistry and oxygen isotopic data consistent with $\mathrm{L}$ chondrite. Estimated petrologic type 3.6 based on $\mathrm{Fa}$ and $\mathrm{Fs}$ spread, and $\mathrm{Cr}_{2} \mathrm{O}_{3}$ content in olivine. L3, S4, W0.

Specimens: Michael Farmer and Moritz Karl - $50 \mathrm{~kg}$, Eric Twelker $7.74 \mathrm{~kg}$, and $A S U 242 \mathrm{~g}$. Based on photographic evidence, it is estimated that the Nigerian government collected on the order of $100 \mathrm{~kg}$ of stones, though this mass is uncertain. Given the size of the fall and area of the fall, the total mass is conservatively given as $160 \mathrm{~kg}$.

\section{Ablaketka $\quad 49^{\circ} 51.27^{\prime} \mathrm{N}, 82^{\circ} 48.89^{\prime} \mathrm{E}$ \\ East Kazakhstan, Kazakhstan \\ Fall: 16 Feb 2018 \\ Classification: Ordinary chondrite (H5)}

History: The fall occurred on the 16 Feb 2018 at 14:43:37 UTS. Residents of Ablaketka, a suburb of Ust-Kamenogorsk, Kazakhstan, watched a bright bolide moving approximately north and accompanied by an explosion. The bolide was recorded by video camera mounted on a locomotive moving NE from Charsk. The first sample was discovered by Mr. Alexander Baranov on February 18, near the Irtysh river, on a field covered by snow $\sim 10-20 \mathrm{~cm}$ deep. The fall left two scratchy traces: the first was $\sim 0.5 \mathrm{~m}$ in length, excavating snow and some soil; the second scratch, $8 \mathrm{~m}$ away, was terminated by a channel in the snow where the stone was found. On Feb. 22, two other pieces were recovered by anonymous finders. Later, about 10 pieces were collected by anonymous persons from Feb to Apr 2018, in a strewn field of $1 \times 5 \mathrm{~km}$, on an azimuth of $20^{\circ}$, with central coordinates of $49^{\circ} 51.27^{\prime} \mathrm{N}, 82^{\circ} 48.89^{\prime} \mathrm{E}$.

Physical characteristics: The stone discovered first is $763 \mathrm{~g}$ and covered by black fusion crust, with only a few small broken parts, displaying light-gray interior. Two smaller individuals are 63.5 and $266.6 \mathrm{~g}$. Later, several individual samples with dark-grey- to black interior were recovered. The masses of individuals are in a range $0.1-1.2 \mathrm{~kg}$. A total collected mass of the meteorite shower is approximately $5.5 \mathrm{~kg}$.

Petrography: The meteorite has brecciated, chondritic texture. Macroscopically, a light-gray chondritic host contains $\mathrm{cm}$-sized clasts of dark-gray chondritic lithology ( $\sim 20 \mathrm{vol} \%$ ). The clasts have sharp contacts with the host. The host is composed of chondrules and chondrule fragments, mineral clasts and fine-grained, recrystallized, 
silicate matrix. The main phases are olivine, pyroxene, FeNi metal; minor phases are devitrified glass, feldspar, troilite; spinel is accessory. Olivine has weak undulatory extinction and planar fractures. Impact melt veinlets and melt pockets are present in the host chondite. The dark lithology has the same texture and composition of the silicates, but is intensive darkened. It has high abundance of tiny inclusions and narrow veinlets of troilite, occurring in the fractures and along the grain boundaries. Several objects in the dark lithology are fine-grained, silicate breccias with troilite matrix. The olivine demonstrates strong undulatory extinction and planar fractures decorated by opaque inclusions.

Geochemistry: Mineral compositions and geochemistry: (N. N. Kononkova, EMP, Vernad): Olivine $\mathrm{Fa}_{19.1 \pm 0.4}(\mathrm{~N}=24)$, Pyroxene $\mathrm{Fs}_{17.1 \pm 0.6} \mathrm{Wo}_{1.4 \pm 0.2}(\mathrm{~N}=23)$. Classification: (C. A. Lorenz, Vernad) ordinary chondrite (H5, monomict breccia) Shock stage is $\mathrm{S} 3$; weathering grade is W0.

Specimens: Two thin sections and two samples of 108.7 and $57.2 \mathrm{~g}$ are on deposit in Vernad; anonymous collectors hold the main mass.

\title{
Alaer $003 \sim 40^{\circ} 20^{\prime} \mathrm{N}, \sim 81^{\circ} 15^{\prime} \mathrm{E}$
}

Xinjiang, China

Find: 2007

Classification: Ordinary chondrite (LL 6)

History: (Ziyao Wang) Purchased by Wang Ziyao from Fan Xingang in Aksu, Xinjiang in 2010. This meteorite was discovered in the Taklimakan region, close to Alaer in 2007.

Physical characteristics: (Wang Ziyao) Dark brown stone of $85.4 \mathrm{~g}$

Petrography: (R. Bartoschewitz, Bart) Brown stainted recrystallized matrix with nearly complete recrystallized chondrules $(0.9-1.8 \mathrm{~mm}$, av. $1.1 \mathrm{~mm})$ and few metal specks.

Geochemistry: Magnetic susceptibility (R. Bartoschewitz, Bart $) \log \chi\left(\times 10^{-}\right.$ $\left.{ }^{9} \mathrm{~m}^{3} / \mathrm{kg}\right)=4.44$

Classification: Ordinary chondrite (LL $\sim 6, \mathrm{~W} 3)$

Specimens: $18.1 \mathrm{~g}$ on deposit at Kiel, Wang Ziyao holds the main mass, and $22 \mathrm{~g}$ with Bart.

\author{
Alatage Mountain 043 (AM 043) $\quad 41^{\circ} 03^{\prime} 22^{\prime \prime} \mathrm{N}, 92^{\circ} 06^{\prime} 00^{\prime \prime} \mathrm{E}$ \\ Xinjiang, China \\ Find: 2013 \\ Classification: Iron meteorite (IVB)
}

History: The meteorite was found in 2013 by a stone hunter in the eastern of Lop Nur, Xinjiang Province.

Physical characteristics: The total mass of the four meteorites is $9.8 \mathrm{~kg}$. The largest one is $7.68 \mathrm{~kg}$. The largest meteorite has an irregular shield shape, the back side with regmalypts and the front side with melting streamlines. 
Petrography: Polished section of the sample observed under the SEM shows extremely fine $(\sim 5 \mathrm{um})$ taenite and kamacite forming an ataxite pattern. No inclusions can be observed in the section

Geochemistry: (ELAN DRC-e Q-ICP-MS) $\mathrm{Ni}=16.7, \mathrm{Co}=0.73$ (in wt $\%$ ), $\mathrm{Ga}=0.22$, $\mathrm{Ge}=2.27, \mathrm{As}=1.35, \mathrm{~W}=3.36, \mathrm{Ir}=36.80, \mathrm{Au}=0.06$ (in ppm)

Classification: Iron Meteorite (ataxite), IVB

Specimens: Main mass with Xinping Wen. One polished section and a $100 \mathrm{~g}$ slice was deposited in IGCAS

\section{Alatage Mountain 044 (AM 044) $\quad 41^{\circ} 04^{\prime} 44.73^{\prime \prime} \mathrm{N}, 92^{\circ} 01^{\prime} 24.76^{\prime \prime} \mathrm{E}$}

Xinjiang, China

Find: 22 Jan 2015

Classification: Ordinary chondrite $(\mathrm{H} \sim 5)$

History: (Ziyao Wang) The meteorite was discovered by Xu Jianjiang (Hami/Xinjiang) in the Gobi Desert about $120 \mathrm{~km} \mathrm{NE}$ of Lop Nur, colose to the Dawadi pass, near Ruoqiang county, Korla city, Bayingol Mongolian Autonomous Prefecture, Xinjiang province on Jan. 22, 2015. Wang Ziyao purchased part of it. Physical characteristics: (Ziyao Wang and R. Bartoschewitz, Bart) Reddishbrown, heavily fractured, irregular rock of $709 \mathrm{~g}$ without fusion crust.

Petrography: (R. Bartoschewitz, Bart) Brown-stained, recrystallized matrix, with well developed chondrules (0.3-0.6 mm) and irregular metallic specks.

Geochemistry: Magnetic susceptibility (R. Bartoschewitz, Bart) $\log \chi\left(\times 10^{-}\right.$ $\left.{ }^{9} \mathrm{~m}^{3} / \mathrm{kg}\right)=4.89$

Classification: Ordinary chondrite $(\mathrm{H} \sim 5, \mathrm{~W} 3)$

Specimens: $23.3 \mathrm{~g}$ on deposit at Kiel, Xu Jianjiang (Hami/Xinjiang) and Wang Ziyao hold the main mass, and $79.2 \mathrm{~g}$ with Bart.

\section{Andila $\quad 12.79^{\circ} \mathrm{N}, 21.20^{\circ} \mathrm{E}$ \\ Ouaddai, Chad \\ Confirmed fall: 2014 Aug 21 \\ Classification: Ordinary chondrite (L6)}

History: According to written report from the prefect of Abdi (Mr. Levy Dappina), on August 21, 2014, around 10 am, a series of strong detonations were heard over a distance of $70 \mathrm{~km}$. Later the prefect got a phone call from the village of Andila (12 $\mathrm{km} \mathrm{W}$ of Abdi) signaling that rocks fell from the sky. A police patrol went to the place and collected, the same day, a series of stones, said to have made a $10 \mathrm{~cm}$ hole in the soil and which produced smoke on impact. It is not clear if several individuals were collected or if the stones mentioned were fragments from a single individual. Based on the $171 \mathrm{~g}$ piece received by J.C. Doumnang from N'Djamena University, and a discussion with Mr. Dappina, the whole individual mass is estimated to about half a $\mathrm{kg}$. The prefect later handed the stones (except the one that was brought to J.C. Doumnang for identification in 2018) to the governor of 
Ouaddaï, together with a report. J.C. Doumnang later brought the stone to $C E R E G E$ where it was classified.

Physical characteristics: The $171 \mathrm{~g}$ fragment is one-third covered with black fusion crust. Fractures reveal a homogeneous, light gray, chondrule-bearing stone with a few faint rust stains. Some troilite aggregates. The material is quite fragile due to intense fracturing.

Petrography: Recrystallized chondritic texture with average plagioclase size $>50$ $\mu \mathrm{m}$, but relatively well-preserved chondrules. Main minerals are olivine, pyroxene, plagioclase, kamacite, troilite. Olivine shows sharp optical extinction. No oxidation products visible in section.

Geochemistry: Olivine $\mathrm{Fa}_{25.5 \pm 0.2}(\mathrm{~N}=3)$, orthopyroxene $\mathrm{Fs}_{21.8 \pm 0.1} \mathrm{Wo}_{1.8 \pm 0.3}(\mathrm{~N}=3)$.

Classification: Ordinary chondrite, L6

Specimens: $124 \mathrm{~g}$ fragment in N'Djamena University, type specimen (42 g) a polished section and a thin section at CEREGE.

\title{
Aridal $017 \quad 25.634^{\circ} \mathrm{N}, 13.193^{\circ} \mathrm{W}$
}

Rio de Oro, Western Sahara

Purchased: 2016 Sep

Classification: Lunar meteorite (feldspathic breccia)

History: Found in Morocco in sandy soil, several meters from the find site for Northwest Africa 10608 (which was found earlier by desert truffle hunters). The stone was purchased by Dr. David Gregory in September 2016 from a Moroccan dealer at the Denver Show.

Physical characteristics: A single grayish stone (590 g) lacking fusion crust but with a glossy external patina. The fresh interior consists of small whitish clasts in a dark-gray matrix.

Petrography: (A. Irving and S. Kuehner, UWS) Breccia composed of angular mineral clasts of anorthite, olivine, orthopyroxene, pigeonite, ferropigeonite, augite, silica polymorph, TI-chromite and ilmenite (with rare inclusions of baddeleyite) in a fine grained matrix of the same minerals plus minor kamacite, troilite and K-bearing glass.

Geochemistry: Olivine $\left(\mathrm{Fa}_{10.1-27.0}, \mathrm{FeO} / \mathrm{MnO}=74-106, \mathrm{~N}=3\right)$, orthopyroxene $\left(\mathrm{Fs}_{20.9} \mathrm{Wo}_{4.5}, \mathrm{FeO} / \mathrm{MnO}=51\right)$, pigeonite $\left(\mathrm{Fs}_{21.7} \mathrm{Wo}_{7.0}, \mathrm{FeO} / \mathrm{MnO}=54\right)$, ferropigeonite $\left(\mathrm{Fs}_{63.1} \mathrm{Wo}_{18.5}, \mathrm{FeO} / \mathrm{MnO}=72\right)$, augite $\left(\mathrm{Fs}_{17.3} \mathrm{Wo}_{40.5}, \mathrm{FeO} / \mathrm{MnO}=46\right)$, anorthite $\left(\mathrm{An}_{95.2-95.4} \mathrm{Or}_{0.5}, \mathrm{~N}=2\right)$.

Classification: Lunar (feldspathic regolith breccia). This stone was found at the same place as Northwest Africa 10608 and probably is paired with it.

Specimens: $10.1 \mathrm{~g}$ including one polished endcut at $U W B ; 10.1 \mathrm{~g}$ at $R O M$; main mass with Gregory.

\author{
Aydar $\quad 27^{\circ} 49^{\prime} 33.10^{\prime \prime} \mathrm{N}, 10^{\circ} 31^{\prime} 0.40^{\prime \prime} \mathrm{W}$ \\ Morocco \\ Find: 2008
}


Classification: Primitive achondrite (Acapulcoite)

History: The stones were found by Mohamed Maskour. They were bought in TanTan in 2017 from Mohamed Elguirah.

Physical characteristics: Dark brown irregular stones. The largest stone weighs 267 g. Cut surface reveals a dark interior.

Petrography: Recrystallized texture with triple junctions. Typical silicate grain size $200 \mu \mathrm{m}$. Main minerals are olivine, orthopyroxene, Ca-pyroxene. No plagioclase was observed. FeNi metal and troilite are found as small blebs in silicates and as euhedral grains to $500 \mu \mathrm{m}$. Chromite to $100 \mu \mathrm{m}$. Abundant $\mu \mathrm{m}$ wide veins filled with opaques and associated weathering products.

Geochemistry: Olivine $\mathrm{Fa}_{10.9 \pm 0.0}, \mathrm{FeO} / \mathrm{MnO}=18.9(\mathrm{~N}=2)$. Orthopyroxene $\mathrm{Fs}_{9.8 \pm 0.0} \mathrm{Wo}_{1.5 \pm 0.3}, \mathrm{FeO} / \mathrm{MnO}=12.6(\mathrm{~N}=2)$. Ca-pyroxene $\mathrm{Fs}_{4.2 \pm 0.0} \mathrm{Wo}_{44.0 \pm 1.7}$, $\mathrm{Cr}_{2} \mathrm{O}_{3} 1.07 \mathrm{wt} \%, \mathrm{FeO} / \mathrm{MnO}=12.6(\mathrm{~N}=2)$. Kamacite $\mathrm{Ni}=5.87 \mathrm{wt} \%, \mathrm{Co}=0.27 \mathrm{wt} \%$ $(\mathrm{N}=2)$

Classification: Achondrite (acapulcoite)

Specimens: $20.3 \mathrm{~g}$ at CEREGE. Main mass with Jean Redelsperger.

\section{Bañobárez $\quad 40^{\circ} 51.04^{`} \mathrm{~N}, 6^{\circ} 36.46^{\prime} \mathrm{W}$}

Salamanca, Spain

Find: 1981

Classification: Iron meteorite (IIIAB)

History: Eduardo Martín González, retired teacher and founder of the Archeological Museum of Lumbrales (Salamanca), got the sample from a farmer, who found it in 1981. He gave it to Angel Rolland (researcher at IAA-CSIC), who gave it in 2008 to Alberto Castro-Tirado (also researcher at IAA-CSIC), who in turn sent it to Jordi Llorca (UPC) for study.

Petrography: (J. Llorca, UPC) The meteorite is a medium octahedrite containing kamacite, taenite and few plessite fields. Kamacite bandwidth is $1.0 \pm 0.2 \mathrm{~mm}$.

Geochemistry: (J. Llorca, UPC) ICP-OES data: $\mathrm{Ni}=80.8, \mathrm{Co}=5.6, \mathrm{P}=1.4, \mathrm{~S}=0.1$ (all mg/g); ICP-MS data: $\mathrm{Cu}=167, \mathrm{Cr}=48, \mathrm{Ge}=42, \mathrm{Ga}=21, \mathrm{Pt}=11, \mathrm{Os}=5.0, \mathrm{Ru}=9.7$, $\mathrm{Ir}=3.6, \mathrm{Au}=1.0(\mathrm{all} \mu \mathrm{g} / \mathrm{g})$

\section{Beryozovka $\quad 52^{\circ} 39.86^{\prime} \mathrm{N}, 76^{\circ} 55.26^{\prime} \mathrm{E}$ \\ Pavlodar, Kazakhstan \\ Find: August 2013 \\ Classification: Ordinary chondrite (H5-6)}

History: A single stone was found in August 2013 by Mr. K. M. Tulendiev $\sim 18 \mathrm{~km}$ north of the village Berezovka (synonym Zhambyl, Pavlodar region, Kazakhstan) on the steppe during hay making. The stone was in a pit of $2 \mathrm{~m}$ in diameter and $\sim 0.7 \mathrm{~m}$ in depth. The sample was moved to Berezovka and remained there in a shed. In 2015, Mr. Aron Kadyrov learned about the stone's location from Mr. Tulendiev and searched for it. He find that the shed was demolished, but eventually found the stone among junk. Several pieces of $\sim 100 \mathrm{~g}$ were separated from the 
main mass. The stone was identified as a meteorite by Dr. V. L. Levin (K. I. Satpaev Institute of Geological Sciences, Almaty, Kazakhstan). In $2016 \mathrm{Mr}$. Kadyrov presented the sample of the meteorite to DMUH.

Physical characteristics: The stone is a pie-shaped oriented meteorite with a flat frontal surface and well-developed regmaglypts on the sides. The color is grayishbrown. Rear side appears faceted. Fusion crust is mostly preserved. Total recovered weight is $35.65 \mathrm{~kg}$.

Petrography: (C. A. Lorenz, Vernad) The slices show a fractured and brecciated interior. In section, the meteorite has a recrystallized chondritic texture corresponding to type 5 and comprises mineral grains, chondrule fragments and uncommon complete chondrules, cm-sized completely recrystallized fragments of type 6 chondrite, and rare carbonaceous chondrite fragments up to $1.5 \mathrm{~mm}$ in size containing fine-grained phillosylicate matrix and different objects. The type- 5 lithology contains shock melt pockets and veinlets. Main phases are olivine, pyroxene, feldspar, Fe,Ni metal, troilite; chromite and phosphate are accessory. The type- 6 clasts are up to $1.5 \mathrm{~cm}$ in size and have sharp borders with the H5 host. Carbonaceous chondrite clasts, up to $1.5 \mathrm{~mm}$ in size, have a fine-grained phyllosilicate-rich matrix enclosing inclusions of serpentine, olivine, pyroxene, sulfides, minor magnetite, and FeNi metal enclosed within the olivine grains. Two olivine chondrules of 25 and $100 \mu \mathrm{m}$ in diameter were found in the carbonaceous clasts.

Geochemistry: Mineral compositions and geochemistry: (N. N.

Kononkova, Vernad.) Olivine $\mathrm{Fa}_{19.1 \pm 0.5}(\mathrm{~N}=18)$; Pyroxene $\mathrm{Fs}_{16.7 \pm 0.3} \mathrm{Wo}_{1.4 \pm 0.2}(\mathrm{~N}=16)$

. Carbonaceous chondrite clasts matrix (defocused beam, $\mathrm{N}=17$ ), wt $\%: \mathrm{Na}_{2} \mathrm{O}$

$0.18 \pm 0.15 ; \mathrm{MgO} 15.5 \pm 2.6 ; \mathrm{Al}_{2} \mathrm{O}_{3} 2.44 \pm 0.18 ; \mathrm{SiO}_{2} 31.2 \pm 2.2 ; \mathrm{K}_{2} \mathrm{O} 0.46 \pm 0.45 ; \mathrm{CaO}$

$0.58 \pm 0.36 ; \mathrm{TiO}_{2} 0.11 \pm 0.3 ; \mathrm{Cr}_{2} \mathrm{O}_{3} 0.39 \pm 0.8 ; \mathrm{MnO} 0.18 \pm 0.11 ; \mathrm{FeO} 29.9 \pm 3.2 ; \mathrm{NiO}$

$2.02 \pm 0.82$.

Classification: H5-6 breccia with CM-chondrite clasts. The meteorite is moderately weathered (W2) and shocked (S3).

Specimens: A sample of $1468 \mathrm{~g}$ and two sections are deposited in Vernad; DMUH holds a sample of $31.7 \mathrm{~kg}$.

\section{Caçapava do Sul $\quad 30^{\circ} 41^{\prime} 34.9^{\prime \prime} \mathrm{S}, 53^{\circ} 39^{\prime} 05.1^{\prime \prime} \mathrm{W}$}

Rio Grande do Sul, Brazil

Find: about 1908

Classification: Iron meteorite (IID)

History: The meteorite was found around 1908 among the remains of an abandoned country seat. The stone drew attention due to its weight and the farmer decided to use it to amuse visitors. Prof. Elver Ubirajara had heard about the mysterious rock since he was a boy. Recently, thinking it to be a meteorite, he spent many years in finding the current owner of the stone, Mr. João A. R. Lopes. When the meteoritic nature was confirmed by the $M N R J / \mathrm{UFRJ}$, it was donated to the local university (UNIPAMPA). 
Physical characteristics: A rough pear-shaped mass of $27 \mathrm{~kg}$, with average dimensions of $27 \times 21 \times 12 \mathrm{~cm}$ and weathered surface.

Petrography: (Zucolotto, M.E., MNRJ, IGEO-UFRJ) The etched sections display a medium Widmanstätten structure, consisting of kamacite lamellae bordered by taenite and plessite fields. The kamacite lamellae are straight, swollen and have an average bandwidth of $0.65 \pm 0.15 \mathrm{~mm}$ and microhardness HV $325 \pm 30$. The kamacite has an indistinct $\varepsilon$-structure. Taenite and plessite cover $30-40 \%$ per area, mainly as comb and net plessite fields and some with martensitic varieties. The broad taenite lamellae have a mean microhardness HV $500 \pm 30$. Schreibersite occurs as small blocks or skeletal crystals, mainly enveloped by swathing kamacite broad lamellae. Troilite is very rare as scattered nodules.

Geochemistry: Bulk composition (INAA - J.T. Wasson, $U C L A$ ) in $\mathrm{wt} \%: \mathrm{Ni}=9.43$, $\mathrm{Co}=0.66$; and in ppm: $\mathrm{Cr}=90, \mathrm{Cu}=307, \mathrm{Ga}=70.8, \mathrm{As}=4.3, \mathrm{~W}=2.8, \mathrm{Ir}=21.6$ and $\mathrm{Au}=0.56$. Presents the highest Ir among IID irons and one of the lowest $\mathrm{Au}$ and $\mathrm{As}$ values. (EPMA - A. Tosi, LABSONDA/IGEO-UFRJ) kamacite $7.32 \pm 0.15 \mathrm{wt} . \%$ $\mathrm{Ni}$ and $0.65 \pm 0.06 \%$ Co content and taenite lamellae $21.5 \pm 0.5 \mathrm{wt} . \% \mathrm{Ni}$ and $0.35 \pm 0.05$ wt. $\%$ Co.

Classification: (J.T. Wasson, UCLA) Iron (IID), medium octahedrite Specimens: 94.8 g $M N R J, I G E O-U F R J$, main mass UNIPAMPA.

\section{Calama $005 \quad 22^{\circ} 23.56^{\prime} \mathrm{S}, 68^{\circ} 38.61^{\prime} \mathrm{W}$ \\ Antofagasta, Chile \\ Find: 25 March 2017 \\ Classification: Carbonaceous chondrite (CO3)}

Physical characteristics: 1 individual sample, $380 \mathrm{~g}$, broken in several pieces, brown-black and covered with brown fusion crust.

Petrography (M. A. Ivanova, Vernad): meteorite consists of fine-grained matrix material, chondrules of Type I and II, and refractory inclusions. Average diameter of chondrules is $0.2 \mathrm{~mm}$. Chondrules consist of olivine, pyroxene and plagioclase mesostasis. The minor phases are spinel, melilite, chromite, sulfides, kamacite, taenite, and tetrataenite.

Geochemistry: Mineral compositions and geochemistry (M. A. Ivanova, Vernad): Olivine (EMPA) is $\mathrm{Fa}_{22.2 \pm 18.4}(\mathrm{~N}=98) \mathrm{mol} \%$, $\mathrm{CaO} 0.24 \pm 0.16 ; \mathrm{MnO} 0.23 \pm 0.13$ (both wt. \%), pyroxene is presented by orthopyroxene $\left(\mathrm{Fs}_{11.6 \pm 10.8} \mathrm{Wo}_{1.8 \pm 1.4}(\mathrm{~N}=20)\right)$, augite $\left(\mathrm{Fs}_{4.5} \mathrm{Wo}_{41.9} \mathrm{En}_{53.5}\right)$, diopside $\left(\mathrm{Fs}_{1.4} \mathrm{Wo}_{50.7}\right)$, and Al-diopside $\left(\mathrm{Fs}_{4.3} \mathrm{Wo}_{51.7}\right)$,

$\mathrm{Al}_{2} \mathrm{O}_{3} 16.3 \mathrm{wt} \%, \mathrm{TiO}_{2} 2.9 \mathrm{wt} \%$. Mesostasis in chondrules is ( $\left.\mathrm{wt} \%\right): \mathrm{SiO}_{2} 50.4$; $\mathrm{TiO}_{2}$ 0.42; $\mathrm{Al}_{2} \mathrm{O}_{3} 22.8 ; \mathrm{Cr}_{2} \mathrm{O}_{3}$ 0.19; $\mathrm{FeO} 3.6 ; \mathrm{MnO} 0.14 ; \mathrm{MgO} 2.80 ; \mathrm{CaO}$ 14.6; $\mathrm{Na}_{2} \mathrm{O} 2.95 ; \mathrm{K}_{2} \mathrm{O} 0.19$.

Classification (M. A. Ivanova, Vernad): Based on the texture, average chondrules size, mineralogy, and mineral chemistry the meteorite is a carbonaceous chondrite of CO3 type. The meteorite shows only minor weathering features.

Specimens: 1 piece $(70 \mathrm{~g})$ and a thin section are deposited in Vernad. The main mass with Mr. Timur Kryachko and Mt. Eugeniy Zakharchuk. 


\section{Calama $008 \quad 22^{\circ} 27^{\prime} 32.7^{\prime \prime} \mathrm{S}, 68^{\circ} 39^{\prime} 12^{\prime \prime} \mathrm{W}$ \\ Antofagasta, Chile \\ Find: 2017 Oct 19 \\ Classification: Ordinary chondrite (L6)}

History: Meteorite (11.23 g) was found 19 October 2017 by the $U r F U$ meteorite expedition-2017 in Chile (Pastukhovich A.Yu., Larionov M.Yu., Kruglikov N.A., Kolunin R.N.) near Chiu-Chiu, Calama region.

Physical characteristics: Total mass is $11.23 \mathrm{~g}$. The meteorite has roughly rounded shape. Exterior of the stone is desert polished. The surface and interior of the meteorite is light to dark brown in color due to Fe-hydroxides. No fusion crust was observed.

Petrography: Classification (V.V. Sharygin, SIGM and UrFU). Petrographic observation of a polished thin section shows very rare chondrules in a coarsemiddle recrystallized matrix. Such chondrules are very poorly delineated and their sizes vary from $500 \mu \mathrm{m}$ to $3 \mathrm{~mm}$. They have porphyritic texture and mainly consist of olivine, low-Ca-pyroxene and plagioclase, and \pm diopside, chromite and blebs of troilite and FeNi-metal. Chromite-plagioclase segregation (300 $\mu \mathrm{m}$, chondrule ?) was also observed in the matrix. Olivine, low-Ca-pyroxene and plagioclase are main minerals in matrix. Plagioclase grains are larger than 50-100 $\mu \mathrm{m}$. This indicates petrologic type 6. Undulatory extinction and irregular fractures in olivine, as well as the absence of opaque shock veins and melt pockets, indicate a shock stage of S2. Majority of grains of FeNi metal (kamacite, taenite, rarely tetrataenite, up to $500 \mu \mathrm{m})$ and troilite $(200-500 \mu \mathrm{m})$ are fresh. Weathering products (goethite and other Fe-Ni-hydroxides) occur as in situ partial alteration of FeNi-metals and troilite and fill microfractures in minerals from matrix and chondrules (weathering grade - W2). Clinopyroxene, chromite, chlorapatite and merrillite $(200-400 \mu \mathrm{m})$ occur locally in the matrix. Single inclusion $(5 \mu \mathrm{m})$ of molybdenite was found in troilite grain.

Geochemistry: EDS-WDS analyses (V. V. Sharygin, SIGM and UrFU). The primary chondrite paragenesis includes olivine $\mathrm{Fa}_{25.59 \pm 0.50}(\mathrm{~N}=27)$, orthopyroxene $\mathrm{Fs}_{21.72 \pm 0.28} \mathrm{Wo}_{1.61 \pm 0.21}(\mathrm{~N}=22)$, plagioclase $\mathrm{Ab}_{83.5} \mathrm{An}_{10.8} \mathrm{Or}_{5.7}(\mathrm{~N}=23)$, Cr-bearing clinopyroxene $\mathrm{En}_{45.3} \mathrm{Fs}_{8.6} \mathrm{Wo}_{46.1}(\mathrm{~N}=6)$, chromite Crt82.3Spl12.4 (N=16), chlorapatite, merrillite and FeNi-metals. Chlorapatite contains $\mathrm{F}$ (up to $0.5 \mathrm{wt} . \%$ ); $\mathrm{Cl}-5.30$ wt.\% (N=3). Merrillite is poor in $\mathrm{FeO}(0.6-0.7$ wt.\%). Composition of metals (in wt.\%): kamacite $(\mathrm{N}=9)-\mathrm{Fe} 93.71 \pm 1.55, \mathrm{Ni}-5.39 \pm 1.01, \mathrm{Co}-$ $0.97 \pm 0.17$; taenite $(\mathrm{N}=17)-\mathrm{Fe} 68.28 \pm 4.53$, Ni 31.24 \pm 4.67 , Co $0.50 \pm 0.09$; tetrataenite $(\mathrm{N}=3)-\mathrm{Fe} 49.22$, Ni 50.29, Co 0.33 . Fe-Ni-hydroxides sometimes contain 22.6-38.3 wt.\% NiO.

Classification: Ordinary chondrite. L6, S2, W2. In chemical composition of olivine and low-Ca pyroxene this meteorite seems to be similar to Calama 006.

Specimens: $8.4 \mathrm{~g}$ cut-off $-U r F U ; 1.25 \mathrm{~g}$ cut-off and thin section - SIGM. 
Calama $009 \quad 22^{\circ} 24.200^{\prime} \mathrm{S}, 68^{\circ} 37.632^{\prime} \mathrm{W}$

Antofagasta, Chile

Find: 2017 Oct 13

Classification: Ordinary chondrite (L6)

History: The $13.5 \mathrm{~kg}$ meteorite was found 13 October 2017 by the $U r F U$ meteorite expedition-2017 in Chile (Pastukhovich A.Yu., Larionov M.Yu., Kruglikov N.A., Kolunin R.N.) near Chiu-Chiu, Calama region.

Physical characteristics: The meteorite has angular shape. Exterior of the stone is desert polished. The interior of the meteorite is light gray in color with red-brown spots due to Fe-hydroxides around metal and troilite. Fusion crust is present. Petrography: Classification (V. V. Sharygin, SIGM and UrFU). Petrographic observation of a polished thin section shows very rare chondrules in a coarse recrystallized matrix. Chondrules are very poorly delineated with apparent size to 1 $\mathrm{mm}$. The porphyritic and olivine- or orthopyroxene-barred chondrules are common and mainly consist of olivine, low-Ca-pyroxene and plagioclase, diopside, and \pm chromite and blebs of troilite and FeNi-metal. Olivine, low-Ca-pyroxene and plagioclase are main minerals in matrix. Plagioclase grains are larger than 50-100 $\mu \mathrm{m}$. This indicates a petrological type of 6 for the meteorite. Undulatory extinction, irregular and planar fractures in olivine, as well as the presence of opaque melt pockets and twinning in low-Ca-pyroxene, troilite and ilmenite, indicate a shock stage of S3/4. Majority of grains of FeNi metal (kamacite, taenite, tetrataenite, up to $1.5 \mathrm{~mm}$ ) and troilite $(100-500 \mu \mathrm{m})$ are fresh. Weathering products (goethite and other Fe-Ni-hydroxides) occur as in situ partial alteration of FeNi-metals and troilite and fill microfractures in minerals from matrix and chondrules (weathering grade $\mathrm{W} 1 / 2$ ). Grains of copper (up to $50 \mu \mathrm{m}$ ) are occasionally found in kamacitetaenite-tetrataenite aggregate. Pentlandite $( \pm \mathrm{Ni}$-rich pyrrhotite, $4.4-6.1 \mathrm{wt} \% \mathrm{Ni}$, up to $10 \mu \mathrm{m}$ ) is rarely observed in troilite. Ni-free pyrrhotite sometimes fills fissures in troilite and seems to be an intermediate phase of troilite alteration to goethite and other Fe-hydroxides. Clinopyroxene, chromite, ilmenite, chlorapatite and merrillite (100-300 $\mu \mathrm{m}$ ) occur locally in the matrix. Opaque melt pockets (up to $100 \mu \mathrm{m}$ ) are rarely located near large FeNi-metal grains and contain abundant metal blebs in fine-grained olivine-pyroxene aggregate.

Geochemistry: EDS-WDS analyses (V. V. Sharygin, SIGM and UrFU). The primary chondrite paragenesis includes olivine $\mathrm{Fa}_{25.68 \pm 0.43}(\mathrm{~N}=40)$, orthopyroxene $\mathrm{Fs}_{21.46 \pm 0.32} \mathrm{Wo}_{1.43 \pm 0.31}(\mathrm{~N}=32)$, plagioclase $\mathrm{Ab}_{83.9} \mathrm{An}_{10.5} \mathrm{Or}_{5.6}(\mathrm{~N}=25)$, Cr-bearing clinopyroxene $\mathrm{En}_{45.8} \mathrm{Fs}_{8.6} \mathrm{Wo}_{45.8}(\mathrm{~N}=2)$, chromite $\mathrm{Crt}_{81.2} \mathrm{Spl}_{13.3}(\mathrm{~N}=11)$, ilmenite $\mathrm{Ilm}_{84.9} \mathrm{Gkl}_{12.9}(\mathrm{~N}=16)$, chlorapatite, merrillite, FeNi-metals and copper. Chlorapatite contains $\mathrm{F}$ (up to 0.9 wt.\%); $\mathrm{Cl}-5.33$ wt.\% $(\mathrm{N}=11)$. Merrillite is poor in $\mathrm{FeO}(0.5$ 0.7 wt.\%, N=11). Composition of metals (in wt.\%): kamacite $(\mathrm{N}=22)-\mathrm{Fe}$ $94.38 \pm 1.59, \mathrm{Ni}-4.77 \pm 1.52, \mathrm{Co}-0.84 \pm 0.24$; taenite $(\mathrm{N}=40)-\mathrm{Fe} 66.78 \pm 3.53, \mathrm{Ni}$ $32.75 \pm 3.49$, Co $0.42 \pm 0.08$; tetrataenite $(\mathrm{N}=16)-\mathrm{Fe} 44.65 \pm 1.24$, Ni $55.07 \pm 1.26$, Co $0.22 \pm 0.05$; copper $(\mathrm{N}=10)-\mathrm{Cu} 93.13 \pm 0.69, \mathrm{Fe} 4.46 \pm 0.66$, Ni $2.37 \pm 0.18$.

Classification: Ordinary chondrite. L6, S3/4, W1/2.

Specimens: $12.512 \mathrm{~kg}$ at $U r F U ; 960 \mathrm{~g}$ at The Catholic University of the North, Antofagasta, Chile; $19 \mathrm{~g}$ and thin section - SIGM. 


\section{Calama $010 \quad 22^{\circ} 24.643^{\prime} \mathrm{S}, 68^{\circ} 34.622^{\prime} \mathrm{W}$ \\ Antofagasta, Chile \\ Find: 2017 Oct 15 \\ Classification: Ordinary chondrite (H6)}

History: The meteorite was found 15 October 2017 by the $U r F U$ meteorite expedition-2017 in Chile (Pastukhovich A.Yu., Larionov M.Yu., Kruglikov N.A., Kolunin R.N.) near Chiu-Chiu, Calama region.

Physical characteristics: The meteorite has roughly rounded shape. Exterior of the stone is desert polished. The surface and interior of the meteorite is light to dark brown in color due to Fe-hydroxides. No fusion crust was observed.

Petrography: Classification (V. V. Sharygin, SIGM and UrFU). Petrographic observation of a polished thin section shows very rare chondrules in a coarse recrystallized matrix. Chondrules are very poorly delineated with apparent size to 2 $\mathrm{mm}$. The POP chondrules are common and mainly consist of olivine, low-Capyroxene and plagioclase, diopside, and \pm chromite and blebs of troilite and FeNimetal. Olivine, low-Ca-pyroxene and plagioclase are main minerals in matrix. Plagioclase grains are larger than 50-100 $\mu \mathrm{m}$. This indicates a petrological type of 6 for the meteorite. Undulatory extinction and irregular fractures in olivine, as well as the presence of opaque shock veins, indicate a shock stage of S3. Majority of grains of FeNi-metal $(100-800 \mu \mathrm{m})$ are mainly represented by kamacite; taenite and tetrataenite are rare. Sizes of troilite grains are 50-300 $\mu \mathrm{m}$. Weathering products (goethite, Fe-Ni-Cl-hydroxide and other Fe-Ni-hydroxides, gypsum) occur as in situ partial alteration of FeNi-metals and troilite and fill microfractures in minerals from matrix and chondrules (weathering grade -W2). Nickel (1-3 $\mu \mathrm{m}$ ) was found in abundant Fe-Ni-Cl-hydroxide-goethite aggregate around tetrataenite. Pentlandite (up to $10 \mu \mathrm{m}$ ) is rarely observed in troilite. Clinopyroxene, chromite, chlorapatite and merrillite (50-300 $\mu \mathrm{m})$ occur locally in the matrix. Opaque shock veins are locally abundant and contain troilite ( $\pm \mathrm{Fe}-\mathrm{Ni}$-metal) blebs in fine-grained olivineorthopyroxene \pm clinopyroxene aggregate.

Geochemistry: EDS-WDS analyses (V. V. Sharygin, SIGM and UrFU). The primary chondrite paragenesis includes olivine $\mathrm{Fa}_{19.33 \pm 0.35}(\mathrm{~N}=29)$, orthopyroxene $\mathrm{Fs}_{16.96 \pm 0.28} \mathrm{Wo}_{1.46 \pm 0.20}(\mathrm{~N}=25)$, plagioclase $\mathrm{Ab}_{83.9} \mathrm{An}_{10.5} \mathrm{Or}_{5.6}(\mathrm{~N}=25), \mathrm{Cr}$-bearing clinopyroxene $\mathrm{En}_{45.9} \mathrm{Fs}_{7.6} \mathrm{Wo}_{46.5}(\mathrm{~N}=3)$, chromite $\mathrm{Crt}_{80.4} \mathrm{Spl}_{14.9}(\mathrm{~N}=15)$, chlorapatite, merrillite, and FeNi-metals. Chlorapatite contains F (up to $1.1 \mathrm{wt} . \%$ ); $\mathrm{Cl}-4.87$ wt.\% $(\mathrm{N}=11)$. Merrillite is poor in FeO (0.5-0.9 wt.\%, $\mathrm{N}=12)$. Composition of metals (in wt.\%): kamacite $(\mathrm{N}=23)$ - Fe 92.85 $\pm 0.41, \mathrm{Ni}-6.60 \pm 0.42, \mathrm{Co}-$ $0.84 \pm 0.09$; taenite $(\mathrm{N}=43)-\mathrm{Fe} 60.31$, Ni 39.38, Co 0.28; tetrataenite $(\mathrm{N}=1)-\mathrm{Fe}$ 50.55, Ni 49.17, Co 0.14. Fe-Ni-Cl-hydroxide (N=5, in wt.\%): FeO - 59.37, NiO 11.91, $\mathrm{CoO}-0.75, \mathrm{Cl}-16.83$.

Classification: Ordinary chondrite. H6, S3, W2.

Specimens: $1189.29 \mathrm{~g}$ (18 pieces) at $U r F U ; 53.8 \mathrm{~g}$ sample and thin section at $S I G M$. 


\section{Calama $014 \quad 22^{\circ} 26.27^{\prime} \mathrm{S}, 68^{\circ} 51.27^{\prime} \mathrm{W}$ \\ Antofagasta, Chile \\ Find: 11 Feb 2018 \\ Classification: HED achondrite (Eucrite, monomict)}

Petrography: Monomict breccia composed of mostly rounded basaltic eucrite clasts in a sparse matrix of related crystalline debris. Minerals are exsolved pigeonite, calcic plagioclase, silica polymorph, ilmenite, chromite and troilite. Geochemistry: Host orthopyroxene $\left(\mathrm{Fs}_{58.6-60.0} \mathrm{Wo}_{2.8-4.1}, \mathrm{FeO} / \mathrm{MnO}=31 \pm 0.6\right.$, $\mathrm{N}=24)$. Clinopyroxene exsolution lamellae $\left(\mathrm{Fs}_{29.5-30.6} \mathrm{Wo}_{40.1-40.4} ; \mathrm{FeO} / \mathrm{MnO}=\right.$ $32 \pm 0.8, \mathrm{~N}=16)$. Plagioclase $\left(\mathrm{An}_{88.2-89.0} \mathrm{Or}_{0.9-1.0}, \mathrm{~N}=10\right)$

\section{Calama $022 \quad 22^{\circ} 18.192^{\prime} \mathrm{S}, 68^{\circ} 33.0557^{\prime} \mathrm{W}$}

Antofagasta, Chile

Find: 2017 Oct 15

Classification: Ordinary chondrite (L6)

History: The meteorite (1 piece $12 \mathrm{~kg}$ and many smaller fragments) was found October 15, 2017 by Timur Kryachko.

Physical characteristics: Total mass is about $32 \mathrm{~kg}$. Many similar meteorite framents were collected in area about 40 square meters. Largest stone (about $12 \mathrm{~kg}$ ) $\sim 12 \%$ covered by fusion crust. Fragments all have different shapes plus very rare fragments of fusion crust. The interior of the meteorite is light brown, whereas the surface is dark brown. Some cracks (up to $0.3 \mathrm{~mm}$ in thickness) are filled by alunite.

Petrography: (Pavel Yu. Plechov, FMMR). The main part of the meteorite contains many chondrules without clear borders. Chondrule sizes vary from 400 to $800 \mu \mathrm{m}$. Some chondrules (about 5\%) have clear barred olivine textures, with plagioclase between the bars. Most of the chondrules consist of $\mathrm{Ol}+\mathrm{Opx}$ with $\mathrm{Cpx}+\mathrm{Pl}$ interstitial. Matrix consists of olivine and low-Ca pyroxene grains (70-200 $\mu \mathrm{m})$ with aggregates of Ca-pyroxene and plagioclase. Plagioclase grains $\left(\mathrm{An}_{10.4-}\right.$ 10.8) are about 40-70 $\mu \mathrm{m}$. These features indicate a petrologic type of 6 for the meteorite. Rare Fe-Ni metal blebs (up to $300 \mu \mathrm{m}$ ) and very rare troilite (up to 30 $\mu \mathrm{m}$ ) occur in matrix. Metal consists of kamacite and taenite (with 35-53 wt.\% of $\mathrm{Ni}$ ). Olivine has no undulatory extinction; this plus the absence of opaque shock veins and melt pockets indicates a shock stage of S1. Weathering products (goethite, "hydrogoethite") occur as veins and in situ alteration of FeNi-metals. Troilite, olivine, pyroxene, chromite, metal are fresh and not weathered (weathering grade W1).

Geochemistry: Mineral composition and Geochemistry: EDS-WDS analyses (Pavel Yu. Plechov, FMMR). The primary chondrite paragenesis includes olivine $\mathrm{Fa}_{25.35 \pm 0.56}(\mathrm{~N}=25)$, orthopyroxene $\mathrm{Fs}_{20.7 \pm 0.7} \mathrm{Wo}_{1.47 \pm 0.29}(\mathrm{~N}=21)$, plagioclase $\mathrm{Ab}_{82.0} \mathrm{An}_{10.6} \mathrm{Or}_{6.7}(\mathrm{~N}=4), \mathrm{Cr}$-bearing clinopyroxene $\mathrm{En}_{46.6} \mathrm{Fs}_{9.1} \mathrm{Wo}_{44.1}(\mathrm{~N}=2)$, chromite $\mathrm{Crt}_{75.6} \mathrm{Spl}_{9.1}(\mathrm{~N}=2)$ and $\mathrm{Cl}$-apatite. No merrillite occurred in the meteorite. 
Composition of kamacite (in wt.\%, N=1): Fe 93.78, Ni 5.25, Co 0.75. Taenite $(\mathrm{N}=4)$ contains 33.85 and $0.46 \mathrm{wt} . \%$ of $\mathrm{Ni}$ and $\mathrm{Co}$, respectively. Troilite is close to ideal FeS.

Classification: (Pavel Yu. Plechov, FMMR). Ordinary chondrite. L6, S1, W1. Specimens: Timur Kryachko, Ilya Kryachko, Mikhail Nepomiluev holds main mass about $32 \mathrm{~kg}$. $60 \mathrm{~g}$ (7 small fragments) and thin section, FMMR.

\section{Calama $023 \quad 22^{\circ} 28.050^{\prime} \mathrm{S}, 68^{\circ} 38.786^{\prime} \mathrm{W}$}

Antofagasta, Chile

Find: 19 Oct 2017

Classification: Ordinary chondrite (H6)

History: Meteorite $(284.04 \mathrm{~g})$ was found 19 October 2017 by the $U r F U$ meteorite expedition-2017 in Chile (Pastukhovich A.Yu., Larionov M.Yu., Kruglikov N.A., Kolunin R.N.) near Chiu-Chiu, Calama region.

Physical characteristics: Total mass is $284.04 \mathrm{~g}$. The meteorite has roughly rounded shape. Exterior of the stone is desert polished. The surface and interior of the meteorite is light to dark brown in color due to Fe-hydroxides. No fusion crust was observed.

Petrography: Classification (Victor V. Sharygin, SIGM and UrFU). Very rare chondrules in a coarse recrystallized matrix. Chondrules are poorly delineated and their sizes vary from $200 \mu \mathrm{m}$ to $2 \mathrm{~mm}$. The largest chondrules $(1-2 \mathrm{~mm})$ are visible macroscopically and represented by PO, POP and RP textural types. They mainly consist of olivine, low-Ca-pyroxene and plagioclase, diopside, and \pm chromite and blebs of troilite and FeNi-metal. Olivine, low-Ca-pyroxene and plagioclase are main minerals in matrix. Clinopyroxene, chromite, ilmenite and merrillite (50-300 $\mu \mathrm{m})$ occur locally in the matrix, chlorapatite was not observed. Large olivine grains from matrix and POP chondrules may contains partially crystallized (silicate-melt?) inclusions (low-Ca-pyroxene + diopside + plagioclase/glass + troilite). Plagioclase grains in the matrix are larger than $50 \mu \mathrm{m}$, indicating a petrologic type of 6 for the meteorite. The absence of opaque shock veins and weak undulatory extinction and irregular fractures in olivine indicate a shock stage of S2. Majority of grains of FeNi metal (kamacite, taenite, tetrataenite, up to $1.5 \mathrm{~mm}$ ) and troilite $(100-500 \mu \mathrm{m})$ are fresh. Pentlandite ( \pm Ni-rich pyrrhotite, up to $50 \mu \mathrm{m}$ ) is rare in troilite on the contact with kamacite. In addition, alabandite + Ni-rich pyrrhotite (?) association $(3 \times 10 \mu \mathrm{m})$ was found in a kamacite-hosted troilite bleb $(70 \mu \mathrm{m})$. Grains of copper (up to $5 \mu \mathrm{m}$ ) were observed in a kamacite-taenite-tetrataenite-troilite aggregate. Weathering products (goethite, Fe-Ni-Cl-hydroxide and other Fe-Ni-hydroxides, Fe-S-hydroxide) occur as in situ partial alteration of FeNi-metals and troilite and fill microfractures in minerals from matrix and chondrules (weathering grade W2). The association of trevorite + akaganeite is common as an alteration product of Ni-rich metal grains (taenite or kamacite + taenite + tetrataenite). Rare Fe-Nihydroxides veins are crosscut the meteorite sample.

Geochemistry: EDS-WDS analyses (Victor V. Sharygin, SIGM and UrFU). The primary chondrite paragenesis includes olivine $\mathrm{Fa}_{19.44 \pm 0.18}(\mathrm{~N}=36)$, orthopyroxene 
$\mathrm{Fs}_{17.28 \pm 0.35} \mathrm{Wo}_{1.45 \pm 0.14}(\mathrm{~N}=36)$, plagioclase $\mathrm{Ab}_{82.5} \mathrm{An}_{11.8} \mathrm{Or}_{5.7}(\mathrm{n}=10)$, Cr-bearing clinopyroxene $\mathrm{En}_{47.4} \mathrm{Fs}_{7.0} \mathrm{Wo}_{45.6}\left(\mathrm{~N}=8, \mathrm{Cr}_{2} \mathrm{O}_{3}-0.83\right.$ wt.\%), chromite $\mathrm{Crt}_{81.5} \mathrm{Spl}_{13.9}(\mathrm{~N}=16)$, ilmenite $\mathrm{Ilm}_{79.4} \mathrm{Gk}_{10.9}(\mathrm{~N}=10)$, merrillite, and FeNi-metals. Merrillite is poor in $\mathrm{FeO}(0.4-0.8$ wt. $\%, \mathrm{~N}=14)$. Composition of metals and sulfides (in wt.\%): kamacite $(\mathrm{N}=27)$ - Fe $92.83 \pm 0.31, \mathrm{Ni}-6.73 \pm 0.13, \mathrm{Co}-0.44 \pm 0.05$; taenite $(\mathrm{N}=12)-\mathrm{Fe} 75.44$, Ni 24.26, Co 0.28; tetrataenite $(\mathrm{N}=14)-\mathrm{Fe} 47.24, \mathrm{Ni}$ 52.59, Co 0.14; pentlandite $(\mathrm{N}=5)$ - Fe 45.99, Ni 20.67, Co 0.27, S 33.05; alabandite $(\mathrm{N}=2)$ - Mn 53.45; Fe 9.12, Ni 1.09, Co 0.14, S - 36.28.

Classification: Ordinary chondrite. H6, S2, W2.

Specimens: $249.36 \mathrm{~g}$ sample - UrFU; $27.25 \mathrm{~g}$ sample and thin section - SIGM.

\section{Calama $024 \quad 22^{\circ} 27.549^{\prime} \mathrm{S}, 68^{\circ} 39.227^{\prime} \mathrm{W}$}

Antofagasta, Chile

Find: 19 Oct 2017

Classification: Ordinary chondrite (H6)

History: Meteorite $(252.95 \mathrm{~g})$ was found 19 October 2017 by the $U r F U$ meteorite expedition-2017 in Chile (Pastukhovich A.Yu., Larionov M.Yu., Kruglikov N.A., Kolunin R.N.) near Chiu-Chiu, Calama region.

Physical characteristics: Total mass is $252.95 \mathrm{~g}$. The meteorite has roughly rounded shape. Exterior of the stone is desert polished. The surface and interior of the meteorite is light to dark brown in color due to Fe-hydroxides. Fusion crust is locally preserved (up to $1 \mathrm{~mm}$ ).

Petrography: Classification (Victor V. Sharygin, SIGM and UrFU). Petrographic observation of a polished thin section shows chondrules in a coarse recrystallized matrix. Chondrules are poorly delineated and their sizes vary from $200 \mu \mathrm{m}$ to 2 $\mathrm{mm}$. The largest chondrules (1-2 $\mathrm{mm})$ are visible macroscopically and represented by PO, POP and RP textural types. The PO and POP chondrules mainly consist of olivine, low-Ca-pyroxene and plagioclase, diopside, and \pm chromite and metalsulfide blebs. Some RP chondrules may contains $\mathrm{SiO}_{2}$ polymorph $(10 \times 100 \mu \mathrm{m})$ In addition to chondrules, a "refractory forsterite-rich object" ( size - $300 \mu \mathrm{m}, \mathrm{RFRO}$ ) was found. It consists of high-Mg forsterite and low-Ca pyroxene phenocrysts, and interstitial augitic clinopyroxene and An-rich plagioclase. Olivine, low-Capyroxene and plagioclase are main minerals in the chondrite matrix.

Clinopyroxene, chromite, chlorapatite and merrillite $(50-300 \mu \mathrm{m})$ occur locally in the matrix. Plagioclase grains in the matrix are larger than $50 \mu \mathrm{m}$, indicating a petrologic type of 6 for the meteorite. The absence of opaque shock veins and weak undulatory extinction and irregular fractures in olivine indicate a shock stage of S2. Majority of grains of FeNi metal (kamacite, taenite, tetrataenite, up to $1 \mathrm{~mm}$ ) and troilite $(100-500 \mu \mathrm{m})$ are fresh. Pyrrhotite $(\mathrm{Ni}-0.4 \mathrm{wt} . \%$, up to $10 \mu \mathrm{m})$ occurs in outer parts of troilite. Grains of copper (up to $10 \mu \mathrm{m}$ ) were observed in kamacitetaenite-tetrataenite-troilite aggregates. Weathering products (goethite, Fe-Ni-Clhydroxides, Fe-chloride-hydrate and gypsum) occur as in situ partial alteration of FeNi-metals and troilite and fill microfractures in minerals from matrix and chondrules (weathering grade - W2). Rare Fe-Ni-hydroxides veins are crosscut the 
meteorite sample. Fusion crust consists of dendritic/skeletal magnetite and olivine and Fe-rich glass. Plagioclase near fusion crust is transformed into maskelynite. Ferich glass contains rare metal-sulfide blebs $(5-10 \mu \mathrm{m})$.

Geochemistry: EDS-WDS analyses (Victor V. Sharygin, SIGM and $U r F U$ ). The primary chondrite paragenesis includes olivine $\mathrm{Fa}_{19.73 \pm 0.36}(\mathrm{~N}=48)$, low-Ca pyroxene $\mathrm{Fs}_{17.24 \pm 0.26} \mathrm{Wo}_{1.22 \pm 0.25}(\mathrm{~N}=26)$, plagioclase $\mathrm{Ab}_{82.8} \mathrm{An}_{11.7} \mathrm{Or}_{5.5}(\mathrm{n}=17), \mathrm{Cr}$-bearing clinopyroxene $\mathrm{En}_{47.3} \mathrm{Fs}_{7.2} \mathrm{Wo}_{45.5}\left(\mathrm{~N}=8, \mathrm{Cr}_{2} \mathrm{O}_{3}-0.87\right.$ wt.\%), chromite $\mathrm{Crt}_{81.7} \mathrm{Spl}_{13.6}(\mathrm{~N}=12)$, merrillite, chlorapatite and FeNi-metals. Chemical variations in RFRO: olivine $\mathrm{Fa}_{4.9-20.3}(\mathrm{~N}=10)$, low-Ca pyroxene $\mathrm{Fs}_{3.1-3.6} \mathrm{Wo}_{0.3-0.4}(\mathrm{~N}=5)$, clinopyroxene $\mathrm{En}_{59.6-62.5} \mathrm{Fs}_{3.2-5.9} \mathrm{Wo}_{30.9-35.1}\left(\mathrm{Cr}_{2} \mathrm{O}_{3}\right.$ - 1.8-2.5 wt.\%, $\mathrm{Al}_{2} \mathrm{O}_{3}-3.3-5.0$ wt.\%, $\mathrm{N}=8)$, plagioclase $\mathrm{An}_{47.3} \mathrm{Ab}_{51.9} \mathrm{Or}_{0.8}(\mathrm{~N}=1)$. Merrillite in matrix is poor in $\mathrm{FeO}$ (0.6-0.9 wt.\%, $\mathrm{N}=11$ ). Chlorapatite contains $\mathrm{F}$ (up to 0.5 wt.\%); $\mathrm{Cl}-5.32$ wt.\%, $\mathrm{Na}_{2} \mathrm{O}-0.5$ wt. $\%(\mathrm{~N}=8)$. Composition of metals (in wt. $\left.\%\right)$ : kamacite $(\mathrm{N}=30)-\mathrm{Fe}$ 93.46 \pm 1.78 , Ni 6.10 \pm 1.78 , Co $0.46 \pm 0.06$; taenite $(\mathrm{N}=9)-$ Fe 67.25, Ni 32.55, Co 0.26; tetrataenite $(\mathrm{N}=6)-\mathrm{Fe} 49.21$, Ni 51.60, Co 0.15; copper $(\mathrm{N}=4)-\mathrm{Cu} 92.66$, Fe 5.05, Ni 2.33. Fe-chloride-hydrate (in wt.\%): FeO 62.6, NiO 2.8, $\mathrm{CoO} 0.5, \mathrm{SO} 3$ $0.5, \mathrm{Cl} 30.1$.

Classification: Ordinary chondrite. H6, S2, W2.

Specimens: $213.8 \mathrm{~g}$ sample - UrFU; $32.51 \mathrm{~g}$ sample and thin section - SIGM

\section{Caleta el Cobre $022 \quad \sim 24^{\circ} 20^{\prime} \mathrm{S}, \sim 70^{\circ} 15^{\prime} \mathrm{W}$ \\ Antofagasta, Chile \\ Find: 2016 Nov \\ Classification: Martian meteorite (Nakhlite)}

History: A single stone was found casually by a tourist family next to their night camp in the Atacama desert. The stone was presented to Rodrigo Martinez from $M M C$, and identified as a meteorite. A sample was then sent by the finder to $C E R E G E$ for classification.

Physical characteristics: A single stone entirely covered by shiny fusion crust. Cut surface reveals a dark greenish coarse-grained interior with a few mm-sized orangish crystals. Magnetic susceptibility measured on several pieces is very homogeneous with $\log \chi\left(\times 10^{-9} \mathrm{~m}^{3} / \mathrm{kg}\right)=3.93 \pm 0.01(\mathrm{~N}=5$, including measurement of the main mass remaining after cutting)

Petrography: Cumulate texture with euhedral elongated augite crystals (typical size $1 \mathrm{~mm}$ by $300 \mu \mathrm{m}$ ) with interstitial plagioclase (typical size $250 \mu \mathrm{m}$, birefringent) and rare fine grained mesostasis. Olivine crystals (up to $2.5 \mathrm{~mm}$ ) are crosscut and surrounded by aqueous alteration products ("iddingsite") and contain melt inclusions (to $100 \mu \mathrm{m}$ ). Thin sulfide veins (pyrrhotite) penetrate the olivine crystals. Other notable minerals: finely exsolved Fe-Ti oxides to $1 \mathrm{~mm}$ in size, with $\mathrm{TiO}_{2} 16.8 \mathrm{wt} \%, \mathrm{Cr}_{2} \mathrm{O}_{3} 2.23 \mathrm{wt} \%, \mathrm{Al}_{2} \mathrm{O}_{3} 1.56 \mathrm{wt} \%, \mathrm{MgO} 0.50 \mathrm{wt} \%, \mathrm{MnO} 0.42 \mathrm{wt} \%$ (mean of 4 analyses with electron beam defocused to $10 \mu \mathrm{m}$ ). Modal abundances (from image analyses of a $2 \mathrm{~cm}^{2}$ polished section): pyroxene $69.0 \mathrm{vol} \%$, plagioclase and mesostatis $27.1 \mathrm{vol} \%$, olivine $2.2 \mathrm{vol} \%$, opaque minerals $1.8 \mathrm{vol} \%$. Magnetic measurements (hysteresis properties of a $360 \mathrm{mg}$ piece) indicate a 
titanomagnetite content of at least $4.3 \mathrm{wt} \%$. None of the silicates show petrological sign of shock.

Geochemistry: Augite $\mathrm{Fs}_{28.4 \pm 2.9} \mathrm{Wo}_{40.0 \pm 0.6}(\mathrm{~N}=20)$, with Fe-rich 10 umthick rims (cores down to $\mathrm{Fs}_{24.5}$, rims up to $\mathrm{Fs}_{33.3}$ ), $\mathrm{FeO} / \mathrm{MnO}=37.4 \pm 3.6$. Plagioclase

$\mathrm{An}_{30.4 \pm 1.0} \mathrm{Ab}_{66.9 \pm 0.8} \mathrm{Or}_{2.8 \pm 0.4}(\mathrm{~N}=7)$. Olivine $\mathrm{Fa}_{70.2 \pm 3.4}\left(\right.$ range $\left.\mathrm{Fa}_{67.2-76.4}\right)$,

$\mathrm{FeO} / \mathrm{MnO}=47.6 \pm 1.6(\mathrm{~N}=7)$. Olivine alteration products (iddingsite) $\mathrm{SiO}_{2} 53.2 \mathrm{wt} \%$, $\mathrm{FeO} 26.1 \mathrm{wt} \%, \mathrm{MgO} 2.5 \mathrm{wt} \%, \mathrm{Al}_{2} \mathrm{O}_{3} 0.56 \mathrm{wt} \%, \mathrm{MnO} 0.32 \mathrm{wt} \%, \mathrm{CaO} 0.21 \mathrm{wt} \%$, $\mathrm{K}_{2} \mathrm{O} 0.18 \mathrm{wt} \%, \mathrm{Na}_{2} \mathrm{O} 0.16 \mathrm{wt} \%$, total $83.3 \mathrm{wt} \%$ (average of 5 analyses with electron beam defocused at $10 \mu \mathrm{m}$ ). The bulk chondrite-normalized REE pattern (V. Debaille, ULB) is similar to that of nakhlites.

Classification: Martian (nakhlite). Minimal weathering. This nakhlite is unusually rich in plagioclase and in titanomagnetite.

Specimens: $21 \mathrm{~g}, 3$ polished sections, 1 thin polished section at CEREGE. Main mass with anonymous finder

\section{Caleta el Cobre $024 \quad \sim 24^{\circ} 20^{\prime} \mathrm{S}, \sim 70^{\circ} 15^{\prime} \mathrm{W}$ \\ Antofagasta, Chile \\ Find: 2018 Apr \\ Classification: Enstatite chondrite (EL6)}

History: The stone was found lying at the surface in the Atacama desert in April 2018.

Physical characteristics: A single dark brown stone. Cut face reveals a dark interior with abundant metal.

Petrography: (J. Gattacceca, Bertrand Devouard, CEREGE) Highly recrystallized chondritic texture. One relict chondrule observed in the $2 \mathrm{~cm}^{2}$ studied section. Main minerals are enstatite, plagioclase (typical size $100 \mu \mathrm{m}$, to $200 \mu \mathrm{m}$ ), kamacite (typical size $100 \mu \mathrm{m}$ ), sulfides (typical size $100 \mu \mathrm{m}$, also present as abundant 5-10 $\mu \mathrm{m}$ mixed sulfides/kamacite grains in the silicates). No olivine. Sulfides include alabandite and troilite.

Geochemistry: Enstatite $\mathrm{Fs}_{0.4 \pm 0.1} \mathrm{Wo}_{1.4 \pm 0.0}(\mathrm{~N}=3)$. Plagioclase $\mathrm{An}_{14.4 \pm 0.5} \mathrm{Ab}_{81.2 \pm 0.5} \mathrm{Or}_{3.8 \pm 0.0}(\mathrm{~N}=2)$. Si in kamacite $1.5 \pm 0.1 \mathrm{wt} \%(\mathrm{~N}=5)$. Magnetic susceptibility $\log \chi\left(\times 10^{-9} \mathrm{~m}^{3} / \mathrm{kg}\right)=5.36$.

Classification: Enstatite chondrite (EL6). EL classification from the Si content of kamacite and the presence of alabandite. Minimal weathering. This meteorite was found $2 \mathrm{~km}$ away from the Caleta el Cobre $025 \mathrm{EH} 3$ meteorite.

Specimens: Type specimen $32 \mathrm{~g}$ at CEREGE. Main mass with ARTMET.

\section{Caleta el Cobre $025 \sim 24^{\circ} 20^{\prime} \mathrm{S}, \sim 70^{\circ} 15^{\prime} \mathrm{W}$}

Antofagasta, Chile

Find: 2018 Apr

Classification: Enstatite chondrite (EH3)

History: The stone was found lying at the surface in the Atacama desert in April 2018. 
Physical characteristics: A single dark-brown stone. Cut face reveals a dark interior with abundant metal and small chondrules.

Petrography: (J. Gattacceca, Bertrand Devouard, CEREGE) Chondrite with welldefined chondrule (average apparent diameter $270 \pm 150 \mu \mathrm{m}, \mathrm{N}=33$ ) with clear mesostasis. Main mineral is enstatite. Abundant kamacite and sulfide in the form of rounded composite grains with typical size $100 \mu \mathrm{m}$. Sulfides display a wide compositional range and include troilite, alabandite, and niningerite.

Geochemistry: Enstatite $\mathrm{Fs}_{2.7 \pm 2.1} \mathrm{Wo}_{0.8 \pm 0.9}$, Fs $\mathrm{PMD}=58 \%(\mathrm{~N}=7)$. Si in kamacite $2.8 \pm 0.2 \mathrm{wt} \%(\mathrm{~N}=5)$. Magnetic susceptibility $\log \chi\left(\times 10^{-9} \mathrm{~m}^{3} / \mathrm{kg}\right)=5.24$.

Classification: Enstatite chondrite (EH3). EH classification from the Si content of kamacite. Minimal weathering. This meteorite was found $2 \mathrm{~km}$ away from the Caleta el Cobre 024 EL6 meteorite.

Specimens: Type specimen $67 \mathrm{~g}$ at CEREGE. Main mass with ARTMET.

\section{Catalina $257 \sim 25^{\circ} 14^{\prime} \mathrm{S}, \sim 69^{\circ} 43^{\prime} \mathrm{W}$}

Antofagasta, Chile

Find: 2010 Apr 14

Classification: Ordinary chondrite (H3-6)

Petrography: (J. Gattacceca, CEREGE) Chondrite with well-defined, packed chondrules. One $3 \mathrm{~mm}$ type 6 clast.

Geochemistry: Type 3 host lithology: olivine $\mathrm{Fa}_{18.2 \pm 1.9}, \mathrm{PMD}=9 \%$, $(\mathrm{N}=6)$, low-Ca pyroxene $\mathrm{Fs}_{16.9 \pm 0.5}(\mathrm{~N}=3)$. Type 6 clast: olivine $\mathrm{Fa}_{19.7}(\mathrm{~N}=1)$, orthopyroxene $\mathrm{Fs}_{17.7} \mathrm{Wo}_{1.4}$.

\section{Catalina $278 \quad 24^{\circ} 55^{\prime} 54.2^{\prime \prime} \mathrm{S}, 69^{\circ} 44^{\prime} 15.9^{\prime \prime} \mathrm{W}$}

Antofagasta, Chile

Find: 2017 Nov 08

Classification: Ordinary chondrite (LL3.4)

Petrography: (J. Gattacceca, CEREGE) Tightly packed chondrules. Clasts with cluster chondrite texture as defined by Metzler (2012). Based on the structural order of the polyaromatic matter assessed by Raman spectroscopy, this meteorite appears to be as metamorphosed as Chainpur (LL3.4). The Raman spectroscopy parameters of interest are FWHM-D $(\mathrm{cm}-1)=155.8 \pm 7.6, \mathrm{ID} / \mathrm{IG}=1.02 \pm 0.04$ (see Bonal et al., 2016, figure 7).

Geochemistry: Olivine $\mathrm{Fa}_{13.9 \pm 9.6}, \mathrm{Fa} \mathrm{PMD}=65 \%$, range $\mathrm{Fa}_{1.1-29.7}(\mathrm{~N}=16), \mathrm{Cr}_{2} \mathrm{O}_{3}$ in ferroan olivine $0.14 \pm 0.11 \mathrm{wt} \%(\mathrm{~N}=15)$. Low-Ca pyroxene $\mathrm{Fs}_{15.1 \pm 6.9} \mathrm{Wo}_{2.1 \pm 1.9}$, range $\mathrm{Fs}_{1.1-22.2}(\mathrm{~N}=10)$.

Classification: LL3.4. Subtype from Raman spectroscopy. Specimens: $182 \mathrm{~g}$ and a polished section at CEREGE. Main mass at MMC.

\section{Catalina $300 \quad 25^{\circ} 14^{\prime} \mathrm{S}, 69^{\circ} 43^{\prime} \mathrm{W}$}

Antofagasta, Chile 
Find: 2018 March

Classification: Carbonaceous chondrite (CV3)

Physical characteristics: Dark stone. Cut surface reveals chondrules and CAIs set in a dark matrix.

Petrography: Chondrules with average size $1350 \mu \mathrm{m}(\mathrm{n}=10)$ and CAIs set in fine grained matrix (45vol\%, from point counting $n=207)$. Abundant sulfides and magnetite, rare metal.

Geochemistry: Olivine $\mathrm{Fa}_{4.7 \pm 6.1}, \mathrm{PMD}=11 \%$, range $\mathrm{Fa}_{0.2-14.9}(\mathrm{n}=7), \mathrm{Cr}_{2} \mathrm{O}_{3}$ in ferroan olivine is $0.58 \pm 0.78 \mathrm{wt} \%(\mathrm{n}=5)$. Low-ca pyroxene $\mathrm{Fs}_{1.0} \mathrm{Wo}_{0.6}(\mathrm{n}=1)$

Classification: Carbonaceous chondrite (CV3, oxidized subgroup)

Specimens: $23 \mathrm{~g}$ and a polished section at CEREGE. Main mass with Luc Labenne

\section{Chasseron $\quad 46^{\circ} 51.07^{\prime} \mathrm{N}, 6^{\circ} 32.32 \mathrm{E}$}

Vaud, Switzerland

Find: 1959

Classification: Pallasite (Main group)

History: Found by Mr. Reto Merlo, Grüningen, Switzerland, during a school excursion in summer 1959 while he was searching for firewood. The finder remembers having found the sample between the hotel and the summit of Chasseron (distance $\sim 160 \mathrm{~m}$ ). The finder glued the sample on a piece of wood and kept it until 2017. After reading about meteorites, he sent the sample to the $N M B E$ (received May 9, 2017) from where it was transferred to UNIL. Physical characteristics: Metallic sponge shape, only slightly rusted, fragment with embedded yellow-green forsterite (olivine) grains. Locally fresh-looking, shiny fusion crust on metal and olivine. $23.5 \times 13 \times 11 \mathrm{~mm}$.

Petrography: Typical pallasitic texture. Forsterite (olivine) grains size is $8-2 \mathrm{~mm}$. Droplet-like troilite grains up to $0.6 \mathrm{~mm}$ embedded in forsterite (olivine). Schreibersite rounded inclusions in Ni-rich iron up to $1.8 \mathrm{~mm}$. Ni-rich iron weathering is relatively low in an outermost zone just $0.1 \mathrm{~mm}$ thick, consistent with weathering grade W1. Bulk density by immersion in isopropanol is $6.17 \mathrm{~g} / \mathrm{cm} 3$. From density calculation, volume fraction of forsterite is $57 \%$ and volume fraction of Ni-rich iron is $43 \%$.

Geochemistry: Forsterite (olivine): microprobed $\mathrm{Fa}_{14.9 \pm 1.1}\left(\right.$ range $\mathrm{Fa}_{13.6-15.9}$ ), Fe/Mn (at.) $34 \pm n=5$. Two analyses of Ni-rich iron (variety kamacite) yielded: $\mathrm{Ni}$ $7.15 \pm 0.21$ wt. $\%$ and Co $1.20 \pm 0.20$ wt. \%. One analysis of schreibersite (XRD checked) yielded the following formula (based on $4 \mathrm{apfu}$ ):

$\left(\mathrm{Fe}_{1.92} \mathrm{Ni}_{0.97} \mathrm{Co}_{0.03}\right)_{2.92} \mathrm{P}_{1.08}$. Stoichiometric troilite crystals checked by XRD. One analysis of chromite (XRD checked) yielded the following formula (based on 4 oxygen atoms):( $\left.\mathrm{Fe}_{0.62} \mathrm{Mg}_{0.35} \mathrm{Mn}_{0.03}\right)_{1.00}\left(\mathrm{Cr}_{1.65} \mathrm{Al}_{0.31} \mathrm{Fe}_{0.02} \mathrm{~V}_{0.02}\right)_{2.00} \mathrm{O}_{4}$. Forsterite (olivine) oxygen isotopes (R. Greenwood, $O U): \delta^{17} \mathrm{O} 1.514 \pm 0.051, \delta^{18} \mathrm{O}$ $3.239 \pm 0.094, \Delta^{17} \mathrm{O}-0.170(\mathrm{n}=2)$. Cosmogenic radionuclides: ( $\AA$. Rosén, GeMSE): Gamma-spectrometry performed in May 2017 showed significant activities of ${ }^{26} \mathrm{Al}$ $(10.8+2.5 /-1.7 \mathrm{dpm} / \mathrm{kg})$ and ${ }^{40} \mathrm{~K}(17.6+9.3 /-4.9 \mathrm{dpm} / \mathrm{kg}$, probably a contamination). No short-lived cosmogenic isotopes were detected. A significant activity 
$(17.0+2.1 /-1.2 \mathrm{dpm} / \mathrm{kg})$ of ${ }^{137} \mathrm{Cs}$ is present as terrestrial contamination, consistent with a first peak of nuclear bomb-produced ${ }^{137} \mathrm{Cs}$ in the years 1957-1959.

Classification: This is a main-group pallasite based on texture, mineralogy and mineral composition including oxygen isotopes. Low degree of weathering. Specimens: $4.8446 \mathrm{~g}$ and one epoxy resin polished section with isolated olivine grains at UNIL; sample number 093320.

\section{Chug Chug $003 \quad 22^{\circ} 29.931^{\prime} \mathrm{S}, 69^{\circ} 8.683^{\prime} \mathrm{W}$}

Antofagasta, Chile

Find: 2018 Apr 5

Classification: Ordinary chondrite (H4)

History: Meteorite (1 piece $8700 \mathrm{~g}$ and 5 small fragments) was found April 5, 2018, by Mikhail Nepomiluev during a meteorite search organized by Timur Kryachko.

Physical characteristics: Total mass is $8918 \mathrm{~g}$. Meteorite has roughly rounded and slightly elongated shape. The surface and interior of the meteorite is light, with dark brown fusion crust up to $2 \mathrm{~mm}$. No fresh glass in fusion crust was observed. Outermost slightly fractured zone is up to $5 \mathrm{~mm}$ width. Cracks (up to $0.1 \mathrm{~mm}$ in thickness) form a network in this zone.

Petrography: (Pavel Yu. Plechov, FMMR). Petrographic observation of a polished section shows that main part of the meteorite contains well-formed chondrules in the matrix. Chondrules sizes vary from 200 to $600 \mu \mathrm{m}$. They have quenched or porphyritic texture, rarely cryptocrystalline, and consist of $\mathrm{Ol}, \mathrm{Ol}+\mathrm{Opx}$, $\mathrm{O} 1+\mathrm{Opx}+\mathrm{Cpx}+\mathrm{Pl}$. Matrix consists of olivine grains (50-150 $\mu \mathrm{m})$ and less low-Capyroxene, which is surrounded by cryptocrystalline matrix with a significant amount of tiny (about $1 \mu \mathrm{m}$ ) round drops of metal (kamacite and taenite), chromite and plagioclase. Plagioclase grains $\left(\mathrm{An}_{18.5-41}\right)$ are less than $5 \mu \mathrm{m}$. These features indicate a petrological type of 4 . Fresh Fe-Ni metal blebs (up to $300 \mu \mathrm{m}$ ) and troilite (up to $20 \mu \mathrm{m}$ ) occurs in matrix. Metal consists of kamacite surrounded by relatively small grains of taenite and troilite on the boundary with matrix. One elongated area $(4 \times 7 \mathrm{~mm})$ in the thin section have sharp boundaries with main part of the meteorite. It also consists of chondrules (about $500 \mu \mathrm{m}$ ) and coarse-grained $(200-400 \mu \mathrm{m})$ Ol-Px matrix. Less metal is in the matrix of the area of a large $(2 \times 2$ $\mathrm{mm}$ ) metal blob. Olivine has no undulatory extinction, and opaque shock veins and melt pockets are absent, indicating a shock stage of S1. Weathering products (goethite, "hydrogoethite") occur as veins and in situ alteration of FeNi-metals and troilite near meteorite surface. Olivine, pyroxene, chromite, metal and troilite are fresh and not weathered (weathering grade W1). Clinopyroxene, chromite and merrillite (up to $200 \mu \mathrm{m}$ ) occur locally in the matrix.

Geochemistry: Mineral composition and Geochemistry: EDS-WDS analyses (Pavel Yu. Plechov, FMMR). The primary chondrite paragenesis includes olivine $\mathrm{Fa}_{19.9 \pm 1.6}(\mathrm{~N}=34)$, orthopyroxene $\mathrm{Fs}_{14.1 \pm 3.5} \mathrm{Wo}_{1.42 \pm 1.26}(\mathrm{~N}=34)$, plagioclase $\mathrm{Ab}_{62.6} \mathrm{An}_{28.8} \mathrm{Or}_{6.7}(\mathrm{~N}=3)$, Cr-bearing clinopyroxene $\mathrm{En}_{48.1} \mathrm{Fs}_{6.4} \mathrm{Wo}_{45.4}(\mathrm{~N}=1)$, chromite $\mathrm{Crt}_{77.8 \pm 5.93} \mathrm{Spl}_{13.2 \pm 0.78}(\mathrm{~N}=3)$ and merrillite. No Cl-apatite occurred in the 
meteorite. Merrillite contain (in wt.\%, N=6) FeO - 0.28-1.71 wt.\%, $\mathrm{MgO}$ - 3.523.83 and $\mathrm{Cl}-0.01-0.02$. Composition of kamacite (in wt.\%, $\mathrm{N}=2$ ): $\mathrm{Fe}$ 93.3-94.0, $\mathrm{Ni}$ 6.2-6.7, Co 0.88 . Taenite contain 39.8 wt.\% of Ni. Troilite is close to ideal FeS. Rare goethite contains 1.9 wt. $\% \mathrm{NiO}$ and 0.4 wt.\% CoO.

Classification: (Pavel Yu. Plechov, FMMR). Ordinary chondrite. H4, S1, W1. Specimens: 8700 g, Mikhail Nepomiluev; 198 g (4 pieces), Timur Kryachko, type specimen at FMMR.

Coya Sur 004 (CS 004) $\quad 22^{\circ} 26.07^{\prime} \mathrm{S}, 6^{\circ} 39.16^{\prime} \mathrm{W}$

Antofagasta, Chile

Find: 13 Oct 2017

Classification: Ordinary chondrite (LL3)

Petrography: Average chondrule diameter $870 \mu \mathrm{m}$

Coyote Dry Lake 349 (CyDL 349) $\quad 35^{\circ} 3.396^{\prime} \mathrm{N}, 116^{\circ} 46.096^{\prime} \mathrm{W}$

California, USA

Find: 2015 Dec 12

Classification: Ordinary chondrite (H6)

History: While hunting for meteorites on a dry-lake, Mark Bittmann, spotted a rusty rock protruding out of the corner of a hummock that was just beginning to be eroded by a small gully. Glancing at the gully he noticed many more fragments protruding out of the surface. Digging deeper into both the small dune and gully, he recovered 20 more equally weathered chondritic fragments. Later, returning with a recovery crew and a metal-detector, another $130 \mathrm{~g}$, sand-encrusted fragment was unearthed.

Physical characteristics: All 22 fragments are very weathered, but have a range of grades and color from yellowish-brown to brownish-black. Some fragments are coated in silt and sand-grains cemented with orange rust. Cut surfaces show finegrained metal/sulfide set in a uniformly brown matrix, faint chondrules barely detectable.

Petrography: (D. Sheikh, FSU) Chondrule boundaries blurred (250-600 $\mu \mathrm{m}, \mathrm{N}=6$ ). Recrystallized coarse-grained matrix with plagioclase $(>60 \mu \mathrm{m})$. Numerous interconnected iron-oxide veins.

Geochemistry: Olivine, $\mathrm{Fa}_{20.1 \pm 0.1}(\mathrm{~N}=26)$; orthopyroxene, $\mathrm{Fs}_{19.3 \pm 0.2} \mathrm{Wo}_{2.3 \pm 0.1}(\mathrm{~N}=25)$. It is noted that the measured Fs and Wo values are slightly higher than the main amounts present in most $\mathrm{H}$ chondrites.

Classification: Ordinary Chondrite (H6)

Specimens: 36 grams at $U C L A$; main mass with Verish.

\author{
Da Qaidam $001 \quad 37^{\circ} 49^{\prime} 2.34^{\prime \prime} \mathrm{N}, 95^{\circ} 23^{\prime} 25.53^{\prime \prime} \mathrm{E}$ \\ Qinghai, China \\ Find: 13 July 2015
}


Classification: Ordinary chondrite (L $\sim 6)$

History: (Ziyao Wang) Ma Yonglu (Lenghu/Qinghai) discovered two stones of about $17.5 \mathrm{~kg}$ while they were doing road maintenance work $4 \mathrm{~km}$ SE of Chaidan Town, Qinghai province, on July 13, 2015. Both stones were purchased by Wang Ziyao.

Physical characteristics: (Ziyao Wang and R. Bartoschewitz, Bart) Two blackbrown stones, one of $16.5 \mathrm{~kg}$ and a second one of about $1 \mathrm{~kg}$, both without fusion crust.

Petrography: (R. Bartoschewitz, Bart) internally brown-stained (in some rim areas, less oxidized) recrystallized matrix with poorly developed chondrules and irregular metal specks up to $1 \mathrm{~mm}$ and much smaller irregular sulfide specks.

Geochemistry: Magnetic susceptibility (R. Bartoschewitz, Bart) $\log \chi\left(\times 10^{-}\right.$ $\left.{ }^{9} \mathrm{~m}^{3} / \mathrm{kg}\right)=4.55$

Classification: Ordinary chondrite (L $\sim 6, \mathrm{~W} 3$ )

Specimens: $22.7 \mathrm{~g}$ on deposit at Kiel, Wang Ziyao holds the main mass, and 168.4 g with Bart.

\title{
Dahongliuxia $\quad 44^{\circ} 10^{\prime} 20^{\prime \prime}, 91^{\circ} 42^{\prime} 30^{\prime \prime}$ \\ Xinjiang, China \\ Find: 1999
}

Classification: Iron meteorite (IIIAB)

History: The meteorite was found in 1999 by a local herder in Balikun County, Xinjiang Province.

Physical characteristics: The total mass of the meteorite is $15.6 \mathrm{~kg}$. It is an ovoid shaped iron meteorite covered with a brown to black layer. One side is smooth and the other side shows regmaglypts.

Petrography: Polished section of the sample observed under the SEM reveals bands of kamacite and taenite interleaved with bandwidth of $1.2 \mathrm{~mm}$ (medium Widmanstätten pattern). No silicate inclusions and other minerals can be found in the section.

Geochemistry: (ELAN DRC-e Q-ICP-MS) Ni=9.4, Co=0.65 (in wt\%). Ga=18.15, $\mathrm{Ge}=34.53$, $\mathrm{Ir}=9.35, \mathrm{~W}=1.71, \mathrm{Au}=0.53$ (in ppm).

Classification: Iron Meteorite (Om), IIIAB

Specimens: Main mass with Peng Wang. One polished section and a $300 \mathrm{~g}$ slice was deposited in IGCAS

This meteorite was mistakenly released on 24 Feb 2018 as NWA 11650. That name will henceforth be a synonym for Dar al Gani 1069.

\author{
Dhofar 1762 (Dho 1762) $\quad 18.888^{\circ} \mathrm{N}, 54.467^{\circ} \mathrm{E}$ \\ Zufar, Oman \\ Purchased: 2018 Feb \\ Classification: Carbonaceous chondrite (CO3)
}


History: Found in Oman in 2010 by an anonymous prospector and subsequently purchased by Martin Goff in February 2018.

Petrography: (A. Irving and S. Kuehner, UWS) Fairly closely packed, wellformed, small chondrules (apparent diameter $220 \pm 130 \mu \mathrm{m}, \mathrm{N}=20$ ), mineral grains and very fine grained CAI are set in a finer grained matrix (orange-brown in thin section).

Geochemistry: Olivine $\left(\mathrm{Fa}_{1.7-60.7}, \mathrm{Cr}_{2} \mathrm{O}_{3}\right.$ in ferroan examples $0.10-0.45$ wt.\%, mean $0.19 \pm 0.13$ wt. $\%, \mathrm{~N}=8)$, orthopyroxene $\left(\mathrm{Fs}_{0.6-36.6} \mathrm{Wo}_{0.9-2.8}, \mathrm{~N}=3\right)$, clinopyroxene $\left(\mathrm{Fs}_{1.3-1.5} \mathrm{Wo}_{38.3-46.0}, \mathrm{~N}=2\right)$.

Classification: Carbonaceous chondrite (CO3).

Specimens: $16.3 \mathrm{~g}$ including one polished thin section at $U W B$; remainder with $\mathrm{Mr}$. M. Goff.

Dhofar 2092 (Dho 2092) $\quad 18^{\circ} 45.30^{\prime} \mathrm{N}, 54^{\circ} 31.15^{\prime} \mathrm{E}$

Zufar, Oman

Find: 15 Feb 2015

Classification: HED achondrite (Eucrite)

Physical characteristics: A 440 g sample supplied by the Sultan Qaboos University, which has a millimeter thick, dark brown fusion crust present on all faces except one cut face. This fusion crust lacks remaglypts instead has a mainly rough surface and angular. There are no contraction cracks present on this sample. This rock has already been cut and there is a clean face already present. Beige colour clasts can be identified on cut face and they are surrounded by a light grey ground mass. The sample is not magnetic.

Petrography (P. Hill, UWO) The sample is breccia containing numerous clasts of coarse-grained plagioclase and pyroxene. The clasts are poorly sorted and are subrounded with one clast reaching up to $2 \mathrm{~cm}$ on the cut face. Pyroxene (low and high $\mathrm{Ca}$ ) and plagioclase make up most of the clasts and the groundmass; however, and free silica was observed in significant quantities. The free silica mainly located at grain boundaries within the fine-grained groundmass. Distinct exsolution lamellae are present within the grains of pyroxene. Troilite and oxides are present within the sample and through EDS analysis the oxides were determined to be ilmenite. No weathering products were observed within the samples suggest a low degree of terrestrial weathering. The sample does show undulose extinction in some of the grains of plagioclase and irregular fractures were observed, but not in high abundance suggesting that the sample is very weakly shocked.

Geochemistry: Mineral composition and geochemistry (M. Beauchamp, P. Hill, $U W O$ ) Plagioclase $\left(\mathrm{An}_{88.4 \pm 0.9}\right)$, Low-Ca Pyroxene ( $\left.\mathrm{Fs}_{59.8 \pm 2.5} \mathrm{Wo}_{3.0 \pm 2.4}\right)$, High-Ca Pyroxene $\left(\mathrm{Fs}_{27.36 \pm 2.1} \mathrm{Wo}_{42.5 \pm 2.3}\right)$ and Free Silica $\left(\mathrm{SiO}_{2} 99.3 \pm 1.3 \mathrm{wt} \%\right)$

Classification: Classificaiton: Eucrite S2, W1.

Specimens: $440 \mathrm{~g}$ type specimen, including polished thin section, are on deposit at $S Q U$. 
Dhofar 2093 (Dho 2093) $\quad 18^{\circ} 34.398^{\prime} \mathrm{N}, 5^{\circ} 8.889^{\prime} \mathrm{E}$

Zufar, Oman

Find: 2014

Classification: HED achondrite (Eucrite)

History: Discovered by an anonymous finder on a desert trip $12 \mathrm{~km} \mathrm{SE}$ of Dauka in 2014.

Physical characteristics: Four gray fragments, partly covered by black-browinish crust ranging from 301.3 to $30.5 \mathrm{~g}$. Cut face shows light-gray matrix with white, black, and light-brown inclusions.

Petrography: (R. Bartoschewitz, Bart) Microscopic examination of a thin section shows holocrystalline hypidiomorphic texture and weak shock effect: undulatory extinction in plagioclase. Magnetic susceptibility $\log \chi\left(\times 10^{-9} \mathrm{~m}^{3} / \mathrm{kg}\right)=2.88$.

Geochemistry: (R. Bartoschewitz, Bart, P. Appel and B. Mader, Kiel) Pyroxene (pigeonite) $\mathrm{Fs}_{56.8 \pm 3.3} \mathrm{Wo}_{7.2 \pm 4.4}, \mathrm{Fe} / \mathrm{Mn} 29.5$ ( $=13$ ); Plagioclase

$\mathrm{An}_{89.4 \pm 4.9} \mathrm{Ab}_{10.2 \pm 4.5}(\mathrm{n}=20) ; \mathrm{SiO}_{2}$; chromite CRAL 80, FFM 98 ( $\left.\mathrm{n}=1\right)$; ilmenite; kamacite $\mathrm{Ni}<0.1$, Co 0.5 wt. $\%(\mathrm{n}=2)$.

Classification: Achondrite (basaltic eucrite, monomict breccia)

Specimens: $20.0 \mathrm{~g}$ on deposit at Kiel, main mass anonymous

\section{Dueodde $\quad 54^{\circ} 59^{\prime} 24^{\prime}{ }^{\prime} \mathrm{N}, 15^{\circ} 04^{\prime} 12^{\prime \prime} \mathrm{E}$}

Denmark

Find: 2017 July 17

Classification: Ordinary chondrite (H5)

History: While cleaning the roof of the annex of their summerhouse in the area of Dueodde, in the southern part of the island of Bornholm, Denmark, the finder noticed a circular hole of approximately $5 \mathrm{~cm}$ in diameter in the roof. Further inspection of the area under the roof revealed the presence of single dark stone with apparent fusion crust. The finder estimates that the meteorite fall must have occurred within the last 2-3 years. Following recovery of the specimen, staff at the NHMD were contacted and the stone delivered.

Physical characteristics: Single $75 \mathrm{~g}$ spherical stone covered by irregular black fusion crust.

Petrography: The petrography was investigated from a polished thick section using backscattered electron imaging and X-ray scans. The meteorite displays an unbrecciated and recrystallized chondritic texture. Plagioclase grains are present and are typically less than $50 \mu \mathrm{m}$ in size. The chondrules typically display obscured outlines. Porphyritic olivine and radial pyroxene chondrules are observed, ranging in sizes from 0.2 to $1 \mathrm{~mm}$ in diameter.

Geochemistry: The mean composition of olivine is $\mathrm{Fa}_{19.4 \pm 0.4}$ with a compositional range of $\mathrm{Fa}_{19.1-19.8}(\mathrm{n}=14)$. The low-Ca pyroxenes have a mean composition of $\mathrm{Fs}_{17.2 \pm 0.5}(\mathrm{n}=15)$, defining a compositional range of $\mathrm{Fs}_{16.4-17.2}$. The mean plagioclase composition is $\mathrm{An}_{12.0 \pm 0.3} \mathrm{Or}_{5.4 \pm 2.0}\left(\mathrm{n}=15\right.$; range $\left.\mathrm{An}_{11.6-12.2}\right)$.

Classification: $\mathrm{H} 5$

Specimens: Main mass and one thick polished section at $N H M D$. 


\section{Dunhuang $001 \quad 40^{\circ} 13^{\prime} 12.56^{\prime \prime} \mathrm{N}, 94^{\circ} 23^{\prime} 55.97 " \mathrm{E}$ \\ Gansu, China \\ Find: 16 Sept 2018 \\ Classification: Ordinary chondrite $(\mathrm{L} \sim 6)$}

History: (Ziyao Wang) Discovered by Liu Binghan (Haixi/Qinghai) in the desert, 25 km WNW of Dunhuang City, Gansu Province, on 16 Sept 2018

Physical characteristics: (Wang Ziyao) Nine dark brown flat eroded stones of totally $1427 \mathrm{~g}$ (main mass $1283 \mathrm{~g}$ ).

Petrography: (R. Bartoschewitz, Bart) Brown stainted recrystallized matrix with well recognizable chondrules (0.6-1.6 mm, av. 0.7 mm) and few metal specks.

Geochemistry: Magnetic susceptibility (R. Bartoschewitz, Bart) $\log \chi\left(\times 10^{-}\right.$ $\left.{ }^{9} \mathrm{~m}^{3} / \mathrm{kg}\right)=4.84$

Classification: Ordinary chondrite (L $\sim 6, \mathrm{~W} 3)$

Specimens: $22.3 \mathrm{~g}$ on deposit at Kiel, Wang Ziyao and Liu Binghan holds the main mass, and $109 \mathrm{~g}$ with Bart.

El Médano $381($ EM 381) 2441'34.9"S, 7007'18.9"W

Antofagasta, Chile

Find: 2017 Nov

Classification: Ordinary chondrite (L5)

Physical characteristics: Dark stone. Cut surface reveals a dark interior with chondrules and abundant and fresh FeNi metal.

Petrography: (J. Gattacceca, CEREGE) Recrystallized chondrite with packed chondrules. The meteorite displays pervasive shock darkening, with abundant troilite and metal veinlets and droplets. Metal and sulfides are often associated and form grains to several $\mathrm{mm}$. Plagioclase-chromite assemblages to several hundreds $\mu \mathrm{m}$ are found. Plagioclase average size is $<50 \mu \mathrm{m}$.

Classification: Ordinary chondrite (L5). Shock darkened.

Specimens: Type specimen at CEREGE. Main mass with ARTMET.

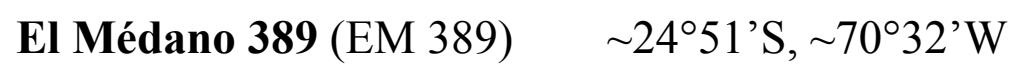

Antofagasta, Chile

Find: 2016 Nov 04

Classification: Carbonaceous chondrite (CO3)

History: The meteorite was found in January 2016 in the Atacama desert by Rodrigo Martinez while he was looking for meteorites

Petrography: (J. Gattacceca, CEREGE) Chondrules (predominantly of type I) and small CAIs set in a fine-grained Fe-rich matrix. Average chondrule apparent size $300 \mu \mathrm{m}(\mathrm{N}=26)$. Opaques are metal and troilite. 
Geochemistry: Olivine in the range $\mathrm{Fa}_{0.5-69.1}$ (mean $\mathrm{Fa}_{16.8 \pm 18.2}, \mathrm{PMD}=89 \%, \mathrm{~N}=25$ ). $\mathrm{Cr}_{2} \mathrm{O}_{3}$ in ferroan olivine $0.11 \pm 0.09$ wt.\% $(\mathrm{N}=22)$. Low-Ca pyroxene $\mathrm{Fs}_{5.5} \mathrm{Wo}_{1.0}$ and $\mathrm{Fs}_{4.8} \mathrm{Wo}_{0.9}(\mathrm{~N}=2)$. Magnetic susceptibility $\log \chi\left(\times 10^{-9} \mathrm{~m}^{3} / \mathrm{kg}\right)=4.42$.

Classification: Carbonaceous chondrite (CO3). Minimal weathering Specimens: $53 \mathrm{~g}$ and a polished section at CEREGE. Main mass at $M M C$.

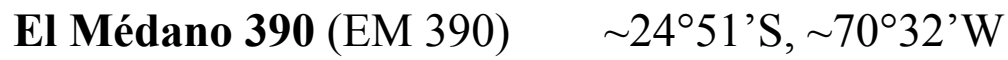

Antofagasta, Chile

Find: 2017 May 17

Classification: Carbonaceous chondrite (CO3)

History: The meteorite was found in May 2017 in the Atacama desert by Rodrigo Martinez while he was looking for meteorites

Petrography: (J. Gattacceca, CEREGE) Chondrules (predominantly of type I) and small CAIs set in a fine-grained Fe-rich matrix. Average chondrule apparent size about $250 \mu \mathrm{m}$. Opaques are metal and troilite.

Geochemistry: Olivine in the range $\mathrm{Fa}_{0.5-34.4}$ mean $\left.\mathrm{Fa}_{18.0 \pm 15.6}, \mathrm{~N}=4\right) . \mathrm{Cr}_{2} \mathrm{O}_{3}$ in ferroan olivine $0.07 \pm 0.01 \mathrm{wt} . \%(\mathrm{~N}=3)$. Low-Ca pyroxene $\mathrm{Fs}_{1.01} \mathrm{Wo}_{4.0}(\mathrm{~N}=1)$. Magnetic susceptibility $\log \chi\left(\times 10^{-9} \mathrm{~m}^{3} / \mathrm{kg}\right)=4.63$.

Classification: Carbonaceous chondrite (CO3). Moderate weathering Specimens: $20 \mathrm{~g}$ and a polished section at CEREGE. Main mass at $M M C$.

El Médano 394 (EM 394) 2451’S, 70³2'W

Antofagasta, Chile

Find: 2018 Apr

Classification: Enstatite chondrite (EL6)

History: The stone was found lying at the surface in the Atacama desert in April 2018.

Physical characteristics: A single dark brown stone. Cut face reveals a dark interior with abundant metal.

Petrography: (J. Gattacceca, Bertrand Devouard, CEREGE) Highly recrystallized chondritic texture. No visible chondrule in the $1.5 \mathrm{~cm} 2$ observed section. Main minerals are enstatite, plagioclase (typical size $100 \mu \mathrm{m}$, to $400 \mu \mathrm{m}$ ), kamacite (typical size $300 \mu \mathrm{m}$, to $\mathrm{mm}$ ), sulfides (typical size $150 \mu \mathrm{m}$, to $400 \mu \mathrm{m}$ ). No olivine. Sulfides include alabandite, oldhamite, troilite.

Geochemistry: Enstatite $\mathrm{Fs}_{0.3 \pm 0.0} \mathrm{Wo}_{1.6 \pm 0.0}(\mathrm{~N}=3)$. Plagioclase

$\mathrm{An}_{15.2 \pm 0.2} \mathrm{Ab}_{80.6 \pm 0.7} \mathrm{Or}_{4.3 \pm 0.5}(\mathrm{~N}=2)$. Si in kamacite $1.4 \pm 0.0 \mathrm{wt} \%(\mathrm{~N}=5)$. Magnetic susceptibility $\log \chi\left(\times 10^{-9} \mathrm{~m}^{3} / \mathrm{kg}\right)=5.51$.

Classification: Enstatite chondrite (EL6). EL classification from the Si content of kamacite and the presence of alabandite. Minimal weathering.

Specimens: Type specimen $41 \mathrm{~g}$ at CEREGE. Main mass with ARTMET. 
Antarctica

Find: 2014 Nov 16

Classification: Carbonaceous chondrite (CK5)

Petrography: The section consists of high abundance of recrystallized matrix.

Complete absence of FeNi-metal. Cr-bearing magnetite is dominant.

Geochemistry: Olivine is $\mathrm{Fa}_{29.5-30.0}$; low-Ca pyroxene is $\mathrm{Fs}_{25.1-26.3} \mathrm{Wo}_{0.6}$;

plagioclase is $\mathrm{An}_{42-71 .}$

Elephant Moraine 14013 (EET 14013) $\quad$ 76 $17.195^{\circ} \mathrm{S}, 1^{\circ} 6^{\circ} 27.181^{\prime} \mathrm{E}$

Antarctica

Find: 2014 Nov 21

Classification: Carbonaceous chondrite (CM2)

Petrography: The section consists of a few small chondrules $(\sim 22 \mathrm{vol} \%,<\sim 100$ $\mu \mathrm{m}$ to $\sim 2 \mathrm{~mm}$ in diameter), CAIs $(\sim 2 \mathrm{vol} \%)$, and matrix $(\sim 76 \mathrm{vol} \%)$ with carbonate, sulfides, and phyllosilicates. Fe-Ni metal is very rare $(<0.1 \mathrm{vol} \%)$. The chondrules have experience varying ${ }^{\circ}$ of aqueous alteration. The matrix occurs as fine-grained rims surrounding chondrules.

Geochemistry: Olivine is $\mathrm{Fa}_{0.3-65.7}$; low-Ca pyroxene is $\mathrm{Fs}_{1.1-4.5} \mathrm{Wo}_{0.7-4.4}$.

Elephant Moraine 14017 (EET 14017) $\quad$ 76º $18.424^{\prime}$ S, 156 $151.991^{\prime} \mathrm{E}$ Antarctica

Find: 2014 Nov 21

Classification: Ordinary chondrite (LL3)

Petrography: Sharply defined chondrules ( $\sim 80$ vol $\%$, average size of $\sim 0.9 \mathrm{~mm})$ with glassy mesostasis are observed. Metal is less than 1 vol\%. Aluminum-rich chondrules are present.

Geochemistry: Olivine is $\mathrm{Fa}_{0.4-53.5}$; low-Ca pyroxene is $\mathrm{Fs}_{1.1-37.1 .5} \mathrm{Wo}_{0-6.2}$.

Elephant Moraine 14018 (EET 14018) $\quad 76^{\circ} 17.125^{\prime} \mathrm{S}, 1^{\circ} 6^{\circ} 29.040^{\prime} \mathrm{E}$

Antarctica

Find: 2014 Nov 21

Classification: Ordinary chondrite (L3)

Petrography: The section has sharply defined chondrules (average size of $\sim 0.6$ $\mathrm{mm}$ ) with some glassy mesostasis. Metal abundance is $\sim 4 \mathrm{vol} \%$.

Geochemistry: Olivine is $\mathrm{Fa}_{0.5-27.1}$; low-Ca pyroxene is $\mathrm{Fs}_{4.9-29.4} \mathrm{Wo}_{0.2-4.0}$.

Elephant Moraine 14049 (EET 14049) $\quad$ 76¹5.926’S, 156³8.370’E

Antarctica

Find: 2014 Dec 12

Classification: HED achondrite (Eucrite) 
Petrography: The section consists of pyroxene and calcic plagioclase $\left(>\mathrm{An}_{80}\right)$ with minor amount of silica and metal. The section is brecciated and contains mineral and lithic fragments.

Geochemistry: Low-Ca pyroxene is $\mathrm{Fs}_{19.4-60.9} \mathrm{Wo}_{1.5-7.9}$; Plagioclase is $\mathrm{An}_{80.2-97.2}$.

Elephant Moraine 14053 (EET 14053) $\quad 7^{\circ} 17.138^{\prime} \mathrm{S}, 156^{\circ} 35.726^{\prime} \mathrm{E}$

Antarctica

Find: 2014 Dec 12

Classification: Ureilite

Petrography: The section consists of large olivine (up to $\sim 2 \mathrm{~mm}$ ) and low-Ca pyroxene (up to $\sim 2 \mathrm{~mm}$ ), and dark interstitial materials including carbon, metal, and sulfides. The high $\mathrm{CaO}$ contents $(0.24 \sim 0.32 \mathrm{wt} \%)$ in olivine are observed.

Geochemistry: Olivine is $\mathrm{Fa}_{4.2-15.3}$; low-Ca pyroxene is $\mathrm{Fs}_{12.5-13.2} \mathrm{Wo}_{4.1-4.6}$.

Elephant Moraine 14056 (EET 14056) $\quad 7^{\circ} 12.637^{\circ} \mathrm{S}, 156^{\circ} 41.497^{\prime} \mathrm{E}$

Antarctica

Find: 2014 Dec 12

Classification: HED achondrite (Eucrite)

Petrography: The section mainly consists of low-Ca pyroxene and plagioclase $\left(>A n_{90}\right)$. This meteorite is unbrecciated and equigranular with grain sizes of $0.3-2$ $\mathrm{mm}$ in diameter.

Geochemistry: Low-Ca pyroxene is $\mathrm{Fs}_{44.8-46.8} \mathrm{Wo}_{1.1-3.3}$; Plagioclase is $\mathrm{An}_{93.1-94.8}$

\section{Elephant Moraine 14064 (EET 14064) $\quad$ 76²15.224’S, 156 35.422’E}

Antarctica

Find: 2014 Dec 12

Classification: Ordinary chondrite (LL3)

Petrography: The section shows sharply defined chondrules ( 70 vol $\%$, average chondrule size of $\sim 0.9 \mathrm{~mm}$ ) with glassy mesostasis. Metal abundance is $\sim<2 \mathrm{vol} \%$. Geochemistry: Olivine is $\mathrm{Fa}_{0.6-28.9}$; low-Ca pyroxene is $\mathrm{Fs}_{3.6-23.6} \mathrm{Wo}_{0.2-7.2}$.

Elephant Moraine 14066 (EET 14066) $\quad 76^{\circ} 15.492^{\prime} \mathrm{S}, 156^{\circ} 36.556^{\prime} \mathrm{E}$ Antarctica

Find: 2014 Dec 12

Classification: HED achondrite (Eucrite)

Petrography: The section mainly consists of pyroxene and plagioclase $\left(>A n_{86}\right)$ with a minor amount of silica and metal. The section is brecciated with mineral and lithic fragments.

Geochemistry: Low-Ca pyroxene is $\mathrm{Fs}_{21.1-56.5} \mathrm{Wo}_{1.3-7.1}$; Plagioclase is $\mathrm{An}_{86.0-}$ 97.2. One olivine was analyzed $\left(\mathrm{Fa}_{26.6}\right)$. 


\section{Elephant Moraine 14068 (EET 14068) $\quad$ 76²14.940’S, $156^{\circ} 36.855^{\prime} \mathrm{E}$}

Antarctica

Find: 2014 Dec 12

Classification: Carbonaceous chondrite (CM2)

Petrography: The section consists of a few small chondrules $(\sim 30$ vol $\%$, up to 1.5 $\mathrm{mm}$ in diameter, the average size of chondrule is $\sim 300 \mu \mathrm{m})$, and matrix $(\sim 70 \mathrm{vol} \%)$. Fe-Ni metal is very rare $(<0.1$ vol\%). The section has abundant hydrated materials, mainly serpentine.

Geochemistry: Olivine is $\mathrm{Fa}_{0.5-35.9}$; low-Ca pyroxene is $\mathrm{Fs}_{0.8-5.5} \mathrm{Wo}_{0.6-2.7}$.

\section{Elephant Moraine 14074 (EET 14074) $\quad$ 76º $15.525^{\prime} \mathrm{S}, 156^{\circ} 34.277^{\prime} \mathrm{E}$}

Antarctica

Find: 2014 Dec 12

Classification: Primitive achondrite (Acapulcoite)

Petrography: The section mainly consists of fine- to medium grained equigranular $(\sim 220 \mu \mathrm{m})$ orthopyroxene and olivine, abundant FeNi metal ( $\sim 14 \mathrm{vol} \%)$, and minor amount of chromite and apatite.

Geochemistry: Olivine is $\mathrm{Fa}_{9.6-11.0}$; low-Ca pyroxene is $\mathrm{Fs}_{10.0-10.6} \mathrm{Wo}_{2.0-2.3}$.

\section{Elmore County}

United States

Find: 1940

Classification: Iron meteorite (IAB-MG)

History: Purportedly found in Alabama, USA. Presented to UCLA by L. Garvie in 2014. The find account provided by Fred Bart is the following: "Many years after leaving medicine, I learned that I practiced in near proximity to the Wetumpka Impact Crater. Not knowing that any fragments of the impact meteorite would have long ago rusted, I asked Greg Dubay to ask his patients if any of them had found fragments of the meteorite and if they would allow me to hunt their property.

Michael Mulgrew politely pointed out that this was an exercise in futility--a fact I failed to convey to Greg Dubay. One of his patients (Mr. Greathouse) relayed that they had a "space rock" in their house that had been found by his father and/or uncle in the 1940s. This farmer family was working the land for most of their lives. One day, they encountered a rock unlike anything they had seen before and brought it home. Sometime in the next decade or so, it was transported to Auburn University, where it was confirmed to be a meteorite and painted with a sealant. Mr. Greathouse gave the piece to Dr Dubay who, in turn, gave it to me. After I received it, I carefully removed the sealant with pure acetone. While he was alive, I asked Dr. Dubay to inquire if he knew the origin of the find or even the name of the farm from where it came. Regrettably, such specifics were forgotten years ago, if they were ever known. All I know is that is was unearthed in Elmore County, Alabama." 
Geochemistry: (J.T. Wasson, $U C L A$ ): INAA data (average of two samples), $\mathrm{Ni}=$ $66.4, \mathrm{Co}=4.48$ (both $\mathrm{mg} / \mathrm{g}), \mathrm{Ga}=89.6, \mathrm{As}=11, \mathrm{~W}=1.25, \mathrm{Ir}=4.1, \mathrm{Au}=1.457$ (all $\mu \mathrm{g} / \mathrm{g}$ ).

Classification: Iron, IAB main group. Chemical composition is similar to Campo del Cielo with which it may be paired.

Specimens: Type specimens at $U C L A$ and $A S U$ (both $>20 \mathrm{~g}$ ).

\section{Entrèves $\quad 45^{\circ} 49.14^{\prime} \mathrm{N}, 06^{\circ} 54.82^{\prime} \mathrm{E}$}

Valle d'Aosta, Italy

Find: May 1987

Classification: Iron meteorite (IAB-MG)

History: A single $20 \times 17 \times 18 \mathrm{~cm}$ mass was found in 1987 by an anonymous bomb-slivers hunter near a moraine at the border of the Brenva glacier, on the Italian side of Mont Blanc, near Entrèves. The sample was subsequently sold to Claude Scavone, a French mineral collector who sold it to Giorgio Tomelleri, an Italian meteorite collector

Physical characteristics: The main mass, weighing $17.3 \mathrm{~kg}$, displays several small fusion pits and is apparently well preserved from rust. A distinct coarse grained Widmanstaetten pattern is visible on sawn and etched surfaces.

Petrography: (V. Moggi Cecchi, G. Pratesi, S. Caporali, UniFi). The $2 \times 2 \mathrm{~cm}$ cut and etched chip examined contains no silicates. Coarse-grained ( $\sim 5 \mathrm{~mm}$ width) equigranular kamacite grains with $120^{\circ}$ grain boundaries can be distinguished, with rare taenite. No Neumann lines are visible. Schreibersite is present as rare laths, up to $150 \mu \mathrm{m}$ in width and to $1 \mathrm{~mm}$ in length, within some kamacite grains and at grain boundaries. Schreibersite is frequently surrounded by iron oxides.

Geochemistry: SEM-EDX spot analyses, all in wt\% (V. Moggi Cecchi, G. Pratesi, S. Caporali, Uni-Fi): schreibersite P19.9 $\pm 0.2 \mathrm{Fe} 44.6 \pm 0.6 \mathrm{Ni35.6} \pm 0.8(\mathrm{~N}=6)$; taenite Fe70.5 $\pm 0.5 \mathrm{Ni} 29.5 \pm 0.2(\mathrm{~N}=6)$; kamacite Fe92.6 $\pm 0.6 \mathrm{Ni} 7.4 \pm 0.2(\mathrm{~N}=5)$. Bulk composition (C. Herd and G. Chen, $U A b$ ) Ni=69.0, $\mathrm{Co}=4.5$ (both $\mathrm{mg} / \mathrm{g}$ ); $\mathrm{Cu}=124$, $\mathrm{Ga}=91, \mathrm{As}=11.2, \mathrm{~W}=1.8, \mathrm{Ir}=3.9, \mathrm{Pt}=9.9, \mathrm{Pd}=3.4, \mathrm{Ru}=7.4, \mathrm{Re}=0.4, \mathrm{Os}=5.2$, (all $\mu \mathrm{g} / \mathrm{g})$. Obtained by ICP-MS using North Chile as standard.

Classification: IAB-MG, coarse octahedrite. Composition is consistent with IAB Complex, Main Group (MG). The composition of this meteorites is extremely similar to that of Campo del Cielo. However Neumann bands are absent whereas they are abundant in Campo del Cielo.

Specimens: A polished chip weighing $28 \mathrm{~g}$ is on deposit at MSN-FI. Main mass with Giorgio Tomelleri.

\section{Erg Chech $001\left(\right.$ EC 001) $\quad 26.0555^{\circ} \mathrm{N}, 2.0797^{\circ} \mathrm{W}$}

Algeria

Find: 2016 Dec

Classification: HED achondrite (Diogenite) 
History: Three similar looking stones (weighing 998, 138, and $130 \mathrm{~g}$ ) were found in December 2016 and January 2017 in the Erg Chech Desert, $235 \mathrm{~km}$ WSW of the village of Tawrirt in the Adrar region of Algeria. The $138 \mathrm{~g}$ stone was subsequently acquired by John Higgins.

Physical characteristics: The $138 \mathrm{~g}$ stone lacks fusion crust and the fresh interior is mostly yellowish-green with sparse opaque grains.

Petrography: (A. Irving and S. Kuehner, UWS) Protogranular aggregate of predominantly orthopyroxene (mean grainsize $0.9 \mathrm{~mm}$ ) plus minor chromite (containing blebs of troilite) and rare taenite (containing minor $\mathrm{Cu}$ ).

Geochemistry: Orthopyroxene $\left(\mathrm{Fs}_{23.1-23.2} \mathrm{Wo}_{1.7-1.8}, \mathrm{FeO} / \mathrm{MnO}=28-33, \mathrm{~N}=3\right)$.

Classification: Diogenite.

Specimens: $21.6 \mathrm{~g}$ including one polished endcut at $U W B$; remainder of the $138 \mathrm{~g}$ stone with Mr. J. Higgins; other stones with the anonymous finders.

\section{Errachidia $\quad 31^{\circ} 41.28102^{\prime} \mathrm{N}, 4^{\circ} 11.16582^{\prime} \mathrm{W}$ \\ Morocco \\ Find: April 2016 \\ Classification: Lunar meteorite (feldspathic breccia)}

History: One piece of $156 \mathrm{~g}$ was found April 2016 about $0.5 \mathrm{~km}$ west of Aoufous in Morocco.

Petrography: (A.-K. Kraemer and A. Bischoff, IfP) Breccia composed of angular anorthite-rich lithic and mineral fragments embedded in a fine-grained clastic matrix. The sample also contains melt spherules and some glassy areas. Observed minerals include anorthite, olivine, low-Ca pyroxene, Ca-rich pyroxene, Al-Tichromite, kamacite, taenit, ilmenite and troilite.

Geochemistry: Mineral compositon and geochemistry: Olivine (mean: $\mathrm{Fa}_{32.6}$, $\mathrm{Fe} / \mathrm{Mn}=96 ; \mathrm{N}=43$ ), low-Ca pyroxene (mean $\mathrm{Fs}_{29.5} \mathrm{Wo}_{7.9}, \mathrm{Fe} / \mathrm{Mn}=59, \mathrm{~N}=40$ ), Ca-rich pyroxene (mean: $\mathrm{Fs}_{15.8} \mathrm{Wo}_{41.4} ; \mathrm{Fe} / \mathrm{Mn}=49 ; \mathrm{N}=11$ ), anorthite (mean $\left.\mathrm{An}_{95.9} \mathrm{Or}_{0.1}, \mathrm{~N}=56\right)$.

\section{Errachidia $002 \quad 31^{\circ} 52^{\prime} 03.4^{\prime \prime} \mathrm{N}, 4^{\circ} 09^{\prime} 55.2^{\prime \prime} \mathrm{W}$}

Draâ-Tafilalet, Morocco

Find: 2017

Classification: HED achondrite (Eucrite, polymict)

History: (H. Chennaoui Aoudjehane, FSAC) Found in a flat area SE of Errachidia called An-Nebch around $8 \mathrm{~km}$ NE of a Jramena village. A single piece of $4.02 \mathrm{~g}$ was collected on 11 Oct 2017 in the field during a systematic prospecting mission by a team of PhD students (FSAC) with $\mathrm{H}$. Chennaoui. This area was selected because of the report of the first sample $17.24 \mathrm{~g}$ that was found there by a hunter on January 2017.

Physical characteristics: (H. Chennaoui Aoudjehane) One stone partially covered by a shiny fusion crust. Fresh pieces showing a low weathering grade. Clear grains of a small size (up to $1 \mathrm{~mm}$ ) are visible through the fusion crust. The fractured face 
shows brecciation with dark and clear areas. Up to $1 \mathrm{~mm}$ sized grains of fresh unoxidized metal are present in the cut portion.

Petrography: (T. Shisseh, FSAC; R. Hewins, S. Pont, MNHNP) Breccia with rock fragments up to $0.7 \mathrm{~mm}$ and lithic clasts up to $1 \mathrm{~mm}$ embedded in a fine grained clastic matrix. Grains of exsolved low-Ca pyroxene with up to $16 \mu \mathrm{m}$ exsolution lamellae, fractured with a $6 \mu \mathrm{m}$ displacement and distorted, are present in the lithic clasts and the matrix. Contains shock veins up to $0.2 \mathrm{~mm}$ with entrained rounded grains of pyroxene, plagioclase and silica. A faulting surface was also observed extending over $1.5 \mathrm{~mm}$. The main minerals are $\mathrm{Ca}$-poor pyroxene, augite and plagioclase, with minor silica, ilmenite, chromite and pure Fe-metal.

Geochemistry: Mineral compositions and geochemistry: (T. Shisseh, H. Chennaoui Aoudjehane, $F S A C$ ) Orthopyroxene compositions are $\mathrm{En}_{37.9} \mathrm{Fs}_{59.5} \mathrm{Wo}_{2.6}$, low-Ca pyroxenes $\mathrm{En}_{32.7} \mathrm{Fs}_{61.8} \mathrm{Wo}_{5.6}$ to $\mathrm{En}_{31.2} \mathrm{Fs}_{59.3} \mathrm{Wo}_{18.3}(\mathrm{~N}=7)$, augite $\mathrm{En}_{51.9} \mathrm{Fs}_{27.2} \mathrm{Wo}_{20.9}$ to $\mathrm{En}_{26.9} \mathrm{Fs}_{29.8} \mathrm{Wo}_{43.4}(\mathrm{~N}=4) \mathrm{Fe} / \mathrm{Mn}=30$ in average. Plagioclase is $\mathrm{An}_{95.2-87.9} \mathrm{Ab}_{4.64-11.7} \mathrm{Or}_{0.12-0.5}(\mathrm{~N}=6)$.

Classification: (H. Chennaoui Aoudjehane, T. Shisseh, FSAC) Polymict eucrite breccia. The meteorite contains anorthite, and pyroxene with $\mathrm{Fe} / \mathrm{Mn}$ ratios matching eucrite, plus typical eucrite textures.

Specimens: The main mass $17.24 \mathrm{~g}$ is held by a private collector. The type specimen consists of $4.4 \mathrm{~g}$ and one polished section in FSAC.

\section{Gandom Beryan $011 \quad 31^{\circ} 52.659^{\prime}$ N, $57^{\circ} 02.920^{\prime} \mathrm{E}$ \\ Kerman, Iran \\ Find: 4 January 2017 \\ Classification: Ordinary chondrite (LL3)}

History: The meteorite was found on 4 January 2017 by the $U r F U$ meteorite expedition-2017 in Iran (Pastukhovich A. Yu., Larionov M. Yu., Kruglikov N.A., Zamyatin D.A.) $5 \mathrm{~km}$ north-east from the middle part of the road Ravar - Dige-eRostam hot springs, Tabas (northern sandy part of the Lut desert).

Physical characteristics: The meteorite has roughly rounded shape. Exterior of the stone is desert polished. The surface and interior of the meteorite is light to dark brown in color due to Fe-hydroxides. Fusion crust is present.

Petrography: Classification (V. V. Sharygin, SIGM and UrFU). The meteorite shows a chondritic texture. The amount of chondrules and their fragments is up to 90 vol.\%. Their sizes vary from $30-50 \mu \mathrm{m}$ to $2 \mathrm{~mm}$ and they are well delineated. They have barred, porphyritic or cryptocrystalline texture and mainly consist of olivine, low-Ca-pyroxene and glassy matrix, diopside, and \pm chromite and blebs of troilite and FeNi-metal. In all chondrules the matrix does not contain fresh glass, now it is fine-devitrified aggregate. Olivine and sometimes low-Ca-pyroxene form skeletal/dendritic crystals and contain silicate-melt inclusions in some chondrules. In addition the crystals of these minerals sometimes show evident zonation. All these indicate the high rate of quenching. The exotic porphyritic chondrules containing olivine, spinel, plagioclase and cryptocrystalline Pl-Cpx matrix (former glass) are very rare. Olivine and low-Ca-pyroxene are main minerals in fine- 
grained matrix; plagioclase was not observed. All petrographic features indicate a petrological type of 3 for the meteorite. Undulatory extinction and irregular fractures in olivine reveal a shock stage of S2. Majority of grains of FeNi-metal $(100-500 \mu \mathrm{m})$ are mainly represented by kamacite, taenite and tetrataenite; but individual grains of kamacite also occur. The Neumann lines were locally observed in kamacite. Rare copper grains (up to $10 \mu \mathrm{m}$ ) were found in tetrataenite-taenitetroilite association. Sizes of troilite grains are 50-200 $\mu \mathrm{m}$. Weathering products (goethite and other Fe-Ni-hydroxides, calcite, Fe-Ni-carbonate) are not very abundant and occur as in situ partial alteration of FeNi-metals and troilite and fill microfractures in all minerals (weathering grade $-\mathrm{W} 2$ ). Pentlandite + Ni-rich pyrrhotite (up to $10 \mu \mathrm{m}$ ) is rarely observed in troilite. Clinopyroxene, chromite and chlorapatite $(50-100 \mu \mathrm{m})$ occur locally in the matrix. Merrillite was found only as rounded inclusions (up to $5 \mu \mathrm{m}$ ) in FeNi-metal and troilite. The fine-grained fusion crust (occasionally vesicular) is up to $100 \mu \mathrm{m}$ and contains zoned olivine, Fe-rich or Fe-poor glass, magnetite and sulfides, rare low-Ca pyroxene. In the chondrules, which were in fusion crust zone, the matrix turned into glass and individual metalsulfide blebs appeared. The blebs consist of troilite, Ni-rich metal and Ni-rich sulfides.

Geochemistry: EDS-WDS analyses (ViV. ctor V. Sharygin, SIGM and UrFU). The primary chondrite paragenesis includes olivine, low-Ca-pyroxene, Cr-bearing clinopyroxene, plagioclase, spinel-supergroup minerals, chlorapatite, merrillite, FeNi-metals and copper. The main minerals vary widely in composition. The averaged olivine is $\mathrm{Fa}_{23.94 \pm 7.36}(\mathrm{~N}=141)$. However its zoned crystals in chondrules show more contrast compositions: core $(\min )-\mathrm{Fa}_{0.80}$, $\operatorname{rim}(\max )-\mathrm{Fa}_{31.53}$; grains from matrix indicate more homogeneous composition - $\mathrm{Fa}_{29.4 \pm 0.61}(\mathrm{~N}=31)$, outlining the LL group. The same situation is with low-Ca-pyroxene: average $\mathrm{Fs}_{14.91 \pm 8.12} \mathrm{Wo}_{1.96 \pm 1.85}(\mathrm{~N}=70)$; chondrules: core $(\min )-\mathrm{Fs}_{1.97}, \operatorname{rim}(\max )-\mathrm{Fs}_{36.93}$; matrix $-\mathrm{Fs}_{19.10 \pm 6.07} \mathrm{Wo}_{1.35 \pm 0.32}(\mathrm{~N}=8)$. Plagioclase is rare: anorthite $\mathrm{An}_{72.9} \mathrm{Ab}_{24.3} \mathrm{Or}_{2.8}(\mathrm{~N}=6)$ occurs in spinel-containing chondrules, whereas albite $\mathrm{Ab}_{93.2} \mathrm{An}_{3.7} \mathrm{Or}_{3.1}(\mathrm{n}=1)$ - in matrix of some POP chondrules. Spinel-supergroup minerals are represented by chromite $\mathrm{Crt}_{87.1} \mathrm{Spl}_{6.5}(\mathrm{~N}=15$, common in matrix), spinel $\mathrm{Spl}_{98.8} \mathrm{Crt}_{0.3}(\mathrm{~N}=6$, in some chondrules) and intermediate chromite-spinel $\mathrm{Crt}_{49.9} \mathrm{Spl}_{47.5}(\mathrm{n}=5$, in some chondrules). Composition of metals (in wt.\%): kamacite $(\mathrm{N}=35)-\mathrm{Fe} 93.16 \pm 0.59, \mathrm{Ni}-5.88 \pm 0.58, \mathrm{Co}-1.00 \pm 0.17$; taenite $(\mathrm{N}=22)-\mathrm{Fe}$ 65.55, Ni 34.02, Co 0.48; tetrataenite $(\mathrm{N}=19)-\mathrm{Fe} 48.43$, Ni 51.25, Co 0.34; copper $(\mathrm{N}=5)-\mathrm{Fe}$ 5.42, Ni 3.02, Cu 91.61. Pentlandite (in wt.\%, $\mathrm{N}=5$ ): $\mathrm{Fe} 46.42$, Ni 19.83 , Co $0.42, \mathrm{~S}-33.49$. Former glasses in chondrules also show contrast compositions: with moderate alkalis $\left(\mathrm{Na}_{2} \mathrm{O}\right.$ 5.3-9.7, $\mathrm{K}_{2} \mathrm{O}$ 2.2-4.1, $\mathrm{SiO}_{2}$ 60.1-77.4, $\mathrm{Al}_{2} \mathrm{O}_{3}$ 10.6-16.3 wt.\%) and nepheline-normative $\left(\mathrm{Na}_{2} \mathrm{O}\right.$ 10.2-15.2, $\mathrm{K}_{2} \mathrm{O}$ 0.2-3.5, $\mathrm{SiO}_{2}$ 47.5-60.7, $\mathrm{Al}_{2} \mathrm{O}_{3}$ 18.0-27.4 wt.\%).

Classification: Ordinary chondrite. LL3, S2, W2. Estimated subtype 3.4/3.9 based on variations of $\mathrm{Fa}$ in olivine and $\mathrm{Fs}$ in low-Ca pyroxene.

Specimens: $2056.6 \mathrm{~g}$ sample - UrFU; $6.53 \mathrm{~g}$ cut-off and thin section - SIGM. 
Ganq $001 \quad 37^{\circ} 29^{\prime} 12.31 " \mathrm{~N}, 92^{\circ} 18^{\prime} 37.84^{\prime \prime} \mathrm{E}$

Qinghai, China

Find: 03 Nov 2013

Classification: Ordinary chondrite (L 6)

History: (Ziyao Wang) Discovered by Liu Binghan (Haixi/Qinghai) in the Gobi desert about $150 \mathrm{~km}$ SE of Huangtouzhen, Haixi Mongolian and Tibetan Autonomous Prefecture, Qinghai province, on Nov. 3, 2013.

Physical characteristics: (Ziyao Wang and R. Bartoschewitz, Bart) One blackbrown almost complete rounded stone of $3907 \mathrm{~g}$ with about $90 \%$ of weathered and eolic eroded fusion crust.

Petrography: (R. Bartoschewitz, Bart) heavy brown stainted recrystallized matrix with poor defined chondrules $(0.4-3 \mathrm{~mm})$ and irregular metal and sulphide inclusions up to $2 \mathrm{~mm}$

Geochemistry: Magnetic susceptibility (R. Bartoschewitz, Bart) $\log \chi\left(\times 10^{-}\right.$ $\left.{ }^{9} \mathrm{~m}^{3} / \mathrm{kg}\right)=4.62$

Classification: Ordinary chondrite (L $\sim 6, \mathrm{~W} 3)$

Specimens: $20.5 \mathrm{~g}$ on deposit at Kiel, Liu Binghan (Haixi/Qinghai) and Wang Ziyao hold the main mass, and $108.5 \mathrm{~g}$ with Bart.

\section{Ganq $002 \quad 37^{\circ} 34^{\prime} 54.59^{\prime \prime} \mathrm{N}, 92^{\circ} 14^{\prime} 45.81^{\prime \prime} \mathrm{E}$}

Qinghai, China

Find: 10 Mar 2014

Classification: Ordinary chondrite (L $\sim 6)$

History: (Ziyao Wang) Discovered by Zhang Qun (Haixi/Qinghai) and Liu Binghan (Haixi/Qinghai) in the Gobi desert about $150 \mathrm{~km}$ SE of Huangtouzhen, Haixi Mongolian and Tibetan Autonomous Prefecture, Qinghai province, on March 10, 2014.

Physical characteristics: (Ziyao Wang and R. Bartoschewitz, Bart) Shield shaped oriented individual of $5340 \mathrm{~g}$. Black, eroded fusion crust presents well-developed flow lines.

Petrography: (R. Bartoschewitz, Bart) weak brown-stained, recrystallized matrix with poorly defined chondrules and irregular metal and sulfide inclusions up to 1 $\mathrm{mm}$

Geochemistry: Magnetic susceptibility (R. Bartoschewitz, Bart $) \log \chi\left(\times 10^{-}\right.$ $\left.{ }^{9} \mathrm{~m}^{3} / \mathrm{kg}\right)=4.95$

Classification: Ordinary chondrite (L $\sim 6, \mathrm{~W} 1)$

Specimens: $20.3 \mathrm{~g}$ on deposit at Kiel, Liu Binghan (Haixi/Qinghai) and Zhang Qun (Haixi/Qinghai) hold the main mass, $150 \mathrm{~g}$ with Wang Ziyao, and $129.6 \mathrm{~g}$ with Bart.

\section{Ganq $003 \quad 37^{\circ} 39^{\prime} 35.17^{\prime \prime N}, 92^{\circ} 15^{\prime} 36.22^{\prime \prime} \mathrm{E}$}

Qinghai, China

Find: 16 Sept 2015 
Classification: Ordinary chondrite (L $\sim 5)$

History: (Ziyao Wang) Discovered by Chen Wie (Haixi/Qinghai) in the desert close to Mangai county, Haixi, Mongolian and Tibetan Autonomous Prefecture on 16 Sept 2015. Liu Binghan (Haixi/Qinghai) purchased the complete stone in 2018. Physical characteristics: (Ziyao Wang) Black-brown stone of $2200 \mathrm{~g}$ without fusion crust

Petrography: (R. Bartoschewitz, Bart) brown stainted recrystallized matrix with well-defined chondrules $(0.4-2 \mathrm{~mm}$, av. $0.7 \mathrm{~mm})$.

Geochemistry: Magnetic susceptibility (R. Bartoschewitz, Bart $) \log \chi\left(\times 10^{-}\right.$ $\left.{ }^{9} \mathrm{~m}^{3} / \mathrm{kg}\right)=4.82$

Classification: Ordinary chondrite (L $\sim 5, \mathrm{~W} 3 / 4)$

Specimens: $20.7 \mathrm{~g}$ on deposit at Kiel, Liu Binghan holds the main mass, $138 \mathrm{~g}$ with Wang Ziyao, and $106 \mathrm{~g}$ with Bart.

\section{Gueltat Zemmour $\quad 25^{\circ} 05^{\prime} 32.0^{\prime} ’ \mathrm{~N}, 12^{\circ} 37^{\prime} 23.9^{\prime} ’ \mathrm{~W}$}

Boujdour, Morocco

Fall: 21 Aug 2018

Classification: Ordinary chondrite (L4)

History: (H. Chennaoui Aoudjehane FSAC, UH2C, Ahmed Bouferra, Mohamed Aid, "Moroccan association of meteorites"). On 21 Aug 212018 around 14:20, many people from the south of Morocco saw a bright fireball. In Smara, Seddiki Mbarek reported the passing of a fireball similar to a yellow-white shining star, moving from the northeast to southwest, in the direction of Gueltat Zemmour. His son Aboubakr also saw the fireball, saying that his sighting only lasted a few seconds before a building blocked the view. Abderrahmane Latifi and his son Adil, also from Smara, both saw a fireball shining red, then yellow, brighter than the Sun, lasting about $3 \mathrm{~s}$. In the fall area, Mahjoub Boutabâa, a nomad, was inside his tent; he heard a sound similar to the explosion of a mine, followed by a few sonic booms that resonated in the entire Oued Labiyad valley, similar to thunder. Abba Essahed, another nomad, and his wife Basaddouk Aicha were out of their tent when they heard a whistling sound almost identical to ocean waves, then five sonic booms akin to the explosion of a mine, making them feel like the mountains would crumble. They saw a very bright fireball comparable to a burning $4 \times 4$ vehicle in terms of size, first red then yellow, more dazzling than the Sun, with a flashing light moving from north to south for a few seconds. There was smoke in the sky directly above them. Aicha was so scared that she went to hide between the water barrels. All testimonies are concordant on the date and the time of August 21 around 14:20. Due to the period of the fall (one day before "Aid El Adha"), hunters could not search immediately. Searching began ten days after the fall and the first pieces were found in mid-September, about three weeks after the fall, a few km to the west of Gueltat Zemmour. A group of hunters: Mohamed Ouicha, Brahim Ouhra, Mbarek Amediaz, Aissa Ouicha and Brahim Ouaadjou found many pieces (5.1, 5.0, 6.3, 7.2, and $15 \mathrm{~g}$ ) in a valley between Wad Awzireft and Wad Labiyad (N25 05'32.0'; W12 37'23.9' ') and north of Koudiat Rjilya. Bigger pieces were 
found SW of this site (N24'53'29.52"; W12 $42^{\circ}$ ' $10.5^{\prime}$ ' ; around $700 \mathrm{~g}$ ) (N24'56'37.06"; W12 40'52.12' '; around $200 \mathrm{~g}$ ). The direction of the fall was NNE-SSW and the strewnfield is about $12 \mathrm{~km}$.

Physical characteristics: A few small pieces totalling around $500 \mathrm{~g}$ and 3 bigger ones ( $5448 \mathrm{~g}, 1374 \mathrm{~g}$ and $426 \mathrm{~g}$ ) not weathered, totally covered by a black matte fusion crust, showing fine grains, very friable material, and easily discernable chondrules. Large pieces are broken and show a gray-colored interior; they are partially covered by fusion crust.

Petrography: (H. Chennaoui Aoudjehane UH2C, L. Folco UniPi) Numerous welldefined chondrules (POP, PO, GPO, PP, RP and glassy chondrules) up to $3 \mathrm{~mm}$. Plagioclase is very small ( $2 \mu \mathrm{m}$ maximum). Mesostasis is microcrystalline. Most metal and sulfides are automorphous grains, um sized, to large grains up to $500 \mathrm{um}$; they occur inside chondrules as small grains, in the mesostasis as small and large grains, and surrounding some chondrules.

Geochemistry: (V. Moggi Cecchi, UniFi, H. Chennaoui Aoudjehane UH2C) Olivine $\mathrm{Fa}_{26.0 \pm 0.7} \mathrm{Fe} / \mathrm{Mn}=51.23(\mathrm{~N}=10)$, low-Ca pyroxene

$\mathrm{Fs}_{20.7 \pm 0.8} \mathrm{Wo}_{1.1 \pm 0.5} \mathrm{Fe} / \mathrm{Mn}=28.51(\mathrm{~N}=7)$, plagioclase $\mathrm{An}_{8.2 \pm 2.4} \mathrm{Or}_{4.8 \pm 0.8}(\mathrm{~N}=3)$, Taenite Ni 50.3 $\pm 2.7(\mathrm{~N}=3)$ Whole-rock trace-element concentrations by ICP-MS (M. D’Orazio, UniPi): V 59, Cr 2799, Ni 10115, Co 399, La 0.329, Ce 0.879, Pr 0.138, Nd 0.645, Sm 0.211, Eu 0.088, Gd 0.284, Tb 0.054, Dy 0.364, Ho 0.081, Er 0.242, Tm 0.035, Yb 0.236, Lu 0.038 (units $=\mu \mathrm{g} / \mathrm{g})$. Cosmogenic radionuclides: $(\AA$. Rosén, B. Hofmann, NMBE, GeMSE): Gamma-spectroscopy performed in October 2018 on a $21.74 \mathrm{~g}$ sample showed the presence of several short-lived cosmogenic radionuclides $\left({ }^{57} \mathrm{Co},{ }^{46} \mathrm{Sc},{ }^{56} \mathrm{Co},{ }^{58} \mathrm{Co},{ }^{7} \mathrm{Be}\left(\mathrm{t}_{1 / 2}=53 \mathrm{~d} ; 84.6 \pm 10 /-7\right.\right.$ $\mathrm{dpm} / \mathrm{kg})$ and ${ }^{48} \mathrm{~V}\left(\mathrm{t}_{1 / 2}=16 \mathrm{~d} ; 17.8 \pm 4.6 /-5.4 \mathrm{dpm} / \mathrm{kg}\right)$. Recalculated to August 21, 2018 the ${ }^{22} \mathrm{Na} /{ }^{26} \mathrm{Al}$ activity ratio is $1.69 \pm 0.1$. These data confirm that it is a fresh fall. The ${ }^{26} \mathrm{Al}$ activity of $55.5 \pm 3.6 /-3.1 \mathrm{dpm} / \mathrm{kg}$ and absence of detected ${ }^{60} \mathrm{Co}(<0.8$ $\mathrm{dpm} / \mathrm{kg}$ ) indicates only a few cm of shielding.

Classification: L4

Specimens: A sample of $20.1 \mathrm{~g}$ provided by the "Moroccan association of meteorites", small pieces totaling $30 \mathrm{~g}$ provided by Aaronson, FSAC. Aaranson: $1374+426$ g; Mohamed Aid: 5448 g; Private collector, Casablanca: $21+7.2+6.3$ $+5.1+5 \mathrm{~g}$.

\section{Gumu $\quad 33^{\circ} 10.17^{\prime} \mathrm{N}, 85^{\circ} 41.86^{\prime} \mathrm{E}$}

Tibet, China

Find, possible fall: June 25, 2018

Classification: Ordinary chondrite (H6)

History: There was a rumor that a meteorite fall had happened in the Qiangtang region of Tibet. A local farmer first found a $0.5 \mathrm{~kg}$ meteorite on June 25, 2018. After that, $14 \mathrm{~kg}$ and $24.5 \mathrm{~kg}$ were collected in the same location on July 1 and Aug $5,2018$.

Physical characteristics: About 20 pieces were found, with a total weight of $\sim 39$ $\mathrm{kg}$. A few of them are covered with black fusion crust, and some show rusty spots. 
Petrography: (N. Wang, Y. Xu and Y. Lin, IGGCAS) It is an equilibrated oridinary chondrite, with homogeneous compositions of olivine and pyroxene. Most of the feldspars are $>50 \mu \mathrm{m}$ in size. The chondrules are very poorly delineated. Fe-Ni metal and troilite, with minor phosphate make up about $20 \%$ of the volume.

Geochemistry: The chemical compositions of olivine and pyroxene are homogeneous across the thin section. Olivine: $\mathrm{Fa}_{19.1 \pm 1.2}(\mathrm{n}=28)$, orthopyroxene: $\mathrm{Fs}_{16.3 \pm 0.9} \mathrm{Wo}_{1.4 \pm 0.4}(\mathrm{n}=34)$

Classification: H6 (N. Wang, Y. Xu and Y. Lin, IGGCAS) Specimens: Main mass with P. Chen.

\section{Hamburg $\quad 42^{\circ} 26.82^{\prime} \mathrm{N}, 83^{\circ} 50.5^{\prime} \mathrm{W}$ \\ Michigan, United States \\ Confirmed fall: 2018 Jan 16 \\ Classification: Ordinary chondrite (H4)}

History: On 16 January 2018 around 20:10 EST, a fireball was observed and reported by hundreds of people from seven states. The fireball was recorded by multiple security cameras and registered as a 2.0 magnitude earthquake. Mike Hankey $(A m M S)$ calculated the trajectory within one hour. A strewnfield map was produced by Marc Fries (JSC) using weather radar data from radar reflections of falling meteorites. The first fragment was found on 18 January 2018 at 7:50 EST by Robert Ward on Strawberry Lake near Hamburg, Michigan. Five other masses, ranging from 17 to $102.6 \mathrm{~g}$, were found later the same day by Ward, Larry Atkins, and Darryl Landry on Strawberry and Bass Lakes. Thirteen additional pieces were found within two weeks of the fall.

Physical characteristics: Most stones are fully covered by fusion crust. Petrography: Metal-rich texture typical of $\mathrm{H}$ chondrites is apparent on cut surfaces. The metal volume estimate is $9 \%$ based on an Fe EDS map. Chondrules are visible in polished section. The average chondrule diameter is $0.4 \pm 0.2 \mathrm{~mm}$ $(\mathrm{N}=26)$. Several chondrules are well-defined and have sharp boundaries. Pyroxenes are mostly orthopyroxene and less abundant clinopyroxene as determined with Raman spectroscopy. Feldspar grains vary in size and measure $3.4 \pm 2.2 \mu \mathrm{m}(\mathrm{n}=64)$ in a representative field of view. Chromites are impact fractured. No chromite veinlets nor opaque veins are observed within the meteorite. Phosphates account for about $0.5 \%$ of the meteorite and occur mainly as merrillite $(0.4 \%)$ and apatite $(0.1 \%)$. In a representative field of view, grain sizes for merrillite average around $100 \mu \mathrm{m}$ and range from $<1 \mu \mathrm{m}$ up to $430 \mu \mathrm{m}(\mathrm{n}=37)$; apatite ranges from $70 \mu \mathrm{m}$ to $310 \mu \mathrm{m}$ with an average of $150 \mu \mathrm{m}(\mathrm{n}=8)$. The meteorite appears very weakly shocked. Melt veins (Fe-sulfide) occur only in close proximity to the fusion crust. Geochemistry: Mineral Compositions and geochemistry: Olivine $\mathrm{Fa}_{18.7 \pm 0.7}(\mathrm{~N}=34)$. The average composition of Ca-poor $\mathrm{px}$ is $\mathrm{Fs}_{16.3 \pm 0.4} \mathrm{Wo}_{1.3 \pm 0.1}(\mathrm{n}=80)$. Feldspar is present with an average composition of $\mathrm{An}_{14.0 \pm 4.0} \mathrm{Ab}_{81.1 \pm 3.0} \mathrm{Or}_{4.8 \pm 1.3}(\mathrm{n}=13)$. Chromite: $\mathrm{TiO}_{2}=2.0 \pm 0.4 \mathrm{wt} \% \mathrm{~V}_{2} \mathrm{O}_{3}=0.8 \pm 0.2 \mathrm{wt} \%(\mathrm{~N}=25)$. Apatite contains about 5 $\mathrm{wt} \% \mathrm{Cl}$ and $<1 \mathrm{wt} \% \mathrm{~F}$. 
Classification: The petrology, mineral chemistry and compositional hetereogeneity of the minerals listed is consistent with $\mathrm{H} 4$ chondrites. The meteorite is classified as $\mathrm{H} 4, \mathrm{~S} 2$, W0.

Specimens: Type specimen $22.8 \mathrm{~g}$ and polished section at $F M N H ; 102.6 \mathrm{~g}$ specimen with Robert Ward; $44.5 \mathrm{~g}$ specimen with Terry Boudreaux.

\section{Hami $007 \quad 42^{\circ} 08^{\prime} 4.55^{\prime \prime} \mathrm{N}, 93^{\circ} 25^{\prime} 31.72^{\prime \prime} \mathrm{E}$}

Xinjiang, China

Find: 2013 Oct 12

Classification: Ordinary chondrite (H5)

History: The meteorite was found during searching for meteorites near Yamasu ore mine on Oct. 12, 2013. Subsequently, it was purchased by Qiang Xu.

Physical characteristics: complete black fusion crust. The bottom on the desert shows rusty spots.

Petrography: (Y. Xu, N. Wang and Y. Lin, IGGCAS) The meteorite consists mainly of olivine, pyroxene, plagioclase, Fe-Ni metal, and troilite, with minor chromite, chlorapatite and whitlockite.

Geochemistry: Mineral compositions and geochemistry: The olivine, pyroxene and plagioclase have homogeneous chemical compositions. Olivine: $\mathrm{Fa}_{18.6 \pm 0.6}(\mathrm{n}=22)$, orthopyroxene: $\mathrm{Fs}_{16.1 \pm 0.6} \mathrm{Wo}_{1.0 \pm 0.7}(\mathrm{n}=27)$. Plagioclase: $\mathrm{An}_{12}(\mathrm{n}=25)$

Classification: $\mathrm{H} 5$ (Y Xu, N. Wang and Y. Lin, IGGCAS), probably paired with Hami 001, Hami 003, and Hami 005

Specimens: the main mass was hold by Q. Xu

\section{Hami $008 \quad 42^{\circ} 27^{\prime} 51.71 " \mathrm{~N}, 93^{\circ} 13^{\prime} 68.47^{\prime \prime} \mathrm{E}$}

Xinjiang, China

Find: 16 Nov 2016

Classification: Ordinary chondrite (L 6)

History: (Ziyao Wang) The meteorite was discovered by Zijian Wang $45 \mathrm{~km} \mathrm{SW}$ of Hami city, Xinjiang province on 16 Nov 2016. Three fragments were discovered within a distance of $1.2 \mathrm{~m}$.

Physical characteristics: (Ziyao Wang) Three brown heavy fractured irregular fragments of $475 \mathrm{~g}, 75.4 \mathrm{~g}$, and $39 \mathrm{~g}$, in total $589.4 \mathrm{~g}$.

Petrography: (R. Bartoschewitz, Bart) brown stainted recrystallized matrix with poor defined chondrules $(0.6-1.2 \mathrm{~mm}$, av. $0.8 \mathrm{~mm})$

Geochemistry: Magnetic susceptibility (R. Bartoschewitz, Bart) $\log \chi\left(\times 10^{-}\right.$ $\left.{ }^{9} \mathrm{~m}^{3} / \mathrm{kg}\right)=4.79$

Classification: Ordinary chondrite (L $\sim 6, \mathrm{~W} 3$ )

Specimens: $21.7 \mathrm{~g}$ on deposit at Kiel, Wang Ziyao holds the main mass, and $64 \mathrm{~g}$ with Bart.

\section{Hermenegildo $\quad 33^{\circ} 39.07^{\prime} \mathrm{S}, 53^{\circ} 14.90^{\prime} \mathrm{W}$}


Rio Grande do Sul, Brazil

Find: 2004

Classification: Ordinary chondrite (H5)

History: After a large fireball was seen at 1997, several masses of meteorites were found near Santa Vitória do Palmar (RS, Brazil). This mass was found in the sand area of the swamp near the beach after light and thunder had been observed by the finder, who reported the phenomena as UFOs. Despite the great enthusiasm of the finder, he probably witnessed an ignis fatuus due to the marsh or ore deposits, with no relation to the meteorite.

Physical characteristics: A single small weathered irregular mass with about 300 $\mathrm{g}$ and lacking fusion crust.

Petrography: (M. E. Zucolotto, MNRJ, IGEO-UFRJ and C. Villaça, IGEO-UFRJ) Polished thin and microprobe sections show a chondritic texture. A variety of textural types of chondrules and chondrules fragments were observed, embedded in a fine to microcrystalline matrix. Plagioclase grains do not exceed $50 \mu \mathrm{m}$ in size. Metal grains include kamacite, zoned taenite and zoneless plessite. Mono crystalline chromite is present. The presence of sets of planar fractures in olivine and undulatory extinction and mosaicism of olivine with no maskelynite indicate a S4 shock stage. Moderately weathered with the entire sample percolated by hydrated ferric oxide veins; the iron metal nickel is partly transformed into oxides indicatives of weathering grade $\mathrm{W} 2$

Geochemistry: A. Tosi, LABSONDA/IGEO-UFRJ) Olivine $\mathrm{Fa}_{19.3 \pm 0.6}(\mathrm{n}=29)$, pyroxene $\mathrm{Fs}_{17.3 \pm 0.9} \mathrm{Wo}_{1.14 \pm 0.15}(\mathrm{n}=9)$

Classification: Ordinary chondrite (H5), shock stage S4, weathering (W2).

Specimens: Two samples (12 $\mathrm{g}$ and $15 \mathrm{~g}$ ) at MNRJ, $10 \mathrm{~g}$ Pulo Martini INPE. Main mass at Museu Tancredo Mello

\section{Hoboksar $001 \quad 46^{\circ} 49^{\prime} 51.07^{\prime \prime} \mathrm{N}, 85^{\circ} 42^{\prime} 4.33^{\prime \prime} \mathrm{E}$ \\ Xinjiang, China}

Find: 2018 Jun

Classification: Ordinary chondrite $(\mathrm{L} \sim 6)$

History: (Ziyao Wang) A 544 g meteorite fragment was discovered by Zijian Wang in Hoboksar Mongol Autonomous County, Tarbagatay Prefecture, Ili Kazak Autonomous Prefecture, Xinjiang province in Jun 2018. In October he searched for missing fragments and discovered some within a distance of $20 \mathrm{~m}$. In November, the main mass of $6.9 \mathrm{~kg}$ was discovered $500 \mathrm{~m}$ away.

Physical characteristics: (Wang Ziyao) Black brown $6 \mathrm{~kg}$ broken individual with fresh black fusion crust and some smaller fragments of $2 \mathrm{~kg}$ that fit to a nearly complete stone. The main mass of $6.9 \mathrm{~kg}$ is a part individual with fresh fusion crust.

Petrography: (R. Bartoschewitz, Bart) recrystallized matrix with poor defined chondrules (0.6-1.2 mm, av. $0.7 \mathrm{~mm}$ ) and irregular metal and sulphide inclusions. Geochemistry: Magnetic susceptibility (R. Bartoschewitz, Bart) $\log \chi\left(\times 10^{-}\right.$ $\left.{ }^{9} \mathrm{~m}^{3} / \mathrm{kg}\right)=4.95$ 
Classification: Ordinary chondrite (L $\sim 6, \mathrm{~W} 1$ )

Specimens: $22.9 \mathrm{~g}$ on deposit at Kiel, Wang Ziyao holds the main mass, and $58 \mathrm{~g}$ with Bart.

\section{Ilafegh $018 \quad 21^{\circ} 20^{\prime} 31.93^{\prime \prime} \mathrm{N}, 0^{\circ} 49^{\prime} 26.02^{\prime \prime} \mathrm{E}$ \\ Adrar, Algeria \\ Find: 2001 Dec \\ Classification: Iron meteorite (IIIAB)}

History: Compact, heavily sculpted mass with well-preserved fusion crust on large parts of the surface and only a thin layer of surface oxidation. The mass shows distinct regmaglypts and heavy ablation of edges. The iron was found by nomadic Berbers in 2001, "probed" for possible gold or silver content with negative results, and left in place, remembered only as a curiosity. Only in 2015, when the finders made contact with Moroccan meteorite dealers, was the iron was recovered from its find location and recognized as an iron Meteorite, and subsequently purchased by S. Buhl.

Physical characteristics: Single mass with regmaglypts and remnants of fusion crust on large parts of the surface. Minor surface oxidation.

Petrography: (J. Gattacceca, CEREGE): The etched section shows octahedrite texture with a $1-2 \mathrm{~mm}$ heat alteration zone. Only three kamacite bands are visible in the section, with average width $1.4 \mathrm{~mm}$. Kamacite is polycristalline and in the epsilon form. Comb, black and net plessite are present. No accessory minerals were observed.

Geochemistry: (M. D'Orazio, DST-PI): Composition of the metal is $\mathrm{Co}=0.44$, $\mathrm{Ni}=7.7$ (both in wt\%), $\mathrm{Cu}=184, \mathrm{Ga}=20.3, \mathrm{Ge}=41, \mathrm{As}=4.9, \mathrm{Mo}=6.5, \mathrm{Ru}=10.8$, $\mathrm{Rh}=1.85, \mathrm{Pd}=3.10, \mathrm{Re}=0.48, \mathrm{Ir}=5.5, \mathrm{Pt}=11.5, \mathrm{Au}=0.67$ (all in ppm). $\mathrm{Ni}$ and $\mathrm{Co}$ have been measured by hand-held XRF following Gemelli et al. 2015; all other elements by ICP-MS following D'Orazio and Folco (2003).

Classification: Iron, IIIAB.

\section{Jeminay $\quad 47^{\circ} 52^{\prime} 59^{\prime \prime} \mathrm{N}, 85^{\circ} 54^{\prime} 22^{\prime \prime} \mathrm{E}$ \\ Xinjiang, China}

Find: 6 June 2017

Classification: Iron meteorite (ungrouped)

History: Found by Ye Erbo Lati and his companions Wang Ziyao and Yang Guang on June 6, 2017, while they were using metal detectors to search for meteorites in the Gobi Desert. The location is near the small town of Qiake'er in the Xinjiang Uyghur Autonomous Region, Altay, Jeminay County, China.

Physical characteristics: The main mass is discoid in shape, approximately $3 \mathrm{~cm}$ thick, with a glossy brown to red-brown exterior.

Petrography: (C. Herd, $U A b)$ Optical and SEM investigation of a $\sim 1 \times 2.5 \mathrm{~cm}$ polished and etched surface reveals a well-developed Widmanstatten pattern consistent with a finest octahedrite $(0.06 \pm 0.02 \mathrm{~mm} ; \mathrm{n}=66)$, but having a matte 
appearance typical of hatched ?-structure. In detail, shock-hatching predominates, grain boundaries are irregular, and low-Ni areas consist of irregular, wavy grains. Inclusions on cut surfaces of other samples were noted, but not available for study. Geochemistry: ICP-MS data, using sample of North Chile (Filomena) as standard (C. Herd and G. Chen $U A b$ ): $\mathrm{Ni}=16.7, \mathrm{Co}=0.93$ (both $\mathrm{wt} \%$ ); $\mathrm{Cu}=341, \mathrm{Ga}=8$, $\mathrm{As}=12, \mathrm{~W}=0.7, \mathrm{Ir}=13.4, \mathrm{Pt}=20.0, \mathrm{Au}=0.99($ all $\mu \mathrm{g} / \mathrm{g})$.

Classification: (C. Herd, $U A b$ ): Ungrouped, finest octahedrite. Although high $\mathrm{Ni}$ and Ir are suggestive of the IVB group, other element concentrations fall outside of known ranges for IVB irons.

Specimens: Type specimen consisting of two representative slices (13.5 $\mathrm{g}$ and 12.8 g) at $U A b$. Wang Ziyao $3 \mathrm{~kg}$, Ye Erbo Lati $1.5 \mathrm{~kg}$, Yang Guang $1.5 \mathrm{~kg}$, Mendy Ouzillou $300 \mathrm{~g}$.

\section{Jiddat al Harasis $846(\mathrm{JaH} 846) \quad 19.839^{\circ} \mathrm{N}, 55.870^{\circ} \mathrm{E}$}

Al Wusta, Oman

Find: 2011

Classification: Ordinary chondrite (type 3 )

History: Found by an anonymous prospector in Oman in 2011.

Petrography: (A. Irving and S. Kuehner, UWS) Separated, well-formed, mediumsized chondrules (apparent diameter $560 \pm 380 \mu \mathrm{m}, \mathrm{N}=40$; some up to $2.2 \mathrm{~mm}$ ) in a finer grained matrix. Minerals are olivine, orthopyroxene, clinopyroxene, sodic plagioclase, silica polymorph, chromite, troilite and altered kamacite. Some chondrules are devoid of olivine and are composed predominantly of orthopyroxene + a silica polymorph.

Geochemistry: Olivine $\left(\mathrm{Fa}_{0.6-38.1} ; \mathrm{Cr}_{2} \mathrm{O}_{3}\right.$ in ferroan olivine $0.04-0.22 \mathrm{wt} . \%$, mean 0.08 wt.\%, sd 0.06 wt.\%, $\mathrm{N}=9$ ), orthopyroxene $\left(\mathrm{Fs}_{1.9-35.2} \mathrm{Wo}_{0.5-4.4}, \mathrm{~N}=3\right.$ ), clinopyoxene $\left(\mathrm{Fs}_{6.0-6.1} \mathrm{Wo}_{43.6-44.8}, \mathrm{~N}=2\right)$. Oxygen isotopes $(\mathrm{K}$. Ziegler, $U N M)$ : analyses of 7 acid-washed subsamples by laser fluorination gave $\delta^{17} \mathrm{O} 4.988,3.882$, 4.098, 4.396, 4.262, 4.762, 4.838; $\delta^{18} \mathrm{O} 8.668,6.724,6.533,7.237,6.999,8.155$, 8.124; $\Delta^{17} \mathrm{O} 0.411,0.332,0.649,0.575,0.567,0.456,0.549$ per mil. Magnetic susceptibility $\log \chi\left(\times 10^{-9} \mathrm{~m}^{3} / \mathrm{kg}\right)=4.91$.

Classification: Ordinary chondrite (OC3). Unusual features of this specimen include chondrules dominated by orthopyroxene + a silica polymorph, oxygen isotopic compositions closer to the TFL than for typical ordinary chondrites with similar degree of desert weathering and extending to very high $\delta^{18} \mathrm{O}$ values. Specimens: Type specimen plus one polished thin section are at $P S F$; main mass with the anonymous finder.

Jiddat al Harasis $1100(\mathrm{JaH} 1100) \quad 19^{\circ} 42.31^{\prime} \mathrm{N}, 56^{\circ} 35.64^{\prime} \mathrm{E}$

Al Wusta, Oman

Find: 15 Feb 2015

Classification: Ureilite 
Physical characteristics: A 365 g sample supplied by the Sultan Qaboos University, which has a millimeter thick, dark brown-grey fusion crust present on all faces except one cut face. This fusion crust lacks remaglypts instead has a mainly rough surface. There are no contraction cracks present on this sample. This rock has already been cut and there is a clean face already present. Elongated grey laths can be identified on the cut face, with most less than $0.5 \mathrm{~cm}$ wide. The sample is also magnetic.

Petrography (P. Hill, UWO) The sample is composed of elongated, round grains of olivine and pyroxene that are $\sim 1-2 \mathrm{~mm}$ wide and occasionally meet at $120^{\circ}$ angles. Olivine ( $\sim 60 \mathrm{vol} \%$ ) is more modally abundant than pyroxene (30 vol $\%)$. The sample is fairly porous with large gaps between pyroxene and olivine grains; however, there is a lack of porosity between grains of the same mineralogy. Instead, iron oxides are present and have filled in most of the fractures. Within some of the larger pores, graphite is observed. The graphite is associated with the olivine grains, with only a few occurrences near the pyroxene. Pyroxene is often associated with small porous inclusions throughout the grains, often running perpendicular to the elongated nature of the grain and a plane of cleavage. These inclusions are often filled with iron oxides. The reduction rims observed in olivine grains are $<50 \mu \mathrm{m}$ thick fitting within the $\mathrm{R} 2$ grade put forward by Wittke et al. (2007). Very little kamacite or taenite are present, most are occurring as small inclusions. Most of the metal has oxidized, ( $>95 \%)$. Overall there is little evidence of the silicate phases weathering and no weathering phase was identified in abundance. Overall, this meteorite has experienced moderate terrestrial weathering with most of the Fe-Ni metal being oxides but no evidence of alteration in the silicates phases. The olivine within these samples does show undulose extinction and the mosaic texture of the olivines suggesting this sample has been highly shocked.

Geochemistry: Mineral composition and geochemistry (M. Beauchamp, P. Hill, $U W O$ ) Olivine $\left(\mathrm{Fa}_{23.9 \pm 1.5}\right)$, low-Ca pyroxene $\left(\mathrm{Fs}_{15.5 \pm 2.8} \mathrm{Wo}_{7.9 \pm 2.9}\right)$

Classification: Classificaiton: Ureilite, S5, W3. Specimens: $365 \mathrm{~g}$ type specimen, including polished thin section, are on deposit at $S Q U$.

Jiddat al Harasis $1101(\mathrm{JaH} 1101) \quad 1^{\circ} 44.58^{`} \mathrm{~N}, 56^{\circ} 41.22^{\prime} \mathrm{E}$

Al Wusta, Oman

Find: 15 Feb 2015

Classification: Ureilite

Physical characteristics: A 460 g sample supplied by the Sultan Qaboos University, which has a millimeter thick, dark brown-gray fusion crust present on all faces except one cut face. This fusion crust lacks remaglypts instead has a mainly rough surface. There are no contraction cracks present on this sample. This rock has already been cut and there is a clean face already present. Rounded grey laths can be identified on the cut face, with most less than $0.5 \mathrm{~cm}$ wide. The sample is also magnetic. 
Petrography (P. Hill, UWO) The sample is a composed of elongated grains of olivine and pyroxene that are $\sim 1-2 \mathrm{~mm}$ wide. Olivine is much more prevalent than pyroxene ( $60 \mathrm{vol} \%$ vs. $30 \mathrm{vol} \%$ ). The olivine grains show mocaicized texture with some grains of olivine exhibiting undulatory extinction suggesting high levels of shock. Pyroxene grains are fractured but still have clear and distinct extinction. The grain boundaries are defined by oxides and graphite. Pyroxene is often associated with small porous inclusions throughout the grains. Though distinct exsolution lamellae are not present, small blebs of a more Ca-rich pyroxene are present throughout the pyroxene grains. In particular, a single grain of pyroxene $(100 \mu \mathrm{m}$ wide) is surrounded by olivine and completely rimmed by iron oxide that in some places is $50 \mu \mathrm{m}$ thick. The olivine contains reduction rims $\sim 50 \mu \mathrm{m}$ thick with significant amounts of graphite remaining. Based on the classification put forward by Wittke et al. (2007), this sample fits in the R2 grade. Though the grain boundaries are defined by iron oxides, kamacite and taenite are present as inclusions within grains where fractures have not exposed the metal to terrestrial weathering. In one occurrence an iron oxide rim surrounds a Fe-Ni core. Overall there is little evidence of the silicate phases weathering; however, several sulfate veins were observed. This sample has been moderately weathered based on the presence of sulfates, oxidization of most Fe-Ni metal, but the absence of any silicate weathering phase. The olivine within these samples does show undulose extinction and the mosaic texture of the olivines suggest higher levels of shock metamorphism.

Geochemistry: Mineral composition and geochemistry (M. Beauchamp, P. Hill, $U W O$ ) Olivine $\left(\mathrm{Fa}_{23.8 \pm 3.0}\right)$, low-Ca pyroxene $\left(\mathrm{Fs}_{15.8 \pm 2.0} \mathrm{Wo}_{7.5 \pm 1.7}\right)$

Classification: Classificaiton: Ureilite, S5, W3.

Specimens: $460 \mathrm{~g}$ type specimen, including polished thin section, are on deposit at $S Q U$.

\section{Jiddat al Harasis $1102(\mathrm{JaH} 1102) \quad 19^{\circ} 25.34^{\prime} \mathrm{N}, 55^{\circ} 53.44^{\prime} \mathrm{E}$}

Al Wusta, Oman

Find: 15 Feb 2015

Classification: Ureilite

Physical characteristics: A 95 g sample supplied by the Sultan Qaboos University, which has a millimeter thick, dark brown-gray fusion crust present on all faces except one cut face. This fusion crust lacks remaglypts on 3 of faces, however a smooth face with small remaglypts is present. There are no contraction cracks present on this sample. This rock has already been cut and there is a clean face already present. Rounded grey laths can be identified on the cut face, with most less than $0.5 \mathrm{~cm}$ wide. The sample is also magnetic.

Petrography (P. Hill, UWO) The sample is a composed of elongated grains of olivine and pyroxene that are $\sim 1-2 \mathrm{~mm}$ wide, often meeting at $120^{\circ}$ angles. The olivine grains show mocaicized texture suggesting a high level of shock. Pyroxene grains are fractured but still have sharp, distinct extinction. The grain boundaries are defined by oxides, and there is little porosity between grain boundaries. 
Pyroxene is often associated with small porous inclusions throughout the grains. Though the grain boundaries are defined by iron oxides, kamacite and taenite are present as inclusions within grains where fractures have not exposed the metal to terrestrial weathering. In one occurrence and iron oxide rim surrounds a Fe-Ni core; however, most of the iron oxide occurs as veins running along grain boundaries. Troilite was also observed as a large $(200 \mu \mathrm{m})$ inclusion within a pyroxene grain. Though diamonds and carbide were not observed within these thin sections, graphite is observed. The reduction rims of olivine grains are $\sim 50 \mu \mathrm{m}$ thick. According to the classification system put forward by Wittke et al. (2007), this sample fits with the R2 grade. Overall there is little evidence of the silicate phases weathering except for the occurrence of several sulphate veins. The olivine within these samples does show undulose extinction and the mosaic texture of the olivines suggest high levels of shock metamorphism.

Geochemistry: Mineral composition and geochemistry (M. Beauchamp, P. Hill, $U W O$ ) Olivine $\left(\mathrm{Fa}_{21.8 \pm 2.0}\right)$, low-Ca pyroxene $\left(\mathrm{Fs}_{16.6 \pm 1.6} \mathrm{Wo}_{6.8 \pm 1.9}\right)$

Classification: Classificaiton: Ureilite, S5, W3.

Specimens: $95 \mathrm{~g}$ type specimen, including polished thin section, are on deposit at $S Q U$.

Jiddat al Harasis 1103 (JaH 1103)

$19^{\circ} 46.660^{`} \mathrm{~N}, 56^{\circ} 32.840^{`} \mathrm{E}$

Al Wusta, Oman

Find: 2014 May 7

Classification: Ureilite

History: The meteorite was found in 2014 during a field trip in the Oman desert.

Physical characteristics: Brownish individual lacking any fusion crust.

Petrography: The meteorite shows a cumulate texture of up to $1.8 \mathrm{~mm}$ sized olivine and pigeonite grains. Olivine appears to be recrystallized to small up to 15 $\mu \mathrm{m}$ sized crystallites and pigeonite grains display patchy compositional zoning. Olivine shows characteristic reduced rims. The meteorite also contains flaky graphite.

Geochemistry: Reduced rims in olivine: $\mathrm{Fa}_{6.4-14.0}$; olivine contains $0.43 \pm 0.08 \mathrm{wt} \%$ $\mathrm{Cr}_{2} \mathrm{O}_{3}$ and $0.29 \pm 0.05 \mathrm{wt} \% \mathrm{CaO}$

\section{Jinchang $001 \quad 38^{\circ} 24^{\prime} 45.56^{\prime \prime} \mathrm{N}, 102^{\circ} 2^{\prime} 48.71^{\prime \prime} \mathrm{E}$}

Gansu, China

Find: 22 Aug 2015

Classification: Ordinary chondrite $(\mathrm{H} \sim 6)$

History: (Ziyao Wang) Discovered by Wang Ziyao in a Gobi desert area of Yongchang county, $15 \mathrm{~km} \mathrm{SW}$ of Jinchang city, Gansu province, on Aug. 22, 2015. Physical characteristics: (Ziyao Wang and R. Bartoschewitz, Bart) Black-brown stone of $813 \mathrm{~g}$ with rusty fusion crust with adhering sand grains.

Petrography: (R. Bartoschewitz, Bart) Brown-stained, recrystallized matrix with poorly defined chondrules and irregular metal and sulfide specks up to $0.6 \mathrm{~mm}$ 
Geochemistry: Magnetic susceptibility (R. Bartoschewitz, Bart $) \log \chi\left(\times 10^{-}\right.$ $\left.{ }^{9} \mathrm{~m}^{3} / \mathrm{kg}\right)=5.27$

Classification: Ordinary chondrite (H 6, W2)

Specimens: $20.5 \mathrm{~g}$ on deposit at Kiel, Wang Ziyao holds the main mass, and 114.8 g with Bart.

\section{Karimati $\quad 25^{\circ} 54^{\prime} \mathrm{N}, 80^{\circ} 10^{\prime} 31^{\prime \prime} \mathrm{E}$ \\ Uttar Pradesh, India \\ Confirmed fall: 28 May 2009 \\ Classification: Ordinary chondrite (L5)}

History: Around noon on 28 May 2009, an eyewitness in the village of Karimati heard a roaring sound and a blackish stone fell near a wall in his house, $1.5 \mathrm{~m}$ away from him. The fall created an $18 \times 15 \mathrm{~cm}$ oval-shaped pit in the ground. Out of fear, villagers put the stone in a water tank for about $72 \mathrm{hrs}$. When the local authorities came to know about it, they took the material in their possession. Dr. H.C. Verma (IIT) visited the place on June 2, following a newspaper report and took possession of the sample. Dr. Verma sent the meteorite to $P R L$, and it was then transferred to GSI.

Physical characteristics: The stone is covered with a black crust, except on one face, where a piece has been broken off by the villagers. The sample has rounded to sub-rounded edges. Regmaglypts are also observed. A few chondrules are visible by naked eye.

Petrography: The meteorite is composed of olivine + orthopyroxene + feldspathic glass (plagioclase composition) $+\mathrm{Fe}-\mathrm{Ni}$ metals (kamacite and taenite) + troilite \pm apatite \pm merrillite. Clinopyroxene is rare. The chondrules are composed of olivine and orthopyroxene, whereas the interstitial spaces (mesostasis) are occupied by feldspathic glass. Chondrule range up to $1000 \mu \mathrm{m}$ in diameter. The shape of chondrules varies from near circular to elliptical to sub-elliptical. Barred olivine (BO), granular pyroxene (GP), porphyritic olivine (PO), porphyritic olivine pyroxene (POP) and granular olivine (GO) chondrules are present. Matrix is relatively fine grained and thoroughly recrystallized in which a feldspathic melt mosaic is disseminated. The feldspathic melt (plagioclase composition) is fine grained $(<50 \mu \mathrm{m})$, patchy and interconnected in the matrix. At places it forms melt veins. The matrix is also brecciated. Troilite forms rim around kamacite grains.

Classification: Ordinary chondrite (L5)

Specimens: Main mass at GSI.

\section{Kumtag $040 \quad 41^{\circ} 53^{\prime} 15.67^{\prime \prime N}, 93^{\circ} 55^{\prime} 44.80^{\prime \prime} \mathrm{E}$}

Xinjiang, China

Find: 18 Aug 2012

Classification: Ordinary chondrite (LL 4)

History: (Ziyao Wang) Discovered by Wang Ziyao in the Gobi Desert, $4 \mathrm{~km}$ E of Yamansu Town, near Hami city in Xinjiang province on Aug. 18, 2012. 
Physical characteristics: (Ziyao Wang and R. Bartoschewitz, Bart) One darkbrown, stone broken into two pieces that fit together, totaling $560 \mathrm{~g}$. No fusion crust recognizable.

Petrography: (R. Bartoschewitz, Bart) Gray, brecciated texture of microcrystalline matrix with well-defined deformed chondrules up to $2 \mathrm{~mm}$ and irregular metal and sulfide specks up to $0.5 \mathrm{~mm}$.

Geochemistry: Magnetic susceptibility (R. Bartoschewitz, Bart $) \log \chi\left(\times 10^{-}\right.$ $\left.{ }^{9} \mathrm{~m}^{3} / \mathrm{kg}\right)=4.44$

Classification: Ordinary chondrite (LL 4, W1)

Specimens: $22.7 \mathrm{~g}$ on deposit at Kiel, Wang Ziyao holds the main mass, and $54.4 \mathrm{~g}$ with Bart.

\section{La'gad $002 \quad 27^{\circ} 14^{\prime} 25.7^{\prime \prime} \mathrm{N}, 8^{\circ} 50^{\prime} 49.5^{\prime \prime} \mathrm{W}$}

Saguia el Hamra, Western Sahara

Find: 2004

Classification: Pallasite (Main group, anomalous)

History: Near the end of 2004, Michael Farmer visited a site at the northeastern corner of Western Sahara in search of a newly discovered pallasite www.meteoriteguy.com/catalog/almahbas.htm. This site yielded several large masses of a deeply weathered pallasite. In addition, Mr. Farmer collected and acquired several small fresh looking pallasite stones from the same site. The relationship between the large weathered masses and fresh stones is unknown. This pallasite is sold and traded under the name "Al Mahbas". The total mass of the weathered material is in the many $\mathrm{kg}$ range, whereas only a few grams, probably $<100 \mathrm{~g}$ of the fresh stones was collected. $A S U$ acquired one of the fresh stones upon which this classification is based.

Physical characteristics: Dark rounded stone with olivine grains visible at the surface.

Petrography: Cut, polished, and etched surface shows the following minerals (areal\%) 70\% metal, 20\% olivine, and 5\% troilite and schreibersite. Olivine grains are small $(<5 \mathrm{~mm})$ and well-rounded. They are light green and glassy clear. One grain contains oriented linear inclusions. Metal composed of approximately 50:50 kamacite:plessite. Kamacite mostly swathing the olivine. Troilite highly shocked and fine grained. Where troilite and olivine are in contact, the olivine appears brecciated and fragments are distributed throughout the troilite. Interior of the plessite composed of $\sim 75 \%$ kamacite and $25 \%$ tetrataenite. Within the plessite, the kamacite is dominated by anhedral grains typically 5 to 15 microns across.

Geochemistry: (J. Wasson, UCLA) Metal composition by INAA (mean of two analyses) $\mathrm{Ni}=76.8 \mathrm{mg} / \mathrm{g}, \mathrm{Co}=5.64 \mathrm{mg} / \mathrm{g}, \mathrm{Cr}=11 \mu \mathrm{g} / \mathrm{g}, \mathrm{Cu}=164 \mu \mathrm{g} / \mathrm{g}, \mathrm{Ga}=20.6 \mu \mathrm{g} / \mathrm{g}$, As $=22.8 \mu \mathrm{g} / \mathrm{g}, \mathrm{Ru}=0.8 \mu \mathrm{g} / \mathrm{g}, \mathrm{W}=0.2 \mu \mathrm{g} / \mathrm{g}, \mathrm{Re}=121 \mathrm{ng} / \mathrm{g}$, and $\mathrm{Ir}=1.42 \mu \mathrm{g} / \mathrm{g}$. The Ni concentration is the lowest PMG ever measured by UCLA; however, in pallasites $\mathrm{Ni}$ is strongly affected by random variations in the kamacite/taenite ratios. Our Ni replicates scattered $(90.5$ and $63.0 \mathrm{mg} / \mathrm{g})$. The $\mathrm{Ir}$ content is the fifth highest in a PMG. No other PMG is closely similar to La'gad 002; the closest is Marjalahti, 
which also has high $\operatorname{Ir}(2.1 \mu \mathrm{g} / \mathrm{g})$ and relatively low $\mathrm{Ni}(88 \mathrm{mg} / \mathrm{g}$, the eighth lowest). (K. Ziegler, $U N M$ ): Analyses of 2 acid-washed olivine subsamples by laser fluorination dual inlet mass spectrometry gave, respectively $\delta^{17} \mathrm{O} 1.68,1.777 ; \delta^{18} \mathrm{O}$ $3.623,3.861 ; \Delta^{17} \mathrm{O}-0.295,-0.262$ per mil (reference TFL slope $=0.528$, values are linearized). (L. Garvie, $A S U$ ) EPMA analyses of eight olivine grains $\mathrm{Fa}_{13.8 \pm 0.1}$, $\mathrm{FeO} / \mathrm{MnO}=42.0 \pm 1.8, \mathrm{Cr}_{2} \mathrm{O}_{3}$ to $0.05 \mathrm{wt} \%$.

Classification: Main-group pallasite-anomalous. This stone is likely paired with NWA 10015, NWA 10023, NWA 10252 and NWA 11720 based on the similar Fa and oxygen isotopic values and the high proportion of plessite. The anomalous designation is based on the high proportion of plessitic metal, similar to that in NWA 10023, 10252 and 11720.

\section{Lop Nur $003 \quad 40^{\circ} 5^{\prime} \mathrm{N}, 90^{\circ} 12^{\prime} \mathrm{E}$ \\ Xinjiang, China \\ Find: 2017 \\ Classification: Ordinary chondrite $(\mathrm{H} \sim 5)$}

History: (Ziyao Wang) Purchased by Wang Ziyao from Zhao Tailu (Shanshan County, Xinjiang) in Shanshan/Xinjiang in 2017. This meteorite was discovered in the Lop Nur dense collection area in 2017. The reported coordinates were read from a map provided by the finder.

Physical characteristics: (Ziyao Wang) Black-brown stone of $27.2 \mathrm{~g}$ with fusion crust and adhering sand grains

Petrography: (R. Bartoschewitz, Bart) recrystallized matrix with poor defined chondrules (0.2-0.9 mm, av. $0.4 \mathrm{~mm})$

Geochemistry: Magnetic susceptibility (R. Bartoschewitz, Bart) $\log \chi\left(\times 10^{-}\right.$ $\left.{ }^{9} \mathrm{~m}^{3} / \mathrm{kg}\right)=4.55$

Classification: Ordinary chondrite $(\mathrm{H} \sim 5, \mathrm{~W} 1)$

Specimens: $5.8 \mathrm{~g}$ on deposit at Kiel, Wang Ziyao holds the main mass, and $5.0 \mathrm{~g}$ with Bart.

\section{Los Vientos 212 (LoV 212) \\ Antofagasta, Chile \\ Find: 2017 Feb 21 \\ Classification: HED achondrite (Eucrite, polymict)}

$24^{\circ} 38.765^{\prime} \mathrm{S}, 69^{\circ} 53.048^{\prime} \mathrm{W}$

History: Found by driving on small hills while meteorite prospecting.

Physical characteristics: Crusted stone. Cut surface reveals light grey interior with darker clasts to $5 \mathrm{~mm}$.

Petrography: (Jérôme Gattacceca, CEREGE) Brecciated igenous rocks with clasts of varied textures (variolitic, subophitic, granulitic) set in a clastic matrix of plagioclase and pyroxene with typical grain size $20 \mu \mathrm{m}$. Some pyroxene in the matrix are zoned, others not, others are exsolved. Other minerals: FeNi metal, troilite, chromite, ilmenite, silica polymorph. 
Geochemistry: Clastic matrix: low-Ca pyroxene $\mathrm{Fs}_{31.2 \pm 8.5} \mathrm{Wo}_{1.2-8.2}, \mathrm{Fe} / \mathrm{Mn}=34.2$ $(\mathrm{N}=7)$, plagioclase $\mathrm{An}_{90.5} \mathrm{Ab}_{8.8} \mathrm{Or}_{0.7}(\mathrm{~N}=1)$. Granulitic clasts: low-Ca pyroxene $\mathrm{Fs}_{56.0 \pm 0.6} \mathrm{Wo}_{4.3 \pm 0.3}, \mathrm{FeO} / \mathrm{MnO}=31.5$, plagioclase $\mathrm{An}_{80.0} \mathrm{Ab}_{18.8} \mathrm{Or}_{1.3}(\mathrm{~N}=2)$. Variolitic clasts: low-Ca pyroxene $\mathrm{Fs}_{56.9} \mathrm{Wo}_{5.0 \pm 1.1}, \mathrm{FeO} / \mathrm{MnO}=35.6(\mathrm{~N}=3)$, plagioclase $\mathrm{An}_{89.0} \mathrm{Ab}_{10.1} \mathrm{Or}_{0.9}(\mathrm{~N}=2)$.

Classification: eucrite-pmict

Specimens: Type specimen at CEREGE. Main mass with Sergey Vasiliev.

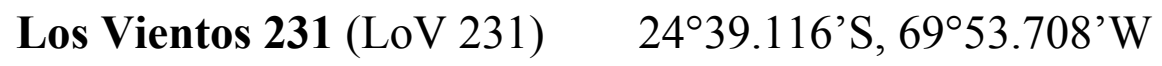

Antofagasta, Chile

Find: 2017 Feb 21

Classification: Ordinary chondrite (LL7)

History: Found by a six-person team who were driving on small hills while meteorite prospecting.

Petrography: (J. Gatacceca, CEREGE) Highly recrystallized texture. Main minerals are olivine, pyroxene (to $200 \mu \mathrm{m}$ ), plagioclase (to $100 \mu \mathrm{m}$ ). No discernible chondrules. FeNi metal is rare. Troilite is almost entirely replaced by terrestrial weathering products.

Geochemistry: Olivine $\mathrm{Fa}_{31.1}(\mathrm{~N}=1)$. Low-Ca pyroxene $\mathrm{Fs}_{25.7 \pm 0.0} \mathrm{Wo}_{2.0 \pm 0.3}(\mathrm{~N}=3)$ Classification: LL7. Type 7 designation from texture, absence of chondrules and low-Ca pyroxene Wo content.

Specimens: Type specimen and polished section at CEREGE. Main mass with Sergey Vasiliev.

\section{Los Vientos 250 (LoV 250) 2441.093'S, 6950.533'W \\ Antofagasta, Chile \\ Find: 8 Feb 2018 \\ Classification: HED achondrite (Diogenite)}

Physical characteristics: Single stone partially covered by fusion crust with flow lines. Sawn surface shows a yellowish-orange interior with abundant opaque grains and metal.

Petrography: Thin section shows pyroxene (95 vol\%) grains up to $6 \mathrm{~mm}$ in the long dimension with abundant shock veins. Chromite spinel grains (3 vol\%) are well scattered through the sample and measure up to $3 \mathrm{~mm}$ and are usually associated to iron oxide. Metal grains (2 vol\%) are in the range 1-3 mm. Olivine occurrence is rare. Additional minerals are Si polymorphs, FeS and Ti-spinel.

Geochemistry: Low-Ca pyroxene composition in the range $\mathrm{Fs}_{23.2-23.5} \mathrm{Wo}_{0.9}$ 1.2 (mean $\mathrm{Fs}_{23.4 \pm 0.2} \mathrm{Wo}_{1.0 \pm 0.2}, \mathrm{~N}=8$ ), with $\mathrm{FeO} / \mathrm{MnO}=26.0 \pm 1.2$. Mean olivine composition is $\mathrm{Fa}_{35.3 \pm 0.2}(\mathrm{~N}=2)$. Chromite exhibits $\mathrm{Cr} /(\mathrm{Cr}+\mathrm{Al})$ values of $0.82 \pm 0.05$ $(\mathrm{N}=8)$. Metal is Ni-free. No plagioclase has been found in the section studied.

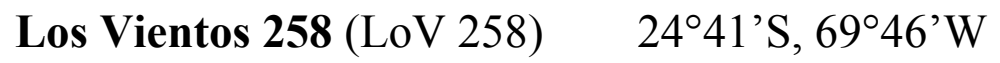


Antofagasta, Chile

Find: 2017 Nov

Classification: Ordinary chondrite (L6)

Petrography: (J. Gattacceca, CEREGE) Brecciated chondrite with abundnat shock veins and $\mathrm{cm}$ size melt pockets. The melt pockets contain abundant $\mu \mathrm{m}$ sized FeS/FeNi eutectic droplets. Plagioclase size $>50 \mu \mathrm{m}$.

Classification: L6. Likely paired with Los Vientos 014.

Specimens: Type specimen at CEREGE. Main mass with ARTMET.

\section{Loulan Yizhi $008 \sim 40^{\circ} 30^{\prime} \mathrm{N}, \sim 89^{\circ} 54^{\prime} \mathrm{E}$ \\ Xinjiang, China \\ Find: 2013 \\ Classification: Ordinary chondrite $(\mathrm{H} \sim 5)$}

History: (Ziyao Wang) Purchased by Wang Ziyao from Bi Chunlin (Tangshan City,Hebei) in Bayingol/Xinjiang in 2013. This meteorite was discovered in the Lop Nur area, close to the ruins of Loulan Yizhi in 2013.

Physical characteristics: (Wang Ziyao) Black-brown stone of $29.3 \mathrm{~g}$

Petrography: (R. Bartoschewitz, Bart) Brown stainted recrystallized matrix with poor defined chondrules $(0.2-1.1 \mathrm{~mm}$, av. $0.4 \mathrm{~mm})$. Metal and sulphide are nearly complet oxidized and vugs are filled with gypsum.

Geochemistry: Magnetic susceptibility (R. Bartoschewitz, Bart) $\log \chi\left(\times 10^{-}\right.$ $\left.{ }^{9} \mathrm{~m}^{3} / \mathrm{kg}\right)=4.77$

Classification: Ordinary chondrite (H 5, W4)

Specimens: $5.9 \mathrm{~g}$ on deposit at Kiel, Wang Ziyao holds the main mass, and $5.9 \mathrm{~g}$ with Bart.

\section{MacKay Glacier 14005 (MCY 14005) $\quad 77^{\circ} 13.981^{\prime}$ S, $158^{\circ} 13.450^{\prime}$ \\ Antarctica \\ Find: 2015 Jan 15 \\ Classification: Enstatite chondrite (EL6)}

History: A $6 \times 5 \times 5 \mathrm{~cm}$ stone was found during the XXX PNRA Italian expedition, 2014-2015

Physical characteristics: Rounded shape, $80 \%$ crusted stone with a broken tip $(6 \times$ $5 \times 5 \mathrm{~mm}$ )

Petrography: (M.Salvini, M. Gemelli, T. Di Rocco, DST-PI) The meteorite consists largely of prismatic (mainly subhedral) or granular enstatite (grain size 0.2-0.4 mm) and Fe-Ni metal, with minor amount of sulfides and plagioclase and a silica phase. Only vague traces of chondritic structure are discernable in thin section: in fact, only two poorly-defined relict chondrules (diameter of 0.8 and 1.5 $\mathrm{mm}$ ) are visible. The sulfide phases are troilite, alabandite, daubreelite and oldhamite. Schreibersite and osbornite (TiN) have also been identified. No olivine has been found. Plotting our average mineral compositions on the diagrams of Zhang et al. (1995), we conclude that the meteorite is an EL6 chondrite. 
Geochemistry: (M.Salvini, M. Gemelli, T. Di Rocco EMP at $I G G-C N R$ Padua) Enstatite ( $\left.\mathrm{Fs}_{0.2-0.8} \mathrm{En}_{98-98.3} \mathrm{Wo}_{1.4-1.5}\right)$, plagioclase $\left(\mathrm{Ab}_{80.5} \mathrm{An}_{15.0} \mathrm{Or}_{4.15}\right)$, metal $(\mathrm{Fe}$ 93.5, Ni 6.6, Si 0.9). Magnetic susceptibility $\log \chi\left(\times 10^{-9} \mathrm{~m}^{3} / \mathrm{kg}\right)=5.51$.

Classification: Enstatite chondrite (EL6)

Specimens: Main mass (172.1g), type specimen and one thin section (MNA-SI).

\section{Mahbas Arraid $\quad 27^{\circ} 38.333^{\prime} \mathrm{N}, 9^{\circ} 28.124^{\prime} \mathrm{W}$}

Guelmim Smara, Western Sahara

Probable fall: 2013 Dec 9

Classification: Ordinary chondrite (LL6)

History: (H. Chennaoui Aoudjehane $F S A C$, H. Lharbi) A fireball fall was reported in the southern part of Morocco by many eyewitnesses from Assa, Zag and Al Mahbes area on 9 December 2013 around 6:30 pm GMT. Ali Houmida, Cheggef, Jdi and many other Moroccan hunters, as well as people from the area, saw the fireball lasting several ". Collection of the testimonies of the fireball and their conciliation indicate a bolide moving from the northeast to southwest. The meteor terminated above the area south of Mahbas Aaraid. Three sonic booms were reported. Meteorite hunters traveled to the expected fall site as indicated by meteor termination. No meteorites were found, and hunting was stopped after a few days. In the beginning of September 2014 (nine months latter after the fireball), Fatma, a young nomad girl found two black stones, totally different from all the other rocks in the area. After news of the find spread, several meteorite hunters returned to the area and collected more pieces. Samples of this meteorite were sent to the Hassan II University of Casablanca Morocco by H. Lharbi on 30 December 2014 for classification. To date, the total known mass is about $5500 \mathrm{~g}$. The meteorite is known under the name of "Al Mahbes" by Moroccan hunters.

Physical characteristics: Many fresh full stones with fusion crust. The interior is brecciated with clear and dark-gray clasts surrounded by lighter-colored regions. Neither troilite nor metal grains are clearly visible.

Petrography: Polished mount shows recrystallized matrix with a few poorly defined barred chondrules. Olivine and opx are fractured ( $\mathrm{mm}$-sized clasts). Plagioclase occurs as irregular grains to $0.1 \mathrm{~mm}$. Troilite and taenite are evenly distributed in grains to $0.1 \mathrm{~mm}$. Accessory chromite and ilmenite.

Geochemistry: Olivine $\mathrm{Fa}_{26 \pm 0.4}(\mathrm{~N}=15)$; $\mathrm{Opx} \mathrm{Fs}_{25 \pm 0.2} \mathrm{Wo}_{2 \pm 0.3}(\mathrm{~N}=11)$; $\mathrm{Cpx}$ $\mathrm{Fs}_{10 \pm 1} \mathrm{Wo}_{44 \pm 1}(\mathrm{~N}=5)$; plagioclase $\mathrm{An}_{10 \pm 0.2} \mathrm{Or}_{5 \pm 1}(\mathrm{~N}=7)$; taenite Ni52. Short-lived cosmogenic isotopes were measured by P. Weber at La Chaux de Fonds (La Vuedes-Alpes underground laboratory, Switzerland; Gonin et al 2003). Measurement was on a sample of $27.23 \mathrm{~g}$ during 6 days in April 2015, 478 days after the fireball. Long- and short-lived cosmogenic radionuclides were measured. The presence and amount of short-lived isotopes support a fall around the time of the witnesses fireball. ${ }^{26} \mathrm{Al} 43.6 \pm 2.2,{ }^{22} \mathrm{Na} 79.4 \pm 4.5 ;{ }^{54} \mathrm{Mn} 47.1 \pm 3.2$, all calculated at 9 December 2013; the other short lived isotopes: ${ }^{57} \mathrm{Co}<1.5 ;{ }^{46} \mathrm{Sc}<1.0 ;{ }^{56} \mathrm{Co}<3.0 ;{ }^{58} \mathrm{Co}$ $<0.8 ;{ }^{51} \mathrm{Cr}<6.2 ;{ }^{48} \mathrm{~V}<1.3$. The ${ }^{22} \mathrm{Na} /{ }^{26} \mathrm{Al}$ ratio calculated at the time of the fireball 1.82 (compared to Mreira 2.4 or Tissint 2.3) is compatible with the estimated time 
of fall. The activity of the cosmogenic isotopes was calculated according to the method described in Weber et al. (2017), with 3-sigma statistical errors. A 10\% additional systematic uncertainty is estimated.

Classification: LL6, brecciated

Specimens: $26 \mathrm{~g}(21.4+3.9+1.6)$ provided by Hamza Lharbi and $24.1 \mathrm{~g}$ provided by Aaras Jonikas to FSAC.

\section{Malotas (b) $\quad 28^{\circ} 56^{\prime} \mathrm{S}, 63^{\circ} 14^{\prime} \mathrm{W}$ \\ Santiago del Estero, Argentina \\ Find, doubtful fall: 22 Jun 1931 \\ Classification: HED achondrite (Eucrite)}

History: On the morning of 22 June 1931, a meteorite fall was reported in the province of Santiago del Estero, Argentina. Samples were given to Professor Juan Olsacher (Cordoba University) who decided to travel to the place of the fall and collect some pieces. Many of the studied pieces were H5 ordinary chondrites, named Malotas. However, during a visit to the small meteorite collection at $M M G$ Cordoba in 2015, Maria Eugenia Varela discovered two very different looking stones labeled as "Malotas, 1931" in the showcase. Recognizing that one of the stones was not an ordinary chondrite, Varela, in agreement with the director of the museum, made a detailed study of these stones. One of the samples was a piece of the ordinary chondrite, Malotas; the second one turned out to be a eucrite. The director of $M M G$-Cordoba provided Varela with a copy of Prof. Olsacher's paper, entitled: The meteorite of Salavina, 1931, in which he clearly indicates that the fall consisted of two different types of rocks (translated Spanish text, see attached pdf): "This meteorite, which represents a case of 'stone rain' is made up of fragments that correspond to two different types based on their composition and structure: one is chondritic, composed by olivine and pyroxene with abundant metallic minerals; the second one is feldspar-rich with scarce presence of the previous components and with an ophitic texture (1). (1) This feldspar-rich meteorite is mainly composed of anorthite with few olivine and troilite grains. As we do not have enough bibliography concerning feldspar-rich meteorites I have left the description for a complementary publication, to the one presented now in which only the chondrite is studied."

Physical characteristics: Single $62.4 \mathrm{~g}$ stone covered by fresh shiny fusion crust. Petrography: The meteorite is a monomict breccia, mainly composed of pyroxene (pigeonite, often with augite lamellae) and plagioclase with coarse- and finegrained domains. Accessory phases include: troilite, silica-polymorphs, ilmenite, chromite, F-apatite, merrillite, and zircon. There are only few shock features, with some minerals showing faint undulatory extinction. One of the thin sections (sample belonging to the $N H M V$ ) show a small melt vein. The state of shock is minimal. The rock is unaltered.

Geochemistry: Mineral composition and geochemistry: (J. Roszjar; NHMV) Electron Microprobe examination of two polished mounts show very similar plagioclase and pyroxene composition for the coarse-and fine-grained areas. Low- 
Ca pyroxene $\mathrm{Fs}_{62.5 \pm 0.8} \mathrm{Wo}_{2.23 \pm 0.9}, \mathrm{n}=96$; Ca-rich Pyroxenes $\mathrm{Fs}_{26.9 \pm 0.9} \mathrm{Wo}_{43.9 \pm 1}, \mathrm{n}=96$. The composition of the rock-forming plagioclase is $\mathrm{An}_{82.1 \pm 3.4}, \mathrm{n}=110$ with minor, secondary anorthitic plagioclase with $\mathrm{An}_{95.7 \pm 2.7}, \mathrm{n}=50$. Spinel composition is chromite $\left(\mathrm{Chr}_{0.74} \mathrm{Her}_{0.18}\right), \mathrm{n}=124$. Oxygen Isotopes ( $\mathrm{R}$. Tanaka, OkaU): A cleaned sample was analyzed by laser fluorination. Replicate analyses are, respectively, $\delta^{17} \mathrm{O}$ of 1.785 and 1.775 permil; $\delta^{18} \mathrm{O}$ of 3.779 and 3.787 permil; $\Delta^{17} \mathrm{O}$ of -0.208 and -0.222 permil.

Classification: Basaltic eucrite, type 4 (based on the chemical composition of spinels and characteristic of pyroxenes that are also diagnostic for this thermal metamorphic classification). Because this meteorite is fresh and the ordinary chondrite Malotas is weathered, this eucrite may actually be the meteorite that fell on June 22, 1931, and the ordinary chondrite may be an unrelated find. This hypothesis remains untested. Henceforth, Malotas (a) will be a synonym for the Malotas H5 chondrite.

Specimens: Type specimen of $20 \mathrm{~g}$ in deposit at the $M L P$ and three thin sections are on deposit at ICATE. One thin section and another sample mount are in deposit at $N H M V$. The main mass is at the $M M G$-Cordoba.

\section{Mangui $\quad 22^{\circ} 2^{\prime} 6 " \mathrm{~N}, 100^{\circ} 10^{\prime} 29^{\prime \prime} \mathrm{E}$ \\ Yunnan, China \\ Confirmed fall: 1 June 2018 \\ Classification: Ordinary chondrite (L6)}

History: A fireball was witness seen around $9 \mathrm{pm}$ in Xishuangbanna region of Yunnan province on June 1, 2018. A piece of meteorite with black fusion crust broke through a farmer's silt-roofed house. A number of pieces of the meteorite varying from 1 to $100 \mathrm{~s}$ of $\mathrm{g}$ have been found by several farmers from Manlun, Manyan, Manlang villages from June 2 to 9 . The largest mass (for which coordinates are provided) weighs $1.28 \mathrm{~kg}$ and was found near the village of Mangui.

Physical characteristics: Most fragments are partially or fully covered with black fusion. Thin black shock-induced melt veins can be seen in the exposed areas of most samples.

Petrography: (S. Hu, Y. Lin and J. Ji, IGGCAS; Q. Zhou, NAOC; Li Ye, PMO) Equilibrated ordinary chondrite texture with poorly defined chondrules and strongly recrystallized matrix. Main minerals are olivine, pyroxene, plagioclase (maskelinized), Fe-Ni metal, and troilite, with minor chromite, chlorapatite and whitlockite. Secondary feldspar grains are 50-100 $\mu \mathrm{m}$ in size. Shock veins and melt pockets are observed.

Geochemistry: Mineral compositions and geochemistry: (S. Hu, Y. Lin and J. Ji, IGGCAS; Q. Zhou, NAOC) The olivine and pyroxene have homogeneous chemical compositions. Olivine $\mathrm{Fa}_{25.0 \pm 0.6}(\mathrm{n}=109)$, orthopyroxene $\mathrm{Fs}_{21.1 \pm 0.3} \mathrm{Wo}_{1.6 \pm 0.3}(\mathrm{n}=66)$.

Classification: Ordinary chondrite, L6 S5 
Specimens: Type specimens at $P M O(45 \mathrm{~g})$ and IGGCAS (35 g). Main mass with various Chinese meteorite collectors.

\author{
Maryborough $\quad 37^{\circ} 05^{\prime} 21^{\prime \prime} \mathrm{S}, 143^{\circ} 44^{\prime} 32^{\prime \prime} \mathrm{E}$ \\ Victoria, Australia \\ Find: 2015 May \\ Classification: Ordinary chondrite (H5)
}

History: Found $2 \mathrm{~km}$ south of Maryborough.

Petrography: (A. Tomkins, Monash) Chondrule edges are indistinct, matrix and chondrule mesostases are strongly recrystallised, and plagioclase grains are up to $50 \mu \mathrm{m}$ across, but mostly less than $20 \mu \mathrm{m}$. Discernible chondrule varieties include $\mathrm{RP}, \mathrm{BO}, \mathrm{PO}, \mathrm{PP}, \mathrm{POP}$. Olivine has sharp optical extinction, there are irregular fractures and no melt pockets. About $20 \%$ of the metal has been destroyed by rusting, whereas most troilite remains.

Geochemistry: (W. Birch, Vic) Microprobe analyses: olivine $\mathrm{Fa}_{19.3 \pm 0.5}(\mathrm{n}=24)$; Low-Ca pyroxene $\mathrm{Fs}_{17.1 \pm 0.5} \mathrm{Wo}_{1.4}(\mathrm{n}=27)$.

Classification: Ordinary Chondrite (H5, S1, W1)

\title{
Mazichuan $\quad 36.693^{\circ} \mathrm{N}, 108.30^{\circ} \mathrm{E}$ \\ Shaanxi, China \\ Confirmed fall: 2016 Sept 16 \\ Classification: HED achondrite (Diogenite)
}

History: On 16 September 2016, at about 15:00 local time, many residents from several villages heard an airplane-like noise followed by three loud sonic booms. A woman in Mazichuan witnessed dusty smoke rising from a hillside $100 \mathrm{~m}$ away from her home. She went to the hillside and picked up a black stone in a hole. She then knocked off a corner of the stone and found greenish crystals inside. The stone was abandoned outside her yard and later picked up by her neighbor. Photos of the stone with black fusion crust and greenish crystals appeared on an amateur meteorite website in November 2016. A Chinese meteorite collector (Wang Ziyao) contacted the holders. He went to the fall site three times (April, June, and September 2017) and did a thorough investigation. Mr. Wang confirmed the fall account. A total of three stones $(1302,1100$, and $880 \mathrm{~g}$ ) were recovered by residents. The largest was bought from a local villager by a meteorite hobbyist (Liu Yukun) on December 1, 2017.

Physical characteristics: The hand specimen is covered with a fresh black fusion crust. Numerous mm-sized greenish crystals can be seen on the exposed section.

Petrography: A monomict breccia mainly consisting of coarse-grained orthopyroxene (a few $\mathrm{mm}$ ) set in a fine-grained matrix of orthopyroxene. Accessory minerals are chromite, sulfide, FeNi metal and silica. Anorthite was not found. 
Geochemistry: Orthopyroxene grains have very homogeneous compositions regardless their grain size. Orthopyroxene $\left(\mathrm{Fs}_{20.9-22.5} \mathrm{Wo}_{1.3-2.2}\right), \mathrm{Fe} / \mathrm{Mn}$ (cation ratio) range from 25.2 to 33.6 with an average of $29.8 \pm 2.1$ ( $\mathrm{n}=32$ ).

Specimens: $36 \mathrm{~g}$ at $P M O$, a $1.3 \mathrm{~kg}$ mass with Mr. Liu Yukun, and two other masses are in the possession of local finders.

\section{Mount Yirtkuq Bulak 001 (MYB 001) 4 4²6’20.91"N, 9042’25.67"E}

XinJiang, China

Find: 01 May 2015

Classification: Ordinary chondrite $(\mathrm{H} \sim 6)$

History: (Ziyao Wang) The meteorite was discovered by Xu Jianjiang (Hami/Xinjiang), one of four meteorite hunters, in the Gobi Desert about $100 \mathrm{~km} \mathrm{~N}$ of Lop Nur, near the Paergang pass in Shanshan county, Tulufan city, Xinjiang province on May 1, 2015.

Physical characteristics: (Ziyao Wang and R. Bartoschewitz, Bart) Dark-brown stone of $540 \mathrm{~g}$, partly covered with fusion crust.

Petrography: (R. Bartoschewitz, Bart) Brown-stained, recrystallized porous matrix with poorly developed chondrules $(0.3-2 \mathrm{~mm})$ and few irregular metal specks.

Geochemistry: Magnetic susceptibility (R. Bartoschewitz, Bart $) \log \chi\left(\times 10^{-}\right.$ $\left.{ }^{9} \mathrm{~m}^{3} / \mathrm{kg}\right)=5.30$

Classification: Ordinary chondrite (H 6, W3)

Specimens: $22.4 \mathrm{~g}$ on deposit at Kiel, Xu Jianjiang (Hami/Xinjiang) holds the main mass, $80 \mathrm{~g}$ with Wang Ziyao, and $63.4 \mathrm{~g}$ with Bart.

\section{Mukundpura $\quad 26^{\circ} 52^{\prime} 52.5^{\prime}{ }^{\prime} \mathrm{N}, 75^{\circ} 39^{\prime} 53.7^{\prime \prime} \mathrm{E}$}

Rajasthan, India

Fall: June 6, 2017

Classification: Carbonaceous chondrite (CM2)

History: A meteorite weighing approximately $2 \mathrm{~kg}$ fell in Mukundpura village at 05:15 hrs on June 6, 2017, on sandy soil in the agricultural field of Mr. Banshi Ram. The impact formed a nearly circular crater of $\sim 40 \mathrm{~cm}$ diameter with a depth of $15 \mathrm{~cm}$. Upon impact, the stone shattered into several large pieces and multiple gram and subgram-sized fragments. Mr. Banshi Ram observed a bright red-yellow colored burning fragment fall with a thundering sound about $100 \mathrm{~m}$ away from his house. They informed the nearby Bhankrota police station, where the large pieces were deposited. The fall of meteorite in the Mukundpura Village was reported in the media. Dr. Anil D. Shukla and Dr. D. Ray visited the fall site two days after the fall and acquired some fragments from the local villagers, with the help of police. Physical characteristics: The meteorite is covered in well-developed matte fusion crust with a few broad regmaglypts. The interior of the stone is dark black with an abundance of small white spots. There are various clasts (angular to rounded and up to $4 \mathrm{~cm}$ across) embedded in the dark colored matrix. 
Petrography: The modal abundances avearged from three thick sections include chondrule $(\sim 15 \%)$, matrix $(\sim 70 \%)$, refractory inclusions $(\sim 2 \%)$, carbonates $(\sim 7 \%)$, Fe-Ni metal $(\sim 2 \%)$, and sulfides ( $\sim 4 \%)$. Matrix is dominated by serpentine with lesser tochilinite.

Geochemistry: The Fa content of granular porphyritic olivine chondrule is uniform and highly forsteritic $(\mathrm{Fa}<1, \mathrm{SD}=0.13, \mathrm{n}=16)$. The $\mathrm{Fa}$ content of barred olivine chondrule is ferroan and composition ranges $\mathrm{Fa}_{32-33}(\mathrm{SD}=0.06, \mathrm{n}=11)$. $\mathrm{The} \mathrm{Cr}_{2} \mathrm{O}_{3}$ of granular and barred olivine is almost indistinguishable and ranges 0.36 to 0.61 $\mathrm{wt} \%$. The Fe/Mn ratios of type IIA chondrule follow the similar trend to the $\mathrm{CO}$ chondrites. The bulk chemical composition of Mukundpura chondrite was analysed using XRF and Q-ICPMS. The major oxides of Mukundpura show a very good match with Murchison and Paris CM carbonaceous chondrite. Following XRF data in $\mathrm{wt} \%: \mathrm{SiO}_{2} 29.5, \mathrm{TiO}_{2} 0.13, \mathrm{Al}_{2} \mathrm{O}_{3}$ 1.3, $\mathrm{Fe}_{2} \mathrm{O}_{3} 32.4, \mathrm{MnO} 0.22, \mathrm{MgO} 17.4, \mathrm{CaO}$ 1.7, $\mathrm{Na}_{2} \mathrm{O} 0.59, \mathrm{~K}_{2} \mathrm{O} 0.05$, and $\mathrm{P}_{2} \mathrm{O}_{5} 0.21$.

Classification: CM2 chondrite fall.

Specimens: Type specimen $30.5 \mathrm{~g}$ at $P R L$. Main mass $\sim 2000 \mathrm{~g}$ with GSI (Based on the report on GSI web portal).

\section{Mürtschenstock $\quad 47^{\circ} 4.285^{\prime} \mathrm{N}, 9^{\circ} 9.023^{\prime} \mathrm{E}$}

Glarus, Switzerland

Find: 2017 Jul 17

Classification: Ordinary chondrite (L6)

History: A black stone was found by Andreas Stucki on the return from a mountain hike during a chance meeting with another independently hiking person, Iris Landolt, who witnessed the discovery.

Physical characteristics: Single stone with dimensions $10.1 \times 5.7 \times 5.0 \mathrm{~cm}$. The stone is nearly completely covered by black matte fusion crust. Small areas of approx. $1 \mathrm{~cm}$ square show exposed interior material, which is of yellowish brown color. The volume based on a shape model is $108.38 \mathrm{~cm}^{3}$, yielding a bulk density of $3.27 \mathrm{~g} / \mathrm{cm}^{3}$.

Petrography: (B. Hofmann, E. Gnos): The rock shows a strongly recrystallized texture with poorly delineated large chondrules well recognizable. Typical size of plagioclase grains is $50-150 \mu \mathrm{m}$. Chromites are up to $0.6 \mathrm{~mm}$ in size, ilmenite is common, up to $0.1 \mathrm{~mm}$. Shock stage is S4 based on partially mosaicized olivine and partially maskelynitized plagioclase. Some shock veins are present and show metal-troilite globules in silicate matrix. Weathering is low (W1), thickness of (hydr)oxide rims on metal ranges from a few $\mu \mathrm{m}$ to $100 \mu \mathrm{m}$.

Geochemistry: Electron microprobe analysis (E. Reusser, ETH) yielded: Olivine $\mathrm{Fa}_{24.4 \pm 0.4}\left(\mathrm{Fa}_{24.0-26.7} ; \mathrm{n}=48\right)$; low-Ca pyroxene $\mathrm{Fs}_{20.6 \pm 0.4} \mathrm{Wo}_{1.5 \pm 0.3}\left(\mathrm{Fs}_{20.2-22.2} \mathrm{Wo}_{0.9-2.1}\right.$; $\mathrm{n}=52)$; high-Ca pyroxene $\mathrm{Fs}_{7.2 \pm 0.8} \mathrm{Wo}_{45.8 \pm 1.0}\left(\mathrm{Fs}_{6.5-8.4} \mathrm{Wo}_{44.3-46.8}, \mathrm{~N}=9\right)$. Cosmogenic radionuclides: (Å. Rosén, Bern/GeMSE): Gamma-spectroscopy performed in August-September 2017 showed no detectable short-lived radionuclides $\left({ }^{22} \mathrm{Na}<0.5\right.$ $\mathrm{dpm} / \mathrm{kg})$, the ${ }^{26} \mathrm{Al}$ activity was $52.2(+4.6,-4.8) \mathrm{dpm} / \mathrm{kg}$. The fall must have occurred before the year 2000 . 
Classification: All available data are consistent with a classification as L ordinary chondrite, petrographic grade 6 , shock stage S4, low weathering (W1).

Specimens: $70 \mathrm{~g}$ and one polished thin section at ETH.

\author{
Nagara $\quad 35^{\circ} 26^{\prime} 33.9^{\prime \prime} \mathrm{N}, 136^{\circ} 46^{\prime} 51.5^{\prime \prime} \mathrm{E}$ \\ Chubu, Japan \\ Find: 2012 Oct \\ Classification: Iron meteorite (IAB-MG)
}

History: A $6.5 \mathrm{~kg}$ piece of iron was discovered by Mr. K. Mitsumura in October, 2012, among stones plowed from his chestnut yard in Nagara, Gifu city, Gifu prefecture, Japan. Mr. Mitsumura took the specimen to his home and kept it till June, 2017. Eventually, he considered that it might be a meteorite and the specimen was brought by his friend Mr. H. Iwasa to S. Kawakami at Gifu Shotoku Gakuen Univ. Then, the specimen was sent to T. Mikouchi, UTok for identification. A second $9.7 \mathrm{~kg}$ mass was found later.

Physical characteristics: The $6.5 \mathrm{~kg}$ mass has an exterior with several rounded ridges and shows a brownish dark surface color coated by rust.

Petrography: Polished sections (all ca. $12 \times 6 \mathrm{~mm}$ in sizes) show no

Widmanstatten pattern and $>95 \%$ of the sections consist of kamacite with veins of terrestrial weathering products (up to $0.5 \mathrm{~mm}$ wide). Rare schreibersite is present at the interior of such weathered veins. Taenite is present as small elongated grains ( $\sim 0.1 \mathrm{~mm}$ wide and $\sim 0.5 \mathrm{~mm}$ long) with less than $0.1 \%$ abundance.

Geochemistry: Electron microprobe analysis (T. Mikouchi, UTok): $\mathrm{Fe}=92.0-94.5$, $\mathrm{Ni}=5.5-6.5$ and $\mathrm{Co}=0.55-0.65$ for kamacite $(\mathrm{N}=549), \mathrm{Fe}=45-54, \mathrm{Ni}=30-38$ and $\mathrm{P}=15$ for schreibersite $(\mathrm{N}=26)$, and $\mathrm{Fe}=61-65, \mathrm{Ni}=34-39$ and $\mathrm{Co}=0.1-0.2$ for taenite $(\mathrm{N}=9)$. LA-ICP-MS data (A. Yamaguchi, NIPR and N. Shirai at Tokyo Met. U.): $\mathrm{Ni}=6.10$, (in $\mathrm{wt} \%$ ); $\mathrm{Ir}=4.25, \mathrm{Ga}=91.6, \mathrm{Ge}=402, \mathrm{Au}=1.58(\mathrm{all} \mu \mathrm{g} / \mathrm{g}$, average of $\sim 5$ $\mathrm{mm}$ long 4 traverses on the section).

Classification: IAB-MG

Specimens: UTok: $52.7 \mathrm{~g}$ slice (after cutting of small chips for polished mounts) and NIPR: 3 polished mounts

\title{
Nanbaxian $001 \quad 37^{\circ} 59^{\prime} 2.07^{\prime \prime} \mathrm{N}, 94^{\circ} 18^{\prime} 6.72 \mathrm{E}$ \\ Qinghai, China \\ Find: 07 June 2016 \\ Classification: Ordinary chondrite (L 4)
}

History: (Ziyao Wang) On June 7, 2016, the farmer Xu Zhong (Huzhu/Qinghai) and his colleges discovered a strange rock while they were on a desert walk about $0.5 \mathrm{~km}$ WNW of Nanbaxian village, Dachaidan Town, Qinghai province. Wang Ziyao purchased the complete stone in 2018.

Physical characteristics: (Ziyao Wang and R. Bartoschewitz, Bart) Reddishbrown, heavily fractured, irregular rock of $3784 \mathrm{~g}$ without fusion crust. 
Petrography: (R. Bartoschewitz, Bart) Brown-stained, microcrystalline matrix with well developed chondrules $(0.2-2 \mathrm{~mm})$ and few metallic specks. Geochemistry: Magnetic susceptibility (R. Bartoschewitz, Bart $) \log \chi\left(\times 10^{-}\right.$ $\left.{ }^{9} \mathrm{~m}^{3} / \mathrm{kg}\right)=4.83$

Classification: Ordinary chondrite (L $\sim 4, \mathrm{~W} 3)$

Specimens: $22.2 \mathrm{~g}$ on deposit at Kiel, Wang Ziyao holds the main mass, and 103.5 g with Bart.

\section{Ngare Sero $\quad 2^{\circ} 37.845^{\prime} \mathrm{S}, 35^{\circ} 53.353^{\prime} \mathrm{E}$}

Arusha, Tanzania

Find: 9 Sept 2017

Classification: Ordinary chondrite (L5)

History: Meteorite (2233 g) was found 9 Sept 2017 by Kirill Vlasov and Artyom Akshintsev while returning to the field camp from Ngare Sero village. The date of fall is unknown.

Physical characteristics: Total mass is $2233 \mathrm{~g}$. Meteorite has roughly rounded shape. The surface and interior is light with dark brown crust up to $2 \mathrm{~mm}$ due to desert weathering. Cracks (up to $0.1 \mathrm{~mm}$ in thickness) occur in external part of meteorite.

Petrography: Classified by P. Yu. Plechov, FMMR. Petrographic observation of a polished section shows clearly delineated chondrules in a coarse recrystallized matrix. Chondrules are relatively rare and their sizes vary from 500 to $1200 \mu \mathrm{m}$. They have quenched or porphyritic texture, rarely cryptocrystalline, and consist of $\mathrm{Ol}+\mathrm{Pl}, \mathrm{Opx}+\mathrm{Pl}, \mathrm{Ol}+\mathrm{Opx}+\mathrm{Cpx}+\mathrm{Pl}$ or $\mathrm{Opx}+\mathrm{Cpx}+\mathrm{Pl}$. Olivine, low-Ca-pyroxene and plagioclase are main minerals in matrix. Plagioclase grains are larger than $50 \mu \mathrm{m}$. This indicates a petrological type of 5 for the meteorite. Olivine has no undulatory extinction, as well as the absence of opaque shock veins and melt pockets indicates a shock stage of S1. Fresh FeNi metals and troilite (up to $200 \mu \mathrm{m}$ ) occurs in recrystallized matrix. Some taenite-kamacite aggregates have thin rims of Fehydroxide. Kamacite-taenite-troilite aggregates (up to $20 \mu \mathrm{m}$ ) are observed in some cryptocrystalline chondrules. Weathering products (goethite, "hydrogoethite") occur as veins and in situ alteration of FeNi-metals and troilite near meteorite surface. Olivine, pyroxene, chromite and troilite are fresh and not weathered (weathering grade - W1). Clinopyroxene, chromite, chlorapatite and merrillite (up to $200 \mu \mathrm{m}$ ) occur locally in the matrix.

Geochemistry: EDS-WDS analyses (P. Yu. Plechov, FMMR). The primary chondrite paragenesis includes olivine $\mathrm{Fa}_{25.24 \pm 0.6}(\mathrm{~N}=28)$, orthopyroxene $\mathrm{Fs}_{22.8 \pm 0.53} \mathrm{Wo}_{1.66 \pm 0.35}(\mathrm{~N}=19)$, plagioclase $\mathrm{Ab}_{80.2} \mathrm{An}_{10.6} \mathrm{Or}_{6.3}(\mathrm{~N}=11)$, Cr-bearing clinopyroxene $\mathrm{En}_{47.8} \mathrm{Fs}_{8.1} \mathrm{Wo}_{43.9}(\mathrm{~N}=6)$, chromite $\mathrm{Crt}_{76.6 \pm 4.5} \mathrm{Spl}_{10.5 \pm 1.3}(\mathrm{~N}=14)$, chlorapatite and merrillite. Chlorapatite contains $\mathrm{Cl}-5.26 \mathrm{wt} . \%(\mathrm{~N}=5)$. Merrillite has wide range in $\mathrm{FeO}$ content (0.54-2.92 wt.\%, $\mathrm{N}=13)$. Composition of kamacite (in wt.\%, $\mathrm{N}=8$ ): $\mathrm{Fe} 92.3 \pm 0.67, \mathrm{Ni}-6.3 \pm 0.7, \mathrm{Co}-1.45 \pm 0.17$. Troilite is close to ideal FeS. Rare goethite contains 4.2-6.8 wt.\% NiO and up to $1.12 \mathrm{wt} . \% \mathrm{CoO}$. Classification: Ordinary chondrite. L5, S1, W1 
Specimens: $2127 \mathrm{~g}$ - Kirill Vlasov; $106 \mathrm{~g}$ cut-offs and thin section - FMMR.

\author{
Northwest Africa 431 (NWA 431) \\ (Northwest Africa) \\ Purchased: 2003 \\ Classification: Ordinary chondrite (H6)
}

Petrography: Chondrules are recrystallized and poorly defined. Olivine exhibits undulose extinction, but lacks planar fractures. Plagioclase grains are $\sim 50 \mu \mathrm{m}$ across. There are chromite-plagioclase assemblages and small chromite veinlets present.

\title{
Northwest Africa 432 (NWA 432) \\ (Northwest Africa) \\ Purchased: 2003 \\ Classification: Ordinary chondrite (H3)
}

Petrography: Chondrules are sharply defined, but none contain isotropic mesostases. Low-Ca pyroxene phenocrysts exhibit prominent polysynthetic twinning. Olivine grains have sharp optical extinction.

Classification: H3 (estimated subtype 3.7/3.8).

\section{Northwest Africa 435 (NWA 435) \\ (Northwest Africa) \\ Purchased: 2003 \\ Classification: Ordinary chondrite (H4)}

Petrography: Chondrules are wel defined. Olivine exhibits sharp optical extinction. Chondrules range in diamter from 0.25 to $2.7 \mathrm{~mm}$.

\section{Northwest Africa 436 (NWA 436)}

(Northwest Africa)

Purchased: 2003

Classification: Ordinary chondrite (L6)

Petrography: Chondrules are recrystallized and poorly defined. Olivine exhibits undulose extinction; some grains contain planar fractures. Plagioclase grains range up to $\sim 120 \mu \mathrm{m}$ across. Weathering veins traverse the sample.

\section{Northwest Africa 438 (NWA 438) \\ (Northwest Africa) \\ Purchased: 2003 \\ Classification: Ordinary chondrite (LL5)}

Petrography: Chondrules are recrystallized, but readily discernable. Olivine exhibits undulose extinction, but lacks planar fractures. Chondrules range up to 1.6 
$\mathrm{mm}$ in diameter. Many metal and troiltie grains are 200-800 $\mu \mathrm{m}$ across. Some troilite grains are polycrystalline.

\section{Northwest Africa 451 (NWA 451) \\ (Northwest Africa) \\ Purchased: 2003 \\ Classification: Ordinary chondrite (H5)}

Petrography: Numerous weathering veins transect the meteorite. A few patches of clay are present. More than $99 \%$ of the metal and troilite grains have been weathered to form oxide. Olivine exhbits undulose extinction. Chondrules are well defined.

\section{Northwest Africa 452 (NWA 452) \\ (Northwest Africa) \\ Purchased: 2003 \\ Classification: Ordinary chondrite (H4)}

Petrography: A few metal grains contain some small, irregular grains of troilite. Chondrules are well defined. Olivine exhbits sharp optical extinction. A few chondrules are surrounded by thin rings of troilite. About $75 \%$ of the metal grains have been weathered to form oxides.

\section{Northwest Africa 453 (NWA 453)}

(Northwest Africa)

Purchased: 2003

Classification: Ordinary chondrite (H4)

Petrography: More than $99 \%$ of the metal and troilite grains have been weathered to form oxide. Some clay is present. Numerous weathering veins transect the meteorite. Chondrules are well defined. Olivine exhibits undulose extinction. Some low-Ca pyroxene phenocrysts exhibit polysynthetic twinning.

\section{Northwest Africa 454 (NWA 454)}

(Northwest Africa)

Purchased: 2003

Classification: Ordinary chondrite (L6)

Petrography: Some metal grains are elongated. A few chondrules are surrounded by troilite-rich rims. Some metal grains contain small, irregular grains of troilite. The troilite is polycrystalline. Chondrules are recrystallized and poorly defined. Some plagiolcase grains are $50-80 \mu \mathrm{m}$ in size. Some olivine grains exhibit weak mosaic extinction.

\section{Northwest Africa 660 (NWA 660)}


(Northwest Africa)

Purchased: 2001

Classification: Ordinary chondrite (H4)

Petrography: The sample contains elongated metal grains; some metal grains contain small, irregular grains of troilite. Some kamacite and troilite grains are polycrytalline. Chondrules are well defined. Olivine exhibits weak mosaic extinction.

\section{Northwest Africa 669 (NWA 669) \\ (Northwest Africa) \\ Purchased: 2001 \\ Classification: Ordinary chondrite (L6)}

Petrography: Chondrules are recrystallized and poorly defined. A few plagioclase grains reach $100 \mu \mathrm{m}$ in diameter. Olivine has undulose extinction. Silicate-rich shock veins $(\sim 50 \mu \mathrm{m} \times 6 \mathrm{~mm})$ including small metal blebs cut across the meteorite. Also present are small metal and sulfide veins. Some coarse metal grains are elongated. The sample was probably shocked and annealed.

\section{Northwest Africa 671 (NWA 671)}

(Northwest Africa)

Purchased: 2001

Classification: Ordinary chondrite (L6)

Petrography: Olivine exhibits weak-to-moderate mosaic extinction. Chondrules are recrystallized and poorly defined. Plagioclase grains range up to $120 \mu \mathrm{m}$ in size. Maskelynite is absent. Metal grains are coarse, averaging $\sim 400 \mu \mathrm{m}$. Some metal grains contain a few small, irregular grains of troilite.

\section{Northwest Africa 674 (NWA 674)}

(Northwest Africa)

Purchased: 2001

Classification: Ordinary chondrite (L5)

Petrography: Chondrules are moderately recrystallized, but readily delineated. Olivine exhibits undulose extinction, but lacks planar fractures. About $95 \%$ of the metal grains have been oxidized.

\section{Northwest Africa 787 (NWA 787)}

(Northwest Africa)

Purchased: 2001 Mar

Classification: Ordinary chondrite (L3-6)

History: Purchased by Michael Farmer in March 2001 from a dealer in Casablanca, Morocco. 
Petrography: (A. Irving and S. Kuehner, UWS; A. Rubin, UCLA) Breccia composed predominantly of type- 6 chondrite clasts exhibiting some remnant chondrules in a recrystallized matrix containing stained metal and merrillite. Sparse, isolated enstatite-rich chondrules are present in this breccia, but no forsterite-rich chondrules were observed in the studied thin section.

Geochemistry: Olivine $\left(\mathrm{Fa}_{24.3-27.3}, \mathrm{~N}=3\right)$, orthopyroxene $\left(\mathrm{Fs}_{20.4} \mathrm{Wo}_{1.1}, \mathrm{~N}=2\right)$, enstatite in one unequilibrated chondrule $\left(\mathrm{Fs}_{2.1} \mathrm{Wo}_{0.7}\right)$, clinopyroxene $\left(\mathrm{Fs}_{1.4} \mathrm{Wo}_{40.3}\right)$. Classification: Ordinary chondrite (L3-6, brecciated). Contains a few isolated enstatite-rich chondrules.

Specimens: $90 \mathrm{~g}$ including one polished thin section at UCLA; $5.89 \mathrm{~g}$ at $P S F$; remainder with Farmer.

\section{Northwest Africa 1088 (NWA 1088) \\ (Northwest Africa) \\ Purchased: 2003 \\ Classification: Ordinary chondrite (H6)}

Petrography: Numerous weathering veins cut across the sample. Some chondrules are readily discernable; others are poorly defined. Some plagioclase grains are between 50 and $80 \mu \mathrm{m}$ across. Olivine exhibits undulose extinction. Some troilite grains are elongated.

\section{Northwest Africa 1089 (NWA 1089)}

(Northwest Africa)

Purchased: 2003

Classification: Ordinary chondrite (H5)

Petrography: There are rapidly cooled, cellular, metal-troilite assemblages. Some metal and sulfide grains are elongated. Chondrules are recrystallized; some are readily discernable; others are poorly defined. Plagioclase grains average $\sim 20 \mu \mathrm{m}$ in size. Olivine exhibits weak mosaic extinction. Some low-Ca pyroxene grains display shock-induced polysynthetic twinning.

\section{Northwest Africa 1091 (NWA 1091) \\ (Northwest Africa) \\ Purchased: 2003 \\ Classification: Ordinary chondrite (L4)}

Petrography: Several chondrules are partly surrounded by troilite-rich rims. Some metal grains contain small, irregular grains of troilite. Troilite is polycrystalline. Some metal and sulfide grains are elongated. Most chondrules are well defined, but some appear recrystallized. Some olivine grains contain planar fractures; a few olivine and low-ca pyroxene grains exhibit weak mosaic extinction. 


\section{Northwest Africa 1095 (NWA 1095)}

(Northwest Africa)

Purchased: 2003

Classification: Ordinary chondrite (L5)

Petrography: Some metal grains are elongated and form parts of discontinuous veins. Small grains of metallic $\mathrm{Cu}$ are present within some metal grains. Some metal grains contain small, irregular grains of troilite. Chondrules are recrystallized, but readily discernable. Plagioclase grains average $\sim 20 \mu \mathrm{m}$ in size. Olivine exhibits weak mosaic extinction. Some low-Ca pyroxene grains exhibit shock-induced polysynthetic twinning.

\section{Northwest Africa 1096 (NWA 1096) \\ (Northwest Africa) \\ Purchased: 2003 \\ Classification: Ordinary chondrite (LL3)}

Petrography: Many chondrules are surrounded by thin rims of troilite \pm metal. A few metal grains contain small, irregular troilite grains. Thin metal and sulfide veins also occur. Thin weathering veins are also present. Chondrules are very sharply defined. Some chondrules contain patches of brown isotropic mesostases. Olivine exhbits weak mosaic ectinction. Some low-Ca pyroxene phenocrysts exhibit polysynthetic twinning.

Classification: LL3 (estimated subtype 3.5).

\section{Northwest Africa 1099 (NWA 1099)}

(Northwest Africa)

Purchased: 2003

Classification: Ordinary chondrite (L4)

Petrography: Metal and sulfide grains are elongated; some metal grains contain small irregular grains of troiite. Chondrules are well defined. A few low-Ca pyroxene grains exhibit polysynthetic twinning. No glassy mesostases occur. Olivine grains exhibit weak mosaic extinction.

\section{Northwest Africa 1108 (NWA 1108) \\ (Northwest Africa) \\ Purchased: 2003 \\ Classification: Ordinary chondrite (L6)}

Petrography: Some metal and sulfide grains are elongated. Metal and sulfide veins are present. Chondrules are poorly defined and very recrystallized. Some plagioclase grains are $50 \mu \mathrm{m}$ in size. A few olivine grains contain planar fractures. 
(Northwest Africa)

Purchased: Aug 2001

Classification: Carbonaceous chondrite (CO3)

History: Purchased by G. and A. Hupe in August 2001 from a dealer in Erfoud, Morocco.

Petrography: (A. Irving and S. Kuehner, UWS) Fairly closely-packed, well-formed small chondrules (apparent diameter $190 \pm 100 \mu \mathrm{m}$ ) together with mineral fragments and fine grained CAI are set in a sparse matrix $(\sim 15$ vol.\%) containing stained kamacite and minor pleonaste.

Geochemistry: Olivine $\left(\mathrm{Fa}_{0.5-39.9}, \mathrm{Cr}_{2} \mathrm{O}_{3}\right.$ in ferroan examples 0.06-0.76 wt.\%, mean $0.19 \pm 0.28$ wt.\%, $\mathrm{N}=7$ ), orthopyroxene $\left(\mathrm{Fs}_{1.4} \mathrm{Wo}_{2.9}\right)$, pigeonite $\left(\mathrm{Fs}_{6.5} \mathrm{Wo}_{5.7}\right)$, clinopyroxene $\left(\mathrm{Fs}_{0.5-9.4} \mathrm{Wo}_{49.3-49.0}, \mathrm{~N}=2\right)$.

Classification: Carbonaceous chondrite (CO3).

Specimens: $21.7 \mathrm{~g}$ including one polished thin section at PSF; $11.3 \mathrm{~g}$ at UCLA; remainder with GHupé.

\section{Northwest Africa 1723 (NWA 1723) \\ Morocco \\ Find: unknown \\ Classification: Ordinary chondrite (L3)}

Petrography: Olivine $\mathrm{Fa}_{22.9}$, range $\mathrm{Fa}_{10-24}$, PMD 6\% $(\mathrm{n}=17)$ with average $0.05 \mathrm{wt} \%$ $\mathrm{CaO}$. Low-Ca pyroxene $\mathrm{Fs}_{19.4}$, range $\mathrm{Fs}_{13-20}$, PMD 15\% (n=15). Plagioclase $\mathrm{Ab}_{95} \mathrm{Or}_{2}(\mathrm{n}=10)$. Metal includes kamacite, taenite and possible tetrataenite. Troilite and chromite are abundant. Contains a $2-\mathrm{cm}$ sized dark inclusion.

\section{Northwest Africa 2424 (NWA 2424) \\ (Northwest Africa) \\ Purchased: 2016 \\ Classification: Martian meteorite (Shergottite)}

Physical characteristics: Fresh, unstained, 20\% covered by dull, smooth fusion crust, remaining surfaces polished by ventifaction. Cut appearance: uniform gray, fine-grained.

Petrography: (P. Warren, UCLA) The rock consists of about $20 \%$ olivine phenocrysts, up to $1 \mathrm{~mm}$ ( $2 \mathrm{~mm}$ as glomerocrysts), in a much finer-grained pyroxene-dominated groundmass. The minor feldspathic phase is entirely maskelynite.

Geochemistry: Mineral compositions and geochemistry: Olivines are zoned from $\mathrm{Fa}_{21.5}$ to $\mathrm{Fa}_{50}$. Groundmass pyroxene compositions fan out from $\mathrm{Fs}_{26} \mathrm{Wo}_{3.7}$ toward $\mathrm{Fs}_{25} \mathrm{Wo}_{28}$ and $\mathrm{Fs}_{44} \mathrm{Wo}_{11}$. Pyroxenes in melt inclusions within olivine are systematically more magnesian, e.g., $\mathrm{Fs}_{21} \mathrm{Wo}_{28}$. Pyroxene $\mathrm{FeO} / \mathrm{MnO}$ (wt.) averages 30.0 \pm 2.4. Maskelynite $(\mathrm{N}=31)$ is $\mathrm{An}_{44-54}$, average $\mathrm{An}_{51.5}$. Bulk composition results from INAA and fused-bead EPMA include $\mathrm{Ga}=14.4 \mathrm{ppm}, \mathrm{Al}_{2} \mathrm{O}_{3}=5.1 \mathrm{wt} \%$; this $\mathrm{Ga} / \mathrm{Al}$ ratio confirms shergottite affinity. 
Classification: Shergottite.

\section{Northwest Africa 2425 (NWA 2425) \\ (Northwest Africa) \\ Purchased: 2016 \\ Classification: Lunar meteorite (feldspathic breccia)}

Physical characteristics: Weathered, red caliche stain, dull, no fusion crust, etched by ventifaction. Cut appearance: near-black matrix with gray clasts.

Petrography: (P. Warren, UCLA) The rock is a thoroughly polymict lunar highland breccia, mostly plagioclase, with clasts up to $10 \mathrm{~mm}$ but dominated by extremely fine-grained chaotic impact debris. A search for regolithic glass spheroids found only one, $0.2 \mathrm{~mm}$, very round and undevitrified.

Geochemistry: Mineral compositions and geochemistry: Plagioclase: $\mathrm{An}_{95.9+} /-1.3$ $(\mathrm{N}=19)$. Low-Ca pyroxene: $\mathrm{Fs}_{33 \pm 8} \mathrm{Wo}_{4.3 \pm 0.9}(\mathrm{~N}=11)$, high-Ca pyroxene:

$\mathrm{Fs}_{17 \pm 4} \mathrm{Wo}_{40 \pm 2}(\mathrm{~N}=7)$. Pyroxene $\mathrm{FeO} / \mathrm{MnO}$ (wt.) averages 55 $\pm 8(\mathrm{~N}=24)$. Bulk composition results from INAA and fused-bead EPMA include $\mathrm{Al}_{2} \mathrm{O}_{3}=27.1 \mathrm{wt} \%$, $\mathrm{Mg} \#=73 \mathrm{~mol} \%, \mathrm{Th}=0.13 \mathrm{ppm}$. The glass spheroid is similarly anorthositic (26 wt $\% \mathrm{Al}_{2} \mathrm{O}_{3}$ ).

Classification: The meteorite is a lunar highland polymict breccia.

\section{Northwest Africa 2426 (NWA 2426) \\ (Northwest Africa) \\ Purchased: 2016 \\ Classification: HED achondrite (Eucrite, cumulate)}

Physical characteristics: Weathered, red caliche stain, $80 \%$ covered by glossy fusion crust showing flow lines and scales. Cut appearance: mottled gray matrix with both lighter and darker gray clasts; one large $(\sim 12 \mathrm{~mm})$ clast with white feldspar and deep brown mafic silicates.

Petrography: (P. Warren, UCLA) The rock is dominated by pyroxene and plagioclase. Its texture is for the most part severely brecciated and fine-grained, but scattered clasts preserve vestiges of a medium-grained (1-3 $\mathrm{mm})$ equigranular igneous texture.

Geochemistry: Mineral compositions and geochemistry: Low-Ca pyroxene: $\mathrm{Fs}_{37+} /-$ 3 , $\mathrm{Wo}_{3.5+} /-0.9(\mathrm{~N}=35)$, high-Ca pyroxene: $\mathrm{Fs}_{15.5 \pm 2} \mathrm{Wo}_{43 \pm 1}(\mathrm{~N}=10)$. Pyroxene $\mathrm{FeO} / \mathrm{MnO}$ (wt.) averages $28 \pm 3$. Plagioclase: $\mathrm{An}_{92.5 \pm 0.8}(\mathrm{~N}=11)$. Scattered tiny metals have $\mathrm{Ni}$ at margin of EPMA detectability: very approximately $0.04 \mathrm{wt} \%$, along with $0.23 \pm 0.16 \mathrm{wt} \% \mathrm{Co}(\mathrm{N}=10)$. Bulk composition results from INAA and fusedbead EPMA include $\mathrm{Al}_{2} \mathrm{O}_{3}=12.5 \mathrm{wt} \%, \mathrm{Mg} \#=62 \mathrm{~mol} \%, \mathrm{Sm}=0.6 \mathrm{ppm}$. Despite obscuration of texture by brecciation, high $\mathrm{Mg} \#$ and low REE indicate cumulate origin.

Classification: Brecciated cumulate eucrite.

Specimens: manifestly paired stones of 254, 232 and $144 \mathrm{~g}$. 


\section{Northwest Africa 2637 (NWA 2637)}

(Northwest Africa)

Purchased: 2004

Classification: Carbonaceous chondrite (CV3)

History: Purchased by Adam Hupé in 2004 from a dealer in Erfoud, Morocco. Petrography: (A. Irving and S. Kuehner, UWS) Granular chondrules (apparent diameter $710 \pm 510 \mu \mathrm{m}, \mathrm{N}=18$ ) plus sparse, amoeboid very fine grained CAI are set in a fine grained matrix (orange-brown in thin section). Andradite garnet was observed in one CAI.

Geochemistry: Olivine $\left(\mathrm{Fa}_{0.6-57.3}, \mathrm{~N}=3\right)$, orthopyroxene $\left(\mathrm{Fs}_{0.7-51.2} \mathrm{Wo}_{0.5-2.5}, \mathrm{~N}=2\right)$, clinopyroxene $\left(\mathrm{Fs}_{0.8} \mathrm{Wo}_{39.0}\right)$.

Classification: Carbonaceous chondrite (CV3).

Specimens: $14.4 \mathrm{~g}$ including one polished thin section at $P S F$; remainder with AHupé.

\section{Northwest Africa 2650 (NWA 2650) \\ Morocco \\ Find: 2004 \\ Classification: HED achondrite (Eucrite, monomict)}

History: A $2224 \mathrm{~g}$ complete stone that measures $189 \mathrm{~mm} \times 112 \mathrm{~mm} \times 82 \mathrm{~mm}$ at the widest points, with fresh crust was purchased in Erfoud, Morocco

Petrography: The meteorite has a texture of brecciated meteorite, with coarsegrained gabbro eucrite fragments and coarse-grained mineral fragments embedded in relatively fine-grained matrix composed of pyroxene and plagioclase fragments. Both shock-induced melt veins and pockets are observed. Mineralogically, this meteorite consists mainly of coarse-grained plagioclase (up to $7 \mathrm{~mm}$ ) and pyroxenes (up to $5 \mathrm{~mm}$ ). Other minerals include silica phase, chromite, ilmenite, troilite, and zircon. Almost all of the pyroxene grains exhibit exsolution lamella. Extensive albite, Carlsbad, and complex igneous twinning occur in plagioclase. Geochemistry: The coarse-grained minerals in the coarse-grained gabbro eucrite fragments have compositions almost identical to those in the relatively fine-grained matrix. Orthopyroxene host $\left(\mathrm{En}_{37.2 \pm 0.7} \mathrm{Fs}_{60.1 \pm 1.5} \mathrm{Wo}_{2.7 \pm 1.3}, \mathrm{Fe} / \mathrm{Mn}=29.9 \pm 0.7 \mathrm{n}=14\right)$, augite exsolution lamella $\left(\mathrm{En}_{29.7 \pm 0.4} \mathrm{Fs}_{26.4 \pm 0.5} \mathrm{Wo}_{43.9 \pm 0.5}, \mathrm{Fe} / \mathrm{Mn}=29.8 \pm 1.8, \mathrm{n}=14\right)$, plagioclase $\left(\mathrm{An}_{90.5 \pm 1.5} \mathrm{Ab}_{9.2 \pm 1.4}, \mathrm{n}=17\right)$, and chromite $(\mathrm{Cr} /(\mathrm{Cr}+\mathrm{Al})=0.79 \pm 0.01, \mathrm{n}=8)$.

\section{Northwest Africa 2730 (NWA 2730)}

(Northwest Africa)

Purchased: 2004

Classification: Ordinary chondrite (L6)

History: A stone of several hundred grams was purchased by Mike Farmer and Jim Strope in 2004 from a dealer in Erfoud, Morocco, and a $24.5 \mathrm{~g}$ specimen was 
submitted to Dr. Ted Bunch for classification. The whereabouts and exact weight of the original main mass are unknown, but the $24.5 \mathrm{~g}$ specimen was acquired by the Planetary Studies Foundation and subsequently classified at the University of Washington.

Petrography: (A. Irving and S. Kuehner, UWS) Mostly recrystallized with rare remnant chondrules. Accessory merrillite and chlorapatite are present.

Geochemistry: Olivine $\left(\mathrm{Fa}_{24.8-24.9}, \mathrm{~N}=3\right)$, orthopyroxene $\left(\mathrm{Fs}_{20.6-20.9} \mathrm{Wo}_{1.5-1.4}, \mathrm{~N}=\right.$ 2), clinopyroxene $\left(\mathrm{Fs}_{8.3-8.5} \mathrm{Wo}_{45.1-44.3}, \mathrm{~N}=2\right)$.

Classification: Ordinary chondrite (L6).

Specimens: The entire specimen including one polished thin section is at PSF.

\section{Northwest Africa 2887 (NWA 2887) \\ (Northwest Africa) \\ Purchased: 2016 \\ Classification: HED achondrite (Eucrite)}

Physical characteristics: Weathered, slight red caliche stain, $90 \%$ covered by dull black fusion crust.

Petrography: (P. Warren, UCLA) The rock is dominated by pyroxene and plagioclase. Its texture is unbrecciated and for the most part fine-grained, but uncommonly heterogeneous: locally coarse (laths up to $4 \mathrm{~mm}$ ), and elsewhere locally equigranular with 120 -degree triple junctions.

Geochemistry: Mineral compositions and geochemistry: Low-Ca pyroxene: $\mathrm{Fs}_{57.5 \pm 0.3} \mathrm{Wo}_{6.3 \pm 0.2}(\mathrm{~N}=7)$, high-Ca pyroxene: $\mathrm{Fs}_{28.8 \pm 0.1} \mathrm{Wo}_{41.5 \pm 0.2}(\mathrm{~N}=3)$. Plagioclase: 5

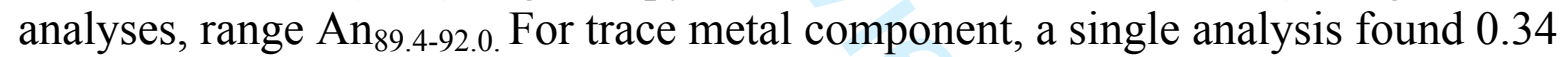
$\mathrm{wt} \% \mathrm{Co}, 0.04 \mathrm{wt} \% \mathrm{Ni}$. Bulk composition results from INAA and fused-bead EPMA include $\mathrm{Al}_{2} \mathrm{O}_{3}=12.5 \mathrm{wt} \%, \mathrm{Mg} \#=40 \mathrm{~mol} \%, \mathrm{Sm}=1.9 \mathrm{ppm}$.

Classification: The meteorite is a eucrite.

Specimens: manifestly paired stones of 570, 275 and $42 \mathrm{~g}$.

\section{Northwest Africa 4669 (NWA 4669)}

(Northwest Africa)

Purchased: 2006

Classification: Carbonaceous chondrite (CV3)

History: In 2006, A. Pani purchased a single stone of $369 \mathrm{~g}$ in Erfoud, Morocco.

Physical characteristics: The stone is a compact rock with a wind-polished surface of grayish-black color. Fusion crust is not present.

Petrography: (F. Brandstätter, $N H M V$ ) The meteorite consists mainly of abundant chondrules (mean diameter $1.05 \mathrm{~mm}$ ), minor AOAs and CAIs set in a fine-grained matrix. CAIs (fine-grained) are sparse and on average small $(<2-3 \mathrm{~mm})$. The matrix is dominated by $\mathrm{Fe}$-rich olivine, magnetite, minor pentlandite, and very rare metal grains. 
Geochemistry: Chondrule olivine $\left(\mathrm{Fa}_{0.2-31.3}\right.$, mean $\left.\mathrm{Fa}_{6.7}, \mathrm{~N}=68\right)$, matrix olivine $\left(\mathrm{Fa}_{8.9-63.8}\right.$, mean $\mathrm{Fa}_{47.3}$, mean $\left.\mathrm{NiO}=0.09 \mathrm{wt} \%, \mathrm{~N}=26\right)$, magnetite $\left(\mathrm{Al}_{2} \mathrm{O}_{3}=1.18\right.$ $\mathrm{wt} \%, \mathrm{Cr}_{2} \mathrm{O}_{3}=0.31 \mathrm{wt} \%, \mathrm{TiO}_{2}=0.15 \mathrm{wt} \%, \mathrm{NiO}=0.05 \mathrm{wt} \%, \mathrm{~N}=10$ )

Classification: Carbonaceous chondrite (CV3)

Specimens: $21.7 \mathrm{~g}$ is on deposit at NHMV. Pani holds the main mass.

\section{Northwest Africa 4838 (NWA 4838)}

(Northwest Africa)

Purchased: 2006

Classification: Carbonaceous chondrite (CV3)

History: Purchased by Marcin Cimala in 2006 from a dealer in Erfoud, Morocco, and subsequently acquired by $R O M$.

Petrography: (A. Irving and S. Kuehner, UWS) Granular chondrules (apparent diameter $500 \pm 300 \mu \mathrm{m}, \mathrm{N}=15)$ and fine-grained CAI are set in a very fine-grained matrix ( 40 vol.\%, black in thin section).

Geochemistry: Olivine $\left(\mathrm{Fa}_{0.1-37.9}, \mathrm{~N}=3\right)$, orthopyroxene $\left(\mathrm{Fs}_{0.4-0.5} \mathrm{Wo}_{1.0-0.9}, \mathrm{~N}=3\right)$, clinopyroxene $\left(\mathrm{Fs}_{1.0} \mathrm{Wo}_{28.0}\right)$, Al-Ti-diopside $\left(\mathrm{Fs}_{0.8} \mathrm{Wo}_{59.0}, \mathrm{Al}_{2} \mathrm{O}_{3}=19.9\right.$ wt.\%, $\mathrm{TiO}_{2}=2.0$ wt.\%).

Classification: Carbonaceous chondrite (CV3).

Specimens: All material from this stone including one polished thin section is at $R O M$.

\section{Northwest Africa 5693 (NWA 5693) \\ (Northwest Africa) \\ Purchased: 2007 \\ Classification: Pallasite}

History: The meteorite was purchased from a local meteorite dealer in Erfoud, Morocco.

Physical characteristics: Brownish, strongly altered individual lacking any fusion crust.

Petrography: The meteorite is composed of large up to $1 \mathrm{~cm}$ sized olivine crystals set into a matrix of iron oxides or hydroxides. Olivine is often crosscut by veins filled with brownish/reddish alteration products. No primary Fe-Ni metal was detected

Geochemistry: Olivine: $\mathrm{Fa}_{13.1 \pm 0.1}\left(\mathrm{Fa}_{12.9-13.4}, \mathrm{FeO} / \mathrm{MnO}=32-46, \mathrm{n}=19\right)$

Classification: Pallasite

\section{Northwest Africa 5908 (NWA 5908)}

(Northwest Africa)

Purchased: 2007

Classification: HED achondrite (Eucrite, brecciated) 
History: The meteorite was purchased from a Moroccan meteorite dealer on the mineral fair in Munich, Germany.

Physical characteristics: Small dark-grayish individual partly covered with fusion crust.

Petrography: The meteorite is a fragmental breccia composed of basaltic clasts and up to $1 \mathrm{~cm}$ sized black impact melt clasts set into a fine-grained clastic matrix. Basaltic clasts and matrix are dominantly composed of exsolved pyroxene and plagioclase grains about 20 to $300 \mu \mathrm{m}$ in size. Few matrix pyroxenes display magmatic zoning. Minor phases include chromite, ilmenite, pyrrhotite, silica and metallic iron.

Geochemistry: low-Ca pyroxene: $\mathrm{Fs}_{42.1 \pm 3.6} \mathrm{Wo}_{2.1 \pm 0.3}\left(\mathrm{Fs}_{34.7-45.8} \mathrm{Wo}_{1.8-3.0}, \mathrm{n}=12\right.$, $\mathrm{FeO} / \mathrm{MnO}=36-42)$; Ca-pyroxene: $\mathrm{Fs}_{27.1 \pm 1.0} \mathrm{Wo}_{40.8 \pm 1.3}\left(\mathrm{Fs}_{26.1-29.0} \mathrm{Wo}_{37.9-42.1}, \mathrm{n}=12\right.$, $\mathrm{FeO} / \mathrm{MnO}=31-36)$; calcic plagioclase: $\mathrm{An}_{87.6 \pm 1.8}\left(\mathrm{An}_{84.1-90.0}, \mathrm{n}=14\right)$

\section{Northwest Africa 6023 (NWA 6023)}

(Northwest Africa)

Purchased: 2009

Classification: Brachinite-an

History: The meteorite was bought from a local meteorite dealer in Erfoud, Morocco.

Physical characteristics: Dark brownish rock with patches of fusion crust. Petrography: A polished surface of the meteorite shows sub-mm sized shiny crystals. It displays an equilibrium texture of 200-300 $\mu \mathrm{m}$ sized olivine (about 90 vol.\%), some Ca-pyroxene and chromite typically showing triple junctions between adjoining crystals. Nearly all olivine crystals are surrounded by fine-grained intergrowths of low-Ca pyroxene and metallic Fe. Strikingly, olivine shows pronounced reduced rims and the meteorite contains flaky graphite. No plagioclase has been detected.

Geochemistry: olivine: $\mathrm{Fa}_{32.3 \pm 0.7}\left(\mathrm{Fa}_{30.4-32.9}, \mathrm{n}=14, \mathrm{CaO}=0.11 \pm 0.02\right.$, $\mathrm{Cr}_{2} \mathrm{O}_{3}=0.03 \pm 0.01, \mathrm{FeO} / \mathrm{MnO}=70 \pm 5$ ); reduced rims in olivine: $\mathrm{Fa}_{12.6-16.2}$; Capyroxene: $\mathrm{Fs}_{11.4 \pm 0.2} \mathrm{Wo}_{43.3 \pm 0.3}\left(\mathrm{Fs}_{11.1-11.8} \mathrm{Wo}_{42.6-43.8}, \mathrm{n}=13, \mathrm{FeO} / \mathrm{MnO}=35 \pm 3\right)$; Oxygen isotopes (K. Ziegler, $U N M$ ): material analyzed by laser fluorination gave: $\delta^{18} \mathrm{O}=5.162,5.226,4.838,5.110 ; \delta^{17} \mathrm{O}=2.445,2.491,2.269,2.402 ; \Delta{ }^{17} \mathrm{O}=-0.281$, $0.268,-0.285,-0.296$ (all per mil)

Classification: Brachinite-anomalous. Mineral chemistry, high abundance of olivine, equilibrium texture and oxygen isotopes are consistent with classification as brachinite, but reduced rims and high graphite-content are typical of ureilites.

\section{Northwest Africa 6263 (NWA 6263)}

(Northwest Africa)

Purchased: 2010

Classification: Ordinary chondrite (L3)

History: Purchased by Carsten Giessler in 2010 from a dealer in Erfoud, Morocco. 
Petrography: (A. Irving and S. Kuehner, UWS) Well-formed, fairly closely packed chondrules (apparent diameter $680 \pm 350 \mu \mathrm{m}, \mathrm{N}=12$ ) are set in a finer matrix (dark brown in thin section).

Geochemistry: Olivine $\left(\mathrm{Fa}_{4.7-26.5}, \mathrm{Cr}_{2} \mathrm{O}_{3}\right.$ in ferroan examples 0.03-0.06 wt.\%, mean $0.05 \pm 0.01 \mathrm{wt} . \%, \mathrm{~N}=5)$, orthopyroxene $\left(\mathrm{Fs}_{9.2-12.9} \mathrm{Wo}_{0.9-1.1}, \mathrm{~N}=2\right)$, subcalcic augite $\left(\mathrm{Fs}_{9.0} \mathrm{Wo}_{32.5}\right)$. Magnetic susceptibility $\log \chi\left(\times 10^{-9} \mathrm{~m}^{3} / \mathrm{kg}\right)=4.46$.

Classification: Ordinary chondrite (L3).

Specimens: 23 g including one polished thin section at $U W B$; remainder with $\mathrm{Mr}$. C. Giessler.

\section{Northwest Africa 6308 (NWA 6308)}

(Northwest Africa)

Purchased: 2009

Classification: Primitive achondrite (Brachinite)

History: The meteorite was bought from a local meteorite dealer in Erfoud, Morocco.

Physical characteristics: Dark-brownish rock without fusion crust.

Petrography: A sawn surface of the meteorite shows up to $1 \mathrm{~mm}$ sized shiny crystals. It displays an equilibrium texture of equally sized olivine (about 80 vol.\%) and orthopyroxene with abundant triple junctions between adjoining crystals. Along the grain boundaries symplectic exsolutions of orthopyroxene and $\mathrm{Fe}-$ sulfides are frequently observed. Minor phases are chromite and some phosphates. Geochemistry: olivine: $\mathrm{Fa}_{28.5 \pm 0.4}\left(\mathrm{Fa}_{27.8-29.2}, \mathrm{n}=20, \mathrm{FeO} / \mathrm{MnO}=63 \pm 4\right)$; low-Ca pyroxene: $\mathrm{Fs}_{25.4 \pm 0.1} \mathrm{Wo}_{2.2 \pm 0.1}\left(\mathrm{Fs}_{25.1-25.6} \mathrm{Wo}_{2.0-2.3}, \mathrm{n}=20, \mathrm{FeO} / \mathrm{MnO}=44 \pm 3\right.$ )

Classification: Mineral chemistry, high abundance of olivine, equilibrium texture and grain size are consistent with classification as brachinite.

\section{Northwest Africa 6827 (NWA 6827) \\ (Northwest Africa) \\ Purchased: 2008 \\ Classification: Carbonaceous chondrite (CO3)}

History: The meteorite was purchased from a local meteorite dealer in Erfoud, Morocco.

Physical characteristics: Twenty five brownish fragments lacking any fusion crust.

Petrography: The meteorite shows a dark brownish interior and is composed of abundant small chondrules, CAIs, and mineral fragments set into a fine-grained matrix. Chondrules are dominantly porphyritic type I and have an apparent mean diameter of about $0.2 \mathrm{~mm}$. Some sulfides and FeNi metal are present in matrix and chondrules.

Northwest Africa 7055 (NWA 7055) 
(Northwest Africa)

Purchased: 2007 Jan

Classification: Carbonaceous chondrite (CK4/5)

History: Purchased by Dr. David Gregory in January 2007 from a dealer at the Tucson Gem and Mineral Show.

Petrography: (A. Irving and S. Kuehner, UWS) Very fresh specimen consisting of separated chondrules (apparent diameter 0.2-2.0 mm) containing $\mathrm{Cr}$-magnetite set within a relatively coarse grained matrix. The texture is transitional between type 4 and type 5 .

Geochemistry: Olivine $\left(\mathrm{Fa}_{30.9-31.1}, \mathrm{~N}=2\right)$, orthopyroxene $\left(\mathrm{Fs}_{25.4-26.3} \mathrm{Wo}_{0.5-0.9}, \mathrm{~N}=\right.$ $3)$, clinopyroxene $\left(\mathrm{Fs}_{9.4-11.2} \mathrm{Wo}_{46.5-42.6}, \mathrm{~N}=2\right)$, one exotic ferroan olivine grain $\left(\mathrm{Fa}_{62.7}\right)$.

Classification: Carbonaceous chondrite (CK4/5).

Specimens: The entire specimen plus a polished thin section and polished thick section at $R O M$.

\section{Northwest Africa 8406 (NWA 8406) \\ (Northwest Africa) \\ Purchased: 2013 Feb \\ Classification: Carbonaceous chondrite (CV3)}

History: Purchased by Marc Jost in February 2013 from a dealer in Rissani, Morocco.

Petrography: (A. Irving and S. Kuehner, UWS) Medium sized, granular chondrules together with some amoeboid, fine to medium grained CAIs in a brown matrix.

Geochemistry: Olivine $\left(\mathrm{Fa}_{0.4-16.8} ; \mathrm{Cr}_{2} \mathrm{O}_{3}\right.$ in ferroan olivine $=0.06-0.28$ wt.\%, mean $0.05 \pm 0.02$ wt. $\%, \mathrm{~N}=7$ ), orthopyroxene $\left(\mathrm{Fs}_{0.8-13.8} \mathrm{Wo}_{0.9-3.5}, \mathrm{~N}=3\right)$, diopside $\left(\mathrm{Fs}_{0.8-}\right.$ $\left.{ }_{0.9} \mathrm{Wo}_{47.3-46.0}\right)$.

Classification: Carbonaceous chondrite (CV3).

Specimens: $24 \mathrm{~g}$ including one polished thin section at $U W B$. The remaining material is held by $S J S$.

\section{Northwest Africa 8671 (NWA 8671)}

(Northwest Africa)

Purchased: 2014 May

Classification: HED achondrite (Eucrite)

History: Purchased in Morocco by Ali and Mohammed Hmani in May 2014 and said to have been found near Foum El Hisn, Morocco.

Physical characteristics: Single stone (164.2 g) lacking fusion crust. The fresh interior is relatively coarse grained and composed of subequal amounts of dark gray pyroxene and beige plagioclase.

Petrography: (A. Irving and S. Kuehner, UWS) This is a very unusual specimen consisting of dispersed, angular domains of coarse, polygranular, birefringent, 
calcic plagioclase separated by regions of recystallized clinopyroxene. The plagioclase domains have irregular geometric shapes with re-entrant angles, and exhibit marginal reaction zones against clinopyroxene composed of ferropigeonite plus fayalitic olivine. The main regions of clinopyroxene contain numerous irregularly distributed, small patches with two distinctly different compositions: one low in $\mathrm{Ca}$ and the other higher in $\mathrm{Ca}$. No high-Ca augite was found. Accessory minerals are silica polymorph, Al-bearing chromite and troilite.

Geochemistry: Low-Ca pyroxene $\left(\mathrm{Fs}_{47.9-49.1} \mathrm{Wo}_{5.5-4.4}, \mathrm{FeO} / \mathrm{MnO}=28-29, \mathrm{~N}=3\right)$, pigeonite $\left(\mathrm{Fs}_{36.3-34.7} \mathrm{Wo}_{10.8-7.6}, \mathrm{FeO} / \mathrm{MnO}=26-28, \mathrm{~N}=3\right)$, ferropigeonite rims $\left(\mathrm{Fs}_{52.5-55.5} \mathrm{Wo}_{21.6-16.5}, \mathrm{FeO} / \mathrm{MnO}=30, \mathrm{~N}=2\right)$, fayalite $\left(\mathrm{Fa}_{82.2-82.9}, \mathrm{FeO} / \mathrm{MnO}=39\right)$, plagioclase $\left(\mathrm{An}_{91.4-93.0} \mathrm{Or}_{0.1}, \mathrm{~N}=2\right)$. Oxygen isotopes (K. Ziegler, $\left.U N M\right)$ : acidwashed subsamples analyzed by laser fluorination gave $\delta^{17} \mathrm{O}=1.736,1.642,1.694$; $\delta^{18} \mathrm{O}=3.847,3.688,3.776 ; \Delta^{17} \mathrm{O}=-0.295,-0.305,-0.300$ per mil

Classification: Eucrite (unbrecciated, partially melted). The overall texture and reaction zones on plagioclase aggregates are unique among eucrites, and indicate that this specimen underwent a partial melting event subsequent to its igneous crystallization.

Specimens: $20.1 \mathrm{~g}$ including one polished thin section at $U W B$. The remainder is held by Hmani Inc.

\section{Reclassification of Northwest Africa 8671 (NWA 8671)}

(Northwest Africa)

Find: 2014

Classification: Eucrite (anomalous). The disequilibrium textures in this specimen are unique among eucrites and the absence of high $\mathrm{Ca}$ augite is very unusual. Furthermore, the oxygen isotope composition plots to more negative $\Delta^{17} \mathrm{O}$ values than for all other analyzed eucrites.

\section{Northwest Africa 8676 (NWA 8676)}

(Northwest Africa)

Purchased: 2014 May

Classification: Carbonaceous chondrite (CO3)

History: Purchased by John Higgins in May 2014 from a dealer in Laayoune, Morocco.

Petrography: (A. Irving and S. Kuehner, UWS) Small chondrules $(230 \pm 130 \mu \mathrm{m}$, $\mathrm{N}=20$ ) and rare, very fine-grained CAI in a sparse, brown matrix (40 vol\%) rich in ferroan olivine and containing sparse altered kamacite, taenite and troilite.

Geochemistry: Olivine $\left(\mathrm{Fa}_{0.7-43.4} ; \mathrm{Cr}_{2} \mathrm{O}_{3}\right.$ in ferroan olivine 0.07-0.19 wt.\%, $\mathrm{N}=7$, orthopyroxene $\left(\mathrm{Fs}_{0.9-6.3} \mathrm{Wo}_{0.9-4.4}, \mathrm{~N}=3\right)$, clinopyroxene $\left(\mathrm{Fs}_{2.0-4.0} \mathrm{Wo}_{47.2-45.2}\right)$.

Classification: Carbonaceous chondrite (CO3).

Specimens: $9 \mathrm{~g}$ including one polished thin section at $U W B$. The remaining material is held by Mr. J. Higgins. 


\section{Northwest Africa 8681 (NWA 8681)}

(Northwest Africa)

Purchased: 2014 Jun

Classification: HED achondrite (Eucrite, brecciated)

History: Purchased by Sergey Vasiliev from a Moroccan dealer at the Ensisheim Show in June 2014.

Physical characteristics: Single stone (590 g) with a light reddish-brown weathered exterior. The interior is dark gray with whitish clasts visible.

Petrography: (A. Irving and S. Kuehner), UWS) Breccia consisting of gabbroic eucrite clasts set in a very dark brown matrix containing related crystal debris plus minor unexsolved pigeonite. Gabbroic eucrite clasts are composed of exsolved pigeonite (commonly with wavy clinopyroxene exsolution lamellae), polycrystalline calcic plagioclase, silica polymorph, chromite, ilmenite and Ni-free metal. Secondary minerals from terrestrial weathering include, barite, calcite and iron hydroxides.

Geochemistry: Orthopyroxene host crystals $\left(\mathrm{Fs}_{61.0-62.2} \mathrm{Wo}_{4.4-2.1}, \mathrm{FeO} / \mathrm{MnO}=32-35\right.$, $\mathrm{N}=3$ ), clinopyroxene exsolution lamellae $\left(\mathrm{Fs}_{26.8-28.3} \mathrm{Wo}_{43.6-42.5}, \mathrm{FeO} / \mathrm{MnO}=30-33\right.$, $\mathrm{N}=3)$, pigeonite $\left(\mathrm{Fs}_{36.2} \mathrm{Wo}_{5.6}, \mathrm{FeO} / \mathrm{MnO}=23\right)$.

Classification: Eucrite (brecciated). Likely paired with NWA 7989, NWA $\underline{8036}, \underline{\text { NWA } 8588}$ and related stones.

Specimens: $24.8 \mathrm{~g}$ including one polished thin section at $U W B$; main mass with Mr. S. Vasiliev.

\section{Northwest Africa 8781 (NWA 8781)}

(Northwest Africa)

Purchased: 2014 Jul

Classification: Carbonaceous chondrite (Ungrouped)

History: Several black stones found together near Foum Zguid, Morocco, in July 2014 were purchased by Rachid Chaoui and were subsequently sold to Stefan Ralew and Darryl Pitt.

Petrography: (A. Irving and S. Kuehner, UWS, L. Garvie, ASU) Composed of very small chondrules $(210 \pm 60 \mu \mathrm{m}, \mathrm{N}=20)$ and small mineral fragments in a black, very fine-grained matrix ( $\sim 50$ vol. $\%)$. No phyllosilicates were detected by powder-XRD in the matrix. No CAI were observed. Minerals are olivine, orthopyroxene, diopside, stained kamacite and troilite.

Geochemistry: Olivine $\left(\mathrm{Fa}_{1.1-70.7}, \mathrm{Cr}_{2} \mathrm{O}_{3}\right.$ in ferroan examples $=0.21-0.40 \mathrm{wt} . \%, \mathrm{~N}$ $=8$ ), orthopyroxene $\left(\mathrm{Fs}_{1.0-7.0} \mathrm{Wo}_{1.2-0.4}, \mathrm{~N}=3\right)$, diopside $\left(\mathrm{Fs}_{2.1} \mathrm{Wo}_{40.8} ; \mathrm{Fs}_{0.4} \mathrm{Wo}_{48.2}\right)$. Oxygen isotopes (K. Ziegler, UNM): analyses of five 1-2 mg acid-washed non homogenized subsamples by laser fluorination gave, respectively, $\delta^{17} \mathrm{O}-10.020$, $9.832,-6.298,-5.897,-4.490 ; \delta^{18} \mathrm{O}-6.085,-5.472,-1.822,-0.895,1.221 ; \Delta^{17} \mathrm{O}-$ $6.807,-6.943,-5.336,-5.424,-5.135$ (all per mil). These compositions plot on the CCAM trend, but distinctly below the trend for $\mathrm{CO}$ chondrites 
Classification: Carbonaceous chondrite (ungrouped). This specimen is not a CV3 chondrite (chondrule size much too small, no CAI), not a CK chondrite (no magnetite), not a $\mathrm{CO} 3$ chondrite (oxygen isotopes are different, no $\mathrm{CAI}$ ), and not a $\mathrm{CM}$ chondrite (matrix apparently anhydrous, oxygen isotopes displaced to more ${ }^{16} \mathrm{O}$-rich compositions). Therefore it seems to be an ungrouped carbonaceous chondrite.

Specimens: $20.1 \mathrm{~g}$ including one polished thin section at $U W B ; 170 \mathrm{~g}$ with Kuntz; other samples with Mr. S. Ralew and DPitt.

\section{Northwest Africa 8791 (NWA 8791) \\ (Northwest Africa) \\ Purchased: 2011 Jun \\ Classification: Rumuruti chondrite (R3-5)}

History: Purchased by Fabien Kuntz in June 2011 from a Moroccan dealer at the Ensisheim Show.

Physical characteristics: Single stone ( $241 \mathrm{~g})$ with an overall light-brown color and composed of small clasts in a finer-grained matrix.

Petrography: (A. Irving and S. Kuehner, UWS) Breccia consisting of equilibrated Type 5 clasts (containing sparse chondrules), separated well-formed, forsteritebearing Type 3 chondrules ((apparent diameter $470 \pm 210 \mu \mathrm{m})$ ) and angular mineral fragments in a finer grained matrix (orange-brown in thin section). Accessory minerals include Ti-V-Al-bearing chromite, troilite, Ni-bearing pyrrhotite and minor altered kamacite.

Geochemistry: Olivine in Type 5 clasts $\left(\mathrm{Fa}_{39.2-39.9}, \mathrm{~N}=3\right)$, magnesian olivine in Type 3 chondrules $\left(\mathrm{Fa}_{3.2-4.5}, \mathrm{~N}=2\right)$, orthopyroxene $\left(\mathrm{Fs}_{2.2-30.3} \mathrm{Wo}_{0.2-3.2}, \mathrm{~N}=3\right)$, augite $\left(\mathrm{Fs}_{27.7} \mathrm{Wo}_{39.2} ; \mathrm{Fs}_{12.1} \mathrm{Wo}_{44.0}, \mathrm{~N}=2\right)$, fayalite $\left(\mathrm{Fa}_{71.4}\right)$.

Classification: R3-5 chondrite.

Specimens: $21.2 \mathrm{~g}$ plus one polished thin section at $P S F ; 21.29 \mathrm{~g}$ at $M G C$; main mass with Kuntz.

\section{Northwest Africa 11112 (NWA 11112) \\ Morocco \\ Find: 2016 \\ Classification: Ungrouped achondrite}

History: Purchased by Ke Zuokai in Dec. 2016 from a Moroccan dealer.

Physical characteristics: The meteorite has a black fusion crust and partially covered by weathering products.

Petrography: A polished mount shows a medium-grained rock (mostly 0.1-1 mm) with granular texture. It is composed of $\sim 47 \%$ olivine and $\sim 42 \%$ pyroxene with intersertally $\sim 4 \%$ plagioclase and $7 \%$ opaque minerals (FeNi metal, sulfide and chromite). No relic chondrules. Not brecciated.

Geochemistry: The compositions of olivine, pyroxene and plagioclase are quite uniform. Olivine $\mathrm{Fa}_{36.4-38.5}, \mathrm{Fe} / \mathrm{Mn}=80.2-108.1$; orthopyroxene $\mathrm{Fs}_{29.1-30.0} \mathrm{Wo}_{2.8-3.2}$, 
$\mathrm{Fe} / \mathrm{Mn}=51.2-63.6$; plagioclase $\mathrm{An}_{52.3-55.7} \mathrm{Ab}_{43.0-46.5} \mathrm{Or}_{1.1-1.3}$; chromite $\mathrm{TiO}_{2}$ 1.51-1.80, $\mathrm{Al}_{2} \mathrm{O}_{3}$ 13.71-14.05, $\mathrm{MgO}$ 3.01-3.72 (all in wt\%); taenite Ni 16.09-21.16 wt\%; kamacite $\mathrm{Ni}$ 6.58-6.93 wt\%.

Classification: Achondrite (ungrouped). No relic chondrules are found in this thin section, and the compositions of plagioclase $\left(\sim \mathrm{An}_{52.3}\right)$ are significantly different from those of chondrites. In this regard, we consider NWA 11112 is not an equilibrated chondrite. The petrography and mineral compositions (olivine, pyroxene, plagioclase, chromite and Fe-Ni metal) in this meteorite are very similar to those of the ungrouped achondrite NWA 3250.

\section{Northwest Africa 11113 (NWA 11113)}

Morocco

Find: 2016

Classification: Carbonaceous chondrite (CO3)

History: Purchased by Ke Zuokai in Dec. 2016 from a Moroccan dealer.

Physical characteristics: The meteorite has a black fusion crust.

Petrography: Abundant small well-defined chondrules $(50-300 \mu \mathrm{m})$ and a few fine-grained CAIs set in fine-grained matrix. Most chondrules appear to be Type I with or without pyroxene, and a small amount of Type II chondrules are present. Metal and sulfide occur within and around the chondrules.

Geochemistry: Olivine $\mathrm{Fa}_{0.6-49.7}, \mathrm{Cr}_{2} \mathrm{O}_{3}$ in ferroan olivine 0.01-0.15 wt.\%, mean $0.0 \pm 0.03$ wt. $\%, N=25$; In detail, the cores of olivine in Type I chondrules are rich in magnesium, and the Fa values are between 0.6-10.6. In comparison, the rims of olivine in some Type I chondrules and the grains in Type II chondrules have higher Fe contents $\left(\mathrm{Fa}_{22.9-49.7}\right) . \mathrm{Cr}_{2} \mathrm{O}_{3}$ in ferroan olivine 0.01-0.15 wt.\%, mean 0.07 \pm 0.03 wt.\%, $\mathrm{N}=25$; orthopyroxene $\mathrm{Fs}_{0.9-4.3} \mathrm{Wo}_{0.7-5.3}$; clinopyroxene $\mathrm{Fs}_{1.0-9.5} \mathrm{Wo}_{30.1-40.0}$, $\mathrm{Fs}_{1.0-6.1} \mathrm{Wo}_{43.1-49.5 \text {. }}$

Classification: $\mathrm{CO} 3$

Northwest Africa 11254 (NWA 11254)

(Northwest Africa)

Purchased: 2016 Oct

Classification: Carbonaceous chondrite (CM, anomalous)

History: Purchased by Dustin Dickens in October 2016 from a dealer in Laayoune, Morocco.

Petrography: (A. Irving and S. Kuehner, UWS ; L. Garvie, ASU) Very small chondrules (apparent diameter $200 \pm 100 \mu \mathrm{m}$ ), mineral fragments and some very fine grained CAI are set in an extremely fine grained matrix ( $\sim 30$ vol.\%, black in thin section) containing pyrrhotite, kamacite, and taenite. No phyllosilicates were detected by X-ray diffraction.

Geochemistry: Olivine $\left(\mathrm{Fa}_{0.8-58.6}, \mathrm{~N}=3\right)$, orthopyroxene $\left(\mathrm{Fs}_{1.8-3.1} \mathrm{Wo}_{1.1-0.4}, \mathrm{~N}=3\right)$, clinopyroxene $\left(\mathrm{Fs}_{1.5-2.0} \mathrm{Wo}_{45.3-42.6}, \mathrm{~N}=2\right)$. Oxygen isotopes (Karen Ziegler, $A S U$ ) of 
acid-washed subsamples: $\delta^{17} \mathrm{O} 0.241,-0.423 ; \delta^{18} \mathrm{O} 9.268,8.823 ; \Delta^{17} \mathrm{O}-4.653,-$ 5.082 (all per mil).

Classification: Carbonaceous chondrite (CM-an). The anomalous character comes from the absence of phyllosilicates, and the oxygen isotopes that plot below the trend for CM chondrites.

Specimens: $3.6 \mathrm{~g}$ including one polished thin section at $U W B$; remainder with $\mathrm{Mr}$. D. Dickens.

\section{Northwest Africa 11303 (NWA 11303)}

(Northwest Africa)

Purchased: 2017 Mar

Classification: Lunar meteorite (feldspathic breccia)

History: Material excavated from a site near Tindouf, Algeria, was purchased by Dustin Dickens in March 2017 from a Mauritanian dealer. The coordinates of the site are unknown.

Physical characteristics: Many small fragments coated by pale reddish-brown terrestrial weathering products. The fresh interiors of the largest fragments exhibit white to beige clasts in a dark gray, fine-grained matrix.

Petrography: (A. Irving and S. Kuehner, UWS) Breccia composed of angular mineral grains of anorthite, olivine, orthopyroxene, exsolved pigeonite, ferroan pigeonite, augite, ilmenite, Ti-chromite and fayalite in a partly vesicular matrix containing minor kamacite and barite.

Geochemistry: Olivine $\left(\mathrm{Fa}_{26.7-47.2}, \mathrm{FeO} / \mathrm{MnO}=75-81, \mathrm{~N}=2\right)$, orthopyroxene $\left(\mathrm{Fs}_{24.1} \mathrm{Wo}_{3.6}, \mathrm{FeO} / \mathrm{MnO}=67\right)$, orthopyroxene host $\left(\mathrm{Fs}_{47.1} \mathrm{Wo}_{3.3}, \mathrm{FeO} / \mathrm{MnO}=68\right)$, ferroan pigeonite $\left(\mathrm{Fs}_{52.4} \mathrm{Wo}_{18.8}, \mathrm{FeO} / \mathrm{MnO}=67\right)$, augite $\left(\mathrm{Fs}_{8.0} \mathrm{Wo}_{44.3}, \mathrm{FeO} / \mathrm{MnO}=\right.$ 35), plagioclase $\left(\mathrm{An}_{96.7-97.4} \mathrm{Or}_{0.2-0.1}, \mathrm{~N}=2\right)$.

Classification: Lunar (feldspathic regolith breccia).

Specimens: $20.0 \mathrm{~g}$ including one polished endcut at $U W B$; remainder with $\mathrm{Mr}$. D. Dickens.

\section{Northwest Africa 11304 (NWA 11304) \\ (Northwest Africa) \\ Purchased: 2017 April \\ Classification: Rumuruti chondrite (R4)}

History: Purchased by Dustin Dickens in April 2017 from a Moroccan dealer.

Petrography: (A. Irving and S. Kuehner, UWS ) The specimen is a breccia mostly composed of well-formed, relatively small chondrules (apparent diameter $250 \pm 150$ $\mu \mathrm{m}, \mathrm{N}=12)$ in a finer grained matrix ( $\sim 30 \mathrm{vol} . \%)$, but some petrologically-similar, angular lithic clasts (including type 3 and type 4 clasts) are also present. Both olivine and orthopyroxene have very magnesian cores, but the predominant mafic minerals are much more ferroan. Other minerals are clinopyroxene, sodic plagioclase, pentlandite, pyrrhotite and magnetite. 
Geochemistry: Olivine (cores $\mathrm{Fa}_{1.0-1.2}$; predominantly $\mathrm{Fa}_{39.1-41.6} ; \mathrm{N}=4$ ), orthopyroxene (core $\mathrm{Fs}_{4.5} \mathrm{Wo}_{0.4}$; mantle $\left.\mathrm{Fs}_{16.3} \mathrm{Wo}_{2.7}\right)$, clinopyroxene $\left(\mathrm{Fs}_{11.0-}\right.$ $\left.{ }_{12.4} \mathrm{Wo}_{45.5-41.7}, \mathrm{~N}=2\right)$, plagioclase $\left(\mathrm{Ab}_{85.7} \mathrm{An}_{12.3} \mathrm{Or}_{14.6}\right)$.

Classification: R4. May contain R3 material.

Specimens: $21.2 \mathrm{~g}$ including one polished thin section at $U W B$; remainder with $\mathrm{Mr}$. D. Dickens.

\section{Northwest Africa 11354 (NWA 11354) \\ Morocco \\ Purchased: Feb. 2017 \\ Classification: Enstatite achondrite (ungrouped)}

History: Purchased by Ke Zuokai in Feb. 2017 from a Moroccan dealer.

Physical characteristics: The meteorite is covered by a dark brown fusion crust.

Petrography: The meteorite is composed of medium-grained $(\sim 0.1-0.5 \mathrm{~mm})$ silicates, metal and sulfide. Enstatite is the dominant silicate phase. Other silicates are olivine, albitic plagioclase and fine-grained diopside $(4-20 \mu \mathrm{m})$. No chondrules or clasts are observed. Some regions of the thin section exhibite triple junctions. Metal and sulfide include kamacite, tanite, troilite, alabandite, daubreelite and schreibersite. Daubreelite often presents as lamellae within troilite.

Geochemistry: Enstatite $\mathrm{Fs}_{0.7-2.0} \mathrm{Wo}_{0.7-1.7}(\mathrm{n}=19), \mathrm{Fe} / \mathrm{Mn}$ (cation ratio)=8.0 3.9 ; minor fine-grained Ca-rich pyroxene $\mathrm{Fs}_{43.1-47.2} \mathrm{Wo}_{43.1-47.2}(\mathrm{n}=9$ ), $\mathrm{Fe} / \mathrm{Mn}$ (cation ratio) $=16.6 \pm 10.8$; plagioclase $\mathrm{Ab}_{71.5-83.6} \mathrm{Or}_{0.01-3.4}(\mathrm{n}=9)$; olivine grains are almost pure fosterite $\mathrm{Fo}_{99.3-100}(\mathrm{n}=18), \mathrm{Fe} / \mathrm{Mn}$ (cation ratio) $=3.1 \pm 1.6$. This meteorite lacks Si content in metal. Kamacite (wt\%) Fe 92.49, Ni 5.99, Co 0.51, P 0.05. Taenite (wt\%) Fe 75.71, Ni 23.55, Co 0.31. Troilite (wt\%) Fe 63.11, S 36.07, Co 0.13, Ti 0.18 , Cr 0.29, V 0.06. Daubreelite (wt\%) Fe 19.69, Cr 36.44, S 44.28, Ni 0.07, Ti $0.01, \mathrm{P} 0.04$, Co 0.05. Sulfide is low in Si (below $0.01 \mathrm{wt} \%$ ).

Classification: Enstatite achondrite ungrouped. The meteorite is chemically similar to equilibrated EL chondrites. However, it is distinguished from EL6 chondrite by the lack of chondrules and presence of olivine. Moreover, this meteorite has low $\mathrm{Si}$ content in metal (below detection limit) and low Ti content in troilite $(0.16 \pm 0.02$ $\mathrm{wt} \%$ ), similar to those in NWA 10519 (Enst achon-ung).

\section{Northwest Africa 11511 (NWA 11511)}

Algeria

Purchased: 2017 Jun

Classification: Ureilite (anomalous)

History: Purportedly found near Adrar, Algeria, and purchased by Darryl Pitt in June 2017 from a dealer in Mauritania.

Petrography: (A. Irving and S. Kuehner, UWS) Protogranular aggregate composed predominantly of mafic silicates (orthopyroxene with subordinate olivine, grainsize $0.2-0.7 \mathrm{~mm}$ ) with accessory sodic plagioclase, tabular graphite and rare 
schreibersite. Both orthopyroxene and olivine have thin reduced, magnesian rims containing minor blebs of Fe metal.

Geochemistry: Orthopyroxene $\left(\mathrm{Fs}_{10.4-10.5} \mathrm{Wo}_{4.5-4.4}, \mathrm{FeO} / \mathrm{MnO}=13\right.$; rim $\mathrm{Fs}_{1.1} \mathrm{Wo}_{0.4}$, $\mathrm{FeO} / \mathrm{MnO}=20 ; \mathrm{N}=3)$, olivine $\left(\mathrm{Fa}_{12.6-12.7}, \mathrm{FeO} / \mathrm{MnO}=22-23 ; \operatorname{rim~Fa}_{6.8}, \mathrm{FeO} / \mathrm{MnO}\right.$ $=10 ; \mathrm{N}=3$ ). Oxygen isotopes (K. Ziegler, $\mathrm{UNM})$ : analyses of acid-washed subsamples by laser fluorination gave, respectively, d17O 2.018, 1.933, 1.622; d18O 6.632, 6.506, 6.128; D17O -1.484, -1.502, -1.614 per mil.

Classification:Ureilite (anomalous). Originally thought to be an orthopyroxenerich lodranite. Oxygen isotopes show this graphite-rich achondrite is a ureilite, different from typical ureilites in being orthopyroxene-rich, containing unusually magnesian olivine and most significantly in containing sodic plagioclase.

Specimens: $20.3 \mathrm{~g}$ including one polished thin section at $U W B$; remainder with DPitt.

\section{Northwest Africa 11517 (NWA 11517)}

Northwest Africa

Purchased: 2017

Classification: Lunar meteorite (feldspathic breccia)

History: Four individuals weighing a total of $88 \mathrm{~g}$ were found near Tindouf, Algeria. John Sinclair purchased the stones from a Moroccan meteorite hunter at the Sainte Marie Aux Mines mineral show in France during June of 2017.

Physical characteristics: Sample is irregular-ovoid shaped and lacks fusion crust. The exterior surface is weathered and light orangish-gray in color. The stone shows brecciated texture composed of dominant light-colored clasts in a dark grey matrix. FeNi grains are present in several slices. Pits from weathering of clasts and vesicles contain orange caliche.

Petrography: Description and classification (A. Love, App) Sample is a polymict breccia composed of 0.5-3 mm irregularly shaped lithic and mineral clasts set within a micro-vesiclar, melt matrix. Clasts are composed of poikilitic gabbro, vitrophyric, anorthosite, crystalline impact melt, and basaltic lithologies. Minerals clasts include olivine, exsolved $\mathrm{Ca}$-rich and $\mathrm{Ca}$-poor pyroxene, baddeleyite, ulvospinel, ilmenite, chromite, FeS, kamacite, taenite, Si-polymorph.

Geochemistry: (A. Love, App) Olivine $\left(\mathrm{Fa}_{18.65-53.01}, \mathrm{FeO} / \mathrm{MnO}=83.29-109.49\right.$, $\mathrm{N}=12)$, Opx $\left(\mathrm{Fs}_{16.43-42.54} \mathrm{Wo}_{2.92-4.47}, \mathrm{FeO} / \mathrm{MnO}=53.16-60.73, \mathrm{~N}=5\right)$, pigeonite $\left(\mathrm{Fs}_{40.64-67.00} \mathrm{Wo}_{5.14-11.16}, \mathrm{FeO} / \mathrm{MnO}=54.93-67.00, \mathrm{~N}=8\right)$; augite $\left(\mathrm{Fs}_{17.44-36.61} \mathrm{Wo}_{19.71-}\right.$ 43.48, $\mathrm{N}=8$ ); pyroxeferroite $\mathrm{Fs}_{70.28} \mathrm{Wo}_{5.8}, \mathrm{FeO} / \mathrm{MnO}=60.5, \mathrm{~N}=1$ ); plagioclase $\mathrm{An}_{95.55-}$ 98.72 $\left.\mathrm{Or}_{0.00-0.27}, \mathrm{~N}=15\right)$.

Classification: Lunar (feldspathic breccia). Textures and mineral compositions indicate this sample is a feldspathic breccia. $\mathrm{FeO} / \mathrm{MnO}$ ratios from olivine and pyroxene and An-content of plagioclase suggest this sample is a lunar feldspathic breccia.

Specimens: J. Sinclair holds the main masses. An individual and a slice weighing $17.6 \mathrm{~g}$ and one polished thin section are on deposit at App. 


\title{
Northwest Africa 11521 (NWA 11521) \\ Moroco
}

Purchased: 2017

Classification: Carbonaceous chondrite (CO3)

History: A single piece with a mass of $50 \mathrm{~g}$ was bought in 2017 from a Moroccan meteorite dealer in Ensisheim, France.

Physical characteristics: Fresh-looking individual with black (fusion) crust.

Petrography: The meteorite shows a chondritic texture of abundant small chondrules, metals, sulfides, CAIs, and mineral fragments embedded into a finegrained greyish and porous matrix. Chondrules are dominantly porphyritic and typically $0.1-0.2 \mathrm{~mm}$ in diameter. Sulfides and FeNi metal are fresh and present in matrix and chondrules.

Geochemistry: Mineral compositions and geochemistry: Olivine (mean: Fa $18.7 \pm 14.6$, range: $\mathrm{Fa}_{0.4-38.4}, \mathrm{n}=33$ ), low-Ca pyroxene (mean $\mathrm{Fs}_{4.8 \pm 3.4} \mathrm{Wo}_{2.7 \pm 2.2}$, range: $\mathrm{Fs}_{0.6}$ $\left.{ }_{11.8} \mathrm{Wo}_{0.4-7.4}, \mathrm{n}=24\right)$

Classification: The textural observations suggest a CO3 classification of this rock. The olivines show undulatory extinction. Thus, the rock is of shock stage S2. The sample is only slightly weathered.

\section{Northwest Africa 11550 (NWA 11550)}

(Northwest Africa)

Purchased: 2017 Jan 29

Classification: HED achondrite (Eucrite)

History: Purchased NWA stone at the Tucson Gem show from Sidi Mohamad Ismaily

Physical characteristics: Exterior surfaces partly covered by black fusion crust; non-crusted surfaces are gray with portions covered by pinkish dust.

Petrography: In thin section dominated by many small mineral clasts, and some lithic clasts

Geochemistry: Low-Ca pyroxene $\mathrm{Fs}_{60.0 \pm 1.7} \mathrm{Wo}_{2.1 \pm 0.8}(\mathrm{~N}=47)$.

Classification: Monomict, largely equilibrated, brecciated eucrite based on texture and mineral composition.

Specimens: Cascadia holds the entire sample, consisting of $157.8 \mathrm{~g}$, a polished thin section, and potted butt.

\author{
Northwest Africa 11551 (NWA 11551) \\ Mauritania \\ Purchased: 2017 Jan 30 \\ Classification: HED achondrite (Howardite)
}


History: Type specimen donated to Cascadia by Mohammed Hmani at the Tucson Gem Show in 2017. Mr. Hmani stated that the meteorite was found in 2014 in Mauritania.

Physical characteristics: Exterior surfaces partly covered by black fusion crust; non-crusted surfaces show obvious breccia texture with variegated clasts up 1o 1 $\mathrm{cm}$ across. Most surfaces appear relatively unweathered but rust spots are present Petrography: In thin section appears to be a roughly 50-50 mixture of diogenitic (coarse orthopyroxene) and eucritic (plagioclase, pyroxene-dominated) clasts. Some eucritic pyroxene shows zoning.

Geochemistry: Diogenitic orthopyroxene $\mathrm{Fs}_{27.1 \pm 3.8} \mathrm{Wo}_{2.4 \pm 1.0}(\mathrm{~N}=182)$, eucritic lowCa pyroxene $\mathrm{Fs}_{41.6 \pm 6.0} \mathrm{Wo}_{3.1 \pm 1.0}(\mathrm{~N}=40)$, augite $\mathrm{Fs}_{26.8 \pm 7.8} \mathrm{Wo}_{41.5 \pm 2.5}(\mathrm{~N}=30)$, uninverted pigeonite $\mathrm{Fs}_{48.8 \pm 5.8} \mathrm{Wo}_{8.0 \pm 2.3}(\mathrm{~N}=36)$, bulk inverted pigeonite

$\mathrm{Fs}_{50.5 \pm 5.4} \mathrm{Wo}_{10.7 \pm 3.0}(\mathrm{~N}=24)$.

Classification: Howardite based on textures and mineral proportions.

Specimens: Cascadia holds $20.0 \mathrm{~g}$ and two polished thin sections and a potted butt. The main mass is held by HMANI Inc. (13 Rue Joules Hardouin Mansart, 92600 Asnieres, France).

\section{Northwest Africa 11552 (NWA 11552) \\ (Northwest Africa) \\ Purchased: 2016 Nov 16 \\ Classification: HED achondrite (Eucrite)}

History: Donated to Cascadia in February, 2017 by Dr. Baecker, who had purchased the sample as an unclassified NWA on eBay.

Physical characteristics: Single oriented cone mostly covered by black fusion crust. Interior cut faces show clastic texture ranging from small fragments to sub$\mathrm{cm}$ subophitic-textured clasts. Brownish rust spots visible.

Petrography: Clastic texture in thin section, including mineral, lithic, and melt breccia clasts. Relatively abundant calcite veining

Geochemistry: Most pyroxene analyses are mixtures of low- and high-Ca

pyroxene. Low-Ca pyroxene $\mathrm{Fs}_{66.1 \pm 1.7} \mathrm{Wo}_{4.9 \pm 0.3}(\mathrm{~N}=6)$; augite $\mathrm{Fs}_{34.1 \pm 2.0} \mathrm{Wo}_{39.4 \pm 2.4}(\mathrm{~N}=9)$.

Classification: Monomict, largely equilibrated, brecciated eucrite based on texture and mineral composition.

Specimens: Cascadia holds $21.5 \mathrm{~g}$, two polished thin sections, and potted butt. Main mass is held Dr. Bastian Baecker (Huntsville, AL)

\section{Northwest Africa 11556 (NWA 11556)}

(Northwest Africa)

Purchased: 2017 Mar

Classification: Carbonaceous chondrite ( $\mathrm{C} 2$, ungrouped)

History: Purchased in Agadir, Morocco by Aziz Habibi in March 2017. 
Physical characteristics: A single, very soft jet black stone (61 g) containing some visible but tiny, clear crystalline objects. During preparation for powder X-ray diffraction by immersing sample fines in methanol a strong organic odor was emitted.

Petrography: (A. Irving and S. Kuehner, UWS; L. Garvie, ASU) Sparse very small, subspherical polycrystalline objects (apparent diameter $180 \pm 150 \mu \mathrm{m}, \mathrm{N}=18$ ) and angular mafic silicate mineral grains are set in a very fine grained matrix. The dominant matrix ( $\sim 90$ vol.\%, jet black in thin section) contains prominent open shrinkage cracks and is composed mainly of indeterminate mafic silicates plus minor chromite, pentlandite and calcite. X-ray diffraction shows the specimen to be dominated by tochilinite and well-crystallized serpentine. No CAI were observed despite a careful search.

Geochemistry: Olivine $\left(\mathrm{Fa}_{1.2-56.0}, \mathrm{Cr}_{2} \mathrm{O}_{3}\right.$ in ferroan examples $0.10-0.40$ wt.\%, mean $0.25 \pm 0.11$ wt. $\%, \mathrm{~N}=8)$, orthopyroxene $\left(\mathrm{Fs}_{0.9-1.0} \mathrm{Wo}_{1.0-3.6}, \mathrm{~N}=3\right)$, clinopyroxene $\left(\mathrm{Fs}_{1.1} \mathrm{Wo}_{42.2} ; \mathrm{Fs}_{0.9} \mathrm{Wo}_{48.0} ; \mathrm{N}=2\right)$. Oxygen isotopes $(\mathrm{K}$. Ziegler, UNM): analyses of untreated subsamples by laser fluorination gave, respectively, $\delta^{17} \mathrm{O}-2.789,-3.797$; $\delta^{18} \mathrm{O} 1.421,-0.319 ; \Delta^{17} \mathrm{O}-3.539,-3.629$ per mil.

Classification: Carbonaceous chondrite (type 2, ungrouped). The paucity of chondrule-like objects, high abundance of matrix, and more 16O-rich bulk oxygen isotope composition distinguish this specimen from $\mathrm{CM}$ chondrites.

Specimens: $12.3 \mathrm{~g}$ including one polished thin section at $U W B$; remainder with $\mathrm{Mr}$. A. Habibi.

\section{Northwest Africa 11558 (NWA 11558)}

(Northwest Africa)

Purchased: 2017 Sep

Classification: Ungrouped achondrite

History: Two identical small samples of an unusual achondrite were sent by two separate Moroccan dealers to Dr. Anthony Irving in January 2017. Later in September 2017 the remainder of one of these specimens was purchased from one of the original dealers by Ben Hoefnagels.

Physical characteristics: The friable specimens (total weight $3.92 \mathrm{~g}$ ) are both composed of abundant white and green grains.

Petrography: (A. Irving and S. Kuehner, UWS; I. Barker and D. Moser, UWO) The specimens have a porphyritic plutonic igneous texture. The mineralogy is dominated by larger grains $(1.6-2.1 \mathrm{~mm})$ of anorthite, strongly zoned clinopyroxene and tridymite (identified by electron backscatter diffraction analysis). More sodic (bytownitic) plagioclase and more ferroan clinopyroxenes occur in the groundmass together with accessory orthopyroxene, ilmenite, ulvöspinel, troilite, cristobalite, minor quartz, rare low-Ni kamacite and rare baddeleyite. Some silica occurs as graphic intergrowths with clinopyroxene in the groundmass, or as very small inclusions accompanied by clinopyroxene within plagioclase.

Geochemistry: Diopside cores $\left(\mathrm{Fs}_{5.1-5.3} \mathrm{Wo}_{38.8-39.0}, \mathrm{FeO} / \mathrm{MnO}=6, \mathrm{~N}=2\right)$, subcalcic augite $\left(\mathrm{Fs}_{37.0} \mathrm{Wo}_{30.9}, \mathrm{FeO} / \mathrm{MnO}=8\right)$, subcalcic ferroaugite $\left(\mathrm{Fs}_{67.3} \mathrm{Wo}_{25.8}, \mathrm{FeO} / \mathrm{MnO}\right.$ 
$=10)$, ferropigeonite $\left(\mathrm{Fs}_{69.7-78.7} \mathrm{Wo}_{22.3-15.4}, \mathrm{FeO} / \mathrm{MnO}=7-10, \mathrm{~N}=2\right)$, plagioclase $\left(\mathrm{An}_{69.2-91.9} \mathrm{Or}_{1.2-0.2}, \mathrm{~N}=3\right)$. Oxygen isotopes (K. Ziegler, $\left.U N M\right)$ : analyses of acidwashed subsamples by laser fluorination gave, respectively, $\delta^{17} \mathrm{O} 2.716,3.020$, $3.525 ; \delta^{18} \mathrm{O} 6.990,7.573,8.530 ; \Delta^{17} \mathrm{O}-0.975,-0.979,-0.979$ per mil.

Classification: Ungrouped achondrite. This material is paired with NWA 11119 on the basis of closely similar mineralogy and identical oxygen isotopic composition. According to the IUGS nomenclature for igneous rocks (Le Maitre et al., 1989), this specimen would be a porphyritic quartz gabbro. However, given that the dominant silica polymorph is tridymite, a more appropriate designation would be tridymite gabbro.

Specimens: $2.56 \mathrm{~g}$ including one polished epoxy mount at $U W B ; 1.36 \mathrm{~g}$ with $\mathrm{Mr}$. B. Hoefnagels.

\section{Northwest Africa 11559 (NWA 11559)}

Algeria

Purchased: 2017 Jun

Classification: Carbonaceous chondrite (CO3)

History: Purportedly found near the Algeria/Libya border and purchased by Darryl Pitt in June 2017 from a dealer in Mauritania.

Petrography: (A. Irving and S. Kuehner, UWS) Small, well-formed chondrules (apparent diameter $420 \pm 190 \mu \mathrm{m}, \mathrm{N}=20$ ) accompanied by sparse angular mineral grains are set in a finer grained matrix ( 40 vol. $\%$, brown in thin section) containing accessory chromite, altered kamacite and pyrrhotite. No CAI were observed.

Geochemistry: Olivine $\left(\mathrm{Fa}_{0.9-41.4}, \mathrm{Cr}_{2} \mathrm{O}_{3}\right.$ in ferroan examples $\left.<0.02 \mathrm{wt} . \%, \mathrm{~N}=8\right)$, orthopyroxene $\left(\mathrm{Fs}_{12.6-23.8} \mathrm{Wo}_{2.1-0.6}, \mathrm{~N}=3\right)$, clinopyroxene $\left(\mathrm{Fs}_{9.1-10.9} \mathrm{Wo}_{46.8-45.9}, \mathrm{~N}=\right.$ 2). Magnetic susceptibility $\log \chi\left(\times 10^{-9} \mathrm{~m}^{3} / \mathrm{kg}\right)=3.02$.

Classification: Carbonaceous chondrite (CO3). The below detection limit $\mathrm{Cr}_{2} \mathrm{O}_{3}$ contents in ferroan olivines indicate a petrologic subtype $>3.6$.

Specimens: $9.5 \mathrm{~g}$ including one polished thin section at $U W B$; remainder with DPitt.

\section{Northwest Africa 11560 (NWA 11560)}

(Northwest Africa)

Purchased: 2017 Oct

Classification: HED achondrite (Diogenite)

History: Purchased in Temara, Morocco by Adam Aaronson in October 2017. Physical characteristics: A single large stone (13060 g) composed predominantly of vitreous yellow-green pyroxene with sparse opaque grains.

Petrography: (A. Irving, UWS and P. Carpenter, WUSL) Protogranular aggregate (mean grainsize $0.9 \mathrm{~mm}$ ) of predominantly orthopyroxene plus minor chromite and rare olivine. Minor calcite accompanied by iron hydroxide occurs along grain boundaries. 
Geochemistry: Orthopyroxene $\left(\mathrm{Fs}_{22.7-23.5} \mathrm{Wo}_{1.5-2.0}, \mathrm{FeO} / \mathrm{MnO}=28-30, \mathrm{~N}=3\right)$, olivine $\left(\mathrm{Fa}_{26.2-26.3}, \mathrm{FeO} / \mathrm{MnO}=44-46, \mathrm{~N}=2\right)$.

Classification: Diogenite.

Specimens: $20.2 \mathrm{~g}$ including one polished thin section at $U W B$; remainder with Aaronson.

\section{Northwest Africa 11561 (NWA 11561)}

(Northwest Africa)

Purchased: 2016 Dec

Classification: Carbonaceous chondrite (CR6)

History: Purchased in December 2016 by Ben Hoefnagels from a French dealer, who had obtained the stone from a source in Niger.

Petrography: (A. Irving and S. Kuehner, UWS) Metamorphic texture. Larger (up to $3.6 \mathrm{~mm}$ ) pyroxene poikiloblasts containing numerous rounded olivine chadacrysts are present within a dominant finer-grained, recrystallized matrix exhibiting triple grain junctions. Pyroxene is mostly orthopyroxene, but blebby inclusions of clinopyroxene are present in places. Accessory phases are chromite, kamacite, Ni-poor taenite and troilite.

Geochemistry: Olivine $\left(\mathrm{Fa}_{29.0-29.8}, \mathrm{FeO} / \mathrm{MnO}=64-67, \mathrm{~N}=3\right)$, orthopyroxene host $\left(\mathrm{Fs}_{23.0-23.6} \mathrm{Wo}_{3.8-3.6}, \mathrm{FeO} / \mathrm{MnO}=40-42, \mathrm{~N}=2\right)$, clinopyroxene exsolution lamellae $\left(\mathrm{Fs}_{10.7-12.0} \mathrm{Wo}_{41.8-39.8}, \mathrm{FeO} / \mathrm{MnO}=28-31, \mathrm{~N}=2\right)$, plagioclase $\left(\mathrm{An}_{42.0-50.3} \mathrm{Or}_{1.6-1.8}, \mathrm{~N}=\right.$ 2). Oxygen isotopes (K. Ziegler, $U N M$ ): analyses of acid-washed subsamples by laser fluorination gave, respectively, $\delta^{17} \mathrm{O}-0.259,-0.450,-0.344 ; \delta^{18} \mathrm{O} 2.659,2.297$, 2.506; $\Delta^{17} \mathrm{O}-1.663,-1.663,-1.667$ per mil.

Classification: Carbonaceous chondrite (CR6). In terms of textural, mineralogical and isotopic characteristics, this specimen is very similar to Tafassasset and NWA 7531.

Specimens: $53.0 \mathrm{~g}$ including one polished thin section at $U W B$; remainder with $\mathrm{Mr}$. B. Hoefnagels.

\section{Northwest Africa 11562 (NWA 11562) \\ (Northwest Africa) \\ Purchased: 2017 Oct \\ Classification: Ungrouped achondrite}

History: Purchased in Temara, Morocco, by Adam Aaronson in October 2017.

Petrography: (A. Irving, UWS and P. Carpenter, WUSL) The specimen consists of coarser, protogranular regions (mean grainsize $0.25 \mathrm{~mm}$ ) composed of forsterite and enstatite with some interstitial diopside, altered kamacite and blade-like cohenite, plus much finer-grained, quench-textured regions with the same mineralogy.

Geochemistry: Olivine $\left(\mathrm{Fa}_{1.0-1.1}, \mathrm{FeO} / \mathrm{MnO}=3-4, \mathrm{~N}=3\right)$, orthopyroxene $\left(\mathrm{Fs}_{0.9-}\right.$ $\left.{ }_{1.0} \mathrm{Wo}_{1.0-2.3}, \mathrm{FeO} / \mathrm{MnO}=2-3, \mathrm{~N}=3\right)$, diopside $\left(\mathrm{Fs}_{0.2-0.9} \mathrm{Wo}_{36.3-40.5}, \mathrm{Al}_{2} \mathrm{O}_{3}=2.0-3.6\right.$ wt. $\%, \mathrm{TiO}_{2}=0.5-0.6 \mathrm{wt} . \%, \mathrm{~N}=3$ ). Oxygen isotopes (K. Ziegler, $\left.U N M\right)$ : analyses 
of acid-washed subsamples by laser fluorination gave, respectively $\delta^{17} \mathrm{O} 1.567$, $1.396,1.479 ; \delta^{18} \mathrm{O} 6.345,6.084,6.291 ; \Delta^{17} \mathrm{O}-1.783,-1.816,-1.843$ per mil.

Classification: Achondrite (ungrouped). The oxygen isotope composition plots between the trends for $\mathrm{CR}$ and $\mathrm{CV}$ chondrites, and far from the trends for most achondrites (except for some ureilites); however, the highly magnesian silicates and evidence for partial melting would seem to preclude any affinity to ureilites.

Specimens: $20.5 \mathrm{~g}$ including one polished thin section at $U W B$; remainder with $\mathrm{Ke}$ Zuokai.

\section{Northwest Africa 11563 (NWA 11563)}

(Northwest Africa)

Purchased: 2016 Jun

Classification: Lunar meteorite (feldspathic breccia)

History: Purchased by Ben Hoefnagels in June 2016 from a Moroccan dealer. Petrography: (A. Irving and S. Kuehner, UWS) Breccia composed of mineral clasts of anorthite, labradorite, olivine, orthopyroxene, pigeonite, subcalcic augite, exsolved pigeonite, ilmenite, fayalite and silica polymorph in a finer grained, partly vesicular matrix containing minor troilite and kamacite.

Geochemistry: Olivine $\left(\mathrm{Fa}_{23.0-37.4}, \mathrm{FeO} / \mathrm{MnO}=94, \mathrm{~N}=2\right)$, orthopyroxene $\left(\mathrm{Fs}_{29.5} \mathrm{Wo}_{4.5}, \mathrm{FeO} / \mathrm{MnO}=45\right)$, pigeonite $\left(\mathrm{Fs}_{29.6} \mathrm{Wo}_{7.4}, \mathrm{FeO} / \mathrm{MnO}=83\right)$, subcalcic augite $\left(\mathrm{Fs}_{22.7} \mathrm{Wo}_{25.1} ; \mathrm{Fs}_{34.2} \mathrm{Wo}_{32.4}, \mathrm{FeO} / \mathrm{MnO}=71-77, \mathrm{~N}=2\right)$, anorthite $\left(\mathrm{An}_{95.0} \mathrm{Or}_{0.2}\right)$, labradorite $\left(\mathrm{An}_{50.9-54.1} \mathrm{Or}_{3.6-1.0}, \mathrm{~N}=2\right)$.

Classification: Lunar (feldspathic regolith breccia).

Specimens: $11.8 \mathrm{~g}$ in the form of a polished endcut at $U W B$; remainder of the stone with Mr. B. Hoefnagels.

\section{Northwest Africa 11564 (NWA 11564)}

(Northwest Africa)

Purchased: 2017 Sep

Classification: Carbonaceous chondrite (CO3)

History: Purchased by Habib Naji in September 2017 from a Mauritanian dealer.

Petrography: (A. Irving and S. Kuehner, UWS) Small, rounded to irregularly shaped chondrules (apparent diameter $165 \pm 80 \mu \mathrm{m}, \mathrm{N}=20$ ) accompanied by sparse angular mineral grains and some very fine grained CAI are set in a finer grained matrix ( $\sim 30$ vol. \%, brown in thin section).

Geochemistry: Olivine $\left(\mathrm{Fa}_{1.2-64.7}, \mathrm{Cr}_{2} \mathrm{O}_{3}\right.$ in ferroan examples $\left.<0.02 \mathrm{wt} \% \%, \mathrm{~N}=7\right)$, orthopyroxene $\left(\mathrm{Fs}_{0.8-27.5} \mathrm{Wo}_{0.8-3.0}, \mathrm{~N}=3\right)$, clinopyroxene $\left(\mathrm{Fs}_{1.5-4.4} \mathrm{Wo}_{43.7-47.7}, \mathrm{~N}=2\right)$.

Classification: Carbonaceous chondrite (CO3). The below detection limit $\mathrm{Cr}_{2} \mathrm{O}_{3}$ contents in ferroan olivines indicate a petrologic subtype $>3.6$.

Specimens: $24.05 \mathrm{~g}$ including one polished thin section at $U W B$; remainder with $\mathrm{H}$. Naji. 


\section{Northwest Africa 11565 (NWA 11565)}

(Northwest Africa)

Purchased: 2017

Classification: Carbonaceous chondrite (CK6)

Petrography: The stone is very recrystallized with a groundmass coarseness of 50$100 \mu \mathrm{m}$, typical of CK6 chondrites. It is rather weathered; $\sim 80 \%$ of the silicates are stained brown. The rock contains $\sim 15$ vol.\% discernable chondrules. Plagioclase grains are approxcimately $50 \mu \mathrm{m}$ in size. The plagioclase is heterogeneous (typical of $\mathrm{CKs}): \mathrm{Ab}_{44.1 \pm 19.4} \mathrm{Or}_{1.5 \pm 1.3} \mathrm{An}_{54.4 \pm 20.6}(\mathrm{n}=9)$ with an overall range $\mathrm{An}_{27.2-85.8 \text {. Also }}$ present are diopside $\left(\mathrm{Fs}_{9.2 \pm 0.6} \mathrm{Wo}_{45.9 \pm 0.9}, \mathrm{n}=4\right)$ and augite $\left(\mathrm{Fs}_{14.8} \mathrm{Wo}_{27.1}, \mathrm{n}=1\right)$. As in some other $\mathrm{CK}$ chondrites, there is more $\mathrm{Ca}$ pyroxene than low-Ca pyroxene. Olivine $\left(\mathrm{Fa}_{30.1 \pm 0.5}, \mathrm{n}=17\right)$ is also in the $\mathrm{CK}$-chondrite range.

\section{Northwest Africa 11570 (NWA 11570) \\ (Northwest Africa) \\ Purchased: March 2017 \\ Classification: Ordinary chondrite (H/L4)}

History: Purchased by Sean Tutorow and Brahim Tahiri from several diferent meteorite hunters in Morocco.

Petrography: The olivine in the rock is fairly homogeneous and is in between the Fa ranges for $\mathrm{H}\left(\mathrm{Fa}_{17.3-20.2}\right)$ and $\mathrm{L}\left(\mathrm{Fa}_{23.0-25.8}\right)$ chondrites, leading to a classification of $\mathrm{H} / \mathrm{L}$. Although the mean low-Ca pyroxene composition $\left(\mathrm{Fs}_{17.6}\right)$ is within the range of $\mathrm{H}$ chondrites, it is heterogeneous and is thus not definitive. Also present is rare Ca-pyroxene: $\mathrm{Fs}_{8.7} \mathrm{Wo}_{21.0}(\mathrm{n}=1)$. The rock has well-defined chondrules. A few low-Ca pyroxene grains exhibit polysynthetic twinning. Chondrules haev devitrified mesostases. There is minor recrystallization apparent in the rock; there is some integration of chondrules with the matrix. Some troilite grains are polycrystalline.

\section{Northwest Africa 11571 (NWA 11571) \\ (Northwest Africa) \\ Purchased: March 2017 \\ Classification: Rumuruti chondrite (R4)}

History: Purchased by Sean Tutorow and Brahim Tahiri from several diferent meteorite hunters in Morocco.

Petrography: Pyroxene is rare, but some low-Ca pyroxene grains exhibit polysynthetic twinning. The only two grains that were analyzed are both $\mathrm{Ca}-$ pyroxene. Opaque phases consist of small grains of metal and both small and large grains of pyrrhotite. Chondrules are well defined; chondrule types include BO, PP, PO, POP, C, GOP and RP. Some

Northwest Africa 11572 (NWA 11572) 
(Northwest Africa)

Purchased: March 2017

Classification: Ordinary chondrite (H4, melt breccia)

History: Purchased by Sean Tutorow and Brahim Tahiri from several diferent meteorite hunters in Morocco.

Petrography: The rock is an impact-melt breccia. Melted regions are very dark; they contain numerous metal-sulfide veins, polycrystalline troilite and rare cellular, rapidly cooled metal-sulfide assemblages. There are rare grains of coarse troilite. Most metal grains are not attached to sulfide grains. Some low-Ca pyroxene grains exhibit polysynthetic twinning, perhaps caused by post-shock quenching. There is strong mosaic extinction evident in the olivine grains.

\section{Northwest Africa 11573 (NWA 11573)}

(Northwest Africa)

Purchased: 2017

Classification: Enstatite achondrite (Aubrite)

History: Three uncrusted stones weighing a total of $107 \mathrm{~g}$ were found in Morocco prior to 2016. J. Donald Cline and John Sinclair acquired the samples from a meteorite dealer at the Tucson Gem and Mineral Show in February of 2017.

Physical characteristics: Specimen is dark orange-brown-colored, has a pitted irregular-shape and lacks fusion crust. Interior shows a brecciated texture composed of light orange-brown crystals, lithic fragments and metallic grains are visible on the cut surface.

Petrography: Description and Classification (A. Love, App): Sample is a breccia composed of angular to subrounded crystals of enstatite (up to $5 \mathrm{~mm}$ ) and cumulate-textured clasts. Clasts show poikolitic texture composed of subhedral to anhedral, polysynthetically twinned enstatite and anhedral plagioclase feldspar set within birefringent, nonstoichiometric albitic glass. Additional minerals are; daubréelite; Si-polymorph; schreibersite; Si-bearing (4.88 wt\%) kamacite; perryite; graphite; niningerite; troilite and oldhamite.

Geochemistry: (A. Love, App) Enstatite $\left(\mathrm{Fs}_{0.2 \pm 0.2} \mathrm{Wo}_{0.4 \pm 0.1}, \mathrm{~N}=18\right)$; plagioclase $\left(\mathrm{An}_{2.4 \pm 0.5} \mathrm{Or}_{5.0 \pm 0.4}, \mathrm{~N}=11\right)$.

Classification: Achondrite (aubrite). Textures, mineralogy and mineral compositions suggest this is a aubrite.

Specimens: PARI holds the main mass. A whole individual and an endcut weighing $20.6 \mathrm{~g}$, a polished mount and thin section are on deposit at App.

\section{Northwest Africa 11574 (NWA 11574)}

(Northwest Africa)

Purchased: 2017

Classification: Ordinary chondrite (L3.10)

History: Purchased by Marcin Cimala in Morocco, 2017. 
Physical characteristics: Single stone with weathered exterior, saw cuts shows many densely packed chondrules set in a dark brown matrix.

Petrography: (C. Agee, $U N M)$ Microprobe examination of a polished mount shows numerous porphyritic chondrules, most with mesostasis or glass. Adundant opaque matrix. Apparent mean chondrule diameter $700 \pm 390 \mu \mathrm{m}, \mathrm{n}=31$, consistent with L.

Geochemistry: (C. Agee, $U N M$ ) All olivine $\mathrm{Fa}_{16.4 \pm 12.5}, \mathrm{Fe} / \mathrm{Mn}=50 \pm 25, \mathrm{n}=40$; Ferroan chondrule olivine $\mathrm{Fa}_{19.7 \pm 11.3}, \mathrm{Fe} / \mathrm{Mn}=55 \pm 23, \mathrm{Cr}_{2} \mathrm{O}_{3}=0.21 \pm 0.21$ (wt\%), $\mathrm{n}=33$; low-Ca pyroxene $\mathrm{Fs}_{13.0 \pm 10.6} \mathrm{Wo}_{1.8 \pm 1.9}, \mathrm{n}=13$.

Classification: Ordinary chondrite (L3.10), type 3.10 based on mean value of $\mathrm{Cr}_{2} \mathrm{O}_{3}$ in ferroan chondrule olivine and the 1-sigma standard deviation, near the boundary between 3.10 and 3.15 in figure 13 (Grossman and Brearley, 2005), however closest to values of Adrar 003 (L/LL3.10) with $\mathrm{Cr}_{2} \mathrm{O}_{3}=0.23 \pm 0.21(\mathrm{n}=47)$. Specimens: $29 \mathrm{~g}$ including a probe mount on deposit at $U N M$, Marcin Cimala holds the main mass.

\section{Northwest Africa 11575 (NWA 11575) \\ Mali \\ Purchased: 2016 Sept \\ Classification: Ungrouped achondrite}

History: Found in June 2016 at the border region of Mali and Algeria; purchased in Mauritania in September 2016.

Physical characteristics: Single stone, $80 \%$ covered by fusion crust. Saw cut reveals a surface of approximately 12 square $\mathrm{cm}$ with a light colored, felsicappearing, aphanitic texture. A single, $\sim 1 \mathrm{~mm}$ wide, dark colored shock melt vein crosscuts this surface. Also present are a few smaller xenoliths which are dark in color, angular, mafic-appearing, and aphanitic; the largest of which is approximately $1 \mathrm{~cm}$ across.

Petrography: (C. Agee, UNM) Microprobe and SEM examinations were performed on a polished probe mount and on the saw-face of the deposit sample respectively. An ophitic texture of pyroxene and plagioclase grains, making up approximately $90-95 \%$ of the model mineralogy, was observed. Pyroxenes were typically $300-500 \mu \mathrm{m}$ in size and showed significant igneous zoning. The pyroxene grains had cores of magnesian pigeonite, outwardly transitioning to a zone of augite, and rimmed by a zone of sub-calcic ferroaugite to ferropigeonite.

Plagioclase grains were oligoclase, mostly lath-like in shape, and typically 200x50 $\mu \mathrm{m}$ in size. We also observed ubiquitous potassium feldspar and silica, which were commonly found in contact with each other, and together made up $\sim 5 \%$ of the modal mineralogy. Opaque phases made up $\sim 1 \%$ of the modal mineralogy; these included ilmenite, iron sulfide, iron metal (nickel not detected), chromite, and iron oxide. The mafic xenolith clasts were basaltic in major mineralogy and lacked Kfeldspar. The shock melt vein composition within the host felsic lithology plots in the andesite field of the TAS diagram, whereas the composition of the fusion crust of the meteorite, plots in the basaltic andesite field. 
Geochemistry: (C. Agee, $U N M$ ) Magnesian pigeonite $\mathrm{Fs}_{28.3 \pm 3.3} \mathrm{Wo}_{7.3 \pm 1.6}$ $\mathrm{Fe} / \mathrm{Mn}=37 \pm 4, \mathrm{n}=5$; augite $\mathrm{Fs}_{24.4 \pm 4.2} \mathrm{Wo}_{30.0 \pm 3.8}, \mathrm{Fe} / \mathrm{Mn}=33 \pm 4$, $\mathrm{n}=21$; sub-calcicferroaugite/ferropigeonite $\mathrm{Fs}_{57.3 \pm 6.2} \mathrm{Wo}_{14.7 \pm 3.7}, \mathrm{Fe} / \mathrm{Mn}=48 \pm 3, \mathrm{n}=19$; plagioclase $\mathrm{Ab}_{81.3 \pm 1.9} \mathrm{An}_{17.4 \pm 2.0} \mathrm{Or}_{1.3 \pm 0.2}, \mathrm{n}=20$; potassium feldspar $\mathrm{Ab}_{4.0 \pm 0.9} \mathrm{An}_{0.4 \pm 0.3} \mathrm{Or}_{95.6 \pm 1.1}$, $\mathrm{n}=23$; shock melt vein (proxy for felsic lithology bulk composition) $\mathrm{SiO}_{2}=59.1 \pm 5.7, \mathrm{Al}_{2} \mathrm{O}_{3}=10.1 \pm 1.5, \mathrm{MgO}=4.5 \pm 1.3, \mathrm{FeO}=15.6 \pm 5.9, \mathrm{CaO}=6.5 \pm 1.5$, $\mathrm{Na}_{2} \mathrm{O}=3.8 \pm 1.2$ (all wt\%), $\mathrm{n}=11$; fusion crust (proxy for meteorite bulk composition) $\mathrm{SiO}_{2}=55.8 \pm 3.7, \mathrm{Al}_{2} \mathrm{O}_{3}=8.2 \pm 3.4, \mathrm{MgO}=6.7 \pm 1.0, \mathrm{FeO}=16.2 \pm 6.2$, $\mathrm{CaO}=7.2 \pm 0.7, \mathrm{Na}_{2} \mathrm{O}=2.6 \pm 0.5, \mathrm{~K}_{2} \mathrm{O}=0.5 \pm 0.2$ (all wt\%), $\mathrm{n}=8$. Oxygen isotopes $(\mathrm{K}$. Ziegler, $U N M)$ : 3 acid-washed fragments analyzed by laser fluorination gave $\delta^{18} \mathrm{O}=$ $4.875,5.583,5.349 ; \delta^{17} \mathrm{O}=3.760,4.137,4.006 ; \Delta^{17} \mathrm{O}=1.186,1.189,1.182$ (linearized, all per mil, TFL slope $=0.528$ ).

Classification: Ungrouped achondrite. Pyroxene compositional core-rim zoning trends are similar to that observed in Martian basalt QUE 94201 (Papike et al. 2009) and Apollo 15 Mare basalts (Kushiro, 1973). Oxygen isotopes plot within the field defined by LL-chondrites, however the 3 measured data points, from different fragments of the meteorite, form a slope of 0.53 , parallel to the mass dependent terrestrial fractionation line. The andesite to basaltic andesite compositions of the shock melt vein and fusion crust of this meteorite plot on the TAS diagram in a broadly similar location to the estimated bulk composition of ungrouped achondrites GRA 06128/GRA 06129; and this meteorite and GRA 06128/06129 have similar oligoclase plagioclase. However, GRA 06128/06129 are not likely related to this meteorite since they are olivine-bearing, lack K-feldspar, and have oxygen isotopes that plot below the TFL.

Specimens: $22.1 \mathrm{~g}$ on deposit at $U N M$, Darryl Pitt holds the main mass.

\section{Northwest Africa 11576 (NWA 11576)}

(Northwest Africa)

Purchased: 2017

Classification: HED achondrite (Eucrite, melt breccia)

History: Reportedly found in Mali, September 2017, purchased in Morocco.

Physical characteristics: Single stone, no fusion crust, exterior shows numerous light colored clasts set in a dark matrix of shock melt.

Petrography: (C. Agee, UNM) Microprobe examination shows basaltic clasts with approximately $60 \%$ pyroxene and $35 \%$ plagioclase grains most in the range 100 $200 \mu \mathrm{m}$. Many pyroxene grains have exsolution lamellae.

Geochemistry: (C. Agee, UNM) Low-Ca pyroxene $\mathrm{Fs}_{62.1 \pm 1.1} \mathrm{Wo}_{2.7 \pm 0.8}$, $\mathrm{Fe} / \mathrm{Mn}=32 \pm 1, \mathrm{n}=7$; high-Ca pyroxene $\mathrm{Fs}_{28.3 \pm 1.6} \mathrm{Wo}_{42.2 \pm 1.8}, \mathrm{Fe} / \mathrm{Mn}=31 \pm 1, \mathrm{n}=6$; plagioclase $\mathrm{An}_{88.9 \pm 0.4}, \mathrm{n}=3$.

Classification: Eucrite-melt breccia, eucrite clasts are consistent with type 6 (Takeda and Graham, 1991) showing clear compositional separation of low and high calcium pyroxenes.

Specimens: $24.5 \mathrm{~g}$ on deposit at $U N M$, Mohamed Aid holds the main mass. 


\section{Northwest Africa 11577 (NWA 11577) \\ Morocco \\ Purchased: 2017 Jan \\ Classification: Iron meteorite (IAB-sHL)}

History: Reportedly found in Algeria by nomads in December 2016, subsequently sold to a Moroccan merchant in Ouarzazate, from whom it was purchased by $\mathrm{J}$. Shea.

Physical characteristics: The main mass consists of two tabular specimens approximately $1 \mathrm{~cm}$ thick, both having dark brown exteriors.

Petrography: (C. Herd, $U A b$ ) SEM investigation of a $\sim 2 \times 3 \mathrm{~cm}$ polished surface reveals a well-developed Widmanstatten pattern consistent with a finest octahedrite $(0.025 \pm 0.005 \mathrm{~mm} ; \mathrm{n}=16)$. Some areas of kamacite are coarser, and some kamacite has been replaced by terrestrial oxidation.

Geochemistry: ICP-MS data, using sample of North Chile (Filomena) as standard (C. Herd and G. Chen $U A b): \mathrm{Ni}=12.5, \mathrm{Co}=0.55$ (both $\mathrm{wt} \%$ ); $\mathrm{Ir}=0.12, \mathrm{Ga}=17$, $\mathrm{Ge}=38, \mathrm{As}=24, \mathrm{~W}=0.38, \mathrm{Re}=0.01, \mathrm{Os}=0.22, \mathrm{Cu}=276, \mathrm{Au}=2.7(\mathrm{all} \mu \mathrm{g} / \mathrm{g})$.

Classification: (C. Herd, $U A b)$ : IAB iron, sHL subgroup according to Wasson and Kallemeyn (2002).

Specimens: Type specimen consisting of two representative slices (16.6 and $2.1 \mathrm{~g}$ ) at $U A b$. Main mass with Shea.

\section{Northwest Africa 11584 (NWA 11584)}

(Northwest Africa)

Purchased: 2017

Classification: Ordinary chondrite (L5)

History: Purchased from a Moroccan meteorite dealer in Tucson, 2017. Reportedly found in Morocco in 2016.

Physical characteristics: Single stone, broken surface shows numerous well defined chondrules, some oxidation present, desert caliche on exterior.

Petrography: (C. Agee, UNM) Microprobe examination shows distinct chondrules throughout, plagioclase grain size is $\sim 20 \mu \mathrm{m}$, a significant portion of the matrix appears to be recrystallized. Kamacite, troilite, and oxidized iron are ubiquitous. Geochemistry: (C. Agee, $U N M$ ) Olivine $\mathrm{Fa}_{24.8 \pm 1.2}, \mathrm{Fe} / \mathrm{Mn}=48 \pm 7$, $\mathrm{n}=11$; low-Ca pyroxene $\mathrm{Fs}_{21.5 \pm 0.5} \mathrm{Wo}_{1.8 \pm 0.2}, \mathrm{Fe} / \mathrm{Mn}=29 \pm 1, \mathrm{n}=6$.

Classification: Ordinary chondrite (L5)

Specimens: $20.5 \mathrm{~g}$ on deposit at $U N M$, DPitt holds the main mass.

\section{Northwest Africa 11585 (NWA 11585)}

North West Africa, Morocco

Purchased: October 2015

Classification: Ureilite 
Petrography: (H. Downes, Birkbeck) Equigranular, anhedral, slightly poikilitic crystals of olivine and low-Ca pyroxene, with minor interstitial metal and very rare carbon. There is no evidence of zoning in minerals. This meteorite does not exhibit a typical ureilite texture, but is more similar to the Hughes Type.

Geochemistry: Mineral compositions and geochemistry: (N. Rai, N.V. Almeida, NHM). All analyses by EPMA. Olivine cores $\mathrm{Fa}_{10.8 \pm 0.6}, 0.45$ to $0.52 \mathrm{wt} \%$ $\mathrm{MnO}, 0.50$ to $0.55 \mathrm{wt} \% \mathrm{Cr}_{2} \mathrm{O}_{3}, 0.14$ to $0.68 \mathrm{wt} \% \mathrm{CaO},<0.01 \mathrm{wt} \% \mathrm{Ni},(\mathrm{N}=32)$. Pyroxene $\mathrm{Fs}_{10.1 \pm 0.8} \mathrm{Wo}_{8.3 \pm 0.6}, 0.38$ to $0.52 \mathrm{wt} \% \mathrm{MnO}, 0.89$ to $0.92 \mathrm{wt} \% \mathrm{Cr}_{2} \mathrm{O}_{3}$, $(\mathrm{N}=11)$.

Classification: Ureilite

Specimens: $14.2 \mathrm{~g}$ on deposit at the NHM. M. Bilet holds the main mass.

\section{Northwest Africa 11586 (NWA 11586)}

Algeria

Purchased: 2016 May

Classification: HED achondrite (Eucrite)

History: Purchased by Miao Bing-an in May 2016 from Morocco.

Physical characteristics: Meteorite has residual visible black fusion crust and weak magnetic properties. Additionally, hand specimen is unbrecciated.

Petrography (B. Miao, C. Zhang, GUT): This meteorite, with weak magnetic properties and residual visible black fusion crust, whose rock structure is dense and section is full of small metal (Fe). Within polished thin section, mineral is slightly broken, rock structure is igneous but not brecciated. The mineral modal abundance is as follows: Low-Ca pyroxene (36.2 vol\%), high-Ca pyroxene (10.6 vol\%), plagioclase (44.3 vol\%), $\mathrm{SiO}_{2}(6.1 \mathrm{vol} \%)$, metal-Fe (0.3 vol\%), chromite $(0.6$ vol\%), other minerals such as ilmenite, troilite, apatite and zircon (1.9 vol\%).

Geochemistry (B. Miao, C. Zhang, GUT, EPMA): Low-Ca pyroxene: $\mathrm{Fe} / \mathrm{Mn}=27.5$

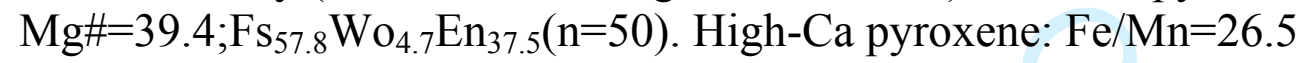

$\mathrm{Mg} \#=51.8 ; \mathrm{Fs}_{28.2} \mathrm{Wo}_{41.5} \mathrm{En}_{30.3}(\mathrm{n}=60)$. Plagioclase: $\mathrm{An}_{90.9} \mathrm{Or}_{0.5} \mathrm{Ab}_{8.62}(\mathrm{n}=29)$.

Classification: Basaltic unbrecciated eucrite

Specimens: $21.8 \mathrm{~g}$ specimen and a polished thin section are deposited in GUT

\section{Northwest Africa 11587 (NWA 11587)}

(Northwest Africa)

Purchased: 2017

Classification: Rumuruti chondrite (R3-5)

History: A single stone weighing $714 \mathrm{~g}$ was found in Morocco prior to 2017. J. Donald Cline and John Sinclair acquired the sample from a meteorite dealer at the Tucson Gem and Mineral Show in February of 2017.

Physical characteristics: Sample is dark brown and has a rounded shape with an irregular surface composed of pits and protrusions. The cut face shows the sample is a breccia composed of 3-4 distinct chondritic lithologies that are light orange to dark-brown in color. 
Petrography: Description and classification (A. Love, App): Sample is a breccia composed of 0.5-4 cm unequilibrated equilibrated chondritic clasts (type 4-5) and a shock-darkened, unequilibrated lithology set within a host of unequilibrated chodrules, fragments and fragmental matrix. Chondrules in host and clasts have an average diameter of $340 \mu \mathrm{m}(\mathrm{n}=81)$. Additional minerals are: apatite, $\mathrm{FeS}$, pentlandite and chromite.

Geochemistry: (A. Love, App) Type 5: Olivine $\left(\mathrm{Fa}_{38.9 \pm 0.3}, \mathrm{Fe} / \mathrm{Mn}=76.1, \mathrm{~N}=6\right)$; high-Ca pyroxene $\left(\mathrm{Fs}_{11.1 \pm 0.9} \mathrm{Wo}_{45.9 \pm 1.0}, \mathrm{~N}=6\right)$; low-Ca pyroxene $\left(\mathrm{Fs}_{30.5 \pm 0.6} \mathrm{Wo}_{4.12} /\right.$ 2.8, $\mathrm{n}=2)$. Type 3: Olivine $\left(\mathrm{Fa}_{13.7 \pm 9.9}\right.$, range $\mathrm{Fa}_{0.4-33.8}, \mathrm{CV}=72 \%, \mathrm{Fe} / \mathrm{Mn}=2.3-85.6$, $\mathrm{N}=36, \mathrm{Cr}_{2} \mathrm{O}_{3}$ in ferroan olivine $0.2 \pm 0.1, \mathrm{~N}=22$ ); low-Ca pyroxene $\mathrm{Fs}_{12.2 \pm 9.7} \mathrm{Wo}_{1.6 \pm 1.2}\left(\mathrm{Fs}_{0.7-31.7} \mathrm{Wo}_{0.3-4.1}, \mathrm{~N}=15\right)$.

Classification: Rumuruti chondrite (R3.2-5, S3, wi-2). Based on $\mathrm{Fa}$ and Fe/Mn compositions of olivines, chondrule diameter and texture this sample is a Rumuruti chondrite breccia. Based on the coefficient of variation of olivines within unequilibrated clast, the petrologic subtype is estimated at 3.2 (Bischoff, 2000). Specimens: PARI holds the main mass. One $20.2 \mathrm{~g}$ endcut and a polished thin section are on deposit at App.

\section{Northwest Africa 11588 (NWA 11588)}

(Northwest Africa)

Purchased: 2017

Classification: Carbonaceous chondrite (CM2)

Physical characteristics: Dark-brown fragments. Cut surface reveals a dark interior with abundant matrix and small chondrules.

Petrography: (J. Gattacceca, H. Pourkhorsandi, CEREGE; L. Bonal, IPAG) Chondrules (average apparent diameter about $200 \mu \mathrm{m}$ ) and chondrules fragments set in a fine-grained iron-rich matrix. Modal abundances: matrix $71 \mathrm{vol} \%$, chondrules and chondrule fragments $29 \%$ (point counting, $N=317$ ). Infrared transmission spectra of matrix grains reveal the presence of phyllosilicates dominating the mineralogical assemblage and are comparable to those of $\mathrm{CM}$ chondrites (Beck et al., 2010. The 3- $\mu \mathrm{m}$ band, whose spectral characteristics are reflecting the chemistry of the phyllosilicate phases and are correlated with the alteration classification scheme of Rubin et al. (2007), shows that this meteorite experienced a relatively low extent of aqueous alteration, comparable to Murray (2.4/2.5).

Geochemistry: Olivine $\mathrm{Fa}_{16.6 \pm 13.1}$, PMD 66\%, range $\mathrm{Fa}_{0.9-37.9}(\mathrm{~N}=5)$. Orthopyroxene $\mathrm{Fs}_{0.8} \mathrm{Ws}_{0.8}$ and FS7.2 $\mathrm{Wo}_{3.8}(\mathrm{~N}=2)$. Magnetic susceptibility $\log \chi\left(\times 10^{-9} \mathrm{~m}^{3} / \mathrm{kg}\right)=$ 4.23 .

Classification: $\mathrm{CM} 2$

Specimens: Type specimen at CEREGE. Main mass with Kuntz.

Northwest Africa 11589 (NWA 11589)

(Northwest Africa) 
Purchased: 2017

Classification: Carbonaceous chondrite (CV3)

Physical characteristics: Dark stone. Cut surface reveals a dark-gray interior with mm-sized chondrules and CAIs.

Petrography: (J. Gattacceca, H. Pourkhorsandi, CEREGE ; Lydie Bonal, IPAG) Chondrules (average apparent diameter $1.1 \mathrm{~mm}, \mathrm{~N}=18$ ), mostly of type I, and CAIs set in a fine-grained iron-rich matrix. Main opaque minerals are troilite and metal. Based on the structural order of the polyaromatic matter assessed by Raman spectroscopy, this meteorite appears to be as metamorphosed as Axtell (3.6/3.7). The Raman spectroscopy parameters of interest are FWHM-D (cm-1)=69.2 \pm 4.0 , ID/IG=1.41 \pm 0.06 (see Bonal et al. 2006, GCA 70:1849-1863, figure 4).

Geochemistry: Olivine $\mathrm{Fa}_{10.0 \pm 10.5}$, PMD 68\% (N=11). Orthopyroxene $\mathrm{Fs}_{1.2 \pm 0.4} \mathrm{Wo}_{0.9 \pm 0.1}(\mathrm{~N}=2)$. Magnetic susceptibility $\log \chi\left(\times 10^{-9} \mathrm{~m}^{3} / \mathrm{kg}\right)=3.91$. Classification: Carbonaceous chondrite (CV3, reduced subgroup). Estimated subtype 3.6/3.7.

Specimens: Type specimen at CEREGE. Main mass with Kuntz.

\section{Northwest Africa 11590 (NWA 11590) \\ (Northwest Africa) \\ Purchased: 2017 \\ Classification: Ordinary chondrite (LL3)}

Physical characteristics: Brownish stone. Cut surface reveals a brownish interior with packed, well-defined, mm-sized chondrules, and cm-sized darker clasts with smooth contours.

Petrography: (J. Gattacceca, H. Pourkhorsandi, CEREGE) Packed chondrules with average size $850 \mu \mathrm{m}(\mathrm{N}=24)$. Contains cm-sized clasts with very closely packed and indented chondrules, and abundant compound chondrules ("cluster chondrite" texture as defined by Metzler 2012, MAPS 47:2193-2217). These clasts are more weathered than the host lithology (W5 against W4).

Geochemistry: Olivine $\mathrm{Fa}_{14.2 \pm 8.8}$, range $\mathrm{Fa}_{4.0-26.6}, \mathrm{PMD} 57 \%(\mathrm{~N}=9), \mathrm{Cr}_{2} \mathrm{O}_{3}$ in ferroan olivine $0.14 \pm 0.14 \mathrm{wt} \%(\mathrm{~N}=9)$. Orthopyroxene $\mathrm{Fs}_{13.4 \pm 8.4} \mathrm{Wo}_{0.9 \pm 0.7}, \mathrm{Fs} \mathrm{PMD}$ $50 \%(\mathrm{~N}=10)$.

Classification: LL3. Contains cm-sized "cluster chondrite" LL3 clasts.

Specimens: Type specimen at CEREGE. Main mass with Kuntz.

\section{Northwest Africa 11591 (NWA 11591)}

Algeria

Purchased: 2016 May 1

Classification: HED achondrite (Eucrite)

History: Purchased by Miao Bing-an in 2016 in Morocco.

Physical characteristics: The fusion crust has been largely weathered from the surface of the sample. In the area where it remains, it is thin $(<0.5 \mathrm{~mm})$ and shiny black. The interior of the stone is medium gray and contains inclusions that range from whitish-gray to black. There are several clasts and small fractures in the stone. 
A small amount of oxidation is present in the interior of the sample. The sample is approximately $6.8 \times 8.6 \times 3.8 \mathrm{~cm}$.

Petrography (L.Huang, B. Miao, H. Chen, GUT): This meteorite is a pigeoniteplagioclase rock with fine to medium grain size. Breccia composed of mineral and lithic fragments set in a fine-grained, generally fragmental matrix. Lithic clasts are generally ophitic with medium-grained pyroxenes and skeletal plagioclase grains (width 30-200 $\mu \mathrm{m}$ ) and a fine-grained recrystallized mesostasis. Pyroxene generally contain pigeonite with augite lamellae (width $\sim 3 \mu \mathrm{m}$ ). A silica phase is commonly present. Accessories are ilmenite, chromite, troilite and rare phosphate (apatite and merrillite.

Geochemistry (L. Huang, B. Miao, H. Chen, GUT): Bulk pyroxene: Fe/Mn=30.2;

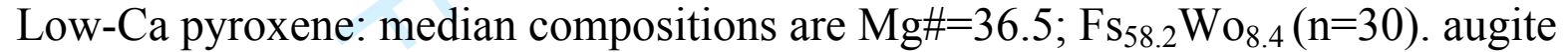
lamellae: median compositions are $\mathrm{Mg} \#=40.1, \mathrm{Fs}_{43.9} \mathrm{Wo}_{26.7}(\mathrm{n}=25)$. Plagioclase: $\mathrm{An}_{86.0} \mathrm{Or}_{0.7}$ to $\mathrm{An}_{92.4} \mathrm{Or}_{0.4}$.

Classification: Basaltic polymict brecciated eucrite

Specimens: $35.1 \mathrm{~g}$ and polished thin section are deposited in GUT

\section{Northwest Africa 11592 (NWA 11592)}

Algeria

Purchased: 2016 May 1

Classification: HED achondrite (Eucrite)

History: Purchased by Miao Bing-an in 2016 in Morocco.

Physical characteristics: This achondrite is covered on two surfaces with a shiny black fusion crust ( $\sim 2 \mathrm{~mm}$ thick). The interior appears massive and is a smokey gray color. Many cracks penetrate the interior of this meteorite. A minute amount of white deposit lines one crack. Dimensions: $2.5 \times 2.0 \times 4.5 \mathrm{~cm}$.

Petrography (L. Huang, B. Miao, H. Chen, GUT): This meteorite is an unbrecciated pigeonite-plagioclase rock with medium grain size. Textures are ophitic with medium-grained ( $\sim 0.3-0.8 \mathrm{~mm})$ pyroxenes and granular to lath-shaped plagioclase grains. Pyroxenes generally contain pigeonite with augite lamellae. It also contains a shock vein (width $\sim 4 \mathrm{~mm}$ ) and a vein associated with formation of medium-grained ferroan olivine $(\sim 50-250 \mu \mathrm{m})$ and sometimes fine-grained ilmenite, chromite and rare very fine-grained phosphate (apatite and merrillite). Accessories are silica, ilmenite, chromite, troilite.

Geochemistry (L. Huang, B. Miao, H. Chen, GUT): Bulk pyroxene: Fe/Mn=31.7; low-Ca pyroxene: median compositions are $\mathrm{Mg} \#=37.5 ; \mathrm{Fs}_{59.9} \mathrm{Wo}_{4.2}(\mathrm{n}=25)$. Augite

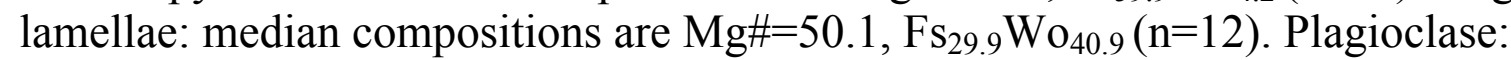
$\mathrm{An}_{87.2-90.0} \mathrm{Or}_{0.5-0.9}$, median $\mathrm{An}_{88.1} \mathrm{Or}_{0.7}(\mathrm{n}=8)$.

Classification: Basaltic unbrecciated eucrite

Specimens: $4.2 \mathrm{~g}$ and polished thin section are deposited in $G U T$

\section{Northwest Africa 11593 (NWA 11593)}

Algeria 
Purchased: 2016 May 1

Classification: HED achondrite (Eucrite)

History: Purchased by Miao Bing-an in 2016 in Morocco.

Physical characteristics: A single stone of $44.37 \mathrm{~g}$, partly covered by fusion crust. Many cracks penetrate the interior of this meteorite. Dimensions: $4.5 \times 4.0 \times 5.0$ $\mathrm{cm}$.

Petrography (L. Huang, B. Miao, H. Chen, GUT): Breccia containing a variety of basaltic lithologies; also contains $<10$ vol\% cumulate lithologies. Cumulate eucrite lithologies are coarse-grained $(\sim 0.5-1 \mathrm{~mm})$ gabbros composed principally of lowCa zoned clinopyroxene and calcic plagioclase. Basaltic breccia lithologies have fine to medium grain size, textures are subophitic to ophitic, and they contain very fine-grained vitrophyric textured basaltic lithologies. A silica phase is commonly present. Accessories are ilmenite, chromite, troilite and rare iron metal.

Geochemistry (L. Huang, B. Miao, H. Chen, GUT): Bulk pyroxene: Fe/Mn=34.9.

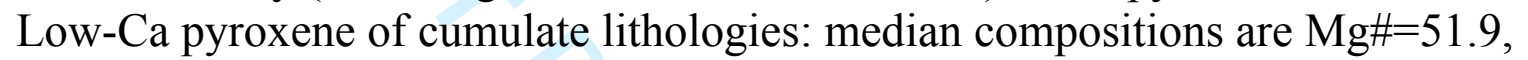
$\mathrm{Fs}_{46.3} \mathrm{Wo}_{3.8}(\mathrm{n}=20)$. Low-Ca pyroxene of basaltic lithologies: median compositions are $\mathrm{Mg} \#=41.5, \mathrm{Fs}_{55.3} \mathrm{Wo}_{5.6}(\mathrm{n}=36)$. High-Ca pyroxene of basaltic lithologies:

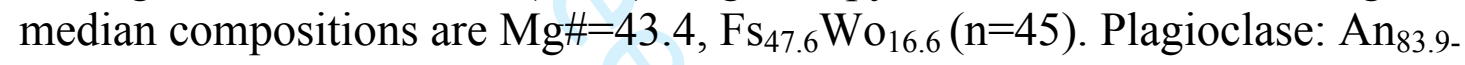
${ }_{92.9} \mathrm{Or}_{0.32-2.63}$, median $\mathrm{An}_{90.1} \mathrm{Or}_{0.9}(\mathrm{n}=25)$.

Classification: Basaltic polymict brecciated eucrite

Specimens: $12.1 \mathrm{~g}$ and polished thin section are deposited in $G U T$

\section{Northwest Africa 11594 (NWA 11594)}

Algeria

Purchased: 2016 May 1

Classification: HED achondrite (Eucrite)

History: Purchased by Miao Bing-an in 2016 in Morocco.

Physical characteristics: On the unbroken surfaces, black fusion crust is partly preserved. The meteorite is a polymict breccia consisting of fragments of mediumto coarse-grained metamorphosed gabbroic rocks and fine-grained subophitic basaltic rocks. Dimensions: $5.5 \times 6.0 \times 7.2 \mathrm{~cm}$.

Petrography (L. Huang, B. Miao, H. Chen, GUT): Breccia containing a variety of basaltic lithologies, with $<10$ vol $\%$ cumulate lithologies. Cumulate eucrite lithologies are coarse grained ( 0.6-1 mm) gabbros composed principally of low$\mathrm{Ca}$ zoned clinopyroxene and calcic plagioclase. Basaltic breccia lithologies are fine grained, have mostly subophitic textures, and contain very fine-grained vitrophyric textured basaltic lithologies. A silica phase is commonly present. Accessories are ilmenite, chromite, troilite and rare iron metal.

Geochemistry (L.Huang, B. Miao, H. Chen, GUT): Bulk pyroxene: Fe/Mn=35.2. Low-Ca pyroxene of cumulate lithologies: median compositions are $\mathrm{Mg} \#=51.7$, $\mathrm{Fs}_{46.6} \mathrm{Wo}_{3.7}(\mathrm{n}=20)$. Low-Ca pyroxene of basaltic lithologies: median compositions

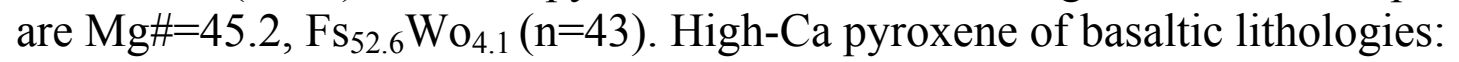

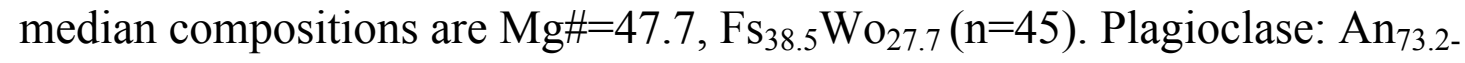
94.1 $\mathrm{Or}_{0.23-2.17}$, median $\mathrm{An}_{88.2} \mathrm{Or}_{0.76}(\mathrm{n}=10)$. 
Classification: Basaltic polymict brecciated eucrite

Specimens: $34.6 \mathrm{~g}$ and polished thin section are deposited in GUT

\section{Northwest Africa 11595 (NWA 11595)}

Algeria

Purchased: 2016 May 1

Classification: HED achondrite (Eucrite)

History: Purchased by Miao Bing-an in 2016 in Morocco.

Physical characteristics: The fusion crust has been largely weathered from the surface of the sample. The meteorite is a polymict breccia consisting of fragments of medium- to coarse-grained metamorphosed gabbroic rocks and medium- to finegrained subophitic basaltic rocks. Dimensions: $4.5 \times 6.0 \times 6.4 \mathrm{~cm}$.

Petrography (L. Huang, B. Miao, H. Chen, GUT): Breccia containing a variety of basaltic lithologies, with $<10$ vol $\%$ cumulate lithologies. Cumulate eucrites lithologies are coarse grained $(\sim 0.4-0.6 \mathrm{~mm})$ gabbros composed principally of low$\mathrm{Ca}$ zoned clinopyroxene and calcic plagioclase. Basaltic breccia lithologies are fine grained, have mostly subophitic textures, and contain very fine-grained vitrophyric textured basaltic lithologies. A silica phase is commonly present. Accessories are ilmenite, chromite, troilite and rare iron metal.

Geochemistry (L. Huang, B. Miao, H. Chen, GUT): Bulk pyroxene: Fe/Mn=34.1. Low-Ca pyroxene cumulate lithologies: median compositions are $\mathrm{Mg} \#=50.9$, $\mathrm{Fs}_{48.0} \mathrm{Wo}_{2.2}(\mathrm{n}=13)$. Low-Ca pyroxene of basaltic lithologies: median compositions are $\mathrm{Mg} \#=42.6, \mathrm{Fs}_{54.6} \mathrm{Wo}_{4.9}(\mathrm{n}=12)$. High-Ca pyroxene of basaltic lithologies: median compositions are $\mathrm{Mg} \#=44.4, \mathrm{Fs}_{45.3} \mathrm{Wo}_{19.1}(\mathrm{n}=28)$. Plagioclase: $\mathrm{An}_{87.2-}$ 95.1 $\mathrm{Or}_{0.41-1.56}$, median $\mathrm{An}_{92.6} \mathrm{Or}_{0.78}(\mathrm{n}=12)$.

Classification: Basaltic polymict brecciated eucrite Specimens: $25.3 \mathrm{~g}$ and polished thin section are deposited in GUT

\section{Northwest Africa 11596 (NWA 11596)}

Algeria

Purchased: 2016 May 1

Classification: HED achondrite (Eucrite)

History: Purchased by Miao Bing-an in 2016 in Morocco.

Physical characteristics: A black fusion crust coveres the surface of the stone. The meteorite is a polymict breccia consisting of fragments of medium- to coarsegrained metamorphosed gabbroic rocks and medium- to fine-grained subophitic basaltic rocks. Dimensions: $3.5 \times 5.0 \times 5.4 \mathrm{~cm}$.

Petrography (L. Huang, B. Miao, H. Chen, GUT): Breccia containing a variety of basaltic lithologies, with $<10$ vol\% cumulate lithologies. Cumulate eucrite lithologies are coarse grained ( 0.3-1 mm) gabbros composed principally of low$\mathrm{Ca}$ zoned clinopyroxene and calcic plagioclase. Basaltic breccia lithologies are fine- to medium-grained, have textures that are mostly subophitic, and contain very 
fine-grained vitrophyric textured basaltic lithologies. A silica phase is commonly present. Accessories are ilmenite, chromite, troilite and rare iron metal.

Geochemistry (L. Huang, B. Miao, H. Chen, GUT): Bulk pyroxene: Fe/Mn=36.6. Low-Ca pyroxene of cumulate lithologies: median compositions are $\mathrm{Mg} \#=51.3$, $\mathrm{Fs}_{46.7} \mathrm{Wo}_{4.2}(\mathrm{n}=10)$. Low-Ca pyroxene of basaltic lithologies: median compositions are $\mathrm{Mg} \#=45.1, \mathrm{Fs}_{52.9} \mathrm{Wo}_{3.8}(\mathrm{n}=8)$. High-Ca pyroxene of basaltic lithologies: median compositions are $\mathrm{Mg} \#=43.8, \mathrm{Fs}_{47.5} \mathrm{Wo}_{16.0}(\mathrm{n}=18)$. Plagioclase: $\mathrm{An}_{79.8-89.0} \mathrm{Or}_{0.5-1.9}$, median $\mathrm{An}_{85.1} \mathrm{Or}_{1.1}(\mathrm{n}=11)$.

Classification: Basaltic polymict brecciated eucrite

Specimens: $16.9 \mathrm{~g}$ and polished thin section are deposited in GUT

\section{Northwest Africa 11597 (NWA 11597)}

Algeria

Purchased: 2016 May 1

Classification: HED achondrite (Eucrite)

History: Purchased by Miao Bing-an in 2016 in Morocco.

Physical characteristics: The interior of the meteorite is medium gray. Dimensions: $2.5 \times 3.0 \times 3.4 \mathrm{~cm}$.

Petrography (L. Huang, B. Miao, H. Chen, GUT): Breccia containing a variety of basaltic lithologies, with $<10$ vol $\%$ cumulate lithologies. Cumulate eucrites lithologies are coarse grained $(\sim 0.3-0.6 \mathrm{~mm})$ gabbros composed principally of low$\mathrm{Ca}$ clinopyroxene and calcic plagioclase. Basaltic breccia lithologies are fine grained, and have textures that are mostly subophitic. A silica phase is commonly present. Accessories are ilmenite, chromite and troilite.

Geochemistry (L. Huang, B. Miao, H. Chen, GUT): Bulk pyroxene: Fe/Mn=35.5. Low-Ca pyroxene of cumulate lithologies: median compositions are Mg\#=45.1, $\mathrm{Fs}_{52.3} \mathrm{Wo}_{2.9}(\mathrm{n}=10)$. Low-Ca pyroxene of basaltic lithologies: median compositions are $\mathrm{Mg} \#=40.8, \mathrm{Fs}_{57.2} \mathrm{Wo}_{3.7}(\mathrm{n}=8)$. High-Ca pyroxene of basaltic lithologies: median compositions are $\mathrm{Mg} \#=43.2, \mathrm{Fs}_{46.6} \mathrm{Wo}_{18.6}(\mathrm{n}=21)$. Plagioclase: $\mathrm{An}_{90.0-91.9} \mathrm{Or}_{0.5-0.7}$, median $\mathrm{An}_{91.9} \mathrm{Or}_{0.7}(\mathrm{n}=6)$.

Classification: Basaltic polymict brecciated eucrite

Specimens: $11.1 \mathrm{~g}$ and polished thin section are deposited in GUT

\section{Northwest Africa 11598 (NWA 11598)}

Algeria

Purchased: 2016 May 1

Classification: HED achondrite (Howardite)

History: Purchased by Miao Bing-an in 2016 in Morocco.

Physical characteristics: No fusion crust.

Petrography (L. Huang, B. Miao, H. Chen, GUT): Breccia containing a variety of diogenite lithologies with $\sim 40$ vol\% eucrite lithologies (mostly basaltic lithologies). Basaltic eucrite detritus (composed of granular pyroxene and plagioclase) as veins 
invade the orthopyroxenitic diogenite lithologies (mainly composed of orthopyroxene). Accessories are silica, ilmenite, chromite, troilite.

Geochemistry (L. Huang, B. Miao, H. Chen, GUT): Bulk pyroxene: $\mathrm{Fe} / \mathrm{Mn}=30.9$;

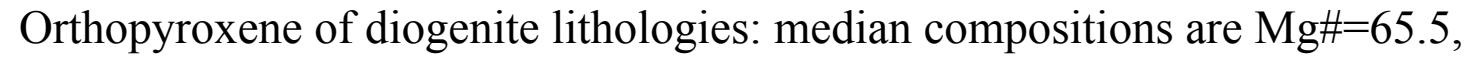
$\mathrm{Fs}_{33.6} \mathrm{Wo}_{2.6}(\mathrm{n}=24)$. Low-Ca pyroxene of basaltic eucrite lithologies: median compositions are $\mathrm{Mg} \#=37.1, \mathrm{Fs}_{61.5} \mathrm{Wo}_{2.2}(\mathrm{n}=15)$. High-Ca pyroxene of basaltic eucrite lithologies: median compositions are $\mathrm{Mg} \#=48.5, \mathrm{Fs}_{30.9} \mathrm{Wo}_{40.0}(\mathrm{n}=12)$. Plagioclase: median $\mathrm{An}_{91.7} \mathrm{Or}_{0.3}(\mathrm{n}=11)$.

Classification: Polymict brecciated howardite Specimens: $1.5 \mathrm{~g}$ and polished thin section are deposited in GUT. The remaining material is held by the anonymous finder.

\section{Northwest Africa 11599 (NWA 11599) \\ Algeria \\ Purchased: 2016 May 1 \\ Classification: HED achondrite (Eucrite)}

History: Purchased by Miao Bing-an in 2016 in Morocco.

Physical characteristics: Single stone with black fusion crust. A saw cut reveals a mosaic of gray, coarse-grained, interlocking crystals.

Petrography (L. Huang, B. Miao, H. Chen, GUT): Unbrecciated cumulate lithologies containing coarse-grained $(\sim 0.5-1.1 \mathrm{~mm})$ gabbros composed principally of low-Ca clinopyroxene and calcic plagioclase ( $20-40 \mathrm{vol} \%)$ with minor chromite (coarse-grained $\sim 0.5-1.0 \mathrm{~mm}$ ). Texture is equigranular with subequal amounts of pyroxene and plagioclase grains.

Geochemistry (L. Huang, B. Miao, H. Chen, GUT): Bulk pyroxene: $\mathrm{Fe} / \mathrm{Mn}=28.6$. Low-Ca pyroxene of cumulate lithologies: median compositions are $\mathrm{Mg} \#=63.1$, $\mathrm{Fs}_{36.0} \mathrm{Wo}_{2.4}(\mathrm{n}=29)$. High-Ca pyroxene of cumulate lithologies: median compositions are $\mathrm{Mg} \#=73.2, \mathrm{Fs}_{14.5} \mathrm{Wo}_{46.0}(\mathrm{n}=23)$. Plagioclase: $\mathrm{An}_{89.4-91.4} \mathrm{Or}_{0.1-0.7}$, median $\mathrm{An}_{90.2} \mathrm{Or}_{0.5}(\mathrm{n}=18)$.

Classification: Gabbroic cumulate eucrite

Specimens: $8.9 \mathrm{~g}$ and polished thin section are deposited in GUT

\section{Northwest Africa 11600 (NWA 11600)}

Algeria

Purchased: 2016 May 1

Classification: HED achondrite (Eucrite)

History: Purchased by Miao Bing-an in 2016 in Morocco.

Physical characteristics: Single stone with black fusion crust. A saw cut reveals a mosaic of gray, coarse-grained, interlocking crystals.

Petrography (L. Huang, B. Miao, H. Chen, GUT): Unbrecciated cumulate lithologies containing coarse-grained $(\sim 0.8-1.5 \mathrm{~mm})$ gabbros composed principally of low-Ca clinopyroxene and calcic plagioclase ( $20-40$ vol\%) with minor 
chromite (coarse-grained $\sim 0.5-0.8 \mathrm{~mm}$ ) and accessory phosphate. Texture is equigranular with subequal amounts of pyroxene and plagioclase grains.

Geochemistry (L. Huang, B. Miao, H. Chen, GUT): Bulk pyroxene: Fe/Mn=29.9. Low-Ca pyroxene of cumulate lithologies: median compositions are $\mathrm{Mg} \#=63.7$, $\mathrm{Fs}_{35.4} \mathrm{Wo}_{2.7}(\mathrm{n}=24)$. High-Ca pyroxene of cumulate lithologies: median compositions are $\mathrm{Mg} \#=73.7, \mathrm{Fs}_{14.6} \mathrm{Wo}_{44.4}(\mathrm{n}=20)$. Plagioclase: $\mathrm{An}_{87.8-91.1} \mathrm{Or}_{0.2-0.7}$, median $\mathrm{An}_{89.7} \mathrm{Or}_{0.5}(\mathrm{n}=26)$.

Classification: Gabbroic cumulate eucrite

Specimens: $16.2 \mathrm{~g}$ and polished thin section are deposited in GUT

\section{Northwest Africa 11601 (NWA 11601)}

Algeria

Purchased: 2016 May 1

Classification: HED achondrite (Eucrite)

History: Purchased by Miao Bing-an in 2016 in Morocco.

Physical characteristics: The meteorite has pitted irregular surfaces and is light to dark gray to pale brown in overall color. Most of the stones have partial coatings of greenish to whitish lichens.

Petrography (L. Huang, B. Miao, H. Chen, GUT): This meteorite is a pigeoniteplagioclase rock with fine to medium grain size. Breccia composed of mineral and lithic fragments set in a fine-grained, generally fragmental matrix. Lithic clasts are generally subophitic with fine to medium-grained $(\sim 0.1-0.4 \mathrm{~mm})$ pyroxenes and skeletal plagioclase grains and a fine-grained mesostasis. A silica phase is commonly present. Accessories are ilmenite, chromite, troilite and rare iron metal. Geochemistry (L. Huang, B. Miao, H. Chen, GUT): Bulk pyroxene: $\mathrm{Fe} / \mathrm{Mn}=32.1$.

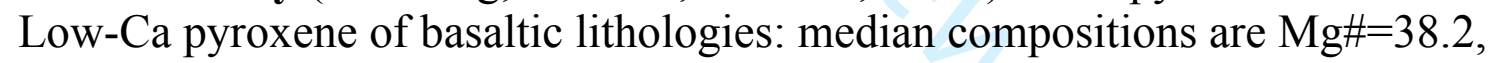
$\mathrm{Fs}_{57.1} \mathrm{Wo}_{5.6}(\mathrm{n}=16)$. High-Ca pyroxene of basaltic lithologies: median compositions are $\mathrm{Mg} \#=37.5, \mathrm{Fs}_{45.5} \mathrm{Wo}_{27.5}(\mathrm{n}=13)$. Plagioclase: $\mathrm{An}_{85.2-87.7} \mathrm{Or}_{0.5-1.3}$, median $\mathrm{An}_{86.3} \mathrm{Or}_{0.9}(\mathrm{n}=14)$.

Classification: Basaltic unbrecciated eucrite

Specimens: $8.4 \mathrm{~g}$ and polished thin section are deposited in GUT

\section{Northwest Africa 11602 (NWA 11602)}

Algeria

Purchased: 2016 May 1

Classification: HED achondrite (Eucrite)

History: Purchased by Miao Bing-an in 2016 in Morocco.

Physical characteristics: Thin, shiny, black fusion crust covers $60 \%$ of the exterior of this sample. No fractures exist. Cleaving this achondrite revealed an interior with coarse-grained matrix containing an even distribution of white laths. The specimen is coherent and weathering is minimal.

Petrography (L. Huang, B. Miao, H. Chen, GUT): This meteorite is an unbrecciated pigeonite-plagioclase rock with medium grain size. With rhyolitic 
structure, it contains fine to medium-grained $(\sim 0.05-0.5 \mathrm{~mm})$ pyroxenes and granular plagioclase grains. Pyroxenes generally contain pigeonite with augite lamellae. Accessories are silica, ilmenite, troilite and rare iron metal.

Geochemistry (L. Huang, B. Miao, H. Chen, GUT): Bulk pyroxene: Fe/Mn=31.7.

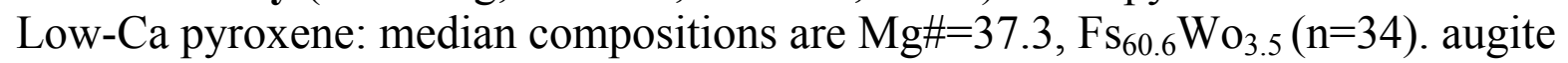

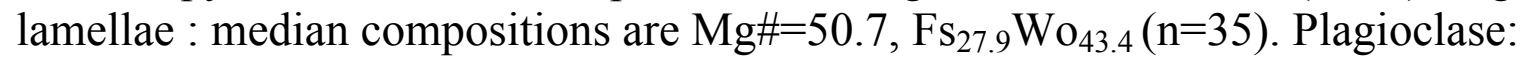
$\mathrm{An}_{88.4-90.2} \mathrm{Or}_{0.1-0.4}$, median $\mathrm{An}_{90.6} \mathrm{Or}_{0.3}(\mathrm{n}=22)$.

Classification: Basaltic unbrecciated eucrite

Specimens: $10.8 \mathrm{~g}$ and polished thin section are deposited in $G U T$

Northwest Africa 11603 (NWA 11603)

Algeria

Purchased: 2016 May 1

Classification: HED achondrite (Eucrite)

History: Purchased by Miao Bing-an in 2016 in Morocco.

Physical characteristics: Black fusion crust covers $40 \%$ of the exterior of this sample. No fractures exist. Cleaving this achondrite revealed an interior with coarse-grained matrix containing an even distribution of white laths.

Petrography (L. Huang, B. Miao, H. Chen, GUT): This meteorite is a unbrecciated pigeonite-plagioclase rock with medium grain size. Ophitic-gabbro texture with fine to medium-grained $(\sim 0.05-0.45 \mathrm{~mm})$ pyroxenes and granular to lath-shaped plagioclase grains. Pyroxenes generally contain pigeonite with augite lamellae. Accessories are silica, ilmenite, troilite.

Geochemistry (L. Huang, B. Miao, H. Chen, GUT): Bulk pyroxene: Fe/Mn=32.7. Low-Ca pyroxene: median compositions are $\mathrm{Mg} \#=36.5, \mathrm{Fs}_{60.5} \mathrm{Wo}_{4.8}(\mathrm{n}=26)$. Augite

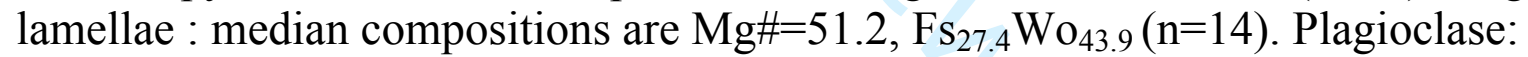

$\mathrm{An}_{89.8-91.5} \mathrm{Or}_{0.1-0.8}$, median $\mathrm{An}_{90.6} \mathrm{Or}_{0.3}(\mathrm{n}=23)$.

Classification: Basaltic unbrecciated Eucrite

Specimens: $4.6 \mathrm{~g}$ and polished thin section are deposited in GUT

\section{Northwest Africa 11604 (NWA 11604)}

Algeria

Purchased: 2016 May 1

Classification: HED achondrite (Howardite)

History: Purchased by Miao Bing-an in 2016 from Morocco.

Physical characteristics: The medium gray matrix contains a variety of clasts and the fusion crust is not found in this small sample.

Petrography (L. Huang, B. Miao, H. Chen, GUT): Breccia containing a variety of basaltic eucrite lithologies ( $\sim 55 \mathrm{vol} \%)$ and cumulate eucrite lithologies ( $30 \mathrm{vol} \%)$, with $\sim 15$ vol\% diogenite lithologies. Basaltic eucrite lithologies mostly have a finegrained recrystallized or subophitic texture. The texture of cumulate eucrite lithologies is equigranular with subequal amounts of pyroxene and plagioclase 
grains. Diogenite lithologies mostly contain orthopyroxene. Accessories are silica, ilmenite, chromite, troilite.

Geochemistry (L. Huang, B. Miao, H. Chen, GUT): Bulk pyroxene: $\mathrm{Fe} / \mathrm{Mn}=30.9$; Orthopyroxene of diogenite lithologies: median compositions are $\mathrm{Mg} \#=66.9$, $\mathrm{Fs}_{32.3} \mathrm{Wo}_{2.3}(\mathrm{n}=10)$. Low-Ca pyroxene of cumulate eucrite lithologies: median compositions are $\mathrm{Mg} \#=54.3, \mathrm{Fs}_{44.8} \mathrm{Wo}_{2.0}(\mathrm{n}=12)$. Low-Ca pyroxene of basaltic eucrite lithologies: median compositions are $\mathrm{Mg} \#=38.8, \mathrm{Fs}_{59.5} \mathrm{Wo}_{2.6}(\mathrm{n}=10)$. High$\mathrm{Ca}$ pyroxene of basaltic eucrite lithologies: median compositions are $\mathrm{Mg} \#=51.6$, $\mathrm{Fs}_{28.1} \mathrm{Wo}_{41.8}(\mathrm{n}=8)$. Plagioclase: median $\mathrm{An}_{92.5} \mathrm{Or}_{0.3}(\mathrm{n}=8)$.

Classification: Polymict brecciated howardite Specimens: $1.2 \mathrm{~g}$ and polished thin section are deposited in GUT

\section{Northwest Africa 11605 (NWA 11605)}

Algeria

Purchased: 2016 May 1

Classification: HED achondrite (Howardite)

History: Purchased by Miao Bing-an in 2016 in Morocco.

Physical characteristics: The medium-gray matrix contains a variety of clasts and fusion crust is not found in this small sample.

Petrography (L. Huang, B. Miao, H. Chen, GUT): Breccia containing a variety of eucrite lithologies with $\sim 10-15$ vol\% of diogenite lithologies. Basaltic eucrite lithologies mostly have a fine-grained recrystallized or subophitic texture and contain very fine-grained vitrophyric textured basaltic lithologies. Diogenites lithologies are mostly a orthopyroxene. The matrices are mostly basaltic detritus. Accessories are silica, ilmenite, chromite, troilite.

Geochemistry (L.Huang, B. Miao, H. Chen, GUT): Bulk pyroxene: $\mathrm{Fe} / \mathrm{Mn}=32.5$; Orthopyroxene of diogenite lithologies: median compositions are $\mathrm{Mg} \#=67.1$, $\mathrm{Fs}_{31.9} \mathrm{Wo}_{3.2}(\mathrm{n}=16)$. Low-Ca pyroxene of basaltic eucrite lithologies: median compositions are $\mathrm{Mg} \#=37.4, \mathrm{Fs}_{60.4} \mathrm{Wo}_{3.6}(\mathrm{n}=14)$. High-Ca pyroxene of basaltic eucrite lithologies: median compositions are $\mathrm{Mg} \#=54.1, \mathrm{Fs}_{26.3} \mathrm{Wo}_{42.7}(\mathrm{n}=8)$. Plagioclase: median $\mathrm{An}_{91.3} \mathrm{Or}_{0.4}(\mathrm{n}=10)$.

Classification: Polymict brecciated howardite Specimens: $0.6 \mathrm{~g}$ and polished thin section are deposited in GUT

\section{Northwest Africa 11606 (NWA 11606)}

Algeria

Purchased: 2016 May 1

Classification: HED achondrite (Howardite)

History: Purchased by Miao Bing-an in 2016 in Morocco.

Physical characteristics: The medium-gray matrix contains a variety of clasts and fusion crust is not found in this small sample.

Petrography (L. Huang, B. Miao, H. Chen, GUT): Breccia containing a variety of eucrite lithologies with $\sim 20 \%-40$ vol\% diogenite lithologies. Basaltic eucrite 
lithologies mostly have subophitic texture. The texture of cumulate eucrite lithologies is equigranular with subequal amounts of pyroxene and plagioclase grains. Diogenites lithologies are mostly orthopyroxene. The matrices are mostly basaltic detritus. A higher $\mathrm{C}$ content chondritic clast (carbonaceous chondrite) was found in this section. Accessories are silica, ilmenite, chromite, troilite.

Geochemistry (L. Huang, B. Miao, H. Chen, GUT): Bulk pyroxene: $\mathrm{Fe} / \mathrm{Mn}=30.1$;

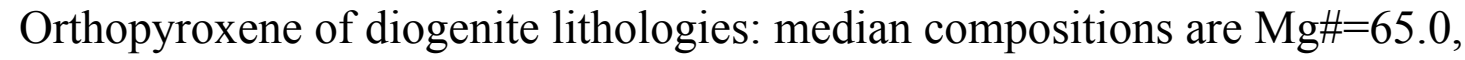
$\mathrm{Fs}_{34.2} \mathrm{Wo}_{2.4}(\mathrm{n}=12)$. Low-Ca pyroxene of cumulate eucrite lithologies: median compositions are $\mathrm{Mg} \#=46.2, \mathrm{Fs}_{53.1} \mathrm{Wo}_{1.3}(\mathrm{n}=8)$. Low-Ca pyroxene of basaltic eucrite lithologies: median compositions are $\mathrm{Mg} \#=36.3, \mathrm{Fs}_{61.9} \mathrm{Wo}_{2.9}(\mathrm{n}=15)$. HighCa pyroxene of basaltic eucrite lithologies: median compositions are $\mathrm{Mg} \#=51.8$, $\mathrm{Fs}_{27.6} \mathrm{Wo}_{42.7}(\mathrm{n}=8)$. Plagioclase: median $\mathrm{An}_{90.5} \mathrm{Or}_{0.4}(\mathrm{n}=10)$.

Classification: Polymict brecciated howardite

Specimens: $1.3 \mathrm{~g}$ and polished thin section are deposited in GUT

\section{Northwest Africa 11607 (NWA 11607) \\ Northwest Africa \\ Purchased: 2017 \\ Classification: Carbonaceous chondrite (CK3-6)}

History: The meteorite was bought from a meteorite dealer in Morocco.

Physical characteristics: Dark grayish to black individual partly covered with fusion crust.

Petrography: The meteorite is a chondritic breccia composed of angular to subrounded type 3-6 clasts set into a more fine-grained clastic matrix. The different clasts are up to $2 \mathrm{~cm}$ in size and vary in color from light greenish to almost black. Matrix dominates over chondrules which are on average about $1.2 \mathrm{~mm}$ in diameter. Ferroan, Ni-rich olivine is the most abundant mineral in all lithologies. While highly unequilibrated in type 3 clasts and matrix it is equilibrated at $\mathrm{Fa}_{29.6}$ in type 5 and 6 lithologies. One type 6 fragment with more Fe-rich olivine $\left(\mathrm{Fa}_{35}\right)$ has been encountered. Less abundant are Ca-pyroxene, low-Ca pyroxene, and intermediate feldspar. Feldspar is up to $200 \mu \mathrm{m}$ in size in type 6 lithologies and often intergrown with Ca-pyroxene. The meteorite containes abundant $\mathrm{Cr}$-bearing magnetite and some FeNi-sulfides.

Geochemistry: Type 3 lithology: olivine: $\mathrm{Fa}_{22.4 \pm 12.7}\left(\mathrm{Fa}_{1.7-34.0}, \mathrm{NiO}=0.3 \pm 0.2\right.$; $\mathrm{FeO} / \mathrm{MnO}=103 \pm 31, \mathrm{n}=32)$; low-Ca pyroxene: $\mathrm{Fs}_{16.3 \pm 8.0} \mathrm{Wo}_{0.9 \pm 0.9}\left(\mathrm{Fs}_{2.9-27.7} \mathrm{Wo}_{0.5-4.1}, \mathrm{n}\right.$ = 15), Ca-pyroxene: $\mathrm{Fs}_{10.9 \pm 4.8} \mathrm{Wo}_{33.8 \pm 6.5}\left(\mathrm{Fs}_{2.4-17.6} \mathrm{Wo}_{23.1-45.1}, \mathrm{n}=9\right)$; type 6 lithology: olivine: $\mathrm{Fa}_{29.6 \pm 0.6}, \mathrm{NiO}=0.4 \pm 0.1, \mathrm{FeO} / \mathrm{MnO}=105 \pm 9, \mathrm{n}=42$; low-Ca pyroxene: $\mathrm{Fs}_{26.9 \pm 0.8} \mathrm{Wo}_{1.1 \pm 0.4}, \mathrm{n}=9$, Ca-pyroxene: $\mathrm{Fs}_{11.6 \pm 2.6} \mathrm{Wo}_{39.3 \pm 6.6}, \mathrm{n}=13$; Fe-rich fragment: olivine: $\mathrm{Fa}_{35.0 \pm 0.3}, \mathrm{NiO}=0.4 \pm 0.1, \mathrm{FeO} / \mathrm{MnO}=143 \pm 13, \mathrm{n}=38$; Ca-pyroxene: $\mathrm{Fs}_{12.6 \pm 1.3} \mathrm{Wo}_{46.5 \pm 1.6}, \mathrm{n}=29$; plagioclase: $\mathrm{An}_{78.8} \mathrm{Ab}_{20.9} \mathrm{Or}_{0.3}, \mathrm{n}=3$; andesitic feldpar: $\mathrm{An}_{35.0} \mathrm{Ab}_{64.3} \mathrm{Or}_{0.7}, \mathrm{n}=3 ; \mathrm{Cr}_{2} \mathrm{O}_{3}$ in magnetite is about $4.9 \mathrm{wt} \%$. 
(Northwest Africa)

Purchased: 2017 Aug

Classification: Ureilite

History: Purchased in Temara, Morocco by Adam Aaronson in August 2017.

Petrography: (A. Irving and S. Kuehner, UWS) Protogranular aggregate (mean

grainsize $1.7 \mathrm{~mm}$ ) composed almost entirely of olivine (with dark, reduced magnesian rims) with minor accessory Fe metal and graphite. Multiple linear deformation boundaries are visible in some olivine grains viewed in cross-polarized light. A thorough search of the thin section revealed no pyroxene.

Geochemistry: Olivine (cores $\mathrm{Fa}_{17.3-17.5}$, rims $\mathrm{Fa}_{7.1-9.4}, \mathrm{~N}=5$ ).

Classification: Ureilite (dunitic).

Specimens: $10.7 \mathrm{~g}$ including one polished thin section at $U W B$; main mass

with Aaronson.

\section{Northwest Africa 11609 (NWA 11609)}

Northwest Africa

Purchased: 2017 Oct

Classification: Ordinary chondrite (L4/5)

History: Purchased by Ben Hoefnagels in October 2017 from a dealer in Marrakech, Morocco.

Petrography: (A. Irving and S. Kuehner, UWS) Fairly well-formed chondrules are present within a recrystallized matrix containing stained metal.

Geochemistry: Olivine $\left(\mathrm{Fa}_{25.5-25.6}, \mathrm{~N}=3\right)$, orthopyroxene $\left(\mathrm{Fs}_{20.9-21.0} \mathrm{Wo}_{1.4-1.5}, \mathrm{~N}=\right.$ 2), clinopyroxene $\left(\mathrm{Fs}_{6.7} \mathrm{Wo}_{46.0}\right)$.

Classification: Ordinary chondrite (L4/5).

Specimens: $21.2 \mathrm{~g}$ including one polished thin section at $U W B$; main mass with Mr. B. Hoefnagels.

\section{Northwest Africa 11610 (NWA 11610) \\ (Northwest Africa) \\ Purchased: 2017 Jun \\ Classification: Carbonaceous chondrite (CO3)}

History: Purchased by Ke Zuokai in June 2017 from Moroccan dealer Habib Naji.

Petrography: (A. Irving and S. Kuehner, UWS) Well-formed small chondrules (apparent diameter $220 \pm 110 \mu \mathrm{m}, \mathrm{N}=18$ ), angular mineral fragments and sparse very fine grained CAI are set in a finer grained matrix (brown in thin section). Geochemistry: Olivine $\left(\mathrm{Fa}_{0.2-41.0}, \mathrm{Cr}_{2} \mathrm{O}_{3}\right.$ in ferroan examples $0.06-0.27$ wt.\%, mean $0.12 \pm 0.08$ wt. \%, $\mathrm{N}=8$ ), orthopyroxene $\left(\mathrm{Fs}_{2.3-23.2} \mathrm{Wo}_{0.8-0.9}, \mathrm{~N}=3\right)$, clinopyroxene $\left(\mathrm{Fs}_{0.7-4.5} \mathrm{Wo}_{42.2-41.0}, \mathrm{~N}=2\right)$. Magnetic susceptibility $\log \chi\left(\times 10^{-9} \mathrm{~m}^{3} / \mathrm{kg}\right)=4.07$. Classification: Carbonaceous chondrite (CO3).

Specimens: $40.1 \mathrm{~g}$ including one polished thin section at $U W B$; main mass with Mr. K. Zuokai. 


\section{Northwest Africa 11611 (NWA 11611)}

(Northwest Africa)

Purchased: 2017 Aug

Classification: Ordinary chondrite (LL(L)3)

History: Purchased in Temara, Morocco, by Aaronson in August 2017.

Petrography: (A. Irving and S. Kuehner, UWS) Fairly closely-packed, well-formed chondrules (apparent diameter $580 \pm 310 \mu \mathrm{m}, \mathrm{N}=18$ ) are set in a finer-grained matrix containing altered kamacite and chlorapatite.

Geochemistry: Olivine $\left(\mathrm{Fa}_{0.8-56.1}, \mathrm{Cr}_{2} \mathrm{O}_{3}\right.$ in ferroan examples $0.05-0.10$ wt.\%, mean $0.08 \pm 0.02 \mathrm{wt} . \%, \mathrm{~N}=8)$, orthopyroxene $\left(\mathrm{Fs}_{0.7-16.0} \mathrm{Wo}_{0.7-0.1}, \mathrm{~N}=3\right)$, clinopyroxene $\left(\mathrm{Fs}_{11.8} \mathrm{Wo}_{37.3} ; \mathrm{Fs}_{8.7} \mathrm{Wo}_{45.2} ; \mathrm{N}=2\right)$. Magnetic susceptibility $\log \chi\left(\times 10^{-9} \mathrm{~m}^{3} / \mathrm{kg}\right)=$ 4.20 .

Classification: Ordinary chondrite (LL(L)3).

Specimens: $22 \mathrm{~g}$ including one polished thin section at $U W B$; main mass with Aaronson.

\section{Northwest Africa 11612 (NWA 11612)}

(Northwest Africa)

Purchased: 2017 Aug

Classification: HED achondrite (Howardite)

History: Purchased in Temara, Morocco, by Aaronson in August 2017.

Petrography: (A. Irving and S. Kuehner, UWS) Breccia composed of angular grains of diogenitic orthopyroxene ( $\sim 60 \mathrm{vol} . \%)$, exsolved pigeonite, bytownitic plagioclase and some polycrystalline diogenite clasts in a finer grained matrix containing silica polymorph, Ti-poor chromite, ilmenite, Ni-free metal, troilite, zircon and minor secondary calcite.

Geochemistry: Orthopyroxene host $\left(\mathrm{Fs}_{52.0} \mathrm{Wo}_{1.3} ; \mathrm{Fs}_{36.8-39.6} \mathrm{Wo}_{1.7-1.6} ; \mathrm{FeO} / \mathrm{MnO}=\right.$ $30-31 ; \mathrm{N}=3)$, clinopyroxene exsolution lamellae $\left(\mathrm{Fs}_{14.4-14.5} \mathrm{Wo}_{45.1-43.1}, \mathrm{FeO} / \mathrm{MnO}=\right.$ $23, \mathrm{~N}=2)$, diogenitic orthopyroxene $\left(\mathrm{Fs}_{34.8} \mathrm{Wo}_{2.5}, \mathrm{FeO} / \mathrm{MnO}=30\right)$, plagioclase $\left(\mathrm{An}_{74.6-83.0} \mathrm{Or}_{1.0-0.6}, \mathrm{~N}=2\right)$.

Classification: Howardite.

Specimens: $24 \mathrm{~g}$ including one polished thin section at $U W B$; main mass with Aaronson.

\section{Northwest Africa 11613 (NWA 11613)}

(Northwest Africa)

Purchased: 2017 Aug

Classification: Ordinary chondrite (H7)

History: Purchased in Temara, Morocco, by Aaronson in August 2017.

Petrography: (A. Irving and S. Kuehner, UWS) Recrystallized and equilibrated specimen lacking chondrule remnants, but containing some orthopyroxene 
poikiloblasts enclosing smaller olivine grains. Primary metal is partly altered and some cross-cutting iron hydroxide veinlets are present.

Geochemistry: Olivine $\left(\mathrm{Fa}_{18.2-18.3}, \mathrm{~N}=3\right)$, orthopyroxene $\left(\mathrm{Fs}_{16.0-16.1} \mathrm{Wo}_{3.5-4.3}, \mathrm{~N}=\right.$ 2), clinopyroxene $\left(\mathrm{Fs}_{6.9-7.2} \mathrm{Wo}_{43.4-41.4}, \mathrm{~N}=2\right)$.

Classification: Ordinary chondrite (H7).

Specimens: $21.5 \mathrm{~g}$ including one polished thin section at $U W B$; main mass

with Aaronson.

\title{
Northwest Africa 11614 (NWA 11614)
}

(Northwest Africa)

Purchased: 2017 Sep

Classification: Rumuruti chondrite (R3-5)

History: Purchased by Gary Fujihara in September 2017 from a Moroccan dealer.

Petrography: (A. Irving and S. Kuehner, UWS) Breccia composed of separated unequilibrated chondrules (apparent diameter $440 \pm 180 \mu \mathrm{m}, \mathrm{N}=15$ ) together with equilibrated type 5 chondrite clasts in a finer grained matrix. Accessory minerals include sodic plagioclase, pentlandite, troilite and Ti-bearing chromite.

Geochemistry: Olivine in equilibrated clasts $\left(\mathrm{Fa}_{40.2-42.4}, \mathrm{~N}=3\right)$, forsterite in type 3 chondrules $\left(\mathrm{Fa}_{2.9-3.3}, \mathrm{~N}=2\right)$, orthopyroxene $\left(\mathrm{Fs}_{1.1-31.0} \mathrm{Wo}_{0.3-2.2}, \mathrm{~N}=3\right)$, clinopyroxene $\left(\mathrm{Fs}_{0.8-10.0} \mathrm{Wo}_{45.8-46.3}, \mathrm{~N}=2\right)$, plagioclase $\left(\mathrm{An}_{9.4-12.0} \mathrm{Or}_{6.4-3.5}, \mathrm{~N}=2\right)$.

Classification: R3-5 chondrite breccia.

Specimens: $25.7 \mathrm{~g}$ including one polished thin section at $U W B$; main mass with Mr. G. Fujihara.

\section{Northwest Africa 11615 (NWA 11615) \\ Northwest Africa \\ Purchased: 2017 Aug \\ Classification: Ordinary chondrite (LL3)}

History: Purchased in Temara, Morocco by Adam Aaronson in August 2017. Petrography: (A. Irving and S. Kuehner, UWS) Closely packed, well-formed chondrules (apparent diameter $710 \pm 420 \mu \mathrm{m}, \mathrm{N}=18$ ) are set in a finer grained matrix containing altered kamacite.

Geochemistry: Olivine $\left(\mathrm{Fa}_{0.7-41.7}, \mathrm{Cr}_{2} \mathrm{O}_{3}\right.$ in ferroan examples $0.06-0.10 \mathrm{wt} . \%$, mean $0.08 \pm 0.02$ wt. $\%, \mathrm{~N}=8)$, orthopyroxene $\left(\mathrm{Fs}_{2.8-21.2} \mathrm{Wo}_{0.2-3.0}, \mathrm{~N}=3\right)$, pigeonite $\left(\mathrm{Fs}_{17.8 \text { - }}\right.$ $\left.{ }_{21.9} \mathrm{Wo}_{20.9-16.2}, \mathrm{~N}=2\right)$. Magnetic susceptibility $\log \chi\left(\times 10^{-9} \mathrm{~m}^{3} / \mathrm{kg}\right)=3.73$.

Classification: Ordinary chondrite (LL3).

Specimens: $32.8 \mathrm{~g}$ including one polished thin section at $U W B$; main mass with Aaronson.

\author{
Northwest Africa 11616 (NWA 11616) \\ (Northwest Africa) \\ Purchased: 2017 Mar
}


Classification: Lunar meteorite

History: Purchased by DPitt in March 2017 from a Mauritanian dealer.

Physical characteristics: A single very fresh specimen (2550.8 g) containing separate olivine gabbro and olivine-free basaltic clasts in a fragmental matrix. Petrography: (A. Irving and S. Kuehner, UWS; S. Valencia and P. Carpenter, $W U S L$ ) Polymict breccia with two different clast types. Olivine gabbro clasts (mean grainsize $1.1 \mathrm{~mm}$ ) consist of equant grains of olivine, zoned clinopyroxene (pigeonite, ferroan pigeonite, subcalcic augite) and maskelynite with accessory chromite, ilmenite, troilite, fayalite, taenite and baddeleyite. A basalt clast has porphyritic texture and is composed mainly of larger prismatic grains (up to $2.5 \mathrm{~mm}$ long) of zoned clinopyroxene and smaller lath-like grains of maskelynite with accessory ilmenite, chromite, troilite, silica polymorph, rare baddeleyite, rare $\mathrm{K}$-feldspar and rare Ni-poor kamacite. The remainder of the breccia consists of related crystalline debris.

Geochemistry: Olivine gabbro clasts: olivine $\left(\mathrm{Fa}_{34.6-59.8}, \mathrm{FeO} / \mathrm{MnO}=83-108, \mathrm{~N}=\right.$ 9), pigeonite $\left(\mathrm{Fs}_{26.4-39.4} \mathrm{Wo}_{9.7-11.7}, \mathrm{FeO} / \mathrm{MnO}=51-70, \mathrm{~N}=6\right)$, ferroan pigeonite $\left(\mathrm{Fs}_{43.4-71.4} \mathrm{Wo}_{15.4-25.0}, \mathrm{FeO} / \mathrm{MnO}=57-94, \mathrm{~N}=3\right)$, subcalcic augite $\left(\mathrm{Fs}_{17.6-28.4} \mathrm{Wo}_{27.2-}\right.$ 35.6, $\mathrm{FeO} / \mathrm{MnO}=51-59, \mathrm{~N}=5$ ), maskelynite (85.2-95.6Or $\left.\mathrm{Or}_{2-2-1}, \mathrm{~N}=10\right)$. Basalt clast: pigeonite $\left(\mathrm{Fs}_{26.2-26.3} \mathrm{Wo}_{6.4-6.5} ; \mathrm{Fs}_{29.1} \mathrm{Wo}_{11.7} ; \mathrm{Fs}_{49.8} \mathrm{Wo}_{19.1} ; \mathrm{FeO} / \mathrm{MnO}=55-73 ; \mathrm{N}\right.$ $=4)$, augite $\left(\mathrm{Fs}_{21.9-25.6} \mathrm{Wo}_{34.7-35.2}, \mathrm{FeO} / \mathrm{MnO}=49-50, \mathrm{~N}=2\right)$, ferropigeonite rims $\left(\mathrm{Fs}_{78.0-78.2} \mathrm{Wo}_{18.9-20.3}, \mathrm{FeO} / \mathrm{MnO}=83-86, \mathrm{~N}=2\right)$, maskelynite $\left(\mathrm{An}_{84.4-86.2} \mathrm{Or}_{3.6-2.0}, \mathrm{~N}\right.$ $=2$ ). Bulk composition (R. Korotev, WUSL) INAA of breccia subsamples gave (in wt.\%) FeO 19.1, $\mathrm{Na}_{2} \mathrm{O} 0.25$ (in ppm) Sc 42.0, Cr 3230, La 13.6, Nd 22.9, Sm 6.73, Eu 0.578, Tb 1.37, Yb 1.37, Lu 0.66, Hf 5.1, Th 2.0.

Classification: Lunar meteorite (polymict breccia with olivine gabbro and basalt clasts). The olivine gabbro lithology is paired with other magnesian gabbro material present in breccia stones such as NWA 773, NWA 2727 and NWA $\underline{3333}$ and also as discrete meteorites such as NWA 2977, NWA 6950 and NWA 8127. The olivine-free basalt lithology differs from the olivine-phyric basalt lithology represented by NWA 3160, and is a new member of the complex NWA 773 clan of lunar meteorites.

Specimens: $20.9 \mathrm{~g}$ including a polished thin section and polished endcut at $U W B$; remainder with DPitt.

\section{Northwest Africa 11617 (NWA 11617)}

(Northwest Africa)

Purchased: 2017 Nov

Classification: Ordinary chondrite (LL(L)3)

History: Purchased in Temara, Morocco, by Aaronson in November 2017.

Petrography: (A. Irving and S. Kuehner, UWS) Closely packed, well-formed chondrules (apparent diameter $680 \pm 420 \mu \mathrm{m}, \mathrm{N}=18$ ) are set in a finer grained matrix containing altered kamacite. 
Geochemistry: Olivine $\left(\mathrm{Fa}_{0.8-48.0}, \mathrm{Cr}_{2} \mathrm{O}_{3}\right.$ in ferroan examples $0.03-1.04$ wt.\%, mean $0.24 \pm 0.40$ wt. $\%, \mathrm{~N}=8)$, orthopyroxene $\left(\mathrm{Fs}_{0.2-21.4} \mathrm{Wo}_{0.4-3.9}, \mathrm{~N}=3\right)$, subcalcic augite $\left(\mathrm{Fs}_{6.9-12.4} \mathrm{Wo}_{30.4-25.0}, \mathrm{~N}=2\right)$. Magnetic susceptibility $\log \chi\left(\times 10^{-9} \mathrm{~m}^{3} / \mathrm{kg}\right)=4.21$.

Classification: Ordinary chondrite (LL(L)3).

Specimens: $21.2 \mathrm{~g}$ including one polished thin section at $U W B$; main mass with Aaronson.

\section{Northwest Africa 11629 (NWA 11629)}

(Northwest Africa)

Purchased: 2017

Classification: Ordinary chondrite (LL4-6)

Petrography: Chondritic breccia consisting of clastic LL4 type matrix and abundant LL6 clasts. Plagioclase grain size in LL6 clasts is about $80 \mu \mathrm{m}$.

Geochemistry: type 4 lithology: olivine: $\mathrm{Fa}_{29.2 \pm 0.3}, \mathrm{n}=12$; pyroxene:

$\mathrm{Fs}_{20.6 \pm 4.6} \mathrm{Wo}_{2.4 \pm 0.8},\left(\mathrm{Fs}_{8.6-24.5} \mathrm{Wo}_{0.5-3.5}, \mathrm{n}=17\right)$; type 6 lithology: olivine: $\mathrm{Fa}_{30.0 \pm 0.5}$, $\mathrm{n}=12$; pyroxene: $\mathrm{Fs}_{24.2 \pm 0.2} \mathrm{Wo}_{3.5 \pm 0.4}, \mathrm{n}=9$

\section{Northwest Africa 11636 (NWA 11636) \\ (Northwest Africa) \\ Purchased: 2017 \\ Classification: Carbonaceous chondrite (CK6)}

Physical characteristics: Sixteen small dark brownish individuals partly covered with fusion crust.

Petrography: The meteorite predominantly consists of fine-grained recrystallized matrix mainly composed of Ni-bearing ferrous olivine. Chondrules are only rarely encountered. Minor phases include intermediate plagioclase, low-Ca pyroxene, and troilite. Cr-rich magnetite is abundant; metal is virtually absent.

Geochemistry: Olivine: $\mathrm{Fa}_{29.5 \pm 0.2}, \mathrm{NiO}=0.4 \pm 0.1, \mathrm{FeO} / \mathrm{MnO}=100 \pm 11, \mathrm{n}=12$; magnetite $\mathrm{Cr}_{2} \mathrm{O}_{3}$ is about $3.8 \mathrm{wt} \%$.

\section{Northwest Africa 11637 (NWA 11637) \\ Morocco \\ Purchased: May 2017 \\ Classification: Iron meteorite (IIAB)}

History: On May 21, 2017, during the China (Hunan) Mineral and Gem Show in Chenzhou, China, Wang Chen purchased 3 individual specimens $(3096$ g, 1100 g, $172.3 \mathrm{~g}$ ) from a Moroccan dealer attending the show.

Physical characteristics: The three pieces have a dark brown exterior with minor rust-colored spots.

Petrography: (C. Herd, $U A b$ ) Optical investigation of a $\sim 4 \times 5 \mathrm{~cm}$ polished and etched surface reveals a well-developed Widmanstatten pattern consistent with a 
coarsest octahedrite $(3.6 \pm 1 \mathrm{~mm} ; \mathrm{n}=6)$. No obvious inclusions were noted. Terrestrial alteration has occurred along fractures connecting to the exterior.

Geochemistry: ICP-MS data, using sample of North Chile (Filomena) as standard (C. Herd and G. Chen $U A b$ ): $\mathrm{Ni}=5.7, \mathrm{Co}=0.47$ (both $\mathrm{wt}^{\mathrm{o}} \%$ ); $\mathrm{Ir}=2.3, \mathrm{Ga}=54, \mathrm{Ge}$ $=137, \mathrm{As}=4.8, \mathrm{~W}=2.5, \mathrm{Re}=0.15, \mathrm{Pt}=18.3, \mathrm{Cu}=139, \mathrm{Au}=0.5(\mathrm{all} \mu \mathrm{g} / \mathrm{g})$.

Classification: (C. Herd, $U A b)$ : IIAB iron, through comparison of bulk composition with data from Wasson et al. (2007).

Specimens: Type specimen consisting of two representative slices (13.5 and 11.8 $\mathrm{g})$ at $U A b$. Wang Chen holds the main mass (2910 g), Wang Ziyao the $1100 \mathrm{~g}$ individual, and Mendy Ouzillou holds the $172.3 \mathrm{~g}$ individual and a $117.5 \mathrm{~g}$ end cut from the main mass.

\section{Northwest Africa 11638 (NWA 11638)}

(Northwest Africa)

Purchased: 2017

Classification: CM2.0

History: The meteorite was purchased from a local meteorite dealer in Morocco. Physical characteristics: Many small and dark-grayish fragments lacking any fusion crust.

Petrography: The meteorite is a carbonaceous chondrite that is composed of chondrule and CAI pseudomorphs set into a fine-grained Fe-rich matrix dominated by phyllosillicates, carbonates and sulfides. It does not contain any anhydrous silicates. Low totals of defocused matrix analyses attest to porosity and the presence of hydrous phases, i.e., phyllosilicates. Abundant mineral phases are up to $50 \mu \mathrm{m}$ sized Ca-carbonates, often fibrous phyllosilicates, and Ni-bearing pyrrhotite. Geochemistry: Mean values of defocused (10 $\mu \mathrm{m}$ beam diameter) matrix analyses (all wt\%; $\mathrm{N}=35$ ): $\mathrm{SiO}_{2}: 26.5, \mathrm{TiO}_{2}: 0.12 ; \mathrm{Na}_{2} \mathrm{O}: 0.19 ; \mathrm{Cr}_{2} \mathrm{O}_{3}: 0.46, \mathrm{MgO}: 20.5$, $\mathrm{MnO}: 0.25, \mathrm{CaO}: 0.68, \mathrm{FeO}: 29.5, \mathrm{Al}_{2} \mathrm{O}_{3}: 2.59$, NiO: $0.69, \mathrm{P}_{2} \mathrm{O}_{5}: 0.14, \mathrm{~S}: 0.38$, Total: 82.0; Ca-carbonates contain about $1 \mathrm{wt} \% \mathrm{FeO}$.

Classification: CM2.0. Subtype following the classification scheme of Rubin et al. (2007)).

\section{Northwest Africa 11639 (NWA 11639)}

(Northwest Africa)

Purchased: 2017

Classification: Ureilite

History: The meteorite was purchased from a local meteorite dealer in Morocco. Physical characteristics: Dark brownish fragment lacking any fusion crust. Petrography: The meteorite displays a characteristic cumulate texture of up to 2 $\mathrm{mm}$ sized olivine and orthopyroxene crystals. Reduced rims in olivine are abundant. The meteorite contains flaky graphite.

Geochemistry: reduced rims in olivine: $\mathrm{Fa}_{3.6-5.8}$; olivine contains $0.32 \pm 0.02 \mathrm{wt} \%$ $\mathrm{Cr}_{2} \mathrm{O}_{3}$ and $0.25 \pm 0.02 \mathrm{wt} \% \mathrm{CaO}$ 


\section{Northwest Africa 11640 (NWA 11640)}

(Northwest Africa)

Purchased: 2017

Classification: Ureilite

History: The meteorite was purchased from a local meteorite dealer in Morocco. Physical characteristics: Dark brownish fragment lacking any fusion crust. Petrography: The meteorite shows a cumulate texture of up to $2.5 \mathrm{~mm}$ sized olivine and pigeonite grains. Olivine crystals appear to consists of smaller subgrains and pigeonite shows patchy compositional zoning. Olivine displays characterisitic reduced rims. The meteorite contains diamond and shows some foliation.

Geochemistry: reduced rims in olivine: $\mathrm{Fa}_{2.0-7.7}$; olivine contains $0.65 \pm 0.04 \mathrm{wt} \%$ $\mathrm{Cr}_{2} \mathrm{O}_{3}$ and $0.35 \pm 0.02 \mathrm{wt} \% \mathrm{CaO}$

\section{Northwest Africa 11641 (NWA 11641) \\ (Northwest Africa) \\ Purchased: 2017 \\ Classification: Ureilite}

History: The meteorite was purchased from a local meteorite dealer in Mali.

Physical characteristics: Dark bownish fragment lacking any fusion crust.

Petrography: The meteorite shows a cumulate texture composed of up to $1 \mathrm{~mm}$ sized olivine and pigeonite grains. Olivine displays characteristic reduced rims and the meteorite contains abundant flaky graphite.

Geochemistry: reduced rims in olivine: $\mathrm{Fa}_{4.7-8.8}$; olivine contains $0.61 \pm 0.02 \mathrm{wt} \%$ $\mathrm{Cr}_{2} \mathrm{O}_{3}$ and $0.34 \pm 0.01 \mathrm{wt} \% \mathrm{CaO}$

\section{Northwest Africa 11643 (NWA 11643) \\ (Northwest Africa) \\ Purchased: 2017 \\ Classification: Ordinary chondrite (LL5)}

Petrography: The meteorite is an ordinary chondrite predominantly composed of densely packed chondrules with a mean diameter of about $1 \mathrm{~mm}$. Matrix and metal are rare; plagioclase grain size is about $20 \mu \mathrm{m}$. Olivine and pyroxene are completely equilibrated.

\section{Northwest Africa 11644 (NWA 11644)}

(Northwest Africa)

Purchased: 2017

Classification: Ureilite

History: The meteorite was purchased from a local meteorite dealer in Morocco. 
Physical characteristics: Two small dark brownish fragments lacking any fusion crust.

Petrography: The meteorite displays a coarse-grained cumulate texture of predominant up to $2 \mathrm{~mm}$ sized olivine grains and less abundant pigeonite. Contains some graphites flakes; olivine displays characteristic reduced rims.

Geochemistry: reduced rims in olivine: $\mathrm{Fa}_{1.6-5.3}$; olivine contains $0.73 \pm 0.02 \mathrm{wt} \%$ $\mathrm{Cr}_{2} \mathrm{O}_{3}$ and $0.33 \pm 0.02 \mathrm{wt} \% \mathrm{CaO}$

\section{Northwest Africa 11645 (NWA 11645)}

(Northwest Africa)

Purchased: 2015

Classification: Ordinary chondrite (LL4-6)

Petrography: Chondritic breccia consisting of LL6 type fragments set into a clastic LL4 type matrix. Plagioclase grain size in LL6 clasts is about $70 \mu \mathrm{m}$.

Geochemistry: type 4 lithology: olivine: $\mathrm{Fa}_{29.7 \pm 0.2}, \mathrm{n}=12$; pyroxene:

$\mathrm{Fs}_{23.4 \pm 2.4} \mathrm{Wo}_{2.3 \pm 0.9},\left(\mathrm{Fs}_{13.0-25.6} \mathrm{Wo}_{0.5-3.5}, \mathrm{n}=21\right)$; type 6 lithology: olivine: $\mathrm{Fa}_{30.0 \pm 0.3}$, $\mathrm{n}=13$; pyroxene: $\mathrm{Fs}_{25.2 \pm 0.1} \mathrm{Wo}_{2.8 \pm 0.2}, \mathrm{n}=13$

\section{Northwest Africa 11647 (NWA 11647)}

(Northwest Africa)

Purchased: 2016

Classification: HED achondrite (Eucrite, polymict)

History: The meteorite was bought from a meteorite dealer at the mineral fair in St. Marie-aux Mines, France.

Physical characteristics: Grayish fragment partly covered with fusion crust.

Petrography: The meteorite is a polymict breccia with basaltic and black melt clasts set into a clastic mineral matrix. Dominant minerals are magmatically zoned low-Ca pyroxenes, Fe-rich low-Ca pyroxenes with augite exsolution lamellae and calcic plagioclase. Minor phases include silica, chromite, FeS, and ilmenite. No metallic $\mathrm{Fe}$ has been found.

Geochemistry: zoned low-Ca pyroxene $\mathrm{Fs}_{26.7 \pm 2.3} \mathrm{Wo}_{2.9 \pm 0.8}\left(\mathrm{Fs}_{23.5-29.2} \mathrm{Wo}_{2.0-5.1}, \mathrm{n}=15\right.$, $\mathrm{FeO} / \mathrm{MnO}=27-33$ ); low-Ca pyroxene host to augite exsolution lamellae:

$\mathrm{Fs}_{43.4 \pm 0.3} \mathrm{Wo}_{2.9 \pm 0.3}\left(\mathrm{Fs}_{43.0-44.1} \mathrm{Wo}_{2.6-3.5}, \mathrm{n}=12, \mathrm{FeO} / \mathrm{MnO}=32-36\right)$; Ca-pyroxene:

$\mathrm{Fs}_{21.0 \pm 0.3} \mathrm{Wo}_{40.0 \pm 0.2}\left(\mathrm{Fs}_{20.3-21.5} \mathrm{Wo}_{40.3-41.1}, \mathrm{n}=13, \mathrm{FeO} / \mathrm{MnO}=26-32\right)$; calcic plagioclase: $\mathrm{An}_{90.7 \pm 1.1}\left(\mathrm{An}_{89.3-92.4}, \mathrm{n}=14\right)$

\section{Northwest Africa 11648 (NWA 11648)}

(Northwest Africa)

Purchased: 2002 Feb

Classification: Ordinary chondrite (L6)

History: A single stone was purchased by Edwin Thompson at the Tucson Gem Show and donated to Cascadia. 
Petrography: About a dozen readily identifiable chondrules are visible in thin section, but their edges grade into surrounding granular regions. Approximately 2\% metal present. Minimal weathering. Shock stage (conventional) estimated from olivine deformation; plagioclase deformed.

Geochemistry: (A. Ruzicka and K. Farley, Cascadia) Olivine ( $\mathrm{Fa}_{24.9 \pm 0.2}, \mathrm{~N}=13$ ), low-Ca pyroxene ( $\mathrm{Wo}_{1.5 \pm 0.3} \mathrm{Fs}_{21.1 \pm 0.2} \mathrm{En}_{77.4 \pm 0.2}, \mathrm{~N}=12$ ), plagioclase

$\left(\mathrm{Ab}_{85.1 \pm 0.6} \mathrm{Or}_{3.2 \pm 0.4} \mathrm{An}_{11.4 \pm 0.8}, \mathrm{~N}=13\right)$.

Classification: L6 chondrite based on texture and mineral chemistry.

Specimens: Cascadia holds the main mass and type specimen, consisting of $28.7 \mathrm{~g}$ in two pieces plus one thin section.

\section{Northwest Africa 11649 (NWA 11649) \\ Morocco \\ Purchased: 2005 \\ Classification: Iron meteorite (IAB-MG)}

History: Purchased from a Moroccan dealer at the 2005 Munich show.

Physical characteristics: The meteorite is oblong, approximately $20 \mathrm{~cm}$ in longest dimension, and is characterized by a dark brown to black, regmaglypted exterior.

Petrography: (C. Herd, $U A b)$ Optical investigation of a $\sim 1.5 \times 3 \mathrm{~cm}$ polished and etched surface reveals $\mathrm{cm}$-scale, equant kamacite grains with Neumann lines.

Sulfide inclusions are present; no other inclusions are visible, although the surface area examined is relatively small. Terrestrial alteration has occured along fractures connecting to the exterior.

Geochemistry: ICP-MS data, using sample of North Chile (Filomena) as standard (C. Herd and G. Chen $U A b$ ): $\mathrm{Ni}=6.7, \mathrm{Co}=0.46$ (both wt\%); $\mathrm{Ir}=3.5, \mathrm{Ga}=88$, As $=12.5, \mathrm{~W}=1.4, \mathrm{Re}=0.31, \mathrm{Pt}=8.6, \mathrm{Cu}=134, \mathrm{Au}=1.3(\mathrm{all} \mu \mathrm{g} / \mathrm{g})$.

Classification: (C. Herd, $U A b$ ): IAB iron, Main Group, through comparison of bulk composition with data from Wasson and Kallemeyn (2002).

Specimens: Type specimen consisting of one representative piece (22.8 g) at $U A b$. Main mass in the Hollis Meteorite Collection.

\section{Northwest Africa 11667 (NWA 11667)}

(Northwest Africa)

Purchased: 2017

Classification: Enstatite chondrite (EL6)

History: The meteorite was bought by the main mass holder from a local meteorite dealer in Morocco.

Physical characteristics: Dark brownish individual with some patches of fusion crust.

Petrography: The meteorite displays a fine-grained, recrystallized texture of predominantly enstatite, less abundant albitic feldspar, often intergrown daubreelite and troilite, and FeNi metal. No chondrules are visible. 
Geochemistry: Feldspar: $\mathrm{An}_{15.3} \mathrm{Ab}_{80.2}, \mathrm{n}=12$; FeNi metal contains about $1.2 \mathrm{wt} \%$ $\mathrm{Si}$

\section{Northwest Africa 11668 (NWA 11668) \\ (Northwest Africa) \\ Purchased: 2018 \\ Classification: Martian meteorite (Shergottite)}

History: The meteorite was purchased in Tindouf, Algeria, from a Sahrawi dealer. Physical characteristics: Four dark-green pieces partly covered with fusion crust. Petrography: The meteorite is a medium grained basalt predominantly composed of up to $1.5 \mathrm{~mm}$ sized pyroxene and plagioclase grains. Pyroxene is intensely fractured pigeonite and augite displaying mottled compositional zoning. Plagioclase is completely transformed into compositionally homogeneous maskelynite. Minor phases are ilmenite, ulvöspinel, pyrrhotite and some Si-rich mesostasis.

Geochemistry: pigeonite: $\mathrm{Fs}_{53.7 \pm 4.4} \mathrm{Wo}_{14.3 \pm 2.4}\left(\mathrm{Fs}_{35.5-59.6} \mathrm{Wo}_{9.7-20.0}, \mathrm{n}=38\right.$, $\mathrm{FeO} / \mathrm{MnO}=33-43)$; augite: $\mathrm{Fs}_{26.2 \pm 7.0} \mathrm{Wo}_{32.2 \pm 3.9}\left(\mathrm{Fs}_{21.1-48.9} \mathrm{Wo}_{20.6-37.8}, \mathrm{n}=32\right.$, $\mathrm{FeO} / \mathrm{MnO}=27-40$ ); feldspar: $\mathrm{An}_{56.8} \mathrm{Ab}_{42.0} \mathrm{Or}_{1.2}, \mathrm{n}=15$

Classification: Martian (basaltic shergottite)

\section{Northwest Africa 11669 (NWA 11669) \\ (Northwest Africa) \\ Purchased: 2013 Dec \\ Classification: Ordinary chondrite (LL3)}

History: Purchased in Rissani, Morocco by Adam Aaronson in December 2013. Petrography: (A. Irving and S. Kuehner, UWS) Closely-packed, well-formed chondrules (apparent diameter $580 \pm 340 \mu \mathrm{m}, \mathrm{N}=18$ ) are set in a recrystallized matrix containing altered kamacite.

Geochemistry: Olivine $\left(\mathrm{Fa}_{0.5-61.4}, \mathrm{Cr}_{2} \mathrm{O}_{3}\right.$ in ferroan examples $0.07-0.18$ wt.\%, mean $0.12 \pm 0.04$ wt.\%, $\mathrm{N}=8)$, orthopyroxene $\left(\mathrm{Fs}_{3.0-14.9} \mathrm{Wo}_{0.3-3.6}, \mathrm{~N}=3\right)$, subcalcic augite $\left(\mathrm{Fs}_{14.5} \mathrm{Wo}_{33.0}\right)$, diopside $\left(\mathrm{Fs}_{1.0} \mathrm{Wo}_{45.9}\right)$. Magnetic susceptibility $\log \chi\left(\times 10^{-9} \mathrm{~m}^{3} / \mathrm{kg}\right)=$ 3.22 .

Classification: Ordinary chondrite (LL3).

Specimens: $26.4 \mathrm{~g}$ including one polished thin section at $U W B$; main mass with Aaronson.

\section{Northwest Africa 11670 (NWA 11670)}

(Northwest Africa)

Purchased: 2017 Mar

Classification: Carbonaceous chondrite (CK4)

History: Purchased in Zagora, Morocco, by Adam Aaronson in March 2017. 
Petrography: (A. Irving and S. Kuehner, UWS) Equilibrated specimen composed of separated, medium-sized chondrules (containing rounded grains of stained $\mathrm{Cr}$ magnetite) set in a finer grained matrix containing Cr-magnetite, chlorapatite and pentlandite.

Geochemistry: Olivine $\left(\mathrm{Fa}_{32.1-32.4}, \mathrm{~N}=3\right)$, orthopyroxene $\left(\mathrm{Fs}_{26.7-29.7} \mathrm{Wo}_{0.8-1.8}, \mathrm{~N}=\right.$ $3)$, clinopyroxene $\left(\mathrm{Fs}_{11.5-14.6} \mathrm{Wo}_{47.6-48.3}, \mathrm{~N}=2\right)$.

Classification: CK4 chondrite.

Specimens: $21 \mathrm{~g}$ including one polished thin section at $U W B$; main mass with Aaronson.

\section{Northwest Africa 11671 (NWA 11671) \\ (Northwest Africa) \\ Purchased: 2017 Aug \\ Classification: Ureilite}

History: Purchased in Temara, Morocco, by Adam Aaronson in August 2017.

Petrography: (A. Irving and S. Kuehner, UWS) Protogranular aggregate (mean grainsize $1.5 \mathrm{~mm}$ ) composed entirely of olivine (with relatively thick dark, reduced magnesian rims). Multiple planar deformation lamellae are visible in some olivine grains viewed in cross-polarized light. A thorough search of the thin section revealed no pyroxene.

Geochemistry: Olivine (cores $\mathrm{Fa}_{20.6-20.8}, \operatorname{rim~} \mathrm{Fa}_{5.8}, \mathrm{~N}=4$ ).

Classification: Ureilite (dunitic).

Specimens: $20.6 \mathrm{~g}$ including one polished thin section at $U W B$; main mass

with Aaronson.

\section{Northwest Africa 11672 (NWA 11672)}

(Northwest Africa)

Purchased: 2017 Jun

Classification: Ordinary chondrite (L3)

History: Purchased by Gary Fujihara in June 2017 from a Moroccan dealer.

Petrography: (A. Irving and S. Kuehner, UWS) Closely packed, well-formed, unequilibrated chondrules (apparent diameter $620 \pm 340 \mu \mathrm{m}, \mathrm{N}=18$ ) are set in a recrystallized matrix containing slightly stained kamacite.

Geochemistry: Olivine $\left(\mathrm{Fa}_{1.8-64.0}, \mathrm{Cr}_{2} \mathrm{O}_{3}\right.$ in ferroan examples $0.02-0.84$ wt.\%, mean $0.19 \pm 0.33$ wt. $\%, \mathrm{~N}=8)$, orthopyroxene $\left(\mathrm{Fs}_{3.3-18.8} \mathrm{Wo}_{0.2-2.0}, \mathrm{~N}=3\right)$, augite $\left(\mathrm{Fs}_{8.7-}\right.$ $\left.{ }_{11.8} \mathrm{Wo}_{41.3-36.7}, \mathrm{~N}=2\right)$. Magnetic susceptibility $\log \chi\left(\times 10^{-9} \mathrm{~m}^{3} / \mathrm{kg}\right)=4.73$.

Classification: Ordinary chondrite (L3).

Specimens: $21.8 \mathrm{~g}$ including one polished thin section at $U W B$; main mass with Mr. G. Fujihara.

Northwest Africa 11674 (NWA 11674)

(Northwest Africa) 
Purchased: 2017 Nov

Classification: Ordinary chondrite (L4)

History: Purchased in Temara, Morocco by Adam Aaronson in November 2017. Petrography: (A. Irving and S. Kuehner, UWS) Closely packed, well-formed chondrules are set in a recrystallized matrix containing stained kamacite and chlorapatite.

Geochemistry: Olivine $\left(\mathrm{Fa}_{24.9-25.3}, \mathrm{~N}=3\right)$, orthopyroxene $\left(\mathrm{Fs}_{20.6-20.8} \mathrm{Wo}_{0.9-0.7}, \mathrm{~N}=\right.$ $3)$, clinopyroxene $\left(\mathrm{Fs}_{6.7-7.6} \mathrm{Wo}_{45.6-44.3}, \mathrm{~N}=2\right)$.

Classification: Ordinary chondrite (L4).

Specimens: $21 \mathrm{~g}$ including one polished thin section at $U W B$; main mass with Aaronson.

\section{Northwest Africa 11675 (NWA 11675)}

(Northwest Africa)

Purchased: 2017 Mar

Classification: Ureilite

History: Purchased by Bob Falls in March 2017 from a dealer in Zagora, Morocco. Petrography: (A. Irving and S. Kuehner, UWS) Protogranular aggregate (mean grainsize $1.9 \mathrm{~mm}$ ) composed of olivine ( $\sim 75 \mathrm{vol} . \%$ ) and pigeonite ( $\sim 25 \mathrm{vol} . \%)$. Olivine grains have relatively thick, dark, reduced magnesian rims associated with Fe metal (extensively altered to hydroxides). Minor secondary gypsum is present. Geochemistry: Olivine (cores $\mathrm{Fa}_{19.6-19.9}$, rim $\mathrm{Fa}_{6.3}, \mathrm{~N}=3$ ), pigeonite $\left(\mathrm{Fs}_{13.0} \mathrm{Wo}_{5.5}\right.$; $\mathrm{Fs}_{15.5} \mathrm{Wo}_{11.7} ; \mathrm{N}=2$ ).

Classification: Ureilite.

Specimens: $22.4 \mathrm{~g}$ including one polished thin section at $U W B$; remainder with $\mathrm{Mr}$. R. Falls.

\section{Northwest Africa 11676 (NWA 11676) \\ (Northwest Africa) \\ Purchased: 2005 \\ Classification: Ordinary chondrite (L6)}

History: Purchased by an anonymous collector from a dealer at the Munich Show in 2005, subsequently acquired by the Hollis Collection and donated to PSF. In 2018 the specimen was acquired by the Kenosha Public Museum, Wisconsin.

Physical characteristics: A single broken stone (1664 g) partly coated with dark brown fusion crust. The fresher interior exhibits some partly weathered shiny metal and indistinct chondrules.

Petrography: (A. Irving and S. Kuehner, UWS) Mostly recrystallized with rare chondrule remnants and altered metal.

Geochemistry: Olivine $\left(\mathrm{Fa}_{26.1-26.2}, \mathrm{~N}=3\right)$, orthopyroxene $\left(\mathrm{Fs}_{20.9-21.2} \mathrm{Wo}_{1.3-1.5}, \mathrm{~N}=\right.$ $3)$, clinopyroxene $\left(\mathrm{Fs}_{7.8-8.3} \mathrm{Wo}_{44.2-44.7}, \mathrm{~N}=2\right)$.

Classification: Ordinary chondrite (L6). 
Specimens: $39.1 \mathrm{~g}$ including one polished thin section at $P S F$; main mass housed at the Kenosha Public Museum, Wisconsin, USA.

\section{Northwest Africa 11677 (NWA 11677) \\ (Northwest Africa) \\ Purchased: 2017 Nov \\ Classification: HED achondrite (Eucrite, polymict)}

History: Purchased by Darryl Pitt in November 2017 from a Mauritanian dealer. Petrography: (A. Irving and S. Kuehner, UWS) Polymict breccia consisting predominantly of eucrite clasts (exhibiting intersertal, subophitic, diabasic and gabbroic textures) and related crystalline debris, together with $\sim 10$ vol.\% of diogenitic orthopyroxene (exhibiting more ferroan reaction rims against the matrix). Other minerals are exsolved pigeonite, calcic plagioclase, silica polymorph, ilmenite, Ti-chromite and troilite.

Geochemistry: Diogenitic orthopyroxene (cores $\mathrm{Fs}_{31.3-33.5} \mathrm{Wo}_{3.6-5.3}, \mathrm{FeO} / \mathrm{MnO}=35$ 36; $\left.\operatorname{rim} \mathrm{Fs}_{53.8} \mathrm{Wo}_{3.8}, \mathrm{FeO} / \mathrm{MnO}=32 ; \mathrm{N}=3\right)$, orthopyroxene host $\left(\mathrm{Fs}_{61.2} \mathrm{Wo}_{1.5}\right.$, $\mathrm{FeO} / \mathrm{MnO}=36)$, clinopyroxene exsolution lamella $\left(\mathrm{Fs}_{26.0} \mathrm{Wo}_{42.9}, \mathrm{FeO} / \mathrm{MnO}=34\right)$, low-Ca pyroxene host $\left(\mathrm{Fs}_{45.1} \mathrm{Wo}_{7.1}, \mathrm{FeO} / \mathrm{MnO}=31\right)$, augite exsolution lamella $\left(\mathrm{Fs}_{22.6} \mathrm{Wo}_{39.1}, \mathrm{FeO} / \mathrm{MnO}=30\right)$, augite $\left(\mathrm{Fs}_{30.1} \mathrm{Wo}_{37.4}, \mathrm{FeO} / \mathrm{MnO}=35\right)$, plagioclase $\left(\mathrm{An}_{88.7-89.1} \mathrm{Or}_{0.3-0.6}, \mathrm{~N}=2\right)$.

Classification: Eucrite (polymict breccia).

Specimens: $21.1 \mathrm{~g}$ including one polished thin section at $U W B$; main mass with DPitt.

\section{Northwest Africa 11678 (NWA 11678)}

(Northwest Africa)

Purchased: 2017 Nov

Classification: Ordinary chondrite (L4)

History: Purchased in Temara, Morocco by Adam Aaronson in November 2017.

Petrography: (A. Irving and S. Kuehner, UWS) Closely packed, well-formed chondrules are set in a recrystallized matrix containing altered kamacite and merrillite.

Geochemistry: Olivine $\left(\mathrm{Fa}_{25.5-25.6}, \mathrm{~N}=3\right)$, orthopyroxene $\left(\mathrm{Fs}_{20.9-21.0} \mathrm{Wo}_{1.4-1.5}, \mathrm{~N}=\right.$ 3), clinopyroxene $\left(\mathrm{Fs}_{7.4-7.7} \mathrm{Wo}_{45.1-45.4}, \mathrm{~N}=2\right)$.

Classification: Ordinary chondrite (L4).

Specimens: $35.4 \mathrm{~g}$ including one polished thin section at $U W B$; main mass with Aaronson.

Northwest Africa 11679 (NWA 11679)

Morocco, Laayoun, (Northwest Africa)

Purchased: May 2014

Classification: Enstatite chondrite (EL6) 
History: Collected by nomads in Western Sahara and purchased by Nicola Castellano at the Genova Mineral Fair in May 2014 from a Moroccan dealer. Physical characteristics: A single piece weighing $98 \mathrm{~g}$ with no fusion crust. Petrography: (V. Moggi Cecchi, G. Pratesi, S. Caporali, UniFi); A fine-grained texture, with a pyroxene-dominant matrix and minor plagioclase is visible in thin section. No relic chondrules are visible. Kamacite and troilite are the main opaque phases, partially weathered to iron oxides. Accessory phases are alabandite and daubreelite as blades in troilite.

Geochemistry: EMP (V. Moggi Cecchi, G. Pratesi, S. Caporali, UniFi) Orthopyroxene $\left(\mathrm{Fs}_{0.3} \mathrm{En}_{98.1} \mathrm{Wo}_{1.6}\right)$, plagioclase $\left(\mathrm{An}_{17.9} \mathrm{Or}_{4.8}\right) ; \mathrm{Si}$ in kamacite $=0.5$ wt. $\%$, Ti in troilite $=6.2 \mathrm{wt} . \%$

Classification: Enstatite chondrite (EL6); strong weathering. EL classification is suggested by the presence of alabandite, An content of plagioclase and Si content of kamacite. Possibly paired with NWA 11413 , NWA 11414, NWA 11415, NWA 11416.

Specimens: A total of $19.6 \mathrm{~g}$ specimen is on deposit at MSN-FI. Castellano holds the main mass.

\section{Northwest Africa 11695 (NWA 11695)}

Algeria

Purchased: 2017

Classification: Lunar meteorite (feldspathic breccia)

History: Purchased from a Moroccan meteorite dealer in 2017. Reportedly found by nomads in Algeria, March 2017.

Physical characteristics: Many identical appearing pieces. Weathered exterior, no fusion crust, saw cut reveals a fragmental breccia with numerous white feldspathic clasts set in a dark-gray ground mass.

Petrography: (C. Agee, UNM) Microprobe examination of a polished mount shows primarily feldspathic clasts and shock melt with lesser amounts of fragmental pyroxene and olivine. Z. Kerensky made 6 thin sections and also identified minor amounts of troilite and Fe-metal.

Geochemistry: (C. Agee, $U N M$ ) Olivine $\mathrm{Fa}_{35.7 \pm 10.2}, \mathrm{Mn} / \mathrm{Fe}=90 \pm 8, \mathrm{n}=15$; pigeonite $\mathrm{Fs}_{30.0 \pm 6.8} \mathrm{Wo}_{10.1 \pm 7.6}, \mathrm{Mn} / \mathrm{Fe}=56 \pm 6, \mathrm{n}=9$; augite $\mathrm{Fs}_{26.7 \pm 15.3} \mathrm{Wo}_{37.0 \pm 7.7}, \mathrm{Mn} / \mathrm{Fe}=54 \pm 11$, $\mathrm{n}=7$; plagioclase $\mathrm{An}_{96.5 \pm 0.5}, \mathrm{n}=7$; Shock melt $(20 \mu \mathrm{m}$ defocused electron beam, proxy for bulk meteorite composition): $\mathrm{SiO}_{2}=43.5 \pm 0.1, \mathrm{TiO}_{2}=0.19 \pm 0.01$, $\mathrm{Al}_{2} \mathrm{O}_{3}=28.9 \pm 0.1, \mathrm{Cr}_{2} \mathrm{O}_{3}=0.11 \pm 0.01, \mathrm{MgO}=6.2 \pm 0.0, \mathrm{FeO}=4.1 \pm 0.1, \mathrm{MnO}=0.06 \pm 0.00$, $\mathrm{CaO}=16.0 \pm 0.1, \mathrm{Na}_{2} \mathrm{O}=0.34 \pm 0.01, \mathrm{~K}_{2} \mathrm{O}=0.02 \pm 0.00, \mathrm{n}=3$ (all wt\%).

Classification: Lunar, feldspathic breccia Specimens: $20.85 \mathrm{~g}$ on deposit at $C S F K$, probe mount at $U N M$, Zsolt Kereszty holds the main mass.

\section{Northwest Africa 11696 (NWA 11696)}

(Northwest Africa) 
Purchased: 2017 Sep

Classification: HED achondrite (Diogenite)

History: Portions of the same disaggregated stone were purchased separately by Habib Naji (540 g) and Rachid Chaoui (2166 g) from a Mauritanian dealer in September 2017. In February 2018 the material obtained by Rachid Chaoui was acquired by Terry Boudreaux and Bob Falls.

Physical characteristics: A disaggregated and quite brittle stone (2706 g); very fresh, mostly dark gray and in part exhibiting a vitreous luster.

Petrography: (A. Irving, UWS and P. Carpenter, WUSL) The fresh specimen is composed predominantly of orthopyroxene with accessory troilite, chromite and minor kamacite. Larger orthopyroxene grains are surrounded by regions of finer grained, crushed grains in a cataclastic texture.

Geochemistry: Orthopyroxene $\left(\mathrm{Fs}_{24.5-25.6} \mathrm{Wo}_{1.9-2.1}, \mathrm{FeO} / \mathrm{MnO}=27-30, \mathrm{~N}=5\right)$.

Classification: Diogenite.

Specimens: $24.8 \mathrm{~g}$ including one polished thin section and one polished mount at $U W B$; remaining material with Mr. T. Boudreaux, Mr. H. Naji and Mr. R. Falls.

\section{Northwest Africa 11697 (NWA 11697)}

(Northwest Africa)

Purchased: 2017 Nov

Classification: Rumuruti chondrite (R3-6)

History: Purchased by Bob Falls in November 2017 from a dealer in Zagora, Morocco.

Petrography: (A. Irving and S. Kuehner, UWS) Breccia composed predominantly of small unequilibrated Type 3 lithic clasts (containing separated chondrules with apparent diameter $560 \pm 350 \mu \mathrm{m}, \mathrm{N}=10$ ) and related debris (including isolated chondrules), plus rare fine grained, recrystallized Type 5 and Type 6 chondrite clasts, in a finer grained matrix. Minerals in addition to olivine and pyroxenes include albitic plagioclase, pentlandite, troilite and minor ilmenite. Metal is absent. No CAI were observed in the studied thin section, but several chondrules were found to contain highly aluminous pyroxenes.

Geochemistry: Olivine in Type 3 clasts and isolated chondrules $\left(\mathrm{Fa}_{1.5-48.4}, \mathrm{~N}=4\right)$, orthopyroxene $\left(\mathrm{Fs}_{1.9-14.5} \mathrm{Wo}_{0.3-2.9}, \mathrm{~N}=3\right)$, augite $\left(\mathrm{Fs}_{10.7} \mathrm{Wo}_{45.4}\right)$, Al-rich subcalcic clinopyroxene in chondrule $\left(\mathrm{Fs}_{3.5} \mathrm{Wo}_{29.2}, \mathrm{Al}_{2} \mathrm{O}_{3}=7.7 \mathrm{wt} . \%, \mathrm{TiO}_{2}=1.0\right.$ wt.\%), Alrich diopside in chondrule $\left(\mathrm{Fs}_{1.8} \mathrm{Wo}_{41.9}, \mathrm{Al}_{2} \mathrm{O}_{3}=7.7\right.$ wt.\%, $\mathrm{TiO}_{2}=0.9$ wt.\%), Alrich low-Ca pyroxene in chondrules $\left(\mathrm{Fs}_{1.9-2.2} \mathrm{Wo}_{2.9-3.2}, \mathrm{Al}_{2} \mathrm{O}_{3}=11.6-13.8\right.$ wt.\%, $\mathrm{TiO}_{2}$ 0.5-0.7 wt.\%; $\mathrm{Fs}_{16.6} \mathrm{Wo}_{3.7}, \mathrm{Al}_{2} \mathrm{O}_{3}=16.0$ wt.\%, $\mathrm{TiO}_{2}=0.4$ wt. $\% ; \mathrm{N}=4$ ), plagioclase $\left(\mathrm{An}_{6.5-10.0} \mathrm{Or}_{2.0-2.9}, \mathrm{~N}=2\right)$.

Classification: R3-6 chondrite. The high content of Mg-Tschermak's component in low-Ca pyroxenes in some chondrules is an anomalous feature of this specimen.

Specimens: $20.5 \mathrm{~g}$ including one polished thin section at $U W B$; remainder with $\mathrm{Mr}$. R. Falls. 


\section{Northwest Africa 11698 (NWA 11698)}

(Northwest Africa)

Purchased: 2017 Feb

Classification: HED achondrite (Eucrite, monomict)

History: Purchased by Bob Falls in February 2017 from a Moroccan dealer at the Tucson Gem and Mineral Show.

Petrography: (A. Irving and S. Kuehner, UWS) Monomict breccia consisting of closely packed, subophitic eucrite clasts in a sparse matrix of related debris.

Minerals are exsolved pigeonite, calcic plagioclase, silica polymorph, ilmenite and troilite.

Geochemistry: Orthopyroxene host $\left(\mathrm{Fs}_{60.8-62.5} \mathrm{Wo}_{3.6-1.8}, \mathrm{FeO} / \mathrm{MnO}=33-35, \mathrm{~N}=3\right)$, clinopyroxene exsolution lamellae $\left(\mathrm{Fs}_{26.3-26.8} \mathrm{Wo}_{44.2-43.7}, \mathrm{FeO} / \mathrm{MnO}=33-35, \mathrm{~N}=2\right)$, plagioclase $\left(\mathrm{An}_{84.5-84.6} \mathrm{Or}_{0.8-0.6}, \mathrm{~N}=2\right)$.

Classification: Eucrite (monomict breccia).

Specimens: $25.5 \mathrm{~g}$ including one polished thin section at $U W B$; remainder with $\mathrm{Mr}$.

R. Falls.

\section{Northwest Africa 11699 (NWA 11699)}

(Northwest Africa)

Purchased: 2017 Oct

Classification: Carbonaceous chondrite (CM2)

History: Purchased by Ben Hoefnagels in October 2017 from a Mauritanian dealer. Petrography: (A. Irving and S. Kuehner, UWS; L. Garvie, ASU) Very small, wellformed granular chondrules (apparent diameter $200 \pm 70 \mu \mathrm{m}, \mathrm{N}=14$ ), mineral fragments and sparse very fine grained CAI are set in a finer grained matrix (deep sepia brown in thin section) containing clay minerals and serpentine (both confirmed by powder X-ray diffraction) plus accessory phosphorian Fe-Ni sulfides. Geochemistry: Olivine $\left(\mathrm{Fa}_{0.4-58.8}, \mathrm{~N}=3\right)$, orthopyroxene $\left(\mathrm{Fs}_{0.6-0.7} \mathrm{Wo}_{1.3-0.8}, \mathrm{~N}=2\right)$, clinopyroxene $\left(\mathrm{Fs}_{0.7-0.8} \mathrm{Wo}_{44.6-40.1}, \mathrm{~N}=2\right)$. Oxygen isotopes $(\mathrm{K}$. Ziegler, $A S U) \delta^{17} \mathrm{O}$ $0.850,-1.728 ; \delta^{18} \mathrm{O} 6.238,5.426 ; \Delta^{17} \mathrm{O}-4.144,-4.593$ (all per mil).

Classification: Carbonaceous chondrite (CM2). The presence of phosphoran $\mathrm{Fe}-\mathrm{Ni}$ sulfides in this specimen is notable and represents only the second such confirmation among northwest African specimens. Phosphorian Fe-Ni sulfides were previously described in CM chondrites by Nazarov et al. (2009) and in CMrelated clasts within polymict eucrite NWA 5232 by Kuehner et al. (LPSC 40, \#2314, 2009).

Specimens: $15.2 \mathrm{~g}$ including one polished thin section at $U W B$; main mass with Mr. B. Hoefnagels.

\section{Northwest Africa 11700 (NWA 11700)}

(Northwest Africa)

Purchased: 2017 Nov

Classification: Ordinary chondrite (H4) 
History: Purchased in Temara, Morocco by Adam Aaronson in November 2017. Petrography: (A. Irving and S. Kuehner, UWS) Closely packed, well-formed, relatively small chondrules are set in a recrystallized matrix containing altered kamacite and merrillite.

Geochemistry: Olivine $\left(\mathrm{Fa}_{18.8-18.9}, \mathrm{~N}=3\right)$, orthopyroxene $\left(\mathrm{Fs}_{15.5-17.0} \mathrm{Wo}_{0.5-1.2}, \mathrm{~N}=\right.$ 3), clinopyroxene $\left(\mathrm{Fs}_{7.5-9.4} \mathrm{Wo}_{40.5-42.4}, \mathrm{~N}=2\right)$.

Classification: Ordinary chondrite (H4).

Specimens: $29.2 \mathrm{~g}$ including one polished thin section at $U W B$; main mass with Aaronson.

\section{Northwest Africa 11701 (NWA 11701)}

(Northwest Africa)

Purchased: 2017 Sep

Classification: HED achondrite (Eucrite, polymict)

History: Purchased by Bob Falls in September 2017 from a Moroccan dealer at the Denver Show.

Petrography: (A. Irving and S. Kuehner, UWS) Breccia composed of intersertal to gabbroic eucrite clasts plus $\sim 5$ vol.\% diogenitic orthopyroxene grains in a quenchtextured melt matrix (partly vesicular with minor calcite infillings). Eucrite clasts consist of exsolved pigeonite and calcic plagioclase (polycrystalline) with accessory silica polymorph, ilmenite, fayalite, troilite, Ni-poor metal and zircon. Geochemistry: Orthopyroxene host $\left(\mathrm{Fs}_{61.4-61.5} \mathrm{Wo}_{2.8-2.0}, \mathrm{FeO} / \mathrm{MnO}=32, \mathrm{~N}=3\right)$, clinopyroxene exsolution lamellae $\left(\mathrm{Fs}_{27.0-27.3} \mathrm{Wo}_{43.4-41.4}, \mathrm{FeO} / \mathrm{MnO}=32-33, \mathrm{~N}=2\right)$, diogenitic orthopyroxene $\left(\mathrm{Fs}_{30.8-32.6} \mathrm{Wo}_{5.4-3.1}, \mathrm{FeO} / \mathrm{MnO}=31-34, \mathrm{~N}=2\right)$, fayalite $\left(\mathrm{Fa}_{84.5-85.6}, \mathrm{FeO} / \mathrm{MnO}=38, \mathrm{~N}=2\right)$, plagioclase $\left(\mathrm{An}_{78.7-82.9} \mathrm{Or}_{1.3-0.6}, \mathrm{~N}=2\right)$.

Classification: Eucrite (melt-matrix polymict breccia).

Specimens: $25.8 \mathrm{~g}$ including one polished thin section at $U W B$; remainder with $\mathrm{Mr}$. R. Falls.

\section{Northwest Africa 11702 (NWA 11702) \\ Mauritania \\ Purchased: 2017 Sep}

Classification: Ordinary chondrite (LL6)

History: Purchased by Jason Bliss in September 2017 from a Mauritanian dealer. Petrography: (A. Irving and S. Kuehner, UWS) Breccia consisting of closely packed clasts with rare remnant chondrules in a dominant recrystallized, poikiloblastic matrix containing stained metal and chlorapatite.

Geochemistry: Olivine $\left(\mathrm{Fa}_{29.7-30.0}, \mathrm{~N}=3\right)$, orthopyroxene $\left(\mathrm{Fs}_{22.9-23.6} \mathrm{Wo}_{2.7-3.9}, \mathrm{~N}=\right.$ $3)$, clinopyroxene $\left(\mathrm{Fs}_{10.6-11.4} \mathrm{Wo}_{42.5-42.2}, \mathrm{~N}=2\right)$.

Classification: Ordinary chondrite (LL6 breccia).

Specimens: $8.4 \mathrm{~g}$ including one polished thin section at $U W B$; remainder with $\mathrm{Mr}$. J. Bliss. 


\section{Northwest Africa 11703 (NWA 11703)}

(Northwest Africa)

Purchased: 2015 Apr

Classification: Lunar meteorite (gabbro)

History: Purchased by Darryl Pitt in April 2015 from a dealer in Erfoud, Morocco. Physical characteristics: A single pale greenish stone (5309.1 g) with thin crosscutting shock veinlets.

Petrography: (A. Irving and S. Kuehner, UWS) Medium grained (grainsize up to $1.4 \mathrm{~mm}$ ) with cumulate texture. Major minerals are olivine, pigeonite, subcalcic augite and calcic plagioclase (predominantly converted to maskelynite with minor birefringent domains); accessory phases include ilmenite, taenite, troilite, Mgmerrillite and baddeleyite. Shock veinlets contain dark glass.

Geochemistry: Olivine $\left(\mathrm{Fa}_{32.1-32.4}, \mathrm{FeO} / \mathrm{MnO}=91-97, \mathrm{~N}=3\right)$, pigeonite $\left(\mathrm{Fs}_{26.0} \mathrm{Wo}_{9.7} ; \mathrm{Fs}_{27.7} \mathrm{Wo}_{5.7} ; \mathrm{FeO} / \mathrm{MnO}=47-60 ; \mathrm{N}=2\right)$, subcalcic augite $\left(\mathrm{Fs}_{16.4-}\right.$ $\left.{ }_{17.5} \mathrm{Wo}_{34.9-32.3}, \mathrm{FeO} / \mathrm{MnO}=46-52, \mathrm{~N}=2\right)$, plagioclase $\left(\mathrm{An}_{89.7-91.2} \mathrm{Or}_{0.8-1.1}, \mathrm{~N}=2\right)$. Classification: Lunar (gabbro). This specimen is texturally and mineralogically identical to NWA 2977, NWA 6950, NWA 8127, and to the olivine gabbro clasts in NWA 773, NWA 2700, NWA 2727 and NWA 3333, and evidently is paired with those lithologies.

Specimens: $20.1 \mathrm{~g}$ including one polished thin section at $U W B$; remainder held by DPitt.

\section{Northwest Africa 11704 (NWA 11704)}

Mauritania

Purchased: 2018 Jan

Classification: HED achondrite (Eucrite, polymict)

History: A batch of about 50 stones with similar appearance found together in Mauritania was purchased by John Higgins in January 2018 from a Mauritanian dealer.

Petrography: (A. Irving and S. Kuehner, UWS) Breccia consisting predominantly of gabbroic eucrite clasts, some ophitic eucrite clasts and sparse clasts of feldspathic diogenite in a finer grained matrix. Minerals include exsolved pigeonite, calcic plagioclase (exhibiting undulose to polycrystalline microstructure), silica polymorph, ilmenite, Ti-chromite, Ti-poor chromite, troilite and minor barite. Feldspathic diogenite clasts consist mainly of relatively ferroan orthopyroxene with subordinate anorthite and minor clinopyroxene.

Geochemistry: Orthopyroxene host $\left(\mathrm{Fs}_{58.5} \mathrm{Wo}_{4.6}, \mathrm{FeO} / \mathrm{MnO}=35\right)$, clinopyroxene exsolution lamella $\left(\mathrm{Fs}_{28.1} \mathrm{Wo}_{41.0}, \mathrm{FeO} / \mathrm{MnO}=34\right)$, orthopyroxene host $\left(\mathrm{Fs}_{44.5} \mathrm{Wo}_{2.2}\right.$, $\mathrm{FeO} / \mathrm{MnO}=30)$, clinopyroxene exsolution lamella $\left(\mathrm{Fs}_{17.8} \mathrm{Wo}_{43.8}, \mathrm{FeO} / \mathrm{MnO}=25\right)$, plagioclase $\left(\mathrm{An}_{86.6} \mathrm{Or}_{0.8}\right)$. Minerals in feldspathic diogenite clast: orthopyroxene $\left(\mathrm{Fs}_{33.2-33.8} \mathrm{Wo}_{1.7-2.4}, \mathrm{FeO} / \mathrm{MnO}=29-32, \mathrm{~N}=2\right)$, clinopyroxene $\left(\mathrm{Fs}_{10.8} \mathrm{Wo}_{45.8}\right.$, $\mathrm{FeO} / \mathrm{MnO}=21)$, anorthite $\left(\mathrm{An}_{95.4} \mathrm{Or}_{0.2}\right)$. 
Classification: Eucrite (polymict breccia).

Specimens: $26.6 \mathrm{~g}$ including one polished thin section at $U W B$; remainder with Mr. J. Higgins.

\section{Northwest Africa 11705 (NWA 11705)}

(Northwest Africa)

Purchased: 2017 Mar

Classification: Ordinary chondrite $(\mathrm{H} 4 / 5)$

History: Purchased by Bob Falls in March 2017 from a dealer in Zagora, Morocco. Petrography: (A. Irving and S. Kuehner, UWS) Very fresh specimen containing some well-formed, relatively small chondrules in a recrystallized matrix containing merrillite, troilite, kamacite and taenite.

Geochemistry: Olivine $\left(\mathrm{Fa}_{15.6-15.7}, \mathrm{~N}=3\right)$, orthopyroxene $\left(\mathrm{Fs}_{14.0-14.1} \mathrm{Wo}_{0.6-0.7}, \mathrm{~N}=\right.$ 3), clinopyroxene $\left(\mathrm{Fs}_{9.9} \mathrm{Wo}_{33.9} ; \mathrm{Fs}_{7.9} \mathrm{Wo}_{47.2} ; \mathrm{N}=2\right)$.

Classification: Ordinary chondrite (H4/5). The mafic silicates in this specimen are unusually magnesian in comparison to those in most $\mathrm{H}$ chondrites.

Specimens: $26.7 \mathrm{~g}$ including one polished thin section at $U W B$; remainder with $\mathrm{Mr}$. R. Falls.

\section{Northwest Africa 11706 (NWA 11706) \\ Morocco \\ Purchased: Sept 2016 \\ Classification: Carbonaceous chondrite (CK5)}

History: A single stone weighing $116 \mathrm{~g}$ was purchased by Hichame Mimaghador at the Erfoud market, Morocco, in 2016.

Physical characteristics: A single stone, almost completely covered by a fusion crust with several contraction cracks.

Petrography: (V. Moggi Cecchi, G. Pratesi, S. Caporali, UniFi): The thin section displays a texture consisting of $\mathrm{mm}$-sized granular olivine-rich chondrules containing magnetite blebs well integrated in a medium grained olivine-rich matrix. A fine grained olivine-augite-plagioclase aggregate $500 \times 250 \mu \mathrm{m}$ wide is visible. Main minerals are olivine, augite, intermediate plagioclase. Opaques are mainly pentlandite and $\mathrm{Cr}$-rich magnetite, with minor altered kamacite.

Geochemistry: (V. Moggi Cecchi, G. Pratesi, S. Caporali, UniFi) Olivine $\left(\mathrm{Fa}_{34.7 \pm 1.3}, \mathrm{Fe} / \mathrm{Mn}=142.0 \pm 8.1, \mathrm{~N}=7\right)$, augite $\left(\mathrm{Fs}_{13.5 \pm 0.4} \mathrm{Wo}_{49.3 \pm 1.3}, \mathrm{Al}_{2} \mathrm{O}_{3}=1.28\right.$ Wt.\%, $\mathrm{N}=6$ ). Feldspar $\mathrm{An}_{55.0} \mathrm{Ab}_{43.9} \mathrm{Or}_{1.1 .}$. Magnetite contains $1.7 \pm 0.1 \mathrm{wt} . \% \mathrm{Cr}_{2} \mathrm{O}_{3}$, 0.26 wt. $\% \mathrm{Al}_{2} \mathrm{O}_{3}, 0.23$ wt.\% $\mathrm{NiO}(\mathrm{N}=3)$. Oxygen isotopes: (I. Franchi, R.

Greenwood, $O U) \delta^{17} \mathrm{O}=-5.76 \%, \delta^{18} \mathrm{O}=-1.91 \%, \Delta^{17} \mathrm{O}=-4.76 \%$.

Classification: Carbonaceous chondrite (CK5). Moderate weathering. Specimens: A total of $19.6 \mathrm{~g}$ specimen, a block $(0.4 \mathrm{~g})$ and a thin section are on deposit at MSN-Fi. $96 \mathrm{~g}$ with Mimaghador. 


\section{Northwest Africa 11707 (NWA 11707)}

Morocco

Purchased: Feb 2016

Classification: Carbonaceous chondrite (CK4)

History: A single stone weighing $470 \mathrm{~g}$ was purchased by Hichame Mimaghador at the Erfoud market, Morocco, in 2016.

Physical characteristics: A single stone covered by fusion crust with contraction cracks.

Petrography: (V. Moggi Cecchi, G. Pratesi, S. Caporali, UniFi): A texture consisting of well-formed, mm-sized granular chondrules containing magnetite can be observed. Chondrules are surrounded by a medium grained, recrystallized matrix with disseminated opaque phases. Main minerals are olivine, orthopyroxene, augite, albitic plagioclase. Opaque phases are mainly represented by troilite and $\mathrm{Cr}$ rich magnetite, with minor kamacite.

Geochemistry: (V. Moggi Cecchi, G. Pratesi, S. Caporali, UniFi): Olivine $\left(\mathrm{Fa}_{28.0 \pm 1.2}, \mathrm{Fe} / \mathrm{Mn}=93.6 \pm 10.1, \mathrm{~N}=8\right)$, orthopyroxene $\left(\mathrm{Fs}_{23.7 \pm 1.0} \mathrm{Wo}_{1.7 \pm 0.2}, \mathrm{Al}_{2} \mathrm{O}_{3}=\right.$ $2.51 \mathrm{Wt} . \%, \mathrm{~N}=4)$; augite $\left(\mathrm{Fs}_{8.8 \pm 1.5} \mathrm{Wo}_{44.1 \pm 1.8}, \mathrm{Al}_{2} \mathrm{O}_{3}=0.51 \mathrm{Wt} \% \%, \mathrm{~N}=4\right)$. Feldspar $\mathrm{An}_{17.2} \mathrm{Or}_{5.3}$. Magnetite contains $2.7 \pm 0.1$ wt. $\% \mathrm{Cr}_{2} \mathrm{O}_{3}, 0.67$ wt. $\% \mathrm{Al}_{2} \mathrm{O}_{3}, 0.55$ wt. $\%$ $\mathrm{NiO}(\mathrm{N}=3)$; Oxygen isotopes: (I. Franchi, R. Greenwood, $O U) \delta^{17} \mathrm{O}=-3.58 \%$, $\delta^{18} \mathrm{O}=0.93 \%$ o, $\Delta^{17} \mathrm{O}=-4.06 \%$

Classification: Carbonaceous chondrite (CK4). Moderate weathering.

Specimens: A total of $20.7 \mathrm{~g}$ specimen is on deposit at MSN-Fi. The main mass is with owner.

\section{Northwest Africa 11708 (NWA 11708) \\ Morocco \\ Purchased: Feb 2016 \\ Classification: Carbonaceous chondrite (CK3)}

History: A stone weighing $1104 \mathrm{~g}$ was purchased by Hichame Mimaghador at the Erfoud market in 2016.

Physical characteristics: A single stone covered by fusion crust.

Petrography: (V. Moggi Cecchi, G. Pratesi, S. Caporali, UniFi): The thin section displays separated, well-formed chondrules (ranging in diameter from 300 to 700 $\mu \mathrm{m}$, mean value $350 \mu \mathrm{m}$ ) of different types, in a fine-grained matrix consisting of Fe-rich olivine and orthopyroxene, coarse grained $\mathrm{Fe}$-rich olivine fragments and scattered opaque phases. Chondrule types are mainly PO, with minor POP and PP types. Main minerals are olivine, orthopyroxene, Ca-rich plagioclase and clinopyroxene. Opaques are mainly consisting of troilite and Cr-magnetite, with minor altered kamacite.

Geochemistry: (V. Moggi Cecchi, G. Pratesi, S. Caporali, UniFi) Olivine in PO and POP chondrules $\left(\mathrm{Fa}_{1.7 \pm 0.2}, \mathrm{Fe} / \mathrm{Mn}=11.4 \pm 1.1, \mathrm{~N}=6\right)$; Olivine in mineral fragments $\left(\mathrm{Fa}_{38.9 \pm 5.3}, \mathrm{Fe} / \mathrm{Mn}=101.7 \pm 10.1, \mathrm{~N}=10\right)$, Orthopyroxene in POP chondrules $\left(\mathrm{Fs}_{2.5 \pm 0.2} \mathrm{Wo}_{2.1 \pm 0.1}, \mathrm{Fe} / \mathrm{Mn}=11.5 \pm 1.2, \mathrm{~N}=15\right)$; Matrix orthopyroxene $\left(\mathrm{Fs}_{43.5 \pm 3.5} \mathrm{Wo}_{1.2 \pm 0.2}, \mathrm{Fe} / \mathrm{Mn}=105.1 \pm 1.2, \mathrm{~N}=5\right)$; fassaite $\left(\mathrm{Fs}_{4.4 \pm 0.4} \mathrm{En}_{56.6 \pm 0.3} \mathrm{Wo}_{39.0 \pm 0.9}\right.$, 
$\mathrm{Al}_{2} \mathrm{O}_{3}=6.0 \mathrm{Wt} . \%, \mathrm{~N}=5$ ). Feldspar $\mathrm{An}_{95.7} \mathrm{Or}_{0.1}$. Oxygen isotopes: (I. Franchi, $\mathrm{R}$. Greenwood, $O U) \delta^{17} \mathrm{O}=-4.75 \%$ o, $\delta^{18} \mathrm{O}=-1.14 \%$, $\Delta^{17} \mathrm{O}=-4.15 \%$.

Classification: Carbonaceous chondrite (CK3). Moderate weathering.

Specimens: A total of $24 \mathrm{~g}$ specimen is on deposit at $M S N-F I$. The main mass is with owner.

\section{Northwest Africa 11709 (NWA 11709) \\ Morocco \\ Purchased: June 2016 \\ Classification: HED achondrite (Eucrite)}

History: A fragmented stone consisting of many pieces totally weighing $180 \mathrm{~g}$ was found by an anonymous person in Western Sahara and purchased 2016 by Enzo Compagnucci.

Physical characteristics: Few fragments are partly covered by fusion crust. Petrography: (V. Moggi Cecchi, G. Pratesi, S. Caporali, UniFi): The thin section reveals a brecciated texture consisting of lithic clasts in a fine grained matrix. Lithic clasts consists of exsolved orthopyroxene crystals with fine pigeonite exsolution lamellae, 4-8 $\mu \mathrm{m}$ in width, augite, calcic plagioclase, ilmenite, chromite, troilite and FeNi metal.

Geochemistry: (V. Moggi Cecchi, G. Pratesi, S. Caporali, UniFi) Eucritic clasts: orthopyroxene host $\left(\mathrm{Fs}_{36.6 \pm 1.1} \mathrm{En}_{60.2 \pm 1.3} \mathrm{Wo}_{3.3 \pm 0.2}, \mathrm{FeO} / \mathrm{MnO}=29.5 ? 0.3, \mathrm{n}=6\right)$; augite $\left(\mathrm{Fs}_{22.6 \pm 1.1} \mathrm{En}_{33.0 \pm 1.2} \mathrm{Wo}_{44.4 \pm 1.4} ; \mathrm{n}=6 ; \mathrm{Fe} / \mathrm{Mn}=28.3 \pm 0.3\right)$; pigeonite exsolution lamellae in orthopyroxene $\left(\mathrm{Fs}_{52.6 \pm 1.2}, \mathrm{En}_{44.4 \pm 1.1} \mathrm{Wo}_{3.0 \pm 0.3} ; \mathrm{n}=5 ; \mathrm{Fe} / \mathrm{Mn}=31.2 \pm 0.3\right)$; Plagioclase An ${ }_{91.7 \pm 1.3} \mathrm{Or}_{0.3 \pm 0.1}, \mathrm{n}=4$;

Classification: Eucrite with medium degree of shock and medium degree of weathering.

Specimens: A total of $20.2 \mathrm{~g}$ specimen and one thin section is on deposit at MSNFi. Cavaglià owns the main mass, now weighing $160 \mathrm{~g}$.

\section{Northwest Africa 11710 (NWA 11710)}

(Northwest Africa)

Purchased: 2017

Classification: HED achondrite (Eucrite)

History: The meteorite was purchased from a Morrocan meteorite dealer at the mineral fair in Munich, Germany.

Physical characteristics: Greyish individual partly covered with fusion crust. Petrography: The meteorite is a medium-grained unbrecciated igneous rock predominantly composed of 100-600 $\mu \mathrm{m}$ sized exsolved pyroxene and calcic plagioclase. Minor phases include silica, ilmenite, troilite, and metallic iron. Geochemistry: low-Ca pyroxene $\mathrm{Fs}_{58.3 \pm 1.3} \mathrm{Wo}_{3.9 \pm 1.6}\left(\mathrm{Fs}_{55.9-59.5} \mathrm{Wo}_{2.1-6.2}, \mathrm{n}=11\right.$, $\mathrm{FeO} / \mathrm{MnO}=29-30)$; Ca-pyroxene: $\mathrm{Fs}_{29.6 \pm 3.1} \mathrm{Wo}_{39.4 \pm 3.9}\left(\mathrm{Fs}_{27.1-38.8} \mathrm{Wo}_{28.1-42.2}, \mathrm{n}=11\right.$, $\mathrm{FeO} / \mathrm{MnO}=27-32)$; calcic plagioclase: $\mathrm{An}_{89.8 \pm 0.5}\left(\mathrm{An}_{88.6-90.5}, \mathrm{n}=13\right)$ 


\title{
Northwest Africa 11712 (NWA 11712)
}

(Northwest Africa)

Purchased: 2017

Classification: HED achondrite (Howardite)

History: The meteorite was purchased from a local meteorite dealer in Morocco.

Physical characteristics: Dark-grayish individual lacking any fusion crust.

Petrography: The meteorite is a breccia composed of abundant up to $1.3 \mathrm{~mm}$ mineral and less abundant basaltic clasts set into a finer grained clastic matrix. Predominant minerals are exsolved pyroxene, diogenetic orthopyroxene (30-35\%) and calcic plagioclase. Accessory phases include silica, chromite, and metallic Fe. The meteorite contains abundant shock melt veins and pockets.

Geochemistry: diogenetic low-Ca pyroxene $\mathrm{Fs}_{33.6 \pm 0.5} \mathrm{Wo}_{2.3 \pm 0.2}\left(\mathrm{Fs}_{33.2-34.6} \mathrm{Wo}_{2.0-2.8}\right.$, $\mathrm{n}=16, \mathrm{FeO} / \mathrm{MnO}=25-31$ ); low-Ca pyroxene host to augite exsolution lamellae: $\mathrm{Fs}_{58.6 \pm 0.7} \mathrm{Wo}_{4.4 \pm 0.7}\left(\mathrm{Fs}_{57.5-59.7} \mathrm{Wo}_{3.2-5.7}, \mathrm{n}=10, \mathrm{FeO} / \mathrm{MnO}=32-35\right)$; Ca-pyroxene: $\mathrm{Fs}_{28.6 \pm 2.0} \mathrm{Wo}_{40.4 \pm 1.9}\left(\mathrm{Fs}_{25.8-31.9} \mathrm{Wo}_{37.4-42.7}, \mathrm{n}=6, \mathrm{FeO} / \mathrm{MnO}=31-38\right)$; calcic plagioclase: $\mathrm{An}_{89.4 \pm 3.1}\left(\mathrm{An}_{82.6-92.9}, \mathrm{n}=17\right)$

\section{Northwest Africa 11720 (NWA 11720)}

(Northwest Africa)

Purchased: 2017 Jan

Classification: Pallasite (Main group, anomalous)

History: A batch of small metal-bearing stones $(230 \mathrm{~g})$ purported to have been found at the same site as NWA 10023 and NWA 10252 was purchased by Mike Bandli in January 2017 from a Moroccan dealer at the Tucson Gem and Mineral Show.

Petrography: (A. Irving and S. Kuehner, UWS) Coarse grained aggregate of olivine and stained plessitic metal with rare accessory schreibersite.

Geochemistry: Olivine $\left(\mathrm{Fa}_{13.3-13.7}, \mathrm{FeO} / \mathrm{MnO}=47-53, \mathrm{~N}=3\right)$.

Classification: Pallasite PMG-an. Likely paired with NWA 10023 and NWA 10252. Anomalous designation based on the presence of the same plessitic metal observed in the larger specimens, plus the fact that these stones were found with the previous specimens and purchased from the same Moroccan dealer.

Specimens: $20.2 \mathrm{~g}$ including one polished endcut at $U W B$; remainder with Mr. M. Bandli.

\author{
Northwest Africa 11721 (NWA 11721) \\ (Northwest Africa) \\ Purchased: 2017 Dec \\ Classification: Rumuruti chondrite (R3)
}


History: A batch of about 10 stones with similar appearance (total $5345 \mathrm{~g}$, the largest stone $2670 \mathrm{~g}$ ) was purchased by Jason Phillips in December 2017 from a Mauritanian dealer.

Petrography: (A. Irving and S. Kuehner, UWS) Breccia composed of angular clasts in a matrix of related debris. Clasts contain well-formed, separated unequilibrated chondrules (apparent diameter $310 \pm 200 \mu \mathrm{m}, \mathrm{N}=15$ ) set in a finer grained metal-free matrix. Accessory minerals are sodic plagioclase, troilite, Tichromite and chlorapatite.

Geochemistry: Olivine $\left(\mathrm{Fa}_{7.0-57.4}, \mathrm{~N}=5\right)$, orthopyroxene $\left(\mathrm{Fs}_{0.7-8.1} \mathrm{Wo}_{0.1}, \mathrm{~N}=2\right)$, pigeonite $\left(\mathrm{Fs}_{32.7} \mathrm{Wo}_{6.6}\right)$, augite $\left(\mathrm{Fs}_{11.7} \mathrm{Wo}_{44.7}\right)$. Magnetic susceptibility $\log \chi\left(\times 10^{-}\right.$ $\left.{ }^{9} \mathrm{~m}^{3} / \mathrm{kg}\right)=3.43$.

Classification: R3 chondrite (breccia).

Specimens: $25.3 \mathrm{~g}$ including one polished thin section at $U W B$; remainder with $\mathrm{Mr}$. J. Phillips.

\section{Northwest Africa 11722 (NWA 11722)}

(Northwest Africa)

Purchased: 2005

Classification: Ordinary chondrite (L3)

History: Purchased by an anonymous collector from a dealer at the Munich Show in 2005, subsequently acquired by the Hollis Collection and then donated to PSF. Petrography: (A. Irving and S. Kuehner, UWS) Well-formed chondrules (apparent diameter $510 \pm 370 \mu \mathrm{m}, \mathrm{N}=15$ ) are set in a finer grained matrix containing altered kamacite, merrillite and taenite.

Geochemistry: Olivine $\left(\mathrm{Fa}_{6.3-43.5}, \mathrm{Cr}_{2} \mathrm{O}_{3}\right.$ in ferroan examples $0.03-0.15$ wt.\%, mean $0.08 \pm 0.05$ wt. $\%, \mathrm{~N}=8)$, orthopyroxene $\left(\mathrm{Fs}_{2.1-19.5} \mathrm{Wo}_{0.3-0.4}, \mathrm{~N}=3\right)$, pigeonite $\left(\mathrm{Fs}_{3.6} \mathrm{Wo}_{17.1}\right)$, augite $\left(\mathrm{Fs}_{7.9} \mathrm{Wo}_{44.7}\right)$.

Classification: Ordinary chondrite (L3).

Specimens: The entire specimen including one polished thin section is at PSF.

\section{Northwest Africa 11723 (NWA 11723) \\ (Northwest Africa) \\ Purchased: 2005 \\ Classification: Ordinary chondrite (L5)}

History: Purchased by an anonymous collector from a dealer at the Munich Show in 2005, subsequently acquired by the Hollis Collection and then donated to PSF. Petrography: (A. Irving and S. Kuehner, UWS) Sparse chondrules occur in a recrystallized matrix containing altered metal.

Geochemistry: Olivine $\left(\mathrm{Fa}_{25.5-25.9}, \mathrm{~N}=3\right)$, orthopyroxene $\left(\mathrm{Fs}_{20.9-21.2} \mathrm{Wo}_{1.8-1.5}, \mathrm{~N}=\right.$ 2), clinopyroxene $\left(\mathrm{Fs}_{6.8-8.1} \mathrm{Wo}_{46.2-44.3}, \mathrm{~N}=2\right)$.

Classification: Ordinary chondrite (L5).

Specimens: The entire specimen including one polished thin section is at PSF. 


\section{Northwest Africa 11724 (NWA 11724)}

(Northwest Africa)

Purchased: 2005

Classification: Ordinary chondrite (L6)

History: Purchased by an anonymous collector from a dealer at the Munich Show in 2005, subsequently acquired by the Hollis Collection and then donated to PSF. Petrography: (A. Irving and S. Kuehner, UWS) Mostly recrystallized with rare remnant chondrules; accessory chlorapatite and merrillite are present. Silicates are extensively stained by weathering products of primary metal and there are crosscutting Fe hydroxide veinlets.

Geochemistry: Olivine $\left(\mathrm{Fa}_{25.2-25.3}, \mathrm{~N}=3\right)$, orthopyroxene $\left(\mathrm{Fs}_{21.6-21.8} \mathrm{Wo}_{1.7-1.1}, \mathrm{~N}=\right.$ 2), clinopyroxene $\left(\mathrm{Fs}_{8.5-12.7} \mathrm{Wo}_{43.8-41.8}, \mathrm{~N}=2\right)$.

Classification: Ordinary chondrite (L6).

Specimens: The entire specimen including one polished thin section is at PSF.

\section{Northwest Africa 11725 (NWA 11725)}

(Northwest Africa)

Purchased: 2005

Classification: Ordinary chondrite (L4)

History: Purchased by an anonymous collector from a dealer at the Munich Show in 2005, subsequently acquired by the Hollis Collection and then donated to PSF. Petrography: (A. Irving and S. Kuehner, UWS) Well-formed, closely packed chondrules are set in a finer grained matrix containing stained metal.

Geochemistry: Olivine $\left(\mathrm{Fa}_{24.9-25.0}, \mathrm{~N}=3\right)$, orthopyroxene $\left(\mathrm{Fs}_{20 . .5-20.6} \mathrm{Wo}_{1.6-4.0}, \mathrm{~N}=\right.$ 2), clinopyroxene $\left(\mathrm{Fs}_{8.6-9.3} \mathrm{Wo}_{44.2-42.7}, \mathrm{~N}=2\right)$.

Classification: Ordinary chondrite (L4).

Specimens: The entire specimen including one polished thin section is at PSF.

\section{Northwest Africa 11726 (NWA 11726)}

(Northwest Africa)

Purchased: 2005

Classification: Ordinary chondrite (L4/5)

History: Purchased by an anonymous collector from a dealer at the Munich Show in 2005, subsequently acquired by the Hollis Collection and then donated to PSF.

Petrography: (A. Irving and S. Kuehner, UWS) Some well-formed chondrules are present within a recrystallized matrix containing stained metal.

Geochemistry: Olivine $\left(\mathrm{Fa}_{24.5-24.7}, \mathrm{~N}=3\right)$, orthopyroxene $\left(\mathrm{Fs}_{20.5-21.8} \mathrm{Wo}_{1.6-1.5}, \mathrm{~N}=\right.$ 2), clinopyroxene $\left(\mathrm{Fs}_{7.9-8.1} \mathrm{Wo}_{44.5-45.1}, \mathrm{~N}=2\right)$.

Classification: Ordinary chondrite (L4/5).

Specimens: The entire specimen including one polished thin section is at PSF. 


\section{Northwest Africa 11727 (NWA 11727)}

(Northwest Africa)

Purchased: 2005

Classification: Ordinary chondrite (L5)

History: Purchased by an anonymous collector from a dealer at the Munich Show in 2005, subsequently acquired by the Hollis Collection and then donated to PSF. Petrography: (A. Irving and S. Kuehner, UWS) Sparse chondrules occur in a recrystallized matrix containing altered metal.

Geochemistry: Olivine $\left(\mathrm{Fa}_{24.7-25.1}, \mathrm{~N}=3\right)$, orthopyroxene $\left(\mathrm{Fs}_{20.9-21.0} \mathrm{Wo}_{1.3-1.6}, \mathrm{~N}=\right.$ 2), clinopyroxene $\left(\mathrm{Fs}_{7.2-7.9} \mathrm{Wo}_{45.2-44.7}, \mathrm{~N}=2\right)$.

Classification: Ordinary chondrite (L5).

Specimens: The entire specimen including one polished thin section is at PSF.

\section{Northwest Africa 11728 (NWA 11728)}

(Northwest Africa)

Purchased: 2005

Classification: Ordinary chondrite (L4)

History: Purchased by an anonymous collector from a dealer at the Munich Show in 2005, subsequently acquired by the Hollis Collection and then donated to PSF. Petrography: (A. Irving and S. Kuehner, UWS) Well-formed, closely-packed chondrules are set in a finer grained matrix containing stained metal.

Geochemistry: Olivine $\left(\mathrm{Fa}_{24.5-24.9}, \mathrm{~N}=3\right)$, orthopyroxene $\left(\mathrm{Fs}_{19.4-20.5} \mathrm{Wo}_{0.5-1.2}, \mathrm{~N}=\right.$ 2), subcalcic augite $\left(\mathrm{Fs}_{21.6} \mathrm{Wo}_{29.1}\right)$, augite $\left(\mathrm{Fs}_{9.6} \mathrm{Wo}_{37.1}\right)$.

Classification: Ordinary chondrite (L4).

Specimens: The entire specimen including one polished thin section is at PSF.

\section{Northwest Africa 11729 (NWA 11729)}

Mauritania

Purchased: 2017 Dec

Classification: HED achondrite (Eucrite, anomalous)

History: Purchased by Rachid Chaoui in December 2017 from a Mauritanian dealer and subsequently acquired by Bob Falls.

Physical characteristics: A single brownish stone (345 g) mostly coated by black fusion crust. The interior exhibits a breccia texture with beige clasts stained light orange by terrestrial weathering.

Petrography: (A. Irving and S. Kuehner, UWS) Breccia composed of closely packed microgabbroic clasts plus some genomict breccia clasts within a sparse matrix of related debris. Minerals are exsolved ferroan pigeonite, calcic plagioclase, silica polymorph, ilmenite Ti-chromite, fayalitic olivine and minor zircon. Terrestrial weathering has resulted in extensive alteration of primary troilite to Fe hydroxides and some cross-cutting hydroxide veinlets. 
Geochemistry: Orthopyroxene host $\left(\mathrm{Fs}_{69.6} \mathrm{Wo}_{1.7}, \mathrm{FeO} / \mathrm{MnO}=29\right)$, clinopyroxene exsolution lamella $\left(\mathrm{Fs}_{33.2} \mathrm{Wo}_{42.1}, \mathrm{FeO} / \mathrm{MnO}=30\right)$, clinopyroxene host $\left(\mathrm{Fs}_{30.9} \mathrm{Wo}_{43.5}\right.$, $\mathrm{FeO} / \mathrm{MnO}=32)$, orthopyroxene exsolution lamella $\left(\mathrm{Fs}_{69.5} \mathrm{Wo}_{1.9}, \mathrm{FeO} / \mathrm{MnO}=36\right)$, fayalite $\left(\mathrm{Fa}_{73.9-74.1}, \mathrm{FeO} / \mathrm{MnO}=47, \mathrm{~N}=2\right)$, plagioclase $\left(\mathrm{An}_{82.3-84.3} \mathrm{Or}_{0.7-0.5}, \mathrm{~N}=2\right)$. Oxygen isotopes (K. Ziegler, UNM): analyses of acid-washed subsamples by laser fluorination gave, respectively, d17O 2.680,3.062, 2.301; d18O 5.540, 6.312, 4.934; D17O -0.245, $-0.271,-0.304$ per mil.

Classification: Eucrite (anomalous monomict breccia, microgabbroic, highly ferroan). The pyroxenes in this specimen are much more ferroan than in any other known eucrites: ferrosilite contents in orthopyroxene are almost 70 mol.\%. The oxygen isotopic composition is also unusual with a higher $\mathrm{d} 18 \mathrm{O}$ than other main group eucrites.

Specimens: $22.5 \mathrm{~g}$ including one polished thin section at $U W B$; remainder with $\mathrm{Mr}$. R. Falls.

\section{Northwest Africa 11730 (NWA 11730)}

(Northwest Africa)

Purchased: 2017

Classification: Ordinary chondrite (LL6, melt breccia)

History: Purchased by Larry Atkins from Morocco in 2017.

Physical characteristics: Single stone, no fusion crust. A saw cut reveals a graycolored breccia with pervasive dark shock melt veins and pockets.

Petrography: (C. Agee, UNM) This meteorite is a breccia of LL6 chondrite clasts set in a network of shock melt veins. Faint chondrules were observed, most metal and sulfide is in the form of finely dispersed blebs.

Geochemistry: (C. Agee, $U N M$ ) Olivine $\mathrm{Fa}_{32.7 \pm 0.2}, \mathrm{Fe} / \mathrm{Mn}=66 \pm 2, \mathrm{n}=8$; low $\mathrm{Ca}$ pyroxene $\mathrm{Fs}_{26.3 \pm 0.2} \mathrm{Wo}_{1.8 \pm 0.3}, \mathrm{Fe} / \mathrm{Mn}=39 \pm 1, \mathrm{n}=7$.

Classification: LL6-melt breccia

Specimens: $34.8 \mathrm{~g}$ including a probe mount on deposit at $U N M$, Larry Atkins holds the main mass.

\section{Northwest Africa 11731 (NWA 11731) \\ (Northwest Africa) \\ Purchased: 2017 \\ Classification: Enstatite chondrite (EL6)}

History: The meteorite was purchased from a local meteorite dealer in Morocco. Physical characteristics: Brownish individual with some patches of black fusion crust.

Petrography: The meteorite displays a recrystallized texture dominated by up to $300 \mu \mathrm{m}$ sized enstatite grains. Less abundant are albitic feldspar and typically intergrown daubreelite and troilite. No chondrules are visible. The meteorite is strongly altered, i.e., metal is completely converted into Fe-oxides and cracks are frequently filled with calcite and barite. 
Geochemistry: feldspar: $\mathrm{An}_{15.8} \mathrm{Ab}_{79.8} \mathrm{Or}_{4.4}, \mathrm{n}=5$

Classification: Although metal is completely oxidized and the Si content could not be determined, the absence of niningerite and alkali sulfides points towards an EL6 classification.

\section{Northwest Africa 11732 (NWA 11732)}

(Northwest Africa)

Purchased: 2017

Classification: Carbonaceous chondrite (CM2.0)

History: The meteorite was purchased from a local meteorite dealer in Morocco. Physical characteristics: Small black individual with some fusion crust.

Petrography: The meteorite is a brecciated carbonaceous chondrite composed of chondrule, mineral and CAI polymorphs set into a fine-grained matrix of phyllosilicates (as indicated by fibrous appearance), carbonates and sulfides including up to $400 \mu \mathrm{m}$ long needles of Ni-bearing pyrrhotite. Carbonates are almost pure calcites and $\mathrm{Mg}-\mathrm{Mn}-\mathrm{Fe}$-rich varieties. The hydrous and porous nature of the matrix is also proven by low analytical totals of defocused $(10 \mu \mathrm{m}$ beam diameter) electron microprobe analyses. Many components are surrounded by flaky phyllosilicates apparently replacing former dust rims. No anhydrous silicates have been found.

Geochemistry: Mean values of defocused ( $10 \mu \mathrm{m}$ beam diameter) matrix analyses (all wt\%; $\mathrm{N}=27$ ): $\mathrm{SiO}_{2}: 27.0, \mathrm{TiO}_{2}: 0.09 ; \mathrm{Na}_{2} \mathrm{O}: 0.11 ; \mathrm{Cr}_{2} \mathrm{O}_{3}: 0.41, \mathrm{MgO}: 19.8$, MnO: 0.18, CaO: 0.25, FeO: 21.7, $\mathrm{Al}_{2} \mathrm{O}_{3}: 2.29$, NiO: 0.79, $\mathrm{P}_{2} \mathrm{O}_{5}:$ 0.03, S: 0.36, Total: 73.01

Classification: CM2.0. Subtype based on the absence of anhydrous silicates.

Northwest Africa 11733 (NWA 11733)

(Northwest Africa)

Purchased: 2017

Classification: Carbonaceous chondrite (CM2)

History: The meteorite was purchased from a local meteorite dealer in Morocco.

Physical characteristics: Small black individual without fusion crust.

Petrography: The meteorite is a brecciated chondrite consisting of small chondrules (mean apparent diameter about $0.3 \mathrm{~mm}$ ), mineral fragments, and less abundant CAIs set into a fine-grained matrix. Chondrules and CAIs are sometimes surrounded by dust rims. Matrix minerals are phyllosilicates (as indicated by low analytical totals), carbonates, and pyrrhotite.

\section{Northwest Africa 11734 (NWA 11734)}

(Northwest Africa)

Purchased: 2017

Classification: HED achondrite (Eucrite, melt breccia) 
History: The meteorite was purchased from a local meteorite dealer in Erfoud, Morocco.

Physical characteristics: Brownish individual with some patches of black fusion crust.

Petrography: The meteorite is a eucrite-melt breccia composed of up to $1 \mathrm{~cm}$ sized light colored basaltic clasts set into dark recrystallized shock melt matrix.

Dominant minerals in the clasts are exolved pyroxene and calcic plagioclase. Minor phases include silica, ilmenite, troilite, chromite, and barite. No metallic Fe has been found.

Geochemistry: low-Ca pyroxene $\mathrm{Fs}_{60.8 \pm 1.0} \mathrm{Wo}_{2.8 \pm 0.5}\left(\mathrm{Fs}_{59.1-62.1} \mathrm{Wo}_{2.1-3.3}, \mathrm{n}=16\right.$, $\mathrm{FeO} / \mathrm{MnO}=30-35)$; Ca-pyroxene: $\mathrm{Fs}_{27.6 \pm 1.5} \mathrm{Wo}_{42.9 \pm 1.5}\left(\mathrm{Fs}_{25.8-32.2} \mathrm{Wo}_{37.9-44.2}, \mathrm{n}=23\right.$, $\mathrm{FeO} / \mathrm{MnO}=29-37)$; calcic plagioclase: $\mathrm{An}_{88.2 \pm 2.4}\left(\mathrm{An}_{83.3-91.0}, \mathrm{n}=21\right)$

\section{Northwest Africa 11735 (NWA 11735)}

(Northwest Africa)

Purchased: 2017

Classification: Ordinary chondrite (L3)

History: The meteorite was purchased from a Moroccan meteorite dealer on the mineral fair in Munich, Germany.

Physical characteristics: Dark brownish individual with some fusion crust.

Petrography: The meteorite displays a chondritic texture with sometimes slightly flattened and well packed chondrules (mean diameter about $0.6 \mathrm{~mm}$ ) in a more fine-grained matrix that contains sulfides and FeNi metal.

Northwest Africa 11736 (NWA 11736)

(Northwest Africa)

Purchased: 2017

Classification: Ordinary chondrite (L3)

History: The meteorite was purchased from a local meteorite dealer in Algeria.

Physical characteristics: Light brownish individual without fusion crust.

Petrography: The meteorite shows a chondritic texture with clearly defined, sometimes deformed packed chondrules (mean diameter about $0.7 \mathrm{~mm}$ ) in a finegrained matrix of mineral and chondrule fragments, FeNi metal and sulfides.

\section{Northwest Africa 11737 (NWA 11737)}

(Northwest Africa)

Purchased: 2017

Classification: Carbonaceous chondrite (CV3)

History: The meteorite was purchased from a local meteorite dealer in Erfoud, Morocco.

Physical characteristics: Sixteen dark grayish individuals with some fusion crust. 
Petrography: The meteorite displays a dark brownish interior and is composed of up to $1.5 \mathrm{~mm}$ sized chondrules (mean diameter about $0.9 \mathrm{~mm}$ ), whitish CAIs, and olivine amoeboids set into a fine-grained almost opaque matrix. Chondrules frequently show brownish staining due to terrestrial weathering; type II chondrules are absent.

\section{Northwest Africa 11738 (NWA 11738)}

(Northwest Africa)

Purchased: 2012

Classification: Ureilite

History: The meteorite was purchased from a local meteorite dealer in Zagora, Morocco.

Physical characteristics: Dark brownish individual without fusion crust.

Petrography: The meteorite shows a cumulus texture of up to $1.5 \mathrm{~mm}$ sized $\mathrm{Cr}$ rich olivine and pigeonite crystals. It is brecciated, contains some flaky graphite and olivine displays pronounced reduced rims.

Geochemistry: reduced rims in olivine: $\mathrm{Fa}_{2.5-6.2}$; olivine contains $0.70 \pm 0.03 \mathrm{wt} \%$ $\mathrm{Cr}_{2} \mathrm{O}_{3}$ and $0.43 \pm 0.02 \mathrm{wt} \% \mathrm{CaO}$

Classification: Ureilite, brecciated

\section{Northwest Africa 11739 (NWA 11739)}

(Northwest Africa)

Purchased: 2017

Classification: Primitive achondrite

History: The meteorite was purchased from a local meteorite dealer in Erfoud, Morocco.

Physical characteristics: Small light brownish individual without fusion crust. Sawn surface reveals mosaic of shiny, fine-grained crystals.

Petrography: The meteorite shows an equigranular equilibrium texture with abundant triple junctions between adjoining mineral grains. It is predominantly composed of 200-300 $\mu \mathrm{m}$ sized Fe-rich olivine (about 60 vol.\%) with less abundant clinopyroxene (about $15 \mathrm{vol} . \%$ ) and intermediate plagioclase (about $20 \mathrm{vol} . \%$ ). Minor phases are FeNi metal and sulfides. No orthopyroxene has been detected. Geochemistry: olivine: $\mathrm{Fa}_{31.2 \pm 0.1}, \mathrm{CaO}=0.08 \pm 0.1, \mathrm{Cr}_{2} \mathrm{O}_{3}=0.01 \pm 0.1, \mathrm{FeO} / \mathrm{MnO}=74-$ 96, n=12; Ca-pyroxene: $\mathrm{Fs}_{11.2 \pm 0.5} \mathrm{Wo}_{43.4 \pm 0.6}, \mathrm{FeO} / \mathrm{MnO}=34-54, \mathrm{n}=12$; feldspar: $\mathrm{An}_{34.0} \mathrm{Ab}_{63.0} \mathrm{Or}_{3.0}, \mathrm{n}=18$

Classification: Olivine-rich primitve achondrite with mineral chemistry close to brachinites. However, small grain sizes and high abundance of plagioclase are contrary to brachinites.

Northwest Africa 11740 (NWA 11740)

(Northwest Africa) 
Purchased: 2014

Classification: Carbonaceous chondrite (CV3)

History: The meteorite was purchased from a local meteorite dealer in Zagora, Morocco.

Physical characteristics: Greyish individual with some fusion crust.

Petrography: Carbonaceous chondrite composed of up to $3 \mathrm{~mm}$ sized chondrules (mean diameter about $1 \mathrm{~mm}$ ), CAIs and olivine amoeboids all set into a finegrained almost black matrix. One CAI about $7 \mathrm{~mm}$ in diameter has been observed. Type II chondrules are absent.

\section{Northwest Africa 11741 (NWA 11741)}

(Northwest Africa)

Purchased: 2016

Classification: HED achondrite (Diogenite)

History: The meteorite was bought from a local meteorite dealer in Zagora, Morocco.

Physical characteristics: Light brownish to orange individual with some patches of fusion crust.

Petrography: The meteorite is a fragmental breccia predominantly composed of blocky up to $4 \mathrm{~mm}$ sized orthoproxene crystals. Minor phases include silica, chromite, troilite and metallic iron. Neither plagioclase nor olivine have been found in the section studied.

Geochemistry: low-Ca pyroxene: $\mathrm{Fs}_{27.5 \pm 0.4} \mathrm{Wo}_{4.0 \pm 0.4}\left(\mathrm{Fs}_{26.7-28.0} \mathrm{Wo}_{3.2-6.8}, \mathrm{n}=22\right.$, $\mathrm{FeO} / \mathrm{MnO}=27-32$ )

\section{Northwest Africa 11742 (NWA 11742)}

(Northwest Africa)

Purchased: 2016

Classification: HED achondrite (Eucrite, melt breccia)

History: The meteorite was bought from a local meteorite dealer in Quarzazate, Morocco.

Physical characteristics: Brownish individual without fusion crust.

Petrography: The meteorite is a melt breccia composed of up to $5 \mathrm{~mm}$ sized basaltic clasts set into shock melted matrix. Basaltic clasts consist of exsolved pyroxenes and often lath shaped calcic plagioclase. The shock melt is mostly recrystallized to plagioclase and pyroxene; some regions show pronounced flow textures. Minor phases include silica, chromite, ilmenite and barite.

Geochemistry: low-Ca pyroxene: $\mathrm{Fs}_{59.9 \pm 0.9} \mathrm{Wo}_{4.0 \pm 1.3}\left(\mathrm{Fs}_{57.4-61.1} \mathrm{Wo}_{2.2-7.6}, \mathrm{n}=14\right.$, $\mathrm{FeO} / \mathrm{MnO}=33-37)$; Ca-pyroxene: $\mathrm{Fs}_{27.8 \pm 1.2} \mathrm{Wo}_{42.1 \pm 1.2}\left(\mathrm{Fs}_{26.2-30.1} \mathrm{Wo}_{39.7-43.8}, \mathrm{n}=16\right.$, $\mathrm{FeO} / \mathrm{MnO}=33-39)$; calcic plagioclase: $\mathrm{An}_{90.6 \pm 0.8}\left(\mathrm{An}_{89.3-91.9}, \mathrm{n}=16\right)$ 


\section{Morocco}

Purchased: May 2014

Classification: Ordinary chondrite $(\mathrm{H} 4)$

History: A small fragment of the studied meteorite weighting $5.0 \mathrm{~g}$ was purchased by I. Baziotis from a dealer in Marrakech, Morocco (May 2014). The stone was a broken fragment partly surrounded by fusion crust. A polished thin section was carefully prepared from the fragment and subsequently examined using optical microscopy to identify the texture and mineralogy.

Physical characteristics: 5.0 g. 1 piece. The color of the meteorite is pale brown. Black fusion crust partly covers the sample. Red-brown oxidation is visible on the exterior surface, around metal grains on cut surfaces and within the matrix.

Petrography (I. Baziotis, $A U A$; A. Bischoff, IfP): Textural observations of the thin section in reflected light showed a chondritic structure. It contains porphyritic and non-porphyritic chondrules, both Type I $(\mathrm{Mg} /(\mathrm{Mg}+\mathrm{Fe})<90)$. ?he porphyritic olivine (PO) chondrules have a rounded to oval shape ranging from 300 to $350 ? \mathrm{~m}$, and from 600 to $1200 ? \mathrm{~m}$ in size. The groundmass consists of olivine (30 vol.\%), low Ca-pyroxene (20 vol.\%), clinopyroxene (5 vol.\%), plagioclase (5 vol.\%) and rare phosphates (apatite). Also, metal-rich material has filled the cracks of the fragment (40 vol\%). Shock veins were not observed. The rock matrix is brecciated.

Geochemistry: Mineral Composition and Geochemistry: Coarse grained rock with euhedral to subhedral olivine with narrow compositional range ( $\left.\mathrm{Fa}_{18.6-19.2}\right)$, embedded in a translucent feldspathic glassy mesostasis $\mathrm{Ab}_{87.6-82.8}$. Olivine $\mathrm{Fa}_{18.9 \pm 0.3}(\mathrm{n}=23)$; Low-Ca Pyroxene Fs ${ }_{17.8 \pm 0.3}(\mathrm{n}=24), \mathrm{Wo}_{0.8 \pm 0.1}(\mathrm{n}=24)$.

Classification (I. Bazioti and A.Pantazidis, $A U A$ ): This meteorite is a $\mathrm{H} 4$ ordinary chondrite. Weathering stage: Minor to slighly moderate oxidation W2, with the major silicates being unaffected.

Specimens: $4.94 \mathrm{~g}$ at $N H M V$.

\section{Northwest Africa 11744 (NWA 11744)}

(Northwest Africa)

Purchased: 2004

Classification: Ordinary chondrite (L5)

History: sample was purchased from a meteorite collector by Dr Mahesh Anand $(O U)$. In July 2016, thin sections of the sample were passed to Craig Walton (UStA) for classification work.

Physical characteristics: A single 1566 g stone. Sample mostly has a dark fusion crust, but in places a light coloured interior is exposed. Sample contains abundant complex shock features, mainly micro-fault shear textures, associated with melt pools.

Petrography: (Craig Walton, UStA) Overall, sample has a chondritic brecciated texture. Main phases are olivine and pyroxene with opaque metal + troilite. Porphyritic olivine, radial pyroxene, barred olivine and porphyritic olivine pyroxene chondrule types are all common, along with rare cryptocrystalline and glassy chondrules. Chondrule average diameter is $370 \pm 220 \mu \mathrm{m}(\mathrm{N}=27)$, up to 1 
$\mathrm{mm}$. Chondrules comprise $\sim 70 \%$ of the sample. Opaque phases are metal (Fe,Ni in exsolution as kamacite and taenite) and sulfide (troilite). Modal abundances of metal and sulfide are $5.8 \%$ and $4.4 \%$, respectively. A recrystallized and shockdarkened matrix of fine-grained lithic fragments comprises $\sim 20 \%$ of the sample area. Some isolated euhedral olivine grains (up to $\sim 1 \mathrm{~mm}$ ) occur embedded in this matrix. Olivine contains planar deformation features and has undulose extinction. Planes across which shear displacement has occurred (microfaults) crosscut chondrule and brecciated matrix textures. Fine-grained silicate melt-pools bearing globules of immiscible metal and sulfide are hosted within some sections of these microfaults. Microfault textures crosscut chondrules, matrix and metal, with individual faults running along the length of the slide. Offset on the faults is on the order of $500 \mu \mathrm{m}$. $\sim 50 \mu \mathrm{m}$-wide shock-melt pools/veins occur within the faults. Geochemistry: Mineral compositions and geochemistry: (Craig Walton, UStA; Mahesh Anand, Sam Hammond, Richard Greenwood, $O U$ ) Microprobe (Cameca SX100 EMPA) (all values given in wt \%). Olivine ( $\mathrm{n}=5): \mathrm{MgO}=38.8, \mathrm{CaO}=0.1$, $\mathrm{MnO}=0.5, \mathrm{FeO}=22.7, \mathrm{SiO}=38.1 . \mathrm{TiO}=0.1$ Pyroxene $(\mathrm{n}=2): \mathrm{Na}_{2} \mathrm{O}=0.1, \mathrm{MgO}$ $=28.5, \mathrm{~K}_{2} \mathrm{O}=0.2, \mathrm{CaO}=1.3, \mathrm{MnO}=0.4, \mathrm{FeO}=14.2, \mathrm{Al}_{2} \mathrm{O}_{3}=0.8, \mathrm{Cr}_{2} \mathrm{O}_{3}=0.4$, $\mathrm{SiO}_{2}=55.1, \mathrm{TiO}_{2}=0.1, \mathrm{P}_{2} \mathrm{O}_{5}=0.1$. Oxygen Isotopes (Laser fluorination $\mathrm{CO}_{2}$ laser, Thermo Scientific MAT 253 gas source mass spectrometer) $\delta^{18} \mathrm{O}=5.139$, $\delta^{17} \mathrm{O}=3.778, \Delta^{17} \mathrm{O}=1.105$ (all in \%o).

Classification: L5 chondrite. Shock stage is $\mathrm{S} 4 / 5$ as evidenced by shear microfault textures, associated shock-melt veins, shock darkening of matrix and undulose extinction and planar deformation features in olivine. Oxygen isotopes are indiscriminate between $\mathrm{L}$ and LL, however Fs (Px) and $\mathrm{Fa}(\mathrm{Ol})$ data along with metal abundances indicate an $\mathrm{L}$ chondrite grouping. Potentially paired with NWA 11745.

Specimens: Main mass at $O U$

Northwest Africa 11745 (NWA 11745)

(Northwest Africa)

Purchased: 2004

Classification: Ordinary chondrite (L5)

History: sample was purchased in Morocco from a meteorite collector by $\mathrm{Dr}$ Mahesh Anand (OU) some years ago. In July 2016, thin sections of the sample were passed to Craig Walton (UStA) for preliminary classification work.

Physical characteristics: One large piece is present in the collection (554.16). Sample predominantly has a light color, but has a dark fusion crust on one side. Sample contains abundant complex shock features - mainly micro-fault shear textures, associated with melt pools.

Petrography: (Craig Walton, UStA) Overall, sample has a chondritic brecciated texture. Main phases are olivine and pyroxene with opaque metal + troilite. Porphyritic olivine, radial pyroxene, barred olivine and porphyritic olivine pyroxene chondrule types are all common, along with rare cryptocrystalline and glassy chondrules. Chondrule average diameter is $0.56 \mathrm{~mm}$, ranging from $1.25 \mathrm{~mm}$ 
to $0.25 \mathrm{~mm}$. Chondrules comprise $\sim 70 \%$ of the sample. Opaque phases are metal ( $\mathrm{Fe}, \mathrm{Ni}$ in exsolution as kamacite and taenite) and sulfide (troilite). A recrystallized and shock-darkened matrix of fine-grained lithic fragments comprises $\sim 20 \%$ of the sample area. Some isolated euhedral olivine grains (up to $\sim 1 \mathrm{~mm}$ ) occur embedded in this matrix. Olivine contains planar deformation features and has undulose extinction. Planes across which shear displacement has occurred (microfaults) crosscut chondrule and brecciated matrix textures. Finegrained silicate melt-pools bearing globules of immiscible metal and sulfide are hosted within some sections of these microfaults. Microfault textures crosscut chondrules, matrix and metal, with individual faults running along the length of the slide. Offset on the faults is on the order of $500 \mu \mathrm{m}$. $~ 50 \mu \mathrm{m}$-wide shock-melt pools/veins occur within the faults.

Geochemistry: Mineral compositions and geochemistry: (Craig Walton, UStA; Mahesh Anand, Sam Hammond, Richard Greenwood, $O U$ ) Microprobe (Cameca SX100 EMPA) Olivine ( $\mathrm{n}=15): \mathrm{MgO}=38.4, \mathrm{MnO}=0.5, \mathrm{FeO}=23.4, \mathrm{SiO}=38.3$ $\mathrm{P}_{2} \mathrm{O}_{5}=0.1$ Pyroxene $(\mathrm{n}=7): \mathrm{MgO}=28.7, \mathrm{CaO}=0.9, \mathrm{MnO}=0.5, \mathrm{FeO}=14.5$, $\mathrm{Al}_{2} \mathrm{O}_{3}=0.2, \mathrm{Cr}_{2} \mathrm{O}_{3}=0.1, \mathrm{SiO}_{2}=55.7, \mathrm{TiO}_{2}=0.2$. Plagioclase: $\mathrm{Na}_{2} \mathrm{O}=10.7, \mathrm{~K}_{2} \mathrm{O}=$ $1.1, \mathrm{CaO}=2.3, \mathrm{FeO}=0.8, \mathrm{Al}_{2} \mathrm{O}_{3}=22.1, \mathrm{SiO}_{2}=65.8 ;$ Plagioclase $\mathrm{Ab}_{76.1} \mathrm{Or}_{7.71} \mathrm{An}_{16.2}(\mathrm{~N}=11)$.. Oxygen Isotopes (Laser fluorination $\mathrm{CO}_{2}$ laser, Thermo Scientific MAT 253 gas source mass spectrometer) $\delta^{18} \mathrm{O}=4.931, \delta^{17} \mathrm{O}=3.693$, $\Delta \Delta^{17} \mathrm{O}=1.129, \Delta^{17} \mathrm{O}$ linear $=1.105($ all \%o $)$.

Classification: L5. Shock stage is $\mathrm{S} 4 / 5$ as evidenced by shear microfault textures, associated shock-melt veins, shock darkening of matrix and undulose extinction and planar deformation features in olivine. Oxygen isotopes are indiscriminate between $\mathrm{L}$ and LL, however Fs (PX) and Fa (Ol) data indicate an L chondrite. Potentially paired with NWA 11744.

Specimens: Main mass at $O U$

Northwest Africa 11746 (NWA 11746)

(Northwest Africa)

Purchased: 2015

Classification: Martian meteorite (Shergottite)

History: Purchased by Jay Piatek from Morocco, 2015.

Physical characteristics: Fusion crusted individual. A saw-cut surface reveals gray-green, fine-grained, basaltic texture.

Petrography: (C. Agee, $U N M)$ Microprobe examination of a polished mount shows an ophitic texture with $\sim 60 \%$ zoned pyroxene and $\sim 30 \%$ maskelynite. Accessory phases include titanomagnetite, ilmenite, Fe-sulfide, and silica.

Geochemistry: (C. Agee, $U N M$ ) Pigeonite $\mathrm{Fs}_{50.5 \pm 14.8} \mathrm{Wo}_{16.9 \pm 6.8}, \mathrm{Fe} / \mathrm{Mn}=37 \pm 3, \mathrm{n}=8$; augite $\mathrm{Fs}_{29.3 \pm 10.4} \mathrm{Wo}_{31.7 \pm 2.8}, \mathrm{Fe} / \mathrm{Mn}=32 \pm 4, \mathrm{n}=12$; maskelynite $\mathrm{Or}_{2.8 \pm 1.1} \mathrm{Ab}_{49.8 \pm 3.9} \mathrm{An}_{47.4 \pm 4.9}, \mathrm{n}=6$.

Classification: Martian (shergottite), pyroxene-phyric. Possibly paired with NWA $\underline{10016 .}$. 
Specimens: $1.9 \mathrm{~g}$ including a probe mount on deposit at $U N M$, Jay Piatek holds the main mass.

\title{
Northwest Africa 11747 (NWA 11747) \\ (Northwest Africa) \\ Purchased: 2011 \\ Classification: HED achondrite (Eucrite)
}

Petrography: Highly brecciated, fine-medium grained sample. Fine grained matrix is composed of fragmented pyroxene and feldspar with three cohesive, coarse grained subophitic/gabbroic clasts. Dark grey to black melt glass pockets, often containting small fragmented clasts of pyroxene, are focused in a darker region of the sample which constitutes approximately $40 \%$ of the thin section. Accessory phases include apatite, merrillite, zircon, ilmenite and rare baddeleyite.

Geochemistry: Mineral compositions and geochemistry: Pyroxene yield bulk $\mathrm{Fe} / \mathrm{Mn}$ ratios of $29.42(\mathrm{~N}=46)$. Pyroxene $\left(\mathrm{En}_{45} \mathrm{Fs}_{46} \mathrm{Wo}_{8} ; \mathrm{N}=43\right)$ with exsolved augite lamella $\left(\mathrm{En}_{35} \mathrm{Fs}_{23} \mathrm{Wo}_{42} ; \mathrm{N}=3\right)$ occur throughout the sample. Feldspar composition averages $\mathrm{An}_{88}(\mathrm{~N}=25)$. Mineral chemistry measured by EMP.

Classification: Eucrite (monomict breccia)

\section{Northwest Africa 11748 (NWA 11748) \\ (Northwest Africa) \\ Purchased: 2011 \\ Classification: HED achondrite (Eucrite)}

Petrography: Sample is brecciated, containing subophitic gabbroic clasts of pyroxene and plagioclase set in a finer grained matrix of the same assemblage. Accessory phases include apatite, merrillite, zircon, ilmenite and rare baddeleyite. Geochemistry: Mineral compositions and geochemistry: Pyroxene yield bulk $\mathrm{Fe} / \mathrm{Mn}$ ratios of $31.49(\mathrm{~N}=34)$. Orthopyroxene $\left(\mathrm{En}_{35} \mathrm{Fs}_{58} \mathrm{Wo}_{7} ; \mathrm{N}=29\right)$ contain abundant exsolution lamella of augite composition $\left(\mathrm{En}_{30} \mathrm{Fs}_{29} \mathrm{Wo}_{41} ; \mathrm{N}=5\right)$. Feldspar yields highly anorthitic compositions averaging $\mathrm{An}_{91}(\mathrm{~N}=20)$. Mineral chemistry measured by EMP.

Classification: Eucrite (monomict breccia)

\author{
Northwest Africa 11749 (NWA 11749) \\ Morocco \\ Purchased: 2016 Oct \\ Classification: HED achondrite (Eucrite, unbrecciated)
}

History: Bought in Munich from a dealer from NWA.

Physical characteristics: Crusted stone. Cut surface reveals a rather orase-grained light grey rock. 
Petrography: Unbrecciated igneous rock with subophitic texture and typical grain size $500 \mu \mathrm{m}$. Main minerals are pyroxene and plagioclase. Troilite (to $40 \mu \mathrm{m}$ ), chromite, ilmenite, silica polymorph, FeNi metal are present.

Geochemistry: Low-Ca pyroxene $\mathrm{Fs}_{63.0 \pm 0.3} \mathrm{Wo}_{2.2 \pm 0.4}(\mathrm{~N}=6)$, augite exsolution $\mathrm{Fs}_{26.4} \mathrm{Wo}_{45.0}(\mathrm{~N}=2), \mathrm{FeO} / \mathrm{MnO} 31.1 \pm 1.7(\mathrm{~N}=8)$. Plagioclase $\mathrm{An}_{88.6} \mathrm{Ab}_{11.1} \mathrm{Or}_{0.3}(\mathrm{~N}=3)$. Classification: Eucrite-unbr Specimens: Type specimen at CEREGE. Main mass with Andreas Gren.

\section{Northwest Africa 11750 (NWA 11750) \\ Morocco \\ Purchased: 2016 May \\ Classification: C3.00-ung}

History: The meteorite was reportedly found near Foum Zguid. It was bought by Pierre-Marie Pelé in Morocco in May 2016 and a subsample was sent to $C E R E G E$ for classification. In view of its scientific interest, the whole stone was eventually given to $C E R E G E$ by Pierre-Marie Pelé.

Physical characteristics: Black irregular stone. Cut surface reveals a dark homogeneous interior.

Petrography: (J. Gattacceca, B. Devouard, H. Pourkhorsandi, CEREGE; L. Bonal, IPAG) Chondrules and mineral fragments (26 vol\%) set in a fine-grained iron-rich matrix ( $74 \mathrm{vol} \%$, modal abundances by point counting $\mathrm{N}=204$ ). Average chondrule apparent size $240 \pm 170 \mu \mathrm{m}(\mathrm{N}=28)$. Opaque are mostly FeNi metal (both in matrix and chondrules) and sulfides with typical grain size about $10 \mu \mathrm{m}$. In the matrix, FeNi metal and sulfides are finely intermixed. Saturation magnetization is $\mathrm{Ms}=2.39 \mathrm{Am} 2 / \mathrm{kg}$ indicating a bulk metal content of at least $1.1 \mathrm{wt} \%$. Hysteresis properties show the significant presence of tetrataenite in the form of cloudy zone (Gattacceca et al., 2014). XRD over a $\sim 1 \mathrm{~cm} 2$ area on a polished section (D. Borschnek, B. Devouard, CEREGE) does not reveal the presence of hydrous phases (serpentine, tochilinite) typically observed in type 2 chondrites using the same experimental setup. The absence of hydrous phases in the fine-grained matrix is confirmed by IR transmission spectra. Raman spectra of the fine-grained matrix reveal the presence of polyaromatic carbonaceous matter characterized by a structural order comparable to type 2 chondrites, thus reflecting a peak metamorphic temperature lower than in the least metamorphosed type 3 chondrites (e.g., Semarkona)

Geochemistry: Olivine $\mathrm{Fa}_{11.9 \pm 17.1}$, range $\mathrm{Fa}_{0.8-45.1}$, PMD 126\% (N=7). Ferroan olivine has $\mathrm{Cr}_{2} \mathrm{O}_{3} 0.24 \pm 0.16 \mathrm{wt} \%(\mathrm{~N}=3)$. Orthopyroxene $\mathrm{Fs}_{3.2 \pm 2.2} \mathrm{Wo}_{3.8 \pm 1.1}(\mathrm{~N}=3)$. Kamacite Fe 91.0, Ni 6.7, Co, $2.3(\mathrm{~N}=1)$. Taenite Fe 67.2, Ni 29.6, Co 0.9 (atom\%). Oxygen isotopic composition (J. Gattacceca, C. Sonzogni, CEREGE) from analysis of a $1.5 \mathrm{mg}$ aliquot of a powdered $40 \mathrm{mg}$ bulk sample is $\delta^{17} \mathrm{O}=3.47$, $\delta^{18} \mathrm{O}=14.3, \Delta{ }^{17} \mathrm{O}=-4.0$, all per mil (linearized, slope 0.5247 , analytical uncertainties $0.08 \%, 0.12 \%, 0.03 \%$ respectively $)$. Magnetic susceptibility $\log \chi\left(\times 10^{-9} \mathrm{~m}^{3} / \mathrm{kg}\right)=$ 4.03 . 
Classification: C3.00-ung. The type 3 derives from the unequilibrated nature, the absence of phyllosilicates and abundance of metal. The subtype 3.00 derives from Raman spectroscopy. The ungrouped designation derives from the oxygen isotopes and petrography.

Specimens: Main mass and type specimen at CEREGE.

\section{Northwest Africa 11751 (NWA 11751)}

(Northwest Africa)

Purchased: 2017

Classification: Carbonaceous chondrite (CO3.05)

Physical characteristics: Dark brown stone. Cut surface reveals an almost featurless dark brown interior.

Petrography: (J. Gattacceca, H. Pourkhorsandi, CEREGE ; Lydie Bonal, IPAG) Small chondrules (average apparent diameter $230 \pm 100 \mu \mathrm{m}, \mathrm{N}=22$ ) set in a finegrained iron-rich matrix. Chondrules $65 \mathrm{vol} \%$, matrix $35 \mathrm{vol} \%$ (point counting, $\mathrm{N}=213$ ). Small CAI are present. Based on the structural order of the polyaromatic matter assessed by Raman spectroscopy, this meteorite appears to be less metamorphosed than Colony (CO 3.1) and more metamorphosed than ALHA 77307 (CO 3.05). The Raman spectroscopy parameters of interest are FWHM-D $(\mathrm{cm}-1)=187.6 \pm 6.0, \mathrm{ID} / \mathrm{IG}=1.00 \pm 0.04$ (see Bonal et al. 2016, GCA 189:312-337, figure 6).

Geochemistry: Olivine $\mathrm{Fa}_{10.4 \pm 10.0}$, range $\mathrm{Fa}_{0.7-25.4}, \mathrm{PMD} 91 \%(\mathrm{~N}=9), \mathrm{Cr}_{2} \mathrm{O}_{3}$ in ferroan olivine $0.35 \pm 0.17 \mathrm{wt} \%(\mathrm{~N}=7)$. Orthopyroxene $\mathrm{Fs}_{3.6 \pm 1.4} \mathrm{Wo}_{3.8 \pm 2.0}(\mathrm{~N}=4)$. Magnetic susceptibility $\log \chi\left(\times 10^{-9} \mathrm{~m}^{3} / \mathrm{kg}\right)=4.69$

Classification: Carbonaceous chondrite (CO3.05). Subtype from Raman spectroscopy and $\mathrm{Cr}_{2} \mathrm{O}_{3}$ content in ferroan olivine.

Specimens: Type specimen at CEREGE. Main mass with Fabien Kuntz.

\section{Northwest Africa 11752 (NWA 11752)}

(Northwest Africa)

Purchased: 2017

Classification: Ordinary chondrite (LL3.05)

Physical characteristics: Dark brown stone. Cut surface reveals packed welldefined chondrules in a brown matrix.

Petrography: (J. Gattacceca, H. Pourkhorsandi, CEREGE; Lydie Bonal, IPAG) Large and closely packed chondrules. Opaques are FeNi minerals and troilite. Based on the structural order of the polyaromatic matter assessed by Raman spectroscopy, this meteorite appears to be as metamorphosed as ALHA 77307 (CO3.05). The Raman spectroscopy parameters of interest are FWHM-D (cm1) $=210.9 \pm 14.8, \mathrm{ID} / \mathrm{IG}=0.95 \pm 0.05$ (see Bonal et al. 2016, GCA 189:312-337, figure $7)$. 
Geochemistry: Olivine $\mathrm{Fa}_{16.8 \pm 5.4}$, range $\mathrm{Fa}_{8.6-24.9}, \mathrm{PMD} 27 \%(\mathrm{~N}=6), \mathrm{Cr}_{2} \mathrm{O}_{3}$ in ferroan olivine $0.45 \pm 0.05 \mathrm{wt} \%(\mathrm{~N}=6)$. Orthopyroxene $\mathrm{Fs}_{10.4 \pm 8.4} \mathrm{Wo}_{1.6 \pm 1.3}(\mathrm{~N}=4)$. Magnetic susceptibility $\log \chi\left(\times 10^{-9} \mathrm{~m}^{3} / \mathrm{kg}\right)=4.00$.

Classification: LL3.05. Subtype from Raman spectroscopy and $\mathrm{Cr}_{2} \mathrm{O}_{3}$ content in ferroan olivine.

Specimens: Type specimen at CEREGE. Main mass with Kuntz.

\section{Northwest Africa 11753 (NWA 11753) \\ Morocco \\ Purchased: $2017 \mathrm{Jul}$ \\ Classification: HED achondrite (Eucrite, polymict)}

Petrography: (K. Metzler, IfP) Fine-grained polymict breccia consisting of mmsized basalt clasts (subophitic and granulitic textures) and mineral fragments set in a fine-grained clastic matrix made of related debris. Breccia-in breccia textures occur. Most pyroxenes exhibit augite exsolution lamellae. Fragments of diogenitic low-Ca pyroxene are admixed ( $<10 \mathrm{vol} \%)$. Accessories are silica polymorph, ilmenite, chromite, troilite, and Cr-Al-spinel.

Geochemistry: Mineral compositions and geochemistry: Eucritic low-Ca pyroxene (host; $\mathrm{n}=9$ ): $\mathrm{Fs}_{47.8 \pm 5.8}$ (39-58); $\mathrm{Wo}_{2.1 \pm 0.6}(1-3)$; mean $\mathrm{Fe} / \mathrm{Mn}$ (at.): 33. Eucritic Capyroxene (exsolution lamellae; $\mathrm{n}=3$ ) $\mathrm{Fs}_{20.2 \pm 4.2}(18-25)$; $\mathrm{Wo}_{42.2 \pm 1.9}$ (40-44). Diogenitic pyroxene $(\mathrm{n}=4): \mathrm{Fs}_{29.5 \pm 1.9} \mathrm{Wo}_{3.7 \pm 0.5}\left(\mathrm{Fs}_{28-31} \mathrm{Wo}_{3-4}\right)$; mean $\mathrm{Fe} / \mathrm{Mn}$ (at.): 36.Plagioclase $(\mathrm{n}=10): \mathrm{An}_{90.6 \pm 2.6}(85-94)$.

\section{Northwest Africa 11754 (NWA 11754) \\ Morocco \\ Purchased: 2017 Jul \\ Classification: Ureilite}

Petrography: (K. Metzler, IfP) Coarse-grained ultramafic rock with metamorphic texture ( $\sim 120^{\circ}$ triple junctions), consisting of olivine, low-Ca pyroxene, and Capyroxene grains with sizes up to $\sim 3 \mathrm{~mm}$. Reverse zoning of olivine with Mg-rich zones at the margins and along cracks can be observed. Metal is concentrated along grain boundaries and cracks and mostly oxidized by terrestrial weathering.

Geochemistry: Mineral compositions and geochemistry: Olivine cores $(\mathrm{n}=4)$ : $\mathrm{Fa}_{11.4 \pm 0.7}$; Olivine rims $(\mathrm{n}=3): \mathrm{Fa}_{6.6 \pm 0.3}$. Low-Ca pyroxene grains $(\mathrm{n}=9)$ : $\mathrm{Fs}_{10.7 \pm 0.2} \mathrm{Wo}_{4.9 \pm 0.2 .}$ Ca-pyroxene grains $(\mathrm{n}=12): \mathrm{Fs}_{6.6 \pm 0.2} \mathrm{Wo}_{36.2 \pm 0.2}$. The concentration of $\mathrm{Cr}_{2} \mathrm{O}_{3}$ in olivine and pyroxene is $0.5 \mathrm{wt} \%$ and $1.1 \mathrm{wt} \%$, respectively.

\section{Northwest Africa 11755 (NWA 11755) \\ Morocco \\ Purchased: 2017 Sep \\ Classification: Ureilite}


Petrography: (K. Metzler, IfP) Coarse-grained ultramafic rock with metamorphic texture $\left(\sim 120^{\circ}\right.$ triple junctions), consisting of olivine and pyroxene grains up to $\sim 5$ $\mathrm{mm}$. Reverse zoning of olivine grains with $\mathrm{Mg}$-rich zones at the margins and along cracks. Graphite and partly oxidized metal are concentrated along grain boundaries. Geochemistry: Mineral compositions and geochemistry: Olivine cores $(\mathrm{n}=12)$ :

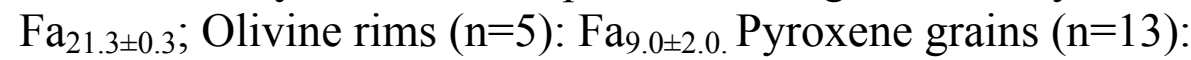

$\mathrm{Fs}_{11.8 \pm 1.5} \mathrm{Wo}_{6.1 \pm 1.8}$. $\mathrm{The} \mathrm{Cr}_{2} \mathrm{O}_{3}$ concentration in olivine and pyroxene is $0.7 \mathrm{wt} \%$ and $1.0 \mathrm{wt} \%$, respectively.

\section{Northwest Africa 11756 (NWA 11756) \\ Morocco \\ Purchased: 2017 Sep \\ Classification: Primitive achondrite (Brachinite)}

Petrography: (K. Metzler, IfP) Olivine-dominated ultramafic rock with metamorphic texture $\left(\sim 120^{\circ}\right.$ triple junctions $)$, consisting of olivine, low-Ca pyroxene, and Ca-pyroxene grains up to $\sim 0.8 \mathrm{~mm}$. Metal is replaced by weathering products which are also concentrated along grain boundaries. Accessories are $\mathrm{Cr}$ Al-spinel (up to $700 \mu \mathrm{m}$ ) and weathered FeS. No plagioclase was found.

Geochemistry: Mineral compositions and geochemistry: Olivine grains $(\mathrm{n}=12)$ : $\mathrm{Fa}_{26.8 \pm 0.2}$, mean $\mathrm{Fe} / \mathrm{Mn}(\mathrm{at})=$.58 ; Low-Ca pyroxene grains $(\mathrm{n}=12)$ :

$\mathrm{Fs}_{22.1 \pm 0.4} \mathrm{Wo}_{2.1 \pm 0.1}$. Ca-pyroxene grains $(\mathrm{n}=5): \mathrm{Fs}_{9.1 \pm 0.2} \mathrm{Wo}_{43.6 \pm 0.5}$. The concentration of $\mathrm{Cr}_{2} \mathrm{O}_{3}$ in olivine and pyroxene is $<0.1 \mathrm{wt} \%$ and $0.3 \mathrm{wt} \%$, respectively. Composition of Cr-spinel ( $\mathrm{n}=2$; at.): $\mathrm{Cr} /(\mathrm{Cr}+\mathrm{Al})=0.78 ; \mathrm{TiO}_{2} 1.4-1.5 \mathrm{wt} \%$. SEM analyses of large thin section areas reveal that this sample is nearly devoid of aluminum.

Classification: Brachinite, based on metamorphic texture, grain size, modal composition, absence of plagioclase, and mineral chemistry.

\section{Northwest Africa 11757 (NWA 11757) \\ Morocco \\ Purchased: 2017 Sep \\ Classification: Ureilite}

Petrography: (K. Metzler, IfP) Coarse-grained ultramafic rock with metamorphic texture $\left(\sim 120^{\circ}\right.$ triple junctions), consisting of olivine and pyroxene grains up to $\sim 2$ $\mathrm{mm}$. Reverse zoning of olivine grains with $\mathrm{Mg}$-rich zones at the margins and along cracks. Metal is concentrated along grain boundaries and partly oxidized by terrestrial weathering.

Geochemistry: Mineral compositions and geochemistry: Olivine cores $(\mathrm{n}=10)$ : $\mathrm{Fa}_{21.0 \pm 0.6}$; Olivine rims $(\mathrm{n}=5): \mathrm{Fa}_{1.4 \pm 0.1}$. Pyroxene grains $(\mathrm{n}=10)$ :

$\mathrm{Fs}_{18.0 \pm 0.3} \mathrm{Wo}_{7.2 \pm 0.2}$. The concentration of $\mathrm{Cr}_{2} \mathrm{O}_{3}$ in olivine and pyroxene is $0.5 \mathrm{wt} \%$ and $0.8 \mathrm{wt} \%$, respectively.

Northwest Africa 11761 (NWA 11761) 
(Northwest Africa)

Purchased: June 2016

Classification: Mesosiderite

History: The meteorite was purchased by an anonymous collector at St. Marie-auxMines, France, mineral show in June 2016 from an anonymous Moroccan dealer. Physical characteristics: The stone of $2258 \mathrm{~g}$ total weight has irregular shape; the surface is reddish-brown; the fusion crust is not preserved.

Petrography: (C. A. Lorenz, Vernad) The meteorite has a brecciated texture; it is composed of lithic and mineral fragments (50 vol\%) embedded into a troilitemetal-silicate melt matrix $(13,22,15 \mathrm{vol} \%$ respectively). The mineral fragments are pyroxene, feldspar and silica; the rock fragments are fine-, medium- and coarsegrained pyroxene-feldspar rocks of equigranular, subophitic or gabbroic textures, fine-grained clastic and melt-matrix breccias and minor dunitic and peridothitic rocks. Coarse-grained pyroxenes contain the fine exsolution lamellae. The rock contains $11 \mathrm{vol} \%$ of fragments of $>2 \mathrm{~mm}$ in size. The matrix has heterogeneous texture varying in troilite, metal and silicates composition. Matrix silicates forming intergrowths with troilite are fine-grained subhedral pyroxene, anhedral feldspar and glass. Metal-and sulfide-poor matrix surrounding large lithic fragments are fine-grained melt rock. Metal forms irregular and globular grains in the troilite matrix and is composed of kamacite with inclusions of taenite. Accessory minerals are silica and chromite.

Geochemistry: Mineral compositions and geochemistry: (Kononkova N. $\mathrm{N}$, Vernad) Matrix pyroxene is $\mathrm{En}_{21.5-56.2} \mathrm{Wo}_{0.5-8.4}(\mathrm{Fe} / \mathrm{Mn}=16$ - 30, at. $)$, feldspar is $\mathrm{An}_{84.7-90.2} \mathrm{Ab}_{9.5-14.6}$; fragments pyroxene is $\mathrm{En}_{20.4-31.0} \mathrm{Wo}_{2.1-15.2}(\mathrm{Fe} / \mathrm{Mn}=23$ - 30, at.); feldspar is $\mathrm{An}_{94.5} \mathrm{Ab}_{5.5}$.

Classification: Mesosiderite of Type 4, anomalous; weathering is minimal. This mesosiderite is unusually troilite-rich and metal-poor.

Specimens: A piece and slices of $103 \mathrm{~g}$ total weight and thick section is on deposit in Vernad. Anonymous collector holds the main mass.

\section{Northwest Africa 11762 (NWA 11762)}

(Northwest Africa)

Purchased: 2014

Classification: HED achondrite (Eucrite, brecciated)

History: The meteorite was purchased from a local meteorite dealer in Zagora, Morocco.

Physical characteristics: Small dark greyish individual with some fusion crust.

Petrography: The meteorite is a fragmental breccia composed of up to $5 \mathrm{~mm}$ sized basaltic clasts and dark impact melt clasts set into a fine-grained clastic matrix. Basaltic clasts and matrix are dominantly composed of exsolved pyroxene and plagioclase grains of variable grain sizes ranging from about 20 to $600 \mu \mathrm{m}$. Several matrix pyroxenes display magmatic zoning. Minor phases include ilmenite, pyrrhotite, silica, apatite, and metallic iron. 
Geochemistry: low-Ca pyroxene $\mathrm{Fs}_{42.3 \pm 10.3} \mathrm{Wo}_{3.9 \pm 1.2}\left(\mathrm{Fs}_{20.9-51.3} \mathrm{Wo}_{2.0-5.7}, \mathrm{n}=15\right.$, $\mathrm{FeO} / \mathrm{MnO}=31$-47); Ca-pyroxene: $\mathrm{Fs}_{25.2 \pm 1.0} \mathrm{Wo}_{39.6 \pm 1.4}\left(\mathrm{Fs}_{23.2-27.6} \mathrm{Wo}_{37.1-42.1}, \mathrm{n}=14\right.$, $\mathrm{FeO} / \mathrm{MnO}=30-35)$; calcic plagioclase: $\mathrm{An}_{90.2 \pm 1.5}\left(\mathrm{An}_{87.5-92.5}, \mathrm{n}=14\right)$

\section{Northwest Africa 11763 (NWA 11763)}

(Northwest Africa)

Purchased: 2014

Classification: HED achondrite (Eucrite, brecciated)

History: The meteorite was purchased from a local meteorite dealer in Zagora, Morocco.

Physical characteristics: Small individual almost completely covered with fusion crust.

Petrography: The meteorite displays a light greyish to whitish interior and is composed of up to $4 \mathrm{~mm}$ sized angular to subrounded basaltic clasts set into a finegrained recrystallized matrix. Dominant minerals in clasts and matrix are exsolved pyroxene and calcic plagioclase. Grain sizes in the matrix are 10 to $40 \mu \mathrm{m}$; plagioclase laths in basaltic clasts are up to $300 \mu \mathrm{m}$ in length. Minor phases are silica, chromite, ilmenite, and troilite. Metallic iron was not detected. Contains some thin shock melt veins.

Geochemistry: low-Ca pyroxene $\mathrm{Fs}_{60.9 \pm 0.5} \mathrm{Wo}_{2.4 \pm 0.3}\left(\mathrm{Fs}_{59.9-61.5} \mathrm{Wo}_{1.9-3.0}, \mathrm{n}=14\right.$, $\mathrm{FeO} / \mathrm{MnO}=31-35)$; Ca-pyroxene: $\mathrm{Fs}_{26.7 \pm 1.3} \mathrm{Wo}_{42.6 \pm 1.4}\left(\mathrm{Fs}_{25.6-31.0} \mathrm{Wo}_{37.3-43.9}, \mathrm{n}=18\right.$, $\mathrm{FeO} / \mathrm{MnO}=30-38)$; calcic plagioclase: $\mathrm{An}_{89.4 \pm 0.5}\left(\mathrm{An}_{88.6-90.2}, \mathrm{n}=13\right)$

\section{Northwest Africa 11764 (NWA 11764)}

(Northwest Africa)

Purchased: 2014

Classification: Carbonaceous chondrite (CK5)

History: The meteorite was purchased from a local meteorite dealer in Zagora, Morocco.

Physical characteristics: Dark greyish to greenish individual with some fusion crust.

Petrography: The meteorite is composed of recrystallized matrix predominantly composed of Fe-rich olivine with several scattered and clearly defined chondrules (mean diameter about $0.8 \mathrm{~mm}$ ). Cr-rich magnetite is abundant. More minor phases include intermediate plagioclase, low-Ca pyroxene, and sulfides. Metal is virtually absent. Plagioclase grain size is about $100 \mu \mathrm{m}$.

Geochemistry: olivine: $\mathrm{Fa}_{33.4 \pm 0.3}, \mathrm{n}=16, \mathrm{FeO} / \mathrm{MnO}=133 \pm 9$; low-Ca

pyroxene: $\mathrm{Fs}_{26.8 \pm 0.2} \mathrm{Wo}_{0.8 \pm 0.1}, \mathrm{n}=9$; feldspar: $\mathrm{An}_{54.9 \pm 3.1} \mathrm{Ab}_{42.9 \pm 2.8}, \mathrm{n}=3$

\section{Northwest Africa 11765 (NWA 11765)}

(Northwest Africa)

Purchased: 2014 
Classification: Carbonaceous chondrite (CK6)

History: The meteorite was purchased from a local meteorite dealer in Erfoud, Morocco.

Physical characteristics: Small individual almost completely covered with fusion crust.

Petrography: The meteorite is largely composed of fine-grained recrystallized matrix mainly consisting of ferrous olivine. Chondrules are only rarely encountered; one CAI was found. Minor phases include plagioclase, low-Ca pyroxene, Ca-pyroxene and sulfides. Cr-rich magnetite is abundant, metal is virtually absent.

Geochemistry: olivine: $\mathrm{Fa}_{31.3 \pm 0.3}, \mathrm{n}=14, \mathrm{FeO} / \mathrm{MnO}=128 \pm 13$; low-Ca

pyroxene: $\mathrm{Fs}_{26.4 \pm 0.3} \mathrm{Wo}_{1.6 \pm 1.4}, \mathrm{n}=10$; feldspar: $\mathrm{An}_{79.8 \pm 0.8} \mathrm{Ab}_{19.9 \pm 0.8}, \mathrm{n}=5$

\section{Northwest Africa 11766 (NWA 11766)}

(Northwest Africa)

Purchased: 2014

Classification: Carbonaceous chondrite (CV3)

History: The meteorite was purchased from a local meteorite dealer in Erfoud, Morocco.

Physical characteristics: Almost black individual without fusion crust.

Petrography: Carbonaceous chondrite composed of separated and clearly defined chondrules (mean apparent diameter about $1.1 \mathrm{~mm}$ ), CAIs (up to $7 \mathrm{~mm}$ ), and olivine amoeboids all set into a fine-grained almost black matrix. Type II chondrules are absent.

\section{Northwest Africa 11767 (NWA 11767)}

(Northwest Africa)

Purchased: 2012

Classification: Lunar meteorite

History: The meteorite was purchased from a local meteorite dealer in Erfoud, Morocco.

Physical characteristics: Many small light greenish fragments lacking any fusion crust.

Petrography: Monomict olivine-rich gabbroic breccia predominantly composed of up to $4 \mathrm{~mm}$ sized Fe-rich olivine, pigeonite, augite and calcic plagioclase. Minor phases include chromite, troilite and K-feldspar. The meteorite contains abundant shock melt veins along which some olivine has been transformed into ringwoodite. Geochemistry: olivine: $\mathrm{Fa}_{31.6 \pm 0.2}\left(\mathrm{Fa}_{31.3-32.0}, \mathrm{n}=13, \mathrm{FeO} / \mathrm{MnO}=85-105\right)$; pigeonite: $\mathrm{Fs}_{25.6 \pm 1.1} \mathrm{Wo}_{10.7 \pm 2.8}\left(\mathrm{Fs}_{23.3-27.7} \mathrm{Wo}_{5.9-16.4}, \mathrm{n}=12, \mathrm{FeO} / \mathrm{MnO}=46-63\right)$; augite: $\mathrm{Fs}_{16.1 \pm 1.0} \mathrm{Wo}_{35.7 \pm 2.1}\left(\mathrm{Fs}_{14.8-17.8} \mathrm{Wo}_{32.1-39.0}, \mathrm{n}=12, \mathrm{FeO} / \mathrm{MnO}=41-52\right)$; calcic plagioclase: $\mathrm{An}_{93.1 \pm 0.8}\left(\mathrm{An}_{91.1-93.9}, \mathrm{n}=10\right)$

Classification: Lunar Olivine-Gabbros. The meteorite might be paired with NWA 773 and paired stones. 


\section{Northwest Africa 11768 (NWA 11768)}

(Northwest Africa)

Purchased: 2013

Classification: Carbonaceous chondrite (CV3)

History: The meteorite was purchased from a local meteorite dealer in Erfoud, Morocco.

Physical characteristics: Five dark greyish fragments without fusion crust.

Petrography: Carbonaceous chondrite composed of chondrules (mean diameter about $1 \mathrm{~mm}$; up to $6 \mathrm{~mm}$ sized), CAIs, and olivine amoeboids all set into a finegrained dark brownish matrix. Type II chondrules are absent. Most chondrules show reddish staining due to terrestrial weathering.

Northwest Africa 11769 (NWA 11769)

(Northwest Africa)

Purchased: 2014

Classification: Rumuruti chondrite (R3)

History: The meteorite was purchased from a local meteorite dealer in Zagora, Morocco.

Physical characteristics: Brownish individual without fusion crust.

Petrography: The meteorite shows an orange interior and is composed of 200 to $800 \mu \mathrm{m}$ sized chondrules (mean about $400 \mu \mathrm{m}$ ) in abundant matrix dominated by Fe-rich olivine. Olivine and low-Ca pyroxene in chondrules are mostly

unequilibrated. Minor phases include pentlandite, sodic plagioclase, and Ti-bearing chromite; no metal has been detected.

Northwest Africa 11770 (NWA 11770)

(Northwest Africa)

Purchased: 2014

Classification: Carbonaceous chondrite (CV3)

History: The meteorite was purchased from a local meteorite dealer in Zagora, Morocco.

Physical characteristics: Dark greyish individual without fusion crust.

Petrography: Carbonaceous chondrite composed of up separated and partly flattened chondrules (mean diameter about $1.2 \mathrm{~mm}$; up to $5 \mathrm{~mm}$ ), CAIs (up to 6 $\mathrm{mm}$ ), and olivine amoeboids all set into a fine-grained dark brownish matrix. Type II chondrules are absent. Most chondrules show reddish staining due to terrestrial weathering.

Northwest Africa 11771 (NWA 11771)

(Northwest Africa) 
Purchased: 2014

Classification: HED achondrite (Diogenite)

History: The meteorite was purchased from a local meteorite dealer in Zagora, Morocco.

Physical characteristics: Greyish individual partly covered with fusion crust Petrography: Monomict breccia predominantly composed of blocky up to $2 \mathrm{~mm}$ sized orthoproxenes and more fine-grained cataclastic interstitial regions. Minor phases include feldspar, olivine, silica, chromite, and FeNi metal. No metallic iron has been detected.

Geochemistry: low-Ca pyroxene: $\mathrm{Fs}_{29.2 \pm 0.3} \mathrm{Wo}_{4.2 \pm 0.2}\left(\mathrm{Fs}_{28.7-29.5} \mathrm{Wo}_{3.9-4.6}, \mathrm{n}=18\right.$, $\mathrm{FeO} / \mathrm{MnO}=27-32$ ); olivine: $\mathrm{Fa}_{37.7}, \mathrm{n}=1, \mathrm{FeO} / \mathrm{MnO}=54$; calcic plagioclase:

$\mathrm{An}_{92.7 \pm 0.4}\left(\mathrm{An}_{92.2-93.4}, \mathrm{n}=5\right)$

Northwest Africa 11772 (NWA 11772)

(Northwest Africa)

Purchased: 2014

Classification: Rumuruti chondrite (R5)

History: The meteorite was purchased from a local meteorite dealer in Zagora, Morocco.

Physical characteristics: Thirty eight small dark greyish fragments partly covered with fusion crust. Sawn surface shows a fresh greyish interior.

Petrography: The meteorite is a R-type breccia composed of up to $8 \mathrm{~mm}$ sized angular to subrounded clasts set into a more fine-grained clastic matrix. Chondrules are $100 \mu \mathrm{m}$ to $1 \mathrm{~mm}$ with an average of about $350 \mu \mathrm{m}$. Fe-rich olivine is the most abundant mineral phase in all lithologies. More minor phases include low-Ca pyroxene, augite, pentlandite, and sodic plagioclase (mean grain size about $40 \mu \mathrm{m}$ ). No metal has been detected. All silicates are compositionally equilibrated.

Geochemistry: olivine: $\mathrm{Fa}_{38.2 \pm 0.3}(\mathrm{n}=13$, FeO/MnO=74-87);low-Ca pyroxene: $\mathrm{Fs}_{30.1 \pm 0.2} \mathrm{Wo}_{1.3 \pm 0.1}(\mathrm{n}=11, \mathrm{FeO} / \mathrm{MnO}=47-58)$; augite: $\mathrm{Fs}_{12.0 \pm 0.3} \mathrm{Wo}_{44.3 \pm 0.4}(\mathrm{n}=10$, $\mathrm{FeO} / \mathrm{MnO}=37-55)$

Classification: R5 according to mineral chemistry and plagioclase grain size.

\section{Northwest Africa 11773 (NWA 11773)}

(Northwest Africa)

Purchased: 2014

Classification: Carbonaceous chondrite (CK6)

History: The meteorite was purchased from a local meteorite dealer in Zagora, Morocco.

Physical characteristics: Dark greyish individual without fusion crust.

Petrography: The meteorite displays a greyish to light greenish interior and is composed of abundant recrystallized matrix dominated by of Fe-rich olivine with only few scattered and poorly defined chondrules (mean diameter about $0.7 \mathrm{~mm}$ ). 
Cr-rich magnetite is abundant. More minor phases include intermediate plagioclase, Ca-pyroxene, and sulfides. No metal has been detected.

Geochemistry: olivine: $\mathrm{Fa}_{33.2 \pm 0.2}, \mathrm{n}=16, \mathrm{FeO} / \mathrm{MnO}=118 \pm 9$; Ca-pyroxene:

$\mathrm{Fs}_{9.7 \pm 1.2} \mathrm{Wo}_{47.2 \pm 3.1}, \mathrm{n}=12$; feldspar: $\mathrm{An}_{64.2} \mathrm{Ab}_{35.0} \mathrm{Or}_{0.8}, \mathrm{n}=2$

\section{Northwest Africa 11774 (NWA 11774) \\ Mauritania \\ Purchased: 2018 Jan \\ Classification: Mesosiderite}

History: Found near Ain Ben Tili, Mauritania, in December 2017 and purchased by Bob Falls in January 2018 from a Moroccan dealer.

Physical characteristics: A single metal-rich mass (439.2 g) with a weathered exterior. The fresh interior consists mainly of metal with $\sim 10$ vol. $\%$ interstitial silicate-rich material.

Petrography: (A. Irving and S. Kuehner, UWS) Composed predominantly of granular kamacite (containing small, irregularly-shaped grains of taenite and rare schreibersite) together with interstitial regions containing calcic plagioclase, orthopyroxene, silica polymorph, chromite, merrillite, ilmenite and troilite.

Geochemistry: Orthopyroxene $\left(\mathrm{Fs}_{30.3-31.7} \mathrm{Wo}_{2.9-3.8}, \mathrm{FeO} / \mathrm{MnO}=18-19, \mathrm{~N}=3\right)$, plagioclase $\left(\mathrm{An}_{85.3-86.9} \mathrm{Or}_{0.5-0.2}, \mathrm{~N}=2\right)$.

Classification: Mesosiderite. This specimen has close textural and mineralogical similarities to the metal-rich nodules in Bondoc, and is unusual because of its very high metal/silicate ratio relative to typical examples.

Specimens: $24.1 \mathrm{~g}$ in the form of a polished slice at $U W B$; remainder with Mr. R. Falls.

\section{Northwest Africa 11775 (NWA 11775) \\ Morocco \\ Purchased: 2018 Jan \\ Classification: Ureilite}

History: The sample was bought in Malaga, Spain, from a Saharawi meteorite dealer

Petrography: (K. Metzler, IfP) Ultramafic rock with metamorphic texture $\left(\sim 120^{\circ}\right.$ triple junctions), consisting of olivine and pyroxene grains up to $\sim 1.5 \mathrm{~mm}$. Reverse zoning of olivine grains with Mg-rich zones at the margins and along cracks. Metal is concentrated along grain boundaries and cracks and partly oxidized by terrestrial weathering.

Geochemistry: Mineral compositions and geochemistry: Olivine cores $(\mathrm{n}=10)$ : $\mathrm{Fa}_{20.1 \pm 0.3}$; Olivine rims $(\mathrm{n}=5): \mathrm{Fa}_{7.7 \pm 2.3}$. Pyroxene grains $(\mathrm{n}=10)$ :

$\mathrm{Fs}_{17.3 \pm 0.3} \mathrm{Wo}_{3.6 \pm 0.1}$. The mean concentration of $\mathrm{Cr}_{2} \mathrm{O}_{3}$ in olivine and pyroxene is 0.5 $\mathrm{wt} \%$ and $0.7 \mathrm{wt} \%$, respectively. 


\section{Northwest Africa 11776 (NWA 11776)}

(Northwest Africa)

Purchased: 2017

Classification: Ordinary chondrite (LL6, anomalous)

History: 1 stone weighing $100.7 \mathrm{~g}$ was found near Laayoune, Morocco, prior to 2017. J. Donald Cline and John Sinclair acquired the sample from a meteorite dealer at the Tucson Gem and Mineral Show in February of 2017.

Physical characteristics: Sample is dark-orange-brown in color, irregularly shaped. The sample lacks fusion crust. The interior of the cut face shows a brecciated texture.

Petrography: Description and Classification (A. Lov, App): Sample is a breccia composed of up to $1 \mathrm{~cm}$ angular recrystallized clasts of chondritic rock with barely discernible chondrules. All FeNi has been oxidized. Sulfides are partly altered. Average diameter of relict chondrules $1314 \mu \mathrm{m}$. Magnetic susceptibility $\log \chi(\times$ $\left.10^{-9} \mathrm{~m}^{3} / \mathrm{kg}\right)=3.88$ (measured with a SM30 instrument).

Geochemistry: (A. Love, App) Olivine $\left(\mathrm{Fa}_{35.7 \pm 0.3}, \mathrm{~N}=8, \mathrm{Fe} / \mathrm{Mn}=69.6 \pm 1.0\right)$, low $\mathrm{Ca}$ pyroxene $\left(\mathrm{Fs}_{26.6 \pm 0.4} \mathrm{Wo}_{1.9 \pm 0.3}, \mathrm{~N}=8\right)$. Oxygen isotopes (K. Ziegler, $\left.U N M\right)$ : analyses of acid-washed subsamples by laser fluorination gave, respectively, $\delta^{17} \mathrm{O}=4.321$, 4.476, 4.508; $\delta^{18} \mathrm{O}=5.841,6.144,6.148 ; \Delta^{17} \mathrm{O}=1.237,1.232,1.262$ (all per mil). Classification: Ordinary Chondrite (LL6-anomalous) S6 W3. The oxygen isotopes are in the LL range. Mean olivine and pyroxene compositions are outside the normal range for LL chondrites, hence the anomalous designation.

Specimens: PARI holds the main mass. One endcut weighing $20.02 \mathrm{~g}$ and a polished thin section are on deposit at App.

\section{Northwest Africa 11777 (NWA 11777)}

(Northwest Africa)

Purchased: 2017

Classification: HED achondrite (Eucrite)

History: One mostly crusted stone weighing $705 \mathrm{~g}$ was found in Morocco prior to 2017. J. Donald Cline and John Sinclair acquired the sample from a meteorite dealer at the Tucson Gem and Mineral Show in February of 2017.

Physical characteristics: Sample is rounded to irregularly shaped and contains many narrow and broad, shallow regmaglypts on $1 / 3$ of its surface. Sample is $\sim 85 \%$ covered by orange brown to dark brown patina, some of which may be original fusion crust. Fractures within the patina show a light-colored interior with finegrained basaltic texture.

Petrography: Description and classification (A. Love, App): Sample is a breccia composed of up to $1 \mathrm{~cm}$, rounded to irregularly shaped, ophitic, cumulate textured clasts and subophitic basaltic textured clasts. Clasts are set within a matrix of granular eucritic debris. Pyroxenes are exsolved. Minerals: orthopyroxene, Capyroxene, ilmenite, phosphates, $\mathrm{SiO}_{2}$ polymorph, FeS.

Geochemistry: (A. Love, App) Eucrite: low-Ca pyroxene $\mathrm{Fs}_{61.2 \pm 0.5} \mathrm{Wo}_{2.5 \pm 0.4}\left(\mathrm{Fs}_{60.2-}\right.$ $\left.{ }_{62.1} \mathrm{Wo}_{2.0-3.2}, \mathrm{FeO} / \mathrm{MnO}=29.4-31.1, \mathrm{~N}=14\right)$; high-Ca pyroxene exsolution lamellae 
$\mathrm{Fs}_{26.4 \pm 0.7} \mathrm{Wo}_{44.2 \pm 0.6}\left(\mathrm{Fs}_{25.1-27.8} \mathrm{Wo}_{42.9-44.9}, \mathrm{FeO} / \mathrm{MnO}=29.6-32.0, \mathrm{~N}=14\right)$; plagioclase $\left(\mathrm{An}_{88.0 \pm 2.0} \mathrm{Or}_{0.0}\left(\mathrm{An}_{85.2-91.9} \mathrm{Or}_{0.0}, \mathrm{~N}=14\right)\right.$;

Classification: HED (equilibrated basaltic eucrite) Original igneous textures within clasts are preserved while major element compositions of pyroxenes within sample are equilibrated. Plagioclase compositions are poorly equilibrated.

Specimens: $P A R I$ holds the main mass. One $20 \mathrm{~g}$ endcut and a polished thin section are on deposit at App.

\section{Northwest Africa 11778 (NWA 11778)}

(Northwest Africa)

Purchased: June 2013

Classification: Ordinary chondrite (H5)

History: Purchased by WrocU, Faculty of Geoengineering, Mining and Geology from Moroccan dealer in Zagora; $120 \mathrm{~g}$ was cut off and analyzed in thin section; $16.5 \mathrm{~g}$ of sample was powdered and for bulk chemistry.

Physical characteristics: Single stone $(767.5 \mathrm{~g})$ covered by black (with slight brown shade) fusion crust, $0.5 \mathrm{~mm}$ thick.

Petrography: (T.A. Przylibski, K. Luszczek, UTWroc) Chondrules (75 vol.\%) include BO, PO, GO, RP, POP, and C types. Chondrules are mostly well defined and often have sharp edges. Chondrule diameters are from 0.2 to $1.2 \mathrm{~mm}$, average $600 \pm 290 \mathrm{um}$. One compound chondrule is $6.7 \mathrm{~mm}$. Devitrified, partly crystallized mesostasis. Opaque minerals (20 vol.\%) include troilite, kamacite and taenite, and rare chromite. Small feldspar grains discernible in electron images. Accessory apatite crystals. Matrix (5 vol.\%) is fine-grained, crystalline and transparent, composed mostly of olivine crystals. Around $25 \%$ of low-Ca pyroxene crystals are twinned, some of them showing inclined extinction. Some olivine and pyroxene crystals show undulatory extinction. Irregular veins and cracks are filled with opaque iron oxides and hydroxides. Some grains of kamacite are weathered on edges.

Geochemistry: (R. Kryza, WrocU; T.A. Przylibski, K. Luszczek, UTWroc) Olivine $\mathrm{Fa}_{18.0 \pm 0.0}(\mathrm{n}=6)$; Pyroxene $\mathrm{Fs}_{13.2 \pm 0.0} \mathrm{Wo}_{1.1}(\mathrm{~N}=12)$; Chromite $(\mathrm{Mg} 0.3 \mathrm{Fe} 2+0.38$ Mn0.03 Zn0.01 Si0.19 Ti0.02 Ca0.01)(Al0.27 Fe3+0.38 Cr1.27)O4; kamacite (5.85 wt\%), taenite (from 40.4 to $52.7 \mathrm{wt} \%$ ). Bulk chemistry (in wt\%): Si 15.69, Al 0.99, Fe 29.74, Mg 12.42, Ca 1.19, Na 0.55, K 0.07, Ti 0.05, P 0.12, Mn 0.22, Cr 0.34, Ni 1.81, C 0.07, S 2.04; (in ppm) Ba 8, Co 961.2, Ga 4.8, Hf 6, Nb 0.3, Rb 1.5, Sr 15.2, V 64, Mo 1.3, Cu 93.5, Pb 0.1, Zn 10, As 0.7, Se 9.1, Zr 253.4, Au 207.3, Y 15.7, La 0.6, Ce 0.8, Pr 0.13, Nd 0.6, Sm 0.19, Eu 0.04, Gd 0.25, Tb 0.05, Dy 0.26 , Ho 0.07, Er 0.18, Tm 0.02, Yb 0.18, Lu 0.02

Classification: Ordinary chondrite, H5, S2, W1.

\section{Northwest Africa 11779 (NWA 11779)}

(Northwest Africa)

Purchased: 2012 
Classification: Ordinary chondrite (L6)

History: Purchased by Tomasz Jakubowski from Moroccan dealer in 2012; $40 \mathrm{~g}$ was cut off and analyzed as one thin section on petrographic microscope in polarized and reflected light, other one thin section was analyzed on microprobe; $16.5 \mathrm{~g}$ of sample was powdered and bulk chemistry was analyzed by ICP MS Physical characteristics: Single specimen with partly preserved fusion crust. Petrography: (T.A. Przylibski, K. Luszczek UTWroc) Rare chondrules, most with gradual transition to matrix. Only a few chondrules have sharp edges. Chondrules are identified as: BO, RP, PO, GO, PP. Some of them have rims up to $0.2 \mathrm{~mm}$ thick. Chondrule diameters are from 0.5 to $1.9 \mathrm{~mm}$, average $1.2 \mathrm{~mm}$. Some chondrules are up to $2.7 \mathrm{~mm}$ in diameter. Mesostasis is crystallized and matrix is corse-grained with olivine crystals up to $0.5 \mathrm{~mm}$ in diameter. Also feldspar crystals are clearly visible and some of them are polysynthetically twinned. Feldspars are from 0.02 to $0.15 \mathrm{~mm}$ large. Opaque minerals (around 10 vol.\%) include troilite, kamacite and taenite, and rare chromite.

Geochemistry: (R. Kryza WrocU; T.A. Przylibski, K. Luszczek, UTWroc) Olivine $\mathrm{Fa}_{24.9}$; pyroxene $\mathrm{Fs}_{19.4} \mathrm{Wo}_{1.3}$; chromite $\left(\mathrm{Mg} 0.12 \mathrm{Fe}^{2+} 0.71 \mathrm{Mn} 0.03 \mathrm{Zn} 0.01\right.$ Ti0.07)(A10.25 $\left.\mathrm{Fe}^{3+} 0.19 \mathrm{Cr} 1.56\right) \mathrm{O} 4$; kamacite (5.7-6.7 wt\%), taenite (up to 29.6 $\mathrm{wt}^{\%} \%$ ), troilite without traces of Ni. Bulk chemistry (in wt\%): Si 19.65, Al 1.19, Fe 18.45, Mg 15.51, Ca 1.52, Na 0.70, K 0.09, Ti 0.07, P 0.10, Mn 0.28, Cr 0.39, Ni 1.20, C 0.07, S 1.61; (in ppm) Ba 66, Co 306.7, Ga 4.6, Hf 13.9, Nb 0.8, Rb 2.1, Sn 18, Sr 24.7, V 74, Mo 0.8, Cu 82.1, Pb 0.2, Zn 20, Se 6.9, Zr 581.3, Au 126.5, Hg 0.11, Y 34.5, La 0.7, Ce 1.4, Pr 0.19, Nd 0.9, Sm 0.28, Eu 0.09, Gd 0.37, Tb 0.06, Dy 0.38 , Ho 0.08 , Er 0.22, Tm 0.03, Yb 0.2, Lu 0.03

Classification: Ordinary chondrite, L6, S1, W1

\section{Northwest Africa 11781 (NWA 11781)}

(Northwest Africa)

Purchased: 2017 Aug 09

Classification: Carbonaceous chondrite (CM2)

History: The meteorite was purchased from a meteorite dealer in Moscow, Russia. Petrography: (S. V. Berzin and K. A. Dugushkina, RAS-UB) Chondrule $\sim 20 \%$, refractory inclusion (CAIs, AOAs, Forsterite rich objects) $\sim 3-5 \%$ and fine-grained matrix. Most chondrules are porphyritic. Average chondrule size is $0.3 \mathrm{~mm}$.

Chondrule mesostasis is rare. The matrix contains phyllosilicates and calcite. CAIs are small (0.05-0.3 mm in size) and very fine grained. The dominant minerals of CAIs are spinel, Ca-pyroxene, hibonite, with accessory perovskite. Some CAIs have forsterite rims. AOAs mainly has spinel-diopside inclusion. Forsterite rich objects have low-Ca pyroxene rims. Refractory forsterite is zoned. Unshocked, low grade of terrestrial weathering (metal partially oxidized).

Geochemistry: Element composition by ICP-MS (ppm): Li 6.05, Sc 2.93, Ti 427, V 55.9, Cr 2307, Mn 1365, Co 236, Ni 2597, Cu 86.0, Zn 129, Ga 6.82, Ge 8.91, As 2.69, Se 4.82, Rb 0.952, Sr 190, Y 1.87, Zr 5.54, Nb 0.431, Mo 1.33, Ag 0.197, Cd 0.151, Sn 1.180, Sb 0.146, Te 0.949, Cs 0.038, Ba 283, La 0.545, Ce 1.33, Pr 
0.194, Nd 0.963, Sm 0.281, Eu 0.104, Gd 0.458, Tb 0.061, Dy 0.409, Ho 0.088, Er 0.267, Tm 0.040, Yb 0.264, Lu 0.041, Hf 0.209, Ta 0.365, W 0.151, Tl 0.053, Pb 7.36, Bi 0.024, Th 0.100, U 0.481.

Classification: CM2 Carbonaceous chondrite

\section{Northwest Africa 11782 (NWA 11782) \\ Mali}

Purchased: 2018

Classification: Ordinary chondrite (L3)

History: Purchased by Abdelhadi Aithiba in Mali.

Physical characteristics: Broken surface reveals numerous, distinct, closely packed chondrules. A single large sulfide grain $~ 5 \mathrm{~mm}$ was also observed.

Petrography: (C. Agee, $U N M)$ microprobe examination of a polished mount of this lithology shows numerous porphyritic chondrules, most with mesostasis or glass. Cl-rich apatite detected.

Geochemistry: (C. Agee, $U N M$ ) Olivine $\mathrm{Fa}_{24.7 \pm 5.5}, \mathrm{Cr}_{2} \mathrm{O}_{3}=0.10 \pm 0.21$ (wt\%), $\mathrm{n}=21$; low-Ca pyroxene $\mathrm{Fs}_{19.8 \pm 5.6} \mathrm{Wo}_{1.2 \pm 0.7}, \mathrm{n}=11$.

Classification: Ordinary chondrite (L3), subtype approximately L3.7 based on Fa and Fa s.d.

Specimens: $39.4 \mathrm{~g}$ including a probe mount on deposit at $U N M$, Abdelhadi Aithiba holds the main mass.

\section{Northwest Africa 11783 (NWA 11783)}

(Northwest Africa)

Purchased: 20 Sep 2014

Classification: Lunar meteorite (feldspathic breccia)

History: Purchased by Jay Piatek from a Moroccan meteorite dealer.

Physical characteristics: Several visually identical appearing pieces. No fusion crust. A saw cut reveals a fragmental breccia with white feldspathic clasts set in a dark gray ground mass.

Petrography: (C. Agee, UNM) This meteorite is a breccia of fragmental pyroxene, olivine, plagioclase grains. There are many domains that are fine-grained and cataclastic with shock melt and vesicles. Fe-metal, chromite, ilmenite, silica, and a phosphate mineral were detected.

Geochemistry: (C. Agee, $U N M$ ) olivine Fa: 28.1 $\pm 4.8, \mathrm{Fe} / \mathrm{Mn}=100 \pm 2$, n=4; clinopyroxene $\mathrm{Fs}_{19.0 \pm 4.4} \mathrm{Wo}_{23.1 \pm 17.6}, \mathrm{Fe} / \mathrm{Mn}=52 \pm 8, \mathrm{n}=3$; plagioclase $\mathrm{An}_{94.6 \pm 1.3}, \mathrm{n}=3$; Shock melt (20 $\mu \mathrm{m}$ defocused electron beam, proxy for bulk meteorite composition): $\mathrm{SiO}_{2}=46.0 \pm 0.4, \mathrm{TiO}_{2}=0.51 \pm 0.02, \mathrm{Al}_{2} \mathrm{O}_{3}=28.0 \pm 0.5$, $\mathrm{Cr}_{2} \mathrm{O}_{3}=0.11 \pm 0.01, \mathrm{MgO}=6.0 \pm 0.2, \mathrm{FeO}=4.7 \pm 0.2, \mathrm{MnO}=0.07 \pm 0.00, \mathrm{CaO}=15.3 \pm 0.1$, $\mathrm{Na}_{2} \mathrm{O}=0.61 \pm 0.03, \mathrm{~K}_{2} \mathrm{O}=0.16 \pm 0.01($ all wt $\%), \mathrm{n}=7$.

Classification: Lunar feldspathic breccia Specimens: $13 \mathrm{~g}$ including a probe mount on deposit at $U N M$, Jay Piatek holds the main mass. 


\section{Northwest Africa 11784 (NWA 11784)}

(Northwest Africa)

Purchased: 27 Jul 2015

Classification: Ureilite

History: Purchased by Jay Piatek from a Moroccan meteorite dealer.

Physical characteristics: Single stone. A saw cut reveals medium grained, gray, pyroxene crystals with a mildly aligned orientation. Very fine, scattered metal and graphite grains are visible throughout.

Petrography: (C. Agee, $U N M$ ) Microprobe examination shows olivine, pigeonite, and augite grains, many in the size range $\sim 1-2 \mathrm{~mm}$. Graphite blades are present up to $\sim 500 \mu \mathrm{m}$ in length. Fine metal blebs and oxidized iron are observed along olivine grain boundaries. Olivines have oxidation-reduction rims.

Geochemistry: (C. Agee, UNM) Olivine $\mathrm{Fa}_{12.0 \pm 3.5}, \mathrm{Fe} / \mathrm{Mn}=25 \pm 8, \mathrm{Cr}_{2} \mathrm{O}_{3}=0.61 \pm 0.02$ $\mathrm{CaO}=0.29 \pm 0.03\left(\mathrm{wt} \%\right.$ ), $\mathrm{n}=6$; olivine cores $\mathrm{Fa}_{14.2 \pm 0.7}, \mathrm{n}=4$; olivine rims $\mathrm{Fa}_{7.7 \pm 0.4}, \mathrm{n}=2$; pigeonite $\mathrm{Fs}_{12.7 \pm 0.3} \mathrm{Wo}_{4.9 \pm 0.0}, \mathrm{Fe} / \mathrm{Mn}=20 \pm 0, \mathrm{n}=6$; augite $\mathrm{Fs}_{8.1 \pm 0.1} \mathrm{Wo}_{35.4 \pm 0.4}$, $\mathrm{Fe} / \mathrm{Mn}=16 \pm 1, \mathrm{n}=5$.

Classification: Ureilite, augite-bearing.

Specimens: $20.2 \mathrm{~g}$ on deposit at UNM, Jay Piatek holds the main mass.

\section{Northwest Africa 11785 (NWA 11785)}

(Northwest Africa)

Purchased: 22 May 2015

Classification: HED achondrite (Eucrite, melt breccia)

History: Purchased by Jay Piatek from a Moroccan meteorite dealer.

Physical characteristics: Single stone, no fusion crust. Exterior shows numerous white clasts set in a dark matrix of shock melt.

Petrography: (C. Agee, UNM) Microprobe examination shows a cataclastic mix of fragmental pyroxene and feldspar grains engulfed in abundant shock melt veins and pools. Accessory chromite and ilmenite were detected.

Geochemistry: (C. Agee, $U N M$ ) Low-Ca pyroxene $\mathrm{Fs}_{53.1} \mathrm{Wo}_{1.9}, \mathrm{Fe} / \mathrm{Mn}=32, \mathrm{n}=1$; high-Ca pyroxene $\mathrm{Fs}_{22.6} \mathrm{Wo}_{44.1}, \mathrm{Fe} / \mathrm{Mn}=28$, n=1; plagioclase $\mathrm{An}_{90.5 \pm 1.3}, \mathrm{n}=2$; Shock melt (20 $\mu \mathrm{m}$ defocused electron beam, proxy for bulk meteorite composition): $\mathrm{SiO}_{2}=46.1 \pm 1.9, \mathrm{TiO}_{2}=0.8 \pm 0.02, \mathrm{Al}_{2} \mathrm{O}_{3}=25.1 \pm 9.9, \mathrm{Cr}_{2} \mathrm{O}_{3}=0.13 \pm 0.10, \mathrm{MgO}=5.0 \pm 3.5$, $\mathrm{FeO}=9.2 \pm 6.0, \mathrm{MnO}=0.28 \pm 0.19, \mathrm{CaO}=14.4 \pm 2.7, \mathrm{Na}_{2} \mathrm{O}=0.66 \pm 0.27, \mathrm{~K}_{2} \mathrm{O}=0.05 \pm 0.01$ (all $\mathrm{wt} \%), \mathrm{n}=5$.

Classification: Eucrite-melt breccia, eucrite pyroxene compositions are consistent with cumulate eucrite.

Specimens: $20 \mathrm{~g}$ including a probe mount on deposit at $U N M$, Jay Piatek holds the main mass.

\section{Northwest Africa 11786 (NWA 11786)}


(Northwest Africa)

Purchased: 30 Aug 2015

Classification: HED achondrite (Eucrite, monomict)

History: Purchased by Jay Piatek from a Moroccan meteorite dealer.

Physical characteristics: Single stone, no fusion crust. Exterior shows, dark gray, fine grained texture.

Petrography: (C. Agee, UNM) (C. Agee, $U N M)$ Microprobe examination shows approximately $60 \%$ pyroxene and $35 \%$ plagioclase. Shock melt veins and mild brecciation are present. Accessory troilite, chromite, silica, and ilmenite were detected.

Geochemistry: (C. Agee, UNM) Low-Ca pyroxene $\mathrm{Fs}_{59.0 \pm 2.1} \mathrm{Wo}_{5.2 \pm 2.5}$, $\mathrm{Fe} / \mathrm{Mn}=31 \pm 1, \mathrm{n}=1$; high-Ca pyroxene $\mathrm{Fs}_{27.6 \pm 1.3} \mathrm{Wo}_{42.4 \pm 1.5}, \mathrm{Fe} / \mathrm{Mn}=31 \pm 3, \mathrm{n}=4$; plagioclase $\mathrm{An}_{89.7 \pm 0.7}, \mathrm{n}=2$.

Classification: Eucrite, monomict

Specimens: $22 \mathrm{~g}$ including a probe mount on deposit at $U N M$, Jay Piatek holds the main mass.

\section{Northwest Africa 11787 (NWA 11787)}

Mauritania

Purchased: 2017

Classification: Lunar meteorite (feldspathic breccia)

History: Purchased in Mauritania by Dustin Dickens from anonymous meteorite hunter.

Physical characteristics: Many visually identical appearing pieces, ranging from several kilograms to sub-gram fragments. Some pieces show a light-gray, remnant fusion crust on one or more faces, with most having no fusion crust. Saw cut reveals a fragmental breccia with white feldspathic clasts set in a dark-gray ground mass.

Petrography: (C. Agee, $U N M)$ This meteorite is a polymict breccia of fragmental feldspathic, troctolitic and mafic lithologies, as well as fragmental pyroxene, olivine, plagioclase grains, and shock melt with vescicles.

Geochemistry: (C. Agee, $U N M$ ) olivine Fa: 25.6 \pm 8.9 , Fe/Mn=95 \pm 18 , n=13; lowCa pyroxene $\mathrm{Fs}_{26.2 \pm 8.8} \mathrm{Wo}_{6.2 \pm 2.5}, \mathrm{Fe} / \mathrm{Mn}=52 \pm 4, \mathrm{n}=7$; subcalcic augite $\mathrm{Fs}_{35.9 \pm 5.8} \mathrm{Wo}_{26.9 \pm 7.9}, \mathrm{Fe} / \mathrm{Mn}=69 \pm 5, \mathrm{n}=2$; augite $\mathrm{Fs}_{14.5 \pm 7.1} \mathrm{Wo}_{41.0 \pm 2.0}, \mathrm{Fe} / \mathrm{Mn}=45 \pm 15$, $\mathrm{n}=3$; plagioclase $\mathrm{An}_{96.2 \pm 0.8}, \mathrm{n}=6$; Shock melt (20_m defocused electron beam, proxy for bulk meteorite composition): $\mathrm{SiO}_{2}=42.9 \pm 0.4, \mathrm{TiO}_{2}=0.11 \pm 0.05$, $\mathrm{Al}_{2} \mathrm{O}_{3}=31.1 \pm 4.0, \mathrm{Cr}_{2} \mathrm{O}_{3}=0.10 \pm 0.05, \mathrm{MgO}=5.1 \pm 3.3, \mathrm{FeO}=3.3 \pm 2.0, \mathrm{MnO}=0.05 \pm 0.05$, $\mathrm{CaO}=16.6 \pm 1.6, \mathrm{Na}_{2} \mathrm{O}=0.37 \pm 0.06, \mathrm{~K}_{2} \mathrm{O}=0.04 \pm 0.00($ all $\mathrm{wt} \%), \mathrm{n}=3$.

Classification: Lunar feldspathic breccia

Specimens: $21.25 \mathrm{~g}$ including a probe mount on deposit at $U N M$, Dustin Dickens holds the main mass.

Northwest Africa 11788 (NWA 11788) 


\section{Mali}

Purchased: 2017

Classification: Lunar meteorite (feldspathic breccia)

History: Purchased in Mali by Dustin Dickens from anonymous meteorite hunter. Physical characteristics: Many dark visually identical appearing pieces, with masses ranging from $\sim 2 \mathrm{~kg}$ to many sub-g fragments, showing no fusion crust. Saw cut reveals a finely fragmental breccia with white feldspathic clasts set in a dark gray ground mass with metal flecks and minor vesiculation appearing throughout. Petrography: (C. Agee, UNM) This meteorite is a breccia of fragmental pyroxene, olivine, plagioclase grains. There are domains that are fine-grained and cataclastic with shock melt and vesicles.

Geochemistry: (C. Agee, $U N M$ ) olivine Fa: 31.2 \pm 9.4 , Fe/Mn=95 \pm 7 , $\mathrm{n}=12$; clinopyroxene $\mathrm{Fs}_{36.0 \pm 10.0} \mathrm{Wo}_{22.2 \pm 8.8}, \mathrm{Fe} / \mathrm{Mn}=60 \pm 5, \mathrm{n}=12$; plagioclase $\mathrm{An}_{93.0 \pm 1.8}, \mathrm{n}=7$; Shock melt (20_m defocused electron beam, proxy for bulk meteorite composition): $\overline{\mathrm{SiO}}_{2}=43.2 \pm 1.7, \mathrm{TiO}_{2}=0.46 \pm 0.20, \mathrm{Al}_{2} \mathrm{O}_{3}=24.2 \pm 3.6$, $\mathrm{Cr}_{2} \mathrm{O}_{3}=0.23 \pm 0.08, \mathrm{MgO}=7.8 \pm 1.4, \mathrm{FeO}=8.0 \pm 3.2, \mathrm{MnO}=0.10 \pm 0.05, \mathrm{CaO}=14.2 \pm 1.4$, $\mathrm{Na}_{2} \mathrm{O}=0.35 \pm 0.23, \mathrm{~K}_{2} \mathrm{O}=0.08 \pm 0.05$ (all wt\%), $\mathrm{n}=4$.

Classification: Lunar feldspathic breccia Specimens: $28.9 \mathrm{~g}$ including a probe mount on deposit at UNM, Dustin Dickens holds the main mass.

\section{Northwest Africa 11789 (NWA 11789)}

Mauritania

Purchased: 2017

Classification: Lunar meteorite (feldspathic breccia)

History: Purchased in Mauritania by Dustin Dickens from anonymous meteorite hunter.

Physical characteristics: Seven black visually identical appearing pieces that fit back together to form a single mass with the largest piece weighing over $2 \mathrm{~kg}$ and the smallest under $45 \mathrm{~g}$. The larger pieces show a black-brown fusion crust and thumb printing. Saw cut reveals a fragmented breccia with white feldspathic clasts set in a black ground mass.

Petrography: (C. Agee, UNM) This meteorite is a polymict breccia of fragmental feldspathic and mafic lithologies, as well as fragmental pyroxene, olivine, plagioclase grains, and shock melt.

Geochemistry: (C. Agee, $U N M$ ) Olivine Fa: 31.7 \pm 6.3 , Fe/Mn=92 \pm 9 , n=10; clinopyroxene $\mathrm{Fs}_{32.2 \pm 10.8} \mathrm{Wo}_{12.9 \pm 8.2}, \mathrm{Fe} / \mathrm{Mn}=54 \pm 8, \mathrm{n}=12$; plagioclase $\mathrm{An}_{96.7 \pm 0.8}, \mathrm{n}=7$; Shock melt (20 $\mu \mathrm{m}$ defocused electron beam, proxy for bulk meteorite composition): $\mathrm{SiO}_{2}=43.6 \pm 0.8, \mathrm{TiO}_{2}=0.19 \pm 0.13, \mathrm{Al}_{2} \mathrm{O}_{3}=27.6 \pm 5.0$, $\mathrm{Cr}_{2} \mathrm{O}_{3}=0.12 \pm 0.08, \mathrm{MgO}=6.7 \pm 4.4, \mathrm{FeO}=4.3 \pm 2.9, \mathrm{MnO}=0.06 \pm 0.04, \mathrm{CaO}=15.7 \pm 2.3$, $\mathrm{Na}_{2} \mathrm{O}=0.34 \pm 0.13, \mathrm{~K}_{2} \mathrm{O}=0.09 \pm 0.05$ (all wt\%), $\mathrm{n}=6$.

Classification: Lunar feldspathic breccia Specimens: $43.15 \mathrm{~g}$ including a probe mount on deposit at $U N M$, Dustin Dickens holds the main mass. 


\section{Northwest Africa 11790 (NWA 11790)}

(Northwest Africa)

Purchased: 2018

Classification: Carbonaceous chondrite (CO3)

History: The meteorite was bought from a local meteorite dealer in Morocco. Physical characteristics: Two black fragments partly covered with fusion crust. Petrography: The meteorite displays a dark interior and is composed of small (mean diameter about $280 \mu \mathrm{m}$ ) chondrules, mineral fragments and CAIs set into an abundant fine-grained Fe-rich matrix. Opaques are mostly Fe,Ni metal. Several components are surrounded by fine-grained dust rims. Matrix constituents include phyllosilicates (low analytical totals), Ca-carbonates, and pyrrhotite.

Northwest Africa 11791 (NWA 11791)

(Northwest Africa)

Purchased: 2017

Classification: Carbonaceous chondrite (CM2)

History: The meteorite was bought from a local meteorite dealer in Morocco. Physical characteristics: Six black fragments partly covered with fusion crust. Petrography: The meteorite is a breccia dominated by fine-grained almost opaque matrix composed of phyllosilicates, Fe-sulfides, Ca-carbonates and rare FeNi metal. Embedded in this matrix are chondrules (mean diameter about $300 \mu \mathrm{m}$ ), chondrule pseudomorphs, mineral fragments, and rare CAIs some of which are surrounded by fine-grained dust rims.

Northwest Africa 11792 (NWA 11792)

(Northwest Africa)

Purchased: 2017

Classification: Carbonaceous chondrite (CM2)

History: The meteorite was bought from a local meteorite dealer in Morocco.

Physical characteristics: Many small dark-grayish to black fragments, some partly covered with fusion crust.

Petrography: Carbonaceous chondrite composed of small (about 0.1-0.6 mm in diameter, mean about $250 \mu \mathrm{m}$ ) chondrules, chondrule pseudomorphs, mineral fragments and rare CAIs many of which surrounded by fine-grained dust rims set into abundant matrix. Matrix is Fe-rich and contains abundant Fe-sulfides and less abundant phyllosilicates and Ca-carbonates. Most of the Fe-rich olivines are strongly corroded.

Northwest Africa 11793 (NWA 11793)

(Northwest Africa) 
Purchased: 2018

Classification: Carbonaceous chondrite (CM2)

History: The meteorite was bought from a local meteorite dealer in Morocco. Physical characteristics: Twelve small almost black fragments lacking fusion crust.

Petrography: Brecciated matrix-dominated carbonaceous chondrite with chondrules (mean diameter about $350 \mu \mathrm{m}$ ), mineral fragments and few CAIs. Some of the components are dust-mantled. Matrix is highly porous and composed of abundant $\mathrm{Ca}$-carbonates and less abundant phyllosilicates and Fe-sulfides. Some rare FeNi metal grains are present.

\section{Northwest Africa 11794 (NWA 11794) \\ (Northwest Africa) \\ Purchased: 2017 \\ Classification: HED achondrite (Eucrite)}

History: The meteorite was bought from a local meteorite dealer in Morocco. Physical characteristics: Eleven greyish fragments partly covered with fusion crust.

Petrography: Unbrecciated fine-grained basaltic rock predominantly composed of exsolved pyroxene and often lath-shaped plagioclase with average grains sizes of about $150 \mu \mathrm{m}$. Minor phases include silica, ilmenite, chromite, and troilite; no metallic iron has been detected. The meteorite contains several shock melt veins. Geochemistry: low-Ca pyroxene: $\mathrm{Fs}_{61.6 \pm 0.6} \mathrm{Wo}_{2.4 \pm 0.5}\left(\mathrm{Fs}_{60.0-62.2} \mathrm{Wo}_{1.8-3.7}, \mathrm{n}=14\right.$, $\mathrm{FeO} / \mathrm{MnO}=31-36)$; Ca-pyroxene: $\mathrm{Fs}_{26.8 \pm 0.8} \mathrm{Wo}_{43.3 \pm 0.9}\left(\mathrm{Fs}_{26.0-29.5} \mathrm{Wo}_{40.0-44.0}, \mathrm{n}=16\right.$, $\mathrm{FeO} / \mathrm{MnO}=29-36)$; calcic plagioclase: $\mathrm{An}_{89.4 \pm 0.6}\left(\mathrm{An}_{88.7-90.4}, \mathrm{n}=13\right)$

\section{Northwest Africa 11795 (NWA 11795)}

(Northwest Africa)

Purchased: 2018

Classification: Primitive achondrite (Lodranite)

History: The meteorite was purchased in Tindouf, Algeria, from a Sahrawi dealer. Physical characteristics: Brownish individual lacking any fusion crust.

Petrography: The meteorite is a breccia predominantly composed of up to $3 \mathrm{~mm}$ sized olivine and orthopyroxene grains (mean diameter about $0.6 \mathrm{~mm}$ ) with related fine-grained mineral debris. More minor phases include Ca-pyroxene, chromite, kamacite, troilite and Ni-bearing pyrrhotite. Ca-pyroxene often ocurrs as fine exsolution lamellae in low-Ca pyroxene and kamacite is mostly altered to Feoxides/hydroxides. No feldspar was found in the section studied.

Geochemistry: olivine: $\mathrm{Fa}_{10.7 \pm 0.1},\left(\mathrm{Fa}_{10.5-10.8}, \mathrm{n}=13, \mathrm{FeO} / \mathrm{MnO}=25 \pm 1\right)$; low-Ca pyroxene: $\mathrm{Fs}_{10.3 \pm 0.1} \mathrm{Wo}_{2.2 \pm 0.2}\left(\mathrm{Fs}_{10.1-10.5} \mathrm{Wo}_{1.9-2.5}, \mathrm{n}=12, \mathrm{FeO} / \mathrm{MnO}=16 \pm 1\right)$; $\mathrm{Ca}-$ pyroxene: $\mathrm{Fs}_{4.6 \pm 0.2} \mathrm{Wo}_{43.6 \pm 0.5}\left(\mathrm{Fs}_{4.3-4.9} \mathrm{Wo}_{42.8-44.9}, \mathrm{n}=12, \mathrm{FeO} / \mathrm{MnO}=11 \pm 1\right) ; \mathrm{Cr}_{2} \mathrm{O}_{3}$ in Ca-pyroxene: $1.06 \pm 0.13$ wt. $\%$ 
Classification: Lodranite according to mineral chemistry, grain size, and absence of feldspar.

\section{Northwest Africa 11796 (NWA 11796) \\ (Northwest Africa) \\ Purchased: 2018 \\ Classification: Ordinary chondrite (L3)}

History: The meteorite was purchased in Tindouf, Algeria, from a Sahrawi dealer. Petrography: The meteorite displays a chondritic texture with slightly flattened and well-packed chondrules (mean diameter about $0.6 \mathrm{~mm}$ ) in a more fine-grained matrix that contains sulfides and FeNi metal.

\section{Northwest Africa 11797 (NWA 11797) \\ (Northwest Africa) \\ Purchased: 2018 \\ Classification: Ordinary chondrite (L6)}

History: The meteorite was purchased in Tindouf, Algeria from a Sahrawi dealer. Physical characteristics: Brownish individual lacking any fusion crust.

Petrography: Plagioclase grain size about $70 \mu \mathrm{m}$

\section{Northwest Africa 11801 (NWA 11801)}

Algeria

Purchased: 2016 May 1

Classification: Lunar meteorite

History: Purchased by Miao Bing-an in 2016 from Morocco.

Physical characteristics: This meteorite is in irregular and brecciated, partially covered with black fusion crust. A saw cut reveals an abundance of fragments set in dark-colored matrix.

Petrography: The section is composed of abundant mineral fragments and a few lithic clasts and glass set in a fine-grained matrix. The mineral fragments, lithic clasts, and glass areas show irregular shapes and variable textures with different sizes (from $\sim 10 \mu \mathrm{m}$ to $\sim 4.1 \mathrm{~mm}$ across). The matrix is made up of fine-grained minerals bound by glassy cement.Mineral fragments include plagioclase, pyroxene, olivine, and minor ilmenite, chromite, silica, Ni-Fe metal, troilite, armalcolite, kfeldspar. The section contains various types of lithic clasts (anorthosite, gabbro, troctolite, and crystalline impact melt breccia). Several glassy spheres and veins were found in the thin section. One anomalously large glassy vein (up to $2.7 \mathrm{~mm}$ thick) that crosscut the section was observed.

Geochemistry: Plagioclase $\mathrm{An}_{91-96} \mathrm{Or}<0.4$; pyroxene $\mathrm{Wo}_{4-36} \mathrm{En}_{7-69}$; olivine $\mathrm{Fo}_{10-}$ 83. The $\mathrm{Fe} / \mathrm{Mn}$ ratios of pyroxene and olivine in this meteorite lie approximately along the lunar line, although the range of values is very large (44-79 for pyroxene and 72-127 for olivine). 
Classification: Lunar (breccia)

Specimens: specimen and a polished thin section are deposited in GUT

\section{Northwest Africa 11802 (NWA 11802) \\ (Northwest Africa) \\ Purchased: 2017 Jun \\ Classification: Ordinary chondrite (L3)}

History: Purchased by Gary Fujihara in June 2017 from a Moroccan dealer. Petrography: (A. Irving and S. Kuehner, UWS) Closely-packed unequilibrated chondrules (apparent diameter $620 \pm 410 \mu \mathrm{m}, \mathrm{N}=18$ ) are set in a sparse fine grained matrix containing stained metal.

Geochemistry: Olivine $\left(\mathrm{Fa}_{0.6-81.6}, \mathrm{Cr}_{2} \mathrm{O}_{3}\right.$ in ferroan examples $0.06-0.28$ wt. $\%$, mean $0.12 \pm 0.06$ wt.\%, $\mathrm{N}=9$ ), orthopyroxene $\left(\mathrm{Fs}_{1.5-23.3} \mathrm{Wo}_{0.2-3.4}, \mathrm{~N}=3\right)$, subcalcic augite $\left(\mathrm{Fs}_{14.6} \mathrm{Wo}_{26.6}\right)$, augite $\left(\mathrm{Fs}_{5.4} \mathrm{Wo}_{45.4}\right)$. Magnetic susceptibility $\log \chi\left(\times 10^{-9} \mathrm{~m}^{3} / \mathrm{kg}\right)=$ 4.76.

Classification: Ordinary chondrite (L3). The ranges of Fa content in olivine and of $\mathrm{Cr}_{2} \mathrm{O}_{3}$ content in ferroan olivine indicate a relatively low petrologic subtype between 3.3 and 3.5 .

Specimens: $20.5 \mathrm{~g}$ including one polished thin section at $U W B$; remainder with $\mathrm{Mr}$. G. Fujihara.

\section{Northwest Africa 11803 (NWA 11803)}

Algeria

Purchased: 2017 Aug

Classification: HED achondrite (Eucrite, brecciated)

History: Purportedly found in Algeria and purchased by Gary Fujihara in August 2017 from a Moroccan dealer.

Petrography: (A. Irving and S. Kuehner, UWS) Breccia composed of large gabbroic eucrite clasts in a very fine grained, vesicular quenched melt matrix containing angular mineral debris. Eucrite clasts (grainsize up to $2 \mathrm{~mm}$ ) consist of exsolved pigeonite, calcic plagioclase, silica polymorph, ilmenite, troilite and $\mathrm{Ni}$ free metal. Pyroxenes in the matrix include both pigeonite and ferropigeonite.

Geochemistry: Gabbroic eucrite clasts: orthopyroxene host $\left(\mathrm{Fs}_{61.1} \mathrm{Wo}_{2.1}, \mathrm{FeO} / \mathrm{MnO}\right.$ $=33)$, clinopyroxene exsolution lamella $\left(\mathrm{Fs}_{27.3} \mathrm{Wo}_{42.4}, \mathrm{FeO} / \mathrm{MnO}=33\right)$, orthopyroxene host $\left(\mathrm{Fs}_{51.7} \mathrm{Wo}_{2.0}, \mathrm{FeO} / \mathrm{MnO}=35\right)$, clinopyroxene exsolution lamella $\left(\mathrm{Fs}_{20.3} \mathrm{Wo}_{44.5}, \mathrm{FeO} / \mathrm{MnO}=26\right)$, plagioclase $\left(\mathrm{An}_{91.3} \mathrm{Or}_{0.2}\right)$. Matrix: pigeonite $\left(\mathrm{Fs}_{44.2} \mathrm{Wo}_{11.6}, \mathrm{FeO} / \mathrm{MnO}=32\right)$, ferropigeonite $\left(\mathrm{Fs}_{70.3} \mathrm{Wo}_{23.1}, \mathrm{FeO} / \mathrm{MnO}=38\right)$, plagioclase $\left(\mathrm{An}_{89.7} \mathrm{Or}_{0.2}\right)$.

Classification: Eucrite (gabbroic, melt matrix breccia). Specimens: $22 \mathrm{~g}$ including one polished thin section at $U W B$; remainder with $\mathrm{Mr}$. G. Fujihara. 


\title{
Northwest Africa 11804 (NWA 11804)
}

(Northwest Africa)

Purchased: 2018 Jan

Classification: Martian meteorite (Shergottite)

History: Purportedly found in the Tanezrouft region of Algeria and purchased by John Higgins in January 2018 from a Mauritanian dealer.

Physical characteristics: A single disaggregated brownish stone (34 grams).

Several of the desert-ablated fragments fit together and some retain partial black fusion crust.

Petrography: (A. Irving and S. Kuehner, $U W S$ ) Diabasic assemblage of mainly zoned clinopyroxene and maskelynite with accessory ilmenite, titanomagnetite, fayalite and pyrrhotite. Inclusions of K-rich glass and silica polymorph are present within pyroxenes.

Geochemistry: Subcalcic augite $\left(\mathrm{Fs}_{26.1-45.0} \mathrm{Wo}_{28.6-30.8}, \mathrm{FeO} / \mathrm{MnO}=31-38, \mathrm{~N}=3\right)$, pigeonite $\left(\mathrm{Fs}_{47.8-59.8} \mathrm{Wo}_{12.7-15.4}, \mathrm{FeO} / \mathrm{MnO}=35-37, \mathrm{~N}=3\right)$, fayalite $\left(\mathrm{Fa}_{76.3}\right.$, $\mathrm{FeO} / \mathrm{MnO}=54)$, maskelynite $\left(\mathrm{An}_{53.1-53.3} \mathrm{Or}_{0.9-1.0}, \mathrm{~N}=2\right)$.

Classification: Shergottite (diabasic). Paired with NWA 2975 and many other stones found in the Tanezrouft area.

Specimens: $6.88 \mathrm{~g}$ including one polished thick section at $U W B$; remainder with Mr. J. Higgins.

\section{Northwest Africa 11805 (NWA 11805) \\ (Northwest Africa) \\ Purchased: 2018 Jan \\ Classification: HED achondrite (Diogenite, polymict)}

History: Purchased by Christopher Colvin in January 2018 from a dealer in Arizona, who had obtained the specimen from a Moroccan source.

Petrography: (A. Irving and S. Kuehner, UWS) Breccia composed predominantly of angular mineral debris plus sparse lithic clasts of diogenite and microgabbroic eucrite. The majority ( $>90 \mathrm{vol} . \%$ ) of the mineral debris is diogenitic (orthopyroxene, olivine, chromite) with minor eucritic exsolved pigeonite, calcic plagioclase, silica polymorph, Ti-chromite and troilite.

Geochemistry: Diogenitic orthopyroxene $\left(\mathrm{Fs}_{23.4-24.5} \mathrm{Wo}_{2.3-2.0} ; \mathrm{Fs}_{17.1} \mathrm{Wo}_{0.9}\right.$; $\mathrm{FeO} / \mathrm{MnO}=27-32 ; \mathrm{N}=3)$, olivine $\left(\mathrm{Fa}_{28.9} ; \mathrm{Fa}_{52.0} ; \mathrm{FeO} / \mathrm{MnO}=44-49 ; \mathrm{N}=2\right)$, orthopyroxene host $\left(\mathrm{Fs}_{44.7} \mathrm{Wo}_{3.5}, \mathrm{FeO} / \mathrm{MnO}=33\right)$, clinopyroxene exsolution lamella $\left(\mathrm{Fs}_{23.0} \mathrm{Wo}_{39.4}, \mathrm{FeO} / \mathrm{MnO}=26\right)$, plagioclase $\left(\mathrm{An}_{88.6-90.3} \mathrm{Or}_{0.5-0.4}, \mathrm{~N}=2\right)$.

Classification: Diogenite (polymict breccia).

Specimens: $21.1 \mathrm{~g}$ including one polished thin section at $U W B$; remainder with $\mathrm{Mr}$. C. Colvin.

\author{
Northwest Africa 11806 (NWA 11806) \\ (Northwest Africa) \\ Purchased: 2005
}


Classification: Ordinary chondrite (H3)

History: Purchased by an anonymous collector from a dealer at the Munich Show in 2005, subsequently acquired by the Hollis Collection and donated to the Planetary Studies Foundation.

Petrography: (A. Irving and S. Kuehner, UWS) Closely packed chondrules (apparent diameter $350 \pm 240 \mu \mathrm{m}, \mathrm{N}=24$ ) are set in a sparse fine grained matrix containing altered metal.

Geochemistry: Olivine $\left(\mathrm{Fa}_{0.8-23.1}, \mathrm{Cr}_{2} \mathrm{O}_{3}\right.$ in ferroan examples 0.02-0.28 wt.\%, mean $0.07 \pm 0.10$ wt.\%, $\mathrm{N}=7$ ), orthopyroxene $\left(\mathrm{Fs}_{1.4-28.6} \mathrm{Wo}_{0.7-1.8}, \mathrm{~N}=3\right)$, subcalcic augite $\left(\mathrm{Fs}_{9.2-30.1} \mathrm{Wo}_{30.9-32.7}, \mathrm{~N}=2\right)$, diopside $\left(\mathrm{Fs}_{1.1} \mathrm{Wo}_{41.7}\right)$. Magnetic susceptibility $\log \chi(\times$ $\left.10^{-9} \mathrm{~m}^{3} / \mathrm{kg}\right)=4.86$.

Classification: Ordinary chondrite (H3).

Specimens: The entire specimen including a polished endcut is at PSF.

\section{Northwest Africa 11807 (NWA 11807)}

(Northwest Africa)

Purchased: 2018 Jan

Classification: Primitive achondrite (Winonaite)

History: Purchased by Darryl Pitt in January 2018 from a Moroccan dealer at the Tucson Gem and Mineral Show.

Petrography: (A. Irving and S. Kuehner, UWS) Metamorphic aggregate (mean grainsize $320 \mu \mathrm{m}$, with triple grain junctions) composed of forsterite, diopside and enstatite with accessory troilite and stained kamacite.

Geochemistry: Olivine $\left(\mathrm{Fa}_{2.9-3.0}, \mathrm{FeO} / \mathrm{MnO}=12-13, \mathrm{~N}=3\right)$, orthopyroxene $\left(\mathrm{Fs}_{2.7-}\right.$ $\left.{ }_{3.0} \mathrm{Wo}_{1.1-1.0}, \mathrm{FeO} / \mathrm{MnO}=8, \mathrm{~N}=2\right)$, clinopyroxene $\left(\mathrm{Fs}_{1.1-1.2} \mathrm{Wo}_{46.4-46.3}, \mathrm{FeO} / \mathrm{MnO}=5\right.$, $\mathrm{N}=2$ ). Oxygen isotopes (K. Ziegler, UNM): analyses of acid-washed subsamples by laser fluorination gave, respectively, $\delta^{17} \mathrm{O} 2.395,2.477,2.360 ; \delta^{18} \mathrm{O} 5.567$, $5.758,5.487 ; \Delta^{17} \mathrm{O}-0.544,-0.563,-0.537$ per mil.

Classification: Winonaite.

Specimens: $20.2 \mathrm{~g}$ including one polished thin section at $U W B$; remainder with DPitt.

\section{Northwest Africa 11808 (NWA 11808)}

(Northwest Africa)

Purchased: 2017 Nov

Classification: Carbonaceous chondrite (CK4)

History: Found by a camel herder in November 2017 near Tarfaya El Hagounia, Morocco and purchased by Mbark Arjdal.

Petrography: (A. Irving and S. Kuehner, UWS) Separated, well-formed. magnetite-bearing granular chondrules (apparent diameter $770 \pm 380 \mu \mathrm{m}, \mathrm{N}=20$ ) and sparse very fine grained CAI are set in a fine grained matrix containing orangestained Cr-magnetite. 
Geochemistry: Olivine $\left(\mathrm{Fa}_{34.3-34.3}, \mathrm{~N}=3\right)$, clinopyroxene $\left(\mathrm{Fs}_{9.3-9.8} \mathrm{Wo}_{46.5-46.0}, \mathrm{~N}=\right.$ 2), orthopyroxene $\left(\mathrm{Fs}_{27.0} \mathrm{Wo}_{3.9}\right)$.

Classification: Carbonaceous chondrite (CK4).

Specimens: $20.2 \mathrm{~g}$ including one polished thin section at $U W B$; remainder with $\mathrm{Mr}$.

M. Arjdal.

\section{Northwest Africa 11809 (NWA 11809)}

(Northwest Africa)

Purchased: 2017 Sep

Classification: Lunar meteorite (feldspathic breccia)

History: Purchased by Fabien Kuntz in September 2017 from a dealer in Erfoud, Morocco.

Petrography: (A. Irving and S. Kuehner, UWS) Breccia composed of angular mineral clasts of anorthite, olivine, orthopyroxene, pigeonite, subcalcic augite and Ti-chromite in a finer grained matrix containing minor barite.

Geochemistry: Olivine $\left(\mathrm{Fa}_{30.0-41.3}, \mathrm{FeO} / \mathrm{MnO}=87-97, \mathrm{~N}=3\right)$, orthopyroxene $\left(\mathrm{Fs}_{35.7} \mathrm{Wo}_{4.2}, \mathrm{FeO} / \mathrm{MnO}=56\right)$, pigeonite $\left(\mathrm{Fs}_{25.2-29.8} \mathrm{Wo}_{7.9-10.1}, \mathrm{FeO} / \mathrm{MnO}=52-63, \mathrm{~N}\right.$ $=2)$, subcalcic augite $\left(\mathrm{Fs}_{15.8} \mathrm{Wo}_{30.1}, \mathrm{FeO} / \mathrm{MnO}=56\right)$, anorthite $\left(\mathrm{An}_{92.0-95.0} \mathrm{Or}_{0.2-0.1}, \mathrm{~N}\right.$ $=2$ ).

Classification: Lunar (feldspathic breccia).

Specimens: $14.11 \mathrm{~g}$ including a polished slice at $U W B$; main mass with Kuntz.

\section{Northwest Africa 11810 (NWA 11810)}

(Northwest Africa)

Purchased: 2005

Classification: Ordinary chondrite (H3)

History: Purchased by an anonymous collector from a dealer at the Munich Show in 2005, subsequently acquired by the Hollis Collection and donated to the Planetary Studies Foundation.

Petrography: (A. Irving and S. Kuehner, UWS) Closely packed chondrules (apparent diameter $400 \pm 160 \mu \mathrm{m}, \mathrm{N}=20$ ) are set in a sparse fine grained matrix containing merrillite, chlorapatite and stained metal. A single crystal clast of forsterite was observed.

Geochemistry: Olivine in chondrules $\left(\mathrm{Fa}_{2.0-37.0} \mathrm{Cr}_{2} \mathrm{O}_{3}\right.$ in ferroan examples 0.030.21 wt. $\%$, mean $0.08 \pm 0.07$ wt. $\%, \mathrm{~N}=7)$, fosterite crystal $\left(\mathrm{Fa}_{0.7}\right)$, orthopyroxene $\left(\mathrm{Fs}_{2.1-31.3} \mathrm{Wo}_{0.6-1.7} \mathrm{~N}=3\right)$, pigeonite $\left(\mathrm{Fs}_{0.4} \mathrm{Wo}_{6.3}\right)$, augite $\left(\mathrm{Fs}_{3.2} \mathrm{Wo}_{35.0} ; \mathrm{Fs}_{6.2} \mathrm{Wo}_{45.5} ; \mathrm{N}\right.$ $=2)$. Magnetic susceptibility $\log \chi\left(\times 10^{-9} \mathrm{~m}^{3} / \mathrm{kg}\right)=5.14$.

Classification: Ordinary chondrite (H3).

Specimens: The entire specimen including a polished endcut is at PSF.

\section{Northwest Africa 11811 (NWA 11811)}

(Northwest Africa) 
Purchased: 2017

Classification: LL5-an

Physical characteristics: Dark stone. Cut surface reveals a gray interior with chondritic texture.

Petrography: (J. Gattacceca, H. Pourkhorsandi, CEREGE) Chondrite with recrystallized texture. Main minerals are olivine, pyroxene, and interstitial plagioclase with typical size about $25 \mu \mathrm{m}$. Presence of merrilite. Opaque minerals are sulfides (including pentlandite), chromite, and rare FeNi metal.

Geochemistry: Olivine $\mathrm{Fa}_{33.5 \pm 0.9}, \mathrm{PMD}=3 \%$, $\mathrm{NiO} 0.17$ wt $\%(\mathrm{~N}=8)$. Orthopyroxene $\mathrm{Fs}_{26.4 \pm 0.2} \mathrm{Ws} 1.6 \pm 0.1(\mathrm{~N}=5)$. Ca-pyroxene $\mathrm{Fs}_{25.1} \mathrm{Wo}_{17.4}(\mathrm{~N}=1)$. Plagioclase $\mathrm{An}_{11.9} \mathrm{Ab}_{80.2} \mathrm{Or}_{7.9}(\mathrm{~N}=2)$. Chromite $\mathrm{Cr} \#=0.91, \mathrm{TiO}_{2} 3.6 \mathrm{wt} \%(\mathrm{~N}=1)$. FeNi metal Fe32.3 $\pm 0.3 \mathrm{Ni} 66.2 \pm 0.3 \mathrm{Co} 1.35 \pm 0.06(\mathrm{~N}=7)$. Magnetic susceptibility $\log \chi\left(\times 10^{-}\right.$ ${ }^{9} \mathrm{~m}^{3} / \mathrm{kg}$ ) = 3.42. Oxygen isotopic composition (J. Gattacceca, C.

Sonzogni, CEREGE) from analysis of one acid-washed $1.5 \mathrm{mg}$ aliquot of a powdered $475 \mathrm{mg}$ bulk sample is $\delta^{17} \mathrm{O}=4.38 \%$, $\delta^{18} \mathrm{O}=5.85 \%$, $\Delta^{17} \mathrm{O}=1.31 \%$ o (linearized, slope 0.5247 , analytical uncertainties $0.08 \%$, $0.12 \%, 0.03 \%$ o respectively).

Classification: LL5-anomalous. The anomalous designation comes from the high Fa content of olivine compared to other equilibrated LL chondrites, and the presence of metal in the form of FeNi2 exclusively.

Specimens: Type specimen at CEREGE. Main mass with Kuntz.

\section{Northwest Africa 11812 (NWA 11812)}

(Northwest Africa)

Purchased: 2017

Classification: Ordinary chondrite (LL3)

Physical characteristics: Greyish stone. Cut surface reveals a dark-gray interior with closely packed well-defined chondrules.

Petrography: (J. Gattacceca, H. Pourkhorsandi, CEREGE) Packed mm-sized chondrules.

Geochemistry: Olivine $\mathrm{Fa}_{23.5 \pm 6.7}$, range 11.9-30.6, $\mathrm{PMD} 23 \%(\mathrm{~N}=5)$.

Orthopyroxene $\mathrm{Fs}_{17.5 \pm 5.3} \mathrm{Wo}_{1.1 \pm 1.0}$, Fs PMD 25\% (N=5).

Classification: LL3. LL than L classification from magnetic susceptibility.

Specimens: Type specimen at CEREGE. Main mass with Fabien Kuntz.

\section{Northwest Africa 11813 (NWA 11813)}

(Northwest Africa)

Purchased: 2017

Classification: Ordinary chondrite (LL3)

Physical characteristics: Greyish stone. Cut surface reveals a dark grey interior with closely packed well-defined chondrules.

Petrography: (J. Gattacceca, H. Pourkhorsandi, CEREGE) Packed chondrules with average apparent diameter $920 \mu \mathrm{m}(\mathrm{N}=36)$. 
Geochemistry: Olivine $\mathrm{Fa}_{18.2 \pm 8.1}$, range 1.1-33.8, $\mathrm{PMD} 33 \%(\mathrm{~N}=13), \mathrm{Cr}_{2} \mathrm{O}_{3}$ in ferroan olivine $0.17 \pm 0.12 \mathrm{wt} \%(\mathrm{~N}=11)$. Orthopyroxene $\mathrm{Fs}_{8.0 \pm 4.1} \mathrm{Wo}_{2.3 \pm 1.8}(\mathrm{~N}=4)$. Classification: LL3. LL than L classification from magnetic susceptibility. Specimens: Type specimen at CEREGE. Main mass with Fabien Kuntz.

\section{Northwest Africa 11814 (NWA 11814)}

(Northwest Africa)

Purchased: 2016

Classification: Ordinary chondrite (H5)

Petrography: Chondrite with plagioclase to $40 \mu \mathrm{m}$ in the matrix. Porosity under the form of pores of with typical size $150 \mu \mathrm{m}$ (maximum $300 \mu \mathrm{m}$ ) amouts to 9 vol\% (point couting, $\mathrm{N}=185$ ) is unusual for an equilibrated ordinary chondrite.

\section{Northwest Africa 11815 (NWA 11815)}

(Northwest Africa)

Purchased: 2017 Dec

Classification: Ureilite

History: Purchased from an Algerian mineral dealer.

Petrography: (K. Metzler, IfP) Coarse-grained ultramafic rock with aligned texture, consisting of olivine and pyroxene grains with sizes up to $\sim 2.5 \mathrm{~mm}$. Reverse zoning of olivine grains with $\mathrm{Mg}$-rich zones at the margins and along cracks. Metal is concentrated along grain boundaries and mostly oxidized by terrestrial weathering.

Geochemistry: Mineral compositions and geochemistry: Olivine cores $(\mathrm{n}=9)$ : $\mathrm{Fa}_{18.3 \pm 0.2}$; Olivine rims $(\mathrm{n}=6): \mathrm{Fa}_{3.6 \pm 1.1}$; Pyroxene grains $(\mathrm{n}=14)$ :

$\mathrm{Fs}_{15.3 \pm 0.3} \mathrm{Wo}_{8.1 \pm 0.3}$. The mean concentration of $\mathrm{Cr}_{2} \mathrm{O}_{3}$ in olivine and pyroxene is 0.7 wt $\%$ and $1.2 \mathrm{wt} \%$, respectively.

\section{Northwest Africa 11828 (NWA 11828)}

(Northwest Africa)

Find: 2017

Classification: Lunar meteorite (feldspathic breccia)

History: Stones were acquired from a Moroccan dealer in 2017.

Physical characteristics: A group of dark- and light-gray stones with no fusion crust.

Petrography: (S. Demidova and D. Badyukov, Vernad): The meteorites are polymict breccias containing numerous mineral fragments and large rounded lithic clasts occuring within a brownish cryptocrystalline to glassy impact-melt matrix; the lithic clast population comprises impact-melt breccias, as well as cataclastic granulitic rocks of anorthositic, noritic, gabbro-noritic and troctolitic compositions; clasts of impact-melt breccias typically show breccia-in-breccia textures; glass fragments and rare spherules are present. 
Geochemistry: feldspar, $\mathrm{An}_{92-98}$; orthopyroxene, $\mathrm{Wo}_{2-5} \mathrm{En}_{60-81}$; clinopyroxene; $\mathrm{Wo}_{5-43} \mathrm{En}_{33-69}$, olivine, $\mathrm{Fo}_{22-93}(\mathrm{FeO} / \mathrm{MnO}=85)$; accessory minerals include: ilmenite (2.6-6.2 wt \% MgO), Cr-rich ulvöspinel, Ti,Al-rich chromite, silica, Caphosphate, armalcolite, baddeleyite, troilite, and abundant FeNi metal; the stones are moderately weathered; carbonate veins and Fe hydroxides are present.

Classification: Lunar, feldspathic regolith breccia

Specimens: 23 type specimens of $662.5 \mathrm{~g}$, and a thin section, Vernad, the main mass with an anonymous keeper

\section{Northwest Africa 11829 (NWA 11829) \\ (Northwest Africa) \\ Purchased: 2018 \\ Classification: Ordinary chondrite (L3)}

History: The meteorite was purchased from a local meteorite dealer in Morocco. Physical characteristics: Dark-grayish individual lacking any fusion crust. Petrography: The meteorite displays a chondritic texture with slightly flattened and well packed chondrules (mean apparent diameter about $0.6 \mathrm{~mm}$ ) in a more fine-grained dark matrix that contains sulfides and FeNi metal.

\section{Northwest Africa 11830 (NWA 11830) \\ (Northwest Africa) \\ Purchased: 2018 \\ Classification: Ordinary chondrite (H4)}

History: The meteorite was purchased from a local meteorite dealer in Morocco. Physical characteristics: Dark brownish individual without fusion crust. Petrography: The meteorite displays a chondritic texture with well defined chondrules (mean diameter about $0.3 \mathrm{~mm}$ ) and fine-grained matrix containing sulfides and abundant FeNi metal. Low-Ca pyroxene is compositionally highly unequilibrated; olivine is equilibrated.

\section{Northwest Africa 11831 (NWA 11831) \\ (Northwest Africa) \\ Purchased: 2018 \\ Classification: Ordinary chondrite (LL3-6)}

History: The meteorite was purchased from a local meteorite dealer in Morocco. Physical characteristics: Dark greyish individual with some fusion crust.

Petrography: The meteorite is a chondritic breccia consisting of clastic LL3 type matrix with embedded up to $2 \mathrm{~mm}$ sized LL6 clasts. Plagioclase grain size in type 6 clasts is about $60 \mu \mathrm{m}$. Chondrules have a mean diameter of about $0.8 \mathrm{~mm}$.

Geochemistry: type 3 lithology: olivine: $\mathrm{Fa}_{14.9 \pm 8.5}\left(\mathrm{Fa}_{5.0-31.9}, \mathrm{n}=11\right)$; pyroxene: $\mathrm{Fs}_{11.9 \pm 7.6} \mathrm{Wo}_{0.8 \pm 0.9}\left(\mathrm{Fs}_{2.0-24.7} \mathrm{Wo}_{0.3-4.6}, \mathrm{n}=21\right)$; type 6 lithology: olivine: $\mathrm{Fa}_{30.7 \pm 0.1}$, $\mathrm{n}=12$; pyroxene: $\mathrm{Fs}_{25.0 \pm 0.2} \mathrm{Wo}_{2.1 \pm 0.6}, \mathrm{n}=13$ 


\section{Northwest Africa 11832 (NWA 11832)}

(Northwest Africa)

Purchased: 2018

Classification: Ordinary chondrite (L, melt rock)

History: The meteorite was purchased from a local meteorite dealer in Morocco. Physical characteristics: Almost black individual partly covered with fusion crust. Petrography: The meteorite displays an almost black interior and consists of abundant and partly recrystallized shock melt regions with characteristic FeNi metal and sufide spherules. Few relict chondrules and mineral fragments are present; no metal or sulfide veins are observed.

\section{Northwest Africa 11833 (NWA 11833)}

(Northwest Africa)

Purchased: 2018

Classification: HED achondrite (Diogenite)

History: The meteorite was purchased from a local meteorite dealer in Morocco. Physical characteristics: Light-grayish individual with patches of fusion crust. Petrography: The meteorite is a breccia mainly composed of up to $2 \mathrm{~mm}$ sized blocky orthopyroxene grains (mean diameter about $0.8 \mathrm{~mm}$ ) and related finegrained mineral debris. Minor phases include calcic plagioclase, chromite, kamacite, and troilite; no nickel free metal has been detected.

Geochemistry: low-Ca pyroxene: $\mathrm{Fs}_{28.8 \pm 0.3} \mathrm{Wo}_{4.1 \pm 0.1}\left(\mathrm{Fs}_{28.3-29.4} \mathrm{Wo}_{3.9-4.3}, \mathrm{n}=14\right.$, $\mathrm{FeO} / \mathrm{MnO}=27-31)$; calcic plagioclase: $\mathrm{An}_{93.0 \pm 0.7}\left(\mathrm{An}_{91.9-93.8}, \mathrm{n}=9\right)$

\section{Northwest Africa 11834 (NWA 11834)}

(Northwest Africa)

Purchased: 2018

Classification: HED achondrite (Howardite)

History: The meteorite was purchased from a local meteorite dealer in Morocco. Physical characteristics: Dark greyish individual without fusion crust.

Petrography: The meteorite is a polymict breccia composed of mineral and lithic clasts (basaltic and impact melt clasts) set into a finer grained clastic matrix. Main minerals are exsolved pyroxene, up to $3 \mathrm{~mm}$ sized diogenetic orthopyroxene (about $15 \%)$ and calcic plagioclase. Minor phases include silica, chromite, ilmenite, barite, $\mathrm{FeS}$, and metallic Fe. It contains abundant shock melt veins.

Geochemistry: pyroxene host to augite lamellae: $\mathrm{Fs}_{59.2 \pm 0.9} \mathrm{Wo}_{3.9 \pm 1.2}\left(\mathrm{Fs}_{58.0-60.5} \mathrm{Wo}_{2.1-}\right.$ $\left.{ }_{6.6}, \mathrm{n}=15, \mathrm{FeO} / \mathrm{MnO}=31-35\right)$; Ca-pyroxene: $\mathrm{Fs}_{15.8 \pm 6.2} \mathrm{Wo}_{43.4 \pm 1.2}\left(\mathrm{Fs}_{11.7-29.0} \mathrm{Wo}_{40.2-44.5}\right.$, $\mathrm{n}=14, \mathrm{FeO} / \mathrm{MnO}=21-38)$; diogenitic pyroxene: $\mathrm{Fs}_{32.4 \pm 0.2} \mathrm{Wo}_{2.2 \pm 0.1}\left(\mathrm{Fs}_{31.8-32.6} \mathrm{Wo}_{2.2-2.5}\right.$, $\mathrm{n}=12, \mathrm{FeO} / \mathrm{MnO}=29-34)$; calcic plagioclase: $\mathrm{An}_{87.4 \pm 2.5}\left(\mathrm{An}_{82.7-90.2}, \mathrm{n}=16\right)$ 
Northwest Africa 11835 (NWA 11835)

(Northwest Africa)

Purchased: 2018

Classification: Ordinary chondrite (L3)

History: The meteorite was purchased from a local meteorite dealer in Morocco. Physical characteristics: Brownish individual with some fusion crust.

Petrography: The meteorite shows a chondritic texture with mostly rounded and well-packed chondrules (mean apparent diameter about $0.7 \mathrm{~mm}$ ) in a fine-grained matrix, that contains sulfides and FeNi metal.

\section{Northwest Africa 11836 (NWA 11836)}

(Northwest Africa)

Purchased: 2018

Classification: Carbonaceous chondrite (CK5)

History: The meteorite was purchased from a local meteorite dealer in Morocco. Physical characteristics: Several greyish to light greenish fragments without fusion crust.

Petrography: The meteorite displays a greyish interior and is predominantly composed of recrystallized olivine-rich matrix with scattered chondrules still being discernable. Cr-rich magnetite is abundant. More minor phases include intermediate plagioclase, low-Ca pyroxene, Ca-pyroxene and troilite; metal was not detected.

Geochemistry: olivine: $\mathrm{Fa}_{31.1 \pm 0.2}, \mathrm{n}=15, \mathrm{FeO} / \mathrm{MnO}=121.5 \pm 7.2$; low-Ca pyroxene: $\mathrm{Fs}_{25.8 \pm 0.2} \mathrm{Wo}_{1.0 \pm 0.2}, \mathrm{n}=13$; Ca-pyroxene: $\mathrm{Fs}_{11.3 \pm 0.7} \mathrm{Wo}_{48.2 \pm 0.3}, \mathrm{n}=6$

\section{Northwest Africa 11837 (NWA 11837) \\ (Northwest Africa) \\ Purchased: 2018 \\ Classification: HED achondrite (Eucrite)}

History: The meteorite was purchased from a local meteorite dealer in Morocco.

Physical characteristics: Brownish individual with some fusion crust.

Petrography: Unbrecciated medium-grained basaltic rock predominantly composed of exsolved pyroxene and often lath-shaped calcic plagioclase with average grains sizes of about $600 \mu \mathrm{m}$. Minor phases include silica, ilmenite, chromite, troilite, and metallic iron.

Geochemistry: low-Ca pyroxene: $\mathrm{Fs}_{62.6 \pm 0.6} \mathrm{Wo}_{6.6 \pm 0.7}\left(\mathrm{Fs}_{61.4-63.2} \mathrm{Wo}_{5.5-8.0}, \mathrm{n}=13\right.$, $\mathrm{FeO} / \mathrm{MnO}=25-29)$; Ca-pyroxene: $\mathrm{Fs}_{34.4 \pm 0.2} \mathrm{Wo}_{39.6 \pm 0.3}\left(\mathrm{Fs}_{34.1-34.8} \mathrm{Wo}_{39.1-40.1}, \mathrm{n}=14\right.$, $\mathrm{FeO} / \mathrm{MnO}=26-30)$; calcic plagioclase: $\mathrm{An}_{88.1 \pm 0.5}\left(\mathrm{An}_{87.2-88.9}, \mathrm{n}=13\right)$

\section{Northwest Africa 11838 (NWA 11838)}

(Northwest Africa)

Find: 2017 
Classification: Ordinary chondrite (LL6)

History: Purchased by Daniel Sheikh in 2018

Petrography: (F. Sheikh, UFla) Chondrules almost absent $(800 \pm 50 \mu \mathrm{m}, \mathrm{N}=3)$.

Fine-grained matrix containing low amounts of metal. Scattered shock melt veins containing FeS and FeO.

Geochemistry: Olivine, $\mathrm{Fa}_{25.2-47.6}$, mean $\mathrm{Fa}_{31.1 \pm 0.7}, \mathrm{~N}=54$; orthopyroxene, $\mathrm{Fs}_{22.5 \text { - }}$ ${ }_{38.6} \mathrm{Wo}_{2.4-6.6}$, mean $\mathrm{Fs}_{27.7 \pm 0.6} \mathrm{Wo}_{3.2 \pm 0.8}, \mathrm{~N}=51$.

Classification: Ordinary Chondrite (LL6).

Specimens: $455.88 \mathrm{~g}$ at UCLA; main mass with Daniel Sheikh.

\section{Northwest Africa 11839 (NWA 11839)}

(Northwest Africa)

Purchased: 2017

Classification: Carbonaceous chondrite (CM2)

History: The meteorite was purchased from a local meteorite dealer in Morocco. Physical characteristics: Several small dark grayish to black fragments some of which partly covered with fusion crust.

Petrography: The meteorite is a brecciated carbonaceous chondrite composed of small chondrules (mean diameter about $250 \mu \mathrm{m}$ ), CAIs, and mineral fragments often surrounded by fine-grained dust rims. The ferroan matrix contains abundant sulfides, almost pure calcite and fibrous phyllosilicates.

\section{Northwest Africa 11840 (NWA 11840) \\ (Northwest Africa) \\ Purchased: 2018 \\ Classification: Carbonaceous chondrite (CM2)}

History: The meteorite was purchased from a local meteorite dealer in Morocco. Physical characteristics: Many small almost black fragments partially covered with fusion crust.

Petrography: Brecciated carbonaceous chondrite with abundant Fe-rich matrix, chondrules (mean diameter $280 \mu \mathrm{m}$ ), chondrule pseudomorphs, CAIs, and mineral fragments. Many components are surrounded by fine-grained dust rims. Matrix consists of abundant carbonates, sulfides and phyllosilicates. No pyroxene has been detected in the thin section studied. Absence of pyroxene and presence of Fe-Mgrich carbonates attest to a high degree of alteration.

Geochemistry: Carbonates contain up to $1.3 \mathrm{wt} \% \mathrm{MgO}$ and $1.0 \mathrm{wt} \% \mathrm{FeO}$

\section{Northwest Africa 11841 (NWA 11841)}

(Northwest Africa)

Purchased: 2018

Classification: Carbonaceous chondrite (CM2)

History: The meteorite was purchased from a local meteorite dealer in Morocco. 
Physical characteristics: Several small black fragments without fusion crust. Petrography: Carbonaceous chondrite consisting of chondrule and CAI pseudomorphs and mostly corroded mineral grains set into abundant fine-grained matrix. Some components are surrounded by dust rims. Ferroan olivine and all pyroxene have been aqueously altered; only few forsteric olivines are unaltered. Matrix contains sulfides, phyllosilicates and some carbonates.

Geochemistry: Carbonates too small to be analyzed

\section{Northwest Africa 11844 (NWA 11844)}

(Northwest Africa)

Purchased: 2018

Classification: Carbonaceous chondrite (CO3)

History: The meteorite was purchased from a local meteorite dealer in Morocco. Physical characteristics: Small dark-brownish individual with some fusion crust. Petrography: The meteorite shows a dark-brownish interior and is composed of abundant small chondrules, CAIs, and mineral fragments some of which surrounded by fine-grained dust rims set into a fine-grained matrix. Chondrules have a mean diameter of about $0.2 \mathrm{~mm}$; few regions show brownish staining due to terrestrial weathering.

\section{Northwest Africa 11845 (NWA 11845)}

(Northwest Africa)

Purchased: 2018

Classification: Carbonaceous chondrite (CV3)

History: The meteorite was purchased from a local meteorite dealer in Morocco. Physical characteristics: Dark greyish individual with patches of fusion crust. Petrography: Carbonaceous chondrite composed of separated well-defined chondrules (mean diameter about $1.0 \mathrm{~mm}$ ), CAIs (up to $4 \mathrm{~mm}$ ), and olivine amoeboids all set into a fine-grained almost black matrix. Type II chondrules are absent. Some chondrules show reddish staining due to terrestrial weathering.

\section{Northwest Africa 11846 (NWA 11846) \\ (Northwest Africa) \\ Purchased: 2018 Jan \\ Classification: HED achondrite (Eucrite, unbrecciated)}

History: Purchased in New York City by Darryl Pitt in January 2018 from a Moroccan dealer.

Petrography: (A. Irving and S. Kuehner, UWS) Ophitic texture. Aggregate of exsolved pigeonite and calcic plagioclase with accessory silica polymorph, Tichromite, ilmenite and troilite. 
Geochemistry: Orthopyroxene host $\left(\mathrm{Fs}_{62.2-62.3} \mathrm{Wo}_{2.2-1.9}, \mathrm{FeO} / \mathrm{MnO}=31-32, \mathrm{~N}=2\right)$, clinopyroxene exsolution lamellae $\left(\mathrm{Fs}_{27.3-27.4} \mathrm{Wo}_{43.5-43.3}, \mathrm{FeO} / \mathrm{MnO}=28-31, \mathrm{~N}=2\right)$, plagioclase $\left(\mathrm{An}_{88.4-89.7} \mathrm{Or}_{0.5-0.6}, \mathrm{~N}=2\right)$.

Classification: Eucrite (unbrecciated).

Specimens: $20.6 \mathrm{~g}$ including one polished thin section at $U W B$; remainder with DPitt.

Northwest Africa 11847 (NWA 11847)

(Northwest Africa)

Purchased: 2018 Jan

Classification: Ordinary chondrite (L5)

History: Purchased in New York City by Darryl Pitt in January 2018 from a Moroccan dealer.

Petrography: (A. Irving and S. Kuehner, UWS) Sparse chondrules occur in a dark, recrystallized matrix containing stained metal. The specimen is cross-cut by shock melt veins with very fine grained quench texture.

Geochemistry: Olivine $\left(\mathrm{Fa}_{24.4-25.2}, \mathrm{~N}=3\right)$, orthopyroxene $\left(\mathrm{Fs}_{20.5-20.6} \mathrm{Wo}_{2.1-2.0}, \mathrm{~N}=\right.$ 3), clinopyroxene $\left(\mathrm{Fs}_{8.1-8.5} \mathrm{Wo}_{44.8-43.8}, \mathrm{~N}=2\right)$.

Classification: Ordinary chondrite (L5, highly shocked).

Specimens: $20.1 \mathrm{~g}$ including one polished thin section at $U W B$; remainder with DPitt.

\section{Northwest Africa 11848 (NWA 11848)}

(Northwest Africa)

Purchased: 2018 Jan

Classification: Primitive achondrite (Lodranite)

History: Purchased by Rachid Chaoui in January 2018 from a dealer in Nouadhibou, Mauritania.

Petrography: (A. Irving and S. Kuehner, UWS) Orthopyroxene-rich breccia composed of large grains (up to $6 \mathrm{~mm}$ ) of low-Ca pyroxene (some exhibiting irregular exsolution lamellae) and orthopyroxenite lithic clasts in a relatively coarse grained matrix of orthopyroxene with accessory clinopyroxene, olivine, chromite, troilite and altered kamacite. Some secondary goethite veinlets are present.

Geochemistry: Orthopyroxene $\left(\mathrm{Fs}_{9.9-10.5} \mathrm{Wo}_{1.5-2.7}, \mathrm{FeO} / \mathrm{MnO}=15-16, \mathrm{~N}=3\right)$, clinopyroxene $\left(\mathrm{Fs}_{4.2-4.8} \mathrm{Wo}_{44.4-41.4}, \mathrm{FeO} / \mathrm{MnO}=10-12, \mathrm{~N}=2\right)$, olivine $\left(\mathrm{Fa}_{10.7-10.8}\right.$, $\mathrm{FeO} / \mathrm{MnO}=25-26, \mathrm{~N}=2)$.

Classification: Lodranite (pyroxenitic breccia).

Specimens: $28.8 \mathrm{~g}$ including one polished thin section at $U W B$; remainder with $\mathrm{Mr}$.

R. Chaoui.

Northwest Africa 11849 (NWA 11849)

(Northwest Africa) 
Purchased: 2018 Jan

Classification: L6-an

History: Purchased by Greg Hupe in January 2018 from a Moroccan dealer at the Tucson Gem and Mineral Show.

Petrography: (A. Irving and S. Kuehner, UWS) Vesicular, metal-poor specimen consisting predominantly of finely recrystallized mafic silicates together with accessory sodic plagioclase, chromite, troilite and stained kamacite. Very sparse remnant chondrules are present, as well as poikiloblastic regions (presumably former chondrules). Small, spherical vesicles are present throughout.

Geochemistry: Olivine $\left(\mathrm{Fa}_{24.4-25.0}, \mathrm{~N}=3\right)$, orthopyroxene $\left(\mathrm{Fs}_{18.9-20.5} \mathrm{Wo}_{4.0-4.4}, \mathrm{~N}=\right.$ 3), clinopyroxene $\left(\mathrm{Fs}_{10.9-11.6} \mathrm{Wo}_{38.6-35.9}, \mathrm{~N}=2\right)$.

Classification: Ordinary chondrite (L6, anomalous, metal-poor, vesicular). The presence of abundant vesicles and the paucity of metal are anomalous features in comparison with typical L6 chondrites. Although this specimen contains rare remnant chondrules, in most other respects it closely resembles NWA 11253, and may be related to or even paired with that heterogeneous meteorite.

Specimens: $20.4 \mathrm{~g}$ including one polished thin section at $U W B$; remainder with GHupé.

\section{Northwest Africa 11850 (NWA 11850) \\ (Northwest Africa) \\ Purchased: 2017 Sep \\ Classification: Carbonaceous chondrite (CV3)}

History: Purchased by Fabien Kuntz in September 2017 from a dealer in Erfoud, Morocco.

Petrography: (A. Irving and S. Kuehner, UWS) Irreglarly shaped to rounded chondrules (mostly granular, some BO; apparent diameter $890 \pm 350 \mu \mathrm{m}, \mathrm{N}=18$ ) and very fine grained, amoeboid CAI are set in a fine grained matrix ( 40 vol. $\%$, black in thin section).

Geochemistry: Olivine $\left(\mathrm{Fa}_{0.6-58.7}, \mathrm{~N}=3\right)$, orthopyroxene $\left(\mathrm{Fs}_{2.5-15.1} \mathrm{Wo}_{0.4-1.5}, \mathrm{~N}=3\right)$, clinopyroxene ( $\left.\mathrm{Fs}_{9.3-12.5} \mathrm{Wo}_{39.6-37.4}, \mathrm{~N}=2\right)$.

Classification: Carbonaceous chondrite (CV3).

Specimens: $21.62 \mathrm{~g}$ including one polished thin section at $U W B$; remainder with Kuntz.

\section{Northwest Africa 11851 (NWA 11851)}

Mali

Purchased: 2018 Mar

Classification: Lunar meteorite (feldspathic breccia)

History: Found in the Azawad region of northern Mali and purchased by Habib Naji in March 2018.

Physical characteristics: A fine grained, medium gray stone (227 g) with macroscopically indistinct clasts; partly coated by light brown weathering products. 
Petrography: (A. Irving, UWS and P. Carpenter, WUSL) Polymict breccia consisting of small mineral clasts (anorthite, olivine, pigeonite, augite, orthopyroxene, ilmenite, Ti-Cr-Mg-Al spinel, troilite, merrillite, kamacite) and small lithic clasts (including gabbroic lithologies and quench-textured melt rocks) in a finer grained matrix.

Geochemistry: Olivine $\left(\mathrm{Fa}_{17.8-23.5}, \mathrm{FeO} / \mathrm{MnO}=90-127, \mathrm{~N}=3\right)$, orthopyroxene $\left(\mathrm{Fs}_{16.1} \mathrm{Wo}_{3.8}, \mathrm{FeO} / \mathrm{MnO}=53\right)$, pigeonite $\left(\mathrm{Fs}_{25.4-37.2} \mathrm{Wo}_{8.1-13.0}, \mathrm{FeO} / \mathrm{MnO}=54-60, \mathrm{~N}\right.$ $=3)$, augite $\left(\mathrm{Fs}_{44.1} \mathrm{Wo}_{35.6}, \mathrm{FeO} / \mathrm{MnO}=62\right)$, plagioclase $\left(\mathrm{An}_{95.5-95.7} \mathrm{Or}_{0.5-0.3}, \mathrm{~N}=2\right)$.

Classification: Lunar (feldspathic breccia).

Specimens: $22 \mathrm{~g}$ including one polished thin section at $U W B$; remainder with $\mathrm{Mr}$. H. Naji.

\section{Northwest Africa 11852 (NWA 11852)}

(Northwest Africa)

Purchased: 2018 Feb

Classification: Ordinary chondrite (LL7)

History: Purchased by Fred Olsen in February 2018 from a Moroccan dealer at the Tucson Gem and Mineral Show.

Petrography: (A. Irving and S. Kuehner, UWS) Triple grain junction texture with no chondrules or chondrule remnants. Relatively fine grained aggregate (mean grainsize $\sim 150 \mu \mathrm{m}$ ) of olivine, orthopyroxene, clinopyroxene, sodic plagioclase, chromite, chlorapatite, taenite and troilite.

Geochemistry: Olivine $\left(\mathrm{Fa}_{29.6-29.8}, \mathrm{~N}=3\right)$, orthopyroxene $\left(\mathrm{Fs}_{23.7-23.8} \mathrm{Wo}_{1.7-2.0}, \mathrm{~N}=\right.$ $3)$, clinopyroxene $\left(\mathrm{Fs}_{10.0-10.4} \mathrm{Wo}_{43.2-42.1}, \mathrm{~N}=2\right)$.

Classification: Ordinary chondrite (LL7).

Specimens: $9.2 \mathrm{~g}$ including one polished thin section at $U W B$; remainder with $\mathrm{Mr}$.

F. Olsen.

\section{Northwest Africa 11853 (NWA 11853)}

(Northwest Africa)

Purchased: 2011 Apr

Classification: Mesosiderite

History: Purchased by Alexandre Debienne in April 2011 from a dealer in Agadir, Morocco.

Physical characteristics: A batch of identical brownish stones (total weight 270

g).

Petrography: (A. Irving and S. Kuehner, UWS) Relatively coarse-grained aggregate of compositionally zoned orthopyroxene, calcic plagioclase, troilite and abundant altered kamacite.

Geochemistry: Orthopyroxene $\left(\mathrm{Fs}_{15.8} \mathrm{Wo}_{1.1}, \mathrm{FeO} / \mathrm{MnO}=33 ; \mathrm{Fs}_{24.7} \mathrm{Wo}_{2.7}\right.$, $\mathrm{FeO} / \mathrm{MnO}=27 ; \mathrm{Fs}_{46.6} \mathrm{Wo}_{2.8}, \mathrm{FeO} / \mathrm{MnO}=31, \mathrm{~N}=3$ ).

Classification: Mesosiderite. 
Specimens: A $21 \mathrm{~g}$ stone polished on one side is at PSF. The remaining material is held by Mr. A. Debienne.

\section{Northwest Africa 11854 (NWA 11854) \\ (Northwest Africa) \\ Purchased: 2005 \\ Classification: Carbonaceous chondrite (CV3)}

History: Purchased by an anonymous collector from a dealer at the Munich Show in 2005, subsequently acquired by the Hollis Collection and then donated to PSF. Petrography: (A. Irving and S. Kuehner, UWS) Granular chondrules (apparent diameter $960 \pm 360 \mu \mathrm{m}, \mathrm{N}=20$ ) and irregularly shaped, very fine grained CAI are set in a fine grained matrix (40 vol.\%, red-brown in thin section).

Geochemistry: Olivine $\left(\mathrm{Fa}_{0.2-41.0}, \mathrm{~N}=3\right)$, orthopyroxene $\left(\mathrm{Fs}_{0.9-1.2} \mathrm{Wo}_{3.8-0.7}, \mathrm{~N}=3\right)$, clinopyroxene $\left(\mathrm{Fs}_{0.9-1.2} \mathrm{Wo}_{39.4-35.3} ; \mathrm{Fs}_{45.1} \mathrm{Wo}_{50.3} ; \mathrm{N}=3\right)$.

Classification: Carbonaceous chondrite (CV3).

Specimens: The entire specimen including one polished thin section is at PSF.

\section{Northwest Africa 11855 (NWA 11855)}

(Northwest Africa)

Purchased: 2017 Oct

Classification: Ordinary chondrite (H6)

History: Purchased by Stefan Ralew in October 2017 from a Moroccan dealer at the Munich Show.

Petrography: (A. Irving and S. Kuehner, UWS) Shock-darkened breccia. Very sparse remnant chondrules occur in a recrystallized matrix containing merrillite, altered kamacite and taenite. Some secondary goethite veinlets are present.

Geochemistry: Olivine $\left(\mathrm{Fa}_{19.9-20.0}, \mathrm{~N}=3\right)$, orthopyroxene $\left(\mathrm{Fs}_{16.9-17.6} \mathrm{Wo}_{1.2-1.1}, \mathrm{~N}=\right.$ 3), clinopyroxene $\left(\mathrm{Fs}_{6.4-9.3} \mathrm{Wo}_{45.9-44.7}, \mathrm{~N}=2\right)$.

Classification: Ordinary chondrite (H6 breccia, shock-darkened).

Specimens: $20.8 \mathrm{~g}$ including one polished slice and one polished thin section at $U W B$; remainder with Mr. S. Ralew.

\section{Northwest Africa 11856 (NWA 11856)}

(Northwest Africa)

Purchased: 2017

Classification: HED achondrite (Eucrite)

History: A single stone weighing $137 \mathrm{~g}$ was found in Morocco prior to 2017. J. Donald Cline and John Sinclair purchased the sample from a meteorite dealer during the 2017 Tucson Gem and Mineral Show.

Physical characteristics: Sample is coated with a patchy, glossy, black, flow-lined fusion crust that covers $\sim 75 \%$ of the irregularly shaped stone. Sample contains a 
number of broad regmaglypts. Areas where fusion crust is absent show a lightcolored, fine-grained granular texture.

Petrography: Description and Classification (A. Love, App): Sample is a matrixsupported breccia composed of dominantly of granular mineral fragments and sparse ( $\sim 8$ vol\%) small (avg. dia. $1150 \mu \mathrm{m})$ angular-subrounded lithic clasts of intergranular to subophitic textured eucrite. Minerals are: birefringent clouded, plagioclase; exsolved pyroxenes; ilmenite; chromite; Fe metal; FeS.

Geochemistry: (A. Love, App) Low-Ca pyroxene host $\mathrm{Fs}_{59.4 \pm 0.6} \mathrm{Wo}_{2.0 \pm 0.2}\left(\mathrm{Fs}_{58.9-}\right.$ $\left.61.3 \mathrm{Wo}_{1.7-2.3}, \mathrm{FeO} / \mathrm{MnO}=30.2-34.5, \mathrm{~N}=12\right)$; high-Ca pyroxene exsolution lamellae $\mathrm{Fs}_{25.8 \pm 0.4} \mathrm{Wo}_{43.4 \pm 0.4}\left(\mathrm{Fs}_{25.1-26.5} \mathrm{Wo}_{42.8-44.0}, \mathrm{~N}=12\right)$; plagioclase $\mathrm{An}_{82.3 \pm 1.4} \mathrm{Or}_{0.6 \pm 0.2}\left(\mathrm{An}_{80.7-}\right.$ 84.0 $\mathrm{Or}_{0.4-1.0} \mathrm{~N}=8$ ).

Classification: HED (eucrite) $\mathrm{FeO} / \mathrm{MnO}$ and Fs compositions of pyroxenes indicate this sample is a basaltic eucrite. Original igneous textures within clasts are preserved while major element compositions of pyroxenes within sample are equilibrated. Minor element compositions of pyroxenes (Al, Ti, $\mathrm{Cr}$ ) are less equilibrated. Plagioclase compositions are unequilibrated.

Specimens: PARI holds the main mass. One endcut weighing $20 \mathrm{~g}$ and a polished thin section are on deposit at $A p p$.

\section{Northwest Africa 11857 (NWA 11857) \\ Northwest Africa \\ Purchased: 2018 \\ Classification: HED achondrite (Diogenite)}

History: One stone weighing $580 \mathrm{~g}$ was found in Morocco prior to 2018. Steve Arnold purchased the sample from a meteorite dealer while at the 2018 Tucson Gem and Mineral Show.

Physical characteristics: The uncrusted sample has a weathered orangish-yellow exterior and yellowish-orange interior that is brecciated and friable. The cut face shows angular-subrounded clasts up to $5 \mathrm{~mm}$ in the long dimension.

Petrography: Description and classification (A. Love, App): Sample is a breccia composed of diogenitic lithic fragments (with average grainsize of $681 \mu \mathrm{m}$ ) and coarser grained $(\sim 1287 \mu \mathrm{m})$ cataclastic clasts mixed within a comminuted host of similar materials. Lithic clasts are composed of interlocking, orthopyroxenes, which share $120^{\circ}$ grain boundaries. Orthopyroxenes contain CPX inclusions and exsolution lamellae (up to $0.5 \mathrm{~mm}$ ) within crystals. Additional minerals are $\mathrm{Si}$ polymorphs, chromite, FeS and rare Ni-free iron metal.

Geochemistry: (A. Love, App) Low-Ca pyroxene host $\mathrm{Fs}_{26.2 \pm 0.6} \mathrm{Wo}_{2.8 \pm 0.7}\left(\mathrm{Fs}_{26.2-}\right.$ $\left.{ }_{28.0} \mathrm{Wo}_{1.8-3.6}, \mathrm{FeO} / \mathrm{MnO}=26.3-27.6, \mathrm{~N}=12\right)$; high-Ca pyroxene inclusions and exsolution lamellae $\mathrm{Fs}_{10.6 \pm 0.5} \mathrm{Wo}_{44.0 \pm 1.2}\left(\mathrm{Fs}_{9.9-11.6} \mathrm{Wo}_{41.9-45.7}, \mathrm{FeO} / \mathrm{MnO}=20.1-21.2\right.$ $\mathrm{N}=6$ )

Classification: HED (monomict diogenite breccia). Textures, FeO/MnO and Fs compositions of pyroxenes indicate this sample is a diogenite.

Specimens: Steve Arnold holds the main mass. One $20 \mathrm{~g}$ endcut, several fragments and a polished thin section are on deposit at App. 


\title{
Northwest Africa 11858 (NWA 11858) \\ Morocco \\ Purchased: 2008 \\ Classification: HED achondrite (Howardite)
}

History: The meteorite was purchased at the Munich show in 2008 from a Moroccan meteorite dealer from Rissani.

Physical characteristics: The main mass is approximately $4 \times 4 \times 10 \mathrm{~cm}$, and is almost completely covered in fusion crust, some of which is dark brown and glassy with flow lines. Minor evidence of terrestrial weathering is present as red-brown straining and adhered dust. Cut surfaces reveal numerous, mostly gray clasts set in a fine-grained, light-gray matrix.

Petrography: (C. Herd and J. Hamilton, $U A b$ ) Well-consolidated breccia of clasts up to $5 \mathrm{~mm}$ in longest dimension of diogenite ( $\sim 25 \mathrm{vol} \%)$, unequilibrated eucrite $(\sim 10$ vol $\%)$, equilibrated eucrite $(\sim 10$ vol $\%)$, cumulate gabbroic eucrite $(\sim 10$ vol\%), and impact melt, as well as grains of anorthitic plagioclase, olivine, chromite, sulfide, and FeNi metal. All plagioclase has been transformed to maskelynite, and strong mosaicism is common. Fine-grained recrystallized areas of some gabbroic eucrite clasts indicate shock melting.

Geochemistry: (C. Herd, $U A b$ ) Diogenite orthopyroxene $\mathrm{Fs}_{27 \pm 3} \mathrm{Wo}_{2.6 \pm 0.8}$, $\mathrm{Fe} / \mathrm{Mn}=32 \pm 2, \mathrm{n}=23$, eucrite pigeonite average $\mathrm{Fs}_{48 \pm 10} \mathrm{Wo}_{6 \pm 3}, \mathrm{Fe} / \mathrm{Mn}=32 \pm 2$, range $\mathrm{Fs}_{32-63}, \mathrm{n}=32$, eucrite augite $\mathrm{Fs}_{40 \pm 10} \mathrm{Wo}_{28 \pm 10}, \mathrm{Fe} / \mathrm{Mn}=32 \pm 1, \mathrm{n}=10$; plagioclase $\mathrm{An}_{88 \pm 3} \mathrm{Ab}_{11 \pm 3} \mathrm{Or}_{0.5 \pm 0.3}, \mathrm{n}=21$; high-Ti $\left(15 \mathrm{wt} \% \mathrm{TiO}_{2}\right)$ and low-Ti $\left(0.8 \mathrm{wt} \% \mathrm{TiO}_{2}\right)$ chromite.

Classification: Achondrite (howardite) Specimens: Type specimen of $21.3 \mathrm{~g}$, including one thin section, is at $U A b$. Main mass at JTESM.

\section{Northwest Africa 11859 (NWA 11859)}

(Northwest Africa)

Purchased: 2017

Classification: Iron meteorite (IAB-MG)

History: Purportedly found in the NWA area. Presented to UCLA by A. Aaronson in 2017.

Geochemistry: (J.T. Wasson, UCLA): INAA data, $\mathrm{Ni}=69.1, \mathrm{Co}=4.54$ (both $\mathrm{mg} / \mathrm{g}), \mathrm{Ga}=94.9, \mathrm{As}=11.8, \mathrm{~W}=1.27, \mathrm{Ir}=3.75, \mathrm{Au}=1.485(\mathrm{all} \mu \mathrm{g} / \mathrm{g})$.

Classification: Iron, IAB main group. Probably paired with Campo del Cielo. Specimens: Type specimen at UCLA.

\author{
Northwest Africa 11860 (NWA 11860) \\ (Northwest Africa) \\ Purchased: 2017
}


Classification: Rumuruti chondrite (R3-5)

History: A single stone weighing $520 \mathrm{~g}$ was found in Morocco prior to 2017. J. Donald Cline and John Sinclair acquired the sample from a meteorite dealer at the Tucson Gem and Mineral Show in February of 2017.

Physical characteristics: Sample is black in color, lacks fusion crust, has a rounded flattened shape with shallow broad regmaglypts. A small cut face shows brecciated texture composed of clasts set within abundant small chondrules, which, are visible through the patina. Sample is non-magnetic and only sulfides appear to reflect light on the surface of the cut face.

Petrography: Description and Classification (A. Love, App) Sample is a breccia composed of up to $3 \mathrm{~cm}$ irregularly shaped, light-orangish to brown-black unequilibrated and equilibrated clasts set within a recrystallized host containing chondrules (avg. dia. $432 \mu \mathrm{m}$ ), fragments and abundant Ni-bearing sulfides and pentlandite. Host contains 40 vol\% well-defined, unequilibrated chondrules set within a recrystallized matrix. Unequilibrated clasts contain chemically-zoned, unequilibrated chondrules set within a fine-grained, opaque matrix. Equilibrated clasts contain olivine, low-Ca pyroxene within a recrystallized matrix containing isolated intergrowths of plagioclase with average size $30 \mu \mathrm{m}$ (Berlin and Stoeffler, 2004).

Geochemistry: (A. Love, App) Host: Olivine $\mathrm{Fa}_{25.0 \pm 14.7}\left(\mathrm{Fa}_{2.1-48.5}\right), \mathrm{Cr}_{2} \mathrm{O}_{3}$ in ferroan olivine $=0.1 \pm 0.1, \mathrm{Fe} / \mathrm{Mn}=74.5 \mathrm{n}=22$, Low Ca Pyroxene: $\mathrm{Fs}_{13.8 \pm 9.2} \mathrm{Wo}_{1.1 \pm 1.0}\left(\mathrm{Fs}_{2.1}\right.$ ${ }_{28.1} \mathrm{Wo}_{0.3-3.1}$ ), $\mathrm{N}=9$. Clast: Olivine $\mathrm{Fa}_{41.8 \pm 0.3}, \mathrm{Fe} / \mathrm{Mn}=94.5 \pm 2.2, \mathrm{n}=5$, low $\mathrm{Ca}$ Pyroxene: $\mathrm{Fs}_{36.0 \pm 0.2} \mathrm{Wo}_{2.2 \pm 0.3}, \mathrm{~N}=3$.

Classification: Rumuruti chondrite (R3-5, S2, wi-2). Based on Fa and Fe/Mn compositions of olivines, chondrule diameter and texture this sample is a Rumuruti chondrite breccia. Based on the coefficient of variation of olivine within unequilibrated clast, the petrologic subtype is estimated at 3.4 (Bischoff, 2000). Based on presence of low-Ca pyroxene and isolated plagioclase intergrowths within equilibrated clasts, these represent type 5 material.

Specimens: PARI holds the main mass. One endcut weighing $20.02 \mathrm{~g}$ and a polished thin section are on deposit at $A p p$.

\section{Northwest Africa 11861 (NWA 11861)}

(Northwest Africa)

Purchased: 2018

Classification: HED achondrite (Eucrite, polymict)

History: A single $538 \mathrm{~g}$ stone was found in Morocco prior to 2018. Steve Arnold purchased the stone from a meteorite dealer during the 2018 Tucson Gem and Mineral Show.

Physical characteristics: Stone is irregularly shaped and $\sim 20 \%$ is covered by a weathered fusion crust. The weathered exterior is light orangish-gray in color and light gray on the cut face. Numerous mineral and rock clasts can be seen on the cut face. 
Petrography: Description and classification (A. Love, $A p p$ ): Sample is a breccia composed of up to $5 \mathrm{~mm}$ angular basaltic and cumulate textured eucritic $(\sim 13$ vol $\%)$ and diogenitic ( $\sim 8 \mathrm{vol} \%)$ lithic clasts and related mineral fragments hosted within a fine-grained comminuted matrix. Eucrite clasts have ophitic to subophitic textures and exsolved pyroxenes. Minerals: Chromite, Ilmenite, FeS, Si Polymorph, phosphate.

Geochemistry: (A. Love, App) Eucrite: Low-Ca pyroxene host

$\mathrm{Fs}_{49.7 \pm 7.6} \mathrm{Wo}_{2.5 \pm 0.7}\left(\mathrm{Fs}_{40.7-65.8} \mathrm{Wo}_{1.7-4.5}, \mathrm{FeO} / \mathrm{MnO}=27.5-32.3, \mathrm{~N}=16\right)$; pigeonite $\left(\mathrm{Fs}_{42.0 \pm 4.4} \mathrm{Wo}_{12.2 \pm 6.7}\left(\mathrm{Fs}_{35.9-48.4} \mathrm{Wo}_{8.5-25.7}, \mathrm{FeO} / \mathrm{MnO}=29.0-31.2, \mathrm{~N}=5\right)\right.$; high-Ca pyroxene exsolution lamellae $\mathrm{Fs}_{23.73 \pm 6.1} \mathrm{Wo}_{42.5 \pm 1.8}\left(\mathrm{Fs}_{16.1-33.5} \mathrm{Wo}_{39.5-44.2}, \mathrm{~N}=9\right)$; plagioclase $\mathrm{An}_{85.8 \pm 7.4} \mathrm{Or}_{0.6 \pm 0.7}\left(\mathrm{An}_{71.5-94.7}, \mathrm{Or}_{0.0-1.6} \mathrm{~N}=10\right)$. Diogenite: Low-Ca pyroxene $\mathrm{Fs}_{26.4 \pm 3.8} \mathrm{Wo}_{2.4 \pm 0.5}\left(\mathrm{Fs}_{21.1-31.8} \mathrm{Wo}_{1.7-3.4}, \mathrm{FeO} / \mathrm{MnO}=27.8-34.7, \mathrm{~N}=10\right)$

Classification: HED (polymict eucrite breccia). $\mathrm{FeO} / \mathrm{MnO}$ and $\mathrm{Fs}$ compositions of pyroxenes indicate this sample is a HED meteorite composed of a mixture of eucrite and diogenite components.

Specimens: Steve Arnold holds the main mass. Three slices and an endcut totaling $20 \mathrm{~g}$ and a polished thin section are on deposit at App.

\section{Northwest Africa 11862 (NWA 11862)}

(Northwest Africa)

Purchased: 2005

Classification: Ordinary chondrite (H4/5)

History: Purchased by an anonymous collector from a dealer at the Munich Show in 2005, subsequently acquired by the Hollis Collection and then donated to PSF. Petrography: (A. Irving and S. Kuehner, UWS) Some well-formed chondrules are set in a recrystallized matrix containing relatively abundant altered metal.

Geochemistry: Olivine $\left(\mathrm{Fa}_{18.9-19.1}, \mathrm{~N}=3\right)$, orthopyroxene $\left(\mathrm{Fs}_{16.3-17.2} \mathrm{Wo}_{1.0-0.7}, \mathrm{~N}=\right.$ 3), clinopyroxene $\left(\mathrm{Fs}_{6.0-6.2} \mathrm{Wo}_{45.0-47.3}, \mathrm{~N}=2\right)$.

Classification: Ordinary chondrite (H4/5).

Specimens: The entire specimen including one polished thin section is at PSF.

\section{Northwest Africa 11863 (NWA 11863)}

(Northwest Africa)

Purchased: 2005

Classification: Ordinary chondrite (L6)

History: Purchased by an anonymous collector from a dealer at the Munich Show in 2005, subsequently acquired by the Hollis Collection and then donated to PSF. Petrography: (A. Irving and S. Kuehner, UWS) Mostly recrystallized with rare remnant chondrules.

Geochemistry: Olivine $\left(\mathrm{Fa}_{25.0-25.8}, \mathrm{~N}=3\right)$, orthopyroxene $\left(\mathrm{Fs}_{20.6-21.2} \mathrm{Wo}_{2.0-2.5}, \mathrm{~N}=\right.$ 3), clinopyroxene ( $\left.\mathrm{Fs}_{9.4-9.9} \mathrm{Wo}_{42.8-42.7}, \mathrm{~N}=2\right)$.

Classification: Ordinary chondrite (L6).

Specimens: The entire specimen including one polished thin section is at PSF. 


\section{Northwest Africa 11864 (NWA 11864)}

(Northwest Africa)

Purchased: 2005

Classification: Ordinary chondrite (L4)

History: Purchased by an anonymous collector from a dealer at the Munich Show in 2005, subsequently acquired by the Hollis Collection and then donated to PSF. Petrography: (A. Irving and S. Kuehner, UWS) Well-formed chondrules are set in relatively coarse grained matrix.

Geochemistry: Olivine $\left(\mathrm{Fa}_{24.8-25.1}, \mathrm{~N}=3\right)$, orthopyroxene $\left(\mathrm{Fs}_{18.9-20.6} \mathrm{Wo}_{1.1-0.5}, \mathrm{~N}=\right.$ $3)$, subcalcic augite $\left(\mathrm{Fs}_{18.1} \mathrm{Wo}_{26.7}\right)$, augite $\left(\mathrm{Fs}_{10.6} \mathrm{Wo}_{36.3}\right)$.

Classification: Ordinary chondrite (L4).

Specimens: The entire specimen including one polished thin section is at PSF.

\section{Northwest Africa 11865 (NWA 11865)}

(Northwest Africa)

Purchased: 2005

Classification: Ordinary chondrite (L6)

History: Purchased by an anonymous collector from a dealer at the Munich Show in 2005, subsequently acquired by the Hollis Collection and then donated to PSF. Petrography: (A. Irving and S. Kuehner, UWS) Mostly recrystallized with rare remnant chondrules.

Geochemistry: Olivine $\left(\mathrm{Fa}_{24.5-25.0}, \mathrm{~N}=3\right)$, orthopyroxene $\left(\mathrm{Fs}_{21.6-21.7} \mathrm{Wo}_{1.6-1.9}, \mathrm{~N}=\right.$ 3), clinopyroxene $\left(\mathrm{Fs}_{8.1-8.9} \mathrm{Wo}_{44.4-43.7}, \mathrm{~N}=2\right)$.

Classification: Ordinary chondrite (L6).

Specimens: The entire specimen including one polished thin section is at PSF.

Northwest Africa 11866 (NWA 11866)

(Northwest Africa)

Purchased: 2018 May

Classification: Martian meteorite (Shergottite)

History: Purchased by Ben Hoefnagels in May 2018 from a dealer in Zagora, Morocco.

Physical characteristics: A group of broken fragments with exterior coatings of black fusion crust $(23.3 \mathrm{~g})$ from an unknown larger stone. The pieces are very fresh and contain gray pyroxene and obvious glassy maskelynite.

Petrography: (A. Irving and S. Kuehner, UWS) Diabasic texture. Igneous assemblage of predominantly zoned clinopyroxene and maskelynite with accessory titanomagnetite, ilmenite, pyrrhotite and rare Fe-merrillite. Low-Ca pyroxene compositions do not extend to very magnesian compositions. 
Geochemistry: Pigeonite $\left(\mathrm{Fs}_{41.8-58.8} \mathrm{Wo}_{11.1-14.4}, \mathrm{FeO} / \mathrm{MnO}=33-36, \mathrm{~N}=4\right)$, subcalcic augite $\left(\mathrm{Fs}_{24.5-47.2} \mathrm{Wo}_{33.9-28.8}, \mathrm{FeO} / \mathrm{MnO}=30-38, \mathrm{~N}=3\right)$, maskelynite $\left(\mathrm{An}_{53.8-54.4} \mathrm{Or}_{0.9}, \mathrm{~N}=2\right)$.

Classification: Martian (shergottite, diabasic). Although the pyroxene compositions are somewhat like those in Shergotty, the trend for low-Ca pyroxenes does not extend to as magnesian values.

Specimens: $4.8 \mathrm{~g}$ in the form of a polished endcut at $U W B$; remainder with Mr. B. Hoefnagels.

\section{Northwest Africa 11868 (NWA 11868)}

(Northwest Africa)

Purchased: 2005

Classification: Ordinary chondrite (L6)

History: Purchased by an anonymous collector from a dealer at the Munich Show in 2005, subsequently acquired by the Hollis Collection and then donated to PSF. Petrography: (A. Irving and S. Kuehner, UWS) Mostly recrystallized with rare remnant chondrules.

Geochemistry: Olivine $\left(\mathrm{Fa}_{24.8-24.9}, \mathrm{~N}=3\right)$, orthopyroxene $\left(\mathrm{Fs}_{20.8-21.8} \mathrm{Wo}_{1.0-0.9}, \mathrm{~N}=\right.$ 3), clinopyroxene $\left(\mathrm{Fs}_{7.2-12.2} \mathrm{Wo}_{45.7-43.1}, \mathrm{~N}=2\right)$.

Classification: Ordinary chondrite (L6).

Specimens: The entire specimen including one polished thin section is at PSF.

\section{Northwest Africa 11869 (NWA 11869)}

(Northwest Africa)

Purchased: 2005

Classification: Ordinary chondrite (L4)

History: Purchased by an anonymous collector from a dealer at the Munich Show in 2005, subsequently acquired by the Hollis Collection and then donated to PSF. Petrography: (A. Irving and S. Kuehner, UWS) Well-formed chondrules are set in relatively coarse grained, shock-darkened matrix.

Geochemistry: Olivine $\left(\mathrm{Fa}_{23.5-23.7}, \mathrm{~N}=3\right)$, orthopyroxene $\left(\mathrm{Fs}_{20.0-20.2} \mathrm{Wo}_{3.4-1.5}, \mathrm{~N}=\right.$ 3 ), subcalcic augite $\left(\mathrm{Fs}_{11.3} \mathrm{Wo}_{27.4}\right)$, augite $\left(\mathrm{Fs}_{12.6} \mathrm{Wo}_{35.6}\right)$.

Classification: Ordinary chondrite (L4, shock-darkened).

Specimens: The entire specimen including one polished thin section is at PSF.

\section{Northwest Africa 11870 (NWA 11870)}

(Northwest Africa)

Purchased: 2005

Classification: Ordinary chondrite (L5)

History: Purchased by an anonymous collector from a dealer at the Munich Show in 2005, subsequently acquired by the Hollis Collection and then donated to PSF. 
Petrography: (A. Irving and S. Kuehner, UWS) Mostly recrystallized with sparse remnant chondrules.

Geochemistry: Olivine $\left(\mathrm{Fa}_{24.7-24.8}, \mathrm{~N}=3\right)$, orthopyroxene $\left(\mathrm{Fs}_{21.4-21.5} \mathrm{Wo}_{1.3-1.2}, \mathrm{~N}=\right.$ $3)$, subcalcic augite $\left(\mathrm{Fs}_{12.0} \mathrm{Wo}_{29.1}\right)$, augite $\left(\mathrm{Fs}_{11.1} \mathrm{Wo}_{43.8}\right)$.

Classification: Ordinary chondrite (L5).

Specimens: The entire specimen including one polished thin section is at PSF.

\section{Northwest Africa 11871 (NWA 11871)}

Algeria

Purchased: 2018 May

Classification: Lunar meteorite (feldspathic breccia)

History: Purportedly found near Tindouf, Algeria and purchased by Joseph Hum in May 2018 from a dealer in Gladstone, Oregon, who obtained it from a Mauritanian source.

Petrography: (A. Irving and S. Kuehner, UWS) Breccia consisting of mineral clasts of anorthite, olivine, exsolved pigeonite, unexsolved pigeonite,orthopyroxene, aluminous subcalcic augite, chromite, ilmenite, troilite and kamacite in a fine grained, partly vesicular matrix.

Geochemistry: Olivine $\left(\mathrm{Fa}_{19.7-51.6}, \mathrm{FeO} / \mathrm{MnO}=92-105, \mathrm{~N}=3\right)$, orthopyroxene host $\left(\mathrm{Fs}_{45.7} \mathrm{Wo}_{3.6}, \mathrm{FeO} / \mathrm{MnO}=55\right)$, clinopyroxene exsolution lamella $\left(\mathrm{Fs}_{24.2} \mathrm{Wo}_{34.6}\right.$, $\mathrm{FeO} / \mathrm{MnO}=54)$, orthopyroxene $\left(\mathrm{Fs}_{47.9} \mathrm{Wo}_{3.6}, \mathrm{FeO} / \mathrm{MnO}=59\right)$, pigeonite $\left(\mathrm{Fs}_{24.8} \mathrm{Wo}_{10.0}, \mathrm{FeO} / \mathrm{MnO}=68\right)$, aluminous subcalcic augite $\left(\mathrm{Fs}_{18.5} \mathrm{Wo}_{23.9}, \mathrm{FeO} / \mathrm{MnO}\right.$ $=56, \mathrm{Al}_{2} \mathrm{O}_{3}=15.4$ wt. $\left.\%, \mathrm{Na}_{2} \mathrm{O}=0.4 \mathrm{wt} . \%\right)$, plagioclase $\left(\mathrm{An}_{94.1-96.4} \mathrm{Or}_{0.3-0.1}\right)$.

Classification: Lunar (feldspathic regolithic breccia).

Specimens: $4.73 \mathrm{~g}$ including a polished endcut at $U W B$; remainder with Mr. J. Hum.

\section{Northwest Africa 11872 (NWA 11872) \\ (Northwest Africa) \\ Purchased: 2007 June \\ Classification: Ordinary chondrite (L4)}

History: Purchased from a moroccan mineral dealer at the meteorite show in Ensisheim, France.

Physical characteristics: Individual with about 50\% fusion crust and deep, welldeveloped regmaglypts. The fusion crust and old fracture planes appear light brown, in contrast to the shock-darkened interior which is exposed on younger fracture planes.

Petrography: Brecciated shock-darkened ordinary chondrite with well-preserved chondrules. Silicates with tiny metal and sulfide veins along cracks and grain boundaries. Shock-deformed apatite with enclosed silicate clasts. One large sulfide grain $(1.2 \mathrm{~cm})$ was found. 


\section{Northwest Africa 11873 (NWA 11873)}

(Northwest Africa)

Purchased: 2008 June

Classification: Ordinary chondrite (LL3-6)

History: Purchased from a Moroccan mineral dealer at the mineral show in SainteMarie-aux-Mines, France

Physical characteristics: Individual without fusion crust. Brecciation texture with differently colored clasts is visible. One fine-grained, gray, melt-rock clast of about $4 \mathrm{~cm}$ stands out.

Petrography: Genomict LL chondritic breccia, consisting of type 3 and type 6 clasts, embedded in a clastic matrix. The matrix also contains intact chondrules. One large melt rock clast occurs, consisting of euhedral igneously zoned olivine grains, embedded in a glassy mesostasis.

Geochemistry: Type 3 clast: Olivine $\mathrm{Fa}_{28.2 \pm 4.1}\left(\mathrm{Fa}_{12.9-32.7} ; \mathrm{n}=20\right)$. Pyroxene $\mathrm{Fs}_{17.2 \pm 6.0} \mathrm{Wo}_{0.8 \pm 0.5}\left(\mathrm{Fs}_{8.0-24.4} \mathrm{Wo}_{0.1-1.7} ; \mathrm{n}=15\right)$. Type 6 clast: Olivine $\mathrm{Fa}_{29.3 \pm 0.4}\left(\mathrm{Fa}_{28.5-30.0}\right.$; $\mathrm{n}=12)$. Pyroxene $\mathrm{Fs}_{23.6 \pm 0.4} \mathrm{Wo}_{1.5 \pm 0.2}\left(\mathrm{Fs}_{23.1-24.2} \mathrm{Wo}_{1.3-1.9} ; \mathrm{n}=9\right)$. Olivine in melt rock clast: $\mathrm{Fa}_{11.6-42.2}(\mathrm{n}=2)$.

\section{Northwest Africa 11880 (NWA 11880) \\ Northwest Africa \\ Purchased: 2018 Jan \\ Classification: Rumuruti chondrite (R3.5-4)}

Petrography: The sample is a breccia, consisting of light and dark clasts. The light clasts display well-defined chondrules surrounded by a fine-grained matrix. The dark clasts show very well-defined chondrules in a dark and glassy matrix. Mean apparent chondrule size is $550 \pm 377 \mu \mathrm{m}$ in the dark clasts, and $694 \pm 316 \mu \mathrm{m}$ in the light clasts. Maximum chondrule size is $2.65 \mathrm{~mm}$. Undulatory extinction is visible in olivine grains. No oxidation rims are seen on sulphides or metal grains.

Geochemistry: As determined by EPMA, Dark Clast olivine compositions are: $\mathrm{Fa}=31.3 \pm 27.6(\mathrm{n}=35)$, coefficient of variation $\mathrm{CV} \%=43.7, \mathrm{Cr}_{2} \mathrm{O}_{3}(\mathrm{wt} \%)=0.14$. Dark clast pyroxene compositions are: High Ca Pyroxene $\mathrm{Fs}_{18.2 \pm 5.5} \mathrm{En}_{52.5 \pm 7.3} \mathrm{Wo}_{29.2 \pm 12}(\mathrm{n}=4)$, Low Ca Pyroxene - Fs $29.6 \pm 5.6 \mathrm{En}_{66.2 \pm 1.2} \mathrm{Wo}_{4.2 \pm 5.1}$, $(\mathrm{n}=5)$. Light Clast olivine compositions are: $\mathrm{Fa}_{40.9 \pm 0.6}(\mathrm{n}=10), \mathrm{CV} \%=1.1$, $\mathrm{Cr}_{2} \mathrm{O}_{3}(\mathrm{wt} \%)=0.08$. Light clast pyroxene compositions are:

$\mathrm{Fs}_{13.7 \pm 3.4} \mathrm{En}_{43.9 \pm 0.7} \mathrm{Wo}_{42.4 \pm 4.0}(\mathrm{n}=6)(\mathrm{V}$. Di Cecco, $R O M)$

Classification: Pyroxenes: Dark/Unequilibrated Clast - High and low Ca pyroxenes occur in roughly equal proportions. Light/Equilibrated Clast - Ca pyroxene is dominant. This follows the summary of Bischoff et al. (2011) for R chondrites. $\mathrm{Fa}_{40.9 \pm 0.6}$ is consistent with reported composition of equilibrated $\mathrm{R}$ chondrites. $\mathrm{CV} \%$ of 43.7 suggests petrologic type 3.5 .

Northwest Africa 11881 (NWA 11881)

(Northwest Africa) 
Purchased: 2018

Classification: Ordinary chondrite (L3)

History: Bought from Mohamed Elguirah in Zagora in 2018.

Petrography: (J. Gattacceca, CEREGE) Packed chondrules with average size about $700 \mu \mathrm{m}$.

Geochemistry: Olivine $\mathrm{Fa}_{7.3-29.1}, \mathrm{Fa} \mathrm{PMD}=32 \% . \mathrm{Cr}_{2} \mathrm{O}_{3}$ in ferroan olivine $0.07 \pm 0.07 \mathrm{wt} \%(\mathrm{n}=7)$.

Classification: L3. L group based on magnetic susceptibility and chondrule size.

\section{Northwest Africa 11886 (NWA 11886)}

(Northwest Africa)

Purchased: 2018 March

Classification: Lunar meteorite

History: Bought in Erfoud, Morocco, in March 2018

Physical characteristics: Cut surface reveals a gray interior with light grey and white clasts.

Petrography: (J. Gattacceca, CEREGE) Igneous fragmental breccia. Main minerals are pyroxene and plagioclase with typical grain size of $300 \mu \mathrm{m}$. Other minerals include olivine, chromite, troilite, silica polymorph (to $200 \mu \mathrm{m}$ ), ilmenite, rare metal.

Geochemistry: Pyroxene $\mathrm{Fs}_{44.4 \pm 6.0} \mathrm{Wo}_{22.9 \pm 9.0}, \mathrm{FeO} / \mathrm{MnO}=67.3 \pm 12.4(\mathrm{~N}=5)$.

Plagioclase $\mathrm{An}_{92.7} \mathrm{Ab}_{7.2} \mathrm{Or}_{0.1}(\mathrm{~N}=2)$. Olivine $\mathrm{Fa}_{87.8}, \mathrm{FeO} / \mathrm{MnO}=109(\mathrm{~N}=1)$.

Classification: Lunar, fragmental breccia

Specimens: $7.5 \mathrm{~g}$ at CEREGE. Main mass with Labenne

\section{Northwest Africa 11887 (NWA 11887)}

(Northwest Africa)

Purchased: 2015

Classification: Mesosiderite

Physical characteristics: Cut surface reveals abundant metal, up to cm size.

Petrography: (J. Gattacceca, CEREGE) Main minerals are pyroxene (to $200 \mu \mathrm{m}$ ), interstitial plagioclase (to $150 \mu \mathrm{m}$ ), kamacite (to $\mathrm{cm}$ ). Other minerals: troilite, silica polymorph, merrillite. Silicate show triple junctions.

Geochemistry: Orthopyroxene $\mathrm{Fs}_{15.9} \mathrm{Wo}_{0.6}, \mathrm{FeO} / \mathrm{MnO}=34.7(\mathrm{~N}=1)$. Plagioclase $\mathrm{An}_{89.9} \mathrm{Ab}_{9.6} \mathrm{Or}_{0.5}(\mathrm{~N}=3)$.

Classification: Mesosiderite

Specimens: $16 \mathrm{~g}$ at CEREGE. Main mass with Labenne

\section{Northwest Africa 11888 (NWA 11888)}

(Northwest Africa)

Purchased: 2018

Classification: HED achondrite (Diogenite) 
Physical characteristics: Dark stone. Cut surface reveals a dark gray interior with a variety of clasts up to $\mathrm{cm}$.

Petrography: (J. Gattacceca, CEREGE) Brecciated igneous rock with fractured lithic and mineral clasts set in a clastic matrix. The dominant mineral is pyroxene (to $500 \mathrm{um}$ ) and plagioclase (to $400 \mu \mathrm{m}$ ). Other minerals: troilite, chromite, silica polymorph, FeNi metal.

Geochemistry: Low-Ca pyroxene shows a wide range of composition from $\mathrm{Fs}_{12.9}$ to $\mathrm{Fs}_{47.4}$, and average $\mathrm{Fa}_{26.6 \pm 10.0} \mathrm{Wo}_{2.8 \pm 1.7}, \mathrm{FeO} / \mathrm{MnO}=30.0 \pm 1.8(\mathrm{n}=15)$. Augite exsolution $\mathrm{Fs}_{25.2} \mathrm{Wo}_{39.2}(\mathrm{n}=1)$. Plagioclase $\mathrm{An}_{86.6} \mathrm{Ab}_{12.9} \mathrm{Or}_{0.5}(\mathrm{~N}=2)$

Classification: Diogenite

Specimens: $30.9 \mathrm{~g}$ and a polished section at CEREGE. Main mass with R. Lenssen.

\section{Northwest Africa 11889 (NWA 11889)}

(Northwest Africa)

Purchased: 2018

Classification: Carbonaceous chondrite (CM2)

Physical characteristics: Dark stone. Cut surface reveals homogeneous dark interior.

Petrography: (J. Gattacceca, CEREGE) Chondrules, some with fine grained dust rim, with average size $180 \pm 70 \mu \mathrm{m}(\mathrm{N}=20)$ set in a abundant fine grained iron rich matrix (61 vol\% by point counting). Opaques are magnetite and troilite. No metal was observed. Low microprobe analyses totals in the matrix indicate the presence of phyllosilicates.

Geochemistry: Olivine $\mathrm{Fa}_{1.0-46.6}, \mathrm{Cr}_{2} \mathrm{O}_{3} 0.25 \pm 0.14$ wt\% $(\mathrm{n}=10)$. Defocalized (10 $\mu \mathrm{m})$ electron microprobe analyses in the matrix yield totals of $75 \mathrm{wt} \%$.

Classification: $\mathrm{CM} 2$

Specimens: $23.5 \mathrm{~g}$ and a polished section at CEREGE. Main mass with R. Lenssen.

\section{Northwest Africa 11890 (NWA 11890) \\ (Northwest Africa) \\ Purchased: Nov 2016 \\ Classification: Ureilite}

Petrography: coarse-grained olivine with reduced rims $(90 \%)$, pyroxene $(<5 \%)$, Fe-rich metal, and carbon phases. Graphite, metal, and sulfide occur in veins around the olivines and pyroxenes. Olivines are up to $2 \mathrm{~mm}$. Si glass, graphite, and diamonds were also identified.

Geochemistry: Mineral Compositions and Geochemistry: Olivine cores, $\mathrm{Fa}_{21.7 \pm 0.1}$; rims $\mathrm{Fa}_{8.2 \pm 4.0}$. Pyroxenes contain a varied texture with lighter and darker spots, which display differing compositions: brighter $\mathrm{Fs}_{9.3 \pm 1.8} \mathrm{Wo}_{30.4 \pm 4.6}$; darker, $\mathrm{Fs}_{12.6 \pm 2.5} \mathrm{Wo}_{9.3 \pm 1.7}$ ).

Classification: Ureilite

Specimens: $20.0 \mathrm{~g}$ including a thin section and polished mount at $U W O$. 


\section{Northwest Africa 11891 (NWA 11891)}

(Northwest Africa)

Purchased: Nov 2016

Classification: Ureilite

Petrography: olivine (90\%) with reduced rims, pyroxene (5\%), metals, and carbon phases. Olivine grains are up to $1.5 \mathrm{~mm}$. Graphite and diamonds were found.

Geochemistry: Mineral Compositions and Geochemistry: Olivine cores, $\mathrm{Fa}_{19.7 \pm 0.1}$; rims, $\mathrm{Fa}_{10.2 \pm 2.0}$. Pyroxene, $\mathrm{Fs}_{16.7 \pm 0.2} \mathrm{Wo}_{7.6 \pm 0.1}$.

Classification: Ureilite

Specimens: $20.1 \mathrm{~g}$ including a thin section and polished mount at UWO.

\section{Northwest Africa 11892 (NWA 11892)}

(Northwest Africa)

Purchased: Nov 2016

Classification: Ureilite

Petrography: olivine (80\%) with reduced rims, pyroxenes $(15 \%)$, metal, sulfides, and carbon phases. Metal grains are present in veins and at the edges of olivine, pyroxene, and carbon grains. Olivines are up to $2.5 \mathrm{~mm}$. Graphite and diamond were found.

Geochemistry: Mineral Compositions and Geochemistry: Olivine cores, $\mathrm{Fa}_{21.6 \pm 0.1}$; rims $\mathrm{Fa}_{8.9 \pm 3.0}$. Pyroxenes contain a varied texture with lighter and darker spots, which display differing compositions: brighter $\mathrm{Fs}_{8.0 \pm 1.8} \mathrm{Wo}_{31.9 \pm 6.8}$; darker $\left.\mathrm{Fs}_{11.5 \pm 2.4} \mathrm{Wo}_{7.9 \pm 1.6}\right)$.

Classification: Ureilite

Specimens: $37.8 \mathrm{~g}$ including a thin section and polished mount at UWO.

Northwest Africa 11893 (NWA 11893)

(Northwest Africa)

Purchased: Feb 2017

Classification: Ureilite

Petrography: The sample has a fine grained brecciated texture. It contains olivine $(\sim 65 \%)$ with reduced rims, pyroxene (pigeonite) ( $\sim 25 \%)$, Metals in veins around grain boundaries, and graphite. Olivines are up to $3 \mathrm{~mm}$. Graphite was found.

Geochemistry: Mineral Compositions and Geochemistry: Olivine core and rim compositions vary, with reduced rims. Cores $\mathrm{Fa}_{16.0 \pm 0.1}\left(\mathrm{Fa}_{15.9-16.1}, \mathrm{~N}=10\right)$; rims $\mathrm{Fa}_{4.5 \pm 2.3}\left(\mathrm{Fa}_{1.9}\right.$ 8.4, $\left.\mathrm{N}=10\right)$. All pyroxenes are clinopyroxene, $\mathrm{Fs}_{13.7 \pm 0.1} \mathrm{Wo}_{7.4 \pm 0.1}\left(\mathrm{Fs}_{13.4-}\right.$ ${ }_{13.8} \mathrm{Wo}_{7.3-7.5}, \mathrm{~N}=10$ ).

Classification: Ureilite

Specimens: $21.0 \mathrm{~g}$ including a thin section and polished mount at UWO. 
Northwest Africa 11894 (NWA 11894)

(Northwest Africa)

Purchased: 2016

Classification: Ureilite

History: Specimen was purchased from a dealer in Dakhla, Western Moroccan Sahara.

Petrography: olivine ( $\sim 80 \%)$ with reduced rims, pyroxene (pigeonite) $(\sim 15 \%)$, metals, and carbon phases. Olivines are up to $1 \mathrm{~mm}$ in size. Graphite and diamonds were found.

Geochemistry: Mineral Compositions and Geochemistry: Olivine cores, $\mathrm{Fa}_{19.2 \pm 0.0}$; rims $\mathrm{Fa}_{12.6 \pm 0.0}$ ). Pyroxenes, $\left.\mathrm{Fs}_{13.7 \pm 0.1} \mathrm{Wo}_{7.4 \pm 0.1}\right)$.

Classification: Ureilite

Specimens: $15.3 \mathrm{~g}$ including a thin section at $U W O$.

\section{Northwest Africa 11895 (NWA 11895)}

Sahara

Find: 1998

Classification: Ordinary chondrite (H3-5)

History: Sample was found 1998 by the Labenne group. The coordinates are unknown.

Petrography: The sample is a breccia and contains fragments of different petrologic type, most fragments are recrystallized (type 5) and some are unequilibrated.

Geochemistry: Olivine compositions are $\mathrm{Fa}_{14.2-21.0}$ (mean $\mathrm{Fa}_{17.6 \pm 2.5}, \mathrm{n}=9$ ), pyroxene compositions are $\mathrm{Fs}_{8.2-20.4} \mathrm{Wo}_{0.8-2.6}\left(\right.$ mean $\left.\mathrm{Fs}_{15.7 \pm 3.4} \mathrm{Wo}_{1.4 \pm 0.5}, \mathrm{n}=11\right)$. The equilibrated clasts have olivine compositions of $\mathrm{Fa}_{19.2 \pm 0.2}(\mathrm{n}=3)$ and pyroxene compositions of $\mathrm{Fs}_{16.9 \pm 0.3} \mathrm{Wo}_{1.3 \pm 0.2}(\mathrm{n}=3)$.

Classification: Brecciated $\mathrm{H}$ chondrite (H3-5) based on mineral compositions and chondrule size. Very weakly shocked (S2), weathering degree: W2

\section{Northwest Africa 11896 (NWA 11896) \\ (Northwest Africa) \\ Purchased: 2017 \\ Classification: Martian meteorite}

History: The Smithsonian specimen was purchased from Mendy Ouzillou, who had purchased it from Stefan Ralew in Ensisheim in 2017.

Physical characteristics: The type specimen has black exterior except for its sawcut face. The saw cut exposes a gray interior that includes black spherules with sharp boundaries along the contact with the matrix and white, gray, and black clasts.

Petrography: Petrographic and microprobe examination of the polished sections show this breccia contains clasts, spherical inclusions, and mineral fragments with a wide range of textures and compositions. The fragmental minerals include 
orthopyroxene, pigeonite, augite, plagioclase, albitic feldspar, potassium feldspar, phosphates, and opaque oxides. The clasts include basaltic (pyroxene-plagioclase) lithic clasts, with varying mineral compositions. This meteorite is texturally similar to Northwest Africa 7034 and 7475.

Geochemistry: Mineral compositions were determined by EPMA at SI. Low-Ca pyroxene are $\mathrm{Fs}_{34 \pm 8} \mathrm{Wo}_{5 \pm 4}, \mathrm{Fe} / \mathrm{Mn}=35 \pm 2, \mathrm{n}=144$. Augite are $\mathrm{Fs}_{24 \pm 7} \mathrm{Wo}_{40 \pm 4}, \mathrm{Fe} / \mathrm{Mn}$ $=32 \pm 3, \mathrm{n}=53$. Collectively, the pyroxenes in Fe-Mn composition space have linear regression slope equal to 0.0289, using the method of Papike et al. (2003) American Mineralogist 88: 469. Plagioclase $\mathrm{Ab}_{55 \pm 10} \mathrm{An}_{43 \pm 10} \mathrm{Or}_{2.4 \pm 0.7}, \mathrm{n}=71$, albitic feldspar $\mathrm{Ab}_{81 \pm 11} \mathrm{An}_{13 \pm 11} \mathrm{Or}_{5.4 \pm 3.2}, \mathrm{n}=19$, and potassium feldspar $\mathrm{Ab}_{20 \pm 13} \mathrm{An}_{2.0 \pm 1.5} \mathrm{Or}_{78 \pm 14}, \mathrm{n}=18$ are all present.

Classification: Martian, basaltic breccia

\section{Northwest Africa 11897 (NWA 11897) \\ (Northwest Africa) \\ Purchased: 2018 \\ Classification: HED achondrite (Eucrite, unbrecciated)}

History: Purchased in March 2018 by Craig Zlimen and Dustin Dickens from a Moroccan meteorite dealer.

Physical characteristics: Fusion crusted exterior; sawcut reveals a fine-grained, gray interior.

Petrography: (C. Agee, $U N M$ ) This meteorite is a porphyritic basalt with pigeonite phenocrysts set in a groundmass of fine-grained quench crystals of pyroxene, plagioclase, silica, chromite, and Fe-sulfide. Pigeonite phenocrysts show igneous zoning.

Geochemistry: (C. Agee, $U N M$ ) pigeonite $\mathrm{Fs}_{45.5 \pm 8.4} \mathrm{Wo}_{5.2 \pm 2.0}, \mathrm{Fe} / \mathrm{Mn}=36 \pm 2, \mathrm{n}=16$. Classification: Unequilibrated, porphyritic eucrite; unbrecciated

Specimens: 8.9 g including a probe mount on deposit at UNM, Craig Zlimen and Dustin Dickens hold the main mass.

\section{Northwest Africa 11898 (NWA 11898) \\ Mali}

Purchased: 2018

Classification: Lunar meteorite (feldspathic breccia)

History: Purchased in March 2018 by Dustin Dickens from a meteorite dealer in Mali.

Physical characteristics: Several identical appearing dark stones covered partially with a milky-green fusion crust. Broken surface reveals a fragmental breccia with numerous white feldspathic clasts set in a dark colored ground mass.

Petrography: (C. Agee, UNM) This meteorite is a breccia of primarily feldspathic clasts, with lesser amounts of olivine and pyroxene fragments. The shock melt composition has a very high alumina content, vesicles occur throughout. 
Geochemistry: (C. Agee, $U N M$ ) olivine $\mathrm{Fa}_{25.0 \pm 6.4}, \mathrm{Fe} / \mathrm{Mn}=90 \pm 5, \mathrm{n}=8$; pigeonite $\mathrm{Fs}_{31.0 \pm 3.6} \mathrm{Wo}_{8.1 \pm 3.6}, \mathrm{Fe} / \mathrm{Mn}=58 \pm 4, \mathrm{n}=6$; high-Ca pyroxene $\mathrm{Fs}_{22.3 \pm 3.7} \mathrm{Wo}_{35.0 \pm 4.7}$, $\mathrm{Fe} / \mathrm{Mn}=52 \pm 5, \mathrm{n}=4$; plagioclase $\mathrm{An}_{96.3 \pm 0.7} \mathrm{Ab}_{3.5 \pm 0.7} \mathrm{Or}_{0.2 \pm 0.0}, \mathrm{n}=6$; Shock melt $(20 \mu \mathrm{m}$ defocused electron beam, proxy for bulk meteorite composition): $\mathrm{SiO}_{2}=43.1 \pm 0.6$, $\mathrm{TiO}_{2}=0.12 \pm 0.03, \mathrm{Al}_{2} \mathrm{O}_{3}=31.3 \pm 1.2, \mathrm{Cr}_{2} \mathrm{O}_{3}=0.03 \pm 0.01, \mathrm{MgO}=2.0 \pm 0.5, \mathrm{FeO}=2.5 \pm 0.5$, $\mathrm{MnO}=0.03 \pm 0.01, \mathrm{CaO}=18.0 \pm 0.4, \mathrm{Na}_{2} \mathrm{O}=0.36 \pm 0.01, \mathrm{~K}_{2} \mathrm{O}=0.04 \pm 0.00$ (all wt\%), $\mathrm{n}=4$.

Classification: Lunar feldspathic breccia

Specimens: $21.6 \mathrm{~g}$ including a probe mount on deposit at $U N M$, Dustin Dickens holds the main mass.

\section{Northwest Africa 11899 (NWA 11899) \\ Mali \\ Purchased: 2017 \\ Classification: HED achondrite (Howardite)}

History: Purchased by Dustin Dickens from Nomad in Mali.

Physical characteristics: Many identically appearing pieces. Fusion crusted exterior. A saw cut reveals a breccia with light and dark clasts set in light-brown matrix.

Petrography: (C. Agee, UNM) This meteorite is a polymict breccia consisting of approximately $60 \%$ diogenite and $40 \%$ eucrite clasts. Accessory Fe-Ni metal, Fesulfide, and silica observed.

Geochemistry: (C. Agee, $U N M$ ) diogenite low-Ca pyroxene $\mathrm{Fs}_{25.1 \pm 2.5} \mathrm{Wo}_{3.6 \pm 2.2}$, $\mathrm{Fe} / \mathrm{Mn}=30 \pm 2, \mathrm{n}=10$; eucrite low-Ca pyroxene $\mathrm{Fs}_{49.7 \pm 5.2} \mathrm{Wo}_{4.8 \pm 2.8}, \mathrm{Fe} / \mathrm{Mn}=29 \pm 2$, $\mathrm{n}=7$; plagioclase AN93.1 $\pm 1.2, \mathrm{n}=7$.

Classification: Howardite

Specimens: $22.8 \mathrm{~g}$ including a probe mount on deposit at $U N M$, Dustin Dickens holds the main mass.

\section{Northwest Africa 11900 (NWA 11900) \\ Western Sahara \\ Purchased: 2017 \\ Classification: Ureilite}

History: Purchased by Dustin Dickens from nomad near Dahkla in Western Sahara.

Physical characteristics: Weathered exteri; saw cut reveals an interior mosaic of green and brown crystals.

Petrography: (C. Agee, $U N M$ ) This meteorite consists of olivine, pigeonite, and augite. Ubiquitous fine-grained $\mathrm{Fe}$-metal is present along grain boundaries. Elongate $(100-200 \mu \mathrm{m})$ grains of graphite are scattered throughout.

Geochemistry: (C. Agee, $U N M$ ) Olivine $\mathrm{Fa}_{22.2 \pm 0.1}, \mathrm{Fe} / \mathrm{Mn}=49 \pm 1, \mathrm{Cr}_{2} \mathrm{O}_{3}=0.36 \pm 0.02$ (wt\%), n=7; pigeonite $\mathrm{Fs}_{18.6 \pm 0.3} \mathrm{Wo}_{4.2 \pm 0.1}, \mathrm{Fe} / \mathrm{Mn}=29 \pm 1, \mathrm{n}=4$; augite $\mathrm{Fs}_{11.7 \pm 0.1} \mathrm{Wo}_{34.9 \pm 0.1}, \mathrm{Fe} / \mathrm{Mn}=22 \pm 1, \mathrm{n}=3$. 
Classification: Augite-bearing ureilite

Specimens: $20.7 \mathrm{~g}$ including a probe mount on deposit at $U N M$, Dustin Dickens holds the main mass.

\author{
Northwest Africa 11901 (NWA 11901) \\ Western Sahara \\ Purchased: 2017 \\ Classification: Primitive achondrite (Lodranite)
}

History: Purchased by Dustin Dickens from nomad near Dahkla in Western Sahara.

Physical characteristics: Many identically appearing pieces. Weathered exterior. A saw cut reveals an interior of coarse green and brown crystals. Opaque grains are scattered throughout.

Petrography: (C. Agee, UNM) This meteorite consists primarily of olivine and Crdiopside; low-Ca pyroxene and plagioclase are absent. Most grains are in the 1-3 $\mathrm{mm}$ size range.

Geochemistry: (C. Agee, $U N M$ ) Olivine $\mathrm{Fa}_{11.0 \pm 0.1}, \mathrm{Fe} / \mathrm{Mn}=38 \pm 5, \mathrm{Cr}_{2} \mathrm{O}_{3}=0.03 \pm 0.01$ ( $\left.w t^{\%}\right), \mathrm{n}=4$; diopside $\mathrm{Fs}_{5.0 \pm 0.6} \mathrm{Wo}_{41.4 \pm 2.9}, \mathrm{Fe} / \mathrm{Mn}=14 \pm 1, \mathrm{Cr}_{2} \mathrm{O}_{3}=1.09 \pm 0.06(\mathrm{wt} \%) \mathrm{n}=7$. Classification: Lodranite, opx-absent Specimens: $22.9 \mathrm{~g}$ including a probe mount on deposit at $U N M$, Dustin Dickens holds the main mass.

\title{
Northwest Africa 11903 (NWA 11903) \\ (Northwest Africa) \\ Purchased: 2016 \\ Classification: Ordinary chondrite (H6)
}

History: Purchased by Dustin Dickens from Fyssal Mesgouri in Agadir, Morocco. Physical characteristics: Weathered exterior. Saw cut reveals an interior with many small opaque grains and a few faint chondrules set in a dark, reddish-brown groundmass.

Petrography: (C. Agee, UNM) Faint, texturally equilibrated chondrules present, abundant Fe-Ni metal and sulfide throughout, plagioclase up to $150 \mu \mathrm{m}$.

Geochemistry: (C. Agee, $U N M$ ) Olivine $\mathrm{Fa}_{19.2 \pm 0.1}, \mathrm{Fe} / \mathrm{Mn}=38 \pm 1, \mathrm{n}=7$; low-Ca pyroxene $\mathrm{Fs}_{17.0 \pm 0.2} \mathrm{Wo}_{1.3 \pm 0.3}, \mathrm{Fe} / \mathrm{Mn}=22 \pm 1, \mathrm{n}=7$.

Classification: Ordinary chondrite, $\mathrm{H} 6$

Specimens: $25 \mathrm{~g}$ including a probe mount on deposit at $U N M$, Dustin Dickens holds the main mass.

\section{Northwest Africa 11904 (NWA 11904)}

(Northwest Africa)

Purchased: 2018

Classification: Carbonaceous chondrite (CV3) 
History: Purchased by Abdelhadi Aithiba in Morocco in 2018.

Physical characteristics: Single stone, dark, weathered, irregular exterior, distinct chondrules up to $\sim 3 \mathrm{~mm}$ and scattered CAIs are visible on the surface; the matrix is dark gray in color.

Petrography: (C. Agee, $U N M$ ) Microprobe examination of a polished mount many irregular shaped porphryritic chondrules, some AOAs observed, fine-grained matrix makes up about $\sim 50 \%$ of this meteorite.

Geochemistry: (C. Agee, UNM) Olivine $\mathrm{Fa}_{2.8 \pm 2.7}$, range $\mathrm{Fa}_{0.5-11.9}, \mathrm{Cr}_{2} \mathrm{O}_{3}=0.25 \pm 0.14$ (wt\%), $\mathrm{CaO}=0.27 \pm 0.13$ (wt\%), $\mathrm{n}=22$; low-Ca pyroxene $\mathrm{Fs}_{4.5 \pm 7.7} \mathrm{Wo}_{1.2 \pm 0.5}, \mathrm{n}=6$.

Oxygen isotopes (K. Ziegler, $U N M): 3$ analyses, with $\delta^{17} \mathrm{O}=-2.359,-2.108,-0.349$ and $\delta^{18} \mathrm{O}=2.317,2.000,4.233$ (linearized values).

Classification: Carbonaceous chondrite (CV3)

Specimens: $20.66 \mathrm{~g}$ including a probe mount on deposit at $U N M$, Abdelhadi Aithiba holds the main mass.

\section{Northwest Africa 11905 (NWA 11905)}

(Northwest Africa)

Purchased: 2017

Classification: LL3.10-5

History: Purchased by Matthew Martin, February 2017, in Tucson from a Moroccan meteorite dealer.

Physical characteristics: Single stone. Saw cuts show a breccia of visually different chondrite clasts set in a host chondrite groundmass. Some of the clasts appear to be texturally equilibrated with less distinct chondrules, whereas others resemble type-3 lithologies with densely packed chondrules. The host groundmass contains numerous large, distinct, well-formed chondrules.

Petrography: (C. Agee, UNM) Microprobe examination of several polished mounts shows three group LL lithologies present: Lithology 1 is LL3.10; Lithology 2 is LL5; and Lithology 3 makes up the host groundmass and is LL3. Lithology 1 has numerous porphyritic chondrules, most with mesostasis or glass and abundant opaque matrix. The apparent mean chondrule diameter in Lithology 1 is $900 \pm 400$ $\mu \mathrm{m}, \mathrm{n}=31$. Lithology 2 has texturally equilibrated chondrules and plagioclase grains up to $25 \mu \mathrm{m}$. Lithology 3 (host) has numerous porphyritic chondrules, many with mesostasis, fine-grained plagioclase also detected.

Geochemistry: (C. Agee, $U N M$ ) Lithology 1: all olivine $\mathrm{Fa}_{14.0 \pm 11.0}, \mathrm{Fe} / \mathrm{Mn}=41 \pm 15$, $\mathrm{n}=44$; ferroan chondrule olivine $\mathrm{Fa}_{17.3 \pm 9.9}, \mathrm{Fe} / \mathrm{Mn}=45 \pm 13, \mathrm{Cr}_{2} \mathrm{O}_{3}=0.32 \pm 0.20$ (wt\%), $\mathrm{n}=35$; low-Ca pyroxene $\mathrm{Fs}_{11.1 \pm 10.0} \mathrm{Wo}_{1.1 \pm 0.8}, \mathrm{n}=12$. Lithology 2: olivine $\mathrm{Fa}_{30.7 \pm 0.6}$, $\mathrm{Fe} / \mathrm{Mn}=60 \pm 2, \mathrm{n}=19$; low-Ca pyroxene $\mathrm{Fs}_{24.9 \pm 0.3} \mathrm{Wo}_{1.3 \pm 0.9}, \mathrm{n}=12$. Lithology 3: olivine $\mathrm{Fa}_{27.9 \pm 3.0}, \mathrm{Fe} / \mathrm{Mn}=57 \pm 15, \mathrm{n}=8$; low-Ca pyroxene $\mathrm{Fs}_{24.3 \pm 1.8} \mathrm{Wo}_{2.1 \pm 3.5}, \mathrm{n}=4$.

Classification: Ordinary chondrite (LL3.10-LL3-LL5), LL-group genomict breccia. Type 3.10 for Lithology 1 is based on the mean value of $\mathrm{Cr}_{2} \mathrm{O}_{3}$ in ferroan chondrule olivine and the 1-sigma standard deviation, (Grossman and Brearley, $\underline{2005)}$, a similar petrologic type to NWA 1756 (LL3.10), NWA 3127 (LL3.10), $\underline{\text { RC }}$ 
$\underline{075}$ (H3.10), and MET 96503 (L3.10). Estimated type for Lithology 3 is 3.8/3.9 based on the scatter in olivine composition.

Specimens: $48.5 \mathrm{~g}$ on deposit at $U N M$, Matthew Martin holds the main mass.

\section{Northwest Africa 11906 (NWA 11906)}

(Northwest Africa)

Purchased: 2018

Classification: Carbonaceous chondrite (CO3.0)

History: Purchased by Matthew Stream from Tindouf, Algeria, April 2018.

Physical characteristics: Single stone, partially covered in dark fusion crust with polygonal cracks. Broken surface reveals gray, fined-grained interior, with many small chondrules visible, scattered small CAIs were observed.

Petrography: (C. Agee, UNM) This meteorite consists of numerous small chondrules set in a fine-grained matrix. The matrix makes up approximately $40 \%$ of this meteorite. Mean apparent chondrule size $200 \pm 175 \mu \mathrm{m}, \mathrm{n}=50$.

Geochemistry: (C. Agee, $U N M$ ) Olivine $\mathrm{Fa}_{14.0 \pm 14.6}, \mathrm{Cr}_{2} \mathrm{O}_{3}=0.37 \pm 0.15$ (wt\%), $\mathrm{n}=18$; low-Ca pyroxene $\mathrm{Fs}_{2.3 \pm 1.2} \mathrm{Wo}_{3.4 \pm 0.5}, \mathrm{n}=4$.

Classification: Carbonaceous chondrite (CO3.0), 3.0 based on the $\mathrm{Cr}_{2} \mathrm{O}_{3}$ content and 1-sigma in olivine (Grossman and Brearley, 2005).

Specimens: $17.3 \mathrm{~g}$ including a probe mount on deposit at $U N M$, Matthew Stream holds the main mass.

\section{Northwest Africa 11907 (NWA 11907)}

(Northwest Africa)

Purchased: 2018

Classification: Ordinary chondrite (LL3)

History: Darryl Pitt purchased from Said Haddany at the Tucson Gem and Mineral Show in January 2018

Physical characteristics: Single stone with fusion crust. Saw cut reveals two distinct lithologies, one lighter and one darker in color. Numerous well formed, closely packed chondrules, some up to $2 \mathrm{~mm}$ in diameter. Average apparent chondrule diameter is $750 \pm 450 \mu \mathrm{m}(\mathrm{n}=16)$. Scattered metal/sulfide grains observed. Petrography: (C. Agee, UNM) Microprobe examination shows many porphyritic chondrules in both lithologies, most with mesostasis and glass.

Geochemistry: (C. Agee, $U N M$ ) All olivine $\mathrm{Fa}_{27.2 \pm 10.5}$, $\mathrm{n}=42$; all low-Ca pyroxene $\mathrm{Fs}_{23.6 \pm 8.3} \mathrm{Wo}_{0.9 \pm 0.5}, \mathrm{n}=12$. Dark lithology: olivine $\mathrm{Fa}_{22.1 \pm 11.4}, \mathrm{Cr}_{2} \mathrm{O}_{3}=0.11 \pm 0.16$, $\mathrm{Fe} / \mathrm{Mn}=51 \pm 17, \mathrm{n}=21$; low-Ca pyroxene $\mathrm{Fs}_{18.2 \pm 0.2} \mathrm{Wo}_{1.1 \pm 0.8}, \mathrm{Fe} / \mathrm{Mn}=21 \pm 1, \mathrm{n}=6$. Light lithology: olivine $\mathrm{Fa}_{32.4 \pm 6.3}, \mathrm{Cr}_{2} \mathrm{O}_{3}=0.05 \pm 0.08, \mathrm{Fe} / \mathrm{Mn}=66 \pm 13, \mathrm{n}=21$; low-Ca pyroxene $\mathrm{Fs}_{29.2 \pm 3.2} \mathrm{Wo}_{1.1 \pm 0.5}, \mathrm{Fe} / \mathrm{Mn}=46 \pm 11, \mathrm{n}=6$.

Classification: Ordinary chondrite (LL3), estimated sub-type LL3.2-3.3 based on mean $\mathrm{Fa}$ content of olivine and the 1-sigma Fa content of olivine, and on mean $\mathrm{Cr}_{2} \mathrm{O}_{3}$ content of ferroan olivine and the 1-sigma $\mathrm{Cr}_{2} \mathrm{O}_{3}$ content of ferroan 
olivine (Grossman and Brearley, 2005). Similar to St. Marys County (LL3.3), GRO 95502 (L3.2) and GRO 95544 (L3.2).

Specimens: $22.7 \mathrm{~g}$ on deposit at $U N M, M M G M$ holds the main mass.

\section{Northwest Africa 11908 (NWA 11908)}

(Northwest Africa)

Purchased: 2018

Classification: Ordinary chondrite (LL3)

History: Darryl Pitt purchased from Said Haddany at the Tucson Gem and Mineral Show in January 2018

Physical characteristics: Single stone. A saw cut reveals numerous distinct, closely packed chondrules, a few up to $4 \mathrm{~mm}$ in diameter, set in a dark brown matrix.

Petrography: (C. Agee, UNM) Microprobe examination reveals many porphyritic chondrules with mesostasis and glass, abundant opaque matrix. Apparent mean chondrule diameter $800 \pm 625 \mu \mathrm{m}, \mathrm{n}=47$.

Geochemistry: (C. Agee, UNM) Olivine $\mathrm{Fa}_{16.8 \pm 8.3}, \mathrm{Cr}_{2} \mathrm{O}_{3}=0.09 \pm 0.05$, $\mathrm{Fe} / \mathrm{Mn}=52 \pm 21, \mathrm{n}=31$; low-Ca pyroxene $\mathrm{Fs}_{12.5 \pm 9.4} \mathrm{Wo}_{1.2 \pm 1.1}, \mathrm{Fe} / \mathrm{Mn}=24 \pm 15, \mathrm{n}=10$. Classification: Ordinary chondrite (LL3), estimated sub-type LL3.2-3.3 based on mean $\mathrm{Fa}$ content of olivine and the 1-sigma Fa content of olivine, and on mean $\mathrm{Cr}_{2} \mathrm{O}_{3}$ content of ferroan olivine and the 1-sigma $\mathrm{Cr}_{2} \mathrm{O}_{3}$ content of ferroan olivine (Grossman and Brearley, 2005). Similar to St. Mary's County (LL3.3), GRO 95502 (L3.2) and GRO 95544 (L3.2).

Specimens: $24.4 \mathrm{~g}$ on deposit at $U N M, M M G M$ holds the main mass.

\section{Northwest Africa 11909 (NWA 11909) \\ (Northwest Africa) \\ Purchased: 2018 \\ Classification: HED achondrite (Diogenite)}

History: Darryl Pitt purchased from Said Haddany at the Tucson Gem and Mineral Show in January 2018

Physical characteristics: Single stone, weathered exterior, coarse grained, green crystals with some iron staining.

Petrography: (C. Agee, $U N M)$ Microprobe examination reveals an orthopyroxenite with minor amounts of chromite and sulfide; plagioclase and olivine are absent. Highly equilibrated and relatively low shock.

Geochemistry: (C. Agee, UNM) low-Ca pyroxene, $\mathrm{Fs}_{23.2 \pm 0.3} \mathrm{Wo}_{1.8 \pm 0.1}$, $\mathrm{Fe} / \mathrm{Mn}=25 \pm 1, \mathrm{n}=10$.

Classification: Diogenite Specimens: $20.1 \mathrm{~g}$ on deposit at $U N M, M M G M$ holds the main mass.

Northwest Africa 11912 (NWA 11912) 
(Northwest Africa)

Purchased: February 2014

Classification: Ordinary chondrite (H5)

History: Purchased by Fred Olsen in 2014

Petrography: (D. Sheikh, FSU) Chondrule boundaries blurred $(500 \pm 80 \mu \mathrm{m}$, $\mathrm{N}=12$ ). Recrystallized matrix with typical plagioclase size about $10 \mu \mathrm{m}$.

Geochemistry: Although the vast majority of olivine and orthopyroxene analyses are clustered, a few grains are unequilibrated. Olivine range $\mathrm{Fa}_{14.1-24.0}$, mean $\mathrm{Fa}_{18.4 \pm 0.3}(\mathrm{~N}=50)$; orthopyroxene, range $\mathrm{Fs}_{15.74-30.36} \mathrm{Wo}_{0.95-3.34}$, mean $\mathrm{Fs}_{19.5 \pm 0.3} \mathrm{Wo}_{1.7 \pm 0.1}(\mathrm{~N}=60)$.

Classification: Ordinary Chondrite (H5)

Specimens: $24.30 \mathrm{~g}$ at $U C L A$; main mass with Fred Olsen.

\section{Northwest Africa 11913 (NWA 11913) \\ (Northwest Africa) \\ Purchased: 2013 \\ Classification: Ordinary chondrite (H5)}

History: Purchased by John A. Shea in 2013 via E-bay from an IMCA member who acquired it in a lot of northwest African meteorites.

Physical characteristics: Dark brown weathering patina with patches of rust covers all exterior surfaces

Petrography: In thin section, chondrules and fragments are delineated by a complex veining of weathering products (oxides and carbonate). Individual grains within chondrules are readily distinguished from mesostasis. Chondrule mesostases are devitrified and generally fine-grained, with feldspar grains typically small $(<10$ microns in diameter), but up to $\sim 40 \mu \mathrm{m}$ across.

Geochemistry: (M. Hutson and A. Ruzicka, Cascadia) Olivine ( $\left.\mathrm{Fa}_{19.8 \pm 0.2}, \mathrm{~N}=16\right)$, low-Ca pyroxene $\left(\mathrm{Fs}_{17.4 \pm 0.2} \mathrm{Wo}_{1.5 \pm 0.1}, \mathrm{~N}=16\right)$.

Classification: H5 chondrite based on chemistry and texture.

Specimens: Cascadia holds $11.4 \mathrm{~g}$ in two pieces, in addition to one polished thin section and a mounted butt.

\section{Northwest Africa 11914 (NWA 11914)}

(Northwest Africa)

Purchased: 2013

Classification: Ordinary chondrite (H5)

History: Purchased by John A. Shea in 2013 via E-bay from Steve Witt who acquired it in a lot of northwest African meteorites

Physical characteristics: Dark reddish brown weathering patina covers all exterior surfaces

Petrography: In thin section, chondrules are readily delineated; mesostases are devitrified. 
Geochemistry: (M. Hutson, A. Cairns, A. Maroni, J. Longfellow, and A. Ruzicka, Cascadia $)$ Olivine $\left(\mathrm{Fa}_{19.2 \pm 0.6}, \mathrm{~N}=33\right)$, low-Ca pyroxene $\left(\mathrm{Fs}_{17.1 \pm 0.8} \mathrm{Wo}_{1.2 \pm 0.3}\right.$, $\mathrm{N}=17$ ).

Classification: H5 chondrite based on chemistry and texture

Specimens: Cascadia holds $17.1 \mathrm{~g}$ in three pieces, in addition to one polished thin section and a mounted butt.

\section{Northwest Africa 11915 (NWA 11915)}

(Northwest Africa)

Purchased: 2013

Classification: Ordinary chondrite (LL4-6)

History: Purchased by John A. Shea in 2013 via E-bay from Mirko Graul who acquired it in a lot of northwest African meteorites.

Physical characteristics: Exterior surface covered by reddish brown weathering patina and patches of black fusion crust. Cut faces show angular dark bluish-gray clasts amid a beige host lithology, along with a few metal and sulfide grains and patches of rust.

Petrography: Three lithologies are readily distinguished in thin section. A lighter colored coarse-grain clast (clast B), approximately $400 \times 600 \mu \mathrm{m}$, with a highly integrated texture is encompassed within a larger clast (clast A) which has a well integrated texture with barely distinguishable chondrules. Clast $\mathrm{A}$ is truncated along the edge of the section, but is approximately $1.2 \mathrm{~cm}$ long. The two clasts are set in a host which has readily delineated chondrules, smaller clasts, and fragments. Approximately $85 \%$ of the opaque grains have been replaced by Fe-hydroxide weathering product. In BSE imaging, host olivine grains appear equilibrated, but many larger host pyroxene grains are zoned. The host is brecciated on a fine scale: a single large (150 micron long) plagioclase feldspar grain, is located adjacent to a chondrule fragment with strongly-zoned low-calcium pyroxene grains with a mesostasis of devitrified glass. A rectangular clast (approximately $1.1 \times 0.8 \mathrm{~mm}$ ) consists of zoned low-calcium clinopyroxene enclosing a large patch of mostly replaced troilite containing silica polymorph rimmed by high-Ca pyroxene. Chondrules, clasts and larger mineral grains in the host are surrounded by a finergrained (typically 5-20 $\mu \mathrm{m}$ across grains) of olivine, low-calcium pyroxene, and plagioclase feldspar grains.

Geochemistry: (M. Hutson and A. Ruzicka, Cascadia) Both host olivine $\left(\mathrm{Fa}_{30.2 \pm 0.4}\right.$, $\mathrm{N}=17)$ and clast $\mathrm{B}$ olivine $\left(\mathrm{Fa}_{30.3 \pm 0.1}\right)$ are equilibrated. Clast $\mathrm{B}$ low-Ca pyroxene $\left(\mathrm{Fs}_{25.6 \pm 0.3} \mathrm{Wo}_{2.0 \pm 0.2}, \mathrm{~N}=9\right)$ is also equilibrated; however host low-Ca pyroxene $\left(\mathrm{Fs}_{19.9 \pm 4.0} \mathrm{Wo}_{1.0 \pm 0.8}, \mathrm{~N}=28\right)$ is variable. Clast $\mathrm{B}$ plagioclase feldspar is albitic $\left(\mathrm{Ab}_{84.5 \pm 1.1} \mathrm{An}_{11.3 \pm 0.6} \mathrm{Or}_{4.2 \pm 0.5}, \mathrm{~N}=3\right)$.

Classification: LL4-6 based on chemistry and texture.

Specimens: Cascadia holds $83.5 \mathrm{~g}$ in two pieces, in addition to one polished thin section and a mounted butt 
Northwest Africa 11916 (NWA 11916)

(Northwest Africa)

Purchased: 2014

Classification: Ungrouped achondrite

History: Bought by Luc Labenne in Agadir in 2014

Physical characteristics: A single brown weathered stone

Petrography: J. Gattacecca, B. Devouard, P. Rochette, CEREGE) Main minerals are finely exsolved, blocky pigeonite crystals, to $1.5 \mathrm{~mm}$, set in plagioclase. LowCa pyroxene is found as small inclusions in pigeonite. Pigeonite shows reduction at grain rims and along cracks. Accessory silica, merrilite, troilite, FeNi metal. Metal and sulfides are finely dispersed in the silicates and finely interspersed.

Geochemistry: Pigeonite $\mathrm{Fs}_{38.7 \pm 3.8} \mathrm{Wo}_{6.9 \pm 2.0}\left(\mathrm{Fs}_{30.6-42.7} \mathrm{Wo}_{5.0-10.2}, \mathrm{~N}=8\right)$, $\mathrm{FeO} / \mathrm{MnO}=23.0 \pm 3.4$ (range 17.0 - 28.5). Plagioclase

$\mathrm{An}_{95.9 \pm 0.1} \mathrm{Ab}_{4.0 \pm 0.1} \mathrm{Or}_{0.1 \pm 0.1}(\mathrm{~N}=4)$. Oxygen isotopic composition (J. Gattacceca, $\mathrm{C}$. Sonzogni, CEREGE) from analysis of two acid-washed $1.5 \mathrm{mg}$ aliquots of a powdered $55 \mathrm{mg}$ bulk sample is $\delta^{17} \mathrm{O}=1.47 \%, \delta^{18} \mathrm{O}=3.68 \% 0, \Delta^{17} \mathrm{O}=-0.46 \%$, and $\delta^{17} \mathrm{O}=1.71 \%$, $\delta^{18} \mathrm{O}=4.09 \%$, $\Delta^{17} \mathrm{O}=-0.44 \%$ (linearized, slope 0.5247 , analytical uncertainties $0.08 \%, 0.12 \%, 0.03 \%$ respectively). Magnetic susceptibility $\log \chi(\times$ $\left.10^{-9} \mathrm{~m}^{3} / \mathrm{kg}\right)=3.91$.

Classification: Ungrouped achondrite. Oxygen isotopes and Ca-pyroxene composition set this achondrite apart from HED achondrites.

Specimens: $2.2 \mathrm{~g}$ at CEREGE. Main mass with Labenne

\section{Northwest Africa 11917 (NWA 11917)}

(Northwest Africa)

Purchased: 2012

Classification: Primitive achondrite (Winonaite)

Petrography: No chondrules are present, but there are irregularly shaped silicate clusters (50-700 $\mu \mathrm{m}$ in size) that may have been derived from recrystallized chondrule fragments. There is no clear igneous texture. Olivine exhibits mildy undulose extinction. Plagioclase also occurs. The rock contains $\sim 20$ vol.\% metal plus sulfide. Opaque phases include kamacite, martensite, taenite, schreibersite, ferroan alabandite, and magnesiochromite. Metal and sulfide grains average $\sim 100$ $\mu \mathrm{m}$ in size. There are some grains of polycrystalline troilite and polycrystalline kamacite. There are rare $8 \times 12-\mu \mathrm{m}$-size patches of daubreelite at the ede of some troilite grains. There are also rare $\sim 40-\mu \mathrm{m}$-size metal grains with martensitic structure, rimmed by cloudy taenite. There is a single thick, elongated band of troilite at the edge of one thin section $(\sim 300 \times 3000 \mu \mathrm{m})$.

Geochemistry: Also present are plagioclase $\left(\mathrm{Ab}_{84.7 \pm 0.2} \mathrm{Or}_{2.7 \pm 0.2} ; \mathrm{n}=5\right)$; kamacite (in wt.\%): $92.5 \mathrm{Fe}, 0.48 \mathrm{Co}, 0.06 \mathrm{P}, 6.8 \mathrm{Ni},<0.03 \mathrm{Si}$; taenite: $73.5 \mathrm{Fe}, 0.17 \mathrm{Co},<0.03$ $\mathrm{P}, 23.5 \mathrm{Ni},<0.03 \mathrm{Si}$; troilite: $62.6 \mathrm{Fe}, 37.0 \mathrm{~S}, 0.35 \mathrm{Cr}$; magnesiochromite: 67.3 $\mathrm{Cr}_{2} \mathrm{O}_{3}, 5.8 \mathrm{MnO}, 12.0 \mathrm{MgO}, 6.2 \mathrm{FeO}$. The O-isotopic composition of replicate samples was analyzed by R. Greenwood $(O U): \delta^{17} \mathrm{O}=1.876 \pm 0.006, \delta^{18} \mathrm{O}=$ $4.541 \pm 0.011, \Delta{ }^{17} \mathrm{O}=-0.486 \pm 0.012$ (per mil); this is in the winonaite range. 
Classification: This is one of the most reduced winonaites, with very little FeO in the mafic silicates. The presence of some grains of polymict troilite and kamacite indicates moderately high shock, but the mildly undulose extinction in the olivine (characteristic of S2) suggests that the rock was shocked and then annealed, and perhaps (mildly) shocked again.

\section{Northwest Africa 11919 (NWA 11919) \\ (Northwest Africa) \\ Purchased: 2018 \\ Classification: Carbonaceous chondrite (CM2)}

History: The meteorite was purchased from a local meteorite dealer in Morocco. Physical characteristics: Eleven dark fragments some of which partly covered with fusion crust.

Petrography: The meteorite is a brecciated carbonaceous chondrite composed of chondrules (mean diameter about $300 \mu \mathrm{m}$ ), chondrule pseudomorphs, CAIs, and mineral fragments set into abundant Fe-rich matrix. Many of the components are heavily corroded and often surrounded by fine-grained dust rims. Compositionally zoned olivine is the only anhydrous silicate present; former pyroxenes are completely altered. The meteorite matrix consists of abundant carbonates, sulfides and phyllosilicates. Carbonates are particularly rich in $\mathrm{Fe}$ and $\mathrm{Mg}$ attesting to a high degree of alteration.

Geochemistry: carbonates contain up to $16.5 \mathrm{wt} \% \mathrm{MgO}$ and $15.6 \mathrm{wt} \% \mathrm{FeO}$

\section{Northwest Africa 11920 (NWA 11920)}

(Northwest Africa)

Purchased: 2018

Classification: Ordinary chondrite (H5, melt breccia)

History: The meteorite was purchased from a local meteorite dealer in Zagora, Morocco.

Physical characteristics: Two small black individuals lacking any fusion crust.

Petrography: The meteorite is strongly brecciated and composed of recrystallized shock melt regions, relict mineral fragments and some preserved clasts (type 5). No pyroxene has been found in the thin section studied.

\section{Northwest Africa 11921 (NWA 11921)}

(Northwest Africa)

Purchased: 2018

Classification: Martian meteorite

History: Reportedly recovered in 2017 at the find site of NWA 7034 and purchased by Said Yousfi in 2018 from a local meteorite dealer in Zagora, Morocco.

Physical characteristics: Six black fragments with shiny crust. 
Petrography: The meteorite is a polymict breccia composed of lithic and mineral clasts set into a very fine-grained clastic matrix. Lithic clasts include basaltic and impact melt types; most abundant mineral phases in clasts and matrix are pyroxene and feldspar, both of highly variable composition. Minor phases are ilmenite, magnetite, Ti,Cr-magnetite, and Cl-apatite. The meteorite's high magnetic susceptibility reflects the high content of Fe-oxides and is in good agreement with data in Gattacceca et al. 2014.

Geochemistry: low-Ca pyroxene: $\mathrm{Fs}_{25.1 \pm 5.5} \mathrm{Wo}_{3.6 \pm 1.1}\left(\mathrm{Fs}_{19.5-33.5} \mathrm{Wo}_{2.0-5.0}, \mathrm{n}=15\right.$, $\mathrm{FeO} / \mathrm{MnO}=30-38)$; pigeonite: $\mathrm{Fs}_{39.9 \pm 12.0} \mathrm{Wo}_{8.0 \pm 2.7}\left(\mathrm{Fs}_{25.6-52.3} \mathrm{Wo}_{5.6-12.4}, \mathrm{n}=10\right.$, $\mathrm{FeO} / \mathrm{MnO}=30-40)$; Ca-pyroxene: $\mathrm{Fs}_{25.8 \pm 10.2} \mathrm{Wo}_{36.7 \pm 6.5}\left(\mathrm{Fs}_{17.4-44.3} \mathrm{Wo}_{26.1-43.3}, \mathrm{n}=15\right.$, $\mathrm{FeO} / \mathrm{MnO}=26-41)$; feldspar: $\mathrm{An}_{40.4 \pm 9.2} \mathrm{Ab}_{56.5 \pm 8.2} \mathrm{Or}_{3.1 \pm 1.9}\left(\mathrm{An}_{29.0-49.3} \mathrm{Ab}_{48.3-66.7} \mathrm{Or}_{2.0-4.4}\right.$, $\mathrm{n}=13$ )

Classification: Martian meteorite (basaltic breccia). Likely paired with NWA 7034 and respective clan members.

\section{Northwest Africa 11922 (NWA 11922)}

(Northwest Africa)

Purchased: 2018

Classification: Ordinary chondrite (L3-6)

History: The meteorite was purchased from a local meteorite dealer in Erfoud, Morocco.

Physical characteristics: Two dark-grayish individuals partly covered with fusion crust.

Petrography: The meteorite is a chondritic breccia consisting of angular to subrounded L 3 and L6 clasts set into type 3 clastic matrix. Plagioclase grain size in L6 clasts is about $60 \mu \mathrm{m}$. Chondrules are mostly flattened and well packed and have a mean diameter of about $0.6 \mathrm{~mm}$.

Geochemistry: type 3 lithology: olivine: $\mathrm{Fa}_{14.9 \pm 9.3}\left(\mathrm{Fa}_{2.5-26.9}, \mathrm{n}=20\right)$; pyroxene: $\mathrm{Fs}_{12.5 \pm 7.4} \mathrm{Wo}_{0.5 \pm 0.1}\left(\mathrm{Fs}_{4.5-21.7} \mathrm{Wo}_{0.3-0.8}, \mathrm{n}=17\right)$; type 6 lithology: olivine: $25.2 \pm 0.3$, $\mathrm{n}=11$; pyroxene: $\mathrm{Fs}_{20.0 \pm 0.3} \mathrm{Wo}_{1.9 \pm 0.2}, \mathrm{n}=12$

\section{Northwest Africa 11923 (NWA 11923)}

(Northwest Africa)

Purchased: 2018

Classification: Ordinary chondrite (H3)

History: The meteorite was purchased from a local meteorite dealer in Erfoud, Morocco.

Physical characteristics: Dark brownish individual lacking any fusion crust. Petrography: The meteorite displays a chondritic texture with well defined and slightly flattened chondrules (mean diameter about $0.4 \mathrm{~mm}$ ) and fine-grained matrix containing sulfides and abundant FeNi metal. 


\section{Northwest Africa 11924 (NWA 11924)}

(Northwest Africa)

Purchased: 2018

Classification: Ordinary chondrite (H3)

History: The meteorite was purchased from a local meteorite dealer in Erfoud, Morocco.

Physical characteristics: Dark brownish individual lacking any fusion crust.

Petrography: The meteorite displays a chondritic texture with small and well separated chondrules (mean diameter about $0.3 \mathrm{~mm}$ ) and fine-grained matrix containing sulfides and abundant FeNi metal.

\section{Northwest Africa 11925 (NWA 11925) \\ (Northwest Africa) \\ Purchased: 2018 \\ Classification: Ordinary chondrite (L3)}

History: The meteorite was purchased from a local meteorite dealer in Erfoud, Morocco.

Physical characteristics: Dark greyish individual with some fusion crust.

Petrography: The meteorite displays a chondritic texture with slightly flattened and well packed chondrules (mean diameter about $0.6 \mathrm{~mm}$ ) in a more fine-grained dark matrix that contains sulfides and FeNi metal.

\section{Northwest Africa 11927 (NWA 11927) \\ (Northwest Africa) \\ Purchased: 2018 \\ Classification: Rumuruti chondrite (R3)}

History: The meteorite was purchased from a local meteorite dealer in Erfoud, Morocco.

Physical characteristics: Light brownish individual lacking any fusion crust. Petrography: The meteorite displays a light brownish to orange interior and is composed of up to $5 \mathrm{~mm}$ sized angular type 3 clasts and matrix. Olivine and lowCa pyroxene are compositionally unequilibrated in clasts and matrix; Fe-rich olivine is the most dominant mineral phase in the matrix. Chondrules have an average diameter of about $400 \mu \mathrm{m}$. More minor phases include low-Ca pyroxene, Ca-pyroxene, sodic plagioclase, sulfides, and Ti-bearing chromite; no metal has been detected.

Geochemistry: Olivine: $\mathrm{Fa}_{26.3 \pm 13.8}\left(\mathrm{Fa}_{1.6-44.9}, \mathrm{n}=26\right)$; low-Ca pyroxene: $\mathrm{Fs}_{18.7 \pm 8.8} \mathrm{Wo}_{1.5 \pm 0.6}\left(\mathrm{Fs}_{8.1-30.5} \mathrm{Wo}_{0.4-2.8}, \mathrm{n}=17\right)$; Ca-pyroxene: $\mathrm{Fs}_{10.3 \pm 3.0} \mathrm{Wo}_{42.1 \pm 5.4}\left(\mathrm{Fs}_{5.2-}\right.$ ${ }_{16.1} \mathrm{Wo}_{31.7-47.3}, \mathrm{n}=12$ )

\section{Northwest Africa 11928 (NWA 11928)}

(Northwest Africa) 
Purchased: 2018

Classification: HED achondrite (Eucrite)

History: The meteorite was purchased from a local meteorite dealer in Erfoud, Morocco.

Physical characteristics: Grayish individual partly covered with fusion crust. Petrography: The meteorite is a fine-grained polymict breccia with basaltic and black melt clasts set into a clastic mineral matrix. Dominant minerals are exsolved pyroxene and calcic plagioclase with average grain sizes of about $200 \mu \mathrm{m}$. Minor phases include silica, chromite, FeS, fayalite, and ilmenite. No metallic Fe has been found.

Geochemistry: low-Ca pyroxene: $\mathrm{Fs}_{35.6 \pm 2.1} \mathrm{Wo}_{6.3 \pm 0.8}\left(\mathrm{Fs}_{32.6-38.8} \mathrm{Wo}_{5.5-8.6}, \mathrm{n}=15\right.$, $\mathrm{FeO} / \mathrm{MnO}=28-33)$; Ca-pyroxene: $\mathrm{Fs}_{45.0 \pm 5.7} \mathrm{Wo}_{31.5 \pm 4.8}\left(\mathrm{Fs}_{43.7-46.1} \mathrm{Wo}_{25.4-36.6}, \mathrm{n}=11\right.$, $\mathrm{FeO} / \mathrm{MnO}=29-35)$; calcic plagioclase: $\mathrm{An}_{93.1 \pm 3.3}\left(\mathrm{An}_{86.4-96.4}, \mathrm{n}=12\right)$

\section{Northwest Africa 11933 (NWA 11933)}

(Northwest Africa)

Purchased: 2018

Classification: HED achondrite (Eucrite)

History: The meteorite was bought from a Moroccan meteorite dealer at a mineral fair in Ensisheim, France.

Physical characteristics: Greyish individual with some patches of fusion crust. Petrography: The meteorite is an unbrecciated medium-grained basalt predominantly composed of exsolved pyroxene and calcic plagioclase with average grain sizes of about $500 \mu \mathrm{m}$. Pyroxene appears brownish in plane polarized light. Minor phases include silica, ilmenite, chromite, troilite, and metallic iron.

Geochemistry: low-Ca pyroxene: $\mathrm{Fs}_{58.5 \pm 0.6} \mathrm{Wo}_{2.8 \pm 0.7}\left(\mathrm{Fs}_{56.9-59.2} \mathrm{Wo}_{2.3-4.5}, \mathrm{n}=11\right.$, $\mathrm{FeO} / \mathrm{MnO}=28-31)$; Ca-pyroxene: $\mathrm{Fs}_{29.1 \pm 1.7} \mathrm{Wo}_{40.5 \pm 1.9}\left(\mathrm{Fs}_{27.7-33.5} \mathrm{Wo}_{36.0-41.9}, \mathrm{n}=10\right.$, $\mathrm{FeO} / \mathrm{MnO}=27-31)$; calcic plagioclase: $\mathrm{An}_{89.9 \pm 0.8}\left(\mathrm{An}_{89.5-90.7}, \mathrm{n}=13\right)$

\section{Northwest Africa 11934 (NWA 11934)}

(Northwest Africa)

Purchased: 2018

Classification: HED achondrite (Eucrite, polymict)

History: The meteorite was bought from a Moroccan meteorite dealer at a mineral fair in Ensisheim, France.

Physical characteristics: Three fragments lacking any fusion crust.

Petrography: The meteorite is a polymict breccia composed of mineral and lithic clasts (basaltic and dark impact melt clasts) set into a fine-grained clastic matrix. Main minerals in clasts and matrix are exsolved pyroxene and calcic plagioclase. Minor phases include up to $2 \mathrm{~mm}$ sized diogenetic orthopyroxene, silica, chromite, ilmenite, and FeS. No metallic Fe has been detected.

Geochemistry: pyroxene host to augite lamellae: $\mathrm{Fs}_{37.6 \pm 9.5} \mathrm{Wo}_{5.3 \pm 1.9}\left(\mathrm{Fs}_{23.0-55.4} \mathrm{Wo}_{3.1-}\right.$ 9.1 $\mathrm{n}=21, \mathrm{FeO} / \mathrm{MnO}=27-37)$; $\mathrm{Ca}$-pyroxene: $\mathrm{Fs}_{34.4 \pm 10.8} \mathrm{Wo}_{36.3 \pm 3.2}\left(\mathrm{Fs}_{25.0-57.8} \mathrm{Wo}_{29.3-41.8}\right.$, 
$\mathrm{n}=19, \mathrm{FeO} / \mathrm{MnO}=27-38)$; diogenitic pyroxene: $\mathrm{Fs}_{29.1 \pm 0.2} \mathrm{Wo}_{3.3 \pm 0.2}\left(\mathrm{Fs}_{28.8-29.4} \mathrm{Wo}_{2.9-3.7}\right.$, $\mathrm{n}=11, \mathrm{FeO} / \mathrm{MnO}=28-35)$; calcic plagioclase: $\mathrm{An}_{91.2 \pm 1.4}\left(\mathrm{An}_{88.6-94.3}, \mathrm{n}=13\right)$

\section{Northwest Africa 11935 (NWA 11935) \\ (Northwest Africa) \\ Purchased: 2018 \\ Classification: Ordinary chondrite (H4)}

History: The meteorite was bought from a Moroccan meteorite dealer at a mineral fair in Ensisheim, France.

Physical characteristics: Brownish individual without fusion crust.

Petrography: The meteorite displays a chondritic texture with slightly flattened chondrules (mean diameter about $0.3 \mathrm{~mm}$ ) in a more fine-grained brownish matrix that contains sulfides and FeNi metal.

\section{Northwest Africa 11936 (NWA 11936) \\ (Northwest Africa) \\ Purchased: 2018 \\ Classification: Ordinary chondrite (H3)}

History: The meteorite was bought from a Moroccan meteorite dealer at a mineral fair in Ensisheim, France.

Physical characteristics: Five dark brownish individuals with some fusion crust. Petrography: The meteorite displays a chondritic texture with slightly flattened and well separated chondrules (mean diameter about $0.4 \mathrm{~mm}$ ) in a more finegrained dark matrix that contains sulfides and FeNi metal.

\section{Northwest Africa 11937 (NWA 11937) \\ (Northwest Africa) \\ Purchased: 2018 \\ Classification: Carbonaceous chondrite (CV3)}

History: The meteorite was bought from a Moroccan meteorite dealer at a mineral fair in Ensisheim, France.

Physical characteristics: Seventeen small dark brownish individuals without fusion crust.

Petrography: Carbonaceous chondrite composed of chondrules (mean diameter about $0.8 \mathrm{~mm}$; up to $4 \mathrm{~mm}$ sized), CAIs, and olivine amoeboids all set into a finegrained dark brownish matrix. Type II chondrules are absent. Few chondrules show reddish staining due to terrestrial weathering.

\section{Northwest Africa 11940 (NWA 11940)}

(Northwest Africa)

Purchased: 2018 
Classification: Ordinary chondrite (L3)

History: The meteorite was bought from a Moroccan meteorite dealer at a mineral fair in Sainte Marie aux Mines, France.

Physical characteristics: Dark brownish individual lacking any fusion crust. Petrography: The meteorite shows a chondritic texture with well separated and partly flattened chondrules (apparent mean diameter about $0.6 \mathrm{~mm}$ ).

\section{Northwest Africa 11941 (NWA 11941) \\ (Northwest Africa) \\ Purchased: 2018 \\ Classification: Ordinary chondrite (H4)}

History: The meteorite was bought from a Moroccan meteorite dealer at a mineral fair in Sainte Marie aux Mines, France.

Physical characteristics: Dark brownish individual lacking any fusion crust. Petrography: The meteorite shows a chondritic texture with small (apparent mean diameter about $0.3 \mathrm{~mm}$ ) well separated chondrules.

\section{Northwest Africa 11944 (NWA 11944) \\ (Northwest Africa) \\ Purchased: 2018 \\ Classification: Ordinary chondrite (LL4)}

History: The meteorite was bought from a Moroccan meteorite dealer at a mineral fair in Sainte Marie aux Mines, France.

Physical characteristics: Dark grayish individual lacking any fusion crust. Petrography: The meteorite shows a chondritic texture with densely packed and partly flattened chondrules (apparent mean diameter about $0.9 \mathrm{~mm}$ ).

\section{Northwest Africa 11945 (NWA 11945) \\ (Northwest Africa) \\ Purchased: 2018 \\ Classification: HED achondrite (Diogenite)}

History: The meteorite was bought from a Moroccan meteorite dealer at a mineral fair in Sainte Marie aux Mines, France.

Physical characteristics: Light brownish individual lacking any fusion crust. Petrography: The meteorite displays a light orange interior and is a monomict breccia predominantly composed of blocky up to $1.5 \mathrm{~mm}$ sized orthoproxenes grains and more fine-grained cataclastic regions. Minor phases include silica, chromite, and FeS. Neither plagioclase, olivine nor metallic iron have been found in the section studied.

Geochemistry: low-Ca pyroxene: $\mathrm{Fs}_{25.5 \pm 0.4} \mathrm{Wo}_{2.7 \pm 0.7}\left(\mathrm{Fs}_{25.0-26.3} \mathrm{Wo}_{1.8-4.0}, \mathrm{n}=15\right.$, $\mathrm{FeO} / \mathrm{MnO}=25-31$ ) 


\section{Northwest Africa 11946 (NWA 11946)}

(Northwest Africa)

Purchased: 2018

Classification: Carbonaceous chondrite (CV3)

History: The meteorite was purchased from a local meteorite dealer in Erfoud, Morocco.

Physical characteristics: Almost black individual without fusion crust.

Petrography: Carbonaceous chondrite composed of chondrules (mean diameter about $1 \mathrm{~mm}$; up to $3 \mathrm{~mm}$ sized), CAIs, and olivine amoeboids all set into a finegrained almost black matrix. Type II chondrules are absent. Several chondrules show reddish staining due to terrestrial weathering.

\section{Northwest Africa 11947 (NWA 11947)}

(Northwest Africa)

Purchased: 2018

Classification: HED achondrite (Eucrite)

History: The meteorite was purchased from a local meteorite dealer in Erfoud, Morocco.

Physical characteristics: Dark greyish individual with some fusion crust.

Petrography: The meteorite is a coarse-grained breccia composed of up to $1 \mathrm{~cm}$ sized basaltic clasts set into a clastic matrix. Mineral phases are predominantly up to $1 \mathrm{~mm}$ sized pyroxene and lath shaped calcic plagioclase. Pyroxene is compositionally zoned with rims being more $\mathrm{Fe}$ - and $\mathrm{Ca}$-rich than cores indicating a low degree of thermal metamorphism. Minor phases include silica, ilmenite, chromite, troilite, zircon and fayalite. No metallic iron has been detected.

Geochemistry: pyroxene cores: $\mathrm{Fs}_{34.2 \pm 4.4} \mathrm{Wo}_{9.3 \pm 3.1}\left(\mathrm{Fs}_{30.0-40.1} \mathrm{Wo}_{6.7-14.3}, \mathrm{n}=12\right.$, $\mathrm{FeO} / \mathrm{MnO}=26-30)$; pyroxene rims: $\mathrm{Fs}_{60.7 \pm 6.7} \mathrm{Wo}_{24.5 \pm 3.2}\left(\mathrm{Fs}_{42.0-66.2} \mathrm{Wo}_{20.5-30.4}, \mathrm{n}=12\right.$, $\mathrm{FeO} / \mathrm{MnO}=29-41)$; calcic plagioclase: $\mathrm{An}_{85.5 \pm 2.5}\left(\mathrm{An}_{81.0-88.7}, \mathrm{n}=13\right)$

\section{Northwest Africa 11948 (NWA 11948)}

(Northwest Africa)

Purchased: 2018

Classification: Ordinary chondrite (LL4-6)

History: The meteorite was purchased from a local meteorite dealer in Erfoud, Morocco.

Physical characteristics: Brownish individual with some patches of fusion crust. Petrography: The meteorite is a chondritic breccia consisting of clastic LL4 type matrix and embedded LL6 clasts. Plagioclase grain size in LL6 clasts is about 60 $\mu \mathrm{m}$. 
Geochemistry: type 4 lithology: olivine: $\mathrm{Fa}_{27.3 \pm 0.2}, \mathrm{n}=12$; pyroxene:

$\mathrm{Fs}_{20.6 \pm 3.8} \mathrm{Wo}_{1.2 \pm 0.7}\left(\mathrm{Fs}_{14.1-23.7} \mathrm{Wo}_{0.5-2.0}, \mathrm{n}=20\right)$; type 6 lithology: olivine: $\mathrm{Fa}_{27.2 \pm 0.2}$, $\mathrm{n}=11$; pyroxene: $\mathrm{Fs}_{22.6 \pm 0.3} \mathrm{Wo}_{3.5 \pm 0.7}, \mathrm{n}=10$

Northwest Africa 11949 (NWA 11949)

(Northwest Africa)

Purchased: 2018

Classification: HED achondrite (Eucrite)

History: The meteorite was purchased from a local meteorite dealer in Laâyoune, Western Sahara.

Physical characteristics: Greyish individual partly covered with fusion crust. Petrography: The meteorite is an unbrecciated basalt predominantly composed of exsolved pyroxene and mostly lath-shaped calcic plagioclase with average grain sizes of about $300 \mu \mathrm{m}$. Several regions of the rock have a pronounced spherulitic appearance due to sprays of pyroxene and plagioclase. Minor phases include silica, ilmenite, chromite, and troilite. No metallic iron has been detected.

Geochemistry: low-Ca pyroxene: $\mathrm{Fs}_{57.7 \pm 0.3} \mathrm{Wo}_{1.9 \pm 0.3}\left(\mathrm{Fs}_{57.1-58.1} \mathrm{Wo}_{1.6-2.9}, \mathrm{n}=14\right.$, $\mathrm{FeO} / \mathrm{MnO}=33-35)$; Ca-pyroxene: $\mathrm{Fs}_{23.7 \pm 0.3} \mathrm{Wo}_{44.6 \pm 0.5}\left(\mathrm{Fs}_{23.3-24.6} \mathrm{Wo}_{43.2-45.1}, \mathrm{n}=14\right.$, $\mathrm{FeO} / \mathrm{MnO}=29-35)$; calcic plagioclase: $\mathrm{An}_{81.8 \pm 2.5}\left(\mathrm{An}_{76.9-84.7}, \mathrm{n}=10\right)$

\section{Northwest Africa 11950 (NWA 11950)}

(Northwest Africa)

Purchased: 2007 Jan

Classification: Ureilite

History: Purchased by D. Gregory in Tucson, Arizona. Type specimen is under the museum number M53446

Petrography: (B. C. Hyde, $R O M, U W O$ ) The meterorite is predominantly olivine and pigeonite with grain size generally ranging from 0.5 to $3 \mathrm{~mm}$. Olivine grains have reduced rims and minor reduction is seen along pigeonite rims. All olivine grains contain $\mathrm{Ca}, \mathrm{Cr}$-rich inclusions likely composed of multiple phases. Metal is abundant along grain boundaries and within reduction rims. Both graphite and diamond are present (as determined by Raman spectroscopy).

Geochemistry: Mineral Compositions and Geochemistry: SEM based quantitative EDS and WDS (B. C. Hyde, UWO, ROM): Olivine cores: $\mathrm{Fa}_{21.1 \pm 0.2}(\mathrm{n}=15)$, $0.58 \pm 0.04 \mathrm{wt} \% \mathrm{Cr}_{2} \mathrm{O}_{3}, 0.28 \pm 0.03 \mathrm{wt} \% \mathrm{CaO}$. Pyroxene cores:

$\mathrm{Fs}_{17.9 \pm 0.1} \mathrm{Wo}_{7.5 \pm 0.1}(\mathrm{n}=15)$. Oxygen isotopes (K. Ziegler, $\left.U N M\right)$ : analyses of acidwashed subsamples by laser fluorination gave $\delta^{17} \mathrm{O}=3.909,3.733 ; \delta^{18} \mathrm{O}=9.183$, $8.885 ; \Delta^{17} \mathrm{O}=-0.940,-0.958$ per mil.

Classification: Ureilite

Specimens: Currently includes the main mass, type material, two thin sections and one thick section. 


\section{Northwest Africa 11951 (NWA 11951)}

(Northwest Africa)

Purchased: 2014 Jan

Classification: Ureilite

History: Purchased by D. Gregory in Tucson, Arizona. Designated as DGR-00114. Type specimen is under the museum number M58189

Petrography: (B. C. Hyde, $R O M, U W O$ ) The meteorite is predominantly olivine and pigeonite. Grains can be as large as $3 \mathrm{~mm}$, but regions of small grains (10s to 100 s of $\mu \mathrm{m}$ ) are also common. Olivine grains have reduced rims. The smallest olivine grains often show reduction throughout the entire grain. Minor reduction is also seen along pigeonite rims. Metal is abundant along grain boundaries and within reduction rims. The meteorite has a high abundance of diamond. Graphite is also present (as determined by Raman spectroscopy). Minor phases include chromite (10s of microns).

Geochemistry: Mineral Compositions and Geochemistry: SEM based quantitative EDS and WDS (B. C. Hyde, UWO, ROM): Olivine cores: $\mathrm{Fa}_{19.6 \pm 0.2}(\mathrm{n}=14)$, $0.72 \pm 0.02 \mathrm{wt}^{0} \% \mathrm{Cr}_{2} \mathrm{O}_{3}, 0.30 \pm 0.01 \mathrm{wt} \% \mathrm{CaO}$. Pyroxene cores:

$\mathrm{Fs}_{17.1 \pm 0.1} \mathrm{Wo}_{5.4 \pm 0.1}(\mathrm{n}=17)$. Oxygen isotopes $(\mathrm{K}$. Ziegler, $U N M)$ : analyses of acidwashed subsamples by laser fluorination gave $\delta^{17} \mathrm{O}=3.861,3.832 ; \delta^{18} \mathrm{O}=$ $8.834,8.670 ; \Delta^{17} \mathrm{O}=-0.803,-0.746$ per mil.

Classification: Ureilite

Specimens: Currently includes the main mass, type material, one thin section and one thick section.

\section{Northwest Africa 11953 (NWA 11953) \\ (Northwest Africa) \\ Purchased: 2018 Feb \\ Classification: HED achondrite (Eucrite, monomict)}

History: Purchased by Jason Phillips in February 2018 from a Moroccan dealer. Petrography: (A. Irving and S. Kuehner, UWS) Highly shocked and partially melted breccia. Gabbroic-textured clasts (some up to $3 \mathrm{~cm}$ across) and related disaggregated debris consist mainly of extremely fine scale polycrystalline aggregates after primary pigeonite (with some exsolution lamellae still preserved) and polycrystalline, microvesicular calcic plagioclase (exhibiting "swirly" microtexture indicative of being previously molten). Accessory minerals are silica polymorph, ilmenite, Ti-chromite, Ti-poor chromite, troilite and minor secondary barite and calcite.

Geochemistry: Orthopyroxene host $\left(\mathrm{Fs}_{57.9-60.6} \mathrm{Wo}_{4.0-2.1}, \mathrm{FeO} / \mathrm{MnO}=31-34, \mathrm{~N}=3\right)$, clinopyroxene exsolution lamellae $\left(\mathrm{Fs}_{26.9-27.3} \mathrm{Wo}_{41.8-42.6}, \mathrm{FeO} / \mathrm{MnO}=30-31, \mathrm{~N}=2\right)$, plagioclase $\left(\mathrm{An}_{89.2-90.4} \mathrm{Or}_{0.9-0.4}, \mathrm{~N}=2\right)$.

Classification: Eucrite (monomict breccia, gabbroic, shock melted).

Specimens: $46.5 \mathrm{~g}$ including one polished thin section at $U W B$; remainder with $\mathrm{Mr}$. J. Phillips. 


\section{Northwest Africa 11954 (NWA 11954)}

(Northwest Africa)

Purchased: 2017 Nov

Classification: Ordinary chondrite (LL3)

History: Purchased by Fabien Kuntz in November 2017 from a dealer in Zagora, Morocco.

Petrography: (A. Irving and S. Kuehner, UWS) Well-formed chondrules (apparent diameter $690 \pm 450 \mu \mathrm{m}$ ) are set in relatively fine grained matrix containing stained metal, merrillite and chlorapatite.

Geochemistry: Olivine $\left(\mathrm{Fa}_{1.3-44.0}, \mathrm{Cr}_{2} \mathrm{O}_{3}\right.$ in ferroan examples $0.06-0.36$ wt.\%, mean $0.12 \pm 0.12$ wt. $\%, \mathrm{~N}=7$ ), orthopyroxene $\left(\mathrm{Fs}_{2.5-15.1} \mathrm{Wo}_{0.4-1.5}, \mathrm{~N}=3\right)$, clinopyroxene $\left(\mathrm{Fs}_{9.3-12.5} \mathrm{Wo}_{39.6-37.4}, \mathrm{~N}=2\right)$. Magnetic susceptibility $\log \chi\left(\times 10^{-9} \mathrm{~m}^{3} / \mathrm{kg}\right)=3.80$. Classification: Ordinary chondrite (LL3). LL classification from magnetic susceptibility.

Specimens: $22.41 \mathrm{~g}$ including one polished thin section at $U W B$; remainder with FKuntz.

\section{Northwest Africa 11955 (NWA 11955)}

(Northwest Africa)

Purchased: 2018 May

Classification: Martian meteorite (Shergottite)

History: Purchased by Ben Hoefnagels in May 2018 from a dealer in Zagora, Morocco.

Petrography: (A. Irving and S. Kuehner, UWS) Inequigranular gabbroic texture (pyroxene up to $3.1 \mathrm{~mm}$, olivine up to $2.3 \mathrm{~mm}$ in length). Composed of homogeneous olivine, zoned pigeonite (prismatically twinned) and maskelynite with accessory Ti-chromite, ilmenite, merrillite, chlorapatite, pyrrhotite and minor baddeleyite.

Geochemistry: Olivine $\left(\mathrm{Fa}_{49.3-49.4}, \mathrm{FeO} / \mathrm{MnO}=51-56, \mathrm{~N}=3\right)$, pigeonite $\left(\mathrm{Fs}_{29.2-}\right.$ $\left.{ }_{45.7} \mathrm{Wo}_{10.4-8.1}, \mathrm{FeO} / \mathrm{MnO}=30-33, \mathrm{~N}=4\right)$, maskleynite $\left(\mathrm{An}_{50.9-55.0} \mathrm{Or}_{2.0-1.9}, \mathrm{~N}=2\right)$.

Classification: Shergottite (gabbroic).

Specimens: $23.4 \mathrm{~g}$ including one polished thin section at $U W B$; remainder with $\mathrm{Mr}$. B. Hoefnagels.

\section{Northwest Africa 11956 (NWA 11956)}

(Northwest Africa)

Purchased: 2015 Oct

Classification: Ureilite

History: Purchased by Marc Jost in October 2015 from a Moroccan dealer at the Munich Show. 
Petrography: (A. Irving and S. Kuehner, UWS) Relatively coarse grained protogranular aggregate composed of subequal amounts of olivine (exhibiting reduced magnesian rims containing fine $\mathrm{Fe}$ metal) and polysynthetically twinned clinopyroxene (both pigeonite and augite). No evidence of microdiamond was found.

Geochemistry: Olivine (cores $\mathrm{Fa}_{10.8-11.0} ;$ rims $\mathrm{Fa}_{0.9-5.3} ; \mathrm{N}=4$ ), low-Ca pyroxene $\left(\mathrm{Fs}_{11.0-11.1} \mathrm{Wo}_{4.6-4.7}, \mathrm{~N}=2\right)$, high-Ca pyroxene $\left(\mathrm{Fs}_{5.8-6.8} \mathrm{Wo}_{37.2-37.1}, \mathrm{~N}=2\right)$.

Classification: Ureilite.

Specimens: $27.8 \mathrm{~g}$ including one polished thin section at $U W B$; remainder with Space Jewels Switzerland.

\section{Northwest Africa 11957 (NWA 11957) \\ (Northwest Africa) \\ Purchased: 2018 Mar \\ Classification: Carbonaceous chondrite (CV3)}

History: Purchased by Fabien Kuntz in March 2018 from a dealer in Zagora, Morocco.

Petrography: (A. Irving and S. Kuehner, UWS) Granular chondrules (apparent diameter $950 \pm 450 \mu \mathrm{m}$ ) and irregularly-shaped, very fine grained CAI are set in a fine grained matrix ( $\sim 40$ vol.\%, deep sepia brown in thin section).

Geochemistry: Olivine $\left(\mathrm{Fa}_{1.2-58.7}, \mathrm{~N}=3\right)$, orthopyroxene $\left(\mathrm{Fs}_{1.1-1.2} \mathrm{Wo}_{0.7-4.2}, \mathrm{~N}=3\right)$, clinopyroxene $\left(\mathrm{Fs}_{0.6} \mathrm{Wo}_{46.7} ; \mathrm{Fs}_{0.8-1.0} \mathrm{Wo}_{50.0-49.5}, \mathrm{~N}=3\right)$.

Classification: Carbonaceous chondrite (CV3).

Specimens: $21.82 \mathrm{~g}$ including one polished thin section at $U W B$; remainder with Kuntz.

\section{Northwest Africa 11958 (NWA 11958)}

(Northwest Africa)

Purchased: 2018 Feb

Classification: HED achondrite (Eucrite, monomict)

History: Purchased by Bob Falls in February 2018 from a Moroccan dealer at the Tucson Gem and Mineral Show.

Physical characteristics: A single stone (2112 g) composed of greenish beige crystalline clasts (up to $5 \mathrm{~cm}$ across) in a patchy, very dark and fine grained matrix. Petrography: (A. Irving and S. Kuehner, UWS) Breccia consisting of ophitictextured eucrite clasts in a sparse, dark matrix composed of related crystalline debris within very fine grained quenched melt. Clasts are composed of exsolved pigeonite and calcic plagioclase (both microcrystalline) with accessory silica polymorph, ilmenite, Ti-chromite, troilite, Ni-free metal and minor secondary barite. Cross-cutting veinlets composed of vesicular glass, quenched crystals and entrained eucrite debris are also present. 
Geochemistry: Orthopyroxene host $\left(\mathrm{Fs}_{58.3-61.4} \mathrm{Wo}_{6.5-3.0}, \mathrm{FeO} / \mathrm{MnO}=30-31, \mathrm{~N}=3\right)$, clinopyroxene exsolution lamellae $\left(\mathrm{Fs}_{26.4-29.1} \mathrm{Wo}_{44.1-41.6}, \mathrm{FeO} / \mathrm{MnO}=32-36, \mathrm{~N}=2\right)$, plagioclase $\left(\mathrm{An}_{86.2-89.8} \mathrm{Or}_{0.8-0.2}, \mathrm{~N}=2\right)$.

Classification: Eucrite (monomict breccia, shock melted).

Specimens: $25.5 \mathrm{~g}$ in the form of a large polished slice at $U W B$; remainder with Mr. R. Falls.

Northwest Africa 11959 (NWA 11959)

(Northwest Africa)

Purchased: 2018 May

Classification: Ordinary chondrite (L4)

History: Purchased by Ben Hoefnagels in May 2018 from a Moroccan dealer.

Petrography: (A. Irving and S. Kuehner, UWS) Well-formed chondrules are set in a relatively coarse grained matrix containing relatively abundant altered metal plus accessory merrillite and chlorapatite.

Geochemistry: Olivine $\left(\mathrm{Fa}_{23.5-23.8}, \mathrm{~N}=3\right)$, orthopyroxene $\left(\mathrm{Fs}_{19.8-20.5} \mathrm{Wo}_{1.3-1.0}, \mathrm{~N}=\right.$ $3)$, clinopyroxene $\left(\mathrm{Fs}_{10.3-10.6} \mathrm{Wo}_{35.7-34.6}, \mathrm{~N}=2\right)$.

Classification: Ordinary chondrite (L4).

Specimens: $26.2 \mathrm{~g}$ including one polished thin section at $U W B$; remainder with $\mathrm{Mr}$. B. Hoefnagels.

\title{
Northwest Africa 11960 (NWA 11960)
}

(Northwest Africa)

Purchased: 2018 Apr

Classification: Primitive achondrite (Lodranite)

History: Purportedly found near Smara, Morocco. Purchased from a nomad by a Moroccan dealer in March 2018 and then sold to Ben Hoefnagels in April 2018.

Petrography: (A. Irving and S. Kuehner, UWS) Relatively coarse grained (1-2.5 $\mathrm{mm}$ ) protogranular aggregate of olvine and clinopyroxene with accessory orthopyroxene, chromite, pyrrhotite, partly altered kamacite and taenite. Minor goethite from terrestrial alteration of metal is present as thin coatings on grain boundaries and in cracks.

Geochemistry: Olivine $\left(\mathrm{Fa}_{10.4-10.6}, \mathrm{FeO} / \mathrm{MnO}=22-23, \mathrm{~N}=3\right)$, clinopyroxene $\left(\mathrm{Fs}_{4.2-}\right.$ $\left.{ }_{4.4} \mathrm{Wo}_{45.1-45.5}, \mathrm{FeO} / \mathrm{MnO}=10-11, \mathrm{~N}=2\right)$, orthopyroxene $\left(\mathrm{Fs}_{9.3-9.6} \mathrm{Wo}_{1.5-2.0}\right.$, $\mathrm{FeO} / \mathrm{MnO}=13-15, \mathrm{~N}=2$ ),

Classification: Lodranite (wehrlitic).

Specimens: $24.1 \mathrm{~g}$ including one polished thin section at $U W B$; remainder with $\mathrm{Mr}$. B. Hoefnagels.

\author{
Northwest Africa 11962 (NWA 11962) \\ (Northwest Africa) \\ Purchased: 2013
}


Classification: Lunar meteorite

History: In 2013, the $N H M V$ acquired a single stone weighing $85.8 \mathrm{~g}$ from a Moroccan dealer.

Physical characteristics: The specimen is a flat and rounded individual with regmaglypts exhibiting a black shiny surface with in places dull brownish areas. A cut and polished face $(3 \mathrm{~cm} 2)$ reveals two different lithologies comprising a finegrained, dark-gray matrix with mainly submillimeter-sized light clasts and a black compact area $(1 \times 0.5 \mathrm{~cm})$ of impact melt.

Petrography: Clast-rich breccia containing fine-grained lithic and mineral clasts, brownish vesicular glass, and brownish to orange glass spherules. In places, the glass exhibits a flow-banded texture with dark-grey to black schlieren. Lithic clasts include basalts, gabbroic lithologies, breccia-within-breccia clasts and granophyric intergrowths of K-feldspar and silica. Mineral clasts include ortho- and clinopyroxenes (some showing exsolution lamellae), olivine, plagioclase (mostly anorthitic), silica, spinel (chromite, ulvöspinel), ilmenite, zircon, troilite and Fe,Ni metal.

Geochemistry: Olivine $\mathrm{Fa}_{38.0 \pm 13.5}(\mathrm{~N}=40)$, plagioclase $\mathrm{An}_{88.2 \pm 11.2} \mathrm{Or}_{0.6 \pm 0.5}(\mathrm{~N}=37)$, orthopyroxene $\mathrm{Fs}_{14.3 \pm 0.4} \mathrm{Wo}_{1.3 \pm 1.3}(\mathrm{~N}=63)$, clinopyroxene $\mathrm{Fs}_{30.8 \pm 9.1} \mathrm{Wo}_{23.4 \pm 13.5}(\mathrm{~N}=$ 43), K-feldspar $\left(\mathrm{An}_{4.5 \pm 1.0} \mathrm{Or}_{73.9 \pm 6.4},(\mathrm{~N}=11) \mathrm{BaO}=1.4-3.4 \mathrm{wt} \%\right)$, exsolved pyroxene: orthopyroxene host $\mathrm{Fs}_{65.5 \pm 4.8} \mathrm{Wo}_{4.0 \pm 1.3}(\mathrm{~N}=19)$ with augite exsolution lamellae $\mathrm{Fs}_{33.0 \pm 2.3} \mathrm{Wo}_{41.6 \pm 2.3}(\mathrm{~N}=20)$. The oxygen isotope composition ( $\mathrm{R}$.

Greenwood, $O U)$ is consistent with a lunar origin $\left(\delta^{17} \mathrm{O}=3.14\right.$ per mil, $\delta^{18} \mathrm{O}=6.03$ per mil, $\Delta^{17} \mathrm{O}=0.01$ per mil).

Classification: Lunar, regolith breccia. Moderate weathering.

Specimens: A mass of $80.8 \mathrm{~g}$ and two thin sections are on deposit at $N H M V$.

\section{Northwest Africa 11963 (NWA 11963)}

(Northwest Africa)

Purchased: 02/12/2015

Classification: Ordinary chondrite (LL6)

Petrography: The rock is brecciated and highly recrystallized. Chondrules are poorly defined. Plagioclase grains average $60 \mu \mathrm{m}$ in size; many of them contain patches of maskelynite. There are black glassy shock veins in the rock. It contains chromite-plagioclase assemblages and polycrystalline troilite.

\section{Northwest Africa 11964 (NWA 11964)}

(Northwest Africa)

Purchased: 2017

Classification: HED achondrite (Howardite)

History: Purchased by Aziz Habibi in Morocco, 2017.

Physical characteristics: Saw cut reveals a fragmental breccia with dark and light clasts set in a gray groundmass. 
Petrography: (C. Agee, $U N M$ ) This meteorite consists of approximately $50 \%$ diogenite and $50 \%$ eucrite clasts.

Geochemistry: (C. Agee, $U N M$ ) diogenite low-Ca pyroxene $\mathrm{Fs}_{25.3 \pm 3.0} \mathrm{Wo}_{2.3 \pm 0.6}$, $\mathrm{Fe} / \mathrm{Mn}=30 \pm 1, \mathrm{n}=10$; eucrite low Ca pyroxene $\mathrm{Fs}_{42.3 \pm 8.4} \mathrm{Wo}_{5.8 \pm 3.2}, \mathrm{Fe} / \mathrm{Mn}=31 \pm 2, \mathrm{n}=9$; eucrite high Ca pyroxene $\mathrm{Fs}_{18.7} \mathrm{Wo}_{42.4}, \mathrm{Fe} / \mathrm{Mn}=27, \mathrm{n}=1$; plagioclase $\mathrm{An}_{89.0 \pm 6.7}, \mathrm{n}=7$. Classification: Howardite

Specimens: $20.0 \mathrm{~g}$ including a probe mount on deposit at $U N M$, Kyle Ke holds the main mass

\section{Northwest Africa 11965 (NWA 11965)}

(Northwest Africa)

Purchased: 2018

Classification: Carbonaceous chondrite (CM2)

History: Purchased by Aziz Habibi in Morocco 2018.

Physical characteristics: Complete individual stone, partially covered with fusion crust. A broken surface reveals black, fined-grained interior; very friable; some CAIs are visible.

Petrography: (C. Agee, $U N M$ ) This meteorite consists of scattered porphyritic chondrules set in a fine grained matrix. The matrix makes up approximately $75 \%$ of this meteorite. Ubiquitous Fe-sulfide, Fe,Ni-sulfide, Fe, Ni-metal, phyllosilicates, and carbonates are present in the matrix.

Geochemistry: (C. Agee, UNM) Olivine $\mathrm{Fa}_{12.5 \pm 17.6}$, range $\mathrm{Fa}_{0.5-44.4}$, $\mathrm{Cr}_{2} \mathrm{O}_{3}=0.32 \pm 0.16, \mathrm{n}=11$; low-Ca pyroxene $\mathrm{Fs}_{3.5 \pm 3.3} \mathrm{Wo}_{3.4 \pm 0.5}, \mathrm{n}=2$. Oxygen isotopes $($ K. Ziegler, $U N M)$ done by laser fluorination $\delta^{17} \mathrm{O}=1.681, \delta^{18} \mathrm{O}=10.426, \Delta{ }^{17} \mathrm{O}=-$ 3.825 , normalized, all permil.

Classification: Carbonaceous chondrite (CM2)

Specimens: $11.5 \mathrm{~g}$ including a probe mount on deposit at $U N M$, Aziz Habibi holds the main mass.

\section{Northwest Africa 11966 (NWA 11966)}

(Northwest Africa)

Purchased: 2015

Classification: Lunar meteorite (feldspathic breccia)

History: Purchased by Jay Piatek on April 27, 2015 from a Moroccan meteorite dealer.

Physical characteristics: Single stone, fresh fusion-crusted exterior. A saw cut reveals a fragmental breccia with many white feldspathic clasts and a large lithic clast set in a dark-gray groundmass.

Petrography: (C. Agee, UNM) This meteorite is a breccia of fragmental pyroxene, olivine, plagioclase grains. There are many domains that are fine-grained and cataclastic with shock melt and vesicles.

Geochemistry: (C. Agee, $U N M$ ) olivine $\mathrm{Fa}_{35.8 \pm 5.0}, \mathrm{Fe} / \mathrm{Mn}=98 \pm 11, \mathrm{n}=10$; pigeonite $\mathrm{Fs}_{33.6 \pm 5.3} \mathrm{Wo}_{12.6 \pm 6.8}, \mathrm{Fe} / \mathrm{Mn}=58 \pm 3, \mathrm{n}=8$; augite $\mathrm{Fs}_{29.0 \pm 2.9} \mathrm{Wo}_{34.8 \pm 4.3}, \mathrm{Fe} / \mathrm{Mn}=57 \pm 1, \mathrm{n}=2$; 
plagioclase $\mathrm{An}_{96.4 \pm 0.9}, \mathrm{n}=7$; Shock melt $(20 \mu \mathrm{m}$ defocused electron beam, proxy for bulk meteorite composition): $\mathrm{SiO}_{2}=43.4 \pm 0.4, \mathrm{TiO}_{2}=0.18 \pm 0.06, \mathrm{Al}_{2} \mathrm{O}_{3}=31.0 \pm 1.2$, $\mathrm{Cr}_{2} \mathrm{O}_{3}=0.06 \pm 0.03, \mathrm{MgO}=3.0 \pm 0.6, \mathrm{FeO}=3.6 \pm 0.8, \mathrm{MnO}=0.05 \pm 0.03, \mathrm{CaO}=17.5 \pm 0.5$, $\mathrm{Na}_{2} \mathrm{O}=0.35 \pm 0.04, \mathrm{~K}_{2} \mathrm{O}=0.07 \pm 0.02$ (all wt\%), $\mathrm{n}=7$.

Classification: Lunar feldspathic breccia

Specimens: $20.1 \mathrm{~g}$ including a probe mount on deposit at $U N M$, Jay Piatek holds the main mass.

\section{Northwest Africa 11967 (NWA 11967) \\ (Northwest Africa) \\ Purchased: 2015 \\ Classification: Ordinary chondrite (LL3)}

History: Purchased by Jay Piatek on June 26, 2015, from a Moroccan meteorite dealer.

Physical characteristics: Single stone, weathered exterior. A saw cut reveals numerous distinct, closely packed chondrules set in a brown groundmass. Mild brecciation was observed.

Petrography: (C. Agee, $U N M$ ) This meteorite has many porphyritic chondrules, most with mesostasis or glass, set in abundant opaque matrix. Apparent mean chondrule size is $800 \pm 500 \mu \mathrm{m}, \mathrm{n}=19$.

Geochemistry: (C. Agee, UNM) Coarse-grained (non-matrix) ferroan olivine $\mathrm{Fa}_{23.3 \pm 8.2}, \mathrm{Cr}_{2} \mathrm{O}_{3}=0.18 \pm 0.22(\mathrm{wt} \%), \mathrm{n}=17$; low-Ca pyroxene $\mathrm{Fs}_{15.5 \pm 11.1} \mathrm{Wo}_{0.8 \pm 0.5}$, $\mathrm{n}=11$.

Classification: Ordinary chondrite LL3 breccia. $\mathrm{Cr}_{2} \mathrm{O}_{3}$ distribution in olivine is similar to Ngawi (LL3.0-3.7) as described in Grossman and Brearley (2005)) with two populations, one resembling Semarkona $\mathrm{Cr}_{2} \mathrm{O}_{3}=0.50 \pm 0.08$ and one resembling Bremervörde $\mathrm{Cr}_{2} \mathrm{O}_{3}=0.04 \pm 0.03(\mathrm{wt} \%)$.

Specimens: $22.4 \mathrm{~g}$ including a probe mount on deposit at UNM, Jay Piatek holds the main mass.

\section{Northwest Africa 11968 (NWA 11968) \\ (Northwest Africa) \\ Purchased: 2015 \\ Classification: Lunar meteorite (feldspathic breccia)}

History: Purchased by Jay Piatek from Adam Aaronson in Morocco, 2015. Physical characteristics: Single stone. Fresh, light green-brown fusion crust covers a significant part of the surface; the remainder of the exterior is a smooth, lustrous black color. Saw cut reveals a fragmental breccia with many white feldspathic clasts set in a dark gray ground mass.

Petrography: (C. Agee, $U N M$ ) This meteorite is a breccia of fragmental pyroxene, olivine, plagioclase grains set in a fine-grained matrix. Fe-metal, chromite, ilmenite, and troilite were detected. 
Geochemistry: (C. Agee, $U N M$ ) Olivine Fa: 37.4 \pm 9.5 , Fe/Mn=92 \pm 5 , n=8; low-Ca pyroxene $\mathrm{Fs}_{36.1 \pm 3.5} \mathrm{Wo}_{5.4 \pm 1.3}, \mathrm{Fe} / \mathrm{Mn}=54 \pm 0, \mathrm{n}=2$; high-Ca pyroxene $\mathrm{Fs}_{19.9 \pm 2.3} \mathrm{Wo}_{33.3 \pm 9.3}, \mathrm{Fe} / \mathrm{Mn}=49 \pm 4, \mathrm{n}=3$; plagioclase $\mathrm{An}_{95.7 \pm 1.4}, \mathrm{n}=4$; Shock melt (20 $\mu \mathrm{m}$ defocused electron beam, proxy for bulk meteorite composition): $\mathrm{SiO}_{2}=43.7 \pm 1.0, \mathrm{TiO}_{2}=0.20 \pm 0.11, \mathrm{Al}_{2} \mathrm{O}_{3}=30.7 \pm 3.6, \mathrm{Cr}_{2} \mathrm{O}_{3}=0.07 \pm 0.05$, $\mathrm{MgO}=4.0 \pm 2.2, \mathrm{FeO}=4.6 \pm 2.4, \mathrm{MnO}=0.07 \pm 0.05, \mathrm{CaO}=16.7 \pm 1.8, \mathrm{Na}_{2} \mathrm{O}=0.34 \pm 0.09$, $\mathrm{K}_{2} \mathrm{O}=0.05 \pm 0.02($ all $\mathrm{wt} \%), \mathrm{n}=12$.

Classification: Lunar feldspathic breccia Specimens: $20.0 \mathrm{~g}$ including a probe mount on deposit at $U N M$, Jay Piatek holds $407.8 \mathrm{~g}$.

\section{Northwest Africa 11969 (NWA 11969) \\ (Northwest Africa) \\ Purchased: 2015 \\ Classification: HED achondrite (Eucrite, unbrecciated)}

History: Purchased by Jay Piatek on December 28, 2015 from a Moroccan meteorite dealer.

Physical characteristics: Single stone, saw cut show medium grained, equigranular, basaltic texture.

Petrography: (C. Agee, $U N M$ ) (C. Agee, $U N M)$ Microprobe examination shows approximately $50 \%$ pyroxene and $50 \%$ plagioclase. Pyroxene grains show exsolution lamellae. Accessory troilite, chromite, silica, and ilmenite were detected. Geochemistry: (C. Agee, UNM) Low-Ca pyroxene $\mathrm{Fs}_{59.4 \pm 1.0} \mathrm{Wo}_{3.5 \pm 1.6}$, $\mathrm{Fe} / \mathrm{Mn}=30 \pm 1, \mathrm{n}=3$; high-Ca pyroxene $\mathrm{Fs}_{33.7 \pm 8.6} \mathrm{Wo}_{35.2 \pm 11.4}, \mathrm{Fe} / \mathrm{Mn}=31 \pm 4, \mathrm{n}=3$; plagioclase $\mathrm{An}_{89.6 \pm 0.3}, \mathrm{n}=3$.

Classification: Eucrite, unbrecciated Specimens: $21 \mathrm{~g}$ including a probe mount on deposit at $U N M$, Jay Piatek holds the main mass.

\section{Northwest Africa 11970 (NWA 11970)}

(Northwest Africa)

Find: 2015

Classification: Primitive achondrite (Lodranite)

History: Acquired from a Moroccan meteorite dealer at the 2016 Tucson Gem and Mineral show.

Physical characteristics: Four similar looking stones, the largest being $87.1 \mathrm{~g}$. The uncut stones are light gray with clumped protruding dark brown/red nodules and scattered visible bright green angular mm-scale minerals. The cut stones show a breccia of $<1 \mathrm{~cm}$-sized silicate grains.

Petrography: Observations from a thin section show a breccia dominated by angular to sub-rounded silicate grains the largest to $1 \mathrm{~cm}$. Minerals dominated by olivine, orthopyroxene, and clinopyroxene; some of the pyroxene is polysynthetically twinned. Minor phases include chromite, troilite, stained 
kamacite and taenite, and some composite metal grains. The metal is dominated by kamacite with minor tetrataenite. Metal grains (to $3 \mathrm{~mm}$ ) are irregularly shaped (holly leaf-like), with polycrystalline structure dominated by $>100 \mu \mathrm{m}$ polygonal grains lacking Neumann bands. Troilite is dominantly single crystal.

Geochemistry: (E.T. Dunham, D. Kornau, T. Goodwyn, T. Fougner, N. Delafuente, and E. Smith, $A S U)$ Olivine $\left(\mathrm{Fa}_{11.5-11.9}(\mathrm{avg}=11.7), \mathrm{FeO} / \mathrm{MnO}=24.0-\right.$ $26.8(\mathrm{avg}=25.9), \mathrm{n}=10)$, orthopyroxene, $\mathrm{Fs}_{11.3} \mathrm{Wo}_{1.8}\left(\mathrm{Fs}_{10.4-12.4} \mathrm{Wo}_{0.1-4.0}\right)$, $\mathrm{FeO} / \mathrm{MnO}=15.1-27.1$ (avg=19.6), $\mathrm{n}=11$; clinopyroxene, $\mathrm{Fs}_{5.1} \mathrm{Wo}_{42.3}\left(\mathrm{Fs}_{4.3-6.1} \mathrm{Wo}_{39.7-}\right.$ 44.5), $\mathrm{FeO} / \mathrm{MnO}=10.8-13.1$ (avg=11.5), $\mathrm{n}=9$.

Classification: Lodranite breccia. This material is likely paired with NWA $\underline{8118}$, NWA 8216, and NWA 8251

Specimens: $180.9 \mathrm{~g}$ including 4 thin sections at $A S U$

\section{Northwest Africa 11973 (NWA 11973) \\ (Northwest Africa) \\ Purchased: Nov 2015 \\ Classification: Ordinary chondrite $(\mathrm{H} \sim 5)$}

History: Purchased by Li Bofang from a Moroccan dealer at the mineral show in Beijing in Nov. 2015.

Physical characteristics: 344.8 g stone, about $70 \%$ of the surface is covered with yellowish oxidized crust; light-gray interior with many chondrules and metal specks. Some cracks are partly filled yellow colored adherent soil-like material. Petrography: (R. Bartoschewitz, Bart, B. Li, Beijing) cut surface shows recrystallized matrix with chondrules of $0.2-1.0 \mathrm{~mm}$ (av. $0.6 \mathrm{~mm}$ ), about $30 \%$ of chondrules are well defined.

Geochemistry: (R. Bartoschewitz, Bart) Magnetic susceptibility $\log \chi\left(\times 10^{-}\right.$ $\left.{ }^{9} \mathrm{~m}^{3} / \mathrm{kg}\right)=5.01$.

Classification: ordinary chondrite $(\mathrm{H} \sim 5)$

Specimens: $25.3 \mathrm{~g}$ on deposit at Kiel, B. Li holds the main mass and $40 \mathrm{~g}$ with Bart.

\section{Northwest Africa 11974 (NWA 11974) \\ (Northwest Africa) \\ Purchased: Feb 2018 \\ Classification: Ordinary chondrite $(\mathrm{H} \sim 5)$}

History: Purchased by Li Bofang from a Morrocan dealer in Tucson in Feb 2017. Physical characteristics: Brown globular stone of $73.7 \mathrm{~g}$. Cut face shows brown matrix with many little chondrules and some tiny metal specks.

Petrography: (R. Bartoschewitz, Bart, B. Li, Beijing) cut surface shows recrystallized matrix with well-defined chondrules $(0.1-0.6 \mathrm{~mm}$, av. $0.3 \mathrm{~mm})$ and sparsely distributed metal specks.

Geochemistry: (R. Bartoschewitz, Bart) Magnetic susceptibility $\log \chi\left(\times 10^{-}\right.$ $\left.{ }^{9} \mathrm{~m}^{3} / \mathrm{kg}\right)=4.69$. 
Classification: ordinary chondrite $(\mathrm{H} \sim 5)$

Specimens: $15.2 \mathrm{~g}$ on deposit at Kiel, B. Li holds the main mass and $10.2 \mathrm{~g}$ with Bart.

\section{Northwest Africa 11975 (NWA 11975)}

(Northwest Africa)

Purchased: Feb. 2017

Classification: Ordinary chondrite $(\mathrm{LL} \sim 5)$

History: Purchased by Li Bofang from a Morrocan dealer in Tucson in Feb 2017. Physical characteristics: Brown globular stone of 73.7 g. Cut face shows brown matrix with chondrules and some tiny metal specks.

Petrography: (R. Bartoschewitz, Bart, B. Li, Beijing) cut surface shows recrystallized matrix with well-defined gray chondrules $(0.6-2.0 \mathrm{~mm})$ and metal specks.

Geochemistry: (R. Bartoschewitz, Bart) Magnetic susceptibility $\log \chi\left(\times 10^{-}\right.$ $\left.{ }^{9} \mathrm{~m}^{3} / \mathrm{kg}\right)=4.41$.

Classification: ordinary chondrite (LL $\sim 5)$

Specimens: $15.8 \mathrm{~g}$ on deposit at Kiel, B. Li holds the main mass and $9.6 \mathrm{~g}$ with Bart.

\section{Northwest Africa 11976 (NWA 11976) \\ (Northwest Africa) \\ Purchased: Nov 2017 \\ Classification: Ordinary chondrite (LL3)}

History: Purchased by Li Bofang from a Morrocan who got a language lecture in Beijing in Nov. 2017.

Physical characteristics: 78.6 g stone covered by $>80 \%$ dark, weak, shiny fusion crust; non-crusted surface is strong eroded and presents well-defined chondrules. Petrography: (R. Bartoschewitz, Bart, B. Li, Beijing) cut surface shows poorly recrystallized matrix with well-defined light- to dark-gray and olive-gray chondrules of 0.4-2.0 mm and very few tiny metal specks.

Geochemistry: (R. Bartoschewitz, Bart, P. Appel and B. Mader, Kiel) Olivine $\mathrm{Fa}_{21.8 \pm 10.5}(\mathrm{n}=28)$; pyroxene $\mathrm{Fs}_{11.4 \pm 9.0} \mathrm{Wo}_{0.9 \pm 0.8}(\mathrm{n}=15)$; Ca-pyroxene $\mathrm{Fs}_{26.9} \mathrm{Wo}_{73.1}(\mathrm{n}=1)$. Kamacite: $\mathrm{Ni}=5.0-6.3, \mathrm{Co}=1.6-1.8 \mathrm{wt} . \%(\mathrm{n}=6)$; tetrataenite, troilite. Magnetic susceptibility (R. Bartoschewitz, Bart) $\log \chi\left(\times 10^{-9} \mathrm{~m}^{3} / \mathrm{kg}\right)=$ 3.95 .

Classification: ordinary chondrite (LL3)

Specimens: $15.8 \mathrm{~g}$ on deposit at Kiel, B. Li holds the main mass and $4.4 \mathrm{~g}$ with Bart.

Northwest Africa 11977 (NWA 11977)

(Northwest Africa) 
Purchased: Feb 2017

Classification: Carbonaceous chondrite (CV3)

History: Purchased by Li Bofang from a Morrocan dealer in Tucson in Feb 2017. Physical characteristics: Dark brown $72.9 \mathrm{~g}$ stone, numerous chondrules are visible on the glossy, desert-varnished surface.

Petrography: (R. Bartoschewitz, Bart, B. Li, Beijing) thin section shows many chondrules $<2.5 \mathrm{~mm}$ of various types (PO, PP, POP, RP), fine-grained, olivine-rich aggregates and CAIs are embedded in a fine-grained, dark brown matrix (roughly 40 vol\%). Fe,Ni-rich metal and troilite are less abundant.

Geochemistry: (R. Bartoschewitz, Bart, P. Appel and B. Mader, Kiel) Olivine: $\mathrm{Fa}_{14.7 \pm 5.0}, \mathrm{Cr}_{2} \mathrm{O}_{3}=0.37 \pm 0.12, \mathrm{CaO}=0.13 \pm 0.07 \mathrm{wt} \%(\mathrm{n}=13)$; low-Ca pyroxene $\mathrm{Fs}_{9.4 \pm 5.6} \mathrm{Wo}_{0.8 \pm 0.7}(\mathrm{n}=14)$; pigeonite $\mathrm{Fs}_{17.3} \mathrm{Wo}_{6.2}(\mathrm{n}=1)$. Feldspar:

$\mathrm{An}_{14.2 \pm 1.3} \mathrm{Or}_{8.0 \pm 0.6}$. Chromite: $\mathrm{CRAL}=96.9 .7 \pm 5.3, \mathrm{FFM}=96.4 .7 \pm 3.2(\mathrm{n}=3)$. Kamacite: $\mathrm{Ni}=4.8-6.6, \mathrm{Co}=0.5-1.8(\mathrm{n}=3)$; taenite: $\mathrm{Ni}=44.1, \mathrm{Co}=0.6(\mathrm{n}=1)(\mathrm{wt} . \%)$; tetrataenite, troilite.

Classification: Carbonaceous chondrite (CV3).

Specimens: $14.6 \mathrm{~g}$ on deposit at Kiel, B. Li holds the main mass and $3.7 \mathrm{~g}$ with Bart.

\section{Northwest Africa 11978 (NWA 11978)}

(Northwest Africa)

Purchased: Nov. 2015

Classification: Ordinary chondrite (L3-6)

History: Purchased by Li Bofang from a Morrocoan dealer at the mineral show in Beijing in Nov. 2015.

Physical characteristics: Brown $1159 \mathrm{~g}$ stone of about $10 \mathrm{~cm}$ in diameter.

Petrography: (R. Bartoschewitz, Bart, B. Li, Beijing) cut surface shows brown matrix with poor to well defined chondrules of $0.3-5 \mathrm{~mm}$ of various types (PO, PP, POP, RP), and dark xenolites. Metal is nearly complete oxidized.

Geochemistry: (R. Bartoschewitz, Bart, P. Appel and B. Mader, Kiel) Olivine $\mathrm{Fa}_{23.2 \pm 2.0}(\mathrm{n}=23)$; pyroxene $\mathrm{Fs}_{17.0 \pm 5.6} \mathrm{Wo}_{1.5 \pm 1.4}(\mathrm{n}=13)$; Ca-pyroxene

$\mathrm{Fs}_{26.9} \mathrm{Wo}_{73.1}(\mathrm{n}=1)$. Magnetic susceptibility (R. Bartoschewitz, Bart $) \log \chi\left(\times 10^{-}\right.$ $\left.{ }^{9} \mathrm{~m}^{3} / \mathrm{kg}\right)=4.48$.

Classification: ordinary chondrite (L3-6)

Specimens: $20.1 \mathrm{~g}$ on deposit at Kiel, B. Li holds the main mass and $67 \mathrm{~g}$ with Bart.

\section{Northwest Africa 11979 (NWA 11979)}

(Northwest Africa)

Purchased: Nov 2015

Classification: Ordinary chondrite $(\mathrm{H} \sim 6)$

History: Purchased by Li Bofang from a Morrocoan dealer at the mineral show in Beijing in Nov. 2015. Later the main mass was donated to Qu Pan. 
Physical characteristics: A $78.1 \mathrm{~g}$ dark brown elongated stone. Cut face shows fine distributed tiny metal specks all over the surface.

Petrography: (R. Bartoschewitz, Bart, B. Li, Beijing) cut surface shows light/dark phases with shock veins, strong recrystallized matrix and poor defined chondrules $(0.2-0.5 \mathrm{~mm}$, av. $0.3 \mathrm{~mm})$. The dark phase is finer grained than the lighter one. Geochemistry: (R. Bartoschewitz, Bart) Magnetic susceptibility $\log \chi\left(\times 10^{-}\right.$ $\left.{ }^{9} \mathrm{~m}^{3} / \mathrm{kg}\right)=5.11$.

Classification: ordinary chondrite $(\mathrm{H} \sim 6)$

Specimens: $16.8 \mathrm{~g}$ on deposit at Kiel, B. Li holds the main mass and $11.2 \mathrm{~g}$ with Bart.

\section{Northwest Africa 11980 (NWA 11980)}

(Northwest Africa)

Purchased: 2017 March

Classification: Ordinary chondrite (L5)

History: Purchased from a local dealer in Quarzazate in 2017.

Physical characteristics: Two stones of 1210 and $160 \mathrm{~g}$. Cut face is gray with some lighter gray chondrules, light brown inclusions, and fresh metal.

Petrography: (R. Bartoschewitz, Bart) Microscopic examination of a thin section shows recrystallized and shocked matrix: mosaicism and planar fractures in olivine, undulatory extinction in plagioclase and maskelynite. Chondrules moderatly developed (0.4-1.8 $\mathrm{mm}$, average $0.9 \mathrm{~mm})$.

Geochemistry: (R. Bartoschewitz, Bart, P. Appel and B. Mader, Kiel) Olivine $\mathrm{Fa}_{22.1 \pm 0}, 6(\mathrm{n}=19)$; pyroxene $\mathrm{Fs}_{19.2 \pm 1.1} \mathrm{Wo}_{0.8 \pm 0.6}(\mathrm{n}=9)$; Ca-pyroxene

$\mathrm{Fs}_{21.4 \pm 3.2} \mathrm{Wo}_{9.6 \pm 2.4}(\mathrm{n}=2)$. Kamacite $\mathrm{Ni}=5.9-6.7, \mathrm{Co}=0.6 \mathrm{wt} . \%(\mathrm{n}=5)$; taenite $\mathrm{Ni}=34.7-$ $36.6, \mathrm{Co}=0.3$ wt.\% $(\mathrm{n}=2)$. Magnetic susceptibility (R. Bartoschewitz, Bart) $\log \chi(\times$ $\left.10^{-9} \mathrm{~m}^{3} / \mathrm{kg}\right)=4.94$.

Classification: Ordinary chondrite (type L5, S3, W1)

Specimens: $20.1 \mathrm{~g}$ on deposit at Kiel, main mass Dieter Janek.

\section{Northwest Africa 11982 (NWA 11982)}

(Northwest Africa)

Purchased: 2005

Classification: Ordinary chondrite (L5)

History: Purchased by an anonymous collector from a dealer at the Munich Show in 2005, subsequently acquired by the Hollis Collection and then donated to PSF. Petrography: (A. Irving and S. Kuehner, UWS) Sparse chondrules are present in a recrystallized matrix containing chlorapatite and merrillite.

Geochemistry: Olivine $\left(\mathrm{Fa}_{24.6-25.5}, \mathrm{~N}=3\right)$, orthopyroxene $\left(\mathrm{Fs}_{20.4-20.7} \mathrm{Wo}_{1.5-1.0}, \mathrm{~N}=\right.$ 3), clinopyroxene $\left(\mathrm{Fs}_{7.3-7.8} \mathrm{Wo}_{44.1-45.5}, \mathrm{~N}=2\right)$.

Classification: Ordinary chondrite (L5).

Specimens: The entire specimen including one polished thin section is at PSF. 


\section{Northwest Africa 11984 (NWA 11984)}

(Northwest Africa)

Purchased: 2005

Classification: Ordinary chondrite (L6)

History: Purchased by an anonymous collector from a dealer at the Munich Show in 2005, subsequently acquired by the Hollis Collection and then donated to PSF. Petrography: (A. Irving and S. Kuehner, UWS) Mostly recrystallized with rare remnant chondrules.

Geochemistry: Olivine $\left(\mathrm{Fa}_{25.0-25.5} \mathrm{~N}=3\right)$, orthopyroxene $\left(\mathrm{Fs}_{21.0-21.2} \mathrm{Wo}_{2.1-1.9}, \mathrm{~N}=3\right)$, clinopyroxene $\left(\mathrm{Fs}_{8.8} \mathrm{Wo}_{44.3} ; \mathrm{Fs}_{13.6} \mathrm{Wo}_{41.7} ; \mathrm{N}=2\right)$.

Classification: Ordinary chondrite (L6).

Specimens: The entire specimen including one polished thin section is at PSF.

\section{Northwest Africa 11986 (NWA 11986)}

(Northwest Africa)

Purchased: 2005

Classification: Ordinary chondrite (L4)

History: Purchased by an anonymous collector from a dealer at the Munich Show in 2005, subsequently acquired by the Hollis Collection and then donated to PSF.

Petrography: (A. Irving and S. Kuehner, UWS) Well-formed, equilibrated chondrules are set in a matrix containing altered metal, chlorapatite and merrillite. Geochemistry: Olivine $\left(\mathrm{Fa}_{24.5-24.7}, \mathrm{~N}=3\right)$, orthopyroxene $\left(\mathrm{Fs}_{19.6-20.9} \mathrm{Wo}_{1.0-1.6}, \mathrm{~N}=\right.$ 3), clinopyroxene $\left(\mathrm{Fs}_{8.3-9.5} \mathrm{Wo}_{45.0-44.5}, \mathrm{~N}=2\right)$.

Classification: Ordinary chondrite (L4).

Specimens: The entire specimen including one polished thin section is at PSF.

\section{Northwest Africa 11991 (NWA 11991)}

(Northwest Africa)

Purchased: 2018 Jan

Classification: Ordinary chondrite (LL3)

History: Found in northwest Africa.

Physical characteristics: In cut slices, interior is light gray to light brown with slight weathering staining, has well defined chondrules and metal contents typical of LL chondrites.

Petrography: (A. Ruzicka, S. Goudy, Cascadia) Thin section shows well-defined chondrules, many with glassy mesostases, and good examples of cluster chondrite texture with little intervening matrix. Multiple bleached chondrules. Analysis in reflected light shows thin section contains roughly $2.3 \%$ metal with $\sim 10 \%$ metal weathered, and roughly $4.2 \%$ troilite; largest slice contains $2.8 \pm 0.3 \%$ metal and $5.4 \pm 0.6 \%$ troilite (area\% values). Based on olivine, shock stage is S5. Matrix is 
composed chiefly of $<10 \mu \mathrm{m}$ grains of ferroan olivine and albitic feldspar, reminiscent of that found in Lut 005 and Lut 006.

Geochemistry: (M. Hutson, Cascadia) Olivine $\left(\mathrm{Fa}_{21.6 \pm 9.9}, \mathrm{~N}=156\right.$; $\left.\mathrm{Cr}_{2} \mathrm{O}_{3} 0.07 \pm 0.05 \mathrm{wt} \%, \mathrm{~N}=126\right)$.

Classification: LL3, estimated subtype 3.5. LL indicated by low metal content and minor weathering. Type 3 indicated by texture, presence of chondrule glass, and variable olivine composition. Subtype estimated as 3.5 based on olivine Fa variability (subtype 3.4-3.6) and chrome content in olivine.

Specimens: A total of $48.4 \mathrm{~g}$ in two slices, a polished thin section, and potted butt are available at Cascadia. Mr. James Tobin holds the main mass.

\section{Northwest Africa 11992 (NWA 11992)}

(Northwest Africa)

Purchased: 2007, Sept

Classification: Ordinary chondrite (LL3)

History: Found in northwest Africa, donated to Cascadia July 2012.

Physical characteristics: In cut slices, exterior is dark gray and shiny, interior is gray with well-defined chondrules and has less metal than typically observed in fresh ordinary chondrites. Minimal weathering staining.

Petrography: (A. Ruzicka, S. Goudy, Cascadia) Thin section shows well-defined chondrules with cluster chondrite texture and little intervening matrix. Glassy chondrule mesostases present but not abundant. Roughly half of low-Ca pyroxene grains show twinning suggestive of clinoenstatite polytype. Shock stage is S4.

Shock effects are heterogeneous with S1 and S4 olivine grains predominant. Some bleached chondrules. Minimal weathering effects.

Geochemistry: (M. Hutson, Cascadia) Olivine ( $\mathrm{Fa}_{30.0 \pm 4.0}, \mathrm{~N}=140$; $\left.\mathrm{Cr}_{2} \mathrm{O}_{3} 0.05 \pm 0.02 \mathrm{wt} \%, \mathrm{~N}=56\right)$, low-Ca pyroxene $\left(\mathrm{Fs}_{20.2 \pm 6.8} \mathrm{Wo}_{1.4 \pm 1.1}, \mathrm{~N}=143\right)$.

Classification: LL3 chondrite, estimated subtype 3.7. Iron contents in olivine and low-Ca pyroxene unusually high for LL3. LL indicated by low metal content and low weathering grade and overlap with LL for olivine $\mathrm{Fa}$ and low-Ca pyroxene Fs. Type 3 indicated by texture, presence of chondrule glass, abundance of clinoenstatite, and variable olivine and pyroxene compositions. Subtype indicators can be best reconciled with subtype of 3.7 to 3.8: Fa contents suggest 3.7-3.9, Fs contents suggest 3.4-3.9, olivine chrome contents suggest 3.5.

Specimens: A total of $22.7 \mathrm{~g}$ in one slice, a polished thin section, and potted butt are available at Cascadia. Mr. McKenzie holds the main mass.

\section{Northwest Africa 11993 (NWA 11993)}

(Northwest Africa)

Purchased: 2006 Sept

Classification: Ureilite

History: Samples were donated to Cascadia by Mr. Fred Olsen in July 2012. 
Physical characteristics: Interior and slightly shiny exterior have a relatively uniform dark-gray color with occasional light reddish-brown patches.

Petrography: (A. Ruzicka, S. Goudy, Cascadia) Composed chiefly of olivine and pigeonite grains roughly $1 \mathrm{~mm}$ across, often separated by fine-grained materials, cracks, or Fe-hydroxide weathering product. No metal or sulfide minerals were found. Shock stage U-S4 (Stöffler et al. 2018)

Geochemistry: (A. Ruzicka, M. Hutson, Cascadia) Cores of olivine grains have ferroan olivine $\left(\mathrm{Fa}_{22.6 \pm 0.7}, \mathrm{~N}=38\right)$ and rims are more magnesian $\left(\mathrm{Fa}_{15.6 \pm 3.6}, \mathrm{~N}=9\right)$. Pigeonite $\left(\mathrm{Fs}_{17.1 \pm 3.8} \mathrm{Wo}_{11.1 \pm 3.5}, \mathrm{~N}=28\right)$ shows less prominent magnesian rims. Classification: Achondrite (ureilite). Silicate grain cores and rims were largely unaffected by weathering, but presence of hydroxide and reddish staining indicates some weathering occurred.

Specimens: A total of $20.6 \mathrm{~g}$ and 1 thin section are on deposit at Cascadia. Mr. McKenzie holds the main mass.

\section{Northwest Africa 11994 (NWA 11994)}

(Northwest Africa)

Purchased: 2017 Jan

Classification: Ordinary chondrite (L3)

Petrography: (K. Metzler, IfP) Ordinary chondrite of low petrologic type consisting of closely packed chondrules and metal grains with very low amounts of interchondrule matrix. Cluster chondrite texture. Chondrule shapes are mostly ellipsoidal with parallel alignment.

Geochemistry: Mineral compositions and geochemistry: The mean olivine composition is $\mathrm{Fa}_{22.1 \pm 3.1}\left(\mathrm{Fa}_{13.0-26.5} ; \mathrm{n}=32\right)$; the mean low-Ca pyroxene composition is $\mathrm{Fs}_{11.6 \pm 5.2} \mathrm{Wo}_{1.0 \pm 1.1}\left(\mathrm{Fs}_{2.0-21.0} \mathrm{Wo}_{0.0-5.0} ; \mathrm{n}=28\right)$

Classification: $\mathrm{L}$ chondrite based on olivine composition with a strong peak at $\sim \mathrm{Fa}_{24}$. $\mathrm{PMD}$ of $\mathrm{Fa}$ values $=14$, i.e. petrologic subtype estimated to be 3.8 .

\section{Northwest Africa 11995 (NWA 11995)}

(Northwest Africa)

Purchased: 2012

Classification: Ordinary chondrite (H6)

Geochemistry: Mineral compositions and geochemistry: The mean olivine composition is $\mathrm{Fa}_{19.5 \pm 0.4}\left(\mathrm{Fa}_{19.0-20.0}\right)$; $\mathrm{n}=15$; the mean low-Ca pyroxene composition is $\mathrm{Fs}_{17.0 \pm 0.3} \mathrm{Wo}_{1.3 \pm 0.2}\left(\mathrm{Fs}_{16.5-17.5} \mathrm{Wo}_{1.0-1.5}\right) ; \mathrm{n}=15$

Classification: $\mathrm{H}$ chondrite based on the mean compostitions of olivine and pyroxene. Petrologic type 6 based on the size of secondary plagioclase $(>50 \mu \mathrm{m})$ and small $\mathrm{Fa}$ and $\mathrm{Fs}$ variations.

\section{Northwest Africa 11996 (NWA 11996)}

(Northwest Africa) 
Purchased: 2017 Apr

Classification: Carbonaceous chondrite (CO3)

History: Bought from an African mineral dealer in Alicante, Spain

Petrography: (K. Metzler, IfP) Chondrules, chondrule fragments, and CAIs, embedded in a fine-grained brownish matrix (about $20 \mathrm{vol} \%$ matrix). The vast majority of chondrules belong to chemical type I. Mean apparent chondrule size: $180 \pm 120 \mu \mathrm{m}(70-930 \mu \mathrm{m} ; \mathrm{n}=95)$.

Geochemistry: Mineral compositions and geochemistry: Random measurements of olivine grains revealed $\mathrm{Fa}_{36.0 \pm 8.0}\left(\mathrm{Fa}_{6.0-40.5}\right) ; \mathrm{n}=25$. Random measurements of low-Ca pyroxene grains revealed $\mathrm{Fs}_{5.7 \pm 3.1} \mathrm{Wo}_{2.8 \pm 1.4}\left(\mathrm{Fs}_{2.0-14.0} \mathrm{Wo}_{1.0-5.0}\right) ; \mathrm{n}=21$.

Classification: $\mathrm{CO}$ chondrite based on CAI occurrence, small chondrule size, chondrule-matrix ratio, and mineral chemistry. Petrologic type 3.7 estimated from chemical variation of olivine and pyroxene (PMD-Fa $=22.2$ ).

\section{Northwest Africa 11997 (NWA 11997)}

Morocco

Purchased: July 2018

Classification: HED achondrite (Howardite)

History: Purchased online by D. Sheikh in July 2018.

Physical characteristics: Single 30.2 gram stone covered by black fusion crust with flow lines, cut surface reveals fine-grained, light-gray interior.

Petrography: (D. Sheikh, FSU) Fine-grained polymict breccia, $<4 \mathrm{~mm}$ clast size for diogenite clasts, $<2 \mathrm{~mm}$ for cumulate and basaltic eucrite clasts. Modal content of howardite (in vol.\%): diogenites, $40 \%$; cumulate eucrites, $25 \%$; basaltic eucrites, $25 \%$; anorthosite clasts, $7 \%$; shock melt zones, $2 \%$; opaques, $1 \%$.

Geochemistry: Eucrite pyroxene: pigeonite $\left(\mathrm{Fs}_{47.5} \mathrm{Wo}_{10.99}\right), \mathrm{n}=25$; augite $\left(\mathrm{Fs}_{35.45} \mathrm{Wo}_{30.2}\right), \mathrm{n}=5 ; \mathrm{FeO} / \mathrm{MnO}=23$. Diogenite pyroxene: orthopyroxene $\left(\mathrm{Fs}_{24.4} \mathrm{Wo}_{5.08}\right), \mathrm{n}=10 ; \mathrm{FeO} / \mathrm{MnO}=18$. Calcic plagioclase $\left(\mathrm{An}_{87}\right), \mathrm{N}=25$. Ilmenite $(\mathrm{Fe} /(\mathrm{Fe}+\mathrm{Mg}))=87.83 \%, \mathrm{~N}=4$. $\mathrm{FeS}$ and $\mathrm{FeO}$ scattered throughout matrix. $\mathrm{SiO}_{2}$-rich glass present.

Classification: Achondrite (Howardite)

Specimens: $8.98 \mathrm{~g}$ at $U C L A, 2.67 \mathrm{~g}$ with C. Anderkin, main mass with D. Sheikh

\section{Northwest Africa 11998 (NWA 11998) \\ Northwest Africa \\ Purchased: 2017 \\ Classification: HED achondrite (Eucrite)}

History: A 2241 g, partially fusion-crusted stone was found near Laayoune, Western Sahara, in 2016. J. Donald Cline and John Sinclair Purchased the stone from Mohamed Ismaily at the Tucson Gem and Mineral show in 2017.

Physical characteristics: Sample is dark blackish brown in color, non-magnetic and has an irregular shape. Clasts are readily visible on the exterior of the sample. 
Clasts range in size from $0.25-2 \mathrm{~cm}$, are light colored, rounded to angular in shape and have very-fine to coarse grained igneous textures.

Petrography: Description and classification (A. Love, App): Sample is a breccia composed of clasts of basaltic-cumulate lithologies contained by a host of similar clastic debris set within a micro-vesicular melted matrix. Clasts are: fine-grained recrystallized basalt (25-50 $\mu \mathrm{m}$ phenocrysts); gabbroic clast with $423-512 \mu \mathrm{m}$ pyroxenes and 348-506 $\mu \mathrm{m}$ plagioclase and rare $(<1 \mathrm{vol} \%)$ diogenite. All pyroxenes are exsolved. Some clasts show breccia in breccia structure. Additional minerals and phases are: ilmenite, chromite, FeS, apatite, rare olivine and rare FeNi metal. Geochemistry: (A. Love, $A p p$ ) Basaltic eucrite: Low-Ca pyroxene host $\mathrm{Fs}_{43.9 \pm 2.2} \mathrm{Wo}_{1.7 \pm 0.1}\left(\mathrm{Fs}_{40.7-46.4} \mathrm{Wo}_{1.5-1.8}, \mathrm{FeO} / \mathrm{MnO}=33.2-37.4\right), \mathrm{N}=8$; high-Ca pyroxene exsolution lamellae $\mathrm{Fs}_{17.5 \pm 0.4} \mathrm{Wo}_{44.6 \pm 0.4}\left(\mathrm{Fs}_{17.0-17.8} \mathrm{Wo}_{44.1-44.8}\right), \mathrm{N}=3$. Cumulate eucrite: Low-Ca pyroxene host $\mathrm{Fs}_{61.7 \pm 2.7} \mathrm{Wo}_{2.1 \pm 0.3}\left(\mathrm{Fs}_{54.1-64.3} \mathrm{Wo}_{1.7-2.6}, \mathrm{FeO} / \mathrm{MnO}=30.5-34.7\right.$ $\mathrm{N}=11)$; high-Ca pyroxene exsolution lamellae $\mathrm{Fs}_{27.3 \pm 1.1} \mathrm{Wo}_{43.0 \pm 0.6}\left(\mathrm{Fs}_{26.1-29.0} \mathrm{Wo}_{42.2-}\right.$ 44.1 $\mathrm{N}=6$ ); plagioclase $\mathrm{An}_{87.2 \pm 3.2} \mathrm{Or}_{0.5 \pm 0.6}\left(\mathrm{An}_{80.3-90.9} \mathrm{Or}_{0.0-2.3} \mathrm{~N}=10\right)$. Diogenite: LowCa pyroxene $\mathrm{Fs}_{23.5} \mathrm{Wo}_{2.6}(\mathrm{FeO} / \mathrm{MnO}=30.15, \mathrm{~N}=1)$; Olivine $\mathrm{Fa}_{54.3 \pm 1.1}, \mathrm{~N}=2$.

Classification: $\mathrm{HED}$ (polymict eucrite breccia). $\mathrm{FeO} / \mathrm{MnO}$ and Fs compositions of pyroxenes indicate this sample is a HED meteorite. Textural and compositional data indicate this is a polymict eucrite melt breccia.

Specimens: PARI holds the main mass. One slice totaling $35.6 \mathrm{~g}$ and a polished thin section are on deposit at App.

\section{Northwest Africa 11999 (NWA 11999)}

(Northwest Africa)

Purchased: 2011 Feb

Classification: HED achondrite (Eucrite)

History: Sample purchased by D. Gregory at the Tucson Gem and Mineral show in 2011. Sample originally named "ROM 057" and is listed in ROM catalogues as LM58492.

Physical characteristics: The meteorite is a flattened fist size sample with a darkblack patchy fusion crust, weathered to expose a light orange surface. The interior of the sample is pale gray with a fine-grained matrix and small crystalline clasts visible.

Petrography: Sample bears gabbroic clasts in a fine-grained fragmented matrix. Clasts range in size from $402-5500 \mu \mathrm{m}$ and are composed of pyroxene and plagioclase. The fragmented matrix is of the same mineralogic composition and grains range in size from microcrystalline to $200 \mu \mathrm{m}$. Exsolution lamellae are visible in pyroxene grains.

Geochemistry: Mineral compositions and geochemistry: There is no difference in chemistry between the clasts and matrix. Low calcium pyroxenes have a composition of $\mathrm{Fs}_{54.9 \pm 3.1} \mathrm{Wo}_{4.8 \pm 3.9} \mathrm{FeO} / \mathrm{MnO}(\mathrm{wt} \%)=29.6 \pm 0.7(\mathrm{n}=22)$. Augite has a composition of $\mathrm{Fs}_{24.3 \pm 1.0} \mathrm{Wo}_{42.8 \pm 1.3} \mathrm{FeO} / \mathrm{MnO}(\mathrm{wt} \%)=29.7 \pm 1.1(\mathrm{n}=19)$. Plagioclase $\mathrm{An}_{91.6 \pm 0.8}\left(\mathrm{An}_{90.3-93.7}, \mathrm{n}=39\right)$ 
Classification: (V. Di Cecco, $R O M$ ) Sample is an equilibrated monomict basaltic eucrite breccia. Lamellae and compositions suggest the sample has experienced thermal equilibration. Mineral chemistry suggests the sample is a basaltic eucrite.

\section{Northwest Africa 12000 (NWA 12000) \\ Morocco \\ Purchased: May 2017 \\ Classification: Iron meteorite (IIAB)}

History: Purchased by Zhang Qingyan in May 2017 from a Moroccan dealer during the China (Hunan) Mineral \& Gem Show in Chenzhou City

Physical characteristics: The main mass is approximately $2 \times 5 \times 10 \mathrm{~cm}$ with a dark brown, regmaglypted exterior. Some rust-red spots and adhered caliche are visible.

Petrography: (C. Herd) A $1 \times 2 \mathrm{~cm}$ polished and etched slice shows an equant texture of kamacite crystals at least as wide as the slice $(1 \mathrm{~cm})$ with interstitial schreibersite. The cm-scale kamacite is replaced by sub-500 $\mu \mathrm{m}$ equant crystals. Neumann lines are present.

Geochemistry: ICP-MS data, using sample of North Chile (Filomena) as standard (C. Herd and G. Chen $U A b): \mathrm{Ni}=5.7, \mathrm{Co}=0.49$ (both wt $\%$ ); $\mathrm{Cu}=123, \mathrm{Ga}=55$, $\mathrm{As}=9.5, \mathrm{Ru}=3.4, \mathrm{~W}=0.7, \mathrm{Ir}=0.03, \mathrm{Pd}=2.5, \mathrm{Pt}=4.5, \mathrm{Au}=1.1(\mathrm{all} \mu \mathrm{g} / \mathrm{g})$.

Classification: Iron, IIAB. Recrystallized texture.

Specimens: Type specimen of $24.3 \mathrm{~g}$ at $U A b$. Zhang Qingyan $103 \mathrm{~g}$, Liang Chengguang $28 \mathrm{~g}$, M. Ouzillou $69.3 \mathrm{~g}$.

\section{Northwest Africa 12001 (NWA 12001) \\ Morocco \\ Purchased: 2018 Feb \\ Classification: Ordinary chondrite (H5)}

History: Purchased from Mohammed Hmani during the Tucson Gem and Mineral show February 2018.

Physical characteristics: Stones have a brownish exterior exposing many small chondrules and some specimens are partially covered with a brownish black crust. Some specimens also display prominent metal veining in positive relief.

Petrography: (C. Herd and L. Tunney, $U A b)$ Optical and microprobe examination of a polished thin section shows well-delineated chondrules of a variety of textural types. No metal veins were observed in the specimen studied. Weathering grade according to Zurfluh et al. (2016).

Geochemistry: (C. Herd and L. Tunney, $U A b$ ) Data obtained by EMP examination of carbon-coated thin section: Olivine $\mathrm{Fa}_{18.3 \pm 0.3}(\mathrm{n}=42)$; Low-Ca Pyroxene $\mathrm{Fs}_{16.0 \pm 0.2} \mathrm{Wo}_{1.2 \pm 0.2}(\mathrm{n}=39)$.

Classification: Ordinary chondrite, H5 
Specimens: Type specimen of $71.8 \mathrm{~g}$, including one thin section, is at $U A b .3$ stones totaling $2108 \mathrm{~g}$ with M. Ouzillou, $911 \mathrm{~g}$ stone with GHupé, and 2 stones totaling $2211 \mathrm{~g}$ with D. Dickens.

\section{Northwest Africa 12002 (NWA 12002) \\ Morocco \\ Purchased: 2017 \\ Classification: Martian meteorite (Shergottite)}

History: Purchased from a France-based Moroccan dealer in 2017, via internet.

Physical characteristics: The main mass is a single, green-gray chip with adhering red-brown dust and fusion crust on one side.

Petrography: (C. Herd and L. Tunney, UAb; E. Walton and H. Jurak, MacEwan $\mathrm{U})$ Optical and microprobe examination of a polished thin section shows low-Ca pyroxene oikocrysts up to $3.5 \mathrm{~mm}$ in longest dimension enclosing equant, rounded olivine and subhedral rounded to euhedral chromite chadacrysts. Assemblages of plagioclase, low- and high-Ca pyroxene, Ti-bearing chromite comprise the nonpoikilitic areas. Shock deformation and transformation effects in host rock minerals include strong mosaicism of olivine and pyroxene, mechanical twinning in pyroxene and complete transformation of plagioclase to maskelynite. Small, isolated pockets of shock melt are heterogeneously distributed throughout the host rock, from which needles of stishovite have crystallized. Thin black veinlets of quenched shock melt cut across and displace igneous minerals.

Geochemistry: (C. Herd and L. Tunney, UAb) Data obtained by EMP examination of carbon-coated thin section: Olivine $\mathrm{Fa}_{39.7 \pm 1.5}\left(\right.$ range $\left.\mathrm{Fa}_{34.1-41.2}\right), \mathrm{FeO} / \mathrm{MnO}=51$ $(\mathrm{n}=35)$; low-Ca pyroxene $\mathrm{Fs}_{29.4 \pm 2.4} \mathrm{Wo}_{10.6 \pm 3.3}\left(\right.$ range $\left.\mathrm{Fs}_{24.6-32.8} \mathrm{Wo}_{2.8-16.6}\right), \mathrm{FeO} / \mathrm{MnO}=$ 30 (n=23); high-Ca pyroxene $\mathrm{Fs}_{18.4 \pm 1.2} \mathrm{Wo}_{33.8 \pm 2.2}$ (range $\mathrm{Fs}_{16.5-20.7} \mathrm{Wo}_{31.2-38}$ ), $\mathrm{FeO} / \mathrm{MnO}=25(\mathrm{n}=9)$; plagioclase $\mathrm{An}_{50.9 \pm 4.8} \mathrm{Or}_{2.8 \pm 1.3}\left(\right.$ range $\left.\mathrm{An}_{40.6-56.9} \mathrm{Or}_{1.4-6.2}\right)$ $(\mathrm{n}=20)$. Chromite range $\mathrm{TiO}_{2}=0.9-17 \mathrm{wt} \%(\mathrm{n}=16)$.

Classification: Martian (shergottite, poikilitic)

Specimens: Type specimen of $3.2 \mathrm{~g}$, including one thin section, at $U A b .1 \mathrm{~g}$ with M. Ouzillou.

\section{Northwest Africa 12004 (NWA 12004) \\ Morocco \\ Purchased: 2018 Mar \\ Classification: Angrite}

History: Found near Touignin in southern Morocco and purchased by Ben Hoefnagels in March 2018 from a Moroccan dealer.

Physical characteristics: A gray stone ( $183 \mathrm{~g})$ lacking fusion crust and with visible sparse vesicles.

Petrography: (A. Irving and S. Kuehner, UWS) Diabasic texture (mean grainsize $0.5 \mathrm{~mm}$ ) with sparse vesicles. Major minerals are zoned calcic olivine (with 
kirschsteinite rims), zoned Al-Ti-augite and anorthite, together with accessory titanomagnetite, troilite, silicoapatite and ferroan rhönite.

Geochemistry: Olivine $\left(\mathrm{Fa}_{36.6-84.1} \mathrm{Ln} 1.4-15.4, \mathrm{FeO} / \mathrm{MnO}=70-95, \mathrm{~N}=7\right)$, clinopyroxene $\left(\mathrm{Fs}_{20.9-46.7} \mathrm{Wo}_{52.0-52.9}, \mathrm{FeO} / \mathrm{MnO}=77-148, \mathrm{TiO}_{2}=1.4-4.2\right.$ wt.\%, $\mathrm{Al}_{2} \mathrm{O}_{3}=7.3-11.6$ wt.\%, $\left.\mathrm{N}=5\right)$, kirschsteinite $\left(\mathrm{Fa}_{62.4} \mathrm{Ln} 33.8, \mathrm{FeO} / \mathrm{MnO}=69\right)$, anorthite $\left(\mathrm{An}_{99.5-99.6} \mathrm{Ab}_{0.3} \mathrm{Or}_{0.0}, \mathrm{~N}=2\right)$. Oxygen isotopes ( $\mathrm{K}$. Ziegler, $\left.U N M\right)$ Analyses of acid-washed subsamples by laser fluorination gave, respectively $\delta^{17} \mathrm{O}$ $2.258,2.197,2.285 ; \delta^{18} \mathrm{O} 4.440,4.284,4.431 ; \Delta^{17} \mathrm{O}-0.086,-0.065,-0.055$ per mil. Classification: Angrite (diabasic).

Specimens: $22.3 \mathrm{~g}$ including one polished thin section at $U W B$; remainder with $\mathrm{Mr}$. B. Hoefnagels.

\section{Northwest Africa 12007 (NWA 12007)}

(Northwest Africa)

Purchased: 2018 Feb

Classification: Ordinary chondrite (LL6)

History: Purchased by Blaine Reed in February 2018 from a Moroccan dealer at the Tucson Gem and Mineral Show.

Petrography: (A. Irving and S. Kuehner, UWS) Mostly recrystallized with regions of hollyleaf-shaped, stained metal grains and accessory chlorapatite. Some rare chondrule remnants were observed.

Geochemistry: Olivine $\left(\mathrm{Fa}_{33.0-33.1}, \mathrm{~N}=3\right)$, orthopyroxene $\left(\mathrm{Fs}_{26.4-26.6} \mathrm{Wo}_{1.9-1.8}, \mathrm{~N}=\right.$ $3)$, clinopyroxene $\left(\mathrm{Fs}_{10.2-11.1} \mathrm{Wo}_{44.9-44.2}, \mathrm{~N}=2\right)$.

Classification: Ordinary chondrite (LL6).

Specimens: $21.2 \mathrm{~g}$ including one polished thin section at $U W B$; remainder with $\mathrm{Mr}$. B. Reed.

\section{Northwest Africa 12008 (NWA 12008) \\ Morocco \\ Purchased: 2018 Aug \\ Classification: Lunar meteorite (basalt)}

History: Purportedly found in Morocco and purchased by Ben Hoefnagels in August 2018 from a dealer in Zagora, Morocco.

Petrography: (A. Irving and S. Kuehner, UWS) Small olivine phenocrysts (stained orange-brown) are set in an intersertal groundmass composed mainly of zoned clinopyroxene and elongate lath-like maskelynite with accessory skeletal ilmenite, Ti-chromite, Cr-Ti-Fe spinel, troilite and minor barite. Olivine contains inclusions of chromite and quenched melt inclusions (surrounded by radial cracks) composed of Al-Ti-bearing augite $+\mathrm{K}$-bearing glass.

Geochemistry: Olivine (cores $\mathrm{Fa}_{36.6-42.3}$, rim $\mathrm{Fa}_{90.0}, \mathrm{FeO} / \mathrm{MnO}=96-99, \mathrm{~N}=3$ ), pigeonite $\left(\mathrm{Fs}_{31.1} \mathrm{Wo}_{14.0}, \mathrm{FeO} / \mathrm{MnO}=50\right)$, subcalcic augite $\left(\mathrm{Fs}_{25.9-71.4} \mathrm{Wo}_{28.6-23.1}\right.$, $\mathrm{FeO} / \mathrm{MnO}=50-75, \mathrm{~N}=5)$, augite $\left(\mathrm{Fs}_{23.2} \mathrm{Wo}_{39.7}, \mathrm{FeO} / \mathrm{MnO}=49\right)$, ferropigeonite 
$\left(\mathrm{Fs}_{80.1} \mathrm{Wo}_{19.5}, \mathrm{FeO} / \mathrm{MnO}=79\right)$, augite in melt inclusion $\left(\mathrm{Fs}_{28.8} \mathrm{Wo}_{50.1}, \mathrm{FeO} / \mathrm{MnO}=\right.$ 71, $\mathrm{Al}_{2} \mathrm{O}_{3}=10.5$ wt.\%, $\left.\mathrm{TiO}_{2}=5.3 \mathrm{wt} . \%\right)$, plagioclase $\left(\mathrm{An}_{86.8-87.6} \mathrm{Or}_{0.4-0.3}, \mathrm{~N}=2\right)$. Classification: Lunar (mare basalt).

Specimens: $25.2 \mathrm{~g}$ including two polished thin sections at $U W B$; remainder with Mr. B. Hoefnagels.

\section{Northwest Africa 12009 (NWA 12009)}

(Northwest Africa)

Purchased: 2017 Jun

Classification: Ordinary chondrite (LL3)

History: Purchased by Marc Jost in June 2017 from a Moroccan dealer at the Ensisheim Show.

Petrography: (A. Irving and S. Kuehner, UWS) Well-formed, fairly closely packed chondrules (apparent diameter $950 \pm 610 \mu \mathrm{m}, \mathrm{N}=24$ ) are set in relatively fine grained matrix containing altered metal, merrillite and chlorapatite.

Geochemistry: Olivine $\left(\mathrm{Fa}_{1.2-98.0}, \mathrm{Cr}_{2} \mathrm{O}_{3}\right.$ in ferroan examples $0.09-0.18$ wt.\%, mean $0.1 \pm 0.04$ wt. $\%, \mathrm{~N}=7$ ), orthopyroxene $\left(\mathrm{Fs}_{2.1-17.0} \mathrm{Wo}_{0.4-2.4}, \mathrm{~N}=3\right)$, subcalcic augite $\left(\mathrm{Fs}_{47.1} \mathrm{Wo}_{31.2}\right)$, augite $\left(\mathrm{Fs}_{9.4} \mathrm{Wo}_{42.5}\right)$. Magnetic susceptibility $\log \chi\left(\times 10^{-9} \mathrm{~m}^{3} / \mathrm{kg}\right)=$ 3.61 .

Classification: Ordinary chondrite (LL3).

Specimens: $22.7 \mathrm{~g}$ including one polished thin section at $U W B$; remainder held by SJS.

\section{Northwest Africa 12010 (NWA 12010)}

(Northwest Africa)

Purchased: 2018 Apr

Classification: Ordinary chondrite (H6)

History: Purchased by Martin Goff in April 2018 from a Moroccan dealer.

Petrography: (A. Irving and S. Kuehner, UWS) Mostly recrystallized with rare chondrule remnants. Accessory minerals include sodic plagioclase, stained kamacite, chromite, merrillite, chlorapatite and troilite.

Geochemistry: Olivine $\left(\mathrm{Fa}_{19.5-19.8}, \mathrm{~N}=3\right)$, orthopyroxene $\left(\mathrm{Fs}_{16.7-17.4} \mathrm{Wo}_{1.5-1.3}, \mathrm{~N}=\right.$ 3), clinopyroxene $\left(\mathrm{Fs}_{5.6-6.7} \mathrm{Wo}_{46.4-45.2}, \mathrm{~N}=2\right)$.

Classification: Ordinary chondrite (H6).

Specimens: $20.1 \mathrm{~g}$ including one polished thin section at $U W B$; remainder with $\mathrm{Mr}$. M. Goff.

\section{Northwest Africa 12011 (NWA 12011)}

(Northwest Africa)

Purchased: 2018 Jan

Classification: HED achondrite (Eucrite, monomict) 
History: Purchased by Darryl Pitt on behalf of the Maine Mineral and Gem Museum in January 2018 from a Moroccan dealer at the Tucson Gem and Mineral Show.

Petrography: (A. Irving and S. Kuehner, UWS) Fresh breccia composed of gabbroic eucrite clasts (grainsize up to $0.8 \mathrm{~mm}$ ) and related mneral debris. Minerals are exsolved pigeonite and calcic plagioclase with accessory silica polymorph, ilmenite, Ti-chromite, troilite and baddeleyite.

Geochemistry: Orthopyroxene host $\left(\mathrm{Fs}_{59.0-59.4} \mathrm{Wo}_{1.8-1.5}, \mathrm{FeO} / \mathrm{MnO}=31-32, \mathrm{~N}=3\right)$, clinopyroxene exsolution lamellae $\left(\mathrm{Fs}_{24.9-26.3} \mathrm{Wo}_{43.3-42.0}, \mathrm{FeO} / \mathrm{MnO}=28-29, \mathrm{~N}=2\right)$, plagioclase $\left(\mathrm{An}_{91.8-92.0} \mathrm{Or}_{0.4-0.3}, \mathrm{~N}=2\right)$.

Classification: Eucrite (monomict gabbroic breccia).

Specimens: $20.2 \mathrm{~g}$ including one polished thin section at $U W B$; remainder at $M M G M$.

\section{Northwest Africa 12013 (NWA 12013)}

(Northwest Africa)

Purchased: 2018

Classification: Carbonaceous chondrite (CV3)

History: A single stone weighing $332.1 \mathrm{~g}$ was found in Morocco and subsequently purchased by Blaine Reed at the 2018 Tucson Gem and Mineral Show.

Physical characteristics: The stone is dark-orangish-brown in color, has a flattened ovoid shape, lacks fusion crust and has a weathered exterior with some chondrules protruding from the surface. The cut face showing the interior of the stone is dark greenish-brown and shows readily discernible dark-colored chondrules and irregular to circular dark-gray metallic grains.

Petrography: Description and classification (Anthony Love, App): Sample contains distinct chondrules (average diameter of $940 \mu \mathrm{m}(\mathrm{N}=24)$, fragments and refractory inclusions (CAIs up to $3 \mathrm{~mm}$ ) all set within an opaque dark brown matrix. Matrix contains FeO-rich olivine, andradite, magnetite framboids and one prominent type B dark inclusion. Type I olivine chondrules are dominant (some of which contain isotropic glass). Sample has $\sim 50 \%$ matrix.

Geochemistry: (A.Love-App) Type I chondrules $\mathrm{Fa}_{3.2 \pm 2.5}\left(\mathrm{Fa}_{0.4-8.2}\right)$, $\mathrm{Fe} / \mathrm{Mn}=37.7 \pm 27.3 \mathrm{n}=17$; Type II chondrules $\mathrm{Fa}_{11.14}, \mathrm{n}=1$ low-Ca pyroxene $\mathrm{Fs}_{1.1 \pm 0.3} \mathrm{Wo}_{1.8 \pm 1.8}\left(\mathrm{Fs}_{0.7-1.5} \mathrm{Wo}_{0.7-5.0}\right), \mathrm{n}=4$; high Ca pyroxene $\mathrm{Fs}_{1.4} \mathrm{Wo}_{26.9}, \mathrm{n}=1$.

Classification: Carbonaceous Chondrite (CV3, S2, high weathering)

Specimens: Blaine Reed holds the main mass. Two sliced fragments weighing $42.38 \mathrm{~g}$ and one polished thin section are on deposit at App.

Northwest Africa 12014 (NWA 12014)

(Northwest Africa)

Purchased: 2017

Classification: Ordinary chondrite (H6)

History: Purchased by Christian Anderkin in 2017. 
Physical characteristics: The sample is a single $43 \mathrm{~g}$ stone with visible weathered fusion crust. There are several weak fractures that span the minor axis of the stone. Petrography: Sample is highly shocked, with no discernable chondrules and many dark shock melt veins. Matrix contains veins of sulfides and oxides amongst small grains of pyroxene and olivine. Plagioclase grains are approximately $55 \mu \mathrm{m}$ across $(\mathrm{N}=2)$.

Geochemistry: Olivine, $\mathrm{Fa}_{17.8-21.8}, \mathrm{~N}=15$, mean $\mathrm{Fa}_{19.6}, \mathrm{~N}=15$; orthopyroxene, $\mathrm{Fs}_{16.8-22.4}$, mean $18.8, \mathrm{Wo}_{1.2-3.7}$, mean 1.9

Classification: $\mathrm{H} 6$

Specimens: $26.9 \mathrm{~g}$ at UCLA. Remainder of mass with C. Anderkin at UF.

\section{Northwest Africa 12015 (NWA 12015) \\ (Northwest Africa) \\ Purchased: 2007 Feb \\ Classification: Ordinary chondrite (LL6)}

Petrography: Chondrules are relatively difficult to recognize. The matrix is mostly recrystilized with fine secondary grains. Weak mosaicism is seen in parts with undulatory extinction. Pervasive shock veins are seen with melt pockets. Minor oxidation is taking place in the metals. (S. Yokoyama, ROM).

\section{Northwest Africa 12016 (NWA 12016) \\ (Northwest Africa) \\ Purchased: 2005 Feb \\ Classification: Ordinary chondrite (LL6)}

Petrography: Very few chondrules are visible. Minor opaque and mostly recrystalized fine grained matrix surrounds chondrule fragments. A series of large dark patches with fine grained crystals are seen. Minor mosaicism is seen in some pyroxenes and undulatory extinction is present in olivines. Opaque shock veins are also seen. $<3 \%$ of oxidation has affected metal. (S. Yokoyama, ROM).

\section{Northwest Africa 12017 (NWA 12017) \\ (Northwest Africa) \\ Purchased: 2008 Feb \\ Classification: Ordinary chondrite (LL4)}

Petrography: Well defined chondrules are seen in the slide surrounded by some opaque and clastic matrix. Minor undulatory extinction is seen with some mosaicism. Shock veins also seen in parts. $>60 \%$ of metal has been affected by oxidation from weathering. (S. Yokoyama, ROM).

Northwest Africa 12018 (NWA 12018)

(Northwest Africa) 
Purchased: 2012 Feb

Classification: Ordinary chondrite (H6)

Petrography: Sample is divided into darker and lighter clasts throughout the slide. Chondrules are difficult to detect as not many of them are intact. A fine grained matrix is observed. Although maskelynite is not observed, undulatory extinction and mosaicism are quite common. Interconnected shock veins are also seen. Not many metal grains are affected by oxidation. However, pervasive and interconnected oxide veins are seen throughout the slide. (S. Yokoyama, ROM).

\section{Northwest Africa 12019 (NWA 12019)}

(Northwest Africa)

Purchased: 2012

Classification: Ordinary chondrite (H4)

Petrography: Some patches of dark coexist with the brown. Chondrules are well defined in a mainly recrystalized matrix. Some opaque shock veins and undulatory extinctions are seen while sharp optial extinctions are still common in many olivine grains. Between 50 and $60 \%$ of $\mathrm{Ni}-\mathrm{Fe}$ metal has been replaced by oxidation. Oxide rims are common as well as oxidation veins. (S. Yokoyama, ROM).

\section{Northwest Africa 12020 (NWA 12020) \\ (Northwest Africa) \\ Purchased: 2017 Dec \\ Classification: Ordinary chondrite (LL6)}

Petrography: (K. Metzler, IfP) Monomict breccia with recrystallized texture, showing some relict BO chondrules. Most secondary feldspar grains are $>50 \mu \mathrm{m}$. Melt pockets and melt veins are present.

Geochemistry: Mineral compositions and geochemistry: The mean olivine composition is $\mathrm{Fa}_{31.3 \pm 0.4}\left(\mathrm{Fa}_{30.7-32.0}\right)$; $\mathrm{n}=13$. The mean low-Ca pyroxene composition is $\mathrm{Fs}_{24.9 \pm 0.4} \mathrm{Wo}_{1.6 \pm 0.1}\left(\mathrm{Fs}_{24.2-25.6} \mathrm{Wo}_{1.3-1.8}\right), \mathrm{n}=13$.

Classification: LL chondrite based on mineral chemistry. Petrologic type 6 based on the equilibrated state of olivine and pyroxene and grain size of secondary plagioclase

\section{Northwest Africa 12180 (NWA 12180)}

(Northwest Africa)

Purchased: 2018

Classification: Carbonaceous chondrite (CV3)

History: The meteorite was bought from a local meteorite dealer in Morocco. Physical characteristics: Dark grayish-greenish individual lacking any fusion crust.

Petrography: Carbonaceous chondrite composed of chondrules (mean diameter about $1 \mathrm{~mm}$; up to $2.5 \mathrm{~mm}$ sized), CAIs (up to $5 \mathrm{~mm}$ sized), and olivine amoeboids 
all set into a fine-grained brownish to almost black matrix. Type II chondrules are absent.

\section{Northwest Africa 12181 (NWA 12181)}

(Northwest Africa)

Purchased: 2018

Classification: Ordinary chondrite (L4, melt breccia)

History: The meteorite was bought from a local meteorite dealer in Morocco.

Physical characteristics: Dark brownish individual lacking any fusion crust.

Petrography: The meteorite is a breccia composed of large regions of recrystallized shock melt containing characteristic FeNi metal and sulfide globules and regions displaying chondritic textures with well preserved chondrules. In chondritic portions olivine is compositionally equilibrated and pyroxene is unequilibrated.

\section{Northwest Africa 12182 (NWA 12182) \\ (Northwest Africa) \\ Purchased: 2017 \\ Classification: HED achondrite (Eucrite, brecciated) \\ History: The meteorite was bought from a local meteorite dealer in Tagant, Mauritania.}

Physical characteristics: Grayish individual partly covered with fusion crust. Petrography: The meteorite is a fine-grained basaltic breccia composed of up to 2 $\mathrm{mm}$ sized basaltic clasts and up to $3.5 \mathrm{~mm}$ sized impact melt clasts set into a basaltic matrix. Mineral phases are predominantly up to $400 \mu \mathrm{m}$ sized pyroxene and lath shaped calcic plagioclase. Pyroxene often shows magmatic zoning attesting to a low degree of thermal metamorphism. Minor phases include silica, ilmenite, chromite, and troilite. No metallic iron has been detected.

Geochemistry: low-Ca pyroxene: $\mathrm{Fs}_{42.3 \pm 8.3} \mathrm{Wo}_{4.1 \pm 1.4}\left(\mathrm{Fs}_{30.5-54.4} \mathrm{Wo}_{1.9-6.3}, \mathrm{n}=11\right.$, $\mathrm{FeO} / \mathrm{MnO}=28-39)$; Ca-pyroxene: $\mathrm{Fs}_{29.6 \pm 2.2} \mathrm{Wo}_{37.5 \pm 5.9}\left(\mathrm{Fs}_{25.8-34.4} \mathrm{Wo}_{23.1-41.6}, \mathrm{n}=10\right.$, $\mathrm{FeO} / \mathrm{MnO}=29-36)$; calcic plagioclase: $\mathrm{An}_{88.8 \pm 4.0}\left(\mathrm{An}_{81.0-93.4}, \mathrm{n}=15\right)$

\section{Northwest Africa 12183 (NWA 12183)}

(Northwest Africa)

Purchased: 2018

Classification: Ureilite

History: The meteorite was bought from a local meteorite dealer in Morocco.

Physical characteristics: Dark-brownish individual lacking any fusion crust.

Petrography: The meteorite shows a characteristic cumulate texture of up to $1 \mathrm{~mm}$ sized olivine and pigeonite grains. Olivine is enriched in $\mathrm{Cr}_{2} \mathrm{O}_{3}$ and displays typical reduced rims. The meteorite contains abundant flaky graphite. 
Geochemistry: reduced rims in olivine: $\mathrm{Fa}_{5.1-11.0}$; olivine contains $0.67 \pm 0.18 \mathrm{wt} \%$ $\mathrm{Cr}_{2} \mathrm{O}_{3}$ and $0.31 \pm 0.02 \mathrm{wt} \% \mathrm{CaO}$

\author{
Northwest Africa 12184 (NWA 12184) \\ (Northwest Africa) \\ Purchased: 2018 \\ Classification: Carbonaceous chondrite (CM2)
}

History: The meteorite was bought from a local meteorite dealer in Algeria.

Physical characteristics: Almost black individual partly covered with fusion crust. Petrography: Brecciated carbonaceous chondrite composed of chondrules (mean diameter about $0.3 \mathrm{~mm}$ ), chondrule pseudomorphs, CAIs, and mineral fragments set into abundant Fe-rich matrix. Many of the components are surrounded by finegrained dust rims. The fine-grained almost black meteorite matrix consists of abundant Ca-carbonates, sulfides and phyllosilicates.

\title{
Northwest Africa 12185 (NWA 12185)
}

(Northwest Africa)

Purchased: 2009

Classification: Ordinary chondrite (LL3)

History: The meteorite was bought from a local meteorite dealer in Morocco. Physical characteristics: Brownish individual partly covered with fusion crust. Petrography: The meteorite shows a chondritic texture of densely packed and partly flattened chondrules, with an apparent mean diameter of about $0.9 \mathrm{~mm}$; low FeNi metal content and low magnetic susceptibility.

\section{Northwest Africa 12186 (NWA 12186)}

(Northwest Africa)

Purchased: 2018

Classification: Pallasite

History: The meteorite was bought from a local meteorite dealer in Algeria. Physical characteristics: Brownish individual lacking any fusion crust.

Petrography: The meteorite is composed of typically $5 \mathrm{~mm}$ sized brownish olivine set into abundant and well preserved FeNi metal matrix. Ni-poor regions contain about $5 \mathrm{wt} \%$ and Ni-rich regions up to $31.6 \mathrm{wt} \% \mathrm{Ni}$.

\section{Northwest Africa 12187 (NWA 12187)}

(Northwest Africa)

Purchased: 2018

Classification: Carbonaceous chondrite (CV3)

History: The meteorite was bought from a local meteorite dealer in Morocco.

Physical characteristics: Dark brownish individual lacking any fusion crust. 
Petrography: Strongly weathered carbonaceous chondrite composed of chondrules (mean diameter about $0.8 \mathrm{~mm}$; up to $2 \mathrm{~mm}$ sized), CAIs, and olivine amoeboids all set into a fine-grained dark brownish matrix. Type II chondrules are absent. Nearly all chondrules show reddish staining due to terrestrial alteration.

\section{Northwest Africa 12188 (NWA 12188) \\ Mauritania \\ Purchased: May 2018 \\ Classification: Ureilite}

History: Found in the Adrar region of Mauritania, February 2018. Purchased by Darryl Pitt in Mauritania, May 2018.

Physical characteristics: Single stone; sandblasted exterior; saw cut reveals an interior mosaic of light- and dark-gray crystals; minor amount iron-staining present. Petrography: (C. Agee, UNM) This meteorite consists of olivine, pigeonite, and augite. Ubiquitous Fe-metal is present along grain boundaries, small $(<100 \mu \mathrm{m})$ metal blebs are scattered throughout, and there are numerous Fe-metal/oxide veinlets crosscutting the silicate grains. Silicates are highly equilibrated both compositionally and texturally, no oxidation reduction rims observed on olivine grains. No graphite or diamond was observed.

Geochemistry: (C. Agee, $U N M$ ) Olivine $\mathrm{Fa}_{12.1 \pm 0.1}, \mathrm{Fe} / \mathrm{Mn}=21 \pm 1, \mathrm{Cr}_{2} \mathrm{O}_{3}=0.50 \pm 0.01$ $(\mathrm{wt} \%), \mathrm{n}=6$; pigeonite $\mathrm{Fs}_{11.1 \pm 1.6} \mathrm{Wo}_{4.5 \pm 0.1}, \mathrm{Fe} / \mathrm{Mn}=15 \pm 1, \mathrm{n}=2$; augite

$\mathrm{Fs}_{6.0 \pm 0.2} \mathrm{Wo}_{36.5 \pm 0.5}, \mathrm{Fe} / \mathrm{Mn}=10 \pm 0, \mathrm{n}=2$.

Classification: Augite-bearing ureilite

Specimens: $22.2 \mathrm{~g}$ including a probe mount on deposit at UNM, DPitt holds the main mass.

Northwest Africa 12189 (NWA 12189)

Algeria

Purchased: May 2018

Classification: Ureilite

History: Found near Tindouf, February 2018. Purchased by Darryl Pitt in Mauritania, May 2018.

Physical characteristics: Single stone; irregular, sandblasted exterior; saw cut reveals an interior mosaic of light and dark gray-brown crystals.

Petrography: (C. Agee, $U N M$ ) This meteorite consists of olivine, pigeonite, graphite and Fe-metal. Ubiquitous, finely disseminated Fe-metal is occupies zones between silicate grain boundaries. Elongate graphite grains up to $1 \mathrm{~mm}$ are present throughout. Significant oxidation reduction rims observed on olivine grains with compositions of $\sim \mathrm{Fa}_{08}$.

Geochemistry: (C. Agee, $U N M$ ) Olivine $\mathrm{Fa}_{21.5 \pm 0.1}, \mathrm{Fe} / \mathrm{Mn}=47 \pm 4, \mathrm{Cr}_{2} \mathrm{O}_{3}=0.54 \pm 0.10$ (wt\%), $\mathrm{n}=5$; pigeonite $\mathrm{Fs}_{17.8 \pm 0.1} \mathrm{Wo}_{4.8 \pm 0.8}, \mathrm{Fe} / \mathrm{Mn}=30 \pm 1, \mathrm{n}=6$.

Classification: Ureilite, graphite-bearing. 
Specimens: $21.1 \mathrm{~g}$ including a probe mount on deposit at $U N M, D P i t t$ holds the main mass.

\section{Northwest Africa 12190 (NWA 12190) \\ Western Sahara \\ Purchased: May 2018 \\ Classification: HED achondrite (Howardite)}

History: Found in Gdeim Ashham, Western Sahara, February 2018. Purchased by Darryl Pitt in Mauritania, May 2018.

Physical characteristics: Single stone; sandblasted exterior; saw cut reveals a brecciated interior with numerous feldspar, pyroxene, and lithic clasts; significant domains of recrystallized shock melt are present.

Petrography: (C. Agee, UNM) This meteorite is a polymict breccia consisting of approximately $50 \%$ diogenite material and $50 \%$ cumulate eucrite material.

Geochemistry: (C. Agee, UNM) Diogenite mineralogy: olivine $\mathrm{Fa}_{45.7}, \mathrm{Fe} / \mathrm{Mn}=49$, $\mathrm{n}=1$, low-Ca pyroxene $25.4 \pm 3.3 \mathrm{Wo}_{2.6 \pm 1.0}, \mathrm{Fe} / \mathrm{Mn}=30 \pm 1, \mathrm{n}=5$; eucrite mineralogy: low-Ca pyroxene $47.8 \pm 4.7 \mathrm{Wo}_{8.7 \pm 9.0}, \mathrm{Fe} / \mathrm{Mn}=31 \pm 1, \mathrm{n}=5$; plagioclase $\mathrm{An}_{92.9 \pm 3.4}$. Classification: Howardite Specimens: $25.3 \mathrm{~g}$ including a probe mount on deposit at UNM, DPitt holds the main mass.

\section{Northwest Africa 12191 (NWA 12191) \\ Western Sahara \\ Purchased: May 2018 \\ Classification: Ureilite}

History: Found in Agmamin region, Western Sahara, February 2018. Purchased by Darryl Pitt in Mauritania, May 2018.

Physical characteristics: Single stone; irregular, weatherd exterior; saw cut reveals an interior mosaic of light-brown and dark-gray crystals.

Petrography: (C. Agee, $U N M$ ) This meteorite consists of olivine, pigeonite, and $\mathrm{Fe}-\mathrm{metal}$. Ubiquitous, finely disseminated Fe-metal is occupies zones between silicate grain boundaries. A carbon polymorph (diamond or graphite) is present. Geochemistry: (C. Agee, $U N M$ ) Olivine $\mathrm{Fa}_{15.4 \pm 0.1}, \mathrm{Fe} / \mathrm{Mn}=36 \pm 1, \mathrm{Cr}_{2} \mathrm{O}_{3}=0.59 \pm 0.01$ (wt\%), $\mathrm{n}=6$; pigeonite $\mathrm{Fs}_{9.8 \pm 2.1} \mathrm{Wo}_{7.0 \pm 1.8}, \mathrm{Fe} / \mathrm{Mn}=14 \pm 4, \mathrm{n}=6$.

Classification: Ureilite Specimens: $20.1 \mathrm{~g}$ including a probe mount on deposit at $U N M, D P i t t$ holds the main mass.

\section{Northwest Africa 12192 (NWA 12192)}

Algeria

Purchased: May 2018

Classification: Carbonaceous chondrite (CK5) 
History: Found north of Tindouf, Algeria, January 2018. Purchased by Darryl Pitt in Mauritania, May 2018.

Physical characteristics: Single stone with irregular weathered surface, saw cut reveals distinct chondrules up to $1 \mathrm{~mm}$ in diameter set in a dark-gray groundmass. Petrography: (C. Agee, UNM) Microprobe examination shows scattered, distinct, texturally equilibrated chondrules set in a fine-grained matrix. Plagioclase grain size up to $50 \mu \mathrm{m}$ in diameter. Abundant chromium-rich magnetite observed throughout, some up to $300 \mu \mathrm{m}$ in diameter.

Geochemistry: (C. Agee, $U N M$ ) Olivine $\mathrm{Fa}_{29.9 \pm 1.0}, \mathrm{Fe} / \mathrm{Mn}=104 \pm 8, \mathrm{NiO}=0.30 \pm .03$ $\left(\mathrm{wt} \%\right.$ ), $\mathrm{n}=13$; low-Ca pyroxene $\mathrm{Fs}_{25.4 \pm 1.0} \mathrm{Wo}_{0.7 \pm 0.0}, \mathrm{Fe} / \mathrm{Mn}=66 \pm 6, \mathrm{n}=6$; plagioclase $\mathrm{Ab}_{69.2 \pm 3.3} \mathrm{An}_{22.1 \pm 6.9} \mathrm{Or}_{8.7 \pm 8.8}, \mathrm{n}=5$.

Classification: Carbonaceous chondrite (CK5) CK chondrite based Fe/Mn of olivine and pyroxenes, and on the presence of chromium-rich magnetite. Petrologic type 5 based on the equilibrated state of olivine and pyroxene, distinct chondrules, and secondary plagioclase size.

Specimens: $9 \mathrm{~g}$ on deposit at UNM, DPitt holds the main mass.

\section{Northwest Africa 12193 (NWA 12193)}

Western Sahara

Purchased: May 2018

Classification: HED achondrite (Eucrite, polymict)

History: Found in Gdeim Asham, Western Sahara, February 2018. Purchased by Darryl Pitt in Mauritania, May 2018.

Physical characteristics: Single stone, with weathered exterior. A saw cut reveals a fragmental breccia of lithic clasts set in a light colored, fine-grained groundmass. Petrography: (C. Agee) Microprobe examination of a polished mount shows a mixture of unequilibrated, zoned pyroxenes and equilibrated pyroxenes with exsolution lamellae, leading to the conclusion that there are at least two distinct eucrite lithologies present.

Geochemistry: (C. Agee, UNM) Pyroxene $\mathrm{Fs}_{48.1 \pm 14.0} \mathrm{Wo}_{10.7 \pm 7.3}, \mathrm{Fe} / \mathrm{Mn}=31 \pm 5, \mathrm{n}=13$;

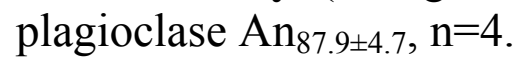

Classification: Polymict eucrite Specimens: $21.9 \mathrm{~g}$ including a probe mounts on deposit at UNM, DPitt holds the main mass.

\section{Northwest Africa 12194 (NWA 12194)}

Algeria

Purchased: May 2018

Classification: HED achondrite (Howardite)

History: Found near Oum El Assel, Algeria, September 2017. Purchased by Darryl Pitt in Mauritania, May 2018.

Physical characteristics: Single stone, with weathered exterior. A saw cut reveals a very dark-gray surface with faint appearing clasts and mineral fragments. 
Petrography: (C. Agee) Microprobe examination of a polished mount shows a fragmental breccia of pyroxene, plagioclase, and lithic clasts. The meteorite consists of approximately $33 \%$ diogenite, $33 \%$ cumulate eucrite, and $33 \%$ basaltic material.

Geochemistry: (C. Agee, $U N M$ ) Pyroxene $\mathrm{Fs}_{38.6 \pm 13.9} \mathrm{Wo}_{7.5 \pm 9.6}, \mathrm{Fe} / \mathrm{Mn}=30 \pm 2, \mathrm{n}=12$; plagioclase $\mathrm{An}_{88.8 \pm 1.3}, \mathrm{n}=5$. Diogenite pyroxenes $\mathrm{Fs}_{25.1 \pm 4.8} \mathrm{Wo}_{3.5 \pm 1.5}, \mathrm{n}=4$.

Classification: Howardite

Specimens: $22.8 \mathrm{~g}$ including a probe mounts on deposit at UNM, DPitt holds the main mass.

\section{Northwest Africa 12195 (NWA 12195)}

Algeria

Purchased: May 2018

Classification: HED achondrite (Eucrite, polymict)

History: Found north of Tindouf, Algeria, February 2018. Purchased by Darryl Pitt in Mauritania, May 2018.

Physical characteristics: Single stone, partial dark fusion-crusted exterior. Saw cut reveals a light-gray fragmental breccia.

Petrography: (C. Agee) Microprobe examination of a polished mount shows a mixture of unequilibrated, zoned pyroxenes with a population of equilibrated pyroxenes with exsolution lamellae and a clear compositional separation between low-Ca and high-Ca pyroxenes, leading to the conclusion that there are at least two distinct eucrite lithologies present.

Geochemistry: (C. Agee, $U N M$ ) Pyroxene $\mathrm{Fs}_{42.6 \pm 11.4} \mathrm{Wo}_{9.8 \pm 9.1}, \mathrm{Fe} / \mathrm{Mn}=30 \pm 2, \mathrm{n}=12$; plagioclase $\mathrm{An}_{87.1 \pm 3.5}, \mathrm{n}=6$.

Classification: Polymict eucrite

Specimens: $12.5 \mathrm{~g}$ including a probe mounts on deposit at $U N M$, DPitt holds the main mass.

\section{Northwest Africa 12196 (NWA 12196)}

Western Sahara

Purchased: May 2018

Classification: Carbonaceous chondrite (CV3)

History: Found near the Algerian-Western Sahara border region, January 2018. Purchased by Darryl Pitt in Mauritania, May 2018.

Physical characteristics: Single stone, weathered exterior. A saw cut reveals numerous distinct chondrules up to $3 \mathrm{~mm}$ in diameter and some CAIs up to $5 \mathrm{~mm}$ in size. Gray colored groundmass makes up about $40 \%$ of this meteorite.

Petrography: (C. Agee, $U N M$ ) Microprobe examination showed many porphyritic chondrules most of which were type I. Abundant fine grained matrix throughout.

Geochemistry: (C. Agee, $U N M$ ) Olivine $\mathrm{Fa}_{4.4 \pm 4.1}, \mathrm{n}=16$; enstatite $\mathrm{Fs}_{1.1 \pm 0.3} \mathrm{Wo}_{0.9 \pm 0.2}$, $\mathrm{n}=8$.

Classification: Carbonaceous chondrite (CV3) 
Specimens: $21.2 \mathrm{~g}$ on deposit at UNM, DPitt holds the main mass.

\author{
Northwest Africa 12197 (NWA 12197) \\ Western Sahara \\ Purchased: May 2018 \\ Classification: Carbonaceous chondrite (CR2)
}

History: Found near the Algerian-Western Sahara border region, March 2018. Purchased by Darryl Pitt in Mauritania, May 2018.

Physical characteristics: Single stone, weathered exterior. A saw cut reveals numerous chondrules up to $1 \mathrm{~mm}$ in diameter many with irregular or decorated rims, and sparse CAIs, but one observed was $\sim 5 \mathrm{~mm}$ in size. Dark, gray-colored groundmass makes up about $30 \%$ of this meteorite, ubiquitous opaques visible.

Petrography: (C. Agee, UNM) Microprobe examination showed many porphyritic chondrules most of which were type I. Many chondrules are metal-rich. Abundant fine grained, hydrous matrix throughout.

Geochemistry: (C. Agee, UNM) Olivine $\mathrm{Fa}_{3.3 \pm 3.3}, \mathrm{Cr}_{2} \mathrm{O}_{3}=0.20 \pm 0.11$ (wt\%), $\mathrm{n}=21$; pyroxene $\mathrm{Fs}_{1.3 \pm 0.4} \mathrm{Wo}_{1.9 \pm 1.3}, \mathrm{n}=10$.

Classification: Carbonaceous chondrite (CR2)

Specimens: $22.3 \mathrm{~g}$ on deposit at UNM, DPitt holds the main mass.

\title{
Northwest Africa 12198 (NWA 12198) \\ Western Sahara \\ Purchased: May 2018 \\ Classification: Ureilite
}

History: Found in Western Sahara, April 2018. Purchased by Darryl Pitt in Mauritania, May 2018.

Physical characteristics: Single stone; irregular, weathered exterior; saw cut reveals an interior mosaic of light brown and dark gray crystals.

Petrography: (C. Agee, $U N M)$ This meteorite consists of olivine, pigeonite, and Fe-metal. Ubiquitous, finely disseminated Fe-metal is occupies zones between silicate grain boundaries. Graphite observed.

Geochemistry: (C. Agee, $U N M$ ) Olivine $\mathrm{Fa}_{21.6 \pm 0.3}, \mathrm{Fe} / \mathrm{Mn}=50 \pm 3, \mathrm{Cr}_{2} \mathrm{O}_{3}=0.49 \pm 0.27$ (wt\%), n=6; pigeonite $\mathrm{Fs}_{9.8 \pm 3.0} \mathrm{Wo}_{9.8 \pm 3.0}, \mathrm{Fe} / \mathrm{Mn}=18 \pm 3, \mathrm{n}=5$.

Classification: Ureilite

Specimens: $20 \mathrm{~g}$ including a probe mount on deposit at UNM, DPitt holds the main mass.

\author{
Northwest Africa 12199 (NWA 12199) \\ Western Sahara \\ Purchased: May 2018 \\ Classification: Primitive achondrite (Acapulcoite)
}


History: Found near the Western Sahara-Mauritania border region, February 2018. Purchased by Darryl Pitt in Mauritania, May 2018.

Physical characteristics: Two identically looking stones with weathered exterior. Saw cut reveals an interior fine-grained mosaic of light-brown and dark-gray crystals.

Petrography: (C. Agee, $U N M$ ) Recrystallized texture with triple junctions. Grain size about $250 \mu \mathrm{m}$. This meteorite consists of olivine, low-Ca pyroxene, and $\mathrm{Fe}-$ metal. No plagioclase detected.

Geochemistry: (C. Agee, $U N M$ ) Olivine $\mathrm{Fa}_{12.0 \pm 0.1}, \mathrm{Fe} / \mathrm{Mn}=22 \pm 1, \mathrm{Cr}_{2} \mathrm{O}_{3}=0.50 \pm 0.01$ (wt\%), n=6; low-Ca pyroxene $\mathrm{Fs}_{10.4 \pm 0.1} \mathrm{Wo}_{4.5 \pm 0.1}, \mathrm{Fe} / \mathrm{Mn}=14 \pm 0, \mathrm{n}=7$.

Classification: Acapulcoite

Specimens: $21.1 \mathrm{~g}$ including a probe mount on deposit at $U N M, D P i t t$ holds the main mass.

\section{Northwest Africa 12200 (NWA 12200)}

Mauritania

Purchased: May 2018

Classification: Carbonaceous chondrite (CK6)

History: Found in the Adrar Region, Mauritania, March 2018. Purchased by Darryl Pitt in Mauritania, May 2018.

Physical characteristics: Single stone with irregular weathered surface. A saw cut reveals numerous faint chondrules up to $2 \mathrm{~mm}$ in diameter set in a dark-gray groundmass. Orange-reddish oxidation staining is visible.

Petrography: (C. Agee, $U N M$ ) Microprobe examination shows recrystallized relict texturally equilibrated chondrules set in a recrystallized matrix. Plagioclase grain size up to $300 \mu \mathrm{m}$ in length. Abundant chromium-rich magnetite observed throughout, some up to $200 \mu \mathrm{m}$ in size. Only trace amounts of pyroxene are present. Melt pockets observed.

Geochemistry: (C. Agee, $U N M$ ) Olivine $\mathrm{Fa}_{27.2 \pm 0.9}, \mathrm{Fe} / \mathrm{Mn}=89 \pm 7, \mathrm{NiO}=0.49 \pm 0.11$, $\mathrm{n}=13$; melt $\mathrm{SiO}_{2}=34.1 \pm 1.9, \mathrm{TiO}_{2}+0.15 \pm 0.02, \mathrm{Al}_{2} \mathrm{O}_{3}=5.7 \pm 1.9, \mathrm{Cr} 2 \mathrm{O} 2=0.63 \pm 0.11$, $\mathrm{MgO}=21.5 \pm 1.0, \mathrm{FeO}=26.6 \pm 3.3, \mathrm{MnO}=0.21 \pm 0.03, \mathrm{CaO}=5.3 \pm 0.3, \mathrm{NiO}=0.34 \pm 0.05$, $\mathrm{Na}_{2} \mathrm{O}=0.65 \pm 0.23, \mathrm{~K}_{2} \mathrm{O}=0.08 \pm 0.00, \mathrm{Fe} / \mathrm{Mn}=128 \pm 10, \mathrm{n}=3$; plagioclase

$\mathrm{Ab}_{52.0 \pm 4.3} \mathrm{An}_{41.6 \pm 7.8} \mathrm{Or}_{6.4 \pm 5.6}, \mathrm{n}=6$.

Classification: Carbonaceous chondrite (CK6)

Specimens: $15.9 \mathrm{~g}$ on deposit at $U N M, D P i t t$ holds the main mass.

\section{Northwest Africa 12201 (NWA 12201)}

Western Sahara

Purchased: May 2018

Classification: HED achondrite (Eucrite, unbrecciated)

History: Found in Western Sahara, March 2018. Purchased by Darryl Pitt in Mauritania, May 2018. 
Physical characteristics: Single stone, weathered exterior. Saw cut reveals a dark gray surface with plagioclase and pyroxene crystals.

Petrography: (C. Agee) Microprobe examination of a polished mount shows a mixture of approximately $50 \%$ pyroxene and $40 \%$ plagioclase. Pyroxenes show a clear compositional separation of low-Ca and high-Ca pyroxene typical of equilibrated basaltic eucrites. No brecciation observed.

Geochemistry: (C. Agee, $U N M$ ) Low-Ca pyroxene $\mathrm{Fs}_{57.1 \pm 1.4} \mathrm{Wo}_{3.8 \pm 2.4}$, $\mathrm{Fe} / \mathrm{Mn}=33 \pm 1, \mathrm{n}=8$; high-Ca pyroxene $\mathrm{Fs}_{26.5 \pm 2.3} \mathrm{Wo}_{41.5 \pm 2.9}, \mathrm{Fe} / \mathrm{Mn}=31 \pm 2, \mathrm{n}=5$; plagioclase $\mathrm{An}_{85.4 \pm 0.8}, \mathrm{n}=6$.

Classification: Unbrecciated basaltic eucrite Specimens: $17.1 \mathrm{~g}$ including a probe mounts on deposit at $U N M, D P i t t$ holds the main mass.

\section{Northwest Africa 12202 (NWA 12202) \\ Western Sahara \\ Purchased: May 2018 \\ Classification: HED achondrite (Eucrite, polymict)}

History: Found in Western Sahara, January 2018. Purchased by Darryl Pitt in Mauritania, May 2018.

Physical characteristics: Single stone, partial dark fusion-crusted exterior. Saw cut reveals a light-gray fragmental breccia.

Petrography: (C. Agee) Microprobe examination of a polished mount shows a fragmental breccia of predominately plagioclase and pyroxene. The pyroxene is mixture of cumulate eucrite compositions with a population of basaltic eucrite compositions leading to the conclusion that there are at least two distinct eucrite lithologies present.

Geochemistry: (C. Agee, UNM) Basaltic eucrite: low Ca pyroxene

$\mathrm{Fs}_{60.9 \pm 1.3} \mathrm{Wo}_{3.3 \pm 2.2}, \mathrm{Fe} / \mathrm{Mn}=31 \pm 1, \mathrm{n}=4$; high $\mathrm{Ca}$ pyroxene $\mathrm{Fs}_{47.9 \pm 6.2} \mathrm{Wo}_{23.2 \pm 2.1}$, $\mathrm{Fe} / \mathrm{Mn}=31 \pm 0, \mathrm{n}=3$. Cumulate eucrite: low $\mathrm{Ca}$ pyroxene $\mathrm{Fs}_{35.9 \pm 1.9} \mathrm{Wo}_{5.0 \pm 2.8}, \mathrm{Fe} / \mathrm{Mn}=$ $29 \pm 2, n=4$.

Classification: Polymict eucrite

Specimens: $20.4 \mathrm{~g}$ including a probe mounts on deposit at UNM, DPitt holds the main mass.

\section{Northwest Africa 12203 (NWA 12203) \\ Algeria \\ Purchased: May 2018 \\ Classification: HED achondrite (Howardite)}

History: Found near Oum El Assel, Algeria, March 2018. Purchased by Darryl Pitt in Mauritania, May 2018.

Physical characteristics: Single stone, with weathered exterior. A saw cut reveals a very dark gray surface with faint appearing clasts and mineral fragments. 
Petrography: (C. Agee) Microprobe examination of a polished mount shows a fragmental breccia of pyroxene, plagioclase, and lithic clasts. The meteorite consists of approximately $50 \%$ diogenite and $50 \%$ eucrite.

Geochemistry: (C. Agee, $U N M$ ) Diogenite pyroxene $\mathrm{Fs}_{28.1 \pm 2.5} \mathrm{Wo}_{2.6 \pm 1.0}$, $\mathrm{Fe} / \mathrm{Mn}=29 \pm 2$, $\mathrm{n}=7$; eucrite pigeonite $\mathrm{Fs}_{47.5 \pm 5.9} \mathrm{Wo}_{6.7 \pm 3.1}, \mathrm{Fe} / \mathrm{Mn}=30 \pm 2$, $\mathrm{n}=6$, eucrite high-Ca pyroxene $\mathrm{Fs}_{29.3 \pm 2.6} \mathrm{Wo}_{39.1 \pm 1.9}, \mathrm{Fe} / \mathrm{Mn}=30 \pm 2, \mathrm{n}=2$, plagioclase $\mathrm{An}_{88.7 \pm 5.6}, \mathrm{n}=5$. Classification: Howardite Specimens: $23.3 \mathrm{~g}$ including a probe mounts on deposit at UNM, DPitt holds the main mass.

\section{Northwest Africa 12210 (NWA 12210)}

(Northwest Africa)

Purchased: 2018 Jun

Classification: Martian meteorite (Shergottite)

History: Purchased by Ben Hoefnagels in June 2018 from a dealer in Zagora, Morocco.

Petrography: (A. Irving and S. Kuehner, UWS) Poikilitic texture. Large pyroxene oikocrysts (up to $6 \mathrm{~mm}$ across) enclose olivine chadacrysts, and are set in a relatively coarse grained groundmass containing olivine, pyroxenes, lath-like maskelynite, abundant phosphates (both merrillite and chlorapatite), Ti-poor chromite and pyrrhotite. Mafic silicates exhibit very limited compositional zoning. Cores of pyroxene oikocrysts are stained orange.

Geochemistry: Olivine $\left(\mathrm{Fa}_{38.1-40.7}, \mathrm{FeO} / \mathrm{MnO}=49-51, \mathrm{~N}=3\right)$, low-Ca pyroxene core $\left(\mathrm{Fs}_{24.7} \mathrm{Wo}_{4.1}, \mathrm{FeO} / \mathrm{MnO}=30\right)$, pigeonite $\left(\mathrm{Fs}_{26.4-30.8} \mathrm{Wo}_{11.0-7.5} ; \mathrm{Fs}_{28.1} \mathrm{Wo}_{16.3}\right.$; $\mathrm{FeO} / \mathrm{MnO}=25-29 ; \mathrm{N}=3)$, subcalcic augite $\left(\mathrm{Fs}_{16.8-18.5} \mathrm{Wo}_{33.7-36.6}, \mathrm{FeO} / \mathrm{MnO}=23-\right.$ $25, \mathrm{~N}=2)$, maskleynite $\left(\mathrm{An}_{53.8-54.4} \mathrm{Or}_{2.1-2.2}, \mathrm{~N}=2\right)$.

Classification: Martian (shergottite, poikilitic).

Specimens: $20.5 \mathrm{~g}$ including one polished thin section at $U W B$; remainder with $\mathrm{Mr}$. B. Hoefnagels.

\section{Northwest Africa 12211 (NWA 12211)}

(Northwest Africa)

Purchased: 2018 Apr

Classification: Ordinary chondrite (H5)

History: Purchased by Thierry Simard in April 2018 from a Moroccan dealer. Petrography: (A. Irving and S. Kuehner, UWS) Sparse chondrules are present within a coarsely recrystallized assemblage containing abundant altered metal (some now replaced by red-brown iron hydroxides) and accessory merrillite.

Geochemistry: Olivine $\left(\mathrm{Fa}_{18.3-18.4}, \mathrm{~N}=3\right)$, orthopyroxene $\left(\mathrm{Fs}_{15.4-15.9} \mathrm{Wo}_{1.0-1.7}, \mathrm{~N}=\right.$ 4), clinopyroxene $\left(\mathrm{Fs}_{5.1-6.6} \mathrm{Wo}_{46.3-45.3}, \mathrm{~N}=2\right)$.

Classification: Ordinary chondrite (H5).

Specimens: $20.6 \mathrm{~g}$ including one polished thin section at $U W B$; remainder with Monsieur T. Simard. 
Northwest Africa 12212 (NWA 12212)

(Northwest Africa)

Purchased: 2005

Classification: Mesosiderite

History: Purchased by an anonymous collector from a dealer at the Munich Show in 2005, subsequently acquired by the Hollis Collection and donated to the Planetary Studies Foundation.

Petrography: (A. Irving and S. Kuehner, UWS) Aggregate of predominantly kamacite (containing wispy regions of taenite), low-Ca pyroxene and anorthite together with accessory Mg-merrillite, schreibersite and minor troilite.

Geochemistry: Low-Ca pyroxene $\left(\mathrm{Fs}_{32.7-32.9} \mathrm{Wo}_{4.2-5.1}, \mathrm{FeO} / \mathrm{MnO}=21-28, \mathrm{~N}=3\right)$, anorthite $\left(\mathrm{An}_{90.8-94.4} \mathrm{Or}_{0.9-0.8}, \mathrm{~N}=2\right)$.

Classification: Mesosiderite.

Specimens: The entire specimen polished on one side is at $P S F$.

\section{Northwest Africa 12215 (NWA 12215)}

(Northwest Africa)

Purchased: 2018 May

Classification: Ordinary chondrite (H5)

History: Purchased by SunChul Lee from a dealer in Florida, who had obtained the specimen from a Moroccan source.

Petrography: (A. Irving and S. Kuehner, UWS) Extensively recrystallized with sparse chondrules, abundant altered metal and accessory merrillite.

Geochemistry: Olivine $\left(\mathrm{Fa}_{19.5-19.9}, \mathrm{~N}=3\right)$, orthopyroxene $\left(\mathrm{Fs}_{16.8-16.9} \mathrm{Wo}_{1.1-1.0}, \mathrm{~N}=\right.$ $3)$, clinopyroxene $\left(\mathrm{Fs}_{5.7-5.9} \mathrm{Wo}_{44.4-45.7}, \mathrm{~N}=2\right)$.

Classification: Ordinary chondrite (H5).

Specimens: $122.1 \mathrm{~g}$ including one polished thin section at $U W B$; remainder with Mr. S. Lee.

\section{Northwest Africa 12217 (NWA 12217)}

(Northwest Africa)

Purchased: 2015

Classification: Ungrouped achondrite

History: Purchased by Jay Piatek in 2015 from a Moroccan meteorite dealer.

Physical characteristics: Single stone, partially covered with black fusion crust. The surface also exposes a fragmental breccia interior with coarse cream-colored grains crosscut by fine dark veinlets. Saw-cut surface reveals light colored angular mineral fragments up to $1 \mathrm{~cm}$ in size bounded by darker gray, fine grained material. A prominent, partially oxidized metal grain was visible in the saw cut surface.

Petrography: (C. Agee, UNM) Backscatter electron mapping gives the approximate modal abundances: $93 \%$ olivine, $4 \%$ pyroxene, with minor chromite, 
troilite, Fe-metal, alkali feldspar, plagioclase, silica, and fluorapatite, primarily occupying boundaries and pockets between the larger olivine grains.

Geochemistry: (C. Agee, $U N M$ ) Olivine $\mathrm{Fa}_{9.6 \pm 2.5}, \mathrm{Fe} / \mathrm{Mn}=36 \pm 3$, $\mathrm{n}=19$; low-Ca pyroxene $\mathrm{Fs}_{19.3 \pm 0.8} \mathrm{Wo}_{2.6 \pm 0.4}, \mathrm{Fe} / \mathrm{Mn}=25 \pm 1, \mathrm{n}=8$; alkali feldspar

$\mathrm{An}_{2.9 \pm 1.1} \mathrm{Or}_{56.2 \pm 10.3} \mathrm{Ab}_{41.0 \pm 10.1}, \mathrm{n}=18$; plagioclase $\mathrm{An}_{43.5 \pm 3.7} \mathrm{Or}_{1.6 \pm 0.3} \mathrm{Ab}_{54.9 \pm 4.0}, \mathrm{n}=4$; apatite $\mathrm{CaO}=46.3 \pm 0.4, \mathrm{MgO}=3.6 \pm 0.0, \mathrm{FeO}=0.27 \pm 0.01, \mathrm{Na}_{2} \mathrm{O}=2.5 \pm 0.1$, $\mathrm{P}_{2} \mathrm{O}_{5}=45.3 \pm 0.7, \mathrm{~F}=0.53 \pm 0.04, \mathrm{Cl}$ (not detected) all $\mathrm{wt} \%, \mathrm{n}=3$; metal $\mathrm{Fe}=97.8 \pm 0.6$, $\mathrm{Ni}=2.1 \pm 0.6$ ( $\mathrm{wt} \%$ ), $\mathrm{n}=5$; chromite $\mathrm{Al}_{2} \mathrm{O}_{3}=3.4 \pm 0.1, \mathrm{Cr}_{2} \mathrm{O}_{3}=64.4 \pm 0.8, \mathrm{MgO}=7.9 \pm 0.4$, $\mathrm{MnO}=0.69 \pm 0.05, \mathrm{FeO}=19.4 \pm 0.3(\mathrm{wt} \%), \mathrm{n}=3$. Oxygen isotopes $(\mathrm{K}$. Ziegler, $U N M)$ : 3 acid-washed fragments analyzed by laser fluorination gave $\delta^{18} \mathrm{O}=3.723,3.869$, $3.660 ; \delta^{17} \mathrm{O}=1.793,1.884,1.796 ; \Delta^{17} \mathrm{O}=-0.173,-0.159,-0.136$ (linearized, all per mil, TFL slope $=0.528$ ).

Classification: Ungrouped achondrite, dunite breccia. The oxygen isotope values of this meteorite plot midway between the eucrite fractionation line (EFL) and the angrite fractionation line (AFL), however its mineralogy is unlike HEDs or angrites; in particular this meteorite possesses alkali feldspar and lacks Ca-rich plagioclase. Furthermore, the $\mathrm{Fe} / \mathrm{Mn}$ of this meteorite is somewhat lower than for HEDs and much lower than angrites. Brachinites have similar oxygen isotope values, however this meteorite has olivine with much lower $\mathrm{Fa}$ and $\mathrm{Fe} / \mathrm{Mn}$ values than brachinites.

Specimens: $20.7 \mathrm{~g}$ including a probe mount on deposit at $U N M$, Jay Piatek holds the main mass.

\section{Northwest Africa 12218 (NWA 12218)}

(Northwest Africa)

Purchased: 2007 Jun

Classification: Carbonaceous chondrite (CO3)

History: Purchased from a moroccan dealer at the meteorite show in Ensisheim, France

Petrography: (K. Metzler, IfP) Chondrules, chondrule fragments, and CAIs are embedded in a fine-grained black matrix (about $20 \mathrm{vol} \%$ matrix). The vast majority of chondrules are type I chondrules. Apparent chondrule sizes mostly between 50 and $300 \mu \mathrm{m}$.

Geochemistry: Mineral compositions and geochemistry: Random measurements of olivine grains revealed $\mathrm{Fa}_{12.3 \pm 16.7}(2.7-77.5) ; n=25$. Random measurements of lowCa pyroxene grains revealed $\mathrm{Fs}_{2.7 \pm 2.4} \mathrm{Wo}_{1.7 \pm 0.9}\left(\mathrm{Fs}_{1.1-11.9} \mathrm{Wo}_{0.8-4.6}\right) ; \mathrm{n}=25$

Classification: CO chondrite based on CAI occurrence, small chondrule size, chondrule-matrix ratio, and mineral chemistry. Petrologic type 3 based on the chemical variation of olivine and pyroxene. Very low petrologic subtype $(<3.5)$ based on the PMD of Fa values (136).

Northwest Africa 12219 (NWA 12219)

(Northwest Africa) 
Purchased: 2007 Jun

Classification: Carbonaceous chondrite (CO3)

History: Purchased from a Moroccan dealer at the meteorite show in Ensisheim, France

Petrography: (K. Metzler, IfP) Chondrules, chondrule fragments, and CAIs are embedded in a fine-grained brownish matrix (about $20 \mathrm{vol} \%$ matrix). The vast majority of chondrules are type I chondrules. Apparent chondrule sizes mostly between 50 and $300 \mu \mathrm{m}$.

Geochemistry: Mineral compositions and geochemistry: Random measurements of olivine grains revealed $\mathrm{Fa}_{21.5 \pm 14.3}(2.5-48.3)$; $\mathrm{n}=23$. Random measurements of lowCa pyroxene grains revealed $\mathrm{Fs}_{3.6 \pm 3.0} \mathrm{Wo}_{1.9 \pm 1.1}\left(\mathrm{Fs}_{1.0-13.9} \mathrm{Wo}_{0.3-4.4}\right) ; \mathrm{n}=24$

Classification: $\mathrm{CO}$ chondrite based on CAI occurrence, small chondrule size, chondrule-matrix ratio, and mineral chemistry. Petrologic type 3 based on the chemical variation of olivine and pyroxene. Low petrologic subtype $(<3.5)$ based on the PMD of Fa values (67).

\section{Northwest Africa 12221 (NWA 12221) \\ (Northwest Africa) \\ Purchased: 2006 June \\ Classification: Carbonaceous chondrite (CV3)}

History: Purchased from a Moroccan dealer at the meteorite show in Ensisheim, France

Petrography: (K. Metzler, IfP) Chondrules, CAIs and AOAs are embedded in a fine-grained dark matrix (about 40 vol\% matrix). Most chondrules have apparent diameters between 0.2 and $1.0 \mathrm{~mm}$, some up to $2.3 \mathrm{~mm}$. Very few type II chondrules. Many chondrules with distinctive fine-grained rims; some chondrules with igneous rims. CAIs with sizes up to $2.3 \mathrm{~mm}$ have been observed.

Geochemistry: Mineral compositions and geochemistry: Random measurements of olivine grains revealed $\mathrm{Fa}_{5.7 \pm 3.0}(0.7-9.6) ; \mathrm{n}=19$. Random measurements of low-Ca pyroxene grains revealed $\mathrm{Fs}_{1.6 \pm 0.7} \mathrm{Wo}_{1.3 \pm 0.8}\left(\mathrm{Fs}_{0.7-3.2} \mathrm{Wo}_{0.4-3.7}\right) ; \mathrm{n}=21$.

Classification: CV chondrite based on CAI and AOA occurrence, size of chondrules and CAIs, chondrule-matrix ratio, and mineral chemistry. Petrologic type 3 based on the chemical variation of olivine and pyroxene.

\section{Northwest Africa 12222 (NWA 12222)}

(Northwest Africa)

Purchased: 2018 Mar

Classification: Martian meteorite (basaltic breccia)

Petrography: (K. Metzler, IfP) Achondritic breccia, consisting of lithic and mineral clasts, set in a fine-grained clastic matrix. The following minerals were identified: Plagioclase, pyroxene (occasionally with exsolution lamellae), Clbearing apatite, ilmenite, and pyrrhotite. Beside complex fine-grained lithologies two different basalt clasts were found. 
Geochemistry: Mineral compositions and geochemistry: Basalt clast \#1: Pyroxene $(\mathrm{n}=5) \mathrm{Fs}_{37.6 \pm 0.2} \mathrm{Wo}_{35.7 \pm 0.4}\left(\mathrm{Fs}_{37.3-37.8} \mathrm{Wo}_{35.2-36.1}\right)$; mean $\mathrm{Fe} / \mathrm{Mn}$ (at.) $=36$. Plagioclase $(\mathrm{n}=6): \mathrm{An}_{55.2 \pm 3.5} \mathrm{Or}_{1.9 \pm 0.3}\left(\mathrm{An}_{50.2-59.3} \mathrm{Or}_{1.3-2.2}\right)$. Basalt clast \#2: Pyroxene $(\mathrm{n}=7)$ $\mathrm{Fs}_{33.5 \pm 3.1} \mathrm{Wo}_{4.0 \pm 0.9}\left(\mathrm{Fs}_{25.6-34.6} \mathrm{Wo}_{1.6-4.6}\right)$; mean $\mathrm{Fe} / \mathrm{Mn}($ at. $)=38$. Plagioclase $(\mathrm{n}=7)$ : $\mathrm{An}_{53.5 \pm 4.2} \mathrm{Or}_{1.9 \pm 0.4}\left(\mathrm{An}_{49.5-60.9} \mathrm{Or}_{1.4-2.7}\right)$.

Classification: Martian (basaltic breccia), based on texture and mineral composition. An values in Feldspar and $\mathrm{Fe} / \mathrm{Mn}$ in pyroxene are typical of Martian basalts. This sample is most probably paired to NWA 7034 and pairings.

\section{Northwest Africa 12223 (NWA 12223)}

(Northwest Africa)

Purchased: 2005 Jun

Classification: HED achondrite (Eucrite, melt breccia)

History: Purchased from a Moroccan dealer at the mineral show in Sainte-Marieaux-Mines, France

Petrography: (K. Metzler, IfP) Melt breccia, consisting of eucritic basalt clasts (ophitic to subophitic textures) with sizes up to several $\mathrm{cm}$ and mineral fragments (pyroxene, plagioclase, silica polymorph), set in a dark matrix. This matrix represents a crystallized melt with subophitic texture consisting of pyroxene and skeletal plagioclase crystals. Melt pockets of partly devitrified glass also occur. Most pyroxene fragments show augite exsolution lamellae. Accessories are ilmenite, chromium spinel, and metal (mostly oxidized).

Geochemistry: Mineral compositions and geochemistry: Low-Ca pyroxene (host; $\mathrm{n}=10): \mathrm{Fs}_{63.3 \pm 0.5} \mathrm{Wo}_{2.4 \pm 0.3}\left(\mathrm{Fs}_{62.6-63.9} \mathrm{Wo}_{.9-2.8}\right)$; mean $\mathrm{Fe} / \mathrm{Mn}$ (at.): 33. Ca-rich pyroxene (exsolution lamellae; $\mathrm{n}=10) \mathrm{Fs}_{27.8 \pm 0.9} \mathrm{Wo}_{43.2 \pm 0.7}\left(\mathrm{Fs}_{27.0-30.2} \mathrm{Wo}_{41.7-44.0}\right)$; Plagioclase $(\mathrm{n}=10): \mathrm{An}_{87.8 \pm 1.7}\left(\mathrm{An}_{84.9-89.9)}\right.$.

\section{Northwest Africa 12224 (NWA 12224)}

(Northwest Africa)

Purchased: 2006 Jun

Classification: HED achondrite (Howardite)

History: Purchased from a Moroccan dealer at the mineral show in Sainte-Marieaux-Mines, France

Petrography: (K. Metzler, IfP) Shock-compacted fine-grained breccia of dark gray appearance, criss-crossed by black shock melt veins up to several $\mathrm{mm}$ in width. It consists of gabbroic and basaltic (ophitic to subophitic) rock fragments and related debris. The majority of pyroxenes is free of exsolution lamellae, but exsolved pyroxenes also occur. Three grains of Fe-rich olivine have been found. This breccia contains devitrified melt droplets as well as clast-rich and clast-poor impact melt rocks. About 10-15\% diogenitic components. Accessories are ilmenite, chromium spinel, troilite, silica polymorph, and Ni-poor metal (mostly oxidized).

Geochemistry: Mineral compositions and geochemistry: Low-Ca pyroxene $(\mathrm{n}=19): \mathrm{Fs}_{35.1 \pm 5.1} \mathrm{Wo}_{5.4 \pm 0.7}\left(\mathrm{Fs}_{29.3-49.7} \mathrm{Wo}_{4.1-7.1}\right)$; mean Fe/Mn (at.): 33. Ca-rich 
pyroxene ( $\mathrm{n}=5) \mathrm{Fs}_{49.3 \pm 2.8} \mathrm{Wo}_{16.8 \pm 3.6}\left(\mathrm{Fs}_{45.4-53.0} \mathrm{Wo}_{13.1-20.5}\right)$; mean $\mathrm{Fe} / \mathrm{Mn}$ (at.): 31. Diogenitic pyroxene ( $\mathrm{n}=3) \mathrm{Fs}_{24.1} \mathrm{Wo}_{1.9}\left(\mathrm{Fs}_{21.8-26.0} \mathrm{Wo}_{1.6-2.1}\right)$ mean $\mathrm{Fe} / \mathrm{Mn}$ (at.): 32. Olivine ( $\mathrm{n}=3$ ) $\mathrm{Fa}_{77.7}\left(\mathrm{Fa}_{76.5-78.6}\right)$; mean Fe/Mn (at.): 47. Plagioclase ( $\mathrm{n}=9$ ):

$\mathrm{An}_{83.8 \pm 3.9}\left(\mathrm{An}_{78.9-92.0}\right)$.

\section{Northwest Africa 12225 (NWA 12225)}

(Northwest Africa)

Purchased: 2007 Jun

Classification: HED achondrite (Eucrite, polymict)

History: Purchased from a Moroccan dealer at the mineral show in Sainte-Marieaux-Mines, France

Petrography: (K. Metzler, IfP) Polymict breccia consisting of gabbroic and basaltic rock fragments and related debris. Partly brown appearance due to oxidized $\mathrm{Ni}$-free metal. This breccia contains clast-rich impact melt rocks. Accessories are ilmenite, chromium spinel, silica polymorph, and traces of merrilite and zircon.

Geochemistry: Mineral compositions and geochemistry: Basalt clast \#1: Pyroxene $(\mathrm{n}=10): \mathrm{Fs}_{26.7 \pm 0.9} \mathrm{Wo}_{43.0 \pm 0.6}\left(\mathrm{Fs}_{25.4-28.4} \mathrm{Wo}_{42.0-43.9}\right)$; mean $\mathrm{Fe} / \mathrm{Mn}$ (at.): 29. Plagioclase $(\mathrm{n}=10): \mathrm{An}_{74.5 \pm 3.9}\left(\mathrm{An}_{71.2-82.4}\right)$. Basalt clast \#2: Pyroxene $(\mathrm{n}=10)$ :

$\mathrm{Fs}_{56.0 \pm 0.5} \mathrm{Wo}_{5.2 \pm 1.1}\left(\mathrm{Fs}_{55.1-56.6} \mathrm{Wo}_{3.7-8.0}\right)$; mean Fe/Mn (at.): 26. Plagioclase ( $\left.\mathrm{n}=10\right)$ : $\mathrm{An}_{87.3 \pm 3.5}\left(\mathrm{An}_{82.6-92.4)}\right.$.

\section{Northwest Africa 12226 (NWA 12226)}

(Northwest Africa)

Purchased: 2007 Dec

Classification: HED achondrite (Eucrite)

History: Purchased from a Moroccan dealer at the mineral show $(\mathrm{CCH})$ in Hamburg, Germany

Petrography: (K. Metzler, IfP) Unbrecciated ophitic basalt with exsolved pyroxene. Accessories are ilmenite, chromium spinel, silica polymorph, Ni-free iron metal, and troilite.

Geochemistry: Mineral compositions and geochemistry: Low-Ca pyroxene $(\mathrm{n}=15): \mathrm{Fs}_{58.3 \pm 1.2} \mathrm{Wo}_{6.7 \pm 1.2}\left(\mathrm{Fs}_{55.5-59.5} \mathrm{Wo}_{5.7-9.1}\right)$; mean $\mathrm{Fe} / \mathrm{Mn}$ (at.): 32. Exsolved Carich pyroxene lamellae $(\mathrm{n}=9)$ : $\mathrm{Fs}_{35.3 \pm 2.6} \mathrm{Wo}_{34.4 \pm 3.3}\left(\mathrm{Fs}_{32.3-39.0} \mathrm{Wo}_{29.4-38.5}\right)$; mean $\mathrm{Fe} / \mathrm{Mn}$ (at.): 34. Plagioclase ( $\mathrm{n}=5): \mathrm{An}_{88.4 \pm 1.8}\left(\mathrm{An}_{86.4-90.5}\right)$.

\section{Northwest Africa 12227 (NWA 12227)}

Algeria

Find: April 2018

Classification: HED achondrite (Howardite)

Petrography: (D. Sheikh, FSU) Fine-grained polymict breccia, $<7 \mathrm{~mm}$ clast size for basaltic eucrite clasts, $<3 \mathrm{~mm}$ for diogenite clasts, $<2 \mathrm{~mm}$ for cumulate eucrite 
clasts. Modal content (in vol.\%): diogenites, $46 \%$; cumulate eucrites, $12 \%$; basaltic eucrites, $35 \%$; anorthosite clasts, $5 \%$; shock melt zones, $1 \%$; opaques, $1 \%$.

Geochemistry: Eucrite pyroxene: pigeonite $\left(\mathrm{Fs}_{44.95} \mathrm{Wo}_{18.29}\right), \mathrm{n}=25$; augite $\left(\mathrm{Fs}_{39.9} \mathrm{Wo}_{34.4}\right), \mathrm{n}=6 ; \mathrm{FeO} / \mathrm{MnO}=28$. Diogenite pyroxene: orthopyroxene $\left(\mathrm{Fs}_{27.4} \mathrm{Wo}_{6.84}\right), \mathrm{n}=10 ; \mathrm{FeO} / \mathrm{MnO}=22$. Calcic plagioclase $\left(\mathrm{An}_{89}\right), \mathrm{N}=25$. Ilmenite $(\mathrm{Fe} /(\mathrm{Fe}+\mathrm{Mg}))=88.42 \%, \mathrm{~N}=2 . \mathrm{FeS}$ and $\mathrm{SiO}_{2}$-rich glass present.

Classification: Achondrite (howardite)

Specimens: 25.65 grams at $U C L A$, main mass with Matthew Stream.

\section{Northwest Africa 12229 (NWA 12229)}

(Northwest Africa)

Purchased: 2017

Classification: Carbonaceous chondrite (CV3)

History: A single stone weighing $675 \mathrm{~g}$ was found in Morocco prior to 2016. J. Donald Cline and John Sinclair acquired the sample from a meteorite dealer at the Tucson Gem and Mineral Show in February 2017.

Physical characteristics: Sample is dark brown in color, rounded to irregular in shape and lacks fusion crust. Sample is coated on the bottom and sides with caliche, some of which contains rounded sand grains. Chondrules and CAIs are visible through the patina. The cut face shows abundant chondrules, CAIs and AOAs.

Petrography: Description and classification (A. Love, App): Sample contains distinct chondrules (average diameter of $743 \mu \mathrm{m}(\mathrm{N}=29)$, fragments and refractory inclusions (CAIs up to $1 \mathrm{~mm}$ ) all set within an opaque dark brown matrix. Matrix contains FeO-rich olivine and has a weak fabric defined by slightly elongated chondrules and oriented amoeboid olivine aggregates. Type I olivine chondrules are dominant. Sample has $40 \%$ matrix.

Geochemistry: (A. Love, App) Olivine $\mathrm{Fa}_{11.3 \pm 15.6}(0.6-49.7), \mathrm{N}=16$; low $\mathrm{Ca}$ pyroxene $\mathrm{Fs}_{1.4 \pm 0.4} \mathrm{Wo}_{1.1 \pm 0.8}\left(\mathrm{Fs}_{0.8-2.2} \mathrm{Wo}_{0.5-3.6}\right), \mathrm{N}=11$.

Classification: CV3. CV based on diameter of chondrules, matrix abundance and ubiquitous CAIs. CV subgroup based on magnetic susceptibility. Type 3 based upon unequilibrated compositions of chondrules.

Specimens: PARI holds the main mass. One endcut weighing $28 \mathrm{~g}$ and a polished thin section are on deposit at App.

\section{Northwest Africa 12230 (NWA 12230)}

(Northwest Africa)

Purchased: 2017

Classification: HED achondrite (Eucrite, polymict)

History: 34 identical stones with a total weight $1024.7 \mathrm{~g}$ were found by a meteorite prospector in Morocco. John Sinclair purchased the specimens at the 2017 Tucson Gem and Mineral Show. 
Physical characteristics: The stones have a dark-brownish-gray weathered exterior that has a granular texture and contains circular pits where clasts have been plucked out. The cut face of the interior is dark greenish-grey and shows a brecciated texture composed of lithic clasts and mineral fragments.

Petrography: Description and classification (A. Love, $A p p$ ): Sample is a polymict breccia composed of rounded to irregular-shaped mineral clasts and sparse lithic clasts (up to $2 \mathrm{~cm}$ ) from cumulate and vitrophyric eucrites, and diogenites ( $\sim 6$ vol\%). Pyroxenes are exsolved. Accessory minerals are FeNi grains, silica polymorph, ilmenite, chromite and olivine.

Geochemistry: (A. Love, App) Pigeonite host: $\mathrm{Fs}_{51.4 \pm 9.8} \mathrm{Wo}_{5.8 \pm 2.3}\left(\mathrm{Fs}_{34.8-65.6} \mathrm{Wo}_{3.6-9.3}\right.$, $\mathrm{FeO} / \mathrm{MnO}=27.4-34.2), \mathrm{N}=8$; high-Ca pyroxene exsolution lamellae:

$\mathrm{Fs}_{26.2 \pm 5.8} \mathrm{Wo}_{40.5 \pm 0.9}\left(\mathrm{Fs}_{20.5-34.8} \mathrm{Wo}_{39.2-41.4}\right), \mathrm{N}=6$; diogenitic orthopyroxene:

$\mathrm{Fs}_{23.5 \pm 4.8} \mathrm{Wo}_{2.7 \pm 1.3}\left(\mathrm{Fs}_{14.2-28.2} \mathrm{Wo}_{0.3-3.8}, \mathrm{FeO} / \mathrm{MnO}=24.4-32.4\right), \mathrm{N}=6$; olivine $\left(\mathrm{Fa}_{43.5 \pm 6.5}\right.$, $\mathrm{FeO} / \mathrm{MnO}=50.0-65.3), \mathrm{N}=3$; plagioclase $\mathrm{An}_{92.0 \pm 2.1}\left(\mathrm{An}_{88.5-94.3}\right), \mathrm{N}=10$.

Classification: Textural and compositional data (Fe/Mn of pyroxenes) indicate this is an HED achondrite. Sample contains $6 \%$ diogenite indicating this is a polymict eucrite breccia. The distinctive granular exterior and magnetic susceptibility suggest these stones are paired.

Specimens: John Sinclair holds the remainder of this material. A polished thin section, epoxy mount and 2 slices weighing $41.5 \mathrm{~g}$ are on deposit at App.

\section{Northwest Africa 12231 (NWA 12231)}

(Northwest Africa)

Purchased: 2018

Classification: HED achondrite (Howardite)

History: Purchased by V. Ouellet in Zagora in 2018.

Physical characteristics: The sample is a small stone with black fusion crust and a light grey, fine-grained interior.

Petrography: The sample is a fine-grained polymict breccia. Modal percentages of lithic fragments (in vol.\%): cumulate eucrite clasts, $40 \%$; basaltic eucrites, $30 \%$; diogenitic clasts, $20 \%$; anorthosite clasts, $5 \%$; opaques, $3 \%$; glass, $2 \%$.

Geochemistry: Eucritic pyroxene: pigeonite $\left(\mathrm{Fs}_{47.1 \pm 4.3} \mathrm{Wo}_{15.8 \pm 4.3}\right), \mathrm{n}=15$; augite $\left(\mathrm{Fs}_{38.9 \pm 3.8} \mathrm{Wo}_{31.2 \pm 3.6}\right), \mathrm{n}=6$. Diogenitic pyroxene: orthopyroxene $\left(\mathrm{Fs}_{18.05}\right.$ $\left.{ }_{ \pm 1.6} \mathrm{Wo}_{4.54 \pm 1.6}\right), \mathrm{n}=9$. Calcic plagioclase $\left(\mathrm{An}_{88.4 \pm 4.2}\right), \mathrm{N}=15$. Ilmenite $(\mathrm{Fe} /(\mathrm{Fe}+\mathrm{Mg}))=$ 90.06\%, N=4. Chromite, FeS and FeO Present. Silica-rich glass present.

Classification: howardite

Specimens: $3 \mathrm{~g}$ part slice at $U C L A .1 \mathrm{~g}$ powdered sample with C. Anderkin.

Remainder of mass with V. Oullet.

\section{Northwest Africa 12234 (NWA 12234)}

Algeria

Find: 2016 March

Classification: Ordinary chondrite (LL3) 
Petrography: (D. Sheikh, FSU) Composed of small to medium-sized chondrules (average $\sim 0.7 \mathrm{~mm}$ ) in a fine-grained matrix. Olivine and pyroxene are both heterogeneous in composition throughout. Low amount of metal present.

Geochemistry: Olivine, $\mathrm{Fa}_{20.8 \pm 9.6}\left(\mathrm{Fa}_{4.5-38.7}, \mathrm{~N}=55\right)$; orthopyroxene $\mathrm{Fs}_{16.2 \pm 5.3} \mathrm{Wo}_{3.2 \pm 0.4}\left(\mathrm{Fs}_{8.2-31.5} \mathrm{Wo}_{2.2-3.8}, \mathrm{~N}=50\right)$.

Classification: Ordinary Chondrite (LL3)

Specimens: 22.13 grams at $U C L A$; main mass with Matthew Stream.

\section{Northwest Africa 12235 (NWA 12235) \\ Morocco \\ Purchased: Jan. 2017 \\ Classification: Ordinary chondrite (H4)}

History: Purchased from S. Tutorow via Ebay. Place of origin is stated as Morocco.

Physical characteristics: Physical Characteristics: One mass of $1740 \mathrm{~g}$. Completely fusion encrusted. Brown fusion crust. Sample cut once for preparing sections and a $21.88 \mathrm{~g}$ slice for Yale.

Petrography: Average chondrule diameter is $0.35 \mathrm{~mm}$. No matrix, but sharp chodrule boundaries. Almost all Fe,Ni metal altered, but considerable volumes of troilite remain.

Classification: Ordinary Chondrite (H4).

Specimens: Main mass is at WesU, Joe Webb Peoples Museum. Several thick sections were prepared as well. YPMNH.

\section{Northwest Africa 12236 (NWA 12236) \\ Morocco \\ Purchased: 2018 Jan \\ Classification: Ordinary chondrite (H7)}

History: Specimen is cataloged at the Royal Ontario Museum as LM58922.

Petrography: Specimen is composed of olivine, pyroxene and minor plagioclase and is devoid of chondrules or relict chondrule rims. Texture is mainly poikilitic, with large olivine oikocrysts (averaging $2383 \mu \mathrm{m}$ in diameter, $\mathrm{n}=12$ ) enclosing small olivine phenocrysts (averaging $104 \mu \mathrm{m}$ in diameter, $\mathrm{n}=26$ ).

Geochemistry: Olivine: $17.9 \pm 1.4, \mathrm{Fe} / \mathrm{Mn}=35.9 \pm 5.5(\mathrm{n}=27)$; Low Ca-pyroxene: $15.0 \pm 0.7 \mathrm{Wo}_{2.1 \pm 1.1}, \mathrm{Fe} / \mathrm{Mn}=23.3 \pm 5.0(\mathrm{n}=24)$; Augite $\mathrm{Fs}_{6.6 \pm 1.0} \mathrm{Wo}_{42.0 \pm 3.6}$, $\mathrm{Fe} / \mathrm{Mn}=15.9 \pm 1.5, \mathrm{Cr}_{2} \mathrm{O}_{3}=1.22 \pm 0.23 \mathrm{wt} \%(\mathrm{n}=7)$. Oxygen Isotopes: (Karen Ziegler, $U N M) \delta^{17} \mathrm{O}=2.937$ permil, $\delta^{18} \mathrm{O}=4.497$ permil, $\delta^{17} \mathrm{O}=0.562$ permil

\section{Northwest Africa 12237 (NWA 12237)}

(Northwest Africa)

Purchased: 2017

Classification: Ureilite 
History: Purchased by Suzanne Morrison from a Moroccan meteorite dealer in Tucson, 2017.

Physical characteristics: Single stone with dark brown, weathered exterior.

Petrography: (C. Agee, UNM) This meteorite consists of olivine, pigeonite, and Fe-metal. Ubiquitous, finely disseminated Fe-metal occupies zones between silicate grain boundaries. Graphite is present.

Geochemistry: (C. Agee, UNM) Olivine $\mathrm{Fa}_{22.0 \pm 0.2}, \mathrm{Fe} / \mathrm{Mn}=50 \pm 3, \mathrm{Cr}_{2} \mathrm{O}_{3}=0.72 \pm 0.18$ (wt\%), n=6; pigeonite $\mathrm{Fs}_{18.8 \pm 0.1} \mathrm{Wo}_{6.0 \pm 0.2}, \mathrm{Fe} / \mathrm{Mn}=32 \pm 2, \mathrm{n}=6$.

Classification: Ureilite

Specimens: $20.2 \mathrm{~g}$ including a probe mount on deposit at $U N M$, Suzanne Morrison holds the main mass.

\section{Northwest Africa 12238 (NWA 12238)}

(Northwest Africa)

Purchased: 2011

Classification: Ordinary chondrite (L5)

History: Purchased by Brad Kern in January 2011 from Morocco.

Physical characteristics: Three identical appearing stones.

Petrography: (C. Agee, UNM) Microprobe examination of a polished mount shows porphyritic chondrules and plagioclase grains up to $25 \mu \mathrm{m}$.

Geochemistry: (C. Agee, $U N M$ ) Olivine $\mathrm{Fa}_{25.5 \pm 0.3}, \mathrm{Fe} / \mathrm{Mn}=50 \pm 1, \mathrm{n}=6$; low-Ca pyroxene $\mathrm{Fs}_{20.3 \pm 0.6} \mathrm{Wo}_{1.8 \pm 1.3}, \mathrm{Fe} / \mathrm{Mn}=28 \pm 1, \mathrm{n}=5$.

Classification: Ordinary chondrite (L5)

Specimens: $30.6 \mathrm{~g}$ including a probe mount on deposit at $U N M$, Brad Kern holds the main mass of $1092.2 \mathrm{~g}$.

Northwest Africa 12239 (NWA 12239)

(Northwest Africa)

Purchased: 2017

Classification: Ordinary chondrite (H5)

History: Purchased by Suzanne Morrison in Tucson, 2017.

Physical characteristics: Many identical appearing stones.

Petrography: (C. Agee, UNM) Microprobe examination of a polished mount shows porphyritic chondrules and plagioclase grains up to $25 \mu \mathrm{m}$.

Geochemistry: (C. Agee, $U N M$ ) Olivine $\mathrm{Fa}_{18.7 \pm 0.3}, \mathrm{Fe} / \mathrm{Mn}=38 \pm 2$, $\mathrm{n}=6$; low-Ca pyroxene $\mathrm{Fs}_{15.4 \pm 0.1} \mathrm{Wo}_{1.4 \pm 1.2}, \mathrm{Fe} / \mathrm{Mn}=22 \pm 2$, $\mathrm{n}=4$, plagioclase $\mathrm{Ab}_{75.2 \pm 11.1}, \mathrm{n}=4$.

Classification: Ordinary chondrite (H5)

Specimens: $20 \mathrm{~g}$ including a probe mount on deposit at $U N M$, Suzanne Morrison holds the main mass.

Northwest Africa 12240 (NWA 12240)

Mauritania 
Find: 2017

Classification: Ordinary chondrite (H4)

Petrography: The rock contains well-defined RP, PP, PO, and BO chondrules ranging from 80 to $1100 \mu \mathrm{m}$ in diameter and averaging $\sim 400 \mu \mathrm{m}$. Opaques include metallic $\mathrm{Fe}-\mathrm{Ni}$, troilite and chromite. There are many shock features: (1) Most troilite grains are polycrystalline. (2) Some kamacite grains contain small irregular grains of troilite, some also containing metallic $\mathrm{Cu}$ (up to $8 \times 30 \mu \mathrm{m}$ in size) adjacent to the trolite. (3) Also present are chromite-plagioclase assemblages and chromite veinlets inside olivine grains. The typical plagioclase grain is $5-10 \mu \mathrm{m}$ in size. All of the low-Ca pyroxene grains are orthorhombic.

\section{Northwest Africa 12241 (NWA 12241)}

Algeria

Purchased: 2018 Aug

Classification: Martian meteorite (Shergottite)

History: Purportedly found in Algeria and purchased by Mohamed Brahim Sueilem in August 2018 from a dealer in Bordj Badji Mokhtar, Algeria.

Physical characteristics: The material (total weight $1150 \mathrm{~g}$ ) comprises multiple broken fragments, some with adhering black fusion crust. The friable pieces are pale green in color and contain white plagioclase but no evident maskelynite.

Petrography: (A. Irving and S. Kuehner, UWS) This fresh specimen has an orthocumulate texture with $\sim 15$ vol.\% lath-like, birefringent plagioclase occurring interstitially to more abundant and coarser grained $(0.2-1.6 \mathrm{~mm}$, mean $0.7 \mathrm{~mm})$ mafic silicate grains (unzoned olivine and patchily zoned low-Ca and high-Ca clinopyroxene with very limited compositional variation). Accessory phases include Mg-merrillite, chlorapatite, ilmenite, Ti-rich chromite, Cr-rich chromite and pyrrhotite (some associated with minor pentlandite). Levels of shock are judged to be low, based on the absence of optical effects in plagioclase and the complete lack of any conversion to maskelynite; the presence of widespread microcracks in mafic silicates is the only clear evidence of shock. Geochemistry: Olivine $\left(\mathrm{Fa}_{32.7-34.3}, \mathrm{FeO} / \mathrm{MnO}=47-48, \mathrm{~N}=4\right)$, pigeonite $\left(\mathrm{Fs}_{25.0-}\right.$ $\left.{ }_{30.4} \mathrm{Wo}_{5.5-10.0}, \mathrm{FeO} / \mathrm{MnO}=28-30, \mathrm{~N}=4\right)$, subcalcic augite $\left(\mathrm{Fs}_{17.1-18.1} \mathrm{Wo}_{32.7-32.4}\right.$, $\mathrm{FeO} / \mathrm{MnO}=23-25, \mathrm{~N}=2)$, aluminous augite inclusion in olivine $\left(\mathrm{Fs}_{17.1} \mathrm{Wo}_{39.5}\right.$, $\left.\mathrm{FeO} / \mathrm{MnO}=28, \mathrm{TiO}_{2}=1.1 \mathrm{wt} . \%, \mathrm{Al}_{2} \mathrm{O}_{3}=6.2 \mathrm{wt} . \%\right)$, plagioclase $\left(\mathrm{An}_{43.0-57.8} \mathrm{Or}_{2.4-}\right.$ ${ }_{1.3}, \mathrm{~N}=3$ ). Oxygen isotopes $(\mathrm{K}$. Ziegler, $U N M)$ : analyses of acid-washed subsamples by laser fluorination gave, respectively, $\delta^{17} \mathrm{O} 2.907,2.635,2.808 ; \delta^{18} \mathrm{O}$ 5.092, 4.620, 4.875; $\Delta^{17} \mathrm{O} 0.218,0.195,0.234$ per mil.

Classification: Martian (shergottite, olivine gabbroic, orthocumulate, low shock). Specimens: $22.4 \mathrm{~g}$ including one polished thin section at $U W B$; remainder held jointly by Naji Ben Faraji and Mohamed Brahim Sueilem.

Northwest Africa 12243 (NWA 12243)

Mauritania 
Purchased: 2018 May

Classification: HED achondrite (Eucrite, polymict)

History: Found in Mauritania and purchased by Darryl Pitt in May 2018 from a Mauritanian dealer.

Petrography: (A. Irving and S. Kuehner, UWS) Fresh breccia specimen consisting of larger angular mineral clasts derived mostly from gabbroic eucrites in a finer matrix of the same phases. Minerals are exsolved pigeonite, anorthite, silica polymorph, unexsolved ferroan pigeonite, minor ( $<10$ vol.\%) diogenitic orthopyroxene, olivine, ilmenite, chromite and troilite.

Geochemistry: Diogenitic orthopyroxene $\left(\mathrm{Fs}_{27.9-29.0} \mathrm{Wo}_{2.1-3.6}, \mathrm{FeO} / \mathrm{MnO}=27-28, \mathrm{~N}\right.$ $=2)$, olivine $\left(\mathrm{Fa}_{42.3-51.0}, \mathrm{FeO} / \mathrm{MnO}=50, \mathrm{~N}=2\right)$, low-Ca pyroxene host in exsolved pigeonite $\left(\mathrm{Fs}_{43.5-45.9} \mathrm{Wo}_{2.6-5.5}, \mathrm{FeO} / \mathrm{MnO}=27-34, \mathrm{~N}=2\right)$, clinopyroxene exsolution lamella $\left(\mathrm{Fs}_{17.7} \mathrm{Wo}_{44.2}, \mathrm{FeO} / \mathrm{MnO}=26\right)$, subcalcic augite $\left(\mathrm{Fs}_{62.5} \mathrm{Wo}_{26.0} \mathrm{FeO} / \mathrm{MnO}=\right.$ 34), anorthite $\left(\mathrm{An}_{91.5-93.9} \mathrm{Or}_{0.6-0.5}, \mathrm{~N}=2\right)$.

Classification: Eucrite (polymict breccia).

Specimens: $20.1 \mathrm{~g}$ including one polished thin section at $U W B$; remainder with DPitt.

\section{Northwest Africa 12244 (NWA 12244) \\ Morocco \\ Purchased: 2018 May \\ Classification: Ordinary chondrite (LL3)}

History: Found near Bir Lahlou, Western Sahara, and purchased by DPitt in May 2018 from a Mauritanian dealer.

Petrography: (A. Irving and S. Kuehner, UWS) Fairly closely-packed, well-formed chondrules (apparent diameter $780 \pm 630 \mu \mathrm{m}, \mathrm{N}=21$ ) are set in a sparse finer grained matrix.

Geochemistry: Olivine $\left(\mathrm{Fa}_{0.3-49.1}, \mathrm{Cr}_{2} \mathrm{O}_{3}\right.$ in ferroan examples 0.07-0.10 wt.\%, mean $0.09 \pm 0.01$ wt.\%, $\mathrm{N}=8$ ), orthopyroxene $\left(\mathrm{Fs}_{2.3-25.2} \mathrm{Wo}_{0.5-3.8}, \mathrm{~N}=3\right)$, pigeonite

$\left(\mathrm{Fs}_{18.0} \mathrm{Wo}_{14.6}\right)$, subcalcic augite $\left(\mathrm{Fs}_{23.7} \mathrm{Wo}_{22.4}\right)$. Magnetic susceptibility $\log \chi\left(\times 10^{-}\right.$ $\left.{ }^{9} \mathrm{~m}^{3} / \mathrm{kg}\right)=3.80$.

Classification: Ordinary chondrite (LL3).

Specimens: $25.6 \mathrm{~g}$ including one polished thin section at $U W B$; remainder with DPitt.

\section{Northwest Africa 12245 (NWA 12245)}

(Northwest Africa)

Purchased: 2018 Apr

Classification: HED achondrite (Diogenite)

History: Purchased by Fred Olsen in April 2018 from a dealer in Zagora, Morocco. Petrography: (A. Irving and S. Kuehner, UWS) Breccia composed predominantly of angular grains of orthopyroxene (exhibiting undulose extinction and containing sparse exsolution lamellae of clinopyroxene), anorthite ( 15 vol.\%, 
polycrystalline), silica polymorph, chromite, troilite and Ni-free metal. Some composite clasts containing both orthopyroxene and anorthite are present, as well as pockets composed of radially-quenched shock melt.

Geochemistry: Orthopyroxene host $\left(\mathrm{Fs}_{34.0-34.1} \mathrm{Wo}_{2.3-2.8}, \mathrm{FeO} / \mathrm{MnO}=27-29, \mathrm{~N}=3\right)$, clinopyroxene exsolution lamellae $\left(\mathrm{Fs}_{14.3-15.6} \mathrm{Wo}_{44.4-42.8}, \mathrm{FeO} / \mathrm{MnO}=24-25, \mathrm{~N}=2\right)$, anorthite $\left(\mathrm{An}_{94.0-95.1} \mathrm{Or}_{0.4-0.3}, \mathrm{~N}=2\right)$.

Classification: Diogenite (noritic breccia, shocked).

Specimens: $16.32 \mathrm{~g}$ including one polished thin section at $U W B$; remainder with the estate of Mr. F. Olsen managed by D. Baldwin.

\section{Northwest Africa 12246 (NWA 12246) \\ Morocco \\ Purchased: 2018 May \\ Classification: HED achondrite (Eucrite, unbrecciated)}

History: Found near Bir Tighisit, southern Morocco and purchased by Darryl Pitt in May 2018 from a Mauritanian dealer.

Petrography: (A. Irving and S. Kuehner, UWS) Fresh gabbroic eucrite composed mostly of zoned low-Ca and high-Ca pyroxene (grainsize up to $1.5 \mathrm{~mm}$ ) and anorthite (as interstitial stubby laths) with accessory silica polymorph, fayalite, ilmenite, chromite and troilite.

Geochemistry: Low-Ca pyroxene cores $\left(\mathrm{Fs}_{30.2-30.7} \mathrm{Wo}_{5.8-6.1}, \mathrm{FeO} / \mathrm{MnO}=26-27, \mathrm{~N}=\right.$ 2), orthopyroxene rims $\left(\mathrm{Fs}_{57.2-62.8} \mathrm{Wo}_{3.9-2.1}, \mathrm{FeO} / \mathrm{MnO}=33-34, \mathrm{~N}=2\right)$, subcalcic augite $\left(\mathrm{Fs}_{39.2} \mathrm{Wo}_{30.3} ; \mathrm{Fs}_{45.7} \mathrm{Wo}_{23.4} ; \mathrm{FeO} / \mathrm{MnO}=31, \mathrm{~N}=2\right)$, fayalite $\left(\mathrm{Fa}_{80.5-82.6}\right.$, $\mathrm{FeO} / \mathrm{MnO}=39-41)$, anorthite $\left(\mathrm{An}_{91.4-92.1} \mathrm{Or}_{0.6-0.8}, \mathrm{~N}=2\right)$.

Classification: Eucrite (unbrecciated, gabbroic).

Specimens: $21.1 \mathrm{~g}$ including one polished thin section at $U W B$; remainder with DPitt.

\section{Northwest Africa 12247 (NWA 12247)}

Algeria

Purchased: 2018 May

Classification: Carbonaceous chondrite (CV3)

History: Found in Algeria and purchased by Darryl Pitt in May 2018 from a Mauritanian dealer.

Petrography: (A. Irving and S. Kuehner, UWS) Well-formed chondrules (apparent diameter $900 \pm 350 \mu \mathrm{m}, \mathrm{N}=21$; some rimmed) and fine grained CAI are set in a fine grained matrix ( $\sim 30 \mathrm{vol} . \%$, orange-brown in thin section) containing minor calcite and barite.

Geochemistry: Olivine $\left(\mathrm{Fa}_{0.3-37.3}, \mathrm{~N}=3\right)$, orthopyroxene $\left(\mathrm{Fs}_{0.7-1.2} \mathrm{Wo}_{0.8-0.7}, \mathrm{~N}=3\right)$, augite $\left(\mathrm{Fs}_{5.0} \mathrm{Wo}_{39.8} ; \mathrm{Fs}_{47.9} \mathrm{Wo}_{50.1} ; \mathrm{N}=2\right)$.

Classification: Carbonaceous chondrite (CV3).

Specimens: $15.8 \mathrm{~g}$ including one polished thin section at $U W B$; remainder with DPitt. 


\section{Northwest Africa 12248 (NWA 12248)}

Mauritania

Purchased: 2018 May

Classification: Lunar meteorite (feldspathic breccia)

History: Found in Mauritania and purchased by Darryl Pitt in May 2018 from a Mauritanian dealer.

Physical characteristics: A small (37.3 g) dark stone lacking fusion crust but coated by a shiny desert patina. The fresh interior conatins small white and beige clasts set in a dark-gray matrix.

Petrography: (A. Irving and S. Kuehner, UWS) Breccia composed of mineral clasts of anorthite, olivine, low-Ca pyroxenes, augite, ilmenite and troilite in a fine grained matrix containing Ni-poor metal and minor barite.

Geochemistry: Olivine $\left(\mathrm{Fa}_{44.5}, \mathrm{FeO} / \mathrm{MnO}=105 ; \mathrm{Fa}_{13.1}, \mathrm{FeO} / \mathrm{MnO}=54 ; \mathrm{N}=2\right)$, low-Ca pyroxene $\left(\mathrm{Fs}_{38.2} \mathrm{Wo}_{4.5}, \mathrm{FeO} / \mathrm{MnO}=59\right)$, pigeonite $\left(\mathrm{Fs}_{32.0-32.5} \mathrm{Wo}_{14.6-9.7}\right.$, $\mathrm{FeO} / \mathrm{MnO}=55-62, \mathrm{~N}=2)$, augite $\left(\mathrm{Fs}_{22.4} \mathrm{Wo}_{43.1}, \mathrm{FeO} / \mathrm{MnO}=51\right)$, anorthite $\left(\mathrm{An}_{94.3}\right.$ ${ }_{95.9} \mathrm{Or}_{0.2}, \mathrm{~N}=2$ ).

Classification: Lunar (feldspathic breccia).

Specimens: $9.5 \mathrm{~g}$ including one polished thin section at $U W B$; remainder with DPitt.

\section{Northwest Africa 12250 (NWA 12250)}

(Northwest Africa)

Purchased: 2018 May

Classification: HED achondrite (Howardite)

History: Found in Algeria and purchased by Darryl Pitt in May 2018 from a Mauritanian dealer.

Petrography: (A. Irving and S. Kuehner, UWS) Breccia composed mainly of mineral clasts derived from gabbroic eucrites, rare lithic clasts of gabbroic eucrite, and $\sim 15$ vol. $\%$ diogenitic orthopyroxene $(\sim 15$ vol. $\%)$ in a finer grained matrix of related debris. Other minerals are olivine, exsolved pigeonite, unexsolved pigeonite, subcalcic augite, calcic plagioclase, ilmenite and troilite.

Geochemistry: Diogenitic orthopyroxene $\left(\mathrm{Fs}_{16.0-18.3} \mathrm{Wo}_{1.3-1.5}, \mathrm{FeO} / \mathrm{MnO}=29-31, \mathrm{~N}\right.$ $=2)$, olivine $\left(\mathrm{Fa}_{52.0-57.1}, \mathrm{FeO} / \mathrm{MnO}=47-50, \mathrm{~N}=2\right)$, orthopyroxene host in exsolved pigeonite $\left(\mathrm{Fs}_{54.3} \mathrm{Wo}_{1.1} ; \mathrm{Fs}_{61.7} \mathrm{Wo}_{1.4} ; \mathrm{FeO} / \mathrm{MnO}=28-29 ; \mathrm{N}=2\right)$, pigeonite $\left(\mathrm{Fs}_{58.0} \mathrm{Wo}_{15.1}, \mathrm{FeO} / \mathrm{MnO}=34\right)$, subcalcic augite $\left(\mathrm{Fs}_{55.6} \mathrm{Wo}_{24.6} \mathrm{FeO} / \mathrm{MnO}=30\right)$, plagioclase $\left(\mathrm{An}_{82.0-89.4} \mathrm{Or}_{0.9-0.3}, \mathrm{~N}=2\right)$.

Classification: Howardite.

Specimens: $12.2 \mathrm{~g}$ including one polished thin section at $U W B$; remainder with DPitt.

Northwest Africa 12262 (NWA 12262) 
(Northwest Africa)

Purchased: $2018 \mathrm{Jul}$

Classification: Martian meteorite (Shergottite)

History: Purchased in Ouarzazate, Morocco by Mohamed Aid in July 2018.

Petrography: (A. Irving and S. Kuehner, UWS; P. Carpenter, WUSL) Mediumgrained with diabasic texture and some small, dark glassy shock melt pockets. Composed predominantly of zoned clinopyroxene and maskelynite with accessory fine grained intergrowths of hedenbergite + fayalite + silica, Fe-Ti oxide (finely exsolved), merrillite and pyrrhotite.

Geochemistry: Subcalcic augite $\left(\mathrm{Fs}_{28.5-30.6} \mathrm{Wo}_{28.2-23.7}, \mathrm{FeO} / \mathrm{MnO}=30, \mathrm{~N}=2\right)$, pigeonite $\left(\mathrm{Fs}_{41.3} \mathrm{Wo}_{15.5}, \mathrm{FeO} / \mathrm{MnO}=31\right)$, ferropigeonite $\left(\mathrm{Fs}_{71.1-83.4} \mathrm{Wo}_{17.1-11.3}\right.$, $\mathrm{FeO} / \mathrm{MnO}=38-45, \mathrm{~N}=4)$, maskelynite $\left(\mathrm{An}_{50.9-52.1} \mathrm{Or}_{2.1}, \mathrm{~N}=2\right)$.

Classification: Martian (shergottite, diabasic)

Specimens: $26.3 \mathrm{~g}$ including one polished thin section at $U W B$; remainder with Aaronson.

\section{Northwest Africa 12265 (NWA 12265) \\ (Northwest Africa) \\ Purchased: 2015 Jan \\ Classification: HED achondrite (Eucrite, melt breccia)}

History: Purchased in Rissani, Morocco by Adam Aaronson in January 2015. Petrography: (A. Irving and S. Kuehner, UWS) Composed predominantly of a vesicular quenched melt rock lithology enclosing sparse remnant clasts of shocked gabbroic eucrite. The fine grained melt rock lithology consists of aluminous pigeonite (with two distinct $\mathrm{Fe} / \mathrm{Mg}$ ratios) and calcic plagioclase with accessory $\mathrm{Ti}$ poor chromite, Cr-ulvöspinel and troilite. Gabbroic eucrite clasts contain recrystallized pigeonite and polycrystalline calcic plagioclase with minor fayalite, silica polymorph, Ti-poor chromite, Ti-chromite, trolite and minor barite.

Geochemistry: Pigeonite $\left(\mathrm{Fs}_{31.2-38.4} \mathrm{Wo}_{12.0-17.1}, \mathrm{FeO} / \mathrm{MnO}=26-28, \mathrm{Al}_{2} \mathrm{O}_{3}=2.3-5.7\right.$ wt.\%, $\mathrm{N}=3$ ), ferroan pigeonite $\left(\mathrm{Fs}_{56.6-58.7} \mathrm{Wo}_{16.8-19.6}, \mathrm{FeO} / \mathrm{MnO}=32-33, \mathrm{Al}_{2} \mathrm{O}_{3}=\right.$ 0.8-1.1 wt.\%), fayalite $\left(\mathrm{Fa}_{80.4-80.8}, \mathrm{FeO} / \mathrm{MnO}=45-47, \mathrm{~N}=2\right)$, plagioclase $\left(\mathrm{An}_{88.0-}\right.$ ${ }_{89.9} \mathrm{Or}_{0.4-0.8}, \mathrm{~N}=2$ ).

Classification: Eucrite (vesicular, shock-melted breccia).

Specimens: $20.6 \mathrm{~g}$ including one polished thin section at $U W B$; remainder with Aaronson.

\section{Northwest Africa 12267 (NWA 12267)}

(Northwest Africa)

Purchased: 2018 Jun

Classification: HED achondrite (Diogenite)

History: Purchased by Craig Zlimen in June 2018 from a Moroccan dealer. Petrography: (A. Irving, UWS and P. Carpenter, WUSL) Breccia composed predominantly of larger and smaller angular clasts of orthopyroxene with accessory 
chromite, Ti-chromite, calcic plagioclase, troilite and rare olivine (as inclusions in orthopyroxene). Some cross-cutting veinlets of goethite are present.

Geochemistry: Orthopyroxene $\left(\mathrm{Fs}_{22.8-28.5} \mathrm{Wo}_{2.9-1.3}, \mathrm{FeO} / \mathrm{MnO}=27-31, \mathrm{~N}=3\right)$, plagioclase $\left(\mathrm{An}_{83.4} \mathrm{Or}_{1.1} ; \mathrm{An}_{96.3} \mathrm{Or}_{0.1} ; \mathrm{N}=2\right)$, olivine $\left(\mathrm{Fa}_{22.6}, \mathrm{FeO} / \mathrm{MnO}=49\right)$.

Classification: Diogenite (breccia).

Specimens: $18.1 \mathrm{~g}$ including one polished thin section at $U W B$; remainder with $\mathrm{Mr}$.

C. Zlimen.

\section{Northwest Africa 12268 (NWA 12268)}

(Northwest Africa)

Purchased: 2018 Jun

Classification: Carbonaceous chondrite (CV3)

History: Purchased by Craig Zlimen in June 2018 from a Moroccan dealer.

Petrography: (A. Irving, UWS and P. Carpenter, WUSL) Well-formed granular chondrules (apparent diameter $950 \pm 450 \mu \mathrm{m}, \mathrm{N}=22$ ) plus amoeboid to irregular, fine grained CAI are set in a very fine grained matrix ( $\sim 30$ vol.\%, black in thin section).

Geochemistry: Olivine $\left(\mathrm{Fa}_{0.8-53.0}, \mathrm{~N}=6\right)$, orthopyroxene $\left(\mathrm{Fs}_{0.7-2.4} \mathrm{Wo}_{1.0-1.2}, \mathrm{~N}=2\right)$, clinopyroxene $\left(\mathrm{Fs}_{1.0-1.7} \mathrm{Wo}_{37.9-38.3}, \mathrm{~N}=2\right)$.

Classification: Carbonaceous chondrite (CV3).

Specimens: $24.5 \mathrm{~g}$ including one polished thin section at $U W B$; remainder with $\mathrm{Mr}$.

C. Zlimen.

Northwest Africa 12269 (NWA 12269)

(Northwest Africa)

Purchased: $2018 \mathrm{Jul}$

Classification: Martian meteorite (Shergottite)

History: Purchased in Ouarzazate, Morocco by Mohamed Aid in July 2018.

Petrography: (A. Irving and S. Kuehner, UWS; P. Carpenter, WUSL) Relatively fine grained with intersertal texture. Composed predominantly of zoned clinopyroxene and maskelynite (some exhibiting a curved, narrow lath-like habit) with accessory fine grained intergrowths of hedenbergite+fayalite + silica, $\mathrm{Fe}-\mathrm{Ti}$ oxides, merrillite and pyrrhotite.

Geochemistry: Subcalcic augite $\left(\mathrm{Fs}_{24.8-41.8} \mathrm{Wo}_{31.4-24.3}, \mathrm{FeO} / \mathrm{MnO}=30-33, \mathrm{~N}=3\right)$, ferropigeonite $\left(\mathrm{Fs}_{66.9-73.0} \mathrm{Wo}_{16.6-16.5}, \mathrm{FeO} / \mathrm{MnO}=41-43, \mathrm{~N}=2\right)$, maskelynite $\left(\mathrm{An}_{51.9-}\right.$ 53.1 $\mathrm{Or}_{1.3-1.2}, \mathrm{~N}=2$ ).

Classification: Martian (shergottite, fine-intersertal)

Specimens: $20.5 \mathrm{~g}$ including one polished thin section at $U W B$; remainder with Mr. Aaronson.

Northwest Africa 12270 (NWA 12270)

Mauritania 
Purchased: 2018

Classification: Lunar meteorite (feldspathic breccia)

History: Purchased by Terry Boudreaux from a meteorite dealer in Mauritania.

Physical characteristics: Six identically appearing pieces, no fusion crust, saw cut reveals a fragmental breccia with numerous white feldspathic clasts set in a dark gray ground mass.

Petrography: (C. Agee, $U N M$ ) This meteorite consists of primarily of fragmental pyroxene, olivine, feldspathic clasts and shock melt. Ubiquitous Ti-rich chromite and ilmenite present, rare Fe-Ni metal detected.

Geochemistry: (C. Agee, $U N M$ ) olivine $\mathrm{Fa}_{42.7 \pm 7.3}, \mathrm{Fe} / \mathrm{Mn}=94 \pm 9$, $\mathrm{n}=14$; pigeonite $\mathrm{Fs}_{37.0 \pm 8.0} \mathrm{Wo}_{17.9 \pm 6.9}, \mathrm{Fe} / \mathrm{Mn}=56 \pm 5, \mathrm{n}=13$; plagioclase $\mathrm{An}_{96.6 \pm 0.4}, \mathrm{n}=6$; Shock melt (20 $\mu \mathrm{m}$ defocused electron beam, proxy for bulk meteorite composition):

$\mathrm{SiO}_{2}=44.8 \pm 1.3, \mathrm{TiO}_{2}=0.31 \pm 0.08, \mathrm{Al}_{2} \mathrm{O}_{3}=26.0 \pm 3.9, \mathrm{Cr}_{2} \mathrm{O}_{3}=0.15 \pm 0.09$, $\mathrm{MgO}=5.0 \pm 2.2, \mathrm{FeO}=5.9 \pm 1.7, \mathrm{MnO}=0.08 \pm 0.03, \mathrm{CaO}=16.1 \pm 1.8, \mathrm{NiO}=0.02 \pm 0.01$, $\mathrm{Na}_{2} \mathrm{O}=0.44 \pm 0.07, \mathrm{~K}_{2} \mathrm{O}=0.08 \pm 0.01\left(\right.$ all $\left.w t^{0} \%\right), \mathrm{n}=7$.

Classification: Lunar feldspathic breccia

Specimens: $29.2 \mathrm{~g}$ including a probe mount on deposit at UNM, Terry Boudreaux holds the main mass.

\section{Northwest Africa 12271 (NWA 12271)}

(Northwest Africa)

Purchased: 2018

Classification: Ureilite

History: Purchased in Guelmim, Morocco, July 2018.

Physical characteristics: Single stone; sandblasted exterior; saw cut reveals an interior mosaic of brown crystals; minor amount iron-staining present.

Petrography: (C. Agee, UNM) This meteorite consists of olivine, pigeonite, and augite. Ubiquitous graphite throughout. Fine grained Fe-metal is present along grain boundaries.

Geochemistry: (C. Agee) olivine $\mathrm{Fa}_{20.8 \pm 0.1}, \mathrm{Fe} / \mathrm{Mn}=44 \pm 1, \mathrm{n}=7$; pigeonite $\mathrm{Fs}_{17.4 \pm 0.2} \mathrm{Wo}_{4.3 \pm 0.2}, \mathrm{Fe} / \mathrm{Mn}=27 \pm 2, \mathrm{n}=5$; augite $\mathrm{Fs}_{10.9 \pm 0.1} \mathrm{Wo}_{34.5 \pm 0.1}, \mathrm{Fe} / \mathrm{Mn}=19 \pm 0, \mathrm{n}=2$.

Classification: Augite and graphite-bearing ureilite Specimens: $20.2 \mathrm{~g}$ including a probe mount on deposit at $U N M$, Zouhair Fettouh holds the main mass.

\section{Northwest Africa 12272 (NWA 12272)}

(Northwest Africa)

Purchased: 2017 Oct

Classification: Ordinary chondrite (LL7)

Petrography: (K. Metzler, IfP) Unbrecciated ordinary chondrite with distinct recrystallization texture. Large areas of secondary feldspar grains (many with sizes $>100 \mu \mathrm{m}$ ) which poikilitically enclose olivine and pyroxene grains. Many plagioclase grains with rectangular outlines due to the alignement along boundaries 
of recrystallized pyroxene crystals. Some metal veins up to $4 \mathrm{~mm}$ are observed. The outlines of a few large chondrules are still dimly visible.

Geochemistry: Mineral compositions and geochemistry: The mean olivine composition is $\mathrm{Fa}_{29.9 \pm 0.3}(29.1-30.5)$; $\mathrm{n}=14$. The mean low-Ca pyroxene composition is $\mathrm{Fs}_{23.9 \pm 0.4} \mathrm{Wo}_{4.1 \pm 0.3}\left(\mathrm{Fs}_{23.3-24.4} \mathrm{Wo}_{3.6-4.5}\right), \mathrm{n}=14$; Mean $\mathrm{CaO}$ concentration in low-Ca pyroxene: $2.1 \pm 0.2(1.9-2.3) \mathrm{wt} \%$. The mean composition of Ca-rich pyroxene is $\mathrm{Fs}_{12.8 \pm 0.9} \mathrm{Wo}_{38.5 \pm 1.9}\left(\mathrm{Fs}_{10.8-13.9} \mathrm{Wo}_{36.7-41.9}\right), \mathrm{n}=16$.

Classification: LL chondrite based on mineral chemistry. Petrologic type 7 based on the equilibrated state of olivine and pyroxene, and large grain size of secondary plagioclase. According to Tait et al. (2014) the transition between types 6 and 7 is a $\mathrm{CaO}$ concentration in low-Ca pyroxene of $1.0 \mathrm{wt} \%$; in this sample the respective value is $2.1 \mathrm{wt} \%$. According to Brearley and Jones (1998) Ca-rich pyroxene in type 6 chondrites has a Wo value of $45 \mathrm{~mol} \%$, which decreases in type 7 chondrites to 45-36 mol\%. In this sample the respective value is $38.5 \mathrm{~mol} \%$.

\section{Northwest Africa 12273 (NWA 12273)}

(Northwest Africa)

Purchased: 2018

Classification: Ungrouped chondrite

History: Purchased by Jay Piatek from a Moroccan meteorite dealer on October 25, 2018.

Physical characteristics: Dense, single individual. Fusion crust absent; abundant bright metal; scattered small chondrules are visible.

Petrography: (C. Agee, UNM) This meteorite consists of approximately $75 \%$ metal in the form of kamacite $(\sim 90 \%)$ and taenite $(\sim 10 \%)$ grains, diameters ranging from 50-500 $\mu \mathrm{m}$. Minor troilite was observed throughout. Silicates occur primarily in the form of chondrules, most are porphyritic, diameters in the range 100-500 $\mu \mathrm{m}$, making up approximately $20 \%$ of this meteorite.

Geochemistry: (C. Agee) Olivine $\mathrm{Fa}_{26.2 \pm 3.3}, \mathrm{Fe} / \mathrm{Mn}=55 \pm 5$, $\mathrm{n}=29$; low-Ca pyroxene $\mathrm{Fs}_{15.3 \pm 1.8} \mathrm{Wo}_{0.7 . \pm 0.2}, \mathrm{Fe} / \mathrm{Mn}=23 \pm 2, \mathrm{n}=11$. Oxygen isotopes (K. Ziegler, UNM): 3 acidwashed fragments analyzed by laser fluorination gave $\delta^{18} \mathrm{O}=5.258,5.613,7.249$; $\delta^{17} \mathrm{O}=3.939,3.971,4.786 ; \Delta^{17} \mathrm{O}=1.163,1.007,0.959$ (linearized, all per mil, TFL slope $=0.528)$.

Classification: Ungrouped chondrite. This is a metal-rich chondrite with oxygen isotopes that overlap with LL-chondrites. The olivine Fa content is consistent with L3, however the low-Ca pyroxene Fs is consistent with H4. The high metal content is inconsistent with ordinary chondrite. Texturally, this meteorite resembles a CB$\mathrm{b}$, however the oxygen isotopes and $\mathrm{Fa}$ and $\mathrm{Fs}$ are unlike $\mathrm{CB}$ chondrites.

Specimens: $23.2 \mathrm{~g}$ including a probe mount on deposit at UNM, Jay Piatek holds the main mass of $240 \mathrm{~g}$.

Northwest Africa 12279 (NWA 12279)

Tindouf, Algeria 
Find: 2016 Oct 10

Classification: Lunar meteorite (feldspathic breccia)

History: The meteorite was purchased in Denver USA by Zhouping Guo and Guobing Zhong on September, 2017 from Adam Aaronson.

Physical characteristics: A single bulk specimen, with sepia fresh fusion crust and low weathering degree. A large impact molten mass was observed in the specimen.

Petrography: This lunar meteorite comprises two petrographic areas: impact melt breccia and anorthosite. The anorthosite includes forsterite, pigeonite, low-Ca pyroxene, plagioclase and minor minerals such as ilmenite, chromite, quartz and other opaque minerals. It has a subophitic structure. The impact-melt breccia contains various types of lithic clasts (anorthosite, olivine-gabbro, gabbro), mineral and glassy fragments. The matrix consists of very fine-grained, cryptocrystalline $(\sim 0.1 \mathrm{~mm})$ and vitric particles with sporadic small vesicles.

Geochemistry (JK. Zhou, HY. Chen, ZP. Xia GUT): Olivine, Fa ${ }_{12.8-46.3} \mathrm{Fo}_{53.8-87.3}$, $\mathrm{FeO} / \mathrm{MnO}=66.3-96.9$ (n=29); Orthopyroxene, $\mathrm{Fs}_{15.5-32.2} \mathrm{Wo}_{2.98-4.22} \mathrm{En}_{64.9-80.3}$, $\mathrm{Fe} / \mathrm{Mn}(\mathrm{mol})=54.5-69.3(\mathrm{n}=3)$; Pigeonite: $\mathrm{Fs}_{23.2-53.1} \mathrm{Wo}_{7.19-23.7} \mathrm{En}_{39.8-57.4}$, $\mathrm{Fe} / \mathrm{Mn}(\mathrm{mol})=51.1-61.7(\mathrm{n}=6) ;$ Augite, $\mathrm{Fs}_{8.42-44.4} \mathrm{Wo}_{31.4-44.1} \mathrm{En}_{18.7-47.5}$, $\mathrm{Fe} / \mathrm{Mn}(\mathrm{mol})=37.1-53.9(\mathrm{n}=14)$; Plagioclase: $\mathrm{An}_{92.9-98.5} \mathrm{Or}_{0.06-0.69}(\mathrm{n}=15)$; Spinel: $\mathrm{MgO}, 20.1 \%-23.6 \% ; \mathrm{Al}_{2} \mathrm{O}_{3}, 59.5 \%-69.2 \%$; $\mathrm{Cr}_{2} \mathrm{O}_{3}, 3.91 \%-6.00 \%$; $\mathrm{FeO}, 6.05 \%$ $7.46 \%$.

Classification: Lunar (anorthositic impact-melt breccia)

Specimens: $30.2 \mathrm{~g}$ including one polished thin section are deposited in GUT; Remaining material with Guobing Zhong and Zhouping Guo.

\section{Northwest Africa 12280 (NWA 12280)}

Tindouf, Algeria

Find: 2016 Oct 10

Classification: Martian meteorite (Shergottite)

History: The meteorite was purchased in Denver USA by Zhouping Guo and Guobing Zhong on September, 2017 from Adam Aaronson.

Physical characteristics: A single bulk specimen, no fusion crust. Coarse-grained absinthe-green minerals can be observed. Weathering is strong.

Petrography: This meteorite is composed of a moderate-grained $(20-100 \mu \mathrm{m})$ assemblage of pyroxene (38 vol\%), olivine (51 vol\%), maskelynite ( $7 \mathrm{vol} \%$ ) and minor opaque minerals such as ilmenite, chromite and spinel. Maskelynite appears to be injected between pyroxene and olivine crysts.

Geochemistry (JK. Zhou, HY. Chen, ZP. Xia GUT): olivine: Fa $35.2-37.3(\mathrm{n}=42)$, $\mathrm{Fe} / \mathrm{Mn}(\mathrm{mol})=48.3-53.3(\mathrm{n}=42)$; pigeonite: $\mathrm{Fs}_{18.8-28.2} \mathrm{Wo}_{6.50-15.4} \mathrm{En}_{56.8-68.6}$, $\mathrm{Fe} / \mathrm{Mn}(\mathrm{mol})=24.5-30.0, \mathrm{Mg} \#=67.1-73.8(\mathrm{n}=15)$; augite: $\mathrm{Fs}_{13.9-20.4} \mathrm{Wo}_{26.4-43.3} \mathrm{En}_{42.8-}$ 53.3, Fe/Mn(mol)=23.6-30.0, Mg\#=70.9-73.3 (n=20); Maskelynite: An $_{49.5-59.9} \mathrm{Or}_{2.29-}$ ${ }_{3.62} \mathrm{Ab}_{36.9-46.9}(\mathrm{n}=17)$; Spinel: $\mathrm{MgO}, 2.85 \%-4.15 \% ; \mathrm{Al}_{2} \mathrm{O}_{3}, 4.08 \%-7.17 \%$; $\mathrm{TiO}_{2}, 12.6 \%-19.6 \% ; \mathrm{Cr}_{2} \mathrm{O}_{3}, 20.1 \%-31.7 \% ; \mathrm{FeO}, 44.3 \%-52.8 \%$.

Classification: Martian (shergottite) 
Specimens: $18.5 \mathrm{~g}$ including one polished thin section are deposited in GUT; Remaining material $60.5 \mathrm{~g}$ with Guobing Zhong and Zhouping Guo.

\section{Northwest Africa 12281 (NWA 12281) \\ (Northwest Africa) \\ Purchased: 2013 \\ Classification: Ordinary chondrite (LL3)}

History: Acquired from Ruben Garcia from 2013 Tucson Gem and Mineral Show. Physical characteristics: Sub-rounded, $30.6 \mathrm{~g}$ meteorite partially covered in fusion crust. Interior is dominantly light colored with well-developed chondrules and mineral fragments supported by a sparse dark matrix. Metals and sulfides finegrained. Weathering is heterogeneous and is dominantly W1 with patches of W3. Petrography: (Bercovici, H., Dolinschi, J., Dunham, E., Franco, G., Garani, J., Loescher, G., $A S U$ ): Visually, the meteorite is estimated to be $80 \%$ chondrules and fragments, $16 \%$ matrix, and $3 \%$ opaques. Chondrules typically 0.2 to $1.5 \mathrm{~mm}$ in diameter, with an estimated mean of $0.8 \mathrm{~mm}$. Range of chondrules types present including PP, PO, POP, BO, and RP. Some PO chondrules contain purple (plane polarized light) glass. Several chondrules are mantled by sulfides with minor metal. The largest chondrule $(9 \times 5 \mathrm{~mm})$ is composed of a barred olivine core, PO mantle, and porphyritic low-Ca pyroxene rim. Another chondrule $(3 \times 4 \mathrm{~mm}$ troilitemantled PO) contains euhedral olivine phenocrysts that have a comb-like overgrowth of olivine (with different BSE brightness) protruding into the devitrified mesostatis. Sulfide is more abundant than metal. Metal and troilite occur in three settings: fine-grained and dispersed in the matrix; rounded grains to 100 microns; and rarely as nodules. The largest nodule $(1.5 \times 1 \mathrm{~mm})$ consists of a troilite with rounded grains of kamacite. This nodule contains a few grains (to 20 $\mu \mathrm{m})$ of native copper. Troilite is polycrystalline with occasional shock twins. Shock grade consistent with $\mathrm{S} 2$ and weathering with $\mathrm{W} 2 / 3$.

Geochemistry: Olivine $\mathrm{Fa}_{20.0 \pm 6.2}$, range $\mathrm{Fa}_{12.0-29.9} \mathrm{Cr}_{2} \mathrm{O}_{3}$ in olivine grain cores is $0.62 \pm 0.07$, range $0.46-0.69, \mathrm{n}=10$. Low-Ca pyroxene $\mathrm{Fs}_{15.7 \pm 7.8} \mathrm{Wo}_{1.4 \pm 1.2}$, range $\mathrm{Fs}_{1.9-}$ ${ }_{33.7} \mathrm{Wo}_{0.2-4.8}, \mathrm{n}=49$. High-Ca pyroxene $\mathrm{Fs}_{17.4 \pm 8.6} \mathrm{Wo}_{16.1 \pm 9.5}$, range $\mathrm{Fs}_{11.0-42.1} \mathrm{Wo}_{5.1-41.2}$, $\mathrm{n}=18$. Feldspar grains, found in only one chondrule, are $A n_{97.9 \pm 2.5} A b_{2.1 \pm 2.5}$, ranging $\mathrm{An}_{95.1-100.0} \mathrm{Ab}_{0.0-4.8}, \mathrm{n}=3$. Metal compositions in $\mathrm{wt} \%$ are Fe78.46 $\pm 14.98 \mathrm{Ni21} .14 \pm 14.91 \mathrm{Co} 0.40 \pm 0.14 \mathrm{n}=5$.

Classification: LL based on he low metal content. Estimated petrologic type of 3.4 based on $\mathrm{Fa}$ and $\mathrm{Fs}$ spread and $\mathrm{Cr}_{2} \mathrm{O}_{3}$ content in olivine grain cores.

Northwest Africa 12282 (NWA 12282)

(Northwest Africa)

Find: 2016

Classification: HED achondrite (Eucrite, polymict)

History: Purchased by Michael Farmer from a Moroccan meteorite dealer at the 2016 Tucson Gem and Mineral show. 
Physical characteristics: Sand-blasted, angular, orange-stained $141.5 \mathrm{~g}$ meteorite with patches of fusion crust. Light-colored clasts to $8 \mathrm{~mm}$ readily visible at the surface of the stone and supported by a gray matrix. Fresh stone with low weathering.

Petrography: (Dillon, S., Dunham, E., Fitch, R., Karageozian, M., Sedler, M., Teichert, Z., ASU): The meteorite is a breccia dominated by angular to sub-rounded grains and clasts to $8 \mathrm{~mm}$. The petrography is based on two slices $(4 \times 3 \mathrm{~cm}$ and 4 $\times 4 \mathrm{~cm}$ ), and a polished mount. The $4 \times 3 \mathrm{~cm}$ slice is light colored with angular to sub-angular clasts to $4 \mathrm{~mm}$ in a poorly sorted clastic matrix dominated by pyroxene and plagioclase. The $4 \times 4 \mathrm{~cm}$ slice is petrographically more varied with a range of clast textures (subophitic, diabasic, granulitic) supported by the eucritic debris. This slice has a prominent $6 \times 5 \mathrm{~mm}$ subangular fine-grained dark clast containing a 3$\mathrm{mm}$ spherical fine-grained clast with similar mineralogy as the bulk eucrite. Accessory minerals include silica, troilite, chromite, and ilmenite. The majority of the pyroxene grains exhibit exsolution lamellae. Plagioclase shows extensive twinning. Pyroxene grains are primarily Ca-poor pyroxene with Ca-rich pyroxene present in exsolution lamellae. Plagioclase grains are primarily anorthite rich end members. The sample has a low shock grade; one melt clast within a pyroxene clast is present and is potentially representative of a previous higher-stage shock event. Geochemistry: Pyroxene without exsolution lamellae is pigeonite

$\mathrm{Fs}_{52.6 \pm 4.4} \mathrm{Wo}_{10.4 \pm 5.6}$, range $\mathrm{Fs}_{43.7-55.8} \mathrm{Wo}_{6.5-22.0}, \mathrm{FeO} / \mathrm{MnO}=33.2 \pm 2.2, \mathrm{n}=8$. Pyroxene with exsolution lamellae is predominantly clinopyroxene $\mathrm{Fs}_{57.2 \pm 0.7} \mathrm{Wo}_{4.4 \pm 0.9}$, range $\mathrm{Fs}_{55.8-58.2} \mathrm{Wo}_{2.7-5.4}, \mathrm{FeO} / \mathrm{MnO}=33.0 \pm 2.1, \mathrm{n}=12$, the lamellae are orthopyroxene $\mathrm{Fs}_{27.3 \pm 0.9} \mathrm{Wo}_{41.9 \pm 0.7}$, range $\mathrm{Fs}_{26.3-28.8} \mathrm{Wo}_{40.7-42.8}, \mathrm{FeO} / \mathrm{MnO}=34.1 \pm 1.7, \mathrm{n}=8$. Plagioclase has composition $\mathrm{An}_{88.5 \pm 6.0}, \mathrm{n}=18$.

Specimens: An original mass of $141.5 \mathrm{~g}$ was acquired by MFarmer; $21.99 \mathrm{~g}$ and one thin section is held by $A S U$. The main mass is held by Michael Farmer.

\section{Northwest Africa 12283 (NWA 12283)}

(Northwest Africa)

Purchased: 2018

Classification: HED achondrite (Eucrite)

History: The meteorite was purchased from a local meteorite dealer in Agadir, Morocco.

Physical characteristics: Grayish fragments without fusion crust.

Petrography: The meteorite is a brecciated medium-grained basalt predominantly composed of exsolved pyroxene and often lath-shaped calcic plagioclase with average grains sizes of about $500 \mu \mathrm{m}$. Minor phases include silica, ilmenite, chromite, and troilite. No metallic iron has been detected.

Geochemistry: low-Ca pyroxene: $\mathrm{Fs}_{60.3 \pm 0.5} \mathrm{Wo}_{2.4 \pm 0.7}\left(\mathrm{Fs}_{59.3-60.9} \mathrm{Wo}_{1.8-4.0}, \mathrm{n}=12\right.$, $\mathrm{FeO} / \mathrm{MnO}=31-34)$; Ca-pyroxene: $\mathrm{Fs}_{26.7 \pm 0.4} \mathrm{Wo}_{43.4 \pm 0.7}\left(\mathrm{Fs}_{25.7-27.4} \mathrm{Wo}_{41.7-44.0}, \mathrm{n}=12\right.$, $\mathrm{FeO} / \mathrm{MnO}=29-33)$; calcic plagioclase: $\mathrm{An}_{89.9 \pm 2.1}\left(\mathrm{An}_{86.4-92.5}, \mathrm{n}=12\right)$ 
Northwest Africa 12284 (NWA 12284)

(Northwest Africa)

Purchased: 2018

Classification: Ordinary chondrite (LL3)

History: The meteorite was purchased from a local meteorite dealer in Erfoud, Morocco.

Physical characteristics: Brownish fragments without fusion crust.

Petrography: The meteorite displays a chondritic texture with mostly rounded chondrules (mean diameter about $0.7 \mathrm{~mm}$ ) in a fine-grained brownish and strongly altered matrix that contains sulfides and rare FeNi metal.

Classification: LL based on magnetic susceptibility.

\section{Northwest Africa 12285 (NWA 12285)}

(Northwest Africa)

Purchased: 2018

Classification: Ordinary chondrite (L3)

History: The meteorite was purchased from a local meteorite dealer in Morocco. Physical characteristics: Dark brownish individual without fusion crust.

Petrography: The meteorite displays a chondritic texture with mostly rounded and well packed chondrules (mean diameter about $0.6 \mathrm{~mm}$ ) in a more fine-grained dark matrix that contains sulfides and FeNi metal.

\section{Northwest Africa 12286 (NWA 12286) \\ (Northwest Africa) \\ Purchased: 2018 \\ Classification: Carbonaceous chondrite (CK5)}

History: The meteorite was purchased from a local meteorite dealer in Morocco. Physical characteristics: Grayish to slightly greenish individual lacking any fusion crust.

Petrography: The meteorite displays a greyish to greenish interior and is predominantly composed of recrystallized Fe-rich olivine dominated matrix with scattered well defined chondrules. Cr-rich magnetite is abundant. More minor phases include intermediate plagioclase, low-Ca pyroxene, Ca-pyroxene and troilite; metal was not detected.

Geochemistry: olivine: $\mathrm{Fa}_{29.9 \pm 0.5}, \mathrm{n}=14, \mathrm{FeO} / \mathrm{MnO}=110.5 \pm 8.4$; low-Ca pyroxene: $\mathrm{Fs}_{25.7 \pm 0.3} \mathrm{Wo}_{0.8 \pm 0.1}, \mathrm{n}=3$; Ca-pyroxene: $\mathrm{Fs}_{7.0 \pm 0.4} \mathrm{Wo}_{47.5 \pm 0.3}, \mathrm{n}=3$

\section{Northwest Africa 12287 (NWA 12287)}

(Northwest Africa)

Purchased: 2018

Classification: Carbonaceous chondrite (CM2)

History: The meteorite was purchased from a local meteorite dealer in Morocco. 
Physical characteristics: Dark grayish individual without fusion crust.

Petrography: Brecciated carbonaceous chondrite composed of small chondrules (mean diameter about $230 \mu \mathrm{m}$ ), CAIs, and mineral fragments most of which are surrounded by fine-grained dust rims. The matrix is Fe-rich and contains abundant sulfides, calcite and phyllosilicates.

\section{Northwest Africa 12288 (NWA 12288) \\ (Northwest Africa) \\ Purchased: 2017 \\ Classification: Pallasite}

History: The meteorite was purchased from a local meteorite dealer in Morocco. Physical characteristics: Many up to $1 \mathrm{~cm}$ sized brownish fragments without fusion crust.

Petrography: The meteorite is composed of about $5 \mathrm{~mm}$ sized subrounded olivine grains surrounded by a matrix of partly layered brownish iron oxides or hydroxides. No pristine FeNi-metal has been found in the two fragments studied.

Geochemistry: olivine: $\mathrm{Fa}_{12.6-13.2}, \mathrm{FeO} / \mathrm{MnO}: 38-48$

\section{Northwest Africa 12289 (NWA 12289)}

(Northwest Africa)

Purchased: 2018

Classification: Ordinary chondrite (L5, melt breccia)

History: The meteorite was purchased from a local meteorite dealer in Errachidia, Morocco.

Physical characteristics: Brownish individual without fusion crust.

Petrography: The meteorite is a melt breccia composed of abundant recrystallized shock melt regions with characteristic FeNi metal and sulfide globules and embedded relict mineral fragments and some preserved chondrules. In chondritic portions olivine and low-Ca pyroxene are compositionally equilibrated and the plagioclase grain size is about $30 \mu \mathrm{m}$.

\section{Northwest Africa 12290 (NWA 12290) \\ (Northwest Africa) \\ Purchased: 2018 \\ Classification: HED achondrite (Eucrite)}

History: The meteorite was purchased from a local meteorite dealer in Layaoune, Morocco.

Physical characteristics: Brownish individual without fusion crust.

Petrography: The meteorite is an unbrecciated medium-grained basalt predominantly composed of exsolved pyroxene and mostly lath-shaped calcic plagioclase with average grains sizes of about $400 \mu \mathrm{m}$. Minor phases include silica, ilmenite, chromite, troilite, barite, and zircon. No metallic iron has been detected. 
The meteorite is strongly shock metamorphosed and crosscut by several shock melt veins. All plagioclase has been transformed into maskelynite.

Geochemistry: low-Ca pyroxene: $\mathrm{Fs}_{60.3 \pm 0.7} \mathrm{Wo}_{2.6 \pm 0.8}\left(\mathrm{Fs}_{58.2-61.1} \mathrm{Wo}_{2.1-5.3}, \mathrm{n}=16\right.$, $\mathrm{FeO} / \mathrm{MnO}=31-35)$; Ca-pyroxene: $\mathrm{Fs}_{26.3 \pm 1.0} \mathrm{Wo}_{42.9 \pm 1.3}\left(\mathrm{Fs}_{25.2-29.4} \mathrm{Wo}_{39.1-44.1}, \mathrm{n}=14\right.$, $\mathrm{FeO} / \mathrm{MnO}=28-37)$; calcic plagioclase: $\mathrm{An}_{89.3 \pm 1.0}\left(\mathrm{An}_{88.1-90.7}, \mathrm{n}=14\right)$

\section{Northwest Africa 12291 (NWA 12291)}

(Northwest Africa)

Purchased: 2018

Classification: Ordinary chondrite (LL3)

History: The meteorite was purchased from a local meteorite dealer in Layaoune, Morocco.

Physical characteristics: Two brownish fragments with some fusion crust.

Petrography: The meteorite shows a chondritic texture with sharply defined, mostly flattened and well packed chondrules (mean diameter about $0.9 \mathrm{~mm}$ ) in a fine-grained matrix, that contains sulfides and FeNi metal.

\section{Northwest Africa 12293 (NWA 12293) \\ (Northwest Africa) \\ Purchased: 2018 \\ Classification: HED achondrite (Eucrite)}

History: The meteorite was purchased from a local meteorite dealer in Layaoune, Morocco.

Physical characteristics: Grayish individual with some fusion crust.

Petrography: Unbrecciated coarse-grained basalt predominantly composed of exsolved pyroxene and often lath-shaped calcic plagioclase with average grains sizes of about $1 \mathrm{~mm}$. Minor phases include silica, ilmenite, chromite, and barite. No metallic iron has been detected. The meteorite contains abundant shock melt veins and pockets.

Geochemistry: low-Ca pyroxene: $\mathrm{Fs}_{60.3 \pm 0.7} \mathrm{Wo}_{2.1 \pm 0.2}\left(\mathrm{Fs}_{59.1-61.3} \mathrm{Wo}_{1.7-2.6}, \mathrm{n}=14\right.$, $\mathrm{FeO} / \mathrm{MnO}=29-33)$; Ca-pyroxene: $\mathrm{Fs}_{26.4 \pm 0.3} \mathrm{Wo}_{42.8 \pm 0.5}\left(\mathrm{Fs}_{25.8-26.8} \mathrm{Wo}_{41.9-43.6}, \mathrm{n}=15\right.$, $\mathrm{FeO} / \mathrm{MnO}=28-32)$; calcic plagioclase: $\mathrm{An}_{90.5 \pm 0.4}\left(\mathrm{An}_{89.8-91.4}, \mathrm{n}=15\right)$

\section{Northwest Africa 12297 (NWA 12297)}

(Northwest Africa)

Purchased: 2015

Classification: Ordinary chondrite $(L \sim 5)$

History: (Ziyao Wang) Purchased by Wang Ziyao from Sbai Mohamed at the mineral show in Beijing in Nov. 2015

Physical characteristics: (Wang Ziyao) Black 110.5 g stone with dark blackbrown fusion crust 
Petrography: (R. Bartoschewitz, Bart) Light-gray recrystallized matrix with well recognizable lighter chondrules $(0.5-1.6 \mathrm{~mm}$, av. $0.8 \mathrm{~mm})$ and irregular metal and sulphide.

Geochemistry: Magnetic susceptibility (R. Bartoschewitz, Bart $) \log \chi\left(\times 10^{-}\right.$ $\left.{ }^{9} \mathrm{~m}^{3} / \mathrm{kg}\right)=5.06$

Classification: Ordinary chondrite (L $\sim 5, \mathrm{~W} 0)$

Specimens: $20.1 \mathrm{~g}$ on deposit at Kiel, Wang Ziyao holds the main mass, and $4.5 \mathrm{~g}$ with Bart.

\section{Northwest Africa 12298 (NWA 12298)}

(Northwest Africa)

Purchased: 2015

Classification: Ordinary chondrite (LL6)

History: (Ziyao Wang) Purchased by Wang Ziyao from Sbai Mohamed at the mineral show in Beijing in Nov. 2015

Physical characteristics: (Wang Ziyao) $87.6 \mathrm{~g}$ Partly with fresh black fusion crust coverd gray-blue elongated stone.

Petrography: (R. Bartoschewitz, Bart) brecciated texture of light gray recrystallized matrix with poor developed chondrules up to $1.2 \mathrm{~mm}$, mineral fragments, tiny sulphide grains that somtimes build larger assemblages, and few metal specks up to $0.5 \mathrm{~mm}$.

Geochemistry: Magnetic susceptibility (R. Bartoschewitz, Bart $) \log \chi\left(\times 10^{-}\right.$ $\left.{ }^{9} \mathrm{~m}^{3} / \mathrm{kg}\right)=3.49$

Classification: Ordinary chondrite (LL $\sim 6, \mathrm{~W} 0)$

Specimens: $18.2 \mathrm{~g}$ on deposit at Kiel, Wang Ziyao holds the main mass, and $19 \mathrm{~g}$ with Bart.

\section{Northwest Africa 12299 (NWA 12299) \\ Morocco \\ Purchased: 2018 \\ Classification: Ordinary chondrite $(\mathrm{H} \sim 6)$}

History: (Ziyao Wang) Purchased by Wang Ziyao from Fayssal Mezgouri during the China (Hubei) Mineral \& Gem Show in Huangshi City in Sept 2018.

Physical characteristics: (Wang Ziyao) A red-purple stone of 4500 grams with some fusion crust.

Petrography: (R. Bartoschewitz, Bart) Gray recrystallized matrix with poor developed chondrules (0.2-0.8 $\mathrm{mm}$, av. $0.4 \mathrm{~mm}$ ), and much homogenous distributed irregular metal and sulphide.

Geochemistry: Magnetic susceptibility (R. Bartoschewitz, Bart) $\log \chi\left(\times 10^{-}\right.$ $\left.{ }^{9} \mathrm{~m}^{3} / \mathrm{kg}\right)=5.39$

Classification: Ordinary chondrite $(\mathrm{H} \sim 6, \mathrm{~W} 0)$

Specimens: $20.8 \mathrm{~g}$ on deposit at Kiel, Wang Ziyao holds the main mass, and $131 \mathrm{~g}$ with Bart. 


\section{Northwest Africa 12300 (NWA 12300)}

(Northwest Africa)

Purchased: 2018

Classification: Ordinary chondrite $(\mathrm{H} \sim 5)$

History: (Ziyao Wang) Purchased by Wang Ziyao from Sadam Sbai during the China (Hunan) Mineral \& Gem Show in Chenzhou City in May 2018.

Physical characteristics: (Wang Ziyao) Gray-black stone of $273 \mathrm{~g}$, metal bumps are recognizable on the surface.

Petrography: (R. Bartoschewitz, Bart) Gray-black recrystallized matrix with poor developed chondrules $(0.2-1.4 \mathrm{~mm}$, av. $0.5 \mathrm{~mm})$, irregular shaped metal and sulphide grains $(<0.1-1.5 \mathrm{~mm})$.

Geochemistry: Magnetic susceptibility (R. Bartoschewitz, Bart) $\log \chi\left(\times 10^{-}\right.$ $\left.{ }^{9} \mathrm{~m}^{3} / \mathrm{kg}\right)=5.12$

Classification: Ordinary chondrite (H $\sim 5, \mathrm{~W} 1)$

Specimens: $21.2 \mathrm{~g}$ on deposit at Kiel, Wang Ziyao holds the main mass, and $38 \mathrm{~g}$ with Bart.

\section{Northwest Africa 12301 (NWA 12301)}

(Northwest Africa)

Purchased: 2017 Apr

Classification: Ordinary chondrite (L5)

History: Discovered by an anonymous person and purchased in Erfoud in April 2017

Physical characteristics: One brown stone with regmaglypts. Cut face shows tiny metal specks.

Petrography: (R. Bartoschewitz, Bart) Microscopic examination of a thin section shows recrystallized and shocked matrix: mosaicism and planar fractures in olivine, undolatory extinction in plagioclase. Chondrules poor developed. Magnetic susceptibility $\log \chi\left(\times 10^{-9} \mathrm{~m}^{3} / \mathrm{kg}\right)=4.04$.

Geochemistry: (R. Bartoschewitz, Bart, P. Appel and B. Mader, Kiel) Olivine $\mathrm{Fa}_{24.3 \pm 0}, 9(\mathrm{n}=32)$; pyroxene $\mathrm{Fs}_{20.4 \pm 1.2} \mathrm{Wo}_{1.5 \pm 0.2}(\mathrm{n}=19)$; Ca-pyroxene $\mathrm{Fs}_{48.8} \mathrm{Wo}_{43.1}(\mathrm{n}=1)$; feldspar $\mathrm{An}_{10.1 \pm 0.3} \mathrm{Or}_{5.9 \pm 1.2}(\mathrm{n}=9)$. Kamacite $\mathrm{Ni}=2.9-5.3, \mathrm{Co}=1.0$ wt.-\% $(n=2)$. Sulfides: troilite, pyrrhotite, and pyrite.

Classification: Ordinary chondrite (type L5)

Specimens: $20.2 \mathrm{~g}$ on deposit at Kiel, main mass Kuntz.

\section{Northwest Africa 12320 (NWA 12320)}

Mauritania

Purchased: 2018 Apr

Classification: Angrite 
History: Two large, dark gray stones (2379 g and $1946 \mathrm{~g}$ ) plus several smaller stones were found together at an unspecified site in Mauritania, and subsequently purchased by Darryl Pitt in April-July 2018 from a dealer in Nouakchott, Mauritania. An additional $14.2 \mathrm{~g}$ of the same material (confirmed by electron microprobe analyses) was purchased by Ben Hoefnagels in October 2018 from a Moroccan dealer.

Petrography: (A. Irving and S. Kuehner, UWS) Diabasic texture (grainsize 0.3-0.5 $\mathrm{mm}$ ) with sparse vesicles containing partial interior coatings of secondary calcite and barite. Major minerals are zoned calcic olivine (with kirschsteinite rims), zoned Al-Ti-augite and anorthite (with slightly more sodic rims), together with accessory titanomagnetite, troilite and silicoapatite. Sparse xenocrysts of magnesian olivine with more ferroan reaction rims are present.

Geochemistry: Olivine $\left(\mathrm{Fa}_{39.6-79.7} \mathrm{Ln} 1.7-14.8, \mathrm{FeO} / \mathrm{MnO}=72-96, \mathrm{~N}=5\right)$, clinopyroxene $\left(\mathrm{Fs}_{20.5-45.9} \mathrm{Wo}_{51.6-52.8}, \mathrm{FeO} / \mathrm{MnO}=68-143, \mathrm{TiO}_{2}=1.5-4.3\right.$ wt.\%, $\mathrm{Al}_{2} \mathrm{O}_{3}=6.9-7.8$ wt. $\left.\%, \mathrm{~N}=4\right)$, kirschsteinite $\left(\mathrm{Fa}_{57.9-63.3} \operatorname{Ln} 36.7-34.3, \mathrm{FeO} / \mathrm{MnO}=\right.$ 68-75, $\mathrm{N}=2$ ), olivine xenocryst (core $\mathrm{Fa}_{13.8} \mathrm{Ln} 0.6$; $\mathrm{rim} \mathrm{Fa}_{35.2} \mathrm{Ln} 1.3 ; \mathrm{FeO} / \mathrm{MnO}=$ $85-103 ; \mathrm{N}=2$ ), anorthite (core $\mathrm{An}_{99.7} \mathrm{Ab}_{0.2} \mathrm{Or}_{0.0} ;$ rim $\mathrm{An}_{96.6} \mathrm{Ab}_{3.3} \mathrm{Or}_{0.1} ; \mathrm{N}=2$ ).

Oxygen isotopes (K. Ziegler, UNM) Analyses of acid-washed subsamples by laser fluorination gave, respectively $\delta^{17} \mathrm{O} 2.268,2.281,2.339 ; \delta^{18} \mathrm{O} 4.416,4.407,4.545$; $\Delta^{17} \mathrm{O}-0.063,-0.046,-0.060$ per mil.

Classification: Angrite (diabasic).

Specimens: $20.6 \mathrm{~g}$ including one polished thin section at $U W B$; remainder with DPitt, except for $14.2 \mathrm{~g}$ with Mr. B. Hoefnagels.

\section{Northwest Africa 12321 (NWA 12321) \\ (Northwest Africa) \\ Purchased: 2004 Oct \\ Classification: Ureilite}

History: Purchased in Erfoud, Morocco by Adam Aaronson in October 2004.

Petrography: (A. Irving and S. Kuehner, UWS) Protogranular aggregate of olivine $(\sim 80$ vol.\%) and pigeonite ( $\sim 20 \mathrm{vol} . \%)$. Olivine exhibits dark reduced, magnesian rims. Minor grain boundary graphite was observed.

Geochemistry: Olivine (cores $\mathrm{Fa}_{21.9-22.2} ;$ rim $\mathrm{Fa}_{7.4} ; \mathrm{N}=3$ ), pigeonite $\left(\mathrm{Fs}_{18.4-}\right.$ ${ }_{18.5} \mathrm{Wo}_{9.3-9.2}, \mathrm{~N}=2$ ).

Classification: Ureilite.

Specimens: $20.1 \mathrm{~g}$ including one polished thin section at $U W B$; remainder with Aaronson.

\section{Northwest Africa 12322 (NWA 12322)}

Niger

Purchased: 2018 Aug

Classification: Carbonaceous chondrite (CV3) 
History: Found in Niger and purchased in Morocco by Adam Aaronson in August 2018.

Petrography: (A. Irving and S. Kuehner, UWS) Well-formed granular and BO chondrules (some composite; apparent diameter $1250 \pm 690 \mu \mathrm{m}$ ) and fine grained CAI (some up to $4.5 \mathrm{~mm}$ across) are set in a fine grained matrix ( 30 vol.\%, orange-brown in thin section).

Geochemistry: Olivine $\left(\mathrm{Fa}_{0.2-45.3}, \mathrm{~N}=3\right)$, orthopyroxene $\left(\mathrm{Fs}_{0.7-0.9} \mathrm{Wo}_{1.1}, \mathrm{~N}=2\right)$, clinopyroxene $\left(\mathrm{Fs}_{2.5} \mathrm{Wo}_{36.0} ; \mathrm{Fs}_{21.4} \mathrm{Wo}_{44.1} ; \mathrm{N}=2\right)$.

Classification: Carbonaceous chondrite (CV3).

Specimens: $42 \mathrm{~g}$ including one polished thin section at $U W B$; remainder with Aaronson.

\section{Northwest Africa 12323 (NWA 12323)}

(Northwest Africa)

Purchased: 2018 Aug

Classification: Martian meteorite (Shergottite)

History: Purchased by Ben Hoefnagels in August 2018 from a dealer in Agadir, Morocco.

Physical characteristics: Single stone (447 g) exhibiting a brown weathered exterior and a fresh interior with obvious glassy maskelynite. The specimen is crosscut by several thin, dark shock melt veinlets.

Petrography: (A. Irving and S. Kuehner, UWS) Gabbroic assemblage of predominantly prismatic, zoned and twinned grains of clinopyroxene (up to $3.6 \mathrm{~mm}$ long) and maskelynite (some as thin, curved laths in sheaf-like groups) with accessory merrillite, ilmenite, ulvöspinel and pyrrhotite, plus minor secondary barite and calcite.

Geochemistry: Orthopyroxene core $\left(\mathrm{Fs}_{22.1} \mathrm{Wo}_{3.1}, \mathrm{FeO} / \mathrm{MnO}=32\right)$, pigeonite $\left(\mathrm{Fs}_{29.9} \mathrm{Wo}_{7.3} ; \mathrm{Fs}_{31.9-76.8} \mathrm{Wo}_{13.7-15.7} ; \mathrm{FeO} / \mathrm{MnO}=31-46 ; \mathrm{N}=5\right)$, subcalcic augite $\left(\mathrm{Fs}_{28.9-39.3} \mathrm{Wo}_{31.7-27.1}, \mathrm{FeO} / \mathrm{MnO}=33-37, \mathrm{~N}=2\right)$, maskelynite $\left(\mathrm{An}_{54.6-54.7} \mathrm{Or}_{0.6-0.5}, \mathrm{~N}\right.$ $=2)$.

Classification: Martian (shergottite, gabbroic).

Specimens: $22.1 \mathrm{~g}$ including one polished thin section at $U W B$; remainder with $\mathrm{Mr}$. B. Hoefnagels.

\section{Northwest Africa 12324 (NWA 12324)}

(Northwest Africa)

Purchased: 2017

Classification: Ordinary chondrite (L3)

History: The meteorite was purchased from a local meteorite dealer in Morocco.

Physical characteristics: Dark brownish individual with some fusion crust.

Petrography: The meteorite displays a chondritic texture with slightly flattened and slightly packed chondrules (mean diameter about $0.6 \mathrm{~mm}$ ) in a more fine- 
grained dark matrix that contains sulfides and FeNi metal. The meteorite contains shock melt veins.

\section{Northwest Africa 12326 (NWA 12326)}

(Northwest Africa)

Purchased: 2016

Classification: HED achondrite (Eucrite, polymict)

History: The meteorite was purchased from a local meteorite dealer in Morocco.

Physical characteristics: Two grayish individuals with fusion crust.

Petrography: The meteorite is a polymict breccia composed of mineral and lithic clasts set into a fine-grained clastic matrix. Lithic clasts are fine- and coarsegrained about 0.2 to $1.5 \mathrm{~mm}$ sized basaltic clasts and dark about 0.1 to $4 \mathrm{~mm}$ sized impact melt clasts. Main minerals in basaltic clasts and matrix are exsolved pyroxene and calcic plagioclase. Minor phases include silica, chromite, ilmenite, and zircon. No metallic Fe has been detected.

Geochemistry: low-Ca pyroxene: $\mathrm{Fs}_{55.1 \pm 0.3} \mathrm{Wo}_{3.4 \pm 0.4}\left(\mathrm{Fs}_{54.5-55.6} \mathrm{Wo}_{2.7-4.2}, \mathrm{n}=14\right.$, $\mathrm{FeO} / \mathrm{MnO}=31-36)$; Ca-pyroxene: $\mathrm{Fs}_{31.6 \pm 5.0} \mathrm{Wo}_{34.9 \pm 5.8}\left(\mathrm{Fs}_{25.2-39.3} \mathrm{Wo}_{26.1-42.2}, \mathrm{n}=16\right.$, $\mathrm{FeO} / \mathrm{MnO}=29-34)$; calcic plagioclase: $\mathrm{An}_{80.0 \pm 1.7}\left(\mathrm{An}_{77.2-82.3}, \mathrm{n}=13\right)$

\section{Northwest Africa 12327 (NWA 12327)}

(Northwest Africa)

Purchased: 2016

Classification: HED achondrite (Eucrite, polymict)

History: The meteorite was purchased from a local meteorite dealer in Morocco. Physical characteristics: Two dark grayish individuals with fusion crust.

Petrography: The meteorite is a coarse-grained basaltic breccia composed of up to $6 \mathrm{~mm}$ sized basaltic and up to $5 \mathrm{~mm}$ sized dark impact melt clasts set into a clastic matrix. Mineral phases are predominantly up to $1.5 \mathrm{~mm}$ sized exsolved pyroxene and lath shaped calcic plagioclase. Several low-Ca pyroxene grains are magmatically zoned indicating a low degree of thermal metamorphism. Minor phases include silica, chromite, and FeNi metal.

Geochemistry: low-Ca pyroxene: $\mathrm{Fs}_{41.1 \pm 4.2} \mathrm{Wo}_{2.6 \pm 0.9}\left(\mathrm{Fs}_{33.3-45.8} \mathrm{Wo}_{1.2-3.8}, \mathrm{n}=21\right.$, $\mathrm{FeO} / \mathrm{MnO}=28$-37); Ca-pyroxene: $\mathrm{Fs}_{21.0 \pm 0.3} \mathrm{Wo}_{41.9 \pm 0.6}\left(\mathrm{Fs}_{20.4-21.7} \mathrm{Wo}_{40.8-42.5}, \mathrm{n}=15\right.$, $\mathrm{FeO} / \mathrm{MnO}=23-27)$; calcic plagioclase: $\mathrm{An}_{91.0 \pm 1.6}\left(\mathrm{An}_{88.5-93.2}, \mathrm{n}=15\right)$

\section{Northwest Africa 12328 (NWA 12328)}

(Northwest Africa)

Purchased: 2017

Classification: Carbonaceous chondrite (CM1)

History: Ruben Garcia and Bob Cucchiara purchased eight similar-looking, partially fusion-crusted meteorite fragments weighting $43.2 \mathrm{~g}$ from Adam Aaronson at the 2017 Denver Gem show. 
Physical characteristics: The largest fragments $20.2 \mathrm{~g}$ and $10.6 \mathrm{~g}$ have one surface with well-developed, ropy fusion crust. Pieces without fusion crust are gray and show slight wind abrasion. Fresh broken surfaces exhibit an earthy fracture, are black and studded with tiny crystalline white flecks and sparse protruding chondrules and nodules. Stones are very soft and can be easily crushed by hand to a fine powder.

Petrography: (L. Garvie, $A S U$ ) Powder x-ray diffraction of the bulk meteorite shows a pattern dominated by serpentine, with low-intensity reflections for sulfides (mackinawite, pentlandite), magnetite, bassanite, carbonates (ankerite, calcite), and very weak reflections possibly from olivine. The 001 serpentine is a well-resolved doublet with maxima at 0.735 and $0.724 \mathrm{~nm}$. Powder XRD from each of the fragments comprising the $43.2 \mathrm{~g}$ shows the double-peaked 001 serpentine reflection. Powder XRD patterns from several nodules and chondrules shows patterns dominated by the $0.735 \mathrm{~nm}$ clay. One chondrule also shows a threecomponent $0.7 \mathrm{~nm}$ reflection and weak $1.4 \mathrm{~nm}$ peak, suggesting the presence of minor chlorite. A polished mount of a $2 \times 1.5 \mathrm{~cm}$ fragment shows two well-defined chondrules ( $1 \mathrm{~mm}$ and $0.9 \mathrm{~m}$ diameter), smaller $(<0.5 \mathrm{~mm})$ chondrules and dark nodules, and sparse sulfide grains $(<100 \mu \mathrm{m})$ set in a dark matrix with abundant fine-grained sulfide. Analysis of a $2 \times 2 \mathrm{~mm}$ region of the section shows that chondrules, relict chondrules, and clay-rich nodules comprise 41 areal\% of the section. Chondrules and relict chondrules have a mean diameter of $331 \mu \mathrm{m}(\mathrm{n}=25)$. An Mg x-ray map shows magnesium "hot-spots" in a few chondrules corresponding to relict olivine grains. BSE images show that the olivine is deeply corroded and partially replaced by clays. Olivine represents $\sim 1$ areal $\%$ of the section, consistent with the weak peaks in the powder XRD profile. No CAIs were found. Some of the fine-grained sulfide may be partially replaced by terrestrial oxides.

Geochemistry: (L. Garvie, ASU) WDX of a few relict olivine grains. BO chondrule with olivine bars largely replaced by clay. One bar has $\mathrm{Fa}_{1.1}$. A $200 \mu \mathrm{m}$ grain in matrix, $\mathrm{Fa}_{18.3}$. The $1 \mathrm{~mm}$ chondrule contains a $0.5 \mathrm{~mm}$ olivine grain, $\mathrm{Fa}_{0.5}$. A $100 \mu \mathrm{m}$ olivine grain in $300 \mu \mathrm{m}$-sized chondrule, $\mathrm{Fa}_{1.4}$.

Classification: Carbonaceous chondrite (CM1). The chondrules to matrix ratio and average diameter of the chondrules are consistent with a $\mathrm{CM}$ chondrite. The predominance of serpentine and only minor amounts of olivine ( $\sim 1$ areal\%), which show extensive alteration to clays, argue for a petrologic grade 1 carbonaceous chondrite.

Specimens: $8.7 \mathrm{~g}$ and one polished mount at $A S U$.

Northwest Africa 12331 (NWA 12331)

(Northwest Africa)

Purchased: March 2018

Classification: Ordinary chondrite (L3) 
Geochemistry: Olivine $\mathrm{Fa}_{25.4 \pm 2.5}, \mathrm{PMD}=8 \%$, range $\mathrm{Fa}_{23.5-29.9 \text {, }}$ $\mathrm{Cr}_{2} \mathrm{O}_{3}=0.02 \pm 0.03 \mathrm{wt} \%, \mathrm{n}=5$. Low-ca pyroxene $\mathrm{Fs}_{14.4 \pm 4.7} \mathrm{Wo}_{0.6 \pm 0.3}$, range $\mathrm{Fs}_{8.9}$ $21.7 \mathrm{Wo}_{0.3-1.1}(\mathrm{n}=4)$.

Specimens: $23.13 \mathrm{~g}$ and a polished section at $C E R E G E$. Main mass with Fabien Kuntz

\section{Northwest Africa 12335 (NWA 12335)}

(Northwest Africa)

Purchased: 2018

Classification: Martian meteorite (Shergottite)

History: Purchased in Guelmim, Morocco by Ali Fettouh in July 2018

Physical characteristics: Six identically appearing pieces with the following weights: $2290 \mathrm{~g}, 1486 \mathrm{~g}, 170 \mathrm{~g}, 20.7 \mathrm{~g}$, and 2 smaller fragments of $4 \mathrm{~g}$. No fusion crust. Broken surface reveals a mix of brown pyroxene grains and black maskelynite domains and shock melt.

Petrography: (C. Agee, $U N M$ ) The two major phases in this meteorite are clinopyroxene and maskelynite. The pyroxene compositions range from pigeonite to sub-calcic augite to ferro-pigeonite with a modal abundance of approximately $60 \%$, and significant core to rim zoning in backscatter electron images.

Maskelynite has modal abundance of approximately $35 \%$.

Geochemistry: (C. Agee, $U N M$ ) Pigeonite $\mathrm{Fs}_{48.0 \pm 10.7} \mathrm{Wo}_{16.8 \pm 6.0}, \mathrm{Fe} / \mathrm{Mn}=35 \pm 3, \mathrm{n}=8$; sub-calcic augite $\mathrm{Fs}_{29.4 \pm 3.9} \mathrm{Wo}_{34.7 \pm 2.1}, \mathrm{Fe} / \mathrm{Mn}=32 \pm 2$, $\mathrm{n}=4$; ferro-pigeonite $\mathrm{Fs}_{81.8 \pm 1.9} \mathrm{Wo}_{13.7 \pm 1.4}, \mathrm{Fe} / \mathrm{Mn}=36 \pm 3, \mathrm{n}=8$ : maskelynite $\mathrm{An}_{48.2 \pm 6.0} \mathrm{Ab}_{49.6 \pm 4.5} \mathrm{Or}_{2.2 \pm 1.7}$, $\mathrm{n}=10$; alkali feldspar $\mathrm{An}_{3.8} \mathrm{Ab}_{24.1} \mathrm{Or}_{72.1}, \mathrm{n}=1$.

Classification: Evolved Martian basalt, pyroxene trends are similar to QUE 94201. Specimens: $20.7 \mathrm{~g}$ including a probe mount on deposit at $U N M$. John Higgins holds the $2290 \mathrm{~g}$ stone; remainder with Ali Fettouh.

\section{Northwest Africa 12337 (NWA 12337) \\ (Northwest Africa) \\ Purchased: 2015 Apr \\ Classification: Ordinary chondrite (LL5)}

Petrography: (Z.G. Guo, UWO) This meteorite comprises 60-70\% chondrules with mean diameter of $800 \mu \mathrm{m}$. Undulatory extinction is present in olivine. There is $\sim 10 \%$ metal found within thin section.

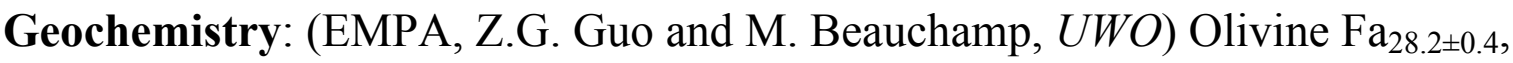
$\mathrm{Fe} / \mathrm{Mn}=53.9 \pm 2.5(\mathrm{~N}=15)$, high-Ca pyroxene $\mathrm{Fs}_{8.5 \pm 0.8} \mathrm{Wo}_{43.6 \pm 3.0} ; \mathrm{Fe} / \mathrm{Mn}=22.5 \pm 2.9$ $(\mathrm{N}=3)$; low-Ca pyroxene $\mathrm{Fs}_{23.1 \pm 0.4} \mathrm{Wo}_{1.3 \pm 0.1}, \mathrm{Fe} / \mathrm{Mn}=31.0 \pm 0.9(\mathrm{~N}=15)$; plagioclase $\mathrm{An}_{12.6 \pm 0.5}(\mathrm{~N}=12)$.

Classification: Ordinary chondrite (LL5, S2)

Specimens: $42.7 \mathrm{~g}$ at $U W O$ including a probe mount 


\section{Northwest Africa 12338 (NWA 12338)}

(Northwest Africa)

Purchased: 2016 Nov

Classification: Ungrouped achondrite

Physical characteristics: The repository specimen has a brown fusion crust surface and a light-gray, fresh interior.

Petrography: (Z.G. Guo, $U W O$ ) This is an unbrecciated basaltic meteorite with fine-grained ophitic texture including $\sim 100 \mu \mathrm{m}$-sized clinopyroxene $(\sim 55 \%)$, and $100-500 \mu \mathrm{m}$-sized plagioclase $(\sim 35 \%)$. Plagioclase is not acicular, as usually found in eucrites, but instead is interstitial between clinopyroxene crystals. Olivine $(\sim 5 \%)$ is found as $\sim 100 \mu \mathrm{m}$ individual crystals. It has a low content $(<1 \%)$ of metal (partially weathered), chromite and troilite. Undulatory (up to mosaic) extinction is observed in plagioclase, with silica veinlets, which indicate a moderate shock stage. Geochemistry: (EMPA, Z.G. Guo and M. Beauchamp, UWO) Olivine $\mathrm{Fa}_{70.0 \pm 0.2 \text {, }}$ $\mathrm{Fe} / \mathrm{Mn}=44 \pm 1(\mathrm{n}=10)$; pigeonite $\mathrm{Fs}_{40.5 \pm 3.3} \mathrm{Wo}_{10.6 \pm 4.1}, \mathrm{Fe} / \mathrm{Mn}=30 \pm 1(\mathrm{n}=20)$; plagioclase $\mathrm{An}_{89.1 \pm 1.4}(\mathrm{n}=10)$; Bulk chemistry (INAA, R. Korotev, WashU) $\mathrm{Na}_{2} \mathrm{O}$ $0.495 \%, \mathrm{CaO} 10.2 \%, \mathrm{FeO} 15.3 \%$, and (all in ppm) Cr 3043, Co 16, Sr 92, Ba 19, La 1.05, Sm 0.74, Hf 0.52, Th 0.10, and U 0.16. (laser fluorination oxygen isotope analyses, A. Alexandre; L. Webb, $U W O$ ) acid-washed bulk rock powder $\Delta^{17} \mathrm{O}=-$ $0.18 \pm 0.01, \delta^{17} \mathrm{O}=1.980 \pm 0.203, \delta^{18} \mathrm{O}=4.16 \pm 0.37(\mathrm{n}=2)$.

Classification: Achondrite, ungrouped. This meteorite is an anomalous eucrite-like basaltic meteorite similar to other specimens which have been found to diverge from the HED oxygen mass fractionation line (similarly to PCA 91007 and Pasamonte, and significantly above the HED line). It has geochemical characteristics of eucrites for major element mineral compositions, and of cumulate eucrites for bulk trace element chemistry. But its mineral sssemblage with the presence of igneous olivines, texture, and its oxygen isotopic composition indicate a different parent body from HED, thus an ungrouped achondrite classification.

Specimens: $24.0 \mathrm{~g}$ at $U W O$ including a probe mount.

\section{Northwest Africa 12339 (NWA 12339) \\ (Northwest Africa) \\ Purchased: 2017 \\ Classification: HED achondrite (Eucrite, polymict)}

Physical characteristics: Stone is partially crusted with black glassy fusion crust. The interior is light gray with brown staining.

Petrography: (Z.G. Guo, UWO) This is a brecciated meteorite, in which the size of clasts varies from $300 \mu \mathrm{m}$ to $8 \mathrm{~mm}$. The metal content is low $(<1 \%)$. A silica polymorph was observed. Other shock features like shock melt veins and sheared pyroxene exsolution lamellae were observed in thin section.

Geochemistry: (EMPA, Z.G. Guo and M. Beauchamp, UWO) The compositions of two pyroxenes vary among clasts and host matrix. Host: low-Ca pyroxene $\mathrm{Fs}_{63.9 \pm 2.0} \mathrm{Wo}_{2.3 \pm 2.0}, \mathrm{Fe} / \mathrm{Mn}=28.6 \pm 0.7(\mathrm{~N}=5)$, high-Ca pyroxene $\mathrm{Fs}_{31.7 \pm 3.7} \mathrm{Wo}_{39.9 \pm 4.2}, \mathrm{Fe} / \mathrm{Mn}=29.0 \pm 1.3(\mathrm{~N}=5)$ and plagioclase $\mathrm{An}_{90.1 \pm 2.7}(\mathrm{~N}=13)$; Clasts: 
low-Ca pyroxene $\mathrm{Fs}_{55.4 \pm 3.9} \mathrm{Wo}_{13.3 \pm 4.8}, \mathrm{Fe} / \mathrm{Mn}=28.6 \pm 0.2(\mathrm{~N}=3)$; high-Ca pyroxene $\mathrm{Fs}_{36.5 \pm 3.8} \mathrm{Wo}_{32.6 \pm 4.5}, \mathrm{Fe} / \mathrm{Mn}=27.8 \pm 0.5(\mathrm{~N}=3)$, and plagioclase (2) $\mathrm{An}_{72.0 \pm 4.2}, \mathrm{~N}=6$. Classification: Polymict eucrite

Specimens: $20.8 \mathrm{~g}$ at $U W O$ including a probe mount.

\section{Northwest Africa 12340 (NWA 12340)}

(Northwest Africa)

Purchased: 2006 Jun

Classification: HED achondrite (Eucrite, polymict)

History: Purchased from a Moroccan mineral dealer at the meteorite show in Ensisheim, France

Petrography: (K. Metzler, IfP) Polymict breccia consisting of eucritic lithic clasts with ophitic, subophitic and granulitic textures, and mineral fragments (eucritic and diogenitic pyroxene, plagioclase). Breccia-in breccia clasts and impact melt rock clasts are also present. These components are set in a fine-grained matrix of related debris, cemented by thin films of intergranular shock-melt. Most eucritic pyroxenes exhibit exsolution lamellae. Percentage of diogenitic components in the investigated sample is below $10 \mathrm{vol} \%$. Accessories are silica polymorph, ilmenite, Cr-spinel, troilite, and metal. Secondary calcite occurs.

Geochemistry: Mineral compositions and geochemistry: Eucritic low-Ca pyroxene: $\mathrm{Fs}_{45.6 \pm 10.1} \mathrm{Wo}_{4.7 \pm 1.6}\left(\mathrm{Fs}_{32-61} \mathrm{Wo}_{3-8}\right)$; mean $\mathrm{Fe} / \mathrm{Mn}$ (at.): 32 ; $\mathrm{n}=15$. Eucritic Ca-rich pyroxene: $\mathrm{Fs}_{27.3 \pm 6.3} \mathrm{Wo}_{40.1 \pm 2.8}\left(\mathrm{Fs}_{15-35} \mathrm{Wo}_{36-44} ; \mathrm{n}=8\right)$. Diogenitic pyroxene: $\mathrm{Fs}_{26.1 \pm 1.8} \mathrm{Wo}_{3.3 \pm 0.5}\left(\mathrm{Fs}_{23-29} \mathrm{Wo}_{2-4}\right)$; mean $\mathrm{Fe} / \mathrm{Mn}$ (at.): 33; $\mathrm{n}=8$. Plagioclase $\mathrm{An}_{91.1 \pm 0.7} \mathrm{Or}_{0.2 \pm 0.2}\left(\mathrm{An}_{90-92} \mathrm{Or}_{0-0.5}\right) ; \mathrm{n}=13$.

\section{Northwest Africa 12341 (NWA 12341) \\ (Northwest Africa) \\ Purchased: 2018 Aug \\ Classification: Ordinary chondrite (L3)}

Petrography: (K. Metzler, IfP) Unshocked ordinary chondrite of low petrologic type, consisting of chondrules (types I and II) of all major textural types (PO, POP, $\mathrm{PP}, \mathrm{BO}, \mathrm{C})$ with small amounts of interchondrule matrix. The apparent mean chondrule size is $470 \mu \mathrm{m}(\mathrm{n}=49)$. Nearly all metal has been oxidized by terrestrial weathering.

Geochemistry: Mineral compositions and geochemistry: The mean olivine composition is $\mathrm{Fa}_{18.4 \pm 12.3}\left(\mathrm{Fa}_{2.3-39.9} ; \mathrm{n}=21\right)$; the mean low-Ca pyroxene composition is $\mathrm{Fs}_{7.3 \pm 6.4} \mathrm{Wo}_{0.7 \pm 0.5}\left(\mathrm{Fs}_{1.3-23.8} \mathrm{Wo}_{0.2-2.4}\right) ; \mathrm{n}=22$.

Classification: L chondrite based on mean apparent chondrule size. Petrologic type based on the chemical variation of olivine and pyroxene. The PMD of Fa values is $67 \%$, indicating that the petrologic subtype is $<3.5$.

Northwest Africa 12342 (NWA 12342) 
(Northwest Africa)

Purchased: 2018 Aug

Classification: HED achondrite (Eucrite, melt breccia)

Petrography: (K. Metzler, IfP) Subrounded eucritic lithic clasts (up to $2 \mathrm{~cm}$ ) with ophitic to subophitic textures and mineral fragments (pyroxene, plagioclase, silica polymorph) embedded in a vesicular crystallized melt. The modal amount of melt is $30-50 \mathrm{vol} \%$. The latter shows a fan-spherulitic texture consisting of skeletal pyroxene and plagioclase crystals with sizes up to $100 \mu \mathrm{m}$. Vesicles can reach sizes of $\sim 400 \mu \mathrm{m}$. Many lithic clasts are surrounded by a thin dark layer of a more finegrained crystallized melt. Most pyroxene fragments show shock-deformed exsolution lamellae. Accessories are silica polymorph, ilmenite, Cr-spinel, and troilite.

Geochemistry: Mineral compositions and geochemistry: Lithic clasts: Low-Ca pyroxene $\mathrm{Fs}_{59.8 \pm 2.0} \mathrm{Wo}_{3.5 \pm 1.4}\left(\mathrm{Fs}_{56.1-62.8} \mathrm{Wo}_{1.8-6.2}\right)$; mean Fe/Mn (at.): 33; n=14. Capyroxene $\mathrm{Fs}_{26.7 \pm 0.4} \mathrm{Wo}_{43.2 \pm 0.4}\left(\mathrm{Fs}_{25.9-27.3} \mathrm{Wo}_{42.7-44.1} ; \mathrm{n}=12\right)$. Plagioclase $\mathrm{An}_{88.9 \pm 1.1} \mathrm{Or}_{0.4 \pm 0.3}\left(\mathrm{An}_{87.9-90.6} \mathrm{Or}_{0-0.8}\right) ; \mathrm{n}=8$.

\section{Northwest Africa 12343 (NWA 12343) \\ (Northwest Africa) \\ Purchased: 2018 Mar \\ Classification: Carbonaceous chondrite (CV3)}

Petrography: (K. Metzler, IfP) Chondrules, CAIs and AOAs are embedded in a fine-grained dark matrix (about 30 vol\% matrix). Most chondrules have apparent diameters between 0.3 and $1.5 \mathrm{~mm}$, some up to $2.2 \mathrm{~mm}$. Several chondrules show reddish staining due to terrestrial weathering. A slight alignement of chondrules is visible. CAIs with sizes of a few $\mathrm{mm}$ are observed.

Geochemistry: Mineral compositions and geochemistry: Random measurements of olivine grains revealed $\mathrm{Fa}_{7.4 \pm 2.7}\left(\mathrm{Fa}_{1.3-12.7} ; \mathrm{n}=26\right) ; \mathrm{n}=23$. Random measurements of low-Ca pyroxene grains revealed $\mathrm{Fs}_{1.8 \pm 0.8} \mathrm{Wo}_{1.6 \pm 1.2}\left(\mathrm{Fs}_{0.8-3.6} \mathrm{Wo}_{0.8-4.4} ; \mathrm{n}=13\right)$.

Classification: CV chondrite based on CAI and AOA occurrence, size of chondrules, chondrule-matrix ratio, and mineral chemistry. Petrologic type 3 based on the chemical variation of olivine and pyroxene.

\section{Northwest Africa 12344 (NWA 12344) \\ (Northwest Africa) \\ Purchased: 2018 Jan \\ Classification: HED achondrite (Eucrite, polymict)}

Petrography: (K. Metzler, IfP) Polymict breccia consisting of eucritic lithic clasts with ophitic textures and mineral fragments (pyroxene, plagioclase, silica polymorph) set in a fine-grained matrix of related debris. The matrix is clastic, bound by intergranular shock-melt, and intermingled with vesicular melt pockets (up to $5 \mathrm{~mm}$ ). The latter show quench tetxtures, consisting of parallel aligned pyroxene and plagioclase skeletal crystals. Both pyroxenes with and without 
exsolution lamellae occur. Iron-rich olivine was found as isolated fragments and in the shape of veins in lithic clasts. Accessories are silica polymorph, ilmenite, $\mathrm{Cr}$ spinel, troilite, and Ni-poor metal.

Geochemistry: Mineral compositions and geochemistry: Low-Ca pyroxene: $\mathrm{Fs}_{64.5 \pm 4.4} \mathrm{Wo}_{2.4 \pm 0.7}\left(\mathrm{Fs}_{55.8-68.4} \mathrm{Wo}_{1.9-4.7}\right)$; mean $\mathrm{Fe} / \mathrm{Mn}$ (at.): 29.8; $\mathrm{n}=13$. Ca-rich pyroxene: $\mathrm{Fs}_{30.8 \pm 1.0} \mathrm{Wo}_{42.5 \pm 0.6}\left(\mathrm{Fs}_{28.4-32.2} \mathrm{Wo}_{41.6-43.4}\right) \mathrm{n}=12$. Olivine: $\mathrm{Fa}_{75.0}\left(\mathrm{Fa}_{74.6-75.7}\right)$; mean Fe/Mn (at.): 44.1; $\mathrm{n}=3$. Plagioclase $\mathrm{An}_{90.6 \pm 2.5} \mathrm{Or}_{0.3 \pm 0.3}\left(\mathrm{An}_{86.9-94.0} \mathrm{Or}_{0.0-0.8}\right) ; \mathrm{n}=8$.

\section{Northwest Africa 12346 (NWA 12346) \\ Morocco \\ Purchased: 2017 Feb \\ Classification: Ordinary chondrite (L5)}

Physical characteristics: Small stone with visible oxidation of the matrix.

Petrography: (P. Reger, UWO) Mostly olivine and pyroxene, recrystallized matrix, with few secondary plagioclase grains (generally smaller than $50 \mu \mathrm{m}$ ), metal abundance $\sim 5 \%$, large chondrules generally easily discernible, smaller ones have fewer sharp boundaries. Mostly PP, RP chondrules, some POP and BO chondrules; mean diameter $\sim 0.5 \mathrm{~mm}$, larger $(>0.5 \mathrm{~mm})$ round, but fractured, smaller ones $(<0.5$ $\mathrm{mm}$ ) can be less rounded; chondrule glass absent. Undulutory extinction in olivine and pyroxene, in addition to planar fractures in olivine (S3). All metal oxidized, but no alteration in silicates visible (W4).

Geochemistry: (EMPA, M. Beauchamp, UWO) Olivine $\left(\mathrm{Fa}_{24.4 \pm 0.3} ; \mathrm{Fe} / \mathrm{Mn}=\right.$ $45.7 \pm 1.6 ; \mathrm{n}=10)$, Orthopyroxene $\left(\mathrm{Fs}_{20.6 \pm 0.4} \mathrm{Wo}_{1.1 \pm 0.1}, \mathrm{n}=10\right)$, Plagioclase $\mathrm{An}_{9.7 \pm 0.7}(\mathrm{n}=6)$. Maximum Ni content in metal phases is $>20 \%$. Bulk rock ICP-MS analysis shows enrichment of incompatible rare earth elements compared to CI chondrites in G701

Classification: Ordinary chondrite L5 (W4, S3)

Specimens: $89.6 \mathrm{~g}$ including a thin section at $U W O$. S. Tutorow holds the main mass.

\section{Northwest Africa 12347 (NWA 12347) \\ Morocco \\ Purchased: 2017 Feb \\ Classification: Ordinary chondrite (H5)}

Physical characteristics: A dark-brown fusion crust covers the outer edges of the hand sample. The sample appears to be at least moderately weathered due to an overall dark brown appearance and the absence of abundant visible metal grains. The largest chondrule visible in the type specimen has a diameter of $3.5 \mathrm{~mm}$. Petrography: (J. Laughton, UWO) Model abundances were determined using a combination of optical microscopy and ImageJ software: 74\% chondrules, $12 \%$ matrix, $6 \%$ oxide, $5 \%$ metal, and 3\% sulphide [ + trace amount of calcium phosphate]. The silicates present include: olivine, orthopyroxene, minor clinopyroxene and plagioclase, and metal $(\sim 5 \%)$. The sulfide present was in the 
form of troilite with $<0.5 \mathrm{wt} . \% \mathrm{Ni}$ content. Chondrule diameters ranged in size from 3.5 to $0.1 \mathrm{~mm}$, with an average diameter of $0.4 \mathrm{~mm}(\mathrm{n}=50)$. Majority of the chondrules could be discerned, but not all had sharp boundaries. Very minor undulatory extinction patterns were observed in olivine and pyroxene crystals, as well as some minor irregular fractures within the crystals. In thin section $\sim 70 \%$ of the metal and troilite has been replaced by a dark red iron oxide (hematite).

Oxidation of silicates has also started along the cracks of some crystals.

Geochemistry: (EMPA, M. Beauchamp, $U W O)$ Olivine $\left(\mathrm{Fa}_{16.7 \pm 0.2} ; \mathrm{Fe} / \mathrm{Mn}=\right.$ $29.2 \pm 1.0 ; \mathrm{n}=10)$, Orthopyroxene $\left(\mathrm{Fs}_{14.6 \pm 0.2} \mathrm{Wo}_{1.7 \pm 0.0} ; \mathrm{Fe} / \mathrm{Mn}=17.8 \pm 0.6 ; \mathrm{n}=11\right)$ and Plagioclase $\mathrm{An}_{8.8 \pm 0.4}(\mathrm{n}=10)$.

Classification: Oridinary chondrite H5, S2, W3.

Specimens: $54.0 \mathrm{~g}$ including a thin section at $U W O$. S. Tutorow holds the main mass.

\section{Northwest Africa 12348 (NWA 12348)}

Morocco

Purchased: 2017 Feb

Classification: Ordinary chondrite (L4)

Physical characteristics: The meteorite has a reddish-brown colored matrix containing chondrules and chondrule fragments.

Petrography: (G. Ibe, $U W O$ ) Chondrules constitute about 75 vol\% of this meteorite and have well-defined rims, up to $2.2 \mathrm{~mm}$ in the thin section. Opaque minerals in the meteorite include $\mathrm{Fe}-\mathrm{Ni}$ metal, troilite, chromite. The amounts of Fe-Ni metal and troilite are $\sim 1 \%$ and $\sim 3 \%$, respectively. Plagioclase is present as a minor phase. Undulatory optical extinction could be observed in olivine. About 60 ? $70 \%$ of the metal and sulfides were oxidized, but no similar features were observed in the silicates.

Geochemistry: (EMPA, M. Beauchamp, $U W O$ ) Olivine $\mathrm{Fa}_{25.0 \pm 2.0}(\mathrm{n}=10)$, low-Ca pyroxenes $\left(\mathrm{Fs}_{20.5 \pm 0.6} \mathrm{Wo}_{1.5 \pm 0.2}, \mathrm{n}=10\right)$, and plagioclase $\mathrm{An}_{12.1 \pm 0.02}$.

Classification: Ordinary Chondrite (L4, S4, W3).

Specimens: $51.3 \mathrm{~g}$ including a thin section at $U W O$. S. Tutorow holds the main mass.

\section{Northwest Africa 12349 (NWA 12349) \\ Morocco \\ Purchased: 2017 Feb \\ Classification: Ordinary chondrite (H4)}

Physical characteristics: A dark-brown to black fusion crust with regmaglypts is observed over approximately $80 \%$ of the exterior. Faint chondrules set in a brownorange matrix are observed on the saw-cut face.

Petrography: (T.V. Kizovski, ROM) In thin section and in backscattered electron view, chondrules are discernible with opaque boundaries set in a semirecrystallized matrix. The chondrules vary in size with an average diameter of 0.4 
$\mathrm{mm}(\mathrm{n}=41)$. The majority of the chondrules are porphyritic and are mainly comprised of pyroxene with varying amounts of olivine and devitrified glass. Barred olivine and cryptocrystalline chondrules are also observed. The matrix is mainly composed of iron oxide with some devitrified glass, pyroxene, terrestrial barite, and rare metal. The stone shows multiple signs of significant desert weathering. Several cracks, approximately $0.03 \mathrm{~mm}$ in width, cross-cut the sample and appear to be filled with terrestrial weathering products. The metals and sulfides in the matrix have been completely oxidized, although there has been no alteration of silicates. Subtle undulatory extinction is observed in the olivine and pyroxene grains. Plagioclase is rare, and only observed in the porphyritic chondrules. Geochemistry: (EMPA, M. Beauchamp, UWO) Olivine $\left(\mathrm{Fa}_{19.5 \pm 0.1} ; \mathrm{Fe} / \mathrm{Mn}=\right.$ $37.6 \pm 1.6 ; \mathrm{n}=10)$, low-Ca pyroxene $\left(\mathrm{Fs}_{16.3 \pm 1.4} \mathrm{Wo}_{1.4 \pm 1.1} ; \mathrm{Fe} / \mathrm{Mn}=25.7 \pm 7.1 ; \mathrm{n}=10\right)$, plagioclase $\left(\mathrm{An}_{8.8 \pm 4.7} \mathrm{Ab}_{78.1 \pm 12.7} \mathrm{Or}_{13.1 \pm 16.5} ; \mathrm{n}=6\right)$.

Classification: Ordinary Chondrite (H4, S2, W4)

Specimens: $23.2 \mathrm{~g}$ including a thin section at $U W O$. S. Tutorow holds the main mass.

\section{Northwest Africa 12350 (NWA 12350) \\ Morocco \\ Purchased: 2006 \\ Classification: Ordinary chondrite (H4)}

History: Purchased in 2006 by Sean Tutorow in Quartzsite, Arizona.

Physical characteristics: Typing specimen has a fusion crust and displays minor weathering.

Petrography: (M. Spencer, UWO) Olivine has irregular fractures. One large barred olivine crystal $(>1 \mathrm{~mm})$ is present in the thin section. Plagioclase displays twinning and undulatory extinction (S2). Pyroxene displays pleochroism in plain polarized light, undulatory extinction. Metal Abundance: Fe-Ni metals (mostly taenite, some kamacite present), Fe-Ni oxides, Fe-sulfides are still present (W1). Most chondrules display sharp boundaries, with an average diameter of $0.27 \mathrm{~mm}(\mathrm{n}=13)$. Geochemistry: (EMPA, M. Beauchamp, UWO) Olivine: $\mathrm{Fa}_{19.1 \pm 0.5}$; $\mathrm{Fe} / \mathrm{Mn}=36.8 \pm 1.5(\mathrm{n}=10)$, Pyroxene: $\mathrm{Fs}_{16.2 \pm 0.1} \mathrm{Wo}_{1.0 \pm 0.3} ; \mathrm{Fe} / \mathrm{Mn}=21.8 \pm 1.1(\mathrm{n}=10)$, Plagioclase: $\operatorname{An}_{10.7 \pm 3.3}(\mathrm{n}=10)$.

Classification: Ordinary Chondrite (H4), Weathering W1, Shock S2

Specimens: $206 \mathrm{~g}$ including a thin section at $U W O$. Main mass with S. Tutorow.

\section{Northwest Africa 12351 (NWA 12351) \\ Morocco \\ Purchased: 2017 Feb \\ Classification: Ordinary chondrite (H5)}

Physical characteristics: Repository specimen has a fresh surface.

Petrography: (C. Zhu, $U W O$ ) Mostly granular pyroxene chondrule $(200-500 \mu \mathrm{m})$ and porphyritic pyroxene chondrules $(200-600 \mu \mathrm{m})$ Mostly barred olivine, radial 
olivine $(200-400 \mu \mathrm{m})$ and porphyritic olivine $(200-300 \mu \mathrm{m})$. Plagioclase grains range from 10 to $40 \mu \mathrm{m}$ in diameter with tabular shape. Olivine and pyroxene grains show undulatory extinction, and some display planar fractures. Fe-Ni metal $(\sim 10-15 \mathrm{vol} \%)$ is slightly oxidized. Other phases found include Fe-Ni-sulfides, and minor chromite and chlorapatite.

Geochemistry: (EMPA, M. Beauchamp, UWO) Olivine ( $\mathrm{Fa}_{18.8 \pm 0.5} ; 18.2-19.6$; $\mathrm{Fe} / \mathrm{Mn}=36.7 \pm 2.0 ; \mathrm{n}=15)$, low-Ca pyroxene $\left(\mathrm{Fs}_{15.9 \pm 1.7} \mathrm{Wo}_{0.5 \pm 0.3} ; \mathrm{Fs}_{13.3-19.2} \mathrm{Wo}_{0.1-1.1}\right.$; $\mathrm{Fe} / \mathrm{Mn}=28.5 \pm 8.8 ; \mathrm{n}=10)$, and plagioclase $\mathrm{An}_{12.2 \pm 2.9}\left(\mathrm{An}_{5.6-17.8} ; \mathrm{n}=10\right)$.

Classification: Ordinary chondrite (H5, S3, W1)

Specimens: $32.2 \mathrm{~g}$ including a polished thin section are on deposit at UWO. S. Tutorow holds the main mass.

\section{Northwest Africa 12352 (NWA 12352) \\ Morocco \\ Purchased: 2017 Feb \\ Classification: Ordinary chondrite (H5)}

Physical characteristics: This sample has a dar-brown fusion crust. The inside shows a dark-gray matrix with a significant number of chondrules and metals.

Petrography: (B. Borba de Carvalho, $U W O$ ) The thin section exhibits numerous chondrules (up to $1.6 \mathrm{~mm}$ ) in a dark matrix of medium-grained silicates, metals, minor oxides and sulfides. Most of the chondrules lack well-delineated edges. Major mineral phases are olivine and low-Ca pyroxene. Minor plagioclase and clinopyroxene were observed. No alteration and strong shock features were observed, except for irregular fractures and undulutory extinction in olivine and pyroxene. Fe-Ni metal phases have minor oxide rims.

Geochemistry: (EMPA, M. Beauchamp, UWO $)$ Olivine $\left(\mathrm{Fa}_{17.7 \pm 0.2} ; \mathrm{Fe} / \mathrm{Mn}=\right.$ $35.6 \pm 1.2 ; \mathrm{n}=10)$, Pyroxene $\left(\mathrm{Fs}_{15.5 \pm 0.2} \mathrm{Wo}_{1.4 \pm 0.1} ; \mathrm{Fe} / \mathrm{Mn}=20.7 \pm 1 ; \mathrm{n}=10\right)$, Plagioclase $\left(\mathrm{An}_{13.1 \pm 0.5} ; \mathrm{n}=10\right)$.

Classification: Ordinary chondrite (H5, S2, W1).

Specimens: $30.2 \mathrm{~g}$ including a thin section at $U W O$. Main mass with S. Tutorow.

\section{Northwest Africa 12353 (NWA 12353) \\ Morocco \\ Purchased: 2017 Feb \\ Classification: Ordinary chondrite (H5)}

Physical characteristics: The repositiory specimen has a very dark interior and contains visible chondrules.

Petrography: (Z. Jiang, $U W O$ ) Porphyritic and radial pyroxene chondrules are found with an average size of $\sim 0.3 \mathrm{~mm}$. There are planar and irregular fractures in olivine and orthopyroxene. Minor plagioclase was found.

Geochemistry: (EMPA, M. Beauchamp, $U W O)$ Olivine $\left(\mathrm{Fa}_{18.7 \pm 0.2} ; \mathrm{Fe} / \mathrm{Mn}=\right.$ $36.8 \pm 1.5 ; \mathrm{n}=15)$, orthopyroxene $\left(\mathrm{Fs}_{16.4 \pm 0.1} \mathrm{Wo}_{1.5 \pm 0.2}, \mathrm{n}=13\right)$, and plagioclase $\left(\mathrm{An}_{12.4 \pm 0.3} ; \mathrm{n}=10\right)$. 
Classification: Ordinary chondrite (H5, S3, W2)

Specimens: $22.7 \mathrm{~g}$ including a thin section at $U W O$. S. Tutorow holds the main mass.

Northwest Africa 12365 (NWA 12365)

(Northwest Africa)

Purchased: April 2018

Classification: Carbonaceous chondrite (CO3)

History: Bought in April 2018 from a mineral dealer in Laayoune, Morocco.

Petrography: (A. Bischoff, IfP) Chondrules, chondrule fragments, and CAIs are embedded in a fine-grained brownish matrix, which has an abundance of about 20$30 \mathrm{vol} \%$. The majority of chondrules belong to chemical type I and the mean apparent chondrule size is about $200 \mu \mathrm{m}$. The sample is strongly weathered. Geochemistry: (K. Klemm; A. Bischoff, IfP) Mineral compositions and geochemistry: Random measurements of olivine grains revealed $\mathrm{Fa}_{8.9 \pm 9.8}\left(\mathrm{Fa}_{0.7-28.6}\right)$; $\mathrm{n}=21$. Random measurements of low-Ca pyroxene grains revealed $\mathrm{Fs}_{7.2 \pm 13.3} \mathrm{Wo}_{1.3 \pm 1.1}\left(\mathrm{Fs}_{0.7-39.9} \mathrm{Wo}_{0.5-4.2}\right) ; \mathrm{n}=15$.

Classification: $\mathrm{CO} 3$ chondrite based on small chondrule size, chondrule-matrix ratio, texture, and mineral chemistry.

\section{Northwest Africa 12366 (NWA 12366) \\ (Northwest Africa) \\ Purchased: April 2018 \\ Classification: Carbonaceous chondrite (CO3)}

History: Bought in April 2018 from a dealer in Zagora, Morocco.

Petrography: (A. Bischoff, IfP) Chondrules, chondrule fragments, and CAIs are embedded in a fine-grained brownish matrix, which has an abundance of about 20$30 \mathrm{vol} \%$. The majority of chondrules contain Fe-poor olivine and low-Ca pyroxene and the mean apparent chondrule size is about $200 \mu \mathrm{m}$.

Geochemistry: (K. Klemm; A. Bischoff, IfP) Mineral compositions and geochemistry: Random measurements of olivine grains revealed $\mathrm{Fa}_{11.9 \pm 14.0}\left(\mathrm{Fa}_{0.4-}\right.$ 49.4); $\mathrm{n}=14$. Random measurements of low-Ca pyroxene grains revealed $\mathrm{Fs}_{2.3 \pm 1.3} \mathrm{Wo}_{1.7 \pm 1.5}\left(\mathrm{Fs}_{1.1-5.7} \mathrm{Wo}_{0.3-5.1}\right) ; \mathrm{n}=12$.

Classification: $\mathrm{CO} 3$ chondrite based on small chondrule size, chondrule-matrix ratio, texture, and mineral chemistry.

\section{Northwest Africa 12367 (NWA 12367)}

(Northwest Africa)

Purchased: April 2018

Classification: Carbonaceous chondrite (CO3)

History: Bought in April 2018 from a dealer in Zagora, Morocco. 
Petrography: (A. Bischoff, IfP) Chondrules, chondrule fragments, and CAIs are embedded in a fine-grained brownish matrix, which has an abundance of about 20$30 \mathrm{vol} \%$. The majority of chondrules belong to chemical type I and the mean apparent chondrule size is about $200 \mu \mathrm{m}$.

Geochemistry: (K. Klemm; A. Bischoff, IfP) Mineral compositions and geochemistry: Random measurements of olivine grains revealed $\mathrm{Fa}_{10.3 \pm 14.5}\left(\mathrm{Fa}_{0.7}\right.$ 43.3); $\mathrm{n}=29$. Random measurements of low-Ca pyroxene grains revealed $\mathrm{Fs}_{6.2 \pm 12.0} \mathrm{Wo}_{1.5 \pm 1.0}\left(\mathrm{Fs}_{0.6-35.9} \mathrm{Wo}_{0.5-4.3}\right) ; \mathrm{n}=15$.

Classification: $\mathrm{CO} 3$ chondrite based on small chondrule size, chondrule-matrix ratio, texture, and mineral chemistry.

\section{Northwest Africa 12368 (NWA 12368) \\ (Northwest Africa) \\ Purchased: 2018 \\ Classification: Lunar meteorite (feldspathic breccia)}

History: Found in the spring of 2017 in the Moroccan Sahara and purchased in Temara by A. Aaronson on July 23, 2018.

Physical characteristics: Single complete stone, no fusion crust; saw cut reveals a fragmental breccia with scattered white feldspathic and mafic clasts set in a darkgray groundmass.

Petrography: (C. Agee, $U N M$ ) This meteorite consists primarily of fragmental pyroxene, olivine, feldspathic and mafic clasts, and shock melt. Ubiquitous Fe-Ti$\mathrm{Cr}$-Al oxides are present, rare kamacite was detected. There appears to be 2 or 3 distinct populations of pyroxenes and olivines, suggesting that this is a polymict breccia.

Geochemistry: (C. Agee, $U N M$ ) olivine $\mathrm{Fa}_{53.3 \pm 11.8}, \mathrm{Fe} / \mathrm{Mn}=96 \pm 7, \mathrm{n}=7$; fayalite $\mathrm{Fa}_{91.8 \pm 2.9}, \mathrm{Fe} / \mathrm{Mn}=91 \pm 8, \mathrm{n}=3$; pigeonite $\mathrm{Fs}_{26.0 \pm 1.3} \mathrm{Wo}_{6.4 \pm 1.3}, \mathrm{Fe} / \mathrm{Mn}=51 \pm 3, \mathrm{n}=7$; subcalcic augite $\mathrm{Fs}_{38.3 \pm 3.2} \mathrm{Wo}_{24.2 \pm 4.3}, \mathrm{Fe} / \mathrm{Mn}=57 \pm 3, \mathrm{n}=2$; ferro-augite $\mathrm{Fs}_{63.9 \pm 4.3} \mathrm{Wo}_{30.4 \pm 7.6}, \mathrm{Fe} / \mathrm{Mn}=92 \pm 21, \mathrm{n}=2$; plagioclase $\mathrm{An}_{95.5 \pm 1.4}, \mathrm{n}=6$; Shock melt (20 $\mathrm{m}$ defocused electron beam, proxy for bulk meteorite composition): $\overline{\mathrm{SiO}}_{2}=44.6 \pm 1.5, \mathrm{TiO}_{2}=0.18 \pm 0.13, \mathrm{Al}_{2} \mathrm{O}_{3}=28.8 \pm 4.2, \mathrm{Cr}_{2} \mathrm{O}_{3}=0.11 \pm 0.14$, $\mathrm{MgO}=4.1 \pm 1.8, \mathrm{FeO}=4.2 \pm 2.1, \mathrm{MnO}=0.07 \pm 0.03, \mathrm{CaO}=16.8 \pm 1.9, \mathrm{NiO}=0.02 \pm 0.02$, $\mathrm{Na}_{2} \mathrm{O}=0.54 \pm 0.14, \mathrm{~K}_{2} \mathrm{O}=0.06 \pm 0.04$ (all wt\%), $\mathrm{n}=3$.

Classification: Lunar feldspathic breccia

Specimens: $20.6 \mathrm{~g}$ including a probe mount on deposit at $U N M$, Adam Aaronson holds the main mass.

\section{Northwest Africa 12369 (NWA 12369)}

(Northwest Africa)

Purchased: 2018

Classification: Ordinary chondrite (H5)

History: Found in the Moroccan western Sahara in May 2018, purchased in Temara by A. Aaronson on August 14, 2018. 
Physical characteristics: Single stone, no fusion crust, saw cut reveals numerous closely packed chondrules in a brown matrix.

Petrography: (C. Agee, $U N M$ ) This meteorite has many porphyritic chondrules, abundant Fe-metal, and plagioclase up to $25 \mu \mathrm{m}$ in size.

Geochemistry: (C. Agee, $U N M$ ) olivine $\mathrm{Fa}_{18.2 \pm 0.2}, \mathrm{Fe} / \mathrm{Mn}=37 \pm 2$, $\mathrm{n}=30$; low-Ca pyroxene $\mathrm{Fs}_{16.0 \pm 0.1} \mathrm{Wo}_{1.5 \pm 0.1}, \mathrm{Fe} / \mathrm{Mn}=21 \pm 1, \mathrm{n}=11$.

Classification: Ordinary chondrite $\mathrm{H} 5$

Specimens: $25 \mathrm{~g}$ including a probe mount on deposit at $U N M$, Adam Aaronson holds the main mass.

Northwest Africa 12381 (NWA 12381)

(Northwest Africa)

Purchased: 2018

Classification: Ordinary chondrite (L $\sim 6)$

History: Purchased at Hamburg Mineral Show Dec. 2018.

Physical characteristics: $21.7 \mathrm{~g}$ dark-brown fragment

Petrography: (R. Bartoschewitz, Bart) recrystallized matrix with poorly defined, but partly metal-rimmed chondrules $(0.5-2 \mathrm{~mm})$ and metal specks

Geochemistry: Magnetic susceptibility (R. Bartoschewitz, Bart $) \log \chi\left(\times 10^{-}\right.$

$\left.{ }^{9} \mathrm{~m}^{3} / \mathrm{kg}\right)=4.85$

Classification: Ordinary chondrite (L $\sim 6, \mathrm{~W} 2)$

Specimens: $4.4 \mathrm{~g}$ on deposit at Kiel, Ben Levi Karsten (Hamburg) holds the main mass, and $3.1 \mathrm{~g}$ with Bart.

Northwest Africa 12382 (NWA 12382)

(Northwest Africa)

Purchased: 2018

Classification: Ordinary chondrite $(\mathrm{H} \sim 5)$

History: Purchased at Hamburg Mineral Show Dec. 2018.

Physical characteristics: $29.0 \mathrm{~g}$ brown fragment

Petrography: (R. Bartoschewitz, Bart) Brown stained, recrystallized matrix with well-defined chondrules (av. $0.5 \mathrm{~mm}$ )

Geochemistry: Magnetic susceptibility (R. Bartoschewitz, Bart $) \log \chi\left(\times 10^{-}\right.$

$\left.{ }^{9} \mathrm{~m}^{3} / \mathrm{kg}\right)=4.70$

Classification: Ordinary chondrite ( $\mathrm{H} \sim 5, \mathrm{~W} 4)$

Specimens: $5.9 \mathrm{~g}$ on deposit at Kiel, Joseph Kaiser (Hamburg) holds the main mass, and $3.9 \mathrm{~g}$ with Bart.

Northwest Africa 12383 (NWA 12383)

(Northwest Africa)

Purchased: 2018

Classification: Ordinary chondrite $(\mathrm{H} \sim 5)$ 
History: Purchased at Hamburg Mineral Show Dec. 2018.

Physical characteristics: 209.65 g dark brown fragment

Petrography: (R. Bartoschewitz, Bart) recrystallized matrix with recognizable chondrules(av. $0.5 \mathrm{~mm}$ ) and metal specks

Geochemistry: Magnetic susceptibility (R. Bartoschewitz, Bart) $\log \chi\left(\times 10^{-}\right.$

$\left.{ }^{9} \mathrm{~m}^{3} / \mathrm{kg}\right)=5.13$

Classification: Ordinary chondrite $(\mathrm{H} \sim 5, \mathrm{~W} 2)$

Specimens: $22.0 \mathrm{~g}$ on deposit at Kiel, W. Kalisch (Berlin) holds the main mass, and $17 \mathrm{~g}$ with Bart.

\section{Northwest Africa 12385 (NWA 12385) \\ Morocco \\ Find: unknown \\ Classification: Ordinary chondrite (H5)}

History: Found in Morocco and purchased by $H M G$ from a dealer (Ke Zuokai) in Changsha, China.

Physical characteristics: About $75 \%$ of the angular stone is covered by a dull, dark fusion crust. The broken surface shows round chondrules.

Petrography: The thin section shows moderately abundant chondrules set in a fine-grained matrix. The chondrules have no clear boundaries and are cracked. The diameter of chondrules are 0.1 to $2.0 \mathrm{~mm}$. Some chondrules are surrounded by opaque minerals. A few fragments of BO chondrules are visible. The opaque phases include metal, troilite and Fe-oxides are about $20 \mathrm{vol} \%$. Oxidation of metal and troilite distributed as minor rims or fine veins. The weathering grade is $\mathrm{W} 1$. Geochemistry: Silicates are equilibrated. Olivine $\mathrm{Fa}_{18.3 \pm 0.1}(\mathrm{PMD}=0.5 \%)$; low-Ca pyroxene $\mathrm{Fs}_{16.4 \pm 0.3} \mathrm{Wo}_{1.6 \pm 0.2} \mathrm{En}_{82.0 \pm 0.3}(\mathrm{PMD}-\mathrm{Fs}=2.0 \%)$;

Classification: Ordinary chondrite. H5

Specimens: $3463 \mathrm{~g}$ is at $H M G$.

The name Nova 054 replaces the discredited name Mercantour. A description of the meteorite may be found under the former name.

The name Nova 055 replaces the discredited name Castenaso. Information originally submitted for this meteorite may be found under the former name.

\section{Nova 020}

(unknown)

Purchased: 2014

Classification: Iron meteorite (IAB-MG)

History: Purportedly found in Licking River, KY, USA. Presented to UCLA by P. Allen in 2014.

Geochemistry: (J.T. Wasson, UCLA): INAA data, $\mathrm{Ni}=65.2, \mathrm{Co}=4.58$ (both $\mathrm{mg} / \mathrm{g}), \mathrm{Ga}=89.5, \mathrm{As}=11.5, \mathrm{~W}=1.27, \mathrm{Ir}=3.93, \mathrm{Au}=1.444(\mathrm{all} \mu \mathrm{g} / \mathrm{g})$.

Classification: Iron, IAB main group. Probably paired with Campo del Cielo. Specimens: Main mass at UCLA. 


\section{Nova 021}

(unknown)

Purchased: 2016

Classification: Iron meteorite (IAB-MG)

History: Purportedly found in Copiapo, Chile. Presented to UCLA by D. Ruiz in 2016.

Geochemistry: (J.T. Wasson, $U C L A$ ): INAA data (average of two samples), $\mathrm{Ni}=$ 64.8, $\mathrm{Co}=4.56$ (both $\mathrm{mg} / \mathrm{g}), \mathrm{Ga}=86.4, \mathrm{As}=10.5, \mathrm{~W}=1.22, \mathrm{Ir}=3.39, \mathrm{Au}=1.43$ (all $\mu \mathrm{g} / \mathrm{g}$ ).

Classification: Iron, IAB main group. Probablypaired with Campo del Cielo. Specimens: Type specimen at $U C L A$.

\section{Nova 022}

(unknown)

Purchased: 2013

Classification: Iron meteorite (IAB-MG)

History: Purportedly found in Redding, CA, USA. Presented to UCLA by J. Utas in 2013.

Geochemistry: (J.T. Wasson, $U C L A$ ): INAA data, $\mathrm{Ni}=63, \mathrm{Co}=4.58$ (both $\mathrm{mg} / \mathrm{g}$ ), $\mathrm{Ga}=91.2, \mathrm{As}=11.4, \mathrm{~W}=1.26, \mathrm{Ir}=3.55, \mathrm{Au}=1.531(\mathrm{all} \mu \mathrm{g} / \mathrm{g})$.

Classification: Iron, IAB main group. Probably paired with Campo del Cielo. Specimens: Type specimen at $U C L A$.

\section{Nova 023}

(unknown)

Purchased: 2014

Classification: Iron meteorite (IAB-MG)

History: Purportedly found on a beach, $7 \mathrm{~km}$ NNE of Sorso, Sardinia, Italy. Reported to $N M B E$ by the finder on 22 Nov 2012. Sample presented to UCLA by B. Hofmann in 2014.

Physical characteristics: The surface of the specimen is mostly metal with little rust, with remnants of an earlier rust, so likely the specimen has been cleaned. However the finder reported that he did not clean the sample.

Geochemistry: (J.T. Wasson, $U C L A$ ): INAA data, $\mathrm{Ni}=64.7, \mathrm{Co}=4.64$ (both $\mathrm{mg} / \mathrm{g}), \mathrm{Ga}=89.1, \mathrm{As}=10.9, \mathrm{~W}=1.29, \mathrm{Ir}=3.47, \mathrm{Au}=1.419(\mathrm{all} \mu \mathrm{g} / \mathrm{g})$.

Classification: Iron, IAB main group. Probably paired with Campo del Cielo. Specimens: Type specimen (22 g) and main mass at $N M B E$. INAA sample ( $2 \mathrm{~g}$ ) at UCLA.

\section{Nova 024}


(unknown)

Purchased: 2010

Classification: Iron meteorite (IAB-MG)

History: Purportedly found in Tucson, AZ, USA. Presented to UCLA by

L. Labenne in 2010.

Geochemistry: (J.T. Wasson, $U C L A$ ): INAA data, $\mathrm{Ni}=68.1, \mathrm{Co}=4.52$ (both $\mathrm{mg} / \mathrm{g}), \mathrm{Ga}=91.2, \mathrm{As}=11.2, \mathrm{~W}=1.25, \mathrm{Ir}=3.8, \mathrm{Au}=1.463(\mathrm{all} \mu \mathrm{g} / \mathrm{g})$.

Classification: Iron, IAB main group. Probably paired with Campo del Cielo. Specimens: Type specimen at $U C L A$.

\section{Nova 025}

(unknown)

Purchased: 2004

Classification: Iron meteorite (IAB-MG)

History: Purportedly found in Blanco, NM, USA. Presented to UCLA by R. Jones in 2004.

Geochemistry: (J.T. Wasson, UCLA): INAA data (average of two samples), $\mathrm{Ni}=$ $67.1, \mathrm{Co}=4.55$ (both $\mathrm{mg} / \mathrm{g}$ ), $\mathrm{Ga}=92.1, \mathrm{As}=11.1, \mathrm{~W}=1.45, \mathrm{Ir}=3.75, \mathrm{Au}=1.482$ (all $\mu \mathrm{g} / \mathrm{g}$ ).

Classification: Iron, IAB main group. Likely paired with Campo del Cielo. Specimens: $2682 \mathrm{~g}$ at $A S U$. INAA sample $(1 \mathrm{~g})$ at $U C L A$.

\section{Nova 026}

(unknown)

Purchased: 2005

Classification: Iron meteorite (IAB-MG)

History: Purportedly found in Randsburg, CA, USA. Presented to UCLA by G. Hupe and T. Irving in 2005.

Geochemistry: (J.T. Wasson, $U C L A$ ): INAA data (average of two samples), $\mathrm{Ni}=$ $69.9, \mathrm{Co}=4.54$ (both $\mathrm{mg} / \mathrm{g}$ ), $\mathrm{Ga}=87.8, \mathrm{As}=10.8, \mathrm{~W}=1.19, \mathrm{Ir}=3.65, \mathrm{Au}=1.462$ (all $\mu \mathrm{g} / \mathrm{g}$ ).

Classification: Iron, IAB main group. Probably paired with Campo del Cielo. Specimens: Type specimen at $U C L A$.

\section{Nova 027}

(unknown)

Purchased: 2005

Classification: Iron meteorite (IAB-MG)

History: Purportedly found in Arispe, Baja California, Mexico. Presented to $U C L A$ by $\mathrm{P}$. Utas in 2005 . 
Geochemistry: (J.T. Wasson, $U C L A$ ): INAA data (average of two samples), $\mathrm{Ni}=$ 65.6, $\mathrm{Co}=4.54$ (both $\mathrm{mg} / \mathrm{g}), \mathrm{Ga}=90.9, \mathrm{As}=11.3, \mathrm{~W}=1.37, \mathrm{Ir}=3.54, \mathrm{Au}=1.465$ (all $\mu \mathrm{g} / \mathrm{g})$.

Classification: Iron, IAB main group. Probably paired with Campo del Cielo. Specimens: Type specimen at UCLA.

\section{Nova 028}

(unknown)

Purchased: 2004

Classification: Iron meteorite (IAB-MG)

History: Purportedly found in Utah, USA.

Geochemistry: (J.T. Wasson, UCLA): INAA data (average of two samples), $\mathrm{Ni}=$ $67.9, \mathrm{Co}=4.5$ (both $\mathrm{mg} / \mathrm{g}$ ), $\mathrm{Ga}=91.6, \mathrm{As}=11.1, \mathrm{~W}=1.26, \mathrm{Ir}=3.92, \mathrm{Au}=1.492$ (all $\mu \mathrm{g} / \mathrm{g}$ ).

Classification: Iron, IAB main group

Specimens: Type specimen at $A S U$.

\section{Ouargla $001 \quad 31^{\circ} 06^{\prime}, 5^{\circ} 11^{\prime}$}

Ouargla, Algeria

Purchased: before 2001

Classification: Ordinary chondrite (H3)

Petrography: Low-Ca pyroxene exhibits polysynthetic twinning. Chondrules are sharply defined but lack glassy mesostases. Many metal grains are elongated and ragged; some contain small silciate grain fragments. Some metal grains contain small, irregular troilite grains. The rock appears to have been shocked and annealed.

Classification: H3 (estimated subtype 3.7).

Note: this name replaces provisional name NWA 736

\section{Ozerki $\quad 52^{\circ} 48.084^{\prime} \mathrm{N}, 38^{\circ} 10.268^{\prime} \mathrm{E}$}

Lipetskaya oblast', Russia

Confirmed fall: 21 June 2018

Classification: Ordinary chondrite (L6)

History: The first meteorite fragments were recovered based on analysis of the fireball observed over the Lipetskaya oblast' in Russia on June 21, 2018, at 01:16:20 UT. The fireball was widely observed from many places in Russia, including Moscow. The scientists from UrFU, UHelsinki, and the Finnish Fireball Network formed a consortium enabling quick prediction and the actual discovery of the meteorite. Collection of the observational data and trajectory analysis were done by Esko Lyytinen, Maria Gritsevich, Nikolai Kruglikov, and Mikhail Larionov. The derived parameters, characterizing the trajectory were clearly 
indicative of a number of meteorite fragments landing on the ground. Using these results, a comprehensive model of the strewn field providing the expected locations of fragments was done by Jarmo Moilanen. The area of the meteorite shower was predicted to be on the line Ozerki - Zlobino - Druzhba, Stanovlyansky district, northwest of Yelets town, Lipetskaya oblast'. The modeling assumed about 100 meteorites (excepting the main body) with total mass $\sim 7 \mathrm{~kg}$. With the support of these data, a meteorite recovery campaign was initiated by Victor I. Grokhovsky. Five samples of the meteorite $(1176 \mathrm{~g})$ were recovered during the $U r F U$ meteorite expedition-2018 (23-27 June 2018; Pastukhovich A.Yu., Yakovlev G.A., Petrova E.V. and volunteer Usenkov A.V.) in the Lipetskaya oblast'. The first sample was found 25 June 2018 by G.A. Yakovlev (59.85 g). The $U r F U$ finds were near Ozerki village. During the next few weeks, more meteorite samples $(>5500 \mathrm{~g})$ were recovered by institutional groups (Vernad - GEOKHI RAS, IA RAS, IDG RAS) and private collectors in the area Ozerki - Zlobino. The total mass and number of stones may increase due to additional finds.

Physical characteristics: The meteorites have roughly rounded shapes, covered by dark fusion crust; the interior is light colored and contains visible opaque shock veins. The samples are fresh, but halos of Fe-hydroxides were already present around metal grains. Some samples contain impact melt.

Petrography: (V.V. Sharygin, SIGM and UrFU, and D.A. Zamyatin, RAS$U B$ and $U r F U$ ): Petrographic observation of a polished section shows very rare chondrules in a coarse recrystallized matrix. Most chondrules are very poorly defined and their apparent sizes vary from $200 \mu \mathrm{m}$ to $1 \mathrm{~mm}$. Only the largest chondrules (up to $4 \mathrm{~mm}$ ) are clearly delineated. The PO, POP and BO chondrules are most common and mainly consist of olivine, plagioclase, low-Ca pyroxene \pm chromite and blebs of troilite and FeNi-metal. Other textural types of chondrules were not observed. Olivine, low-Ca-pyroxene, plagioclase, FeNi-metal and troilite are the main minerals in the matrix. Clinopyroxene, chromite, chlorapatite and merrillite (100-300 $\mu \mathrm{m})$ occur locally in the matrix. Plagioclase grains are larger than 50-100 $\mu \mathrm{m}$. Shock features include undulatory extinction, irregular and planar fractures (three directions) in olivine and the presence of opaque shock veins. The presence of impact melt suggests shock stage S5. Grains of FeNi metal (up to 1 $\mathrm{mm}$ ) are represented by both individual phases (kamacite, taenite or plessite) and their intergrowths (kamacite + taenite, kamacite + plessite + tetrataenite, kamacite + tetrataenite + pentlandite $) \pm$ troilite. Grains of troilite $(100-500 \mu \mathrm{m})$ are spongy, fine-grained aggregates of micrometer-sized individuals $(<5 \mu \mathrm{m})$. Some pentlandite grains occur in such aggregates, especially on contacts with FeNi-metal. Opaque shock veins (up to $100 \mu \mathrm{m}$ in thick) are not very abundant and contain metalsulfide intergrowths in fine-grained low-Ca pyroxene matrix. The thickness of fusion crust is up to $600 \mu \mathrm{m}$. It is highly vesicular and zoned in texture: the outmost zone is a cryptocrystalline aggregate of skeletal crystals of zoned olivine and magnetite (up to $5 \mu \mathrm{m}$ ) and glass (44-47 wt.\% $\mathrm{SiO}_{2}$ ); the inner zone is represented by larger skeletal crystals of newly formed olivine + glass + minor magnetite. Both zones contain rounded relics of initial olivine and chromite and newly formed $\mathrm{Ni}$ - 
rich metal-sulfide globules $(10-20 \mu \mathrm{m})$. Magnetic susceptibility $\log \chi\left(\times 10^{-9} \mathrm{~m}^{3} / \mathrm{kg}\right)$ $=4.8$ (analyst Yakovlev G.A.).

Geochemistry: SEM-EDS-WDS (V. V. Sharygin, SIGM and $U r F U$ ) and EMPAWDS (D.A. Zamyatin, $R A S-U B$ and $U r F U$ ). Olivine $\mathrm{Fa}_{25.6 \pm 0.3}(\mathrm{~N}=51)$, low-Ca pyroxene $\mathrm{Fs}_{21.4 \pm 0.2} \mathrm{Wo}_{1.6 \pm 0.3}(\mathrm{~N}=44)$, plagioclase $\mathrm{Ab}_{83.5} \mathrm{An}_{10.5} \mathrm{Or}_{6.0}(\mathrm{~N}=35), \mathrm{Cr}-$ bearing clinopyroxene $\mathrm{En}_{45.8} \mathrm{Fs}_{9.0} \mathrm{Wo}_{45.2}\left(\mathrm{Cr}_{2} \mathrm{O}_{3}-0.8-1.0 \mathrm{wt} . \%\right.$, $\left.\mathrm{N}=6\right)$, chromite $\mathrm{Crt}_{82.8} \mathrm{Spl}_{12.1}(\mathrm{~N}=17)$, chlorapatite, merrillite, FeNi-metals, troilite and pentlandite. Chlorapatite contains $\mathrm{F}$ (up to 0.5 wt.\%); $\mathrm{Cl}-5.5, \mathrm{Na}_{2} \mathrm{O}-0.5$ wt.\% $(\mathrm{N}=11)$. Merrillite is poor in $\mathrm{FeO}$ (0.3-0.7 wt.\%, $\mathrm{N}=8)$. Composition of metal (in wt.\%): kamacite $(\mathrm{N}=32)-\mathrm{Fe} 93.2 \pm 1.4, \mathrm{Ni}-5.89 \pm 1.44, \mathrm{Co}-0.92 \pm 0.13$; taenite $(\mathrm{N}=20)-$ Fe 68.6 \pm 5.1 , Ni 31.0 \pm 5.1 , Co 0.36 \pm 0.09 ; tetrataenite $(\mathrm{N}=3)-\mathrm{Fe} 48.5$, Ni 51.2, Co 0.16 . Composition of sulfides (in wt.\%): pentlandite ( $\mathrm{N}=10$, in association with troilite) - Fe 50.6 \pm 0.8 , Ni 15.4 \pm 1.0 , Co $0.15 \pm 0.02, \mathrm{Cu} 0.61 \pm 0.26, \mathrm{~S} 33.6 \pm 0.3$; heazlewoodite ( $\mathrm{N}=10$, from fusion crust) $-\mathrm{Fe} 2.62 \pm 0.46$, Ni $71.4 \pm 0.8$, Co $0.11 \pm 0.01, \mathrm{Cu} 0.37 \pm 0.09, \mathrm{~S} 25.6 \pm 0.5$.

Classification: Ordinary chondrite. L6, S5, W0.

Specimens: $U r F U$ - $798.6 \mathrm{~g}$ (3 samples: 550.4, 188.3, 59.9 g); Vernad - $603 \mathrm{~g} \mathrm{(3}$ samples); SIGM - $31.7 \mathrm{~g}$ fragment; RAS-UB - $15 \mathrm{~g}$ fragment; UHelsinki - $15 \mathrm{~g}$ fragment.

\section{Parauapebas $\quad 6^{\circ} 3.373^{\prime} \mathrm{S}, 4^{\circ} 53.347^{\prime} \mathrm{W}$ \\ Para, Brazil \\ Probable fall: 2013 Dec 09 \\ Classification: Ordinary chondrite (H4-5)}

History: (D. Cunha, D. Atencio, A.L.R. Moutinho) On 9 December 2013, about 7:00 p.m. local time, a meteorite fell in the city of Parauapebas, located in the eastern part of Pará State, Brazil. A witness reported seeing the bolide traveling from NE to SW direction. A stone hit the roof of a house with a loud noise that was heard by the resident Mrs. Maria. She claims that a meteorite fragment hit and injured her right shoulder but her son says that she was hit only by roof debris. Her son, I. S. Lima, collected the stone on the roof. The stone broke in two pieces during the impact. One piece is lost but the other remaining $62 \mathrm{~g}$ piece was kept by Mrs. Maria for some years and then donated to the Institute of Geosciences, USP. Another $210.3 \mathrm{~g}$ oriented mass was observed to fall by other city residents while they were in front of their house. Meire C. Rosa, her husband Paulo T. Nunes, her mother Rosa C. Santos and neighbors heard loud thunder and a noise when the stone penetrated the wooden beam of their house, embedding itself in the ground. Meire immediately collected the stone and noticed it was still warm. In early 2018, she contacted Andre L.R. Moutinho, who was already part of the classification team of the $62 \mathrm{~g}$ fragment. The $210.3 \mathrm{~g}$ stone was purchased by Andre L. R. Moutinho.

Physical characteristics: The $210.3 \mathrm{~g}$ mass is almost fully crusted, and measures $5.7 \times 5.3 \times 4.2 \mathrm{~cm}$, and shows orientation features such as nose-cone shape and roll-over lips. A small portion of secondary crust is also present. The $62 \mathrm{~g}$ fragment 
measuring $4.8 \times 3.5 \times 2.6 \mathrm{~cm}$ shows the gray interior with two distinct lithologies: type- 4 clast with an abundance of chondrules showing sharply defined edges; and type- 5 clast, in which the edges of the chondrules are beginning to merge with the surrounding groundmass. Shock melt veins indicate it is a breccia.

Petrography: (C.V.N. Villaça and M.E. Zucolotto, MNRJ/UFRJ; D. Cunha, D. Atencio, IGc/USP). Polished thin sections were examined by MEV/EDS and electron microprobe. There are some black shock-induced melt veins, one broader crossing the entire thin section. Olivine, ortho and clinopyroxene were observed with abundant Fe-Ni-metal and sulfide. Mineralogy dominated by forsterite, enstatite, iron, troilite, and tetrataenite. Albite, chromite, diopside, augite, pigeonite, taenite, and merrillite are minor components. Texturally there are at least two lithologies. One exhibits an abundance of chondrules with well-defined margins and no visible plagioclase, consistent with petrologic type 4 chondrites. The other lithology displays chondrules with outlines blurring into the groundmass as evidence of an increasing recrystallization. In at least one clast some small plagioclase of about $20 \mu \mathrm{m}$ confirmed a type- 5 lithology. The presence of shock veins and large olivine with undulatory extinction, fractures and some weakly mosaicism infer a shock state $\mathrm{S} 4$

Geochemistry: (A.A. Tosi, Labsonda/UFRJ, A.L.R. Moutinho) Microprobe analysis yields: Type 4 - olivines $\left(\mathrm{Fa}_{19.3 \pm 0.4}, \mathrm{PMD}=2.22, \mathrm{n}=30\right)$; low-Ca pyroxenes $\left(\mathrm{Fs}_{17.3 \pm 0.9} \mathrm{Wo}_{0.9 \pm 0.3}, \mathrm{PMD}=9.16, \mathrm{n}=23\right)$; high-Ca pyroxene $\mathrm{Fs}_{10.4 \pm 2.6} \mathrm{Wo}_{28.3 \pm 11.5}$, PMD 29.2, $\mathrm{n}=12$. Type 5 - olivines $\left(\mathrm{Fa}_{18.9 \pm 0.4} \mathrm{PMD}=1.96, \mathrm{n}=\right.$ 45); low-Ca pyroxenes $\left(\mathrm{Fs}_{16.9 \pm 0.6} \mathrm{Wo}_{1.6 \pm 0.9}, \mathrm{PMD}=5.44, \mathrm{n}=19\right)$; high-Ca pyroxene $\mathrm{Fs}_{15.1 \pm 2.5} \mathrm{Wo}_{16.0 \pm 2.5}$, PMD $\left.22.7 \mathrm{n}=7\right)$. Metal: iron $(\mathrm{Ni}=6.15 \pm 0.32 \mathrm{wt} . \%, \mathrm{Co}=$ $0.42 \pm 0.02$ wt. $\%, \mathrm{n}=9)$, tetrataenite $(\mathrm{Ni}=52.9 \pm 2.3$ wt. $\%, \mathrm{Co}=0.09 \pm 0.08$ wt. $\%, \mathrm{n}$ $=3)$, taenite $(\mathrm{Ni}=37.1 \mathrm{wt} . \%, \mathrm{Co}=0.12 \mathrm{wt} . \%, \mathrm{n}=1)$, troilite $(\mathrm{Ni}=0.11 \pm 0.11$ wt.\%, $\mathrm{n}=9)$ and chromite $(\mathrm{Cr} /(\mathrm{Cr}+\mathrm{Al})=0.928 \pm 0.003, \mathrm{n}=3)$. Cathodoluminescence also gives results comparable to breccia H4-5. (D. Atencio, D. Cunha, A. L. R. Moutinho, Laboratório de Microscopia Eletrônica/IGc/USP) SEM/EDS analysis yields: olivines $\left(\mathrm{Fa}_{19.6 \pm 1.1}, \mathrm{PMD}=9.9, \mathrm{n}=6\right)$; low-Ca pyroxenes $\left(\mathrm{Fs}_{16.6 \pm 0.7} \mathrm{Wo}_{0.0 \pm 0.0}, \mathrm{PMD}=3.43, \mathrm{n}=15\right)$. plagioclase $\left(\mathrm{Ab}_{83.0 \pm 2.0} \mathrm{An}_{12.2 \pm 1.3} \mathrm{Or}_{4.8 \pm 0.8}, \mathrm{n}=5\right)$. Metal: tetrataenite $(\mathrm{Ni}=49.16 \pm 2.75 \mathrm{wt} . \%, \mathrm{n}=3)$, troilite $(\mathrm{Fe}=61.49 \pm 1.66$ wt. $\%, \mathrm{~S}=37.46 \pm 1.02 \mathrm{wt} . \%, \mathrm{n}=10)$ and chromite $(\mathrm{Cr} /(\mathrm{Cr}+\mathrm{Al})=0.85, \mathrm{n}=2)$ Classification: It is a genomict breccia with the clasts and matrix from the same compositional group but different petrologic types, H4-5, S4, W0

Specimens: IGc/USP: $34.8 \mathrm{~g}$, MNRJ: $4 \mathrm{~g}$; Andre L. R. Moutinho: $210.3 \mathrm{~g}$ main mass and $13.08 \mathrm{~g}$ slice.

\author{
Qira $001 \quad 37^{\circ} 34^{\prime} 22.01^{\prime \prime} \mathrm{N}, 80^{\circ} 23^{\prime} 33.46 " \mathrm{E}$ \\ Xinjiang, China \\ Find: 2012 \\ Classification: Ordinary chondrite $(\mathrm{H} \sim 5)$
}


History: (R. Bartoschewitz, Bart) Discovered by Bi Chunlin in the desert in Hotan county/Xinjiang in 2012 and was purchased by Wang Ziyao, who donated it to R. Bartoschewitz.

Physical characteristics: (R. Bartoschewitz, Bart) Red-brown 8.03 g stone with adhering desert sand.

Petrography: (R. Bartoschewitz, Bart) Red-brown stainted recrystallized matrix with nearly complete recrystallized chondrules (av. $\sim 1.0 \mathrm{~mm}$ ). Metal and sulfide completely oxidiezed, vugs partly filled with gypsum.

Geochemistry: Magnetic susceptibility (R. Bartoschewitz, Bart $) \log \chi\left(\times 10^{-}\right.$ $\left.{ }^{9} \mathrm{~m}^{3} / \mathrm{kg}\right)=4.59$

Classification: Ordinary chondrite (H $\sim 5, \mathrm{~W} 5)$

Specimens: $1.70 \mathrm{~g}$ on deposit at Kiel, main mass of $5.8 \mathrm{~g}$ with Bart.

\section{Rabt Sbayta $010 \quad 24.135944,-14.763139$}

Rio de Oro, Western Sahara

Find: 2017 Dec 8

Classification: Martian meteorite (basaltic breccia)

History: Found on 12/08/2017 by Zaid Oualguirah in the same area as NWA 7034. Purchased by Jean Redelsperger from Mohamed Elguirah in Zagora, Morocco, in 2018.

Physical characteristics: Three identical appearing pieces $4.8 \mathrm{~g}, 0.7 \mathrm{~g}$, and $0.6 \mathrm{~g}$, two pieces preserve patches of original glassy fusion crust, the remaining exterior surfaces have a shiny black luster. Saw cut reveals a breccia with numerous dark and light colored fragmental crystals and polycrystalline lithologies, scattered spherules and spherical objects, set in a dark gray, fine-grained groundmass, some opaques visible.

Petrography: (C. Agee, $U N M$ ) Microprobe examination of the polished deposit sample shows a polymict breccia with fragmental feldspar and pyroxene grains, set in a very fine-grained groundmass composed of feldspar, pyroxene, oxides, and phosphates.

Geochemistry: (C. Agee, $U N M$ ) Low-Ca pyroxene $\mathrm{Fs}_{36.0 \pm 10.0} \mathrm{Wo}_{5.0 \pm 2.3}$, $\mathrm{Fe} / \mathrm{Mn}=36 \pm 4, \mathrm{n}=10$; high-Ca pyroxene $\mathrm{Fs}_{23.5 \pm 2 .} \mathrm{Wo}_{40.3 \pm 6.5}, \mathrm{Fe} / \mathrm{Mn}=32 \pm 1, \mathrm{n}=2$; plagioclase $\mathrm{Ab}_{58.4 \pm 6.5} \mathrm{An}_{38.5 \pm 8.0} \mathrm{Or}_{3.2 \pm 1.6}, \mathrm{n}=12$.

Classification: Martian (basaltic breccia). Paired with NWA 7034 pairing group Specimens: $1.3 \mathrm{~g}$ including a probe mount on deposit at UNM Jean Redelsperger holds the main mass.

Ramlat as Sahmah $530(\operatorname{RaS} 530) \quad 20^{\circ} 34.104^{\prime} \mathrm{N}, 55^{\circ} 43.342^{\prime} \mathrm{E}$

Al Wusta, Oman

Find: 20 Apr 2014

Classification: Ureilite

History: The meteorite was found on 20 April 2014 during a field trip in the Oman desert. 
Physical characteristics: Brownish individual lacking any fusion crust. Petrography: The meteorite is dominantly composed of $<1 \mathrm{~mm}$ sized olivine grains, sometimes poikilitically enclosing augite crystals. Olivine shows abundant reduced rims; the meteorite contains flaky graphite and Cr-bearing sulfides. Cracks are often found to be filled with barite likely due to intense terrestrial weathering. Geochemistry: Reduced rims in olivine: $\mathrm{Fa}_{1.4-4.5}$; olivine contains $0.28 \pm 0.03 \mathrm{wt} \%$ $\mathrm{Cr}_{2} \mathrm{O}_{3}$ and $0.22 \pm 0.02 \mathrm{wt} \% \mathrm{CaO}$; augite contains $1.58 \pm 0.03 \mathrm{wt} \% \mathrm{Cr}_{2} \mathrm{O}_{3}$ and $3.37 \pm 0.03$ wt $\% \mathrm{Al}_{2} \mathrm{O}_{3}$

Classification: Ureilite, augite-bearing

\section{Ramlat Fasad $057 \quad 19^{\circ} 0.580^{\prime} \mathrm{N}, 53^{\circ} 20.587^{\prime} \mathrm{E}$ \\ Zufar, Oman \\ Find: 31.01 .16 \\ Classification: Martian meteorite (Shergottite)}

History: A single fragment was found during a search for meteorites.

Physical characteristics: Pale/greyish brown to greenish brown, platy fragment, $3 \times 4 \times 0.7 \mathrm{~cm}$, without fusion crust. Wind-ablation on small sides.

Petrography: (Felix Mechelke, Bern, B. Hofmann, NMBE) The meteorite consists of pigeonite and augite (together $48 \mathrm{vol} \%$, typical length $0.5-1 \mathrm{~mm}$ ), olivine ( 35 vol. \%, typical size $0.6 \mathrm{~mm}$ ) and plagioclase completely transformed to maskelynite (11 vol.\%, typical size $0.3 \times 0.7 \mathrm{~mm}$ ). Accessories (6 vol\%) comprise pyrrhotite and spinel. Weathering effects: Minor replacement of silicates by terrestrial calcite along fractures, staining of silicates with iron hydroxides near surface.

Geochemistry: (Felix Mechelke, Bern, B. Hofmann, NMBE) Olivine rim compositions are $\mathrm{Fa}_{40.8 \pm 3.5}(\mathrm{~N}=43)$. Dark brown, partially altered cores are $\mathrm{Fa}_{35.1 \pm 1.9}(\mathrm{~N}=21)$. Pigeonite compositions are $\mathrm{Fs}_{29.8 \pm 3.1}(\mathrm{~N}=44$, range 27.3-43.4), $\mathrm{Wo}_{8.8 \pm 1.7}$ (range 6.3-13.6), mean $\mathrm{FeO} / \mathrm{MnO}=28.0 \pm 1.4$; augite compositions are $\mathrm{Fs}_{23.1 \pm 2.8}$ (range 20.1-29.2, $\mathrm{N}=8$ ) $\mathrm{Wo}_{31.0 \pm 5.3}$ (range 20.3-36.4). Maskelynite has $\mathrm{An}_{53.0 \pm 1.5}$ (range 48.5-55.7) $\mathrm{Or}_{1.7 \pm 0.4}($ range 1.2-2.8, $\mathrm{N}=29$ ). Bulk Fe/Mn $(\mathrm{XRF})$ is $35.5 \pm 2.7(\mathrm{~N}=5)$. Oxygen isotopes (R. Greenwood, $O U$ ): $\delta^{17} \mathrm{O} 3.031, \delta^{18} \mathrm{O} 5.257$, $\Delta^{17} \mathrm{O} 0.297$.

Classification: Based on petrography, mineral compositions, bulk Fe/Mn and oxygen isotopes this is a strongly shocked shergottite.

Specimens: All at $N M B E$

\section{Realicó $\quad 35^{\circ} 1^{\prime} \mathrm{S}, 64^{\circ} 19^{\prime} \mathrm{W}$}

La Pampa, Argentina

Find: 1970

Classification: Ordinary chondrite (L5)

History: The meteorite was found around 1970 by a farmer while plowing a rockfree field in the Realicó area, La Pampa Province, Argentina. The only mass, with a weight of $12 \mathrm{~kg}$ approximately, was kept at the farm. This find was little known until 2015, when an amateur astronomer (J.C. Spagnotto, Observatorio El Catalejo 
de Santa Rosa, La Pampa, Argentina) learned about the meteorite. Spagnotto sent a sample to ICATE and the stone was identified as a chondrite.

Petrography: The stone is highly altered. Different types of chondrules are visible: $\mathrm{BO}, \mathrm{RP}$ and POP with apparent diameter ranging from 600 microns to $1.5 \mathrm{~mm}$. The shock features record by crystals such as: undulatory extinction, planar fractures, melt pockets and the presence of polycrystalline troilite indicate a state of shock S3 or S4. A strong degree of oxidation of metal suggest an alteration stage W3.

Geochemistry: Mineral composition: EMP analyses, Olivine, $\mathrm{Fa}_{25.4 \pm 0.5}(\mathrm{n}=51)$; pyroxene, $\mathrm{Fs}_{21.1 \pm 0.5} \mathrm{Wo}_{1.2 \pm 0.2}(\mathrm{n}=28)$.

Classification: Ordinary chondrite (L5).

Specimens: Type specimen of $47.5 \mathrm{~g}$ is on deposit at the $M L P$ and three thin sections are on deposit at ICATE, Argentina. The main mass remains at the farm.

Reckling Peak 14003 (RKP 14003) $\quad 76^{\circ} 12.744^{\prime} \mathrm{S}, 158^{\circ} 32.205^{\prime} \mathrm{E}$

Antarctica

Find: 2014 Nov 10

Classification: Ordinary chondrite (H3)

Petrography: The section shows sharply defined chondrules ( $\sim 0.4 \mathrm{~mm}$ in average) with some glassy mesostasis. Metal abundance is $\sim 13 \mathrm{vol} \%$. The section appears to be brecciated.

Geochemistry: Olivine composition is $\mathrm{Fa}_{0.4-36.4}$; low-Ca pyroxene is $\mathrm{Fs}_{15.5-}$ ${ }_{40.6} \mathrm{Wo}_{0.3-2.7}$

Reckling Peak 14005 (RKP 14005) $\quad 76^{\circ} 15.954^{\prime} \mathrm{S}, 158^{\circ} 07.073^{\prime} \mathrm{E}$

Antarctica

Find: 2014 Nov 16

Classification: Carbonaceous chondrite (CM2)

Petrography: The section consists of relatively small chondrules ( $\sim 16$ vol $\%$, $\sim<100 \mu \mathrm{m}$ to $\sim 1.6 \mathrm{~mm}$ in diameter, the average size of chondrule is $\sim 300 \mu \mathrm{m}$ ) and matrix $(\sim 84 \mathrm{vol} \%)$. Single hibonite crystal is observed in matrix. Fe-Ni metal is very rare $(<0.1 \mathrm{vol} \%)$. The section has abundant hydrated materials, mainly serpentine. The matrix occurs as fine-grained rims surrounding chondrules.

Geochemistry: Olivine is $\mathrm{Fa}_{0.4-57.5}$; low-Ca pyroxene is $\mathrm{Fs}_{1.0-3.7} \mathrm{Wo}_{0.9-3.4}$.

Reckling Peak 14006 (RKP 14006) $\quad 76^{\circ} 16.344^{\prime} \mathrm{S}, 158^{\circ} 08.727^{\prime} \mathrm{E}$

Antarctica

Find: 2014 Nov 16

Classification: Ordinary chondrite (H3)

Petrography: The section shows sharply defined chondrules ( $\sim 70$ vol\%, average size of $\sim 0.3 \mathrm{~mm}$ ) with glassy mesostasis. Metal abundance is $\sim 10 \mathrm{vol} \%$.

Geochemistry: Olivine is $\mathrm{Fa}_{0.5-39.9}$; low-Ca pyroxene is $\mathrm{Fs}_{4.1-42.7} \mathrm{Wo}_{0.1-5.3}$. 


\section{Renchen $\quad 48^{\circ} 35^{\prime} 17.9988^{\prime}{ }^{\prime} \mathrm{N}, 8^{\circ} 0^{\prime} 00.00^{\prime} \mathrm{E}$ \\ Baden-Wurttemberg, Germany \\ Confirmed fall: 10 July 2018 \\ Classification: Ordinary chondrite (L5-6)}

History: (Pavel Spurný, $C z A S$ ). A -13.4 maximum absolute magnitude bolide was observed by many of eyewitnesses, mainly over western Germany, eastern France, Belgium, Netherlands, Luxembourg, and Switzerland, and recorded photographically and photoelectrically by instruments of the Czech and German parts of the European Fireball Network on July 10, 2018, at 21:29:49-53 UT. Based on these instrumental data, precise results on atmospheric trajectory, heliocentric orbit and fragmentation history were quickly determined. From this analysis (done at $C z A S$ ), it was evident that this event terminated with a multiple meteorite fall in Baden-Württemberg, close to the Rhine river. The impact area for a possible range of meteorite masses was thus modeled and sent to a German colleague (Dieter Heinlein) shortly after the fall. As a result, all of the meteorites were found in the predicted location for a given mass during dedicated searches.

Physical characteristics: (D. Heinlein). A total mass of $999 \mathrm{~g}$ was recovered within the predicted fall site. The first meteorite $(12 \mathrm{~g})$ was discovered two weeks after the fall. The largest meteorite fragment had a mass of $955 \mathrm{~g}$ and was found in a field. close to a small impact pit. Two of the meteorites ( 5 and $6 \mathrm{~g}$ ) were caught by a hail net covering a fruit plantation.

Petrography: (M. Patzek and A. Bischoff, IfP): Based on the study of the thin sections, Renchen is brecciated. Highly recrystallized fragments (type 6) coexist with areas showing a chondritic texture (type 5) and fragments of impact melt. Olivine shows weak mosaicism indicating shock stage S4. Opaque phases include metals (kamacite, taenite), troilite, and chromite. Other accessory phases include $\mathrm{Cl}$-apatite and merrillite. Several shock veins cross the meteorite.

Geochemistry: (M. Patzek and A. Bischoff, IfP): The mean composition of olivine is $\mathrm{Fa}_{26.0 \pm 0.5}$ with a compositional range of $\mathrm{Fa}_{24.6-27.1}(\mathrm{n}=59)$. The low-Ca pyroxene has a mean composition of $\mathrm{Fs}_{21.9 \pm 0.4} \mathrm{Wo}_{1.4 \pm 0.3}(\mathrm{n}=21)$, varying between $\mathrm{Fs}_{21.1-}$ 22.6. Mean plagioclase is $\mathrm{An}_{9.8 \pm 0.6} \mathrm{Or}_{3.9 \pm 2.6}\left(\mathrm{n}=10\right.$; range $\left.\mathrm{An}_{8.6-10.7}\right)$. O-isotopes (A. Pack; UGött): The mean oxygen isotope composition of two analyzed fragments is: $\delta^{17} \mathrm{O}=3.63 \%$, $\delta^{18} \mathrm{O}=4.74 \%, \Delta^{17} \mathrm{O}=1.12 \%$ (relative to a reference line with a slope of 0.5305 ). The data are consistent with those of other $\mathrm{L}$ chondrites.

Classification: L chondrite breccia (L5-6, S4, W0)

Specimens: Type Specimens: $20.9 \mathrm{~g}$, IfP; the main masses are with the finders.

\section{Sahara 98063}

Sahara

Find: 1998

Classification: Ordinary chondrite (H3) 
History: Sample was found 1998 by the Labenne-group. The coordinates are unknown.

Petrography: The sample is an unequilibrated chondrite, with chondrules of about $500 \mu \mathrm{m}$. Metal abundance is about $10 \mathrm{vol} \%$.

Geochemistry: Olivine compositions are $\mathrm{Fa}_{6.7 .-0-26.3}\left(\right.$ mean $\mathrm{Fa}_{19.6 \pm 4.3}, \mathrm{n}=21$ ), pyroxene compositions are $\mathrm{Fs}_{2.1-17.5} \mathrm{Wo}_{0.2-5.4}$ (mean $\mathrm{Fs}_{12.3 \pm 5.7} \mathrm{Wo}_{1.3 \pm 1.2}, \mathrm{n}=20$ ).

Classification: $\mathrm{H} 3$ chondrite based on the high metal abundance and many silicate analyses in the H-group field.

\title{
San Juan de Allende $\quad 26.94^{\circ} \mathrm{N}, 105.30^{\circ} \mathrm{W}$ \\ Chihuahua, Mexico \\ Find: 1995 Aug \\ Classification: Ordinary chondrite (L3)
}

History: An approximately $200 \mathrm{~g}$ single invidual was found by Ruben Jurado, sold to Edwin Thompson, with portions donated to Cascadia in 2004 and 2008.

Petrography: Well-defined chondrules, some glass-bearing, with an average diameter of $0.77 \pm 0.34 \mathrm{~mm}(\mathrm{~N}=59)$, are present in a chondrite with an estimated metal content of 3.5 area\%.

Geochemistry: Chondrules contain unequilibrated olivine $\left(\mathrm{Fa}_{22.1 \pm 7.5}, \mathrm{~N}=99\right)$ and low-Ca pyroxene $\left(\mathrm{Wo}_{0.9 \pm 0.8} \mathrm{Fs}_{11.6 \pm 8.2}, \mathrm{~N}=52\right.$ ). A subset of olivine point analyses, obtained with longer count times for better minor element data, gives a $\mathrm{Cr}_{2} \mathrm{O}_{3}$ content of $0.08 \pm 0.08 \mathrm{wt} \%(\mathrm{~N}=13)$.

Classification: L3 chondrite based on mean chondrule size, metal abundance, olivine and pyroxene compositions. Subtype estimated as 3.4-3.6, based on olivine Fa content and $\mathrm{Cr}_{2} \mathrm{O}_{3}$ content (latter suggests $\sim 3.2$ ).

Specimens: Type sample at Cascadia consists of four pieces totaling to $20.2 \mathrm{~g}$ as well as a polished thin section. A $113 \mathrm{~g}$ main mass specimen is held by a person wishing to remain anonymous.

\author{
San Juan 085 (SJ 085) ～25²2’29.4"S, 6948’55.3"W \\ Antofagasta, Chile \\ Find: 2017 Nov \\ Classification: Carbonaceous chondrite (CK6)
}

Physical characteristics: Dark stone. Cut surface reveals an homogeneous darkgray interior.

Petrography: (J. Gattacceca, CEREGE) Recrystallized chondrite with poorly defined large chondrules. Silicates are olivine, Ca-pyroxene, and plagioclase (average size $>50 \mu \mathrm{m}$ ). No orthopyroxene was observed. Abundant sulfides (including pentlandite) and magnetite. No metal was observed.

Geochemistry: Olivine $\mathrm{Fa}_{33.2 \pm 0.4}$, NiO $0.48 \pm 0.06 \mathrm{wt} \%$ (N=4). Ca-pyroxene $\mathrm{Fs}_{12.7 \pm 0.0} \mathrm{Wo}_{47 \pm 0.2}(\mathrm{~N}=2)$. Plagioclase $\mathrm{An}_{31.9} \mathrm{Ab}_{64.1} \mathrm{Or}_{4.0}(\mathrm{~N}=1)$. Magnetite contains 3.7 $\mathrm{wt} \% \mathrm{Cr}_{2} \mathrm{O}_{3}$.

Classification: Carbonaceous chondrite (CK6) 
Specimens: Type specimen at CEREGE. Main mass with ARTMET.

San Juan 086 (SJ 086)

$$
25^{\circ} 37^{\prime} 55^{\prime \prime} \mathrm{S}, 69^{\circ} 46^{\prime} 16^{\prime \prime} \mathrm{W}
$$

Antofagasta, Chile

Find: November 9, 2017

Classification: Ordinary chondrite (L6, melt breccia)

History: The meteorite was found in 2017 during a field trip in the Atacama desert. Physical characteristics: Dark brownish individual without fusion crust.

Petrography: The meteorite is composed of regions displaying chondritic textures with relict chondrules and mean plagioclase size about $60 \mu \mathrm{m}$, and large melt regions characterized by recrystallized shock melt containing characteristic FeNi metal and sulfide globules. Chondritic portions are crosscut by shock melt veins.

San Juan 089 (SJ 089) $\quad 25^{\circ} 26.488^{\prime} \mathrm{S}, 69^{\circ} 53.232^{\prime} \mathrm{W}$

Antofagasta, Chile

Find: 18 Jan 2017

Classification: Ordinary chondrite (H5)

History: Charles and Nick Lucas used low-grade magnets attached to poles, while walking north-south lines about 5 mapart in the area where the meteorite was found, which is a relatively flat area with a 2 to 3 degree down slope to the north.

Physical characteristics: Dark-brown weathering patina covers entire exterior. Patches of beige caliche occur on top of the patina on one side of the samples Petrography: The overall texture is fairly well integrated in thin section, although chondrules are readily identifiable. Chondrule mesostases are fine grained and include devitrified glass. Numerous chromite-plagioclase regions and several grains of metallic copper are present. BSE imaging shows that olivine and low-calcium pyroxene grains are coarse and equilibrated. Both chondrule mesostases and chromite-plagioclase objects extend into feldspathic veins which invade surrounding ferromagnesian. Feldspathic mesostases experienced segregation of sodic, potassic, and calcic components and display different gray levels in BSE images. Tiny chromite grains are concentrated in albitic patches in mesostases. One barred olivine chondrule was examined in detail. It contained a potassium feldspar grain adjacent to a grain with a perthitic intergrowth of albite and orthoclase. In the same chondrule were regions of chromite-albitic plagioclase intergrowths and small patches of calcic feldspathic glass.

Geochemistry: (M. Hutson and A. Ruzicka, Cascadia) Olivine $\left(\mathrm{Fa}_{19.9 \pm 0.5}, \mathrm{~N}=34\right)$ and low-Ca pyroxene $\left(\mathrm{Fs}_{17.8 \pm 0.7} \mathrm{Wo}_{1.3 \pm 0.2}, \mathrm{~N}=27\right)$ grains are equilibrated. Feldspathic material occurs in three roughly defined compositions (sodic $\mathrm{Ab}_{79.8} \mathrm{An}_{15.0} \mathrm{Or}_{5.2}$, $\mathrm{N}=13$; calcic $\mathrm{Ab}_{26.4} \mathrm{An}_{73.2} \mathrm{Or}_{0.4}, \mathrm{~N}=4$; potassic $\mathrm{Ab}_{10.6} \mathrm{An}_{4.2} \mathrm{Or}_{85.2}, \mathrm{~N}=5$ ).

Classification: $\mathrm{H} 5$ based on chemistry and texture.

Specimens: Cascadia holds $17.3 \mathrm{~g}$ in three pieces, in addition to one polished thin section and a mounted butt. 


\author{
San Juan 100 (SJ 100) $\quad 25^{\circ} 29.875^{\prime}$ 'S, 6944.639’W \\ Antofagasta, Chile \\ Find: 14 Feb 2018 \\ Classification: Ureilite
}

Physical characteristics: Sawn surface shows a dark interior with scattered graphite flakes. Stone low weathered.

Petrography: Thin section shows typical ureilitic texture with interlocked olivine (40 vol\%) and pyroxene (60 vol\%) grains of $0.8-3 \mathrm{~mm}$ in size. Common graphite aggregates up to $1 \mathrm{~mm}$ are observed.

Geochemistry: Olivine cores in the range $\mathrm{Fa}_{11.4-13.1}$ (mean $\mathrm{Fa}_{11.9 \pm 0.5}, \mathrm{~N}=18$ ), with $\mathrm{FeO} / \mathrm{MnO}=21.6 \pm 3.2$ and $\mathrm{Cr}_{2} \mathrm{O}_{3}=0.68 \pm 0.07$. Pyroxene in the range $\mathrm{Fs}_{10.0-11.6}$ (mean $\mathrm{Fs}_{10.6 \pm 0.5} \mathrm{Wo}_{4.4 \pm 0.3}, \mathrm{~N}=20$ ).

\title{
San Pedro de Urabá
}

$$
8^{\circ} 16^{\prime} 44.39^{\prime \prime} \mathrm{N}, 76^{\circ} 22^{\prime} 41.67^{\prime \prime} \mathrm{W}
$$

Antioquia, Colombia

Confirmed fall: 16 Feb 2017

Classification: Ordinary chondrite (L6)

History: At 5:30 pm local time on 16 February 2017, a large fireball with sonic booms was observed in northern Colombia. A meteorite subsequently impacted near Mr. Orlando Cuevas on the edge of a soccer field in San Pedro de Urabá, Turbo district, Antioquia State, Colombia. The event was widely reported in the local media, e.g., www.minuto30.com/fotos-en-zona-rural-de-turbo-aseguran-quecayo-un-meteorito/340813/, which shows a picture of the $3768 \mathrm{~g}$ stone from the soccer field. Two other stones are known to have fallen, one much larger than the one that was collected in the soccer field, but their whereabouts are unknown. Michael Farmer acquired the $3768 \mathrm{~g}$ stone from Mr. Cuevas.

Physical characteristics: Single regmaglypted stone covered with matte black fusion crust. Interior is a light greenish-gray and friable. Cut surface shows poorly defined chondrules and even distribution of small metal/troilite grains. Only a single thin shock vein is visible.

Petrography: (L. Garvie, ASU) SEM observation of a polished mount shows scattered poorly defined chondrules (BO, RP, and $\mathrm{PO}$ ) largely integrated with the matrix. All silicates heavily fractured. Feldspar grains typically $>50 \mu \mathrm{m}$, with many around 100 to $200 \mu \mathrm{m}$. Troilite anhedral and dominantly single crystal to $400 \mu \mathrm{m}$. Rare anhedral Ca-Na-Mg phosphates to $60 \mu \mathrm{m}$. Chromite to $600 \mu \mathrm{m}$ is anhedral to subhedral and heavily fractured. Three Fe-Ni metal types present: kamacite, dominantly single crystal (to $0.5 \mathrm{~mm}$ ) with a frosty etch and weakly defined Neumann bands; tetrataenite, rare, commonly contiguous to kamacite; and, equant $\mathrm{Ni}$-zoned grains with Ni-rich rims and dark-etched cores, some showing kamacite spindles. Native $\mathrm{Cu}$ is rare and occurs as $<10$ micron grains at kamacite/troilite boundary. Scattered and rare melt pockets to $60 \mu \mathrm{m}$. 
Geochemistry: Olivine $\mathrm{Fa}_{25.1 \pm 0.3}, \mathrm{FeO} / \mathrm{MnO}=47.6 \pm 1.8, \mathrm{n}=9$; low Ca pyroxene $\mathrm{Fs}_{21.4 \pm 1.2} \mathrm{Wo}_{1.5 \pm 0.2}, \mathrm{FeO} / \mathrm{MnO}=28.4 \pm 1.3, \mathrm{n}=10$; Feldspars $\mathrm{Or}_{13.4 \pm 0.3} \mathrm{Ab}_{67.3 \pm 67.3 \pm 0.5}, \mathrm{n}=3$ and $\mathrm{Or}_{5.4 \pm 0.1} \mathrm{Ab}_{84.4 \pm 0.02}, \mathrm{n}=2$.

Classification: Ordinary chondrite L6, S3, W0

Specimens: $32 \mathrm{~g}$ at $A S U$. Main mass with MFarmer.

Sauceda de la Borda $\quad 22^{\circ} 49^{\prime} 15.73 " \mathrm{~N}, 102^{\circ} 31^{\prime} 28.99^{\prime \prime} \mathrm{W}$

Zacatecas, Mexico

Find: October, 2016

Classification: Iron meteorite (IAB-MG)

History: Found October, 2016, by Mr. Alejandro Cortes while he was clearing brush along a dirt road about $2 \mathrm{~km}$ southwest of Sauceda de la Borda. About 1/4 of the stone was sticking out of the ground. Purchased by Michael Farmer from the finder in December 2016.

Physical characteristics: A relatively unweathered and beautifully regmaglypted $41 \mathrm{~kg}$ stone, measuring approximately $37 \times 19 \times 18 \mathrm{~cm}$. A $17 \times 12 \mathrm{~cm}$ cut and etched slice shows a well-developed Widmanstätten pattern and an abundance of graphite/troilite nodules, one $8 \times 2 \mathrm{~cm}$. A well-developed heat-affected zone is present around the whole of the slice. Layered fusion crust and beads of ablated material are present within some of the regmaglypts.

Petrography: (L. Garvie, ASU) A $13 \times 10 \mathrm{~cm}$ slice demonstrates a variable structure typical of many group IAB octahedrites, with half of the section rich in decomposed cohenite and the other half showing a well-developed coarse octahedral structure with few inclusions - the two structural types are separated by an elongated graphite/troilite nodule. Etched sections display a coarse Widmanstätten pattern with short but bulky kamacite lamellae, typically $2 \mathrm{~mm}$ wide. Kamacite shows two generations of Neumann bands: the first are strongly developed, whereas the second are partially annealed and decorated with sub- $\mu \mathrm{m}$ sized precipitates. Taenite/plessite occupies $\sim 4$ areal $\%$, most commonly as ribbons and wedge-shaped blocks separating kamacite lamellae. Plessite shows dominantly pearlitic, comb, and net structures. Decomposed cohenite is present in abundance as smoothly rounded elongated grains (to $4 \mathrm{~cm}$ ), within and following kamacite lamellae, enveloping schreibersite grains, and around graphite/troilite nodules. In a polished mount, all of the cohenite is decomposed to graphite and ferrite. Schreibersite occurs in three forms: as large grains (to $8 \mathrm{~mm}$ ) with enveloping decomposed cohenite and surrounded by swathing kamacite; as grain boundary veinlets; and as rhabdites and sub-micron precipitates. The troilite/graphite nodules are entirely mantled by a structurally complex rim $(<5 \mathrm{~mm}$ thick) of troilite, graphite, schreibersite, and decomposed cohenite. Deep emerald green crystals, possibly krinovite or kosmochlor, occur in several of the graphite nodules. Geochemistry: (J.T. Wasson, UCLA), INAA, $\mathrm{Ni}=69.9$ and $\mathrm{Co}=4.76$ (both $\mathrm{mg} / \mathrm{g}), \mathrm{Ga}=77.4, \mathrm{As}=15.3, \mathrm{Ir}=2.14$, and $\mathrm{Au}=1.59(\mathrm{all} \mu \mathrm{g} / \mathrm{g})$. 
Classification: Found only a few km from the iron Zacatecas (1792), though Sauceda de la Borda is structurally and chemically distinct. It is a member of the IAB main group and there are no other Mexican irons that are chemically similar. Specimens: $1.2 \mathrm{~kg}$ at $A S U$. The rest with MFarmer.

Sayh al Uhaymir $605(\mathrm{SaU} 605) \quad 20^{\circ} 32^{\prime} 37.51^{\prime \prime} \mathrm{N}, 56^{\circ} 40^{\prime} 35.98^{\prime \prime} \mathrm{E}$

Al Wusta, Oman

Find: 2014 Nov 5

Classification: Ordinary chondrite (L4)

Physical characteristics: Single crusted stone

Petrography: (J. Gattacceca, CEREGE) Chondrite with packed and well-defined chondrules. Opaque are FeNi metal and troilite.

Geochemistry: Olivine $\mathrm{Fa}_{24.2 \pm 1.1}$, PMD $3.9 \%(\mathrm{~N}=8)$, range $\mathrm{Fa}_{22.8 \text { - }}$

26.1. Orthopyroxene $\mathrm{Fs}_{16.2 \pm 6.5} \mathrm{Wo}_{1.0 \pm 0.8}$, Fs PMD 34\%, range $\mathrm{Fs}_{4.8-24.0}(\mathrm{~N}=10)$.

Classification: L4. Olivine is not completely equilibrated but the Fa PMD is below $5 \%$, indicating a type 4 .

Specimens: Type specimen at CEREGE. Main mass with anonymous finder.

Sayh al Uhaymir 606 (SaU 606) $\quad 20^{\circ} 20.555^{`} \mathrm{~N}, 56^{\circ} 49.427^{\prime} \mathrm{E}$

Al Wusta, Oman

Find: 12.02 .17

Classification: Ordinary chondrite (H5)

History: A complete, fresh-looking individual was found during a search for meteorites on February 12, 2017. The area (to a distance of 1-2 km) was searched again in February 2018 but no paired meteorites were found.

Physical characteristics: Complete individual fully covered with black, slightly crackled fusion crust. Bulk density based on a shape model is $3.43 \mathrm{~g} / \mathrm{cm} 3$.

Petrography: ( $\AA$. Rosén, Bern, E. Gnos, $M H N G E$, B. Hofmann, $N M B E$ ) The meteorite shows a brecciated chondritic texture with well-preserved chondrules. Minor and accessory minerals include chromite, ilmenite and native copper. Typical grain size of plagioclase is $10-20 \mu \mathrm{m}$. Mean chondrule size is $0.47 \pm 0.21$ $\mathrm{mm}(\mathrm{n}=128)$. Minor rust staining in silicates close to metal grains. Iron hydroxide rims around metal reach up to $20 \mu \mathrm{m}$.

Geochemistry: (Å. Rosén, Bern and B. Hofmann, $N M B E$ ) Olivine compositions are $\mathrm{Fa}_{18.1 \pm 0.3}(\mathrm{n}=48)$, pyroxene compositions are $\mathrm{Fs}_{16.3 \pm 0.4}, \mathrm{Wo}_{0.9 \pm 0.2}(\mathrm{n}=29)$.

Cosmogenic radionuclides: ( $\AA$. Rosén, GeMSE): Gamma-spectroscopy performed in April 2017 showed detectable activities of short-lived radionuclides indicating decay during $\sim 5$ years. At end of measurement, ${ }^{26} \mathrm{Al}$ was $39.1(+4.2,-3.8),{ }^{22} \mathrm{Na}$ was $17.9(+2.7,-1.3)$ and ${ }^{54} \mathrm{Mn}$ was $1.1(+0.3,-0.2) \mathrm{dpm} / \mathrm{kg}$.

Classification: Based on mineral compositions and mean chondrule size this is an equilibrated $\mathrm{H} 5$ chondrite. Cosmogenic isotope data indicate a fall around the year 2012.

Specimens: All at $N M B E$ 


\section{Scarham Creek $\quad 34.319,-85.979$}

Alabama, United States

Find: 2015

Classification: Ordinary chondrite (H3)

History: Chris Whitten found this meteorite on his family's five-acre property near the Scarham Creek, Alabama, in May 2015. He had often searched the property for meteorites for over a decade with no results, however in 2015 a fire burned and cleared some of the wooded area on the property, after which, a new search produced the meteorite find. The sample, when spotted, stood out as different from local rocks and was slightly magnetic. The sample was sent to his aunt, Dana Jenkerson, for identification. KD Meteorites purchased the main mass.

Physical characteristics: Single stone, no fusion crust, weathered exterior. Petrography: (C. Agee, UNM) Microprobe examination shows numerous porphyritic chondrules, most with mesostasis or glass. Much of the metal has been oxidized by terrestrial weathering.

Geochemistry: (C. Agee, $U N M$ ) Olivine $\mathrm{Fa}_{14.7 \pm 4.0}, \mathrm{Fe} / \mathrm{Mn}=43 \pm 14$, $\mathrm{n}=33$; low-Ca pyroxene $\mathrm{Fs}_{10.8 \pm 8.8} \mathrm{Wo}_{0.9 \pm 0.5}, \mathrm{Fe} / \mathrm{Mn}=26 \pm 18, \mathrm{n}=8$.

Classification: Ordinary chondrite (H3), estimated subtype 3.7-3.9 based on mean Fa content of olivine and the 1-sigma Fa content of olivine.

Specimens: $10.5 \mathrm{~g}$ on deposit at $U N M$, Chris Whitten holds 3 grams, KD Meteorites holds the main mass.

\section{Scottsdale $\quad 33^{\circ} 42.358^{\prime} \mathrm{N}, 111^{\circ} 56.006^{\prime} \mathrm{W}$ \\ Arizona, United States \\ Find: 1 April 2017 \\ Classification: Ordinary chondrite (LL3)}

History: Mr. Todd Dziuk found an unusual looking rock at the edge of a dry wash on 1 April 2017 in a desert area of Scottsdale, Arizona. He noticed that the underside had a rusty appearance and the stone felt slightly heavier than the average rock: power sanding of a corner of the stone revealed numerous bright shiny metallic spots. Extensive searching has not revealed further meteorites. Physical characteristics: Single $210.8 \mathrm{~g}$ stone with broad, shallow regmaglypts. Fusion crust well preserved - light orange stained on the surface in contact with the soil and covered with matte grey fusion crust above the ground. Interior shows tightly packed, well-defined chondrules separated by a dark matrix and scattered metal/troilite grains.

Petrography: SEM observation of a polished mount shows a wide range of chondrule types including PO, PP, BO, RP, POP, and RPT (interleaved radial pyroxene and troilite), with many olivines and pyroxenes zoned. Many chondrules show rims of troilite and more rarely by $\mathrm{Fe}-\mathrm{Ni}$ metal/troilite. Large range of chondrule sizes with the largest (PO) to $6 \times 4 \mathrm{~mm}$, though the majority are $<1 \mathrm{~mm}$ diameter. Opaques dominated by troilite, with sparse kamacite and high-Ni metal. 
Kamacite, to $500 \mu \mathrm{m}$, is single crystal. Many grains have well-developed curved Neumann bands. Troilite as polycrystalline grains to $500 \mu \mathrm{m}$, some grains with twin lamellae. The troilite rimming chondrules often has a "fizzy" texture. Native copper occurs in structurally complex grains of troilite/kamacite/tetrataenite. Scattered melt pockets to 100 microns. Weathering W1 based on minor oxidation of the metal.

Geochemistry: (L. Garvie, $A S U$ ) Olivine $\mathrm{Fa}_{27.9 \pm 7.8}, \mathrm{Fa}_{0.7-31.3}, \mathrm{Cr}_{2} \mathrm{O}_{3}=0.06 \pm 0.05$, range $0.01-0.3 \mathrm{wt} \%, \mathrm{FeO} / \mathrm{MnO}=58.3 \pm 8.6, \mathrm{CaO}=0.12 \pm 0.11 \mathrm{wt} \%, \mathrm{n}=29$. Low $\mathrm{Ca}$ pyroxene $\mathrm{Fs}_{15.9 \pm 10.2} \mathrm{Wo}_{1.7 \pm 2.0}, \mathrm{FeO} / \mathrm{MnO}=21.6 \pm 6.8$. High $\mathrm{Ca}$ pyroxene has a wide range of compositions, e.g., $\mathrm{Fs}_{9.5} \mathrm{Wo}_{30.8}, \mathrm{Fs}_{15.6} \mathrm{Wo}_{41.7}, \mathrm{Fs}_{20.0} \mathrm{Wo}_{44.0}$, and $\mathrm{Fs}_{22.6} \mathrm{Wo}_{23.9}$. Classification: LL3 ordinary chondrite, with estimated 3.6 subtype based on Fa range and $\mathrm{Cr}_{2} \mathrm{O}_{3}$ content.

\section{Sierra Gorda 009} $22^{\circ} 30.10^{\prime} \mathrm{S}, 6^{\circ} 08.20^{\prime} \mathrm{W}$

Antofagasta, Chile

Find: 2017 Oct 21

Classification: Ungrouped chondrite

History: Three pieces ( $30 \mathrm{~g}, 70 \mathrm{~g}$, and $140 \mathrm{~g}$ ) of one meteorite were found in the the desert by Mr. T. V. Kryachko, Mr. M. E. Nepomiluev, and an anonymous finder.

Physical characteristics: All samples of the meteorite have a brown fusion crust Petrography: M. A. Ivanova (Vernad) Meteorite has chondritic texture and consists of FeNi-metal (22-25 vol\%), chondrules, and their fragments; matrix is absent. Chondrules, up to $2 \mathrm{~mm}$, are PP, BO, POP, $\mathrm{SiO}_{2}$-bearing and Al-rich with clear bounaries; PP chondrules are dominant; the main minerals are pyroxene, Fe,Ni-metal, olivine and sulfides (troilite and daubreelite), schreibersite; accessory minerals are silica, Mg-chromite, anorthite, spinel, graphite and PGE-metal nuggets.

Geochemistry: Mineral compositions and geochemistry: M. A. Ivanova (Vernad), V. V. Kozlov (Oxford Instruments OM \& Gatan Inc., Moscow Office) Olivine: $\mathrm{Fa}_{0.47 \pm 0.10}(\mathrm{~N}=74)$, orthopyroxene: $\mathrm{Fs}_{1.42 \pm 0.39} \mathrm{Wo}_{0.88 \pm 0.63}(\mathrm{~N}=42),(\mathrm{N}=42)$, FeO-rich Opx: $\mathrm{Fs}_{9.33-32.48} \mathrm{Wo}_{0.23-4.74}$; diopside: $\mathrm{Fs}_{1.34 \pm 0.57} \mathrm{Wo}_{47.32 \pm 2.31}$; plagioclase varies in composition: $A n_{32.28-95.04} \mathrm{Ab}_{4.96-65.99}$; mesostasis in chondrules is enriched in $\mathrm{K}_{2} \mathrm{O}$ (up to $9.18 \mathrm{wt} \%$ ) and $\mathrm{TiO}_{2}$ (up to $6.53 \mathrm{wt} \%$ ); kamacite contains $5.07 \mathrm{wt} \% \mathrm{Ni}, 0.43$ wt $\% \mathrm{Co}, \mathrm{Co} / \mathrm{Ni}-0.09$, Si and $\mathrm{Cr}$ are below detection $(<0.03 \mathrm{wt} \%)$; taenite: $\mathrm{Ni}-32.3$ $\mathrm{wt} \%, \mathrm{Co}-0.25 \mathrm{wt} \%$; troilite is enriched in $\mathrm{Cr}$ (up to $2.4 \mathrm{wt} \%$ ); $\mathrm{MgO}$-chromite is almost pure $\mathrm{MgO}$-end member. Oxygen isotopic compositions: $(O U) \delta^{17} \mathrm{O} 3.763$, $3.736 ; \delta^{18} \mathrm{O} 6.263,6.169 ; \Delta^{17} \mathrm{O} 0.506,0.528$ permil.

Classification: ungrouped Fe,Ni-metal-rich chondrite with affinities to "Gchondrites" (GRO 95551 and NWA 5492, Weisberg et al., 2015) in texture and mineral chemistry. Oxygen isotopic compositions are between those of ordinary and enstatite chondrites.

Specimens: 3 pieces of the meteorite $(6.3,16.2$, and $18 \mathrm{~g})$, polished and thin sections are deposited in Vernad. Anonymous finder, Mt. M. E. Nepomiluev 
(ramatahatta2@mail.ru), and Mt.T.V.Kryachko (bredfild@mail.ru) hold the main mass of the meteorite.

\section{Sokoto $\quad 13^{\circ} 0.3^{\prime} \mathrm{N}, 5^{\circ} 14.85^{\prime} \mathrm{E}$ \\ Sokoto, Nigeria \\ Confirmed fall: 10 Jan 2008 \\ Classification: Iron meteorite (IIIAB)}

History: According to a newspaper report (THISDAY, January 11, 2008), a meteorite fell after a loud explosion and fireball at approximately 10:00 pm local time (2100h UTC) on 10 Jan 2008. The meteorite reportedly destroyed the roof of the house of Malam Bello Mohammed, in Mana Village, South Sokoto Local Government Council (on the outskirts of the city of Sokoto), and was found almost $2 \mathrm{~m}$ deep in the ground. The main mass reportedly weighs approximately $30 \mathrm{~kg}$, and was broken into pieces with a hammer. A $240 \mathrm{~g}$ specimen was secured by Nigerian resident Eugene Egbe, who provided it to Twelker. Other pieces are reportedly with the Geological Survey Laboratory in Kaduna.

Physical characteristics: The meteorite is irregularly shaped, with deep regmaglypts, and a red-brown exterior.

Petrography: (C. Herd, $U A b)$ Optical investigation of a $\sim 2 \times 3 \mathrm{~cm}$ polished and etched surface reveals a well-developed Widmanstatten pattern consistent with a medium octahedrite $(0.9 \pm 0.2 \mathrm{~mm} ; \mathrm{n}=22)$. No obvious inclusions were noted. Terrestrial alteration has occured along fractures connecting to the exterior.

Geochemistry: ICP-MS data, using sample of North Chile (Filomena) as standard (C. Herd and G. Chen $U A b$ ): $\mathrm{Ni}=8.6, \mathrm{Co}=0.51$ (both wt\%); $\mathrm{Ir}=1.7, \mathrm{Ge}=41$, As $=16.8, \mathrm{~W}=1.1, \mathrm{Re}=0.16, \mathrm{Pt}=6.8, \mathrm{Cu}=158, \mathrm{Au}=1.6(\mathrm{all} \mu \mathrm{g} / \mathrm{g})$.

Classification: (C. Herd, $U A b$ ): IIIAB iron, through comparison of bulk composition with data from Wasson and Richardson (2001).

Specimens: Type specimen consisting of one representative slice (21.9 g) at $U A b$. A $240 \mathrm{~g}$ mass with Twelker.

\section{Stewart Valley 024 (StV 024) $\quad 36^{\circ} 14.991{ }^{\prime} \mathrm{N}, 116^{\circ} 10.319^{\prime} \mathrm{W}$ \\ Nevada, USA \\ Find: 2017 Jan 07 \\ Classification: Ordinary chondrite (H3)}

Petrography: Chondrules are very sharply defined, but none appear to contain isotropic glassy mesostases, suggesting a high type-3 classification. Chondrule types include, PO, PP, POP, BO, RP and C. Olivine grains exhibit sharp optical extinction, indicative of shock stage S1. However, some of the troilite grains are polycrystalline, suggesting the rock was shocked and then annealed. Consistent with this is the occurrence of small irregular troilite grains within some grains of metallic Fe-Ni, probably a feature formed by localized shock melting. There are no observable crystalline plagioclase grains. More than $95 \%$ of the metal and troilite grains have been transformed into limonite and goethite by terrestrial weathering. 


\section{Sueilila $002 \quad 24.732^{\circ} \mathrm{N}, 14.319^{\circ} \mathrm{W}$ \\ Rio de Oro, Western Sahara \\ Find: 2014 May 25 \\ Classification: Martian meteorite (Shergottite)}

History: (H. Chennaoui-Aoudjehane, FSAC) A party of four experienced meteorite hunters (A. Bouferra, Z. Balli, A. Oubedda and M. Ouicha) had camped at a place on their way back from Sbayta to Boujdour in southern Morocco on May 24, 2014, and the next day searched there for meteorites. Mr. Zaid Balli found a $1467 \mathrm{~g}$, mostly buried rock which appeared different from the background desert soil, but without fusion crust and somewhat weathered. Although following its excavation this specimen did not seem promising, they decided to search for more material anyway, and found several identical pieces within a few meters of the first stone. A sample sent to UWS was soon confirmed to be a shergottite. On October 17, 2014 Dr. H. Chennaoui-Aoudjehane (accompanied by M. Aoudjehane, A. Bouragaa and A. Bouferra) traveled to the find site to collect information on the coordinates. Independently, other metorite hunters visited the site during June and July, and found additional pieces. One $560 \mathrm{~g}$ stone was purchased by Darryl Pitt in August 2014 and a $124 \mathrm{~g}$ stone was purchased by Steve Arnold in October 2014.

Physical characteristics: (A. Irving, UWS and A. Bouragaa) The buried portion of the largest $(1467 \mathrm{~g})$ stone was coated by pale brown clay, but the small area that was exposed is brown with dark phenocrysts visible. The cut interiors of both the $560 \mathrm{~g}$ and $124 \mathrm{~g}$ stones have a mottled brown appearance with larger, deep brown phenocrysts. All stones have a weathering rind up to several millimeters thick. Petrography: (A. Irving and S. Kuehner, UWS) Porphyritic texture. Euhedral to subhedral, zoned olivine phenocrysts (up to $2.2 \mathrm{~mm}$ ) are set in a finer grained groundmass composed predominantly of zoned, prismatically-twinned pyroxene and maskelynite with accessory olivine, chromite, ilmenite, Mg-bearing merrillite and pyrrhotite. Olivine phenocrysts contain numerous tiny inclusions of chromite, as well as sparse melt inclusions (now composed of low-Ca pyroxene, silica polymorph, plagioclase, merrillite, pentlandite and glass) surrounded by radial cracks. Cores of olivine grains are orange in thin section, whereas olivine rims, groundmass olivine and groundmass pyroxene are all pale tan in color. Terrestrial weathering effects include orange staining of groundmass opaque minerals and minor thin calcite veinlets.

Geochemistry: Olivine phenocrysts (cores $\mathrm{Fa}_{19.1-20.4}, \mathrm{~N}=3$, rims $\mathrm{Fa}_{36.9-39.5}, \mathrm{~N}=2$, $\mathrm{FeO} / \mathrm{MnO}=48-55)$, groundmass olivine $\left(\mathrm{Fa}_{47.2-48.7}, \mathrm{FeO} / \mathrm{MnO}=53-57, \mathrm{~N}=2\right)$, orthopyroxene cores $\left(\mathrm{Fs}_{16.5-21.7} \mathrm{Wo}_{2.2-3.5}, \mathrm{FeO} / \mathrm{MnO}=25-27, \mathrm{~N}=2\right)$, pigeonite $\left(\mathrm{Fs}_{32.2-34.1} \mathrm{Wo}_{11.0-11.5}, \mathrm{FeO} / \mathrm{MnO}=36, \mathrm{~N}=3\right)$, plagioclase $\left(\mathrm{An}_{60.9-62.1} \mathrm{Or}_{1.4-1.9}, \mathrm{~N}=3\right)$. Classification: Martian (shergottite, olivine-phyric). Specimens: $21 \mathrm{~g}$ including one polished thin section and one polished slice at $U W B ; 20 \mathrm{~g}$ at $F S A C$. The remaining material is held by DPitt, Mr. S. Arnold, Mr. A. Bouragaa and Mr. A. Bouferra. 


\section{Taltal $001 \quad 25^{\circ} 29^{\prime} 19^{\prime}$ 'S, $70^{\circ} 02^{\prime} 28^{\prime}$ 'W \\ Antofagasta, Chile \\ Find: 2016 Jan 14 \\ Classification: Ordinary chondrite (H3)}

History: The meteorite was found in 2016 during a field trip in the Atacama desert. Physical characteristics: Dark brownish individual without fusion crust. Petrography: The meteorite shows a chondritic texture with often slightly flattened and loosely packed chondrules (mean diameter about $0.4 \mathrm{~mm}$ ) in a more fine-grained matrix that contains sulfides and abundant FeNi metal.

\section{Tintigny $\quad 49.683786^{\circ} \mathrm{N}, 5.532957^{\circ} \mathrm{E}$ \\ Luxembourg, Belgium \\ Probable fall: Feb. 1971 \\ Classification: HED achondrite (Eucrite, polymict)}

History: (S. de Foestraets, S. Liégeois and V. Debaille): In February 1971, Mr. Eudore Schmitz was working in his barn in the village of Tintigny late in the afternoon, when he heard a noise from the roof of the building. Going upstairs, he found a hole in a tile and a black stone on the floor. The school teacher of the children, Albert Rossignon, confirmed it was a meteorite and kept the stone, hoping for further investigation. The teacher later started religious seminary and became a priest. While he kept the meteorite, and showed it from time to time to visitors and children, the story never spread. Schmitz died in 2006. In 2017, after reading a popular article about Belgian expeditions in Antarctica to find meteorites, Priest Rossignon contacted Vinciane Debaille at $U L B$. After several contacts, the Schmitz's 3 children, Jean-Paul, Joseph and Rita Schmitz, and his widow, Madam Germaine Mathu, donated the meteorite to RBINS. Some parts of the meteorite are now missing due to the handling of the stone by various people over the years, but Priest Rossignon confirms that the fusion crust was initially complete with even a piece of the tile stuck on it. Cracks were already visible at that time. The fusion crust is still fresh and shiny with preserved flow lines. The punched tile was recovered from the roof in 2018 , as the hole was simply covered by another transparent tile.

Physical characteristics: A single stone partly covered with fresh glassy fusion crust. Light-gray brecciated interior hosts darker (dark-gray to black) clasts.

Petrography: Brecciated ophitic to sub-ophitic texture. Contains low-Ca and highCa pyroxene (both with exsolution lamellae), plagioclase, silica polymorph, chromite, troilite, metal. Clasts with melted and variolitic $(1 \mathrm{~mm})$ textures are present.

Geochemistry: Low-Ca pyroxenes $\mathrm{Fs}_{33.4-56.5} \mathrm{Wo}_{2.6-9.4}$, average $\mathrm{Fs}_{41.5 \pm 8.5} \mathrm{Wo}_{6.3 \pm 2.8} \mathrm{FeO} / \mathrm{MnO}=30.6 \pm 4.4(\mathrm{n}=6)$. Ca-pyroxene $\mathrm{Fs}_{30.1-70.0} \mathrm{Wo}_{10.9-38.4}$, average $\mathrm{Fs}_{51.4 \pm 12.3} \mathrm{Wo}_{22.2 \pm 9.3} \mathrm{FeO} / \mathrm{MnO}=34.7 \pm 3.7(\mathrm{n}=8)$. Average plagioclase 
$\mathrm{An}_{84.1} \mathrm{Or}_{0.5}(\mathrm{n}=4)$. Oxygen isotopes (R. Greenwood, $\left.O U, \mathrm{UK}\right): \Delta^{17} \mathrm{O}:-0.246 \pm 0.003$ per mil; $\delta^{18} \mathrm{O}=3.756 \pm 0.041$ per mil $(n=2)$.

Classification: Achondrite (eucrite, polymict).

Specimens: Main mass and type specimen at RBINS. One polished section at Laboratoire G-TIME (ULB).

\section{Troy Dry Lake $\quad 34^{\circ} 48.53^{\prime} \mathrm{N}, 116^{\circ} 33.05^{\prime} \mathrm{W}$}

California, United States

Find: 2017 Apr 7

Classification: Ordinary chondrite (L6)

History: A single 16.3 g stone was found by Mark Bittmann on April 7, 2017, while he was searching for meteorites on a dry lake. Subsequent searches resulted in no additional fragments or other meteorites.

Physical characteristics: Dark-brown, flat shield-shaped fragment with moderately rounded edges, and patches of relict fusion-crust that have developed a desert-polished patina.

Petrography: (D. Sheikh, FSU) Chondrule boundaries blurred. Recrystallized coarse-grained matrix with plagioclase $(60 \mathrm{um})$. Interconnected shock melt veins.

Geochemistry: Olivine, $\mathrm{Fa}_{24.7 \pm 0.2}(\mathrm{~N}=26)$; orthopyroxene, $\mathrm{Fs}_{22.3 \pm 0.2} \mathrm{Wo}_{2.7 \pm 0.2}(\mathrm{~N}=25)$. Classification: Ordinary Chondrite (L6)

Specimens: 3.5 grams at UCLA; main mass with Verish.

\section{Turpan $001 \quad 42^{\circ} 33^{\prime} 43.03^{\prime \prime} \mathrm{N}, 89^{\circ} 00^{\prime} 36.02^{\prime \prime} \mathrm{E}$ \\ Xinjiang, China}

Find: 26 Nov 2013

Classification: Ordinary chondrite (L 6)

History: (Ziyao Wang) The meteorite was discovered by Zijian Wang $45 \mathrm{~km}$ SSW of Turpan City in Gaochang District on 26 Nov 2013.

Physical characteristics: (Ziyao Wang) Reddish brown heavy fractured wind eroded irregular rock of $1500 \mathrm{~g}$ without fusion crust.

Petrography: (R. Bartoschewitz, Bart) brown stainted recrystallized matrix with poor defined chondrules $(0.6-1.8 \mathrm{~mm}$, av. $0.7 \mathrm{~mm})$.

Geochemistry: Magnetic susceptibility (R. Bartoschewitz, Bart) $\log \chi\left(\times 10^{-}\right.$

$\left.{ }^{9} \mathrm{~m}^{3} / \mathrm{kg}\right)=4.81$

Classification: Ordinary chondrite (L 6, W3)

Specimens: $21.9 \mathrm{~g}$ on deposit at Kiel, Wang Ziyao and Zijian Wang holds the main mass, and $108 \mathrm{~g}$ with Bart.

\section{Twayla $001 \sim 26^{\circ} 50.2^{\prime} \mathrm{N}, \sim 9^{\circ} 11.6^{\prime} \mathrm{W}$}

Saguia el Hamra, Western Sahara

Find, doubtful fall: 2014 Dec

Classification: HED achondrite (Howardite) 
History: The family of nomad Lahsen Duine spent the winter of 2014/2015 in the Wad La'waj Talli area, nearly $70 \mathrm{~km}$ NE of Bir Lahlou. Short before the end of he year, they were awakened by lightning, followed by a curious whistling noise. In March 2015, the children discovered two black stones on the ground, in the grassy scrub, while they were catching their goats. The stones were discovered within several hundred $\mathrm{m}$ of each other. The bigger stone was purchased in Ouarzazate in March 2017.

Physical characteristics: Two nearly complete individuals of 318 and approximately $150 \mathrm{~g}$ with glossy black fusion crust. Cut face shows a breccia with fragmental light and darker clasts set in a light-gray groundmass. Some fractures show penetrating fusion curst; no traces of terrestrial fillings are recognizable.

Petrography: (R. Bartoschewitz, Bart) Polymict breccia with at least three distinct lithologies: diogenite, eucrite and anorthosite. Ubiquitous iron-nickel metal, Fesulfide and chromite present.

Geochemistry: (R. Bartoschewitz, Bart, P. Appel and B. Mader, Kiel) Diogenite pyroxene $\mathrm{Fs}_{35.0 \pm 0.7} \mathrm{Wo}_{5.2 \pm 1.4}, \mathrm{Fe} / \mathrm{Mn}=27, \mathrm{n}=8$; chromite CRAL 78.3 \pm 1.2 , FFM 88.6 \pm 3.6 . Eucrite pyroxene $\mathrm{Fs}_{40.0 \pm 1.8} \mathrm{Wo}_{9.4 \pm 2.5}, \mathrm{Fe} / \mathrm{Mn}=30, \mathrm{n}=15$; plagioclase $\mathrm{An}_{89.7 \pm 1.5} \mathrm{Or}_{0.3 \pm 0.1}, \mathrm{n}=14$; olivine $\mathrm{Fa}_{38.5}, \mathrm{Fe} / \mathrm{Mn}=34, \mathrm{n}=1$. Anorthositic clast $\mathrm{An}_{93.0 \pm 0.8} \mathrm{Or}_{0.3 \pm 0.1}, \mathrm{n}=5$. Matrix pyroxene $\mathrm{Fs}_{35.0 \pm 3.2} \mathrm{Wo}_{5.6 \pm 0.9}, \mathrm{Fe} / \mathrm{Mn}=28, \mathrm{n}=9$; plagioclase $\mathrm{An}_{91.9 \pm 2.1} \mathrm{Or}_{0.3 \pm 0.1}, \mathrm{n}=2$; olivine $\mathrm{Fa}_{46.3 \pm 12.7}, \mathrm{Fe} / \mathrm{Mn}=51, \mathrm{n}=2$; chromite CRAL 79.4 \pm 2.0, FFM 90.4 \pm 0.4 ; kamazite Ni 0.0-2.0, Co 0.2-0.7 wt-\%, $\mathrm{n}=15$, troilite.

Classification: HED achondrite (howardite)

Specimens: $20.0 \mathrm{~g}$ on deposit at Kiel, main mass with Dieter Janek, $21.5 \mathrm{~g}$ Bart.

\section{Weiya $001 \quad 41^{\circ} 43^{\prime} 1.04^{\prime \prime} \mathrm{N}, 94^{\circ} 14^{\prime} 30.94^{\prime \prime} \mathrm{E}$}

XinJiang, China

Find: 20 Aug 2012

Classification: Ordinary chondrite (L $\sim 6)$

History: (Ziyao Wang) Discovered by Wang Ziyao in the Gobi Desert, $12 \mathrm{~km} \mathrm{SW}$ Weiya and $36 \mathrm{~km} \mathrm{SE}$ of Yamansu, near Hami city in Xinjiang province on Aug. 20, 2012.

Physical characteristics: (Ziyao Wang and R. Bartoschewitz, Bart) Dark, graybrown, flat stone of $201 \mathrm{~g}$ without fusion crust.

Petrography: (R. Bartoschewitz, Bart) Gray, porous, strongly recrystallized matrix with poorly defined chondrules and irregular metal and sulfide specks up to 0.5 $\mathrm{mm}$.

Geochemistry: Magnetic susceptibility (R. Bartoschewitz, Bart $) \log \chi\left(\times 10^{-}\right.$ $\left.{ }^{9} \mathrm{~m}^{3} / \mathrm{kg}\right)=4.83$

Classification: Ordinary chondrite (L $\sim 6, \mathrm{~W} 4)$

Specimens: $21.1 \mathrm{~g}$ on deposit at Kiel, Wang Ziyao holds the main mass, and $23.2 \mathrm{~g}$ with Bart. 


\section{Willcox Playa $011 \quad 32^{\circ} 7.0^{\prime} \mathrm{N}, 109^{\circ} 53.3^{\prime} \mathrm{W}$}

Arizona, United States

Find: 2018 Jan 22

Classification: Ordinary chondrite (L6)

History: A single stone was recovered by Mr. Chris McDoniel while he was searching for an old aircraft crash site. Field ID\#: UU180122A

Petrography: (D. Sheikh, FSU) Chondrule boundaries blurred $(900 \pm 100 \mu \mathrm{m}$, $\mathrm{N}=4)$. Recrystallized coarse-grained matrix with plagioclase $(60 \mu \mathrm{m})$.

Interconnected shock melt veins.

Geochemistry: Olivine, $\mathrm{Fa}_{25.1 \pm 0.2} \mathrm{~N}=49$; orthopyroxene, $\mathrm{Fs}_{22.4 \pm 0.2} \mathrm{Wo}_{3.0 \pm 0.1}, \mathrm{~N}=51$.

Classification: Ordinary Chondrite (L6)

Specimens: 8.2 grams at UCLA; main mass with Robert Verish.

\section{Wood River $\quad 50^{\circ} 08^{\prime} \mathrm{N}, 106^{\circ} 13^{\prime} \mathrm{W}$ \\ Saskatchewan, Canada \\ Find: Unknown \\ Classification: Ordinary chondrite (H5)}

History: Found many years ago by the owners father along the banks of the Wood River in southern Saskatchewan. The find area is bounded by provincial highways \#58 to the west; \#363 to the north, \#718 to the south and on the east by the Old Wives Lake bird sanctuary. The nearest villages are Coderre and Courval.

Petrography: Chondrules are readily delineated with much recrystallization. Chondrule glass is absent. Weak undulatory extinction visible in the olivine grains. Geochemistry: Olivine composition determined by two methods: $\mathrm{Fa}_{19.3 \pm 1}$ (by X-ray diffraction, $R O M$ ) and $\mathrm{Fa}_{20.0 \pm 1.2} \%$ (EMPA, $\mathrm{n}=28$ ); $\mathrm{Fs}_{16.6 \pm 0.9} \mathrm{Wo}_{1.1 \pm 0.1}$ (EMPA, $\mathrm{n}=9$ ). No zoning was visible in olivine or pyroxene grains.

\section{Wuerhe $\quad 46^{\circ} 01^{\prime} 01.1^{\prime \prime} \mathrm{N}, 86^{\circ} 16^{\prime} 34.4^{\prime \prime} \mathrm{E}$ \\ Xinjiang, China \\ Find: 2018 July 25 \\ Classification: Ordinary chondrite (L5)}

History: Three small meteorites were first found by three anonymous people who were looking for wind-erosed stones of quartz in the desert. These meteorites were confirmed by Pengli Chen. On return back to this area, 10 more pieces were found within 3 days. On September 17-19, 2018, the meteorite hunting team organized by P. Chen searched this area again, and they found 10 other pieces. Counting an additional 3 pieces recovered by anonymous finders, a total of 26 meteorites were collected in this new meteorite strewn field. The total mass is about $6 \mathrm{~kg}$, and the largest piece is $1.3 \mathrm{~kg}$. All of these pieces were completely covered by fusion crust. Physical characteristics: Two of the meteorites were studied. Black melt veins and pockets can be seen in the exposed surfaces of samples.

Petrography: (Y. Xu, N. Wang and Y. Lin, IGGCAS) Microscopic observations of a polished mount reveal a chondritic texture with recrystallized matrix. Chondrules 
are readily delineated. It consists mainly of olivine, pyroxene, plagioclase, $\mathrm{Fe}-\mathrm{Ni}$ metal, and troilite. Plagioclase size is less than $50 \mu \mathrm{m}$. Ringwoodite and wadsleyite were found within and close to shock veins and pockets.

Geochemistry: Olivine and pyroxene have homogeneous chemical compositions. Olivine $\mathrm{Fa}_{24.6 \pm 0.9}(\mathrm{n}=12)$, orthopyroxene $\mathrm{Fs}_{20.7 \pm 0.8} \mathrm{Wo}_{1.6 \pm 0.2}(\mathrm{n}=11)$, plagioclase: $\operatorname{An}_{11}(\mathrm{n}=5)$

Classification: L5 (Y Xu, N. Wang and Y. Lin, IGGCAS)

Specimens: 2 slices with a total mass of $136 \mathrm{~g}$ in $I G G C A S, 3$ pieces with a total mass of $\sim 400 \mathrm{~g}$ by P. Chen. Others with anonymous owners.

\section{Xinjiang 009 \\ Xinjiang, China \\ Purchased: 2013 \\ Classification: Ordinary chondrite (L $\sim 6)$}

History: (Ziyao Wang) Purchased by Wang Ziyao from an ornamental stone dealer in Hami/Xinjiang in 2013. He reportet that he got this stone from another ornamental stone dealer, and that it was found in the desert of Xinjiang.

Physical characteristics: (Wang Ziyao) Black-brown stone of $60.3 \mathrm{~g}$ with adhering sand grains.

Petrography: (R. Bartoschewitz, Bart) recrystallized matrix with poor defined chondrules (1.0-1.6 mm, av. $1.0 \mathrm{~mm})$

Geochemistry: Magnetic susceptibility (R. Bartoschewitz, Bart) $\log \chi\left(\times 10^{-}\right.$

$\left.{ }^{9} \mathrm{~m}^{3} / \mathrm{kg}\right)=4.32$

Classification: Ordinary chondrite (LL $\sim 6, \mathrm{~W} 4)$

Specimens: $12.3 \mathrm{~g}$ on deposit at Kiel, Wang Ziyao holds the main mass, and $11 \mathrm{~g}$ with Bart.

\section{Xinjiang 010}

Xinjiang, China

Purchased: 2013

Classification: Ordinary chondrite (LL $\sim 6)$

History: (Ziyao Wang) Purchased by Wang Ziyao from an ornamental stone dealer in Hami/Xinjiang in 2013. He reportet that he got this stone from another ornamental stone dealer, and that it was found in the desert of Xinjiang.

Physical characteristics: (Wang Ziyao) Black stone of $71.5 \mathrm{~g}$ with fusion crust Petrography: (R. Bartoschewitz, Bart) recrystallized matrix with poor defined chondrules (1.0-1.6 mm, av. $1.0 \mathrm{~mm}$ )and irregular metal and sulphide specks.

Geochemistry: Magnetic susceptibility (R. Bartoschewitz, Bart $) \log \chi\left(\times 10^{-}\right.$ $\left.{ }^{9} \mathrm{~m}^{3} / \mathrm{kg}\right)=4.37$

Classification: Ordinary chondrite (LL $\sim 6, \mathrm{~W} 3)$

Specimens: $15.6 \mathrm{~g}$ on deposit at Kiel, Wang Ziyao holds the main mass, and $15 \mathrm{~g}$ with Bart. 


\author{
Xinjiang 011 \\ Xinjiang, China \\ Find: 2014 \\ Classification: Ordinary chondrite $(\mathrm{L} \sim 6)$
}

History: (Ziyao Wang) Purchased by Wang Ziyao from Han Yongfeng Urumqi/Xinjiang in 2015. The anonymous finder reported to Han Yongfeng that this meteorite was discovered in the Lop Nur area in 2015.

Physical characteristics: (Wang Ziyao) Brown stone of $89.5 \mathrm{~g}$ without fusion crust

Petrography: (R. Bartoschewitz, Bart) recrystallized matrix with poor defined chondrules (0.4-1.5 mm, av. 0.6 mm)

Geochemistry: Magnetic susceptibility (R. Bartoschewitz, Bart $) \log \chi\left(\times 10^{-}\right.$

$\left.{ }^{9} \mathrm{~m}^{3} / \mathrm{kg}\right)=4.70$

Classification: Ordinary chondrite (L $\sim 6, \mathrm{~W} 4)$

Specimens: $18.5 \mathrm{~g}$ on deposit at Kiel, Wang Ziyao holds the main mass, and $14.2 \mathrm{~g}$ with Bart.

\title{
Yangguanzhen $001 \quad 39^{\circ} 48^{\prime} 04^{\prime \prime} \mathrm{N}, 94^{\circ} 18^{\prime} 56^{\prime \prime} \mathrm{E}$
}

Gansu, China

Find: 2015

Classification: Ordinary chondrite (LL $\sim 6)$

History: (R. Bartoschewitz, Bart) Discovered by Liu Binghan in the desert near near Aksay/Gansu in 2015 and was purchased by Wang Ziyao, who donated it to R. Bartoschewitz.

Physical characteristics: (R. Bartoschewitz, Bart) Brown $8.66 \mathrm{~g}$ stone.

Petrography: (R. Bartoschewitz, Bart) Brown stainted recrystallized matrix with nearly complete recrystallized chondrules $(0.6-1.0 \mathrm{~mm})$ and few metal and sulphide specks, vugs partly filled with gypsum.

Geochemistry: Magnetic susceptibility (R. Bartoschewitz, Bart $) \log \chi\left(\times 10^{-}\right.$

$\left.{ }^{9} \mathrm{~m}^{3} / \mathrm{kg}\right)=4.08$

Classification: Ordinary chondrite (LL $\sim 6, \mathrm{~W} 3)$

Specimens: $1.77 \mathrm{~g}$ on deposit at Kiel, main mass of $6.5 \mathrm{~g}$ with Bart.

\section{Yucca $045 \quad 34^{\circ} 45.894^{\prime} \mathrm{N}, 114^{\circ} 14.101^{\prime} \mathrm{W}$}

Arizona, United States

Find: 27 Mar 2011

Classification: Ordinary chondrite (H3-6)

History: Found with metal detector 6 inches below the surface in the Yucca DCA by Jim Wooddell March 27, 2011. Entire stone donated to Cascadia.

Physical characteristics: Exterior is covered with weathered fusion crust, with large patches of reddish-brown rust. 
Petrography: In thin section, two distinct lithologies that appear lighter and darker in plane-polarized light are visible and have irregular and gradational boundaries. The light lithology is coarser grained and contains chondrules with boundaries integrated into their surroundings. Feldspar is variable in size, most slightly below $50 \mu \mathrm{m}$ in diameter, but with a few larger grains. The dark lithology is dominated by fragmental material containing numerous small cryptocrystalline chondrules; BSE imaging shows that this lithology contains a mix of equilibrated silicates, with a fairly large ( $25-35$ area \%) admixture of type 3 material (both magnesian and iron-rich olivine and pyroxene grains). This lithology also contains occasional coarse feldspar grains (most below $50 \mu \mathrm{m}$ in diameter), coarse phosphate grains, and numerous chromite-plagioclase assemblages. FeNi carbide with some accompanying magnetite, alumnious low-calcium and high-calcium pyroxenes, and silica polymorph are present in the dark lithology. Along two edges of the section abutting the dark lithology is a small patch of shock melt (opaque in planepolarized light) containing metal and troilite blebs, and a coarse-grained clast lacking chondrules.

Geochemistry: (M. Hutson and A. Ruzicka, Cascadia) Light lithology olivine $\left(\mathrm{Fa}_{19.6 \pm 0.6}, \mathrm{~N}=7\right)$ and low-Ca pyroxene $\left(\mathrm{Fs}_{17.6 \pm 0.9} \mathrm{Wo}_{1.3 \pm 0.2}, \mathrm{~N}=5\right)$ are consistent with a type 5 or 6 designation. Dark lithology olivine $\left(\mathrm{Fa}_{17.7 \pm 9.2}, \mathrm{~N}=35\right)$ and low-Ca pyroxene $\left(\mathrm{Fs}_{15.4 \pm 6.5} \mathrm{Wo}_{1.2 \pm 1.0}, \mathrm{~N}=33\right)$ compositions reflect the large admixture of type 3 material. Fragments in this lithology include a partial chondrule containing dusty forsteritic olivine grains $\left(\mathrm{Fa}_{0.7 \pm 4.0}\right)$ and aluminum-rich diopside $(6 \mathrm{wt} \%$ $\mathrm{Al}_{2} \mathrm{O}_{3}$ ) set in anorthitic glass $\left(\mathrm{An}_{92} \mathrm{Or}_{0}\right)$, as well as a fragment of a ferroan chondrule with olivine $\left(\mathrm{Fa}_{34-51}\right)$ and low-Ca pyroxene $\left(\mathrm{Fs}_{24} \mathrm{Wo}_{5}\right)$ set in a Na-K-rich glass. The coarse-grained clast at the edge of the section consists of equilibrated olivine $\left(\mathrm{Fa}_{19.3 \pm 0.3}, \mathrm{~N}=10\right)$, low-Ca pyroxene $\left(\mathrm{Fs}_{16.9 \pm 0.2} \mathrm{Wo}_{1.5 \pm 0.4}, \mathrm{~N}=9\right)$, and feldspar $\left(\mathrm{Ab}_{78.2 \pm 1.4} \mathrm{An}_{14.4 \pm 0.7} \mathrm{Or}_{7.4 \pm 0.8}, \mathrm{~N}=2\right)$.

Classification: H3-6 finely intermixed genomict breccia. Light lithology is type 56 material as implied by medium to coarse feldspar grain sizes and equilibrated olivine and pyroxene; dark lithology contains type 3 material as implied by common presence of magnesian and zoned olivine. Paired with Buck Mountain Wash (synonymous with Yucca 002) on the basis of mineralogy, mineral chemistry, and texture.

Specimens: Cascadia holds $38.0 \mathrm{~g}$ in two pieces, in addition to one polished thin section and a mounted butt.

\section{Yucca $046 \quad 34^{\circ} 46.009^{\prime} \mathrm{N}, 114^{\circ} 14.415^{\prime} \mathrm{W}$}

Arizona, United States

Find: 12 Oct 2011

Classification: Ordinary chondrite (H3-5)

History: Found by Jim Wooddell on October 23, 2011 while searching with a metal detector. Mr. Wooddell donated the entire stone to Cascadia in September, 2013.

Physical characteristics: Brownish weathering patina occurs on exterior surfaces. 
Petrography: In thin section, the sample appears to be relatively integrated, with many chondrule fragments and only a few complete chondrules present; distinct lithic clasts typical of a breccia are absent. The material between chondrule fragments is comprised of individual grains and smaller fragments. BSE imaging shows that most of meteorite consists of fragmental material, with an abundance of magnesian and iron-rich olivine and pyroxene grains, many of which show zoning, and which abut equilibrated fragments with typical H-chondrite compositions. Laths of a silica polymorph were observed, as well as several small chromiteplagioclase objects and merrillite grains up to $250 \mu \mathrm{m}$ across. Feldspathic material consists of glass of variable compositions and crystalline plagioclase feldspar grains typically $20-40 \mu \mathrm{m}$ across; no grains larger than $50 \mu \mathrm{m}$ across were observed. A single fragment $70 \mu \mathrm{m}$ across appears to be a typical type 3 matrix lump, found adjacent to a crystalline feldspar grain. Both high- and low-Ca aluminous pyroxenes (up to $6.7 \mathrm{wt} \% \mathrm{Al}$ ) were observed in three chondrule fragments and as single grains, sometimes with anorthitic glass, and sometimes with forsteritic olivine $(\mathrm{Fa}<5)$, and spinel. In addition to these small spinel grains, an isolated zoned spinel grain (100 $\mu \mathrm{m}$ across) was observed. Fe-Ni carbide was found, along with magnetite; in one clast magnetite appears to be replacing troilite. Geochemistry: (M. Hutson and A. Ruzicka, Cascadia) Sample contains a wide range of compositions of olivine $\left(\mathrm{Fs}_{20.1 \pm 6.0}, \mathrm{~N}=51\right)$, low-Ca pyroxene $\left(\mathrm{Fs}_{15.6 \pm 5.3} \mathrm{Wo}_{1.2 \pm 1.0}, \mathrm{~N}=61\right)$.

Classification: Metal abundance ( 9 area\%) is consistent with an $\mathrm{H}$ chondrite. Although the chemistry and abundance of zoned grains suggests a type 3 designation, the presence of medium-sized crystalline feldspar, coarse phosphate, and chromite-plagioclase objects suggests a higher petrographic type. The thin section studied strongly resembles the "main" lithology found in the Buck Mountain Wash chondrite, suggesting that this is an H3-5 finely intermixed genomict breccia. Paired with Buck Mountain Wash(synonymous with Yucca 002) on the basis of mineralogy, mineral chemistry, and texture.

Specimens: Cascadia holds $9.0 \mathrm{~g}$ in two pieces, in addition to a polished thin section and a mounted butt.

\section{Yucca $047 \quad 34^{\circ} 49.281^{\prime} \mathrm{N}, 114^{\circ} 16.655^{\prime} \mathrm{W}$}

Arizona, United States

Find: 24 Nov 2011

Classification: Ordinary chondrite (H3-6)

History: Found with metal detector in the Yucca DCA by Jim Wooddell November 24, 2011. Entire stone donated to Cascadia.

Physical characteristics: Exterior is covered with a brownish weathering patina. Petrography: In thin section, the sample appears to be a relatively integrated mixture of chondrules, chondrule fragments, and indistinct fragments of chondritic clasts. The section appears dark due to the size of small fragments and individual grains that make up material interstitial to chondrules and larger fragments. One discrete lighter colored clast is visible, which lacks discernable chondrules, and 
contains coarse grains of feldspar (most slightly below $50 \mu \mathrm{m}$ in diameter, but a few larger grains). BSE imaging shows that the meteorite contains a mix of equilibrated silicates, with a fairly large ( $25-35$ area $\%)$ admixture of type 3 material (both magnesian and iron-rich olivine and pyroxene grains). Free copper, an FeNi carbide, and magnetite are present, as well three grains of low-Ca pyroxene with relatively high (3.6-5.8 $\left.\mathrm{wt} \% \mathrm{Al}_{2} \mathrm{O}_{3}\right)$ alumina content, similar to those reported for Buck Mountain Wash. Small chromite-plagioclase objects are present. Opaques show minor weathering ( $\sim 5-10 \%$ replacement by Fe-hydroxides). Shock effects in olivine are variable from sharp optical extinction to a recrystallized clast, but most olivine grains in the section show undulose extinction with one set of planar fractures, indicating a shock stage of S3.

Geochemistry: (M. Hutson and A. Ruzicka, Cascadia) Host lithology olivine $\left(\mathrm{Fa}_{19.2 \pm 7.0}, \mathrm{~N}=50\right)$ and low-Ca pyroxene $\left(\mathrm{Fs}_{13.1 \pm 5.4} \mathrm{Wo}_{0.9 \pm 0.8}, \mathrm{~N}=47\right)$ compositions reflect the large admixture of type 3 material. A clast of higher type material has equilibrated olivine $\left(\mathrm{Fa}_{19.1 \pm 1.2}, \mathrm{~N}=8\right)$ and low-Ca pyroxene $\left(\mathrm{Fs}_{17.0 \pm 0.7} \mathrm{Wo}_{1.8 \pm 1.0}\right.$, $\mathrm{N}=10$ ) compositions.

Classification: H3-6 finely intermixed genomict breccia. Type 5-6 lithology implied by medium to coarse feldspar grain sizes and equilibrated olivine and pyroxene; type 3 lithology implied by common presence of magnesian and zoned olivine. Paired with Buck Mountain Wash (synonymous with Yucca 002) on the basis of mineralogy, mineral chemistry, and texture.

Specimens: Cascadia holds $11.3 \mathrm{~g}$ in a single piece, in addition to one polished thin section and a mounted butt.

\section{Yucca $048 \quad 34^{\circ} 49.402^{\prime} \mathrm{N}, 114^{\circ} 16.573^{\prime} \mathrm{W}$}

Arizona, United States

Find: 23 Sep 2012

Classification: Ordinary chondrite (H3-6)

History: Found with metal detector in the Yucca DCA by Troy Ball September 12, 2012. Entire stone donated to Cascadia.

Physical characteristics: Exterior is covered with weathered fusion crust, with large patches of reddish-brown rust.

Petrography: In thin section two distinct lithologies that appear lighter and darker in plane-polarized light are visible and have irregular and gradational boundaries. The light lithology is coarser grained and contains chondrules with boundaries integrated into their surroundings. Feldspar is variable in size, most slightly below $50 \mu \mathrm{m}$ in diameter, but with a few larger grains. The dark lithology is dominated by fragmental material with only 8 complete chondrules visible; BSE imaging shows that this lithology contains a mix of equilibrated silicates, with a fairly large $(\sim 25$ 35 area \%) admixture of type 3 material (both magnesian and iron-rich olivine and pyroxene grains). This lithology also contains a large coarse-grained clast with anhedral olivine grains up to $1 \mathrm{~mm}$ across, a shock melt clast with zoned olivine grains set in a ferromagnesian glass, and a small ( $\sim 70 \mu \mathrm{m}$ across $)$ chondrule that contains numerous spinel grains $[(\mathrm{Mg} 0.7 \mathrm{Fe} 0.3) \mathrm{Al} 2 \mathrm{O} 4]$ and ilmenite set in a $\mathrm{Ca}$, 
$\mathrm{Mg}$, Al-rich glass and surrounded by a shell of Al-rich low-Ca pyroxene. Abundant FeNi carbide with some accompanying magnetite is found only in the dark lithology.

Geochemistry: (M. Hutson and A. Ruzicka, Cascadia) Light lithology olivine $\left(\mathrm{Fa}_{19.3 \pm 0.8} \mathrm{~N}=8\right)$ and low-Ca pyroxene $\left(\mathrm{Fs}_{16.8 \pm 0.7} \mathrm{Wo}_{1.7 \pm 0.8}\right)$ are consistent with a type 5 or 6 designation. Dark lithology olivine $\left(\mathrm{Fa}_{17.6 \pm 9.6}, \mathrm{~N}=22\right)$ and low-Ca pyroxene $\left(\mathrm{Fs}_{13.9 \pm 4.8} \mathrm{Wo}_{1.4 \pm 0.7}, \mathrm{~N}=30\right)$ compositions reflect the large admixture of type 3 material. The coarse-grained clast in the dark lithology consists of highly equilibrated olivine $\left(\mathrm{Fa}_{19.0 \pm 0.1}, \mathrm{~N}=8\right)$, low-Ca pyroxene $\left(\mathrm{Fs}_{16.7 \pm 0.1} \mathrm{Wo}_{1.4 \pm 0.1}, \mathrm{~N}=5\right)$, diopside $\left(\mathrm{Fs}_{5.4 \pm 0.3} \mathrm{Wo}_{47.3 \pm 0.2}, \mathrm{~N}=5\right)$, and feldspar $\left(\mathrm{Ab}_{70.7 \pm 3.5} \mathrm{An}_{24.2 \pm 4.5} \mathrm{Or}_{5.1 \pm 1.4}, \mathrm{~N}=8\right)$. Classification: H3-6 finely intermixed genomict breccia. Light lithology is type 56 material as implied by medium to coarse feldspar grain sizes and equilibrated olivine and pyroxene; dark lithology contains type 3 material as implied by common presence of magnesian and zoned olivine (dark lithology). Paired with Buck Mountain Wash (synonymous with Yucca 002) on the basis of mineralogy, mineral chemistry, and texture.

Specimens: Cascadia holds $19.3 \mathrm{~g}$ in multiple pieces, in addition to one polished thin section and a mounted butt.

\section{Yucca $049 \quad 34^{\circ} 49.033^{\prime} \mathrm{N}, 114^{\circ} 16.238^{\prime} \mathrm{W}$}

Arizona, United States

Find: 01 May 2014

Classification: Ordinary chondrite (H3-5)

History: Found by Troy Ball on May 1, 2013. The entire stone was donated to Cascadia in September, 2013.

Physical characteristics: Stone entirely coated with weathered fusion crust, which varies in color from dark brown to reddish brown. Two small $(<1 \mathrm{~cm}$ across $)$ patches of crust are missing, revealing a lighter colored reddish-stained interior. Petrography: In thin section, the sample appears to be relatively integrated, with many chondrule fragments and only a few complete chondrules present; distinct lithic clasts typical of a breccia are absent. The material between chondrule fragments is comprised of individual grains and smaller fragments. BSE imaging shows that most of meteorite consists of fragmental material, with an abundance of magnesian and iron-rich olivine and pyroxene grains, many of which show zoning, and which abut equilibrated fragments with typical H-chondrite compositions. Notable petrographic features include a fragment with zoned Fe-rich low-Ca pyroxene and coarse (up to $80 \mu \mathrm{m}$ across) equant silica polymorph with veins and a partial rim of olivine; a large ( $\sim 750 \mu \mathrm{m}$ across $)$ chromite-plagioclase region that encloses olivine; and an irregular fragment ( $\sim 200 \mu \mathrm{m}$ across) that contains elongate chromite-plagioclase pseudomorphs and Ca-pyroxene set in plagioclase. Coarse merrillite (up to $200 \mu \mathrm{m}$ across) and Fe-Ni carbide was observed Geochemistry: (M. Hutson and A. Ruzicka, Cascadia) Sample contains a wide range of compositions of olivine $\left(\mathrm{Fa}_{19.5 \pm 5.1}, \mathrm{~N}=71\right)$, low-Ca pyroxene $\left(\mathrm{Fs}_{15.6 \pm 6.4} \mathrm{Wo}_{1.2 \pm 1.1}, \mathrm{~N}=44\right)$. The pyroxene-silica fragment has zoned $\mathrm{Fe}$-rich low-Ca 
pyroxene $\left(\mathrm{Fs}_{33.5 \pm 6.3} \mathrm{Wo}_{1.8 \pm 0.3}, \mathrm{~N}=7\right)$; the surrounding shell and vein olivine are somewhat higher in iron $\left(\mathrm{Fs}_{26.9 \pm 5.3}, \mathrm{~N}=19\right)$ than the host olivine.

Classification: Although the chemistry and abundance of zoned grains suggests a type 3 designation, the presence of medium-sized crystalline feldspar, coarse phosphate, and chromite-plagioclase objects suggests a higher petrographic type. The thin section studied strongly resembles the "main" lithology found in the Buck Mountain Wash chondrite, suggesting that this is an H3-5 finely intermixed genomict breccia. Paired with Buck Mountain Wash (synonymous with Yucca 002) on the basis of mineralogy, mineral chemistry, and texture.

Specimens: Cascadia holds the entire type specimen and main mass, which consists of $18.4 \mathrm{~g}$ in two pieces, in addition to a polished thin section and a mounted butt

\section{Yucca $050 \quad 34^{\circ} 48.036^{\prime} \mathrm{N}, 114^{\circ} 15.346^{\prime} \mathrm{W}$}

Arizona, United States

Find: 25 Nov 2011

Classification: Ordinary chondrite (H3-5)

History: Found by Wendy Wooddell on November 25, 2011

Physical characteristics: Sample has weathered fusion crust on one side. All other exterior faces are broken surfaces which are medium-brown from weathering, showing numerous small protruding dark bumps, which appear to represent weathered metal grains

Petrography: In thin section, the sample appears to be relatively integrated, with many chondrule fragments and only a few complete chondrules present; distinct lithic clasts typical of a breccia are absent. The material between chondrule fragments is comprised of individual grains and smaller fragments. BSE imaging shows that most of meteorite consists of fragmental material, with about a $10 \%$ admixture of magnesian and iron-rich olivine and pyroxene grains, many of which show zoning, and which abut equilibrated fragments with typical H-chondrite compositions. Numerous chromite-plagioclase objects were observed, including a fragment containing chromite-plagioclase pseudomorphs after a lath-shaped phase set in a feldspathic matrix. Throughout the section, feldspathic material consists of glass of variable compositions and crystalline plagioclase feldspar grains typically 20-40 $\mu \mathrm{m}$ across; a few grains up to $70 \mu \mathrm{m}$ across were observed. Also present are merrillite grains, some up to $250 \mu \mathrm{m}$ across. Fe-Ni carbide was found, along with ilmenite and magnetite.

Geochemistry: (M. Hutson and A. Ruzicka, Cascadia) Sample contains a range of compositions of olivine $\left(\mathrm{Fa}_{18.7 \pm 2.2}, \mathrm{~N}=31\right)$ and low-Ca pyroxene $\left(\mathrm{Fs}_{15.2 \pm 3.6} \mathrm{Wo}_{1.6 \pm 0.8}\right.$, $\mathrm{N}=44$ ).

Classification: Meteorite is an H-chondrite, based on chemistry. Although the chemistry and abundance of zoned grains suggests a type 3 designation, the presence of medium-sized crystalline feldspar, coarse phosphate, and chromiteplagioclase objects suggests a higher petrographic type. The thin section studied strongly resembles the "main" lithology found in the Buck Mountain Wash 
chondrite, which is an H3-5 finely intermixed genomict breccia. Paired with Buck Mountain Wash (synonymous with Yucca 002) on the basis of mineralogy, mineral chemistry, and texture.

Specimens: Cascadia holds $23.4 \mathrm{~g}$ in one piece, in addition to three polished thin sections. Wendy Wooddell retained a $3.8 \mathrm{~g}$ end piece.

\section{Yucca $051 \quad 34^{\circ} 48.990^{\prime} \mathrm{N}, 114^{\circ} 16.151^{\prime} \mathrm{W}$}

Arizona, United States

Find: 11 Nov 2011

Classification: Ordinary chondrite (H6)

History: Found $5 \mathrm{~cm}$ below the surface by Jim Wooddell on November 11, 2011 while searching with a metal detector. Mr. Wooddell donated the main mass to Cascadia.

Physical characteristics: Sample is almost entirely covered with weathered fusion crust. A rusted light-colored interior is visible in two small patches where crust is missing.

Petrography: In thin section, the sample appears to be well integrated and relatively coarse-grained, containing chondrules with boundaries that blend into their surroundings. Crystalline plagioclase feldspar grains are typically $30-40 \mu \mathrm{m}$ across, although a few grains $>100 \mu \mathrm{m}$ across were observed. Metallic copper and several chromite-plagioclase objects are present.

Geochemistry: (M. Hutson and A. Ruzicka, Cascadia) The major silicate phases are equilibrated, including olivine $\left(\mathrm{Fa}_{19.9 \pm 0.5}, \mathrm{~N}=20\right)$, low-Ca pyroxene $\left(\mathrm{Fs}_{17.3 \pm 0.5} \mathrm{Wo}_{1.4 \pm 0.4}, \mathrm{~N}=16\right)$, diopside $\left(\mathrm{Fs}_{6.4 \pm 2.0} \mathrm{Wo}_{46.4 \pm 1.4}, \mathrm{~N}=5\right)$, and plagioclase feldspar $\left(\mathrm{Ab}_{77.7 \pm 1.7} \mathrm{An}_{15.1 \pm 1.4} \mathrm{Or}_{7.2 \pm 1.1}, \mathrm{~N}=8\right)$

Classification: Mineral chemistry indicates an equilibrated $\mathrm{H}$ chondrite. A petrographic grade of 6 (near the $5 / 6$ border) is suggested by the sample?s wellintegrated texture and the sizes of the plagioclase feldspar grains.

Specimens: Cascadia holds $21.7 \mathrm{~g}$ in one piece, in addition to a polished thin section. Jim Wooddell holds a $3.8 \mathrm{~g}$ end cut and a polished thin section.

\section{Yucca $052 \quad 34^{\circ} 45.800^{\prime} \mathrm{N}, 114^{\circ} 14.349^{\prime} \mathrm{W}$}

Arizona, United States

Find: 27 Mar 2011

Classification: Ordinary chondrite (H3-5)

History: Found with metal detector by Jim Wooddell, March 27, 2011. Cut in 3 pieces. Jim Wooddell retained 1.2 gram slice, with remainder donated to Cascadia. Physical characteristics: Exterior is covered with weathered brown fusion crust. Petrography: In plane-polarized light, the thin section consists of a main lithology composed mainly of chondrule fragments, two light and two dark clasts, and a clast with a well-defined boundary that contains delineated chondrules in a dark matrix. BSE imaging of the main lithology shows that it contains a mix of equilibrated silicates, with a fairly large ( $25-35$ area $\%)$ admixture of type 3 material (both 
magnesian and iron-rich olivine and pyroxene grains). Feldspathic material is mainly glass, although small (less than $20 \mu \mathrm{m}$ in diameter) feldspar grains are present. This lithology includes grains of silica polymorph, FeNi carbide, aluminous high-Ca pyroxene, chlor-apatite and merrillite. One large chondrule fragment shows signs of aqueous alteration before it was incorporated into the main lithology. This fragment consists of olivine grains that have irregular Fe-rich rims set in a pyroxene-normative glass that has rims and cross-cutting veins of $\mathrm{Fe}$-rich glass. One of the two dark clasts is along the edge of the section and is dark due to the presence of an extensive web of weathering veins. The other dark clast is shock-blackened and contains numerous parallel veins of partially weathered metal and troilite. BSE imaging of the two light clasts shows that they consist of equilibrated silicates. Minerals present include phosphate grains, including a single large (> $300 \mu \mathrm{m}$ across) grain of merrilite, plagioclase feldspar (less than $20 \mu \mathrm{m}$ in diameter), and chromite-plagioclase assemblages. BSE imaging of the clast with well-defined chondrules shows that it consists of unequilibrated chondrules (rather than fragments) set in an Fe-rich, fine-grained matrix typical of a type 3 chondrite (hereafter referred to as the type 3 clast).

Geochemistry: (M. Hutson and A. Ruzicka, Cascadia) Light clast olivine $\left(\mathrm{Fa}_{18.9 \pm 1.6}, \mathrm{~N}=14\right)$ and low-Ca pyroxene $\left(\mathrm{Fs}_{17.4 \pm 1.0} \mathrm{Wo}_{1.1 \pm 0.7}, \mathrm{~N}=10\right)$ are consistent with an $\mathrm{H}$ chondrite of petrographic type 5 . The dark clasts were not analyzed. The main lithology has olivine $\left(\mathrm{Fa}_{17.0 \pm 6.1}, \mathrm{~N}=47\right)$ and low-Ca pyroxene $\left(\mathrm{Fs}_{15.4 \pm 5.0} \mathrm{Wo}_{1.4 \pm 0.9}, \mathrm{~N}=56\right)$ compositions suggestive of petrographic type 3, but petrographically resembles the "main lithology" of Buck Mountain Wash, which is a fragmental mixture of equilibrated and type 3 material. The type 3 clast has olivine $\left(\mathrm{Fa}_{18.3 \pm 8.1}, \mathrm{~N}=44\right)$ and low-Ca pyroxene $\left(\mathrm{Fs}_{11.5 \pm 8.1} \mathrm{Wo}_{1.1 \pm 1.1}, \mathrm{~N}=35\right)$ compositions consistent with a type 3 designation. The large chondrule fragment which appears to have been aqueously altered has olivine grains with cores of $\mathrm{Fa}_{16.3 \pm 2.3}, \mathrm{~N}=10$, consistent with an H-group precursor. The irregular rims on these grains are $\mathrm{Fa}_{43.4 \pm 3.4,} \mathrm{~N}=7$.

Classification: H3-5 genomict breccia. Paired with Buck Mountain Wash (synonymous with Yucca 002) on the basis of mineralogy, mineral chemistry, and texture.

Specimens: Cascadia holds $19.8 \mathrm{~g}$ in two pieces, in addition to one polished thin section and a mounted butt. Jim Wooddell holds a $1.2 \mathrm{~g}$ slice.

\section{Yuli $\quad 41.3^{\circ} \mathrm{N}, 86.25^{\circ} \mathrm{E}$}

Xinjiang, China

Find: 2015 Dec

Classification: Iron meteorite (IAB-MG)

History: The anonymous finders stated that the object was found in the neighborhood of Yuli County. The finders are unwilling to release the exact coordinates. The original informal name given to this meteorite was Mulei; this was based on a misunderstanding between the finders and the buyer. This is the name it is cataloged under in several collections. 
Petrography: The meteorite is a coarsest octahedrite.

Geochemistry: Composition (based on duplicate INAA): Co, $4.67 \mathrm{mg} / \mathrm{g} ; \mathrm{Ni}, 60.9$ $\mathrm{mg} / \mathrm{g}$; Ga, $92.1 \mu \mathrm{g} / \mathrm{g} ; \mathrm{Ge}, 444 \mu \mathrm{g} / \mathrm{g} ;$ As, $11.7 \mu \mathrm{g} / \mathrm{g} ; \mathrm{W}, 1.33 \mu \mathrm{g} / \mathrm{g}$; Ir $3.78 \mu \mathrm{g} / \mathrm{g} ; \mathrm{Au}$, $1.396 \mu \mathrm{g} / \mathrm{g}$. The meteorite is assigned to IAB-MG.

Classification: The meteorite is assigned to the IAB-MG. The $\mathrm{Au}$ and $\mathrm{Ni}$ are below and Co somewhat above the IAB-MG range. It could be designated IAB-MG-An, but this feature might also be produced by large sampling errors resulting in an anomalously high kamacite/taenite ratio. Most of the data for Yuli fall in the Campo del Cielo range, but the exceptionally low $\mathrm{Ni}$ and $\mathrm{Au}$ and somewhat elevated Co differ by too much for this assignment and there is good evidence that it was discovered in China.

Specimens: Type specimen at PMO. Main mass at SSTM. Only one mass is known.

\section{New Dense Collection Areas (DCA)}

24 Dense Collection Areas have been approved in 2018.A list of all DCAs can be found at https://www.lpi.usra.edu/meteor/DenseAreas.php.

\section{Listing of institutes and collections}

An up-to-date index of collections and approved repositories (next to a green check mark) cited in the Meteorite Bulletin can be found here:

https://www.lpi.usra.edu/meteor/MetBullAddresses.php?grp=country

\section{Bibliography}

- A. Bischoff, N. Vogel, and J. Roszjar (2011) The Rumuruti chondrite group. Chemie der Erde 71, 101-133. (link)

- Beck P., Quirico E., Montes-Hernandez G., Bonal L., Bollarda J., Orthous-Daunay F.R., Howard K.T., Schmitt B., Brissaud O., Deschamps F., Wunder B., and Guillot S. (2010) Hydrous mineralogy of CM and CI chondrites from infrared spectroscopy and their relationship with low albedo asteroids. Geochim. Cosmochim. Acta 74, 4881-4892 (link)

- Bischoff, A. (2000) Mineralogical characterization of primitive, type-3 lithologies in Rumuruti chondrites. Meteorit. Planet. Sci. 35 699-706 (link)

- Bonal L., Quirico E., Flandinet L., and Montagnac G. (2016) Thermal history of type 3 chondrites from the Antarctic meteorite collection determined by Raman spectroscopy of their polyaromatic carbonaceous matter. Geochim. Cosmochim. Acta 189 312-337. (link)

- Brearley A.J. and Jones R.H. (1998) Chondritic meteorites. in Planetary Materials, Reviews in Mineralogy v. 36, J.J. Papike Ed., Mineralogical Society of America, 3398. ISBN 0-939950-46-4; ISBN13 978-0-939950-46-1 (link)

- D'Orazio M. and Folco L. (2003) Chemical analysis of iron meteorites by inductively coupled plasma-mass spectrometry. Geostand. Newslett. 27, 215-225. (link)

- Gattacceca J., Suavet C., Rochette P., Weiss B.P., Winklhofer M., Uehara M. Friedrich J.M. (2014) Metal phases in ordinary chondrites: magnetic hysteresis properties and implications for thermal history. Meteorit. Planet. Sci. 49 652676/ (link) 
- Grossman J.N. and Brearley A.J. (2005) The onset of metamorphism in ordinary and carbonaceous chondrites. Meteorit. Planet. Sci. 40, 87-122. (link)

- Grossman J.N. and Brearley A.J. (2005) The onset of metamorphism in ordinary and carbonaceous chondrites. Meteorit. Planet. Sci. 40, 87-122. (link)

- H. Takeda and A.L. Graham (1991) Degree of equilibration of eucritic pyroxenes and thermal metamorphism of the earliest planetary crust. Meteoritics 26 129134. (link)

- Metzler K. (2012) Ultrarapid chondrite formation by hot chondrule accretion? Evidence from unequilibrated ordinary chondrites. Meteorit. Planet. Sci. 47, 2193-2217 (link)

- Nazarov M.A., Kurat G., Brandstaetter F., Ntaflos T., Chaussidon M., and Hoppe P. (2009) Phosphorus-bearing sulfides and their associations in CM chondrites. Petrology 17, 101-123. (link)

- Rubin A.E., Trigo-Rogriguez J.M., Huber H., and Wasson J.T. (2007) Progressive aqueous alteration of CM carbonaceous chondrites. Geochim. Cosmochim. Acta 71, 2361-2382 (link)

- Stöffler D., Hamann C., and Metzler K. (2018) Shock metamorphism of planetary silicate rocks and sediments: Proposal for an updated classification system. Meteorit. Planet. Sci. 53, 5-49. (link)

- $\quad$ Tait A.W., Tomkins A.G., Gode B.M., Wilson S.A., Wilson, and Hasalova P. (2014) Investigation of the $\mathrm{H} 7$ ordinary chondrite, Watson 012: Implications for recognition and classification of Type 7 meteorites. Geochim. Cosmochim. Acta 134 175-196. (link)

- Wasson J.T. and Richardson J.W. (2001) Fractionation trends among IVA iron meteorites: contrasts with IIIAB trends. Geochem. Cosmochim. Acta 65, 951-970. (link)

- Wasson J.T. and Kallemeyn, G.W. (2002) The IAB iron-meteorite complex: A group, five subgroups, numerous grouplets, closely related, mainly formed by crystal segregation in rapidly cooling melts. Geochim. Cosmochim. Acta 66 24452473 (link)

- Weber P., Hofmann B.A., Tolba T., and Vuilleumier J.-L. (2017) A gamma?ray spectroscopy survey of Omani meteorites. Meteorit. Planet. Sci. 52, 1017-1029. (link)

- Weisberg M.K., Ebel D.S., Nakashima D., Kita N.T., and Humayun M. (2015) Petrology and geochemistry of chondrules and metal in NWA 5492 and GRO 95551: A new type of metal-rich chondrite. Geochim. Cosmochim. Acta 167, 269285

\section{Acknowledgments}

These meteorites were reviewed and approved by the Meteorite Nomenclature Committee of the Meteoritical Society. In 2018, committee members were Audrey Bouvier (Chair of the Nomenclature Committee), Jérôme Gattacceca (Meteorite Bulletin Editor), Francis McCubbin (Meteorite Bulletin Deputy Editor), Jeffrey Grossman (Meteorite Bulletin Database Editor), Trevor Ireland (Vice-President of the Meteoritical Society), Emma Bullock, Hasnaa Chennaoui-Aoudjehane, Vinciane Debaille, Laurence Garvie, Knut Metlzer, Massimo D’Orazio, Tasha Dunn, Mitsumi Komatsu. 